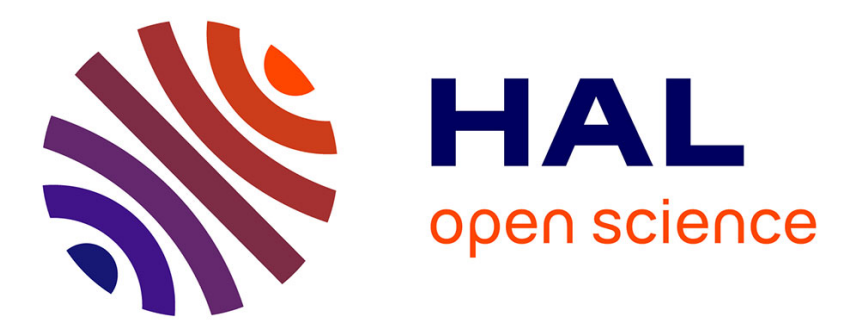

\title{
A reappraisal of the metamorphic history of EH3 and EL3 enstatite chondrites
}

Eric Quirico, Michèle Bourot-Denise, C. Robin, Gilles Montagnac, Pierre Beck

\section{To cite this version:}

Eric Quirico, Michèle Bourot-Denise, C. Robin, Gilles Montagnac, Pierre Beck. A reappraisal of the metamorphic history of EH3 and EL3 enstatite chondrites. Meteoritics and Planetary Science, 2011, 46 Supplement (S1), pp.A195. 10.1111/j.1945-5100.2011.01221.x . insu-00683399

\section{HAL Id: insu-00683399 \\ https://hal-insu.archives-ouvertes.fr/insu-00683399}

Submitted on 28 May 2021

HAL is a multi-disciplinary open access archive for the deposit and dissemination of scientific research documents, whether they are published or not. The documents may come from teaching and research institutions in France or abroad, or from public or private research centers.
L'archive ouverte pluridisciplinaire HAL, est destinée au dépôt et à la diffusion de documents scientifiques de niveau recherche, publiés ou non, émanant des établissements d'enseignement et de recherche français ou étrangers, des laboratoires publics ou privés. 


\section{Abstracts}

\section{2}

RAMAN, FTIR, AND MÖSSBAUER SPECTROSCOPY OF OLIVINES FROM THE D'ORBIGNY METEORITE

Y. A. Abdu ${ }^{1}$, M. E. Varela ${ }^{2}$ and F. C. Hawthorne ${ }^{1}$. ${ }^{1}$ Department of Geological Sciences, University of Manitoba, Winnipeg, Manitoba R3T 2N2, Canada. E-mail: abdu@cc.umanitoba.ca. ${ }^{2}$ ICATE-CONICET, Av. España 1512 Sur, CP J5402DSP, San Juan, Argentina.

Introduction: Olivine is a major basaltic mineral in high-temperature volcanic rocks and in stony meteorites. The D'Orbigny angrite contains large olivine crystals (up to $\sim 1 \mathrm{~cm}$ in size) that are present throughout the rock, mimicking xenocrysts or forming olivinite (polycrystalline olivine) [1-3]. The olivinites are of particular interest, as very little is known about them. Here, we study three types of D'Orbigny olivines: honey olivine $(\mathrm{HOl})$, green olivine $(\mathrm{GOl})$, and olivinite $(\mathrm{POl})$, by Raman, Fourier-transform infrared (FTIR), and Mössbauer spectroscopy, and compare their structural and spectral features.

Experimental: Raman spectroscopy measurements were done using a LabRAM ARAMIS confocal microscope (Horiba Jobin Yvon). A x100 objective microscope was used to focus the laser beam $(532 \mathrm{~nm}$ excitation line) on the sample to a size of $\sim 1 \mu \mathrm{m}$. FTIR spectra over the range (4000-400 $\mathrm{cm}^{-1}$ ) were collected on $\mathrm{KBr}$ sample pellets using a Bruker Tensor 27 FTIR spectrometer. Transmission Mössbauer spectra were acquired at room temperature (RT) using a ${ }^{57} \mathrm{Co}(\mathrm{Rh})$ point source.

Results and Discussion: The chemical compositions of the three olivines were determined by electron microprobe analysis (EMPA). The $\mathrm{Mg} \#[=\mathrm{Mg} /(\mathrm{Mg}+\mathrm{Fe})]$ is 0.64 for $\mathrm{HOl}, 0.85$ for $\mathrm{GOl}$, and 0.90 for POl.

Raman and FTIR spectroscopy.

The main feature of Raman spectra of olivines is a doublet with peaks located at $\sim 850 \mathrm{~cm}^{-1}\left(\kappa_{1}\right)$ and $820 \mathrm{~cm}^{-1}\left(\kappa_{2}\right)$ due to the asymmetric and symmetric vibrations, respectively, of the $\mathrm{SiO}_{4}$ group [4]. For D'Orbigny olivines, the $\kappa_{1}$ and $\kappa_{2}$ peaks occur at $850,820 \mathrm{~cm}^{-1}$ for $\mathrm{HOl}$; $854,823 \mathrm{~cm}^{-1}$ for $\mathrm{GOl} ; 856,824 \mathrm{~cm}^{-1}$ for $\mathrm{POl}$, reflecting variability in composition. Using the $\omega\left(=\kappa_{1}-\kappa_{2}\right)-\mathrm{Mg} \#$ relation [3], the estimated $\mathrm{Mg} \#$ for $\mathrm{HOl}, \mathrm{GOl}$, and $\mathrm{POl}$ are 0.70(4), 0.82(4), and 0.93(4), respectively, in close agreement with EMPA results. Similarly, the FTIR spectra of the three olivines do not show any differences except the composition dependence of the bands positions. Furthermore, no structural $\mathrm{OH}$ bands are observed in the $\mathrm{OH}$-stretching region $\left(3800-3000 \mathrm{~cm}^{-1}\right)$ for all samples.

Mössbauer spectroscopy.

The RT Mössbauer spectra of the $\mathrm{HOl}$ and GOl consist of two asymmetric absorption peaks, resulted from the overlap of two quadrupole doublets due to the presence of $\mathrm{Fe}^{2+}$ at the M1 and M2 octahedral sites. On the other hand, the spectrum of POl shows an additional weak doublet $\left(\mathrm{CS}=0.35 \mathrm{~mm} \mathrm{~s}^{-1}, \mathrm{QS}=0.30 \mathrm{~mm} \mathrm{~s}^{-1}\right.$, relative area $=2 \%$ ) which is attributed to $\mathrm{Fe}^{3+}$. Because $\mathrm{Fe}^{3+}$ could indicate a laihunite-like material similar to that observed in partially oxidized metasomatized mantle olivines [5], olivinites may record the increasing oxidizing conditions prevailing during the late metasomatic event that affect angrite formation.

References: [1] Kurat G. et al. 2001. Abstract \#1753. 32nd Lunar and Planetary Science Conference. [2] Kurat G. et al. 2004. Geochimica et Cosmochimica Acta 68:1901-1921. [3] Varela M. E. et al. 2005. Meteoritics \& Planetary Science 40:409-430. [4] Mouri T. and Enami M. 2008. Journal of Mineralogical and Petrological Sciences 103:100-104. [5] Banfield J. F. et al. 1992. American Mineralogist 77:977-986.

\section{4 \\ A PRELIMINARY STUDY OF MAGMATIC VOLATILES IN ANGRITES}

F. A. J. Abernethy ${ }^{1}$, A. Verchovsky ${ }^{1}$, I. A. Franchi ${ }^{1}$ and M. M. Grady ${ }^{1,2}$. ${ }^{1}$ PSSRI, The Open University, Walton Hall, Milton Keynes MK7 6AA, UK. ${ }^{2}$ Department Mineralogy, The Natural History Museum, London SW7 5BD, UK. E-mail: F.A.J.Abernethy@open.ac.uk.

Introduction: Angrites are a rare group of achondrites notable for their critical silica undersaturation, lack of shock metamorphic features and ancient crystallization ages [1-3]. They are subdivided into plutonic or basaltic angrites on the basis of their inferred petrogenesis. Similar studies to those already carried out on HEDs and shergottites [4, 5] could be used to shed light on magmatic processes on the angrite parent body (APB). Here we present results from carbon and nitrogen stepped combustion experiments on the plutonic angrites Angra dos Reis (AdoR) and NWA 2999, and the basaltic angrite D'Orbigny.

Results: Magmatic volatiles are released once the silicate matrix starts to become plastic, usually between 800 and $1000{ }^{\circ} \mathrm{C}$; depending on the $\mathrm{CRE}$ age of the specimen, it is possible for this to be mixed with a spallogenic component, the effects of which can be removed following correction. We have not yet applied this correction to the data but expect it to have little effect on either carbon or nitrogen abundance, and to reduce isotopic composition by $<2 \%$. We also discount NWA 2999 from further consideration, because it contained large amounts of terrestrial organic carbon which swamped all other potential carbon-bearing components.

\begin{tabular}{lllll}
\hline Name & $\begin{array}{l}\text { Total } \mathrm{C} \\
(\mathrm{ppm})\end{array}$ & $\begin{array}{l}\delta^{13} \mathrm{C} \\
(\%)\end{array}$ & $\begin{array}{l}\text { Magmatic C } \\
(\mathrm{ppm})\end{array}$ & $\begin{array}{l}\delta^{13} \mathrm{C} \\
(\%)\end{array}$ \\
\hline AdoR & 801 & -21.6 & 63 & -21.4 \\
NWA 2999 & 6990 & -29.5 & - & - \\
D'Orbigny & 199 & -8.8 & 9 & -11.4 \\
Name & Total N & $\delta^{15} \mathrm{~N}$ & Magmatic $\mathrm{N}$ & $\delta^{15} \mathrm{~N}$ \\
& $(\mathrm{ppm})$ & $(\%)$ & $(\mathrm{ppm})$ & $(\% \mathrm{o})$ \\
AdoR & 15 & +8 & 3 & -2 \\
NWA 2999 & 84 & -2 & - & - \\
D'Orbigny & 12 & +8 & 1 & +14 \\
\hline
\end{tabular}

Discussion: The overall ${ }^{13} \mathrm{C}$-enrichment of D'Orbigny compared to AdoR is partly the result of carbonates $\left(\delta^{13} \mathrm{C}\right.$ approximately 0 to $\left.+5 \%\right)$ lining vugs in the meteorite. There are also differences in magmatic carbon and nitrogen between the two meteorites. AdoR has a higher abundance of the component, with lighter isotopic composition. This is consistent with the petrogeneses of the meteorites, with D'Orbigny having outgassed during its ascent: McCoy et al. [6] calculated that the vesicles present in D'Orbigny could have formed from as little as approximately $12.5 \mathrm{ppm} \mathrm{CO}_{2}$ dissolved in the magma. Stepped combustion analyses of further angrites should reveal whether there is a relationship between sample petrogenesis and magmatic volatiles.

References: [1] Mittlefehldt D. W. et al. 1998. In Planetary materials, Reviews in Mineralogy 36, Chapter 4. Mineralogical Society of America. [2] Mittlefehldt D. W. and Lindstrom M. M. 1990. Geochimica et Cosmochimica Acta 54:3209-3218. [3] Mittlefehldt D. W. et al. 2002. Meteoritics \& Planetary Science 37:345-369. [4] Grady M. M. et al. 1997. Meteoritics \& Planetary Science 32:863-868. [5] Grady M. M. et al. 2004. International Journal of Astrobiology 3:117-124. [6] McCoy T. J. et al. 2006. Earth and Planetary Science Letters 246:102-108. 
5211

\section{PETROGRAPHIC EVIDENCE OF SHOCK METAMORPHISM IN CR2 CHONDRITE GRO 03116}

N. M. Abreu. Earth Science, Pennsylvania State University-DuBois, PA, USA. E-mail: abreu@psu.edu.

Introduction: CR chondrites are primitive meteorites that show a broad range of aqueous alteration features from nearly pristine [1] to fully altered [2]. However, evidence of any thermal metamorphism group is only beginning to emerge $[3,4]$. To better understand the effects and the source of metamorphism in the CR parent body, an SEM/EPMA/TEM study of GRO 03116 is underway. GRO 03116 was chosen because Oisotopic analyses suggest that it has only suffered limited aqueous alteration [5], while Raman spectra shows that its organic materials have undergone some thermal metamorphism [4]. GRO 03116 is an Antarctic CR2 find of terrestrial weathering type C. Thus, disentangling its record of asteroidal versus terrestrial aqueous alteration merits careful analysis petrologic analysis.

Results and Discussion: GRO 03116 contains porphyritic type I and fewer type II chondrules, abundant opaques, a heterogeneous matrix and CAIs. These components are well defined texturally. Fine-grained layered assemblages and ferryhydrite veins, characteristic of terrestrial aqueous alteration [6], are also present. Chondrule mesostasis is often dendritic. Chondrule forsterites show no systematic enrichment in fayalite content at their edges. However, some chondrules exhibit complex chemical zoning. Opaques range from nearly intact kamacite grains with variable amounts of $\mathrm{Ni}$ and $\mathrm{Co}$ and terrestrial ferrihydrite haloes to terrestrial alteration assemblages. Matrix is significantly less abundant (approximately $21 \mathrm{vol} \%$ - this study, $24.3 \mathrm{vol} \%$ - [5]) than in other CR chondrites ( $>30 \mathrm{vol} \%$ [7]) and clastic in texture. Matrix is rich in opaques, particularly magnetite and sulfides. In addition, some matrix regions appear very compact and have a foliation fabric. Some chondrules show signs of plastic deformation along the direction of matrix foliation. These chondrules display substantial textural integration with the surrounding metal and matrix. In addition, porous melt veins (approximately $200 \mu \mathrm{m}$ across) containing angular olivine fragments and abundant, rounded, micronsized magnetite and pentlandite grains have been identified.

Conclusion: GRO 03116 has undergone pervasive terrestrial weathering that overprinted its original record. However, preservation of some kamacite suggests limited preterrestrial aqueous alteration. TEM studies will be conducted to better establish the extent of alteration. The following petrologic observations also indicate limited thermal metamorphism, certainly lower than in CR GRA 06100 [3]: minor integration of chondrules and matrix, preservation of some mesostasis, low Fe content in type I chondrules, Coheterogeneity in metal, and presence of magnetite. Thus, preterrestrial alteration of GRO 03116 was probably dominated by shock metamorphism, as indicated by its deformed chondrules, comminuted matrix, and melt veins [8].

Acknowledgments: Funded by NNX11AH10G and AAS grants to NMA and conducted at the MRI-Penn State.

References: [1] Abreu N. M. and Brearley A. J. 2010. Geochimica et Cosmochimica Acta 74:1146-1171. [2] Weisberg M. K. and Huber H. 2001. Meteoritics \& Planetary Science 42:1495-1503. [3] Abreu N. M. and Stanek G. L. 2009. Abstract \#2393. 40th Lunar and Planetary Science Conference. [4] Briani et al. 2010. Meteoritics \& Planetary Science 45:5234. [5] Schrader D. L. et al. 2011. Geochimica et Cosmochimica Acta 75:308-325. [6] Zolensky M. E. and Gooding J. L. 1986. [7] Weisberg M. K. et al. 1993. Geochimica et Cosmochimica Acta 57:1567-1586. [8] Sharp T. G. and DeCarli. 2006. In MESS II. pp. 653-677.
5021

BAJADA DEL DIABLO, PATAGONIA, ARGENTINA: THE IMPACT OF A SPLIT COMET?

R. D. Acevedo, C. Prezzi, M. J. Orgeira, J. Rabassa, H. Corbella, J. F. Ponce, O. Martinez, C. Vasquez, I. Subias, M. Gonzalez and M. Rocca. Conicet, Argentina. E-mail: acevedo@cadic-conicet.gob.ar.

An impact origin has been proposed for the almost 200 circular structures found in Bajada del Diablo $\left(42^{\circ} 46^{\prime}\right.$ to $43^{\circ} \mathrm{S}$ and $67^{\circ} 24^{\prime}$ to $45^{\prime}$ W), Patagonia, Argentina [1], some of them partially obliterated by erosion or sediment accumulation, which range in diameter from $100 \mathrm{~m}$ up to $400 \mathrm{~m}$.

The circular structures have been identified in two rock types: the Quiñelaf eruptive complex (Miocene basalts) and Pampa Sastre Formation (Pliocene basaltic conglomerate).

Geological and geomorphological strong evidences of involvement on both geologic entities more over the Pleistocene pediment gravels and sands has been interpreted as the cause of the impact but lack either pieces of the impacting body like meteorites or definitive signs of shock metamorphism. Only samples of breccias randomly spread were collected. In neither case, however, was detected there any evidence of coesite. Most important diagnostic elements collected so far are tiny $\mathrm{Fe}-\mathrm{Ni}$ lawrencitebearing microspheres of 300 microns in average diameter, presumably originated by the impact, buried under post-impact sedimentary coverage.

To strengthen the hypothesis of impact through the geophysical survey magnetometric record of magnetic anomalies were performed. The magnetic anomalies show a circular pattern with magnetic lows in the circular structure's floors. Furthermore in the circular structure's rims, high-amplitude, conspicuous and localized (short wavelength) anomalies are observed. Such large amplitude and short wavelength anomalies are not detected out of the circular structures. For all used frequencies, the electromagnetic profiles show lower apparent electrical conductivities in the circular structure's floor, while the rims present notably higher values. Curvature attributes, analytic signal, horizontal gradient and Euler solutions were calculated for the magnetic data. 2.5 D magnetic models were developed across each one of the studied circular structures. Our results suggest that in the circular structure's floors up to $12 \mathrm{~m}$ of Pampa Sastre conglomerate would have been removed. On the contrary, the circular structure's rims exhibit high-amplitude, localized magnetic anomalies and higher apparent electrical conductivities, which would be related to the anomalous accumulation of basalt boulders and blocks remanently magnetized. The fact that such high-amplitude anomalies are not present out of the surveyed circular structures supports this hypothesis. The geomorphological, geological and geophysical features of the studied circular structures can only be explained by means of an extra-terrestrial projectile impact.

Even $\mathrm{Fe}-\mathrm{Ni}$ microspheres with lawrencite and breccias have been found inside craters, nevertheless, the absence of direct evidences of a disintegrated asteroid opens the possibility to consider the event of a split comet with an ice nucleus hitting the patagonian surface in Pleistocene times.

The authors acknowledge the financial support by Conicet and DGPCyT-ME Provincia del Chubut (Argentinean Agencies) and National Geographic/Waitt and The Planetary Society.

Reference: [1] Acevedo R. D. et al. 2009. Geomorphology 110:58-67. 
5022

\section{METEORITE IMPACT CRATERS IN SOUTH AMERICA: A BRIEF REVIEW}

R. D. Acevedo, M. Rocca, J. Rabassa and J. F. Ponce. Conicet, Argentina. E-mail: acevedo@cadic-conicet.gob.ar.

Introduction: The first enumeration of impact craters sites in South America is presented here. Proximately twenty proven, suspected and disproven structures have been identified by several sources in this continent until now. But everyone events proposed here aren't really produced by impacts at all. About some of them reasonable doubts exist. Brazil leading the record containing almost half detected and following it in the list Argentina. In Bolivia, Peru, Chile, and Colombia only a few were observed. The rest of countries are awaiting for new discoveries.

The following possible and confirmed meteorite impact structures have been reported for this continent, country by country:

ARGENTINA. Campo del Cielo (S 2730', W 61 $42^{\prime}$ ). Impact crater strewn field. At list twenty craters with an age of about 4000 years over sandy-clay sediments of Quaternary-Recent age. The impactor was an iron-nickel Apollo-type asteroid (meteorite type IA) and plenty of meteorite specimens survived the impact. Bajada del Diablo (S 42 $45^{\prime}$, W $\left.67^{\circ} 30^{\prime}\right)$. A very remarkable site of a new very large meteorite impact craters field. Almost 200 structures were identified there. Age is estimated between 0.13 and $0.78 \mathrm{Ma}$. Other possible craters are Rio Cuarto (S $32^{\circ} 52^{\prime}$, W $64^{\circ} 14^{\prime}$ ), Islas Malvinas (S $51^{\circ} 00^{\prime}$, W $62^{\circ} 00^{\prime}$ ), Salar del Hombre Muerto (S $25^{\circ} 12^{\prime}$, W $66^{\circ} 55^{\prime}$ ), Antofalla (S $26^{\circ} 15^{\prime}$, W $68^{\circ} 00^{\prime}$ ), La Dulce (S $38^{\circ} 14^{\prime}$, W 59 $12^{\prime}$ ), and General San Martin (S 38 $00^{\prime}, \mathrm{W} 63^{\circ} 18^{\prime}$ ).

BOLIVIA. Iturralde (S $12^{\circ} 35^{\prime}$, W $67^{\circ} 38^{\prime}$ ). On Quaternary alluvial

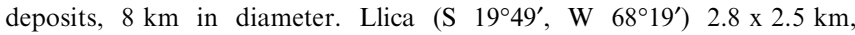
without specific geological information.

BRASIL. Araguainha Dome (S 16 $46^{\prime}$, W 52 $59^{\prime}$ ). This is so far the largest well stated impact crater in South America. It is a $40-\mathrm{km}$ diameter crater in Paleozoic sediments of the Paraná Basin. Serra da Cangalha (S $8^{\circ} 05^{\prime}, \mathrm{W} 46^{\circ} 51^{\prime}$ ). Total diameter of multiple rings has been estimated at $12 \mathrm{~km}$; in Paleozoic sediments in the Parnaiba Basin. Vergeao (S $26^{\circ} 50^{\prime}$, W $52^{\circ} 10^{\prime}$ ). It is a 12.4 kilometer-diameter circular depression located on Cretaceous basalts and Jurassic/Triassic sandstones of the Sao Bento Group of Paraná Basin. Vista Alegre (S 2557', W 52 $41^{\prime}$ ). It is a $9.5 \mathrm{~km}$-wide circular structure in the Parana State, and it is located on the Cretaceous basalts of the Serra Geral formation. Riachao (S 7 $42^{\prime}$, W 46 $38^{\prime}$ ). $4.0 \mathrm{~km}$ diameter in sedimentary rocks of the Paranaiba Basin (sandstones from the Pedra de Fogo Formation). $200 \mathrm{Ma}$. Other possible craters are Gilbues (S $10^{\circ} 10^{\prime}$, W 45 $14^{\prime}$ ), Sao Miguel do Tapuio (S 5³8',

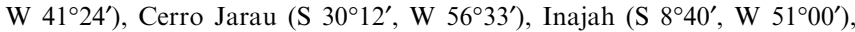
Piratininga (S $22^{\circ} 28^{\prime}, \mathrm{W} 49^{\circ} 09^{\prime}$ ), and Colonia (S $23^{\circ} 52^{\prime}, \mathrm{W} 46^{\circ} 42^{\prime}$ ).

COLOMBIA. Rio Vichada (N $4^{\circ} 30^{\prime}$, W $69^{\circ} 15^{\prime}$ ). Has a diameter of $50 \mathrm{~km}$ and it is the largest possible impact structure ever reported in the continental South America. Rocks exposed there include Precambrian meta-sedimentary and granitoid bodies with an extensive sedimentary Tertiary cover.

CHILE. Monturaqui (S 2356 ${ }^{\prime}, \mathrm{W}$ 68 $17^{\prime}$ ) is emplaced in Jurassic granite rocks, overlain by a thin Tertiary-Quaternary ignimbrite sheet. The impacting asteroid was metallic: an iron-nickel object; $1 \mathrm{Ma}$.

PERU. Carancas (S 16 $40^{\prime}$, W $69^{\circ} 02^{\prime}$ ). H4-5 type ordinary chondrite fall on September 15, 2007 digging a crater of $15 \mathrm{~m}$.

\section{8}

NWA 6588: NEW ANOMALOUS LL6 CHONDRITE

C. B. Agee. Institute of Meteoritics, University of New Mexico, Albuquerque, NM, USA. E-mail: agee@unm.edu.

Introduction: NWA 6588 was found in Morocco in 2008, with TKW of $2730 \mathrm{~g}$. A freshly broken surface reveals a dark gray, polycrystalline rock, with numerous cleavage faces, macroscopic evidence of relict chondrules is obscure, overall appearance resembles a fine grained peridotite hand sample. It shows moderate desert weathering with some iron oxide staining. Currently, NWA 6588 is the only meteorite with the classification LL6-an.

NWA 6588: This new meteorite consists of $65 \%$ olivine: $(\mathrm{Fa} 33.3 \pm 0.2 \mathrm{Fe} / \mathrm{Mn}=68 \pm 3), 25 \%$ low-Ca pyroxene: $(\mathrm{Fs} 27.0 \pm 0.2$ Wo2.2 $\pm 0.1 \mathrm{Fe} / \mathrm{Mn}=40 \pm 2$ ), 5\% albitic feldspar: $\left(\mathrm{Or}_{6.6} \mathrm{Ab}_{84.1} \mathrm{An}_{9.3}\right)$, $3 \%$ sulfides: pentlandite $(\mathrm{Fe}=17.6 \mathrm{Ni}=22.8 \quad \mathrm{Co}=2.1 \quad \mathrm{Si}=2.0$ $\mathrm{S}=55.3$, atom $\%)$, stoichiometric pyrite $\left(\mathrm{FeS}_{2}\right), \quad 1 \%$ chromite $(\mathrm{Fe} \#=0.90, \mathrm{Cr} \#=0.87),<1 \%$ Fe-oxide,$<1 \%$ phosphate consistent with the whitlockite group $\left(\mathrm{Fe} \#=0.27, \mathrm{Na}_{2} \mathrm{O}=2.4 \mathrm{wt} \%\right.$ ). Silicate grain size is variable $(5-300 \mu \mathrm{m})$ and relict chondrules are present. Albitic feldspar grain sizes reach up to $150 \mu \mathrm{m}$.

Discussion: This is an equilibrated, oxidized, ordinary chondrite with very uniform olivine, pyroxene, and plagioclase compositions. Fayalite ( $\mathrm{Fa}$ ), ferrosilite (Fs) content and $\mathrm{Fe} / \mathrm{Mn}$ of olivines are extremely high, Fa and Fs are slightly above, or at the upper limits, recommended by [1] for LL chondrites. Although relict chondrules ranging from 500 $2000 \mu \mathrm{m}$ are observable in BSE, olivines and pyroxenes within chondrule domains are chemically indistinguishable from matrix olivines and pyroxenes. This indicates that chemical equilibrium has been reached while textural equilibrium in this meteorite was not completed. Finely disseminated pyrite and pentlandite $(<1 \mu \mathrm{m})$ are found throughout NWA 6588, also common are larger pockets $(>100 \mu \mathrm{m})$ of pyrite and pentlandite in grain boundary contact with each other, often occupying silicate-bounded triple junctions, suggesting exsolution from monosulfide phase. Pyrite shows fine lamellae. Fe-Ni metal is absent in NWA 6588, however minor amount of low-Ni, Fe-oxide is present and this is presumably the product of desert weathering which has replaced the original minor amounts of primary metal. A few large $(>200 \mu \mathrm{m})$ irregular shaped phosphates with inclusions and intergrowths were observed.

Metamorphic Temperatures: The chromite/olivine geothermometer [2] gives a metamorphic equilibration temperature of approximately $740{ }^{\circ} \mathrm{C}$, which is about $50{ }^{\circ} \mathrm{C}$ above the average for LL6. Therefore, peak metamorphic temperatures for the NWA 6588 parent body were presumably higher than $740{ }^{\circ} \mathrm{C}$ and perhaps very near the silicate solidus. This introduces the question of why there are no known "LLachondrites" in existence (not LL-impact melt, of which a few examples exist). It seems remarkable that the LL chondrite parent body(s) reached peak temperatures just below the silicate solidus, but never exceeded it, and never produced partial melts or magmas that would be recognizable as primitive achondrites with oxygen isotope values consistent with being derived from an LL chondrite precursor.

References: [1] Grossman J. and Rubin A. 2006. Meteoritical Society Nomenclature Committee White Paper. [2] Wlotzka F. 2005. Meteoritics \& Planetary Science 40:1673-1702. 
5478

NWA 6601: INSIGHT INTO THE ORIGIN OF "PURE" IRON METAL IN EUCRITES

C. B. Agee. Institute of Meteoritics, University of New Mexico. E-mail: agee@unm.edu.

Introduction: NWA 6601 is an equilibrated, brecciated, main series eucrite that is permeated with shock melt and possesses nearly pure Femetal grains. Here we explore the origin of "pure" Fe-metal in this new meteorite and implications for processes on eucrite parent body.

NWA 6601: This new eucrite consists of $\sim 60 \%$ total pyroxene, low$\mathrm{Ca}$ pyroxene (Fs61.2 \pm 0.9 Wo3.7 $\pm 1.0 \mathrm{Fe} / \mathrm{Mn}=29 \pm 1$ ), high-Ca pyroxene (Fs26.6 $\pm 1.0 \mathrm{Wo} 44.5 \pm 0.8 \mathrm{Fe} / \mathrm{Mn}=30 \pm 2$ ), and $\sim 30 \%$ plagioclase $\left(\mathrm{Or}_{0.5} \mathrm{Ab}_{10.1} \mathrm{An}_{89.4}\right)$, with ubiquitous silica polymorph, $\mathrm{Cr}$ spinel, ilmenite, troilite, and Fe-metal. Pyroxene shows exsolution lamellae and planar parting, fracturing, and microfaultings. Plagioclase often occurs as laths, some transitioning into impact melt pools or veins. Pyroxene and plagioclase grains are up to 500 microns in size and can appear texturally equilibrated, though some areas resemble fine grained cataclastite. Numerous impact melt veins and pools are present; some larger than 500 microns across, containing abundant silica grains, troilite crystallites and rafted basaltic clasts.

Fe-metal in NWA 6601: Fe-metal grains are conspicuous in NWA 6601 because of their relatively large size, uneven distribution, making up much less than $1 \%$ by volume. The Fe-metal occurs as distinct, equant or irregular clast-like grains, some up to $1 \mathrm{~mm}$, found almost exclusively in cataclastic domains. The largest metal grain observed in our sample was bounded on all sides by plagioclase, though most are in multiphase contact. Troilite is commonly found nearby though rarely sharing a grain boundary with metal. None of the large metal grains occur in the impact melt veins or in the equilibrated domains of pyroxene and plagioclase. The metal is nearly pure iron, with $\mathrm{Ni}, \mathrm{Si}$, Mo below EMPA detection limits, low level of Co is detectable $(\sim 0.04 \mathrm{wt} \%)$.

Formation of "Pure" Fe-metal in Eucrites: Low-Ni metal $(<1 \mathrm{wt} \%$ $\mathrm{Ni}$ ) is not uncommon in eucrites [e.g., 1,2], however its origin and formation may not be uniform in Vesta. In contrast to NWA 6601, Camel Donga possesses "pure" Fe-metal that is very fine grained (5$20 \mu \mathrm{m})$, abundant $(\sim 2 \mathrm{wt} \%)$, and finely dispersed in silicate crystals, and hypothesized to have formed by impact induced reduction of pyroxene facilitated through $S_{2}$ volatile loss by breakdown of troilite [3]. We also see evidence for impact related formation of "pure" iron metal in NWA 6601. However troilite consumption and $\mathrm{S}_{2}$-loss does not seem to be consistent with the ubiquity of this phase in the impact melt veins and pools which are swamped with troilite crystallites, while troilite is nearly absent in texturally equilibrated domains. In NWA 6601, the large, "pure" metal grains and most of the associated troilite give the appearance of being intruded into the texturally equilibrated domain as part of the impact process, not as in situ melt or reduction productssimply because of the paucity of feedstock troilite in equilibrated domains. Future work to determine the trace concentration and distribution of $\mathrm{Ni}, \mathrm{Co}, \mathrm{Mo}$, and other siderophile elements may help shed more light on the origin of "pure" Fe-metal in NWA 6601 and impact processes on Vesta.

References: [1] Seddiki A. et al. 2007. Abstract \#1049. 38th Lunar and Planetary Science Conference. [2] Wittmann et al. 2011. Abstract \#1984. 42nd Lunar and Planetary Science Conference. [3] Palme H. et al. 1988. Meteoritics 23:49-57.

\section{8 \\ ZIRCONIUM-HAFNIUM EVIDENCE FOR SEPARATE SYNTHESIS OF LIGHT NEUTRON-RICH NUCLEI}

W. M. Akram ${ }^{1}$, M. Schönbächler ${ }^{1}$, P. Sprung ${ }^{2}$, N. Vogel ${ }^{2}$. ${ }^{1}$ SEAES, The University of Manchester, M13 9PL. E-mail: waheed.akram@postgrad. manchester.ac.uk. ${ }^{2}$ Institute for Geochemistry and Petrology, ETH Zürich, Switzerland.

Introduction: Hafnium has four stable non-radiogenic isotopes $\left({ }^{177,178,179,180} \mathrm{Hf}\right)$ that are produced by an $\mathrm{s}-/ \mathrm{r}$-process combination. These isotopes have recently been shown to be homogeneously distributed in various carbonaceous chondrites, CAIs and the Earth [1] and this is in line with the observed s-/r-process homogeneity of $\mathrm{Sm}$ and $\mathrm{Nd}$ isotopes. In contrast, nucleosynthetic anomalies have been reported for lighter (s-/r-process) isotopes in bulk carbonaceous chondrites and their components (Ba, Ni, $\mathrm{Cr}$, and $\mathrm{Ti}$ ). This was interpreted as evidence for the synthesis of neutron-rich isotopes (via the r-process) in two distinct astrophysical sites for the light $(Z \leq 56)$ and heavy $(Z>56)$ nuclei, respectively [1]. Zirconium $(Z=40)$ and $\mathrm{Hf}(Z=72)$ are both of similar refractory and geochemical nature, and therefore coincident isotope measurements of the two elements provide a good way of studying variable s-/r-process nucleosynthetic sites for light and heavy nuclei. Here we thus report new $\mathrm{Zr}$ high precision data for the samples previously analyzed for $\mathrm{Hf}$ isotopes.

Analytical Technique and Results: In this study, a terrestrial andesite (AGV-2), three carbonaceous chondrites (Murchison [CM2], Dar al Gani 137 [CO3], Dar al Gani 275 [CK4/5]) and four CAIs from Allende (CV3) were analyzed. Sample preparation and digestion is described in [1] and the $\mathrm{Zr}$ fractions obtained from [1] were passed through an additional anion exchange column to ensure adequate removal of $\mathrm{Zr}$ from the sample matrix. All five $\mathrm{Zr}$ isotopes $\left({ }^{90,}, 91,92,94,96 \mathrm{Zr}\right)$ were measured on a $\mathrm{Nu}$ Plasma MC-ICPMS using the same analytical procedure as outlined in $[3,4]$.

Both Murchison and the CAIs display ${ }^{96} \mathrm{Zr}$ excesses, which are consistent with the values that we reported previously [3]. Furthermore, the new CAI data show identical ${ }^{96} \mathrm{Zr}$ enrichments $\left(\varepsilon^{96} \mathrm{Zr}=2.0 \pm 0.2\right)$ to those previously measured [2,3], suggesting that most CAIs formed from a reservoir with a homogeneous $\mathrm{Zr}$ composition, which is clearly distinct to that of the terrestrial standard. This is analogous to the relatively constant positive shifts observed in CAIs for the neutron-rich nuclide ${ }^{50} \mathrm{Ti}$ $\left(\varepsilon^{50} \mathrm{Ti} \sim+9\right)$ [5]. As ${ }^{50} \mathrm{Ti}$ is synthesized in supernovae, this link potentially indicates a common astrophysical origin for the two isotopes.

In conclusion, our data shows well-resolved ${ }^{96} \mathrm{Zr}$ excesses, in good agreement with previous data, but no variation for r-process dominated $\mathrm{Hf}$ isotopes (data obtained from the same sample digestion). Since both elements are produced through a combination of the s- and r-process, this new data further substantiate the decoupling of nucleosynthetic components. This is consistent with at least two distinct stellar sources for the production of neutron-rich isotopes that operated in different mass regimes.

References: [1] Sprung P. et al. 2010. Earth and Planetary Science Letters 295:1-11. [2] Schönbächler M. et al. 2003. Earth and Planetary Science Letters 216:467-481. [3] Akram. M W. et al. 2011. Abstract \#1908. 42nd Lunar and Planetary Science Conference. [4] Schönbächler M. et al. 2004. The Analyst 129:32-37. [5]. Leya I. et al. The Astrophysical Journal 702:1118-1126. 


\section{9}

H, C AND N ISOTOPE SYSTEMATICS OF CI, CM AND CR CHONDRITES: CLUES TO THE ORIGIN OF WATER

C. M. O'D. Alexander ${ }^{1}$, R. Bowden ${ }^{2}, \quad M$. Fogel $^{2}$ and K. Howard ${ }^{3}$. ${ }^{1}$ DTM, Carnegie Institution of Washington, Washington, DC 20015, USA. E-mail: alexander@dtm.ciw.edu. ${ }^{2} \mathrm{GL}$, Carnegie Institution of Washington, Washington, DC 20015, USA. ${ }^{3}$ IARC, The Natural History Museum, London SW7 5BD, UK.

Introduction: One explanation for the $\mathrm{O}$ isotopic composition of inner solar system bodies is that there was a massive influx of ${ }^{16} \mathrm{O}$-poor water ice from the outer solar system. This ice should have been very D-rich, like comets, and would have been accompanied by abundant organic matter. The $\mathrm{O}$ isotopic compositions of secondary minerals in chondrites suggest that the altering fluids were ${ }^{16} \mathrm{O}$-poor. Thus, one might expect these fluids to have been D-rich and, if alteration occurred in a closed system, the water content to be correlated to that of the organics, particularly the insoluble organic matter (IOM), and the bulk $\mathrm{O}$ isotopes. Previously, we showed in a suite of CMs that $\mathrm{H}$ contents and isotopic compositions correlated with petrologic indicators of alteration [1]. However, the $\mathrm{H}$ is not D-rich. Also, the $\mathrm{O}$ isotopic compositions of the CMs do not correlate with $\mathrm{H}$ content. We have since extended this bulk $\mathrm{H}$ (and $\mathrm{C}$ and $\mathrm{N}$ ) work to additional CMs, as well as Orgueil and CRs.

Methods: The meteorites were crushed to $<106 \mu \mathrm{m}$, and aliquots stored in a desiccator for days to weeks prior to analysis. The $\mathrm{H}, \mathrm{C}$ and $\mathrm{N}$ abundances and isotopes were determined as described in [2].

Results: The bulk $\mathrm{H}$ results for the new CMs are consistent with our earlier results, although Bells $(\delta \mathrm{D} \approx 300 \%$ ), like Essebi, is clearly an outlier. Orgueil $(\delta \mathrm{D} \approx 80 \%)$ is isotopically heavier than CMs, particularly CM1s, but is still much lighter than cometary water. The CRs generally have bulk $\mathrm{H}$ isotopic compositions that are heavy $(\delta \mathrm{D} \approx 580-760 \%)$ and exhibit a limited range of $\mathrm{H}$ contents $(\sim 0.4-0.6 \mathrm{wt} \%)$. The one exception is the CR1 GRO $95577(1.3 \mathrm{wt} \%$ and $270 \%$ ). There is no apparent correlation between bulk $\mathrm{H}$ contents and $\mathrm{O}$ isotopes.

Discussion: The bulk $\mathrm{H}$ measurements are a mix of components primarily organics and hydrated silicates. The IOM is the most abundant known organic component. Subtraction of the D-rich IOM H from the bulks significantly reduces the $\delta \mathrm{D}$ values of the residual $\mathrm{H}$. If it is assumed that the range of IOM $\delta \mathrm{D}$ values is due to isotopic exchange and the most D-rich IOM composition is subtracted, the residual $\delta \mathrm{D}$ values are reduced still further (CI $-180 \%$, CMs -170 to $-370 \%$, CRs 130 $550 \%$, Bells -40 to $120 \%$, Essebi $70 \%$ ). The range within CMs and CRs may at least partially reflect isotopic fractionation associated with $\mathrm{Fe}$ oxidation and/or the presence of an as yet unidentified H-bearing phase $[1,2]$. The $\mathrm{H}$ contents of $\mathrm{CMs}$ or CRs do not correlate with IOM contents or bulk $\mathrm{O}$ isotopes, suggesting that alteration did not occur in closed systems. Redistribution of water within the parent bodies could also produce some $\mathrm{H}$ isotopic fractionation.

Conclusions: There is little evidence for comet-like water in the CIs and CMs. The CRs are isotopically heavier, but this could be largely due to the oxidation of $\mathrm{Fe}$. At present, it seems that the water accreted by the CI, CM and CR chondrites formed or re-equilibrated in the inner solar system. This may have significant implications for the transport and the isotopic evolution of materials in the nebula.

References: [1] C. M. O’D. Alexander et al. 2011. Abstract \#1869. 42nd Lunar and Planetary Science Conference. [2] C. M. O’D. Alexander et al. 2010. Geochimica et Cosmochimica Acta 74:4417.
5084

A STUDY OF THE MINERALOGY AND TEXTURES OF BASALT FINES FROM APOLLO 12 REGOLITH SAMPLE 12023-, 155

L. Alexander ${ }^{1,2}$, J. F. Snape ${ }^{2,3}$, I. A. Crawford ${ }^{1,2}$, K. H. Joy ${ }^{3,4,5}$ and R. Burgess ${ }^{6}$. ${ }^{1}$ Birkbeck College, London, UK. E-mail: 1.alexander@ bbk.ac.uk. ${ }^{2} \mathrm{CPS}$ at UCL-Birkbeck, London. ${ }^{3}$ Department of Earth Sciences, UCL, London, UK. ${ }^{4}$ CLSE, The Lunar and Planetary Institute, USRA, Houston, TX 77058, USA. ${ }^{5}$ The NASA Lunar Science Institute. ${ }^{6}$ SEAES, University of Manchester, UK.

Introduction: The Apollo 12 (A12) mission landed in the Eastern region of Oceanus Procellarum (OP). Crater size-frequency distribution measurements [1] indicate that some of the youngest lava flows on the Moon occur within OP and it is possible that young basalt fragments may have been incorporated into the regolith at the A12 landing site. This study aims to search for such fragments by examining basaltic fines from the A12 landing site and determining their composition. Samples will be dated using Ar-Ar dating. Here we present initial petrographic results for 12023-, 155, consisting of 12 fines $\sim 2 \mathrm{~mm}$ in diameter.

Methods: Each individual grain was split in two with the larger split retained for petrographic analysis and the smaller split allocated for radiometric dating. Textures were examined using a JEOL JXA-8100 electron microprobe with an Oxford Instrument INCA energy dispersive system (EDS) to produce backscattered electron (BSE) images and elemental maps. Modal mineralogies were obtained from BSE images and elemental maps using imaging software to identify the phases. Bulk compositions were calculated using multiple raster beam analyses (RBA) across the samples that were corrected for differences in phase densities in accordance with [2]. These methods have been previously tested on known samples [3] and found to be in good agreement.

Results: Initial results indicate that these fines are generally typical of A12 low-Ti basalts [4], composed of pyroxene (48-60\%), plagioclase $(30-45 \%)$ and ilmenite $(3-4 \%)$, with varying amounts of olivine $(0-12 \%)$ and accessory silica and $\mathrm{Cr}$-spinel. Traces of $\mathrm{Fe}-\mathrm{Ni}$ metal and troilite occur rarely $(<1 \%)$. Textures are varied but are mainly holocrystalline and subophitic. Highly zoned pyroxenes are the major mafic phase, which often enclose anhedral olivines. Plagioclase varies from fine subhedral laths to larger blocky textures, occasionally intrafasciculate, up to $1 \mathrm{~mm}$ in length. Ilmenite commonly occurs as fine laths of varying lengths (50$600 \mu \mathrm{m})$ and also as irregular grains in some samples, while spinels are small $(<100 \mu \mathrm{m})$ and zoned. Silica is present as randomly distributed, anhedral crystals. We have also identified a coarse-grained pyroxene cumulate in which ilmenite is absent.

Discussion: Most basalt fines have modal mineralogies consistent with pigeonite or feldspathic A12 basalt classifications [4], but exceptions are noted, which may require contributions from separate lava flows. Detailed mineral compositions (ongoing) will complete the chemical analysis of these samples. Results can then be evaluated together with $\mathrm{Ar}-\mathrm{Ar}$ dating to understand the petrogenesis and source regions of basaltic grains collected at the A12 site.

References: [1] Hiesinger H. et al. 2003. Journal of Geophysical Research 108, E7. [2] Warren P. H. 1997. Abstract \#1497. 28th Lunar and Planetary Science Conference. [3] Snape J. F. et al. 2011. Abstract \#2011. 42nd Lunar and Planetary Science Conference. [4] Papike J. J. et al. 1998. Lunar samples. Reviews in Mineralogy, vol. 36, pp 5-1 - 5-234. 


\section{0}

MAGNESIUM ISOTOPES IN THE ULTRAREFRACTORY CAIS EFREMOVKA 101.1: EVIDENCE OF OPEN SYSTEM BEHAVIOR J. Aléon ${ }^{1}$, J. Marin-Carbonne ${ }^{2}$ K. D. McKeegan ${ }^{2}$ and A. El Goresy ${ }^{3}{ }^{1}$ CSNSM, CNRS/IN2P3/University of Paris Sud, Bat 104, 91405 Orsay Campus, France. ${ }^{2}$ Earth and Space Science department, UCLA, Los Angeles, CA, USA. ${ }^{3}$ Bayerisches Geoinstitut, Bayreuth, Germany. E-mail: Jerome.Aleon@csnsm.in2p3.fr.

Efremovka 101.1 is an unusual compact type A calcium-aluminumrich inclusion (CAIs) with several lithological subunits that may once have been individual free-floating CAIs in the early solar nebula [1]. All subunits share an ultrarefractory rare earth elements abundance pattern and an ultrarefractory fassaitic clinopyroxene enriched in $\mathrm{Sc}$ and $\mathrm{Zr}$ is locally observed [1]. E101.1 is surrounded by a well preserved WarkLovering rim (WLR) and a partially preserved olivine-rich accretionary rim (AR) In order to understand the formation of this enigmatic object and the implications for isotopic fluctuations in the nebular gas we started an exhaustive isotopic study of E101 using the UCLA IMS1270 ion microprobe combining $\mathrm{O}$ [2], $\mathrm{Mg}$ (this work) and $\mathrm{Si}$ isotopes [3]. Eighty five high precision $\mathrm{Mg}$ isotope analyses, spatially associated with $\mathrm{O}$ isotope analyses whenever possible, were obtained using a approximately $15 \mathrm{nA}$, approximately $30 \mu \mathrm{m} \mathrm{O}^{-}$beam and simultaneous detection of ${ }^{24} \mathrm{Mg},{ }^{25} \mathrm{Mg},{ }^{26} \mathrm{Mg}$ and ${ }^{27} \mathrm{Mg}$ on four Faraday cups.

$\mathrm{Mg}$ isotopic analyses of E101.1 reveal a highly complex distribution of both $\mathrm{Mg}$ isotopic mass fractionation and ${ }^{26} \mathrm{Mg}$ excesses due to ${ }^{26} \mathrm{Al}$ decay. As a whole, different regions in the CAIs have different $\mathrm{Mg}$ isotope systematics. This includes the rims, the different lithological subunits of possible xenolithic origin, as well as different domains defined using $\mathrm{O}$ isotopes [2]. $\delta^{25} \mathrm{Mg}$ values range from $-6.5 \%$ (melilite in subinclusion 1) to $+6.7 \%$ (spinel clusters in the host) with values in the rims clustering around $0 \%$. Initial ${ }^{26} \mathrm{Al} /{ }^{27} \mathrm{Al}$ ratios in subcomponents range from supracanonical $\left(7.1 \times 10^{-5}\right.$ in the $\mathrm{Sc}-\mathrm{Zr}$-fs $)$ down to $1.8 \times 10^{-5}$ in disturbed melilite from the host. Olivine in the AR is enriched in ${ }^{26} \mathrm{Mg}$ by approximately $0.16 \%$. A more complete description of the isotope systematics will be presented at the meeting. To summarize, $\mathrm{Mg}$ isotopes suggest open-system behavior of $\mathrm{Mg}$ with evaporation as well as incorporation of light $\mathrm{Mg}$ possibly from the digestion of xenolithic inclusions or by direct condensation from nebular gas. The lightest components, sinuous fragments and possibly subinclusion 1, can probably be considered as xenoliths. E101.1 likely had an initial canonical ${ }^{26} \mathrm{Al} /{ }^{27} \mathrm{Al}$ ratio but secondary disturbances resulted in both higher and lower than canonical ratios in specific components. $\mathrm{Mg}$ isotopes in the WLR are in agreement with previous studies suggesting a younger age although some spinels having a larger ${ }^{26} \mathrm{Mg}$ excess may be derived from the interior. Comparison with $\mathrm{O}$ isotopes will also be presented at the meeting.

References: [1] El Goresy et al. 2002. Geochimica et Cosmochimica Acta 66:1459-1491. [2] Aléon et al. 2010. Meteoritics \& Planetary Science 45:A7. [3] Marin-Carbonne et al. 2011. This conference.
5244

3-D IMAGING OF MINERAL INCLUSIONS IN EXTRATERRESTRIAL CHROMITE USING SYNCHROTRON RADIATION X-RAY TOMOGRAPHIC MICROSCOPY

C. Alwmark ${ }^{1,2}$, B. Schmitz ${ }^{1}$ S. Holm ${ }^{1,2}$ F. Marone ${ }^{3}$ M. Stampanoni ${ }^{3,4}$. ${ }^{1}$ Department of Geology, University of Lund, Sölveg. 12, SE-22362 Lund, Sweden, E-mail: carl.alwmark@geol.lu.se. ${ }^{2}$ Institute for Geochemistry and Petrology, ETH Zürich, Clausiusstrasse 25, CH-8092 Zürich. ${ }^{3}$ Swiss Light Source, Paul Scherrer Institute, 5232 Villigen, Switzerland ${ }^{4}$ Institute for Biomedical Engineering, University and ETH Zürich, CH-8092 Zürich.

Introduction: In ordinary chondrites, chromite is the only common mineral that survives long-term exposure on Earth. However, a recent study [1] showed that chromite grains from ordinary chondrites contain small inclusions of the matrix minerals of the meteorite and that information about the silicate matrix of the original meteorite can be derived from these inclusions. Thus, the inclusions are an important tool in classification of fossil extraterrestrial chromite used for characterizing the past influx of material to Earth (e.g., [2]), but have, due to their small nature, previously been difficult to locate. The main aim of this study is to, with the help of synchrotron radiation X-ray tomographic microscopy (SRXTM), establish a fast and nondestructive protocol in which chromite grains can be scanned and inclusions located. SXRTM will also allow quantitative and morphological studies of both host chromite grains and inclusions in three dimensions.

Material and Methods: 385 chromite grains from eight equilibrated (type 4-6) ordinary chondrites of different groups (H, L, LL) were searched for inclusions. The grains were stacked in capillaries and then analyzed using SRXTM at the TOMCAT beamline of the Swiss Light Source at the Paul Scherrer Institute [3]. An automatic sample exchanger integrated at the beamline [4] enabled unattended measurements over the course of $26 \mathrm{~h}$. The tomographic reconstructions were performed using a highly optimized routine based on a Fourier transform method and a gridding procedure [5].

Results and Discussion: Analysis of the reconstructed data reveals that inclusions are readily distinguished down to a resolution of $<1 \mu \mathrm{m}$ and that almost $2 / 3$ of all grains contained one or more inclusions. The number of inclusions within each chromite grain varies from $1->300$. The average number of inclusions in samples of petrographic type 4 is about twice as high (33 inclusions per grain) compared to types 5 and 6 (15 inclusions per grain, respectively). The size of the inclusions range from $<1$ to $2.4 \times 10^{5} \mu^{3}$. A comparison of the average size of the largest inclusion from each chromite grain shows that petrographic type 6 chromite grains have an average inclusion size $>20$ times larger than that of grains from type 4 and 5 .

Conclusion: The method applied in this study to identify inclusions in chromite grains, using SRXTM, allows for large amounts of grains to be analyzed in a short time with high spatial and contrast resolution. The method is nondestructive and does not affect the properties of the material analyzed, i.e., grains of interest can be recollected and further studied. Furthermore, the size and frequency distribution of inclusions can be used to assess the petrographic type of the precursor meteorite.

References: [1] Alwmark C. and Schmitz B. 2009. Geochimica et Cosmochimica Acta 73:1472-1486. [2] Schmitz B. et al. 2003. Science 300:961-964. [3] Stampanoni M. et al. 2006. Proceedings of SPIE 6381. [4] Mader K. et al. 2011. Journal of Synchrotron Radiation 18:117-124. [5] Marone F. et al. 2010. Proceedings of SPIE 7804. 


\section{7}

\section{SEARCH FOR Q IN SARATOV (L4)}

Sachiko Amari ${ }^{1}$ and Jun-ichi Matsuda ${ }^{2}$. ${ }^{1}$ Laboratory for Space Sciences and the Physics Department, Washington University, St. Louis, MO 63130-4899, USA. E-mail: sa@wuphys.wustl.edu. ${ }^{2}$ Department of Earth and Space Science, Graduate School of Science, Osaka University, Osaka 560-0043, Japan

Introduction: Q, for quintessence, carries most of heavy noble gases in primitive meteorites [1]. It is most likely carbonaceous matter [2], however the exact nature remains elusive for over three decades. On the other hand, Q-gases in meteorites from various compositional types have been extensively studied [3, 4]. Since Q is less susceptible to thermal metamorphism than presolar diamond [3], meteorites with a higher metamorphic grade $(\geq 3.7)$ contain $\mathrm{Q}$ but not diamond. For this reason, we chose Sararov (L4) to study Q and its noble gases.

Experimental: We started from $\sim 7.2 \mathrm{~g}$ of Saratov. First it was alternately treated HF-HCL and HCL to remove silicates. Then sulfur was extracted with $\mathrm{CS}_{2}$. Noble gases in bulk Saratov and the HF-Hcl residue $\mathrm{AC}$ were analyzed by step-wise heating, and were already reported [5]. The $\mathrm{Ne}$ data points of $\mathrm{AC}$ lie on a mixing line between spallogenic $\mathrm{Ne}$ and Ne-Q in Saratov on a Ne 3-isotope plot. AC was subjected to colloidal separation, yielding the colloidal fraction $\mathrm{AE}$, and the noncolloidal fraction AF. AE is black, while AF is dark brown, suggesting that essentially all carbonaceous matter in $\mathrm{AC}$ was concentrated in $\mathrm{AE}$ (Fig. 1). AE was further subjected to two successive colloidal separations, yielding the colloidal fractions AG and AI, and the non-colloidal fraction AJ. Since AE is most likely devoid of spallogenic Ne, we will be able to precisely determine $\mathrm{Ne}-\mathrm{Q}$ in Saratov from the daughter fractions of AE. If we assume all $\mathrm{Xe}$ in $\mathrm{AC}$ is concentrated in $\mathrm{AE}$, from the mass balance, the ${ }^{132} \mathrm{Xe}$ concentration of AE is calculated to be $20,100 \times 10^{-10} \mathrm{~cm}^{3}$ $\mathrm{STP} / \mathrm{g}$. If further separations are successful, the Xe concentrations of one or two of the daughter fractions (AG, AI, and AJ) can be even higher. We will report noble gas data on these colloidal and non-colloidal fractions.

References: [1] Lewis R. S. et al. 1975. Science 190:1251-1262. [2] Ott U. et al. 1981. Geochimica et Cosmochimica Acta 45:1751-1788. [3] Huss G. R. et al. 1996. Geochimica et Cosmochimica Acta 60:3311-3340. [4] Busemann H. et al. 2000. Meteoritics \& Planetary Science 35:949-973. [5] Matsuda J. et al. 2010. Meteoritics \& Planetary Science 45:361-372.
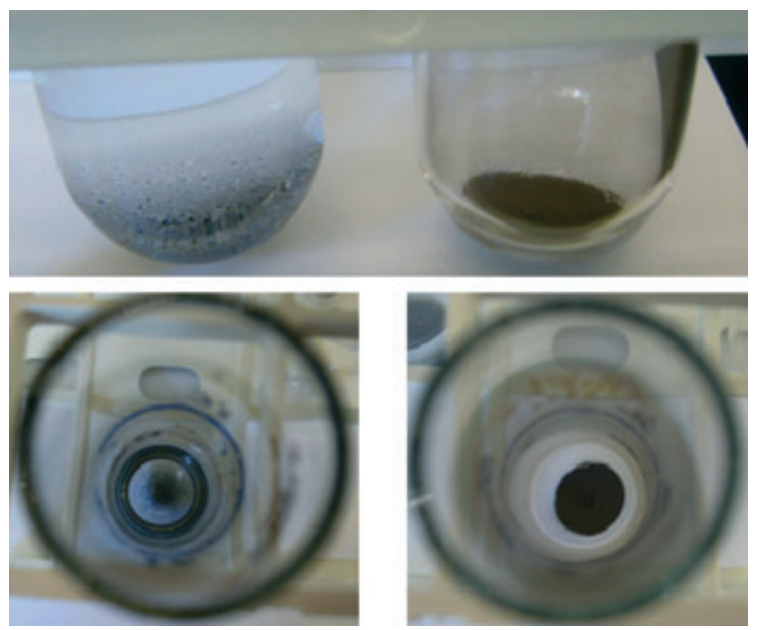

Fig. 1. Saratov fractions AE (left) and AF (right).
5196

\section{LEAD ISOTOPIC AGE OF THE QUENCHED ANGRITE NORTHWEST AFRICA 1296}

Y. Amelin ${ }^{1}$ and A. J. Irving ${ }^{2}$. ${ }^{1}$ Research School of Earth Sciences, The Australian National University, Canberra, ACT, Australia. E-mail: yuri.amelin@anu.edu.au. ${ }^{2}$ Department of Earth and Space Sciences, University of Washington, Seattle, WA 98195 , USA.

Introduction: Northwest Africa 1296 [1] is a quenched angrite with similar composition to the quenched angrites D'Orbigny and Sahara 99555 that were dated with the $\mathrm{Pb}-\mathrm{Pb}$ method at $4564.42 \pm 0.12 \mathrm{Ma}$ [2] and between 4564.58-4564.86 Ma [3, 4], respectively (dates calculated with ${ }^{238} \mathrm{U} /{ }^{235} \mathrm{U}=137.88$ ). Here we describe the $\mathrm{Pb}$-isotopic systematics of the angrite NWA 1296. Determination of $\mathrm{U}-\mathrm{Pb}$ ratios is in progress.

Techniques: NWA 1296 is a very fine-grained rock, therefore separation of minerals by hand picking, as was done for other coarsergrained angrites, was impractical. Instead, we focused on separation of minerals on the basis of solubility in acids. Various size fractions of crushed sample were gently leached in $0.5 \mathrm{M} \mathrm{HNO}$ or $0.3 \mathrm{M} \mathrm{HBr}$ (first leachates) in order to extract phosphate minerals, then leached in hot concentrated $\mathrm{HNO}_{3}$ and $\mathrm{HCl}$ (second leachates), dissolved and analyzed following [5].

Results: Residues after acid leaching of NWA 1296 whole rock contain 9-17 ppb $\mathrm{Pb}$ - lower than in D'Orbigny (18-30 ppb) and Sahara 99555 (27-34 ppb). The measured ${ }^{206} \mathrm{~Pb} /{ }^{204} \mathrm{~Pb}$ ratios are between 280 and 1150 (330-4990 after blank subtraction). The six most radiogenic analyses with blank-corrected ${ }^{206} \mathrm{~Pb} /{ }^{204} \mathrm{~Pb}>1000$ yield consistent ${ }^{207} \mathrm{~Pb} /{ }^{206} \mathrm{~Pb}$ dates with a weighted average of $4564.20 \pm 0.45 \mathrm{Ma}, \mathrm{MSWD}=0.89$ $\left({ }^{238} \mathrm{U} /{ }^{235} \mathrm{U}=137.88\right)$. These analyses yield uniform ${ }^{232} \mathrm{Th} /{ }^{238} \mathrm{U}$ model ratios of $8.87 \pm 0.05(2 \mathrm{SD})$. Less radiogenic residue analyses are more dispersed, probably due to the presence of two nonradiogenic $\mathrm{Pb}$ components.

The first and second leachates contained 200-600 ppb and 23$75 \mathrm{ppb} \mathrm{Pb}$, respectively. Lead in the leachates (with ${ }^{206} \mathrm{~Pb} /{ }^{204} \mathrm{~Pb}$ up to 174 in the first leachates and up to 725 in the second leachates) is sufficiently radiogenic to attempt age calculation. The ${ }^{232} \mathrm{Th} /{ }^{238} \mathrm{U}$ model ratios in the leachates are uniform $(3.78 \pm 0.04$ in the first leachates, $3.70 \pm 0.15$ in the second leachates) and distinct from the value in the residues, suggesting that most $\mathrm{U}$ in the rock is contained in one moderately soluble mineral, or in two minerals with different solubility but similar $\mathrm{Th} / \mathrm{U}$ ratios. A combined $\mathrm{Pb}-\mathrm{Pb}$ isochron for the first and second leachates yields $4565.13 \pm 0.55 \mathrm{Ma}$, MSWD $=92$. The isochron passes between the points for primordial $\mathrm{Pb}$ and modern crustal $\mathrm{Pb}$, implying the presence of two nonradiogenic $\mathrm{Pb}$ components.

Discussion: The residue model age of $4564.20 \pm 0.45 \mathrm{Ma}$ gives a minimum estimate of the crystallization age. This value is consistent with the ages of D'Orbigny and Sahara 99555. The distribution of $\mathrm{Th} / \mathrm{U}$ model ratios between soluble and insoluble minerals is also similar among these meteorites. These observations further support their close genetic connection.

Interpretation of the leachate isochron date depends on identification of the principal U-carrying mineral in NWA 1296. If $U$ is contained in a Ca-phosphate such as merrillite, apatite or silico-apatite, then the isochron date corresponds to the closure of radiogenic $\mathrm{Pb}$ migration in this mineral. Comparison of the residue and leachate dates indicates very rapid cooling, which can be estimated quantitatively if the dates are better constrained, and the grain size of the U-carrying mineral is determined.

References: [1] Jambon A. et al. 2005. Meteoritics \& Planetary Science 40:361-375. [2] Amelin Y. 2008. Geochimica et Cosmochimica Acta 72:221-232. [3] Connelly J. N. et al. 2008. Geochimica et Cosmochimica Acta 72:4813-4824. [4] Amelin Y. 2008. Geochimica et Cosmochimica Acta 72:4874-4885. [5] Amelin Y. et al. 2010. Earth and Planetary Science Letters 300:343-350. 
5197

U-Pb SYSTEMATICS OF THE ULTRAMAFIC ACHONDRITE NORTHWEST AFRICA 5400

Y. Amelin ${ }^{1}$ and A. J. Irving ${ }^{2}$. ${ }^{1}$ Research School of Earth Sciences, The Australian National University, Canberra, ACT, Australia. E-mail: yuri.amelin@anu.edu.au. ${ }^{2}$ Department of Earth and Space Sciences, University of Washington, Seattle, WA 98195, USA.

Introduction: Northwest Africa 5400 is a metal-bearing ultramafic achondrite petrologically similar to brachinites, but with oxygen isotopic composition indistinguishable from that of the Earth [1]. A previous attempt to determine the formation age of this meteorite using the ${ }^{53} \mathrm{Mn}-{ }^{53} \mathrm{Cr}$ system [2] yielded an internal isochron with zero slope and elevated $\varepsilon\left({ }^{53} \mathrm{Cr}\right)$ of 0.44 , corresponding to the age younger than $4541 \mathrm{Ma}$. Here we report U-Pb data for NWA 5400.

Techniques: A portion of coarsely crushed meteorite was leached in warm $7 \mathrm{M} \mathrm{HCl}$ to remove weathering products (mostly iron hydroxides). Three fractions of clinopyroxene with variable abundance of inclusions and turbidity, one fraction of olivine and one fraction of orthopyroxene were hand-picked from this acid-washed material. These fractions, and three whole rock fractions and their leachates were analyzed using the procedures of [3].

Results: Acid-washed minerals and rocks contain between 0.13 $0.72 \mathrm{ppb} \mathrm{U}$ and between $1.2-7.7 \mathrm{ppb} \mathrm{Pb}$. Their $\mathrm{Pb}$ isotopic compositions, with ${ }^{206} \mathrm{~Pb} /{ }^{204} \mathrm{~Pb}$ between 9.35 and 13.69 , plot along the mixing line between primordial $\mathrm{Pb}$ [4] and modern terrestrial crustal $\mathrm{Pb}$. The least radiogenic $\mathrm{Pb}$ isotopic compositions are indistinguishable, within error, from primordial $\mathrm{Pb}$. The ${ }^{206} \mathrm{~Pb} /{ }^{204} \mathrm{~Pb}$ ratios are correlated with ${ }^{238} \mathrm{U} /{ }^{204} \mathrm{~Pb}$. If interpreted as an isochron, this correlation corresponds to the date of $1570 \mathrm{Ma}$.

The whole rock contains 13-32 ppb of acid leachable U, and 49$173 \mathrm{ppb}$ of acid leachable $\mathrm{Pb}$. The most easily soluble $\mathrm{Pb}$ in whole rocks is similar to modern terrestrial crustal $\mathrm{Pb}$; the $\mathrm{Pb}$ extracted with hot concentrated acids is slightly less radiogenic. No correlation between ${ }^{206} \mathrm{~Pb} /{ }^{204} \mathrm{~Pb}$ and ${ }^{238} \mathrm{U} /{ }^{204} \mathrm{~Pb}$ ratios is observed for the leachates.

$\mathrm{On}$ a $\mathrm{Pb}-\mathrm{Pb}$ isochron diagram, all residue and leachate data points plot along a single line that yields a date of $4478 \pm 55 \mathrm{Ma}$ if interpreted as a single-stage isochron.

Discussion: The U-Pb isotopic systematics of NWA 5400 are explained by mixing between initial primordial $\mathrm{Pb}$ and modern crustal $\mathrm{Pb}$ introduced by terrestrial weathering. Acid-soluble uranium was also accumulated during weathering. The linear arrays in $\mathrm{U}-\mathrm{Pb}$ and $\mathrm{Pb}-\mathrm{Pb}$ isotopic space are mixing lines with no chronological significance. This meteorite contains chlorapatite, a potentially suitable mineral for $\mathrm{U}-\mathrm{Pb}$ dating, but it would be necessary to completely remove weathering products by a nonacidic treatment before dating of apatite can be attempted.

Although the U-Pb data reported here do not constrain the crystallization age of NWA 5400, they rule out a terrestrial origin: the initial $\mathrm{Pb}$ isotopic composition is far more primitive than the least radiogenic terrestrial $\mathrm{Pb}$ [5]. Instead, these data are consistent with origin from a body that either differentiated very early, or escaped an extensive loss of volatile elements. We suggest that the evolution of the NWA 5400 parent body differed from that of the Earth, Moon and parent bodies of angrites and eucrites, but was possibly akin to evolution of some of the parent bodies of iron meteorites, where the most primitive $\mathrm{Pb}$ is found.

References: [1] Irving A. J. et al. 2009. Abstract \#2332. 40th Lunar and Planetary Science Conference. [2] Shukolyukov A. et al. 2010. Abstract \#1492. 41st Lunar and Planetary Science Conference. [3] Amelin Y. et al. 2010. Earth and Planetary Science Letters 300:343-350. [4] Tatsumoto M. 1973. Science 180:1279-1283. [5] Frei R. and Rosing M. T. 2001. Chemical Geology 181:47-66.
5089

AN ANISOTROPY OF MAGNETIC SUSCEPTIBILITY STUDY OF THE STAC FADA MEMBER SUEVITE: CONSTRAINTS ON THE IMPACT CRATER LOCATION

K. Amor, J. Taylor, S. P. Hesselbo and C. MacNiocaill. Department of Earth Sciences, University of Oxford, Parks Road, Oxford, OX1 3PR. E-mail: kena@earth.ox.ac.uk.

The Stac Fada Member (SFM) of the 1.177Ga [1] Stoer Group sediments (NW Scotland) has recently been reinterpreted as a proximal ejecta blanket [2]. Field observations of suevite injected between bedding planes of the underlying sandstones suggest a strong component of lateral movement during ejecta emplacement, consistent with the notion of fluidized deposition by surge-type flows rather than simple ballistic sedimentation. If this is the case then the ejecta may be expected to retain a fabric or lineation indicative of flow direction, which when measured at several dispersed geographical locations may point towards the impact crater. Anisotropy of magnetic susceptibility (AMS) has been used to determine directional information in geological materials subjected to flow or stress.

We measured the AMS and frequency dependence of susceptibility of 99 cores taken from 20 oriented blocks at four locations in the SFM in NW Scotland. All locations show an increasing frequency dependence of susceptibility towards the top of the deposit indicating an increasing proportion of fine-grained magnetic particles, which is consistent with an ejecta blanket origin. A weak anisotropy was detected of $1-2 \%$, which is also a characteristic of pyroclastic flow deposits. The data shows a lineation radiating away from a common center. Coupled with observations of other directional field data which is in good agreement with the AMS lineations, we are able to propose a location for the impact crater in the middle of the North Minch Basin between Stornoway and the Stoer Peninsula.

References: [1] Parnell J. et al. 2011. Journal of the Geological Society 168:349-358. [2] Amor K. et al. 2008. Geology 36:303-306. 
5008

ON THE POSSIBILITY OF A LATE PLEISTOCENE EXTRATERRESTRIAL IMPACT: LA-ICP-MS ANALYSIS OF THE BLACK MAT AND USSELO HORIZON SAMPLES

A. V. Andronikov, D. S. Lauretta, I. E. Andronikova and R. J. Maxwell. Department of Planetary Sciences, Lunar and Planetary Laboratory, The University of Arizona, Tucson, AZ, 85721 USA. E-mail: andron@lpl.arizona.edu.

Introduction: A dark layer of organic-rich material contemporaneous with the onset of the Younger Dryas (YD) cooling (12.9 ka) has been identified in North America (black mat; BM) and Western Europe (Usselo Horizon; UH). The following main hypotheses on the origin of this layer exist: (1) it formed by water-transported organic material; (2) it is the result of a heavy deposition of algae in a shallow fresh-water reservoir; (3) it formed in response to periods of spring-fed stream activation when groundwater oxidized organic material; (4) it resulted from wood fires and decomposition of charred wood; or (5) it resulted from the impact of a comet or asteroid $[1,2]$. Most BM sequences contain a thin $(2-5 \mathrm{~cm})$ basal pitch-black layer likely corresponding to the lower YD boundary (LYDB). The UH sequences are represented by dark charcoal-rich layers within aeolian sands [3].

Discussion: Trace element concentrations in the BM (Arizona) and UH (Holland and France) samples were studied using LA-ICP-MS. Trace element compositions of the LYDB layers which directly underlie the $\mathrm{BM}$, and the $\mathrm{BM}$ itself are different: the BM displays trace element concentrations similar to the average continental crust, while the LYDB layers are strongly enriched in REE (up to $800 \times \mathrm{CI}$ chondrite) and relatively depleted in $\mathrm{Ta}, \mathrm{Nb}, \mathrm{Zr}$, and $\mathrm{Hf}($ down to $30 \times \mathrm{CI}$ ). Such a difference in compositions can point to a sharp change in the conditions of sedimentation just before LYDB layer deposition. LYDB, BM and UH samples display 2-5 times higher concentrations of PGEs than the sediments underlying BM sequences. Additionally, LYDB samples display a positive correlation between $\mathrm{Ni}$ and $\mathrm{Ir}$ accompanied by an Os-to-Ir ratio of $1: 1$ and overall higher concentrations of both Os and Ir. In contrast, UH samples do not display any correlation between Os and Ir, and $\mathrm{BM}$ samples have an Os-to-Ir ratio of 1:2, which is more typical for terrestrial sediments [4]. Overall, UH samples display trace element characteristics that are a mixture between those typical of the BM and the LYDB. Trace element distributions and relations for samples of LYDB may be consistent with incorporation of the material of ET origin and could, therefore, support the hypothesis that an impact occurred shortly before the beginning of the YD cooling $12.9 \mathrm{ka}$.

References: [1] Firestone R. B. et al. 2007. Proceedings of the National Academy of Science 104:16016-16021. [2] Haynes C. V. et al. 2010. Proceedings of the National Academy of Science 107:4010-4015. [3] Kaiser K. et al. 2009. Boreas 38:591-609. [4] Agiorgitis G. and Wolf R. 1984. Chemical Geology 42:277-286.
5248

ASTEROID IMPACT VARIATIONS OF NRM AND REM IN TARGET BASALTS OF LONAR CRATER, INDIA

Md. Arif ${ }^{1}$, N. Basavaiah ${ }^{1}$, S. Misra ${ }^{2}$ and K. Deenadayalan ${ }^{1}$. ${ }^{1}$ Indian Institute of Geomagnetism, Navi Mumbai-410218, India. E-mail: mdarifkrl@gmail.com. ${ }^{2}$ School of Geological Sciences, University of KwaZulu Natal, Durban-4000, South Africa.

Introduction: Around $50 \mathrm{ka}$ old Lonar crater (dia. approximately $1.8 \mathrm{~km}$ ), India, is completely excavated in approximately $65 \mathrm{Ma}$ Deccan Traps by an oblique impact of a chondritic asteroid that stuck the preimpact target from the east at an angle of $30-45^{\circ}[1,2$ and references therein]. We report here some rock magnetic experimental results on the possible relationships between variations of natural remanent magnetization (NRM) and REM (=NRM/saturation isothermal remanent magnetization [SIRM]) of the target basalts with the direction of impact.

Experimental Procedures: Oriented drill cores collected from around the Lonar crater rim and adjoining areas were cut into two to three specimens of $2.2 \mathrm{~cm}$ height in the laboratory. The NRM and SIRM imparted at $1 \mathrm{~T}$ by a Molspin pulse magnetizer, were measured using a Molspin and JR-6 spinner magnetometers.

Results: The unshocked target basalts $(n=44)$ at $\geq 2 \mathrm{~km}$ E of crater rim have mean NRM of approximately $3.87 \mathrm{Am}^{-1}$ [standard deviation (SD): $1.24 \mathrm{Am}^{-1}$ ]. The shocked targets [2] between approximately $1-2 \mathrm{~km}$ uprange of crater to the west $(n=207)$ show a general increase in average NRM to approximately $11.24 \mathrm{Am}^{-1}$ (SD: $\left.9.58 \mathrm{Am}^{-1}\right)$. The targets from N, NW, W, and SW sectors of crater rim $(n=203)$ and an eastern sector $(n=27)$ show average NRM of approximately $5.62 \mathrm{Am}^{-1}$ (SD: $2.22 \mathrm{Am}^{-1}$ ) and approximately $3.89 \mathrm{Am}^{-1}$ (SD: $1.28 \mathrm{Am}^{-1}$ ), respectively. The targets from SE $(n=51)$ and $\mathrm{S}(n=34)$ sectors show a wide variation of NRM with an average of approximately $18.2 \mathrm{Am}^{-1}$ (SD: 14.3 $\mathrm{Am}^{-1}$ ). A few samples in the N, SE and S sectors, however, show very high NRM (approximately 100-350 $\mathrm{Am}^{-1}$ ). The REM of unshocked target basalts $(n=7)$ are $<0.79 \%$ with an average of approximately $0.42 \%$. The basalts from around the crater rim $(n=45)$ except the western rim sector $(n=12)$ have REM mostly $<1.5 \%$ with an average of approximately $0.40 \%$, and only $22 \%$ of shocked basalts show higher REM with an average of approximately $3.67 \%$. Further it is found that samples with still higher REM occur only on the western rim sector and to the west adjacent to the crater in the uprange. The samples from this area $(n=39)$ have $36 \%$ data of REM between 1.97 and $12.65 \%$ with an average of approximately $5.10 \%$; the rest of the samples, however, have REM $<1.5 \%$ with an average of approximately $0.49 \%$.

Discussions: The NRM of shocked basalts is in general increases due to impact. Although the NRM does not show much change over the unshocked target either in the downrange (no increment) or uprange (approximately one and half time increment) on the east-west plane of impact, a significant increase (approximately 5 times) is seen oblique to the plane and direction of impact. The shocked targets at approximately 1.5 to $2 \mathrm{~km}$ west of the crater rim in uprange also show a significant increase (approximately 3 times) in NRM. Most of very strongly shocked target basalts (approximately 71\%) from around the crater rim and adjacent to the west of the rim have an average REM of approximately $0.44 \%$, which is very similar to unshocked targets; the rest of the sample set, mostly from the western sector of the crater rim and to the west adjacent to the crater in uprange show an average REM of approximately $5.10 \%$ with values up to approximately 12 times higher over the unshocked targets.

References: [1] Fredriksson et al. 1973. Science 180:862-864. [2] Misra et al. 2010. Geological Society of America Bulletin 122:563-574. [3] Sengupta et al. 1997. Revista de Fisica Aplicada e Instrumentacao 12:1-7. 
5137

IMPACT EJECTA TEMPERATURE PROFILE ON THE MOON WHAT ARE THE EFFECTS ON THE Ar-Ar DATING METHOD?

N. Artemieva ${ }^{1,2}$ and V. A. Fernandes ${ }^{3}$. ${ }^{1}$ Planetary Science Institute E-mail: artemeva@psi.edu. ${ }^{2}$ Institute for Dynamics of Geospheres, Moscow. ${ }^{3}$ Museum of Natural History, Berlin.

Introduction: The ${ }^{40} \mathrm{Ar} /{ }^{39} \mathrm{Ar}$ method is widely used for the acquisition of impact related cooling ages. Several of the Apollo and lunar meteorite samples dated (e.g., [1]) have demonstrated that there is a decoupling between $\mathrm{K}-\mathrm{Ar}$ reset ages and their shock related petrographic features: partial to total resetting of the K-Ar system occurs even at low shock pressures. This suggests that the Apollo samples, after an impact event, were kept in a warm environment which permitted the partial to total resetting of the $\mathrm{K}-\mathrm{Ar}$ clock. Here we present preliminary model results for the formation and evolution of shock-heated ejecta blanket produced during a large impact event on early and modern Moon, and its effects on pre-existing cold rock fragments.

Numerical Model: We model impact cratering on the Moon with the 3D hydrocode SOVA [2] complemented by the ANEOS equation of state for geological materials [3]. We use two thermal profiles within the target: cold modern Moon with a thermal gradient of $2 \mathrm{~K} \mathrm{~km}^{-1}$ and hot ancient Moon with a thermal gradient of $15 \mathrm{~K} \mathrm{~km}^{-1}$ [4]. To define ejecta distribution on the surface, we use a method of ballistic continuation on a sphere [5]. The evolution of post-depositional temperature profile within the ejecta blanket is estimated via one-dimensional thermal conductivity equation.

Results: At any distance from the crater ejecta are a mixture of materials compressed to various shock pressures (from partial vaporization, $\mathrm{P}>150 \mathrm{GPa}$, to weakly compressed fragments) and excavated from various depths. At distal sites, melt and highly compressed materials from the crust prevail; near-crater ejecta consist mainly from unshocked materials. At the current cold Moon, antipodal ejecta have a temperature range of 400-600 K, while proximal ejecta have only slightly elevated $T$ of $250-300 \mathrm{~K}$. If the early Moon was substantially hotter (thermal gradient of $15 \mathrm{~K} \mathrm{~km}^{-1}$ during the first 0.2 $0.5 \mathrm{Gyr}$ ), an average ejecta temperature reaches $600 \mathrm{~K}$ at distances $>2400 \mathrm{~km}$ from a crater center, and is above $400 \mathrm{~K}$ at smaller distances.

Ejecta cooling time $\tau$ after ejecta deposition depends strongly on its thickness $H\left(\tau \sim H^{2}\right)$, which, in turn, increases quickly with a projectile size $D_{p r}$ increase $\left(H \sim D_{p r}{ }^{3}\right)$. Thus, gas losses due to an elevated post-deposition temperature is not as important for small impacts (with fast cooling rate), but may lead to a partial or total resetting of the K-Ar system in large, basin-forming impacts.

Considering plagioclase closure temperature is $<573 \mathrm{~K}$ [e.g., 8], a rock fragment kept under this temperature for $>20$ years within an ejecta blanket [9] will likely have its K-Ar systematics reset and little to no petrographic shock related features.

Future work: (1) high-resolution model of an impact, resulting in a map of ejecta distribution around the crater (thickness $H$ and temperature $T$ as a function of distance and azimuth); (2) cooling time as a function of $T$ and $H$; (3) Ar diffusion and estimates of gas loss.

References: [1] Fernandes et al. 2008. Workshop Early Solar System Impact Bombardment, Abstract \#3028. [2] Shuvalov. 1999. Shock Waves 9:381-390. [3] Thompson and Lauson. 1972. SC-RR-61 0714. Sandia National Laboratories p. 119. [4] Spohn et al. 2001. Icarus 149:54-65. [5] Dobrovolskis. 1981. Icarus 47:203-219. [6] Cassata et al. 2010. Geochimica et Cosmochimica Acta 73:6600-6612. [7] Shuster et al. 2010. Earth and Planetary Science Letters 290:155-165.

\section{5}

MODELING THE FORMATION OF THE GLOBAL K/Pg LAYER

N. Artemieva ${ }^{1,2}$ and J. Morgan ${ }^{3}$. ${ }^{1}$ Planetary Science Institute. E-mail: artemeva@psi.edu. ${ }^{2}$ Institute for Dynamics of Geospheres, Moscow. ${ }^{3}$ Imperial College, London.

Introduction: The $\mathrm{K} / \mathrm{Pg}$ boundary $[1,2]$ is widely recognized as a global ejecta layer from the Chicxulub impact crater [3]. However, some properties of this layer (thickness, shocked quartz distribution, chemical composition) are inconsistent with the idea that ejecta was transported around the globe purely on a ballistic path.

Numerical Model: We model the Chicxulub impact with the 3D hydrocode SOVA [4] complemented by the ANEOS equation of state for geological materials [5]. In three separate stages we model: (1) the impact and initial ejection of material [6]; (2) the ballistic continuation of ejecta on a spherical earth [7]; and (3) ejecta re-entry into the atmosphere (at an altitude of $200 \mathrm{~km}$ re-entering tracers are replaced by real particles with a size-frequency distribution in accordance with their maximum shock pressure; the interaction of these particles with the atmosphere is modeled using a multi-phase approximation).

Results: Up to distances of $1000-1500 \mathrm{~km}$ from the crater, ballistic ejecta is deposited rapidly. At larger distances, the mass of ejecta and atmosphere are comparable, hence, their interaction becomes significant. Re-entering ejecta create strong winds in the upper atmosphere and these winds disperse small fragments (molten spherules and shocked quartz grains of $<1 \mathrm{~mm}$ in diameter) preferentially downrange. For two-three hours after the impact, these dispersed ejecta travel up to a few thousand $\mathrm{km}$ from their re-entry site (final deposition through the dense lower atmosphere or ocean may take days or weeks). This mechanism is much more intense than observed for volcanic aerosols in stable atmospheric flows. Similar phenomena (atmospheric skidding) has been suggested for re-entering ejecta after the Shoemaker-Levy 9 Comet impact on Jupiter [8], but has never been modeled before.

Conclusions: Numerical modeling of the interaction of ejecta with the atmosphere allows us to reproduce the constant thickness of the distal $\mathrm{K} / \mathrm{Pg}$ layer, as well as the presence of low-velocity ejecta from the crystalline basement (shocked quartz) around the globe.

References: [1] Alvarez L. et al. 1980. Science 208:1095-1108. [2] Smit J. 1999. Annual Review of Earth and Planetary Science 27:75-113. [3] Hildebrand A. R. et al. 1991. Geology 19:867-871. [4] Shuvalov V. 1999. Shock Waves 9:381-390. [5] Thompson S. L. and Lauson H. S. 1972. SC-RR-61 0714. Sandia Nat. Lab. 119 p. [6] Artemieva N. and Morgan J. 2009. Icarus 201:768-780. [7] Dobrovolskis A. 1981. Icarus 47:203-219. [8] Colgate S. A. and Petschek A. G. 1985. LA-UR-84-3911. Los Alamos Nat. Lab. 


\section{0}

POST-FALL SURFACE MODIFICATION FEATURES OF IRON METEORITES FOUND BY THE OPPORTUNITY ROVER AND THEIR IMPLICATIONS FOR MARTIAN WEATHERING PROCESSES

J. W. Ashley ${ }^{1}$, M. P. Golombek ${ }^{2}$, P. R. Christensen ${ }^{1}$, S. W. Squyres ${ }^{3}$, T. J. $\mathrm{McCoy}^{4}$, C. Schröder ${ }^{5}$, I. Fleischer ${ }^{6}$, J. R. Johnson ${ }^{7}$, K. E. Herkenhoff ${ }^{7}$ and T. J. Parker ${ }^{2}$. ${ }^{1}$ Mars Space Flight Facility, Arizona State University. ${ }^{2}$ Jet Propulsion Laboratory, California Institute of Technology, Pasadena, CA, USA. ${ }^{3}$ Department of Astronomy, Cornell University, Ithaca, NY, USA. ${ }^{4}$ Department of Mineral Sciences, National Museum of Natural History, Smithsonian Institution, Washington, DC, USA. ${ }^{5}$ Center for Applied Geoscience, Eberhard Karls University, Tübingen, Germany. ${ }^{6}$ Institut für Anorganische und Analytische Chemie, Johannes Gutenberg-Universität, Mainz, Germany. ${ }^{7}$ Astrogeology Science Center, U. S. Geological Survey, Flagstaff, AZ, USA. E-mail: james.ashley@ser.asu.edu.

Introduction: Including Heat Shield Rock [1] (officially renamed Meridiani Planum [2]), a total of six large, iron meteorites have been found to date by the Opportunity rover at Meridiani Planum, Mars. In order of discovery, the five additional meteorites have been unofficially named Block Island, Shelter Island, Mackinac Island, Oileán Ruaidh, and Ireland [3]. In addition to Pancam and Navcam imaging, surface targets on Block Island, Shelter Island and Oileán Ruaidh have been examined using two or more of the rover arm instruments (Alpha-Particle X-ray Spectrometer, Mössbauer Spectrometer, and Microscopic Imager).

Observations: These meteorites show a remarkable variety of surface textures, morphologic features, and coatings that convey their post-fall alteration history, and thus provide insights into Martian weathering processes. Among the more notable of these are (1) a marked dichotomy in surface roughness on Block Island suggestive of partial burial and exposure, (2) a large pit lined with delicate iron protrusions suggestive of inclusion removal on Block Island by a gentle process, (3) expression of the Widmanstätten pattern in four of the meteorites, suggestive of differential mechanical abrasion, (4) a spatially discontinuous iron oxiderich coating elevated in $\mathrm{Mg}$ and $\mathrm{Zn}$ relative to its substrate, and (5) largescale cavernous weathering in two of the meteorites. In addition, the presence of regmaglypts on several of these meteorites suggest that some areas altered much less than other areas since their atmospheric passage and arrival on Mars.

Discussion: Cross-cutting relationships between the oxide coating and Widmanstätten pattern, together with elevated $\mathrm{Mg}$ and $\mathrm{Zn}$ abundances, show the coating to be the result of post-fall processes and not a fusion crust. If the coating is the result of water exposure (possibly ice), a sequence of two periods of abrasion with oxide production between them is indicated. The morphologies of iron protrusions in the Block Island cavity are similarly suggestive of water exposure (possibly acidic) more than of wind abrasion, but the type of inclusive mass removed is unknown. The cavernous weathering features in Shelter Island and Mackinac Island may also have been caused by acidic corrosion. However, the extent to which mechanical abrasion may contribute to the final appearance of these meteorites remains an unknown, but clearly present (and potentially testable), component of their alteration history.

References: [1] Schröder C. et al. 2008. Journal of Geophysical Research 113:doi:10.1029/2007JE002990. [2] Connolly H. C. et al. 2006. Meteoritical Bulletin \#90, Meteoritics \& Planetary Science 41:1383-1418. [3] Ashley J. W. et al. 2011. Journal of Geophysical Research 116: doi:10.1029/2010JE003672.

\section{8}

GEOLOGIC MAPPING, MORPHOLOGY, AND DATING OF THE KING CRATER REGION, LUNAR FARSIDE

J. W. Ashley, ${ }^{1}$ B. R. Hawke ${ }^{2}$, N. DiCarlo ${ }^{1}$, A. C. Enns ${ }^{1}$, H. Hiesinger ${ }^{3}$, M. S. Robinson ${ }^{1}$, H. Sato ${ }^{1}$, E. J. Speyerer ${ }^{1}$, C. H. van der Bogert ${ }^{3}$, R. V. Wagner $^{1}$, K. E. Young ${ }^{1}$ and the LROC Science Team. ${ }^{1}$ Lunar Reconnaissance Orbiter Camera, School of Earth and Space Exploration, Arizona State University, Tempe, AZ 85287-3603. ${ }^{2}$ Hawaii Institute of Geophysics and Planetology, University of Hawaii, Honolulu, HI. ${ }^{3}$ Institut für Planetologie, Westfälische Wilhelms-Universität, Münster, Germany. E-mail: james.ashley@asu.edu.

Introduction: Since its discovery on the lunar farside, King crater $\left(5.0^{\circ} \mathrm{N}\right.$ and $\left.120.5^{\circ} \mathrm{E}\right)$ has been a site of fascination to the lunar science community; and recently, as a Constellation site of interest, become appealing to the goals of human space exploration. The crater-forming impact interacted in a complex way with pre-existing topography, and a variety of impact processes can be explored in the morphology of the resulting features. In particular, the King crater impact melt pond ( $\sim 17 \mathrm{~km}$ diameter; located in nearby Al-Tusi crater) provides opportunities to study large volumes of once-molten material of dominantly anorthositic composition. The Lunar Reconnaissance Orbiter Camera (LROC) Wide Angle Camera (WAC) and Narrow Angle Camera (NAC) [1] imaged King crater from a nominal $50 \mathrm{~km}$ altitude at pixel scales of 100 meters and 0.5 meter, respectively. Images were used to create detailed geologic maps for the region at both scales, with the NAC emphasis on the melt pond. Digital elevation models (DEMs) were also derived from both WAC [2] and NAC [3] images for the area.

Discussion: The high-resolution NAC images are particularly useful for augmenting previous mapping efforts [e.g., 4], showing fine details within the main melt pond that raise questions on melt pond dynamics and evolution. These include both positive and negative relief features, craters with anomalous morphologies (involving hummocky floors, irregular outlines, and boulder associations), and complex flow morphologies. NAC images also show (1) interactions of melt with slump blocks in the crater wall terraces, (2) anomalous mounds and other morphologic complexities in the crater floor melt deposits, and (3) details of deceleration lobes in the distal ejecta blanket. Crater counts on the extended ejecta blanket [e.g., 5] show that King crater is likely to be $\sim 1$ $\mathrm{Ga}$ in age.

Based on pre-existing topography assumptions, an $175 \mathrm{~m}$ depth was estimated for the main melt pond using the NAC DEM, which also reveals kilometer-scale topographic depressions, particularly across the central and eastern portions of the pond. Vertical displacements within these depressions from their inferred original equipotential surfaces range from 15 to 20 meters. The perimeters of these downwarped zones correlate with the occurrence of negative relief features $(\sim 10$ to $100 \mathrm{~m}$ in length). The sagging may have occurred as the result of contraction and/ or compaction within the melt both during and following cooling. Some negative relief features in the melt pond appear to show evidence of collapse into subsurface voids, and are likely the result of melt withdrawal, indicating some mechanism for local drainage while significant volumes of melt remained mobile.

References: [1] Robinson M. S. et al. 2010. Space Science Review 150:81-124. [2] Scholten et al. 2011. Abstract \#2046. 42nd Lunar and Planetary Science Conference. [3] Tran T. et al. 2010. ASPRS CaGIS Specialty Conference. [4] Wilhelms D. E. and El-baz F. 1977. USGS, Map I-948. [5] Ashley et al. 2011. Abstract \#2437. 42nd Lunar and Planetary Science Conference. 
5083

\section{NOBLE GASES IN COSMIC SPHERULES AND MICROMETEORITES FROM THE TRANSANTARCTIC MOUNTAINS}

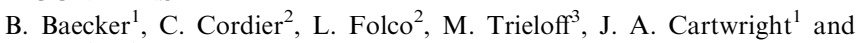
U. Ott ${ }^{1} .{ }^{1}$ Max Planck Institute for Chemistry, Joh.-J.-Becher-Weg 27, D-55128 Mainz, Germany. E-mail: b.baecker@mpic.de. ${ }^{2}$ MNA, Siena, Italy. ${ }^{3}$ University of Heidelberg, Germany.

Introduction: At MPI Mainz we have initiated a comprehensive survey of noble gases in micrometeorites. While $\mathrm{He}$ and $\mathrm{Ne}$ in micrometeorites are generally dominated by solar contributions, the inventory of heavy primordial noble gases is not as well characterized. We hope to fill this gap with particular focus on the diagnostic isotopic composition of Xe. While our study will eventually include material from a variety of collections (e.g., the CONCORDIA collection [1]), our first results are from "large" PNRA (Programma Nazionale delle Ricerche in Antartide, Italy) micrometeorites collected from the Victoria Land, Transantarctic Mountain micrometeorite traps [2].

Samples and Analytics: 51 micrometeorites (MM's)-26 cosmic spherules (CS), 11 scoriaceous (Sc), 14 unmelted (Un) - in the 600$800 \mu \mathrm{m}$ size range were extracted from traps $\# 45 \mathrm{~b}$ and $\mathrm{c}$ on the top of Miller Butte, and split into aliquots for combined petrographic and noble gas analyses. For the latter, aliquots were heated using a $\mathrm{CO}_{2}$ laser. The analyses were performed using our multicollector $\mathrm{Nu}$ Instruments noble gas mass spectrometer [3].

Preliminary Results: At present, we have obtained noble gas results for three CS and one Sc micrometeorites. Our $\mathrm{Ne}$ and Ar results (corrected for isobaric interferences, but not extraction blank) are listed in Table 1.

As found in previous studies (e.g., $[4,5])$ noble gas abundances in $\mathrm{CS}$ are mostly low and essentially have a terrestrial air signature (e.g., $\left.{ }^{20} \mathrm{Ne} /{ }^{22} \mathrm{Ne} \sim 9.8,{ }^{21} \mathrm{Ne} /{ }^{22} \mathrm{Ne} \sim 0.029\right)$. In contrast, the Sc micrometeorite shows ${ }^{20} \mathrm{Ne} /{ }^{22} \mathrm{Ne}$ and (even clearer) ${ }^{21} \mathrm{Ne} /{ }^{22} \mathrm{Ne}$ ratios higher than air, indicative of the presence of solar and spallogenic Ne. Using the production rates for IDPs from table 3 in [6], the spallogenic ${ }^{21} \mathrm{Ne}$ of $\sim 1.6 \times 10^{-9} \mathrm{cc} / \mathrm{g}$ would be produced at $1 \mathrm{AU}$ by SCR primaries in $\sim 0.17 \mathrm{Ma}$, for production by GCR primaries, the inferred exposure would be $\sim 4 \mathrm{Ma}$. The concentration of trapped (solar) ${ }^{20} \mathrm{Ne}$ of $\sim 5 \times 10^{-7} \mathrm{cc} / \mathrm{g}$ is about an order of magnitude lower than the geometric mean for Un micrometeorites from Yamato Mountains and Dome Fuji, but distinctly higher than in CS (see table 4 in [4]). The low ${ }^{40} \mathrm{Ar} /{ }^{36} \mathrm{Ar}$ ratio for the $\mathrm{Sc}$ micrometeorite $(\sim 128)$ indicates the presence of trapped ${ }^{36} \mathrm{Ar}$, at an abundance of $\sim 6 \times 10^{-8} \mathrm{cc} / \mathrm{g}$. This is most likely "planetary" type Ar, carried primarily by phase Q [7].

Acknowledgments: We acknowledge support by DFG through SPP 1385 (Project OT 171/5-1) and PNRA.

References: [1] Duprat J. et al. 2007. Advances in Space Research 39:605-611. [2] Rochette P. et al. 2008. Proceedings of the National Academy of Sciences 105:18206-18211. [3] Ott U. et al. 2010. Meteoritics \& Planetary Science 45:A158. [4] Osawa T. et al. 2010. Meteoritics \& Planetary Science 45:1320-1339 [5] Marty B. et al. 2005. Meteoritics \& Planetary Science 40:881-894. [6] Kehm K. et al. 2006. Meteoritics \& Planetary Science 41:1199-1217. [7] Lewis R. S. et al. 1975. Science 190:1251-1262.

Table 1. Ne and Ar results for MM's. Sizes are given in $\mu \mathrm{m}$. Weights are given in $\mu \mathrm{g}$, noble gas amounts - not corrected for blank - in $10^{-12} \mathrm{cc}$ units. Uncertainties in the last digits.

\begin{tabular}{llllllll}
\hline Sample & Size & Weight & ${ }^{22} \mathrm{Ne}$ & ${ }^{20} \mathrm{Ne}^{/ 22} \mathrm{Ne}$ & ${ }^{21} \mathrm{Ne}^{/ 22} \mathrm{Ne}$ & ${ }^{36} \mathrm{Ar}$ & ${ }^{40} \mathrm{Ar}^{36} \mathrm{Ar}$ \\
\hline CS 45c21 & $720 \times 556$ & 337.9 & 0.41 & $9.11(37)$ & $0.029(2)$ & 0.40 & $319(17)$ \\
CS 45c17 & $630 \times 476$ & 126.3 & 0.16 & $9.34(1.01)$ & $0.038(6)$ & 2.36 & $280(9)$ \\
CS 45c16 & $465 \times 324$ & 105.1 & 0.21 & $8.85(85)$ & $0.027(4)$ & 0.98 & $292(10)$ \\
Sc 45b22 & $268 \times 191$ & 9.2 & 0.49 & $10.46(45)$ & $0.062(4)$ & 1.04 & $128(5)$ \\
\hline
\end{tabular}

5365

CONSTRAINTS ON COSMIC-RAY EXPOSURE HISTORY OF THE VACA MUERTA MESOSIDERITE FROM NOBLE GAS ANALYSIS OF NEUTRON-IRRADIATED SAMPLES

K. Bajo ${ }^{1,2}, \mathrm{H}$. Sumino ${ }^{2}$ and K. Nagao ${ }^{2}$. ${ }^{1}$ Natural History Sciences, Hokkaido University. E-mail: bajo@ep.sci.hokudai.ac.jp. ${ }^{2}$ Geochemical Research Center, Graduate School of Science, University of Tokyo.

Introduction: Mesosiderites have unique and interesting textures, and their formation mechanism and histories have been investigated from petrological, mineralogical, geochemical, and chronological points of view. Bajo and Nagao [1] proposed a complex cosmic-ray exposure history and extreme depletion of volatile elements such as noble gases and halogens of Vaca Muerta. Here we present halogen and $\mathrm{Ba}$ concentrations of Vaca Muerta and discuss on cosmic-ray exposure condition based on noble gas analysis of neutron-irradiated samples?

Experimental: Two samples of different textures, one is metal-silicate mixture (VM-B) and another eucritic pebble (VM-P) [1], were investigated. Measured $\mathrm{Ar}, \mathrm{Kr}$, and $\mathrm{Xe}$ isotope compositions of neutronirradiated samples were decomposed into each component derived from nuclear reactions of several elements $(\mathrm{K}, \mathrm{Ca}, \mathrm{Cl}, \mathrm{Br}, \mathrm{I}, \mathrm{Ba}$ and $\mathrm{U})$ and intrinsic noble gas components in order to determine the ${ }^{39} \mathrm{Ar}-{ }^{40} \mathrm{Ar}$ age and concentrations of the parent elements. The Hb3gr interlaboratory ${ }^{39} \mathrm{Ar}-{ }^{40} \mathrm{Ar}$ age standard ([2], [3]) was used to determine conversion factors of $\mathrm{Cl}, \mathrm{K}$ and $\mathrm{Ca}$ to relevant $\mathrm{Ar}$ isotopes and neutron fluence. Bromine, I, $\mathrm{Ba}$ and $\mathrm{U}$ concentrations were determined from concentrations of neutron-produced $\mathrm{Kr}$ and $\mathrm{Xe}$ isotopes, neutron capture cross sections, and the neutron fluence. The halogen concentrations were calculated from neutron-produced ${ }^{38} \mathrm{Ar},{ }^{80} \mathrm{Kr}$, and ${ }^{128} \mathrm{Xe}$ after subtracting cosmogenic and trapped components from measured concentrations of these isotopes.

Results and Discussion: Bromine and I concentrations for VM-P were less than $10 \mathrm{ppb}$ and lower than those of VM-B $(<5 \mathrm{ppb})$, which is consistent with volatile depletion in VM-P suggested by [1]. However, the low $\mathrm{Cl}$ concentrations and abundant cosmic-ray-produced noble gases allow us to obtain upper limits of $\mathrm{Cl}$ concentrations to be $50 \mathrm{ppm}$. Cosmogenic ${ }^{131} \mathrm{Xe} /{ }^{126} \mathrm{Xe}$ ratio determined for the samples suggests the shielding depth (in the parent body) to be $150 \mathrm{~g} \mathrm{~cm}^{-2}$. Ba concentration determined for the neutron-irradiated VM-P sample was $2.7 \mathrm{ppb}$. Adopting the estimated shielding depth and the cosmic-ray exposure age of $140 \mathrm{Myr}$ [1], $2.5 \times 10^{-12} \mathrm{~cm}^{3} \mathrm{STP} / \mathrm{g}$ of cosmogenic ${ }^{131} \mathrm{Xe}$ is estimated under $4 \pi$ irradiation geometry [4]. This estimation agrees well with the observed value of $(4 \pm 3) \times 10^{-12} \mathrm{~cm}^{3} \mathrm{STP} \mathrm{g}^{-1}$ [1]. This indicates that the cosmogenic ${ }^{131} \mathrm{Xe}$ was mainly produced in meteoroid during transit from its parent asteroid to the Earth, and the progenitor of Vaca Muerta had been located in a deep layer of the parent body.

References: [1] Bajo K. and Nagao K. 2011. Meteoritics \& Planetary Science 46:556-573. [2] Turner G. et al. 1971. Earth and Planetary Science Letters 10:227-234. [3] Roddick J. C. 1983. Geochimica et Cosmochimica Acta 47:887-898. [4] Hohenberg C. M. et al. 1978. Proceedings, 9th Lunar and Planetary Science Conference. pp. 2311-2344. 
5487

\section{TRACE ELEMENT GEOCHEMISTRY OF ENSTATITE CHONDRITES: THE SO-CALLED "REFRACTORY LITHOPHILE" ELEMENTS}

J. A. Barrat ${ }^{1}$, B. Zanda ${ }^{2}$, C. Bollinger ${ }^{1}$ and C. Liorzou ${ }^{1}{ }^{1}{ }^{1}$ Université Européenne de Bretagne, UBO-IUEM, CNRS UMR 6538, Place Nicolas Copernic, 29280 Plouzané Cedex, France. E-mail: barrat@univ-brest.fr. ${ }^{2}$ MNHN \& CNRS UMR7202, 61, rue Buffon, 75005 Paris, France.

We report on the trace element abundances of 15 enstatite chondrites (EL3: MAC 88136, EL6: Eagle, Hvittis, Pillistfer, ALHA 81021, LON 94100, EH3: Kota Kota, Sahara 97096, Sahara 97123, Sahara 97158, EH4: Indarch, EH IMB: Abee, Galim-b, Saint Sauveur, EH impact melt: LAP 02225), the unique enstatite meteorite Itqiy and two ordinary chondritic materials associated with enstatite meteorites (Galim-a and a Cumberland falls chondritic clast). Our ICP-MS procedure (adapted from [1]) allows the determination of abundances and ratios of refractory elements (e.g., REEs $+\mathrm{Y}, \mathrm{Th}, \mathrm{U}, \mathrm{Nb}, \mathrm{Ta}, \mathrm{Sr}, \mathrm{Ba}$ ) in chondrites with a good reproducibility $(<3 \%$ for abundances, $<1.5 \%$ for ratios such as $\mathrm{La} / \mathrm{Sm}, \mathrm{Eu} / \mathrm{Eu}^{*}, \mathrm{Zr} / \mathrm{Hf}$ or $\mathrm{Nb} / \mathrm{Ta}$ ). In agreement with previous studies (e.g., [2-4]), our OC, EL3, EH3, EH4, and some of our EH-IMB (Abee and Saint Sauveur) samples display basically chondritic abundances and ratios. The situation is clearly different for all the other EC samples. The EL6 samples are all light REE depleted and display a small negative Eu anomaly $\left((\mathrm{La} / \mathrm{Lu}) \mathrm{n}=0.64-0.77, \mathrm{Eu} / \mathrm{Eu}^{*}=0.75-0.89\right)$. Their patterns are alike those of an oxidized dark inclusion from Sahara 97158, and the LAP 02225 impact melt. Furthermore, Galim-b display a more pronounced light REE depletion $((\mathrm{La} / \mathrm{Lu}) \mathrm{n}=0.30-0.40)$, a small negative $\mathrm{Yb}$ anomaly and a deep negative $\mathrm{Eu}$ anomaly $\left(\mathrm{Eu} / \mathrm{Eu}^{*}=0.18\right.$ $0.29)$. Finally, Itqiy displays a strong light REE depletion ( $(\mathrm{La} /$ $\mathrm{Lu}) \mathrm{n}=0.12)$ and a deep negative Eu anomaly $\left(\mathrm{Eu} / \mathrm{Eu}^{*}=0.034\right)$, in agreement with [5]. The light REE depletion shown by the impact melted $\mathrm{ECs}$ is accompanied by $\mathrm{Nb}, \mathrm{Sr}, \mathrm{Eu}$, and $\mathrm{Ba}$ depletions. Striking correlations between $\mathrm{La} / \mathrm{Lu}, \mathrm{Nb} / \mathrm{Ta}$, and $\mathrm{Eu} / \mathrm{Eu}^{*}$ are obtained. We performed a series of leaching experiments on various ECs (EL3, EH3, EH-IMB, EL6) in order to constrain the concentrations of trace elements in leachable sulfides and in silicates (residues). The proportions of REEs and $\mathrm{Nb}$ (among others) in the leachable phases (mainly sulfides) are clearly affected by the metamorphic history of the meteorites. For example, the $\mathrm{Nb}$ budget is largely controlled by the sulfides in EL6 chondrites, not in EL3s.

The ranges of abundances and ratios of the so-called "lithophile refractory" elements in ECs can be explained by impact-induced mobilization of sulfides and possibly plagioclase [2]. Vaporization and losses of some of these elements upon impact cannot be directly rejected, and could account for some non-chondritic values inferred for planetary bodies (e.g., the $\mathrm{Nb} / \mathrm{Ta}$ ratio of the Earth).

References: [1] Barrat J. A. et al. 2008. Meteoritics \& Planetary Science 43:1759-1775. [2] Rubin et al. 2009. Geochimica et Cosmochimica Acta 73:1523-1537. [3] Kallemeyn G. W. and Wasson J. T. 1985. Geochimica et Cosmochimica Acta 49:261-270. [4] Kallemeyn G. W. and Wasson J. T. 1985. Geochimica et Cosmochimica Acta 50:2153-2164. [5] Patzer A. et al. 2001. Meteoritics \& Planetary Science 36:1495-1505.
5088

SIGNIFICANCE OF OPAL IN UREILITES-DELIVERY OF $\mathrm{H}_{2} \mathrm{O}$ TO THE INNER SOLAR SYSTEM?

A. D. Beard ${ }^{1}$, H. Downes ${ }^{1,2}$, K. T. Howard ${ }^{2}$. ${ }^{1}$ Birkbeck/UCL Research School of Earth Sciences, Birkbeck, University of London, Malet Street, London WC1E 7HX, U.K. E-mail: a.beard@ucl.ac.uk. ${ }^{2}$ Department of Mineralogy, Natural History Museum, Cromwell Rd, London, UK.

Introduction: Numerous fragments of opal $\left(\mathrm{SiO}_{2} \cdot \mathrm{nH}_{2} \mathrm{O}\right)$ have previously been reported in five internal chips of polymict ureilite EET 83309 [1]. The largest fragments are $>300 \mu \mathrm{m}$ in the longest dimension and show clear banding. The bands are terminated by the adjacent olivine clasts, indicating that the opal clasts were not formed in situ. Opal is also found in contact with ureilitic olivine, forming a single clast with a terrestrial weathering rim around both minerals. It also occurs as thin $(10 \mu \mathrm{m})$ rims completely surrounding suessite $\left(\mathrm{Fe}_{3} \mathrm{Si}\right)$ grains, and can also contain inclusions of schreibersite. It shows no relationship whatsoever with terrestrial weathering rims. These petrographic observations indicate that the opal is (a) extraterrestrial in origin, and (b) native to the ureilite parent body. Suessite and schreibersite are considered to be the products of reduction during shock metamorphism. Therefore formation of the opal occurred after the formation of suessite but before the formation of the regolith represented by polymict ureilites.

Opal Composition: Opal is hydrated silica containing 10-30 wt \% $\mathrm{H}_{2} \mathrm{O}$. XRD analysis suggests that the opal in EET 83309 is of the amorphous opal-A variety, although it appears to have undergone some recrystallization to a $\mathrm{SiO}_{2}$ phase. The largest opal clast has a composition of approximately $65.5 \mathrm{wt} \% \mathrm{SiO}_{2}, 6 \mathrm{wt} \% \mathrm{FeO}$ and $<1 \mathrm{wt} \% \mathrm{MgO}$. In contrast, terrestrial opals only contain up to $1.0 \mathrm{wt} \% \mathrm{FeO}$ and $0.06 \mathrm{wt} \%$ $\mathrm{MgO}$ [2]. The origin of the water or ice which reacted with Si-rich minerals to form the opal is unknown but must be extra-terrestrial in origin, possibly due to impact of an icy body such as a comet on the surface of the ureilite parent body.

The identification of hydrated silica (opal) by the Mars Exploration Rover (MER) Spirit in the Gusev crater, Mars [3] and presence of opal in ureilite EET 83309 has implications for the presence of water and its delivery by cometary bodies during the early formation of the Inner solar system.

References: [1] Beard A. D. et al. Abstract \#5027, 72nd Annual Meteoritic Society Meeting. [2] Gaillou E. et al. 2008. Ore Geology Reviews 34:113-126. [3] Rice et al. Icarus 205:375-395. 


\section{9}

PETROLOGIC AND TEXTURAL DIVERSITY IN ONE OF THE LARGEST SAMPLES OF THE VESTAN REGOLITH

A. W. Beck, H. Y. McSween and C. E. Viviano. Planetary Geosciences Institute, Department of Earth and Planetary Sciences, University of Tennessee, Knoxville, TN 37996. E-mail: abeck3@utk.edu.

Introduction: With the upcoming arrival of Dawn, it will be beneficial to understand the scale of heterogeneity expected in the Vestan regolith. This will help in data interpretation from Dawn, and also in understanding regolith maturity. The best-suited samples for investigating regolith diversity are howardites [1]. Here, we examine the PCA02' paired howardite group, collectively among the largest samples from the Vestan megaregolith ( $\sim 1 \mathrm{~m}$ diameter, pre-atmospheric size) [2]. Along with reporting mineralogical diversity, we examine the scale of textural diversity using a new method where 8 x-ray maps are combined into a single image, allowing us to characterize distributions of specific subtypes of HEDs.

Methods: We have selected 6 PCA02' howardites for this study: PCA 02009(P9), PCA 02013(P13), PCA 02014(P14), PCA 02015(P15), PCA 02018(P18) and PCA 02019(P19). Mineral chemistries and the 8element $\mathrm{x}$-ray maps were acquired using EMP. X-ray maps were merged and classified using image-processing software [3].

Results and Discussion: Pyroxene compositions range from $\mathrm{Wo}_{1}$ $\mathrm{En}_{90} \mathrm{Fs}_{9}$ to $\mathrm{Wo}_{8} \mathrm{En}_{28} \mathrm{Fs}_{64}$, spanning the entire range of HEDs, including subtypes of eucrite and diogenite [4,5]. The majority of olivines fall within the diogenite range $\left(\mathrm{Fo}_{75-65}\right)$ [1]. However, groups of $\mathrm{Fo}_{80-90}$ and $\mathrm{Fo}_{44-60}$ olivine also occur. Plagioclase compositions are like those in basaltic and cumulate eucrites [1]. All samples contain metal, some of which is likely exogenic based on $\mathrm{Co} / \mathrm{Ni}$ ratios [6].

X-ray maps reveal distinctive olivine-phyric $(0.2-1 \mathrm{~mm})$ basaltic clasts, with fine-grained groundmass $(\sim 10 \mu \mathrm{m})$ of $\mathrm{Ol}+\mathrm{Pyx}+$ Plag. These are not typical eucrite basalts, as they contain olivine. Groundmass olivine is Fe-rich $\left(\sim \mathrm{Fo}_{50}\right)$. Olivine xenocrysts are $\sim \mathrm{Fo}_{70}$ and $\sim \mathrm{Fo}_{90}$, both of which are zoned, the rims being in equilibrium with the groundmass $\left(\mathrm{Fo}_{50}\right)$. Several xenoliths of harzburgitic diogenite occur in these basaltic clasts. These clasts also contain $\sim 80 \mu \mathrm{m}$ pieces of metal, some of which have exogenic $\mathrm{Co} / \mathrm{Ni}[6]$.

We propose that these clasts are impact melts, not a new Vestan basalt. We hypothesize that they were generated from the melting of a polymict howardite, rich in a harzburgitic component, or the melting of a yet undiscovered Vestan norite $(\mathrm{Opx}+\mathrm{Ol}+\mathrm{Plag})$. We favor the melting of a howardite rich in harzburgitic material given the xenocrysts and xenoliths observed in the melt clasts. The large metal grains in these melts suggest that the impactor was metal-rich (e.g. OC or Iron).

Modes among the samples vary considerably. Diogenite orthopyroxene $\left(\mathrm{En}_{90-66}\right)$ is the most dominant phase, ranging from $34-89 \%$. Cumulate eucrite pyroxene $\left(\sim \mathrm{En}_{64-45}\right)$ ranges from 2 to $12 \%$, and basaltic eucrite pyroxene $\left(\mathrm{En}_{44-30}\right)$ ranges from 3 to $22 \%$. Olivine ranges from 1 to $10 \%$ and is correlated to the abundance of melt clasts. Melt clasts are $\leq 15 \%$, though they comprise $33 \%$ of $\mathrm{P} 15$. All samples are exceptionally rich in metal, typically containing $1 \%$, with P18 containing an impressive $20 \%$ metal.

The grain size of diogenitic material is primarily $\geq 300 \mu \mathrm{m}$, whereas cumulate eucrite material is $\sim 100 \mu \mathrm{m}$ and basaltic eucrite material is 80 $\leq 3 \mu \mathrm{m}$ matrix material. This grain size distribution is important to Dawn VIR data interpretation, as spectral absorptions in the near infrared occur due to volumetric scattering, and fine-grained textures promote multiple scattering and would relatively increase reflectance [7]. From our textural observations, we anticipate the basaltic matrix material will disproportionately dominate the Dawn reflectance spectra of the regolith.

References: [1] McSween et al. 2011. Space Science Reviews. doi:10.1007/s11214-010-9637-z. [2] Welten et al. 2009 Meteoritics \& Planetary Science 44:A216. [3] ENVI 4.2, ITT Visual Information Solutions 2006. Boulder, Colorado. [4] Mayne et al. 2009. Geochimica et Cosmochimica Acta 73:794-819. [5] Beck and McSween. 2010. Meteoritics \& Planetary Science 45:850-872. [6] Moore et al. 1969. In Meteoritical Research 738-748, Springer-Verlag, NY, USA. [7] Hapke. 1993. Introduction to the theory of reflectance and emittance spectroscopy, Cambridge University Press, NY, USA.

\section{1}

CO-EVOLUTION OF CHONDRITIC ORGANICS AND MINERALS DURING IMPACT METAMORPHISM

P. Beck ${ }^{1}$, H. Yabuta ${ }^{2}$, T. Atou ${ }^{3}$, E. Quirico ${ }^{1}$, Z. Yoldi-Martinez ${ }^{1}$, A. Guillot $^{1}$, L. Bonal ${ }^{1}$, G. Montagnac ${ }^{4}$, B. Schmitt ${ }^{1}$ and G. MontesHernandez ${ }^{5}$. ${ }^{1}$ UJF-Grenoble 1/CNRS-INSU, Institut de Planetologie et d'Astrophysique de Grenoble (IPAG), France, E-mail: pierre.beck@ obs.ujf-grenoble.fr. ${ }^{2}$ Department of Earth and Space Science, Osaka University, Japan. ${ }^{3}$ Secure Material Center Materials and Structures Laboratory, Tokyo Institute of Technology, Japan. ${ }^{4}$ Laboratoire de Sciences de la Terre. ENS Lyon, France. ${ }^{5}$ Institut des Sciences de la Terre (IsTERRE), UJF-Grenoble 1, France.

Introduction: Chondritic organic matter might have evolved on its parent body where subjected to shock metamorphism [1,2]. If the effects of shock waves on minerals have been studied in details from the experimental and observational approaches [3,4] little is known on their effect on the carbonaceous component. Here, we experimentally exposed fragments of matrix of the Murchison chondrite to shock loading up to $40 \mathrm{GPa}$. We describe the effects on the mineralogy as well as on the organic component.

Methods: Samples of Murchison meteorite (CM) were obtained through loan by the Museum of Natural History (Chicago). The sample was powdered and pressed to obtain $1 \mathrm{~cm}$ in diameter and $1 \mathrm{~mm}$ thick pellets. Pellets were then incorporated in a steel container and shocked to pressure of approximately 5-10-20 and $40 \mathrm{GPa}$, using a single-stage propellant gun (5-10-20 GPa) and a two-stage light gas gun (40 GPa) at the Tokyo Institute of Technology. Samples were then recovered and powdered. IR spectra were measured using the Hyperion microscope at IPAG, and Raman spectra were obtained using the Labram HR at ENS Lyon.

Mineralogical Effect: Infra red spectra were obtained in the 215 micron region, and provide insights into the nature of the silicate phase present. As shock pressure increases the $3 \mu \mathrm{m}$ band $\left(-\mathrm{OH}\right.$ and $\left.\mathrm{H}_{2} \mathrm{O}\right)$ appears to decrease in intensity with regard to the $10 \mu \mathrm{m}$ band, indicative of a progressive dehydration of the phyllosilicate phase. For the sample shocked at pressure of 5-20 GPa, minor effects are observed on the $10 \mu \mathrm{m}$ band, which remains broad and structureless, which reveals the presence of a poorly crystalline phyllosilicate [5]. However, in the case of the sample shocked at a pressure of $40 \mathrm{GPa}$, the $10 \mu \mathrm{m}$ band clearly shows structure, which we interpret by the presence of well crystalline phyllosilicates.

Impact on the Structure of the IOM: Raman spectra were directly measured on the powdered sample, and analyzed following [6]. When plotted in a FWHM vs Band G diagram, the samples shocked at 5, 10 and $20 \mathrm{GPa}$ do not appear distinct from the initial samples. However, in the case of the sample shocked at $40 \mathrm{GPa}$, a clear distinction is seen with regard to the other samples. A metamorphic trend is observed, which is distinguishable from long duration thermal metamorphism. The origin of this trend and its relation with those observed in chondritic samples will be discussed.

References: [1] Quirico et al. 2009. Meteoritical Society Meeting, [2] Yabuta et al. 2010. Meteoritics \& Planetary Science 1446-1460. [3] Ahrens T. J. 1980. Science 207:1035-1041. [4] Gillet P. et al. 2007. Mineralogy of shocked meteorites. GSA Monograph, edited by E. Ohtani. [5] Beck et al. 2010. Geochimica et Cosmochimica Acta 74:4881-4892. [6] Bonal et al. Geochimica et Cosmochimica Acta 70:1849-1863. 
5131

HOW DO ACCRETIONARY RIMS AROUND CHONDRULES INFLUENCE THE FORMATION OF CHONDRITES?

E. Beitz ${ }^{1}$, C. Güttler ${ }^{1}$, R. Weidling ${ }^{1}$, J. Blum ${ }^{1}$, D. C. Hezel ${ }^{2}$ and A. Pack ${ }^{3}{ }^{1}$ Institut für Geophysik und extraterrestrische Physik, TU Braunschweig. ${ }^{2}$ National History Museum, London. ${ }^{3}$ Göttingen University, Germany, E-mail: e.beitz@tu-bs.de.

Up to $80 \%$ of the volume of chondric meteorites consists of chondrules. These mm-sized spherules are high-temperature inclusions and most of these are surrounded by a dusty layer. These rims are hypothesized to have formed by accretion of dust grains in a dense region of the solar nebula [1]. It is likely that these rims would influence collisional interaction between the chondrules [2]. We will present new experiments to study the dynamic behavior of dust-coated chondrule analogues.

To produce chondrule rims we used two experimental methods forming rims with different morphologies and porosities around artificial chondrules. One method uses the aerodynamic levitation of millimetersized glass beads (our chondrule analogues) in a gas flow, enriched with $\mu$ m-sized, monodisperse, spherical $\mathrm{SiO}_{2}$ dust. Here, the artificial chondrules accrete very even, highly porous rims with a porosity of $82 \%$. The second method aims at producing dust rims, formed in multiple lowvelocity collisions of chondrules with dust aggregates of the same size. Here, dust from the dust aggregates is transferred to the surface of the solid chondrule. This is realized by shaking a glass bead for a defined time in a dust filled container. These collisions also tend to form rims but less even and with a significantly lower porosity of $60 \%$. To constrain the influence of the different rim structures on the collision behavior, and thus on the formation of larger bodies in the solar nebula, we set up a multiple collision experiment for studying free particle-particle collisions under microgravity conditions [3].

Results: In November 2010 we performed four experiments under microgravity conditions at the Bremen drop tower. Here, we varied the rim porosity (see above) and the particle size ( 2 and $3 \mathrm{~mm}$ glass beads). It turned out that dust coated particles are much more cohesive in collisions compared with non-coated particles, and even dust aggregates of the same size are less likely to stick to each other at the same velocities. For the conducted experiments, we found a correlation between the sticking probability, the rim morphology and the chondrule size. Additionally, we observed the formation of clusters of several tens of chondrules as a result of subsequent sticking collisions [4].

Further Steps: Based on the hypothesis that the chondrules could be still very hot when they start accreting their dust rims, sintering effects and changes in the rim morphology should be taken into account. Therefore we improved our aerodynamical levitation experiment by attaching a laser to heat the chondrules while accreting their rims. For these experiments we exchanged the glass beads by artificial chondrules of realistic compositions, and powdered San Carlos olivine was used to simulate the dust of the early solar nebula. We will present the resultant influence of the chondrule composition, the rim material, and the temperature on the morphology and porosity of the rims based on nano$\mathrm{CT}$ and micro-CT analysis of the produced samples.

References: [1] Metzler K. et al. 1992. Geochimica et Cosmochimica 56:2873-2897. [2] Ormel C. et al. 2007. Astronomy \& Astrophysics 679:1588-1610. [3] Weidling R. et al. 2011. arXiv eprint 1105:3909. [4] Beitz E. et al. 2011. arXiv eprint 1105:3897.
5285

ELEMENTAL MAPPING OF A SULFIDE NODULE

G. K. Benedix ${ }^{1,2}$, I. C. Lyon ${ }^{3}$, M. Schönbächler ${ }^{3}$, T. Henkel ${ }^{3}$ and D. Rost $^{3}$. ${ }^{1}$ Impacts \& Astromaterials Research Centre, (IARC) Dept. of Mineralogy, The Natural History Museum, Cromwell Road, London, SW7 5BD, UK. E-mail: g.benedix@nhm.ac.uk. ${ }^{2}$ IARC/Dept. of Earth Science and Engineering, Imperial College London, South Kensington Campus, London, SW7 2AZ, UK. ${ }^{3}$ School of Earth, Atmospheric and Environmental Sciences, University of Manchester, Manchester M13 9PL.

Introduction: Sulfide occurs predominantly as troilite $(\mathrm{FeS})$ nodules, bars or dumbbells in iron meteorites [1]. These nodules form when the immiscible sulfide liquid is entrained in crystals of $\mathrm{Fe}$, $\mathrm{Ni}$ metal as they solidify. Therefore the crystallization timing of the Fe, Ni metal could be recorded in these nodules. Recent studies $[2,3]$ determined the timing of crystallization of IAB iron meteorites using the ${ }^{205} \mathrm{~Pb}^{205} \mathrm{Tl}$ and ${ }^{107} \mathrm{Ag}_{-}{ }^{107} \mathrm{Pd}$ decay systems. In the studies, both metal and sulfide were analyzed. The elements in the metal produced a correlation that was interpreted as an isochron, whilst the sulfide data were erratic and no correlation was found. A study of the trace elements in sulfide was undertaken last year [4] to elucidate why the sulfide phase exhibit disturbed $\mathrm{Pd}-\mathrm{Ag}$ and $\mathrm{Pb}-\mathrm{Tl}$ isotope systematics. Elemental variation did not deviate from a mean for a traverse across a nodule, with the exception of a few elements: $\mathrm{Ni}, \mathrm{Pb}, \mathrm{Ag}$, and $\mathrm{Cu}$. In this study we extend those results to elemental mapping of the sulfide to ascertain the nature of these element variations.

Samples and Analytical Techniques: The sulfide (FeS) nodule from [4] was analyzed using the UKCAN TOF-SIMS facility at the University of Manchester. The section was analyzed with a pulsed $\mathrm{Au}^{+}$beam focused to a spot size of $\sim 1$ micrometer rastered over selected $\sim 200 \mu \mathrm{m}$ areas of the sample. Positive secondary ions were detected with a mass resolution of $\sim 3500$. High-resolution, back-scattered electron images were acquired using the Zeiss EVO 15LS variable pressure SEM at The Natural History Museum. Mineral phases were identified by EDS analysis on the same instrument.

Results and Discussion: The $\mathrm{FeS}$ nodule is rimmed almost continuously by schreibersite. TOF analyses were obtained from areas within the sulfide, at the sulfide- schreibersite and the sulfide-metal boundaries. Initial results show $\mathrm{Cr}$ streaks within the nodule itself and unrelated to the boundaries. These are attributable to microscopic inclusions of daubreelite, consistent with [1]. Further analysis reveals hotspots of $\mathrm{Ni}, \mathrm{Mn}$, and $\mathrm{Cu}$ in the $\mathrm{FeS}$. These hotspots tend to occur close to the contacts with schreibersite or metal. Analysis by SEM reveal that $\mathrm{MnS}$ and $(\mathrm{Fe}, \mathrm{Ni}) \mathrm{S}$ may be the source of these hotspots. The $\mathrm{Cu}$ is associated with the MnS. The boundaries between FeS/schreibersite and $\mathrm{FeS} /$ metal also contain crushed (most likely sample preparation-derived) and weathered material. The erratic behavior of $\mathrm{Ni}, \mathrm{Pb}$ and $\mathrm{Ag}$ through the FeS nodule may be attributable to the presence of microscopic inclusions of sulfides of those cations. Further analysis will be undertaken to determine the distribution and abundance of these inclusions. The presence of these microscopic sulfides may offer clues to the crystallization history of the FeS nodule during cooling of the parent body as a whole.

References: [1] Buchwald V. F. 1975. Handbook of iron meteorites. [2] Nielsen S. G. et al. 2006. Geochimica et Cosmochimica Acta 70:26432657. [3] Woodland et al. 2005. Geochimica et Cosmochimica Acta 69:2153-2163. [4] Benedix G. K. et al. 2010. Meteoritics \& Planetary Science 45:A13. 
5333

HYDROTHERMAL SYNTHESIS OF CUBANITE: IMPLICATIONS FOR FLUIDS ON COMET 81P/WILD 2 AND THE CI-CHONDRITE PARENT BODY

E. L. Berger ${ }^{1}$, D. S. Lauretta ${ }^{1}$ and L. P. Keller ${ }^{2} .{ }^{1}$ Lunar \& Planetary Laboratory, Tucson, AZ 85721, USA, E-mail: elberger@lpl.arizona.edu. ${ }^{2}$ NASA Johnson Space Center, Houston, TX 77573, USA.

Introduction: The low-temperature form of $\mathrm{CuFe}_{2} \mathrm{~S}_{3}$, cubanite, has been identified in the CI-chondrite and Stardust collections [1]. We report the first synthesis of cubanite under hydrothermal conditions relevant to the CI-chondrite parent body.

An aqueous system can be described by the interplay between $\mathrm{pH}$, temperature, oxygen and sulfur fugacities $\left(f \mathrm{O}_{2}, f \mathrm{~S}_{2}\right)$. These four variables are interrelated; the specification of three of them de facto determines the fourth [2]. Temperatures of $50150^{\circ} \mathrm{C}, \mathrm{pH}$ of $7-10$ and $\log f \mathrm{O}_{2}>10^{-55}$ $10^{-70}$ on the CI-chondrite parent body have been predicted from oxygen isotope measurements [3] and modeling of hydrous asteroids [4]. The presence of cubanite confers a temperature maximum, as it undergoes an irreversible phase transition at $210^{\circ} \mathrm{C}[5,6]$.

Methods: $\mathrm{We}$ form $\mathrm{Cu}-\mathrm{Fe}$ sulfides by controlling temperature, $\mathrm{pH}$ and $f \mathrm{O}_{2}$. Experiments are run at 100,150 , and $200{ }^{\circ} \mathrm{C}$ over the $\mathrm{pH}$ range $7-10$. Oxygen fugacity is held at the iron-magnetite and quartz-fayalitemagnetite buffers.

Compositions of the experimental products are obtained via EMPA on the Cameca SX-50 at LPL. Crystal structure is determined using FIBTEM techniques at JSC on the FEI Quanta 3D 600 dual-beam FIB and the JEOL 2500SE 200kV FE-STEM.

Results and Discussion: Cubanite forms under aqueous conditions, in the presence of iron and magnetite, at a $\mathrm{pH}$ of 9 and temperatures of 150 and $200{ }^{\circ} \mathrm{C}$. While only a minor sulfide constituent at $150{ }^{\circ} \mathrm{C}$, it is the chief product at $200{ }^{\circ} \mathrm{C}$ with an average composition (at.\%) of $\mathrm{Cu}_{15.7} \mathrm{Fe}_{33.6} \mathrm{~S}_{50.7}$.

Two FIB sections were prepared, each containing numerous cubanite grains. Measurements of SAED patterns obtained at multiple orientations from individual grains are consistent with cubanite [5]. TEM analyses reveal extensive twinning. The experimentally produced cubanite, like the CI-chondrite cubanite [1], has a superstructure, likely based on cation ordering. There are superstructure reflections present along both a* and $\mathrm{c}^{*}$.

The successful synthesis of cubanite, under aqueous conditions, aids in refining the parameters $\left(\mathrm{T}, \mathrm{pH}, f \mathrm{O}_{2}\right.$ and $\left.f \mathrm{~S}_{2}\right)$ of the time-varying lowtemperature fluids which existed on the CI-chondrite parent body.

Acknowledgments: This work was supported by NASA grants: NNX08AW48H (ELB) and NNX09AC60G (DSL PI).

References: [1] Berger E. L. et al. 2011. Geochimica et Cosmochimica Acta 75:3501-3513. [2] Barnes H. L and Kullerud G. 1961. Economic Geology 56:648-688. [3] Clayton R. N and Mayeda T. K. 1999. Geochimica et Cosmochimica Acta 63:2089-2104. [4] Zolensky M. E. et al. 1989. Icarus 78:411-425. [5] Fleet M. E. 1971. Zeitschrift fur Kristallographie 132:276-287. [6] Cabri L. J. et al. 1973. Canadian Mineralogist 12:33-38.
5428

3D CHEMICAL ANALYSIS OF THE GUJBA CB CHONDRITE: COMBINING $\mu$-XRF, EDS, EBSD, RAMAN, LA-ICP-MS, AND AFM/ MFM

J. Berlin ${ }^{1}$, A. Käppel ${ }^{1}$, B. K. Hansen ${ }^{1}$, T. Salge ${ }^{1}$, S. Scheller ${ }^{1}$, M. Falke ${ }^{1}$, D. Goran ${ }^{1}$, I. Nemeth ${ }^{1}$, R. Chemnitzer ${ }^{1}$, R. Tagle ${ }^{1}$, U. Waldschläger ${ }^{1}, \mathrm{H}$. M. Chappell ${ }^{2}$, R. H. Jones ${ }^{2}$ and J. I. Goldstein ${ }^{3}$. ${ }^{1}$ Bruker Nano GmbH, Schwarzschildstrasse 12, 12489 Berlin, Germany. ${ }^{2}$ Department of Earth and Planetary Sciences, University of New Mexico, Albuquerque, NM 87131, USA. ${ }^{3}$ Department of Mechanical and Industrial Engineering, University of Massachusetts, Amherst, MA 01003, USA. E-mail: jana.berlin@bruker-nano.de.

Recent technological developments have significantly improved the possibilities to display the $3 \mathrm{D}$ structure of meteorites [e.g., 1]. Here, we present a 3D chemical study of the CB chondrite Gujba combining micro$\mathrm{X}$-ray fluorescence $(\mu-\mathrm{XRF})$ and energy dispersive X-ray spectrometry (EDS)/SEM with serial sectioning (manual grinding/polishing) to display not only the shape of $3 \mathrm{D}$ structures in the $\mu \mathrm{m}$ to $\mathrm{mm}$ range, but also to reveal their chemical composition. The time needed for data acquisition is minimized by using modern SDD technology with high input count rates. Furthermore, element maps are acquired as hyperspectral images (also called PTS: position-tagged spectroscopy) - a format that allows later offline-evaluation and quantification.

The Figure below shows some of our first results, emphasizing the different kind of information obtained with $\mu$-XRF and EDS/SEM. The $\mu$-XRF map of a complete 2D section of the sample was obtained within $1 \mathrm{~h}$ using an M4 Tornado. The green rectangle indicates the EDS acquisition site shown beneath. For this image, nine layers were mapped with a Quantax EDS system and reconstructed in 3D with Amira ${ }^{\circledR}$ software (section depth: $5 \pm 2 \mu \mathrm{m}$; total time spent: $20 \mathrm{~h}$ ).

Using the information from $\mu$-XRF and EDS as a basis, selected regions of interest are examined with additional techniques, such as electron backscatter diffraction (EBSD), laser-ablation inductively coupled plasma mass spectrometry (LA-ICP-MS), Raman spectroscopy and atomic/magnetic force microscopy (AFM/MFM). The main goal of this project is to examine physical and chemical interactions between large metal and silicate clasts and impact melt matrix in CB meteorites. We aim to increase the quality of the analytical work by combining the information obtained with different techniques.

Reference: [1] Ebel D. S. et al. 2008. Meteoritics \& Planetary Science 43:1725-1740.

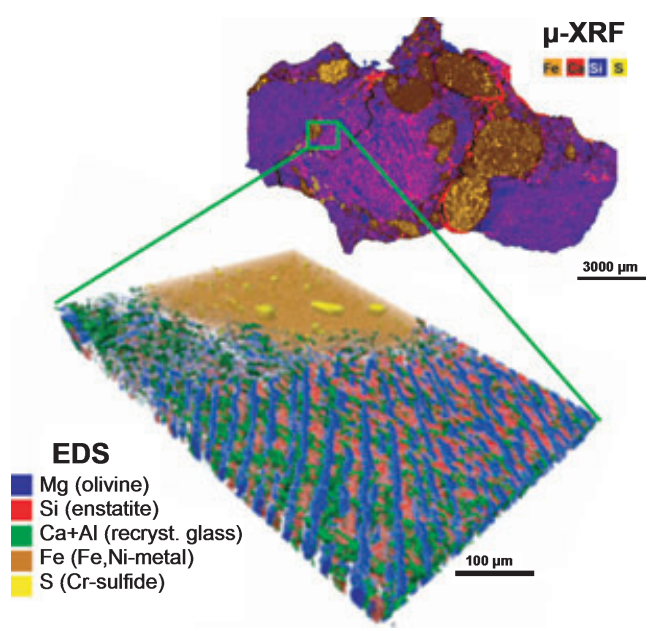


5147

\section{EFFECTS OF METEOROID SHAPE ON THE PRODUCTION RATES OF COSMOGENIC NUCLIDES}

J. Beňo ${ }^{1}$ and J. Masarik ${ }^{1}{ }^{1}$ Faculty of Mathematics, Physics and Informatics, Comenius University, Mlynska dolina F1, Sk-84248, Bratislava, Slovakia, E-mail: beno.juraj@gmail.com.

Introduction: The shape of the meteoroid irradiated in the space can be one of the factors influencing the production of the cosmogenic nuclides. Numerical simulations were done for spherical, cylindrical and elliptical geometries of irradiated object. Simulations show that statistically important differences in particle fluxes dependent on the shape of irradiated object and position of sample within it can be found. Results of simulations were compared with experimental data for St. Severin and other objects that had non-spherical shapes in space during their exposure times.

Simulations: The accurate modeling of the production processes is prerequisite for the physical interpretation of measured depth profiles of cosmogenic nuclides. Productions rates of cosmogenic nuclides are dependent on incident particle fluxes, the meteoroid's preatmospheric shape and size, and its bulk chemical composition. Size effects [1] and bulk chemical composition [2] effects were studied in details and therefore we concentrate our attention in this paper on effects of the parent body shape.

In this work the nucleon spectra are calculated by using the LCS/ MCNP [3]. Having calculated particle fluxes the production rates of cosmogenic nuclides are calculated by folding these spectra with experimental and theoretical cross sections of nuclear reactions leading to the production of particular nuclide.

The geometries investigated in this work were sphere with radius 27 and $45 \mathrm{~cm}$ which is that of a sphere with volume equal to the real St. Severin [5] and Knyahinya, respectively. Further ellipsoid with various ratios between semiaxes and with equal mass were also simulated. Model objects were divided into concentric shells with thickness $2.5 \mathrm{~cm}$. In all shells proton and neutron fluxes were calculated. For the sphere particle fluxes were averaged over the whole volume of the shell. In the case of ellipsoid fluxes were calculated for cylinders with radius $1 \mathrm{~cm}$ and their axis identical with coordinate system axis. The last division of ellipsoid was motivated by the actual location of measured samples [4].

Conclusions: Production rates for the ellipsoids were lower than those for spheres. The differences between the ellipsoids and sphere varied with the nuclide. The shape of irradiated body influences the number of particles escaping from the investigated body without participating in particle cascade development. This can lead to the change of absolute value and the shape of differential particle fluxes. As in the case of ellipsoid there are some regions with larger and smaller curvature than is the curvature of sphere, there are also different numbers of escaping particles in this regions.

References: [1] Bhandari N. et al. 1993. Geochimica et Cosmochimica Acta 57:2361-2375. [2] Masarik J. and Reedy R. C. 1994. Geochimica et Cosmochimica Acta 58:5307-5317. [3] Prael R. E. and Lichtenstein H. 1989. Los Alamos Report LA-UR-89-3014. [4] Englert P. and Herr W. 1980. Earth and Planetary Science Letters 47:361-372. [5] Graf Th., Baur H. and Signer P. 1990. Geochimica et Cosmochimica Acta 54:2511-2520.
5053

COSMOGENIC NOBLE GASES IN CHONDRULES FROM CV AND CR CHONDRITES

U. Beyersdorf ${ }^{1}$, M. Trieloff ${ }^{2}$, U. Ott ${ }^{1}$ and J. Bennett ${ }^{3}$. ${ }^{1}$ Max-PlanckInstitut für Chemie, Joh.-J.-Becherweg 27, D-55128 Mainz, Germany. E-Mail: uta.beyersdorf@mpic.de. ${ }^{2}$ University of Heidelberg, Germany. ${ }^{3}$ ANSTO, Lucas Heights, NSW 2234 Australia.

Introduction: Previous work [1-4] has provided hints for preirradiation of chondrules. While the evidence for pre-irradiation in parent body regoliths seems strong [4], significant uncertainties exist concerning a solar nebula setting. We have started a project to search for such effects in primitive meteorites. Here, we present data of two chondrules from Allende (CV3) and one chondrule from El Djouf 001 (CR2).

Experimental: Chondrules were separated using the freeze-thaw technique similar to [5]. INAA was used to determine abundances of important target elements. Noble gas measurements were performed on the same material analyzed by INAA thus eliminating problems of sample inhomogeneity. Data for bulk/matrix samples at identical shielding will be obtained later. Here, we compare our chondrule results with literature data for the bulk meteorites. Production rates were derived following [6].

Results and Discussion: Data for neon are summarized in Table 1. Concentration of ${ }^{21} \mathrm{Ne}$ in chondrules is higher compared to the matrix because of the higher magnesium contents. Allende chondrules yielded cosmic-ray exposure (CRE) ages of $4.9 \mathrm{Ma}$, which agree with previous analyses $[3,4]$. Since this is similar to the CRE age of Allende matrix [1, $3,4]$ there is no evidence for pre-irradiation. The same is true for El Djouf 001, where the 7.2 Ma CRE age of our chondrule is in the range of ages previously reported for El Djouf $001[7,8]$.

In future work we will concentrate on CR3 chondrites of highly primitive nature and only incipient alteration [9].

Acknowledgment: We thank the DFG (OT171/6-1) for financial support.

References: [1] Das J. P. and Murty S. V. S. 2009. Meteoritics \& Planetary Science 44:1797-1818. [2] Polnau E. et al. 2001. Geochimica et Cosmochimica Acta 65:1849-1866. [3] Eugster O. et al. 2007. Meteoritics \& Planetary Science 42:1351-1371. [4] Roth A. S. G. et al. Meteoritics \& Planetary Science. In press. [5] Grossman L. 2010. Meteoritics \& Planetary Science 45:7-20. [6] Eugster O. 1988. Geochimica et Cosmochimica Acta 52:1649-1662. [7] Bischoff A. et al. 1993. Geochimica et Cosmochimica Acta 57:1587-1603. [8] Weber H. W. and Schultz L. 1991. Meteoritics \& Planetary Science 26:406. [9] Abreu N. M. and Brearley A. J. 2008. Abstract \#2013. 31st Lunar and Planetary Science Conference.

Table 1. Production rate and concentration of cosmogenic neon, T21 CRE ages, shielding parameter $\left({ }^{22} \mathrm{Ne} /{ }^{21} \mathrm{Ne}\right)_{\mathrm{c}}, \mathrm{Mg}$ and $\mathrm{Fe}$ contents of chondrules from Allende (A) and El Djouf 001 (E). Uncertainties in the last digits are given in parentheses. $\mathrm{P} 21$ and ${ }^{21} \mathrm{Ne}_{\mathrm{c}}$ are given in $10^{-8} \mathrm{cc}\left(\mathrm{g}^{-1} \mathrm{Ma}\right)$ and $10^{-8} \mathrm{cc} \mathrm{g}^{-1}$, respectively.

\begin{tabular}{llllllll}
\hline & $\begin{array}{l}\text { Weight } \\
\text { mg }\end{array}$ & $\begin{array}{l}\mathrm{Mg} \\
\text { wt\% }\end{array}$ & $\begin{array}{l}\mathrm{Fe} \\
\text { wt\% }\end{array}$ & P21 & ${ }^{21} \mathrm{Ne}_{\mathrm{c}}$ & $\begin{array}{l}{ }^{21} \mathrm{Ne} / \\
\mathrm{Ne}\end{array}$ & $\begin{array}{l}\mathrm{T} 21 \\
\mathrm{Ma}\end{array}$ \\
\hline A-Ch3 & 5.26 & 19.3 & 15.9 & 0.530 & $2.62(10)$ & $1.07(1)$ & 4.9 \\
A-Ch6 & 1.17 & 20.6 & 16.0 & 0.499 & $2.45(11)$ & $1.07(2)$ & 4.9 \\
E-Ch4 & 7.83 & 19.6 & 12.8 & 0.335 & $2.12(9)$ & $1.20(2)$ & 7.2 \\
\hline
\end{tabular}




\section{8}

\section{IMPACT OF METEOR SCIENCE OUTREACH IN NEPAL}

S. Bhattarai. St. Xavier's College, Kathmandu, Nepal. E-mail: chikursharma@gmail.com.

The Chinese record of meteor shower dates back thousands of years from now, but the modern meteor science is still in its infancy in Asia. The South Asian countries, especially Nepal, lack the documentation of the shower details and meteorite strikes despite the fact that there might have been a number of potential meteorite strikes in these nations. Meteor outreach that has been carried out in Nepal since 2007 will be explained and its impact upon the societies will be analyzed. Major meteor shower analysis done so far from this part in optical domain will also be discussed in short. This paper also tries to present some of the evidence for meteorite falls in Nepal so far. Possible collaborations for the tracking of meteorites/fireballs will be discussed in detail.
5393

SPECTRAL SIGNATURES OF MARTIAN METEORITES AND WHAT THEY CAN TELL US ABOUT ROCKS ON MARS

J. L. Bishop ${ }^{1}$, M. Parente ${ }^{2}$ and V. E. Hamilton ${ }^{3}$. ${ }^{1}$ SETI Institute, Mountain View, CA, USA. E-mail: jbishop@seti.org. ${ }^{2}$ Brown University, Providence, RI. ${ }^{3}$ Southwest Research Institute, Boulder, CO, USA.

Introduction: Martian meteorites provide important compositional information about crustal rocks and enable ground truthing of orbital data. Coordinated VNIR and mid-IR spectral analyses of the meteorites and mineralogy of Mars are important in order to fully understand the composition of the surface. Hamilton et al. [1] used TES data to identify several olivine and orthopyroxene-bearing regions near Nili Fossae, Ganges, Argyre and Hellas that are consistent with ALHA77005, Chassigny and ALH 84001 meteorites, as well as clinopyroxene-rich locations in parts of the Valles Marineris region and Syrtis Major resembling Nakhla-type meteorites. More recently, VNIR spectra collected by OMEGA and CRISM have been used to document mafic outcrops on Mars [2,3]. Here we present a summary of the VNIR and mid-IR spectral properties of several Martian meteorites correlated with their compositions. These data are compared to regions on Mars where mafic outcrops with similar spectral characteristics are observed.

Spectral Properties of Martian Meteorites: We have acquired VNIR reflectance and thermal emissivity spectra of several Martian meteorites including: ALH 84001, EETA79001, Los Angeles, Dar al Gani 670, and NWA 1068. These samples contain pyroxenes ranging from orthopyroxene to pigeonite to augite, feldspar (and maskelynite), fayalitic and fosteritic olivine, silica, and glass. We are comparing the mineral modeling results of the two spectral regions with each other and with the meteorite petrology from other studies. Gaussian modeling is used for the electronic absorptions in the VNIR region spectra based on code developed by Sunshine et al. [4, 5] and adapted by Parente et al. [6]. Gaussian modeling of Martian meteorite spectra has revealed the presence of pyroxene, olivine and glass. Modeling of the thermal emission spectra is based on vibrational absorptions [7] and modeling of Martian meteorite spectra has shown the presence of feldspar, pyroxene, olivine and glass $[1,8]$.

Coordinating Spectral Properties of Meteorites with the Surface of Mars: Mafic outcrops are observed across the surface of Mars in nondusty areas where the caprock has been eroded. Spectral analyses of Martian meteorites are assisting in interpreting these mafic outcrops. Many of these surface mafic outcrops are small; thus, the better spatial resolution of THEMIS and CRISM has enabled improved resolution of these mafic regions. CRISM can detect features due to olivine and pyroxene as they have $\mathrm{Fe}$ excitation bands. TES (and sometimes THEMIS) can detect these minerals as well as feldspar.

References: [1] Hamilton V. E. et al. 2003. Meteoritics \& Planetary Science 38:871-885. [2] Mustard J. F. et al. 2005. Science 307:1594-1597. [3] Mustard J. F. et al. 2009. Journal of Geophysical Research 114, doi:10.1029/2009JE003349. [4] Sunshine J. M. et al. 1990. Journal of Geophysical Research 95:6955-6966. [5] Sunshine J. M. et al. 1993. Icarus 105:79-91. [6] Parente M. et al. 2011. Planetary and Space Science 59:423442. [7] Ramsey M. S. and Christensen P. R. 1998. Journal of Geophysical Research 103:577-596. [8] Hamilton V. E. et al. 1997. Journal of Geophysical Research 102:25593-25603. 


\section{7}

WHEN A CM GROUP METEORITE DECOMPOSES AT THE SEA FLOOR, WHAT WILL REMAIN?

K. Bjärnborg and B. Schmitz. Dept. of Geology, Lund University, Lund, Sweden. E-mail: karolina.bjarnborg@geol.lu.se.

Introduction: Recently our group has successfully recovered relict chromite grains (ca. 63-150 $\mu \mathrm{m}$ ) from unmelted meteorites and micrometeorites that have fallen and decomposed on ancient sea floors, up to $470 \mathrm{Myr}$ old, e.g. [1]. We dissolve $30-100 \mathrm{~kg}$ of slowly formed (ca. $2 \mathrm{~mm} \mathrm{kyr}^{-1}$ ) marine limestone in $\mathrm{HCl}$ and $\mathrm{HF}$ acid. This can yield an almost clean assemblage of resistant chromite from ordinary chondrites. Noble gas analyses of the chromite grains indicate that they mainly originate from micrometeorites [2]. Because carbonaceous chondritic material, primarily CM-group material, makes up a significant fraction of the micrometeorite flux today, we are testing if there are resistant minerals in this type of meteorite that can be recovered from ancient sediments. For different chondrite groups different oxide minerals have been reported. Chromite dominates the oxide fraction in ordinary chondrites, in enstatite chondrites oxides are rare, chromian spinel dominates in $\mathrm{R}$ chondrites, whereas in carbonaceous chondrites a wide range of oxide minerals occur, e.g. magnetite, chromite and different spinel group varieties [3, 4]. In CM2 meteorite Murchison spinel is primarily found in CAIs and as inclusions in olivine, chromite grains are found in matrix and in CAIs [5]. Here we further evaluate what is the case for CM meteorites, with focus on large spinel grains.

Methods: Two four-gram samples of CM2 chondrite Acfer 331 were treated in strong HF acid for $24 \mathrm{~h}$. All spinel grains $>63 \mu \mathrm{m}$ were recovered from the residue under an optical microscope. The grains were mounted in epoxy, polished flat and analyzed with SEM-EDS, see [1].

Results: The samples contained 17 and 20 spinel grains $>63 \mu \mathrm{m}$, respectively. Most grains are $63-150 \mu \mathrm{m}$, a few grains reach ca. $250 \mu \mathrm{m}$. Pink and colorless grains dominate, but red and blue grains also exist. The grains are all rich in $\mathrm{Al}$ and $\mathrm{Mg}$ whereas $\mathrm{Cr}$ and $\mathrm{Fe}$ contents are low: $\mathrm{Mg} 13.2-14.6$ at $\%, \mathrm{Al} 24.8-28.3 \mathrm{at} \%, \mathrm{Cr}$ up to 3.4 at $\%$ and $\mathrm{Fe}$ up to 1.2 at $\%$. Ti and $\mathrm{V}$ contents are mostly low, but Ti occurs up to 0.1 at $\%$ and $\mathrm{V}$ up to $0.2 \mathrm{at} \%$. The grains richer in $\mathrm{Cr}$ are mostly pink or red, whereas colorless grains generally are poorer in $\mathrm{Cr}$. The grains are often heterogeneous in composition; in backscatter mode they often show patchy or gradational variations, a few grains show zoning. The withingrain variations reach maximum 1.5 at $\%$ for $\mathrm{Cr}$ and $0.3 \mathrm{at} \%$ for $\mathrm{Fe}$.

Conclusions: A CM meteorite of one gram being dissolved on the sea floor may thus leave behind ca. four to five relict spinel grains $>63 \mu \mathrm{m}$. These grains can be recovered from sediment samples with the same methods we use for recovering ordinary chondritic chromite. As a comparison, chromite makes up $0.25 \mathrm{wt} \%$ of ordinary chondrites [6], but this includes chromite grains in all size fractions. Apparently our approach of recovering ancient spinels can be extended to reveal the flux variations through Earth history of several of the meteorite types that fall on Earth today.

References: [1] Cronholm A. and Schmitz B. 2010. Icarus 208:36-48. [2] Heck P. R. et al. 2008. Meteoritics \& Planetary Science 43:517-528. [3] Rubin A. E. 1997. Meteoritics \& Planetary Science 32:231-247. [4] Simon S. B. et al. 1994. Geochimica et Cosmochimica Acta 58:1313-1334. [5] Fuchs L. H. et al. 1973. Smithsonian Contributions to Earth Sciences 10:1-39. [6] Keil K. 1962. Journal of Geophysical Research 67:4055-4061.

\section{2}

SECONDARY PROCESSES WITHIN THE PARIS CM CHONDRITE

I. Blanchard ${ }^{1,2}, \quad$ M. Gounelle ${ }^{1}$ M. Bourot-Denise ${ }^{1}$ and A. Kearsley ${ }^{3} .{ }^{1}$ LMCM, UMR 7202, CNRS \& MNHN, CP 52, 57 rue Cuvier, 75005 Paris, France. E-mail: gounelle@mnhn.fr. ${ }^{2}$ IPGP, PRES SPC, Université Paris-Diderot, 4 Place Jussieu, 75005 Paris. ${ }^{3}$ IARC, Department of Mineralogy, Natural History Museum, London SW7 5BD, UK.

Introduction: The Paris meteorite is a pristine rock whose petrography, oxygen isotopic and bulk chemical compositions are compatible with a classification as a $\mathrm{CM}$ chondrite [1,2]. Based on petrography and mineral compositions it was initially classified as a 3.0 chondrite $[2,3]$. This classification is however ambiguous as it has been known for a while that hydrothermalism and thermal metamorphism might be unrelated within CM chondrites [4]. The goal of the present work is to place Paris on two distinct alteration scales (hydrothermalism and thermal metamorphism) proposed for $\mathrm{CM}$ chondrites by [5-7].

Methods: We investigated the Paris polished blocks 2010-05 and 2010-07 prepared at MNHN. X-ray mapping was done at NHM London [8]. Standard SEM and EMPA techniques were performed in Paris itself.

Results: As previously noted, Paris is a breccia whose alteration properties vary at the $\mathrm{cm}$-scale $[1,2]$. Matrix is made of a mixture of abundant phyllosilicates, tochilinite and other minerals that cannot be resolved individually by the techniques we have used so far. Chondrule mesostasis is usually turned into phyllosilicates. Paris has a Fe-Ni metal abundance of about $1 \%$. Metal is predominantly kamacite with $\mathrm{Ni}$ $<10 \mathrm{wt} \%$ and $\mathrm{Co}<0.5 \mathrm{wt} \%$. Metal grains often have an oxidized rim. Iron sulfides are pyrrhotite and pentlandite. The latter appears as rims around or lamellae within the former. PCPs are abundant as clumps, and have elevated $\mathrm{S} / \mathrm{SiO}_{2}(0.7)$ and $\mathrm{FeO} / \mathrm{SiO}_{2}$ (4.1) ratios. Olivine mean $\mathrm{Cr}_{2} \mathrm{O}_{3}$ content is $0.37 \mathrm{wt} \% \quad(\sigma=0.08)$. Lacking matrix analyses with $\mathrm{S}$ $<2 \mathrm{wt} \%$, we were not able to calculate Paris Mineralogical Alteration Index [9].

Discussion: All these properties place Paris among one of the least altered CM chondrite on the hydrothermal scale designed by [7]. On that scale, it is at least a 2.6 rock, possibly a 2.7 or 2.8 given the chemical composition of PCPs. Paris cannot be considered as a (hydrothermal) type 3.0 given the large abundance of secondary minerals such as phyllosilicates or tochilinite. Metal and sulfide compositions, as well as their petrographic relationships, place Paris in between the groups A and B defined by [6] on the basis of the thermal metamorphism experienced. It shows features indicative of both an absence of heating and a moderate amount of heating. Thermal metamorphism temperatures of group B are below $700^{\circ} \mathrm{C}$ [5]. We strongly recommend that two different scales are used for hydrothermal alteration and thermal metamorphism experienced by $\mathrm{CM}$ parent-bodies, be they cometary or asteroidal [10]. We propose that Paris is a CM 2.7/2.8 A/B chondrite, the number referring to hydrothermal alteration and the letter to thermal metamorphism.

References: [1] Zanda B. et al. 2011. Abstract \#2040. 42nd Lunar and Planetary Science Conference. [2] Bourot-Denise M. et al. 2010. Abstract \#1683. 41st Lunar and Planetary Science Conference. [3] Zanda B. et al. 2010. Abstract \#5312. Meteoritics \& Planetary Science 45 (Suppl). [4] Akai J. 1990. Proceedings of the NIPR Symposium on Antarctic Meteorites. 3rd 55-68. [5] Nakamura T. 2005. Journal of Mineralogical and Petrological Sciences 100 260-272. [6] Kimura M. et al. 2011. Meteoritics \& Planetary Science 46:431-442. [7] Rubin A. E. et al. 2007. Geochimica et Cosmochimica Acta 71:2367-2382. [8] Kearsley A. T. et al. 2011. Meteoritics \& Planetary Science, This conference. [9] Browning L. B. et al. 1996. Geochimica et Cosmochimica Acta 60:2621-2633. [10] Gounelle M. 2011. Elements 7:29-34. 
5275

\section{HETEROGENEOUS SHOCK IN POROUS CHONDRITES: IMPLICATIONS FOR ALLENDE MAGNETIZATION}

P. A. Bland ${ }^{1}$, A. R. Muxworthy ${ }^{1}$, G. S. Collins ${ }^{1}$, J. Moore ${ }^{1}$, T. M. Davison $^{2}$ and F. J. Ciesla ${ }^{2}$. ${ }^{1}$ IARC, Dept. Earth Science \& Engineering, Imperial College London, SW7 2AZ, UK. E-mail: p.a.bland@ imperial.ac.uk. ${ }^{2}$ Dept. Geophysical Science, University of Chicago, 5734 South Ellis Av., Chicago, IL 60430, USA.

Introduction: Paleomagnetic data from Allende has been interpreted as suggesting an internally generated field within the parent asteroid, and a liquid metallic core [1-3] i.e., the $\mathrm{CV}$ parent body is a differentiated object [3,4]. Impact-generated fields were considered as a possible mechanism but excluded [3] based on the absence of macroscopic shock textures in Allende (S1) [5].

Results and Discussion: Our paleomagnetic analysis of Allende is consistent with previous studies [3], but we also find evidence to suggest that the strong intrinsic magnetic anisotropy may be coincident with a pervasive uniaxial crystallographic fabric delineated by oriented matrix grains that was plausibly impact-induced [6]. Based on EBSD analysis we can quantify pre-compaction porosity, and by modeling impact-induced porosity reduction [7] we can define the continuum shock pressure and temperature that Allende must have experienced. Assuming a (conservative) initial temperature of $300 \mathrm{~K}$, the fabric/porosity data would be consistent with P (shock) of $1.7 \mathrm{GPa}$ and $\mathrm{T}$ (final) of $553 \mathrm{~K}$ (below the Curie $\mathrm{T}$ for pyrrhotite, the principle magnetic carrier). But note that these are continuum P\&T estimates - valid at large scales. At sub- $\mu \mathrm{m}$ scales in porous materials - relevant to matrix-shock is highly heterogeneous, with temperature excursions of $2-3 \times$ above background commonly observed [8-10], i.e., pyrrhotites should have experienced temperature excursions of 180-430 K above their Curie T. Our own 2D meso-scale numerical simulations of shock waves propagating through chondrule/ matrix mixtures support this: matrix experiences much higher peak shock P\&T than chondrules, and shows significant heterogeneity in shock effects. There is evidence for this in Allende: TEM studies of matrix indicate high shock $P$ [11] and $T$ [12]. High continuum $T$ [13] is not effective in recording an impact-induced field: cooling is too slow. High transient $\mathrm{T}$ is, as matrix pyrrhotites would equilibrate rapidly with the lower continuum $\mathrm{T}$.

Our P (shock) estimates allow us to constrain the position of Allende within the parent body with respect to various impact scenarios: 1.7 GPa pressures are encountered in the breccia lens. The magnitude of an impact generated field can be estimated based on scaling relations derived from experimental data [14]. Allende would have been proximal to a significant impact generated field. In summary, heterogeneous shock effects in sub- $\mu \mathrm{m}$ porous matrix may be an explanation for some aspects of chondrite magnetization. This process has implications for lithification of pristine chondrites [15] and for the survival of pre-solar grains.

References: [1] Butler R. F. 1972. Earth and Planetary Science Letters 17:120-128. [2] Funaki M. and Wasilewski P. 1999. Meteoritics \& Planetary Science 34:A39. [3] Weiss B. P. et al. 2010. Space Science Reviews 152:341-390. [4] Elkins-Tanton L. T. et al. 2011. Earth and Planetary Science Letters 305:1-10. [5] Scott E. R. D. et al. 1992. Geochimica et Cosmochimica Acta 56:4281-4293. [6] Bland P. A. et al. 2011. Nature Geoscience 4:244-247. [7] Davison T. M. et al. 2010. Icarus 208:468-481. [8] Kieffer S. W. 1971. JGR 76:5449-5473. [9] Baer M. R. and Trott W. M. 2002. AIP Conference Proceeding 620:517-520. [10] Sharp T. G. and DeCarli P. S. 2006. MESS II:pp. 653-677. [11] Müller W. F. 1978. 9th Lunar and Planetary Science Conference pp. 609-610. [12] Brenker F. E. and Krot A. N. 2004. American Mineralogist 89:12801289. [13] Folco L. et al. 2004. Geochimica et Cosmochimica Acta 68:23792397. [14] Crawford D. A. and Schultz P. H. 1999. International Journal of Impact Engineering 23:169-180. [15] Bland P. A. et al. 2007. Meteoritics \& Planetary Science 42:1417-1427.
5220

IN SITU SIMS OXYGEN ISOTOPE MEASUREMENTS OF ZONED OLIVINES IN THE TAGISH LAKE METEORITE CHONDRULES A. Blinova ${ }^{1}$, R. Stern ${ }^{1,2}$ and C. D. K. Herd ${ }^{1}{ }^{1}$ Department of Earth and Atmospheric Sciences, University of Alberta, Edmonton, Canada. ${ }^{2}$ Canadian Centre for Isotopic Microanalysis (CCIM), University of Alberta, Edmonton, Canada. E-mail: blinova@ualberta.ca.

Introduction: The Tagish Lake (TL) meteorite is a brecciated carbonaceous Type 2 chondrite consisting of several lithological variations [e.g., 1, 2] with different chondrule abundances [3]. CM-like specimen TL5 b contains the most abundant number of chondrule-like objects up to $500 \mu \mathrm{m}$ diameter set in a phyllosilicate-rich matrix. A large $(2000 \mu \mathrm{m}$ diameter), porphyritic type I (Fe-poor) chondrule dominated by pyroxene and olivine $\left(\mathrm{Fo}_{95-96}\right)$ set in a slightly altered, Al-rich mesostasis was found in $5 \mathrm{~b}$ during processing [1]. The more altered specimen TL11i contains less chondrule-like objects and is dominated by altered matrix. This specimen contains a porphyritic chondule with zoned olivines [1]. The largest olivine has a euhedral shape and exhibits oscillatory zoning $\left(\mathrm{Fo}_{70-79}\right)$. Other olivine grains within this chondrule exhibit normal zoning from $\mathrm{Mg}$-rich cores $\left(\mathrm{Fo}_{79-99}\right)$ to $\mathrm{Fe}$-rich rims $\left(\mathrm{Fo}_{61-74}\right)$.

Methods: Oxygen analyses were obtained using a Cameca IMS1280 ion microprobe at the CCIM, utilizing a approximately $12 \mu \mathrm{m}$ diameter ion $\mathrm{Cs}$ ion probe, and olivine reference materials for IMF correction $\left(\mathrm{Fo}_{75}\right.$ and $\left.\mathrm{FO}_{93}\right)$. A Zeiss EVO 15 scanning electron microscope was utilized for microstructural characterization.

Discussion of Results: Here we present first three-oxygen isotope results on olivines obtained from the CCIM facility.

Chondrule in TL5b: We have analyzed a total of 14 distinct olivine grains within this chondrule, with one to four analyses on each olivine. All data form a cluster just to the left of CCAM line on a three-O isotopic plot, with the average $\Delta^{17} \mathrm{O}=-2.6( \pm 0.5)$ and mean in $\delta^{17} \mathrm{O}$ and $\delta^{18} \mathrm{O}$ of -1.7 and $1.7 \%$, respectively.

Chondrule in TL11i: We analyzed seven zoned olivine grains (total 14 analyses). The average of eleven analyses on zoned grains $\left(\mathrm{Fo}_{60-79}\right)$ is $\Delta \Delta^{17} \mathrm{O}=-1.73( \pm 0.69)$. In contrast to the TL5b data, these analyses are more ${ }^{17,18} \mathrm{O}$-enriched and make a scattered cluster (mean $\delta^{17} \mathrm{O}=1.0 \pm 0.6 \%$ and $\delta^{18} \mathrm{O}=5.3 \pm 0.2 \%$ ) that fall on the CCAM line. Three other grains have ${ }^{16} \mathrm{O}$-enriched relict cores. One of these olivine grains contains a relict core with diffuse boundaries and shows the most extreme Fo and $\Delta^{17} \mathrm{O}$ differences in the core and rim amongst these olivine grains: core $\left(\mathrm{Fo}_{98}\right)$ has $\Delta^{17} \mathrm{O}=-8.6( \pm 0.68)$, and the rim $\left(\mathrm{Fo}_{71}\right)$ has $\Delta^{17} \mathrm{O}=-1.9( \pm 0.62)$. This core is more ${ }^{16} \mathrm{O}$-enriched than data for TL from $[4,5]$, but not as extreme as the data on "enriched" grains by [6]. It appears that the relict cores from these three olivine grains condensed in the ${ }^{16} \mathrm{O}$-enriched nebular environment, whereas the rims formed later in a ${ }^{16} \mathrm{O}$-depleted environment, perhaps concurrently with other zoned olivine grains during melting and formation of this chondrule.

References: [1] Blinova A. et al. 2009. Abstract \#2039. 40th Lunar Prospector Spacecraft. [2] Izawa M. R. M. et al. 2010. Planetary and Space Science 58:1347-1364. [3] Blinova et al. 2010. Meteoritics \& Planetary Science 45:A17. [4] Leshin et al. 2001. Abstract \#1843. 32nd Lunar Prospector Spacecraft. [5] Russell et al. 2010 Geochimica et Cosmochimica Acta, 74:2484-2499. [6] Engrand et al. 2001. Abstract \#1568. 32nd Lunar Prospector Spacecraft. 
5204

\section{CHROMITE AND ILMENITE FRACTIONATION TRENDS IN HOWARDITES}

J. S. Boesenberg. Earth and Planetary Sciences, American Museum of Natural History, Central Park West, New York, NY 10024, USA.

E-mail: bosenbrg@amnh.org.

Introduction: At the 42nd Lunar and Planetary Science Conference, [1] presented pyroxene $\mathrm{Ti}-\mathrm{Cr}$ and $\mathrm{Fe}-\mathrm{Mg}$ data from 11 howardites that suggests many howardites do not consist of unrelated rock fragments that were subsequently lithified, but rather contain diogenitic and eucritic fragments that form continuous and extensive fractional crystallization trends. Up to six types of patterns in pyroxene have been found so far. Here, we report on the chromite and ilmenite phases within most of those howardites to determine whether they are in agreement with this fractionation relationship. These minor phases are important because the timing of chromite and ilmenite crystallization prior to, during or after pyroxene formation is the primary cause of any change in the $\mathrm{Ti}-\mathrm{Cr}$ versus Fe-Mg pattern observed in the pyroxene fractionation trends.

Results and Discussion: Oxides from eight of the 11 howardites used in [1] were analyzed by electron microprobe. These include grains from lithic clasts and mineral fragments from Winterhaven, Bialystok, Le Teilleul, Petersburg, Bholghati, Chaves, Luotolax and Zmenj. The Fe-MgTi-Cr-Al systematics within the chromites vary moderately between samples in modal abundance and compositional range when plotted in a $\mathrm{Fe}_{2} \mathrm{TiO}_{4}-\mathrm{FeCr}_{2} \mathrm{O}_{4}-\mathrm{FeAl}_{2} \mathrm{O}_{4}$ ternary. Chromite $\mathrm{Ti}$ concentrations are the most variable and increase with $\mathrm{Fe} /(\mathrm{Fe}+\mathrm{Mg})$. Typically $\mathrm{Al}$ decreases with FFM and $\mathrm{Cr}$ is either somewhat steady or decreases. Bholghati, Luotolax, Zmenj and Le Teilleul all contain chromites with wide compositional ranges that include very Mg-rich (FFM approximately 64 72) grains. These Mg-rich grains are consistent with derivation from the diogenitic component of the howardites based on the high proportion of diogenitic pyroxene previously measured in those samples [1-3]. Very Ferich chromites $(\mathrm{FFM}>0.9)$ found in many samples contain large variations in their concentrations of $\mathrm{Al}, \mathrm{Cr}$ as well as $\mathrm{Ti}$, possibly reflecting metamorphic alteration towards ulvospinel [4].

Compared to the compositional variations found in the chromites, the ilmenites are remarkably uniform. The only significant variation between the different howardite ilmenites is their range of $\mathrm{Fe} /(\mathrm{Fe}+\mathrm{Mg})$ ratios, with Winterhaven having the most Mg-rich (FFM approximately 86-95) and Bialystok the most Fe-rich (FFM approximately 95-97). All eight howardites have very low minor/trace concentrations in elements such as $\mathrm{Al}, \mathrm{Cr}$ and $\mathrm{Ca}$, with only Bialystok having mildly variable $\mathrm{Al}$ and Cr.

Conclusions: The distribution of chromite compositions is consistent with a fractional crystallization model to produce the full array of lithologies measured in the pyroxene. The ilmenites, though containing little trace element evidence measurable by electron microprobe, are also consistent with fractionation based on their range of FFM ratios.

The one unusual sample in this study is $\mathrm{Zmenj}$, which shows a more convincing continuous compositional fractionation trend in chromite than pyroxene, possibly indicating the section analyzed is unrepresentative of this howardite.

References: [1] Boesenberg J. S. and Erb I. R. 2011. Abstract \#1017. 42nd Lunar and Planetary Science Conference. [2] Mittlefehldt D. W. et al. 1998. Planetary Materials, Reviews in Mineralogy edited by J. J. Papike, pp. 4-1-4-195. [4] Mittlefehldt D. W. 1994. Geochimica et Cosmochimica Acta 58:1537-1552. [3] Arai T. et al. 1998. Antarctic Meteorite Research 11:71-91.
5496

POSSIBLE DEBRIS OF HIGHLY EVOLVED LITHOLOGIES IN HOWARDITES: ADDITIONNAL EVIDENCE OF K-RICH AREAS ON 4 VESTA?

M. Bohn ${ }^{1}$, A. Yamaguchi ${ }^{2}$, A. Jambon ${ }^{3}$, J. A. Barrat ${ }^{1}$, C. Bollinger ${ }^{1}$ and O. Boudouma ${ }^{3}$. ${ }^{1}$ Université Européenne de Bretagne, UBO-IUEM, CNRS UMR 6538, Place Nicolas Copernic, 29280 Plouzané Cedex, France. E-mail: barrat@univ-brest.fr. ${ }^{2}$ NIPR and Graduate University for Advanced Sciences, Tachikawa, Tokyo 190-8518, Japan. ${ }^{3}$ Université Pierre et Marie Curie- Paris 6, 4 place Jussieu, 75252 Paris cedex 05, France.

4 Vesta will be soon studied by the Dawn space probe, and is generally believed to be the parent body of the HED meteorites [1] During the last forty years, these rocks have been used to constrain the composition and the history of this large asteroid. It is generally assumed that its surface exposes, in addition to a variety of breccias (e.g., howardites), basaltic rocks (eucrites) and ultramafic cumulates. The rocks exposed on 4 Vesta are certainly not restricted to the known HED lithologies. Recently, the studies undertaken on impact glasses found in howardites have shown that K-rich areas, with possibly felsites or granites, are probably exposed on 4 Vesta $[2,3]$. We have found unusual clasts in the howardites where K-rich glasses were found (NWA 1664 and NWA 1769). These low-Mg clasts display a fine grained-subophitic texture. They are generally made of assemblages of silica-fayalitic olivinehedenbergite (pyroxferroite breakdown materials (PBM) ?), partly destabilized low-Mg pyroxenes, ilmenites, silica, troilites, merrillites, Clapatites, barian K-feldspars (hyalophane), with accessory baddeleyites and zircons. In two cases, high $\mathrm{Ca}$ pyroxenes displaying fine exsolution lamellae of low-Ca pyroxenes have been observed. REE abundances of PBMs are very high. They range from $10 \times \mathrm{CI}$ to about $100 \times \mathrm{CI}$ for HREEs. Merrillites too $(>10,000 \times$ CI for LREEs, $>4000 \times$ CI for the HREEs) and apatites, which are frequent, are unsurprisingly rich in REEs too. Thus, these clasts are undeniably REE-rich, P-rich, and K-rich. One may suggest these "KREEPy" clasts are debris of areas of mesostasis from eucritic lithologies. Such an explanation is not satisfactory because: (1) these clasts and mesostases from equilibrated or unequilibrated eucrites are texturally distinct; (2) ilmenites in the clasts display lower $\mathrm{Mg}$ abundances than those found in eucritic mesostasis; (3) hyalophane has never been described in eucrites; K-feldspars are exceptional in eucrites, and are $\mathrm{Ba}$ poor. Thus, these clasts are more likely remnants of notpreviously-described highly-evolved lithologies.

References: [1] McCord T. B. et al. 1970. Science 168:1445-1447. [2] Barrat J. A. et al. 2009. Meteoritics \& Planetary Science 44:359-374. [3] Barrat J. A. et al. 2009. Geochimica et Cosmochimica Acta 73:5944-5958. 


\section{1}

\section{HOW HETEROGENEOUS IS ORGUEIL REALLY?}

C. Bollinger ${ }^{1}$, B. Zanda ${ }^{2}$, F. Moynier ${ }^{3}$, J. A. Barrat ${ }^{1}$ and C. Liorzou ${ }^{1}{ }^{1}$ Université Européenne de Bretagne, UBO-IUEM, CNRS UMR 6538, Place Nicolas Copernic, 29280 Plouzané Cedex, France. E-mail: barrat@univ-brest.fr. ${ }^{2}$ MNHN \& CNRS UMR7202, 61, rue Buffon, 75005 Paris, France. ${ }^{3}$ Dept. of Earth and Planetary Sciences and McDonnell Center for the Space Sciences, Washington University in St. Louis, USA.

We have obtained six chips of Orgueil prepared from five different stones, and weighing between $600 \mathrm{mg}$ to more than $1 \mathrm{~g}$. This sampling has been complemented by a sample of Ivuna and Alais. The samples have been analyzed by ICP-AES (Ti, Al, Fe, Mn, Mg, Ca, Na, P, Cr, Co, $\mathrm{Ni}$ ) and ICP-MS (40 minor and trace elements) using procedures adapted from [1]. They allows the determination of abundances and ratios of trace elements in chondrites with a good reproducibility $(<3 \%$ for abundances, $<1.5 \%$ for ratios such as $\mathrm{La} / \mathrm{Sm}, \mathrm{Eu} / \mathrm{Eu}^{*}, \mathrm{Zr} / \mathrm{Hf}$ or $\mathrm{Nb} / \mathrm{Ta}$ ). At first glance, our samples of Orgueil display only small range of abundances. Among the 46 elements determined, only 4 show RSDs $>10 \%(\mathrm{Na}, \mathrm{K}$, $\mathrm{Rb}, \mathrm{U}$ ), and 10 show RSDs between 5 and $10 \%(\mathrm{Mn}, \mathrm{Ca}, \mathrm{P}, \mathrm{Sr}, \mathrm{Nb}, \mathrm{Cs}$, $\mathrm{La}, \mathrm{Ta}, \mathrm{W}$, and $\mathrm{Pb}$ ). In the case of $\mathrm{W}$, the RSD is close to the expected analytical reproducibility. In the other cases, the RSDs reflect real small heterogeneities of the meteorite: for example, the results obtained for $\mathrm{Mn}$ by ICP-AES are confirmed by ICP-MS $(0.17-0.21 \mathrm{wt} \%$ ); Na (ICP-AES, $0.29-0.59 \mathrm{wt} \%$ ) is strongly correlated with K (ICP-MS, 365-677 ppm). The range of concentrations is reduced when one of the samples is removed. The later seems abnormal. It displays the lowest alkali abundances and is slightly more light REE enriched than the other chips. The average composition calculated using the five other chips is very close to the previously compiled values for CI chondrites or high quality ID analyses (e.g., [2-5]), and can be used as a CI normalization reference. Possible interlaboratory biases can be eliminated if the analyses are adjusted to the same international standard values. Unsurprisingly, Ivuna displays major and trace element abundances alike the Orgueil average. To the opposite, our Alias chips is unusual and exhibits a convex REE pattern when normalized to our Orgueil average.

The $\mathrm{Cu}$ and $\mathrm{Zn}$ isotopic compositions have been determined on the same powders at Saint Louis. ${ }^{\mathrm{T}}{ }^{66} \mathrm{Zn}$ and ${ }^{\mathrm{T}}{ }^{65} \mathrm{Cu}$ values range respectively from 0.42 to 0.54 per $\mathrm{ml}$, and from 0.02 to 0.24 per $\mathrm{ml}$, in agreement with previous studies $[6,7]$.

References: [1] Barrat J. A. et al. 2008. Meteoritics \& Planetary Science 43:1759-1775. [2] Anders E. and Grevesse N. 1989. Geochimica et Cosmochimica Acta 53:197-214. [3] Lodders K. et al. 2009. In LandoltBörnstein, new series, vol. VI/4B. [4] Beer H. et al. 1984. Physical Review 30:464-478. [5] Münker et al. 2003. Science 301:67-84. [6] Luck J. M. et al. 2003. Geochimica et Cosmochimica Acta 67:143-151. [7] Luck J. M. et al. 2005. Geochimica et Cosmochimica Acta 69:5351-5363.
5125

STRUCTURE OF PRIMITIVE POLYAROMATIC CARBONACEOUS MATTER: IDPS VERSUS CHONDRITES.

L. Bonal ${ }^{1 *}$, E. Quirico ${ }^{1}$ and G. Montagnac ${ }^{2}$. ${ }^{1}$ Institute of Planetology and Astrophysics of Grenoble (IPAG), UJF-Grenoble 1 / CNRS-INSU, France. ${ }^{2}$ Laboratoire de Sciences de la Terre-ENS Lyon, France. *E-mail: lydie.bonal@obs.ujf-grenoble.fr.

Resolving the origin(s) and initial characteristics of the organic components, and determining whether primitive cosmomaterials accreted similar organic precursors are key points in cosmochemistry. As the structure of polyaromatic carbonaceous matter (PCM) is controlled by (i) the alteration episode of the hosting rock and (ii) the organic precursor [e.g., 1,2], it is a powerful tracer to distinguish pre-accretion heterogeneities from post-accretion modifications. Interplanetary Dust Particles (IDPs) collected in the Earth's stratosphere are fragments of primitive asteroids and comets that are potentially unsampled by meteorites. By characterizing the structure of the PCM in IDPs and comparing it to primitive chondrites, we want to better constrain and understand (i) the heterogeneities within and among IDPs; (ii) the asteroid-comet continuum in terms of organic precursor.

Seventeen clustered IDPs were received in small drops of silicon oil on glass plates. Preliminary Raman spectra $\left(\lambda_{\text {laser }}=514 \mathrm{~nm}\right)$ were directly acquired with no further sample preparation (i.e., no hexane rinsing). Extreme care was taken to avoid any laser damage. Further Raman characterizations correlated to chemical and isotopic maps are planned after the IDP extractions and specific sample preparations. The preliminary Raman examination already reveals clear differences among our IDPs. Three main types of Raman spectra were obtained: (i) Group I: presence of the typical D and G carbon bands superimposed onto a large fluorescence background (3/17); (ii) Group II:D and G carbon bands with no significant fluorescence background (9/17); (iii) Group III: absence of carbon bands but presence of mineral bands (olivine and hematite, 2/17). No identifying Raman features were obtained on 3 IDPs. Spectral parameters of the carbon bands were obtained through our usual analytical treatment [e.g., 2]. Fluorescence spectra of Group I IDPs were obtained.

IDPs from Groups I and II are all characterized by the presence of PCM. The raw spectra exhibit variable spectral valleys, relative intensities and widths of the $\mathrm{D}$ and $\mathrm{G}$ bands. This reflects a variable structural order of the PCM. The broad carbon bands and shallow spectral valleys are typical of poorly structurally ordered PCM. Raman spectra of the PCM in type 1 and 2 chondrites (CI, CM, CR) are typically characterized by a high fluorescence background, in contrast to type 3 chondrites. Thus the absence of significant fluorescence background in the Raman spectra of Group II IDPs clearly differentiates them from CI, CM, and CR. The Raman spectra of the IDPs do not match either with type 3 chondrites. Indeed, the $\mathrm{D}$ and $\mathrm{G}$ bands spectral parameters do not follow the trends as defined by the type 3 chondrites. These differences can be interpreted in terms of variable organic precursors and/or thermal events (nebular, parent body, atmospheric entry) between IDPs and chondrites.

This preliminary study reveals a real structural variability of the PCM in our IDPs collection, similarly to previous observation $[3,4]$. Further Raman characterization (mapping, multi-wavelength analysis) on the extracted dusts will allow us to refine the differences compared to chondrites.

References: [1] Wopenka and Pasteris 1993. American Mineralogist 78:553. [2] Bonal et al. 2006. Geochimica et Cosmochimica Acta 70:1849. [3] Wopenka 1988. Earth and Planetary Science Letters 88:221. [4] Quirico et al. 2005. Planetary and Space Science 53:1443. 
5251

THERMAL DEGRADATION OF N-RICH POLYMERIC SOLIDS: CONSTRAINTS ON THE FORMATION OF INSOLUBLE ORGANIC MATTER

J-Y. Bonnet ${ }^{1}$, E. Quirico ${ }^{1}$, A. Buch ${ }^{2}$, C. Szopa ${ }^{3}$, N. Fray ${ }^{4}$, H. Cottin ${ }^{4}$ and R. Thissen ${ }^{1}$. ${ }^{1}$ Institut de Planetologie et d'Astrophysique de Grenoble, France. E-mail: bonnetjy@obs.ujf-grenoble.fr. ${ }^{2}$ Ecole Centrale Paris, France. ${ }^{3}$ LATMOS, Guyancourt, France. ${ }^{4}$ LISA Univ. Paris XII France.

Introduction: Insoluble organic matter (IOM) in carbonaceous chondrites and stratospheric IDPs is the end-product of a complex history that took place in the presolar molecular cloud and/or in the solar nebula, followed by the action of various post-accretional processes [1,2]. Nitrogen is a minor element in IOM, with abundance ranging from $\sim 1$ up to $20 \mathrm{wt} \%$ in some IDPs [1,3]. N-bearing heterocycles and cyanide have been identified, while amino groups were not $[4,5]$. The polyaromatic structure of IOM suggests a formation process through thermal degradation of one or several organic precursors, which were possibly nitrogen rich. To examine this scenario, N-rich polymeric solids were thermally degraded at various temperatures, simulating short duration ( $\sim$ hour) thermal processes in the solar nebula.

Experiments: Analogs selected for this study were tholins (SA90) and $\mathrm{HCN}$ polymers that are N-rich polymeric organic solids [6]. They were heated at 300, 500700 and $1000^{\circ} \mathrm{C}$ under an argon atmosphere. Combustion analysis, infrared and Raman micro-spectroscopy (244 and $514 \mathrm{~nm}$ excitations) were subsequently performed to measure the $\mathrm{N} / \mathrm{C}$ ratio, chemical composition (including nitrogen speciation) and the degree of organization of the carbonaceous skeleton of the thermally processed samples.

Results: The $\mathrm{N} / \mathrm{C}$ ratio was found to dramatically decrease upon heating. However, at $500{ }^{\circ} \mathrm{C}$, the $\mathrm{N} / \mathrm{C}$ ratio and the abundance of amine functional groups were still large with respect to chondritic IOM, while alkyl groups and almost all cyanides had disappeared. This suggests that the bulk chondritic IOM could not be formed from an N-rich compound like a HCN polymer. $514 \mathrm{~nm}$ Raman spectra exhibit the first order carbon bands $\mathrm{G}$ and $\mathrm{D}$ and point to very disordered carbon materials. The width of the G band (FWHM-G) was found to decrease upon heating and its position $\omega_{\mathrm{G}}$ to increase, and both parameters linearly correlate. This evidences the presence of structural changes during the carbonization process, i.e., aromatization and growth of the polyaromatic units. This also supports the idea that IOM experienced thermal processing in the solar nebula, as this linear correlation between FWHM$\mathrm{G}$ vs $\omega_{\mathrm{G}}$ was previously reported for $\mathrm{OM}$ in Antarctic micrometeorites [7]. The presence of materials similar to thermally processed tholins or $\mathrm{HCN}$ polymer in the N-rich fraction of stratospheric IDPs OM cannot be excluded. Hence, Raman spectra, using 514 and $244 \mathrm{~nm}$ excitations, are similar for both types of materials. A better understanding of nitrogen speciation in stratospheric IDPs is necessary to go further. A study of kinetic effects on day/month time scale is under way.

References: [1] Alexander CMOD et al. 2007. Geochimica et Cosmochimica Acta 71:4380-4404. [2] Huss G. et al. 2003. Geochimica et Cosmochimica Acta 67:4823-4848. [3] Aléon J. 2003. Geochimica et Cosmochimica Acta 67:3773-3783. [4] Remusat L. et al. 2005. Geochimica et Cosmochimica Acta 69:3919-3932. [5] Quirico et al. 2008. IAU proceedings 251:409-416. [6] Quirico et al. 2008. Icarus 198:218-231. [7] Dobrica et al. Meteoritics \& Planetary Science, in press.
5161

LONG, STRANGE TRIPS: PARTICLE TRAJECTORIES IN A MARGINALLY GRAVITATIONALLY UNSTABLE SOLAR NEBULA A. P. Boss ${ }^{1}$, C. M. O'D. Alexander ${ }^{1}$ and M. Podolak ${ }^{2}$. ${ }^{1}$ DTM, Carnegie Institution, 5241 Broad Branch Road NW, Washington, DC 20015, USA.

${ }^{2}$ Tel Aviv University, Israel. E-mail: boss@dtm.ciw.edu.

Introduction: The formation of the solar system's gas giant planets appears to require that the solar nebula was massive enough at some phase to become at least marginally gravitationally unstable (MGU), whether the giant planets formed by core accretion [1] or disk instability [2]. Previous models have shown that such a MGU disk is capable of the rapid radial transport of gas and small particles both inward and outward $[3,4]$, as well of the rapid homogenization of isotopic spatial heterogeneity associated with isotopic injection from, e.g., supernova shock waves $[5,6]$. The previous models used a color field equation to follow the trajectories of gas particles and dust grains small enough to remain coupled to the gas. Here we present the first results of MGU disk models where the particles are represented as finite-size bodies that interact with the disk gas by gas drag [7], while moving on Keplerian orbits, subject to the gravity of the protosun and of the simultaneously evolving MGU protoplanetary disk.

Models: Three new sets of 3D MGU disk models with particle sizes ranging from $1 \mathrm{~mm}$ to $1 \mathrm{~m}$ have been computed, or are currently underway, for disks with sizes of either 1 to $10 \mathrm{AU}$ or 10 to $40 \mathrm{AU}$. The particles orbit in the disk midplanes. In order to speed the disk evolutions, the initial disk temperature distributions are held fixed, while the disk gas evolves temporally in three spatial dimensions, allowing the $10 \mathrm{AU}$ disk models to be followed approximately $1000 \mathrm{yr}$ in time and the $40 \mathrm{AU}$ disk models for approximately 10,000 yr.

Results: For the $10 \mathrm{AU}$ disks, particles with sizes of $1 \mathrm{~mm}, 1 \mathrm{~cm}$, or $10 \mathrm{~cm}$ that are initially close together can remain close together for $100 \mathrm{~s}$ of years, exhibiting "closed system" behavior, while clumps of $1 \mathrm{~m}$-sized bodies tend to disperse on these time scales. For all sizes, a large fraction of the trajectories move either outward to the outer grid boundary, or inward to the inner grid boundary, on the same time scales. Individual particles can move inward and outward in the disk repeatedly on eccentric orbits, sampling a wide range of nebular densities and temperatures. For the $40 \mathrm{AU}$ disks, particles initially distributed throughout the disk's radial extent tend to either migrate to the outer disk boundary, or hover on orbits around $25 \mathrm{AU}$, with the smaller particles often migrating to the inner disk boundary at $10 \mathrm{AU}$, over time scales of 1000 s of years.

Conclusions: These models imply that MGU disks may explain the cosmochemical facts that small, refractory grains can be transported outward to great distances [8], and can even make long, strange trips back and forth throughout the nebula [9].

References: [1] Chambers J. E. 2008. Icarus 198:655. [2] Boss A. P. 2010. The Astrophysical Journal 725:L145. [3] Boss A. P. 2007. The Astrophysical Journal 660:1707. [4] Boss A. P. 2008. Earth and Planetary Science Letters 268:102. [5] Boss A. P. et al. 2010. The Astrophysical Journal 708:1268. [6] Boss A. P. and Keiser S. A. 2010. The Astrophysical Journal 717:L1. [7] Haghighipour N. and Boss A. P. et al. 2003. The Astrophysical Journal 583:996. [8] Brownlee D. E. et al. 2006. Science 314:1711. [9] Simon J. I. et al. 2011. Science 331:1157. 
5156

THE USE OF AUGER SPECTROSCOPY FOR IN SITU ELEMENTAL CHARACTERIZATION WITH SUBMICROMETER SPATIAL RESOLUTION: APPLICATIONS AND OUTLOOK

M. Bose. Laboratory for Space Sciences and Physics Department, Washington University, St. Louis, MO 63130, USA. E-mail: mbose@ physics.wustl.edu.

Introduction: Auger spectroscopy is well established in the field of material science and in the semiconductor industry [1], but its use for geological applications has been limited [2], largely because of sample charging issues. Recently, however, the technique has found widespread application in the elemental characterization of presolar grains [3-6] and in the analysis of residues in impact craters from the Stardust mission to comet Wild 2 [7-8], where favorable analysis conditions mitigate most sample charging problems. I will review the development of this technique for the quantitative elemental analysis of submicrometer presolar silicate grains and outline future prospects for characterizing the small volumes of residue possibly remaining in impact craters from the $\mathrm{Al}$ foils of the Stardust interstellar dust collector [9-10].

Principles of Auger Spectroscopy: This technique has the capability to perform elemental characterizations at a high spatial resolution (approximately $15 \mathrm{~nm}$ ). Such a high spatial resolution can be achieved because of the small primary electron beam size that is attained, and the small analytical volume from which characteristic Auger electrons are measured.

Other advantages include routine nondestructive measurements, detection of all elements in the periodic table except $\mathrm{H}$ and $\mathrm{He}$, and no need for special sample preparation.

Quantitative Silicate Grain Analysis: Elemental sensitivity factors and their associated uncertainties were obtained by measuring pyroxene and olivine silicate grain standards with varying $\mathrm{mg \# s}$ [11]. These elemental factors have been used to determine the elemental composition of hundreds of presolar silicate grains identified in primitive meteorites [e.g., 3-6]. Characterization of numerous silicate grains has shown that the majority exhibit nonstoichiometric elemental compositions. In addition, new presolar phases namely magnesiowüstite [12] and $\mathrm{SiO}_{2}$ [e.g., 6] grains could be identified.

Measurement of Residues from ISPE Impact Craters: In addition to cometary samples, the Stardust mission also sampled dust from the contemporary interstellar medium. The Al foils from this collector are being searched to identify impact craters [e.g., 9] that may contain residue corresponding to interstellar dust. Auger spectroscopy can be useful for the analysis of such residue, which is expected to present in only minor amounts. The first such analyses have recently been carried out $[13,14]$ and results will be discussed at the meeting.

References: [1] Watts J. F. and Wolstenhome J. 2003. An Introduction to Surface Analysis by XPS \& AES Chichester: Wiley \& Sons. [2] Hochella M. F., Jr. et al. 1986. American Mineralogist 71:1247-1257. [3] Floss C. and Stadermann F. J. 2009. Geochimica et Cosmochimica Acta 73:2415-2440. [4] Vollmer C. et al. 2009. Geochimica et Cosmochimica Acta 73:7127-7149. [5] Bose M. et al. 2010. The Astrophysical Journal 714:1624-1636. [6] Nguyen A. et al. 2010. The Astrophysical Journal 719:166-189. [7] Stadermann F. J. et al. 2007. Abstract \#1334. 38th Lunar and Planetary Science Conference. [8] Stadermann F. J. and Floss C. 2008. Meteoritics \& Planetary Science 43:A147. [9] Stroud R. M. et al. 2011. Abstract \#1753. 42nd Lunar and Planetary Science Conference. [10] Floss C. et al. 2011. Abstract \#1576. 42nd Lunar and Planetary Science Conference. [11] Stadermann F. J. et al. 2009. Meteoritics \& Planetary Science 44:1033-1049. [12] Floss C. et al. 2008. The Astrophysical Journal 672:1266-1271. [13] Stroud R. M. et al. 2011. This conference. [14] Floss C. et al. 2011. This conference volume.

\section{1}

\section{NON-EQUILIBRIUM PRESOLAR CONDENSATES IN PRIMITIVE METEORITES}

M. Bose, C. Floss, and F. J. Stadermann. Laboratory for Space Sciences and Physics Department, Washington University, St. Louis, MO 63130. E-mail: mbose@physics.wustl.edu.

Introduction: The compositions of dust grains forming in O-rich environments under equilibrium conditions are well understood, predicting the formation of abundant (Mg-rich) forsterite and enstatite as well as some $\mathrm{Fe}$ metal [1]. However, elemental characterization of presolar silicate grains identified in primitive meteorites has shown that the majority of the grains are Fe-rich [e.g., 2, 3]. This is most likely due to their formation in dynamic stellar environments, where equilibrium conditions are not maintained. In addition, presolar $\mathrm{SiO}_{2}$ grains have recently been identified in meteorites $[2,4,5] ; \mathrm{SiO}_{2}$ grains have been dubbed "mythical condensates" [1], and can only condense under nonequilibrium conditions [6].

Auger characterization has shown that many presolar silicates have non-stoichiometric elemental compositions [2,3] and TEM work suggests that at least some presolar silicates are amorphous aggregates with variable compositions [e.g., 7]. Here we present data for additional presolar silicates identified in the ALHA77307 CO3.0 chondrite and explore the possible origins of these grains.

Results and Discussion: Seventy-eight O-anomalous grains were identified in the ALHA77307 matrix. Elemental characterization of 46 of these grains shows that 43 exhibit non-stoichiometric silicate grain compositions. More than half of the grains have compositions intermediate between olivine and pyroxene; more than half are also $\mathrm{Fe}$ rich, although a significant fraction do have Mg-rich compositions.

We also found two composite grains in ALHA77307. One of these consists of two silicate sub-grains with different elemental compositions; the oxygen isotopic composition is uniformly ${ }^{17} \mathrm{O}$-rich over both subgrains. The other one consists of a number of $\sim 50-70 \mathrm{~nm}$ sized subgrains that co-exist as a loose aggregate; elemental spectra indicate that some of the sub-grains are Ca-rich. Some heterogeneity appears to be present in the $\mathrm{O}$ isotopic composition of this composite grain, but it is not clear if the variations are statistically significant. Finally, we have also found a Mg-oxide grain in ALHA77307. Comparison to a periclase standard indicates that the $\mathrm{Mg} / \mathrm{O}$ ratio of the grain is $1.0 \pm 0.1$. This is the first reported occurrence of this grain type in the presolar grain inventory.

Extensive study of presolar graphite and $\mathrm{SiC}$ has shown that equilibrium condensation models adequately explain the formation of these grains [e.g., 8]. In contrast, the presence of large numbers of nonstoichiometric grains in the presolar silicate inventory suggests that condensation of presolar silicates more often occurred under nonequilibrium conditions. We will discuss possible scenarios for the formation of these grains. In addition, we will evaluate the conditions under which the composite grains found in ALHA77307 may have formed.

References: [1] Lodders K. and Fegley B. 1999. In: AGB stars, IAU Symposium No. 191. [2] Floss C. and Stadermann F. J. 2009. Geochimica et Cosmochimica Acta 73, 2415. [3] Bose M. et al. 2009. The Astrophysical Journal 714, 1624. [4] Bose M. et al. 2010. Abstract \#1812. 41st Lunar and Planetary Science Conference. [5] Nguyen A. N. et al. 2007. The Astrophysical Journal 656, 1223. [6] Ferrarotti A. S. and Gail H. -P. 2001. Astronomy \& Astrophysics 371, 133. [7] Stroud R. M. et al. 2009. Abstract \#1063. 40th Lunar and Planetary Science Conference. [8] Bernatowicz T. et al. 2006. In: Meteorites and the Early solar system II, pp. 109-126. 


\section{0}

C AND N ION IMAGING IN THE EH3 CHONDRITE SAH 97159

M. Bose ${ }^{1}$, X. Zhao ${ }^{2}$, C. Floss ${ }^{1}$, F. J. Stadermann ${ }^{1}$ and Y. Lin $^{2}$. ${ }^{1}$ Laboratory for Space Sciences and Physics Department, Washington University, St. Louis, MO 63130, USA. E-mail: mbose@physics.wustl.edu. ${ }^{2}$ Laboratory of Earth's Deep Interior, Institute of Geology and Geophysics, Chinese Academy of Sciences, Beijing 100029, China.

Introduction: The insoluble organic matter (IOM) in numerous primitive meteorites, notably the $\mathrm{CR}$ chondrites, is ${ }^{15} \mathrm{~N}$-rich in bulk. Carbon and $\mathrm{N}$ isotopic imaging shows that discrete hotspots with large enhancements in ${ }^{15} \mathrm{~N}$ are often present in this matter; some of these also have associated anomalous $\mathrm{C}$ isotopic compositions [1-3]. Coordinated NanoSIMS and TEM analyses of $\mathrm{C}$ - and $\mathrm{N}$-anomalous hotspots show that the isotopic hotspots are often associated with carbonaceous grains with variable morphologies [3, 4].

To date, $\mathrm{C}$ and $\mathrm{N}$ ion imaging measurements have been reported for a variety of carbonaceous chondrites [e.g., 5], but no enstatite chondrites have been explored as yet. We recently identified presolar silicate and C-anomalous grains in the paired EH3 chondrites SAH 97096 and SAH 97159 [6], indicating that high temperatures conditions in the nebular phase did not destroy stardust materials completely in these meteorites. Indeed, fifteen C-anomalous grains were identified in SAH 97159 on the basis of $\mathrm{C}$ and $\mathrm{O}$ imaging [6], of which six are most likely SiC grains. We, therefore, decided to explore whether $\mathrm{N}$-anomalous material may have survived in this meteorite.

Experimental: We carried out NanoSIMS $\mathrm{C}$ and $\mathrm{N}$ ion imaging searches in the matrix of a thin-section of SAH 97159. Instrument tuning and standard measurements were carried out on terrestrial kerogen standards [7].

Results and Discussion: We identified five additional C-anomalous grains in the SAH 97159 matrix, with ${ }^{12} \mathrm{C} /{ }^{13} \mathrm{C}$ ratios from $52-110$; the ${ }^{14} \mathrm{~N} /{ }^{15} \mathrm{~N}$ ratios range from $190-441$. On the basis of the isotopic compositions and the ${ }^{12} \mathrm{C}^{-} /{ }^{28} \mathrm{Si}^{-}$elemental ratios two grains may be $\mathrm{SiC}$, while the others may be carbonaceous material intermixed with silicates in varying proportions.

Nitrogen-anomalous hotspots were absent in the area analyzed. This is consistent with the $\mathrm{N}$ isotopic compositions of bulk IOM in enstatite chondrites [8]. In contrast, the most pristine CR chondrites, MET 00426 and QUE 99177 contain large isotopic anomalies both in bulk and in the form of localized hotspots [2]. The CO chondrite ALHA77307 shows only localized hotspots, at levels that are consistent with the lack of bulk ${ }^{15} \mathrm{~N}$ enrichment in the IOM of this meteorite [3]. It has been suggested that the material from which the enstatite chondrites formed experienced high temperature processing in the solar nebula [9]; the lack of $\mathrm{N}$ isotopic anomalies in SAH 97159 is consistent with this scenario.

References: [1] Busemann H. 2006. Science 312:727. [2] Floss C. and Stadermann F. J. 2009. The Astrophysical Journal 697:1242. [3] Bose M. et al. 2010. Meteoritics \& Planetary Science 45:A19. [4] Floss C. et al. 2011. Abstract \#1455. 42nd Lunar and Planetary Science Conference. [5] Davidson J. et al. 2009. Abstract \#1853. 40th Lunar and Planetary Science Conference. [6] Bose M. et al. 2010. POS-NIC XI 138. [7] Hayes J. M. et al. 1983. In Earth's earliest biosphere: Its origin and evolution. pp. 93-134. [8] Alexander C. M. O'D. et al. 2007. Geochimica et Cosmochimica Acta 71:4380. [9] Huss G. et al. 2003. Geochimica et Cosmochimica Acta 67:4823.

\section{5}

U-PB CHRONOLOGY OF TWO LUNAR IMPACT MELT BRECCIAS

A. Bouvier ${ }^{1}$, M. Wadhwa ${ }^{1}$, R. L. Korotev ${ }^{2}$, W. K. Hartmann ${ }^{3}$. ${ }^{1}$ Arizona State University, Tempe, AZ, USA. ${ }^{2}$ Washington University in St. Louis, St. Louis, MO, USA. ${ }^{3}$ Planetary Science Institute, Tucson, AZ, USA. E-mail: audrey.bouvier@asu.edu.

Introduction: Current understanding of lunar impact history is largely based on Ar-Ar studies of impact breccias and glass spherules from the Apollo lunar samples, and more recently from lunar meteorites, which have been interpreted to show a dearth of $>4$ Ga ages (e.g., [1, 2]). This has led to the hypothesis of a late heavy bombardment (LHB) in the inner solar system at $3.9 \pm 0.1 \mathrm{Ga}$. However, recent $\mathrm{Ar}-\mathrm{Ar}$ and $\mathrm{U}-\mathrm{Pb}$ studies of impact melts from Apollo samples and meteorites have yielded ages $>4.0 \mathrm{Ga}$ (e.g., [3-5]), calling into question the LHB hypothesis. Large-scale impacts such as the Imbrium event could be responsible for resetting the isotope systematics of various radiometric chronometers to different degrees in samples from different locations on the Moon. Since Ar has a lower (approximately $700 \mathrm{~K}$ ) blocking temperature than $\mathrm{Pb}$ (approximately $1300 \mathrm{~K}$ ) in silicates [6, 7], the U-Pb chronometer may be able to provide more robust "primary" impact melt ages. Therefore, we present here the U-Pb chronology of two feldspathic impact melt breccias: 65015,60 (from the Apollo 16 Descartes landing site) and Shişr 166 (a lunar meteorite).

Results: One bulk-rock sample (approximately $20 \mathrm{mg}$ ) for 65015 and four mineral separates (approximately $10-40 \mathrm{mg}$ each) for 65015 and Shişr 166 were processed for U-Pb dating. Leaching (3 steps for 65015 and 5-7 steps for Shişr 166), dissolution, and $\mathrm{Pb}$ extraction methods were similar to [8]. $\mathrm{Pb}$ isotopic compositions and $\mathrm{U} / \mathrm{Pb}$ of 65015 mineral residues were analyzed by MC-ICPMS and quadrupole ICPMS respectively at ASU. The 65015 residues have corrected $206 \mathrm{~Pb}$ / $204 \mathrm{~Pb} \approx 9351-43,501$. We obtain a U-Pb Concordia upper intercept age of $3,929 \pm 11 \mathrm{Ma}(\mathrm{MSWD}=3$ ) and a consistent, but more precise, $\mathrm{Pb}-\mathrm{Pb}$ isochron age of $3935 \pm 2 \mathrm{Ma}(\mathrm{MSWD}=12)$, using ${ }^{238} \mathrm{U} /{ }^{235} \mathrm{U}=137.84$. The Shişr 166 residues have ${ }^{206} \mathrm{~Pb} /{ }^{204} \mathrm{~Pb} \approx 60-250$ and give a $\mathrm{Pb}-\mathrm{Pb}$ isochron age of $3567 \pm 5 \mathrm{Ma}(\mathrm{MSWD}=0.05)$.

Discussion: The $3935 \mathrm{Ma}$ age for 65015 is consistent with, but more precise than, previous estimates using the $\mathrm{Ar}-\mathrm{Ar}$ and $\mathrm{Rb}-\mathrm{Sr}$ systems [9], but younger than a previously reported approximately $3.99 \mathrm{Ga} \mathrm{Pb}-\mathrm{Pb}$ age [10]. Based on geochemistry and $\mathrm{Ar}-\mathrm{Ar}$ chronometry, it was recently suggested that the Descartes terrains could be approximately $3.87 \mathrm{Ga}$ ejecta from Imbrium instead of Nectaris basins [11]. The age of the 65015 breccia is older and may suggest that either it formed during a different event or the Imbrium impact event is older than previously thought (i.e., approximately $3.9 \mathrm{Ga}$ based Ar-Ar chronometry of Apollo 15 and 16 breccias $[1,11])$. The younger approximately $3.57 \mathrm{Ga}$ age of Shişr 166 is recognized in other lunar meteorites by $\mathrm{Ar}-\mathrm{Ar}$ chronometry [3] and suggests a protracted history for the formation of impact melt breccias on the surface of the Moon.

References: [1] Dalrymple G. B. and Ryder G. 1991. Geophysical Research Letters 18:1163-1166. [2] Cohen B. A. et al. 2000. Science 290:1754-1756. [3] Cohen B. A. 2008. LPI Contribution 1439:27-28. [4] Fernandes V. A. et al. 2008. LPI Contribution 1439:30-31. [5] Grange M. L. et al. 2011. Geochimica et Cosmochimica Acta 75:2213-2232. [6] Cherniak D. J. 2001. Chemical Geology 177:381-397. [7] Reiners P. W. 2009. Geochimica et Cosmochimica Acta 73:3612-3629. [8] Bouvier A. and Wadhwa M. 2010. Nature Geoscience 3:637-641. [9] Papanastassiou D. A. and Wasserburg G. J. 1972. Earth and Planetary Science Letters 17:52-63. [10] Nunes P. D. et al. 1973. Proceedings, 4th Lunar Science Conference 2:1797-1822. [11] Norman M. D. et al. 2010. Geochimica et Cosmochimica Acta 74:763-783. 
5120

A NEW METEORITE TYPE: FORSTERITE CHONDRITE WITH EH-3 AFFINITY: SAHARA 97158

M. Boyet ${ }^{1}$, A. El Goresy ${ }^{2}$, M. Miyahara ${ }^{3}$ and A. Gannoun ${ }^{1}{ }^{1}{ }^{1}$ Université Blaise Pascal, Lab. Magmas et Volcans, UMR CNRS 6524, BP 10448, F-63000 Clermont-Ferrand, France. E-mail: M.Boyet@opgc.univbpclermont.fr. ${ }^{2}$ Bayerisches Geoinstitut, Universitat Bayreuth, 95440 Bayreuth, Germany. ${ }^{3}$ Institute of Mineralogy, Petrology and Economic Geology, Graduate School of Science, Tohoku University, Sendai, Japan.

Introduction: A large black clast was encountered inside a slab of the EH-3 chondrite Sahara 97158. The clast is poor in metals and sulfides. It has an unusual mineralogy and texture. It is barren of chondrules and depleted in metal-sulfide clasts that are characteristic of unequilibrated EH-3 or EL-3 chondrites. It consists of $40-60 \mathrm{vol} \%$ of large olivine crystals (up to $80 \mu \mathrm{m}$ in diameter, $\left(\mathrm{Fo}_{0.995}-\mathrm{Fa} 0{ }_{0025}\right)$, up to 25 vol. \% prismatic enstatite crystals $\left(\mathrm{En}_{0.99}-\mathrm{Fs}_{0.003} \mathrm{Wo}_{0.001}\right)$, minor contents of glass and some 4-7 vol. \% oldhamite, FeNi-metal and other sulfides. The silicate assemblage depicts igneous-like texture and feigns an unusual olivine-rich achondrite but is entirely different from any aubritic meteorite. The sulfide and metal alloy assemblage consists of oldhamite, niningerite, djerfisherite, sphalerite, schreibersite, troilite, caswellsilverite and metallic FeNi. The sulfide-metal inventory is very similar to that of EH-3 chondrites. Djerfisherite is depleted in K (3.36 wt. \%) compared to other EH-3s: (8.40 wt. \% in Qingzhen). Presence of the clast inside an EH-3 host indicates origin from a chondrule-free proto-asteroid that accreted and was destroyed prior to accretion of the host EH-3 Sahara 97158.

Results and Discussion: The clast was subjected to an endogenous heating event, partial melting and melt migration prior to its accretion with the other constituents of the EH-3 Sahara 97158 host. The preaccretion heating event led to considerable melt migration of sulfides, specifically of troilite along with FeNi metal. There is no evidence of shock features like high-pressure phase transformation of olivine to ringwoodite or wadsleyite and enstatite to majorite or akimotoite so that a dynamic event is not responsible for the partial melting and sulfide-melt migration.

REE abundances were measured by LA-ICPMS in several oldhamite crystals in this clast in conjunction with the REE measurements in oldhamite grains in the Sahara 97158 EH-3 host and in an idiomorphic oldhamite in a chondrule in the same EH-3 host. REE patterns in oldhamite in the clast, in the host EH-3 chondrite and the one idiomorphic oldhamite in a chondrule in the EH-3 host display very similar patterns (e.g., Fig. 6 in [1]). All REE patterns display the mainstream type pattern of Sahara 97158 and Sahara 97072 with positive $\mathrm{Eu}$ and $\mathrm{Yb}$ anomalies [1].

We consider these findings as a strong hint that all the oldhamite crystals in the EH-3 host, chondrule and in the clast emerged from the same nebular reservoir. Oxygen isotopic investigations are planed and will be conducted after completion of the mineralogical investigations on olivine and enstatite in the clast and enstatite in the host EH-3 chondrite.

Reference: [1] Gannoun A. et al. 2011. Geochimica et Cosmochimica Acta 75:3269-3289.
5086

ON THE OCCURRENCE OF CUBANITE IN THE KAIDUN METEORITE

F. Brandstätter ${ }^{1}$ and A. Ivanov ${ }^{2}$. ${ }^{1}$ Naturhistorisches Museum, Burgring 7, 1010 Wien, Austria. E-mail: franz.brandstaetter@nhm-wien.ac.at. ${ }^{2}$ Vernadsky Institute of Geochemistry and Analytical Chemistry, Kosygin St. 19, Moscow 119991, Russia.

Introduction: Kaidun is a unique polymict microbreccia containing a large variety of oxidized and reduced chondritic and achondritic rocks. Its host was originally classified as CR-like material [1, 2]. However, it turned out that the carbonaceous chondrite (CC) lithologies dominating Kaidun contain numerous $\mathrm{C} 1$ and $\mathrm{C} 2$ materials which apparently are not known from other meteorites [3]. In addition, the isotopic and petrologic characteristics of most of these $\mathrm{CC}$ clasts are outside or intermediate between the established limits of CI, CM, and CR chondrites [4]. Here we report on the occurrence of a large individual cubanite grain found in a CC clast from section Kaidun \#01.3.d1. Cubanite is a very rare mineral phase in meteorites. Up to date it has only been described from the CI chondrites Alais, Orgueil and Ivuna [5, 6], and from the Stardust collection [7]

Results: The host lithology of cubanite is a CI-like clast being chondrule-free and having a phyllosilicate- and magnetite-rich matrix. Magnetite typically occurs as framboids (up to $50 \mu \mathrm{m}$ in size), plaquettes and spherulites. The second abundant opaque phase is pyrrhotite forming up to $30 \mu \mathrm{m}$ large plates and laths. In places, subrounded isolated grains of dolomite and olivine (rarely exceeding $50 \mu \mathrm{m}$ in size) are present. Cubanite occurs as a single subhedral grain, $\sim 100 \mu \mathrm{m}$ in diameter, terminated in part by crystal faces. Electron microprobe analyses performed along a line from rim to core revealed that the cubanite is chemically homogenous. Its average composition (15 analyses, in $\mathrm{wt} \% \pm 2 \sigma$ ) is near stoichiometric with $22.9 \pm 0.4 \mathrm{Cu}, 41.1 \pm 0.4 \mathrm{Fe}$, $36.5 \pm 0.6 \mathrm{~S}$ [formula $\mathrm{Cu}_{16.3} \mathrm{Fe}_{33,5} \mathrm{~S}_{50.2}$ ] and agrees, within error, with those given for cubanite in Orgueil [8].

Implications: Upon heating above $210{ }^{\circ} \mathrm{C}$, orthorhombic $\mathrm{CuFe}_{2} \mathrm{~S}_{3}$ undergoes an irreversible phase transition to a high-temperature cubic form [9]. Cubanite found in CI chondrites is believed to be a hydrothermal alteration product [5] and it was shown that it is present in these meteorites as low-temperature orthorhombic $\mathrm{CuFe}_{2} \mathrm{~S}_{3}$ [8]. Thus, we predict that the Kaidun cubanite is orthorhombic. This assumption implies that (i) cubanite in Kaidun formed at low temperatures by precipitating from an aqueous fluid, (ii) after the formation of cubanite its host clast did not experience temperatures above $210^{\circ} \mathrm{C}$, (iii) cubanite formed at a later stage than pyrrhotite, as suggested by [8].

References: [1] Ivanov A. V. et al. 1986. Meteoritika 45:3-19. In Russian. [2] Ivanov A. V. 1989. Geochemistry International 26:84-91. [3] Zolensky M. E. and Ivanov A. V. 2003. Chemie der Erde 63:185-246. 1997. [4] MacPherson G. J. et al. 2009. Geochimica et Cosmochimica Acta 73:5493-5511. [5] Bullock E. S. et al. 2005. Geochimica et Cosmochimica Acta 69:2687-2700. [6] Macdougall J. D. and Kerridge J. F. 1977. Science 197:561-562. [7] Berger E. L. et al. 2009. Abstract \#1892. 40th Lunar and Planetary Science Conference. [8] Berger E. L. et al. 2010. Abstract \#1160. 41st Lunar and Planetary Science Conference. [9] Caye R. et al. 1988. Mineralogical Magazine 52:509-514. 
5364

\section{FORMATION OF CA-PHOSPHATES DURING AQUEOUS ALTERATION OF TYPE IIA CHONDRULES IN THE Y-791198 CM2 CARBONACEOUS CHONDRITE}

A. J. Brearley. Department of Earth and Planetary Sciences, MSC032040, 1 University of New Mexico, Albuquerque, NM87131, USA. E-mail: brearley@unm.edu.

Introduction: The CM2 chondrites are a primitive group of meteorites that record a complex history of early solar system processes [1]. The CM2s show evidence of significant aqueous alteration that has resulted in the replacement of primary nebular phases by a variety of secondary alteration products [1]. An understanding the nature of the alteration reactions provides important insights into the composition of aqueous fluids and elemental mass transfer during alteration. To address the issue of elemental mobility during alteration, we have examined the behavior of $\mathrm{Ca}$ and $\mathrm{P}$ in type IIA chondrules [2,3] that results in the formation of $\mathrm{Ca}$-phosphates during aqueous alteration.

Method: Several type IIA chondrules and their associated finegrained rims from the weakly altered CM2 chondrite, Y-791198 were characterized by SEM/BSE imaging and elemental X-ray mapping by EPMA. Selected regions from the interface between these chondrules and their fine-grained rims were extracted using FIB techniques and were studied in detail by TEM.

Results and Discussion: X-ray maps of type IIA chondrules show that, in all cases, a narrow zone of $\mathrm{Ca}$ and $\mathrm{P}$ enrichment is present at the interface between altered mesostasis and the fine-grained rim [2]. The zone of $\mathrm{Ca}$ and $\mathrm{P}$ enrichment has been characterized in detail using TEM. These observations show that the $\mathrm{Ca}$ and P-bearing phase is wellcrystallized apatite, but with a highly unusual morphology. The apatite occurs as continuous, elongate, but curved plates, that extend for several microns, but are clearly single crystals. In some locations, the apatite crystals resemble a wishbone with straight segments that may be $2-3 \mu \mathrm{m}$ in length, linked by a short, curved segment. The apatites range from $<0.1-0.8 \mu \mathrm{m}$ in width. The TEM data show that the apatites are not located exactly at the interface between the chondrule and fine-grained rim, but are contained completely within the altered chondrule mesostasis, 2-3 $\mu \mathrm{m}$ from the edge of the chondrule. The altered mesostasis consists of fine-grained phyllosilicates and material that appears to be amorphous. EDS analysis of this material show that it is $\mathrm{SiO}_{2^{-}}$(approximately 40 $\mathrm{wt}^{\mathrm{O}} \%$ ) and $\mathrm{FeO}$-rich $(40 \mathrm{wt} \%)$, but also contains significant concentrations of $\mathrm{Al}_{2} \mathrm{O}_{3}$, (approximately $7 \mathrm{wt} \%$ ) $\mathrm{MgO}$ (approximately $8 \mathrm{wt} \%$ ) and, notably, $\mathrm{K}_{2} \mathrm{O}$ (approximately $4 \mathrm{wt} \%$ ).

The occurrence of apatite in Y-791198 type IIA chondrules is remarkably similar to textures observed in partially altered glass in a type IIA chondrule in the CR2 chondrite EET 92015 [3]. We have argued that $\mathrm{Ca}$ and $\mathrm{P}$ leached from chondrule mesostasis during aqueous alteration are precipitated as apatite at a geochemical reaction front on the edge of the chondrule. Precipitation is promoted by an increase in $\mathrm{pH}$, stabilizing apatite [4]. The Y-791198 phosphates appear to share a similar origin, except that the degree of alteration in Y-791198 is much more extensive. This suggests that early formed phosphates are stable to relatively advanced degrees of alteration, but do eventually undergo dissolution in more heavily altered CM2s [4].

References: [1] Brearley A. J. 2006. In 'MESS II' (Eds Lauretta, D. S. and McSween, H. Y. Jr). p. 587. [2] Brearley A. J. and Chizmadia, L. J. 2005. Abstract \#2176. 36th Lunar Prospector Spacecraft. [3] Brearley A. J. and Burger P. V. 2009. Meteoritics \& Planetary Science 72:5150. [4] Brearley A. J. 2006. Abstract \#2074. 37th Lunar Prospector Spacecraft.
5030

URANIUM ISOTOPE COMPOSITIONS OF MINERAL SEPARATES FROM A SINGLE REFRACTORY INCLUSION

G. A. Brennecka and M. Wadhwa. School of Earth and Space Exploration, Arizona State University. E-mail: brennecka@asu.edu

Introduction: Recent work on bulk calcium-aluminum rich inclusions (CAIs) has shown variability in the ${ }^{238} \mathrm{U} /{ }^{235} \mathrm{U}$ ratio, which has major implications for the accuracy of $\mathrm{Pb}-\mathrm{Pb}$ dating $[1,2]$. While ${ }^{238} \mathrm{U} /{ }^{235} \mathrm{U}$ variability among CAIs is clear, there have been no recent high-precision measurements of the uranium isotope compositions of minerals within individual CAIs. The ${ }^{238} \mathrm{U} /{ }^{235} \mathrm{U}$ ratios of various bulk fragments and residues of the coarse-grained Allende CAI, Egg-2 were previously reported by Chen and Wasserburg (1981), with an average value of $137.8 \pm 0.5$ [3]. This value is identical, within the reported uncertainty, to the previously accepted "terrestrial" ${ }^{238} \mathrm{U} /{ }^{235} \mathrm{U}$ ratio of 137.88. We revisit this sample with modern instrumentation and greatly improved precision to determine if uranium isotopic variability exists between mineral phases in a single CAI.

Samples and Methods: Mineral separates of the Allende CAI, Egg-2 were provided by G. J. Wasserburg. These mineral separates were obtained by a combination of heavy liquid and magnetic separation. The phases were divided into three groups: $3.3 \mathrm{~g} \mathrm{~mL}^{-1}$ sink - magnetic (89 mg), $3.3 \mathrm{~g} \mathrm{~mL}^{-1}$ sink - less magnetic $(38 \mathrm{mg})$, and $3.1 \mathrm{~g} \mathrm{~mL}^{-1}$ float $(55 \mathrm{mg})$, representing high-Fe pyroxene, high-Mg pyroxene, and plagioclase-rich separates, respectively. These separates were fully digested using $\mathrm{HF}, \mathrm{HNO}_{3}$, and $\mathrm{HCl}$. An aliquot $(\sim 1 \%)$ of each sample solution was reserved for trace element analyses by quadrupole ICPMS at Arizona State University (ASU). Uranium was separated from the remaining sample solutions for measurement of the ${ }^{238} \mathrm{U} /{ }^{235} \mathrm{U}$ ratio, using the procedure outlined in [4]. Uranium isotopes were measured on a Thermo Neptune MC-ICPMS at ASU following the ${ }^{236} \mathrm{U}-{ }^{233} \mathrm{U}$ double spike procedure discussed in [1].

Results and Discussion: The REE patterns of the mineral separates demonstrate that Egg-2 is a non-group II CAI. The $\mathrm{Th} / \mathrm{U}$ ratios of all phases are indistinguishable from chondritic $\mathrm{Th} / \mathrm{U}$. The measured ${ }^{238} \mathrm{U} /{ }^{235} \mathrm{U}$ ratios of the high-Fe pyroxene $(137.784 \pm 0.034)$, high-Mg pyroxene $(137.787 \pm 0.034)$, and plagioclase-rich $(137.793 \pm 0.034)$ fractions are indistinguishable from one another. However, all mineral separates are distinct from their previously assumed ${ }^{238} \mathrm{U} /{ }^{235} \mathrm{U}$ value of 137.88 [3]. The reported uncertainty (2SD) is based on multiple runs of the SRM950a standard at the same concentrations as the samples, and includes the analytical uncertainty, and uncertainties in the compositions of the spike and the SRM950a standard [5].

This work confirms that no variability in the ${ }^{238} \mathrm{U} /{ }^{235} \mathrm{U}$ ratios exists between individual minerals in CAIs at the current level of analytical precision. However, if large $\mathrm{Th} / \mathrm{U}$ fractionation is found between mineral phases in other CAIs, variability in ${ }^{238} \mathrm{U} /{ }^{235} \mathrm{U}$ could exist from the decay of ${ }^{247} \mathrm{Cm}$ to ${ }^{235} \mathrm{U}\left(\mathrm{t}_{1 / 2} \sim 15.6 \mathrm{Ma}\right)[1]$.

References: [1] Brennecka et al. 2010. Science 327:449-451. [2] Amelin et al. 2011. Earth and Planetary Science Letters 300:343-350. [3] Chen and Wasserburg 1981. Analytical Chemistry 53:2060-2067. [4] Weyer et al. 2008. Geochimica et Cosmochimica Acta 72:345-359. [5] Richter et al. 2010. IJMS. 295:94-97. 
5070

INCORPORATION OF ${ }^{7} \mathrm{Be},{ }^{10} \mathrm{Be},{ }^{14} \mathrm{C},{ }^{26} \mathrm{Al},{ }^{36} \mathrm{Cl},{ }^{41} \mathrm{Ca}$, And ${ }^{53} \mathrm{Mn}$ INTO EARLY SOLAR SYSTEM MATERIALS IN THE SOLAR WIND IMPLANTATION MODEL

G. E. Bricker ${ }^{1}$ and M. W. Caffee ${ }^{2}$. ${ }^{1}$ Dept. of Physics, Purdue University North Central, Westville, IN. 46391. ${ }^{2}$ Primelab, Dept. of Physics, Purdue University, West Lafayette, IN. 47907.

Introduction: Numerous studies have indicated the incorporation of the short-lived radionuclides (SLRs) $\left(\mathrm{T}_{1 / 2}<5 \mathrm{Myr}\right){ }^{7} \mathrm{Be},{ }^{10} \mathrm{Be},{ }^{26} \mathrm{Al},{ }^{36} \mathrm{Cl}$, ${ }^{41} \mathrm{Ca},{ }^{53} \mathrm{Mn}$, and ${ }^{60} \mathrm{Fe}$ into early solar system materials, including calcium aluminum inclusions (CAIs), in amounts above background levels, although the mechanism for this incorporation is a matter of considerable debate (cf.[1]). Bricker \& Caffee [2] proposed a solar wind implantation model for incorporation of ${ }^{10} \mathrm{Be}$ in CAI precursor materials. It is known that ${ }^{10} \mathrm{Be}$ is currently produced in the atmosphere of the Sun through the spallation of oxygen with energetic protons. This spallogenic ${ }^{10} \mathrm{Be}$ in entrained in the solar wind and implanted in solar system materials exposed to the solar wind. Nishiizumi \& Caffee [3] detected solar-windimplanted ${ }^{10} \mathrm{Be}$ in Apollo 17 trench samples. Bricker and Caffee proposed that the ${ }^{10} \mathrm{Be}$ incorporated into CAIs was created in the proto-solar atmosphere, rather than in-situ in CAIs (cf. Lee et al. [4] \& Gounelle et al. [5]) by the same mechanism we observe now, bombardment of $\mathrm{O}$ by solar energetic protons and He nuclei. In their solar wind implantation model, Bricker \& Caffee proposed that some fraction of this outward flowing ${ }^{10} \mathrm{Be}$ is incorporated into the inward flowing material which drops from the funnel flow accreting onto the proto-Sun and they were able to account for ${ }^{10} \mathrm{Be}$ concentrations found in CAIs. Other SLRs: ${ }^{7} \mathrm{Be},{ }^{14} \mathrm{C}$, ${ }^{26} \mathrm{Al},{ }^{36} \mathrm{Cl},{ }^{41} \mathrm{Ca}$, and ${ }^{53} \mathrm{Mn}$ can also be produced through solar energetic particle reactions. We now consider the incorporation these short lived radionuclides $\left({ }^{7} \mathrm{Be},{ }^{10} \mathrm{Be},{ }^{14} \mathrm{C},{ }^{26} \mathrm{Al},{ }^{36} \mathrm{Cl},{ }^{41} \mathrm{Ca}\right.$, and $\left.{ }^{53} \mathrm{Mn}\right)$ into early solar system materials in accordance with the solar wind implantation model.

Results: We consider experimentally determined ${ }^{10} \mathrm{Be}$ and ${ }^{14} \mathrm{C}$ [6] contemporary spallation production rates scaled to $\mathrm{x}$-ray luminosities scene in T-Tauri stars and ancient enhanced energetic particle fluxes [7] and theoretically determined ${ }^{7} \mathrm{Be},{ }^{10} \mathrm{Be},{ }^{14} \mathrm{C},{ }^{26} \mathrm{Al},{ }^{36} \mathrm{Cl},{ }^{41} \mathrm{Ca}$, and ${ }^{53} \mathrm{Mn}$ production rates scaled to ancient energetic particle fluxes at the protoSun. Using this enhanced production value in conjunction with an accepted refractory mass inflow rates at $.06 \mathrm{AU}$ from the proto-Sun we model the concentrations of SLRs in CAI precursors. The model predictions are consistent with measurements of ${ }^{7} \mathrm{Be},{ }^{10} \mathrm{Be},{ }^{36} \mathrm{Cl},{ }^{41} \mathrm{Ca}$, and ${ }^{53} \mathrm{Mn}$ found in CAIs. The model fails to produce the ${ }^{26} \mathrm{Al}$ content by a factor of 15 . We predict the content of ${ }^{14} \mathrm{C}$ in CAIs will be $3.7 \times 10^{12}$ ${ }^{14} \mathrm{C} \mathrm{g}^{-1}$ with an isotopic ration of ${ }^{14} \mathrm{C} /{ }^{12} \mathrm{C}$ of $2.2 \times 10^{-9}$.

References: [1] Gounelle M., Chaussidon M., and Montmerle T. 2007. C.R. Geoscience 339:885. [2] Bricker G. E. and Caffee M. W. 2010. The Astrophysics Journal 725:443. [3] Nishiizumi K. and Caffee M. W. 2001. Science 294:352. [4] Lee T., Shu F. H., Glassgold A. E., and Rehm K. E. 1998. The Astrophysics Journal 506:898. [5] Gounelle M., Shu F. H., Shang H., Glassgold A. E., Rehm K. E., and Lee T. 2006. The Astrophysics Journal 640:1163. [6] Jull A. J. T., Lal D., and Donahue D. J. 1995. Earth and Planetary Science Letters 136:693. [7] Feigelson E. D., Garmire G. P., and Pravdo S. H. 2002. The Astrophysics Journal 572:335.
5262

AMORPHOUS GEL IN THE NAKHLITES: PRODUCT OF A RAPIDLY COOLED HYDROTHERMAL FLUID

J. C. Bridges and L. J. Hicks. Dept. of Physics \& Astronomy, Space Research Centre, University of Leicester, LE1 7RH, UK. E-mail: j.bridges@le.ac.uk.

Introduction: It has been demonstrated that the nakhlites' alteration assemblage includes an hydrated silicate gel. This is within the central part of veins, surrounded by smectite-(serpentine) and siderite in some cases [1]. High resolution TEM has shown the non crystalline nature of the gel [1]. It formed during rapid cooling in the latter stages of the hydrothermal event. In related work we show that the fluid was derived through dissolution of host nakhlite by a dilute brine [2]. Here we use more nakhlites to investigate the variation of composition of secondary minerals with depth and constrain the nature of the hydrothermal event.

Samples and Methods: We have analyzed polished sections of Lafayette, NWA 998, Governador Valadares, Nakhla, NWA 817 , Y000593, Y000749, Miller Range 03346, NWA 5790 by SEM-EDX, FIBSEM and TEM. EDX totals are normalized to $100 \%$ anhydrous.

Results: Most of the nakhlite veins are found to include the amorphous gel. No pure gel analyses have yet been made in NWA 998 due to its small vein sizes or from NWA 5790 and Y000749 due to their terrestrial weathering, which is shown for instance, by the presence of pure $\mathrm{Ca}$ carbonate and jarosite.

The average $\mathrm{Mg} /(\mathrm{Mg}+$ total $\mathrm{Fe})$ numbers of the gel are: Lafayette 0.38 , GV 0.33, Nakhla 0.25, NWA 817 0.22, Y000593 0.15, Miller Range 033460.14 . Based on Fe K XANES analyses, the $\mathrm{Fe}^{3+} /$ total $\mathrm{Fe}$ ratio of the gel is approximately $0.7-1.0$ [3]. In Lafayette, the $\mathrm{Fe} / \mathrm{Si}$ wt ratios of the gel 1.0-1.6 are in the same range as those of the phyllosilicate 1.0-1.6. Likewise the normalized $\mathrm{Na}_{2} \mathrm{O}+\mathrm{K}_{2} \mathrm{O}$ contents of the Lafayette gel $\leq 0.8$ wt $\%$ overlap those of the phyllosilicate $\leq 0.9 \mathrm{wt} \%$. The highest gel $\mathrm{Na}_{2} \mathrm{O}+\mathrm{K}_{2} \mathrm{O}$ contents seen so far $\leq 2.5 \mathrm{wt} \%$, are in Nakhla. Al/Si wt ratios are similar in the phyllosilicate and gel $\leq 0.1$.

Discussion: The variation of gel $\mathrm{Mg \#}$ is not reflected in a parallel variation in the $\mathrm{Mg} \#$ of the surrounding olivine. Nakhlite olivine has

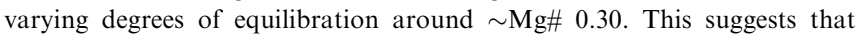
the gel compositional fractionation is a function of variation in the composition of the fluid as it migrated up through the nakhlites rather than being controlled by the composition of the adjacent phases.

The gel Mg\# compositions are mostly consistent with the predicted stratigraphy of the nakhlites which envisage NWA 998 then Lafayette at the base and Miller Range 03346 near the top [4]. However, the low abundance of gel in NWA 998 compared to Lafayette, which has a high abundance of secondary minerals and was closest to the fluid source, suggests that the nakhlites have preserved both lateral and vertical traces of the brine's passage and culmination. The presence of amorphous gel, with a composition similar to the Lafayette phyllosilicate, in most of the nakhlite veins demonstrates that the secondary assemblage formed in a short hydrothermal event, cooling too rapidly for phyllosilicate to continue crystallizing.

References: [1] Changela H. G. and Bridges J. C. 2010. Meteoritics \& Planetary Science 45:1847-1867. [2] Schwenzer S. P. and Bridges J. C. 2011. Meteoritics \& Planetary Science, this volume. [3] Hicks L. J. et al. 2011. Meteoritics \& Planetary Science, this volume. [4] Mikouchi T. et al. Abstract \#1865. 37th Lunar and Planetary Science Conference. 
5405

THE "BASAL ONAPING INTRUSION": POTENTIAL ROOF ROCKS OF THE SUDBURY IGNEOUS COMPLEX

D. T. M. Brillinger ${ }^{1}$, R. A. F. Grieve ${ }^{1,2}$, G. R. Osinski ${ }^{1}$ and D. E. Ames ${ }^{2}$. ${ }^{1}$ Department of Earth Sciences, University of Western Ontario, London, ON, Canada. ${ }^{2}$ Earth Sciences Sector, Natural Resources Canada, Ottawa, ON, Canada.

Introduction: The origin of the Sudbury Igneous Complex (SIC) as the remnant of the coherent impact melt sheet at the Sudbury impact structure is now generally accepted. Hindering its early recognition were the fact that is was differentiated, due to its great thickness, and is not flat-lying but somewhat funnel-shaped, due to almost immediate postimpact folding and thrusting. It also has no apparent foot and roof rocks, which are relatively finer-grained melt rocks, with high lithic and mineral clast contents, as observed at the base and top of coherent melt sheets at other impact structures. Here, we present the results of field, petrographic and geochemical studies of the so-called Onaping Intrusion, and discuss its relationship to the SIC.

Results: The so-called basal Onaping Intrusion occurs at approximately $50 \%$ of the upper contact of the Granophyre of the SIC and the overlying breccias of the Onaping Formation [1]. Near Joe Lake, in the North Range of the SIC, this lithology has a fine-grained (0.2$0.5 \mathrm{~mm}$ ) igneous-textured matrix, consisting mainly of interlocking intergrowths of plagioclase, quartz, K-feldspar, amphibole, clino-pyroxene and biotite. It also contains $>5$ to approximately $50 \%$ mineral and lithic clasts, with corroded margins indicative of partial melting, corresponding to the lithologies of the original target area. Some quartz grains, usually within granitoid or quartzite clasts, exhibit partially annealed, decorated PDFs. Locally, clast content decreases and matrix grain-size increases toward the contact with the underlying Granophyre of the SIC. Samples closer to the Granophyre exhibit fine-grained granophyric matrix textures and the contact with the Granophyre can be described as gradation over a few meters. X-ray fluorescence analyses of the Onaping Intrusion at Joe Lake show that it is average composition is more siliceous (approximately $67 \%$ ) than the average of the currently exposed SIC (approximately 63\%) and the North Range Offset dikes (approximately 59.2-61.9\%) [2, 3].

Discussion: The matrix texture and the presence of clasts with PDFs demonstrate that the basal Onaping Intrusion conforms to the descriptive definition of an impact melt rock. This, together with the geochemical similarities to the SIC and the field relations suggest that the basal Onaping Intrusion may be the roof rocks of the SIC. The early failure to recognize the basal Onaping Intrusion as the potential roof rocks of the SIC may be attributable to the fact that it only occurs at $50 \%$ of the SIC's upper contact with the Onaping Formation. Its absence is likely due to its destruction as a coherent lithology and local removal by explosive melt-fuel-coolant interactions (MFCI) between incoming sea-water and the superheated proto-SIC impact melt within the Sudbury impact structure [4]. These interactions led to the formation of the bulk of the lithologies of the Onaping Formation as a series of impact melt-related "phreatomagmatic" breccias, containing vitric proto-SIC clasts and only a minor component of impact-related shocked materials.

References: [1] Ames D. E. et al. 2008. Economic Geology 103:10571077. [2] Ames D. E. et al. 2002. Economic Geology 97:1541-1562. [3] Carter W. M. et al. 2009. Geological Survey of Canada Open File 6134. [4] Grieve R. A. F. 2010. Meteoritics \& Planetary Science 45:759-782.
5403

TEM STUDY OF MATRIX IN THE CO3 CHONDRITE ALHA77307: CLUES ABOUT THE FIRST STAGES OF METAMORPHISM IN CHONDRITES

C. E. Brunner and A. J. Brearley. Department of Earth and Planetary Sciences, MSC03-2040, 1 University of New Mexico, Albuquerque, NM 87131, USA. E-mail: cbrunner@unm.edu, brearley@unm.edu

Introduction: The fine-grained matrices of carbonaceous chondrites are sensitive recorders of both nebular and asteroidal processes in the early solar system. The matrices of type 3.0 carbonaceous chondrites consist of a complex assemblage of very fine-grained, unequilibrated materials (silicates, sulfides, oxides, etc.) that are particularly susceptible to modification by aqueous alteration and thermal metamorphism [1]. Only in very pristine chondrites (type 3.0) is matrix minimally altered, preserving a primary nebular record. We are examining matrix silicates in the most pristine CO3 chondrite, ALHA77307 (type 3.0), as part of study to investigate the formation and metamorphism of crystalline silicates in type 3.0 carbonaceous chondrite matrices.

Experimental: Electron-transparent sections of ALHA77307 matrix were prepared using focused ion beam (FIB) techniques. The chemical and microstructural properties of the FIB sections where characterized in detail using a JEOL 2010F FEG/STEM.

Results and Discussion: All FIB sections of ALHA77307 matrix are composed largely of extremely fine-grained materials $(<100 \mathrm{~nm}$ in size). Each FIB section contains similar silicate assemblages that occur in clusters surrounded by amorphous matrix material, consistent with previous observations [1]. The FIB sections are dominated by an amorphous silicate groundmass in which nanoparticles of iron oxides, $\mathrm{Fe}$ and $\mathrm{Fe}, \mathrm{Ni}$ sulfides $(<200 \mathrm{~nm})$, olivine and pyroxene grains are embedded [1]. In addition, our new studies have also revealed what appears to be a population of distinct, amorphous ferromagnesian grains that were not recognized in the previous study [1].

ALHA77307 contains a population of Mg-rich olivine and pyroxene grains that are probably primary in origin [1]. Each FIB section contains several distinct, fine-grained $(>400 \mathrm{~nm})$ subhedral to anhedral forsterite grains that usually occur in clusters, but also occur as isolated crystals. Dark field STEM imaging of the olivine and amorphous $\mathrm{Mg}-\mathrm{Fe}$ silicate grains shows distinct Fe-rich rims on these grains which are usually 10$30 \mathrm{~nm}$ in width. STEM EDS analysis and STEM line profiles across these grains confirm the Fe-enrichment in their rims. The olivine core compositions lie in the range $\mathrm{FO}_{78-99}$ while $\mathrm{Fe}$-enriched rims range from $\mathrm{Fo}_{40-80}$. Fine-grained $(>400 \mathrm{~nm})$ low-Ca pyroxenes $\left(\mathrm{En}_{75-99}\right)$ show no evidence of Fe-enrichment at their grain edges.

We interpret the presence of narrow Fe-rich rims on olivine and amorphous silicate grains as representing the very first stages of metamorphic equilibration in the matrix of ALHA77307. These features were not recognized previously by conventional TEM studies [1]. Nevertheless, the effects are so limited and subtle that it further confirms the highly pristine nature of this meteorite. However, it does demonstrate that ALHA77307 has not completely escaped metamorphism, consistent with the arguments of [1], but provides the first glimpse of the process of metamorphism in the finest grain fraction of chondritic materials.

References: [1] Brearley A. J. 1993. Geochimica et Cosmochimica Acta 57:1521-1550. [2] Brearley A. J. and Jones R. H. 1998. In Planetary materials 3-1:3-398. [3] Brearley A. J. 1995. In Chondrules and the Protoplanetary Disk. pp. 137-151. 


\section{8}

\section{A POSSIBLE NEW IMPACT SITE NEAR NALBACH (SAARLAND, GERMANY)}

E. Buchner ${ }^{1,3}$, W. Müller ${ }^{2}$ and M Schmieder ${ }^{3}$. ${ }^{1}$ Rieskrater-Museum Nördlingen, Eugene-Shoemaker-Platz 1, 86720 Nördlingen, Germany. E-mail: elmar.buchner@geologie.uni-stuttgart.de. ${ }^{2}$ Diefflerstr. 217, 66809 Nalbach/Saar, Germany. ${ }^{3}$ Institut für Planetologie, Universität Stuttgart, Herdweg 51, 70174 Stuttgart, Germany.

Introduction: The two impact craters Nördlinger Ries and the Steinheim Basin (S Germany) represent the only confirmed impact structures in Germany. Although a great number of possible impact structures in many parts of Germany were proposed in the past, none of them was confirmed. We suggest a new possible impact site in the German state of the Saarland (W Germany) on the base of possible impact glasses and potential traces of an iron meteorite in melt particles that are distributed within an area of some square kilometers in the surroundings of the village of Nalbach. The possible impact melt particles were recently discovered by one of the authors (W. Müller).

Petrographic and Geochemical Analyses: Externally, the melt particles exhibit a bluish to black color and are often aerodynamically deformed (tear- or spindle-shaped). The particles contain a homogenous, schlierenand vesicle-textured glassy melt groundmass; roundish sandstone particles are incorporated into the melt matrix. Geochemically, the melt matrix yielded a Si-, Al-, and K-rich mixed silicatic composition. We have not detected shocked quartz grains with PDFs so far, however, some of the melt particles show conspicuous ballen-textured $\alpha$-cristobalite (Fig. 1A) and contain spherule-shaped iron droplets (Fig. 1B). Preliminary geochemical SEM-EDX analyses showed that the Fe droplets commonly contain higher amounts of $\mathrm{Ni}$ (up to $\sim 10 \mathrm{wt} \%$ ), together with variable amounts of $\mathrm{S}, \mathrm{P}$, $\mathrm{Ni}$, and $\mathrm{Co}$. We also detected metallic fragments that exhibit a composition close to taenite, troilite, and schreibersite as known from meteorites. Further analyses are in progress.

Conclusions: Ballen $\alpha$-cristobalite, detected in some melt particles, was recently suggested as an impact-diagnostic feature [1]. Externally and internally, the Nalbach melt particles closely resemble impact glasses from Wabar [2,3] and other terrestrial impact sites by their color, texture, composition, and content of metallic particles. The metallic droplets and fragments in the melt particles may be interpreted as the molten and fragmented remnants of an iron meteorite. The melt particles are distributed over some $\mathrm{km}^{2}$ that could represent an (eroded or covered) impact crater or an area affected by a larger meteoritic airburst.

References: [1] Ferrière L. et al. 2009. European Journal of Mineralogy 21:203-217. [2] Schmieder M. and Buchner E. 2009. Abstract volume of the 1st AICAC Conference, Amman, Jordan, CD-ROM. [3] Schmieder M. et al. 2011. This volume.

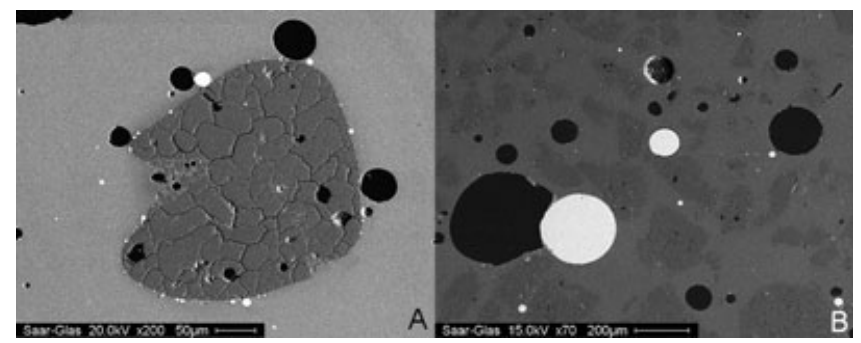

Fig. 1. Nalbach glass. A: Ballen $\alpha$-cristobalite and B: Fe-Ni droplets (white) in a vesicular glassy melt matrix (backscattered electron images).
5135

MAPPING OF MICROSTRUCTURAL DEFORMATION IN EXPERIMENTAL IMPACT CRATERS FORMED IN SANDSTONE

E. Buhl ${ }^{1,2}$, M. H. Poelchau ${ }^{1}$, T. Kenkmann ${ }^{1}$ and G. Dresen ${ }^{2} .{ }^{1}$ A.-L.-Univ. Freiburg, Germany, E-mail: e.buhl@gmx.de. ${ }^{2}$ German Research Center for Geosciences, Potsdam, Germany.

Introduction: As part of the MEMIN project experimental hypervelocity impacts into porous sandstone were conducted at the ErnstMach-Institut (EMI), Germany [1]. In combination with real-time investigations of the ejection process and ultrasound signals, spatially resolved impact damaging of the target was systematically investigated with a scanning electron microscope (SEM). As first result four distinct subsurface zones can be discriminated by their deformation mechanisms.

Methods: A $2.5 \mathrm{~mm}$ sphere of high alloyed steel impacted into a $20 \mathrm{~cm}$ sandstone cube with a velocity of $4.8 \mathrm{~km} \mathrm{~s}^{-1}$. Acceleration was achieved by a two stage light gas gun (Space Gun, EMI) [2, 3]. The sandstone has a porosity of ca. $22 \%$ and a quartz content of $94 \mathrm{wt} \%$. The sub-rounded quartz grains are cemented by quartz and clay mineral coatings. For investigations the impacted block was bisected parallel to the impact direction and thin sections were prepared directly underneath the crater floor. For a detailed mapping ca. 300 SEM photomicrographs $(160 \times$, BSE) were merged to a high resolution image of the crater subsurface.

Results: The formed crater has a diameter of $5.76 \mathrm{~cm}$ and a depth of $1.1 \mathrm{~cm}$. Deformation gradually decreases with increasing distance from the crater floor. The outermost zone $\left(>3.6\right.$ projectile diameters $\left(\varnothing_{p}\right)$ from the impact center) of apparent deformation is dominated by concussion fractures which are well known from natural impacts [4]. These intragranular fractures occur on grain-grain contacts and have a preferred radial orientation. Macroscopic radial fractures as described for cratering in basalt [5] were not detected. The second zone of deformation (2.9$3.6 \varnothing_{\mathrm{p}}$ from the impact center) is characterized by localized compactional shear bands in which grain comminution along with shearing and compaction occurs. This zone has a hemispherical appearance beneath the crater floor with a thickness of ca. $2.2 \mathrm{~mm}$. In the third zone (1.7-2.9 $\varnothing_{\mathrm{p}}$ from the impact center) the compaction and grain crushing is pervasive. Pore space is completely crushed and filled with small quartz fragments and clay mineral aggregations. This zone has a maximum thickness of $3.1 \mathrm{~mm}$. The innermost zone (1.4-1.7 $\varnothing_{\mathrm{p}}$ from the impact center) of deformation in contact to the crater floor has a maximum thickness of $0.9 \mathrm{~mm}$. In addition to the deformation described for zone three, tensional fractures occur sub-parallel to the crater floor. These fractures are up to $1.2 \mathrm{~mm}$ long and formed new pore space in the compacted host rock. Macroscopic spall fractures are traceable laterally at distances $>5 \varnothing_{\mathrm{p}}$ from the impact center

Conclusion: The sub-surface deformation in shocked porous sandstone can be differentiated into four different zones by the occurrence of different deformation types. A more detailed analysis of deformational modes is planned to give further insights into deformation mechanisms in porous targets during shock loading.

References: [1] Kenkmann T. et al. 2011. Meteoritics \& Planetary Science, in press. [2] Schäfer F. et al. 2006. ESA SP-612. [3] Poelchau M. H. 2011. Abstract \#1824. 42nd Lunar and Planetary Science Conference. [4] Kieffer S. W. 1971. Journal of Geophysical Research 76:5449-5473. [5] Polanskey C. A. and Ahrens T. J. 1990. Icarus 87:140-155 
5437

OXYGEN ISOTOPES IN PYROXENES FROM TWO VIGARANO CAIS VARY INDEPENDENTLY OF $\boldsymbol{f} \mathrm{O}_{2}$

E. S. Bullock ${ }^{1}$, G. J. MacPherson ${ }^{2}$, A. N. Krot ${ }^{2}, K$. Nagashima $^{2}$, T. Ushikubo $^{3}$ and N. T. Kita ${ }^{3}$. ${ }^{1}$ US National Museum of Natural History, Smithsonian Institution, Washington, D.C., 20560 USA. E-mail: BullockE@si.edu. ${ }^{2}$ Hawai'i Institute of Geophysics and Planetology, University of Hawai'i at Mãnoa, Honolulu, HI 96822, USA. ${ }^{3}$ Department of Geosciences, University of Wisconsin, Madison, WI 53706, USA.

Introduction: A hallmark of Ca-Al-rich inclusions (CAIs) is enrichment in most of their constituent minerals in ${ }^{16} \mathrm{O}$, taken to be the isotopic signature of the gas in which they formed. Other minerals that are ${ }^{16} \mathrm{O}$-poor likely underwent later exchange with a different isotopic reservoir after formation [e.g., 1]. However, recent work [2] has shown that some CAIs sampled multiple distinct reservoirs during their primary formation, either as a result of temporal variations in the composition of the gas or because the CAIs were transported to different isotopicallydistinct regions, before being accreted into the parent body. Analyses of the isotopic and chemical compositions of pyroxene from two different Vigarano CAIs (F6; a compact type A, and F8; a fluffy type A) sheds new light on the environment in which the CAIs underwent isotopic exchange.

Methods: Minerals were analyzed with a JEOL 8900 electron probe at the Smithsonian Institution. Oxygen isotopes were analyzed using the University of Hawai'i Cameca ims 1280, in multicollector mode (see [3]). $\mathrm{Al}-\mathrm{Mg}$ isotopes were analyzed using the University of Wisconsin Cameca ims 1280 (see [4]).

Results and Discussion: High-precision $\mathrm{Al}-\mathrm{Mg}$ dating of $\mathrm{F} 6$ and $\mathrm{F} 8$ [4] gives initial ${ }^{26} \mathrm{Al} /{ }^{27} \mathrm{Al}$ ratios of $(5.3 \pm 0.3) \times 10^{-5} \quad$ (F8) and $(4.2 \pm 0.4) \times 10^{-5}(\mathrm{~F} 6)$, corresponding to a approximately $200,000 \mathrm{yr}$ time difference. The Ti-rich pyroxene in $\mathrm{F} 6$ plots on the ${ }^{26} \mathrm{Al}$ isochron and is ${ }^{16} \mathrm{O}$-rich $\left(\Delta^{17} \mathrm{O}=-20.6 \pm 1.6 \%\right.$ and $\left.-23.8 \pm 2.0 \%\right)$; similar pyroxene in F8 plots on the F8 ${ }^{26} \mathrm{Al}$ isochron but is consistently ${ }^{16} \mathrm{O}$-poor $\left(\Delta^{17} \mathrm{O}=-2.6\right.$ to $\left.-4.1 \pm 1.5 \%\right)$. The pyroxene in $\mathrm{F} 8$ rims (replaces) perovskite, which also is ${ }^{16} \mathrm{O}$-poor, so the pyroxene must postdate perovskite exchange. Pyroxene in both F6 and F8 contains on average approximately $60 \% \mathrm{Ti}^{3+}$. Pyroxene in the Wark-Lovering rim (WL) of both inclusions is ${ }^{16} \mathrm{O}$-rich $\left(\Delta^{17} \mathrm{O}\right.$ approximately -22 to $-23 \pm 2.8 \%$ o $)$. F6 is almost uniformly ${ }^{16} \mathrm{O}$-rich, even melilite. Melilite in $\mathrm{F} 8$, however, is variably depleted in ${ }^{16} \mathrm{O}$.

During the time of F6 and F8 evolution, O-isotopes in their environs varied greatly yet $f \mathrm{O}_{2}$ did not. This is hard to reconcile with CAIs transport from dust-poor to relatively dust-rich environments [2]. Low $f \mathrm{O}_{2}$ persisted during the formation of the WL rims [5], yet these two CAIs solidified 200,000 yr apart. Either the rims themselves formed at different times, or else all rims formed late at a time that postdated the formation of F6.

References: [1] Clayton R. N. et al. 1977. Earth and Planetary Science Letters 34:209-224. [2] Yurimoto H. et al. 2008. Reviews in Mineralogy and Geochemistry vol. 68:141-187. [3] Makide K. et al. 2009. Geochimica et Cosmochimica Acta 73:5018-5050. [4] MacPherson G. J. et al. 2010. Abstract \#2356. 41st Lunar and Planetary Science Conference. [5] Simon S. et al. 2007. Geochimica et Cosmochimica Acta 71:3098-3118.
5224

NORTHWEST AFRICA 6698: A HIGH TEMPERATURE "DIORITIC" ACHONDRITE WITH OXYGEN ISOTOPE COMPOSITION IN THE LL CHONDRITE FIELD

T. E. Bunch ${ }^{1}$, A. J. Irving ${ }^{2}$, J. H. Wittke ${ }^{1}$ and D. Rumble III ${ }^{3}$. ${ }^{1}$ Geology Program, SESES, Northern Arizona University, Flagstaff, AZ 86011, USA, E-mail: tbear1@cableone.net ${ }^{2}$ Earth \& Space Sciences, University of Washington Seattle, WA 98195, USA. ${ }^{3}$ Geophysical Laboratory, Carnegie Institution, Washington, DC 20015, USA.

Petrography: NWA 6698 is a small (38.4 g), fresh stone with unusual mineralogical and isotopic characteristics. It is a fine to medium grained $(<2 \mathrm{~mm})$ "dioritic" assemblage of relatively sodic plagioclase $(69$ vol \%), pyroxenes $(27 \mathrm{vol} \%)$ and accessory taenite, chromite $(\mathrm{cr} \#=90)$, chlorapatite and troilite. Plagioclase displays simple albite and Carlsbad twinning, is mostly equigranular, and contains included pyroxene chadacrysts. Two pyroxenes (augite and unexsolved pigeonite) are present as grains of highly variable size (up to $2 \mathrm{~mm}$ ), and contain included plagioclase chadacrysts. Sparse pockets of brown, glassy late stage residuum with microlites occur between the other phases. Undulatory extinction in plagioclase and some pyroxenes implies mild shock levels.

Silicate Compositions: Most plagioclase ranges in composition from $\mathrm{An}_{22.4} \mathrm{Or}_{2.0}$ to $\mathrm{An}_{36} \mathrm{Or}_{2.2}$; a few zoned grains have cores of $\mathrm{An}_{52-56} \mathrm{Or}_{2.1}$ and rims of $\mathrm{An}_{36} \mathrm{Or}_{2.1}$, and albite in residuum pockets is $\mathrm{An}_{10} \mathrm{Or}_{5.7}$. Both augite $\left(\mathrm{Fs}_{18.5-27.0} \mathrm{Wo}_{36.7-41.2} ; \mathrm{FeO} / \mathrm{MnO}=14-17, \quad \mathrm{Al}_{2} \mathrm{O}_{3}=2.2 \mathrm{wt} \%\right.$, $\mathrm{Na}_{2} \mathrm{O}=1.2 \mathrm{wt} \% ; \mathrm{TiO}_{2}=1.2 \mathrm{wt} \% ; \mathrm{Cr}_{2} \mathrm{O}_{3}=1.6 \mathrm{wt} \%$ ) and pigeonite $\left(\mathrm{Fs}_{34.4-39.7} \mathrm{Wo}_{8.6-14.5} ; \mathrm{FeO} / \mathrm{MnO}=20, \mathrm{Al}_{2} \mathrm{O}_{3}\right.$ up to $\left.3.2 \mathrm{wt} \%\right)$ are notably aluminous. Residuum glass contains (in wt \%): $\mathrm{SiO}_{2} 70, \mathrm{Al}_{2} \mathrm{O}_{3} 13.8, \mathrm{FeO}$ 2.8, $\mathrm{CaO} 2.1, \mathrm{Na}_{2} \mathrm{O} 9.4$ and $\mathrm{K}_{2} \mathrm{O} 2.9$.

Oxygen Isotopes: Results obtained by laser fluorination of acidwashed material $\left(\delta^{18} \mathrm{O}=4.80,4.73 ; \delta^{17} \mathrm{O}=3.72,3.75 ; \Delta^{17} \mathrm{O}=1.199\right.$, 1.265 per mil, respectively) plot well above the TFL in the field for LL chondrites [1].

Discussion: Several aspects of this achondrite imply that it is a rapidly cooled, high temperature igneous rock. The coexistence of pigeonite and subcalcic augite is possible only at temperatures above $1175^{\circ} \mathrm{C}$ in Fe-free systems at 1 bar [2], but even in Fe-bearing systems the coexistence of these two pyroxenes would indicate temperatures probably above $900-1000^{\circ} \mathrm{C}$, which are consistent also with the elevated aluminum contents of both pyroxenes. The partly glassy intergranular residuum is consistent with rapid quenching of a partly crystallized magmatic liquid. The possibility that NWA 6698 might represent an impact melt rock is not in concert with the overall texture and complete lack of included clasts. Although the oxygen isotopic composition and sodic plagioclase suggest affinities with LL chondrites, the pyroxene compositions and especially their very low $\mathrm{FeO} / \mathrm{MnO}$ ratios are quite unlike those for pyroxenes in LL chondrites.

References: [1] Clayton R. et al. 1991. Geochimica et Cosmochimica Acta 55:2317-2337. [2] Longhi J. and Bertka C. 1996. American Mineralogist 81:685-695. 


\section{5}

ACAPULCOITE NORTHWEST AFRICA 6557 AND PROPOSED ESTABLISHMENT OF THE ACAPULCOITE-LODRANITE CLAN AS A SINGLE METEORITE CLASS

T. E. Bunch ${ }^{1}$, A. J. Irving ${ }^{2}$, J. H. Wittke ${ }^{1}$ and D. Rumble $\mathrm{III}^{3}$. ${ }^{1}$ Geology Program, SESES, Northern Arizona University, Flagstaff, AZ 86011, USA, E-mail: tbear1@cableone.net ${ }^{2}$ Earth \& Space Sciences, University of Washington Seattle, WA 98195, USA. ${ }^{3}$ Geophysical Laboratory, Carnegie Institution, Washington, DC 20015, USA.

Petrography: NWA 6557 is a very fresh $68 \mathrm{~g}$ crusted achondrite with a mean grainsize (gs) of $240 \mu \mathrm{m}$ exhibiting equigranular metamorphic texture with triple grain junctions. It is composed of 38 vol\% orthopyroxene $\left(\mathrm{Fs}_{12.5} \mathrm{Wo}_{2.1}, \mathrm{FeO} / \mathrm{MnO}=12\right), 35$ vol\% olivine $\left(\mathrm{Fa}_{13.9}, \mathrm{FeO} / \mathrm{MnO}=16\right), 12 \mathrm{vol} \%$ sodic plagioclase $\left(\mathrm{An}_{6.8} \mathrm{Or}_{5.5}\right), 7 \mathrm{vol} \%$ subcalcic augite $\left(\mathrm{Fs}_{8.1} \mathrm{Wo}_{36.8}\right)$, and $8 \quad$ vol\% chromite $($ cr\# $=87]+$ kamacite $(\mathrm{Ni}=6.2 \mathrm{wt} \%)$.

Oxygen Isotopes: Results obtained by laser fluorination of acidwashed NWA 6557 material $\left(\delta^{18} \mathrm{O}=3.05,2.79 ; \quad \delta^{17} \mathrm{O}=0.64,0.55\right.$; $\Delta^{17} \mathrm{O}=-0.962,-0.913$ per mil, respectively) plot within the relatively broad field for acapulcoites and lodranites.

Nomenclature Issues: Since the falls of Acapulco (mean gs $170 \mu \mathrm{m}$ ) and Lodran (mean gs $580 \mu \mathrm{m}$ ), more than 45 separate related meteorites have been found in Antarctica, Africa and elsewhere. There is a continuous range in mean grainsize among specimens assigned the names acapulcoite and lodranite (based on the arbitrary division at $340 \mu \mathrm{m}$ proposed by [1]), and grainsizes range widely even within single specimens of some of these achondrites (e.g., $50-1700 \mu \mathrm{m}$ with a mean of $550 \mu \mathrm{m}$ in NWA 4448). In the column chart below the apparent peak in the 500$550 \mu \mathrm{m}$ range is an artifact of grainsize estimates reported simply as " $0.5 \mathrm{~mm}$ " by some classifiers, rather than a precise value.

Proposal: We suggest that the term acapulcoite-lodranite clan be applied to all of the members of this group (which oxygen isotopes and CRE ages suggest derive from different portions of the same parent body [2]). The terms acapulcoite and lodranite could still be used informally for members at either end of the range, but it seems imprudent to attempt to arbitrarily assign a specific grainsize at which the name changes. The term transitional has also been used for specimens of "intermediate" grainsize, but this also seems quite arbitrary and of limited usefulness.

References: [1] McCoy T. et al. 1996. Geochimica et Cosmochimica Acta 60:2861-2908. [2] Irving A. et al. 2007. Abstract \#1338. 38th Lunar and Planetary Science Conference; Eugster O. and Lorenzetti S. 2005. Geochimica et Cosmochimica Acta 69:2675-2685.

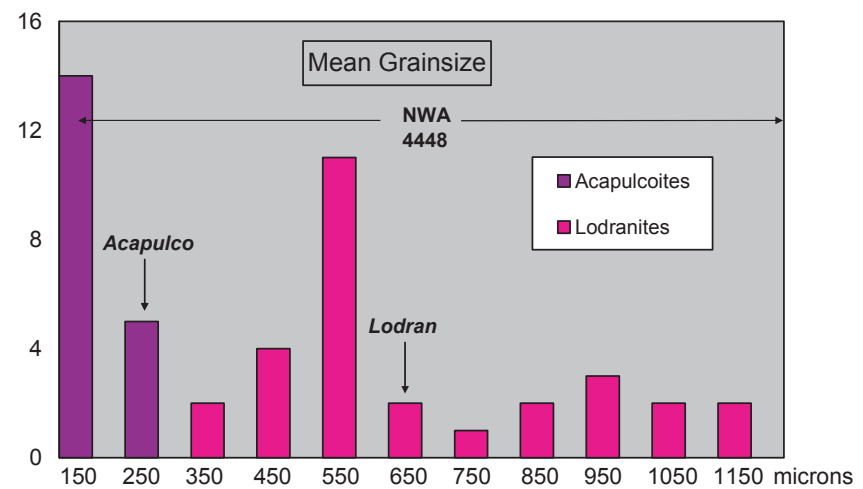

5345 ICE IMPACTS ON AEROGEL AND STARDUST AI FOIL

M. J. Burchell ${ }^{1}$, M. J. Cole ${ }^{1}$, M. C. Price ${ }^{1}$ and A. T. Kearsley ${ }^{2}{ }^{1}{ }^{1}$ School of Physical Science, University of Kent, Canterbury, CT2 7NH, UK, E-mail: m.j.burchell@kent.ac.uk. ${ }^{2}$ Impacts \& Astromaterials Research Centre (IARC), Department of Mineralogy, Natural History Museum (NHM), London SW7 5BD, UK.

Introduction: The NASA Stardust mission to comet Wild 2 returned a dust collector to Earth in 2006 (see [1] for first results and [2] for a recent summary). The main collection substrate was $\mathrm{SiO}_{2}$ aerogel, with a smaller area of aluminum (Al) foil. As a low density, highly porous material, aerogel can retain much relatively unprocessed material from many types of hypervelocity particles [3] within tracks of diverse shape, instead of as the highly disrupted and partially melted residue found in $\mathrm{Al}$ foil impact craters. Stardust flew close to the comet nucleus $(236 \mathrm{~km})$ at $6.1 \mathrm{~km} \mathrm{~s}^{-1}$, and captured numerous mineral particles in aerogel tracks [45] and foil craters [6-7]. So far, it has been assumed that no impact features were produced by ice, as modeling suggests small ice grains should have rapidly sublimated following release into the coma, disappearing before reaching Stardust (see [2] for discussion of dust transit time to the collector). However, live-time data recorded during the fly-by show a late burst of impacts, as if a larger ejected boulder broke up after leaving the comet surface [8]. Might this have still been carrying ice? Images from comet Hartley 2, taken by the EPOXI mission in 2010, show $\mathrm{cm}$-scale ice-rich grains above the comet surface [9]. We therefore ask how we might recognize if ice did impact on Stardust, and have made analogue experimental impacts on aerogel and $\mathrm{Al}$ foil at $6.1 \mathrm{~km} \mathrm{~s}^{-1}$.

Method: We used the Kent two stage light gas gun [10]. Modifications to breech and launch tube mounts for active cooling and pre-cooling of the launch tube allow mm-size solid ice projectiles to be fired. Projectiles fragment due to shock during acceleration, and a spreading shower of fragments proceeds to the target.

Discussion: Our preliminary results show ice impacts at appropriate velocity (higher than previous work [11]), by particles similar in size to Stardust impactors. Foils and Al support plates show shallow craters lined by collapsed vesicles. Broad projectile dispersion has so far yielded fewer impacts on aerogel, the first tracks are currently being characterized; they show no terminal grain or fine projectile fragments lining the wall, consistent with loss of a volatile impactor. Given the polydisperse size distribution of grains striking the targets, we do not yet have a calibration for track vs. impactor size, but this is being investigated. In summary, we can now fire ice at Stardust speeds in the gun, and have impacts corresponding to those that might have occurred from ice grains from comet Wild 2 impacting onto the Stardust collector.

References: [1] Brownlee D. E. et al. 2006. Science 314:1711-1716. [2] Burchell M. J. and Kearsley A. T. 2009. Planetary and Space Science 57:1146-1161. [3] Burchell M. J. et al. 2006. Annual Review of Earth and Planetary Sciences 34:385-418. [4] Hörz F. et al. 2006. Science 314:17161719. [5] Burchell et al. 2008. Meteoritics \& Planetary Science 43:23-40. [6] Kearsley A. T. et al. 2008. Meteoritics \& Planetary Science 43:41-74. [7] Price M. C. et al. 2010. Meteoritics \& Planetary Science 45:1409-1428. [8] Tuzzolino A. J. et al. 2006. Science 304:1776-1780. [9] A'Hearn M. F. et al. 2011. Abstract \#2516. 42nd Lunar and Planetary Science Conference. [10] Burchell M. J. et al. 1999. Meas. Sci. Technol. 10:41-50. [11] Yano H. et al. 1999 Abstract \#1961. 30th Lunar and Planetary Science Conference. 
5506

\section{SYNCHROTRON FOURIER TRANSFORM INFRARED SPECTROSCOPY ON FRAGMENTS OF INTER-PLANETARY DUST PARTICLES (IDPS)}

H. Busemann ${ }^{1}$, S. Bajt ${ }^{2}$, N. H. Spring ${ }^{1}$ and L. R. Nittler ${ }^{3}$. ${ }^{1}$ SEAES, University of Manchester, UK. E-mail: henner.busemann@manchester. ac.uk. ${ }^{2}$ DESY, Hamburg, Germany. ${ }^{3}$ DTM, Carnegie Institution of Washington DC, USA.

Introduction: IDPs are among the most primitive materials available for laboratory analysis and contain pristine material essentially unaltered since solar system formation [1]. As $>80 \%$ may originate from comets [2], they allow the study of comets in the laboratory without sample return [3]. Our ultimate goal will be the (destructive) examination of the heavy noble gases in IDPs to assess the volatile contents in comets [4]. Before we will perform various correlated analyses including Raman spectroscopy [5] to identify the material that is most likely of cometary origin.

Here we present preliminary FTIR results from a first set of IDP fragments. The IDP analyses yield large spectral variations in the organic matter.

Experimental: Without any sample preparation, several fragments (typically 3-4 to up to $10 \mu \mathrm{m}$ diameter) were split off bulk IDPs on NASA glass slides and transferred onto $\mathrm{KBr}$ windows. All particles were analyzed with the FTIR microscope at the ALS Berkeley $(\sim 600$ $4000 \mathrm{~cm}^{-1}$ ) in mapping mode with diffraction-limited spatial resolution [6]. The IDPs were not pressed to allow removal for future NanoSIMS analysis.

Results and Discussion: Four IDPs from collector L2036 clusters 4 and 20 show the typical aliphatic hydrocarbon signatures observed earlier [e.g., 7,8]. Two of those may also indicate primary amine functionality, as one IDP from L2006. Both Raman [5] and FTIR spectroscopy of L2011 cluster 1 IDP fragments suggest only little OM, consistent with their nonchondritic-porous, metal-rich nature. A fragment from a L2005 cluster 26 IDP did not yield IR bands for OM above the detection limit, whereas another fragment shows the typical Raman parameters indicative of disordered C. Comet Grigg-Skjellerup collection IDPs were analyzed so far as two fragments of a L2054 cluster 1 IDP. Whilst one fragment does not yield IR results for OM above detection limit, the other shows potentially the signature of $\mathrm{NO}_{2}$ compounds, but no aliphatic hydrocarbon bands. Raman spectroscopy on a different fragment indicates particularly disordered OM. One IDP from L2006 suggests carbonate.

These analyses will be supplemented by SEM and NanoSIMS examinations of both the fragments analyzed here by FTIR and their main IDP masses to determine chemistry and mineralogy, and the presence of isotopically anomalous organic matter and presolar grains. The preliminary tentative detection of carbonate, $\mathrm{NO}_{2}$, primary amine and widespread aliphatic hydrocarbon $\left(\mathrm{CH}_{2}\right.$ and $\mathrm{CH}_{3}$ stretch functionality in the IDPs is consistent with their primitive, essentially unheated character and their origin from a variety of parent bodies including comets, where the $\mathrm{OM}$ did not experience homogenization and destruction.

References: [1] Bradley J. P. 2003. Treatise on Geochemistry vol. 1:689-711. [2] Nesvorný D. et al. 2010. The Astrophysical Journal 713:816-836. [3] Busemann H. et al. 2009. Earth and Planetary Science Letters 288:44-57. [4] Busemann H. et al. 2010. Abstract \#1947. 41st Lunar and Planetary Science Conference. [5] Spring N. H. and Busemann H. 2011. Meteoritics \& Planetary Science 46: this volume. [6] Bajt S. et al. 2009. Meteoritics \& Planetary Science 44:471-484. [7] Matrajt G. et al. 2005. Astronomy \& Astrophysics 433:979-995. [8] Flynn G. J. et al. 2003. Geochimica et Cosmochimica Acta 67:4791-4806.
5289

INSIGHTS ON THE THERMAL HISTORY OF THE PARIS CM CHONDRITE BASED ON THE PHASE RELATIONS OF ITS OPAQUE ASSEMBLAGES

C. Caillet Komorowski ${ }^{1}$, O. Boudouma ${ }^{2}$, B. Reynard ${ }^{3}$, B. Van de Moortèle $^{3}$ and A. El Goresy ${ }^{4}$. ${ }^{1}$ Muséum National d'Histoire Naturelle, LMCM, UMR CNRS 7202, Paris, France. E-mail: ccaillet@mnhn.fr. ${ }^{2}$ UPMC-ISTEP, Paris. ${ }^{3}$ ENS - Laboratoire de Géologie de Lyon, UMR CNRS 5276. ${ }^{4}$ Bayerishes Geoinstitut, Universität Bayreuth, Germany.

We studied the characteristic features of FeNi metals and sulfides in matrix, around clasts and in chondrules of the primitive Paris CM chondrite [1] to explore its thermal history. Two polished thin sections were optically studied and at the FESEM. EMPA and Raman spectra were obtained on selected minerals.

Paris is a primitive microbrecciated chondrite with well-preserved chondrules containing large quantities of $\mathrm{FeNi}$ metal globules, heterogeneously distributed refractory inclusions, altered clasts (PCP), phenocrysts, $\quad 150-300 \mu \mathrm{m}$ rounded metal grains surrounded by accretionary rims and a fine-grained matrix locally altered. The opaque minerals consist of FeNi metal, FeNi sulfides, rare phosphides and oxides. $\mathrm{X}$-ray mapping shows the ubiquitous presence of sulfur-bearing components in matrix. Here, we describe sulfides: mainly pyrrhotite, pentlandite $(\mathrm{Pn})$, other FeNi-sulfides, and very rare stoichiometric troilite. They occur as (1) tiny matrix grains, (2) ubiquitous large grains of pyrrothite containing blebs or flames of $\mathrm{Pn}$, and (3) associated with Fe-rich olivine as isolated entities plastered around clasts or chondrules. No $\mathrm{Hg}$ minerals were found [2].

Chemical compositional of sulfides in matrix and chondrule rims is identical suggesting similar formation conditions. Pn crystals have up to $1.35 \mathrm{wt} \% \mathrm{Co}$. Only sulfides associated with $\mathrm{Fe}$-rich olivines contain $\mathrm{Cr}$. The $\mathrm{Ni}$ content of metal ranges between 4 and $15 \mathrm{wt} \%$ and is positively correlated with Co content $(0.17-0.6 \mathrm{wt} \%)$. $\mathrm{P}$ is up to $0.4 \mathrm{wt} \%$ and $\mathrm{Cr}$ up to $1 \mathrm{wt} \%$. Tetrateanite contains $1.7 \mathrm{wt} \%$ Co. Kamacite is far more abundant than taenite.

Other opaques in matrix include tiny grains of $(\mathrm{FeNi})_{3} \mathrm{P}$ and a $100 \mu \mathrm{m}$ idiomorphic Pn grain. These grains formed before accretion on the Paris parent body. Presence of Pn limits the upper temperature bound of heating of bulk Paris to $<610^{\circ} \mathrm{C}$ in agreement with [3]. Several broken sulfates, PCP-rich clumps, carbonates (about $40 \mu \mathrm{m}$ ) were incorporated and further altered on the parent body. Gypsum surrounds some carbonates.

Idiomorphic (hexagonal or trigonal) and rounded silica inclusions, up to $4 \mu \mathrm{m}$ large, are present in large isolated metallic globules in the matrix, often surrounded by accretion rims, or occur in metal of chondrules. We identified cristobalite and silica glass. $\mathrm{SiO}_{2}$ glass was found as hexagonal inclusion in kamacite. High temperature silica polymorph demonstrates that a high thermal event at high $f \mathrm{O}_{2}$ (probably formation by oxidation of Si-bearing $\mathrm{FeNi}$; [4]) followed by rapid quenching prior to accretion of the metal in the meteorite. Presence of glass, cristobalite, and large fragments of Pn strongly suggests that only low temperature metamorphism occurred in the meteorite after accretion. FIB-TEM of these idiomorphic inclusions are in progress.

References: [1] Bourot-Denise M. et al. 2010. Abstract \#1683. 31st Lunar and Planetary Science Conference. [2] Caillet Komorowski C. et al. 2010. Meteoritics \& Planetary Science 45:A28. [3] Kimura M. et al. 2011. Meteoritics \& Planetary Science 46:431-442. [4] Hewins and Zanda B. 1992. Meteoritics 27:233. 
5242

\section{SERS INVESTIGATION OF NUCLEOBASES DEPOSITED ON EVAPORITE MINERALS: A TEST CASE FOR ANALOG MARS REGOLITH}

S. Caporali ${ }^{1,2}$, V. Moggi-Cecchi ${ }^{1}$, M. Pagliai ${ }^{2}$, G. Pratesi ${ }^{3}$, V. Schettino ${ }^{2,4}$. ${ }^{1}$ Museo di Scienze Planetarie, Provincia di Prato, Via Galcianese, 20/h, I-59100 Prato, Italy. ${ }^{2}$ Dipartimento Chimica, Università di Firenze, Via della Lastruccia, 3, I-50019, Sesto Fiorentino, Italy. E-mail: stefano.caporali@unifi.it. ${ }^{3}$ Dipartimento di Scienze della Terra, Università di Firenze, Via La Pira, 4, I-50121, Florence, Italy. ${ }^{4}$ European Laboratory for NonLinear Spectroscopy (LENS) Università di Firenze, Via Carrara 1, I-50019, Sesto Fiorentino, Italy.

Introduction: Traditionally, Micro-Raman spectroscopy is one of the primary analytical techniques for the detection of minerals and organic molecules in the laboratory. However, in owe of its versatility and capability to interrogate samples without preliminary purification or concentration treatments, it is one of the frontrunners for the next generation of in situ instrument designed to explore solar system bodies. In particular the Raman capability to unambiguously detect organic molecules and biomarkers might result of great importance to understand if and how primitive life generates in extraterrestrial environments (e.g., Mars). The technique's analytical sensitivity towards organic molecules can be further enhanced by means of the interaction with coin metals nanoparticles [1]. This approach, named Surface Enhanced Raman Scattering (SERS) has proved to be able to detect traces amount of nucleobases adsorbed on Terrestrial [2] or Martian [3, 4] substrates. Even if these studies have shown the suitability of SERS to detect tiny amounts of biomarkers in magmatic rocks, other substrates such as evaporites should result more prone to host biomolecules. According to orbital and rover observations [5, 6] as well as the presence of tiny amounts of evaporitic minerals in nakhlites (Martian meteorites) [7], Martian evaporites are considered to be constituted by carbonates, sulfates and clay minerals. In the present study we focused on the identification of nucleobases on Martian analog material in order to test the SERS response on such kind of mineral substrates.

Experimental and Results: Two different nucleobases (adenine and hypoxanthine) were deposited on crushed samples made of carbonates (calcite, dolomite, magnesite and siderite) and sulfates (anhydrite and gypsum). Then the Raman spectra were collected under air at room temperature on every single mineral phase. Without depositing $\mathrm{Ag}$ nanoparticles (Ag-nps) only the bands attributable to the substrate were observed. However, the addition of Ag-nps enhanced the intensity of the Raman bands of the two nucleobases allowing their unambiguous identification and assessing the SERS capability to deal with subpicogram amounts of nucleobases.

Acknowledgments: The authors would like to thank Regione Toscana for financial support of the project LTSP through the found POR FSE 2007-2013 (Obiettivo 2,Asse IV).

References: [1] K. Kneipp et al. (eds). 2006. Surface-Enhanced Raman Scattering: Physics and Applications. [2] M. Muniz-Miranda et al. 2010. Journal Raman Spectroscopy 41:12-15. [3] El Amri C. et al. 2004. Journal Raman Spectroscopy 35:170-177. [4] S. Caporali et al. 2011. Abstract \#1401. 42nd Lunar and Planetary Science Conference. [5] Bibring J.-P. et al. 2005. Science 307:1576-1581. [6] Chevrier V. and Math P. E. 2007. Planetary and Space Science 55:289-314. [7] Bridges J. C. and Grady M. M. 2000. Earth and Planetary Science Letters 176:267-279.

\section{5}

Ar-Ar CRYSTALLIZATION AGES OF SHERGOTTITE RBT 04262 MINERAL SEPARATES

J. A. Cartwright ${ }^{1}$ and R. Burgess ${ }^{2}$. ${ }^{1}$ Max Planck Institute for Chemistry, J.-J.-Becher-Weg 27, 55128, Mainz, Germany. ${ }^{2}$ SEAES, University of Manchester, Oxford Road, Manchester, M13 9PL, UK. E-mail: julia.cartwright@mpic.de

Introduction: For most chronometers including Ar-Ar, the crystallization ages of shergottites show groupings at $165-180 \mathrm{Ma}$, $375 \mathrm{Ma}$ and 474-900 Ma [1-3]. However, crystallization ages determined by $\mathrm{Pb}-\mathrm{Pb}$ dating suggest ages of $\sim 4 \mathrm{Ga}$, with [4] citing isotopic resetting as the cause for younger ages in other chronometers. In addition, the presence of trapped ${ }^{40} \mathrm{Ar}$ components, including terrestrial atmosphere, has been suggested as a cause for elevated ages in both nakhlites and shergottites [5]. Having observed evidence for trapped air in previously reported Xe analysis of Roberts Massif 04262 (RBT 04262) [6], here we report apparent ages based on the Ar-Ar technique for RBT 04262 mineral separates, to assess whether and to what extent trapped air can account for variable ages.

Meteorite description and Methodology: Enriched shergottite RBT 04262 shows two distinctive lithologies. The first has coarse-grained pyroxene oikocrysts and rare olivine, whilst the second is fine-grained with pyroxene, olivine and maskelynite [7]. Previous research reported crystallization ages of $225 \pm 21 \mathrm{Ma}$ (Lu-Hf, [8]), $167 \pm 6 \mathrm{Ma}$ (Rb-Sr, [9]) and $174 \pm 14 \mathrm{Ma}(\mathrm{Sm}-\mathrm{Nd}$, [9]). As in [6], a small chip of RBT 04262,14 (2nd lithology) was crushed and mineral separates hand-picked into 8 aliquots: 4 of olivine-pyroxene (Olpx), 3 of maskelynite (Mask) and 1 of opaque (Opq). Following neutron irradiation, the aliquots were analyzed for Ar isotopes using a laser step-heating technique on an MS1 noble gas mass spectrometer (Manchester).

Results and Discussion: RBT 04262 showed an average apparent $\mathrm{K}-\mathrm{Ar}$ age of $\sim 321.0 \pm 0.3 \mathrm{Ma}$, with total $\mathrm{Ar}-\mathrm{Ar}$ ages ranging between 310-1355 Ma. The Olpx aliquots yield older apparent ages (625 \pm 19 , $644 \pm 3,723 \pm 17,1355 \pm 47 \mathrm{Ma})$ than the Mask $(309 \pm 1,340 \pm 2$, $348 \pm 3 \mathrm{Ma})$ and Opq $(575 \pm 31 \mathrm{Ma})$, and show overall increasing age with temperature during stepped heating. These apparent ages are older than previously reported Lu-Hf and Sm-Nd ages of RBT 04262, and may suggest the presence of a trapped ${ }^{40} \mathrm{Ar}$ component. In addition, correcting for ${ }^{40} \mathrm{Ar}_{\mathrm{K}}$ (assumed age $174 \mathrm{Ma}$ [9]) does not significantly reduce the ${ }^{40} \mathrm{Ar}$ content, leaving a putative trapped ${ }^{40} \mathrm{Ar} /{ }^{36} \mathrm{Ar}$ of $\sim 1000$. There is a clear correlation in the Mask between ${ }^{40} \mathrm{Ar}$ and $\mathrm{K}$, and correlation in Olpx between ${ }^{40} \mathrm{Ar}$ and $\mathrm{Cl}$. This may suggest multiple trapped components including an aqueous Cl-bearing phase or Cl-rich melt inclusions in olivine. In addition, there is no obvious overall correlation between ${ }^{40} \mathrm{Ar}$ concentration and mineral separate, which may suggest its location within a minor phase (e.g., melt/fluid inclusion).

References: [1] Borg L. et al. 2001. Abstract \#1144. 32nd Lunar and Planetary Science Conference. [2] Nyquist L. E. et al. 2001. Space Science Reviews 96:105-164. [3] Symes S. J. K. et al. 2008. Geochimica et Cosmochimica Acta 72:1696-1710. [4] Bouvier A. et al. 2008. Earth and Planetary Science Letters 266:105-124. [5] Bogard D. et al. 2009. Meteoritics \& Planetary Science 44:905-923. [6] Cartwright J. A. et al. 2010. Meteoritics \& Planetary Science 45:1359-1379. [7] Mikouchi T. et al. 2008. Abstract \#2403. 39th Lunar and Planetary Science Conference. [8] Lapen T. J. et al. 2008. Abstract \#2073. 39th Lunar and Planetary Science Conference. [9] Shih C.-Y. et al. 2009. Abstract \#1360. 40th Lunar and Planetary Science Conference. 


\section{2}

"SOLAR-WIND-RICH" HOWARDITES: TRUE REGOLITH VS. CMIMPLANTED COMPONENTS

J. A. Cartwright ${ }^{1}$, D. W. Mittlefehldt ${ }^{2}$, J. S. Herrin ${ }^{2}$, S. Herrmann ${ }^{1}$ and U. Ott ${ }^{1}$. ${ }^{1}$ Max Planck Institut für Chemie, J.-J.-Becher-Weg 27, 55128, Mainz, Germany. ${ }^{2}$ NASA/Johnson Space Center, Houston, Texas, USA. E-mail: julia.cartwright@mpic.de.

Introduction: Howardite, eucrite and diogenite meteorites (collectively HED) likely originate from asteroid 4 Vesta [1], one of two asteroids targeted by NASA's Dawn mission. Many howardites (polymict breccias of E and D material) contain "regolithic" features, including impact-melt clasts, fragmental breccia clasts, and carbonaceous chondrite fragments. True regolithic nature can be determined through noble gas analysis, as solar wind (SW) is implanted into the upper-most surfaces of solar system bodies. While previous work [2] suggested that high siderophile element contents (e.g., Ni of $300-1200 \mu \mathrm{g} \mathrm{g}^{-1}$ ) were regolith indicators, we found no obvious correlation between SW and these indicators in our initial howardite noble gas analyses [3]. We observed CM-like fragments in a number of our howardites, whose textures suggest late addition to the breccia assemblage [4]. As typical CMs contain mixtures of SW (in matrix) and planetary (in clasts) components [5], we investigate the dominance of such components in SW-rich howardites. This will help determine the extent of implanted SW in HED grains vs. SW and planetary gases from CM fragments, and allow better understanding of regolith processes.

Results: Noble gas analysis was performed using furnace stepheating on an MAP 215-50 noble gas mass spectrometer. Our results are shown in Table 1. Only 4 howardites show potential SW (e.g. GCR-SW mixing ${ }^{20} \mathrm{Ne} /{ }^{22} \mathrm{Ne} 1-13.75,{ }^{21} \mathrm{Ne} /{ }^{22} \mathrm{Ne} 0.85-0.03$ [6]), of which 3 contain $\mathrm{CM}$ fragments. A pure CM clast (PRA CM) was analyzed to assess $\mathrm{CM}$ dominance compared to bulk PRA. LEW (no CM) has the highest ${ }^{20} \mathrm{Ne}_{\mathrm{t}}$ concentration and SW-Ne ratios. The CM-rich samples show typical mixes of SW (seen in $\mathrm{Ne}$ ) and planetary (seen in $\mathrm{Xe}$ ) components as observed for $\mathrm{CM}$ Murchison $\left({ }^{20} \mathrm{Ne} /{ }^{22} \mathrm{Ne} \sim 9,{ }^{136} \mathrm{Xe} /{ }^{132} \mathrm{Xe} \sim 0.322\right)$. Our estimated CM fractions (from petrological analysis [4]) are roughly consistent with observed heavy noble gas concentrations. Significant late addition of $\mathrm{CM}$ (with matrix) to howardite assemblages may thus overprint latent SW components. The effects are thus not as strong with low CM fractions (e.g. MET) - possibly due to less incorporated matrix. Elevated Xe concentrations may help identify potential CM-rich howardites.

References: [1] Drake M. J. 2001. Meteoritics \& Planetary Science 36:501-513. [2] Warren P. H. et al. 2009. Geochimica et Cosmochimica Acta 73:5918-5943. [3] Cartwright J. A. et al. 2011. Abstract \#2655. 42nd Lunar and Planetary Science Conference. [4] Herrin J. S. et al. 2011. Abstract \#2806. 42nd Lunar and Planetary Science Conference. [5] Nakamura T. et al. 1999. Geochimica et Cosmochimica Acta 63:257-273. [6] Grimberg A. et al. 2008. Geochimica et Cosmochimica Acta 72:626645 .

Table 1. "SW-rich" Howardite noble gas results.

\begin{tabular}{|c|c|c|c|c|c|}
\hline $\begin{array}{l}\text { Noble } \\
\text { Gas (cc/g) }\end{array}$ & $\begin{array}{l}\text { LEW } \\
85313\end{array}$ & $\begin{array}{l}\text { MET } \\
00423\end{array}$ & $\begin{array}{l}\mathrm{SCO} \\
06040^{*}\end{array}$ & $\begin{array}{l}\text { PRA } \\
04401\end{array}$ & $\begin{array}{l}\text { PRA } \\
\text { CM }\end{array}$ \\
\hline${ }^{22} \mathrm{Ne}\left(10^{-8}\right)$ & $36.7(8)$ & $19.6(6)$ & $2.7(2)$ & $6.6(3)$ & $4.9(2)$ \\
\hline${ }^{20} \mathrm{Ne} /{ }^{22} \mathrm{Ne}$ & $9.68(4)$ & $8.90(1)$ & $1.61(2)$ & $3.22(4)$ & $3.57(3)$ \\
\hline${ }^{21} \mathrm{Ne} /{ }^{22} \mathrm{Ne}$ & 0.157 (1) & $0.272(2)$ & $0.814(7)$ & $0.670(6)$ & 0.567 (4) \\
\hline${ }^{20} \mathrm{Ne}_{\mathrm{t}}\left(10^{-8}\right)$ & 347 (22) & $169(9)$ & $2.3(2)$ & $16.9(11)$ & $14.3(9)$ \\
\hline${ }^{132} \mathrm{Xe}_{\mathrm{t}}\left(10^{-10}\right)$ & $2.1(1)$ & $0.64(2)$ & $5.1(2)$ & 39.9 (13) & $78.0(27)$ \\
\hline${ }^{129} \mathrm{Xe} /{ }^{132} \mathrm{Xe}$ & $1.023(3)$ & $1.004(8)$ & $0.992(2)$ & $1.046(3)$ & $1.042(3)$ \\
\hline${ }^{136} \mathrm{Xe} /{ }^{132} \mathrm{Xe}$ & $0.334(1)$ & $0.358(3)$ & $0.334(1)$ & $0.324(1)$ & $0.323(1)$ \\
\hline $\mathrm{CM}$ fraction & - & $<1 \%$ & $\sim 10 \%$ & $\sim 60 \%$ & $\sim 100 \%$ \\
\hline
\end{tabular}

Errors in parentheses. $t=$ trapped. ${ }^{*}$ incomplete analysis.

\section{1}

METEORITE BENDEGÓ, A BRAZILIAN COMICS SUPER HERO

W. P. Carvalho and D. C. Rios. Instituto de Geociências, Universidade Federal da Bahia. E-mail: wilton@atarde.com.br.

Introduction: Comics are mainly written for children, but everyone knows that many grown-up readers are fond of such a visual storytelling medium. Because comics' colorful pages, characters expressions and the funny dialogue balloons attract, at a first glance, most people for its contents, that medium has been used in Brazil as a non-technical educational tool to make meteoritics more popular among kids and adults. The Bendegó iron, the most known meteorite in the Brazilian collection, is the super hero who interacts visually with school children to tell its rich history and scientific facts regarding the entrance, fall, finding, analysis and classification of meteorites.

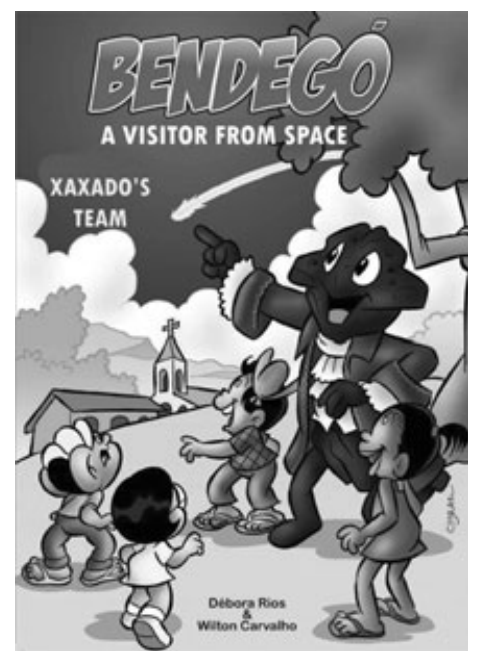

Results: Starting in October 2008 the authors and a team of nine professors and students of geology executed a 2-year academic project to identify, recover and register meteorites in Brazil. That project has opened a communication channel to teach high school students and laymen about the importance of meteorites to science and how to distinguish space rocks from terrestrial ones. A 16-page comics titled Bendegó: a visitor from space [1] was designed and 10,000 full color copies were printed in Portuguese and freely distributed to people who attended educational events such as lectures, geology congresses, astronomy parties and scientific expositions. Children and adults have enjoyed Bendegó's comics so much that other 10,000 copies are being printed to attend demand. Looking forward to make Bendegó's comics international, an English version has been set up and hopefully will be followed by other languages.

Discussion: In every country there is one meteorite which is the master-piece and the most popular among all, as Bendegó in the 60specimen Brazilian collection. "Comics have a lot of communication power in development and education." [2], so why not use that characteristic to design and execute information campaigns about meteoritics?

Acknowledgments: We are grateful to Fundação de Apoio à Pesquisa do Estado da Bahia (FAPESB) and Conselho Nacional de Desenvolvimento Cientifico e Tecnológico (CNPQ) for the financial support granted.

References: [1] Rios D. C. and Carvalho W. P. 2009. Bendegó: um visitante do espaço. Editora Cedraz, Salvador. 16 p. [2] Packalém L. and Odoi F. 2004. Comics with an attitude... A Guide to Use of Comics in Development Information. Hakapainos. 97 p. 
5334

\section{NATURAL HISTORY MUSEUM (LONDON) METEORITE COLLECTION DIGITIZATION PROJECT}

D. J. Cassey, D. A. Smith and C. L. Smith. Department of Mineralogy, The Natural History Museum, London, SW7 5BD, UK, E-mail: deboc@nhm.ac.uk

Introduction: The Meteorite Collection housed at the Natural History Museum (NHM) in London is one of the world's leading collections encompassing some 1975 individual meteorites as 4845 registered specimens with associated preparations (e.g., thin sections, powders) and curatorial documentation. The collection is a heavily used resource for scientists around the world, for example over the last five years the Museum has carried out 297 individual loans, of 1156 specimens to 93 individual borrowers. A key strategy of the NHM is to increase access to the collections and enhance the value of the collections through the integration new scientific data for each specimen. With the implementation of the KeEMu database system [1] we have initiated a number of projects to provide more detailed information on the Meteorite Collection, which be made available to scientists, historians and members of the public.

Digitization Strategy: The first element of the project was a thorough audit of the carbonaceous chondrites and achondrite meteorites. This work included weighing of all samples (including fragments, chips and powders at the milligram level), re-housing specimens into conservation grade storage, photographing all specimens and associated "permanent labels" - the permanent label is the original paper label that is created on specimen registration and all information relating to that specimen is added to the label as and when curatorial action occurs. All of this data is now entered into the EMu database adding to the level of information readily available for each specimen. Importantly this work has provided up to date holdings information for scientifically valuable samples, which we use when evaluating requests for material for study [2].

The second element of the project (still ongoing) is the digitization of all information relating to fall meteorites held in the collection (the NHM currently holds 682 of the 1095 recognized fall meteorites). This includes a full audit, photography of all specimens and permanent labels and digitization of all locally held paper records and archives including register entries, correspondence, newspaper/magazine articles, paper-held data records, chemical data and scans of thin sections.

A third element is the acquisition of compositional imagery of thin sections by automated analytical electron microscopy, which will allow for the "virtual loan" of specimens [3].

Value to the community: The NHM's significant investment in a holistic approach to collections digitization provides unrivalled levels of information on each registered specimen, considerably enhancing the collection for present and future users. In return we expect users of the collection to provide us with information obtained during their studies. Using this model, meteorite collections enhancement is a dual responsibility of both institutions and collections users. We encourage the community to consider this approach to ensure the sustainability of limited resources for the continued benefit of all.

References: [1] Sendino C. 2009. Collections: A Journal for Museum and Archives Professionals 5:149-158. [2] Smith C. L. and Welzenbach L. C. 2006. Meteoritics \& Planetary Science 41:A164. [3] Kearsley A. T. et al. 2011. This volume.

\section{9}

DO WE HAVE ANY IRONS RELATED TO THE IIIAB GROUP BY CRYSTALLIZATION AT THE FE-FES EUTECTIC?

Nancy L. Chabot ${ }^{1}$. ${ }^{1}$ Applied Physics Laboratory, Johns Hopkins Rd, Laurel, MD, 20723, USA, E-mail: Nancy.Chabot@jhuapl.edu.

Introduction: Most previous modeling work on the crystallization of iron meteorites has focused on the fractional crystallization of a molten metallic liquid up to the Fe-FeS eutectic composition [e.g. 1-5]. These models have had success at explaining the trends of irons in major magmatic groups, even if there remains disagreement on the details such as the bulk S composition [e.g., 3-5]. There is general agreement that the trends observed in these iron meteorite groups resulted from fractional crystallization, and these fractional crystallization models end when the eutectic composition is reached, even though liquid remains.

Many irons are from silicate-bearing, or non-magmatic groups, or are ungrouped irons whose chemical compositions are not explained by simple fractional crystallization. This project aims to model the expected composition of irons formed at the eutectic in the Fe-Ni-S system and to compare the model results to these irons. Here I present results of such modeling for the IIIAB group, the largest magmatic group of iron meteorites.

Modeling Approach: At the eutectic composition, both FeNi solid metal and FeS troilite crystallize. For the calculations in this model [6], it is assumed that the troilite would be pure FeS and that all Ni and siderophile trace elements would partition completely into the crystallizing solid metal, similar to the approach of [7]. The eutectic composition of the Fe-Ni-S system was expressed mathematically as a function of the liquid $\mathrm{Ni}$ and $\mathrm{S}$ contents. Published parameterizations of the solid metal/ liquid metal partition coefficients as a function of the liquid $\mathrm{S}$ content were used for 17 siderophile elements [8,9].

Results: For the IIIAB group, the predicted eutectic solid metal composition was calculated and a mixing line was determined between the pre-eutectic solid metal and the eutectic solid metal. Irons with a component of the IIIAB eutectic solid metal are characterized by: highly elevated concentrations of $\mathrm{Sb}$; $\mathrm{Au}$, As, and Co contents near those of late crystallizing IIIAB irons; elevated $\mathrm{Ni}$ contents with values up to $\sim 30 \mathrm{wt} \%$; low $\mathrm{Ga}, \mathrm{Ge}$, and Ir concentrations. Iron meteorites with compositions near these predictions include the sHH subgroup of the IAB complex [10] and the Twin City duo, as previously noted by [7] based on similar modeling with fewer trace elements. The sHL subgroup of the IAB complex [10] also falls intriguingly close to the IIIAB eutectic mixing line calculation. Current work is further evaluating the feasibility that these meteorites may be genetically related to the IIIAB group.

References: [1] Jones J. H. and Drake M. J. 1983. Geochimica et Cosmochimica Acta 47:1199-1209. [2] Haack H. and Scott E. R. D. 1993. Geochimica et Cosmochimica Acta 57:3457-3472. [3] Wasson J. T. 1999. Geochimica et Cosmochimica Acta 63:2875-2889. [4] Chabot N. L. 2004. Geochimica et Cosmochimica Acta 68:3607-3618. [5] Wasson J. T. et al. 2007. Geochimica et Cosmochimica Acta 71:760-781. [6] Chabot N. L. 2010. Meteoritics \& Planetary Science. Abstract \#5021. [7] Kracher A. 2002. 33rd Lunar and Planetary Science Conference. Abstract \#1421. [8] Chabot et al. 2003. Meteoritics \& Planetary Science 38:1425-1436. [9] Chabot et al. 2007. Meteoritics \& Planetary Science 42:1735-1750. [10] Wasson J. T. and Kallemeyn G. W. 2002. Geochimica et Cosmochimica Acta 66:2445-2473. 
5504

\section{NANOGLOBULE ABUNDANCES IN IOM EXTRACTS: CORRELATION WITH PARENT BODY PROCESSING}

H. G. Changela ${ }^{1,2}$, R. M Stroud ${ }^{2}$, B. T. De Gregorio ${ }^{3}$, L. R. Nittler ${ }^{4}$, C. M. O'D. Alexander ${ }^{4}$ and G. D. Cody ${ }^{4} .{ }^{1}$ George Washington University, Department of Physics, Washington, District of Columbia 20052, USA. ${ }^{2}$ Naval Research Laboratory, 4555 Overlook Ave. SW, Washington, District of Columbia 20375, USA. E-mail: changela@anvil.nrl.navy. mil. ${ }^{3}$ Johnson Space Center, Houston, Texas 77058, USA. ${ }^{4}$ Carnegie Institution of Washington, Washington, District of Columbia 20015, USA.

Introduction: Nanoglobules are a form of Insoluble Organic Matter (IOM) found in chondrites [1], IDPs [2] and Comet 81P/Wild2 [3]. Some nanoglobules exhibit large ${ }^{15} \mathrm{~N}$ and $\mathrm{D}$ enrichments relative to solar values, indicating that they likely originated in the ISM or the outskirts of the protoplanetary disk [4]. Recent studies of samples from the Tagish Lake meteorite with varying levels of hydrothermal alteration suggest that nanoglobule abundance decreases with increasing hydrothermal alteration [5]. The aim of the present study is to further constrain the morphologies of IOM from a range of primitive chondrites in order to determine any correlation between morphology and petrographic grade and chondrite class.

Methods: We are performing a comprehensive scanning transmission electron microscopy (STEM) survey of IOM residues prepared by methods described in [3]. STEM annular dark-field (ADF) imaging surveys were performed with NRL's JEOL 2200FS on residues from Murchison (CM2), EET 92042 (CR2) and QUE 97008 (L3). IOM textures were classified as either having a 'globular' morphology, i.e. spherical, non-porous regions approximately $50-1000 \mathrm{~nm}$ in size; 'fluffy', i.e. porous material with fine scale heterogeneity below $50 \mathrm{~nm}$; and 'dense-irregular', i.e. uniformly dense, non-spherical material $>50 \mathrm{~nm}$. ADF images were taken of each chondrite, sampling approximately $100 \mu \mathrm{m}^{2}$ of IOM. The different morphologies were manually identified from the images and masked using Adobe Photoshop. Area fractions were then obtained using Image $\mathbf{J}$ software. Because some small globules $(<100 \mathrm{~nm})$ are difficult to distinguish from the background of fluffy IOM, our results reflect a lower limit on globular abundances.

Results and Discussion: Globular to fluffy and dense irregular to fluffy IOM fractions in these chondrites show distinctive variations. Murchison contains the highest abundance of nanoglobules (approximately 7\%), followed by EET 92042 (approximately 5\%) and then QUE 97008 (approximately 1\%). The abundances of dense irregular IOM fractions are similar for Murchison and EET 92402, but have much higher abundance (approximately 20\% of measured IOM) in QUE 97008. We will also present new analyses of residues from types 1, 2 and $3 \mathrm{CR}$ and other chondrites to identify any links between nanoglobule abundances and petrographic grade. It has been shown that isotopic enrichment within the IOM is not just localized to globular species, but to some 'fluffy' components as well [6]. It is not clear whether some of the 'dense irregular' morphologies of IOM contain similar enrichments. Coordinated analyses of these extracts and in situ IOM studies will enable us to further constrain the nature of these morphologically distinct components and how they vary with petrographic grade or chondrite class.

References: [1] Nakamura-Messenger $\mathrm{K}$. et al. 2006. Science 314:1439-1442. [2] Flynn G. J. et al. 2003. Geochimica et Cosmochimica Acta 4791-4806. [3] De Gregorio B. T. et al. 2010. Geochimica et Cosmochimica Acta 74:4454-4470. [4] Alexander C. M. O'D. et al. 2007. Geochimica et Cosmochimica Acta 71:4380-4403. [5] Herd C. D. K. et al. 2011. Science, in press. [6] De Gregorio et al. 2010. Abstract \#2108. 41st Lunar and Planetary Science Conference.
5401

ABUNDANCES OF Cl, F, H, AND S IN APATITES FROM SNC METEORITES

M. B. Channon, J. W. Boyce, E. M. Stolper and J. M. Eiler. California Institute of Technology, Pasadena, CA 91125, USA. E-mail: mchannon@ gps.caltech.edu.

Introduction: Magmatic volatiles influence igneous phase equilibria, eruptive behavior, atmospheric composition, and climate. Several lines of evidence suggest that Mars is rich in halogens and $\mathrm{S}$ relative to Earth. However, magmatic volatile abundances and their effects on magmatic processes on Mars are controversial. Here we examine igneous apatites in SNC meteorites as indicators of magmatic volatile abundances.

Methods: We measured $\mathrm{Cl}, \mathrm{F}, \mathrm{H}$ (reported as $\mathrm{wt} \% \mathrm{H}_{2} \mathrm{O}$ ), and $\mathrm{S}$ in 25 apatites from 3 basaltic shergottites (JaH 479, NWA 856, and NWA 2986), 1 lherzolitic shergottite (NWA 1950), and 1 nakhlite (NWA 998) using Caltech's 7f SIMS. We compared our data to literature data for volatiles in SNC apatites [1-8].

Results: Our measurements of $\mathrm{Cl}, \mathrm{F}$, and $\mathrm{H}$ in $\mathrm{SNC}$ apatites are similar to previous data, but the increased size of the collective data set sheds light on systematic variations. Our 3 basaltic shergottites have $0.33-$ $0.95 \mathrm{wt} \% \mathrm{H}_{2} \mathrm{O}$ (literature data: $0.18-0.64 \mathrm{wt} \%$ [1-2]), those from the lherzolitic shergottite have $0.07-0.27 \mathrm{wt} \%$ (the only previously measured apatite from such a sample has $0.43 \mathrm{wt} \%$ [3]); and those from the nakhlite have $0.07-0.11 \mathrm{wt} \%$. Data are also available for apatites from Chassigny (0.21 wt\% [4]) and ALH 84001 (0.08 wt\% [2] and $0.22 \mathrm{wt} \%$ [4]). Apatites from basaltic shergottites appear to be on average $\mathrm{H}_{2} \mathrm{O}$-rich relative to apatites from other lithologies. S contents are variable $(<0.01-$ $0.82 \mathrm{wt} \%$; previous measurements are $<0.01-0.08 \mathrm{wt} \%$ [5-8]). As with $\mathrm{H}_{2} \mathrm{O}, \mathrm{S}$ contents are lower in the nakhlite $(<0.01 \mathrm{wt} \%)$ and lherzolitic shergottite $(0.02-0.04 \mathrm{wt} \%)$ than in the basaltic shergottites $(0.05-$ $0.82 \mathrm{wt} \%)$.

Discussion: The $\mathrm{H}$ in apatites from basaltic shergottites spans a range similar to apatites from terrestrial mafic igneous rocks. The higher $\mathrm{H}$ and $\mathrm{S}$ contents of basaltic shergottites relative to other SNC rock types may indicate higher initial volatile contents or lower extents of degassing prior to apatite crystallization.

Based on our results, apatites in SNCs, particularly basaltic shergottites, are significantly richer in S (by approximately $2 \times$ ) than those in mafic terrestrial igneous rocks. $\mathrm{S}$ is typically incorporated into apatite as $\mathrm{SO}_{4}{ }^{2-}$, and thus is expected to be more strongly partitioned into igneous apatite at higher $f \mathrm{O}_{2}$ (i.e., where sulfate dominates over sulfide in basaltic melts). As expected, terrestrial S-rich apatites are found in lavas that are oxidized $\left(\triangle \mathrm{QFM}+2\right.$ to $+6 \log$ units). Estimates of $f \mathrm{O}_{2}$ in shergottite parent magmas $(\triangle \mathrm{QFM}-4$ to $+0.5 \log$ units) would indicate that $\mathrm{S}^{2-}$ was the primary sulfur species. The high $\mathrm{S}$ contents of SNC apatite despite low $f \mathrm{O}_{2}$ could reflect (1) high total magmatic S contents; (2) apatite crystallized late when $f \mathrm{O}_{2}$ levels rose above estimates; or (3) some $\mathrm{S}$ in SNC apatites is present as sulfide in the halogen site.

References: [1] Leshin L. A. 2000. Geophysical Research Letters 27:2017-2020. [2] Greenwood J. P. et al. 2008. Geophysical Research Letters 35:L05203. [3] Guan Y. et al. 2003. Abstract \#1830. 34th Lunar and Planetary Science Conference. [4] Boctor N. Z. et al. 2003. Geochimica et Cosmochimica Acta 67:3971-3989. [5] Harvey R. P. et al. 1993. Geochimica et Cosmochimica Acta 57:4769-4783. [6] Xirouchakis D. et al. 2002. Geochimica et Cosmochimica Acta 66:1867-1880. [7] Greenwood J. P. et al. 2003. Geochimica et Cosmochimica Acta 67:22892298. [8] Treiman A. H. and Irving A. J. 2008. Meteoritics \& Planetary Science 43:829-854. 


\section{8}

INTERACTION BETWEEN IMPACT MELT MATRIX AND METAL PARTICLES IN THE WEATHERFORD CBa METEORITE

H. M. Chappell ${ }^{1}$, R. H. Jones ${ }^{1}$ and J. I. Goldstein ${ }^{2}{ }^{1}{ }^{1}$ University of New

Mexico, Albuquerque, NM 87131, USA. E-mail: hchappel@unm.edu.

${ }^{2}$ University of Massachusetts, Amherst, MA 01003, USA.

Introduction: The $\mathrm{CB}$ meteorites, which are subdivided into $\mathrm{CBa}$ and $\mathrm{CBb}$ subgroups, are metal rich members of the $\mathrm{CR}$ clan [1]. Metal and silicate material in $\mathrm{CB}$ meteorites possibly formed by condensation from a vapor plume generated during a major impact event [2]. Weatherford is a $\mathrm{CBa}$ that has chondrule-like silicate particles and metal particles which are highly deformed, especially compared to the elliptical particles found in the CBa meteorite Gujba. Weatherford, like all the CBa meteorites, also contains a silicate-dominated impact melt matrix which may have been injected at a much later time than when the large particles formed and accreted [3, 4]. The purpose of this study is to investigate the extent to which reheating during injection of impact melt affected the physical nature of pre-existing metal particles.

Results: We examined two thin sections of Weatherford (UNM 259 and 260) using reflected light microscopy, SEM and EDS analysis. We observed abundant evidence of physical interaction between the impact melt matrix and metal particles. Metal particles of all sizes have very fine droplets of $\mathrm{Fe}, \mathrm{Ni}$ metal dissociating from their edges. In places, the impact melt has infiltrated into and begun to disaggregate the metal particles along grain boundaries. Embayments up to $500 \mu \mathrm{m}$ in size within the metal particles appear to be areas in which metal-rich silicate material, consistent in composition with the impact melt, has invaded the grains.

Many metal grains in $\mathrm{CBa}$ meteorites are sulfide-rich and contain Cr-rich troilite $(\mathrm{FeS})$ as tiny blebs within the body of the metal particles, as well as arcuate troilite along grain boundaries [1, 5]. In Weatherford, we observed that some sulfide-rich metal grains, up to $300 \mu \mathrm{m}$ across, entrained in the impact melt, have inclusions with a Fe-Ni-S eutectic texture [6]. The inclusions are rounded, ranging in size from 5-50 $\mu \mathrm{m}$, and contain smaller, somewhat elongated, blebs of $\mathrm{Fe}, \mathrm{Ni}$ metal in a $\mathrm{FeS}$ matrix. These inclusions may be the same as what Rubin [7] described as "cellular troilite" in Gujba.

Discussion: Deformation and disassociation of metal particles by an invading silicate-rich impact melt matrix, and reheating of entrained metal particles to the eutectic temperature of $\mathrm{Fe}-\mathrm{Ni}-\mathrm{S}(1223 \mathrm{~K})$ provides evidence that there was significant interaction between the melt and preexisting metal particles. Reheating from this process may also have produced two-phase metal particles [8]. Late formation of impact melt (e.g., at $4.2 \mathrm{Ga}$ [4]) may have had a significant effect on the chemical and isotopic properties of CBa meteorites.

References: [1] Weisberg M. K. et al. 2001. Meteoritics \& Planetary Science 36:401-418. [2] Campbell A. et al. 2002. Geochimica et Cosmochimica Acta 66:647-660. [3] Barber D. J. and Hutchison R. 1991 Meteoritics 26:83-95. [4] Marty B. et al. 2010. Geochimica et Cosmochimica Acta 74:6636-6653. [5] Mason B. and Nelen J. 1968. Geochimica et Cosmochimica Acta 32:661-664. [6] Scott E. R. D. 1982. Geochimica et Cosmochimica Acta 46:813-823. [7] Rubin A. E. et al. 2003. Geochimica et Cosmochimica Acta 67:3283-3298. [8] Goldstein J. I. et al. 2011. This conference.
5408

COMPOSITIONS OF SINGLE CHONDRULES IN THE CR2 CHONDRITE NWA 801 BY MEDICAL X-RAY MICRO-COMPUTED TOMOGRAPHY

C. R. J. Charles ${ }^{1}$, P. J. A. McCausland ${ }^{2}$. J. $\mathrm{Umoh}^{3}$, D. Holdsworth ${ }^{3}$, D. W. Davis ${ }^{1}$, D. Gregory ${ }^{4}$, K. Tait ${ }^{4}$ and I. Nicklin ${ }^{4}$. ${ }^{1}$ Jack Satterly Geochronology Laboratory, Department of Geology, University of Toronto, Toronto, ON, Canada. E-mail: christopher.charles@ utoronto.ca. ${ }^{2}$ Department of Earth Sciences, University of Western Ontario, London, ON, Canada. ${ }^{3}$ Robarts Imaging Institute; University of Western Ontario, London, ON, Canada. ${ }^{4}$ Department of Natural History, Royal Ontario Museum, Toronto, ON, Canada.

Introduction: CR2 chondrites are some of the most primitive and pristine meteorites that contain abundant mm-sized silicate and "armored" chondrules. Armored chondrules show rims of thin $\mathrm{Fe}-\mathrm{Ni}$ metal on their surfaces or in their interiors, which are thought to be remnants of melting events in a dusty nebular environment [1]. X-ray computed tomography (CT) is an ideal technique to nondestructively map the compositions of such chondrules in $3 \mathrm{D}$, due to variable attenuation of $\mathrm{X}$-rays in different silicates and metal. Synchrotron radiation has been very successful in this respect (i.e., [2]-[4]). However the new generation of "medical" laboratory-based CT scanners also provide excellent spatial resolutions $(<50 \mu \mathrm{m})$, high sample throughput, and efficient image acquisition and reconstruction algorithms. Here we report the mineral compositions of chondrules separated from the CR2 chondrite NWA 801 using a GE eXplore speCZT scanner.

Experimental: Five hundred and twenty-three individual mm-sized chondrules and chondrule fragments were extracted from $12 \mathrm{~g}$ of NWA 801 (loaned by the Royal Ontario Museum) using automated freeze-thaw disaggregation [5]. All objects were sieved and grouped by size onto glass mounts with up to 12 objects/mount. 3D renderings were obtained for all objects along with enstatite, olivine, augite, $\mathrm{Si}$ and $\mathrm{Fe}$ "standards" through 900 views at $16 \mathrm{~ms}$ exposure $(110 \mathrm{kVp}, 32 \mathrm{~mA})$ at $50 \mu \mathrm{m} /$ voxel. CT grayscale numbers were converted to Houndsfield units (HU) and all final images were manipulated and examined by GE Microview and custom MatLab scripts.

Results: Histograms of HU-values versus number of voxels show one or more distinct peaks throughout the range of 2886 (Si) $<\mathrm{HU}<25427$ (Fe; saturated). These peaks correspond to the chemical compositions of the objects, which are approximately constrained by the standards. To quantify the compositions of the chondrules obtained by CT imaging, 14 fragments that span the range of HU values, together with the olivine, enstatite and augite "standards" were analyzed by an SX-50 electron microprobe (EMP) to obtain their mineral chemistries and structures. The fragments showed high forsteritic olivine with variable amounts of mesostasis containing low-Ca pyroxene, and $\mathrm{Fe}$-oxide veins and nodules.

References: [1] Rubin A. E. 2010. Geochimica et Cosmochimica Acta 74:4807-4828. [2] Ebel D. S. and Rivers M. 2007. Meteoritics \& Planetary Science 39:531-544. [3] Fredrich J. M., Wignarajah D. P., Chaudary S., Rivers M. L., Nehru C. E. and Ebel D. S. 2008. Earth and Planetary Science Letters 275:132-180. [4] Uesugi M., Uesugi K. and Oka M. 2011. Earth and Planetary Science Letters 299:359-367. [5] Charles C. R. J. 2011. Review of Scientific Instruments, in press. 


\section{0}

\section{TEXTURES OF SINGLE CHONDRULES IN CR2 CHONDRITE NWA 801 BY MEDICAL X-RAY MICRO-COMPUTED TOMOGRAPHY}

C. R. J. Charles ${ }^{1}$, P. J. A. McCausland ${ }^{2}$. J. $\mathrm{Umoh}^{3}$, D. Holdsworth ${ }^{3}$, D. W. Davis ${ }^{1}$, D. Gregory ${ }^{4}, K$. Tait ${ }^{4}$ and I. Nicklin ${ }^{4} .{ }^{1}$ Jack Satterly Geochronology Laboratory, Department of Geology, University of Toronto, Toronto, ON, Canada. E-mail: christopher.charles@ utoronto.ca. ${ }^{2}$ Department of Earth Sciences, University of Western Ontario, London, ON, Canada. ${ }^{3}$ Robarts Imaging Institute; University of Western Ontario, London, ON, Canada. ${ }^{4}$ Department of Natural History, Royal Ontario Museum, Toronto, ON, Canada.

Introduction: "Armored" chondrules contain one or more concentric rings of $\mathrm{Fe}-\mathrm{Ni}$ metal on their exterior surfaces or throughout their interiors. These chondrules are found in the CR2-class of carbonaceous chondrites, and the 3D distributions of metal and silicates inside these chondrules are important to constrain the nebular environment where they formed. Petrography suggests that many CR2 chondrules are remnants of precursor chondrules that were incorporated into dustballs, which were remelted in one or more episodes in a dusty nebular setting [1]. X-ray computed tomography (CT) is an ideal way to test this idea, since 3D renderings of chondrule textures can be obtained nondestructively. Synchrotron radiation has been very successful for imaging chondrule textures due to its very high spatial resolutions (approximately $1 \mu \mathrm{m}$; i.e., [2]-[4]). However new laboratory-based medical CT scanners have received less attention, although these instruments offer high resolutions, rapid data acquisition and processing times, the ability to image large numbers of samples, and are more widely available than synchrotron facilities. Here we investigate the use of two General Electric CT scanners, the Locus and eXplore speCZT, for mapping the 3D textures of single chondrules removed from the CR2 chondrite NWA 801.

Experimental: Automated freeze thaw disaggregation was used to extract 523 individual $\mathrm{mm}$-sized chondrules and chondrule fragments from $12 \mathrm{~g}$ of NWA 801 [5]. All objects were sieved and grouped by size onto glass mounts with up to 12 objects/mount. 3D renderings were obtained for all objects along with enstatite, olivine, augite and $\mathrm{Fe}$ "standards" on the eXplore speCZT through 900 views at $16 \mathrm{~ms}$ exposure $(110 \mathrm{kVp}, 32 \mathrm{~mA})$ at $50 \mu \mathrm{m} /$ voxel. One mount containing several large chondrules was imaged on the Locus through 900 views at a $4500 \mathrm{~ms}$ exposure $(80 \mathrm{kVp}, 0.45 \mathrm{~mA})$ at $20 \mu \mathrm{m} /$ voxel. CT grayscale numbers from both scanners were converted to Houndsfield units (HU) and all final images were manipulated and examined by GE Microview and custom MatLab scripts.

Results: Twelve percent of chondrules contain distinct multiple $\mathrm{Fe}$ $\mathrm{Ni}$ rims and $75 \%$ showed only a single Fe-Ni rim usually on the exterior surface of the chondrule. A total of $13 \%$ of the objects were either "metallic" chondrules or large Fe-Ni blebs of approximately $1 \mathrm{~mm}$ or less in diameter. This suggests that enveloping compound chondrules [1] in NWA 801 formed at about the same frequently as the large Fe-Ni blebs, but that both these objects formed less frequently as noncompound chondrules. Thus the majority of NWA 801 chondrules are not products of compound mergers.

References: [1] Rubin A. E. 2010. Geochimica et Cosmochimica Acta 74:4807-4828. [2] Ebel D. S. and Rivers M. 2007. Meteoritics \& Planetary Science 39:531-544. [3] Fredrich J. M., Wignarajah D. P., Chaudary S., Rivers M. L., Nehru C. E. and Ebel D. S. 2008. Earth and Planetary Science Letters 275:132-180. [4] Uesugi M., Uesugi K. and Oka M. 2011. Earth and Planetary Science Letters 299:359-367. [5] Charles C. R. J. 2011. Review of Scientific Instruments, in press.

\section{1}

\section{DATING OF SECONDARY MINERALS IN NAKHLITES}

B. L. A. Charlier ${ }^{1}$, A. W. Needham ${ }^{2}$ and M. M. Grady ${ }^{2,3}$. ${ }^{1}$ Department of Earth and Environmental Science, The Open University, Walton Hall, Milton Keynes, MK7 6AA, UK. ${ }^{2}$ PSSRI. The Open University, Walton Hall, Milton Keynes, MK7 6AA, UK. ${ }^{3}$ Department of Mineralogy, The Natural History Museum, Cromwell Road, London SW7 5BD, UK. E-mail: m.m.grady@open.ac.uk.

Introduction: Nakhlites are a group of Martian clinopyroxenites, currently 13 in number, that have a tightly-constrained crystallization age of $1320 \pm 40 \mathrm{Ma}$ [1]. They are thought to emanate from either a single lava flow, or closely-allied series of flows [2], and following their crystallization, have experienced aqueous alteration. Secondary alteration products include clay minerals, carbonates, sulfates and halite, which form complex assemblages and intergrowths. It is not clear what the relationship is between the different minerals: they may have formed by gradual evaporation following a single episode of fluid incursion [3], or over an extended period of time following several episodes of aqueous activity. The latter case is supported by spectral data from widespread regions of the Martian surface, where overlying units of secondary minerals have been employed to produce a relative chronology [4]. Direct measurement of the age of the secondary products in the meteorites has been difficult - the minerals are fine-grained and not easy to separate from their host silicates. Where results have been obtained, they indicate formation ages of around $600 \mathrm{Ma}$ for phyllosilicates [5]. We have used $\mathrm{Rb}$-Sr dating of Nakhla and Y 000593 in an attempt to determine a series of ages for different components within the meteorites.

Technique: We carried out a sequence of leaches on samples of between 200 and $500 \mathrm{mg}$ powdered whole rock meteorite. The sequence was designed to remove increasingly insoluble components, and started with cold water, followed by hot water, cold then hot $0.025 \mathrm{M} \mathrm{HCl}$, cold then hot $0.25 \mathrm{M} \mathrm{HCl}$, then hot $2.5 \mathrm{M} \mathrm{HCl}$, hot $6 \mathrm{M} \mathrm{HCl}$ and finally hot $\mathrm{HNO}_{3}-\mathrm{HCl}$. Each leachate was split, with one aliquot analyzed by ICPMS to determine $\mathrm{Rb}$ and $\mathrm{Sr}$ abundances, and the remainder taken for $\mathrm{Sr}$ isotope analysis by TIMS.

Results: Were a bit bizarre, to say the least, with a maximum ${ }^{87} \mathrm{Sr} /{ }^{86} \mathrm{Sr}$ ratio in the cold water leachate from Nakhla of 0.7574 . Ratios of this magnitude are more usually associated with evolved rocks rather than soluble components within basalts. The ${ }^{87} \mathrm{Sr} /{ }^{86} \mathrm{Sr}$ decreased with increasing acid strength, converging on a value around 0.702 , compatible with an age of $>1000 \mathrm{Ma}$.

Discussion: Our modeling of the components extracted by sequential leaching suggests that halite will be dissolved in cold water, phyllosilicates will start to give up their loosely-bound ions in weak $\mathrm{HCl}$, with those more firmly bound dissolving out in stronger $\mathrm{HCl}$. Carbonates (mainly siderite) will be removed by hot $0.25 \mathrm{M} \mathrm{HCl}$, then the silicates will start to be attacked by $6 \mathrm{M} \mathrm{HCl}$. Duplicate measurements from two separate splits of Nakhla gave elevated values of ${ }^{87} \mathrm{Sr}{ }^{86} \mathrm{Sr}$ in the cold water extract thought to be associated with halite. Further interpretation will be forthcoming in the fullness of time.

References: [1] Nyquist L. E. et al. 2001. Space Science Reviews 96:105-164. [2] Treiman A. H. 2005. Chemie der Erde 65:203-270. [3] Bridges J. C. and Grady M. M. 2001. Earth and Planetary Science Letters 176:267-279. [4] Bibring J.-P. et al. 2006. Science 312:400-404. [5] Swindle T. D. et al. 2000. Meteoritics \& Planetary Science 35:107-115. 
5254

CARBONS IN ACAPULCO AND LODRAN AT THE NANOSCALE: COMPARISON WITH EXPERIMENTAL ANALOGS

E. Charon ${ }^{1,2}$, J. Aléon ${ }^{2}$ and J. N. Rouzaud ${ }^{1}$. ${ }^{1}$ Laboratoire de Géologie de l'Ecole Normale Supérieure, UMR CNRS 8538, 24 rue Lhomond 75005 Paris France. ${ }^{2}$ Centre de Spectrométrie Nucléaire et de Spectrométrie de Masse, CNRS/IN2P3 UMR 8609, 91405 Orsay Campus France. E-mail: charon@geologie.ens.fr.

Introduction: Acapulcoites and Lodranites (A-L) are very primitive partially differentiated objects and can bring essential insights on planetesimal accretion and differentiation within the first 1-2 Myr of the solar system [1]. Here, we study the origin and evolution of carbons in A-L as tracers of early differentiation stages. The study is focused on the relationship between carbons and Fe-based metal. We coupled, on the same micrometer-sized grains, structural characterization by Raman $\mu$ spectrometry and mapping and High Resolution TEM on Focused Ion Beam (FIB) sections. The carbon phases of A-L are systematically associated with metal and are partially graphitized [2]. In order to show the possible catalytic role of iron on the graphitization, experimental analogs have been produced using blends of $10 \%$ saccharose-based chars analogous to the insoluble organic matter (IOM) of chondrites and $90 \%$ iron powder, heated between 650 and $1600{ }^{\circ} \mathrm{C}$ and compared to carbons in Acapulco and Lodran.

Results: Raman spectra corrected from polishing effects reveal as strong structural heterogeneity at the micrometer scale (defect bands of various intensities). Raman mapping shows that carbons in contact with metal are more graphitized, suggesting a catalytic effect of metal on the graphitization of organic matter, thus allowing formation of graphite at temperature $<1200{ }^{\circ} \mathrm{C}$. Experimental analogues completely reproduce the range of structural heterogeneity and relationships with metal observed in Acapulco and Lodran.

HRTEM results specify iron-carbon interactions from the micrometer to the nanometer scales. Again micro- and nano-structures observed in Acapulco and Lodran are correctly reproduced in our pyrolysis experiments. Furthermore, the degree of order of carbons and the morphology of metal nano-inclusions in carbons suggest different mechanisms of graphitization as a function of temperature. At low temperature $\left(650^{\circ} \mathrm{C}\right)$, iron diffusion is limited (solid-solid interactions) and mesoporous turbostratic carbons and carbides can be formed. At higher temperature, iron becomes liquid and its diffusion is favored. In both cases 'catalytic' graphitization is favored by the presence of iron and sensu stricto graphite can be obtained at temperatures as low as $1200{ }^{\circ} \mathrm{C}$.

Conclusion: Our experimental study shows that carbon structural heterogeneities in Acapulco and Lodran meteorites result from the catalytic graphitization by $\mathrm{Fe}-\mathrm{Ni}$ metal of an organic precursor having the typical structure of chondritic IOM and probably not from the preservation of preaccretional heterogeneities [3].

References: [1] Touboul et al. 2009. Earth and Planetary Science Letters 284:168-178. [2] Charon et al. 2009. Meteoritics \& Planetary Science 44:A48. [3] El Goresy A. et al. 2005. Geochimica et Cosmochimica Acta 69:4535-4556.
5374

O ISOTOPIC CONSTRAINTS ON THE ORIGIN OF Mg-RICH OLIVINES FROM ALLENDE CHONDRULES: PLANETARY OR NOT ?

M. Chaussidon ${ }^{1}$, A. Gurenko ${ }^{1}$, T. H. Luu ${ }^{1}$, C. Rollion-Bard ${ }^{1}$. ${ }^{1}$ CRPGCNRS, BP 20, 54501 Vandoeuvre-lès-Nancy, France. chocho@crpg. cnrs-nancy.fr.

Introduction: If chondrites parent bodies accreted late, $\approx 4-5 \mathrm{Myr}$ after the condensation/crystallization of type B CAIs (when ${ }^{26} \mathrm{Al} /{ }^{27} \mathrm{Al}=5.2 \times 10^{-5}$ ) as imposed by chondrule ages, there are several evidences that planetesimal accretion (up to sizes of $\approx 1000 \mathrm{~km}$ ) started very early, perhaps nearly at the same time than CAIs formed [1]. Developments of high precision $\mathrm{Mg}$ isotopic analysis by multi-collector ion microprobe allow to determine both the slopes $\left({ }^{26} \mathrm{Al} /{ }^{27} \mathrm{Al}\right)$ and the initial $\left(\delta^{26} \mathrm{Mg}_{0}\right)$ of ${ }^{26} \mathrm{Al}$ isochrons in chondrules and CAIs. This can be used to test the homogeneous distribution of ${ }^{26} \mathrm{Al}$ and $\mathrm{Mg}$ isotopes in the accretion disk at the time of formation of CAIs [2] and to try to calculate model ${ }^{26} \mathrm{Al}$ ages (under the assumption of an homogeneous distribution) for chondrule precursors [2] or for minerals devoid of $\mathrm{Al}$ such as $\mathrm{Mg}$-rich olivines in chondrules [3]. This suggests that some of the chondrule precursors and $\mathrm{Mg}$-rich olivines were formed very early, within $1 \mathrm{Myr}$ or less than CAIs [2,3]. One possibility would be that these Mg-rich olivines are precursors of type I chondrules and could be the fragments of the mantle of pre-existing disrupted planetesimals [4]. In the present study we explore what constraints could be derived on the origin of $\mathrm{Mg}$-rich olivines in Allende (either in type I chondrules or isolated in the matrix) from high precision measurements of their $\mathrm{O}$ isotopic compositions and from their major and trace elements concentrations.

Experimental: The $\mathrm{O}$ isotopic compositions were measured with the CRPG-CNRS (Nancy) ims 1270 and ims 1280HR2 multi-collector ion microprobes. In each olivines numerous (from 10 to 25) spots were made in order to reach an analytical precision on the level of $\pm 0.01-0.02 \%$ o $(2 \sigma)$ on ${ }^{17} \mathrm{O}$ excesses relative to mass fractionation (i.e., $\Delta^{17} \mathrm{O}$ ).

Results and Discussion: Mg-rich olivines in type I chondrules from Allende cluster around a few modes (less than 10) of $\Delta^{17} \mathrm{O}$ values ranging from -7.5 to $0 \%$. They do not show a continuum of $\Delta^{17} \mathrm{O}$ values contrary to bulk $\Delta^{17} \mathrm{O}$ values for Allende type I chondrules [5]. Bulk $\Delta^{17} \mathrm{O}$ values are likely the result of variable isotopic exchange with the nebular gas during chondrule melting. In most cases $\mathrm{Mg}$-rich olivines appear relict (predating chondrule melting) from their $\Delta^{17} \mathrm{O}$ values. The most populated mode for Mg-rich olivines is at $\Delta^{17} \mathrm{O}=-5.56 \pm 0.09 \%$. Within a given mode of $\Delta^{17} \mathrm{O}$ values, a large range of mass fractionation (up to $\approx 6 \%$ or for $\delta^{18} \mathrm{O}$ values) is observed. This is consistent with previous (but less precise) measurements [4]. Correlations between $\Delta^{17} \mathrm{O}, \delta^{18} \mathrm{O}$, major and trace element concentrations, allow to better constrain the origin of the olivines and to test the possibility that they originate from a restricted number of planetesimals which differentiated very early in the disk.

References: [1] Dauphas N. and Chaussidon M. 2011. Annual Review of Earth and Planetary Sciences 39:351-386. [2] Villeneuve J. et al. 2009. Science 325:985-988. [3] Villeneuve J. et al. 2011. Earth and Planetary Science Letters 301:107-116. [4] Libourel G. and Chaussidon M. 2011. Earth and Planetary Science Letters 301:9-21. [5] Clayton R. N. et al. 1983. In "Chondrules and their Origins" LPI 37-43. 
5467

Ca ISOTOPIC ANALYSIS AS A TOOL FOR EXPLORING GENETIC RELATIONSHIPS AMONG ACHONDRITES

H. -W. Chen ${ }^{1}$, T. Lee ${ }^{1,2}$, D. -C. Lee ${ }^{1}$ and J. -C. Chen ${ }^{1}{ }^{1}$ Institute of Earth Sciences, Academia Sinica. E-mail: typhoon@asiaa.sinica.edu.tw. ${ }^{2}$ Institute of Astronomy and Astrophysics, Academia Sinica.

Introduction: Lord Kelvin would probably put meteoritics as a "stamp collection" science. In this paper we aim to evaluate the potential of helping this endeavor by using the widespread ${ }^{48} \mathrm{Ca}$ anomalies.

Method: $\mathrm{Ca}$ was extracted by conventional cation exchange. For each run $2 \mu \mathrm{g} \mathrm{Ca}$ was analyzed by double filament TIMS. Four runs were binned to yield one grand mean that is shown in Fig. 1. NIST SRM 915a was used as the normal standard. Two standard deviations of the population $(2 \sigma)$ consisting of 18 such grand means were measured over 6 months to test our long term reproducibility. They were found to be 0.87 , 0.13 , 4.6, and $0.42 \varepsilon$ for ${ }^{40} \mathrm{Ca} /{ }^{44} \mathrm{Ca},{ }^{43} \mathrm{Ca} /{ }^{44} \mathrm{Ca},{ }^{46} \mathrm{Ca} /{ }^{44} \mathrm{Ca}$, and ${ }^{48} \mathrm{Ca} /{ }^{44} \mathrm{Ca}$ after correcting for instrumental fractionation by normalizing ${ }^{42} \mathrm{Ca} /{ }^{44} \mathrm{Ca}$ to 0.31221 . A plagioclase sample from the Uzen volcano, Japan, was processed the same way as meteorites samples. Fig. 1 plots the deviation of ${ }^{48} \mathrm{Ca} /{ }^{44} \mathrm{Ca}$ relative to the normal which is represented by the average of all 18 grand means of SRM 915a. Each symbol is the grand mean of 4 runs. The two Uzen results agree with each other and normal within $0.2 \varepsilon$. For each sample we take the long term reproducibility or $2 \sigma$ of 4 runs which is greater. To further test our ability to resolve epsilon level of isotope shift.

Ca-48 anomalies: Two to four meteorites from each of the five classes of achondrites were studied. Meteorites in the same class seem to share the same characteristic $\varepsilon^{48} \mathrm{Ca}$ isotopic shift. Ureilite, diogenite, angrites, eucrites, and Martian meteorites show $\varepsilon^{48} \mathrm{Ca}$ of $-2.0,-1.6,-1.2$, -0.9 and $-0.1 \varepsilon$ respectively. Previously $\delta \mathrm{O}$ were used most successfully in classifying meteorites. Our preliminary data show that $\varepsilon^{48} \mathrm{Ca}$ is probably as effective as $\delta \mathrm{O}$. Moreover, $\mathrm{e}^{48} \mathrm{Ca}$ may carry different record than $\delta \mathrm{O}$. An interesting example is ureilite. Among achondrite, ureilite was thought to be related to carbonaceous chondrites, indeed, their $\delta \mathrm{O}$ fall on the same 1:1 line. However, all four ureilites have $\varepsilon^{48} \mathrm{Ca}$ around $-2 \pm 0.5 \varepsilon$ while the only whole rock $\varepsilon^{48} \mathrm{Ca}$ data for carbonaceous chondrite seems to show effect of opposite direction $(+5 \pm 2 \varepsilon)[1]$.

Reference: [1] Chen J. H. et al. 2011. Abstract \#2440. 42nd Lunar and Planetary Science Conference.

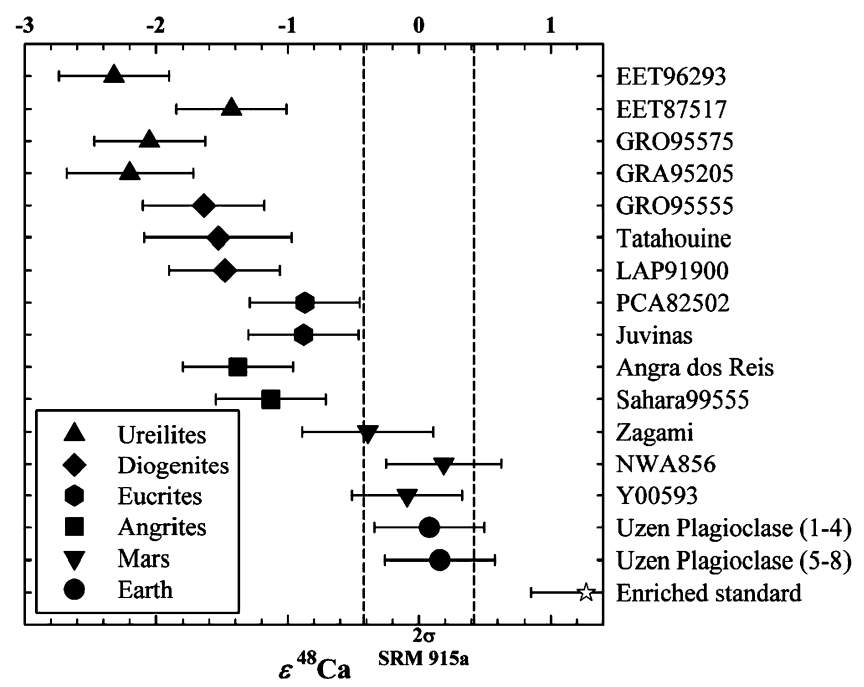

Fig. 1.
5002

PLANAR DEFORMATION FEATURES IN QUARTZ FROM THE XIUYAN CRATER, CHINA

M. Chen ${ }^{1}$, C. Koeberl ${ }^{2}$, W. Xiao ${ }^{1}$, X. Xie ${ }^{1}$ and D. Tan ${ }^{1} .{ }^{1}$ Guangzhou Inst. Geochem., Chinese Academy of Sciences, Guangzhou 510640, China. E-mail: mchen@gig.ac.cn. ${ }^{2}$ Depart. Lithos. Res., Univ. Vienna, Althanstrasse 14, A-1090 Vienna, Austria.

Introduction: The 1.8-km-diameter Xiuyan crater is located in the Liaodong Peninsula in northeastern China. The crater is exposed in a Proterozoic metamorphic rock complex composed of granulite, hornblendite, gneiss, tremolite marble, and marble. A drilling positioning at the center of the crater reveals the geological structure and stratum of the crater [1]. The bottom at the center of the crater covers about $100 \mathrm{~m}$ thick lacustrine sediments underlain by $188 \mathrm{~m}$ thick crater-fill breccia. A layer of impact breccia composed of clasts of granulite, gneiss, hornblendite and fragments of glass as well as clastic matrix, occurs at the lower part of the breccia units in the depth interval from 260 to $295 \mathrm{~m}$. We have examined abundant planar deformation features (PDFs) in quartz from the impact breccia.

Results and Discussion: Quartz with multiple PDFs was identified in the fine-grained matrix and within the clast of granulite. One to five sets of PDFs are observed in a single quartz crystal. A quantity of 70 quartz grains from five thin sections made from the clasts of granulite in the impact breccia recovered at the depth of $290 \mathrm{~m}$ were measured in detail by using a Universal stage to determine the orientations of PDFs relative to the optical axis. Total 13 forms of PDFs correspond to rational crystallographic orientations of quartz have been indexed, and these orientations of PDFs include the forms with miller indices of e $\{10 \overline{1} 4\}$, $\omega\{10 \overline{1} 3\}, \pi\{10 \overline{1} 2\}, \xi\{11 \overline{2} 2\}, \mathrm{r}, \mathrm{z}\{10 \overline{1} 1\}, \mathrm{s}\{11 \overline{2} 1\}, \rho\{21 \overline{3} 1\},\{22 \overline{4} 1\},\{31 \overline{4} 1\}$, $\mathrm{t}\{40 \overline{4} 1\}, \mathrm{x}\{511\}, \mathrm{m}\{100\}$ and $\mathrm{a}\{110\}$. PDFs parallel to the basal $(0001)$ plane is absent. $44 \%$ of the quartz grains contain 3 sets of PDFs, and other $40 \%$ contain 2 sets of PDFs. Of all the PDFs measured, $11.2 \%$ of them, which did not correspond to rational crystallographic orientations of quartz, were not indexed. The most abundant PDFs are rhombohedron forms of $\pi\{102\}, \omega\{103\}$, and $r / z\{101\}$ with frequency of $33.5 \%, 22.3 \%$ and $9.6 \%$, respectively. The predominant occurrence of PDFs $\{102\}+\{103\}$ and the absence of PDFs parallel to (0001) indicate a high intensity shock of the samples [2-4]. A predominant PDF form of $\{102\}$ in quartz suggests a shock pressure $>20 \mathrm{GPa}$ [4]. The occurrence of PDFs in quartz from the impact breccia provides crucial evidence for shock metamorphism of target rocks and confirms the impact origin of this crater.

Acknowledgments: This research was supported by CAS (Kzcx2yw-140), NNSF of China (40872041) and President Foundation of CAS. The University of Vienna provided partial assistance to M.C. during a research visit in Vienna.

References: [1] Chen M. et al. 2010. Chinese Science Bulletin 55:1777-1781. [2] Robertson P. and Grieve R. A. F. 1977. In Impact and explosion cratering, by Roddy D. J. et al. Pergamon Press, New York. 687-702. [3] Stöffler D. and Langenhorst F. 1994. Meteoritics \& Planetary Science 29:155-181. [4] Grieve R. A. F. et al. 1996. Meteoritics \& Planetary Science 31:6-35. 
5325

A NEW METEORITE FIND IN BOU AZARIF, ALNIF AREA (SOUTH MOROCCO)

H. Chennaoui Aoudjehane ${ }^{1,2}$, A. Jambon ${ }^{2}$, N. Larouci ${ }^{1,2}$ and $\mathrm{H}$. Gako ${ }^{1}$ ' ${ }^{1}$ Laboratoire GAIA, Equipe patrimoine géologique du Maroc, Université Hassan II Casablanca Faculté des Sciences Ain Chock BP 5366 Maârif Casablanca Maroc. E-mail: h.chennaoui@fsac.ac.ma. ${ }^{2}$ UPMC-PARIS 6, ISTEP 4 place Jussieu, 75005 Paris France. CNRS UMR 7193.

During a field trip in the south of Morocco in December 2010, which aimed to collect the coordinates of two recent falls in the MoroccoAlgerian border, we visited a minerals and fossils store in Alnif (South of Morocco). The dealer Mohamed Bouiri showed us a sample of a badly weathered meteorite that he claimed to know the exact provenance. Then he called the finder of the meteorite Hassan Belaid that agreed to accompany us to the find place a few kilometers from Alnif. We collected many samples with the coordinates $\mathrm{N} 31^{\circ} 09.386^{\prime}$; W $005^{\circ} 09.157^{\prime}$, in less than one hour, on a hillside. The finder estimates that he collected about $100 \mathrm{~kg}$ of this meteorite that is regularly discovered after rain showers. The fall is old as the samples are recovered by sediments that are eroded by the rain. The weathering grade provides evidence of this old age.

We collected SEM images (UPMC ISTeP) to see the size of minerals, their layout, and the mode. Listed minerals are olivine, pyroxene, plagioclase, weathered kamacite, taenite less weathered than kamacite, troilite, phosphates (chlorapatite and merilite).

EMP analyses (SX100- UPMC ISTeP) on olivines, pyroxenes and plagioclases: Olivine Fa 18.2; Pyroxene Fs 17.9, Wo 2.3; that allowed us to classify the meteorite as an ordinary chondrite H5. The shock intensity is $\mathrm{S} 3$ and the weathering grade is $\mathrm{W} 4$. The magnetic susceptibility is $\log \chi=4.75$.

By searching in the Meteoritical Bulletin database, we found that the name "Alnif" has already been given to an $8 \mathrm{~kg}$ sample, purchased in April 1992 by A. Carion in a mineral shop in Rissani, Morocco. The seller said it had been collected with trilobites near Oum-Jrane, $60 \mathrm{~km}$ south of Alnif. Microprobe analyses of Alnif meteorite yield: olivine, Fa19.3; pyroxene Fs17.3, Wo1.7; plagioclase, An10.6; that corresponds to an ordinary $\mathrm{H} 5$ chondrite, shock stage $\mathrm{S} 2$; weathering grade $\mathrm{W} 2$; submitted by the MNHN Paris, in 1998.

Even for an ordinary chondrite, it is important to avoid the NWA nomenclature whenever possible. This meteorite will have a proper geographic name that could be "Bou Azarif" (Bou Izarif) as the hill is named in the topographic $1 / 100,000$ map of Alnif. It will be added to meteorites that have been submitted by a Moroccan French team, as Benguerir [1], Al Haggounia [2], Tamdakht [3], with a proper geographic name.

It could be interesting to measure the terrestrial age of this meteorite and try to find its history, if it is a fossil meteorite, how deep are the samples, to compare it with the Alnif meteorite in order to assess whether they are paired stones.

References: [1] Chennaoui Aoudjehane H. et al. 2006. Meteoritics \& Planetary Science 41:231-238. [2] Chennaoui Aoudjehane et al. H. 2007. Abstract \#5329. Meteoritics \& Planetary Science 42. [3] Chennaoui Aoudjehane H. et al. 2009. Abstract \#5038. Meteoritics \& Planetary Science 44
5078

THE PATHS OF ICE GRAINS IN THE SOLAR NEBULA: IRRADIATION AND THE FORMATION OF ORGANICS

F. J. Ciesla ${ }^{1}$, S. A. Sandford ${ }^{2}$ and R. M. Mastrapa ${ }^{2} .{ }^{1}$ Department of the Geophysical Sciences, University of Chicago, 5734 South Ellis Avenue, Chicago, IL 60637. E-mail: fciesla@uchicago.edu. ${ }^{2}$ NASA-Ames Research Center, Moffett Field, CA 94035.

Introduction: Organic compounds have been found in a number of meteorites, leading many to suggest that Earth's prebiotic material was delivered through the accretion of cometary and chondritic-like planetesimals. While formation of organic molecules during aqueous alteration of a parent body can occur [1], the $\mathrm{D} / \mathrm{H}$ ratios in these compounds have led many to investigate a low temperature origin [2]. Among the favored pathways is the UV irradiation of ice-mantled grains, where photons break up molecules in solid grains, leaving behind reactive ions and radicals, which form more complex species. Most studies have considered an interstellar setting for such reactions [e.g., 3, 4]. Here we explore whether ice-mantled grains in the outer solar nebula could have absorbed enough UV photons to contribute significantly to the organic inventory of the solar system.

Model: We have developed a particle-tracking model that calculates vertical and radial paths of dust particles through a diffusive protoplanetary disk $[5,6]$. This allows us to determine the amount of time that particles spend at different locations throughout an evolving disk. By assuming an incident flux of UV radiation on the disk, we can then determine the flux wherever the particles reside and determine an irradiation history (number of incident UV photons) for each particle.

Results: We tracked the motions of 5000 ice grains released from the midplane of a disk where temperatures were $<50 \mathrm{~K}$-low enough for molecular species like $\mathrm{H}_{2} \mathrm{O}, \mathrm{CO}_{2}, \mathrm{NH}_{3}$, and $\mathrm{CH}_{3} \mathrm{OH}$ to be frozen onto grain surfaces - for a period of $10^{6}$ years. We find that, because the UV flux is rapidly attenuated through the disk as small dust particles readily absorb the incident radiation, ices only see significant fluences of UV photons when lofted to high altitudes in the disk. Thus only small, $<10 \mu \mathrm{m}$ grains, are exposed to large fluences of UV photons. However, in many cases, these fluences can be large enough to lead to the production of significant amounts of organic molecules.

Discussion: Throop (2011) [7] suggested organics could have been produced in the outer solar nebula if the Sun formed in an Orion-like setting, such that a nearby massive star would have irradiated the disk at $10^{6} \times$ the current interstellar UV flux $\left(\mathrm{G}_{0}\right)$. In that model, UV photons were assumed to be evenly distributed among all ice particles at a given location in the disk, and as such, high UV fluxes were necessary to produce high yields of organics. We find that turbulence would have lofted small grains to high altitudes, exposing many to enough UV radiation that significant organic synthesis could have occurred, even at low incident UV fluxes $\left(<10^{3} \mathrm{G}_{0}\right)$, such as those expected for a young star irradiating its own disk or one in a small cluster. This suggests organic synthesis can occur in most protoplanetary disks, meaning organic compounds should be commonly found in young planetary systems.

References: [1] Peltzer E. T. et al. 1984. Advances in Space Research 4:69-74. [2] Sandford S. A. et al. 2001. Meteoritics \& Planetary Science 36:1117-1133. [3] Bernstein M. P. et al. 2002. Nature 416:401-403. [4] Muñoz Caro G. M. et al. 2002. Nature 416:403-405. [5] Ciesla F. J. 2010. The Astrophysical Journal 723:514-529. [6] Ciesla F. J. 2011. The Astrophysical Journal. Submitted. [7] Throop H. B. 2011. Icarus 212:885895. 
5082

\section{PETROGRAPHY AND CHEMISTRY OF DJERFISHERITE IN ENSTATITE CHONDRITES}

P. L. Clay ${ }^{1}$, H. Busemann ${ }^{1}$ and A. El Goresy ${ }^{2}$. ${ }^{1}$ SEAES, University of Manchester, M13 9PL, UK. ${ }^{2}$ BGI, Universität Bayreuth, Germany. E-mail: Patricia.Clay@manchester.ac.uk.

Introduction: Establishing a precise chronology of events in the early solar system is crucial for understanding its evolution from parent molecular cloud to the planetary bodies. Various chronometers can be applied to date formation, thermal resets, and differentiation. While short-lived chronometers date events in the first few tens of Ma with a high chronological resolution, they require calibration with a long-lived chronometer for absolute ages. However, mineral phases that can be dated simultaneously with both a long- and a short-lived system are rare. The K-Cl-bearing sulfide djerfisherite $[1,2]$, found in several E-chondrites, offers such an opportunity with the application of short (I-Xe)-and longlived (Ar-Ar, Rb-Sr) chronometers. It is a good target for these isotopic systems, as correlated release of ${ }^{129} \mathrm{Xe}^{*}$ excess and ${ }^{40} \mathrm{Ar} *$ has previously been shown for St. Mark's (EH5), where djerfisherite is one suspected carrier of $\mathrm{Ar}$ and $\mathrm{Xe}[3,4]$. Here, we present initial petrographic and chemical analyses of djerfisherite grains that will be extracted from two $\mathrm{E}$ chondrites for correlated I-Xe and Ar-Ar analysis, with a further subsection reserved for $\mathrm{Rb}-\mathrm{Sr}$ analysis.

Petrography \& Mineral Chemistry: Petrography, texture, and mineral chemistry of several djerfisherite grains have been investigated using reflected light microscopy, BSE imaging, element mapping, and quantitative EPMA analysis in ALHA77295 (“A77”, EH3), and Sahara 97096 ("S97", EH3). Djerfisherite in A77 is present in several forms: matrix grains, included in metal-troilite and accreted around metaltroilite, idiomorphic grains, as well as larger veins; forms previously described for oldhamite distribution in A77 [5]. Chemically, these grains are relatively indistinct, with consistent mineral-chemical formulae, represented by: $(\mathrm{K}, \mathrm{Na})_{5.6-6.1}(\mathrm{Fe}, \mathrm{Ni}, \mathrm{Cu})_{25.4-27}, \mathrm{~S}_{27} \mathrm{Cl}_{1.0-1.4}$, consistent with previous studies. BSE images reveal chemically unzoned and pristine grains. S97 contains less idiomorphic djerfisherite than A77, and shows the characteristic Qingzhen 'breakdown' reaction into porous troilite and other phases, previously described in Qingzhen and Y-691[6].

Results \& Discussion: Idiomorphic djerfisherite in A77 is pristine, primary djerfisherite from nebular condensation, whilst the more common porous 'broken down' djerfisherite of S97 is disturbed, due most likely to a thermal event at $\sim 2 \mathrm{Ga}$ [7]. However, chemically, there is minimal difference between the two pristine djerfisherite populations in both meteorites. Evidence for further disturbances of other sulfide phases, such as eutectic melt textures, as reported in paired SAH 97072 [8], are encountered in S97, again supporting a more complex history for this sample related to thermal metamorphic episodes. Moreover, these textural features could provide additional evidence that these EH3s originated from and evolved in different parent bodies [5].

References: [1] Fuchs L. H. 1966. Science 153:166-167. [2] Lin Y. and El Goresy A. 2002. Meteoritics \& Planetary Science 20:577-599. [3] Busemann, H. et al. 2002. Abstract. Meteoritics \& Planetary Science 37:A28. [4] Busemann H. et al. 2002. Geochimica et Cosmochimica Acta 66: A112. [5] Gannoun A. et al. 2011. Geochimica et Cosmochimica Acta 75:3269-3289. [6] El Goresy A. 1985. Meteoritics 20:639. [7] Torigoye N. and Shima M. 1993. Meteoritics 28:515-527. [8] Lehner S. W. et al. 2010. Meteoritics \& Planetary Science 45:289-303.

\section{8}

\section{IODINE AND XENON SYSTEMATICS OF GRA 06129}

J. L. Claydon ${ }^{1}$, S. A. Crowther ${ }^{1}$, C. K. Shearer ${ }^{2}$ and J. D. Gilmour ${ }^{1}{ }^{1}$ School of Earth, Atmospheric and Environmental Sciences, University of Manchester, Oxford Road, Manchester, M13 9PL, UK, E-mail: jennifer.claydon@postgrad.manchester.ac.uk. ${ }^{2}$ Institute of Meteoritics, Department of Earth and Planetary Sciences, University of New Mexico, Albuquerque, NM 87131, USA.

Introduction: GRA 06129 (GRA 9) is an ancient ungrouped achondrite that has experienced a series of processes on its parent asteroid [1]. Our previous analyses of unirradiated GRA 9 [1] showed excess ${ }^{129} \mathrm{Xe}$ from decay of ${ }^{129} \mathrm{I}$, either in situ or inherited, and ${ }^{131-136} \mathrm{Xe}$ from fission of ${ }^{244} \mathrm{Pu}$. Excess ${ }^{129} \mathrm{Xe}$ was thought to be hosted in a minor phase. An I-Xe age of $\sim 75 \mathrm{Ma}$ after Shallowater and an evolved trapped component have been reported [2].

Experimental: Whole rock fragments $(\sim 1 \mathrm{mg})$ of GRA 9 were neutron irradiated $\left(\sim 10^{19} \mathrm{n} \mathrm{cm}^{-2}\right)$ converting the stable isotope ${ }^{127}$ I to ${ }^{128} \mathrm{Xe}$. Following irradiation the samples were laser step-heated at high resolution and $\mathrm{Xe}$ isotopes analyzed using RELAX [3,4]. ${ }^{131} \mathrm{Xe}$ is produced in the irradiation from $\mathrm{Ba}$ or Te and ${ }^{131-136} \mathrm{Xe}$ are produced by neutron-induced fission of ${ }^{235} \mathrm{U}$, allowing some mineralogical information about phases releasing gas at any temperature to be determined. A correlation between ${ }^{129} \mathrm{Xe}$ and ${ }^{127} \mathrm{I}$ during step-heating of a sample would indicate that some of the ${ }^{129} \mathrm{Xe}$ had been derived from in situ decay of ${ }^{129} \mathrm{I}$. When this is the case, the ratio of excess ${ }^{129} \mathrm{Xe}$ and ${ }^{127} \mathrm{I}$ corresponds to the closure age of the material $[5,6]$.

Results: Three samples of GRA 9 have been analyzed producing 116, 64 and 60 individual releases respectively. High concentrations of excess ${ }^{128} \mathrm{Xe}$ (corresponding to $10-30 \mathrm{ppm}$ of I) released at low temperatures are attributed to Antarctic contamination [7]. Excess ${ }^{131} \mathrm{Xe}$ released at higher temperatures corresponds to $\mathrm{Ba}$ concentrations of $10-50 \mathrm{ppm}$, consistent with an origin in plagioclase [1].

A series of distinct high temperature releases were observed; each with contributions from Ba- and I-derived Xe alongside fission xenon and a trapped component. The systematics suggest an "original" phase with a consistent $\mathrm{I} / \mathrm{Ba}$ ratio overprinted by contributions from a later phase containing iodine alongside $\mathrm{U} / \mathrm{Pu}$. Given the mineralogy of GRA 9, this may be attributed to the addition of ( $\mathrm{U} / \mathrm{Pu}$-bearing) apatite to (Ba-rich) plagioclase.

Small ${ }^{129} \mathrm{Xe}$ excesses were observed alongside these releases, but no overall correlation with I-derived ${ }^{128} \mathrm{Xe}$ was observed. This discordant behavior suggests endmember models involving either inheritance of excess ${ }^{129} \mathrm{Xe}$ during secondary phase formation after complete decay of ${ }^{129} \mathrm{I}$, or production of distinct secondary phases in a series of separate events distributed through time. In neither case can a closure age be reliably determined.

References: [1] Shearer C. K. et al. 2010. Geochimica et Cosmochimica Acta 74:1172-1199. [2] Bajo K. et al. 2010. Abstract. Meteoritics \& Planetary Science 45:A5089. [3] Gilmour J. D. et al. 1994. Review of Scientific Instruments 65:617-625. [4] Crowther S. A. et al. 2008. Journal of Analytical Atomic Spectrometry 23:921-1044. [5] Brazzle R. H. et al. 1999. Geochimica et Cosmochimica Acta 63:739-760. [6] Gilmour J. D. et al. 2006. Meteoritics \& Planetary Science 41:19-31. [7] Heumann K. G. et al. 1987. Geochimica et Cosmochimica Acta 51:25412547. 


\section{0}

\section{OXYGEN AND NITROGEN ISOTOPES IN THE EARTH AND THE} SUN

R. N. Clayton. Enrico Fermi Institute, Department of Chemistry, Department of the Geophysical Sciences, University of Chicago, IL, USA. E-mail: r-clayton@uchicago.edu.

By analogy with $\mathrm{CO}$, it has been suggested that photochemical selfshielding may have been important for $\mathrm{N}_{2}$ in the early solar system [1]. Self-shielding in $\mathrm{CO}$ has led to an enrichment on Earth, relative to the Sun and solar nebula, in the rare heavy isotopes of oxygen by about $6 \%$ [2]. Nitrogen isotope measurements in the Genesis solar wind collectors imply an enrichment of ${ }^{15} \mathrm{~N}$ on Earth of about $40 \%$. [3]. A similar enrichment in the heavy nitrogen isotope was not seen in ammonia on Jupiter [4], implying that the photochemical process operated in the inner solar system, and also implying that the young Sun was the source of UV radiation. An obvious question is: why is the nitrogen isotope effect so much larger than that in oxygen? A possible solution lies in the nature of the photolysis products in the two cases:

$\mathrm{CO}\left({ }^{1} \Sigma\right) \rightarrow \mathrm{C}\left({ }^{3} \mathrm{P}\right)+\mathrm{O}\left({ }^{3} \mathrm{P}\right)$ (both atoms in the ground state) [5] (1)

$\mathrm{N}_{2}\left({ }^{1} \Sigma\right) \rightarrow \mathrm{N}\left({ }^{4} \mathrm{~S}\right)+\mathrm{N}\left({ }^{2} \mathrm{D}\right)$ (one atom in an excited state) [6]. (2)

The subsequent "trapping reactions" are much faster for the excited $\mathrm{N}\left({ }^{2} \mathrm{D}\right)$ than those for the ground-state atoms $\left(\mathrm{C}\left({ }^{3} \mathrm{P}\right), \mathrm{O}\left({ }^{3} \mathrm{P}\right)\right.$ and $\left.\mathrm{N}\left({ }^{4} \mathrm{~S}\right)\right)$. The first (and rate-limiting) step in trapping is probably reaction with the abundant molecular hydrogen:

$\mathrm{N}+\mathrm{H}_{2} \rightarrow \mathrm{NH}+\mathrm{H}(3)$

$\mathrm{O}+\mathrm{H}_{2} \rightarrow \mathrm{OH}+$ H. (4)

It was shown [7] that a depletion of about a factor of five in ${ }^{12} \mathrm{C}^{17} \mathrm{O} /{ }^{12} \mathrm{C}^{16} \mathrm{O}$ and ${ }^{12} \mathrm{C}^{18} \mathrm{O} /{ }^{12} \mathrm{C}^{16} \mathrm{O}$ can be found in a molecular cloud, implying almost complete photodissociation of the rarer isotopologues in the cloud interior. If the same degree of dissociation applies also to ${ }^{14} \mathrm{~N}^{15} \mathrm{~N}$ in the solar nebula, then the atom ratio ${ }^{15} \mathrm{~N}\left({ }^{2} \mathrm{D}\right) /{ }^{17} \mathrm{O}$ is near unity. The implication is that the larger "isotopic anomaly" observed for terrestrial nitrogen relative to oxygen is not due to the primary photochemical process, and likely reflects the greater "trapping" reactivity of $\mathrm{N}\left({ }^{2} \mathrm{D}\right)$ relative to $\mathrm{O}\left({ }^{3} \mathrm{P}\right)$. The observed ratio of isotope effects is consistent with a trapping reaction temperature of about $1400 \mathrm{~K}$, and is inconsistent with lower temperatures, due to the large activation energy (and consequent large temperature dependence) for the oxygen trapping reaction.

Note that there is no large isotope effect in carbon, since its lower ionization potential leads to rapid isotope "scrambling":

$\mathrm{C}+\mathrm{H}^{+} \rightarrow \mathrm{C}^{+}+\mathrm{H}$, and (5)

${ }^{12} \mathrm{C}^{+}+{ }^{13} \mathrm{C} \rightarrow{ }^{12} \mathrm{C}+{ }^{13} \mathrm{C}^{+}$. (6)

There are, of course, many measurements of ${ }^{15} \mathrm{~N} /{ }^{14} \mathrm{~N}$ ratios in solar system materials that are greater than the terrestrial atmospheric value, usually associated with high $\mathrm{D} / \mathrm{H}$ ratios in organic materials. These have been interpreted as the result of low-temperature ion-molecule reactions $[8,9]$ either in the solar nebula or in the precursor molecular cloud.

References: [1] Clayton R. N. 2002. Nature 415:860. [2] McKeegan K. D. et al. 2011. Science 332. in press. [3] Marty B. et al. 2011. Science 332. in press. [4] Owen T. et al. 2001. The Astrophysical Journal 553:L77. [5] Gaydon A. G. and Penney W. G. 1945. Proceedings of the Royal Society 183:374. [6] Walter C. W. et al. Physical Review A 50:2930. [7] Sheffer Y. et al. 2002. The Astrophysical Journal 574:L171. [8] Terzieva R. and Herbst E. 2000. Monthly Notices Royal Astronomical Society 317:563. [9] Charnley S. B. and Rodgers S. D. 2002. The Astrophysical Journal 569:L133.

\section{4}

\section{THE EFFECTS OF PLANETESIMAL COLLISIONS}

G. S. Collins ${ }^{1}$, T. M. Davison ${ }^{1,2}$ and F. J. Ciesla ${ }^{2}$. ${ }^{1}$ Impacts and Astromaterials Research Centre, Dept. Earth Science and Engineering, Imperial College London, SW7 2AZ, UK. E-mail: g.collins@imp-erial. ac.uk. ${ }^{2}$ Dept. of Geophysical Sciences, The University of Chicago, 5734 South Ellis Avenue, Chicago IL 60430, USA.

Introduction: As planetesimals are expected to form via gentle aggregation of smaller bodies, the first generation of large solid bodies likely possessed significant $(>50 \%)$ porosity [1-3]. The compaction of a substantial volume of pore space can dramatically decrease the peak shock pressure and increase the post-shock temperature of impactprocessed materials $[4,5]$.

Critical to understanding the consequences of planetesimal collisions in the early solar system is quantification of (1) the disruption threshold for weak, porous first-generation planetesimals; (2) the evolution of planetesimal porosity with time through impact-induced compaction; and (3) the redistribution, retention and cooling of heated material in subcatastrophic collisions. Here we simulate sub-catastrophic planetesimal collisions to late times, quantifying momentum transfer and compaction as well as the ejection and redistribution of impact heated material.

Method: We used the 2D iSALE hydrocode [6,7] to simulate subcatastrophic head-on collisions between planetesimals. ANEOS-derived equation of state tables for dunite were used to represent the non-porous planetesimal material. Pore-space compaction was modeled using the improved epsilon-alpha porosity model [6,8]. Material strength was modeled according to [7] with parameters for weak rock. Target planetesimals $100 \mathrm{~km}$ and $500 \mathrm{~km}$ in diameter with a uniform initial temperature $(300 \mathrm{~K})$ and initial porosities of $0-50 \%$, were modeled. Impacting planetesimals with diameters $0.1-0.3$ times target planetesimal diameter, and with identical material properties, collided with the target at $0.1-7 \mathrm{~km} \mathrm{~s}^{-1}$. The gravity field was updated periodically during the calculation using a self-gravity algorithm, inspired by [9].

Results: The compaction of pore space during a planetesimal collision increases shock heating and reduces ejection velocities relative to the non-porous case. Hence, if planetesimals are sufficiently porous, collisions can cause localized heating and, even in large sub-catastrophic collisions, the vast majority of the heated material is retained on the target planetesimal. As the energy of the collision approaches the disruption threshold, the fate of the heated material changes considerably, with important implications for post-collision thermal evolution. In small collisions, the small volume of heated material is buried beneath an insulating lens of breccia in the crater. In larger collisions, the heated material is localized within the impacted hemisphere, forming a deep plug beneath the impact site. Close to the disruption threshold, the heated material is distributed in a thin layer over the entire surface of the planetesimal. Secondary compaction occurs across the surface of the rearside, which may reduce the potential for subsequent impacts to further heat the planetesimal.

References: [1] Wurm G. et al. 2001. Physical Review E 64:046301. [2] Cuzzi et al 2008. The Astrophysical Journal 687:1432-1447. [3] Johansen et al. 2007. Nature 448:1022-1025. [4] Zel'dovich, Y. B. and Raizer, Y. P. Physics of shock waves and high-temperature hydrodynamic phenomena. Academic Press, New York. 1966. [5] Davison T. M. et al. 2010. Icarus 208:468-481. [6] Wünnemann K., Collins G. S. and Melosh H. J. 2006. Icarus 180:514-527. [7] Collins G. S., Melosh H. J., and Ivanov B. A. 2004. Meteoritics \& Planetary Science 39:217-231. [8] Collins G. S., Melosh H. J. and Wünnemann K. 2010. International Journal of Impact Engineering, in press. [9] Barnes J. E. \& Hut P. 1986. Nature 324:446-449. 
5313

ALLENDE 3509 HC-2: A COMPACT TYPE A-'F' INCLUSION WITH A SNAKE-LIKE MORPHOLOGY

H. C. Connolly Jr. ${ }^{1,2,3}$, J. R. Beckett ${ }^{4}$, G. R. Huss ${ }^{5}$, K. Nagashima ${ }^{5}$, E. D. Young ${ }^{6}$, K. Ziegler ${ }^{6}, \mathrm{C} . \mathrm{Ma}^{4}$ and G. R. Rossman ${ }^{4}$. ${ }^{1}$ Dept. Physical Sci., Kingsborough Community College of CUNY, Brooklyn NY 11235 \& Dept. Earth \& Envir. Sci., The Graduate Center of CUNY, New York, NY 10016, USA. ${ }^{2}$ Dept. Earth \& Planet. Sci., AMNH, New York, NY 10024, USA. ${ }^{3}$ LPL, Univ. of Arizona, Tucson, AZ 85721, USA. ${ }^{4}$ Div. of Geol. \& Planet. Sci., Caltech, Pasadena, CA 91125, USA. ${ }^{5}$ Hawai'i Institute of Geophysics \& Planetology, Univ. of Hawai'i at Mãnoa, Honolulu, HI 96822, USA. ${ }^{6}$ Dept. of Earth \& Space Sci., UCLA, Los Angeles, CA 90095, USA. E-mail: chondrule@haroldconnolly.com.

Introduction: Calcium-, aluminum-rich inclusions (CAIs) are the first rocks to form in the solar system. Some inclusions are igneous and a very few of these (called $\mathrm{F}$ inclusions) experienced significant mass dependant fractionation in oxygen [1], and in some cases, other isotopic systems (FUN inclusions). Increasing the number and types of $\mathrm{F}$ inclusions will provide additional constraints on CAI formation and their formation environments. We report here on a Compact Type A (CTA) inclusion from Allende with an overall snake-like morphology, is an F inclusion, and initial ${ }^{26} \mathrm{Al} /{ }^{27} \mathrm{Al}$ that was canonical.

Analytical Methods: The entire inclusion, HC-2, was recovered from a slab of Allende 3509 (USNM). Major and minor element abundances were determined with the Cameca SX-50 at the LPL UAz. Oxygen isotopes and $\mathrm{Al}-\mathrm{Mg}$ isotopic systematics were analyzed for on the Cameca 1280 ion microprobe at the Univ. of Hawai'i, Mãnoa [2]. Si isotopes were analyzed by LA-MC-ICPMS at UCLA.

Results: HC-2 has an overall morphology that is snake-like, wrapping throughout a $1.5 \mathrm{~cm}$ thick (total width is approximately $0.75 \mathrm{~cm}$ ) section of the meteorite, inspiring it to be nicknamed The Snake. It has a Wark-Lovering rim on both sides, although the rim is thicker on one side. The inclusion has experienced some brittle deformation. HC-2 is dominated by spinel $(\mathrm{Ti}=0.14-0.37 ; \mathrm{V}=0.44-0.64 ; \mathrm{Cr}=0.08-0.14$; all $\mathrm{wt} \%)$ and melilite $(\mathrm{Ak}=$ approximate 4 to approximate 50$)$. Perovskites are numerous, ranging in size from sub-micron to approximately $0.5 \mathrm{~mm}$. There appear to be two smaller (approximately $400 \mu \mathrm{m}$ ) CAIs that are mineralogically layered included.

SIMS analysis on spinel and melilite yield an isochron with an initial ${ }^{26} \mathrm{Al} /{ }^{27} \mathrm{Al}$ of $(4.9 \pm 0.2) \times 10^{-5}$. $\quad \hat{A}^{25} \mathrm{Mg}$ (variations from the standard in stable $\mathrm{Mg}$ isotope) range from approximately 5 to $13 \%$. Oxygen isotopes: Melilites have $\delta^{18} \mathrm{O}-\delta^{17} \mathrm{O}-$ values that span approximately $10 \%$ and plot on the CCAM line near the TF line. Two perovskite grains are isotopically different with $\delta^{18} \mathrm{O}, \delta^{17} \mathrm{O}$ of (in \%o) approximately $-28,-31$ in one and $-40,-44$ in the other. Overall, the spinels are ${ }^{16} \mathrm{O}$-rich and show a clear mass fractionation of $4 \% \mathrm{amu}^{-1}$, $\left(\delta^{18} \mathrm{O}=-34\right.$ to $-42 \% ; \delta^{17} \mathrm{O}=-41$ to $-45 \%$ ) with the overall trend to the right of the CCAM line.

Conclusion: HC-2 is clearly igneous. The reason for its unusual shape is not clear, but it must have formed while plastic. Based on the stable isotope data, HC-2 experienced considerable mass-dependent isotopic fractionation, most likely while molten and while ${ }^{26} \mathrm{Al}$ was present. We will compare our data to other ' $\mathrm{F}$ ' inclusions to place new constraints on CAI formation and the environment in which they formed.

References: [1] Bullock E. S. et al. 2011. Abstract \#2312. 42nd Lunar and Planetary Science Conference. [2] Kentaro et al. 2009. Geochimica et Cosmochimica Acta. [3] Shahara and Young. 2009. Earth and Planetary Science Letters.
5054

MAJOR AND TRACE ELEMENTS AND OXYGEN ISOTOPES IN DIFFERENTIATED COSMIC SPHERULES RELATED TO VESTALIKE ASTEROIDS

C. Cordier ${ }^{1}$, L. Folco ${ }^{1}$, C. Suavet ${ }^{1}$, P. Rochette ${ }^{2}$ and C. Sonzogni ${ }^{2} .{ }^{1}$ MNA, Siena, Italy. E-mail: cordier@unisi.it. ${ }^{2}$ CEREGE, Aix-en-Provence, France.

Cosmic spherules (CSs) are extraterrestrial particles $<2 \mathrm{~mm}$ in size, captured by the Earth's gravitational field and melted during atmospheric entry. Generally, CSs have geochemical affinities with chondritic material [1]. The ongoing investigation of large and unbiased collections of CSs from the South Pole Water Well and the Transantarctic Mountains (TAM) have, however, led to the identification of new types, including nine differentiated CSs [2, 3]. Here, we define the geochemical characteristics of this new type of CSs from the TAM collection [4] in terms of major $(\mathrm{n}=11$, EPMA), trace element $(\mathrm{n}=6$, LA-ICP-MS) and oxygen isotope $(\mathrm{n}=15$, IRMS) compositions.

The 11 differentiated CSs have high $\mathrm{Fe} / \mathrm{Mg}$ ratios (0.6-1.6) and homogeneous $\mathrm{Fe} / \mathrm{Mn}$ ratios $(31 \pm 4)$. Oxygen isotope compositions $\left(\delta^{18} \mathrm{O}=14.37 \%\right.$ to $20.12 \%$ and $\Delta^{17} \mathrm{O}=-0.47 \%$ to $\left.-0.65 \%\right)$ suggest a Vesta-like parent body. Systematic variations in refractory major and trace elements result from differences of the mineralogy of the spherule precursors. Type-1 spherules $(n=4)$ are akin to bulk eucrites, with high $\mathrm{CaO}$ and $\mathrm{Al}_{2} \mathrm{O}_{3}$ contents, flat REE patterns and high REE contents (La/ $\mathrm{Yb}_{\mathrm{N}}=0.4$ to $1.0, \mathrm{REE}_{\mathrm{N}}=8.5$ to $14 \times \mathrm{CI}$ ). They derive from finegrained precursors with typical eucritic mineralogy. Type-2 spherules $(\mathrm{n}=4)$ have lower $\mathrm{CaO}$ and $\mathrm{Al}_{2} \mathrm{O}_{3}$ contents, higher $\mathrm{MgO}, \mathrm{FeO}$, and $\mathrm{Sc}$ contents, and strong LREE depletions $\left(\mathrm{La} / \mathrm{Yb}_{\mathrm{N}}<0.2\right)$ relative to eucrites. These compositions suggest large amounts of pigeonite in their precursors. Type- 3 spherules $(n=2)$ have flat REE patterns with chondritic REE abundances $\left(\mathrm{REE}_{\mathrm{N}}=1.5\right.$ to $\left.2.2 \times \mathrm{CI}\right)$, and are enriched in $\mathrm{Co}\left(>50 \mu \mathrm{g} \mathrm{g}^{-1}\right)$ and $\mathrm{Ni}\left(>17 \mu \mathrm{g} \mathrm{g}^{-1}\right)$ relative to eucrites. Their composition suggests they derive from howardite-like precursors rich in orthopyroxene. The mineralogies proposed for the differentiated precursors are consistent with those expected for micrometeoroids deriving from the regolith of Vesta-like asteroids [5].

The combination of elemental and isotopic data shows that the elemental ratios and contents $(\mathrm{Fe} / \mathrm{Mg}$ and $\mathrm{Fe} / \mathrm{Mn}, \mathrm{REEs}, \mathrm{Ni}$ and $\mathrm{Co}, \mathrm{V}$, $\mathrm{Zn}$ ) previously proposed to identify the differentiated CSs and their parent body [3] are suitable, provided that the mineralogical control is understood. Differentiated CSs deriving from pigeonite-rich precursors (Type 2) tend to have slightly lower $\mathrm{Fe} / \mathrm{Mg}$ and $\mathrm{Fe} / \mathrm{Mn}$ ratios and higher $\mathrm{V}$ contents than basaltic eucrites, whereas large amount of opx in spherule precursor may result in lower $\mathrm{Fe} / \mathrm{Mg}$ and higher $\mathrm{Ni}$ and $\mathrm{Co}$ contents.

The relative frequency of Vesta-like micrometeorites in the TAM micrometeorite collection $(1.6 \%)$ is consistent with the proportion amongst meteorites of eucrites and howardites $(1.8 \%)$, which are the main constituents of the regoliths of Vesta-like asteroids.

Acknowledgments: This work was supported by the PNRA and by the EU-funded ORIGINS Marie Curie Action.

References: [1] Engrand C. and Maurette M. 1998. Meteoritics \& Planetary Science 33:565-580. [2] Taylor S. et al. 2007. Meteoritics \& Planetary Science 42:155-304. [3] Cordier et al. 2011. Geochimica et Cosmochimica Acta 75:1199-1215. [4] Rochette P. et al. 2008. Proceedings of the National Academy of Sciences 105:18206-18211. [5] Burbine T. H. et al. 2009. Meteoritics \& Planetary Science 44:1331-1341. 
5055

\section{THE GEOCHEMICAL FRACTIONATION RECORDED BY CHONDRITIC GLASS COSMIC SPHERULES DURING ATMOSPHERIC ENTRY}

C. Cordier $^{1}$, L. Folco ${ }^{1}$, C. Suavet ${ }^{1}$, P. Rochette ${ }^{2}$ and C. Sonzogni ${ }^{2}$. ${ }^{1}$ MNA, Siena, Italy. E-mail: cordier@unisi.it. ${ }^{2}$ CEREGE, Aix-en-Provence, France.

Introduction: Cosmic spherules (CSs) are extraterrestrial particles $<2 \mathrm{~mm}$ in size, captured by the Earth's gravitational field and melted while penetrating through the atmosphere. The diversity in quench textures and chemistry among stony CSs has been ascribed to variable peak temperatures and evaporative loss during atmospheric entry [1], with glass CSs forming under very high temperature regimes. Here, we combine the major $(\mathrm{n}=187$, EPMA) and trace element $(\mathrm{n}=76$, LAICP-MS) and oxygen isotope $(\mathrm{n}=6$, IRMS) compositions obtained on glass CSs from the Transantarctic Mountains [2] with literature data $[1,3-6]$ to discuss the processes occurring during their atmospheric entry.

Results: A total of $31 \mathrm{CSs}$ closely resemble the $\mathrm{FeO}-$ poor $(\mathrm{Fe} /$ $\mathrm{Si}<0.06)$, Mg-rich $(\mathrm{Mg} / \mathrm{Si}>0.9)$, colorless, glass CAT-like spherules [3]. 45 "high Ca-Al" $\mathrm{CSs}$ have high $\mathrm{CaO}, \mathrm{Al}_{2} \mathrm{O}_{3}$ and $\mathrm{TiO}_{2}$ contents $\left(\mathrm{CaO}+\mathrm{Al}_{2} \mathrm{O}_{3}>9 \mathrm{wt} \%, \mathrm{Ti} / \mathrm{Si}=0.005 \pm 0.002\right)$, but higher $\mathrm{FeO}(\mathrm{Fe} /$ $\mathrm{Si}>0.6)$ and lesser $\mathrm{MgO}$ contents $(\mathrm{Mg} / \mathrm{Si}<0.9)$ than CAT-like CSs. The 393 other chondritic CSs form the normal group. From normal group to high $\mathrm{Ca}-\mathrm{Al}$ group, through CAT-like group, $\mathrm{CaO}, \mathrm{Al}_{2} \mathrm{O}_{3}, \mathrm{TiO}_{2}$ and REE contents increase, whereas $\mathrm{SiO}_{2}, \mathrm{MgO}$ and volatile element contents decrease. Siderophile and chalcophile elements show a different behavior: their depletion increases from the normal group, to the high CAT-like group, through the high $\mathrm{Ca}-\mathrm{Al}$ group.

Atmospheric Entry Processes: Chemical differences between the three groups of CSs record variations in the degree of heating and evaporation. Based on progressive enrichment in major $(\mathrm{Ca}, \mathrm{Al}, \mathrm{Ti})$ and trace $(\mathrm{Zr}$, REEs) refractory elements relative to moderately refractory elements $(\mathrm{Mg}$, $\mathrm{Si})$ and volatile elements $(\mathrm{Rb}, \mathrm{Na}, \mathrm{Zn}, \mathrm{Pb})$, we propose that the mass lost by evaporation increases from the normal group (40-50 wt $\%$ mass loss), through the CAT-like group (50-60 wt\%), to the high Ca-Al group (80$90 \mathrm{wt} \%)$. However, siderophile and chalcophile elements in CSs appear to be not governed only by evaporation process. As there is no correlation between siderophile and chalcophile element contents, the processes governing their abundances during melting and melt evolution are, however, different. Siderophile elements probably record the mechanical extraction of metal (plus sulfide) beads resulting from immiscibility between metallic and silicate liquids under reducing conditions [7]. Chalcophile elements may record the extraction of chromite relict grains. The relative importance of evaporation vs. segregation is controlled by interdependent factors: parent body, precursor mineralogy and thermal and redox conditions during atmospheric entry.

Acknowledgments: This work was supported by the PNRA and by the EU-funded ORIGINS Marie Curie Action.

References: [1] Taylor S. et al. 2000. Meteoritics \& Planetary Science 35:651-666. [2] Rochette P. et al. 2008. Proceedings of the National Academy of Sciences 105:18206-18211. [3] Alexander C. M. O. et al. 2002. Geochimica et Cosmochimica Acta 66:173-183. [4] Taylor S. et al. 2005. Geochimica et Cosmochimica Acta 69:2647-2662. [5] Yada T. et al. 2005. Geochimica et Cosmochimica Acta 69:5789-5804. [6] Cordier C. et al. 2011. Geochimica et Cosmochimica Acta 75:1199-1215. [7] Brownlee D. E. et al. 1997. Meteoritics \& Planetary Science 32:157-175.
5450

THE OLD WOMAN, CALIFORNIA, METEORITE

C. M. Corrigan ${ }^{1}$, R. S. Clarke Jr. ${ }^{1}$, H. Plotkin ${ }^{2}$ and T. J. McCoy ${ }^{1}$. ${ }^{1}$ Smithsonian Institution, NMNH, P.O. Box 37012, Washington, D.C., 20013-7012, USA. E-mail: corriganc@si.edu. ${ }^{2}$ University of Western Ontario, London, ON, Canada.

Introduction: The Old Woman meteorite, discovered in March 1976, by two prospectors searching for a fabled lost Spanish gold mine in mountains approximately $270 \mathrm{~km}$ east of Los Angeles, achieved legendary status among meteorite collectors.

History of the Old Woman Meteorite: The question of the ownership of the meteorite gave rise to $4 \mathrm{yr}$ of disputes. Ultimately, ownership of the meteorite was transferred to the Smithsonian under the powers of the 1906 Antiquities Act, a ruling upheld in a U.S. District Court and a U.S. Court of Appeals. After additional debate, the Smithsonian removed a $0.5 \mathrm{~m}^{2}$ cut for study and curation, and for disbursement of specimens to qualified researchers. The main mass was then returned to California on long-term loan to the Bureau of Land Management way station in Barstow. The Old Woman meteorite litigation served as an important test case for the ownership and control of meteorites found on federal lands.

Scientific Importance of the Old Woman Meteorite: The Old Woman meteorite, a $2753 \mathrm{~kg}$ group IIAB meteorite, is the second largest meteorite (approximately $0.8 \times 0.9 \times 1.0 \mathrm{~m}$ ) ever found in the United States. The large slice retained at the Smithsonian reveals distinct regions that show the transition between hexahedral IIA and octahedral IIB iron meteorite structures, a greater understanding of the solid-state transformation that occurs during cooling. The slice is comprised of a polycrystalline mass with grain sizes ranging from $<1 \mathrm{~cm}$ to $>40 \mathrm{~cm}$ in maximum dimension, with a strongly bimodal distribution. At high temperature, each grain was an independently oriented austenite crystal. Seven large grains comprise approximately $85 \%$ of the surface area and comprise the hexahedral structure within the meteorite, with the remaining $15 \%$ of the surface sampling the octahedral polycrystalline regions up to $20 \mathrm{~cm}$ in maximum dimension with grain sizes of $1-3 \mathrm{~cm}$. More rarely, smaller grains are found completely enclosed (at least in two-dimensions) within the larger grains.

Within the larger grains (hexahedrite, Region 1), kamacite is the only $\mathrm{Fe}, \mathrm{Ni}$ metallic phase identified. Taenite was not seen. Numerous schreibersite grains up to several $\mathrm{cm}$ in length suggest remnant Widmanstätten, similar to Zacatecas [1]. Shock-melted sulfide inclusions contain troilite and daubreelite. Within Region 2, which might be called a coarsest octahedrite in isolation, smaller parent austenite grains are converted completely to kamacite and lack any remnant Widmanstätten pattern [2]. Thin ribbons of schreibersite and sulfides are seen. Traverses across schreibersites yield identical compositions of unzoned schreibersite in both remnant Widmanstatten pattern of the hexahedral structure in Region 1, and at kamacite-kamacite grain boundaries in Region 2.

If sampled separately, the individual lithologies of Old Woman might well have been classified as three distinct meteorites. Instead, we now recognize that transitional textural types do occur in close proximity and can provide clues to unraveling the unique combinations of chemistry and nucleation and cooling history [3] that produce these textures.

References: [1] Buchwald V. 1975. Zacatecas (1792), in Handbook of meteorites. Berkeley and Los Angeles: University of California Press, 3:1361. [2] Clarke R. S., Jr. 1979/2011, personal communication. [3] Yang and Goldstein. 2005. Meteoritics \& Planetary Science 40:239. 
5424

ELEMENTS MAGAZINE: A NEW FORM OF OUTREACH FOR THE METEORITICAL SOCIETY

C. M. Corrigan ${ }^{1}$, H. Y. McSween Jr. ${ }^{2}$ and E. R. D. Scott ${ }^{3}$. ${ }^{1}$ Smithsonian Institution, NMNH, Washington, D.C. 20560, USA. E-mail: corriganc@si.edu. ${ }^{2}$ University of Tennessee, Knoxville, TN, USA. ${ }^{3}$ HIGP, University of Hawai'i at Mãnoa, Honolulu, HI, USA.

Introduction: In 2010, the Meteoritical Society joined Elements magazine, a bimonthly magazine of mineralogy, geochemistry, and petrology, published jointly by 16 professional societies [1]. Elements offers a great way for the Meteoritical Society to inform its members and others about society activities. The Meteoritical Society's first "Society News" page appeared in the October, 2010, issue of the magazine [2]. Three more have since been published, one in an issue highlighting current research in cosmochemistry [3].

Elements Magazine: Elements is read by approximately 15,000 geoscientists and students. Each issue provides invited, peer reviewed articles to the greater, international geochemical science population. Each issue is organized around an individual, relevant, topic in the geosciences. A guest editor compiles a list of invited authors to cover relevant topics around the issue's main theme. The Impact Factor of Elements has tripled since its launch in 2005 and has climbed from an initial 1.562 to 3.569 in 2009.

The Meteoritical Society and Elements Magazine: On a society scale, Elements provides the members, council and officers of the Meteoritical Society with a broad stage on which to publicize events, meeting announcements and reports, award winners, society news, membership and other information both to its fellow members and to the broader community.

On an even broader scale, Elements provides the Meteoritical Society with the opportunity to engage with, and link itself to, the broader geochemical and petrologic communities. With issues such as the February, 2011 issue on cosmochemistry (ed. Dante Lauretta) [3], current hot topics in our field were presented in a fashion both attractive and informative to scientists not necessarily intimately familiar with the details of our research. The February, 2010 issue on mineral evolution (ed. Robert Hazen) [4], linked meteoritical research to the ever-expanding research climate surrounding the topics of biogeochemistry. The August 2009 issue featured an article on mineral magnetism in extraterrestrial materials by Pierre Rochette [5], and February 2009 issue (ed. John Delano) focused on the Moon [6]. Many other issues [7-13] have featured articles written by our members, as well.

In addition, the Meteoritical Society has agreed to increase its contribution to Elements from the June, 2011 issue forward, by providing two pages per issue. Every other issue (roughly) will alternate between two society news pages and a new feature called CosmoElements which will be akin to a one page news article highlighting a hot scientific topic (i.e., the June 2011 page will cover the status of water in meteorites to align itself with the theme of the issue, Global Water Sustainability).

References: [1] http://www.elementsmagazine.org/index.htm. [2] Richet, Henderson, Neuville, eds. 2010. Elements 6, 5, 327. [3] Lauretta D., ed. 2011. Elements 7:1. [4] Hazen, ed. 2010. Elements 6:1. [5] Feinberg, Harrison, eds. 2009. Elements 5, 4, 223. [6] Delano, ed. 2009. Elements 5, 1, 223. [7] Bodnar, ed. 2005. Elements 1, 1, 39. [8] Harlow and Davies, eds. 2005. Elements 1, 2, 85 \& 97. [9] Valley, ed. 2006. Elements 2:4. [10] McSween, ed. 2006. Elements 2:3. [11] Brenan and Mungall, eds. 2008. Elements 4:4. [12] Hochella, ed. 2008. Elements 4:6. [13] Mandeville, ed. 2010. Elements 6:2.
5104

PRELIMINARY INVESTIGATIONS INTO THE TOMOGRAPHY OF PALLASITES: IMPLICATIONS FOR PALLASITE FORMATION

A. L. Couch and R. G. Mayne. ${ }^{1}$ Monnig Meteorite Collection, Texas Christian University, TCU Box 298830, Fort Worth, TX 76129 E-mail: amanda.couch@tcu.edu.

Introduction: Pallasites consist almost entirely of coarse olivine $\left((\mathrm{Mg}, \mathrm{Fe})_{2} \mathrm{SiO}_{4}\right)$ and $\mathrm{FeNi}$ metal. They are traditionally thought to have formed at the core-mantle boundary of a differentiated parent body [1], where the olivine from the mantle would mix with molten metal in the core. However, there are several problems with this theory. The cooling rate of pallasites is much slower than that of any irons [2]. The density difference between the olivine and metal is so great that one would expect to find only a small volume of pallasites, as the zone of mixing would be very limited in a core-mantle formation [3]. However, for every 12 iron meteorites, we have roughly one pallasite [4].

Other formation models theorize that pallasites could have formed i) near the center $[5,6]$, ii) near the surface $[7,8]$, or iii) at contact zones between an olivine mantle and isolated metallic pools [9]. Model (i) states that pallasites formed close to the center of the parent body, which essentially negates the problem with density difference. Model (ii) requires an external heat source, which would melt the surface of the chondritic parent body, causing differentiation. Model (iii) proposed that partial crystallization of a melt yielded the olivine, and the remaining melt was forced out by liquid metal pools.

Here we present the preliminary results of a tomographical study of pallasite. The goal of this study is to examine the relationship between the olivine and the metal in terms of models for pallasite petrogenesis.

Methods: Pallasites are usually sliced thinly to allow light to shine through the olivine crystals. In order to see the $3 \mathrm{D}$ relationship between the olivine and the metal in pallasites, it is important that we use samples that are still in 'block' form. For the purposes of this preliminary work, we analyzed the sample Fukang from the Monnig Meteorites Collection at TCU. The tomography scans were taken at the High Resolution X-Ray CT Facility at the University of Texas at Austin using a BIR ACTIS scanner. The scans are to be analyzed using the program Blob3D. Characteristics that we will be looking at include the interconnectivity of the olivine and metal, along with the grain size, shape, and orientation of the olivine.

Future Work: We are currently researching other meteorite collections to find more suitable pallasite samples for tomographic analysis. We plan to scan and analyze samples from the main group and Eagle Station pallasites. These two groups are suggested to derive from different parent bodies [10]. We will use tomography to assess any differences between the formations.

References: [1] Yang J. et al. 2010. Geochimica et Cosmochimica Acta 74:4471-4492. [2] Mittlefehldt D. W. et al. 1998. Planetary materials (ed. J. J. Papike) 36:4-1-4-195. [3] Scott E. R. D. 1977. Geochimica et Cosmochimica Acta 41:693-710. [4] Scott E. et al. 2010. www.psrd.hawaii.edu/June10/pallasites-origin.html [5] Buseck P. R. and Goldstein, J. I. 1968. Science 159:300-302. [6] Wahl W. 1965. Geochimica et Cosmochimica Acta 29:177-181. [7] Mittlefehldt D. W. 1980. Earth and Planetary Science Letters 51:29-40. [8] Davis A. M. and Olsen, E. J. 1991. Nature 353:637-640. [9] Urey H. C. 1956. The Astrophysical Journal 124:623-637. [10] Scott E. R. D. 1977. Geochimica et Cosmochimica Acta 41:349-360. 


\section{2}

\section{MAGNETIC STUDY OF PARIS METEORITE}

C. Cournede ${ }^{1}$, J. Gattacceca ${ }^{1}$, B. Zanda ${ }^{2}$ and P. Rochette ${ }^{1}$. ${ }^{1}$ CEREGE, CNRS/Université Aix-Marseille, Aix-en-Provence, France. ${ }^{2}$ LMCM, Museum national d'Histoire naturelle, CP52, 61 rue Buffon, 75005 Paris, France.

The Paris meteorite is a unique carbonaceous chondrite find (CM2/ 3) with almost no traces of terrestrial weathering [1]. Indeed, this $\mathrm{CM}$ chondrite is characterized by the co-existence of $\mathrm{FeNi}$ metal and magnetite, the latter mineral being formed during aqueous alteration on the parent body.

Magnetic measurements offer a non-destructive method to quantify the respective abundances of metal and magnetite over a wide range of spatial scales ( $\mathrm{mm}$ to decimeter). Moreover the comparison of the magnetization obtained for metal-rich and magnetite-rich samples may provide constraints on the chronology of the parent body alteration, and brecciation of this meteorite.

We conducted a magnetic study of several oriented samples of this meteorite, with masses ranging from several $\mathrm{mg}$ up to $17 \mathrm{~g}$. Preliminary rock magnetism results show that Paris has a magnetic mineralogy that is notably different from that of other $\mathrm{CM}$ chondrites, with a significant contribution of $\mathrm{FeNi}$ metal which confirms that Paris suffered less aqueous alteration than other $\mathrm{CM}$ chondrites. We will discuss the smallscale variability of the magnetic mineralogy. We will also discuss the results of measurements of anisotropy of magnetic susceptibility, a proxy to the petrofabric intensity and orientation [2], in relation with the brecciation history of the meteorite.

Paleomagnetic data show that despite having being exposed to an artificial strong magnetic field (magnet), a pre-terrestrial magnetization, apparently carried by magnetite, is still preserved in the meteorite. Because crystallization of magnetite likely occurred only several Myr after the formation of the solar system [3], i.e., after the possible existence of strong solar and nebular magnetic fields, the homogeneity of the magnetization within the meteorite may indicate the existence of an internally generated field at that time. This would imply a partially differentiated parent body with a convecting metallic core, as recently proposed for the parent body of $\mathrm{CV}$ chondrites [4,5]. However, the brecciation history of Paris meteorite needs to be better understood before this conclusion can be confirmed.

References: [1] Zanda et al. 2010. Meteoritics \& Planetary Science 45:222-222. [2] Gattacceca et al. 2005. Earth and Planetary Science Letters 234:351-368. [3] Petitat and Gounelle. 2010. Abstract \#1673, Lunar and Planetary Science Conference. [4] Carporzen et al. 2011. Proceedings of the National Academy of Sciences 108:6386-6389. [5] Elkins-Tanton et al. 2011. Earth and Planetary Science Letters 305:1-10.
5293

THE LIFE MARKER CHIP-EXTRACTING POLAR AND NONPOLAR BIOMARKERS FROM THE MARTIAN SOIL USING A SURFACTANT-BASED SOLVENT

R. W. Court ${ }^{1}$, O. A. Baki ${ }^{1}$, M. R. Sims $^{2}$, D. Cullen ${ }^{3}$ M. A. Sephton ${ }^{1}$. ${ }^{1}$ Dept. of Earth Science and Engineering, Imperial College London. E-mail: r.court@imperial.ac.uk. ${ }^{2}$ Space Research Centre, University of Leicester. ${ }^{3}$ Cranfield Health, Cranfield University.

Introduction: The Life Marker Chip (LMC) is designed to detect the chemical evidence of past or present Martian life [1]. To achieve this it will use an antibody-based detection system, but the organic solvents best suited to extracting organic matter from the Martian soil are incompatible with the LMC antibodies. Instead, an aqueous solvent must be used, but water-based solvents are typically ineffective at dissolving the nonpolar organic compounds of most interest to the LMC. A surfactant-based solution of 20:80 (vol:vol) methanol:water with $1.5 \mathrm{mg} \mathrm{ml}^{-1}$ of polysorbate 80 solves this dilemma, with the surfactant forming micelles that promote the dissolution of nonpolar molecules [2]. Here, we report on the ability of this surfactant solution to dissolve organic standards spiked on to analogues to the Martian soil, and on the tendency of common spacecraft materials to adsorb these standards from solution.

Results: Addition of $1.5 \mathrm{mg} \mathrm{ml}^{-1}$ of the surfactant polysorbate 80 to a 20:80 (vol:vol) methanol:water solution results in an increase in solubility of approximately two orders of magnitude for the standards hexadecane, phytane, squalene, anthracene, pyrene, stigmasterol and coprostane, resulting in solubilities in the range of $\sim 1-50 \mu \mathrm{g} \mathrm{ml}^{-1}$. Tests on a range of Martian analogs, including the palagonitic tephra JSC Mars-1 [3], goethite and hematite, demonstrates its ability to extract organic compounds spiked on to their surfaces without debilitating adsorption. The addition of iron compounds such as iron (III) sulfate, iron (III) perchlorate and iron (III) chloride to JSC Mars-1 similarly has no significant deleterious adsorption or chemical effects. However, the addition of the clay-rich Jurassic Blue Lias shale to JSC Mars-1 results in significant adsorption of the standards and a greatly reduced efficiency of extraction of the standards. Further testing is required to determine whether this applies to clay minerals in Martian environments.

The ability of common spacecraft material to adsorb standards dissolved in the surfactant solution has also been investigated. Passage of solvent through $0.2 \mu \mathrm{m}$ syringe filters shows that polytetrafluoroethylene (PTFE) is least prone to adsorb the standards from surfactant solution, while cellulose acetate adsorbs the most. Polyethersulfone, polypropylene and nylon filters show intermediate levels of adsorption. The fluoropolymer Viton ${ }^{\mathrm{TM}}$ shows a small adsorption effect, but not one large enough to significantly inhibit the operation of the LMC.

References: [1] Sims M. R. et al. 2005. Planetary and Space Science 53:781-791. [2] Court R. W. et al. 2010. Planetary and Space Science 58:1470-1474. [3] Allen C. C. et al. 1998. In Space '98: Proceeding of the Conference of American Society of Civil Engineers. 
5295

\section{VOLATILE PRODUCTION FROM ABLATING MICROMETEORITES -EVIDENCE FROM STEPPED PYROLYSIS-FTIR}

R. W. Court, M. A. Sephton. Dept. of Earth Science and Engineering, Imperial College London. E-mail: r.court@imperial.ac.uk.

Introduction: Pyrolysis-Fourier transform infrared (FTIR) spectroscopy has recently been used to simulate the atmospheric entry of micrometeorites and to quantify the production of water, carbon dioxide, methane and sulfur dioxide produced by the thermal processing of their organic and mineral phases [1-4]. However, these attempts to quantify micrometeoritic volatile contributions to the atmospheres of terrestrial planets have assumed that the entire infalling micrometeorite flux is heated to the same temperature. In reality, micrometeorites of a range of varying masses, densities and mineralogies enter the atmosphere at a range of velocities and angles, experiencing variable degrees of heating. As such, a more sophisticated simulation of atmospheric entry should account for variations in volatile production with temperature. Here, we have subjected samples of five carbonaceous chondrites-CI1 Orgueil, CM1 ALH 88045, CM2 Cold Bokkeveld, CM2 Murchison and CV3 Mokoia - to stepped pyrolysis and used FTIR to identify and quantify the volatiles produced at each temperature step.

Results: The yields of water, carbon dioxide and sulfur dioxide at temperature steps from $250{ }^{\circ} \mathrm{C}$ to $1000{ }^{\circ} \mathrm{C}$ have been quantified. The yields of the volatiles, and their patterns of release, can be related to the degree of aqueous alteration experienced by the chondrite on the meteorite parent body. The relatively strongly aqueously altered chondrites Orgueil and Cold Bokkeveld show prominent low-temperature yields of sulfur dioxide and water that are absent or greatly reduced in Murchison and Mokoia. The CM1 chondrite ALH 88045 shows patterns of volatile release more closely related to the considerably less aqueously altered CM2 Murchison and CV3 Mokoia, an anomaly interpreted in terms of terrestrial alteration while this meteorite was exposed on the Antarctic ice sheet $[3,5,6]$. Carbon dioxide release is more pronounced at higher temperatures, representing the stability of carbon dioxide sources such as carbonates, and the carbon dioxide yield is lower in samples having experienced less aqueous alteration, reflecting the lower abundance of carbonates and organic matter in chondrites such as Mokoia. Combination of these data, revealing volatile production across a range of temperatures, with temperature models of infalling micrometeorites can produce superior estimates of the micrometeoritic contributions to the volatile budgets of terrestrial planets.

References: [1] Court R. W. and Sephton M. A. 2011. Geochimica et Cosmochimica Acta 75:1704-1717. [2] Court R. W. and Sephton M. A. 2009. Earth and Planetary Science Letters 288:382-385. [3] Court R. W. and Sephton M. A. 2009. Geochimica et Cosmochimica Acta 73:35123521. [4] Court R. W. and Sephton M. A. 2009. Analytica Chimica Acta 639:62-66. [5] Shima M., Shima M., and Hintenberger H. 1973. Earth and Planetary Science Letters 19:246-249. [6] Sephton M. A. et al. 2004. Meteoritics \& Planetary Science 39:747-754.
5228

TIC AND RUTILE WITHIN A SUPERNOVA GRAPHITE

T. K. Croat ${ }^{1}$, M. Jadhav ${ }^{1,2}$, E. Lebsack ${ }^{1}$ and T. J. Bernatowicz ${ }^{1}$. ${ }^{1}$ Laboratory for Space Sciences and Physics Department, Washington University, St. Louis, MO 63130, USA. E-mail: tkc@wustl.edu. ${ }^{2}$ HIGP, University of Hawaii at Mãnoa, Honolulu, HI, USA.

Introduction: The origin of rutile grains $\left(\mathrm{TiO}_{2}\right)$ found within presolar graphites (e.g., primary condensate or from secondary alteration) is unclear, given that the host graphites form in C-rich environments and rutile likely in O-rich ones [1]. The Orgueil graphite OR1dm3-6 has ${ }^{12} \mathrm{C}$, ${ }^{15} \mathrm{~N}$ and ${ }^{18} \mathrm{O}$ excesses indicative of a supernova $(\mathrm{SN})$ origin $[2,3]$, and also contains hundreds of Ti-rich internal grains, both $\mathrm{TiC}$ and rutile [4]. Detailed studies of the chemistry, phase, $\mathrm{O}$ isotopic composition and spatial distribution of Ti-rich grains within g6 $\mathrm{SN}$ graphite strongly suggest that the rutile grains do not form secondarily (e.g., via oxidation of existing TiCs), but rather are also a primary SN condensate and are indicative of compositional changes (mixing) in SN ejecta.

Experimental Methods: After isotopic studies [2, 3], OR1d3m-6 was ultramicrotomed and studied in TEM [4]. Subsequent NanoSIMS measurements of ${ }^{16} \mathrm{O},{ }^{18} \mathrm{O},{ }^{28} \mathrm{Si},{ }^{29} \mathrm{Si}$, and ${ }^{30} \mathrm{Si}$ were made on $\mathrm{TiC}$ and rutile-containing graphite sections.

Results and Discussion: Chemical compositions (EDXS) and images were collected from 118 separate Ti-rich internal grains within $7 \mathrm{~g} 6$ graphite cross-sections. Due to their unusually high O content, SN TiCs cannot be distinguished from rutile grains on that basis, so 76 Ti-rich grains were identified by their distinctive electron diffraction patterns (TiC, $\mathrm{FCC}, \mathrm{a}=4.4 \AA$ and $\mathrm{TiO}_{2}$ tetragonal, $\mathrm{a}=4.9 \AA, \mathrm{c}=3.1 \AA$ ). Fifty-two rutile grains and $13 \mathrm{TiCs}$ were found, along with 4 Ti-rich grain patterns that may indicate a different Ti-oxide polymorph. Three SiC and two kamacite grains were also found. The TiCs fall into two groups based on size and composition, with the larger ones (80-130 nm) showing significantly higher $\mathrm{V}$ and lower $\mathrm{Al}$ content (avg. $\mathrm{V} / \mathrm{Ti}$ and $\mathrm{Al} / \mathrm{Ti}$ at. ratios of 0.30 and 0.01 as opposed to 0.15 and 0.40 respectively from smaller $(<30 \mathrm{~nm})$ TiCs. The clear increase in $\mathrm{V} / \mathrm{Ti}$ ratio vs. grain size is consistent with large TiCs experiencing extended growth times at lower $\mathrm{T}$ and thus incorporating more $\mathrm{V}$ (since $\mathrm{VC}$ is less refractory than $\mathrm{TiC}$ ). The high $\mathrm{Al}$ content in some small carbides (not seen previously among SN TiCs) may indicate formation of the structurally-similar $\mathrm{Ti}_{3} \mathrm{AlC}$ phase (4.2 $\AA$ cubic). Other than having somewhat higher O content, the rutile grains are similar in size and composition to the small TiCs. Analysis of several central cross-sections show TiCs predominantly clustered towards the outside of the graphite's surface, whereas rutile grains are spread throughout its volume. Thus, oxidation of existing TiCs to form rutile after encapsulation in graphite is unlikely to occur, which gives credence to primary condensation of rutile in SN ejecta. NanoSIMS O isotopic images of a $\mathrm{g} 6$ cross-section containing two large $\mathrm{TiC}$ show ${ }^{16} \mathrm{O} /{ }^{18} \mathrm{O}$ of $156 \pm 33$ in the carbides and $315 \pm 23$ in the graphite ( $2 \sigma$ errors). The ${ }^{18} \mathrm{O}$ counts from small $\mathrm{TiCs}$ and rutiles were insufficient to allow a separate determination of their isotopic composition. Along with more extreme $\mathrm{O}$ anomalies in $\mathrm{TiC}$ than in graphite, $\mathrm{O}$ isotopic dilution towards normal nearer the graphite's surface is also present, effects similar to those seen in [5].

References: [1] Croat T. K. 2007. Meteoritics \& Planetary Science 42:A34. [2] Croat T. K. et al. 2009. 40th Lunar Prospector Spacecraft A2175. [3] Jadhav M. et al. 2006. New Astronomy Review 50:591-595. [4] Jadhav M. et al. 2008. 39th Lunar Prospector Spacecraft A1047. [5] Stadermann F. J. et al. 2005 Geochimica et Cosmochimica Acta 69:177188. 
5354

PB-PB AGES OF APOLLO 15 ZIRCONS

C. A. Crow ${ }^{1}$, K. D. McKeegan ${ }^{1}$ and D. J. Taylor ${ }^{1}$. ${ }^{1}$ Department of Earth and Space Sciences, University of California Los Angeles, USA. E-mail: ccrow@ucla.edu.

Introduction: Crystallization ages of lunar zircons provide insight into the timing of early magmatic and/or impact events on the Moon. We report new $\mathrm{Pb}-\mathrm{Pb}$ ages for Apollo 15 zircons and previously unreported zircon ages for Apollo 17. We also report on the REE, Ti temperatures, and U/Th data for Apollo 15 zircons.

Sample: The ages reported here are for zircons from two Apollo 15 samples, 15405 and 15455. Both are impact-melt breccias. 15405 is comprised mainly of dark lithology KREEP basalt [1]. 15455 is a mixture of dark basalt and light anorthosite lithologies [2]. These samples were chosen for their enrichment in KREEP, REE, and U concentrations.

Method: Individual zircons were separated from crushed clasts $(4.25 \mathrm{~g}$ of $15405,75,3.91 \mathrm{~g}$ of 15405,9 , and $2.10 \mathrm{~g} \mathrm{15455,226)}$ and analyzed using the UCLA Cameca ims-1270 ion microprobe. U-Pb and $\mathrm{Pb}-\mathrm{Pb}$ ages, $\mathrm{REE}$, and trace element data were collected for all Apollo 15 zircons.

Results: The $\mathrm{Pb}-\mathrm{Pb}$ ages of the Apollo 15 zircons show a peak at approximately $4.33 \mathrm{Ga}$, which is also present in Apollo 17 [3] and Apollo 14 data [4]. Meyers et al. 1996 suggest that the peaks in U-Pb age distribution of lunar zircons correlate with episodes of KREEP magmatism and zircon crystallization. These Apollo 15 ages, when analyzed in conjunction with other reported zircon ages from two other Apollo landing sites (14 and 17), provide strong empirical support for a widespread (possibly global) zircon-forming event at approximately $4.33 \mathrm{Ga}$. The REE patterns of the Apollo 15 zircons reported herein are similar to those reported in [5].

References: [1] Meyer C. 2006, Lunar Sample Compendium, 15455, (http://curator.jsc.nasa.gov/lunar/compendium.cfm). [2] Meyer C. 2004. Lunar Sample Compendium, 15405, (http://curator.jsc.nasa.gov/lunar/ compendium.cfm). [3] Taylor et al. 2009. Earth and Planetary Science Letters 279:157-164. [4] Meyers et al. 1996. Meteoritics \& Planetary Science 31:370-387. [5] Nemchin et al. 2010. American Mineralogist 95:273-283.

\section{Apollo 15 Zircon Pb-Pb Ages}

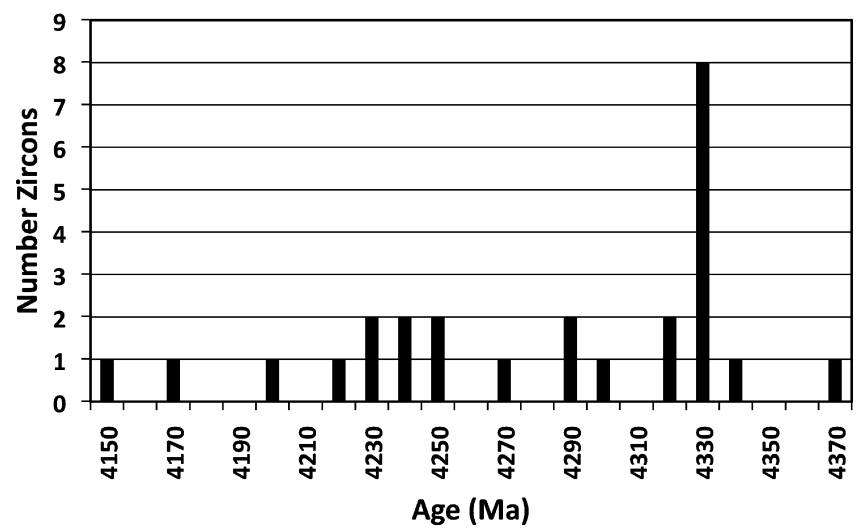

Fig. 1. Pb-Pb ages for zircons from Apollo samples 15405,9, 15405,75, and 15455,226
5328

ABUNDANCE AND ISOTOPIC COMPOSITION OF SOLAR WIND XENON COLLECTED BY THE GENESIS MISSION

S. A. Crowther and J. D. Gilmour. SEAES, University of Manchester, UK. E-mail: sarah.crowther@manchester.ac.uk.

Introduction: One of the major objectives of NASA's Genesis mission is to determine the elemental and isotopic noble gas composition of the present-day solar wind [1]. However concentrations of implanted noble gases in the Genesis collector targets are very low, and the most sensitive techniques and instruments are required to make precise and accurate measurements. Our work focuses on measuring the concentration and isotopic composition of solar wind Xe implanted in CZ-Si targets from the bulk collector array, using the RELAX mass spectrometer [2, 3].

Experimental: The implanted solar wind is extracted by UV laser ablation (Nd:YAG, $355 \mathrm{~nm}, \sim 350 \mathrm{~mJ}$ ). The laser beam covers and ablates the whole surface of the square samples typically $\sim 3-5 \mathrm{~mm}$ in dimension, without the need to raster over the surface. Preliminary experiments suggested that $\sim 30$ laser shots extracted all the solar wind $[4,5]$, however extractions of gas from a further 120 laser shots show a systematic enrichment in $\mathrm{Xe}$ in the flight samples relative to the non-flight samples. The "excess" Xe in these releases amounts to approximately $55 \%$ of the total $\mathrm{Xe}$ in these releases, and if this excess is assumed to be solar wind it accounts for $\sim 15 \%$ of the total. Our preferred extraction protocol now uses $\sim 150$ laser shots to extract all the implanted solar wind.

Instrumental mass discrimination can best be corrected for if sample sizes are matched to those of calibration aliquots. The predicted 2-year fluence for solar wind $\mathrm{Xe}$ implanted into the bulk collector arrays is $\sim 3 \times 10^{6}$ atoms $\mathrm{Xe} \mathrm{cm}^{-2}$ [1]. RELAX calibration aliquots contain $1.2 \times 10^{5}$ atoms of atmospheric ${ }^{132} \mathrm{Xe}$ [3]. Therefore we have analyzed approximately square samples, with typical areas of $\sim 0.09-0.25 \mathrm{~cm}^{2}$, such that the expected amount of ${ }^{132} \mathrm{Xe}$ closely matches that in the air calibration aliquots. Our approach, which tests the reproducibility of the data through a large number of separate analyses, is complementary to the analysis of gas released from large areas of collector material, which can be analyzed at higher precision on conventional instruments. Notably, the expected $\sim 400$ atoms of ${ }^{124} \mathrm{Xe}$ per sample make determination of the ${ }^{124} \mathrm{Xe} /{ }^{132} \mathrm{Xe}$ ratio challenging.

Results: Blank Xe, as measured by the same protocol in non-flight samples, is isotopically consistent with air, but the concentration varies by a factor of $\sim 20$. Variations in Xe concentration are also observed in flight samples. The isotope ratios measured in the flight samples correlate with the reciprocal of the measured concentration suggesting a variable concentration blank mixes with a uniform concentration implanted solar wind component.

16 flight and 15 non-flight samples have been analyzed using the 150 -shot extraction protocol. From the distribution of Xe concentrations measured in these samples we calculate the concentration of the implanted solar wind to be $1.20(9) \times 10^{6}$ atoms ${ }^{132} \mathrm{Xe}^{-2}$. The isotopic composition calculated from this concentration is consistent with the solar wind measured in the young lunar regolith [6], there is no evidence of fractionation.

References: [1] Burnett D. S. et al. 2003. Space Science Reviews 105:509-534. [2] Gilmour J. D. et al. 1994. The Review of scientific instruments. 65:617-625. [3] Crowther S. A. et al. 2008. Journal of Analytical Atomic Spectrometry 23:938-947. [4] Crowther S. A. and Gilmour J. D. 2010. Meteoritics \& Planetary Science 45. Abstract \#5335. [5] Crowther S. A. and Gilmour J.D. 2011. Abstract \#1969. 42nd Lunar and Planetary Science Conference. [6] Wieler R. 2002. Reviews in Mineralogy \& Geochemistry 47:21-70. 
5378

THE HYPERVELOCITY ARTIFICIAL METEOROID EXPERIMENT (HAM•E): A MISSION CONCEPT STUDY

D. C. Cullen ${ }^{1}$, P. C. E. Roberts ${ }^{2}$ and HAM•E Cranfield MSc Group Design Project Team ${ }^{2}$. ${ }^{1}$ Cranfield Health. ${ }^{2}$ Space Research Centre, Cranfield University, Bedfordshire, UK. E-mail: d.cullen@cranfield.ac.uk

The hypervelocity atmospheric entry of natural bodies - meteoritic, asteroidal and cometary-has relevance to the evolution of the solar system. Relevance includes contribution to early planetary inventories of volatile and organic materials and including possible lithopanspermia questions and the current threat of Near Earth Objects. Present understanding of such events is limited given for all observed natural events the detailed boundary conditions are not known-e.g., the size, shape, detail composition of bodies prior to atmospheric entry. Observational data is limited since for natural bodies of significant mass, no event has been predicted with sufficient notice to allow deployment of the best observational techniques currently available.

The scientific community's current understanding of hypervelocity atmospheric entry of natural bodies is from extensive modeling, hypervelocity laboratory experiments and meteorite record interpretation. Absence of detailed observations of natural events means this large body of work suffers poor validation and for the meteorite record, uncertainty in pre-entry conditions.

To address the absence of relevant data, we propose the Hypervelocity Artificial Meteoroid Experiment (HAM•E) which aims to enter, from space, into the Earth's atmosphere fully characterized bodies (artificial meteoroids) representative of natural bodies at velocities relevant to natural events and at known times and locations allowing the deployment of the best available observational techniques. Resultant data would enable validation and refinement of existing models and laboratory experiments and a re-interpretation of the existing meteorite record.

A preliminary $\mathrm{HAM} \bullet \mathrm{E}$ mission study has been performed to assess feasibility. Requirements were: enter four artificial meteoroid bodies into the Earth's atmosphere at velocities greater than $11 \mathrm{~km} \mathrm{~s}^{-1}$; entry times and locations to be compatible with a single observation campaign; entry profiles to be compatible with two bodies undergoing ablation only and two bodies undergoing both fragmentation and ablation. Various mission concepts were proposed and trade-off studies perform to result in a baseline mission. The mission comprised the following key points: Payload of two $236 \mathrm{~kg}$ basalt spheres to undergo fragmentation and two $25 \mathrm{~kg}$ spheres, one each of basalt and sedimentary rock for ablation only. Dedicated Soyuz launch with a Fregat upper stage on a lunar free-return trajectory. Sequenced release of artificial meteoroids on return leg with atmospheric entry of meteoroids above the Woomera Prohibited Area, Australia at $11.1 \mathrm{~km} \mathrm{~s}^{-1}$, separated in a $70 \mathrm{~min}$ period and 6.3 days after launch. Spacecraft design included meteoroid containment and release mechanisms, propulsion system, instrumentation for monitoring meteoroid integrity and sub-systems for power and communication. Resultant spacecraft mass of $1564 \mathrm{~kg}$ including payload.

A detailed science case including more refined payload definition and an observational campaign case comprising space-borne, air-borne and ground based techniques and including resultant meteorite campaign is the subject of on-going studies.

In summary we have proposed the HAM•E concept and produced a preliminary mission design that implements $\mathrm{HAM} \bullet \mathrm{E}$.
5092

QUANTIFICATION OF THE POST-IMPACT THERMAL EVOLUTION OF PLANETESIMALS

T. M. Davison ${ }^{1}$, F. J. Ciesla ${ }^{1}$ and G. S. Collins ${ }^{2}$. ${ }^{1}$ Department of the Geophysical Sciences, The University of Chicago, 5734 South Ellis Avenue, Chicago IL 60637, USA. ${ }^{2}$ Department of Earth Science and Engineering, Imperial College London, London, SW7 2AZ, UK. E-mail: tdavison@uchicago.edu.

Introduction: Impacts between planetesimals were common in the early solar system. In some cases, these collisions were energetic enough to cause significant changes to the planetesimal materials; evidence for shock metamorphism, alteration and melting are found in meteorites. While short-lived radionuclides $\left({ }^{26} \mathrm{Al}\right)$ can account for most of the heating observed in the meteoritic record, hypervelocity impacts have also been invoked as a possible heat source [1-3]. Planetesimals are thought to have formed through low velocity accretionary collisions of smaller bodies [4-6], a process which is expected to have created planetesimals with significant pore space; a recent observational study supports this theory [7]. Numerical modeling has shown that localized heating in porous planetesimals could be higher than previously realized [8]. Here we quantify the post-impact cooling of a planetesimal and compare the thermal evolution to that inferred for primitive meteorites $[9,10]$.

Modeling: We simulate hypervelocity planetesimal collisions using the iSALE hydrocode $[11,12]$ for a range of planetesimal impact scenarios: parameters investigated include porosity, impact velocity, target and impactor sizes and impact angle. A recent addition to the iSALE model is a self-gravity algorithm [13-15], allowing simulations to extend to later times than in [8]. After the resulting planetesimal has relaxed to its final shape, the density, porosity and temperature structure is extracted from the hydrocode model. This information is passed into a finite volume thermal evolution model to track the redistribution and loss of the deposited impact energy, allowing us to determine the cooling rates throughout the planetesimal. The thermal model solves the heat diffusion equation and accounts for the porosity of the material by altering the thermal diffusivity according to [16].

Results: We find peak temperatures of $700-1500 \mathrm{~K}$ and cooling rates in the range $0.1-100 \mathrm{~K} \mathrm{Myr}^{-1}$ are easily achieved. These values are consistent with those inferred from chondritic meteorites [10]. Cooling rates for initially porous material are lower than for fully consolidated bodies-for a $50 \%$ porous parent body that was impacted at $4 \mathrm{~km} \mathrm{~s}^{-1}$, cooling rates are $\sim 1 \mathrm{~K} \mathrm{Myr}^{-1}$, but in an equivalent collision into a nonporous parent body, cooling rates are typically $>10 \mathrm{~K} \mathrm{Myr}^{-1}$. We are determining the full range of thermal histories that would be achieved in collisions and will present results from our full parameter study, and a comparison with the predictions of recent radiogenic thermal models [17].

References: [1] Rubin A. E. 1995. Icarus 113:156-167. [2] Wasson J. T. et al. 1987. Meteoritics \& Planetary Science 22:525-526. [3] Cameron A. G. W. et al. 1990. 21st Lunar and Planetary Science Conference. pp. 155-156. [4] Cuzzi J. N. et al. 2008. The Astrophysical Journal 687:1432-1447. [5] Johansen A. et al. 2007. Nature 448:1022-1025. [6] Teiser J. and Wurm G. 2009. Monthly Notices of the Royal Astronomical Society 393:1584-1594. [7] Bland P. A. et al. 2011. Nature Geoscience 4:244-247. [8] Davison T. M. et al. 2010. Icarus 208:468-481. [9] Taylor G. J. et al. 1987. Icarus 69:1-13. [10] Kleine T. et al. 2008. Earth and Planetary Science Letters 270:106-118. [11] Wünnemann K. et al. 2006. Icarus 180:514-527. [12] Collins G. S. et al. 2004. Meteoritics \& Planetary Science 39:217-231. [13] Collins G. S. et al. 2011. 42nd Lunar and Planetary Science Conference. [14] G. S. Collins et al. This meeting. [15] Barnes J. E. and Hut P. 1986. Nature 324:446-449. [16] Warren P. H. 2011. Meteoritics \& Planetary Science 46:53-78. [17] Harrison K. P. and Grimm R. E. 2010. Geochimica et Cosmochimica Acta 74:5410-5423. 
5319

TEXTURAL AND COMPOSITIONAL VARIATIONS OF CRBEARING SPINEL MINERALS IN REDUCED CV3 CHONDRITES

J. Davidson, D. S. Lauretta, and D. L. Schrader. LPL, Department of Planetary Sciences, University of Arizona, Tucson, AZ 85721-0092, USA. E-mail: davidson@1pl.arizona.edu.

Introduction: The CV3 and CK chondrites have been hypothesized to originate from a single parent body [1]. During our ongoing study of the links between opaque phases from $\mathrm{CV} 3 / \mathrm{CK}$ chondrites we found less $\mathrm{Cr}$ in $\mathrm{Fe}$, Ni metal grains of reduced $\mathrm{CV} 3\left(\mathrm{CV} 3_{\text {Red }}\right)$ chondrites than expected when compared to a more oxidised CK3 [2]. Since the CV3/CK chondrites have approximately bulk solar abundance of $\mathrm{Cr}$ [1], $\mathrm{Cr}$ must be present in different phases for each group/sub-group. $\mathrm{Cr}$ in oxidized CV3s is most abundant in magnetites but these are absent in $\mathrm{CV}_{\text {Red }}$ [2]. Instead $\mathrm{Cr}$ appears to be present, with variable abundance, in the spinel phases of $\mathrm{CV} 3_{\text {Red }}$. Here we investigate the partitioning of $\mathrm{Cr}$ between different phases in unequilibrated $\mathrm{CV} 3_{\text {Red }}$ chondrites.

Analytical: Polished sections of the reduced CV3 chondrites RBT 04133 [3], MET 01017, and Dhofar 1612 were studied via (1) optical microscopy, (2) X-ray mapping, and (3) major/minor elemental analysis of individual mineral phases with the LPL's Cameca SX-50 electron probe microanalyzer (EPMA).

Results: Cr-bearing spinel grains in RBT 04133, MET 01017, and Dhofar 1612 are located exclusively in FeO-rich (type II) chondrules. However, not all FeO-rich chondrules observed are spinel-bearing. Chromium-composition appears to correlate with morphology, with the most euhedral grains being generally the most Cr-rich; RBT 04133 (euhedral; 46.6-52.4 wt.\% $\mathrm{Cr}_{2} \mathrm{O}_{3}$ ), Dhofar 1612 (rounded, euhedral; 28.641.4 wt. $\% \mathrm{Cr}_{2} \mathrm{O}_{3}$ ), and MET 01017 (subhedral; 18.2-34.9 wt.\% $\mathrm{Cr}_{2} \mathrm{O}_{3}$ ). Cr-bearing spinels in MET 01017 have Fe-rich rims (increasing from approximately $22 \mathrm{wt} . \% \mathrm{Fe}_{2} \mathrm{O}_{3}$ in the core to approximately $33 \mathrm{wt} . \%$ at the rim). No zoning was seen in Cr-bearing spinels from RBT 04133 or Dhofar 1612.

Discussion: Chromites from unequilibrated chondrites are reported to be entirely euhedral, with subhedral chromites only present in equilibrated chondrites [4]. The presence of subhedral spinels in MET 01017 may suggest that it has experienced higher degrees of thermal metamorphism than the other CV3s analyzed. This is consistent with a classification of CV3.7 for MET 01017 determined by Raman spectral analysis of its insoluble organic matter [5]. Therefore, we suggest that spinel morphology may also be an indicator of the degree of thermal metamorphism experienced by unequilibrated $\mathrm{CV} 3_{\text {Red }}$ chondrites.

Zoning in the Cr-bearing spinels of MET 01017, the most thermally altered CV3, suggests mobilization of elements with increasing degrees of heating. Cr-bearing spinels appear to become less $\mathrm{Cr}$-rich with increasing parent body thermal alteration. The Cr-composition of $\mathrm{FeO}$-rich olivine grains in carbonaceous chondrites is known to decrease with increasing metamorphic grade [6], forming Cr-rich rims and chromites. This appears to contradict our results. We will determine the Cr-composition of coexisting silicate phases to address this issue.

Acknowledgments: This work was funded by NASA Grant NNX10AH50G (DSL PI).

References: [1] Greenwood R. G. et al. 2010. Geochimica et Cosmochimica Acta 74:1684. [2] Davidson J. et al. 2011. Abstract \#1886. Lunar and Planetary Science Conference. [3] Davidson J. et al. 2009. Meteoritics \& Planetary Science 44:A57. [4] Johnson C. A. and Prinz M. 1991. Geochimica et Cosmochimica Acta 55:893. [5] Busemann H. et al. 2007. Meteoritics \& Planetary Science 42:1387. [6] Grossman J. N. and Brearley A. J. 2005. Meteoritics \& Planetary Science 40:87.

\section{7}

LATE ACCRETION TO THE MOON AND VESTA

James M. D. Day ${ }^{1}$ and Richard J. Walker ${ }^{2}$. ${ }^{1}$ Geosciences Research Division, Scripps Institution of Oceanography, La Jolla, CA 92093-0244, USA, E-mail: jmdday@ ucsd.edu. ${ }^{2}$ Department of Geology, University of Maryland, College Park, MD 20740, USA.

Introduction: We present new highly siderophile element (HSE: Os, $\mathrm{Ir}, \mathrm{Ru}, \mathrm{Pt}, \mathrm{Pd}$, and $\mathrm{Re}$ ) abundance and ${ }^{187} \mathrm{Os} /{ }^{188} \mathrm{Os}$ data for low-Ti mare basalt meteorites, Apollo 12 samples, and diogenite meteorites to explore the late accretion histories of the Moon and asteroid (4) Vesta, the probable diogenite parent body. Moon/Vesta comparisons are potentially useful because both bodies are considered to have witnessed magma ocean differentiation, are relatively volatile depleted (e.g., low ${ }^{87} \mathrm{Sr} /{ }^{86} \mathrm{Sr}$ ), and because recent work has identified the possibility of massive (D $=100$ 's to 1000 's $\mathrm{km}$, respectively) impactors striking the Moon and Earth subsequent to the major phases of core formation [1]. Thus, interpretation of HSE signatures for differentiated asteroids like Vesta may provide complementary information on the timing, size distribution and distribution within the solar system of potential late accretion impactors to bodies such as the Moon [2-4], Earth [5], Mars [6], and the angrite parent body [7].

Results and Discussion: Apollo 12, 15, 17 mare basalts, and mare basalt meteorites have HSE abundances $\left(\sim 1 \times 10^{-3}\right.$ to $1 \times 10^{-7} \times \mathrm{CI}$ chondrite) that extend the abundance range measured for lunar picritic glass bead interiors $\left(\sim 1 \times 10^{-3}\right.$ to $1 \times 10^{-5} \times \mathrm{CI}$ [2]), and exhibit relatively flat chondrite-relative HSE patterns. Current HSE abundance data for lunar derivative melts and pristine crustal rocks support their derivation from a relatively homogenized lunar mantle with HSE abundances $<20 \times$ lower than for the terrestrial or Martian mantles, consistent with stochastic late accretion [1]. Diogenite meteorites have chondritic relative abundances of the HSE and close-to-chondritic ${ }^{187} \mathrm{Os} /{ }^{188} \mathrm{Os}[8]$, and span a range of HSE concentrations from close to terrestrial upper mantle values, to HSE concentrations observed in lunar mare basalts. If it is assumed that the HSE characteristics of most diogenites are reflections of processes internal to Vesta, then their generally chondritic natures, suggests chondritic additions following core formation, but prior to completion of mantle crystallization [8]. If interpretations of stochastic late accretion are valid [1], Vesta, and other asteroids, are smaller targets than Earth, the Moon, or Mars, so the largest early impactors to strike them may have been $\mathrm{D}<100 \mathrm{~km}$. Consequently, perhaps only a portion of the Vesta was affected by late accretion, such that some HSE signatures are more consistent with regional rather than global effects. Planetesimals like Vesta, therefore, may be expected to retain compositional heterogeneity that records their magnitude and frequency of late accretion and provides a complementary record to that retained in terrestrial, lunar, and Martian rocks and achondritic meteorites.

Acknowledgments: This research was partially supported by NASA LASER grant (NNX11AG34G).

References: [1] Bottke W. F. et al. 2010. Science 330:1527. [2] Walker R. J. et al. 2004. Earth and Planetary Science Letters 224:399. [3] Day J. M. D. et al. 2007. Science 315:217. [4] Day J. M. D. et al. 2010. Earth and Planetary Science Letters 289:595. [5] Walker R. J. 2009. Chemie der Erde 69:101. [6] Brandon A. D. et al. 2010. Geochimica et Cosmochimica Acta 74:A117. [7] Riches A. J. V. et al. 2010. Meteoritics \& Planetary Science 45:A171. [8] Day J. M. D. et al. 2010. Abstract \#1942. 41st Lunar and Planetary Science Conference. 


\section{5}

HIGH PRESSURE MINERALS IN RC106 REVEAL ITS DEPTH IN ITS PARENT BODY

P. S. De Carli, Z. Xie, R. Trickey, C. A. Weaver, T. G. Sharp. Poulter Laboratories, SRI International.

The present work is based on the study of a $1.3 \mathrm{~mm}$-wide melt vein in the L6 chondrite RC106. This vein contains an assemblage of high pressure phases that constrain the pressure history of the vein as it solidified and cooled to a temperature low enough for survival of the phases. Cooling rate calculations constrain the temperature history of the vein $[1,2]$. Thus the pressure-time history is constrained. Since shock pressures are relieved by rarefaction waves from the nearest free surface, shock duration is a measure of the depth of the meteorite in its parent body at the time of the vein-forming shock. Preliminary calculations with the Autodyn ${ }^{\circledR}$ hydrocode indicate the RC106 must have been at least 10 $\mathrm{km}$ below the surface of its parent. More refined heat flow and hydrocode calculations are in progress.

References: [1] Langenhorst F. and Poirier J. P. 2000. Earth and Planetary Science Letters 184:37-55. [2] Sharp T. G. and De Carli P. S. 2006. MESS II, eds. Lauretta and McSween, The University of Arizona Press. pp. 653-677.

\section{2}

\section{METEORITE CURATION AND RESEARCH IN BELGIUM}

Walter De Vos. Royal Belgian Institute of Natural Sciences, Brussel, Belgium.

The Royal Belgian Institute of Natural Sciences (RBINS) has held meteorites in its collections since the nineteenth century. Among them are four meteorites that are documented falls in Belgium between 1855 and 1934. In the last few years a new interest in meteorites arose for different reasons. In 2006 a newly discovered mass of the Mont Dieu iron meteorite, weighing $435 \mathrm{~kg}$, was purchased. It is now part of the permanent exhibit of the museum. In 2009, a collection of 361 pieces was acquired from a private collector, including many Saharan meteorites found after 1995.

At present, the RBINS holds 511 pieces from 307 different meteorites, 72 of which, all from The Sahara, have not yet officially been described.

In a separate development, an agreement was signed between Belgian and Japanese scientists to search for meteorites in Antarctica from 2009 to 2014, in the larger neighborhood of the Belgian Princess Elisabeth Station, which was opened in 2009 near the Sor Rondane Mountains. The first field season yielded 635 new meteorites, the second one 218 new meteorites; several ureilites and at least one carbonaceous chondrite were identified on macroscopic inspection. After a first characterization at the Japanese National Institute of Polar Research, half the collected meteorites will be transferred to the RBINS in Belgium for curation.

Starting in 2001, two universities in Brussels carry out research on meteorites and impact structures. Several master students have been at work, notably on the IIE iron Mont Dieu, and on chondrites from The Sahara. One Ph.D. work was concluded this year, and ongoing research at $\mathrm{Ph} . \mathrm{D}$. and postdoc level includes research on meteorites from the RBINS collection, both chondrites and achondrites.

At the RBINS itself a fifth Belgian meteorite, Hautes Fagnes, a LL5 chondrite, was studied in collaboration with other institutions, and at present analytical facilities are being upgraded to produce accurate microanalyses. Research themes include classification of chondrites from The Sahara, magnetic susceptibility measurements, and bulk analyses on chondrites in collaboration with university laboratories. In addition, occasionally genuine meteorites are submitted for identification by the public, and some research has started on these.

Given the perspective of a growing collection through the Antarctic prospection program, the RBINS is gradually improving its research infrastructure and its curation facilities for meteorites. 


\section{0}

\section{MANTLE OVERTURN AS REVEALED BY NAKHLITES: WHAT HAPPENED AFTER?}

V. Debaille ${ }^{1}$ and A. D. Brandon ${ }^{2}$. ${ }^{1}$ Département des Sciences de la Terre et de l'Environnement, Université Libre de Bruxelles, Brussels, Belgium. E-mail: vinciane.debaille@ulb.ac.be. ${ }^{2}$ Department of Earth and Atmospheric Sciences, University of Houston, Houston, USA.

During early stages of differentiation, terrestrial planets may undergo extensive melting, resulting in deep and global magma oceans (MO). For Mars, after its solidification, the contrast of density between early formed Mg-rich MO cumulates located at the bottom of the Martian mantle, and later Fe-rich MO cumulates at the top of the mantle is expected to induce mantle overturn [1]. During the overturn, deep cumulates are brought up to shallower depths and can melt by adiabatic decompression, with large degrees of partial melting of up to $60-65 \%$ [2]. While shergottites record the progressive crystallization of the magma ocean [3], nakhlites provide the first geochemical evidence that a mantle overturn occurred [2]. Following the mantle overturn, the large volumes of magma produced above the upwelling cumulates may have resurfaced the whole planet, hence creating an early Martian crust [4], and potentially generating the Martian crustal dichotomy [2].

Using the hypothesized mineralogy of the Martian mantle [5], the evolution of the $\mathrm{Fe} / \mathrm{Mg}$ ratio during the crystallization of the Martian magma ocean results in $\mathrm{Mg \#}>80$ for cumulates deeper than $13 \mathrm{GPa}$. Consecutively to the mantle overturn, the direct product of $60 \%$ partial melting of these cumulates is magmas with $\mathrm{Mg \#}>79$. These high Mg\#'s are comparable to terrestrial Archaean komatiites, despite that the Martian mantle is, as a whole, slightly more enriched in Fe (or depleted in $\mathrm{Mg}$ ) compared to the terrestrial mantle $(\mathrm{Mg} \#$ of 75 and 81 respectively for the Martian and terrestrial bulk silicate mantle).

However, high Mg\# magmas are not apparent in the geological record of Mars, not only in orbiting spacecraft and lander data [6], but also in Martian meteorite parental magma models [7]. This implies that this primordial komatiite-like crust has been altered and/or covered by following eruptive episodes and sediment deposits, and is not exposed anymore in the present crust of Mars. Mineral segregation within thick komatiite lava flows during crystallization could also lead to biased observations from spacecrafts.

References: [1] Elkins-Tanton et al. 2003. Meteoritics \& Planetary Science 38:1753-1771. [2] Debaille et al. 2009. Nature Geoscience 2:548552. [3] Debaille et al. 2007. Nature 450:525-528. [4] Elkins-Tanton et al. 2005. Journal of Geophysical Research 110: E12S01. [5] Bertka and Fei. 1997. Journal of Geophysical Research 102:5251-5264. [6] McSween et al. 2009. Science 324:736-739. [7] Usui et al. 2008. Geochimica et Cosmochimica Acta 72:1711-1730.

\section{1}

\section{MAGMA MUSH ON MARS}

C. D. Deering, S. Kuehner and O. Bachmann. Dept. of Earth and Space Sciences, University of Washington, Mailstop 351310, Seattle, WA 981951310, USA. E-mail: cdeering@u.washington.edu.

Introduction: Magmatic differentiation on Earth appears to be governed by the extraction of interstitial melt from long-lived magma mushes [1]. Recent numerical models [2] indicate that effective melt extraction from crystal-rich reservoirs is confined to a "most probable" crystallinity window (approximately 50-70 vol.\%) due to (1) low crystal settling rates at low crystallinity (as magma convects) and (2) the extremely slow melt extraction rate at high crystallinity (low permeability). A key factor in such models is that cooling, and hence crystallization rates, are slow enough for a given reservoir to remain within the optimal crystallinity window long enough for the melt extraction to occur. However, Mars is known to have a significantly lower thermal gradient (1/4 to $1 / 3)$ than Earth [3]. Consequently, the ability of magmas to remain at the optimal window of melt extraction for any appreciable length of time on Mars against this low thermal gradient may be severely restricted.

To assess differentiation processes on Mars, we performed a detailed examination of the texture and composition of pyroxene grains in the Martian meteorite NWA 5298 [4]. NWA 5298 is coarse-grained and composed predominately of large euhedral to subhedral elongated pyroxene grains (up to $4 \mathrm{~mm}$ in length) and interstitial subhedral laths of maskelytanized plagioclase. BSE images reveal a complex textural and chemical zoning pattern in the pyroxenes, consisting of a well defined augite core that is irregularly truncated and bounded by a distinctly more ferroan mantle (sometimes discontinuous) of augite and/or pigeonite, which in turn is also irregularly truncated by an even more Fe-rich rim. The core-mantle and mantle-rim textural discontinuities are consistent with dissolution surfaces formed during magma recharge events, a common feature found in igneous rocks on Earth. Pyroxene-only modes indicate that the first recharge occurred when the crystal fraction was at least $25 \%$ and the second recharge was at least $65 \%$. This evaluation indicates that crystal-rich mushes exist on Mars, and are maintained at intermediate crystallinities by hot recharge.

Using the MELTS software [5], we examined whether or not the most evolved Mars meteorites (Los Angeles) represent extracted liquids from a more mafic mush. We modeled the crystallization path and melt evolution of NWA 5298 at 1 kbar, QFM-3 and anhydrous. Results show that the Los Angeles meteorite composition can be produced when this mush reaches $50-70 \%$ crystals. Relative to Earth, however, separation of even more evolved melts at higher crystal fractions is severely hindered on Mars by its lower thermal gradient and resultant higher degree of undercooling. This ensures that the probability of generating any significant amounts of evolved liquid (analogous to terrestrial basalticandesite) on Mars is extremely low.

References: [1] Sinton J. M. and Detrick R. S. 1992. Journal of Geophysical Research 97:197-216. [2] Dufek J. and Bachmann O. 2010. Geology 38:687-690. [3] Solomon S. C. and Head J. W. 1990. Journal of Geophysical Research 95:11073-11083. [4] Irving A. J. and Kuehner S. M. 2008. Abstract \#5332. Meteoritical Society Annual Meeting. [5] Ghiorso M. S. and Sack R. O. 1995. Contributions to Mineralogy and Petrology 119:197-212. 


\section{9}

ISOTOPIC RATIO AND ORIGIN OF HYDROGEN IN IRON METEORITES

C. Defouilloy ${ }^{1}$, R. Duhamel ${ }^{1}$, F. Robert ${ }^{1}$. ${ }^{1}$ LMCM, CNRS-MNHN Paris, France. E-mail: cdefouilloy@mnhn.fr.

Introduction: We generally see Iron meteorites as pieces of differentiated bodies, whether from a totally segregated core or from droplets. Yet, Irons present some inconsistencies with the classically assumed chronology of the solar system. Their parent bodies are thought to come from the differentiation of a chondritic ancestor, but the Hf-W chronometer situates the core segregation in less than a Myr after the CAIs [1]. One way to explore the story of Iron meteorite is to trace the hydrogen's. Indeed, The $\mathrm{D} / \mathrm{H}$ ratio being significantly different between the protosolar hydrogen $\left((\mathrm{D} / \mathrm{H})_{\mathrm{H} 2}=20 \pm 5 \times 10^{-6}[2]\right)$ and hydrogen coming from water reduction during planetesimal differentiation ((D/ $\left.\mathrm{H})_{\text {condritic water }}=150 \times 10^{-6}[2]\right)$ it can be used as an indicator of the origin of hydrogen in the iron meteorites. Therefore, an analytical technique for measuring the $\mathrm{D} / \mathrm{H}$ ratio of Iron meteorites using ion microprobe has been developed.

Method: hydrogen isotopic composition is determined with the CAMECA IMS $3 \mathrm{f}$ at the National Museum of Natural History in Paris [3]. Samples are mounted either in epoxy and Au-coated or as polished thin sections and sputtered with a primary positive $\mathrm{Cs}^{+}$beam. All slits are kept wide open. Under these conditions, the mass resolution is $<300$ (note that with the $\mathrm{Cs}^{+}$primary beam, the possible mass interference between $\mathrm{H}_{2}^{-}$is $\mathrm{D}^{-}$is negligible). To reduce to a minimum the contribution of absorbed water at the surface of the sample, we use a large ion spot $(500 \mu \mathrm{m})$ combined with a $150 \mu \mathrm{m}$ aperture field, so that the ions from the center of the "crater" are selectively collected. The intensity of the primary beam varies from $1 \mathrm{nA}$ to $40 \mathrm{nA}$ in order to keep as much as possible a secondary beam around $10^{5} \mathrm{cps}$ for $\mathrm{H}$ and 10 for D. Data were collected in peak jumping mode, with counting time of 5 and $15 \mathrm{~s}$. for $\mathrm{H}^{-}$and $\mathrm{D}^{-}$, respectively.

Results: A total of seven meteorites have been studied as well as a terrestrial metallic sample from Disko Island. $\mathrm{D} / \mathrm{H}$ have been calibrated with an internal standard of our own, made by rusting a sample with a water whose $\mathrm{D} / \mathrm{H}$ was precisely known. Corrected from this instrumental fractionation, $\mathrm{D} / \mathrm{H}$ ratios lie between $92 \pm 5 \times 10^{-6}$ measured in Mont Dieu and $126 \pm 12 \times 10^{-6}$ in Coahuila. $\mathrm{H}$ concentrations have been calibrated by comparing the IMS secondary beam responses to those obtained on an $\mathrm{H}$-implanted golden sample. [H] varies from $0.48 \pm$ $0.12 \mathrm{ppb}$ to $119 \pm 100 \mathrm{ppb}$. There is no evident correlation between $\mathrm{D} / \mathrm{H}$ ratio and $[\mathrm{H}]$ between samples. Though, a correlation has been noticed internally for one particular sample. This last observation is thought to be a proof of spallation, which is not noticeable on any other sample. A particular care has been taken to assess terrestrial pollution and minimize any possible source of additional contamination during the measures.

Analyses of additional elements parallel to $\mathrm{H}$ showed a correlation between $\mathrm{H}$ and $\mathrm{O}$ and $\mathrm{H}$ and $\mathrm{C}$, which indicates two possible carriers of $\mathrm{H}$ in Irons, respectively water (terrestrial contamination) and residual organic carbon (?).

References: [1] Kleine T. et al. 2005. Geochimica et Cosmochimica Acta 69:5805-5818. [2] Deloule E. and Robert F. 1995. Geochimica et Cosmochimica Acta 59:4695-4706. [3] Defouilloy C. et al. 2010. Abstract \#1340. 41st Lunar and Planetary Science Conference.

\section{4}

THERMAL HISTORIES OF CHONDRULES IN VERY LARGE PLANETESIMAL BOW SHOCKS: DID MARS MAKE CHONDRULES?

S. J. Desch and M. A. Morris. School of Earth and Space Exploration, Arizona State University, Tempe, AZ, USA. E-mail: steve.desch@asu.edu

Introduction: It is widely accepted that the majority of chondrules formed by passage through solar nebula shocks [1,2], but the source of the shocks is debated, with either planetesimal bow shocks [3] or shocks driven by gravitational instabilities [4] favored [5]. Previous studies of bow shock models have indicated that the shocked region would be comparable in size to the planetesimal, approximately $10^{3} \mathrm{~km}$, and so chondrules would necessarily pass through the region in $<10^{3} \mathrm{~s}$, and experience cooling rates $>10^{3} \mathrm{~K} \mathrm{~h}^{-1}$, inconsistent with the porphyritic textures of most chondrules [6,7]. Additionally, the observed mass of chondrules can be produced only by assuming large numbers of $2000 \mathrm{~km}$ diameter planetesimals or other extreme input parameters [7, 8].

The majority of extant chondrules formed at approximately $2 \mathrm{Myr}$ after the birth of the solar nebula [9]. Modeling of planetary growth [10] indicates that planetary embryos the size of Mars can grow in approximately $2 \mathrm{Myr}$, and recent $\mathrm{Hf}-\mathrm{W}$ isotopic systematics indicate Mars did, in fact, form and differentiate within 2 Myr [11]. If true, then Mars formed in the presence of nebular gas and chondrules. Recent dynamical simulations further suggest Mars formed at $<1 \mathrm{AU}$ and by $2 \mathrm{Myr}$ was ejected into an eccentric orbit $(\mathrm{e}>0.25)$ centered near its current orbit at 1.5 AU [12]. A proto-Mars with $a=1.5 \mathrm{AU}$ and perihelion 1.0 AU would move at up to $7 \mathrm{~km} \mathrm{~s}^{-1}$ relative to local gas at aphelion at $2.0 \mathrm{AU}$ and could drive a bow shock capable of melting chondrules. So did Mars form chondrules?

Results: We have conducted preliminary calculations using the methodology of [7], including heating by $\mathrm{H}_{2}$ recombinations [13], to study bow shocks around planetesimals, but assuming a $3400 \mathrm{~km}$ radius planetesimal (Mars). We find that chondrule cooling rates are 10$100 \mathrm{~K} \mathrm{~h}^{-1}$ assuming an opacity equal to a solids-to-gas ratio of 0.13 in $300 \mu \mathrm{m}$ radius chondrules (equivalent to a solids-to-gas ratio 0.01 in $20 \mu \mathrm{m}$ particles), so that the optical depths through the shocked region exceed unity. These cooling rates are consistent with the porphyritic textures of most chondrules [14].

Discussion: If Mars formed as a planetary embryo at $1 \mathrm{AU}$ and was ejected into its present orbit within $2 \mathrm{Myr}$, it would have driven very large planetesimal bow shocks capable of melting chondrules in a manner consistent with constraints. We note that the presence of a magma ocean on an early formed Mars (heated by ${ }^{26} \mathrm{Al}$ ) could introduce $\mathrm{Na}$ and other volatiles into the gas in the chondrule-forming region around the planet, possibly explaining the high inferred $\mathrm{Na}$ vapor pressures during chondrule formation [15]. We estimate that Mars alone could shock approximately $10^{26} \mathrm{~g}$ of chondrules per Myr. We plan more detailed numerical simulations to test the provocative possibility that Mars formed chondrules.

References: [1] Desch S. J. et al. 2011. Meteoritics \& Planetary Science (submitted). [2] Desch S. J. et al. 2010. The Astrophysical Journal 725:692. [3] Hood L. L. 1998. Meteoritics \& Planetary Science 33:97. [4] Boss A. P. and Durisen R. H. 2005. The Astrophysical Journal Letters 621:137. [5] Desch S. J. et al. 2005. CPPD 341:849. [6] Ciesla F. J. et al. 2004. Meteoritics \& Planetary Science 29:1809. [7] Morris M. A. et al. 2010. Abstract \#293. 31st Lunar and Planetary Science Conference. [8] Hood L. L. et al. 2009. Meteoritics \& Planetary Science 44:327. [9] Villeneuve et al. 2009. Science 325:985. [10] Weidenschilling S. J. 2000. SSRv 92:295. [11] Dauphas N. and Pourmand A. 2011. Abstract \#1040. 42nd Lunar and Planetary Science Conference. [12] Hansen B. M. S. 2009. The Astrophysical Journal 703:1131. [13] Morris M. A. and Desch S. J. 2010. The Astrophysical Journal 722:1474. [14] Desch S. J. and Connolly H. C. 2002. Meteoritics \& Planetary Science 37:183. [15] Alexander C. M. O’D. et al. 2008. Science 320:1617. 
5391

\section{SPHERULES IN CHICXULUB EVENT BED DEPOSITS FROM NE MEXICO: CLUES FROM TRACE ELEMENTS}

A. Deutsch ${ }^{1}$, P. Schulte ${ }^{2}$, S. Ebert ${ }^{2}$, and J. Berndt ${ }^{3}$. ${ }^{1}$ Institut f. Planetologie, WWU Münster, D-48149 Münster, Germany. ${ }^{2}$ GeoZentum Nordbayern, Universität Erlangen, D-91054 Erlangen, Germany. ${ }^{3}$ Institut f. Mineralogie, WWU Münster, D-48149 Münster, Germany. E-mail: deutsca@uni-muenster.de.

Introduction: The Chicxulub impact ("K-Pg") event bed is characterized by its diversity in terms of thickness, mineralogical content, geochemical characteristics, and of processes that caused this enormous diversity [1, 2]. In this context, the amount of Chicxulub ejecta and of the local component, and the contribution of projectile matter to the event bed turned out to be much more complicated than previously thought; this development is mainly due to spatial high-resolution in situ analysis by laser-ablation-mass spectrometry (LA-ICP-MS) [3]

Specifically, the Chicxulub event bed around the Gulf of Mexico contains different types of spherules, composed either of calcium carbonates or silicate glass and their alteration products, or of complicated-textured carbonate and silicate compositions [4]. Compared to the Si-Al-rich ejecta spherules present elsewhere in the Gulf of Mexico area [1, 2], the event bed in NE Mexico contains unusually, Fe-, Mg-, and $\mathrm{K}$-rich (nearly) isotropic spherules [5]. To constrain the origin of these materials in the La Popa area, we performed a series of micro-chemical investigations using electron microprobe and LA-ICP-MS.

Methods: The particles were characterized by electron optical methods, and than analyzed in $>100 \mu \mathrm{m}$-thick thin sections. Spot size of the LA-ICP-MS at the WWU was between 50 and $100 \mu \mathrm{m}$. Details of the analytical methods are given in [3].

Results: All analyzed glass spherules are hydrated yielding analytical totals of 88 to wt $\%$; we distinguish Fe-Mg-, and a Si-Al-K-rich particles. The first group is composed of up to $28 \mathrm{wt} \% \mathrm{FeO}$ and $12 \mathrm{wt} \% \mathrm{MgO}$ the $\mathrm{Si}-\mathrm{Al}-\mathrm{K}$-rich particles contain up to $48 \mathrm{wt} \% \mathrm{SiO}_{2}, 25 \mathrm{wt} \% \mathrm{Al}_{2} \mathrm{O}_{3}$, and 8 $\mathrm{wt} \% \mathrm{~K}_{2} \mathrm{O}$. The particles may consist entirely of one of these phases or both phases occur within one particle as blobs or sharply separated schlieren; mixed phases have not been observed.

The spherules have generally low trace element contents; rare earth elements $(\mathrm{REE})$ are sub-chondritic $(0.1 \times \mathrm{C} 1)$ to chondritic with flat $\mathrm{C} 1$ normalized distribution patterns. The lack of $\mathrm{Ce}$ and $\mathrm{Eu}$ anomalies indicates a closed water-rock system during diagenesis. The $\mathrm{Ni} / \mathrm{Cr}$ ratio is approximately 1 in the Fe-Mg-rich, and approximately 0.8 in the Si-Al-Krich spherules.

Outlook: Bulk material from the K-Pg event beds usually display REE abundances and distribution patterns similar to that one of the upper continental crust (e.g., [6]). Our high-resolution in situ analyses allow for the first time constraining the presence of materials with obviously different precursor lithologies.

References: [1] Schulte P. et al. 2010. Science 327:1214-1218. [2] Schulte P. et al. 2011. Sedimentology, in press. [3] Berndt J. et al. 2011. Geology 39:279-282. [4] Deutsch A. et al. 2010. Meteoritics \& Planetary Science 45:A45. [5] Schulte P. and Kontny A. 2005. GSA Special Paper 384:291-221. [6] Martinez-Ruiz F. et al. 2006. Chemical Geology 232:1-11.

We appreciate funding by the DFG.
5298

AL HAGGOUNIA 001: A WEATHERED EL6 CHONDRITE

A. Devaux ${ }^{1}$, A. Jambon ${ }^{1}$, O. Boudouma ${ }^{1}$, D. Badia ${ }^{1}$, E. Charon ${ }^{2}$, J. N. Rouzaud ${ }^{2}$ and J.-A. Barrat ${ }^{3}$. ${ }^{1}$ UPMC-PARIS 6, ISTEP 4 place Jussieu, 75005 Paris France. CNRS UMR 7193. ${ }^{2}$ Laboratoire de Géologie de l'ENS, UMR CNRS 8538, 24 rue Lhomond, 75005 Paris, France ${ }^{3}$ UBO I.U.E.M., Place Nicolas Copernic, 29280 Plouzané Cedex, France. CNRS UMR 6538

Introduction: Al Haggounia (AHa) 001 is a significant find (several tons), the strewnfield of which extends over nearly $50 \mathrm{~km}$ [1]. The fall was dated $23 \mathrm{ka}$ B.P. by ${ }^{14} \mathrm{C}$; therefore it is not a fossil meteorite as confirmed by field evidences [1]. Its classification is however controversial: AHa 001 was classified as an aubrite [2] but paired stones were classified as EL3, EL6, EL7, enstatite achondrite and aubrite [2, 3]. One obvious reason for this disagreement resides in its severely weathered state and possibly in the variability among such a large number of stones of varied sizes. This can be illustrated in its magnetic susceptibility $(\log (\chi))$, which varies from 2.8 - an aubrite like value - up to 4.9 as for a slightly weathered enstatite chondrite (fresh $5.5 \pm 0.2$ ). The present study is based on the observation of a large number of hand specimens, five polished sections and one thin section. Two types of rocks are typical: (1) brownish, heavily rusted samples with fractures filled of iron oxide-hydroxides and (2) bluish samples or remnant cores of several $\mathrm{cm}$ to $\mathrm{dm}$ surrounded by variable amounts of brownish material and limited by oxide-hydroxide filled fractures.

Results: Faint chondrules about $1 \mathrm{~mm}$ in size are quite rare (less than 0.2 per $\mathrm{cm}^{2}$ on the average) and therefore not found in every section. Bulk chemical composition is of no help to discriminate between E chondrite and aubrite, as metal and sulfide have been heavily weathered when not leached away. The blue-grey sections in particular exhibit a porosity of about $40 \%$ as a result of leaching of all sulfides and metal. Brownish fractions however have some preserved sulfide and metal cores. Minor components in sulfides are useful in determining the type and metamorphic grade [4]. This approach however in hampered by the small size of preserved relict crystals. The $\mathrm{Si}$ content of kamacite $(1 \mathrm{wt} \%)$ favors an EL type. The organization degree of carbon as obtained from Raman spectra has been used in order to characterize EH3 and EL3 [5]. As proposed by [6] we used the width of the $G$ band as a reliable parameter of graphitization and not the classical ID/IG affected by polishing artifacts [6]. In AHa the carbon component is well ordered since its FMWH G $\left(19 \mathrm{~cm}^{-1}\right)$ is close to the canonical graphite value. In contrast matrix carbon in type 3 exhibits a broad $\mathrm{G}$ band (FMWH-G $>60 \mathrm{~cm}^{-1}$ ) and well developed D1 and D2 defect bands characteristic of a still disordered carbon [5]. It follows that all matrix carbon in AHa was subject to a significant metamorphism.

Conclusions: From the texture and carbon crystallinity the metamorphic grade is as high as a type 6. According to the presence of ghost chondrules, an aubrite classification is not justified. Search for preserved sulfides will help confirming this diagnosis.

References: [1] Chennaoui-Aoudjehane H. et al. 2007. Meteoritics \& Planetary Science 42:5329. [2] Meteoritical Bulletin 84, 87, 89-91, 94, 96. [3] Irving A. et al. 2006. Meteoritics \& Planetary Science 41:5264. [4] Zhang et al. 1995. Journal of Geophysical Research 100:9417. [5] Quirico E. et al. 2011. Geochimica et Cosmochimica Acta 75:3088. [6] Ammar and Rouzaud J.-N. 2011. Journal of Raman Spectroscopy, in press. 
5413

CRYSTALLINE SILICATES IN COMET 81P/WILD 2 FROM THE STARDUST TRACK 81

E. Dobrică and A. J. Brearley. Department of Earth and Planetary Sciences, MSC03-2040, University of New Mexico, Albuquerque, NM 87131-0001, USA. E-mail: edobrica@unm.edu.

Introduction: Previous studies of Stardust samples collected from comet $81 \mathrm{P} /$ Wild 2 have reported abundant crystalline phases, presumably formed in the asteroid belt $[1,2]$. In this study, we describe the mineralogical composition of one ultramicrotome section from track 81 (C2092.7.81.1.10) examined by transmission electron microscopy (TEM).

Results and Discussion: The microtome section is a polycrystalline aggregate dominated by pyroxenes and plagioclase feldspar. Due to chattering from the microtome sample preparation, it is not possible to determine the exact grain size of the individual phases, but we estimate that the grains are typically submicron to micron in size. We identified low-Ca and Ca-rich pyroxenes $\left(n=12, \mathrm{En}_{80-95} \mathrm{Wo}_{1-8} ; n=5, \mathrm{En}_{52-63}\right.$ $\left.\mathrm{Wo}_{32-38}\right)$, plagioclase $\left(n=21, \mathrm{An}_{45-85} \mathrm{Ab}_{13-55} \mathrm{Or}_{0-2}\right)$ and rare, nanometric sulfides. No olivines were found. Low-Ca pyroxenes are enriched in $\mathrm{Cr}_{2} \mathrm{O}_{3}$ (up to $1.8 \mathrm{wt} \%$; avg. $1.1 \mathrm{wt} \%$ ), $\mathrm{MnO}$ (up to $2.4 \mathrm{wt} \%$; avg. $1.3 \mathrm{wt} \%$ ) and also contain significant $\mathrm{Al}_{2} \mathrm{O}_{3}$ (up to $3.8 \mathrm{wt} \%$; avg. $2.6 \mathrm{wt} \%$ ). Ca-rich pyroxenes contain lower amounts of $\mathrm{Cr}_{2} \mathrm{O}_{3}$ (up to $0.9 \mathrm{wt} \%$, avg. $0.5 \mathrm{wt} \%$ ) and $\mathrm{MnO}$ (up to $0.8 \mathrm{wt} \%$, avg. $0.6 \mathrm{wt} \%$ ) but have $\mathrm{TiO}_{2}$ contents up to $1.3 \mathrm{wt} \%$ (avg. $1.1 \mathrm{wt} \%$ ). The low-Ca pyroxene compositions are extremely unusual having $\mathrm{Mn} / \mathrm{Fe}$ ratios up to $70 \times \mathrm{CI}$, higher that the value of olivines measured previously in Stardust samples [2] $(\mathrm{Mn} / \mathrm{Fe}$ ratios up to $50 \times \mathrm{CI})$. Low-Ca pyroxene $\mathrm{Cr}_{2} \mathrm{O}_{3}$ and $\mathrm{MnO}$ contents correlate positively with $\mathrm{Al}_{2} \mathrm{O}_{3}$, a relationship that is also observed in low-iron, manganese-enriched (LIME) minerals from interplanetary dust particles (IDP) [3]. The LIME silicates have generally been interpreted as condensates from a gas of solar composition [3]. The compositions of Ca-rich pyroxenes in this particle are also distinct from those found in Kool grains from other Stardust particles [1]. The $\mathrm{Al}_{2} \mathrm{O}_{3}$ and $\mathrm{TiO}_{2}$ contents are higher and $\mathrm{CaO}$ and $\mathrm{Cr}_{2} \mathrm{O}_{3}$ lower in the Ca-rich pyroxenes analyzed in this study. However, the average concentrations of $\mathrm{Na}_{2} \mathrm{O}(2.1 \mathrm{wt} \%$ ) and $\mathrm{MnO}$ (avg. $0.5 \mathrm{wt} \%$ ) are comparable in both studies.

Although the assemblage enstatite, clinopyroxene, and plagioclase feldspar does occur in some plagioclase-bearing chondrules in carbonaceous chondrites [4], the fine-grained character, textural relationships, and unusual compositions of the pyroxenes make this particle quite distinct from any known chondritic material. The ambiguous textural relationships between the different phases due to microtoming also prevent any definitive statement about whether the particle is igneous in origin. However, the compositions of the pyroxenes in this particle share similarities to terrestrial igneous pyroxenes that accept $\mathrm{Al}^{3+}$ into the tetrahedral sites, creating a charge imbalance that requires the simultaneous entry $\mathrm{of}^{3+}, \mathrm{Ti}^{4+}$, or $\mathrm{Fe}^{3+}$ in the octahedral sites [5]. Irrespective of the exact formation mechanism of this particle, it is clear that the pyroxene mineral chemistry is indicative of another unique type of material in comet $81 \mathrm{P} /$ Wild 2 that has not been sampled by any known primitive meteorite, but was present within the feeding zone for the accretion of the Jupiter Family comets.

References: [1] Joswiak D. J. et al. 2009. Meteoritics \& Planetary Science 44:1561-1588. [2] Brownlee D. E. et al. 2009. Cosmic Dust 157. [3] Klöck W. et al. 1989. Nature 339. [4] Scott E. R. D. and Krot A. N. 2005. Meteorites, comets and planets. Oxford. [5] Campbell I. H. and Borley G. D. 1974. Contributions to Mineralogy and Petrology 47:281-297.
5452

MICROSTRUCTURAL STUDY OF THE TEXTURE AND MINERALOGY OF TIESCHITZ WHITE MATRIX

E. Dobrică and A. J. Brearley. Department of Earth and Planetary Sciences, MSC03-2040, 1University of New Mexico, Albuquerque, NM 87131-0001, USA. E-mail: edobrica@unm.edu.

Introduction: Tieschitz is an unequilibrated ordinary chondrite (UOC) classified as petrologic type H/L3.6 [1]. This meteorite displays several unique features, notably two types of matrices, termed white and black matrix $[2,3]$. The white matrix is enriched in $\mathrm{Na}, \mathrm{K}$, and $\mathrm{Al}[2-5]$ and has been interpreted as a remobilized component of chondrules, i.e., mesostasis glass which had been leached out and redeposited by a fluid phase [4, 5]. Previous studies have reported the presence of plagioclase and nepheline [6] as the major components of white matrix. However, there are still significant uncertainties regarding the origin of white matrix that remain unresolved. Here we report new observations on white matrix carried out using focused ion beam (FIB)/transmission electron microscopy (TEM) techniques.

Sample and Methods: A variety of TEM techniques were used to examine two FIB sections of white matrix using a JEOL 2010F FEG TEM/Scanning TEM operating at $200 \mathrm{kV}$.

Results and Discussion: White matrix in Tieschitz is found as veins often surrounded by black matrix. Our TEM observations are in agreement with previous work [7], showing that white matrix is an intergrowth of two or more phases. The intergrowth is composed of an amorphous phase which could be a glass or a crystalline phase that was amorphized during the electron beam irradiation. Compositionally, this phase has similarities to both albite and nepheline. It is enriched in $\mathrm{SiO}_{2}(\sim 60 \mathrm{wt} \%), \mathrm{Al}_{2} \mathrm{O}_{3}$ $(\sim 29 \mathrm{wt} \%)$, and $\mathrm{Na}_{2} \mathrm{O}(\sim 7 \mathrm{wt} \%)$ with minor amounts $(<1 \mathrm{wt} \%)$ of $\mathrm{MgO}$, $\mathrm{K}_{2} \mathrm{O}, \mathrm{CaO}$, and $\mathrm{FeO}$. The second phase is crystalline and occurs as elongate, oriented arrays that define a lamellar intergrowth with the amorphous phase. The composition and the crystal structure of these lamellae are consistent with a Ca-rich pyroxene $\left(\mathrm{En}_{28} \mathrm{Wo}_{43}\right)$. These pyroxene lamellae are typically $\sim 30 \mathrm{~nm}$ wide and range $400 \mathrm{~nm}$ to $\sim 2 \mu \mathrm{m}$ in length. Veins of olivine $\left(\mathrm{Fo}_{56}\right)$ were also identified within the intergrowth.

Our new observations confirm the complexity of white matrix in Tieschitz $[3,7,8]$, but differ somewhat from the observations in previous studies. We have identified the presence of Ca-rich pyroxene, which was observed by [7], but not characterized. Unlike [7], we have not observed a twinned, beam sensitive phase, but instead have found an amorphous material with a composition close to albite or nepheline. A possibility remains that the amorphization could be the result of FIB sample preparation and requires further investigation. None of our observations provide definitive evidence of the involvement of an aqueous fluid in the formation of the white matrix. Nevertheless, the clear evidence of leaching of Na-Al-bearing mesostasis in chondrules [8], indicates that fluid mass transport must have played an important role in the formation of the white matrix.

References: [1] Sears D. W. et al. 1980. Nature 287:791. [2] Christophe Michel-Levy M. 1976. Earth and Planetary Science Letters 30:143-150. [3] Dobrică E. and Brearley A. J. 2011. Abstract \#1889. 42nd Lunar and Planetary Science Conference. [4] Ashworth J. R. 1977. Earth and Planetary Science Letters 35:25. [5] Hutchison R. et al. 1979. Nature 280:116. [6] Ashworth J. R. 1981. Proceedings of the Royal Society of London. Series A 374:179-1974. [7] Alexander C. M. O. et al. 1989. Earth and Planetary Science Letters 95:187. [8] Hutchison R. et al. 1998. Meteoritics \& Planetary Science 33:1169-1179. 


\section{6}

\section{MÖSSBAUER STUDY OF Fe-Ni PHASES IN MESOSIDERITES}

E. Dos Santos ${ }^{1}$, R. B. Scorzelli ${ }^{1}$ and M. E. Varela ${ }^{2}{ }^{1}$ Centro Brasileiro de Pesquisas Físicas, RJ, Brazil. ${ }^{2}$ ICATE-CONICET, San Juan, Argentina.

Following previous studies [1] on the applications of Mössbauer spectroscopy to the meteoritic $\mathrm{Fe}-\mathrm{Ni}$ metal structures, the present work focuses on the study of $\mathrm{Fe}-\mathrm{Ni}$ alloys in mesosiderites. The metal from this enigmatic rocks has the lowest cooling rates yet determined for meteorites.

We are performing a systematic study on the following mesosiderite: Vaca Muerta, Mincy, Crab Orchard, Estherville, and Hainholz. Thin $\sim 500 \mu \mathrm{m}$ slices are cut from the metal-rich areas and then polished to thicknesses of $\sim 150 \mu \mathrm{m}$.

The Mössbauer spectra of untreated bulk samples exhibit overlapping of $\mathrm{Fe}-\mathrm{Ni}$ phases, with kamacite being the dominant phase. This abundance makes the detection of the taenite phases difficult, which contains in its microstructure the intergrowth of tetrataenite and antitaenite [2]. In order to determine the presence of tetrataenite/ antitaenite intergrowth that have been observed in ataxites, octahedrites and metal particles of chondrites [3,4] and better define the degree of ordering of tetrataenite, we are analyzing taenite fractions extracted from these mesosiderites by etching the metallic samples with $\mathrm{HCl} / \mathrm{HNO}_{3}$.

The corresponding Mössbauer hyperfine parameters obtained from the analysis of the spectra, although preliminary, seem to support the reported very slow cooling rate of this unique group of meteorites.

References: [1] Scorzelli R. B. and Souza Azevedo I. 2001. Meteoritics \& Planetary Science 36/9:A185. [2] Rancourt D. G. and Scorzelli R. B. 1995. Journal of Magnetism and Magnetic Materials 150:30-36. [3] Danon J., Scorzelli R. B., Azevedo I. S., and Michel-Levy M. C. 1979. Nature 281:469-471. [4] Danon J., Scorzelli R. B., Azevedo I. S., Curvello W., Albertsen J. F., and Knudsen J. M. 1979. Nature 277:283-284.
5085

EARLY SOLAR SYSTEM PROCESSES REVEALED BY AN INTEGRATED STUDY OF UREILITE METEORITES

H. Downes ${ }^{1,2,3}$, C. L. Smith ${ }^{2}$, A. D. Beard ${ }^{1,2}$ and A. J. Ross ${ }^{2,3}$. ${ }^{1}$ Deptartment of Earth and Planetary Sciences, Birkbeck University of London, Malet St, London WC1E 7HX UK. E-mail: h.downes@, ucl.ac.uk. ${ }^{2}$ Deptartment of Mineralogy, Natural History Museum, Cromwell Road, London, UK. Centre for Planetary Sciences at ${ }^{3} \mathrm{UCL} /$ Birkbeck, UCL, Gower St. London, UK.

Introduction: Far from being "bizarre" and "perplexing," ureilite meteorites provide us with a wealth of information about processes in the early solar system which have important implications for the formation of the Earth and other terrestrial planets. The ureilite parent body (UPB) was formed by accretion from a chondritic precursor (as seen in the distribution of oxygen isotope values), which was rich in carbon (ureilites contain up to $6 \mathrm{wt} \% \mathrm{C}$, which exceeds typical values in carbonaceous chondrites). The UPB then underwent differentiation, forming an Fe-rich core and ultramafic silicate mantle, but did not achieve the magma ocean stage experienced by many other planets and asteroids (i.e., its mantle did not homogenize in terms of $\mathrm{mg \#}$ and $\delta^{18} \mathrm{O}$ ) [1]. Thus it provides a window into pre-magma ocean processes not available from any other source. Partial melting of the mantle of the UPB (heated by short-lived radioactive isotopes) produced magmatic activity, as revealed by basalt melt droplets, melt inclusions, LREE-depletion in the silicate mantle phases, and some regions of pyroxene-rich mantle. Extensive magmatic differentiation may also have resulted in small volumes of evolved magmas that formed granitoid-like material [2].

Impact and Reaccretion: Ureilites also yield information about impact processes in the early solar system, as many of them are polymict regolith breccias [3]. The original UPB was disrupted by a major impact while it was undergoing partial melting [1]. This produced extensive reduction of silicates to metal in the presence of graphite [4], and also caused the formation of $\mathrm{Fe}-\mathrm{Ni}$ silicides [5]. If incorporated into larger accreting bodies, such silicides may have contributed to strongly reduced cores in early formed terrestrial planets. Graphite was converted to diamonds by this shock event [6]; thus not all of the carbon accreted to terrestrial planets and asteroids was in the form of the low-temperature organic component found in carbonaceous chondrites. Following the disruptive impact, a daughter planetesimal rapidly reformed by reaccretion, producing a rubble-pile asteroid which is the body sampled by present-day ureilite meteorites. On the surface regolith of this asteroid, impact gardening occurred, with accretion of late veneers of chondritic and other material, including many unusual "orphan" clasts that have no equivalent in our meteorite collections [1]. These clasts are more variable than those found in regolith breccias from the HED parent body. A late addition of water formed opal (hydrated silica) in some areas of the regolith of the ureilite asteroid [7].

References: [1] Downes H. et al. 2008. Geochimica et Cosmochimica Acta 72:4825-4844. [2] Downes H. et al. 2009. Abstract \#5036. 72nd Annual Meteoritical Society Meeting. [3] Goodrich C. et al. 2004. Chemie der Erde 64:283-327. [4] Warren P. H. and Huber H. 2006. Meteoritics \& Planetary Science 41:835-849. [5] Smith C. L. et al. 2010. Abstract \#5221. 73rd Annual Meteoritical Society Meeting. [6] Ross A. J. et al. 2011. Meteoritics \& Planetary Science 46:364-378. [7] Beard A. D. et al. This conference. 
5020

GEBEL KAMIL: THE IRON METEORITE THAT FORMED THE KAMIL CRATER IN EGYPT

M. D'Orazio ${ }^{1}$, L. Folco ${ }^{2}$, A. Zeoli ${ }^{2}$ and C. Cordier ${ }^{2}$. ${ }^{1}$ Dipartimento di Scienze della Terra, Università di Pisa, Italy. E-mail: dorazio@dst.unipi.it. ${ }^{2}$ MNA, Siena, Italy.

The 45 m-diameter Kamil impact crater (eastern Sahara, Egypt) was formed $<5000$ years ago within Cretaceous quartz-arenites of the Gilf Kebir Formation [1, 2]. Most of the pristine features of the impact structure are exceptionally well preserved, including thousands of fragments of the iron meteorite impactor, the Gebel Kamil meteorite. With the exception of a single $83 \mathrm{~kg}$ regmaglypted individual showing remnants of the fusion crust, all specimens of Gebel Kamil are explosion fragments (shrapnel) weighing from $<1 \mathrm{~g}$ to $34 \mathrm{~kg}$. The shrapnel shapes vary from flattened, twisted and jagged to rounded and equidimensional. Gebel Kamil is an ungrouped Ni-rich (about 20 wt $\%$ Ni) ataxite characterized by high $\mathrm{Ge}$ and $\mathrm{Ga}$ and relatively low $\mathrm{Ir}$ contents $(\sim 120$, $\sim 50$ and $\sim 0.5 \mu \mathrm{g} \mathrm{g}^{-1}$, respectively), and by a fine-grained duplex plessite metal matrix. The metal matrix often contains spindles of kamacite (15$25 \mu \mathrm{m}$ in width) nucleated onto small schreibersite crystals, and arranged in a micro-Widmannstätten pattern. Accessory minerals are, in order of decreasing abundance, schreibersite, troilite, daubréelite, and native copper. Schreibersite and troilite are typically enveloped in swathing kamacite. Terrestrial weathering is extremely low and confined to the portions closest to the external surface. Shrapnel fragments are invariably cross-cut by curvilinear shear bands formed during the explosive impact on Earth. These structures are not observed in the individual specimen which detached from the main body prior to impact. In terms of structure and bulk chemical composition Gebel Kamil is similar to the Morradal ungrouped $\mathrm{Ni}$-rich ataxite [3].

Systematic search around the crater revealed that meteorite fragments have a highly asymmetric distribution, with greater concentrations in the southeast sector. A broad maximum in meteorite concentration occurs in the $125^{\circ}-160^{\circ} \mathrm{N}$ sector at about $200 \mathrm{~m}$ from the crater rim. The mass of meteorite specimens $>10 \mathrm{~g}$ recovered in the field is $1700 \mathrm{~kg}$, whereas the total mass inferred from the density map compiled in this study is $3400 \mathrm{~kg}$. Field data indicate that the iron bolide approached the Earth's crust from the northwest $\left(305^{\circ}-340^{\circ} \mathrm{N}\right)$, traveling along a moderately oblique $\left(30^{\circ}-\right.$ $45^{\circ}$ ) trajectory, following [4]. Upon hypervelocity impact, the projectile was disrupted into thousands of fragments. Shattering was accompanied by some melting of the projectile and of the quartz-arenite target rocks, which also suffered variable degree of shock metamorphism (formation of planar fractures, mosaicism, and planar deformation features in quartz). Interestingly, some of this melted and shocked material stuck onto the shrapnel surfaces and was occasionally preserved from the erosive action of wind-driven sand.

Acknowledgments: Supported by EC (Marie Curie Actions-RTNs ORIGINS project), Fondazione Cassa di Risparmio di Torino, Banca Monte dei Paschi di Siena S.p.A., Fondazione Monte dei Paschi di Siena, and Telespazio S.p.A.

References: [1] Folco L. et al. 2010. Science 329:804. [2] Folco L. et al. 2010. Geology 39:179-182. [3] Buchwald V. F. 1975. Handbook of iron meteorites, vol. 3. Berkeley, USA: University of California Press. 1416. [4] Melosh H. J. 1989. Impact cratering. A geologic process, Oxford Monographs on Geology and Geophysics. Oxford: Oxford University Press. $245 \mathrm{p}$.
5507

METAMORPHISM IN THE NEWLY RECOVERED MASON GULLY (H5) ORDINARY CHONDRITE AND ITS CORRELATION TO ANOMALOUS PROPERTIES

K. A. Dyl ${ }^{1,2}$, G. K. Benedix ${ }^{2}$, P. A. Bland ${ }^{1}$, M. C. Towner ${ }^{1}$ and P. Spurný ${ }^{3}{ }^{1}$ Department of Earth Science and Engineering, Imperial College London, SW7 2AZ, UK. E-mail: kdyl@imperial.ac.uk. ${ }^{2}$ Department of Mineralogy, Natural History Museum, London SW7 5BD, UK. ${ }^{3}$ Astronomical Institute of the Academy of Sciences, Fričova 298, CZ-251 65 Ondřejov Observatory, Czech Republic.

Introduction: Mason Gully (MG) is a new H5 ordinary chondrite recovered by the Desert Fireball Network in November 2010 [1]. The presence of discernable chondrules and the major element composition of olivine, pyroxene, and plagioclase support this classification. It is characterized by unusually high porosity and has an unusual modal composition for an $\mathrm{H}$ chondrite with orthopyroxene being more abundant than olivine. We used the EVO 15LS variable pressure scanning electron microscope at the NHM to examine reaction textures, compositional variation, and phase relationships in this meteorite, specifically in porous areas associated with plagioclase. These observations identify several ways to constrain metamorphic conditions and may explain the unusual properties of this new meteorite fall.

Reaction and Formation of Pyroxene: The primary metamorphic reaction observed is qualitatively characterized as follows (in terms of major elements):

$2 \mathrm{Mg}_{2} \mathrm{SiO}_{4}+\mathrm{CaAl}_{2} \mathrm{Si}_{2} \mathrm{O}_{8} \rightarrow \mathrm{Mg}_{2} \mathrm{Si}_{2} \mathrm{O}_{6}+\mathrm{CaMgSi}_{2} \mathrm{O}_{6}+\mathrm{MgAl}_{2} \mathrm{O}_{4}$ olivine + anorthite $\rightarrow$ low-Ca px + high-Ca px + spinel.

Evidence for this reaction includes: low-Ca pyroxene replacing olivine, spinel (chromite) formation along grain boundaries, and pyroxene growth at plagioclase-olivine phase boundaries.

Conditions of Metamorphism: The temperature and oxygen fugacity associated with metamorphism can be constrained via this reaction. Preliminary calculations using olivine-chromite $\mathrm{Fe}-\mathrm{Mg}$ exchange and twopyroxene thermometry suggest more oxidizing conditions at lower temperature (IW-1.5 to IW-0.3) [2]. Further investigation is required to determine what this difference means for the evolution on the parent body.

The Na-rich composition of plagioclase in ordinary chondrites is also a consequence of the reaction we have identified [3, 4]. Further work will determine to what extent the $\mathrm{Na} / \mathrm{Ca}$ content varies and the role of other processes invoked to explain Na-rich plagioclase (i.e., impact melting [5], fluid phase [4]).

Relationship to Bulk Properties: Several unusual bulk properties of Mason Gully are consistent with this reaction.

Modal Abundances.

The production of pyroxene is one explanation for the modal mineralogy of Mason Gully; the observation of $38 \%$ orthopyroxene is significantly higher than the $26 \%$ expected in $\mathrm{H}$ chondrites [3].

High Porosity.

The reaction identified above is commonly observed in granulitefacies metamorphism (high-temperature, low-moderate pressure). There is evidence for the presence of $\mathrm{CO}_{2}$-rich fluids in these reaction assemblages [6], which may explain the high porosity observed in Mason Gully.

References: [1] Towner M. et al. 2011. Meteoritics \& Planetary Science 46. This conference. [2] Benedix G. K. et al. 2005. Geochimica et Cosmochimica Acta 69:5123-5131. [3] McSween H. Y. and Bennett M. E. 1990. Icarus 90:107-116. [4] Kovach H. A. and Jones R. H. 2010. Meteoritics \& Planetary Science 45:246-264. [5] Rubin A. E. 2003. Geochimica et Cosmochimica Acta 67:2695-2709. [6] Selverstone J. 1982. Contributions to Mineralogy and Petrology 79:28-36. 
5359

SEQUENTIAL ACCRETION AND ANNEALING OF SEPARATE SILICATE AND METAL LAYERS IN A CR CHONDRITE CHONDRULE

D. S. Ebel ${ }^{1}$ and M. R. Downen ${ }^{2}$. ${ }^{1}$ Department of Earth and Planetary Sciences, American Museum of Natural History, Central Park West at 79th St, New York, NY 10024, USA. E-mail: debel@amnh.org. ${ }^{2}$ Geography and Geology, Western Kentucky University, 1906 College Heights Blvd. \#31066, Bowling Green, KY 42101-1066, USA.

Introduction: Computed tomography (CT) reveals the 3D shapes and metal abundances in spherical, FeO-poor, metal-rimmed chondrules in $\mathrm{CR}$ chondrites [1]. Hypotheses for their formation [2,3] include a mechanical segregation process [4], or vaporization and recondensation of $\mathrm{Fe}-\mathrm{Ni}$ alloy during cooling. The latter scenario predicts that siderophile element abundances were gradually established during multiple melting events, due to reduction of FeO-bearing olivine and evaporation of $\mathrm{S}$ from sulfides. Repeated heating advanced this process, coarsened silicate grains, and promoted a spherical shape of the resulting chondrules [5-9]. Here, an alternative model is proposed, based on texture and chemistry of a large chondrule in Acfer 139 (CR2). This is an extreme example of similar layering observed in $\mathrm{CT}$ images of $\mathrm{CR}$ chondrite chondrules.

Methods: Acfer 139-t2-ch1 was imaged by synchrotron (APS) and conventional CT (AMNH). Serial thick sections to the core of this approximately $3.5 \mathrm{~mm}$ chondrule provided detailed chemical and textural information on four discrete, concentric, metal layers surround, sulfidefree, almost metal-free silicate layers.

Results: The innermost approximately $1 \mathrm{~mm}$ diameter, metal-free, pyroxene-free, $\mathrm{Mg} \# 98$ core has an annealed barred olivine texture. It is surrounded by many non-connected $5-20 \mu \mathrm{m}$ metal beads. Next is a 400-600 $\mu \mathrm{m}$ enstatite-bearing, Mg\#98, porphyritic olivine layer, with a nearly continuous metal shell up to $150 \mu \mathrm{m}$ thick. The next silicate layer, 300-500 $\mu \mathrm{m}$, has olivine/pyroxene approximately $0.7, \mathrm{Mg} \# 98$ in both, with a thin, up to $100 \mu \mathrm{m}$ thick, near-continuous metal shell. The outermost silicate layer is less continuous, $200-400 \mu \mathrm{m}$ thick, with modal pyroxene $>$ olivine, surrounded by a thin metal shell.

Discussion: This texture seems inconsistent with multiple episodes of heating at $>1800 \mathrm{~K}$ for durations sufficient to evaporate and recondense metal from successive layers [5-9], leaving interior layers intact. But it is consistent with independent accretion and rapid annealing of alternating layers of silicate and metal particles in a reducing, cooling disk environment, as in [10].

References: [1] Ebel D. S. et al. 2008. Meteoritics \& Planetary Science 43:1725-1740. [2] Connolly H. C. Jr. et al. 2001. Geochimica et Cosmochimica Acta 65:4567-4588. [3] Campbell A. J. et al. 2005. In Chondrites and the protoplanetary disk, edited by Krot A. N. et al. Astronomical Society of the Pacific Conference Series 341:407-431. [4] Wasson J. T. \& Rubin A. E. 2010. Geochimica et Cosmochimica Acta 74:2212-2230. [5] Kong P. et al. 1999. Geochimica et Cosmochimica Acta 63:2637-2652. [6] Zanda B. et al. 2002. Abstract \#1852. 33rd Lunar and Planetary Science Conference. [7] Humayun M. et al. 2002. Abstract \#1965. 33rd Lunar and Planetary Science Conference. [8] Varley L. R. et al. 2003. Abstract \#1899. 34th Lunar and Planetary Science Conference. [9] Humayun M. et al. 2010. Abstract \#8004. Chondrules: Their role in early solar system history. [10] Weisberg et al. 1992. Meteoritics 27(suppl.):A306.

Support: NASA-COS-NNX10AI42G, NSF-EAR-0959384(DSE); NSFEAR-0622171, DoE-Geo-DE-FG02-94ER 14466 (APS).
5500

THERMODYNAMIC STABILITY OF LOW-IRON MANGANESEENRICHED OLIVINE IN THE SOLAR NEBULA

D. S. Ebel ${ }^{1,3}$ and M. K. Weisberg ${ }^{1,2,3}$. ${ }^{1}$ Department of Earth and Planetary Sciences, American Museum of Natural History, Central Park West at 79th St, New York, NY 10024, USA. E-mail: debel@amnh.org. ${ }^{2}$ Kingsboroough College, CUNY, Brooklyn, NY. ${ }^{3}$ Graduate Center of the City University of New York, NY, USA.

Introduction: A significant fraction of olivine and Ca-poor pyroxene grains in primitive extraterrestrial materials are very low in $\mathrm{Fe}$, and $\mathrm{Mn}$ enriched. These LIME silicates (primarily olivine) have $\mathrm{wt} \% \mathrm{MnO} / \mathrm{FeO}$ $>0.1$, and usually $<1.0 \mathrm{wt} \% \mathrm{FeO}$. They occur in Allende (CV3) silicates [1], interplanetary dust particles [2], Type I chondrules [3] and amoeboid olivine aggregates $[4,5]$ in $\mathrm{CR}$ chondrites, and Wild 2 cometary particles $[6,7]$. Here, we clarify the reduction/oxidation conditions in which the $\mathrm{Mn}$ component (tephroite, Mn-Ol) of olivine solid solution may be may be more thermodynamically stable relative to the Fe component (fayalite, $\mathrm{Fe}-\mathrm{Ol}$ ), in vapors of solar composition, and in vapors enriched in a dust of CI chondritic composition [8,9].

Methods: (1) Reactions involving $\mathrm{O}_{2}$ (e.g., $2 \mathrm{Mn}+\mathrm{O}_{2}=2 \mathrm{MnO}$ ) in a $\mathrm{G}_{\mathrm{rxn}}=R \operatorname{Tn}\left(f \mathrm{O}_{2}\right)$ vs. temperature $(\mathrm{T})$ were plotted on the classic nomogram [10, Fig 14-4]. Previous results for CI-dust enriched systems [8] were used to compute the stability $\left(\mathrm{G}_{\mathrm{rxn}}\right)$ of olivines with mole fractions 0.1 and $0.5 \mathrm{Mn}-\mathrm{Ol}$ and $\mathrm{Fe}-\mathrm{Ol}$, relative to solar, $100 \times \mathrm{CI}$ and $1000 \times \mathrm{CI}$ chemical systems at total pressure $\left(\mathrm{P}^{\mathrm{tot}}\right) 10^{-3}$ and $10^{-6}$ bar, vs. T. These curves illustrate the relative stability of $\mathrm{Mn}-\mathrm{Ol}$ and $\mathrm{Fe}-\mathrm{Ol}$, by their intersections with $R \operatorname{Tn}\left(f \mathrm{O}_{2}\right)$ calculated for each chemical system. (2) A $\mathrm{Mn}-$, Ni- olivine solid solution model [11] was integrated into the code for vapor-solid-liquid equilibria in cooling gases $[8,12]$, to predict $\mathrm{MnO} / \mathrm{FeO}$ in silicates in particular chemical systems.

Results: In vapor of solar composition LIME olivines are stable at $\mathrm{T}$ where olivine is in equilibrium with vapor, and some $\mathrm{Si}$ remains in the vapor. In systems enriched $>50 \times$ in $\mathrm{CI}$ dust $[8,9]$, $\mathrm{Fe}-\mathrm{Ol}$ becomes stable at $\mathrm{T}$ higher than $\mathrm{Mn}-\mathrm{Ol}$.

Discussion: In chemical systems where $\mathrm{Mn}-\mathrm{Ol}$ becomes stable at higher $\mathrm{T}$ than $\mathrm{Fe}-\mathrm{Ol}$, significant forsteritic olivine $\left(\mathrm{Mg}_{2} \mathrm{SiO}_{4}\right)$ has condensed at even higher $\mathrm{T}$. This is consistent with textures in AOA olivine with Mn-rich rims [4]. LIME olivines are sensitive indicators of oxygen fugacity. In chemical systems investigated here, they are only stable in reduced vapors (solar composition). Silicate melts are not stable in such systems except at $\mathrm{P}^{\text {tot }}>10^{-2}$ bar [9, plate 7], consistent with most (but not all) LIME olivine occurring in melt-free primitive materials.

Acknowledgments: Work was supported by NASA-COSNNX10AI42G (DSE), NASA-COS-NNG06GG44G (MKW).

References: [1] Rubin A.E. 1984. American Mineralogist 69:880-888. [2] Klöck et al. 1989. Nature 339:126-128. [3] Weisberg et al. 1995. Proceedings of Symposium Antarctic Meteorites 8:11-32. [4] Weisberg M. K. et al. 2004. Meteoritics \& Planetary Science 39:1641-1653. [5] Sugiura N. et al. Meteoritics \& Planetary Science 44:559-572. [6] Zolensky et al. 2006. Science 314:1735-1739. [7] Nakamura et al. 2008. Science 321:1664-1667. [8] Ebel D. S. and Grossman L. 2000. Geochimica et Cosmochimica Acta 64:339-366. [9] Ebel 2006. In Meteorites \& the early solar sytem II, 253-277. [10] Darken L. S. and Gurry R. W. 1953. Physical Chemistry of Metals. McGraw Hill. [11] Hirschmann M. M. and Ghiorso M. S. 1994. Geochimica et Cosmochimica Acta 58:4109-4126. [12] Ghiorso M. S. and Sack R. O. 1995. Contributions to Mineralogy and Petrology 119:197-212. 
5290

INTER-ELEMENT FRACTIONATION PROCESSES BETWEEN TARGET MELTS, PROJECTILE MATTER AND SHOCKED QUARTZ IN CRATERING EXPERIMENTS

M. Ebert ${ }^{1}$, L. Hecht ${ }^{1}$, A. Deutsch ${ }^{2}$, T. Kenkmann ${ }^{3}$. ${ }^{1}$ Museum für Naturkunde, Invalidenstrasse 43, D-10115 Berlin, Germany, E-mail: Matthias.Ebert@mfn-berlin.de. ${ }^{2}$ Univ. Münster, Wilhelm-Klemm-Str. 10; D-48149 Münster. ${ }^{3}$ Univ. Freiburg, Albertstr. 23b, D-79104 Freiburg, Germany.

Introduction: The detection of meteoritic components in impactderived rocks is of great diagnostic value for confirming an impact origin [1]. The processes of mixing projectile matter into target and impactite materials are far from being understood. We present results of hypervelocity cratering experiments using a natural sandstone target and iron meteorite matter as projectile.

Experiments have been performed at the two-stage acceleration facilities of the Fraunhofer Ernst-Mach-Institute (Freiburg, GER). Our results are based on experiment \#3298 [2] using a Campo del Cielo meteorite sphere projectile $(\varnothing 10 \mathrm{~mm})$ accelerated to $\sim 4.5 \mathrm{~km} \mathrm{~s}^{-1}$ ( $\mathrm{E}_{\text {kin }}$ $\sim 43 \mathrm{~kJ})$ and as target a $50 \times 50 \times 50 \mathrm{~cm}$ block of Seeberger Sandstone. Ejecta material was captured with a catcher system [3] and analyzed by an electron microprobe.

Results: The ejecta fragments show metamorphic shock features (PDFs in Qtz, diaplectic glass, lechatelierite) and partial melting of the clay-bearing sandstone matrix, which involves Qtz too. Droplets of projectile have only entered the sandstone melt.

Projectile residues occur as spheres, spheroids or partly molten fragments in the ejecta. The residues are enriched in $\mathrm{Ni}$ and $\mathrm{Co}$ and depleted in Fe compared to the Campo del Cielo meteorite. The spheres vary strongly in composition $(\mathrm{Ni}: 5.6-17.3 \mathrm{wt} \%, \mathrm{Co}: 0.4-1.3 \mathrm{wt} \%$, Fe: 77.7-94.0 wt\%). Enrichment of $\mathrm{Ni}$ and Co versus Fe correlates negatively with the sphere-size.

Sandstone melt consists of $\mathrm{SiO}_{2}$ (51-94 wt\%), (1.9-17 wt\%), $\mathrm{FeO}$ $(4.5-32 \mathrm{wt} \%)$, and $\mathrm{NiO}(0.01-0.9 \mathrm{wt} \%)$. Components of this mixture are Qtz, clay-bearing sandstone matrix (with $\mathrm{FeO}=3.2 \mathrm{wt} \%$ ) and up to $\sim 20 \%$ projectile matter. The $\mathrm{Fe} / \mathrm{Ni}$-ratio of the sandstone melt is generally below the projectile ratio.

Shocked quartz with PDFs, diaplectic glass and lechatelierite contain slightly $\mathrm{FeO}(<1.7 \mathrm{wt} \%)$ and $\mathrm{NiO}(<0.08 \mathrm{wt} \%)$. The average $\mathrm{FeO}$ content of the high-shocked Qtz is $0.58 \mathrm{wt} \%$.

Discussion: Our analyses suggest inter-element fractionation between projectile and target in different impact stages. (A) After shock compression with formation of PDFs in Qtz, diaplectic glass or lechatelierite, and during early unloading, $<1 \%$ of projectile matter is added to the glass phases without detectable fractionation. (B) Later, when waste heat triggers melting of the sandstone, molten projectile is mixed with the sandstone melt and significant element fractionation occurs. $\mathrm{Fe}$ is selectively enriched in the silicate melt; $\mathrm{Ni}$ and $\mathrm{Co}$ are enriched over $\mathrm{Fe}$ in coexisting projectile spherules. Comparable processes have been reported in natural impactites [4-6]. The increase of fractionation with decreasing spheres-size shows that the fractionation of $\mathrm{Fe}, \mathrm{Ni}$, and $\mathrm{Co}$ occurs during solution of the metal spheres in the silicate melt due to differences in chemical reactivity [6].

References: [1] Koeberl C. 1998. Geological Society Special Publication 140. pp. 133-153. [2] Poelchau M. et al. 2011. Abstract \#1824. 42nd Lunar and Planetary Science Conference. [4] Domke I. et al. Abstract \#1605. 41st Lunar and Planetary Science Conference. [3] Reiser F. et al. 2011. Abstract \#1733. 42nd Lunar and Planetary Science Conference. [4] Mittlefehldt D. W. et al. 1992. Meteoritics 27:316-370. [5] Gibbons R. V. et al. 1976. Proceedings, 7th Lunar Science Conference. pp. 863-880. [6] Kelly W. R. et al. 1974. Geochimica et Cosmochimica Acta 38:533-543.
5079

ALMAHATA SITTA MS-17 EL-3 CHONDRITE FRAGMENT: CONTRASTING OLDHAMITE ASSEMBLAGES IN CHONDRULES AND MATRIX AND SIGNIFICANT OLDHAMITE REE-PATTERNS A. El Goresy ${ }^{1}$, M. Boyet ${ }^{2}$ and M. Miyahara ${ }^{3}{ }^{1}$ Bayerisches Geoinstitut, Universität Bayreuth, 95447 Bayreuth, Germany, E-mail: Ahmed. Elgoresy@uni-bayreuth.de. ${ }^{2}$ Université Blaise Pascal, Lab. Magmas et Volcans, UMR CNRS 6524, BP 10448, F-63000 Clermont-Ferrand, France. ${ }^{3}$ Department of Earth and Planetary Materials Science, Graduate School of Science, Tohoku University, Sendai 980-8578, Japan.

Introduction: Almahata Sitta $\mathrm{CT}_{3} 2008$ is considered a secondgeneration polymict asteroid dominated by ureilites [1]. The impact also delivered OCs, EH, and EL chondrites. MS-17 preliminarily classified as shocked EL-3/4 chondrite [1] is full of chondrules and FeNi clasts. We report detailed petrographic studies, mineral chemistries, and REE abundances of oldhamite $(\mathrm{CaS})$ in chondrules and matrix in two large polished thin sections from its unweathered interior.

Results and Discussion: MS-17 is a chondrule-rich EL-3 conglomerate with a matrix dominated by fluffy accreted lithologies. The matrix is full of individual oblong FeNi metal objects containing prismatic crystals of enstatite and rarely diopside both grown from the metal outer surfaces into their interiors. FeNi metal objects have fluffy accretionary rims of idiomorphic troilite $(\mathrm{FeS})$ with daubreelite exsolution lamellae, alabandite, and sphalerite crystallites. Chondrules are exceptionally enriched in large amoeboid stoichiometric $\mathrm{CaS}$ grains with minor $\mathrm{Mg}, \mathrm{Mn}$, and Fe-contents ( $1.34 \mathrm{~mol} \% \mathrm{MgS}, 1.55 \mathrm{~mol} \% \mathrm{MnS}$, and $0.89 \mathrm{~mol} \% \mathrm{FeS}$ ). CaS engulfs stoichiometric enstatite (En) crystals barren of $\mathrm{MnO}$, poor in $\mathrm{CaO}$ $(0.63 \mathrm{wt} \%)$, and $\mathrm{FeO}(0.76 \mathrm{wt} \%)$. In contrast to $\mathrm{CaS}$ in chondrules, every $\mathrm{CaS}$ in matrix contains $(\mathrm{FeNi})_{3} \mathrm{P}$ inclusions hinting to different $f \mathrm{O}_{2}$ conditions. We found no metal-troilite eutectic textures or shock-induced phase transitions of En to majorite or akimotointe. We reclassify MS-17 as a pristine EL-3 chondrite. En in FeNi clasts is barren of MnO but hosts higher $\mathrm{FeO}(1.19 \mathrm{wt} \%)$ than in chondrule En $(<0.8 \mathrm{wt} \%)$. Metal clasts enclose in their interiors four different graphite types, no shock twinning or inversion to diamond thus refuting origin of the metal clasts by shock melting. LA-ICPMS studies of 11 oldhamites in different settings reveal CI-normalized REE patterns with prominent negative $\mathrm{Eu}$ anomalies without $\mathrm{Yb}$ depletions. MS-17 is an unshocked EL-3 conglomerate of CaS-rich chondrules and FeNi- enstatite-graphite clasts decorated with FeS fluffy rims prior to accretion. Enstatite compositions point to separate nebular sources of chondrules and metal-bearing clasts.

Conclusions: MS-17 contains two different CaS populations: Grains with $(\mathrm{Fe}, \mathrm{Ni})_{3} \mathrm{P}$ inclusions in the matrix, and clear amoeboid grains in chondrules. CaS in MS-17 EL-3 depicts REE patterns (negative Eu anomaly) different from the mainstream pattern in EH-3 (positive Eu and $\mathrm{Yb}$ anomalies [2]). Presence of $(\mathrm{Fe}, \mathrm{Ni})_{3} \mathrm{P}$ in $\mathrm{CaS}$ in matrix is strongly suggestive of condensation of schreibersite at $1439 \mathrm{~K}$ and $\mathrm{C} / \mathrm{O}$ ratio $>0.83$ before condensation of CaS [3]. The two different settings of CaS in chondrules and matrix strongly suggest two different $f \mathrm{O}_{2}$ at their condensations.

References: [1] Bischoff A. et al. 2010. Meteoritics \& Planetary Science 45:10-11:1638-1656. [2] Gannoun et al. 2011. Geochimica et Cosmochimica Acta 75:3269-3289. [3] Petaev M. I. and Wood J. A. 2000. Abstract \#1608. Meteoritics \& Planetary Science 31. 


\section{2}

LOW-T MAGNETIC PROPERTIES OF 5 CM2 CHONDRITES: COMPARISON WITH THE SIGNATURE OF CRONSTEDTITE AND WITH PRELIMINARY RESULTS FROM THE PARIS CHONDRITE A. Elmaleh ${ }^{1}$, S. C. Tarantino ${ }^{2}$, M. Zema ${ }^{2}$, B. Zanda ${ }^{3}$, B. Devouard ${ }^{4}$ and M. Fialin ${ }^{5}$. ${ }^{1}$ Inst. de Minéralogie et de Physique des Milieux CondensésUPMC-CNRS, Paris, France. E-mail: agnes.elmaleh@impmc.upmc.fr. ${ }^{2}$ Dip. di Scienze della Terra e dell'Ambiente, U. Pavia, Italy. ${ }^{3}$ Museum National d'Histoire Naturelle-CNRS, Paris, France. ${ }^{4}$ Laboratoire Magmas et Volcans, Clermont-Ferrand, France. ${ }^{5}$ CAMPARIS, Paris, France.

Here we report on the low-T (2-300 K) magnetic properties of samples of the Fe-rich serpentine cronstedtite (characterized by EMPA, SCXRD), and of a series of CM2 chondrites in which the mineralogy of phyllosilicates (mostly serpentines) has been extensively studied and related to the degree of alteration, as summarized by [1]. Our conclusions $[2,3]$ are used to interpret the low-T magnetic signal of Paris, newly discovered and considered as a possibly very pristine CM2 [4].

Using single crystals, it is possible to define the low- $\mathrm{T}$ magnetic signature of cronstedtite, and to suggest that the $\mathrm{Mg}$ content modifies this signature by lowering the $T$ of the susceptibility peak $\left(T_{p}, \approx 7 \mathrm{~K}\right)$. A few results on powder samples also suggest that $T_{p}$ increases, relative to single crystals,in an assembly of interacting fine grain particles. Turning to CM2, the low-T properties of Murray are explained by a mixture of small amounts of stoichiometric magnetite, and of an Fe-rich serpentine close to reference cronstedtite. We observe an evolution of $\mathrm{T}_{\mathrm{p}}$ among the CM2 (including Cold Bokkeveld, Migheis, Murchison, and Nogoya) that correlates with the Mineralogical Alteration Index [5] and the $\mathrm{FeO} / \mathrm{SiO}_{2}$ content of PCPs [1]. Deviations from the signature of reference cronstedtite are observed, in particular in Cold Bokkeveld (lowest $T_{p}$ ). In this meteorite, [6] measured a high (up to $8.8 \mathrm{at} \%$ ) Mg content in Fe-rich serpentines by TEM-EDS. Although the effects of other parameters (Al, Fe valence, tochilinite layers, grain size) need to be more completely assessed, our results suggest the influence of the $\mathrm{Mg}$ content, and would pinpoint differences in the composition of $\mathrm{Fe}$-serpentines with the degree of alteration, rather than only mixing between minerals of fixed compositions. Our approach can also be used for evaluating changes in mineralogy, possibly linked to alteration, in $\mathrm{mm}$-size clasts from a meteorite. Direct application is proposed for the Paris breccia, in which we selected fragments from zones showing different degrees of alteration according to the criteria of [4]. First, all samples show low-T susceptibility peaks in a range compatible with that of reference cronstedtite. Second, the less altered and the more altered zones have contrasting low-T magnetic signatures, with a shift in the position of the peak, from high $\mathrm{T}_{\mathrm{p}}$, to a lower one, comparable to what is found in Murray. This would be consistent with the interpretation of Paris as a CM2 breccia partially affected by a low degree of alteration and preserving in some regions a more pristine material, presumably characterized by finer grain and/or Mg-poorer Fe-serpentine.

References: [1] Rubin A. E. et al. 2007. Geochimica et Cosmochimica Acta 71:2361-2382. [2] Elmaleh A. et al. 2010. Abstract \#GP43B-1052. Fall meeting 2010 AGU. [3] Elmaleh A. et al. Meteoritics \& Planetary Science (submitted). [4] Zanda et al. 2011. Abstract \#2040. 27th Lunar and Planetary Conference. [5] Browning L. et al. 1996. Geochimica et Cosmochimica Acta 60:2621-2633. [6] Zega T. and Buseck P. 2003. Geochimica et Cosmochimica Acta 6 7:1711-1721.

\section{6}

MARTIAN SOIL ANALOGS FROM ANTARCTIC DRY VALLEYS: ELEMENTAL ABUNDANCES AND MINERALOGY SIGNAL WEATHERING PROCESSES

P. Englert ${ }^{1}$, J. L. Bishop ${ }^{2,3}$, L. D. Hunkins ${ }^{2,3,4}$ and C. Koeberl ${ }^{5,6}$. ${ }^{1}$ University of Hawaii, Mānoa, HI, USA. penglert@ hawaii.edu. ${ }^{2}$ SETI Institute, Mountain View, CA, USA. ${ }^{3}$ NASA Ames Research Center, Moffet Field, CA, USA. ${ }^{4}$ University of South Florida, FL, USA. ${ }^{5}$ Natural History Museum, Vienna, Austria. ${ }^{6}$ Department of Lithospheric Research, University of Vienna, Vienna, Austria.

Introduction: The only Martian samples returned to date are meteorites. They provide important clues about the mineralogy of Mars; however they are not consistent with most of the planet's composition. Studies of soil and sediment alteration in cold and dry environments on Earth can contribute to understanding Martian surface properties. Antarctic Dry Valley soils exhibit complex chemical weathering in a low temperature and dry environment [1,2]. We present here analyses of elemental abundances and mineralogy of sediments from this area in order to contribute towards our understanding of Mars. Our study focuses on elemental abundances and visible/near-infrared (VNIR) reflectance spectroscopy for comparison with data collected by orbiters and rovers on Mars [e.g., 3-6]. Coordinated evaluation of chemistry and mineralogy of meteorites and Antarctic Dry Valley soils will provide insights into the weathering processes that occurred on Mars.

Samples: We characterized 24 samples, collected directly adjacent to Lakes Fryxell, Vanda, and Brownsworth by geochemical analysis and VNIR spectroscopy. This analysis complements results obtained previously for Lake Hoare sediment cores $[2,7]$.

Chemistry: The soils from each of the lakes show distinct major and minor elemental abundance patterns and $\mathrm{C}$ concentrations indicating that each lake environment may represent moderately different soil formation and weathering conditions. The majority of the samples have a chemical index of alteration of 55, indicating predominantly physical weathering.

$\mathrm{K} / \mathrm{Th}$ ratios for the majority of large Martian domains range from about 4000 to $7000 \mathrm{~K} \mathrm{Th}^{-1}$ ratios in the sample suite range from 522 to 5077, and are below 3000 for all but three samples, with Lake Vanda soils clearly distinguished by ratios systematically below 2000. The low spread of $\mathrm{K} / \mathrm{Th}$ ratios for both Mars and the soil samples for this study indicate little loss of $\mathrm{K}$ through aqueous processes. More detailed analyses may provide model insight into the observation that Martian $\mathrm{K} / \mathrm{Th}$ ratios do not seem to support aqueous alteration contrary to evidence of significant water/rock interaction [8].

Spectra: VNIR spectral analyses indicate orthopyroxene/ clinopyroxene ratios of approximately 40/60 for most samples with higher pyroxene abundances near Vanda and Brownsworth. Weak clay bands are observed for most samples with differences observed among the three sites. The Fryxell samples exhibit $\mathrm{CH}$ and carbonate bands, consistent with their presence in the chemical analyses. Mid-IR spectra show the presence of quartz and feldspar.

References: [1] Gibson E. K. et al. 1983. Journal of Geophysical Research 88:A912-A928. [2] Bishop J. L. et al. 1996. Geochimica et Cosmochimica Acta 60:765-785. [3] Gellert R. et al. 2004. Science 305:829-832. [4] Boynton W. V. et al. 2007. Journal of Geophysical Research 112, doi:10.1029/2007 JE002887. [5] Bibring J.-P. et al. 2005. Science 307:1576-1581. [6] Murchie S. L. et al. 2009. Journal of Geophysical Research 114, doi:10.1029/2009 JE003342. [7] Bishop J. L. et al. 2001. Geochimica et Cosmochimica Acta 65:2875-2897. [8] Taylor G. J. et al. 2006. Journal of Geophysical Research 111, doi:10.1029/ 2006JE002676. 
5301

REDUCTION VERSUS SULFIDATION IN ORDINARY AND ENSTATITE CHONDRITES FROM SPECIATION OF IRON AMONG SILICATES, METALS AND SULFIDES

T. J. Fagan and C. Niki. Department of Earth Sciences, Waseda University, 1-6-1 Nishiwaseda, Shinjuku, Tokyo 169-8050, Japan, E-mail: fagan@waseda.jp.

Introduction: Variations in oxidized vs. metallic iron are used to distinguish LL, L, and $\mathrm{H}$ ordinary chondrites and the enstatite chondrites $[1,2]$. These variations reflect spatial and temporal differences in oxygen fugacity in the solar nebula. Oxidized iron in these meteorites is concentrated in silicates, and metallic iron occurs in Fe, Ni-metals; however, iron also occurs in troilite (and other sulfides in the enstatite chondrites). Sulfur fugacity may have varied independently. This study uses $\mathrm{Fe}$-speciation to address sulfidation in addition to oxidation and reduction during formation of ordinary and enstatite chondrites.

Analytical Methods: In this project, we use a mass balance model developed previously to understand reactions that transfer iron among silicates, metals and sulfides [3, 4]. We use two data sets to constrain the speciation of iron in ordinary and enstatite chondrites: (1) mineral modes and compositions determined from thin sections of six meteorites [St. Marks (EH5), Lewis Cliff 88180 (EH5), Northwest Africa 974 (E6), Bensour (LL6), Mt. Tazerzait (L5) and Tamdakht (H5); and (2) a set of wet chemical analyses of $\mathrm{H}(n=31), \mathrm{L}(n=57), \mathrm{LL}(n=17)$ and enstatite $(n=4)$ chondrites compiled by E. Jarosewich [5, 6].

For data set (1), mineral compositions were analyzed using the JEOL JXA-8900 electron microprobe (EPMA) at Waseda University. Elemental maps were also collected by EPMA. The maps were opened in graphics files and grids were digitally overlain on the maps. The mineral present at each grid node was identified manually by inspection of the elemental maps. The modes and compositions were combined with molar volume data to calculate the distribution of $\mathrm{Fe}$ among major minerals.

E. Jarosewich and others determined whole rock analyses in data set (2). They used aliquots of powdered samples to determine the abundances of $\mathrm{FeO}, \mathrm{FeS}$ and $\mathrm{Fe}^{0}$ from their analyses.

Results: Iron speciation for each group of samples can be summarized from two numbers: the percentage of $\mathrm{Fe}$ as metal and the percentage as sulfide The percentage of $\mathrm{Fe}$ as silicate is not independent. Most LL samples from data set (2) fall in a range from $0-20 \% \mathrm{Fe}$ as metal and $10-25 \%$ as sulfide. The $\mathrm{L}$ samples have $20-40 \% \mathrm{Fe}$ as metal and $10-25 \%$ as sulfide. The $\mathrm{H}$ samples have $50-70 \% \mathrm{Fe}$ as metal, 10 $15 \%$ as sulfide. Results from ordinary chondrites in data set (1) fall within these ranges. Enstatite chondrites from data sets (1) and (2) have nearly no $\mathrm{Fe}$ as $\mathrm{FeO}$, and from 60 to $80 \% \mathrm{Fe}$ as metal.

The LL, L, and $\mathrm{H}$ chondrites are well-separated from each other and may show a slight decrease in sulfidation from LL and $\mathrm{L}$ to $\mathrm{H}$ groups. The enstatite chondrites appear to be more sulfidized than the $\mathrm{H}$ chondrites.

References: [1] Van Schmus W. R. and Wood J. A. 1967. Geochimica et Cosmochimica Acta 31:747-765. [2] Weisberg M. K. et al. 2006. In Meteorites and the Early solar system II, edited by Lauretta D. S. and McSween H. S., pp. 19-52. [3] Fagan T. J. et al. 2008. Abstract \#1604. 39th Lunar and Planetary Science Conference. [4] Fagan T. J. and Wakai H. 2010. Abstract \#5229. 73rd Annual Meteoritical Society Meeting. [5] Jarosewich E. 1990. Meteoritics \& Planetary Science 25:323337. [6] Jarosewich E. 2006. Meteoritics \& Planetary Science 41:13811382 .
5340

SHOCK METAMORPHIC EFFECTS IN ROCKS AND MINERALS FROM THE NEWLY CONFIRMED LUIZI IMPACT STRUCTURE (DEMOCRATIC REPUBLIC OF CONGO)

L. Ferrière ${ }^{1}$, F. R. T. Lubala $^{2}$, G. R. Osinski ${ }^{3}$ and P. K. Kaseti ${ }^{2} . \quad{ }^{1}$ Natural History Museum, Burgring 7, A-1010 Vienna, Austria, E-mail: ludovic.ferriere@nhm-wien.ac.at. ${ }^{2}$ Department of Geology, University of Lubumbashi, P.O. Box 1825, Lubumbashi, Democratic Republic of Congo. ${ }^{3}$ Departments of Earth Sciences/Physics \& Astronomy, University of Western Ontario, 1151 Richmond Street, London, Ontario N6A 5B7, Canada.

Introduction: The Luizi structure, $\sim 17 \mathrm{~km}$ in diameter, centered at $10^{\circ} 10^{\prime} 13.5^{\prime \prime} \mathrm{S}$ and $28^{\circ} 00^{\prime} 27.0^{\prime \prime} \mathrm{E}$, on the Kundelungu Plateau of the Katanga province, lies in an underexplored region of the southeastern Democratic Republic of Congo. Luizi exhibits, from the periphery to the center of the structure, a rim elevated up to $\sim 300-350 \mathrm{~m}$ above the crater interior, an annular depression, an intermediate ring with a diameter of $\sim 5.2 \mathrm{~km}$, and an $\sim 2-\mathrm{km}$-wide circular central ring around a central depression [1]. In 1990, Dumont [2] was the first to suggest the structure was of impact origin. Evidence for an impact origin was found recently by Ferrière et al. [1,3].

Here, we report on geological field observations and petrographic examination of rock samples collected during our 2010 field campaign at the Luizi structure.

Results and Discussion: The structure formed in tabular massive arkosic sandstone beds, of centimeter to decimeter thickness, with intercalated laminated argillaceous sandstones. Up to $40 \mathrm{~cm}$ in size, welldeveloped shatter cones formed in arkosic sandstone were observed. Shatter cones mostly occur in situ and a few were also found in float samples along rivers. They appear to be restricted only to the inner $3.2 \mathrm{~km}$ of the structure.

Monomict lithic impact breccia dikes, up to $\sim 2 \mathrm{~m}$ in thickness, and crosscutting sandstone beds, were mapped up to $\sim 3 \mathrm{~km}$ from the center of the structure.

Quartz grains with up to five sets of planar deformation features (PDFs) were identified under the universal stage microscope. The dominance of PDF orientations parallel to $\omega\{\}$ and the occurrence of a few PDFs parallel to $\pi\{\}$ allow us to estimate that the more heavily shocked exposed target rocks have experienced peak shock pressure slightly above $20 \mathrm{GPa}$. In addition, rare shock deformations in feldspar, notably in perthitic alkali feldspar grains, in which PDFs oblique to the perthite exsolution lamellae were observed. Kink banding in muscovite grains is also common in most investigated samples.

Conclusion: The Luizi structure displays characteristic of complex impact craters, i.e., an elevated structural rim, an annular depression, and a central uplifted zone. For the first time, unique evidence of shock metamorphism, in the form of shatter cones and multiple sets of PDFs in quartz and feldspar grains, is described, confirming the meteorite impact origin of the structure, the first one to be confirmed in Central Africa.

Acknowledgments: Field campaign was supported by the National Geographic Society/Waitt Grants Program (grant 69-09).

References: [1] Ferrière L. et al. 2011. Geology, doi: 10.1130/ G31990.1. [2] Dumont P. 1990. Bulletin de la Société Belge de Géologie 99:57-65. [3] Ferrière L. et al. 2011. Abstract \#1637. 42nd Lunar and Planetary Science Conference. 


\section{2}

\section{AUGER ANALYSIS OF IMPACT CRATERS FROM THE STARDUST INTERSTELLAR FOILS}

C. Floss, C. Allen, A. Ansari, S. Bajt, N. Bassim, H. A. Bechtel, J. Borg, F. Brenker, J. Bridges, D. E. Brownlee, M. Burchell, M. Burghammer, A. L. Butterworth, P. Cloetens, A. M. Davis, G. J. Flynn, D. Frank, Z. Gainsforth, E. Grün, P. R. Heck, J. K. Hillier, P. Hoppe, L. Howard, G. R. Huss, J. Huth, B. Hvide, A. Kearsley, A. J. King, P. Kotula, B. Lai, J. Leitner, L. Lemelle, H. Leroux, L. R. Nittler, R. C. Ogliore, W. J. Ong, F. Postberg, M. C. Price, S. A. Sandford, J. A. Sans Tresseras, S. Schmitz, T. Schoonjans, K. Schreiber, G. Silversmit, A. Simionovici, R. Srama, T. Stephan, J. Stodolna, R. M. Stroud, S. R. Sutton, R. Toucoulou, M. Trieloff, P. Tsou, A. Tsuchiyama, T. Tyliczszak, B. Vekemans, L. Vincze, A. J. Westphal, M. E. Zolensky and $>29,000$ Stardust@home dusters. ISPE author affiliations are at http:// www.ssl.berkeley.edu/ westphal/ISPE/.

Introduction: The interstellar tray from NASA's Stardust mission collected particles for 229 days during two exposures prior to the spacecraft encounter with comet $81 \mathrm{P} /$ Wild 2 , and tracked the interstellar dust stream for all but 34 days of that time. Aluminum foils from this collector are being scanned using high resolution SE imaging and the first impact craters located on the foils have recently been identified [1, 2]. Because the number of impact craters found on the foils appears to be significantly higher than estimates made based on interstellar flux calculations [e.g., 3] (extrapolation of the crater densities on the foils examined to date suggests that as many as $\sim 1000$ craters may be present on the total exposed interstellar foil area [1]), the Science Council for the Interstellar Preliminary Examination Team (ISPE) recently approved Auger Nanoprobe, SEM-EDX, and FIB-TEM analysis on several of these craters. Here we report the results of our Auger Nanoprobe analysis on three impact craters from the interstellar foils.

Experimental: The Auger measurements were done with the PHI 700 Auger Nanoprobe at Washington University, using a $10 \mathrm{kV} 10 \mathrm{nA}$ beam rastered over the crater interiors. Spectrum acquisition consisted of three cycles over an energy range of $30-2130 \mathrm{eV}$ for a total acquisition time of 2 min. Based on measurements of a $\sim 200 \mathrm{~nm}$ crater from a cometary side foil, these parameters were deemed sufficient for determining residue compositions on the crater bottoms if such residue is present.

Results and Discussion: Two impact craters from foil I1061N (036@33 and 188@24, diameters of $350 \mathrm{~nm}$ and $650 \mathrm{~nm}$, respectively) and one crater from foil $1033 \mathrm{~N}\left(11 \_175\right.$, diameter of $\left.1.1 \mu \mathrm{m}\right)$ were analyzed with the Auger Nanoprobe. In addition to $\mathrm{C}, \mathrm{O}$, and $\mathrm{Al}$, all craters show the presence of Si. Crater 36@33 also shows evidence for $\mathrm{Mg}$ and $\mathrm{Fe}$, while crater 11175 also appears to contain a minor amount of Na. Spectra taken on areas of foil adjacent to the craters do not show evidence for these elements, although $\mathrm{Fe}$ is a common inclusion in Stardust foil. The elements observed in these crater residues are in broad agreement with results of SEM-EDX spectra from three other interstellar impact craters [4] and may suggest the presence of residue of extraterrestrial origin. Although $\mathrm{Na}$ is present in the residue of one crater, the absence of $\mathrm{Ce}$ and $\mathrm{Zn}$ suggests that these are not solar cell glass impacts; FIB-TEM analyses are planned to verify this.

References: [1] Stroud R. M. et al. 2011. Abstract \#1753. 42nd Lunar and Planetary Science Conference. [2] Floss C. et al. 2011. Abstract \#1576. 42nd Lunar and Planetary Science Conference. [3] Landgraf M. et al. 1999. Planetary Space Science 47:1029-1050. [4] Stroud R. M. et al. 2011. This conference.

\section{0}

FE-XANES MEASUREMENTS ON ANHYDROUS CLUSTER IDPS G. J. Flynn ${ }^{1}$, A. Lanzirotti ${ }^{2}$, S. Wirick ${ }^{2}$ and S. R. Sutton ${ }^{2}{ }^{1}{ }^{1}$ Department of Physics, SUNY-Plattsburgh, Plattsburgh NY 12901, USA. E-mail: george.flynn@plattsburgh.edu. ${ }^{2}$ CARS, University of Chicago, Chicago, IL 60637, USA.

Introduction: Chondritic porous interplanetary dust particles (CP IDPs), extremely primitive early solar system material [1], are, most likely, not a complete, representative sample of their parent body. A typical $\sim 10 \mu \mathrm{m} \quad \mathrm{CP}$ IDP is an aggregate of $>10^{4}$ submicron grains. But stratospheric collections include many larger non-chondritic, monomineralic grains, mostly olivine, pyroxene and sulfide, that have finegrained, chondritic material (i.e., small bits of CP IDPs) adhering to their surfaces. This indicates at least some of the non-chondritic grains on the stratospheric collectors are fragments from the same parent as the CP IDPs. Cluster IDPs, typically $\sim 100$ times the mass of $10 \mu \mathrm{m}$ CP IDPs containing some large mono-mineralic grains, sample the parent body at a much larger scale than $10 \mu \mathrm{m}$ CP IDPs. Fe is a major element $(\sim 18.5 \%)$ in chondritic meteorites, and the Fe-oxidation state distinguishes various meteorite groups [2].

Measurements: We have begun a project to systematically determine the $\mathrm{Fe}$ oxidation states of anhydrous cluster IDPs by $\mathrm{Fe} \mathrm{X}$-ray Absorption Near-Edge Structure (XANES) spectroscopy. We previously reported that cluster IDP L2009R2 is dominated by $\mathrm{Fe}$ with a valance near $\mathrm{Fe}^{2+}$ [3] In addition, the absence of large amounts of Fe-metal in L2009R2 suggests that even at the size scale of cluster IDPs the anhydrous IDP parent body is different from the Wild 2 particles analyzed by Ogliore et al. [4], which exhibited significant Fe-metal $(24 \%-14 \%+6 \%)$.

Results: We have now performed Fe-XANES on $>75 \%$ of the area of the anhydrous cluster IDP L2009R1, a Zn-depleted cluster ( $\mathrm{Zn} /$ $\mathrm{Fe}=0.20 \mathrm{x} \mathrm{CI}$ ) that likely experienced $\mathrm{Zn}$-loss due to significant atmospheric entry heating. The average spectrum of L2009R1 (shown in Figure 1) plots between $\mathrm{Fe}^{2+}$ and $\mathrm{Fe}^{-3+}$, but is significantly closer to $\mathrm{Fe}^{3+}$ than was L2009R2. Again, we see no evidence for significant Femetal in this anhydrous cluster IDP, indicating that both large cluster IDPs examined thus far differ significantly from the Wild 2 particles measured by Ogliore [4].

References: [1] Ishii H. et al. 2008. 319:447-450. [2] Urey H. C. and Craig, H. 1953. Geochimica et Cosmochimica Acta 4:36-82. [3] Flynn G. J. et al. 2011. Abstract \#2521. 42nd Lunar and Planetary Science Conference. [4] Ogliore R. et al. 2010. Earth and Planetary Science Letters 296:278-286.

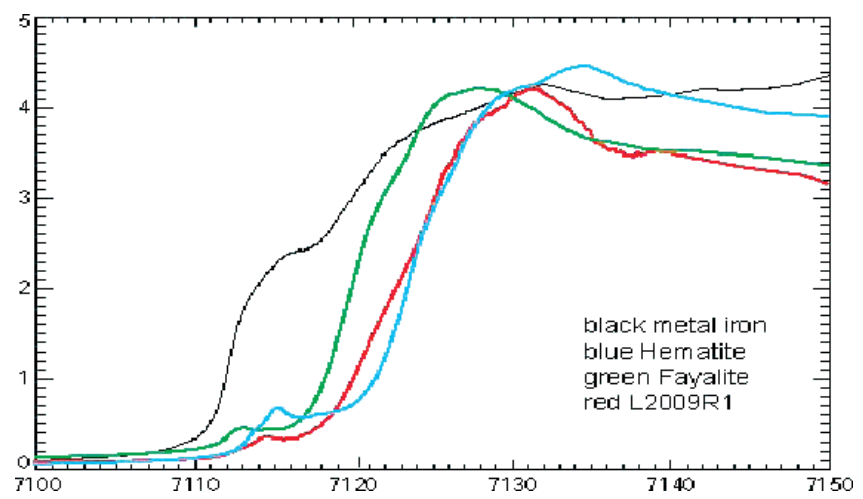

Fig. 1. Fe-XANES spectrum summed over the entire analyzed area of cluster IDP L2009R1 compared to the spectra of Fe-metal, fayalite $\left(\mathrm{Fe}^{2+}\right)$, and hematite $\left(\mathrm{Fe}^{3+}\right)$ standards. 


\section{7}

\section{THE KAMIL CRATER, EGYPT}

L. Folco ${ }^{1}$, M. Di Martino ${ }^{2}$, A. El Barkooky ${ }^{3}$, M. D’Orazio ${ }^{4}$, A. Lethy ${ }^{5}$, S. Urbini ${ }^{6}$, I. Nicolosi ${ }^{6}$, M. Hafez ${ }^{5}$, C. Cordier ${ }^{1}$, M. van Ginneken ${ }^{1}$, A. Zeoli ${ }^{1}$, A. M. Radwan ${ }^{5}$, S. El Khrepy ${ }^{5}$, M. El Gabry ${ }^{5}$, M. Gomaa ${ }^{5}$, A. A. Barakat ${ }^{7}$, R. Serra ${ }^{8}$ and M. El Sharkawi ${ }^{3}{ }^{1}$ MNA, Siena, Italy. E-mail: folco@unisi.it. ${ }^{2}$ INAF, Pino Torinese, Italy. ${ }^{3}$ Department of Geology, Cairo University, Giza, Egypt. ${ }^{4}$ DST, Pisa, Italy. ${ }^{5}$ NRIAG, Helwan, Egypt. ${ }^{6} \mathrm{INGV}$, Roma, Italy. ${ }^{7}$ EMRA, Cairo, Egypt. ${ }^{8} \mathrm{DF}$, Bologna, Italy.

We report the detection in southern Egypt of a rayed impact crater $45 \mathrm{~m}$ in diameter on a Cretaceous sandstone target. The ejecta rays highlight the exceptional freshness of the structure. The crater, identified by V. De Michele during a Google Earth survey in 2008, is named Kamil crater after the nearby peak Gebel Kamil. A geophysical expedition undertaken in February 2010 revealed that the crater is bowl shaped and has an upraised rim ( $\sim 3 \mathrm{~m}$ above pre-impact surface) typical of simple craters [1]. The true crater floor depth is $16 \mathrm{~m}$ and is overlain by a $\sim 6 \mathrm{~m}$ thick crater-fill material. Morphometric parameters agree with those predicted by models [2] for explosive impact craters generated by an iron meteorite $1.3 \mathrm{~m}$ in diameter (equivalent to $9.1 \times 10^{3} \mathrm{~kg}$ ) impacting at a velocity of $3.5 \mathrm{~km} \mathrm{~s}^{-1}$, assuming an average meteoroid entry velocity and entry angle of $18 \mathrm{~km} \mathrm{~s}^{-1}$ and $45^{\circ}$, respectively. Centimeter-scale masses of scoriaceous impact melt glass occur in and close to the crater, and indicate local shock pressures $>60 \mathrm{GPa}$ [1]. We identified over 5000 iron meteorite specimens totaling $\sim 1.71$ tons in the crater and surrounding area during systematic searches. They consist of $<34 \mathrm{~kg}$ shrapnel produced by the explosion of the impactor upon hypervelocity collision with the target, except one individual fragment of $83 \mathrm{~kg}$. This indicates that the Kamil crater was generated by an impactor that landed nearly intact without substantial fragmentation in the atmosphere. The meteorite, named Gebel Kamil, is classified as an ungrouped Ni-rich ataxite (Ni $\sim 20 \mathrm{wt} \%$, Ga $\sim 50 \mu \mathrm{g} \mathrm{g}^{-1}$, Ge $\sim 120 \mu \mathrm{g} \mathrm{g}{ }^{-1}$, Ir $\sim 0.5 \mu \mathrm{g} \mathrm{g}^{-1}$; data by ICP-MS analyses following [3]). Magnetic anomaly data show no evidence of buried meteorites larger than some tens of centimeters.

Based on systematic meteorite search, the estimated total mass of the impactor is of the order of $5-10 \times 10^{3} \mathrm{~kg}$, corresponding to a preatmospheric mass of $\sim 20-40 \times 10^{3} \mathrm{~kg}$ [4]. According to geophysical models [4], iron masses $<3 \times 10^{6} \mathrm{~kg}$ normally fragment upon impact with the Earth's atmosphere, thereby reducing the energy of the impact at the Earth's surface. The present statistics, which include the recently discovered Whitecourt crater [5] and the Kamil crater, suggest however that $\sim 35 \%$ of the iron meteorites in the above mass range are not disrupted in the atmosphere. Details of this work are published in [6, 7].

Acknowledgments: Supported by EC (Marie Curie Actions-RTNs ORIGINS FP6 project), Fondazione Cassa di Risparmio di Torino, Banca Monte dei Paschi di Siena S.p.A., Fondazione Monte dei Paschi di Siena, and Telespazio S.p.A.

References: [1] Melosh H. J. 1989. Impact cratering. A geologic process, Oxford Monographs on Geology and Geophysics. Oxford: Oxford University Press. 245 pp. [2] Collins G. S. 2005. Meteoritics \& Planetary Science 40:817-840. [3] D'Orazio M. and Folco L. 2003. Geostandards Newsletter 27:215-225. [4] Bland, P. A. and Artemieva N. A. 2006. Meteoritics \& Planetary Science 41:607-631. [5] Herd C. D. K. et al. 2008. Geology 36:955-958. [6] Folco L. et al. 2010. Science 329:804. [7] Folco L. et al. 2010. Geology 39:179-182.
5018 SHOCKED QUARTZ AT THE KAMIL CRATER (EGYPT)

L. Folco ${ }^{1}$ and M. D'Orazio ${ }^{2} .{ }^{1}$ MNA, Siena, Italy. E-mail: folco@ unisi.it. ${ }^{2}$ Dipartimento di Scienze della Terra, Pisa, Italy.

Impact melt glass occurs at the recently discovered Kamil crater in southwestern Egypt - a rayed crater, $45 \mathrm{~m}$ in diameter on a Cretaceous sedimentary (mainly quartz-arenites) target $[1,2]$. The glass forms centimeter-scale masses scattered up to a distance of $100 \mathrm{~m}$ from the crater rim, or is stuck to the surface of some of the thousand shrapnel specimens of the meteorite that formed the crater, the Gebel Kamil iron. It mainly consists of vesicular, brown to colorless siliceous glass and $\mathrm{Fe}-\mathrm{Ni}$ droplets entraining clasts of quartz-arenites, testifying to melting of the target and - to some extent-of the projectile during the impact, as a result of the local attainment of shock pressure $>60 \mathrm{GPa}$.

Shock deformation features in the quartz grains of the quartzarenite clasts embedded in an impact melt mass stuck to the surface of one Gebel Kamil shrapnel were studied by optical microscopy. They vary from clast to clast, ranging from undulose extinction associated with pervasive planar fractures (PF) spaced 15-20 $\mu \mathrm{m}$ apart, to blocky undulose extinction associated with undecorated planar deformation features (PDF), typically spaced 3-5 $\mu \mathrm{m}$ apart. Multiple sets of PDF may occur in individual crystals, sometimes with differently oriented sets occurring in different parts of the crystal.

The crystallographic orientation of 28 PDF planes in four quartz grains $<100 \mu \mathrm{m}$ in size was determined using a U-stage and indexed following [3]. Critical sets of PDF include those parallel to the rhombohedra $\{10 \overline{1} 3\}$ and $\{10 \overline{1} 2\}$, with less abundant PDF parallel to the trigonal dipyramid $\{11 \overline{2} 2\}$ (Fig. 1). This assemblage indicates shock pressures $>20 \mathrm{GPa}$, according to [3].

The occurrence of PDF in bedrock quartz crystals embedded in shock melt provide strong evidence that the Kamil crater was produced by a hypervelocity impact.

Acknowledgments: Supported by Università di Pisa (Fondi di Ateneo per la Ricerca), Banca Monte dei Paschi di Siena S.p.A., Fondazione Monte dei Paschi di Siena.

References: [1] Folco L. et al. 2010. Science 329:804. [2] Folco L. et al. 2010. Geology 39:179-182. [3] Stöffler and Langenhorst 1994. Meteoritics \& Planetary Science 29:155-181.

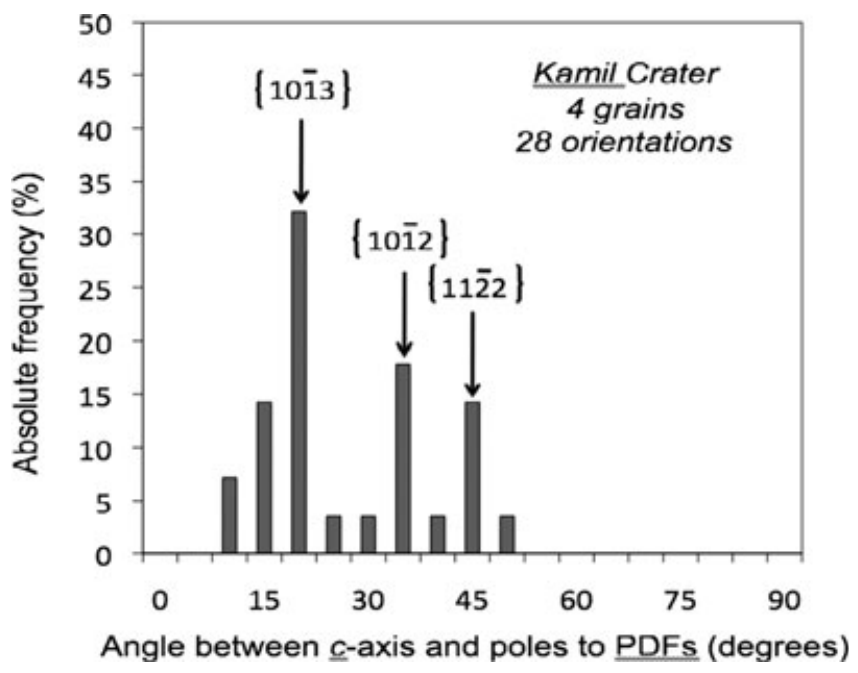

Fig. 1. Histogram (binned at $5^{\circ}$ ) showing PDF orientation in quartz crystals embedded in the Gebel Kamil impact melt. 
5177

THE BRITISH AND IRISH METEORITE SOCIETY (BIMS): PREPARING FOR A FALL

M. Ford and M. P. Smith. BIMS, 27 The Street, Rushington, West Sussex, BN16 3PA, UK. E-mail: britishmeteorite@gmail.com.

About Us: The British and Irish Meteorite Society is a group of over 100 UK and Irish meteorite researchers, curators and collectors. The Society was formed in 2004 by Mark Ford, Dave Harris, and a number of likeminded enthusiasts. With a clear focus on sample research, collection and curation, we are the only such group in the UK and one of only a handful of similar groups worldwide.

The aim of the society is to bring together all those involved with meteorites in the UK under one roof, with a view to educating the general public, furthering the interests of meteorite study, and preserving any new or old UK meteorite material for science. It is also an ideal place to share our interests and meet other people with a similar passion for space rocks.

Members are distributed over the whole of Britain; our main form of communication is via the Internet. Until recently we used an email based mailing list, we have now begun using a Web-based forum. New members are always welcome and may join by completing the form on our website.

Outreach: BIMS members are well informed and committed to promoting an interest in meteorites. We give talks and presentations, put on exhibitions and help with identification of suspected meteorites found by the public. Our members have been involved in the Brighton Science Festival and have given presentations to groups of children from nursery to secondary school age. In 2005 the Society was able to donate a substantial meteorite collection to the South Downs Planetarium. In the past 2 years our members have also had a number of articles published in Meteorite. Members regularly meet up and have organized visits to the Natural History Museum, Manchester Museum, and Open University. Our website is frequently updated and contains numerous useful resources. We cover the basics of identifying meteorites and classification; have sections on British and Irish meteorites, in addition to a growing number of articles written by our members.

New Falls: After the Almahata Sitta fall, 45 students and staff volunteered from the University of Khartoum to begin the search for material [1]. Following the Buzzard Coulee fall many Royal Astronomical Society of Canada members and ConocoPhillips Canada employees aided in the search [2]. The success of these searches validates the use of volunteers in the recovery of new material. Given the size of our membership and their geographical spread across the UK and Ireland it is hoped that in the event of a new meteorite fall in the area we would play a key role in the location and recovery of material, assisting and working with other organizations to preserve as much material as possible for science.

References: [1] Shaddad M. H. et al. 2010. Meteoritics \& Planetary Science 45:1557-1589. [2] Hildebrand A. R. et al. 2009. Abstract \#2505. 40th Lunar and Planetary Science Conference.

Additional Information: For additional information on the British and Irish Meteorite Society please visit our website at http://www. bimsociety.org or email the chairman Mark Ford at britishmeteorite@ gmail.com.
5525

A BI-MODAL DISTRIBUTION OF ALHA77307 MATRIX OLIVINE: EVIDENCE FOR FINE-GRAINED MIXING FROM MULTIPLE RESERVOIRS IN THE CO FORMATION ZONE

D. Frank ${ }^{1}$, M. Zolensky ${ }^{2}$, A. Brearley ${ }^{3}$ and L. Le ${ }^{1}$. ${ }^{1}$ ESCG/NASA Johnson Space Center, Houston, TX 77058, USA. E-mail: david.r.frank@ nasa.gov. ${ }^{2}$ ARES/NASA Johnson Space Center, Houston, TX 77058, USA. ${ }^{3}$ University of New Mexico, Albuquerque, NM, USA.

Introduction: The CO 3.0 chondrite ALHA77307 is thought to be the least metamorphosed of all the $\mathrm{CO}$ chondrites [1]. As such, the finegrained $(<30 \mu \mathrm{m})$ olivine found in its matrix is a valuable resource for investigating the $\mathrm{CO}$ formation environment since its compositions should be primary. In the $\mathrm{CO}$ matrix, we indeed find a wide range of major element compositions $\left(\mathrm{Fa}_{0.5-71}\right)$. However, more importantly, we find that the olivines make up two compositionally distinct populations $\left(\mathrm{Fa}_{0.5-5}\right.$ and $\left.\mathrm{Fa}_{21-71}\right)$. Grains from both populations are found within an extremely close proximity and we see no obvious evidence of two distinct lithologies within our samples. Therefore, we conclude that the olivine grains found in the ALHA77307 matrix must have crystallized within two unique formation conditions and were later mixed at a very fine scale during the accretion epoch. Here, we propose a possible explanation based on $\mathrm{Cr}$ and $\mathrm{Mn}$ concentrations in the olivine.

The $\mathbf{F a}_{21-71}$ Reservoir: We propose that this population consists of fragmental chondrules. We obtained 35 EPMA measurements of olivine found in type IIA chondrules from ALHA77307, which exhibit remarkable compositional similarities to the $\mathrm{Fa}_{21-71}$ found in matrix. Both groups of olivines begin at around $20 \mathrm{wt} . \% \mathrm{FeO}$ and their $\mathrm{Mn} / \mathrm{Fe}$ trends are nearly identical (see figure below). Although the $\mathrm{Cr}$ content in the matrix grains is lower and not positively correlated with $\mathrm{FeO}$ like in the chondrules, mild metamorphic effects could explain this. $\mathrm{Cr}$ is more mobile and will respond faster to metamorphism in smaller grains, dropping to lower values [2].

$\mathrm{Fa}_{0.5-5}$ : It has been suggested that very low $\mathrm{FeO}$ olivines enriched in Mn could have condensed from the solar nebula [3]. As shown, many of our grains in this population are indeed enriched in $\mathrm{MnO}$. However, we note that an elevated amount of $\mathrm{MnO}$ is not a requirement for forsterite to have condensed from a gas and suggest that the majority of this population has such an origin.

References: [1] Brearley A. J. and Jones R. H. 1997. Reviews in Mineralogy, vol. 36. pp. 3-38. [2] Grossman J. and Brearley A. J. 2005. Meteoritics \& Planetary Science 40:87-122. [3] Klöck et al. 1989. Nature 339:126-128.

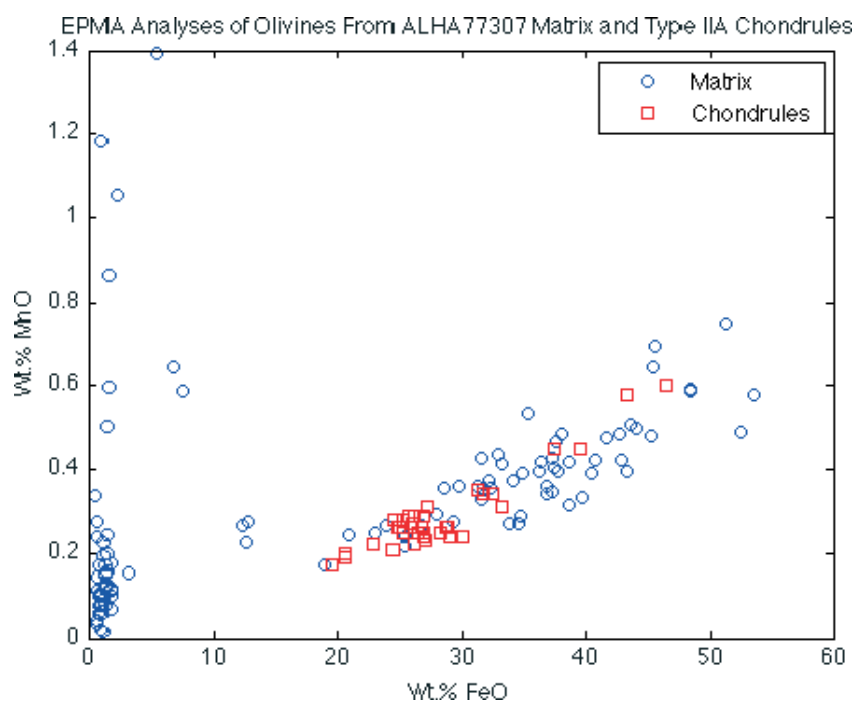




\section{6}

NORTHWEST AFRICA 5492, A UNIQUE UNGROUPED CHONDRITE

P. Friend ${ }^{1}$, J. Zipfel ${ }^{2}$, M. Gellissen ${ }^{1,3}$, R. Kleinschrodt ${ }^{1}$, C. Muenker ${ }^{1}$, T. Schulz ${ }^{1}$, U. Ott ${ }^{4}$, S. Herrmann ${ }^{4}$, A. Pack $^{5}$, A. Stracke ${ }^{6}$ and H. Palme ${ }^{2}$. ${ }^{1}$ Institut für Geologie und Mineralogie, Universität zu Köln, Zülpicherstrasse 49b, D-50674 Köln, Germany. E-mail: piafriend13@googlemail.com. ${ }^{2}$ Sektion Meteoritenforschung, Senckenberg Forschungsinstitut und Naturmuseum Frankfurt/Main, Germany. ${ }^{3}$ Institut für Geowissenschaften, Universität Kiel, Kiel, Germany. ${ }^{4}$ Max Planck Institut für Chemie, Mainz, Germany. ${ }^{5}$ Institut für Mineralogie, Universität Göttingen, Göttingen, Germany. ${ }^{6}$ Institut für Mineralogie, Universität Münster, Münster, Germany.

Northwest Africa (NWA) 5492 is an unusual chondritic meteorite briefly described by $[1,2]$. Here we report additional mineralogical, chemical and isotope data.

Texture and Mineralogy: NWA 5492 is brecciated consisting of large and well defined chondrules, chondrule fragments and various clasts and has apparently no matrix. FeNi metal and sulfides occur as large grains in the chondrule interstitials and as small grains within clasts. The mineralogy of NWA 5492 is very reduced with almost pure enstatite $(\mathrm{Fs}<0.5 \%)$ as dominant mineral followed by forsteritic olivine $(\mathrm{Fa}<0.3 \%)$. The degree of reduction is much stronger than in $\mathrm{CH}-$ chondrites, even exceeding $\mathrm{E}$ chondrites. The low $\mathrm{CaO}$ in opx and the high $\mathrm{CaO}$ in coexisting cpx, as well as the extremely low Fa and Fs contents would imply temperatures at $500{ }^{\circ} \mathrm{C}$ or lower [3]. An extremely long duration of low temperature metamorphism would be required to produce the observed unzoned minerals.

The bulk chemistry has high $\mathrm{Fe}$ and $\mathrm{Ni}$, indicating excess metal similar to $\mathrm{CH}$. $\mathrm{Mg} / \mathrm{Si}$ is $\mathrm{CI}$-chondritic. Refractory elements are neither enriched nor depleted, different from $\mathrm{CH}$ and $\mathrm{E}$ chondrites. Volatiles are strongly depleted similar to $\mathrm{CH}$ but different from $\mathrm{E}$ chondrites.

Oxygen isotopes on two bulk samples and four sub-mg samples of two macroscopically distinguishable clasts are uniform within error limits. On a $\delta^{17} \mathrm{O}$ versus $\delta^{18} \mathrm{O}$ diagram all data plot between LL and EC, above the terrestrial fractionation line, in an area not occupied by another meteorite group [2].

$\mathrm{W}$-isotopes of pure metal phases exhibit $\varepsilon_{\mathrm{W}}$ of about -2.5 to -2.9 , reflecting very early metal separation. Metal-silicate aggregates separated magnetically have $\mathrm{Hf} / \mathrm{W}$ ratios of up to 12 with variable $\varepsilon_{\mathrm{W}}(+0.7$ to +4.5$)$, indicating isotopic disequilibrium between metal phases and aggregates and later, local metal-silicate equilibration, perhaps a result of extensive low temperature metamorphism.

Rare gases were determined on a $167 \mathrm{mg}$ aliquot. Low abundances of trapped $\mathrm{Ar}, \mathrm{Kr}$, and $\mathrm{Xe}$ are consistent with low volatile elements contents. Cosmic ray exposure ages are around $8 \mathrm{Ma}$, concordant for ${ }^{3} \mathrm{He},{ }^{21} \mathrm{Ne}$, and ${ }^{38} \mathrm{Ar}$.

In summary, NWA 5492 is a brecciated chondritic meteorite with no obvious differences in chemistry, mineralogy, and isotopes among various lithic clasts. The metal-rich meteorite has extremely reduced mineralogy, excess metal, a strong depletion of volatiles and an oxygen isotopic composition close to but different from O and E chondrites. NWA 5492 does not fit into existing classification schemes.

References: [1] Friend P. et al. 2011. Abstract \#1095. 42nd Lunar and Planetary Science Conference. [2] Weisberg M. K. et al. 2011. Abstract \#1198. 42nd Lunar and Planetary Science Conference. [3] Lindsley D. H. 1983. American Mineralogist 68:477-493. [4] Clayton R. N. 2004. Treatise on Geochemistry, vol. 1. p. 129.

\section{5}

CARBON MINERALOGY-A COMPREHENSIVE APPROACH FOR THE PLANETARY SCIENCES

Marc Fries. Planetary Science Institute, Tucson, AZ, USA. E-mail: fries@psi.edu.

Carbonaceous materials compose a mineral system independent from, and complimentary with, traditional mineralogy when it comes to studies of geologic/geochemical systems. A broad suite of carbonaceous materials occur in rocks which can be analyzed to quantitatively define limits on the pressure, temperature, chemical environment, shock processing, and oxygen fugacity histories of a given lithology. These phases range from graphite, diamond, macromolecular carbon (MMC)*, vitreous carbon, amorphous carbon and even exotic phases such as buckminsterfullerenes, and graphite whiskers. Each of these phases forms under and retains properties of a distinctly limited range of physical and chemical conditions, describing a range of values for a given lithology to produce useful constraints on the formation and alteration history of that lithology. At this point, technology and methodologies in existence are sufficiently mature to define a comprehensive new field of "carbon mineralogy" capable of quantitatively describing the formation/alteration of extraterrestrial materials based on their carbonaceous phases. By assembling expertise and previous work with new work intended to fill gaps, it should be possible to produce a body of work which will serve as a comprehensive reference on the structure, chemical composition, and phase identities of the carbonaceous materials expected for any given pressure, temperature, and chemical environment that a parent lithology was subjected to. Conversely, for any given extraterrestrial lithology, the pressure, temperature, and chemical environment can be described in terms of the fine structure of the carbonaceous material found within. While considerable work has been done on carbonaceous materials in terrestrial settings, additonal work needs to be done for extraterrestrial carbon found in meteorites, cometary materials, IDPs, etc., the majority of which formed by condensation from hot, diffuse gases.

Pressure: Carbon phases such as amorphous carbon, diamond, lonsdalite, and others constrain a range of temperature and pressure limits for a given lithology. For most extraterrestrial materials this may be most pertinent to shock processing.

Thermal Metamorphic History: MMC exists across a range of submicron structure that describes the thermal alteration history of a given material. The presence and microstructure of diamond, crystalline graphite, aliphatic compounds, and other phases also place limits on the thermal history of a given lithology.

Chemistry: Carbonaceous phases can be sensitive indicators of the chemical environment of their parent lithology, as seen in the direct measurement of heteroatomic composition of $\mathrm{MMC}$, the presence and structure of vitreous carbon, the chemical composition of aliphatic compounds, and other features.

Techniques: Instrumental techniques already exist to measure carbon properties, such as Raman spectroscopy, TEM, SEM/EDS, FTIR, mass spectroscopic techniques, XRD, and others. Quantifying and crosscorrelating these results into a noninstrument-specific format will allow generic description of extraterrestrial carbonaceous materials.

*MMC is defined here as a reduced, primarily carbon solid spanning a continuous range of structural ordering between amorphous carbon and graphite.

References for this abstract are omitted with apologies, as they are too numerous for the abstract format. 
5390

MINERAL INCLUSIONS IN MONAHANS AND ZAG HALITES: EVIDENCE OF THE ORIGINATING BODY

M. Fries ${ }^{1}$, M. Zolensky ${ }^{2}$ and A. Steele ${ }^{3}$. ${ }^{1}$ Planetary Science Institute, Tucson, AZ, USA. ${ }^{2}$ NASA Johnson Space Center, Houston, TX, USA. ${ }^{3}$ Geophysical Laboratory, Carnegie Institute of Science, Washington, DC, USA. E-mail: fries@psi.edu.

Halite grains from Monahans (H5) and Zag (H3-6) are remarkable relicts of aqueous processes in the ancient solar system. Previous work has revealed their age of approximately $4.5 \mathrm{Ga}$ through multiple isotope systems, and fluid-bearing inclusions indicate that these grains have experienced a very mild shock/thermal history [1-3]. Their appearance as discrete grains within an $\mathrm{H}$-chondrite matrix free of aqueous alteration implies that the salt grains are xenogenic to the Monahans/Zag parent body (or bodies). We report the results of Raman imaging analyses of opaque inclusions within the halite grains, which have not been previously described. These results reveal unique insights into the nature of the originating body of the salt grains, as they cannot have been heated in excess of $25^{\circ} \mathrm{C}$ since being encased in halite.

Examination of Monahans and Zag halite grains with Raman imaging reveals the presence of small $(<10 \mu \mathrm{m})$, primarily metal grains with oxidation/hydroxylation products and minor phases inconsistent with H-chondrite mineralogy that must arise from the halite grains' originating body. The grains are generally subrounded and composed largely of metal, magnetite and another phase which is probably finegrained lepidocrocite $(\mathrm{FeO}(\mathrm{OH}))$. Identification of lepidocrocite carries an element of uncertainty at the time of this writing due to broad spectral features shared with clays and glassy phases, but in advance of data from other techniques the identifcation as lepidocrocite is reasonable given the inclusions' apparent history as largely metallic clasts altered by interaction with a brine. Minor phases include forsteritic olivine (Fo $\approx 98)$ [4], macromolecular carbon (MMC), pyroxenes, and a feldspar grain with Raman spectral affinity to anorthoclase. One inclusion features aliphatic material with Raman spectral features consistent with a mixture of shortchain aliphatic compounds.

The minerals and compounds discovered thus far within Monahans/ Zag halites are indicative of an originating body at least partly composed of equilibrated igneous materials (high Fo olivine, possibly the metal) which was subjected to aqueous alteration (the halite parent brine) and containing a light organic component (the short-chain aliphatic compounds). Finally, this material was ejected from the originating body with little or no disruption, as evidenced with the presence of fluid inclusions. An actively geysering body similar to modern Enceladus [2] may be a reasonable analog in this respect. Also, the originating body should have been within close proximity to the $\mathrm{H}$ chondrite parent in order to generate the number of halite grains seen in Monahans and Zag. Other candidates for Monahans/Zag halite parent bodie(s) may include a young Ceres with its possible liquid ocean [3], Main Belt comet(s) [4], or some long-destroyed body.

References: [1] Bogard D., Garrison D., and Masarik J. 2001. Meteoritics \& Planetary Science 36:107-122. [2] Whitby J. et al. 2000. Science 288:1819-1821. [3] Zolensky M. E. et al. 1999. Science 285, 1:3771379. [4] Kuebler K. et al. 2006. Geochimica et Cosmochimica Acta 70, 24:6201-6222. [5] Postberg F. et al. 2009. Nature 459:1098-1101. [6] Rivkin A. et al. 2006. Icarus 185:563-567. [4] Hsieh and Jewitt. 2006. Science 312:561-563.

\section{2}

THERMAL RELEASE AND DIFFUSION OF HELIUM-4 IMPLANTED INTO LUNAR SIMULANTS

X. $\mathrm{Fu}^{1,2}, Y . Z^{1}$, Y. Zheng ${ }^{1}$, H. $\mathrm{He}^{3}$ and Z. Ouyang ${ }^{1}{ }^{1}$ National Astronomical Observatories, Beijing 100012, China. E-mail: fuxh@ bao.ac.cn. ${ }^{2}$ Graduate University of Chinese Academy of Science, China. ${ }^{3}$ Institute of Geology and Geophysics, China.

Introduction: Noble gases and various isotopes in lunar samples and meteorites provide unique and extensive information about a broad range of fundamental questions [1, 2]. Diffusion properties of noble gases are important in the interpretation of such noble gases data. In addition, ${ }^{3} \mathrm{He}$ in the lunar soil is one of the most valuable resources as a fusion fuel. Knowledge of trapping mechanisms and diffusion characteristics of implanted noble gases will enable the optimization of the processes to extract ${ }^{3} \mathrm{He}$ from lunar soil [3]. Here we report ${ }^{4} \mathrm{He}$ implantation and extraction experiments. ${ }^{4} \mathrm{He}$ release pattern and diffusion mechanism in lunar simulants grains were studied.

Experiments: We performed an experiment of solar wind ions implantation and ${ }^{4} \mathrm{He}$ extraction. High-Ti mare soil simulant (CAS-1) [4], highland lunar soil simulant (NAO-1) [5], and Panzhihua ilmenite are irradiated in the experiments. The energy of ${ }^{4} \mathrm{He}$ ions is $50 \mathrm{keV}$. The irradiation dose is $5 \times 10^{16}$ ion $\mathrm{cm}^{-2}$, five times of irradiation saturation dose [6, 7]. After implantation, the samples were analyzed in noble gas mass spectrometry. The samples were heated from 200 to $1500{ }^{\circ} \mathrm{C}$, with $100{ }^{\circ} \mathrm{C}$ stepwise interval.

Results: Lunar simulants, CAS-1 and NAO-1, display similar ${ }^{4} \mathrm{He}$ release patterns in curve shape as lunar soil, but release temperatures are little lower. This is probably a consequence of long-term diffusion after implantation in lunar soil grains. Panzhihua ilmenite displays a different helium release pattern with lunar simulants. Helium release temperatures of Panzhihua ilmenite are higher than those of lunar simulants. Based on the data, we calculated the helium diffusion coefficient $(D)$ and activation energy $\left(E_{a}\right)$ using the equation in [8]. In the Arrhenius plots, we note variation of diffusion parameters of CAS-1 and NAO-1 as temperatures were increasing. It is not consistent with simple thermally activated volume diffusion. The variation could be attributed to physical transformation during high temperature. Activation energy of ${ }^{4} \mathrm{He}$ in Panzhihua ilmenite is apparently higher than $E_{a}$ of CAS-1 and NAO-1, especially at low temperature range. This result confirms that ilmenite is more retentive for noble gas than other lunar materials.

References: [1] Wieler R. 2002. Reviews in mineralogy and Geochemistry, vol. 47. pp. 21-70. [2] Haskin L. and Warren P. 1997. Lunar Sourcebook. [3] Kulhman. 2004. Space resources roundtable VI. pp. 6044. [4] Zheng et al. 2009. Advances in Space Research 43:448-453. [5] Li et al. 2009. Journal of Aerospace Engineering 22:53-57. [6] Futagami et al. 1990. Earth and Planetary Science Letters 101:63-67. [7] Borg et al. 1980. The ancient sun: Fossil record in the earth, moon and meteorites. pp. 431-461. [8] Fulda C. and Lippolt H. 2000. Mathematical Geology 32:3147. 
5240

ALTERATION HISTORY IN THE CI CHONDRITE PARENT BODY INFERRED FROM Mn-Cr DATING OF CAROBNATES

W. Fujiya ${ }^{1}$, N. Sugiura ${ }^{1}$ and Y. Sano ${ }^{2}$. ${ }^{1}$ Earth and Planetary Science, The University of Tokyo, Tokyo, Japan. ${ }^{2}$ Atmosphere and Ocean Research Institute, The University of Tokyo, Tokyo, Japan. E-mail: fujiya@eps.s.u-tokyo.ac.jp.

Introduction: CI chondrites are the most compositionally primitive rocks among the solar system materials. However, they experienced pervasive aqueous alteration, during which chemical and isotopic compositions and mineralogy of their constituents were dramatically changed [1]. For a better understanding of the geological history in the CI chondrite parent asteroid, we investigated ${ }^{53} \mathrm{Mn}-{ }^{53} \mathrm{Cr}$ ages (half-life: 3.7 Myr) of carbonates to determine the timescale of aqueous alteration. Here we report Mn-Cr ages of dolomite in the Orgueil and Y980115 CI chondrites.

Samples and Analytical Methods: For Orgueil, many but small (< approximately $20 \mu \mathrm{m}$ in diameter) dolomite grains were found in a polished thin section. Four relatively large (approximately $40 \mu \mathrm{m}$ ) grains were chosen for the isotope measurements. For Y980115, most dolomite grains are associated with numerous tiny (submicron-size) magnetite grains, which can result in contamination during the isotope measurements. An exception, for which we obtained $\mathrm{Mn}-\mathrm{Cr}$ data, is a large (approximately $300 \mu \mathrm{m}$ ) dolomite grain with a clearly distinct morphology, which might have replaced a primordial CAI. Isotope measurements were performed by the NanoSIMS installed at The University of Tokyo. We used a synthetic calcite standard [2] and analytical methods are described elsewhere [3].

Results and Discussion: Three out of four dolomite grains in Orgueil have initial ${ }^{53} \mathrm{Mn} /{ }^{55} \mathrm{Mn}$ ratios of $(3.24-3.54) \times 10^{-6}$. Combining these data, we obtain the absolute age of $4563.8+0.7 /-0.7 \mathrm{Ma}$ for crystallization of dolomite in Orgueil. However, one dolomite grain, which cannot be distinguished from the others by their morphologies, shows a significantly younger age of $<4556 \mathrm{Ma}$. One dolomite grain in Y980115 shows a slightly higher initial ${ }^{53} \mathrm{Mn} /{ }^{55} \mathrm{Mn}$ ratio of $(4.03 \pm$ $0.46) \times 10^{-6}$. This corresponds to the absolute age of $4564.8+0.6 /$ $-0.7 \mathrm{Ma}$.

The absolute age of three dolomite grains is comparable to those of carbonates in CM chondrites [4]. This indicates that CI and CM chondrite parent bodies accreted around the same time and initial abundances of ${ }^{26} \mathrm{Al}$ are similar as heat sources in these asteroids. Therefore, they experienced heating by the ${ }^{26} \mathrm{Al}$ decay to similar extent, although difference in chemical properties of them (such as water abundances) probably resulted in different thermal histories. The younger dolomite in the present study has a comparable ${ }^{53} \mathrm{Mn}$ abundance to the youngest breunnerites analyzed by Petitat et al. [5], indicating a prolonged duration of aqueous alteration for at least $8 \mathrm{Myr}$.

The dolomite grain in Y980115 is slightly older than those in Orgueil and Ivuna [3]. The initial ${ }^{53} \mathrm{Mn} /{ }^{55} \mathrm{Mn}$ ratiois consistent with those of Kaidun carbonates [6]. Based on the strange morphology, it may have originated from another pre-existing planetesimal and have not precipitated in the CI chondrite parent body.

References: [1] Brearley A. J. 2006. In Meteorites and the early solar system II:587-624. [2] Sugiura N. et al. 2010. Geochemical Journal 44:e11e16. [3] Fujiya W. et al. 2011. Abstract \#1397. 42nd Lunar and Planetary Science Conference. [4] Fujiya W. et al. 2011. In review. [5] Petitat M. et al. 2009. Abstract \#1657. 40th Lunar and Planetary Science Conference. [6] Petitat M. et al. 2011. Meteoritics \& Planetary Science $46: 275-283$.

\section{8}

XENOLITHS IN CARBONACEOUS AND ORDINARY CHONDRITES C. Funk ${ }^{1,3}$, A. Bischoff ${ }^{2}$ and J. Schlüter ${ }^{3}$. ${ }^{1}$ Institut für Geologie und Mineralogie, Zülpicher Straße 49b, 50674 Köln, Germany. ${ }^{2}$ Institut für Planetologie, Wilhelm-Klemm-Straße 10, 48149 Münster, Germany. ${ }^{3}$ Mineralogisch-Petrographisches Institut, Grindelallee 48, 20146 Hamburg, Germany. E-mail: claudia_funk@gmx.net.

Introduction: Xenoliths in meteorites are rock fragments of different lithologies genetically not related to the host rock [1]. In this study we systematically searched for xenoliths in primitive carbonaceous and ordinary chondrites in order to find: (1) fragments of known meteorite classes, but occurring as exotic clasts in other chondrite classes, (2) materials with mineralogical and textural properties of chemically unprocessed material, (3) unknown or unusual materials, and (4) igneoustextured lithologies that clearly underwent differentiation processes.

Results: The investigated samples include 26 thin sections of 23 carbonaceous chondrites and 31 thin sections of 23 ordinary chondrites. Xenoliths are present in Acfer 114 (CR2), Sahara 98645 (H3), Dhajala (H3.8), Tanezrouft 039 (L3), Sahara 98035 (L/LL3), Adrar 003 (L/LL3.1), and Krymka (LL3.1).

In Acfer 114 an optically dark fragment was found and interpreted as a clast of primitive accretionary material consisting predominantly of olivine grains with varying chemical compositions different to olivines in the Acfer/El Djouf host meteorite [2]. The xenolith in Sahara 98645 has affinities to CI and CM chondrites. The fragment largely consists of finegrained phyllosilicate-rich matrix. Coarse-grained constituents include several pyrrhotites, minor amounts of pentlandite and troilite, some small clusters of framboidal and isolated magnetites, carbonates (mainly dolomite), and some olivine and pyroxene grains. The Dhajala meteorite comprises a fragment that mainly consists of an extremely fine-grained and porous matrix in which several multiphase-aggregates and rounded objects are embedded. The rounded objects show diameters of 10-30 $\mu \mathrm{m}$ and are very similar to the "accretionary objects" found within the finegrained fragment BK13 in the Krymka LL3.1 chondrite [3]. Within Tanezrouft 039 (L3) an LL-chondritic xenolith was found. Another fragment in the same thin section predominantly consist of a kind of $\mathrm{Fe}$, $\mathrm{Ni}$-metal groundmass in which chemically almost homogeneous low-Capyroxenes, sulfides, unidentified Fe-oxides, silica, and feldspar is embedded. Olivine is completely absent. The mineral assemblage and the texture of the clast lead to the assumption that this fragment might originate from a mesosideritic parent body. Sahara 98035 contains an extremely fine-grained fragment with several unusual multi-phase aggregates and very few microchondrules. In addition Kakangari chondrite-like xenoliths were found within the Adrar 003 and the Krymka meteorite.

Future investigations by transmission electron microscopy and secondary ion mass spectrometry will allow to clearly define the origin of some of the observed xenoliths.

References: [1] Bischoff et al. 2006. In Meteorites and the early solar system II, edited by Lauretta D. S. and McSween H. Y. pp. 679-712. [2] Bischoff et al. 1993. Geochimica et Cosmochimica Acta 57:1587-1603. [3] Semenenko et al. 2001. Meteoritics \& Planetary Science 36:1067-1085. 


\section{0}

\section{METAMORPHIC INDICATORS IN STARDUST}

Z. Gainsforth ${ }^{1}$, A. L. Butterworth ${ }^{1}$, D. E. Brownlee ${ }^{2}$, G. R. Huss ${ }^{3}$, D. Joswiak ${ }^{2}$, K. Nagashima ${ }^{3}$, R. C. Ogliore ${ }^{3}$, T. Tyliczszak ${ }^{4}$, A. J. Westphal ${ }^{1}$. ${ }^{1}$ U. C. Berkeley, USA. ${ }^{2}$ Hawaii Institute of Geophysics and Planetology, University of Hawaii at Mãnoa, USA. ${ }^{3}$ Astronomy Department, University of Washington, USA. ${ }^{3}$ Astronomy Department, University of Washington, USA. ${ }^{4}$ Advanced Light Source, Lawrence Berkeley National Laboratory, USA. E-mail: zackg@ssl.berkeley.edu.

Introduction: Stardust cometary particles are important for understanding solar system formation because comet Wild 2 most likely preserved mineralic components without additional thermal or aqueous alteration. Evidence of thermal alteration of a Stardust particle would be a significant discovery as it would indicate that the particle resided on a large parent body before being transported to the comet, implying that at least some parent bodies formed during or before large-scale solar mixing and formation of KBOs. On the other hand, absence of thermal metamorphism allows for more accurate dating of particle formation using ${ }^{26} \mathrm{Al}$ and other systems.

Evaluating Metamorphism: Very few indicators of low-grade metamorphism can currently be applied to Stardust samples. Most involve unidirectional changes in properties of the constituents. One promising indicator for the particle Iris (from Stardust track C2052,2,74), which has many characteristics of a type II chondrule [1], is the $\mathrm{Fe} / \mathrm{Mg}$ partitioning between olivine and chromite [2]. At temperatures near the liquidus, the assemblage might be expected to be in equilibrium, and rapid cooling quenches the high-temperature equilibrium values. [2] found that the $K_{\mathrm{D}}=(\mathrm{Fe} / \mathrm{Mg})^{\mathrm{sp}} /(\mathrm{Fe} / \mathrm{Mg})^{\text {oliv }}$ in chondrules from unmetamorphosed chondrites such as Semarkona and ALHA77307 reflects a temperature of approximately $1400{ }^{\circ} \mathrm{C}$. With small degrees of metamorphism, the $K_{\mathrm{D}}$ values increase as the system tries to equilibrate to lower temperature. Although one cannot determine the metamorphic temperature from the $K_{\mathrm{D}}$ s of type 3.1-4.0 meteorites because full lowtemperature equilibrium was not achieved, it is still possible to roughly estimate the metamorphic grade. Iris olivine is $\mathrm{Fo}_{64}$. Iris chromite contains $6.0 \mathrm{wt} \% \mathrm{MgO}, 28.4 \mathrm{wt} \% \mathrm{FeO}$ and $16.9 \mathrm{wt} \% \mathrm{Al}_{2} \mathrm{O}_{3}$. This gives $\ln \left(K_{\mathrm{D}}\right)$ for spinel/olivine of approximately 1.5. Chondrules from type 2 and 3.0-3.1 chondrites have $K_{\mathrm{D}} \mathrm{s}$ of 1-2, while those of type 3.3-3.5 have $K_{\mathrm{D}}$ s of 2-4 [2]. The $K_{\mathrm{D}}$ of Iris suggests that the system was quenched at high temperature and has experienced little thermal metamorphism since.

Other potential indicators of metamorphic grade include $\mathrm{Ca}$ and $\mathrm{Cr}$ distribution in Fe-rich olivine [3, 4], degree of devitrification of mesostasis glass [5], $\mathrm{Al} / \mathrm{Mg}$ ratio in igneous feldspar [6]. However, these indicators presently give disparate results, probably because they were developed for size scales of 10 to $100 \mathrm{~s}$ of $\mu \mathrm{m}$, while Iris features are on the scale of a few $\mu \mathrm{m}$ to hundreds of $\mathrm{nm}$. Careful work to calibrate nucleation and growth of phases at the nanometer scale in artificial and natural chondrules will be necessary to apply these indicators to Iris and other Stardust particles.

References: [1] Gainsforth Z. et al. 2010. Meteoritics \& Planetary Science 45:A60. [2] Johnson C. A. and Prinz M. 1991. Geochimica et Cosmochimica Acta 55:893-904. [3] McCoy T. J. et al. 1991. Geochimica et Cosmochimica Acta 55:601-619. [4] Grossman J. N. and Brearley A. J. 2005. Meteoritics \& Planetary Science 40:87-122. [5] Huss G. R. et al 2006. In Meteorites and the early solar system II. pp. 567-586. [6] LaTourette T. and Wasserburg G. J. 1998. Earth and Planetary Science Letters 158:91-108.

\section{3}

EQUILIBRATION OF FELDSPAR IN PETROLOGIC TYPE 4-6 L AND LL CHONDRITES: METAMORPHIC CONDITIONS ON CHONDRITE PARENT BODIES

J. Gallegos and R. H. Jones. Department of Earth and Planetary Sciences, University of New Mexico, Albuquerque, NM 87131, USA. E-mail: buhhby@unm.edu.

Introduction: Examination of feldspar compositions in petrologic type 4-6 ordinary chondrites (OCs) provides insights into early geologic history and metamorphic processes on $\mathrm{OC}$ parent bodies [1]. Understanding compositional equilibration of feldspar through diffusion helps establish better criteria for metamorphic conditions. Also, feldspar can potentially record interactions with fluids as well as shock effects, which may have played a significant role in alteration of these chondrites.

Kovach and Jones [1] studied three LL chondrites (of petrologic types 4,5 , and 6), and observed significant changes in feldspar compositions that indicate progressive equilibration during metamorphism. In contrast, feldspar in $\mathrm{H}$ chondrites has similar compositions in petrologic types 4, 5, and 6. The goals of our study are twofold. First, we have studied more LL chondrites to determine whether the sequence of progressive equilibration observed by [1] applies more broadly to the LL group. Second, we have studied L chondrites to determine whether they record development of feldspar that is more similar to the $\mathrm{H}$ or LL groups.

Methods: We studied three LL chondrites: Sevilla (LL4), Olivenza (LL5) and Beeler (LL6) and two L chondrites: Kramer Creek (L4) and Roy (1933) (L5). We analyzed feldspar that is located within relict chondrules, similar to [1]. All the samples chosen for this study have low shock levels and low degrees of terrestrial weathering. We analyzed feldspar using a JEOL 8200 electron microprobe. All grains analyzed are $>10 \mu \mathrm{m}$ in diameter.

Results: LL chondrites. Feldspar compositions in Sevilla (LL4) are similar to Bjurböle (L/LL4) [1] and Bo Xian (LL4) [2] - they have highly varying $\mathrm{An} / \mathrm{Ab}$ ratios and low Or contents. Olivenza (LL5) is similar to Tuxtuac (LL5) [1] with a narrower range of $\mathrm{An} / \mathrm{Ab}$ ratios and higher Or content than in type 4. Beeler (LL6) is similar to St. Séverin (LL6) [1]feldspar in both is homogeneous and albitic $\left(\mathrm{An}_{10} \mathrm{Ab}_{85} \mathrm{Or}_{5}\right)$. Alteration textures in anorthitic feldspar were observed in Sevilla, similar to those in Bjurböle and Bo Xian [1-3]. We did not see exsolution in Olivenza comparable to that in Tuxtuac $[1,4]$. Also, Beeler did not exhibit alkali redistribution that was observed in St. Séverin [1], where it is probably attributable to shock.

L chondrites. Feldspar compositions in Kramer Creek (L4) and Roy (1933) (L5) are similar to LL chondrites of the same petrologic types and are much more similar to $\mathrm{LL}$ than to $\mathrm{H}$ chondrites. Kramer Creek exhibits alteration textures and ablitization similar to Bo Xian and Bjurböle [1, 2], and we observed exsolution in Roy (1933) similar to that in Tuxtuac $[1,4]$.

Discussion: Progressive equilibrium is recorded by feldspar in LL chondrites, and the general trends described by [1] are observed more broadly in a larger number of samples. Feldspar in L chondrites shows similar trends to LL chondrites, indicating similar metamorphic environments for both parent bodies.

References: [1] Kovach H. A. and Jones R. H. 2010. Meteoritics \& Planetary Science 45:246-264. [2] Jones R. H. and Brearley A. J. 2010. Abstract \#2133. 41st Lunar and Planetary Science Conference. [3] Jones R. H. and Brearley A. J. 2010. Abstract \#5276. 73rd Meteoritical Society. [4] Jones R. H. and Brearley A. J. 2011. This meeting. 
5246

RECOGNITION OF REDEFORMED PLANAR DEFORMATION FEATURES (PDFs) IN LARGE IMPACT STRUCTURES

A. A. Garde ${ }^{1}$ and A. Y. Glikson ${ }^{2}{ }^{1}$ Geological Survey of Denmark and Greenland. E-mail: aag@geus.dk. ${ }^{2}$ Australian National University.

Introduction: Planar deformation features (PDFs) in quartz constitute a unique criterion for recognition of shock metamorphism. PDFs residing in the brittle upper crust may preserve their initial $2-5 \mu \mathrm{m}$ spacing, although superposed tectonic deformation associated with rebound and formation of a central uplift and recrystallization/annealing by hydrothermal activity triggered by the impact may result in bent, wavy and decorated PDFs. High shock pressures can penetrate into ductile, midcrustal environments where PDFs are subject to crystal-plastic redeformation and high-temperature hydrothermal alteration associated with postimpact processes. In large exhumed impact structures it is therefore appropriate to distinguish primary shock features from secondary deformation and recrystallization of the PDFs.

Results: Optical Universal stage studies of quartz PDFs in the recently discovered Maanitsoq structure, southern West Greenland and the Warburton structure, below the Cooper Basin, northwest South Australia, as well as in the established Sudbury, Vredefort, Manicouagan, Charlevoix, and Azuara impact structures, indicate widespread deformation, recrystallization, coarsening, fluid inclusion decoration, and obliteration of planar microstructures. The planar microstructures contain elements diagnostic of primary PDFs, including multiple intersecting planar sets, prevalence of unique Miller indices (i.e., $\{10-11\},\{10-12\}$, $\{10-13\}$ ), narrow planar spacings (approximately $2-5 \mu \mathrm{m}$ ) and submicron lamella width. Quartz grains may be bent and show undulose extinction, with curved or kinked PDFs preserving near-original crystallographic orientations. Gradual annihilation of planar sets is widespread, increasing the apparent spacing of remaining PDFs. In addition, closely spaced PDFs with en echelon overlaps have merged, resulting in small lateral links not seen in pristine PDFs. TEM studies indicate existence of individual quartz lamellae about or less than one micron in width, consistent with PDFs, and resolves existence of both deformed subgrain boundaries as well as relic contiguous planar crystallographic boundaries. Lamellae in large exhumed impact structures may thus retain critical diagnostic features consistent with PDFs, with two or more orientations being common, preserving the specific crystallographic orientations characteristic of shock metamorphism.

Discussion: Less than perfectly preserved quartz PDFs are rarely described in the literature or are denoted as metamorphic deformation lamellae (MDLs) and/or planar fractures (PFs), commonly considered unrelated to impacting. However, MDLs and/or PFs may form following initial shock metamorphic effects of target materials, during superposed deformation associated with rebound and formation of a central uplift, and in some instances younger orogenic events. MDLs normally constitute coarser elongate, spindle-shaped panels a few microns across displaying small shifts in their crystallographic orientation. Planar features (PFs) are commonly even more widely spaced than MDLs. We are unaware MDL or PF sets dominated by shock-diagnostic Miller indices, submicron planar quartz microstructures, interplanar spacings down to less than 2-4 $\mu \mathrm{m}$ and multiple intersecting planar sets, relics of which allow a distinction of PDFs from MDLs and PFs in deformed and hydrothermally altered rocks.

\section{9}

BEYOND VREDEFORT, SUDBURY, AND CHICXULUB

A. A. Garde ${ }^{1}$, B. A. Ivanov ${ }^{2}$ and I. McDonald ${ }^{3}{ }^{1}$ Geological Survey of Denmark and Greenland, Denmark. E-mail: aag@geus.dk. ${ }^{2}$ Institute for Dynamics of Geospheres, Moscow, Russia. ${ }^{3}$ I. McDonald, Cardiff University, U.K.

Introduction: The largest known impact structures on the Earth (VSC-Vredefort, Sudbury, and Chicxulub) [1, 2] were originally formed as impact craters with diameters of $180-200 \mathrm{~km}$. On other terrestrial planets we observe much larger craters and impact basins up to thousands of $\mathrm{km}$ in diameter. The largest of these structures have been formed during the late heavy bombardment period [3], and nowadays the main asteroid belt delivers only smaller projectiles to planetary crossing orbits. However, Mead (D approximately $270 \mathrm{~km}$ ), the largest crater on Venus, is younger than approximately $0.5 \mathrm{Ga}$ [4], and a priori we can assume that remnants of structures larger than VSC may be found on Earth.

Motivation: Recent research of the Maniitsoq structure, West Greenland [5] opens the problem of the crust and mantle structure under craters larger than VSC. The subsurface dynamic history (shock level, deformation, and uplift) under Vredefort-scale craters has previously been modeled [2]. Here we present the similar modeling for craters larger than Vredefort but smaller than impact basins [6].

Modeling: The same 2-D hydrocode as in $[2,6]$ is used with ANEOS-based equations of state for crust ("granite") and mantle ("dunite"). The thermal gradient is assumed as in [7]. The projectile strikes the surface vertically with the velocity of $15 \mathrm{~km} \mathrm{~s}^{-1}$. The projectile diameter varies from $32 \mathrm{~km}$ downward to $14 \mathrm{~km}$ modeled earlier [2]. Simulated largest craters have diameters approximately $400-480 \mathrm{~km}$ at the poorly expressed rim crest and more well-defined apparent diameter $D_{\text {a }}$ approximately $320 \mathrm{~km}$ (at the level of preimpact surface). The new feature, not observed in Vredefort modeling but in giant basin modeling [6], is the fate of the impact melt volume in the transient cavity. The collapse of a Vredefort-sized transient cavity lifts the bottom of the impact melt volume upward, while in larger craters the crater collapse compresses it into a subvertical neck near the center. In above-Vredefortsize modeling the central melt neck is a norm both for "cold" and for "hot" targets (see [7] for definitions). At the assumed erosion level of $20-25 \mathrm{~km}$ as at Maniitsoq, the central melted neck after the largest modeled impact has a diameter of 40 ("cold" target) up to $90 \mathrm{~km}$ ("hot" target). For smaller projectiles the "melted neck" diameter decreases toward zero when the whole melted zone is located above the erosion level.

Preliminary Results: Although strongly model depended, a quantitative structural change is expected for larger-than-Vredefort impact craters. The presence of impact melt in deeply eroded crust may therefore constitute field evidence that a giant impact structure was created here. Model-derived estimates for rock strain, shock level and variations in preimpacting crustal depth can be used for comparison with field data.

References: [1] Grieve R. and Therriault A. 2000. Annual Review of Earth and Planetary Sciences 28:305-338. [2] Ivanov B. A. 2005. Solar System Research 39:381-409. [3] Hartmann W. K. et al. 2000. In: Origin of the Earth and Moon. Tucson: The University Arizona Press. pp. 493512. [4] Phillips R. J. et al. 1992. GSA Special Papers 465:29-49. [5] Garde A. A. 2010. Abstracts and Proceedings Geological Society, Norway, 1:57-58. [6] Ivanov B. A. et al. 2010. GSA Special Paper 465:2949. [7] Ivanov B. A. and Melosh H. J. 2003. Geology 31:8 69-872. 
5258

POLYPHASE MICROBRECCIA INJECTITES IN THE MANIITSOQ STRUCTURE, WEST GREENLAND

A. A. Garde ${ }^{1}$ and B. Dyck ${ }^{2}$. ${ }^{1}$ Geological Survey of Denmark and Greenland, Copenhagen, Denmark. E-mail: aag@geus.dk. ${ }^{2}$ Lund University, Sweden.

Introduction: In large impacts, the short-lived modification from transient cavity to final crater requires temporary strength degradation of the host rocks. Whereas the process of strength degradation is not well understood, it has been possible to model the crater reorganization observed in large impact structures by employing acoustic fluidization of $100 \mathrm{~m}$-scale individual blocks [1]. Evidence of the repeated compression and dilation that is inherent in such modeling of impact crater modification is found in a regional network of polyphase microbreccia dykes with internal dyke-in-dyke and fold structures in Archean orthogneisses in the Maniitsoq region of West Greenland (interpreted as a giant, deeply eroded Archean impact structure [2]).

Observations: Along and outside the margins of the central melt body of the Maniitsoq structure there is a $>30 \mathrm{~km}$ wide zone containing numerous steep, radial dykelets of fluidized quartz-plagioclase-biotite microbreccia derived from thorough in situ crushing of the host quartzofeldspathic orthogneiss. The microbreccias and their host rocks display intense crushing of quartz, plagioclase and biotite, loss of twinning in plagioclase and $\mathrm{K}$-feldspar (Kfs) exsolution, as well as $\mathrm{Kfs}$ films partially surrounding plagioclase and/or quartz clasts. Microclast shapes and distribution patterns indicate intense compression - but no simple shear as observed in crustal shear zones. The dykes vary in thickness from $<1 \mathrm{~cm}$ to about $5 \mathrm{~m}$, forming up to $c .10 \%$ of the local rock volume. Multiple dyke-in-dyke injection and repeated within-dyke compressional folding are common; internal structures and orientations of rare inclusions of rock fragments suggest almost complete final compressional collapse. The microbreccias are accompanied by coarse-grained, monomineralic, $1-3 \mathrm{~cm} \mathrm{Kfs}$ veins, both interior, ptygmatically folded veins and veins along the margins of individual injectite phases. Such veins are confined to the dykes themselves, and the vein Kfs does not display evidence of crushing or shock metamorphism (contrary to quartz and plagioclase in microbreccia and host rocks).

Some of the microbreccia dykes have been superimposed by ductile simple shear and form part of a second-order, $100 \mathrm{~m}$-scale network of metre-thick shear zones in several directions.

Discussion: Radial zones of intense crushing of regional extent, fluidised microbreccias with compressional textures, and associated second-order shear zone systems are expected if rarely described in the floors of large impact structures; the Maniitsoq microbreccias may be related to breccia dykes and pseudotachylites in the floors of, e.g., Vredefort and Popigai [3]. The internal structures of the microbreccias at Maniitsoq and their Kfs veins indicate repeated but very short-lived compression and dilation (overlapping in time with fluidization and the Kfs component being mobile). Such oscillatory deformation from a central point is known from earthquakes and is also inherent in acoustic fluidisation modeling of impact crater modification.

References: [1] Melosh H. J. and Ivanov B. A. 1999. Annual Review of Earth and Planetary Sciences 27:385-415. [2] Garde A. A. 2010. Abstracts and Proceedings of the Geological Society, Norway, 1:57-58. [3] Reimold W. U. 1995. Earth-Science Reviews 39:247-265.
5394

EXPERIMENTAL PARTIAL MELTING OF THE LAPAZ ICEFIELD 03639 R4 CHONDRITE

K. G. Gardner-Vandy and D. S. Lauretta. Lunar and Planetary Laboratory, University of Arizona. E-mail: kgardner@lpl.arizona.edu.

Introduction: As part of an ongoing study of the petrology, geochemistry and formation conditions of the FeO-rich primitive achondrites [1-3], we perform partial melting experiments of FeO-rich chondritic meteorites. We recently reported that melting experiments of a CK5 chondrite yield compositions similar to those in several ungrouped primitive achondrites, but we also concluded that a more FeO-rich chondritic precursor is needed to yield brachinite compositions [3]. From this, we have begun similar experiments on R4 chondrite LaPaz Icefield (LAP) 03639

Methods: We obtained a thin section and chip (2.7 g) of LAP 03639 from the Meteorite Working Group. We used an approximately $250 \mathrm{mg}$ chip in each experiment to capture textural changes across the experimental conditions. Experiments were run in a Deltech gas-mixing furnace with an oxygen sensor and type $\mathrm{R}$ thermocouple. Thus far, we have completed and analyzed two experiments, R1 and R2. Temperature for both was $1250{ }^{\circ} \mathrm{C}$, and each ran for 4 days. The oxygen fugacity for $\mathrm{R} 1$ was IW-1 and $\mathrm{R} 2$ was $\mathrm{IW}+1$, controlled with a 1 -bar $\mathrm{CO}+\mathrm{CO}_{2}$ gas mixture. We choose $f \mathrm{O}_{2}$ values based on our previous analyses of the thermodynamic environment of $\mathrm{FeO}$-rich primitive achondrite meteorites [2]. No attempt was made to retain volatiles.

Starting Material: LAP 03639 is an R4 chondrite [4-5]. It has chondrules and chondrule fragments $\leq 1.1 \mathrm{~mm}$ across. The matrix is made of olivine, pyroxene and plagioclase with sulfides containing $0-30 \mathrm{wt} \%$ Ni. Olivine is $\mathrm{Fa}_{38.8} \pm 0.2$, low-Ca pyroxene is $\mathrm{Fs}_{6-26}$, high-Ca pyroxene is $\mathrm{Fs}_{10-12}$, and plagioclase is $\mathrm{An}_{5-13}$.

Experimental Results: Both the R1 and R2 experiments resulted in significant silicate melting to the extent that homogeneous $\left(\mathrm{Fa}_{33.5} \pm 0.8\right.$ and $\mathrm{Fa}_{39.8} \pm 0.9$, respectively) anhedral to subhedral olivine is the only silicate mineral remaining. These grains are surrounded by silicate melt with $\mathrm{Mg \#} 28.0 \pm 1.1$ and $20.8 \pm 0.8$, respectively. Significant $\mathrm{S}$ loss occurred, but there are several rounded Ni-rich sulfides in each charge; R1 sulfides have $8.5-40 \mathrm{wt} \% \mathrm{Ni}$, and R2 sulfides have $63-70 \mathrm{wt} \% \mathrm{Ni}$. $\mathrm{R} 1$ contains taenite with up to $39 \mathrm{wt} \% \mathrm{Ni}$. R2 has a few grains of $\mathrm{Fe}$, Ni-metal with $60-79 \mathrm{wt} \% \mathrm{Ni}$. The original chondritic texture remains only in small regions with relict barred olivine grains.

Discussion: Olivine compositions generated in the R1 experiment resulted in olivine with compositions matching those of brachinites $\left(\mathrm{Fa}_{32-35}\right)$ [1]. Those for the R2 experiment, however, resulted in compositions more oxidized than the starting material. This is likely due to the wide range of intrinsic oxygen fugacities $\left(f \mathrm{O}_{2}\right)$ calculated for the $\mathrm{R}$ chondrites (FMQ-1 to FMQ-3.5) [5], and proves that LAP 03639 formed at more reducing conditions than $\mathrm{IW}+1$. These initial results indicate that brachinites could form from the partial melting of an R-chondrite at IW-1.

Acknowledgments: Research supported by NASA grants NNX09AQ88H and NNX10AH50G (DSL) and UA/Sloan Foundation Indigenous Graduate Partnership Fellowship (KGGV).

References: [1] Gardner K. G. et al. 2007. Abstract \#2086. 38th Lunar and Planetary Science Conference. [2] Gardner-Vandy K. G. et al. 2009. Abstract \#2520. 40th Lunar and Planetary Science Conference. [3] Gardner-Vandy et al. 2011. Abstract \#1935. 42nd Lunar and Planetary Science Conference. [4] Kallemeyn G. W. et al. 1996. Geochimica et Cosmochimica Acta 60:2243-2256. [5] Righter K. and Neff K. E. 2007. Polar Science 1:25-44. 
5227

DIAMOND, BUCKY-DIAMOND, GRAPHITE-DIAMOND, AISILICATE, AND STISHOVITE IN THE GUJBA CB CHONDRITE L. A. J. Garvie ${ }^{1}$, P. Németh ${ }^{2}$, P. R. Buseck ${ }^{3,4}$. ${ }^{1}$ Center for Meteorite Studies, Arizona State University, AZ, USA. ${ }^{2}$ Institute of Nanochemistry and Catalysis, Chemical Research Center/Hungarian Academy of Sciences, H-1025 Budapest, Hungary. ${ }^{3}$ School of Earth and Space Exploration, Arizona State University, AZ, USA. ${ }^{4}$ Department of Chemistry and Biochemistry, Arizona State University, AZ, USA.

Introduction: Gujba is a coarse-grained carbonaceous chondrite dominated by silicate globules, large metal globules, and dark interstitial matrix [1]. It shows evidence of shock stage S2 [1]. Features indicating higher shock pressures are evident in the matrix, consisting of fine-grained metal and silicate that resembles the shock veins in ordinary chondrites. Several high-pressure (HP), shock-produced phases occur. These include majorite, wadsleyite, and coesite [2]. The wadsleyite and majorite indicate maximum localized pressures and temperatures of approximately $19 \mathrm{GPa}$ and $2000{ }^{\circ} \mathrm{C}$ [2]. There are no prior reports of diamonds in Gujba, but they were described from Bencubbin, where some of the diamonds are thought to have formed via solid-state transformation of pre-existing graphitic material from an intense shock event [3]. As part of our ongoing study of the fine-grained materials in primitive meteorites, we used transmission electron microscopy (TEM), selected-area electron diffraction (SAED), and electron energy-loss spectroscopy (EELS) to investigate the acid-insoluble material from Gujba.

Methods: The $\mathrm{HF} / \mathrm{HCl}$ residue was separated by acid digestion of several millimeter-sized pieces of the dark interstitial matrix. The acid removed most silicates, sulfides, and metals, leaving a black powder. A droplet of the residue in suspension with water was dried on a Cu TEM grid coated with lacy-C. TEM data were acquired where electrontransparent areas of the residue protruded over the holes of the grid. High-resolution (HRTEM) and SAED data were acquired with Tecnai F20 and JEOL JEM 4000EX electron microscopes. EELS data were acquired with a GATAN 766 DigiPEELS spectrometer attached to a Philips 400-ST TEM.

Results and Discussion: TEM images of the acid residue show abundant carbonaceous aggregates and lath-shaped stishovite. Stishovite laths to $1 \mu \mathrm{m}$ long are common. A combination of HRTEM, SAED, and EELS measurements from multiple carbonaceous aggregates show a range of crystallinities and structures that include amorphous to poorly graphitized carbon, well-ordered graphite, rounded to euhedral diamonds, and clumps of nanodiamonds. Hollow, amorphous carbonaceous nanoglobules similar to those in primitive meteorites [4] also occur. A crystalline phase with composition $\mathrm{Al}_{1.95 \pm 0.2} \mathrm{SiO}_{4.98 \pm 0.5}$ occurs in the residue, but its cell constants do not match those of any known $\mathrm{Al}$ silicate. Prominent in the HRTEM images are $50 \mathrm{~nm}$ particles that show various proportions of parallel 0.350 and $0.206 \mathrm{~nm}$ fringes that presumably reflect highly local regions of graphite and diamond structure, respectively. Associated with the graphite-diamond are rare buckydiamond composed of a nanodiamond core surrounded by curved graphite layers. The unique HP mineral assemblage in Gujba may be the result of its unusual macroscopic structure composed of mm-sized spheres surrounded by silicates. Shockwaves traveling through materials of highly variable densities produce heterogeneous and localized shock pressures and temperatures [5].

References: [1] Rubin A. E. et al. 2003. Geochimica et Cosmochimica Acta 67:3283. [2] Weisberg M. K., Kimura M. 2010. Meteoritics \& Planetary Science 45:873. [3] Mostefaoui S. et al. 2002. Earth and Planetary Science Letters 204:89. [4] Garvie L. A. J., Buseck P. R. 2004. Earth and Planetary Science Letters 224:431. [5] Stöffler et al. 1991 Geochimica et Cosmochimica Acta 55:3845.

\section{4}

EMPLACEMENT TEMPERATURE OF THE IMPACT BRECCIA AT HAUGHTON IMPACT CRATER

J. Gattacceca ${ }^{1}$, G. R. Osinski ${ }^{2}$, Y. Quesnel ${ }^{1}$ and P. Rochette ${ }^{1}$. ${ }^{1}$ CEREGE, CNRS, Aix-Marseille University, Aix-en-Provence, France. E-mail: gattacceca@cerege.fr. ${ }^{2}$ Department of Earth Sciences, University of Western Ontario, London, Ontario, Canada.

Introduction: The Haughton impact structure is a well-preserved Eocene structure $23 \mathrm{~km}$ in diameter, located on Devon Island in the Canadian Arctic Archipelago [1]. A remarkable feature is the abundance of allochthonous impactites derived from the sedimentary target rock (Paleozoic dolomites and limestones). These impactites were originally interpreted to be lithic or fragmental breccias [2] but have since been interpreted as impact melt breccias/clast-rich impact melt rocks [3, 4]. In this work, we estimate the emplacement temperature of the impact breccia, using a paleomagnetic method known as "conglomerate test." This consists in sampling oriented samples in several clasts in the breccia, and determine their paleomagnetic direction using thermal demagnetization. The direction will be scattered for emplacement at low temperature, and clustered for emplacement at high temperature.

We sampled a total of 20 oriented clasts in the impact breccia at two sites located $3 \mathrm{~km}$ apart. The paleomagnetic measurements show that all clasts give an identical paleomagnetic direction that is unblocked to at least $\sim 350^{\circ} \mathrm{C}$. This direction $(\mathrm{D}=, \mathrm{I}=$ a95) is well in agreement with the direction of the magnetic field during Eocene or younger times.

Most of the clasts consist of Paleozoic carbonate rocks whose main ferromagnetic mineral is pyrrhotite (as evidenced by our rock magnetism analyses) that has a Curie temperature of $350^{\circ} \mathrm{C}$. As a consequence, these clasts can provide only a lower limit for the emplacement temperature. However, some clasts consist of Precambrian basement rocks, and among this population a few ones are dominated by magnetite that has a Curie temperature of $585^{\circ} \mathrm{C}$. These latter clasts show two components of magnetization: one unblocked up to $\sim 350^{\circ} \mathrm{C}$ with the above-mentioned Neogene direction, and a second one unblocked between $\sim 350{ }^{\circ} \mathrm{C}$ and $580{ }^{\circ} \mathrm{C}$ which is random from clast to clast indicating that the magnetization was not completely reset by heating.

Although additional data are needed from magnetite-bearing clasts, these preliminary results suggest that when the impact melt rocks were emplaced, its temperature was clearly above $350^{\circ} \mathrm{C}$ and probably not much higher. This is of interest for reconstructing the $\mathrm{P}-\mathrm{T}$ history of the melt rocks and for cratering processes in general. The presence of shockmelted quartz clearly indicates that initial temperatures following decompression were $>1850^{\circ} \mathrm{C}[3,4]$ and possibly higher. These high temperatures occurred before emplacement of the impactites in to their final resting place (i.e., the outcrop locations we see at the present-day). The co-existence in the impact melt breccias of carbonate melts with liquidus temperatures of $\sim 500-600{ }^{\circ} \mathrm{C}$ [4], and clasts that were not heated above $350-400{ }^{\circ} \mathrm{C}$ seems to indicate that the temperature in the breccia was heterogeneous at small scale, and that the high clast content of these melt rocks have ensured rapid cooling. This is in agreement with a previous study showing temperature sensitive fossil biological signatures preserved in the same clasts [5].

References: [1] Osinski G. R. et al. 2005. Meteoritics \& Planetary Science 40:1759-1776. [2] Redeker H. J. and Stöffler D. 1988. Meteoritics 23:185-196. [3] Osinski G. R. and Spray J. G. 2001. Earth and Planetary Science Letters 194:17-29. [4] Osinski G. R. et al. 2005. Meteoritics \& Planetary Science 40:1789-1812. [5] Lindgren P. et al. 2006. Abstract \#1028. Lunar and Planetary Science Conference. 


\section{5}

OTISH BASIN: DISCOVERY OF NEARLY 2,1 GA SHOCKED ROCKS POTENTIALLY OWNING TO A D > 500 KM IMPACT STRUCTURE, QUÉBEC, CANADA

S. Genest ${ }^{1}$, F. Robert ${ }^{1}$ and N. Goulet ${ }^{2}$. ${ }^{1}$ Groupe Omégalpha Inc., Canada. E-mail: groupeomegalpha@bellnet.ca. ${ }^{2}$ Université du Québec à Montréal, Canada. ${ }^{1-2}$ Groupe de Recherche sur les Impacts Météoritiques de l'Institut Hubert-Reeves (GRIM-IHR).

The Otish Basin (OB) is located in northern Québec, Canada, and is only accessible by air transportation. The palaeoproterozoic sediments of the OB are known as the Otish Group (OG). They are mostly detrital in composition with minor carbonated material and lay unconformably on the archaean basement of the Superior Province, being limited to the south by the Grenville Province. The sedimentary pile of the OG has been studied by the first author in the 80 s while performing a basin analysis of the entire OB [1]. Roughly, the OB covers an area of about $5200 \mathrm{~km}^{2}$ and its thickness averages an estimated $2.4 \mathrm{~km}$. Four fining upward megacycles have been characterized, two of which, the older ones from the Indicator Formation, express reducing sedimentary environments. The two younger megacycles of the Peribonca Formation are well characterized by the oxidizing conditions of sedimentation. The evolution of the OB has been disturbed by an event which has been responsible for a widespread gabbro emplacement. Thought to be intrusive up to the $80 \mathrm{~s}$, more recent works showed that the gabbro has been emplaced in an extrusive manner according to their distribution and their physical relationship with the sediments. Shocked derived material (glass and PDFs) are observed in the sediments under the gabbro sheet and inside the large enclaves found in the gabbro itself. That discovery led to a preliminary re-examination of the thin sections already studied for the basin analysis in the $80 \mathrm{~s}$. By paying attention to the shock metamorphism effects, preliminary results allow us to postulate that the overall OB has been subjected to a major impact shockwave between the Indicator and the Peribonca times. Some poorly defined PDFs have been observed up to $1.06 \mathrm{~km}$ in depth from drill hole samples located in the center of the basin. The oxidized sediments of the Peribonca Formation are now thought to be intimately associated with the impact event, being coeval to the gabbro emplacement. According to the scale of the event we are facing, our working hypothesis is as followed (1) the OB has been subjected to the shockwave stress associated with a major impact event some $2.1 \mathrm{Ga}$ ago for which the postulated radius of the relevant structure is well over $250 \mathrm{~km}$; (2) since the Mistassini Basin (MB) shows sedimentary and structural similarities with the $\mathrm{OB}$ and because of the geomorphic characteristics of the $\mathrm{MB}$ showing analogy with the multiring impact structures, we consider $\mathrm{MB}$ as an element of our puzzling huge structure; and (3) the gabbro suite is interpreted as a melt sheet which is potentially linked to a large igneous province observed in the Grenville Province to the south, i.e., a gabbro-related anorthosite suite. For future reference, we propose to temporarily call this hypothetical structure the Mistassini-Otish Impact Structure.

Reference: [1] Genest S. 1989. Ph.D. thesis, Université de Montréal, Canada.
5314

THE ATMOSPHERIC ENTRY OF I-TYPE SPHERULES

M. J. Genge. Department of Earth Science and Engineering, Impact and Astromaterials Research Centre, Imperial College London, Exhibition Road, London SW7 2AZ, UK. E-mail: m.genge@imperial.ac.uk.

Introduction: Micrometeorites (MMs) are extraterrestrial dust particles $<2 \mathrm{~mm}$ in size recovered from the surface of the Earth and represent that fraction of the interplanetary dust population to survive atmospheric entry. The only iron-dominated particles found amongst MMs are I-type cosmic spherules (CSs) that occur as spheres dominated by wustite and/or magnetite, sometimes containing FeNi metal beads [1]. These particles, like silicate dominated (S-type) cosmic spherules, are formed by extensive melting during atmospheric entry. I-type spherules are present in small abundances of approximately $2 \%$ in Antarctic collections [1].

The absence of unmelted metallic MMs and the small abundance of I-type spherules is problematic due to the presence of ordinary chondritelike materials amongst MMs [2] and the large abundance of metal on these meteorite parent bodies. A numerical model of the entry heating of iron micrometeoroids is presented to evaluate whether the abundance of I-type spherules is a result of atmospheric heating or the nature of their parent bodies.

Numerical Methods: The numerical simulations used in this study are based on those of Love and Brownlee [3] and involve the approximation of the equations of motion and heat transfer of particles to determine temperature and mass loss through evaporation. Unlike previous studies [4], oxidation of metallic particles is included and was simulated by considering the mass of atmospheric oxygen encountered whilst particles were at supra-solidus temperatures. Mass loss of oxygen from molten particles was also calculated due to the evaporation of iron oxide liquids that wet the surface of the iron liquid droplets.

Results: Simulation results indicate that iron metal particles are more likely to reach their solidus temperatures $(1809 \mathrm{~K})$ than silicate particles with equivalent entry parameters due to their higher densities. Iron micrometeoroids are, however, likely to survive entry heating with less mass loss than S-type spherules due to the significantly lower evaporation rates of iron oxide liquids [5]. Contrary to observation, the abundance of I-type spherules would be expected to be enhanced compared with the abundance of metal on their parent asteroids. Metal bead survival is predicted to be greatest for particles that decelerate at the highest altitudes, or large particles that experience significant mass loss of oxide liquids from their surfaces.

Conclusion: The low abundance of I-type spherules amongst cosmic spherules is not a consequence of their atmospheric entry and suggests that either metal-poor parent bodies are common, or iron micrometeoroids have a larger mean size than silicates. The low abundance of metal beads amongst I-type spherules suggests that large, high velocity (cometary) iron micrometeoroids are relatively uncommon.

References: [1] Genge M. J. et al. 2008. Meteoritics \& Planetary Science 43:497-515. [2] Genge M. J. 2008 Geology 36:687-690. [3] Love S. G. and Brownlee. D. E. 1990. Icarus 89, 26-43. [4] Yada T. et al. 1996. Abstract \#1732. 28th Lunar and Planetary Science Conference. [5] Wang J. et al. 1994. Abstract \#1459. 25th Lunar and Planetary Science Conference. 
5312

\section{TEM-EELS STUDY OF TITANIUM OXIDATION STATE IN METEORITIC HIBONITES}

M. Giannini ${ }^{1}$, T. Boffa Ballaran ${ }^{1}$, F. Langenhorst ${ }^{2}$ and A. Bischoff . $^{3}$ ${ }^{1}$ Bayerisches Geoinstitut, Universität Bayreuth, D-95447 Bayreuth. E-mail: Mattia.Giannini@uni-bayreuth.de. ${ }^{2}$ Institut für Geowissenschaften, Universität Jena, D-07749 Jena. ${ }^{3}$ Institut für Planetologie, Universität Münster, D-48149 Münster.

Introduction: Besides corundum, hibonite is the most Al-rich phase found in CAIs. It is considered to be one of the first oxide phases that condensed from the hot solar nebula [1]. The formula of hibonite usually deviates from the ideal end-member composition $\left(\mathrm{CaAl}_{12} \mathrm{O}_{19}\right)$ due to the presence of variable amounts of minor element such as $\mathrm{Ti}, \mathrm{Mg}, \mathrm{Fe}, \mathrm{Si}$, and V [2]. Since Ti can occur in different oxidation states, it appears the ideal candidate for providing insight into the oxygen fugacity $\left(f \mathrm{O}_{2}\right)$ at which such mineral has formed and evolved in the solar nebula [3].

Experimental Methods: Three CAIs within two petrographic thin sections of chondrites Acfer 182 (CH; PL01036) and one of Dar al Gani (DAG) 203 (CO3; PL96274) were analyzed with a JEOL JXA-8200 electron microprobe. Electron-transparent sections of one hibonite grain from each CAI have been prepared using a FEI Quanta 3D FEG focused ion beam. Hibonites with the same compositions as the selected meteoritic crystals were also synthesized from oxide mixtures at $1430{ }^{\circ} \mathrm{C}$ for $12 \mathrm{~h}$ both in air and under controlled $f^{\mathrm{O}_{2}}$ in a gas mixing furnace using a $\mathrm{CO} /$ $\mathrm{CO}_{2}$ flux. Thin sections of synthetic hibonite pellets were subsequently thinned down to less than $100 \mathrm{~nm}$ by aid of a Gatan DuoMill 600 argon ion milling machine. The valence state of $\mathrm{Ti}$ was measured both for meteoritic and synthetic hibonites by means of electron energy loss spectroscopy (EELS) using a $200 \mathrm{keV}$ Philips CM20 scanning TEM, equipped with a Gatan PEELS 666 parallel electron spectrometer. The quantification technique used is based on the spectral features and intensities of the $\mathrm{L}_{3,2}$ white lines of Ti [4].

Results and Discussion: Chemical compositions and $\mathrm{Ti}^{3+}$ contents of meteoritic hibonites are reported in the following Table.

The content of $\mathrm{Ti}^{3+}$ in hibonite grains from CAIs is very similar with respect to the synthetic samples prepared at $-\log f \mathrm{O}_{2}=11$ (e.g., Acfer 182-3's analogous has $35 \%$ of $\mathrm{Ti}^{3+}$ ), indicating that this condition may reflect the redox environment in which hibonite has formed. However, even hibonites synthesized in air still have quite large amount of $\mathrm{Ti}^{3+}$ (e.g., $20 \%$ for Acfer 182-3's analogous), suggesting that its concentration does not only depend on the redox conditions at which hibonite formed, but also on its crystal chemistry. Even at very oxidizing conditions the substitution mechanism $\mathrm{Mg}^{2+}+\mathrm{Ti}^{4+}=2 \mathrm{Al}^{3+}$ is not unique and some $\mathrm{Ti}^{3+}=\mathrm{Al}^{3+}$ substitution takes place.

Acknowledgments: We are grateful for the financial support provided by the Deutsche Forschungsgemeinschaft (BO 2550 4-1, LA 830 14-1).

References: [1] Grossman L. 1972. Geochimica et Cosmochimica Acta 86:597-619. [2] Simons S. B. et al. 1997. Meteoritics \& Planetary Science 32:259-269. [3] Ihinger P. D. et al. 1986. Earth and Planetary Science Letters 78:67-79. [4] Stoyanov E. et al. 2007. American Mineralogist 92:577-586.

\begin{tabular}{lll}
\hline Sample & Composition & $\mathrm{Ti}^{3+} / \Sigma \mathrm{Ti}(\%)$ \\
\hline Acfer 182-2 & $\mathrm{CaA}_{11.51} \mathrm{Ti}_{0.80} \mathrm{Mg}_{0.69} \mathrm{O}_{19}$ & 20 \\
Acfer 182-3 & $\mathrm{CaA}_{11.45} \mathrm{Ti}_{0.36} \mathrm{Mg}_{0.23} \mathrm{O}_{19}$ & 35 \\
DaG 203-1 & $\mathrm{CaA}_{11.52} \mathrm{Ti}_{0.75} \mathrm{Mg}_{0.78} \mathrm{O}_{19}$ & 25 \\
\hline
\end{tabular}

5045

ALH 84001: THE KEY TO UNLOCKING SECRETS ABOUT MARS-15 YEARS AND COUNTING

E. K. Gibson ${ }^{1}$, D. S. McKay ${ }^{1}$, K. L. Thomas-Keprta ${ }^{2}$ and S. J. Clemett ${ }^{2}$. ${ }^{1}$ ARES, NASA/JSC, Mail Code KA, Houston, TX 77058, USA. ${ }^{2}$ ESCG at NASA/JSC, Mail Code JE23, Houston, TX 77058, USA. E-mail: everett.k.gibson@nasa.gov.

From the December 27, 1984, discovery of ALH 84001, and its subsequent identification as a sample of Mars in 1993, mystery and debate has surrounded the meteorite [1]. With the realization that the ALH 84001 sample was a orthopyroxenite and one of the oldest SNC meteorites ( $4.09 \mathrm{Ga})$ [2] available to study, important and critical information about the Martian hydrosphere and atmosphere along with the early history and evolution of the planet could be obtained by studying the unique carbonate globules $(\sim 3.9 \mathrm{Ga})$ in the sample [3]. The initial work showed the carbonate globules were deposited within fractures and cracks in the host-orthopyroxene by low-temperature aqueous fluids [4]. Ideas that the carbonates were formed at temperatures [5] approaching $800{ }^{\circ} \mathrm{C}$ were ruled out by later experiments [6].

The 1996 announcement by McKay et al. [7] that ALH 84001 contained features which could be interpreted as having a biogenic origin generated considerable excitement and criticism. The NASA Administrator Dan Golden said the 1996 ALH 84001 announcement "saved" NASA's Mars planetary exploration program and injected \$6 billion dollars over five years into the scientific research and analysis efforts [8]. All of the original four lines of evidence for possible biogenic features within ALH 84001 offered by McKay et al. have withstood the test of time. Criticism has been directed at the interpretation of the 1996 analytical data. Research has expanded to other SNC meteorites. Despite the numerous attacks on the ideas, the debate continues after 15 years. The 2009 paper by ThomasKeprta et al. [9] on the origins of a suite of magnetites within the ALH 84001 has offered strong arguments that some of the magnetites can only be formed by biogenic processes and not from thermal decomposition or shock events which happened to the meteorite.

NASA's Astrobiology Institute was formed from the foundation laid by the ALH 84001 hypothesis of finding life beyond the Earth. The strong astrobiology outreach programs have expanded because of the work done on the Martian meteorites. Despite the criticism on the biogenic-like features in ALH84001, the meteorite has opened a window into the early history of Mars. Clearly low-temperature fluids have left their signatures within the ALH 84001 meteorite and subsequent cratering events on Mars have been recorded on observable features within the meteorite. The 15 years of detailed study on ALH 84001 and its unique carbonate globules have clearly shown formational and secondary processes at work on Mars. Now we need a well-documented Mars sample return mission.

References: [1] Meyer C. 1996. Mars Meteorite Compendium-1996. NASA-JSC. [2] Lapen T. J. et al. 2010. Science 3328:347-351. [3] Borg L. et al. 1999. Science 286:90-94. [4] Romanek C. S. et al. 1994. Nature 372:655-657. [5] Harvey R. P. and H. Y. McSween 1996. Nature 382:4951. [6]Valley J. W. et al. 1997. Science 275:1633-1638. [7] McKay D. S. et al. 1996. Science 273:924-930. [8] Sawyer K. 2006. The rock from Mars. Randon House. 416 p. [9] Thomas-Keprta K. L. et al. 2009. Geochimica et Cosmochimica Acta 73:6631-6677. 
5371

LA-ICPMS TRACE ELEMENT COMPOSITION OF METAL AND SULFIDE IN 3 ORDINARY CHONDRITE FALLS

M. D. Giscard ${ }^{1,2}$, S. J. Hammond ${ }^{3}$, G. K. Benedix ${ }^{1,2}$, P. A. Bland ${ }^{1,2,4}$ and N. W. Rogers ${ }^{3}$. ${ }^{1}$ Impacts and Astromaterials Research Centre, Department of Earth Science and Engineering, Imperial College, London, UK. E-mail: m.giscard10@imperial.ac.uk. ${ }^{2}$ Impacts and Astromaterials Research Centre, Department of Mineralogy, Natural History Museum, London, UK. ${ }^{3}$ Department of Earth Sciences, The Open University, Milton Keynes, UK. ${ }^{4}$ Department of Applied Geology, Curtin University of Technology, Perth, Australia.

Introduction: Trace element compositions are a particularly powerful tool for the study of geochemical processes. Indeed, variations of compositions can span several orders of magnitude [1] and the elements themselves have a wide range of condensation temperatures and geochemical behaviors [2]. Chondrites show different trace elements depletion patterns; their cause is still subject to debate [2-4]. Some elemental concentrations might vary with petrological type, this would mean that the trace element composition is also influenced by metamorphism [4-6]. We hope to contribute to these debates by using LA-ICPMS to provide quantitative trace element abundances for chondritic metal and sulfide. Our first test samples were three equilibrated ordinary chondrite falls: Butsura (H6), Ogi (H6), and Mezo-Madaras (L6 fragment of L3.7-6 breccia).

Method: In situtrace elements measurements have been performed using the LA-ICPMS at the Open University with LASER settings of 80 $\mu \mathrm{m}$ spot size, 10 passes $5 \mu \mathrm{m}$ deep on each point and a 180-200 s washout time between points. Furthermore, $\mathrm{Fe}, \mathrm{Ni}$, and $\mathrm{Co}$ concentrations have been measured by Electron Micropobe at the Natural History Museum and $\mathrm{Fe}$ concentration used as an internal standard for LAICPMS measurements. The CI composition and volatility sequence used for interpreting the trace elements data is from [7].

Results and Discussion: Average Co concentration in kamacite are $5.0 \pm 0.2 \mathrm{mg} \mathrm{g}^{-1}$ in Butsura, $5.0 \pm 0.8 \mathrm{mg} \mathrm{g}^{-1}$ in Ogi and $8.4 \pm 0.2$ $\mathrm{mg} \mathrm{g}^{-1}$ in Mezo-Madaras, which is consistent with the litterature [8, 9 , 10]. 19 trace elements were above detection limits in metal and sulfide grains: four moderately volatile/normally depleted elements $(\mathrm{Cu}, \mathrm{Ag}, \mathrm{Ga}$, and $\mathrm{Ge})[3,4]$ and 15 more refractory elements (Re, Os, W, Ir, Mo, Ru, $\mathrm{V}, \mathrm{Pt}, \mathrm{Rh}, \mathrm{Pd}, \mathrm{Cr}, \mathrm{P}, \mathrm{Mn}, \mathrm{As}$, and $\mathrm{Au}$ ). Individual metal and sulfide grains show a depletion pattern when elements are ordered in a volatility sequence. Individual kamacite grains show a similar pattern while variations in the $\mathrm{Ni}$-rich metal trace element composition seems correlated to the difference in $\mathrm{Ni}$ concentration. Sulfide composition is the most widespread - it could be caused by matrix effects related to the choice of standard. We will also discuss the effect of proximity to the fusion crust on trace elements results.

Acknowledgments: The first author has been funded by the Marie Curie Research Training network Origin's.

References: [1] Shaw D. M. 2006. In Trace elements in magmas-A theoretical treatment. p. 2. [2] Bland P. A. et al. 2005. Proceedings of the National Academy of Sciences 102:13755-13760. [3] Larimer J. W. and Anders E. 1967. Geochimica et Cosmochimica Acta 31:1239-1270. [4] Wasson J. T. and Chou C.-L. 1974. Meteoritics \& Planetary Science 9:6984. [5] Keays R. R. et al. 1970. Geochimica et Cosmochimica Acta 35: 337-363. [6] Takahashi H. et al. 1978. Geochimica et Cosmochimica Acta 42:1859-1869. [7] Lodders K. 2003. The Astrophysical Journal 591:12201247. [8] Afiattalab F. and Wasson J. T. 1979. Geochimica et Cosmochimica Acta 44:431-446. [9] Sears D. W. and Axon H. J. 1976. Nature 260:34-35. [10] Kallemeyn G. W. et al. 1989. Geochimica et Cosmochimica Acta 53:2747-2767.

\section{5}

VARIATION IN DISTAL IMPACT EJECTA WITH DISTANCE FROM THE SOURCE CRATER: A MODEL

B. P. Glass ${ }^{1}$ and B. M. Simonson ${ }^{2}$. ${ }^{1}$ Department of Geological Sciences, University of Delaware, Newark, DE 19716, USA. E-mail: bglass@ udel.edu. ${ }^{2}$ Geology Department, Oberlin College, Oberlin, OH, 44074, USA. bsimonso@ oberlin.edu.

Introduction: We have used information from six Phanerozoic ejecta/spherule layers to construct a model on how distal impact ejecta vary with distance from the source crater. The model appears to be consistent with theoretical studies of the cratering process and observational data from most Phanerozoic and late Precambrian distal ejecta layers, but not from older ( $>2.48 \mathrm{Ga})$, especially Archean spherule layers.

Impact Spherules: Distal impact ejecta layers consist primarily of spherules. There are two types of impact spherules: microtektites which do not contain primary crystallites and microkrystites which do. These two types combined with two mechanisms of formation (melt droplets versus condensate droplets) result in four possible categories of impact spherules: (1) microtektites produced as melt droplets, (2) microtektites formed as condensate droplets, (3) microkrystites formed as melt droplets, and (4) microkrystites formed as condensate droplets.

The Model: As an ejecta layer thins away from the source crater, the average degree of shock metamorphism increases and therefore the percent melt (spherules) increases. Beyond $\sim 10$ crater diameters an ejecta layer consists primarily of spherules and beyond $\sim 100$ crater diameters an ejecta layer is essentially a spherule layer containing little, if any, unmelted ejecta $>125 \mu \mathrm{m}$. Thus, an ejecta layer from a crater $>\sim 200 \mathrm{~km}$ in size will contain unmelted, as well as melted ejecta, globally. On average the petrographic and chemical homogeneity of melt-produced microtektites increases with distance from the source crater (i.e., the number of relict inclusions, vesicles, and schlieren decreases away from the source crater) [1]. The ratio of spherule fragments to whole spherules increases toward the source crater probably due to fragmentation caused by thermal stress as they land in water and/or to collisions between the microtektites in the atmosphere. The latter mechanism is supported by an increase in number of high-velocity impact pits per unit surface area on the microtektites toward the source crater [2]. The average size of the microtektites appears to increase toward the source crater beyond $\sim 100$ crater diameters, but closer in that trend reverses due to the increase in fragmentation. The maximum size of shocked-quartz grains increases toward the source crater, but the average degree of shock metamorphism, as indicated by the average number of sets of PDFs, increases away from the crater [3].

Precambrian Spherule Layers: Spherule layers from at least 11 impacts $>2.4 \mathrm{Ga}$ have been identified. The aggregate thicknesses of these layers are generally greater than the thicknesses of the Phanerozoic ejecta layers, but none of these older layers contain shocked-mineral grains except for trace amounts in four layers that were probably formed by just two impacts. Thus, these spherule layers do not fit the Phanerozoic model. This may be because the target rocks were mafic and/or because of much larger impact sizes as suggested by the large sizes of some of the spherules and the greater thicknesses of the spherule layers.

References: [1] Folco et al. 2010. Geology 38:211-214. [2] Prasad et al. 2010. Meteoritics \& Planetary Science 45:990-1006. [3] Morgan et al. 2006. Earth and Planetary Science Letters 251:264-279. 


\section{8}

ASH SHUTBAH: A NEW IMPACT CRATER IN SAUDI ARABIA

E. Gnos ${ }^{1}$, A. Al-Wagdani ${ }^{2}$, A. Mahjub ${ }^{2}$, A. A. Al-Solami ${ }^{2}$, S. H. Allah ${ }^{2}$, A. Matter ${ }^{3}$, B. A. Hofmann ${ }^{4}$, M. Schmieder ${ }^{5}$ and E. Buchner ${ }^{5}{ }^{1}$ Natural History Museum, Geneva. E-mail: edwin.gnos@ville-ge.ch. ${ }^{2}$ Saudi Geological Survey, Jeddah, Kingdom of Saudi Arabia. ${ }^{3}$ Institut für Geologie, Universität Bern, Switzerland. ${ }^{4}$ Natural History Museum Bern, Switzerland. ${ }^{5}$ Institut für Planetologie, University of Stuttgart, Germany.

Introduction: Although Saudi Arabia covers an area of more than two million square kilometers, only the three small, 3300 -year-old Wabar impact craters located in sand dunes of the Rub al Khali have been confirmed on the ground $[1,2]$. Three additional circular features, the $\sim 5 \mathrm{~km}$ Jabal Rayah, the $\sim 2.5 \mathrm{~km}$ Ash Shutbah, and the $\sim 17 \mathrm{~km}$ Wadi $\mathrm{Na}$ 'am have been proposed as possible impact craters $[3,4]$.

Ash Shutbah: The Ash Shutbah impact crater is located in flat lying limestones of the Middle Jurassic Tuwayq Mountain Formation at $21^{\circ} 37^{\prime} \mathrm{N} / 45^{\circ} 39^{\prime} \mathrm{E}, \sim 50 \mathrm{~km} \mathrm{~S}$ of the village of $\mathrm{Al}$ Haddar. It is located at the edge of the Jabal Tuwayq escarpment. A set of circular features is easily recognizable on satellite images, and two branches of the upper Wadi Ash Shutbah follow the outermost circular feature. The morphological expression as revealed by Shuttle radar data (SRTM) is very weak [4]. Nonetheless, due to lithologic contrast, a dark central area is clearly distinguishable on satellite images.

Field Observations: A 4-day exploration in the Ash Shutbah area in May 2011 confirmed that the morphological expression of the Ash Shutbah impact crater has been strongly reduced by desert alteration and wind erosion. Only one possible shatter cone was found in massif sandstone of the central uplift. The weakly expressed central uplift consists of strongly folded, creamy calcareous sandstone of the Dhruma formation containing a $60-\mathrm{cm}$ thick, black weathering, massif sandstone bed. This marker bed has been located in the escarpment outcrop at ca. $130 \mathrm{~m}$ below the plateau, indicating a minimal uplift of ca. $140 \mathrm{~m}$, consistent with estimates obtained on complex impact craters of similar size [5]. Outward dipping radial folds occur in the external part of the central uplift. The outer parts of the crater consist of deformed Tuwayq Mountain limestone sheets forming circular rings of hills and depressions.

Conclusions: The presence of folded beds and a possible shatter cone confirm that the Ash Shutbah circular feature is a complex meteorite impact crater that is younger than the middle Jurassic limestones of the lower Tuwayq Mountain formation. The presence of an escarpment and a characteristic marker bed provide a unique situation to determine minimum uplift directly.

References: [1] Philby H. S. J. B. 1933. The Geographical Journal 81:1-21. [2] Wynn J. C. 2002. Journal of Environmental \& Engineering 7:143-150. [3] Janjou D., Wyns R., Halawani M. A. and Memesh A. 2004. Saudi Geological Survey Data-file report SGS-DF-2004-3. [4] Schmieder M., Buchner E., Hofmann B. A. and Gnos E. 2009. Abstract, The first Arab Impact Cratering and Astrogeology Conference, Amman, Jordan, 84-85. [5] Grieve R. A. F. 1987. Annual Reviews in Earth and Planetary Science 15:245-270.
5338

EL'GYGYTGYN IMPACT CRATER'S FIREBALL LAYER: SPHERULES, IMPACT MELTS, METEORITIC COMPONENT

S. Goderis ${ }^{1}$, M. Elburg ${ }^{2}$, F. Vanhaecke ${ }^{3}$, Ph. Claeys ${ }^{1}$, A. Wittmann ${ }^{4}$, and the El'gygytgyn Scientific Party. ${ }^{1}$ Vrije Universiteit Brussel, Earth System Science, Department of Geology, B-1050 Brussels, Belgium. E-mail: steven.goderis@vub.ac.be, phclaeys@vub.ac.be. ${ }^{2}$ Ghent University, Department of Geology \& Soil Sciences, B-9000 Ghent, Belgium. E-mail: marlina.elburg@ugent.be. ${ }^{3}$ Ghent University, Department of Analytical Chemistry, B-9000 Ghent, Belgium. E-mail: frank.vanhaecke@ugent.be. ${ }^{4}$ Lunar \& Planetary Institute, Houston, TX 77058, USA. E-mail: wittmann@1pi.usra.edu.

Introduction: The $18 \mathrm{~km} \varnothing$ El'gygytgyn impact crater in NE Siberia formed 3.6 Ma ago in a target of $83.2-89.3 \mathrm{Ma}$ old, $\sim 620 \mathrm{~m}$ thick, rhyolitic, andesitic, and dacitic volcanics [1, 2]. An International Continental Drilling Program (ICDP) project recovered drill cores from an inner-crater continuous section of impactite deposits that contain scarce, pristine impact melt particles and glass spherules [3, 4]. These deposits allow studying the mixing of target rock components and the meteoritic projectile.

Samples and Methods: Three glassy impact melt rocks that were collected near the W' rim of the crater and 17 samples from the ICDPLake Drilling Hole D1 were investigated. Powdered bulk samples were characterized for major and trace element abundances, including the platinum group elements (PGE), using ICP-OES and ICP-MS. The compositions of three glassy impact melt rocks, $11 \sim \mathrm{mm}$-size glassy melt particles, and seven glass spherules in suevitic rocks of the upper $\sim 10 \mathrm{~m}$ of impactite drill core were determined with electron microprobe X-ray analysis.

Results: Major and trace element concentrations for bulk rock samples are in good agreement with previously published data [3,5] and indicate a moderately homogeneous rhyo-dacitic drill core. A single sample at $391.6 \mathrm{~m}$ has a basaltic-trachyandesitic composition and higher than normal concentrations of $\mathrm{Ti}, \mathrm{Fe}, \mathrm{Mn}, \mathrm{Mg}, \mathrm{Ca}, \mathrm{Sc}, \mathrm{V}, \mathrm{Cr}, \mathrm{Co}, \mathrm{Ni}$, and $\mathrm{Zn}$. Although major and trace elements show typical crustal values and ratios (e.g., subchondritic $\mathrm{Ni} / \mathrm{Cr}$ of $\sim 0.30-0.60$ ), PGE abundances revealed the presence of a meteoritic component $(\sim 0.05 \mathrm{wt} \%)$ in three samples. The 11 glassy melt particles and the three glassy melt rocks have rhyolitic compositions. In contrast, six spherules range in composition from rhyolitic, dacitic, trachytic, and andesitic.

Discussion: Scaling laws suggest the depth of the melt zone at El'gygytgyn was $1.5-2.6 \mathrm{~km}$ [6]. Nonetheless, the compositions of glassy melt particles and impact melt rocks indicate melt-mixtures solely derived from the $\sim 620 \mathrm{~m}$ thick volcanic succession of rhyolites, dacites, and andesites [5]. In contrast, five of the six spherules record contribution from a mafic target component [7] that is known from regional outcrops and clasts in the ICDP drill core [3,4]. Thus, the impact melts in suevite deposits appear homogenized but the spherules that presumably condensed from the vapor plume record heterogeneities of an extended target section. A diluted projectile component was detected in a spherulebearing drill core sample and two melt rock samples.

References: [1] Gurov et al. 2007. Meteoritics \& Planetary Science 42:307-319. [2] Gurov and Gurova. 1991. Geological structure and rock composition of impact structures, Naukova Dumka Press, Kiev, pp. 160. [3] Raschke et al. 2011. Abstract \#1299. 42nd Lunar and Planetary Science Conference. [4] Wittmann et al. 2011. Abstract \#2792. 42nd Lunar and Planetary Science Conference. [5] Gurov et al. 2005. GSA Special Paper 384:391-412. [6] Grieve and Cintala. 1992. Meteoritics 27:526-538. [7] Adolph and Deutsch. 2009. Abstract \#1116. 40th Lunar and Planetary Science Conference. 
5067

\section{THERMAL HISTORY OF METAL PARTICLES IN CB} CHONDRITES

J. I. Goldstein ${ }^{1}$, R. H. Jones ${ }^{2}$, P. G. Kotula ${ }^{3}$ and J. R. Michael ${ }^{3}$. ${ }^{1}$ University of Massachusetts, Amherst, MA 01003, USA. E-mail: jig0@ecs.umass.edu. ${ }^{2}$ University of New Mexico, Albuquerque, NM. ${ }^{3}$ Sandia National Laboratory, Albuquerque, NM, USA.

Introduction: The CB chondrites are part of the "CR clan," a group of metal-rich chondrites that include $\mathrm{CH}, \mathrm{CB}$ and $\mathrm{CR}$ groups $[1,2]$. $\mathrm{CB}$ chondrites have more metal than $\mathrm{CH}$ and much larger metal particles: both groups may have formed from a similar type of impact event [3]. Many metal grains in $\mathrm{CH}$ chondrites show evidence for reheating in the form of two-phase metal particles [4]. In general such textures are not observed in metal from $\mathrm{CBa}$ chondrites. Plastically deformed and two phase metal particles have been observed in $\mathrm{CBb}$ chondrites [5]. The purpose of this study is to obtain microstructural and microchemical information at the $\mathrm{nm}$ to $\mu \mathrm{m}$ scale for two-phase metal particles in $\mathrm{CBa}$ Gujba and $\mathrm{CBb} \mathrm{HaH} 237$ chondrites in order to understand the detailed thermal histories of $\mathrm{CB}$ chondrites.

Method: Metal particles were observed optically and selected particles were thinned using focused ion beam techniques for transmission electron microscopy (TEM). Chemistry at the nm level was obtained by $\mathrm{X}$-ray analysis in the TEM and structure analysis was performed using electron backscatter diffraction (EBSD) in the scanning electron microscope (SEM).

Results: We observed one metal particle in Gujba that contained about 30 grains $\sim 0.1 \mathrm{~mm}$ in size with different orientations. Each grain had a two phase microstructure containing $\mu \mathrm{m}$ sized taenite in a recrystallized kamacite matrix. Kamacite and taenite contain 5.5 and $10.5 \mathrm{wt} \% \mathrm{Ni}$ respectively. We estimate, using the calculation procedures of [6], that this metal particle was reheated to $\sim 675^{\circ} \mathrm{C}$ and cooled to $\sim 550{ }^{\circ} \mathrm{C}$ in a month or less. A two phase metal particle in $\mathrm{HaH} 237$ was plastically deformed at temperatures above $500{ }^{\circ} \mathrm{C}$, and reheated at about $640{ }^{\circ} \mathrm{C}$. It has a microstructure similar to grains investigated by Meibom et al. [5]. The taenite and kamacite have a close packed bcc-fcc orientation relationship indicating the exsolution of taenite in kamacite at the reheating temperature. Grain boundary diffusion at the $\mathrm{nm}$ level indicates cooling to $\sim 500-550^{\circ} \mathrm{C}$ in a month or less.

Discussion: Evidence of reheating in $\mathrm{CB}$ metal particles is consistent with a process in which discrete metal and silicate particles, which possibly originated in an impact plume, were accreted onto a parent body and later subjected to a secondary heating event. Initial formation of metal could have included both condensation of solid metal and solidification of liquid metal. Later heating could be the same as the one in which impact-melt matrix was injected into pre-existing metal and silicate components [7]. This may have reheated metal particles selectively, allowing for plastic deformation and formation of taenite from a kamacite matrix in some metal particles.

References: [1] Weisberg M. K. et al. 2001. Meteoritics \& Planetary Science 36:401-418. [2] Krot A. N. et al. 2002. Meteoritics \& Planetary Science 37:1451-1490. [3] Krot A. N. et al. 2005. Nature 436:989-992. [4] Goldstein J. I. et al. 2007. Meteoritics \& Planetary Science 42:913-933. [5] Meibom A. et al. 2005. Meteoritics \& Planetary Science 40:1377-1391. [6] Smith B. A. and Goldstein J. I. 1977. Geochimica et Cosmochimica Acta 41:1061-1072. [7] Weisberg M. K. and Kimura M. 2011. Meteoritics \& Planetary Science 45:873-884.
5066

PRELIMINARY INVESTIGATION INTO THE USE OF SURFACE MODIFICATION TECHNIQUES TO DETECT ORGANIC MATERIALS IN METEORITES

M. D. Goodyear ${ }^{1,2}$, I. Gilmour ${ }^{1}$ and V. K. Pearson ${ }^{1,2}$. ${ }^{1}$ Planetary and Space Sciences Research Institute, The Open University, Milton Keynes, MK7 6AA, UK. ${ }^{2}$ Department of Chemical and Analytical Sciences, The Open University, Milton Keynes, MK7 6AA, UK. E-mail: m.d.goodyear@open.ac.uk.

Introduction: Many carbonaceous chondrites (CCs) display evidence of aqueous and/or thermal alteration of their component minerals [1]. In addition, CCs also contain up to ca. 5\% carbon [2], much of which is organic, insoluble, involatile and unreactive, and known as insoluble organic material (IOM). It is not known if there is a causal connection between the mineral alteration, and formation or modification of organic materials [3]; however, by understanding the relationships between them, any connections (chemical or physical) could be established.

Analytical Constraints: Organic analysis of CCs generally requires extraction or demineralization; mineralogy is often studied in polished surfaces or thin section. None of these methods is suitable for this study, as all spatial information would either be lost, or compromised (e.g. by mechanical redistribution). Scanning electron microscopy (SEM), and Raman spectroscopy are useful complementary techniques, requiring minimal surface preparation and yielding information in situ, on both the mineralogy (from SEM) and organic content (from Raman) of a region under study. It is essential the sample be accurately aligned and imaged, so the same area is studied using both methods.

Experimental: We present preliminary work directed towards the application of molecular tags, whereby more-easily detectable atoms or functional groups (i.e. those not typically present in untreated meteorites) are attached to the IOM.

Ozonolysis: To increase its reactivity to tagging, IOM is pre-treated with ozone, to introduce oxygen-containing functional groups, such as carboxylic acids or ketones. The resulting compounds can then be converted into a range of derivatives containing the chosen tag. Conditions for ozonolysis were developed by following the reaction of polycyclic aromatic hydrocarbon (PAH) standards, typical of those believed to make up the IOM backbone [4], on inert mineral supports, e.g. silica or sandstone. Samples of Murchison were ozonized and examined by SEM and Raman spectroscopy, the latter incorporating image processing to ensure in each case, the same sample region was being studied.

Molecular Tagging: Reaction conditions have been developed to enable the introduction of a range of molecular tags into ozonolysisgenerated oxygen-containing functional groups, initially using model compounds again supported on inert minerals. Using these methods, standard acids (carboxylic and amino-), alcohols, phenols, aldehydes, and ketones have been tagged with fragments containing fluorine or chlorine; work to apply this methodology to meteorites is ongoing.

Results: Good-quality, aligned and focused images were obtained using both SEM and Raman, allowing mapping of elemental abundance and organic content. The derivatization of IOM by ozonolysis (indicated by the changes observed in the Raman signal), shows that the IOM can next be subjected to molecular tagging.

References: [1] Brearley A. J. 2005. Treatise on Geochemistry vol. 1, 1.09:247-268. [2] Sephton M. 2002. Natural Product Reports 19:292-311. [3] Zega T. J. et al. 2010. Geochimica et Cosmochimica Acta 74:5966-5983. [4] Derrene S. and Robert F. 2010. Meteoritics \& Planetary Science 45:1461-1475. 
5512

\section{SLOPE 1, OR NOT SLOPE 1, IS THAT THE QUESTION?}

R. C. Greenwood ${ }^{1}$, I. A. Franchi ${ }^{1}$ and C. T. Pillinger ${ }^{1}$. ${ }^{1}$ PSSRI, Open University, Walton Hall, Milton Keynes MK7 6AA, UK. E-mail: r.c.greenwood@open.ac.uk.

CCAM v. Slope 1 Line: The process involved in producing the non mass-dependent oxygen isotope variation observed in primitive meteorites is poorly understood. While self-shielding of $\mathrm{CO}$ [1-3] is currently favoured, alternative scenarios have been proposed [4]. An important aspect of this problem relates to the significance of various reference lines on oxygen isotope plots. The most widely used, the carbonaceous chondrite anhydrous mineral line (CCAM), has a slope of 0.94 and was derived from analyses of Allende mineral separates [5, 6]. However, its significance has been questioned and instead a line of slope 1 proposed as more representative of the primordial variation [7]. The fact that a highly ${ }^{17,18} \mathrm{O}$-enriched phase in Acfer 094 plots on the slope 1 line lends support to this proposal [8].

High-Precision Studies: Important insights into the relative significance of the slope 1 and CCAM lines are provided by laser fluorination studies. Bulk CR chondrites define a line of slope 0.70 , with less aqueously altered samples plotting close to the slope 1 line [9]. This suggests that the composition of pristine $\mathrm{CR}$ precursor material may be given by the point at which the CR mixing line intersects the slope 1 line. The acapulcoite-lodranite clan and winonaites also form distinct linear arrays plotting between the slope 1 and CCAM lines. Winonaites define a slope 0.54 line, with the less evolved chondrule-bearing samples plotting at the lower $\delta^{18} \mathrm{O}$ values, i.e., closer to the slope 1 line. Acapulcoite and lodranite analyses define a shorter array, with a steeper slope (0.61). Prior to metamorphism the clan may have experienced aqueous alteration, so that their precursor material would also have had a composition closer to the slope 1 line.

Discussion: While the primary significance of the slope 1 line is supported by the evidence discussed above, the relevance of the CCAM line is based on the fact that a significant range of meteorite groups, and their constituents, fall either on, or close to it $[5,6]$. The suggestion that the lower slope of the CCAM simply reflects late stage disturbance of the slope 1 line requires further evaluation [7]. Based on analyses of Acfer 094 chondrules, a further distinct slope 1 line has recently been proposed [10]. This again indicates that near-slope 1 variation is a characteristic feature of a wide range of primitive materials. Slope values between $\sim 0.6$ and 1.8 have been measured in $\mathrm{CO}$ photodissociation experiments [11], as a result there is currently a debate about the extent to which CO selfshielding was responsible for the slope 1 anomaly observed in primitive meteorites [12].

References: [1] Clayton R. N. 2002. Nature 415:860-861. [2] Yurimoto H. and Kurimoto K. 2004. Science 305:1763-1766. [3] Lyons J. R. and Young E. D. 2005. Nature 435:317-320. [4] Dominguez G. 2010. The Astrophysical Journal 713:L59-L63. [5] Clayton R. N. et al. 1977. Earth and Planetary Science Letters 34:209-224. [6] Clayton R. N. and Mayeda T. K. 1999. Geochimica et Cosmochimica Acta 63:2089-2104. [7] Young E. D. and Russell S. S. 1998. Science 282:452-455. [8] Sakamoto N. et al. 2007. Science 317:231-233. [9] Schrader D. L. et al. 2011. Geochimica et Cosmochimica Acta 75:308-325. [10] Ushikubo T. et al. 2011. Abstract \#1183. 42nd Lunar and Planetary Science Conference. [11] Chakraborty S. et al. 2008. Science 321:1328-1331. [12] Lyons J. R. 2011. Abstract \#2780. 42nd Lunar and Planetary Science Conference.
5337

REFLECTANCE SPECTRA OF CARBONACEOUS CHONDRITES UNDER CONTROLLED ATMOSPHERE

F. Grisolle ${ }^{1}$, P. Beck ${ }^{1}$, E. Quirico ${ }^{1}$, B. Schmitt ${ }^{1}$ and F. Moynier ${ }^{2} .{ }^{1}$ UJFGrenoble 1/CNRS-INSU, Institut de Planetologie et d'Astrophysique de Grenoble (IPAG), France. E-mail: pierre.beck@obs.ujf-grenoble.fr. ${ }^{2}$ Department of Earth and Planetary Sciences, Washington University, St. Louis, MO, USA.

Introduction: Carbonaceous chondrites are fine-grained mixtures of silicates, organics and metals. Their connection with asteroid has been studied over the last decades using similarities in the near-infrared reflectance spectra. One of the particularities of carbonaceous chondrites among other chondrites group, is the presence of hydrated silicates, testifying of the action of water at some time of their formation history. Here, we report on the NIR spectra of four carbonaceous chondrites (Tagish Lake [ungrouped CC], Orgueil [CI1], Murchison [CM2] and Allende [CV3]). The specificity of this study is that we pay particular attention to the $3-\mu \mathrm{m}$ region and then, spectra were measured under controlled atmosphere in order to avoid contamination by indigenous water

Reflectance Spectroscopy: Infrared reflectance spectra were measured using the spectrogonio radiometer available at the Laboratoire de Planétologie de Grenoble [1]. This instrument enables bi-directionnal reflectance spectra measurements with a good photometric accuracy (below $1 \%$ ). Spectra were measured in the $0.5-4.6 \mu \mathrm{m}$ range with a sampling interval of $20 \mathrm{~nm}$. Because ambient atmospheric water might adsorb on the surface of the sample and perturb the $3-\mu \mathrm{m}$ band, spectra were acquired within the SERAC environmental cell [2]. Samples were heated up to $80^{\circ} \mathrm{C}$ under vacuum $\left(\mathrm{P}=10^{-3} \mathrm{mbar}\right)$ to remove adsorbed water. Spectra were measured under normal illumination incidence, and with an observation angle of $30^{\circ}$.

The 3-micron Band: - $\mathrm{OH}$ (and $\mathrm{H}_{2} \mathrm{O}$ ) For all three samples, a spectrum was measured after and prior to submitting the sample to a controlled atmosphere. All spectra reveal the presence of a $3-\mu \mathrm{m}$ band, but which is much weaker for Allende (Band Depth (BD) of $11 \%$ ) and stronger for Orgueil, (BD of $60 \%$ ) and Murchison (BD $=47 \%)$. In the case of Orgueil and Murchison, a significant evolution is observed after heating to $80^{\circ} \mathrm{C}$ and exposure to vacuum, suggesting that a significant amount of indigenous water was lost. This observation confirms previous work performed using transmission spectroscopy [3]. We show here, the shape, band maximum and band center evolves as adsorbed and mesopore water is removed and dry condition reflectance spectra are needed for a good comparison with asteroid observation.

Organics Signature: $\mathrm{CH}_{2}$ and $\mathrm{CH}_{3}$. The $3-\mu \mathrm{m}$ region is also precious since traces or organics can be observed around $3.4 \mu \mathrm{m}$ [4]. These signatures are clearly detected in the case of Orgueil, Tagish Lake and Murchison, but hardly for Allende. This difference can be explained by the fact that the proportion of organic mater in Allende is smaller than the other two samples, and that the object was slightly metamorphosed.

References: [1] Brissaud et al. 2004. Applied Optics 43:1926-1937. [2] Pommerol et al. 2009. Icarus 204:114-136. [3] Beck at al. 2010. Geochimica et Cosmochimica Acta 74:8881. [4] Moroz et al. 1998. Icarus 134:253-258. 
5308

MICROSTRUCTURAL HETEROGENEITY OF METAL IN CHINGA METEORITE: STUDY USING MÖSSBAUER SPECTROSCOPY WITH A HIGH VELOCITY RESOLUTION

V. I. Grokhovsky ${ }^{1}$, M. I. Oshtrakh ${ }^{1}$, K. A. Uymina ${ }^{1}$, M. V. Goryunov ${ }^{1,2}$ and V. A. Semionkin ${ }^{1,2}$. ${ }^{1}$ Faculty of Physical Techniques and Devices for Quality Control, Ural Federal University, Ekaterinburg, 620002, Russian Federation. E-mail: grokh47@mail.ru. ${ }^{2}$ Faculty of Experimental Physics, Ural Federal University, Ekaterinburg, 620002, Russian Federation.

Introduction: Iron meteorite Chinga was classified as ataxite IVB with about $16.8 \mathrm{wt} . \%$ of $\mathrm{Ni}$. The main part of metal consisted of dispersive multi-phase structure that was not optically resolved. It is well know the presence of macroscopic selective reflection zones (Schliren bands) in ataxites IVB [1]. Previously it was conclude that the origin of these zones is due to the martensite transformation peculiarities [2]. Recently we have observed another macroscopic zone in a peripheral part of the Chinga meteorite fragments. Therefore, we used the high velocity resolution Mössbauer spectroscopy for comparative study of microstructural peculiarities of this new found zone and internal part of Chinga metal.

Experimental: The typical relief zone along the periphery of the Chinga meteorite fragment was clearly seen after mechanical cutting of the fragment. Its width varies from approximately $0.5 \mathrm{~cm}$ to approximately $1.5 \mathrm{~cm}$. Samples of metal powder were prepared from this zone and internal part of Chinga fragment for Mössbauer study. Mössbauer spectra were measured using automated precision Mössbauer spectrometric system with a high velocity resolution [3] at room temperature in 4096 channels.

Results and Discussion: Mössbauer spectra of studied samples from different parts of Chinga fragment were similar asymmetrical six lines patterns. However, these spectra appeared to be slightly different that may be seen in difference between Mössbauer spectra of studied parts of Chinga fragment. Measured Mössbauer spectra were better fitted using nine magnetic sextets. These sextets may be a result of the presence of kamacite $\alpha-\mathrm{Fe}(\mathrm{Ni}, \mathrm{Co})$, martensite $\alpha_{2}-\mathrm{Fe}(\mathrm{Ni}, \mathrm{Co})$, secondary kamacite $\alpha^{\prime}-\mathrm{Fe}(\mathrm{Ni}, \mathrm{Co})$, taenite $\gamma-\mathrm{Fe}(\mathrm{Ni}, \mathrm{Co})$ and tetrataenite $\gamma-\mathrm{FeNi}$ phases with $\mathrm{Ni}$ variations. Comparing this result with the SEM data we can suggest microstructural heterogeneity of Chinga metal. Moreover, areas of Mössbauer spectra components as well as their hyperfine parameters were different for different metal samples. This fact may be explained as variation of microstructural heterogeneity in various parts of Chinga fragment as a result of phase composition variations after ablative heat alteration or terrestrial pre-corrosion alteration.

Acknowledgment: This work was supported in part by the Federal Grant-in-Aid Program «Human Capital for Science and Education in Innovative Russia» (Governmental Contracts No. P1154 and No 14.740.11.1006).

References: [1] Axon H. J. and Smith P. L. 1972. Mineralogical Magazine 38:736-755. [2] Grokhovsky V. I. et al. 2008. Meteoritics \& Planetary Science 43:A50. [3] Semionkin V. A. et al. 2010. Bulletin of the Russian Academy of Sciences: Physics 74:416-420.
5031

\section{ISOTOPIC AND RAMAN ANALYSES OF LOW-DENSITY ORGUEIL CARBON GRAINS}

Evan Groopman ${ }^{1}$, Brigitte Wopenka ${ }^{2}$ and Ernst Zinner ${ }^{1}$. ${ }^{1}$ Physics Department and ${ }^{2}$ Department of Earth and Planetary Sciences, Washington University, St. Louis MO, USA. E-mail: eegroopm(a) physics.wustl.edu.

Introduction: We present isotopic and Raman spectroscopic data for 20 low-density presolar grains from the OR1d (size $>1 \mu \mathrm{m}, \rho=1.75$ $1.92 \mathrm{~g} \mathrm{~cm}^{-3}$ ) size/density separate of the Orgueil CI chondrite [1]. OR1d contains substantial insoluble organic material (IOM) of solar composition, from which the grains must be physically separated via micromanipulation prior to analysis. Some IOM coating may remain on a grain's surface, yielding isotopic compositions that are less anomalous than the true values. Correlated SEM and isotope images allow us to locate uncoated areas on the grains from which we may infer their true isotopic compositions.

Results: We measured ${ }^{12,13} \mathrm{C},{ }^{14,15} \mathrm{~N},{ }^{16,18} \mathrm{O},{ }^{28,29,30} \mathrm{Si},{ }^{24,25,26} \mathrm{Mg}$, and ${ }^{27} \mathrm{Al}$ isotopes with the NanoSIMS. Inferred ${ }^{26} \mathrm{Al} /{ }^{27} \mathrm{Al}$ ratios in 10 grains are between 0.012 and 0.45 , in the range of $\mathrm{SiC} \mathrm{X}$ grains [2]; additional ${ }^{18} \mathrm{O}$ excesses in these grains confirm their supernova origin. 18 grains have ${ }^{15} \mathrm{~N}$ excesses. ${ }^{12} \mathrm{C} /{ }^{13} \mathrm{C}$ ranges from 7 to 306 , with 9 grains being isotopically heavy, 5 isotopically light, and 6 of solar composition.

$\mathrm{N}$ and $\mathrm{O}$ isotope images of 6 grains reveal regions of large isotopic anomalies amidst regions of roughly solar composition. From SEM images we may verify the presence of IOM adhered to the grains' surfaces. Four grains with ${ }^{16} \mathrm{O} /{ }^{18} \mathrm{O}$ ratios of $162,169,304$, and 139, all highly enriched in ${ }^{18} \mathrm{O}$ with respect to solar, showed regions of much more extreme ${ }^{18} \mathrm{O}$ enrichment with ${ }^{16} \mathrm{O} /{ }^{18} \mathrm{O}$ of $6,14.4,40$, and 9 , respectively, in the ion images. The whole-grain ${ }^{16} \mathrm{O} /{ }^{18} \mathrm{O}$ ratios differ by 1-2 orders of magnitude from the ratios in uncoated areas of the grains. ${ }^{16} \mathrm{O}$ and ${ }^{18} \mathrm{O}$ concentrations are spatially anti-correlated in one grain, implying that low ${ }^{16} \mathrm{O} /{ }^{18} \mathrm{O}$ regions are representative of the uncoated grain, while solar-valued regions must be IOM on the surface. ${ }^{14} \mathrm{~N} /{ }^{15} \mathrm{~N}$ values also correlate with the presence of IOM (whole grain:uncoated area): (141:20), (256:27), (209:30), (261:111) in the same four grains.

There exists a correlation between Raman and isotopic data. Based on 1st and 2nd order Raman spectra [3], 11 grains are Allende-type (i.e., glassy carbon), 3 are graphitic, and 6 are kerogen-type [4]. All grains with unambigous SN signatures were found to have Allende- or graphitic Raman spectra. Kerogen-type grains all have isotopically normal $\mathrm{C}$ and $\mathrm{O}$, but generally have excesses in ${ }^{15} \mathrm{~N}$.

Discussion: Whole-grain isotope ratios of OR1d carbon grains represent lower limits of isotopic anomalies due to the presence of solarcomposition IOM on the grains' surfaces. Isotope images allow us to distinguish between regions covered with IOM and those of the true grain, helping us determine the isotopic composition without sputtering too much of the grain away. These grains will be ultramicrotomed for subsequent Raman, TEM, XANES and NanoSIMS analyses of their interiors, therefore preserving as much of the grain is of utmost importance.

References: [1] Jadhav M. et. al. 2006. New Astronomy Reviews 50:591-595. [2] Zinner E. et al. 2010. Proceedings of Science (NIC XI) 148. [3] Wopenka B. et al. This conference. [4] Wopenka B. et al. 2011. Abstract \#1162. 42nd Lunar and Planetary Science Conference. 
5172

LUNAR SPINEL-RICH ROCKS BY REACTION BETWEEN PICRITIC MAGMA AND ANORTHOSITIC CRUST, AND IMPLICATIONS FOR M3 OBSERVATIONS

J. Gross and A. H. Treiman. Lunar and Planetary Institute, Houston, TX, USA. E-mail: gross@lpi.usra.edu.

Introduction: The lunar crust contains some of the most important and accessible clues to the history of the Moon's chemical evolution [e.g., 1-3]. The $\mathrm{M}^{3}$ near-infrared mapping spectrometer on the Chandrayaan-1 spacecraft [4-6], recently detected a new rock type on the rim of the Moscoviense basin - rock rich in $(\mathrm{Mg}, \mathrm{Fe}) \mathrm{Al}_{2} \mathrm{O}_{4}$ spinel, with less than $5 \%$ mafic silicate minerals (olivine and pyroxene). No such material had been described from lunar rocks. Here, we describe a rock fragment rich in Mg-Al spinel from lunar meteorite Allan Hills (ALH) A81005. The fragment is not identical to the $\mathrm{M}^{3}$ rock (it contains approximately $20 \%$ olivine + pyroxene) but is similar enough to be relevant.

Sample: Antarctic meteorite ALH A81005, is a polymict, anorthositic regolith breccia from the lunar highlands [7-9]. It is composed of anorthosites, granulites, isolated mineral fragments, impactites, impact glasses, and mare basalts set in an glassy, agglutinitic matrix [10]. Thin section ALHA81005,9 contains a single rock fragment, $350 \times 150 \mu \mathrm{m}$, with approximately $30 \mathrm{vol} \%(\mathrm{Mg}, \mathrm{Fe}) \mathrm{Al}_{2} \mathrm{O}_{4}$ spinel-enough that an outcrop of it would be easily detected by $\mathrm{M}^{3}$. The rest of this clast is approximately $15 \%$ olivine, approximately $6 \%$ low-Ca pyroxene, and $49 \%$ plagioclase. The fragment is so fine-grained $(30-50 \mu \mathrm{m})$ that it reasonably could represent a larger rock body; it is not spinel-rich merely by chance.

Discussion and Implications: The clast is so rich in spinel that it could not have formed by melting a peridotitic mantle or a basaltic lunar crust. The clast's small grain size and its apparent disequilibrium between spinel and pyroxene suggest rapid crystallization at low pressure. A likely origin for its bulk composition is assimilation of anorthosite by a picritic magma. This model explains the petrographic and chemical features of our clast, and is consistent with the regional setting of the spinel-rich regions identified by $\mathbf{M}^{3}$. The spinel-rich area near Moscoviense is associated with two other unusual spectral signatures-rock rich in olivine, and rock rich in orthopyroxene (the "OOS" association [5]). A picritic intrusion into the anorthositic crust, as suggested here, could produce such rocks as cumulate dunite and pyroxenite, in addition to its spinel-rich cumulates. All of these rocks, igneous and metasomatic, could have been brought to the Moon's surface in the impact that produced the Moscoviense basin. Other spinel-bearing rocks of such a picritic intrusion, like the rock fragment described here, may not be detectable by $\mathrm{M}^{3}$ because of their high abundance of mafic minerals. The strength of the NIR spinel signature is masked significantly if the rock contains more than $5 \%$ mafic minerals [4]. Thus spinel-rich rocks might be common in the lunar crust but not yet detectable. However, with the advent of high spectral and high spatial resolution instruments, there is every expectation that similar exposures of materials might be found across the entire surface of the Moon [5].

References: [1] Taylor G. J. 1982. Planetary Science: A Lunar Perspective LPI. [2] Demidova et al. 2007. Petrology 15:386-407. [3] Isaacson et al. 2011. Journal of Geophysical Research 116:E00G11. [4] Pieters C. M. 2010. Abstract \#1854. 41st Lunar and Planetary Science Conference. [5] Pieters C. M. 2011. Journal of Geophysical Research 116:E00G08. [6] Lal et al. 2011. Abstract \#1339. 42nd Lunar and Planetary Science Conference. [7] Treiman A. H. and Drake M. J. 1983. Geophysical Research Letters 10:783-786. [8] Goodrich C. A. et al. 1984. Journal of Geophysical Research 89:C87-C94. [9] Korotev R. L. 2005. Chemie der Erde 65:297-346. [10] Gross J. and Treiman A. H. 2010. Abstract \#2180. 41st Lunar and Planetary Science Conference.
5123

MAGMATIC ORIGIN OF CALCITE FROM THE LUNA-20 REGOLITH SAMPLE

J. Götze ${ }^{1}$, A. Gucsik ${ }^{2,3}$, U. Kempe ${ }^{1}$, U. Ott ${ }^{2}$, P. Hoppe ${ }^{2}$, Sz. Nagy ${ }^{4}$, Sz. Bérczi $^{5}$ and M. Veres ${ }^{6}$. ${ }^{1}$ Institut für Mineralogie, TU Bergakademie Freiberg, Brennhausgasse 14, Freiberg, D-09596, Germany. ${ }^{2}$ MaxPlanck- Institut für Chemie, Joh.-J.-Becher-Weg 27, D-55128 Mainz, Germany. ${ }^{3}$ Konkoly Observatory, Hungarian Academy of Sciences, Konkoly-Thege út 15-17, Budapest, H-1121, Hungary. E-mail: ciklamensopron@yahoo.com. ${ }^{4}$ Petrology and Geochemistry, Eötvös Lorand University, Pázmány Péter sétány $1 / \mathrm{c}$, Budapest, H-1117, Hungary. ${ }^{5}$ Department of Material Physics, Institute of Physics, Eötvös University, Pázmány P. s. 1/a, Budapest, H-1117, Hungary. ${ }^{6}$ Research Institute for Solid State Physics and Optics, Konkoly-Thege M. út 29-33, Budapest, H-1121, Hungary.

Introduction: Direct investigations concerning the composition and structure of the Moon started with the space missions of Russia (formerly Soviet Union; Luna missions from 1959 to 1976) and the U.S. (Ranger and Surveyor missions in 1964 and 1968). The Apollo missions between 1969 and 1972 as well as Luna 16, 20, and 24 (1971-1976) returned $382 \mathrm{~kg}$ of geological material. Their investigation revealed that the mineral compositions of lunar rocks are much simpler than those of terrestrial rocks. Plagioclase is the most abundant mineral of the lunar highland rocks (enriched in $\mathrm{Ca}$ and $\mathrm{Al}$ ), whereas pyroxene, plagioclase and ilmenite are predominant in mare basalts (pyroxene together with plagioclase $75-90 \%$ ). Olivine and the high-temperature silica phases (tridymite and cristobalite) mostly occur with contents between 1 and $10 \%[1-5]$.

Sample and Experimental Procedure: We report the existence of small inclusions of calcium carbonate $\left(\mathrm{CaCO}_{3}-\right.$ calcite $)$ in a clast of granitic rock composition from lunar regolith that was returned by the Luna 20 space mission. Their presence was proved by a combination of high-resolution cathodoluminescence (CL) microscopy and spectroscopy, SEM-EDS, micro-Raman spectroscopy and elemental analysis by NanoSIMS.

Results and Discussion: Based on isotope data obtained by NanoSIMS, an origin from a meteorite impact or from an in situ formation process on the surface of the Moon appears highly unlikely. The inclusion of calcite in quartz, intergrown with $\mathrm{K}$-feldspar and displaying an undamaged igneous texture, indicates a magmatic origin of carbonate.

Conclusion: Our observations point to a scenario, where granite crystallized during fractionation from a KREEP-rich melt and where the carbonate inclusion is a residue of immiscible $\mathrm{CO}_{2}$-rich parts (carbonatite?) of this melt. Consequently, our observations provide the evidence for the presence of volatiles during lunar crust formation.

References: [1] Smith J. V. 1974. American Mineralogist 59:231-243. [2] Smith J. V. and Steel I. M. 1976. American Mineralogist 61:1059-1116. [3] Taylor S. R. 1982. Planetary science: A lunar perspective. Houston, Texas: Lunar and Planetary Institute. [4] Taylor S. R. 2008. The origin and evolution of the Moon in a planetary context. Golden Jubilee Memoirs Geological Society of India 66:13-50. [5] Shearer C. K. and Papike J. J. 1999. Magma evolution of the Moon. American Mineralogist 84:1469-1494. 
5121 CATHODOLUMINESCENCE PROPERTIES OF THE FORSTERITE
FORMED UNDER SUPER COOLING: AN IMPLICATION FOR
METEORITICS

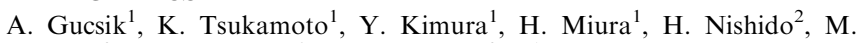
Kayama $^{2}$, K. Ninagawa ${ }^{3}$ and Y. Endo ${ }^{2}$. ${ }^{1}$ Department of Earth and Planetary Materials Science, Graduate School of Science, Tohoku University, Sendai 980-8578, Japan. E-mail: ciklamensopron@yahoo.com. ${ }^{2}$ Research Institute of Natural Sciences, Okayama University of Science, 1-1 Ridai-cho, Okayama, 700-0005, Japan. ${ }^{3}$ Department of Applied Physics, Okayama University of Science, 1-1 Ridai-cho, Okayama, 7000005, Japan.

Purpose: In this study, cathodoluminescence (CL) microscopy and spectroscopy of the experimentally grown forsterite spherule has been characterized to understand more about the mechanism of the crystal growth under the rapid cooling conditions, which may be applied to the study of the meteoritic forsterite.

Samples and Experimental Procedure: Forsterite crystals with $1 \mathrm{~mm}$ in diameter were grown as a chondrule analogue by the aero-acoustic levitation u floating method [1,2] and gas jet levitation methods [3]. Since levitated melt crystallizes under very high super cooling due to homogeneous nucleation, the growth rate of crystallization is quite larger compared with that near equilibrium temperature. The lab-chondrule is well reproduced the texture of natural chondrule. CL spectroscopy was made by a SEM-CL system at Okayama University of Science (Okayama, Japan).

Results and Discussion: In previous CL studies of the meteoritic forsterite samples in Allende and Murchison, a significant blue center (between 400 and $500 \mathrm{~nm}$ ) was observed [4-6]. However, these authors were not able to identify the defect-related centers of the CL emission between 400 and $500 \mathrm{~nm}$ in the synthetic and meteoritic forsterite samples. In our samples, the red CL color and its heterogeneous distribution were observed in, which show two broad emission bands at around $630 \mathrm{~nm}$ in red region and over $720 \mathrm{~nm}$ in red-IR region. The former band can be assigned to impurity center of divalent $\mathrm{Mn}$ ion as an activator [4-6]. The latter one shows a magnificent red emission in a wide range of wavelength responsible for trivalent $\mathrm{Cr}$ ions [4], which possess two components of $\mathrm{Cr}$ activator and structural defect caused by interstitial $\mathrm{Cr}$ ions. The color $\mathrm{CL}$ image of experimentally grown forsterite exhibits significant blue luminescence in the main branches of the interior structure of lab-chondrule, which reflects an order of crystallization. CL spectra from the blue luminescent area give a characteristic broad band emission at around $450 \mathrm{~nm}$, which is associated with a relatively small concentration of $\mathrm{Al}, \mathrm{Ca}, \mathrm{Ti}$ refractory elements [4]. A new $\mathrm{CL}$ band centered at $480 \mathrm{~nm}$ (blue/green CL color) might be assigned to a microdefect-related center, which was formed due to the rapid growth from super cooled melt of forsterite.

References: [1] Tsukamoto et al. 1999. Antarctic Meteorites 24:179181. [2] Tsukamoto et al. 2001. Abstract \#1846. 32nd Lunar and Planetary Science Conference. [3] Nagashima et al. 2006. Journal of Crystal Growth 293:193-197. [4] Steele 1986. American Mineralogist 71:966-970. [5] Vasconcellos M. A. Z. and Steele I. M. 1996. Abstract \#1359. 27th Lunar and Planetary Science Conference. [6] Benstock et al. 1997. American Mineralogist 82:310-315.
5397

IRON AND NICKEL ISOTOPIC COMPOSITIONS OF PRESOLAR SiC Z GRAINS AND A MYSTERIOUS SiC GRAIN OF UNKNOWN ORIGIN

F. Gyngard and L. R. Nittler. Department of Terrestrial Magnetism, Carnegie Institution of Washington, Washington, DC 20015, USA. E-mail: fgyngard@dtm.ciw.edu.

Introduction: Approximately $1 \%$ of presolar $\mathrm{SiC}$ grains are of Type $\mathrm{Z}$, characterized by comparatively large ${ }^{30} \mathrm{Si} /{ }^{29} \mathrm{Si}$ ratios [1]. These grains are thought to have formed in the ejecta of low-metallicity AGB stars, and as such represent good tracers of galactic chemical evolution. Here we report new isotopic measurements of the $\mathrm{Fe}-\mathrm{Ni}$ compositions of three $\mathrm{Z}$ grains, as well as one accidentally measured $\mathrm{SiC}$ grain of unknown type with the largest $\mathrm{Fe}$ isotopic anomaly ever observed.

Experimental: A residue (IH6) composed mostly of $\mathrm{SiC}$ and $\mathrm{Si}_{3} \mathrm{~N}_{4}$ grains from the Indarch enstatite chondrite (EH4) was automatically mapped [2] for ${ }^{12,13} \mathrm{C},{ }^{12} \mathrm{C}^{14} \mathrm{~N},{ }^{12} \mathrm{C}^{15} \mathrm{~N}$, and ${ }^{28,29,30} \mathrm{Si}$ isotopes in multicollection with the Carnegie NanoSIMS 50L. After SEM imaging, candidate $\mathrm{Z}$ grains were selected for further studies based on their physical separation from nearby grains. Two instrumental setups (consisting of two magnetic field steps each) were used to measure the $\mathrm{Fe}-\mathrm{Ni}$ isotopic compositions. In the first, ${ }^{54,56,57} \mathrm{Fe}$ and ${ }^{58,60,61,61} \mathrm{Ni}$ were measured in one magnetic field step, and ${ }^{52} \mathrm{Cr}$ and ${ }^{28} \mathrm{Si}_{2}$ (for locating the grains) in the other step. The other setup consisted of ${ }^{28} \mathrm{Si},{ }^{52} \mathrm{Cr},{ }^{54,56,57} \mathrm{Fe}$, and ${ }^{58,60} \mathrm{Ni}$ in the first magnetic field step and ${ }^{61,62} \mathrm{Ni}$ in the second.

Results: The candidate $\mathrm{Z}$ grains chosen for study were selected based on their enrichments in ${ }^{30} \mathrm{Si}$ relative to the mainstream correlation line, as has been done previously [3-5]. Due to extreme difficulty in relocating many of the grains in the NanoSIMS, only three $\mathrm{Z}$ grains were able to be successfully analyzed for their $\mathrm{Fe}-\mathrm{Ni}$ compositions. Two of the grains have isotopically normal $\mathrm{Fe}$ and $\mathrm{Ni}$, within $2 \sigma$ uncertainties. This is not unexpected as many $\mathrm{Z}$ grains analyzed previously [5] have shown no anomalies in $\mathrm{Fe}$ and $\mathrm{Ni}$, though this may be simply due to poor statistics from low $\mathrm{Fe}-\mathrm{Ni}$ elemental concentrations. $\mathrm{Z}$ grain f3-2-14-6 has a large enrichment in ${ }^{54} \mathrm{Fe}\left(\delta^{54} \mathrm{Fe} /{ }^{56} \mathrm{Fe}=620 \pm 230 \%\right.$ ) - its Ni (as well as other $\mathrm{Fe}$ ) isotopic compositions are normal. As observed before [5, 6], ${ }^{54} \mathrm{Fe}$ excesses are unexpected; grains believed to come from low-metallicity stars are predicted to have ${ }^{54} \mathrm{Fe}$ deficits [6]. In an attempt to locate and measure a Type $\mathrm{C} \mathrm{SiC}$ grain (e2-3-4 [7]), we inadvertently measured a nearby $\mathrm{SiC}$ grain that had not been analyzed for its $\mathrm{C}, \mathrm{N}$, or $\mathrm{Si}$ isotopic compositions; however, it has an $\mathrm{Fe}$ composition of $\delta^{54} \mathrm{Fe} /{ }^{56} \mathrm{Fe}=$ $2200 \pm 150 \%$ and $\delta^{57} \mathrm{Fe} /{ }^{56} \mathrm{Fe}=1860 \pm 230 \%$. The grain's Ni isotopic composition is solar within $2 \sigma$ errors. This grain was not measured during the automated measurements; yet, re-analysis of the overview ion image taken of the area containing the grain during automated scanning reveals it to have a ${ }^{12} \mathrm{C} /{ }^{13} \mathrm{C}$ ratio of approximately 30 . Unfortunately, its $\mathrm{N}$ and $\mathrm{Si}$ compositions are not diagnostic enough to definitively assign it to a given grain type, due to low count rates in the ROI of the grain. More measurements of $\mathrm{Z}$ grains and the Type $\mathrm{C}$ grain are planned for the future.

References: [1] Hoppe P. et al. 1997. The Astrophysical Journal 487:L101. [2] Gyngard F. et al. 2010. The Astrophysical Journal 717:107. [3] Zinner E. et al. 2007. Geochimica et Cosmochimica Acta 71:4786. [4] Nittler L. R. and Alexander C. M. O.D. 2003. Geochimica et Cosmochimica Acta 67:4961. [5] Hynes K. M. et al. 2009. Meteoritics \& Planetary Science 44:A96. [6] Marhas K. K. et al. 2008. The Astrophysical Journal 689:622. [7] Gyngard F. et al. 2010. Meteoritics \& Planetary Science 73:5242. 
5271

THE MARIBO CM FALL AND ITS POSSIBLE RELATIONSHIP TO COMET ENCKE AND THE TAURID METEOR COMPLEX

H. Haack ${ }^{1}$, R. Michelsen ${ }^{1}$, G. Stober ${ }^{2}$, D. Keuer ${ }^{2}$, W. Singer ${ }^{2}$ and I. Williams ${ }^{3}$. ${ }^{1}$ Natural History Museum of Denmark, University of Copenhagen. E-mail: hh@snm.ku.dk. ${ }^{2}$ Institute of Atmospheric Physics, Rostock University, Germany. ${ }^{3}$ Astronomy Unit, Queen Mary, London E1 4NS, UK.

Introduction: The Maribo meteorite is the first carbonaceous chondrite with an instrumental record of its fall [1]. The orbit of Maribo is also the only meteorite orbit to fall within the Taurid complex, a massive swarm of objects including comet Encke, several other large NEOs as well as fireball producing objects. If Maribo is genetically related to these objects it implies that $\mathrm{CM}$ chondrites are related to comets.

CMs and Comets: Short period comets remain active for approximately $10 \mathrm{ky}$. After the comet has depleted its source of volatiles the now extinct cometary nucleus remains in the inner solar system as a member of the near Earth population. NEOs have dynamical lifetimes of ca. $100 \mathrm{My}$ and are likely sources of meteorites. We should therefore expect to see samples of extinct nuclei in our collections of meteorites. Studies comparing the available information on chemistry and petrology of comets and chondrites have suggested that CI and CM chondrites could represent samples of extinct cometary nuclei $[2,3]$.

Recent astronomical observations and space missions to comets have provided new data supporting a connection to chondrites. $\mathrm{D} / \mathrm{H}$ ratios in water detected in the tails of Halley and Hyakutake are ca. $30 \times 10^{-5}$ [5]. This is within error of the $\mathrm{D} / \mathrm{H}$ ratios measured in $\mathrm{CM}$ chondrites $\left(18-20 \times 10^{-5}\right)$ [6]. Spitzer Space telescope observations of the Deep Impact ejecta from comet 9P/Tempel 1 gave the first data on the mineralogy in the interior of comets. The observations demonstrated the presence of crystalline silicates, carbonates and phyllosilicates [7] The crystalline silicates (olivine and pyroxene) require annealing temperatures up to $1400 \mathrm{~K}$ and thus demonstrate the presence of materials processed at high temperatures. The detection of carbonates and phyllosilicates implies that liquid water was present in the comet at some point in time.

The Age of the Taurid Complex: Peaks in the cosmic ray exposure ages of $\mathrm{CM}$ chondrites suggest their parent body suffered several large disruption events $0.2,0.6$, and $1.5 \mathrm{My}$ ago [8]. This is difficult to reconcile with the proposed origin of the Taurid complex. It has been suggested that the source of the Taurid complex was a large comet disruption event 20-30 ky ago [9]. If the CM chondrites come from the Taurid complex its age must be at least a few million years.

Conclusion: The petrology of $\mathrm{CM}$ chondrites and the orbit determined for Maribo suggest that $\mathrm{CM}$ chondrites are related to the Taurid complex and comet Encke.

References: [1] Haack et al. 2009. Abstract \#5085. Meteoritical Society Conference. [2] Lodders K. and Osborne R. 1999. Space Science Review 90:289-297. [3] Weisberg M. K. and Connolly H. C. 2008. Abstract \#1981. 49th Lunar and Planetary Science Conference. [5] Irvine et al. 1998. In Protostars and Planets IV, pp. 1159-1200. [6] Zinner E. 1988. In Meteorites and the Early solar system, pp. 956-983. [7] Lisse C. M. et al. 2006. Science 313:635-640. [8] Nishiizumi. 2009. Abstract \#5358. Meteoritical Society Conference. [9] Asher D. J. et al. 1993. Monthly Notices of the Royal Astronomical Society 264:93-105.
5009

HIGH ABUNDANCE OF STARDUST IN THE CO3.0 CHONDRITE LAPAZ ICEFIELD 031117

P. Haenecour ${ }^{1,2}$ and C. Floss ${ }^{1,3}$. ${ }^{1}$ Laboratory for Space Sciences. ${ }^{2}$ Department of Earth and Planetary Sciences. ${ }^{3}$ Department of Physics, Washington University, St. Louis, MO 63130, USA. E-mail: haenecour@ wustl.edu.

Introduction: Presolar silicate grains have been found in high abundance only in a few highly primitive meteorites, including the carbonaceous chondrites Acfer 094 (C ungrouped), ALHA77307 (CO3.0), QUE 99177 (CR3.0), and MET 00426 (CR3.0) [e.g. 1-3]. The recent discovery of a possible new pristine CO3.0 chondrite, LaPaz 031117 [4] has led us to carry out a reconnaissance study in order to evaluate the abundance of presolar grains in this meteorite. Here we report the results for our first search of carbon- and oxygen-bearing presolar grains in LAP 031117.

Experimental: Using the Cameca NanoSIMS 50 at Washington University, we carried out raster ion imaging $\left({ }^{12,13} \mathrm{C}^{-}\right.$and $\left.{ }^{16,17,18} \mathrm{O}^{-}\right)$in the matrix material of a thin-section of LAP 031117 . A focused primary $\mathrm{Cs}^{+}$ beam of $\sim 1 \mathrm{pA}(\sim 100 \mathrm{~nm}$ in diameter) was rastered over surface areas of $10 \times 10 \mu \mathrm{m}^{2}$ (256 pixels) and the ${ }^{12,13} \mathrm{C}^{-}$and ${ }^{16,17,18} \mathrm{O}^{-}$secondary ions, as well as secondary electrons were collected in multi-collection mode. All measurements were made within the same matrix area and the total area mapped was $1250 \mu \mathrm{m}^{2}$.

Results and Discussion: To date we have identified six oxygenanomalous grains, all of which are ${ }^{17} \mathrm{O}$-rich relative to solar $\left({ }^{17} \mathrm{O} /{ }^{16} \mathrm{O}_{\text {solar }}=3.8 \times 10^{-4}\right.$ and $\left.{ }^{18} \mathrm{O} /{ }^{16} \mathrm{O}_{\text {solar }}=2.0 \times 10^{-3}\right)$, with ${ }^{17} \mathrm{O} /{ }^{16} \mathrm{O}$ ratios between $5.01 \times 10^{-4}$ and $1.39 \times 10^{-3}$. Three grains also exhibit moderate ${ }^{18} \mathrm{O}$ depletions. Based on the classification proposed by [5], the six ${ }^{17} \mathrm{O}$-rich grains belong to group 1 and are likely to have formed in the envelopes of low- to intermediate-mass red giant or AGB stars with closeto-solar or slightly lower-than-solar metallicity.

We also found two C-anomalous grains in LAP 031117. One is ${ }^{13} \mathrm{C}$-rich $\left({ }^{12} \mathrm{C} /{ }^{13} \mathrm{C}=65.7 \pm 0.9\right)$ and is likely to be a mainstream $\mathrm{SiC}$ grain. The other one is ${ }^{12} \mathrm{C}$-rich, with a ${ }^{12} \mathrm{C} /{ }^{13} \mathrm{C}$ ratio of $101.4 \pm 2.4$.

Based on the limited area measured to date, we estimate an abundance of oxygen-bearing grains of $173 \pm 58 \mathrm{ppm}$ (without correcting for detection efficiency), comparable to the range of abundances observed in other primitive carbonaceous chondrites (up to $220 \mathrm{ppm}$ ) [1-3]. These preliminary results suggest that this meteorite indeed did not undergo significant secondary processing, such as aqueous alteration and thermal processing metamorphism, and may be a promising new source of presolar silicate grains. However, other matrix areas in LAP 031117 will need to be investigated in order to ensure that the high abundance found in our initial survey is indeed representative of the whole meteorite. Auger electron spectroscopy will then be used to obtain the elemental compositions of the grains identified.

References: [1] Floss C. and Stadermann F. J. 2009. Geochimica et Cosmochimica Acta 73:2415-2440. [2] Nguyen et al. 2010. The Astrophysical Journal 719:166-189. [3] Bose et al. 2010. The Astrophysical Journal 714:1624-1636. [4] Chizmadia L. J. and Cabret-Lebrónt E. 2009. Abstract \#2031. 40th Lunar and Planetary Science Conference. [5] Nittler L. R. et al. 1997. The Astrophysical Journal 483:475-495. 


\section{0}

\section{HYDROGEN ISOTOPES IN THE NAKHLITES: MAGMATIC AND ATMOSPHERIC MARTIAN RESERVIORS VERSUS TERRESTRIAL CONTAMINATION}

L. J. Hallis ${ }^{1}$ and G. J. Taylor ${ }^{1}{ }^{1} \mathrm{HIGP} / \mathrm{SOEST}$, NASA Astrobiology Institute, University of Hawaii, Honolulu, HI 96822 USA. E-mail: lydh@higp.hawaii.edu.

Introduction: Erosion of Mars' atmosphere via solar wind has led to the preferential loss of the lighter hydrogen isotope from the atmosphere, producing a deuterium enrichment-the present-day Martian $\delta \mathrm{D}$ of $4200 \%$ [1-2] is approximately $5 \times$ higher than that of VSMOW. This enrichment has been detected in a number of primary and secondary phases in the Martian meteorites (e.g., [3-4]). However, varying degrees of terrestrial contamination, and the possible presence of a second magmatic Martian hydrogen isotope reservoir, with a $\mathrm{D} / \mathrm{H}$ ratio similar to VSMOW (e.g., [5]), have resulted in wide $\mathrm{D} / \mathrm{H}$ variations both between and within individual meteorites. The purpose of this study is to determine the role of these three hydrogen isotope reservoirs in the formation, and subsequent alteration, of primary and secondary phases in the nakhlite group of Martian meteorites.

Methods: Polished thin-sections of MIL 03346 and 090030 were prepared at the NASA Johnson Space Centre without the use of water, and thin-sections of Nakhla were prepared in the same way at the University of Hawaii (UH). We utilised the JEOL JSM-5900LV scanning electron microscope at $\mathrm{UH}$ to produce high-resolution backscatter electron images. Secondary-phase major-element chemistry was determined with a JEOL JXA-8500F electron microprobe, also at UH. D $\mathrm{H}$ and $\mathrm{Si}$ isotopic compositions were analyzed in situ with the UH Cameca ims 1280 ion microprobe, with a Cs + primary beam.

Results and Initial Conclusions: $\mathrm{D} / \mathrm{H}$ ratios for iddingsite-like alteration veins in Nakhla are widely variable $(-98$ to $+1165 \%$ o $)$, even between analyses from the same alteration vein. The iddingsite alteration veins in MIL 090030,23 (an interior thin-section) are much less variable, with a range of $134-311 \%$. Petrological analysis also suggests the alteration veins in Nakhla are more heterogeneous than those in MIL 090030. [6] reported the composition of alteration veins in Nakhla as an assemblage of silica gel, siderite and evaporites. Amorphous silica gel is more susceptible to hydrogen exchange with the atmosphere than minerals with structurally bound water, hence areas with lower $\mathrm{D} / \mathrm{H}$ ratios are probably those rich in silica gel. MIL 03346,174, a thin-section with terrestrially exposed surfaces, contains iddingsite-like alteration veins with $\mathrm{D} / \mathrm{H}$ ratios entirely within the terrestrial region $(-240$ to $-69 \%$ ). However, as this thin-section was prepared with water, and exposed to atmospheric conditions since its preparation in 2006, we aim to analyze newly prepared exterior thin-sections of MIL 090030 to confirm this result. We also aim to analyze apatite grains in Nakhla and compare their $\mathrm{D} / \mathrm{H}$ ratios with those of $\mathrm{ALH} 84001$ apatites. These comparisons should help to determine if a separate magmatic hydrogen isotope reservoir did affect these samples, and may give some insight into the effect of shock on $\mathrm{D} / \mathrm{H}$ ratios.

References: [1] Bjoraker G. L. et al. 1989. Geochimica et Cosmochimica Acta. [2] Owen T. 1992. Mars (Eds. H. H. Kieffer et al.). [3] Sugiura N. and Hoshino H. 2000. Meteoritics \& Planetary Science. [4] Boctor N. Z. et al. 2003. Geochimica et Cosmochimica Acta. [5] Leshin L. A. et al. 1996. Geochimica et Cosmochimica Acta. [6] Changela H. G. and Bridges J. C. 2011. Meteoritics \& Planetary Science.
5299

COMBINED CL, OC, AND EBSD IMAGING SHOWS DIFFERENT TYPES OF PDFS IN QUARTZ

M. F. Hamers, G. M. Pennock and M. R. Drury. Faculty of Geosciences, Department of Earth Sciences, Utrecht University, Budapestlaan 4, 3584 CD, Utrecht, The Netherlands. E-mail: hamers@, geo.uu.nl.

Introduction: Planar deformation features (PDFs) in quartz are essential evidence for the impact origin of suspected structures or stratigraphic levels. They can be observed in a light microscope, but can be confused with other (sub) planar microstructures, especially tectonic deformation lamellae (DL). We use scanning electron microscope cathodoluminescence (SEM-CL) and orientation contrast (OC) imaging and electron backscatter diffraction (EBSD) to identify and characterize PDFs in quartz.

Method and Samples: SEM-CL images were acquired in an FEI Nova Nanolab 600 SEM with a panchromatic Gatan PanaCL detector (Gatan UK, Oxford, UK). Red, green, and blue filters were used to record color filtered images, which were combined into a colour composite image. OC images and EBSD data were collected in a Philips XL30 SFEG SEM with a Nordlys CCD camera and Oxford Instruments HKL Channel 5 software. Quartz grains containing PDFs were studied in samples from the Ries, Popigai, Rochechouart, and Vredefort impact structures.

Results and Discussion: $C L$ : PDFs show two types of CL behavior: red luminescence and non-luminescence. Red luminescent PDFs occur in all impact structures, but the non-luminescent type only in the younger samples (Ries and Popigai). Red luminescent PDFs are narrow (100$200 \mathrm{~nm}$ apparent thickness) and straight, while non-luminescent PDFs are often slightly curved features of varying thickness (100-350 nm apparent thickness). Non-luminescent PDFs are more closely spaced and always occur in multiple sets per grain, while red luminescent PDFs can occur as single sets. Some partly red, partly non-luminescent PDFs are also observed.

$O C$ and EBSD: In some Ries and Popigai samples red luminescent PDFs are visible as thin grey lamellae in OC images. Band contrast maps suggest that the material within the PDFs gives a weaker diffraction pattern than the host quartz. In grains from Vredefort and Rochechouart, red luminescent PDFs are not visible in OC images, and they do not show up in band contrast maps. All grains with red-luminescent PDFs contain small scale Dauphiné twins that are often parallel to or bounded by the PDFs. Grains with non-luminescent PDFs give no diffraction pattern from within the PDFs and very weak patterns from the host quartz. OC images of these grains often show a submicron-scale blocky structure of lighter and darker grey domains, parallel to PDFs, that might be very small Dauphiné twins.

Discussion: Grains with non-luminescent PDFs are interpreted to have suffered high shock pressure, because the PDFs always occur in multiple closely spaced sets and the weak diffraction patterns from the host quartz suggest a disordered crystal structure. The non-luminescent (parts of) PDFs might contain amorphous material, as they show no diffraction patterns and are only present in relatively young impact structures. Red luminescent PDFs might contain disordered, but not completely amorphous $\mathrm{SiO}_{2}$, or might represent annealed or recrystallized material.

Conclusions: SEM-CL and OC imaging and EBSD data reveal different types of PDFs that are probably related to shock level and the presence of amorphous material within the PDFs. 
5190

FORMATION OF $\mathrm{TiO}_{2}$ NANOPARTICLES IN A CAI-LIKE OBJECT FROM AN AOA IN THE ALHA77307 CO3.0 CARBONACEOUS CHONDRITE

Jangmi Han and Adrian J. Brearley. Department of Earth and Planetary Sciences, MSC03-2040, 1University of New Mexico, Albuquerque, NM 87131, USA. E-mail: jmhan@unm.edu.

Introduction: Amoeboid olivine aggregates (AOAs) consist of forsterite and lesser amounts of refractory, Ca-Al-rich phases such as Al,Ti-bearing pyroxene, \pm anorthite, \pm spinel, and rarely melilite. AOAs probably represent aggregates of nebular gas-solid condensates [1, 2]. The refractory component of AOAs shows evidence of gas-solid reactions that can constrain the origin and thermal history of the AOAs. Here, we report microstructural studies of an AOA from the CO3.0 chondrite, ALHA77307, aimed at understanding the origin of AOA and the relationship between the refractory component and forsterite.

Methods: SEM/BSE imaging, X-ray mapping, and FIB-TEM were used to study the AOA. TEM observations were made on two FIB sections from a CAI-like object within the AOA [3].

Results: The AOA (approximately $350 \mu \mathrm{m}$ in size) contains CAI-like nodules with a spinel-rich core, a layer of intergrown spinel and diopside, and a diopside rim [3]. The boundary between the spinel core and the diopside layer is highly irregular and embayed indicating a reaction relationship. One FIB section (C) was cut from the spinel core to the diopside rim and a second (D) from a complex spinel-diopside intergrowth in the spinel core.

Both FIB sections are highly compact with no evidence of pores. The spinel core regions consist of a dense aggregate of submicron to microsized grains with an equilibrated grain boundary microstructure, i.e., $120^{\circ}$ triple junctions. The spinel in both FIB sections exhibits a complex intergrowth texture with irregularly shaped Al,Ti-rich diopside grains (11$32 \mathrm{wt} \% \quad \mathrm{Al}_{2} \mathrm{O}_{3} ; 2-17 \mathrm{wt} \% \quad \mathrm{TiO}_{2}$ ), often partially enclosed by spinel. Within the FIB D section, numerous nanoparticles, $<100 \mathrm{~nm}$ in size, decorate the grain boundaries between spinel and diopside. The nanoparticles are elongated, faceted to subrounded and are usually crystallographically oriented relative to the spinel. They also exhibit a complex striated texture indicative of a disordered intergrowth of phases. EDS and EFTEM analyses show that the inclusions contain only $\mathrm{Ti}$ and O. FFT analysis of HRTEM images of several particles are consistent with the presence of two $\mathrm{TiO}_{2}$ polymorphs, rutile, and anatase.

Discussion: To our knowledge, this is the first documented occurrence of nanoparticles of rutile and anatase within any CAI-like object. Ti oxides are not predicted equilibrium condensate phases; instead, $\mathrm{Ti}$ is incorporated into perovskite or Al,Ti-rich diopside. In this AOA, the formation of $\mathrm{TiO}_{2}$ is clearly related to the reaction of spinel to form Al,Ti-rich diopside, a process that may be the result of disequilibrium back-reaction of spinel with the nebular gas [3]. Metastable formation of $\mathrm{TiO}_{2}$ may have occurred during reaction between spinel, diopside, and gas under conditions where the gas was supersaturated in $\mathrm{Ti}$, favoring the condensation of simple oxide phases onto the spinel, by coherent nucleation and growth. Alternatively, $\mathrm{TiO}_{2}$ could have exsolved at high temperatures from a Ti-saturated, Al-rich diopside.

References: [1] Chizmadia L. J. et al. 2002. Meteoritics \& Planetary Science 37:1781-1796. [2] Krot A. N. et al. 2004. Chemie der Erde 64:185239. [3] Han J. and Brearley A. J. 2011. Abstract \#1673. 42nd Lunar and Planetary Science Conference.
5388

EVIDENCE FOR FORMATION OF Al-Ti PYROXENE BY REACTION BETWEEN PEROVSKITE AND MELILITE IN A CAILIKE OBJECT FROM AN AOA IN THE ALHA77307 CO3.0 CHONDRITE

J. Han and A. J. Brearley. Department of Earth and Planetary Sciences, MSC03-2040, 1University of New Mexico, Albuquerque, NM 87131, USA. E-mail: jmhan@unm.edu.

Introduction: Amoeboid olivine aggregates (AOAs), the most common type of refractory inclusion in carbonaceous chondrites, consist mainly of olivine and lesser amounts of refractory components such as spinel, Al,Ti-bearing diopside, and anorthite. Rarely, melilite and perovskite occur in AOAs [1, 2]. We have studied a fine-grained, perovskite-bearing CAI-like object in an AOA in the pristine $\mathrm{CO} 3$ chondrite ALHA77307 (CO3.0) in an effort to constrain the origins and thermal histories of refractory materials in AOAs. In addition, we hope to constrain the relationship between the refractory component and olivine in AOAs.

Method: The AOA was studied using SEM/BSE imaging and elemental X-ray mapping and then sectioned using FIB technique. The section was subsequently in detail by TEM techniques to characterize its microstructure and microchemistry.

Results and Discussion: The irregularly shaped AOA (approximately $300 \mu \mathrm{m}$ in size) investigated in this study consists of approximately $35 \mathrm{vol} \%$ of a dense CAI-like refractory object enclosed by porous olivine grains. The CAI-like object contains numerous nodules consisting of spinel rimmed by a continuous layer of Al,Ti-bearing diopside. Minor perovskite grains, $<3 \mu \mathrm{m}$ in size, are present along grain boundaries between the spinel core and diopside rim.

The FIB section was prepared across a region of two neighboring CAI-like nodules where two approximately $2 \mu \mathrm{m}$ perovskite grains occur between the spinel core and diopside rim. The FIB section consists of perovskite, diopside, spinel, and melilite with highly unequilibrated grain boundary microstructures. The perovskite grains are irregular in shape and completely surrounded by a layer $0.2-0.4 \mu \mathrm{m}$ wide consisting of finegrained diopside and minor spinel. The melilite is completely separated from the perovskite by this layer. Melilite has a high density of defects and dislocations and shows evidence of pores in localized areas.

Perovskite and spinel are nearly pure $\mathrm{CaTiO}_{3}$ and $\mathrm{MgAl}_{2} \mathrm{O}_{4}$, whereas melilite is highly gehlenitic, but compositional homogeneous. In contrast, pyroxene shows large variations in chemical composition, with $\mathrm{Al}_{2} \mathrm{O}_{3}$ contents from 0 to $29 \mathrm{wt} \%$, and $\mathrm{TiO}_{2}$ contents from 0 to $16 \mathrm{wt} \%$. Significantly, pyroxene grains in contact with perovskite have the highest $\mathrm{TiO}_{2}$ contents, whilst those furthest away from perovskite contain $<5 \mathrm{wt} \% \mathrm{TiO}_{2}$.

Unlike another CAI-like object that we have studied in ALHA77307 [3], this inclusion contains unequivocal compositional and textural evidence for a direct reaction between perovskite and melilite to form Al-Ti-rich pyroxene. The high degree of compositional and textural disequilibrium demonstrates that this reaction occurred under highly dynamic conditions, possibly during fast cooling. One possible scenario is that reaction of perovskite with the gas during melilite condensation stabilized Al-Ti pyroxene, until a reaction rim of sufficient thickness developed to isolate the perovskite from the gas phase. Melilite was then able to condense, as a stable phase.

References: [1] Krot A. N. et al. 2004. Chemie der Erde 64:185-239. [2] Chizmadia L. J. et al. 2002. Meteoritics \& Planetary Science 37:17811796. [3] Han J. and Brearley A. J. 2011. Abstract \#1673. 42nd Lunar and Planetary Science Conference. 


\section{3}

\section{PROGRESSIVE AQUEOUS ALTERATION OF CR CHONDRITES}

E. R. Harju ${ }^{1}$, A. E. Rubin ${ }^{2}$ and J. T. Wasson ${ }^{1,2}$. ${ }^{1}$ Department of Earth \& Space Science, UCLA, L.A., CA 90095-1567, USA. E-mail: harju@ucla.edu. ${ }^{2}$ IGPP, UCLA, L.A., CA 90095-1567, USA.

Although CR chondrites have experienced little or no thermal metamorphism, they have undergone varying degrees of parent-body aqueous alteration. O-isotopic measurements of $\mathrm{CR}$ chondrites show increasing $\delta^{17} \mathrm{O}$ and $\delta^{18} \mathrm{O}$ in more aqueously altered meteorites. However, these measurements have limitations because of terrestrial weathering and sample heterogeneity. What is needed is a petrologic scale for aqueous alteration in $\mathrm{CR}$ chondrites analogous to the one constructed for $\mathrm{CM}$ chondrites [1]. We examined $20 \mathrm{CR}$ chondrites and used petrographic and compositional parameters to draft a preliminary $\mathrm{CR}$ alteration index.

The scale ranges from 2.0 (most altered, equivalent to petrologic type 1) to 3.0 (negligibly altered, roughly equivalent to that of LL3.0 Semarkona). With increasing $\mathrm{CR}$ chondrite alteration, metallic $\mathrm{Fe}-\mathrm{Ni}$ grains develop rinds of magnetite, S-bearing oxides and hydroxides, and other OH-bearing phases. Chondrule mesostases have been altered to phyllosilicate in some $\mathrm{CR}$ chondrites. Mafic silicate phenocrysts in chondrules in Renazzo, Al Rais, and A-881595 show incipient alteration.

QUE 99177 and MET 00426 were classified as type 3.0 by [2]; most of the CR chondrites we studied appear to have similar degrees of aqueous alteration to these meteorites, clustering around type 2.9-3.0. These samples can be distinguished from one another by the presence in some chondrules of transparent isotropic glass (QUE 99177, MET 00426, EET 92062, LAP 02342, Gao-Guenie [b], GRV 021710 [2]) and by TEM studies [2] indicating the extent of matrix hydration.

Renazzo and A-881595 contain $\geq 50 \mu \mathrm{m}$-size metal grains that have been partially converted to magnetite; small metal grains have been largely to completely converted to magnetite. Chondrule phenocrysts show incipient alteration along grain surfaces.

In CR-an Al Rais most metal grains not embedded deep within chondrules have substantial alteration rinds of magnetite and a S-bearing oxide. Many of the chondrules contain polysynthetically twinned low-Ca pyroxene grains displaying alteration along twin boundaries. Al Rais is more altered than Renazzo or A-881595.

GRO 95577 is similar to CM2.0 chondrites. All of the metal is nearly or completely oxidized; mafic-silicate phenocrysts have all been converted to phyllosilicate [3]. We found that some of the former metal grains consist of at least nine distinct nested layers of phyllosilicate. Because similarly sized metal grains in CR LAP 02342 are compositionally uniform, this suggests that the layering in GRO 95577 represents episodic alteration of an initially homogeneous substrate.

We used thermogravimetric analysis to examine the differences in bulk indigenous water among relatively unaltered $\mathrm{CR}$ chondrites. Preliminary results indicate that some less-altered samples contain about $1 \mathrm{wt} \%$ less water than Renazzo (5.67 wt\%, [4]). Correction for volatiles in weathering products is required.

Further development and application of this index will enhance our understanding of parent-body aqueous alteration process on the $\mathrm{CR}$ parent body and offer a guide to sample selection in future studies of CR chondrites.

References: [1] Rubin A. E. et al. 2007. Geochimica et Cosmochimica Acta 71:2361-2382. [2] Abreu N. M and Brearley A. J. 2010. Geochimica et Cosmochimica Acta 74:1146-1171. [3] Weisberg M. K. and Huber H. 2007. Meteoritics \& Planetary Science 42:1495-1503. [4] Wiik H. B. 1956. Geochimica et Cosmochimica Acta 9:279-289.
5165

\section{NANOCRYSTALLINE P-BEARING PENTLANDITE AND CHROMIUM NITRIDES FROM CM2 CHONDRITES Y-791198 and Y-793321}

D. Harries ${ }^{1}$ and F. Langenhorst ${ }^{2}$. ${ }^{1}$ Bayerisches Geoinstitut, Universität Bayreuth, Bayreuth, Germany. E-mail: dennis.harries@uni-bayreuth.de. ${ }^{2}$ Institut für Geowissenschaften, Friedrich-Schiller-Universität Jena, Jena, Germany.

Introduction: Phosphorous-bearing $\mathrm{Fe}, \mathrm{Ni}$ sulfides are characteristic accessory phases in CM2 chondrites, but enigmatic in their mineralogy and origin. Extensive textural and microanalytical data has been provided by [1], and a TEM study by [2] has shown that the material is nanocrystalline, but its structure could not be identified. We have prepared several FIB sections of P-bearing sulfides from two selected CM2 chondrites: Yamato(Y)-791198 is an unbrecciated rock with a rather low level of aqueous alteration and no significant secondary heating [3]. On the contrary, Y-793321 is a regolith breccia and has experienced an episode of thermal metamorphism at $250-500{ }^{\circ} \mathrm{C}$ [4]. This choice is intended to draw conclusions about the thermal stability and the origin of P-bearing sulfides and associated phases.

Results: TEM shows that grains in both meteorites are not homogeneous internally. Besides the nanocrystalline sulfide $(<20 \mathrm{~nm})$ we have found in certain grains myriads of $<100 \mathrm{~nm}$ sized flakes of chromium nitrides. SAED derived d-values are consistent with $\mathrm{CrN}$ (carlsbergite) in $\mathrm{Y}-791198$ and $\mathrm{CrN}+\mathrm{Cr}_{2} \mathrm{~N}$ in $\mathrm{Y}-793321$. The presence of chromium and nitrogen has been confirmed by EDX and EELS. This is the first reported observation of chromium nitrides in chondritic meteorites.

SAED patterns of the nanocrystalline sulfides in Y-791198 show two broad rings corresponding to d-values of 0.30 and $0.18 \mathrm{~nm}$. In Y-793321 the principal rings occur at the same positions, but splitting occurs and additional, weaker rings join. The corresponding d-values of strong and weak rings in Y-793321 match to a pentlandite structure. HR-TEM imaging shows that the nanocrystallites in Y-793321 are larger (close to $20 \mathrm{~nm}$ ) and better defined than in Y-791198. Hence, the difference in SAED patterns is due to better crystallinity in Y-793321, which we interpret as a result of thermal annealing. This is supported by the development of a lattice preferred orientation (LPO) of nanocrystallites in Y-793321, which is evident by circumferential intensity variations along the diffraction rings. The LPO is likely a transitional state during recrystallization toward a single pentlandite crystal. Chemical shift of $\mathrm{P}$ $\mathrm{K} \alpha$ determined by EMPA-WDS suggests a mix of phosphate and phosphide and EELS indicates abundant oxygen in the sulfide portion. While phosphide is likely incorporated into the pentlandite structure, phosphate groups probably reside on the large internal boundary surfaces. The same likely applies to lithophile trace elements measured by [1].

Conclusions: Comparison of Y-791198 and Y-793321 shows that the nanocrystalline sulfide is thermally not stable and undergoes recrystallization during processing at temperatures below $500^{\circ} \mathrm{C}$. However, the refractory nitrides must have formed at elevated temperatures in excess of $500{ }^{\circ} \mathrm{C}$. This implies a complex, multistage formation history. Scenarios will be discussed.

Acknowledgments: NIPR is gratefully acknowledged for providing meteorite samples.

References: [1] Nazarov M. A. et al. 2009. Petrology 17:101-123. [2] Devouard B. and Buseck P. R. 1997. Meteoritics \& Planetary Science 32:A34. [3] Nakamura T. 2005. Journal of Mineralogical and Petrological Sciences 100:260-272. [4] Nakamura T. 2006. Earth and Planetary Science Letters 242:26-38. 
5166

SULFIDES IN CM AND CM/CI-LIKE CHONDRITES AND THEIR RECORD OF THERMAL METAMORPHISM: A FIB-TEM STUDY D. Harries ${ }^{1}$ and F. Langenhorst ${ }^{2}{ }^{1}$ Bayerisches Geoinstitut, Universität Bayreuth, Bayreuth Germany. E-mail: dennis.harries@uni-bayreuth.de. ${ }^{2}$ Institut für Geowissenschaften, Friedrich-Schiller-Universität Jena, Jena, Germany.

Introduction: Assemblages containing iron- and nickel-rich monosulfides such as troilite $(\mathrm{FeS})$, pyrrhotite $\left(\mathrm{Fe}_{1-\mathrm{x}} \mathrm{S}\right)$ are interesting proxies for physicochemical conditions of formation, alteration, and metamorphism of planetary materials. Fe deficiency in pyrrhotite is directly related to $f \mathrm{~S}_{2}$ and temperature [1] and can be linked indirectly to $f \mathrm{O}_{2}$ if certain buffer assemblages are present, for example, magnetite plus metallic iron [2]. The results of Fe deficiency are various crystallographic superstructures [3]. To explore the effect of $f S_{2}$ and temperature on sulfide mineralogy, we have sampled sulfide grains from two CM2 chondrites (Y-791198, Y-793321), two anomalous, metamorphic CM/CI-like chondrites (Y-86720, B-7904), and one anomalous CI chondrite (Y-980115) by FIB and studied them by analytical TEM.

Results: Yamato-791198 and Y-793321 contain troilite with abundant pentlandite and pyrrhotite exsolution. In both cases pyrrhotite occurs as strongly twinned $6 \mathrm{C}$ superstructure (approximately $\mathrm{Fe}_{0.92} \mathrm{~S}$ ). In Y-793321, which has experienced mild metamorphism [4], the textures of the sulfide assemblages are significantly coarser than in Y-791198.

The anomalous CM/CI-like chondrites Y-86720 and B-7904 show intimate associations of troilite with metallic $(\mathrm{Fe}, \mathrm{Ni})$ and only traces of $6 \mathrm{C}$-pyrrhotite as narrow $(<100 \mathrm{~nm})$ rims at grain margins. In Y-86720 we have found pseudomorphs of troilite and metal after euhedral, hexagonal crystals - likely former pyrrhotite. Both contain well crystallized anhydrite, but only B-7904 contains magnetite, which is absent in Y-86720.

In Y-980115 euhedral sulfide crystals similar to 4C-pyrrhotite $\left(\mathrm{Fe}_{0.875} \mathrm{~S}\right)$ in typical CI chondrites [5] are composed of polycrystalline troilite and pentlandite and are likely pseudomorphs as well. Also here, 6C-pyrrhotite occurs at grain margins and rims of internal voids. However, $(\mathrm{Fe}, \mathrm{Ni})$ metal is absent.

Conclusions: Compared to pristine Y-791198 the thermal overprint on Y-793321 had only minor effects on sulfide assemblages, mostly leading to coarsening through annealing at temperatures below $150^{\circ} \mathrm{C}$.

Y-86720 and B-7904 experienced more severe heating and low $f \mathrm{~S}_{2}$ at the iron-troilite buffer. Preservation of magnetite in B-7904 points to peak temperatures below $570{ }^{\circ} \mathrm{C}$ and absence in $\mathrm{Y}-86720$ suggest a higher metamorphic grade. The magnetite + troilite + metal assemblage suggests reducing conditions with $\log f \mathrm{O}_{2}$ below -25.8 in case of B-7904. 6C-pyrrhotite and anhydrite in both meteorites cannot be in equilibrium with this assemblage and likely formed during cooling.

Conversion of pyrrhotite to troilite in Y-980115 likely indicates similar low $f \mathrm{~S}_{2}$ conditions as in B-7904. However, metal did not form, possibly due to shorter heating and kinetic inhibition.

Acknowledgments: NIPR is gratefully acknowledged for providing meteorite samples.

References: [1] Rau H. 1976. Journal of Physics and Chemistry of Solids 37:425-429. [2] Whitney J. A. 1984. American Mineralogist 69: 69-78. [3] Harries D. et al. 2011. American Mineralogist 96:716-731. [4] Nakamura T. 2006. Earth and Planetary Science Letters 242:26-38. [5] Berger E. L. et al. 2011. Geochimica et Cosmochimica Acta 75:35013513.
5182

THE SIGNIFICANCE OF Li ISOTOPE VARIATIONS IN CO CHONDRITES: BULK-ROCK, INTER AND INTRACHONDRULE DIFFERENCES

J. Harvey ${ }^{1,2}$, M. A. Fehr ${ }^{1}$, J. L. Sharrock ${ }^{3}$, I. J. Parkinson ${ }^{1}$ and R. H. James ${ }^{4}{ }^{1}$ Department of Earth and Environmental Sciences, The Centre for Earth, Planetary, Space and Astronomical Research (CEPSAR), The Open University, Milton Keynes, UK. E-mail: feejh@leeds.ac.uk. ${ }^{2}$ School of Earth \& Environment, University of Leeds, Leeds, UK. ${ }^{3}$ School of Earth Sciences, Grant Institute, University of Edinburgh, Edinburgh, UK. ${ }^{4}$ National Oceanography Centre, Southampton, UK.

The crystallization of chondrule phenocrysts has the potential to create distinctive ${ }^{7} \mathrm{Li} /{ }^{6} \mathrm{Li}$ profiles $[1,2]$ due to the more rapid diffusion of ${ }^{6} \mathrm{Li}$ compared to ${ }^{7} \mathrm{Li}$ in the presence of a compositional gradient. In well characterized samples it may be possible to extract cooling rates from the diffusion profiles. The mobility of $\mathrm{Li}$ during silicate weathering also results in significant fractionation of ${ }^{6} \mathrm{Li}$ from ${ }^{7} \mathrm{Li}$, with ${ }^{7} \mathrm{Li}$ preferentially entering the solution (e.g., [3]); this is a process that modifies the ${ }^{7} \mathrm{Li} /{ }^{6} \mathrm{Li}$ ratio of both terrestrial and meteoritical material $[3,4]$. The interpretation of lithium isotope diffusion profiles and fractionation patterns may therefore provide the means to quantify the effects of both primary and secondary processes in chondrites.

We will present data for intra- and interchondrule $\delta^{7} \mathrm{Li}$ variation (differences in ${ }^{7} \mathrm{Li} /{ }^{6} \mathrm{Li}$ relative to NIST $\mathrm{L}-\mathrm{SVEC}$ ), determined by ion microprobe and MC-ICP-MS respectively. This is complemented by bulk data for Ornans (CO3.3)and Lancé (CO3.4). The aims of this study are (1) to assess the extent to which $\mathrm{Li}$ isotope diffusion profiles in chondrule phenocrysts are preserved (2) to examine the extent of ${ }^{7} \mathrm{Li} /{ }^{6} \mathrm{Li}$ fractionation of bulk-rock and chondrules, in addition to intra-chondrule $\delta^{7} \mathrm{Li}$ variations in the context of aqueous alteration. High $\mathrm{Mg \#}(>0.99)$ in chondrule cores suggest that primitive geochemical compositions may have been retained. In contrast, lower rim $\mathrm{Mg \#}(\leq 0.80)$ suggest diffusive exchange with matrix during cooling or subsequent secondary alteration. However, the same $\mathrm{Mg} \#$ gradients are also observed close to fractures in the interior of chondrule phenocrysts and thus are unlikely to be primary, suggesting that $\mathrm{Li}$ isotope fractionation during chondrule cooling may have been overprinted. Bulk-rock $\delta^{7} \mathrm{Li}$ values for Ornans $(4.0 \pm 0.4)$ and Lancé $(2.9 \pm 0.1)$ are marginally heavier than previously reported [5].

References: [1] Parkinson et al. 2007. Earth and Planetary Science Letters 257:609-621. [2] Beck et al. 2004. Geochimica et Cosmochimica Acta 68:2925-2933. [3] Kisakurek et al. 2004. Chemical Geology 212:2744. [4] Sephton et al. 2006. Meteoritics \& Planetary Science 41:1039-1043. [5] Seitz et al. 2007. Earth and Planetary Science Letters 260:582-596. 


\section{0}

Mg ISOTOPIC COMPOSITION OF THE SOLAR WIND BY SIMS ANALYSIS OF GENESIS TARGETS

V. S. Heber ${ }^{1}$, A. J. G. Jurewicz ${ }^{2}$, P. Janney ${ }^{2}$, M. Wadhwa ${ }^{2}$, K. D. McKeegan $^{1}$ and D. S. Burnett ${ }^{3}$. ${ }^{1}$ Department of Earth and Space Sciences, UCLA, Los Angeles, CA, USA. E-mail: heber@ess.ucla.edu. ${ }^{2}$ Arizona State University, Tempe, AZ, USA. ${ }^{3}$ CalTech, Pasadena, CA, USA.

Introduction: In order to deduce the isotopic compositions in the solar nebula of volatile elements, e.g., noble gases, $\mathrm{O}$, and $\mathrm{N}$, from analyses of the solar wind (SW), we must understand the magnitude of massdependent fractionation between the SW and the photosphere. With the exception of evaporation effects in CAI's, mass-dependent fractionation of $\mathrm{Mg}$ isotopes is small, as evidenced by $\mathrm{Mg}$ isotopic compositions of terrestrial igneous and meteoritic samples which agree within $\sim 1 \%$ o [1]. Thus, if we assume that the "terrestrial" $\mathrm{Mg}$ isotopic composition is also representative of the solar photosphere, we can use measurements of the SW captured by Genesis to test models of isotopic fractionation in formation of SW. For example, if the inefficient Coulomb-drag model [2] is correct, we would expect that the $\mathrm{Mg}$ isotopic composition in the $\mathrm{SW}$ is $\sim 10 \%$ per amu [2] lighter than the terrestrial composition.

Experimental and Results: Fragments of a silicon target from the passive collector, a ${ }^{25,26} \mathrm{Mg}$ implant (Si target) and a terrestrial magnetite (containing low abundance of $\mathrm{Mg}$-bearing inclusions) were measured in depth-profiling mode with the UCLA Cameca ims 1270 . $100 \times 100 \mu \mathrm{m}^{2}$ craters were sputtered with a $\mathrm{O}_{2}{ }^{+}$beam in $\mathrm{O}_{2}$ atmosphere $(1.2 \mathrm{E}-5$ torr). $7.5 \mathrm{keV}$ impact energy and a field aperture of $40 \times 40 \mu \mathrm{m}^{2}$ were applied to improve depth resolution. $\mathrm{Mg}$ isotopes and ${ }^{28} \mathrm{Si}$ were collected simultaneously on electron multipliers $(\mathrm{Mg})$ and a Faraday cup ( $\mathrm{Si}$ ). A mass resolving power of 2100 sufficiently resolved ${ }^{24} \mathrm{MgH}$ from ${ }^{25} \mathrm{Mg}$. Data are corrected for deadtime, blank and instrumental mass fractionation.

The ${ }^{25,26} \mathrm{Mg}$ implant standard was homogeneously irradiated $\left({ }^{25} \mathrm{Mg} /{ }^{26} \mathrm{Mg}\right.$ from three areas $\sim 5 \mathrm{~cm}$ apart agree within $\left.3 \%\right)$. The ${ }^{25} \mathrm{Mg} /{ }^{26} \mathrm{Mg}$ ratio was calibrated by solution MC-ICPMS at ASU to be $0.9571 \pm 0.0051(2 \sigma$ stdev $)$. Uncertainties in the Genesis data are $\sim 5 \%$ $(1 \sigma)$. Due to unresolved issues with deadtime corrections, we only report $\delta^{26} \mathrm{Mg} /{ }^{25} \mathrm{Mg}$ calibrated against the implant standard: $0.2 \pm 4.1 \%$ (4 runs, $1 \sigma$ stdev). This preliminary result agrees with $[3,4]$. Thus, at present the isotopic fractionation in SW can neither be confirmed nor excluded. Future analyses that include more standards, a better control on the deadtime and more Genesis analyzes will reduce our uncertainties.

References: [1] Young et al. 2004. Reviews in mineralogy and geochemistry, vol. 55. pp. 197-230. [2] Bodmer et al. 2000. Journal of Geophysical Research 105:47-60. [3] Rieck et al. 2010. Abstract \#2391. 41st Lunar and Planetary Science Conference. [4] Humayun et al. 2011. Abstract \#1211. 42nd Lunar and Planetary Science Conference.

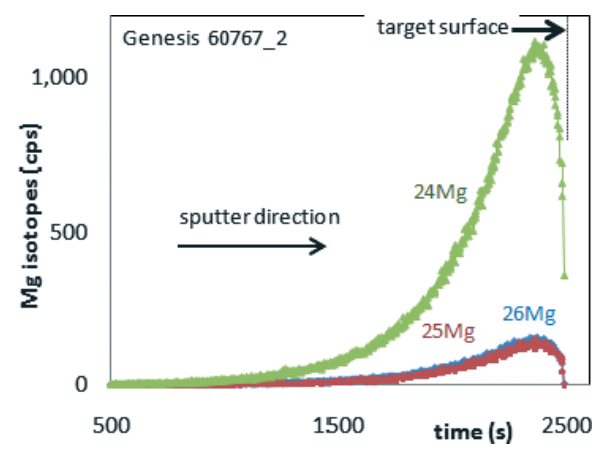

Fig. 1. Backside depth profile of $\mathrm{SW} \mathrm{Mg}$ isotopes. This mode produces a complete profile, avoiding surface contamination and transient sputter effects otherwise encountered when sputtering from the front side. Isotopic ratios were calculated from the sum of the counting rates over the complete profile.
5372

\section{ATOM-PROBE TOMOGRAPHY OF METEORITIC AND} SYNTHETIC NANODIAMONDS

P. R. Heck ${ }^{1,2}$, M. J. Pellin ${ }^{2,3,4,5}$, A. M. Davis ${ }^{1,2,3,5}$, D. Isheim ${ }^{6}$, D. N. Seidman $^{6}$, J. Hiller ${ }^{4}$, A. Mane ${ }^{7}$ J. Elam ${ }^{7}$, M. R. Savina ${ }^{2,4}$, T.

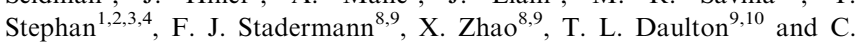
Floss ${ }^{8,9}$. ${ }^{1}$ Robert A. Pritzker Center for Meteoritics and Polar Studies, Department of Geology, The Field Museum, Chicago, IL, USA. E-mail: prheck@fieldmuseum.org. ${ }^{2}$ Chicago Center for Cosmochemistry. ${ }^{3}$ Department of the Geophysical Sciences, University of Chicago, Chicago, IL, USA. ${ }^{4}$ Materials Science Division, Argonne National Laboratory, Argonne, IL, USA. ${ }^{5}$ Enrico Fermi Institute, University of Chicago, Chicago, IL, USA. ${ }^{6}$ Northwestern University Center for AtomProbe Tomography, Department of Materials Science \& Engineering, Northwestern University, Evanston, IL, USA. ${ }^{7}$ Energy Systems Division, Argonne National Laboratory, Argonne, IL, USA. ${ }^{8}$ Laboratory for Space Sciences. ${ }^{9}$ Physics Department. ${ }^{10}$ Center for Materials Innovation, Wash. University, St. Louis, MO, USA.

Introduction: Because of their small size (approximately $3 \mathrm{~nm}$ [1]) meteoritic nanodiamonds [2] are the least understood component found in acid residue containing presolar grains. Their abundance is higher than for any other presolar grains [2], and the knowledge of their origins is therefore of great interest. Isotope anomalies in trace elements have been observed from bulk analyses of nanodiamond residue (e.g., [2]), but bulk $\mathrm{C}$ and $\mathrm{N}$ isotopic ratios are normal [3]. This and other observations have led to suggestions that only a small fraction of nanodiamonds is presolar (e.g., [4]). Another possibility is that the anomalies are carried by an associated abundant disordered non-diamond $\mathrm{sp}^{2} \mathrm{C}$ phase [5]. Carbon isotope analyses of individual nanodiamonds would test if ${ }^{12} \mathrm{C} /{ }^{13} \mathrm{C}$ ratios in any diamonds are anomalous. The atom probe tomograph (APT [6]) is currently the only instrument that can analyze the $\mathrm{C}$ isotopic compositions of individual nanodiamonds. The first APT results on Allende DM [7] nanodiamond residue (ADM) were recently presented $[8,9]$. To correct for a potential instrumental bias, we have now prepared synthetic nanodiamond samples as analytical standards for the APT.

Methods: We used a dual-beam focused ion-beam (FIB) microscope to prepare APT samples of ADM [8]. In another approach, we deposited meteoritic or synthetic nanodiamonds directly on presharpened microtips for APT, and embedded them in a stable matrix using atomic-layer deposition [10] of $\mathrm{W}$ or $\mathrm{Cu}$. APT analyses were performed with a Cameca LEAP4000XSi tomograph equipped with a picosecond UV laser [6].

Results: Direct deposition sample preparation eliminates the need for FIB lift-out work and improves sample stability. We succeeded in APT analyses of ADM and obtained well-defined C peaks in time-offlight mass spectra. Our 3D-tomographic reconstructions exhibit isolated and clustered nanodiamonds. The $\mathrm{C}$ isotopic compositions of different APT tips of the same aliquot are identical within analytical uncertainties. At the meeting, we will present more data that will help to address the origin of the diamonds and also present APT data of synthetic nanodiamonds.

References: [1] Daulton T. L. et al. 1996. Geochimica et Cosmochimica Acta 60:4853-4872. [2] Lewis R. S. et al. 1987. Nature 326:160-162. [3] Russell S. S. et al. 1996. Meteoritics \& Planetary Science 31:343. [4] Dai Z. R. et al. 2002. Nature 418:157-159. [5] Stroud R. M. et al. 2010. Meteoritics \& Planetary Science 73:5352S. [6] Seidman D. N. and Stiller K. 2009. MRS Bull. 34:717-721. [7] Lewis R. S. et al. 1989. Nature 339:117-121. [8] Heck P. R. et al. 2011. Abstract \#2112. 42nd Lunar and Planetray Science Conference. [9] Stadermann F. J. et al. 2011. Abstract \#2134. 42nd Lunar and Planetary Science Conference. [10] Elam J. W. et al. 2006. Applied Physics Letters 89:053124. 


\section{1}

MODELING THE THERMAL CONSTITUTION AND THERMAL EVOLUTION OF ASTEROIDS CONSIDERING SINTERING PROCESSES AND HEATING BY ${ }^{26} \mathrm{Al}$ AND ${ }^{60} \mathrm{Fe}$

S. Henke ${ }^{1}$, H. -P. Gail ${ }^{1}$, M. Trieloff ${ }^{2}$, W. Schwarz ${ }^{2}$ and T. Kleine ${ }^{3}$. ${ }^{1}$ Institut für theoretische Astrophysik ZAH Heidelberg. E-mail: shenke@ ita.uni-heidelberg.de. ${ }^{2}$ Institut für Geowissenschaften Heidelberg. ${ }^{3}$ Institut für Planetologie, Westfälische Wilhelms- Universität Münster.

We present models for the internal constitution and thermal evolution of planetesimals of about $100 \mathrm{~km}$ radius, assuming spherical symmetry.

We solve the one dimensional heat conduction equation and the hydrostatic pressure equation. Heating in our model is due to ${ }^{26} \mathrm{Al},{ }^{60} \mathrm{Fe}$ and long living isotopes as well as the potential energy released during shrinking of the bodies during compaction. We chose the new measured ${ }^{60} \mathrm{Fe}$-halflife (2.6 Ma) reported by [1].

We implemented compaction processes by pressure induced cold compaction based on [2], and by sintering due hot isostatic pressing based on the theory of [3] and [4]. A porosity dependent heat conductivity based on results of laboratory measurements $[5,6]$ is used.

We show results of model calculations for the first $200 \mathrm{Ma}$ of evolution after body formation and compare this to data for the cooling history of H-chondrites. We fit the initial parameters (time of body formation after CAI formation, environmental temperature, heat conduction of the chondritic material) to data of $\mathrm{H}$-chondrites.

The high-porosity layer that remains on the surface in our model causes much higher temperatures in the outer layers of the body than in the analytic model of Miyamoto [7], thus layers of certain petrologic type are much closer to the surface.

References: [1] Rugel F. et al. 2009. Physical Review Letters 103:07250. [2] Güttler C. et al. 2009. The Astrophysics Journal 701:130. [3] Kakar A. K. and Chaklader A. C. D. 1967. Journal of Applied Physics 38:3223. [4] Rao A. S. and Chaklader A. C. D. 1972. Journal of the American Ceramic Society 55:596. [5] Yomigida K. and Matsui T. 1983. Journal of Geophysical Research 88:9513. [6] Krause M. et al. 2011. arXiv 1101.2586. [7] Miyamoto M. et al. 1981. 12th Lunar and Planetary Science Conference p. 1145.

\section{1}

\section{R-PROCESS ELEMENTS IN PRESOLAR SIC GRAINS}

T. Henkel ${ }^{1}$, D. Rost ${ }^{1}$ and I. C. Lyon ${ }^{1}$. ${ }^{1}$ The University of Manchester, SEAES, Oxford Road, Manchester, M13 9PL, UK. E-mail: torsten.henkel@manchester.ac.uk.

Introduction: R-process elements are of special interest in presolar grains. It is still unclear how many sites have contributed and/or if the $\mathrm{r}$-process is universal $[1,2]$ which would allow dating $\mathrm{r}$-process material $[3,4]$. Analyzing r-process material in presolar grains however is a major challenge as the abundances of these elements are very low $(\leq \mathrm{ppm})$ and the grains very small (few micrometers in size) resulting in less than $10^{6}$ atoms per element per grain.

Dating single grains would help establish the grains' lifetime in the interstellar medium (ISM). This is of special interest as the grains are bombarded by cosmic radiation during their travel through the ISM which limits their theoretical lifetime through sputtering [5] and might implant material [6] - although the latter could also happen during the formation of the grains or while incorporated into the forming solar system.

Traditionally secondary ion mass spectrometry (SIMS) has been used for in situ measurements of single grains but ionization efficiencies are too small to analyze isotope ratios of trace elements. This can be overcome by using laser post-ionization of secondary neutrals which improves the instrument sensitivity significantly.

Experimental: We used our time-of-flight SIMS instrument [7] which has an $F_{2}$-excimer laser $(157 \mathrm{~nm}, 7.9 \mathrm{eV})$ for nonresonant ionization of secondary neutrals. Relative sensitivity factors for major elements have already been determined from silicate glass standards [7]. Sensitivity improvements from postionization by up to 2 orders of magnitudes have been achieved.

We have been able to detect heavy element impurities $(\mathrm{Pd}, \mathrm{Ag}, \mathrm{Sn}$ and $\mathrm{Pt}$ ) in gold foil which are typically on the order of a few ppm only. The improved sensitivity allowed the determination of all the major isotope ratios of these elements demonstrating the instrument capabilities.

Results: So far one presolar $\mathrm{SiC}$ grain $(1.5 \mu \mathrm{m}$ in size $)$ has been analyzed which was located with time-of-flight SIMS and confirmed with energy dispersive X-ray analysis. The abundances of heavy elements above iron in the grain were below the detection limits. The grain has a $\delta^{30} \mathrm{Si}$-value of $20 \pm 50 \%$ with an $\mathrm{Al}$ abundance of approximately $1.5 \%$ relative to $\mathrm{Si}$ and other light elements well below $1 \%$. The grain is therefore not of supernova origin and most likely a mainstream grain which might explain the very low or absent heavy elements.

Outlook: The postionization is now working reliably and has been proven capable of detecting heavy elements in low abundances. We are currently locating more presolar grains in our acid-residue and gently separated samples which we will analyze using laser postionization to search for r-process patterns. More results will be presented at the conference.

References: [1] Qian Y.-Z. and Wasserburg G. J. 2000. Physics Reports 333:77-108. [2] Otsuki K. et al. 2003. New Astronomy 8:767-776. [3] Cowan J. J. and Sneden C. 2006. Nature 440:1151-1156. [4] Frebel A. et al. 2007. The Astrophysical Journal 660:L117-L120. [5] Jones A. P. et al. 1994. The Astrophysical Journal 433:797-810. [6] Lyon I. C. et al. 2007. Meteoritics \& Planetary Science 42:373-385. [7] Henkel T. et al. 2007. Review of Scientific Instruments 78:055107. 


\section{0}

BADDELEYITE OCCURRENCES IN MARTIAN METEORITES AND THE POTENTIAL FOR IN SITU GEOCHRONOLOGY OF ZAGAMI C. D. K. Herd ${ }^{1}$ and T. J. McCoy ${ }^{2} .{ }^{1}$ Department of Earth and Atmospheric Sciences, 1-26 Earth Sciences Building, University of Alberta, Edmonton, AB, Canada T6G 2E3. ${ }^{2}$ Department of Mineral Sciences, Smithsonian National Museum of Natural History, 10th \& Constitution NW, Washington, DC 20560-0119, USA. E-mail: herd@ ualberta.ca.

Introduction: Baddeleyite $\left(\mathrm{ZrO}_{2}\right)$ is of interest for its potential use in $\mathrm{U}-\mathrm{Pb}$ geochronology of Martian meteorites [1]. Although it is more common in Martian meteorites than previously recognized, small grain sizes require in situ approaches for analysis, i.e., ion probe mass spectrometry. The primary step, therefore, is locating and documenting baddeleyite grains in thin section. Here we report on the results of this process applied to Zagami, especially the dark, mottled lithology (DML) [2]. Our observations have implications for the occurrence of baddeleyite in Martian meteorites, and the potential for application of in situ U-Pb geochronology methods to this meteorite.

Methods: Thin sections reflecting the range of Zagami lithologies were examined, including normal Zagami and DML (USNM 6545-2), DML (UH 218) and a late-stage melt pocket (LSMP; UH 233). The Particle Search method, which maps Zr hotspots by WDS (Cameca SX100 electron microprobe; University of Alberta) is the most efficient method for locating baddeleyite grains. Grain locations were then verified, documented and measured using the FEI Nova NanoSEM (Smithsonian).

Results: A total of 481 grains were located in the three thin sections; 320 in DML (specimens UH 218 and USNM 6545-2), 95 in Normal Zagami (USNM 6545-2) and 66 in the LSMP (UH 233). These occurrences correspond to frequencies of 1 grain approximately every $1.3 \mathrm{~mm}^{2}$ in DML, 1 every $0.6 \mathrm{~mm}^{2}$ in Normal Zagami, and 1 every $0.5 \mathrm{~mm}^{2}$ in the LSMP. In each case, baddeleyite was found in association with late-stage crystallization products. Most commonly it is found within fine-grained, silica-rich mesostasis pockets, within coarse mesostasis (specific to LSMP) or enclosed within titanomagnetite.

Implications: The primary factor influencing baddeleyite occurrence is buildup of $\mathrm{Zr}$ in late-stage melt [3]. Baddeleyite may be expected to occur in any Martian basalt that started with sufficient $\mathrm{Zr}$ content in the parental melt, and within which $\mathrm{Zr}$ remained incompatible in all other igneous minerals through the course of crystallization. Accordingly, baddeleyite is common in DML, an evolved, incompatible elementenriched lithology within an already $\mathrm{Zr}$-rich rock [3]. That baddeleyite is relatively common in normal Zagami was unexpected, but its occurrence in association with late-stage crystallization phases is consistent with buildup of $\mathrm{Zr}$ as crystallization proceeds.

There is no lack of baddeleyite grains in Zagami. Based on our observations, the best places to find grains of sufficient size and abundances for in situ U-Pb geochronology are (1) within coarse mesostasis in LSMP (average grain size $3.6 \pm 2.4 \mu \mathrm{m}, n=26$ ); and (2) enclosed within titanomagnetite $(4.5 \pm 3.2 \mu \mathrm{m}, n=51)$. The largest grain found in LSMP coarse mesostasis is $14 \times 9 \mu \mathrm{m}$. The largest grains found enclosed in titanomagnetite are $10 \times 8 \mu \mathrm{m}$ and $22 \times 6 \mu \mathrm{m}$ (where $6 \mu \mathrm{m}$ is the maximum width).

References: [1] Herd C. D. K. et al. 2007. Abstract \#1664. 38th Lunar and Planetary Science Conference. [2] McCoy T. J. et al. 1999. Geochimica et Cosmochimica Acta 63:1249-1262. [3] Herd C. D. K. and McCoy T. J. 2011. Abstract \#1801. 42nd Lunar and Planetary Science Conference.
5291

\section{NOMCOM REVIEW OF TYPE SPECIMEN REPOSITORIES}

C. D. K. Herd ${ }^{1}$, C. L. Smith ${ }^{2}$, J. N. Grossman ${ }^{3}$, M. K. Weisberg ${ }^{4}$ and L. Welzenbach ${ }^{5}$. ${ }^{1}$ Department of Earth and Atmospheric Sciences, University of Alberta, Edmonton, AB, Canada T6G 2E3. E-mail: herd@ualberta.ca. ${ }^{2}$ Department of Mineralogy, Natural History Museum, London, SW7 5BD, UK. ${ }^{3}$ NASA Headquarters, Mail Stop 3V71, 300 E Street SW, Washington, DC 20546, USA. ${ }^{4}$ Earth and Planetary Sciences, Kingsborough College, City University of New York, Brooklyn, NY 11235, USA. ${ }^{5}$ Department of Mineral Sciences, National Museum of Natural History, Smithsonian Institution, Washington, DC 20560, USA.

The Issue: The Nomenclature Committee (NomCom) Guidelines for Meteorite Nomenclature [1] require that "type specimens of all new meteorites are preserved in collections that make material available for research" and that "type specimens must be deposited in institutions that have well-curated meteorite collections and long-standing commitments to such curation." Despite the fact that the terms "well-curated" and "longstanding" are vague, NomCom has, to date, approved nearly 90 different repositories to house type specimens, some of which, in retrospect, appear not to have been good choices. Because type specimens are in many cases the only materials, from a specific meteorite, available for research, NomCom has decided to re-evaluate its current policies. The goal is to assure that all type specimens will be available to future researchers and that they will be curated in an appropriate manner.

The Plan: To begin the evaluation process, NomCom has developed a simple questionnaire for curators of existing repositories to complete online. The questionnaire will serve several goals: to update the contact information for repositories, including individuals with curatorial responsibility and those with institutional oversight; to invite respondents to participate in the development of a "best practices" document; and to create an archive of any collections policies they may have. The information collected will be made available as a community resource. The 'best practices' document will eventually include a set of minimum guidelines that we expect any collection to meet if acting as a type specimen repository.

Community Response: Over the next few weeks, individuals with curatorial responsibility for type specimen repositories will receive a letter by email with a link to a page where they can provide the requested contact information, as well as share any policy or procedure documents. We ask that the community assist NomCom in this endeavor, and we welcome any feedback as the process continues.

Reference: [1] Guidelines for Meteorite Nomenclature, 2011. http:// meteoriticalsociety.org/bulletin/nc-guidelines.htm. 


\section{9}

STUDIES ON PRISTINE SAMPLES OF BUZZARD COULEE

R. K. Herd ${ }^{1,3}$, C. Samson ${ }^{1}$, C. Fry ${ }^{1}$, D. Melanson ${ }^{1}$, P. J. A. McCausland ${ }^{2}$ and R. Ernst ${ }^{1}$. ${ }^{1}$ Department of Earth Sciences, Carleton University, Ottawa, Ontario, Canada K1S 5B6. ${ }^{2}$ Department of Earth Sciences, University of Western Ontario, London, Ontario, Canada N6A 5B7. ${ }^{3}$ Earth Sciences Sector, Natural Resources Canada, Ottawa, Ontario, Canada K1A 0E8. E-mail: herd@nrcan.gc.ca.

Introduction: Eighteen individual samples of Buzzard Coulee, an H4 chondrite that fell south of Lloydminster, Saskatchewan, on November 20, 2008 were obtained by the National Meteorite Collection of Canada. Their masses range from $109.14 \mathrm{~g}$ to $8.80 \mathrm{~g}$. Most are fully to almost-fully crusted and were collected before the winter of 2008-2009.

Methodology: To document this suite of samples in their "as received" condition, and to study varying masses of the same meteorite fall, they have first been characterized using independent non-destructive techniques, prior to any mineralogical and textural work. Each fragment, weighed and photographed upon acquisition, has been tracked with checks on weights and with photographs at all stages. Here we report the results from laboratory measurements of bulk volume and density (by silica beads, 3D laser imaging, and X-ray micro-CT scans), grain volume, grain density and porosity (by helium pycnometry), and magnetic susceptibility (in different laboratories and using different instruments).

Results: The silica bead method [1] gave sample volumes yielding an average density of $3.49 \pm 0.06 \mathrm{~g} \mathrm{~cm}^{-3}$ for the sample suite. The volumes from the $3 \mathrm{D}$ laser imaging method gave a slightly more consistent average suite density of $3.46 \pm 0.03 \mathrm{~g} \mathrm{~cm}^{-3}$. X-ray micro-CT scan data used to calculate the sample volumes yielded an average density of $3.41 \pm 0.04 \mathrm{~g} \mathrm{~cm}^{-3}$. These values agree with other findings using the bead method (3.26-3.45 g cm${ }^{-3}$ for six fragments [2]) and 3D laser imaging ( $3.5 \mathrm{~g} \mathrm{~cm}^{-3}$ for one $151.7 \mathrm{~g}$ fragment [3]), and are typical for $\mathrm{H}$ chondrites [4]. The fragments have an average porosity of $6.14 \pm 1.5 \%$ and a grain density of $3.69 \pm 0.03 \mathrm{~g} \mathrm{~cm}^{-3}$, similar to reported values from other $\mathrm{H}$ chondrites within a standard deviation of the mean [5]. Since the 3D laser density values were more consistent, they were divided into the volume magnetic susceptibility readings to give the mass magnetic susceptibility $(X)$ of each sample. The average log of $X$ was $5.2 \pm 0.056 \times 10^{-9} \mathrm{~m}^{3} \mathrm{~kg}^{-1}$, typical of an $\mathrm{H}$ chondrite [6-8]. The magnetic fabric defines an oblate ellipsoid. The laser based density measurements plotted vs. magnetic susceptibility, and vs. porosity, show that the data cluster within 2 standard deviations of the average, forming a single population, unlike the bimodal distribution reported previously [2].

Summary: These studies define a methodology for preliminary examination of new meteorite falls and finds [8], or of specimens from sample return missions to space. Digitized data is preserved on samples for archival and curatorial purposes, and can be used to select subsets of them for further research. Variations in their physical properties may be linked to internal variations such as concentrations of metallic minerals or zones of shock. The X-ray micro-CT scans in particular may be used to define metal concentrations and to calculate bulk metal contents. Portions of the meteorites where polished thin-sections would yield critical details of mineralogy and texture can be pre-selected.

References: [1] Macke R. J. et al. 2009. Planetary and Space Science doi: 10.1016/ j.pss.2009.11.006. [2] Hildebrand A. R. et al. 2009. Abstract \#1893. 40th Lunar and Planetary Science Conference. [3] Walton E. L. et al. 2009. Abstract \#2072. 40th Lunar and Planetary Science Conference. [4] Consolmagno G. J. and Britt D. T. 1998. Meteoritics \& Planetary Science 33:1231-1241. [5] Consolmagno G. J., Britt D. T. and Macke R. J. 2008. Chemie der Erde 68:1-29. [6] Rochette P. et al. 2003. Meteoritics \& Planetary Science 38:251-268. [7] Rochette P. et al. 2009. Elements 5:223-228. [8] Smith D. L. et al. 2006. Meteoritics \& Planetary Science 41:355-373.
5508

TIME-RESOLVED STUDIES OF HYPERVELOCITY VERTICAL IMPACTS INTO POROUS PARTICULATE TARGETS: EFFECTS OF PROJECTILE DENSITY ON EARLY-TIME COUPLING AND CRATER GROWTH

B. Hermalyn and P. H. Schultz. Brown University, Providence, RI 02912-1846, USA. E-mail: Brendan_Hermalyn@brown.edu.

Introduction: The depth and duration of energy and momentum coupling in an impact shape the formation of the crater and distribution of ejected material. Metrics for crater growth (e.g., crater diameter and depth) and ejecta velocity are usually approached from late-stage parameters $[1,2]$. Implicit in most of these studies is a reliance on the point-source approximation, which collapses the initial details of energy and momentum coupling into a single parameter [3]. This approximation breaks down at early times while the projectile is still transferring its energy and momentum to the target, however [4]. The flow-field inside the transient crater is reflected in the ejecta velocity distribution.

In this study, we present new experimental measurements of the ejecta distribution and crater growth from early- to main-stage flow to examine the role of impactor density on the depth and duration of coupling. The technique employed allows expression of the ejecta velocities as a function of both time and launch position for the first time, as well as direct comparison to and benchmarking of hydrocodes.

Experimental Methodology: A suite of hypervelocity vertical impact experiments into Ottowa quartz sand was performed at the NASA Ames Vertical Gun Range using a variety of different projectile materials ranging in density from $0.9 \mathrm{~g} / \mathrm{cc}$ to $7.8 \mathrm{~g} / \mathrm{cc}$. A non-intrusive, high-speed $(15,000 \mathrm{fps})$ imaging technique (particle tracking velocimetry or PTV) tracks the motion of individual sand grains during excavation and allows temporally and spatially resolved measurements of the ejecta velocities.

Results and Analysis: The earliest stages of excavation result in highspeed ejecta derived from the upper surface near the impact point. Dense projectiles penetrate deeper and couple later in the target, whereas underdense projectiles couple quickly relatively closer to the surface. Crater growth metrics for both the depth and diameter of the crater demonstrate non-proportional crater growth, where the crater first exhibits more relatively downward growth before transitioning to relatively outward expansion. This flow-field center evolution manifests in migrating ejection angles for much of crater formation. The temporal nature of the data also enables validation of computational parameters (e.g., EOS) using the CTH hydrocode.

Conclusions: The effect of projectile density on both the depth and duration of coupling has implications for planetary impacts, affecting models of ballistic ejecta emplacement and source depths of ejecta from primary craters (e.g., Apollo samples). Temporal (rather than spatial) description of the ejecta dynamics allows a physical understanding for ejecta produced by artificial impacts, such as the Deep Impact mission and the recent LCROSS impact. Investigation of the time-resolved growth of the crater is essential for comparison to the azimuthally varying ejecta velocities from oblique impact events.

References: [1] Housen et al. 1983. Journal of Geophysical Research 88:2485-2499. [2] Barnouin-Jha et al. 2007. Icarus 188:506-521. [3] Holsapple and Schmidt. 1987. Journal of Geophysical Research 92:63506376. [4] Hermalyn and Schultz 2010. Icarus 209:866-870. 
5158

\section{PHOTOPHORESIS ON CHONDRULES-NEW EXPERIMENTAL} RESULTS

A. Hesse, J. Teiser and G. Wurm. Faculty of Physics, University Duisburg-Essen, Essen, Germany. E-mail: alexander.hesse@stud.unidue.de.

Introduction: Photophoresis, as a fourth light-induced force alongside radiation pressure, Poynting-Robertson drag and the Yarkovski effect, exceeds these by orders of magnitude at conditions found in a protoplanetary disk $[1,2]$.

In contrast to the aforementioned forces, photophoresis is not based on a direct acceleration by photons: Gas molecules of the surrounding atmosphere stick to the surface of a unidirectionally illuminated particle. They take on the different temperatures along the illumination axis and therefore leave the surface at the corresponding thermal velocities. This results in a net momentum transfer that is directed away from the light source (e.g., the central star of the protoplanetary disk).

With its inherent pressure- and particle-size-dependence, it not only qualifies as an additional mechanism for particle transport, but also provides an effective way of sorting. These properties make photophoresis an important aspect in explaining the occurrence of size-sorted chondrules. In a first campaign of microgravity experiments, the efficiency of photophoresis acting on chondrules could clearly be shown. A followup project was initialized to shed light on more detailed aspects of photophoretic acceleration, including rotational properties.

Experiment: To quantify the effect, particularly with regard to chondrules, experiments were carried out at the ZARM drop tower in Bremen, providing approximately $9 \mathrm{~s}$ of microgravity with a residual acceleration of less than $10^{-6} \mathrm{~g}$.

Chondrules originating from the L/LL4-class chondrite Bjurböle are exposed to an intense laser beam in the microgravity environment of the drop capsule at varying ambient pressures. Their motion is observed with two synchronized cameras aligned perpendicular to each other and the incident direction of the laser beam. In the recorded videostreams the three-dimensional motion of the chondrules can be obtained and rotational frequencies can be determined.

Results: The results for the linear motion are consistent with the outcome of the initial study [3] and the measured accelerations of approximately $10^{-4} \mathrm{~m} \mathrm{~s}^{-2}$ are in accordance with the estimates of model forces [4]. Moreover for certain rotating chondrules transverse components in the acceleration could be detected, which hints at a process similar to the Yarkovski effect but with photophoretic momentum transfer. These results are the first experimental data on the interplay between particle rotation, photophoresis and material transport.

References: [1] Wurm G. and Krauss O. 2006. Icarus 180:487-495. [2] Wurm G. and Haack H. 2009. Meteoritics \& Planetary Science 44:689-699. [3] Wurm G. et al. 2010. Icarus 208:482-491. [4] Rohatschek H. 1995. Journal of Aerosol Science 26:717-734.

\section{4}

TYPE II CHONDRULE ORIGIN IN O AND C CHONDRITES

R. H. Hewins ${ }^{1,2}$ and B. Zanda ${ }^{1,2}$. ${ }^{1}$ MNHN \& CNRS, 75005 France. ${ }^{2}$ Deptartment of Earth and Planet Sciences, Rutgers University, Piscataway, NJ, USA. E-mail: hewins@rci.rutgers.edu.

Relict grains are widespread in chondrules: many Type I chondrules contain olivine not crystallized from their associated Al-rich melts [1], including grains with very low $\Delta^{17} \mathrm{O}[2,3]$ and GOA with several specific $\mathrm{O}$ isotope compositions closer to TFL than solar [4]. This suggests chondrule formation with various dust/gas ratios and relatively inefficient recycling. Addition of $\mathrm{SiO}$ to the rims of many chondrules changes them from IA to IAB to IB, perhaps in regions of higher dust/gas ratio generating high $\mathrm{P}_{\mathrm{SiO}}$ during chondrule heating, with higher $\Delta^{17} \mathrm{O}$ [5]. The Nancy open-system chondrule model [1, 3-5] has recently been extended to Type II chondrules formed from Type I material by adding $\mathrm{Fe}$ and $\mathrm{O}$ from the gas [6]. We use our recent extensive EMP data [7] for Type II chondrules in Paris and other CM chondrites to develop the Nancy-type II chondrule model.

Type II chondrules contain abundant $\mathrm{Na}, \mathrm{FeO}$, and $\mathrm{S}$, consistent with formation in large dusty clumps yielding high $\mathrm{P}_{\mathrm{Na}}$, etc. The common survival of relict forsterite of Type I composition in Type II chondrules in both CC and OC strongly supports a Type I precursor. Oxidation of Fe from metal in Type I chondrules [6] would explain the jump in FeO from Type IA olivine composition to the most magnesian Type IIA chondrule olivines. However, Type II chondrules in both CC and OC have positively correlated $\mathrm{FeO}$ and $\mathrm{MnO}$, consistent with condensation rather than oxidation [7]. Olivine in Type II chondrules in Paris and other CMs extends up to $\mathrm{Fa}_{70}$, more ferroan than in OC. The OC chondrules experienced less condensation of $\mathrm{FeO}$, possibly because of lower $\mathrm{P}_{\mathrm{H}_{2} \mathrm{O}}$, and we find $f \mathrm{O}_{2}$ from QFI about $1 / 2 \log$ unit lower.

Type II chondrule olivine in $\mathrm{CMs}$ has an $\mathrm{Fe} / \mathrm{Mn}$ ratio like that in $\mathrm{CO}$ [8], micrometeorites and Wild 2 [9], but distinct from that in OC [7] and therefore comes from a different reservoir. The occurrence of IIA-IIB chondrules in both Paris and Semarkona indicates Fe- and Si-enrichment paths in both reservoirs. CR chondrites, on the other hand, contain Type IIs with intermediate $\mathrm{Fe} / \mathrm{Mn}$ and $\mathrm{O}$ isotope characteristics split between CM-CO and OC reservoirs [8, 10], and thus indicate changing redox conditions related to variations in dust/gas ratios and/or ice contents.

OC contain clusters of chondrules, both Type I and Type II, with similar $\Delta^{17} \mathrm{O}$, consistent with recycling side by side. This suggests turbulent mixing on a local scale before and during transient heating events. In CC, Type II chondrules are much less abundant and formed at higher $\mathrm{f}_{\mathrm{O} 2}$ than in $\mathrm{OC}$ while Type I chondrules have $\Delta^{17} \mathrm{O}$ mostly closer to solar than those in OC. Thus Type IIs were moved to a domain containing abundant dust, including Type Is, themselves formed in several oxygen isotopic reservoirs. This assemblage was not recycled before accretion.

References: [1] Libourel G. et al. 2006. Earth and Planetary Science Letters 251:232-240. [2] Yurimoto H. and Wasson J. T. 2002. Geochimica et Cosmochimica Acta 66:4355-4363. [3] Jones R. H. 2004. Geochimica et Cosmochimica Acta 68:3423-3438. [4] Libourel G. and Chaussidon M. 2011. Earth and Planetary Science Letters 301:9-21. [5] Chaussidon M. et al. 2008. Geochimica et Cosmochimica Acta 72:1924-193. [6] Villeneuve J. 2010. Ph.D., CRPG Nancy. [7] Hewins R. H. et al. 2011. Abstract \#1914. 42nd Lunar and Planetary Science Conference. [8] Berlin J. et al. 2009. Meteoritics \& Planetary Science 46:513-533. [9] Dobricá E. 2010. Ph.D., Univ. Paris Sud. [10] Connolly H. C. Jr. and Huss G. R. 2010. Geochimica et Cosmochimica Acta 74:2473-2483. 


\section{5}

\section{D STRUCTURE OF CARBONACEOUS CHONDRITES}

D. C. Hezel ${ }^{1,2}$, P. Elangovan ${ }^{1,3}$, R. L. Abel ${ }^{1,4}$, L. E. Howard ${ }^{1}$ and R. Armstrong ${ }^{1}$. ${ }^{1}$ Natural History Museum, Department of Mineralogy, Cromwell Road, SW7 5BD, London, UK. ${ }^{2}$ Department of Geology and Mineralogy, University of Cologne, Zülpicher Str. 49b, 50674 Cologne, Germany. ${ }^{3}$ Department of Electronic Engineering, University of Surrey, Guildford, GU2 7XH, Surrey, UK. ${ }^{4}$ Department of Earth Science and Engineering, Imperial College, London, SW7 2AZ, UK. E-mail: dominik.hezel@uni-koeln.de.

Introduction: A number of important conditions of the solar nebula prevailing during the formation of chondrite components can only be learned from petrographic data. Component modal abundances for accurate mass balance calculations, chondrule size distributions to determine sorting processes, component spatial distributions for parent body agglomeration processes, distribution of metal and sulfide between chondrules and matrix to study their origin and formation, orientation of chondrules to study the place of impregnation of their paleomagnetic field and many more are examples of cosmochemical questions where petrographic data are mandatory.

So far petrographic studies were mostly restricted to $2 \mathrm{D}$ slices. Such measurements often provide inaccurate results, or are entierly unsuitable for a given problem. Micro computer-aided tomography ( $\mu$-CT) is an emerging technique that has the potential to solve these shortcomings and provide accurate $3 \mathrm{D}$ petrographic measurements. In recent years, much progress was made in scanning and quantifying rocks and meteorites [16]. First, the quality of the scans became much more accurate, with resolutions down to $<1 \mu \mathrm{m}$. Second, powerful computer hard-and software now allows to reduce the tens of GB of data a single scan produces. We developed a plugin for ImageJ especially designed to quantify meteorite $\mu$-CT data. We are continuously adding new tools to this plugin to make it a standard software package for reducing meteorite $\mu$-CT data.

Technique: We used the Metris X-Tek HMX ST $225 \mathrm{CT}$ at the Natural History Museum, London for scanning. Density is the prime information stored in the voxels (3D equivalent of pixels) of a tomographic scan. Segmenting chondrules from matrix is in particular difficult as both have similar densities and, hence, overlap greatly in gray values. ImageJ is a widely used, public domain image analyses tool. We developed a plugin - called PhaseQuant - that, among other features, employs statistical methods to automatically segment and quantify components whose gray values overlap, such as chondrules and matrix.

Results: We scanned a range of carbonaceous chondrites (Allende, Renazzo, Al Rais, etc.) and are producing a set of standard 3D images and videos of the samples. We currently apply PhaseQuant to quantify the data sets to determine component 3D modal abundances, size as well as their spatial distributions. In addition, sulfide and metal abundances between chondrules and matrix are studied. The results will be presented at the meeting.

References: [1] Ebel D. S. and Rivers M. L. 2007. Meteoritics \& Planetary Science 42:1627-1646. [2] Ebel D. S. et al. 2008. Meteoritics \& Planetary Science 43:1725-1740. [3] Friedrich J. M. et al. 2008a. Planetary and Space Science 56:895-900. [4] Friedrich J .M. 2008b. Computers and Geosciences 34:1926-1935. [5] Friedrich J. M. et al. 2008c. Earth and Planetary Science Letters 275:172-180. [6] Hezel D. C. et al. 2010. Earth and Planetary Science Letters 296:423-433.
5245

XANES AND FERROUS-FERRIC RATIOS IN THE NAKHLITE SECONDARY PHASES

L. J. Hicks ${ }^{1}$, J. C. Bridges ${ }^{1}$ and S. J. Gurman ${ }^{2}$. ${ }^{1}$ Space Research Centre, University of Leicester, Leicester LE1 7RH, UK. E-mail: j.bridges@ le.ac.uk. ${ }^{2}$ Department of Physics \& Astronomy, University of Leicester, Leicester LE1 7RH, UK.

Introduction: X-ray absorption near edge structure (XANES) observes spectra created by the energies of the $3 d$ orbitals in transition metals like iron [1]. Our aim is to report observations and analyses of the Fe K XANES in standards and nakhlite samples in order to gain a calibration between the XANES and the ferrous-ferric ratio of the measured material. This will allow us to constrain the oxidation state, and how it changes, within the nakhlite alteration minerals such as phyllosilicate [2].

Method and Samples: We have used the I18 Beamline for Microfocus Spectroscopy at the UK Diamond Synchrotron. This enabled a spatial resolution of $2 \times 2.5 \mu \mathrm{m}$, measuring energy spectra ranging from 6900 to $7500 \mathrm{eV}$ with a resolution of $0.25 \mathrm{eV}$, but concentrating on the XANES region from 7092 to $7122 \mathrm{eV}$ with an energy resolution of $0.1 \mathrm{eV}$. The nakhlite samples have also been analyzed with Scanning Electron Microscopy at $15 \mathrm{kV}$. The nakhlite samples are NWA 817, Y000593, Y000749, Nakhla, NWA 5790, Governador Valadares, Lafayette, NWA 998, and Miller-Range 03346, along with standards of goethite, haematite, nontronite, magnetite, augite, pyrrhotite, San Carlos olivine, and Barwellolivine.

The pre absorption edge centroid position was calculated by measuring the average position of the normalized intensity summed over the XANES prepeak region subtracted from a curve-fitting baseline. Thus, the calculated position is greatly influenced by the structure of the $L_{2}$ and $L_{3}$ edges observed as prepeak features, with the $L_{3}$ edge typically consisting of two significant peaks which vary in intensity between the left peak (approximately $7112.2 \mathrm{eV}$ ) and the right peak (approximately $7113.7 \mathrm{eV})$ attributed to the presence of $\mathrm{Fe}^{2+}$ and $\mathrm{Fe}^{3+}$ respectively [3].

Results and Conclusions: Barwell L6 olivine $\left(\mathrm{Fe}^{3+} / \Sigma \mathrm{Fe}=0\right)$ has $\mathrm{K}$ edge positions and calculated pre-edge centroid positions of 7118.8 and $7112.3 \mathrm{eV}$. With an increased oxidation state, as seen in hematite and goethite $\left(\mathrm{Fe}^{3+} / \Sigma \mathrm{Fe}=1\right)$, the edge positions and pre-edge centroid were 7122.2 and $7114.7 \mathrm{eV}$ respectively. There is a clear shift seen in these and other standards, in the pre-edge features and edges associated with increasing ferrous-ferric ratios.

XANES measurements across veins, observed in the nakhlites, showed increases in energy for the K-edge and centroid position towards the central gel feature of the vein. Across one particular Lafayette vein, ten points each $2 \mu \mathrm{m}$ apart were analyzed, the K-edge and centroid increased from 7118.6 and $7112.1 \mathrm{eV}$, in the surrounding olivine, to 7121.0 and $7114.8 \mathrm{eV}$ in the central gel of the vein. Based on these observations, the central gel feature has K-edge and centroid positions between those of the magnetite and hematite standards, indicating an approximate $\mathrm{Fe}^{3+} / \Sigma \mathrm{Fe}$ ratio between 0.67 and 1.0. Observing the XANES spectra throughout these measurements, there is also a distinct change in the pre-edge structure in the outer-vein material from that of the surrounding olivine. This outermost vein material is phyllosilicate, measuring a ratio $\mathrm{Fe}^{3+}$ / $\Sigma \mathrm{Fe} \approx 0.4$ and becomes more ferric with each measurement towards the central gel of the vein. Other vein minerals found in Lafayette include siderite, where XANES suggests a $\mathrm{Fe}^{3+} / \Sigma \mathrm{Fe}$ ratio between 0 and 0.2 .

By observing the approximate normalized intensities of the two preedge peaks, a left/right peak intensity ratio reveals a trend from a high left peak changing to an increasing right peak associated with an increased $\mathrm{Fe}^{3+} / \Sigma \mathrm{Fe}$ ratio from 0 to 1 , as measured in the standards. Such a trend is also observed in the veining features from the surrounding olivine into the vein and to the central gel, again indicating an increase in oxidation state towards the centre of the vein.

References: [1] Bunker G. 2010. In Introduction to XAFS. p. 137. [2] Changela H. G. and Bridges J. C. 2011. Meteoritics \& Planetary Science 45:1847-1867. [3] Cressey G. et al. 1993. Physics and Chemistry of Minerals 20:111-119. [4] Marion G. M. et al. 2003. Geochimica et Cosmochimica Acta 67:4251-4266. 


\section{4}

\section{NEUTRON CAPTURE RECORDS OF MESOSIDERITES}

H. Hidaka ${ }^{1}$ and S. Yoneda ${ }^{2}$. ${ }^{1}$ Department of Earth \& Planetary Systems Science, Hiroshima University, Higashi-Hiroshima 739-8526, Japan. E-mail: hidaka@hiroshima-u.ac.jp. ${ }^{2}$ Department of Science and Engineering, National Museum of Nature and Science, Tokyo 169-0073, Japan.

Introduction: Mesosiderites are stony-iron meteorites composed of approximately equal fractions of metallic $\mathrm{Fe}-\mathrm{Ni}$ and silicates. The silicates have mostly basaltic, gabbroic, and pyroxenitic components, and are petrologically similar to eucrites and howardites. The cosmic-ray exposure ages of mesosiderites are intermediate between those of stony and iron meteorites, and vary from $<10$ to $340 \mathrm{Ma}$ [1], and they are long enough to study their exposure history from the accumulation of the cosmogenic products [2-4]. Our interest is to use the neutron irradiation records in mesosiderites (1) to determine the dependence of the thermal neutron production rate on the chemical composition of the target, and (2) to investigate a possibility of the initial irradiation record of mesosiderite silicates on the parent body before formation of the mesosiderites.

Experiments: Six mesosiderites, Dalgaranga, Estherville, Morristown, NWA 1242, NWA 2932, and Vaca Muerta, were used in this study. Silicate inclusions, each with a mass of $0.2-0.3 \mathrm{~g}$, collected from each sample were digested individually using an $\mathrm{HF}-\mathrm{HClO}_{4}$ mixture. The sample was then evaporated to dryness and dissolved in $1 \mathrm{~mL}$ of $2 \mathrm{M}$ $\mathrm{HCl}$. The $\mathrm{Sm}$ and $\mathrm{Gd}$ fractions were chemically separated using a conventional ion exchange method [5]. The isotopic compositions of $\mathrm{Sm}$ and Gd were determined using a VG54-30 thermal ionization mass spectrometer equipped with seven Faraday cup collectors.

Results and Discussion: All six samples showed significant ${ }^{150} \mathrm{Sm} /{ }^{149} \mathrm{Sm}$ and ${ }^{158} \mathrm{Gd} /{ }^{157} \mathrm{Gd}$ isotopic shifts from neutron capture reactions corresponding to neutron fluences of $(1.3-21.8) \times 10^{15} \mathrm{n} \mathrm{cm}^{-2}$. Among the six mesosiderites used in this study, the thermal neutron capture record of the Vaca Muerta silicates differed from those of the other mesosiderites, and showed a larger fluence of neutrons than expected based on its cosmic-ray exposure age. The thermal neutron fluence of the Vaca Muerta silicates was $0.67 \times 10^{16} \mathrm{n} \mathrm{cm}^{-2}$, i.e., larger than the expected value $\left(1.51 \times 10^{16} \mathrm{n} \mathrm{cm}^{-2}\right)$. The parameter for the degree of neutron thermalization, $\varepsilon_{\mathrm{Sm}} / \varepsilon_{\mathrm{Gd}}$, also revealed a significant difference between the thermal neutron production conditions in Vaca Muerta (0.76) and the other mesosiderites (0.93-1.20). These results suggest a two-stage irradiation occurred for the silicates of Vaca Muerta on the surface of the parent body (>50 Ma) and during its transit to earth $(138 \mathrm{Ma})$. Our model is consistent with the results of the ${ }^{81} \mathrm{Kr}-\mathrm{Kr}$ CRE ages of the Vaca Muerta pebble, which were estimated to be $>60 \mathrm{Ma}$ in the first CRE age before the second-stage irradiation [6].

References: [1] Terribilini D. et al. 2000. Meteoritics \& Planetary Science 35:617-628. [2] Begemann F. et al. 1976. Geochimica et Cosmochimica Acta 40:353-368. [3] Albrecht A. et al. 2000. Meteoritics \& Planetary Science 35:975-986. [4] Welten K. et al. 2001. Meteoritics \& Planetary Science 36:939-946. [5] Hidaka H. and Yoneda S. 2007. Geochimica et Cosmochimica Acta 71:1074-1086. [6] Bajo K. and Nagao K. 2011. Meteoritics \& Planetary Science, in press.
5511

DEEP IMPACT SITE AS IMAGED BY STARDUST NEXT ON COMET 9P/TEMPEL 1 REVEALS A STRONG SURFACE AND EXTENSIVE EJECTA BLANKET

A. R. Hildebrand ${ }^{1}$, M. I. Ibrahim ${ }^{1}$, R. D. Cardinal $^{1}$ and L. Maillet ${ }^{1}$. ${ }^{1}$ Department of Geosciences, University of Calgary, Calgary, AB, T2N 1N4, Canada. E-mail: ahildebr@ucalgary.ca.

Introduction: The Stardust NexT spacecraft imaged much of $\sim 6 \mathrm{~km}-$ diameter comet Tempel 1's surface during its February 14, 2011, flyby. The spacecraft captured the crater created during the Deep Impact mission when a "smart" $370 \mathrm{~kg}$ projectile impacted its surface at $10.2 \mathrm{~km} \mathrm{~s}^{-1}$ on July 4, 2005 [1]. One of the Deep Impact mission goals was to assess the surface physical characteristics of Tempel 1 through study of the size and shape of the resulting crater, however, the ejected gas and dust plumes were sufficiently opaque and long lasting to prevent the Deep Impact flyby spacecraft from imaging the impact site during its 800 s-long viewing window necessitating another imaging effort.

Imaged Crater and Ejecta Blanket: The impact location was projected using Deep Impact smart projectile imagery coupled with telemetered guidance data [1]. The impact site reported by the Stardust NexT team at the post-flyby briefing was a relatively subdued $100-150 \mathrm{~m}$ diameter feature at the predicted impact position. However, the impact actually occurred $\sim 400 \mathrm{~m}$ north (comet's reference frame) where a $\sim 20 \mathrm{~m}$ diameter bright spot occurs with two associated bright rays. The impact location is confirmed by the position of the initial light captured by the Deep Impact flyby spacecraft; it is further confirmed by both the position of the shadow generated by the impact ejecta plume and the path of specular reflections from the comet's surface of the glowing projectile as it traversed a dust layer near the comet.

The impact feature is a relatively dramatic bright albedo feature on Tempel 1's surface, and is the greatest visible change in appearance of the comet's surface between the two flybys. The impact was oblique with an elevation angle to the surface of $\sim 30^{\circ}[1,2]$; at low impact angles both natural and artificial craters develop asymmetric ejecta blankets. The $\sim 130^{\circ}$ chevron-shaped ejecta blanket aligns to the impact direction with ejecta spanning a broad " $\mathrm{V}$ " open downrange. The two rays defining the edges of the chevron extend $\sim 150 \mathrm{~m}$; this $\sim 7$ ratio is typical of the crater diameter to ray lengths observed at other oblique impact sites.

Implications for Tempel 1 Surface and Cratering Processes on Small Bodies: The $\sim 20 \mathrm{~m}$ crater size and ejecta blanket characteristics imply a surface strength of $>\sim 10 \mathrm{kPa}$ near the strong end of previous estimates [2]. The $\sim 20 \mathrm{~m}$ size also implies an $\sim 10^{5} \mathrm{~kg}$ ejecta volume near the small end of proposed ejecta volumes [2]. The extensive ejecta blanket (that survived a perihelion passage before the second imaging attempt) indicates ejecta excavation at velocities of order $1 \mathrm{~mm} \mathrm{~s}^{-1}$ for a large fraction of the crater's ejecta despite the $10.2 \mathrm{~km} \mathrm{~s}^{-1}$ impactor speed and a relatively strong impacted surface at odds with current expectations; this also implies that regolith generation by hypervelocity impacts on small bodies is an efficient process. The $\sim 1.5 \mathrm{~km}$-thick, comet-shrouding dust layer inferred from reflections of the glowing projectile near impact explains the initial diffuse light signature recorded by the Deep Impact flyby spacecraft; light from the compact (subpixel size) ejecta cone was scattered to form a broad symmetric shape several pixels across.

References: [1] A'Hearn et al. 2005. Science 310:258-264. [2] Holsapple K. A. and Housen K. R. 2007. Icarus 187:345-356. 
5363

\section{IMPACT CRATERING EXPERIMENTS ON POROUS SINTERED TARGETS OF DIFFERENT STRENGTHS}

K. Hiraoka ${ }^{1}$, S. Hakura ${ }^{1}$, A. M. Nakamura ${ }^{1}$, A. Suzuki ${ }^{2}$, and S Hasegawa $^{3}$. ${ }^{1}$ Earth and Planetary Science, Kobe University, 1-1 Rokkodai-cho, Nada-ku, Kobe, 657-8501 Japan. E-mail: amnakamu@ kobe-u.ac.jp. ${ }^{2}$ Center for Planetary Science (CPS), Kobe University, ${ }^{3}$ ISAS/JAXA, 3-1-1 Yoshinodai, Chuo-ku, Sagamihara, Kanagawa 2525210, Japan.

Introduction: In order to derive from crater morphologies or the dimensions on the surface of asteroids some information on the physical properties of the surface or the impacting bodies, it is important to understand the impact cratering process and derive scaling laws. Extensive studies on cratering have been performed experimentally and theoretically in the past decades, however, there're still not enough laboratory data of cratering on porous targets. In this laboratory study, we changed the strength of the targets while we kept the porosity constant.

Experiments: The targets were sintered glass beads of $40 \%$ porosity [1]. The static tensile strength of the targets was measured by Brazillian disc test [2] and between 6.5 and $640 \mathrm{kPa}$. These values were similar to or lower than those of carbonaceous chondrite meteorites [3]. Low-velocity impact experiments (velocities of 240 and $344 \mathrm{~m} \mathrm{~s}^{-1}$ ) were performed using a small He gas-gun at Kobe University. Projectiles were glass and nylon spheres of $1 / 8$ inches and were shot vertically to the targets. We also performed high-velocity impact experiments $\left(2.94-3.46 \mathrm{~km} \mathrm{~s}^{-1}\right)$ for the strongest targets using a two-stage light-gas gun at the Institute of Space and Astronautical Science (ISAS). Projectiles were $0.94 \mathrm{~mm}$ glass and $1.18 \mathrm{~mm}$ alumina spheres and were shot horizontally.

Results: The depth-diameter ratio of the craters was less than 0.2 for the craters on two weakest targets (strengths of 6.5 and $38 \mathrm{kPa}$ ). Others had the ratio larger than 0.3 . The crater diameter increased with decreasing strength of the targets for the low-velocity shots. However, the diameter was less dependent on the strength for the weaker targets. The change of the dependence probably indicates the strength-gravity transition of the cratering process. We calculated the crater volume from the vertical cut profile of the craters and applied a crater scaling law that covers both strength and gravity regimes [4] to the low-velocity results to derive the scaling parameters. We found that thus derived scaling parameters reproduced the crater volumes of the high-velocity experiments.

Summary: We performed impact cratering experiments of constant porosity but different strength using sintered glass beads targets. It was shown that there was a change in dependence of the crater diameter on target strength. We derived the crater scaling parameters for this porous material that covers both strength and gravity regimes of cratering.

References: [1] Setoh M. et al. 2010. Icarus 205:702-711. [2] Nakamura A. M. et al. 2007. Journal of Geophysical Research 112:E02001. [3] Tsuchiyama A. et al. 2009. 72nd Annual Meeting of the Meteoritical Society. pp. 5189. [4] Holsapple K. 1993. Annual Review of Earth Planetary Sciences 21:333-73.
5441

THE OMANI-SWISS METEORITE SEARCH PROJECT-RECENT CAMPAIGNS AND OUTLOOK

B. A. Hofmann ${ }^{1,3}$, E. Gnos ${ }^{2}$, U. Eggenberger ${ }^{3}$, F. J. Zurfluh ${ }^{3}$, S. Boschetti ${ }^{4}$ and A. Al-Rajhi ${ }^{5}{ }^{1}$ Natural History Museum Bern, Bern, Switzerland. E-mail: beda.hofmann@geo.unibe.ch. ${ }^{2}$ Muséum d'histoire naturelle de la Ville de Genève, Genève, Switzerland. ${ }^{3}$ Institut für Geologie, Universität Bern, Bern, Switzerland. ${ }^{4}$ Institut für Geophysik, ETH Zurich, Zurich, Switzerland. ${ }^{5}$ Directorate General of Minerals, Ministry of Commerce and Industry, Muscat, Oman.

Introduction: A total of approximately 5500 meteorite samples recovered during ten field campaigns are attributable to approximately 690 fall events. We find that a significant part of the surface of Oman is suitable for meteorite search and that more than two-thirds of all meteorites found belong to large strewn fields containing several hundreds to thousands stones. The 40-day 2009/2010 field campaign was conducted mainly along a transsect from NW to SE, from the interior of Oman to the coast. The 23-day 2011 search campaign was conducted along a SW$\mathrm{NE}$ axis in the central part of the Oman desert. Main aims were the continuation of systematic searches by foot in a variety of areas, test searches in unsampled areas and the conduction a geomagnetic survey in the JaH 091 strewn field.

Systematic Searches by Foot: During the 2009/2010 and 2011 field campaigns we completed systematic foot searches on a total of 21 quadrangles of $500 \times 500 \mathrm{~m}$ each (a total of $5.25 \mathrm{~km}^{2}$ ), covering all major areas and soil types of interior Oman suited for meteorite search. We found at least 12 different meteorites (48 stones) on this surface, corresponding to an apparent fall density of 2.3 meteorites $\mathrm{km}^{-2}$. This is significantly higher than estimated find densities from searches by car $\left(1.0 \mathrm{~km}^{-2}\right)$ where small meteorites (few g) are likely missed.

JaH 091 Strewn Field: In the large $(51.2 \times 7.2 \mathrm{~km})$ JaH 091 strewn field [1] we performed a geomagnetic survey of the largest impact sites, after recovery of all visible fragments. Only at the largest impact site a significant unrecognized mass (several $100 \mathrm{~kg}$ of fragments) was discovered. Future work on the data will constrain the buried mass, allowing a determination of the total mass of the $\mathrm{JaH} 091$ meteorite fall on the ground (estimated at approximately $3000 \mathrm{~kg}$ ).

Outlook: Future field work will aim to continue the quantification of meteorite densities in different areas of the Omani desert.

Reference: [1] Russell S. S., Folco L., Grady M., Zolensky M. E., Jones R., Righter K., Zipfel J., and Grossman J. N. 2004. Meteoritics \& Planetary Science 39:A215-A272. 
5427

MAGNETIC SIGNATURE OF NWA 5029: A BASALTIC SHERGOTTITE RELATED TO NWA 480/1460

V. H. Hoffmann ${ }^{1,2}$, T. Mikouchi ${ }^{3}$, M. Torii ${ }^{4}$, M. Funaki ${ }^{5}$ and R. Hochleitner ${ }^{6}$. ${ }^{1}$ Department of Geosciences, University of Tuebingen, Sigwartstr. 10, 72076 Tuebingen, Germany. ${ }^{2}$ Department of GeographyEnvironmental Sciences, University of Munich, Munich, Germany. ${ }^{3}$ Department of Earth and Planetary Sciences, University of Tokyo, Tokyo, Japan. ${ }^{4}$ Department Geosph.-Biosph.-Syst. Sci., Okayama University of Science, Okayama, Japan. ${ }^{5}$ NIPR, Tokyo, Japan. ${ }^{6}$ Mineralogical State Collection, Munich, Germany. E-mail: hoffmann.viktor@gmx.net.

Introduction: Northwest Africa (NWA) 5029 is a small, unpaired $14.67 \mathrm{~g}$ partly fusion-crusted stone which was found in Morocco in 2003 and classified as a basaltic shergottite [1] (diabasic-mafic-intermediate shergottite [2]). Here we report its magnetic properties and compare the data with NWA 480/1460, a diabasic/basaltic shergottite with similar mineralogy and texture $[3,4]$. The opaque minerals have been identified and investigated by optical microscopy and EMPA.

Samples and Investigations: We used several chips and fragments of the type mass (approximately $1.4 \mathrm{~g}$ ) of NWA 5029 for the magnetic experiments. For comparison, chips of NWA 480 (plus fragments) and NWA 1460 (both paired) were used. First magnetic data of NWA 480/ 1460 have been published by [5], whereby NWA 1460 corresponds to the sample of this study.

Results: The natural remanent magnetization (NRM) of NWA 5029 could be attributed to impact shock related processes on Mars, eventually resulting from the last and ejecting event, representing an SRM (shock remanent magnetization). The NWA 480/1460 NRM is most likely of terrestrial origin, an IRM which might be due to remagnetization by the use of hand magnets already in field by the nomads. The magnetic susceptibility (MS) values of the three stones are in the range which was found by [6] for Martian meteorites, and typical for diabasic/basaltic shergottites. The degree of anisotropy of magnetic susceptibility (AMS) is quite low in all cases (P between 1.071 and 1.090) despite the high degree of shock which should be reflected by the AMS. In the low-temperature range all three samples show essentially identical behavior: two magnetic transitions are detected in the range of $40-60 \mathrm{~K}$ (ilmenite and/or chromite) and at $120 \mathrm{~K}$ (magnetite) in both FC and ZFC IRM curves.

Discussion and Conclusion: The magnetic remanences (NRM) in these shergottites are carried by pure magnetite $\left(\mathrm{Fe}_{3} \mathrm{O}_{4}\right)$ with a Curietemperature $\left(\mathrm{T}_{\mathrm{c}}\right)$ of about $575^{\circ} \mathrm{C}$. In agreement with other data, the magnetite is apparently exsolved (magnetite with ilmenite lamellae), that is, high-temperature oxidized titano-magnetite. Minor effects are related to substituted magnetite with a $\mathrm{T}_{\mathrm{c}}$ of about $525^{\circ} \mathrm{C}$. Fe-sulfides such as monoclinic pyrrhotite $\left(\mathrm{Fe}_{7} \mathrm{~S}_{8}\right)$ could not be detected. The magnetic signature and texture of all three stones is very similar which leads to our hypothesis that the three rocks are very likely launch paired. We can conclude that the high-temperature oxidation occurred on the surface of Mars either during the primary formation processes or as a result of shock (at least $35 \mathrm{GPa}$ ) from the event which ejected the meteorites from the Mars surface into space.

References: [1] Mikouchi T. and Barrat J. A. 2009. Abstract \#5344. 72nd Annual Meteoritical Society Meeting. [2] Irving A. 2011. http:// www.imca.cc/mars/martian-meteorites.htm. [3] Barrat J. A. et al. 2002. Meteoritics \& Planetary Science 37:487-499. [4] Irving A. and Kuehner S. 2003. Abstract \#1503. 34th Lunar and Planetary Science Conference. [5] Rochette P. et al. 2005. Meteoritics \& Planetary Science 40/4:529-540. [6] Rochette P. et al. 2009. Meteoritics \& Planetary Science 44:405-427.

\section{6}

INCIPIENT PHYLLOSILICATE STRUCTURES IN SYNTHETIC MgSILICATES HYDRATED IN A HUMID CHAMBER

E. J. Hoffman ${ }^{1}$, L. A. Veblen ${ }^{1}$ and D. R. Veblen ${ }^{2} .{ }^{1}$ Physics Department, Morgan State University, Baltimore, MD 21251, USA. E-mail: eugene. hoffman@morgan.edu. ${ }^{2}$ Department of Earth and Planetary Sciences, Johns Hopkins University.

Introduction: In reactions with water synthetic $\mathrm{Mg}$-silicate smokes from the Goddard Space Center Astrochemistry Lab [1] undergo a color change and produce proto-phyllosilicates. These products appear to be the same whether hydration occurs during immersion in liquid water [2] or by exposure to a humid atmosphere [3]. The products have been detected by X-ray diffraction [3] and by electron microscopy [2, 3], although the protocrystalline structures disintegrate very easily under the electron beam.

We report here continuing efforts to characterize humid-chamber reaction products under the electron microscope.

Experimental: Synthetic Mg-silicate smoke was obtained, stored, pre-treated with flowing dry $\mathrm{N}_{2}$, and incubated over deoxygenated deionized water as described [3]. Incubation temperatures were $17-20{ }^{\circ} \mathrm{C}$.

A Philips EM420 electron microscope was used with several sample preparation methods, including suspension in ethanol and dropping onto a holey carbon grid [3]. Imaging and electron diffraction conditions were also subject to much experimentation. Experience with similarly beamlabile material [4] served as a guide.

Results and Discussion: Images and diffraction patterns were obtained more reliably although further work is warranted to examine the incipient structures more thoroughly before they fall apart.

Acknowledgment: This work was supported in part by an NSF HBCU-UP grant to Morgan State University and an NSF GeoEd grant to EJH.

References: [1] Nuth III, J. A. et al. 2000. Journal of Geophysical Research 105:10,387-10,396. [2] Chizmadia, L. J. et al. 2006. Abstract \#2187. 37th Lunar and Planetary Science Conference. [3] Hoffman E. J. et al. 2011. Abstract \#2526. 42nd Lunar and Planetary Science Conference. [4] Stubbs J. C. et al. 2009. Geochimica et Cosmochimica Acta 73:1563-1576. 


\section{4}

SHOCK BAROMETRY OF THE SILJAN IMPACT STRUCTURE, SWEDEN

S. Holm ${ }^{1}$, C. Alwmark ${ }^{1}$, W. Alvarez ${ }^{2}$ and B. Schmitz ${ }^{1}{ }^{1}$ Department of Geology, Lund University, Sölvegatan 12, 22362 Lund, Sweden, E-mail: sanna.holm@geol.lu.se. ${ }^{2}$ Department of Earth and Planetary Science, University of California, 307 McCone Hall, Berkeley, CA 94720-4767, USA.

Introduction: The Siljan structure in south-central Sweden is the largest verified impact structure in Europe with an estimated diameter of $\sim 50 \mathrm{~km}$ [1]. The structure is dated to $377 \pm 2 \mathrm{Ma}$ [2] and strongly eroded, exposing a surface that was originally located beneath the crater floor [1]. Despite its size, Siljan is poorly studied, and basic facts about the original morphology of the structure remain elusive. Information about the distribution of shocked quartz and shock barometry are lacking. Previous studies of microscopic shock metamorphic features from the structure are limited both with respect to the number of localities and the amount of data presented (e.g., [3, 4]). Here we present the first detailed characterization of the shock level distribution across the Siljan structure by extensive studies of shock metamorphic features in quartz.

Materials and Methods: Three hundred thin sections from seventy three localities across predominantly the central part of the Siljan structure were searched for shock metamorphic features in quartz with optical and u-stage microscopy (see [5] and [6] for details on technique). Measured optic axes and poles perpendicular to planar deformation feature (PDF) planes in individual quartz grains were determined and indexed using a stereographic projection template.

Results and Discussion: Out of 73 localities, 21 display microscopic shock metamorphic features (planar features; PFs, and PDFs). The PDF orientation and frequency pattern reveals that the samples have been subjected to different shock pressures. The most strongly shocked samples record pressures of 16-20 GPa, although locally in the samples higher pressures causing melting were attained, and occur at the center of the structure. Outwards from the geographical center pressures decrease in circular envelopes to $\sim 5 \mathrm{GPa}$ at $10 \mathrm{~km}$ distance. Observations of shatter cones outside of the zone with shocked quartz extend the total shocked area to approximately $30 \mathrm{~km}$ in diameter.

The deep erosion level of the structure prevents any assumptions about the original size of Siljan based on present morphological features. However, with the distribution of shocked quartz across the structure defined, we can estimate the original dimensions based on calculations in [7] (and references therein). Using the relationships summarized in [7], we conclude that the Siljan impact structure can have been as large as $90 \mathrm{~km}$ in diameter before erosion.

References: [1] Grieve R. A. F. 1988. In Deep drilling in crystalline bedrock. The Deep Gas Drilling in the Siljan Impact Structure, Sweden and Astroblemes vol.1. Springer Verlag, pp. 328-348. [2] Reimold W. U. et al. 2005. Meteoritics \& Planetary Science 40:591-607. [3] Svensson N.-B. 1971. Nature Physical Science 229:90-92. [4] Tamminen J. and Wickman F. E. 1980. GFF 102:275-278. [5] Engelhardt W. von, and Bertsch W. 1969. Contributions to Mineralogy and Petrology 20:203-234. [6] Ferrière L. et al. 2009. Meteoritics \& Planetary Science 44:925-940. [7] Therriault A. M. et al. 1997. Meteoritics \& Planetary Science 32:71-77.
5323

PILOT DIGITALIZATION PROJECT FOR THE METEORITE COLLECTION AT THE ROBERT A. PRITZKER CENTER FOR METEORITICS AND POLAR STUDIES

J. L. Holstein ${ }^{1}$, E. A. Pelker ${ }^{1}$ and P. R. Heck ${ }^{1,2}$. ${ }^{1}$ Robert A. Pritzker Center for Meteoritics and Polar Studies, Department of Geology, The Field Museum, 1400 S. Lake Shore Drive, Chicago, IL 60605, USA. E-mail: jholstein@fieldmuseum.org. ${ }^{2}$ Chicago Center for Cosmochemistry, The University of Chicago, Chicago, IL, USA.

Introduction: Digitalization of a research and educational meteorite collection is an important part of collection management. It will allow specimens to be examined remotely by researchers before a loan request is submitted. Through our website it will also serve to educate the public about the classification and components of meteorites. It also has the potential of evolving into an interactive exhibit and applications for handheld electronic devices. Other benefits include the reduced handling of fragile specimens, preserving a record of the appearance of a meteorite before cutting, and the potential to calculate the bulk density of individual meteorite specimens. We are undertaking a pilot project to digitalize selected specimens from the Robert A. Pritzker Center for Meteoritics and Polar Studies' meteorite collection using three techniques: basic 2D digital photography, rotational (3D) digital photography, and 3D laser scans. The resulting macroscopic visual representations will also increase the visibility of our collection to a global audience of scientists, students, and the general public. The goal of this pilot project is to test three different digitalization techniques on selected specimens of different meteorite groups. This will form the basis for a proposal to digitize a larger fraction of our collection.

Materials and Methods: With over 8,000 meteorite specimens in the collection, we selected representative examples of the most important meteorite groups for this pilot study. Chosen were examples of ordinary chondrites (H, L, LL, R, E), carbonaceous chondrites, primitive and differentiated achondrites including Martian and lunar meteorites, pallasites, mesosiderites, and irons. Digital photographs of new specimens will be obtained before accession into our collection. This basic form of digitalization will also be used for all meteorites that we selected from our collection. A subgroup of cut specimens that show internal texture are placed on a turntable and are photographed at regular rotational increments. The set of images are combined into an animation that will be displayed on a webpage. For a third subgroup of specimens we will use a desktop 3-D laser scanner. This results in geometric models of the specimens that will later be placed on a webpage allowing users to visually study the specimens remotely. The models will be calibrated and allow us to calculate the bulk density of a specimen. This data can then be used to study the physical properties of meteorites, e.g., [1, 2].

Acknowledgments: The Robert A. Pritzker Center for Meteoritics and Polar Studies is supported by the Tawani Foundation.

References: [1] Consolmagno G. J. et al. 2006. Meteoritics \& Planetary Science 41:331-342. [2] Macke R. J. et al. 2011. Meteoritics \& Planetary Science 46:311-326. 
5395

THE PLANETESIMAL SHOCK MODEL FOR CHONDRULE FORMATION: A MORE QUANTITATIVE ASSESSMENT

L. L. Hood ${ }^{1}$ and S. J. Weidenschilling ${ }^{2}$. ${ }^{1}$ Lunar and Planetary Lab, University of Arizona, Tucson, Arizona 85721, USA. E-mail: lon@ lpl.arizona.edu. ${ }^{2}$ Planetary Science Institute, 1700 E. Ft. Lowell, Tucson, Arizona 85719, USA.

Introduction: Gas dynamic shock waves in a low-temperature nebula are currently considered to be a plausible mechanism for providing the repetitive transient heating events that were apparently responsible for chondrule formation (e.g., [1]). Possible meteoritic constraints on the sources of chondrule-forming shocks include (1) isotopic evidence that chondrule formation most probably began 1-1.5 Myr after the formation of CAIs and continued for several Myr; (2) coexistence of chondrules in chondrites with products of later parent-body processes (e.g., igneous rock fragments); and (3) inferences that chondrule formation regions were large (more than several hundred $\mathrm{km}$ across) but also relatively localized to explain observed differences in properties of chondrules from different chondrite groups.

One class of nebular shocks that can potentially satisfy the above constraints is shocks generated by planetesimals passing through Jovian resonances, assuming that Jupiter formed approximately $1 \mathrm{Myr}$ after CAIs [2-4]. Included are both planetesimal bow shocks produced by bodies ejected into eccentric orbits and impact vapor plume shocks produced by high-velocity collisions between planetesimals. Here, we employ an improved planetesimal accretion and orbital evolution code that includes the damping effects of collisions and mutual gravitational scattering between embryos. The goal is to provide more accurate estimates of potential chondrule formation efficiency by both planetesimal shock mechanisms.

Planetesimal Evolution Code and Results: A population of several hundred discrete bodies with diameters between approximately 750 and $2000 \mathrm{~km}$ and a total mass of approximately 1 Earth mass is initially distributed in 20 radial zones from 2 to 4 AU. In addition, a background population of smaller bodies is represented in the model by a statistical continuum. A symplectic N-body integrator is applied to calculate the orbital evolution of the large bodies. The accretion rate of discrete bodies is tracked and is used to estimate the collision rate with smaller bodies. After integration times of $250 \mathrm{kyr}$ or more, only approximately $1 \%$ of the discrete bodies have e $>0.3$ (corresponding to midplane crossing velocities $\left.v_{\mathrm{m}}>5 \mathrm{~km} \mathrm{~s}^{-1}\right)$. A few bodies rarely reach $\mathrm{e}>0.4 \quad\left(v_{\mathrm{m}}\right.$ $>7.5 \mathrm{~km} \mathrm{~s}^{-1}$ ). The total cumulative area of the midplane that would be traversed by these bodies and their associated bow shocks over a period of several Myr is $<1 \%$ of the total area. The code output shows that bodies with e $>0.2$ and diameters of 1000-3000 km typically gained mass at rates of $10^{16}-10^{17} \mathrm{~g}$ year ${ }^{-1}$ during the interval from 200 to $250 \mathrm{kyr}$. The corresponding impact interval for $1 \mathrm{~km}$ sized bodies (mass approximately $10^{15} \mathrm{~g}$ ) is days to weeks. Implications of these results for chondrule formation efficiency in planetesimal-generated shocks will be presented at the conference.

References: [1] Desch S. J. et al. 2005. In Chondrites and the Protoplanetary Disk, Krot A. N. et al. eds., Astronomical Society Pacific Conference Series, vol. 341. pp. 849-872. [2] Hood L. L. 1998. Meteoritics \& Planetary Science 33:97-107. [3] Weidenschilling S. J. et al. 1998 Science 279:681-684. [4] Hood L. L. et al. 2009. Meteoritics \& Planetary Science 44:327-342.
5241

I-Xe AND Ar-Ar DATING OF ENSTATITE METEORITES

J. Hopp ${ }^{1}$, M. Trieloff ${ }^{1}$, U. Ott ${ }^{2}$ and O. V. Pravdivtseva ${ }^{3}$. ${ }^{1}$ Institute of Geosciences, University Heidelberg, INF 234-236, D-69120 Heidelberg, Germany. E-mail: jens.hopp@geow.uni-heidelberg.de. ${ }^{2}$ MPI for Chemistry Mainz, Joh.-J.-Becher-Weg 27, D-55128 Mainz, Germany. ${ }^{3}$ Laboratory for Space Sciences, Physics Department, Washington University, 1 Brookings Drive, St. Louis, MO 63130-4899, USA

Introduction: The decay of short-lived ${ }^{129} \mathrm{I}$ into ${ }^{129} \mathrm{Xe}\left(\mathrm{t}_{1 / 2}=15.7 \mathrm{Ma}\right)$ allows resolution of small timescales during early formation history of meteorite parent bodies. Few available data suggest enstatite meteorites are promising candidates to apply this chronometer, thus improving our understanding of the chronology of enstatite meteorite parent body formation and metamorphism. We report first results of a still ongoing I-Xe and Ar-Ar dating study of a suite of aubrites and enstatite chondrites (EH and EL groups, different petrological types).

Results: We focussed on meteorites with low weathering stages. In addition we measured some meteorites for which data already exist, because these are the first I-Xe results obtained at Institute of Geosciences, Heidelberg. So far we analyzed whole rock samples (80$250 \mu \mathrm{m}$ ) of the aubrites ALH 84024, Bishopville, Cumberland Falls, Mayo Belwa, Norton County, and Peña Blanca Spring. Because of low xenon and iodine concentrations, observed low ${ }^{129} \mathrm{Xe} /{ }^{132} \mathrm{Xe}$ excess from ${ }^{129} \mathrm{I}$-decay and problems with ${ }^{128} \mathrm{Xe}$-memory precision was lowered and hence, most aubrites revealed no significant age information except for Peña Blanca Spring. For this meteorite we yielded a relatively well-defined isochron $\left(1040-1300{ }^{\circ} \mathrm{C}\right)$ in ${ }^{129} \mathrm{Xe} /{ }^{132} \mathrm{Xe}$ versus ${ }^{128} \mathrm{Xe} /{ }^{132} \mathrm{Xe}$ space of about $4.2 \pm 4.5(2 \sigma) \mathrm{Ma}$ younger than the enstatite achondrite Shallowater which was used as age reference $(4562.3 \pm 0.4(1 \sigma) \mathrm{Ma},[2])$. This age is similar though less precise to a previously reported (recalculated) I-Xe age of Peña Blanca Spring [1]. Analyses of enstatite separates from some of the aubrites with a higher sensitivity mass spectrometer at Washington University, St. Louis, are in progress.

Because of their much higher xenon and iodine concentrations measurements of chondrites are more significant. Up to now we analyzed mm-sized chips of the enstatite chondrites Abee (EH4) and EET 96135 $(\mathrm{EH} 4 / 5)$ and for both obtained well-defined isochrons. Abee is calculated to be $0.7 \pm 0.9(2 \sigma)$ Ma younger than EET 96135 and $0.2 \pm 0.7(2 \sigma)$ Ma younger than Shallowater. The latter age difference again agrees with previous data [1]. The observed range in ${ }^{129} \mathrm{Xe} /{ }^{132} \mathrm{Xe}$ ratios of extractions defining the isochrons of both meteorites are very different (2.7-7.8, Abee; 1.37-1.86, EET 96135). EET 96135 shows a much higher concentration of trapped ${ }^{132} \mathrm{Xe}\left(2.4 \mathrm{E}-9 \mathrm{~cm}^{3} \mathrm{STP} \mathrm{g}{ }^{-1}\right)$ than Abee $\left(8.5 \mathrm{E}-10 \mathrm{~cm}^{3} \mathrm{STP} \mathrm{g}^{-1}\right)$ explaining its lower ${ }^{129} \mathrm{Xe}$ excess.

Before measurement $\mathrm{Xe}$ is routinely separated from $\mathrm{Ar}$ at a cold trap (about 10\% Ar remaining in Xe fraction). For both chondrites this argon fraction was used for simultaneous Ar-Ar dating. Absolute age evaluation still await standard measurements, but results indicate an approx. 0.3 Ga lower partial "plateau" age for Abee compared with EET 96135.

References: [1] Podosek F. A. 1970. Geochimica et Cosmochimica Acta 34:341-365. [2] Gilmour J. D. et al. 2009. Meteoritics \& Planetary Science 44:573-580. 
5188

MOLECULE CHEMISTRY IN SUPERNOVA EJECTA: INSIGHTS FROM PRESOLAR SiC GRAINS

P. Hoppe ${ }^{1}$ and E. Zinner ${ }^{2} .{ }^{1}$ Max Planck Institute for Chemistry, Particle Chemistry Department, 55128 Mainz, Germany. E-mail: peter.hoppe@ mpic.de. ${ }^{2}$ Laboratory for Space Sciences and Physics Department, Washington University, St. Louis, MO 63130, USA.

Introduction: Primitive solar system materials contain small quantities of presolar grains that formed in the winds of evolved stars or in the ejecta of stellar explosions [1]. Of particular importance are $\mathrm{SiC}$ grains from type II supernovae (SNeII), the $\mathrm{X}$ grains, characterized by isotopically light $\mathrm{Si}$ [2], and the "unusual" and $\mathrm{C}$ (subsequently designated U/C) grains, characterized by heavy Si [3-5]. Many of the isotopic signatures of these grains can be satisfactorily reproduced by ad hoc SN mixing models. In this approach, matter from different layers in a SNII [e.g., 6] is mixed in variable proportions. With ad hoc SN mixing, however, it is not possible to explain the isotope data of $\mathrm{Fe}$ in $\mathrm{X}$ grains [7] and of S in U/C grains [3-5]. While it cannot be completely ruled out that predictions for certain isotope abundances in current SN models [6] are in error, it is more likely that the problems with $\mathrm{Fe}$ and $\mathrm{S}$ point toward element fractionation processes during or prior to $\mathrm{SiC}$ formation. Here we will explore the possible role of molecule chemistry in SN ejecta in this respect.

$\mathrm{S}$ and $\mathbf{F e}$ in Supernova Grains: The U/C grains have isotopically light S [3-5]. Because Si- and S-isotopic signatures (i.e., heavy or light Si and $\mathrm{S}$ ) correlate very well in the interior of SNeII [6], ad hoc mixing models fail to account for heavy $\mathrm{Si}$ together with light $\mathrm{S}$. Most $\mathrm{X}$ grains have higher than solar ${ }^{57} \mathrm{Fe} /{ }^{56} \mathrm{Fe}$ but solar ${ }^{54} \mathrm{Fe} /{ }^{56} \mathrm{Fe}$ [7]. The light $\mathrm{Si}$ of $\mathrm{X}$ grains requires significant contributions from the $\mathrm{Si} / \mathrm{S}$ zone, which should lead to higher than solar ${ }^{54} \mathrm{Fe} /{ }^{56} \mathrm{Fe}$ in $\mathrm{X}$ grains, contrary to observation.

Discussion: Models based on chemical kinetics predict formation of various molecules in the ejecta of massive $\mathrm{SNe}$ [8]. Molecules such as $\mathrm{CO}$ and $\mathrm{SiO}$ have been observed in the ejecta of SN1987A [9]. According to the models by [8], $13-34 \%$ of the ejected matter is in the form of molecules. The molecule inventory depends strongly on the degree of mixing. If $\mathrm{H}$ from the outer $\mathrm{SN}$ layers mixes with matter from interior layers, then $\mathrm{H}_{2}, \mathrm{H}_{2} \mathrm{O}, \mathrm{OH}, \mathrm{N}_{2}, \mathrm{O}_{2}, \mathrm{CO}, \mathrm{CO}_{2}, \mathrm{SO}$, and $\mathrm{SiO}$ dominate. If no mixing occurs, $\mathrm{O}_{2}, \mathrm{SiS}$, and $\mathrm{CO}$ dominate. In this latter case about $25 \%$ of all molecules are $\mathrm{SiS}$ that formed in the $\mathrm{Si} / \mathrm{S}$ zone. Ad hoc $\mathrm{SN}$ mixing shows that it is possible to produce heavy $\mathrm{Si}$ even if matter from the $\mathrm{Si} / \mathrm{S}$ zone is admixed. If $\mathrm{SiS}$ molecules from the $\mathrm{Si} / \mathrm{S}$ zone are incorporated efficiently by the growing $\mathrm{SiC}$ grains, while elemental $\mathrm{S}$ or other $\mathrm{S}$ molecules are not, it would be possible to condense $\mathrm{SiC}$ grains with light $\mathrm{S}$ and heavy $\mathrm{Si}$. The $\mathrm{Fe}$-isotopic signature of $\mathrm{X}$ grains requires preferential trapping of $\mathrm{Fe}$ from the $\mathrm{He} / \mathrm{C}$ and $\mathrm{He} / \mathrm{N}$ zones. According to the models of molecule chemistry in $\mathrm{SN}$ ejecta by [8], some $(\mathrm{FeS})_{2}$ dust precursors form in the $\mathrm{Si} / \mathrm{S}$ zone which may prevent $\mathrm{Fe}$ from the $\mathrm{Si} / \mathrm{S}$ zone to be trapped by the growing $\mathrm{SiC} \mathrm{X}$ grains. Clearly, more detailed work is needed to follow up on these ideas.

References: [1] Zinner E. 2007. In Meteorites, comets, and planets, edited by Davis A. M. Treatise on Geochemistry, vol. 1.02. [2] Lin J. et al. 2010. The Astrophysical Journal 709:1157-1176. [3] Hoppe P. et al. 2010. The Astrophysical Journal 719:1370-1384. [4] Gyngard F. et al. 2010. Meteoritics \& Planetary Science 45:A72. [5] Hoppe P. and Fujiya W. 2011. Abstract \#1059. 42nd Lunar and Planetary Science Conference. [6] Rauscher T. et al. 2002. The Astrophysical Journal 576:323-348. [7] Marhas K. et al. 2008. The Astrophysical Journal 689:622-645. [8] Cherchneff I. and Lilly S. 2008. The Astrophysical Journal 683:L123L126. [9] Wooden D. et al. 1993. The Astrophysical Journal 88:477-507.
5096

SIDEROPHILE ELEMENT PATTERNS OF SULFIDE-METAL ASSEMBLAGES FROM THE ALMAHATA SITTA POLYMICT BRECCIA

M. Horstmann ${ }^{1}$, M. Humayun ${ }^{2}$ and A. Bischoff ${ }^{1}$. ${ }^{1}$ Institut für Planetologie, WWU Münster, 48149 Münster, Germany. E-mail: marianhorstmann@uni-muenster.de. ${ }^{2}$ NHMFL \& Deptartment of EOAS, Florida State University, Tallahassee, FL 32310, USA.

Introduction: Among the meteorites recovered from the strewn field of asteroid $2008 \mathrm{TC}_{3}$ in the Nubian Desert, two unique rocks were discovered dominated by sulfide-metal intergrowths with ureilitic material attached [1]. The samples MS-158 and MS-166 were studied by optical and electron optical microscopy (SEM, EPMA). Siderophile element abundances were determined in situ on polished thin sections applying LA-ICP-MS at Florida State University following the procedures of [2].

Results: MS-158 is dominated by large sulfide domains, often sprinkled with small $(<15 \mu \mathrm{m})$ metal inclusions, rounded to irregularly shaped zoned metal grains (cores $\sim 7-10 \mathrm{wt} \% \mathrm{Ni}$ ) with sharp Ni-rich rims (up to $\sim 35 \mathrm{wt} \% \mathrm{Ni}$ ) and reduced ureilitic material attached or enclosed, suggesting a genetic relationship. MS-166 contains similar areas but is additionally characterized by metal grains with minor, irregular zoning $(\sim 7-23 \mathrm{wt} \% \mathrm{Ni})$ surrounded by small, Ni-rich metal grains $(\sim 30 \mathrm{wt} \%$ $\mathrm{Ni}$ ) embedded in an intergrowth of FeS, a kind of Fe-oxide and minor metal. Siderophile element patterns in MS-158 metal cores are enriched in refractory siderophile elements ( $\mathrm{W}$ to $\mathrm{Rh} ; 2-10 \times \mathrm{CI}$, Ni-norm.) and rims are depleted showing nearly complementary patterns $(0.02-0.6 \times \mathrm{CI}$, Ninorm.) for these elements. $\mathrm{Ga}, \mathrm{Ge}$, and $\mathrm{Sn}$ show lower abundances in the rims with respect to the cores. $\mathrm{Pd}$ and $\mathrm{Sb}$ are enriched in the rims. $\mathrm{Au}$ and As abundances are similar in both regions. Metal in MS-166 is in some cases more strongly enriched in refractory siderophiles $(\sim 2-40 \times \mathrm{CI}$, Ni-norm.). Ni-zoning for siderophile element patterns was not reflected as prominently in grains embedded in the Fe oxide-bearing assemblage. Only one measurement exhibited similar depleted abundances as found in MS158. The range of measured Ir concentrations spans almost three orders of magnitude in the metal grains.

Discussion: Siderophile element patterns indicate that the metal grains in the assemblages formed via in situ fractional crystallization. The cores of the metals crystallized early in this process as indicated by the high abundances of refractory siderophile elements. The sharp Ni-rich rims depleted in the latter elements crystallized from a more evolved liquid with higher $\mathrm{S}$ content. The texture on thin section level supports in situ fractional crystallization. Using the determined core compositions, values for solid metal-liquid metal partition coefficients (D) were estimated [3, 4] with the assumption that the original liquid had a chondritic relative pattern for refractory siderophiles. From the estimated $\mathrm{D}$ values, the range of original $\mathrm{S}$ content of the melts is estimated as 15$25 \%$ S. However, the range of volatile element abundances in calculated liquid compositions is too large to be matched with any specific class of bulk carbonaceous chondrite material. The observed range for $\mathrm{Ir}$ is similar to IIIAB iron meteorites [5].

References: [1] Bischoff A. et al. 2010. Meteoritics \& Planetary Science 45:1638-1656. [2] Humayun M. et al. 2007. Geochimica et Cosmochimica Acta 71:4609-4627. [3] Chabot N. L. and Jones J. H. 2003. Meteoritics \& Planetary Science 38:1425-1436. [4] Chabot N. L. et al. 2009. Meteoritics \& Planetary Science 44:505-519. [5] Wasson J. T. 1999. Geochimica et Cosmochimica Acta 63:2875-2889. 


\section{7}

RARE EARTH ELEMENT (REE) ABUNDANCES OF SULFIDES FROM E CHONDRITE LITHOLOGIES OF THE ALMAHATA SITTA POLYMICT BRECCIA

M. Horstmann ${ }^{1}$, M. Humayun ${ }^{2}$ and A. Bischoff ${ }^{1}$. ${ }^{1}$ Institut für Planetologie, WWU Münster, 48149 Münster, Germany. E-mail: marianhorstmann@uni-muenster.de. ${ }^{2}$ NHMFL \& Department of EOAS, Florida State University, Tallahassee, FL 32310, USA.

Introduction: A large variety of different $\mathrm{E}$ chondrite types was found among the Almahata Sitta samples recovered from the Nubian Desert of northern Sudan [1]. We report REE abundances in oldhamite and keilite from selected samples determined by laser ablation-ICP-MS at FSU with a UP193FX excimer laser system using USGS and NIST standards for calibration [2].

Samples and Methods: In situ measurements (10 or $20 \mu \mathrm{m}$ spots) of REE abundances of oldhamite and keilite were conducted on polished thin sections of samples previously described [1]: oldhamite: MS-14 (EH3, $\mathrm{n}=1)$, MS-17 (EL3/4, $\mathrm{n}=2)$, MS-52 (EL 6, $\mathrm{n}=5)$; keilite: MS-159 $($ EL IMR, $\mathrm{n}=3$ ), MS-D (EL 6, $\mathrm{n}=8$ ).

Results: Oldhamite: MS-14 oldhamite exhibits a pattern with flat HREE $(\sim 30 \times \mathrm{CI})$, LREE decreasing from La to $\mathrm{Nd}(\sim 35-60 \times \mathrm{CI})$, and positive $\mathrm{Eu}(\sim 60 \times \mathrm{CI})$ and $\mathrm{Yb}(\sim 70 \times \mathrm{CI})$ anomalies. In MS-17, oldhamite grains both show similar patterns with flat HREE $(\sim 85-$ $140 \times \mathrm{CI})$, slightly bowl-shaped LREE $(\mathrm{La} / \mathrm{Ce} \sim 1.1, \mathrm{La} / \mathrm{Sm} \sim 0.9 ; \sim 90$ $120 \times \mathrm{CI})$, and negative Eu anomalies $(\sim 50 \times \mathrm{CI})$. All oldhamite grains in MS-52 are characterized by bow-shaped patterns (LREE $\sim 85-200, \mathrm{La} / \mathrm{Sm}$ $\sim 0.5$; HREE $\sim 220-120 \times \mathrm{CI}, \mathrm{Gd} / \mathrm{Lu} \sim 1.7$ ) with pronounced negative $\mathrm{Eu}$ anomalies $(\sim 25-30 \times C I)$. Different grains within the same section show very homogenous REE compositions. In comparison with $\mathrm{MS}-17, \mathrm{Eu}$ anomalies are more pronounced in $\mathrm{MS}-52\left(\mathrm{Eu} / \mathrm{Eu}^{*}=0.15\right.$ vs. 0.45 , calculated as $\mathrm{Eu}_{\mathrm{N}} /\left(\sqrt{ } \mathrm{Sm}_{\mathrm{N}} \times \mathrm{Gd}_{\mathrm{N}}\right)$; normalized to $\left.\mathrm{CI}\right)$. Keilite: REE patterns of keilite in MS-159 and MS-D are similar between different grains and characterized by LREE depletions $(\leq \sim 4 \times \mathrm{CI})$ relative to HREE (around $\sim 10-20 \times \mathrm{CI})$ and negative Eu anomalies $(\leq \sim 1.5 \times \mathrm{CI})$.

Discussion: REE patterns gained for oldhamite in the different samples are very similar to patterns reported in the literature for $\mathrm{E}$ chondrite and aubrite oldhamite [e.g., 3, 4]. The oldhamite in MS-14 indicates that its main REE signature originates from nebular processes because magmatic processes fail to generate positive anomalies in Eu and $\mathrm{Yb}$ [e.g., 3-5]. Patterns found for MS-17, although of type EL3/4, and MS-52 are similar to patterns found in EL6 (equilibrated) chondrites [4] and may result from prolonged thermal metamorphism and reequilibration of oldhamite with surrounding phases [3-5]. Keilite REE patterns share some similarity with alabandite REE patterns from the literature [e.g., 6]. Both minerals have been previously interpreted to form by igneous processes $[6,7]$.

References: [1] Bischoff A. et al. 2010. Meteoritics \& Planetary Science 45:1638-1656. [2] Gaboardi M. and Humayun M. 2009. Journal of Analytical Atomic Spectrometry 24:1188-1197. [3] Crozaz G. and Lundberg L. L. 1995. Geochimica et Cosmochimica Acta 59:3817-3831. [4] Floss C. and Crozaz G. 1993. Geochimica et Cosmochimica Acta 57:40394057. [5] Lodders K. and Fegley B. Jr. 1993. Earth and Planetary Science Letters 117:125-145. [6] Wheelock M. M. et al. 1994. Geochimica et Cosmochimica Acta 58:449-458. [7] Keil K. 2007. Chemie der Erde-Geochemistry 67:37-54.
5256

MODAL MINERALOGY OF CR CHONDRITES BY PSD-XRD: ABUNDANCE OF AMORPHOUS Fe-SILICATE

K. T. Howard ${ }^{1}$, G. K. Benedix ${ }^{1}$, P. A. Bland $d^{1,2,3}$ and D. L. Schrader ${ }^{4} .{ }^{1}$ Mineralogy Dept., Impacts and Astromaterials Research Centre (IARC), The Natural History Museum, London, UK. ${ }^{2}$ IARC, Dept. of Earth Sci. \& Eng., Imperial College, London, UK. ${ }^{3}$ Dept. of Applied Geology, Curtin University of Technology, Perth, Australia. ${ }^{4}$ Univ. of Arizona, Lunar \& Planetary Laboratory, Tucson, USA. E-mail: kieren.howard@nhm.ac.uk.

Introduction: Position sensitive detector X-ray diffraction (PSD$\mathrm{XRD)}$ allows us to quantify the mineralogy of bulk CR chondrite powders down to the nano-scale for all phases $>1 \mathrm{wt} \%$.

Samples and Method: We studied 7 CRs. Weathering complicates interpretations in 3 finds, so we report on: GRO 03116; GRA 06100; MET 00426 and Al Rais (fall). Data were collected by PSD-XRD and quantified using a pattern stripping technique [2]. Here each mode is for an $80 \mathrm{mg}$ sample, < in [2]. Cu radiation induces Fe- fluorescence that is used to quantify the Fe-rich amorphous component [2]. XRD data were crosschecked using Co radiation. Average values are reported in volume $\%$.

Results and Discussion: Olivine (35\%) and pyroxene (40\%) exist in nearly equal volumes in all samples. In samples like MET 00426, these phases show no evidence for peak broadening in XRD patterns: a common feature of even minor aqueous alteration. Our total anhydrous silicate $(\mathrm{ol}+$ pyx) abundance $(75 \%)$ is less than reported in petrographic studies $(85-95 \%)$ [3,4]. XRD shows CRs typically contain 3-4\% metal, $1-2 \%$ magnetite and rare carbonate $(<1 \%)$. Al Rais has a distinct mineralogy reflecting a greater volume of dark inclusions [3] and more advanced aqueous alteration, it contains abundant magnetite (17\%) and only rare metal. XRD patterns for Al Rais show obvious diffraction peaks from crystalline serpentine $(20 \%)$. All other samples show no evidence for diffraction from phyllosilicate, indicating it is not present in well crystalline form. Transmission electron microscopy (TEM) also shows only rare nano-crystalline phyllosilicate in MET 00426 [1]; if this contributed significantly $(>1 \%)$ to the bulk composition, XRD would resolve it in broad reflections.

Meteorite Dark Matter: Subtraction of crystalline phases in pattern fitting reveals large residual X-ray counts in all samples but Al Rais. These residual counts are fluorescence from Fe-rich $\mathrm{X}$-ray amorphous material, typically: $\mathrm{FeNiS}, \mathrm{Fe}_{x} \mathrm{O}_{x}, \mathrm{FeOOH}\left( \pm_{x} \mathrm{H}_{2} \mathrm{O}\right)$ and potentially also $\mathrm{Fe}, \mathrm{Mg}$ phyllosilicates. Modeling the residual component in XRD patterns, using background intensities [2] of these phases, fails to account for the required counts or requires unrealistic abundances ruled out by mass balance calculations. TEM shows that amorphous Fe-silicate is common in the matrices of MET 00426 and QUE 99177 (CR) [1]. Modeling the XRD residuals with large volumes $(\leq 15 \%)$ of this amorphous Fe-silicate [1] and less FeNiS (5-10\%) and $\mathrm{Fe}_{x} \mathrm{O}_{x} /$ FeOOH $\left( \pm_{x} \mathrm{H}_{2} \mathrm{O}\right)(\leq 10 \%)$ yields good agreements with bulk compositions. Petrographic studies counting amorphous material as silicate also explains discrepancies with XRD estimates of total silicate volume.

Conclusion: Amorphous Fe-silicate can be a significant component of CRs. It appears to be destroyed by the progression of aqueous alteration, hence its absence in Al Rais. The question remains: is it a pristine product of the solar nebular [1], or a proto-alteration product of early stage parent body processing?

References: [1] Abreu N. M and Brearely A. J. 2010. Geochimica et Cosmochimica Acta 74:1146-1171. [2] Howard K. T. et al. 2010. Geochimica et Cosmochimica Acta 74:5084-5097. [3] Weisberg M. K. et al. 1993. Geochimica et Cosmochimica Acta 57:1567-1586. [4] Schrader D. L. et al. 2011. Geochimica et Cosmochimica Acta 75:308-325. 
5062

REE MICRODISTRIBUTIONS IN NWA 4898: A HIGH-AI MARE BASALT

W. Hsu ${ }^{1,2}$, Y. Guan ${ }^{3}$, S. $\mathrm{Li}^{2}$ and Y. Wang ${ }^{1}$. ${ }^{1}$ Purple Mountain Observatory, Nanjing, China. E-mail: wbxu@pmo.ac.cn. ${ }^{2}$ China University of Geosciences, Wuhan, China. ${ }^{3}$ GPS, Caltech, Pasadena, CA 91125 , USA.

Introduction: Lunar meteorites extend diversity of lunar materials and yield complementary information on the formation and geological evolution of the Moon. Among them, NWA 4898 is a unique high-Al, low-Ti mare basalt [1]. It is much younger $(3.6 \mathrm{Ga})$ than Apollo 14 high-Al basalts (3.95-4.33 Ga) and was derived from an extremely LREEdepleted mantle source [2]. Here we report ion microprobe analyses of REEs in NWA 4898 and provide additional constraints on its petrogenesis.

Results: NWA 4898 exhibits an intergranular texture, composed of fine-grained, chemically zoned pyroxenes and anorthite (maskelynite). Olivine occurs as megacrysts and often contains chromite and melt inclusions. Several shock-induced melt veins are visible in the sections. Pronounced normal REE zoning was observed in olivine, pyroxenes, and maskelynite, indicating shock metamorphism did not modify REE microdistributions in NWA 4898. Using the average REEs of maskelynite cores and appropriate partition coefficients for calcic plagioclase [3], we estimate the REE contents of the initial melt that anorthite crystallized from. The calculated melt has slightly lower REEs $(20 \%)$ than the bulk sample analyzed by [1]. Melt inclusions in olivine are $40 \%$ higher than the bulk.

Discussion: REE abundances of the calculated initial melt are generally compatible with the bulk composition within analytical uncertainties (relatively large due to low REEs in maskelynite, especially HREEs). This suggests that NWA 4898 evolved in a close system and largely reflects geochemical characteristics of its source region. However, melt inclusions trapped in olivine $\left(\mathrm{Fo}_{70}\right)$ megacrysts do not represent the initial melt. They are enriched in $\mathrm{CaO}(13-16 \mathrm{wt} \%)$ and $\mathrm{Al}_{2} \mathrm{O}_{3}(13-15 \%)$ but depleted in $\mathrm{MgO}(1.3-4.5 \%)$, significantly more evolved $[\mathrm{Mg} /$ $(\mathrm{Mg}+\mathrm{Fe})=0.27$ relative to the bulk sample $(0.46)$.

Acknowledgment: This work was supported by Chinese National Natural Science Foundation (grant nos. 40773046, 10621303) and by the Minor Planet Foundation of China.

References: [1] Greshake A. et al. 2008. Abstract \#1631. 29th Lunar and Planetary Science Conference. [2] Gaffney A. et al. 2008. Abstract \#1877. 29th Lunar and Planetary Science Conference. [3] Snyder G. A. et al. 1992. Geochimica et Cosmochimica Acta 56:3809-3823.

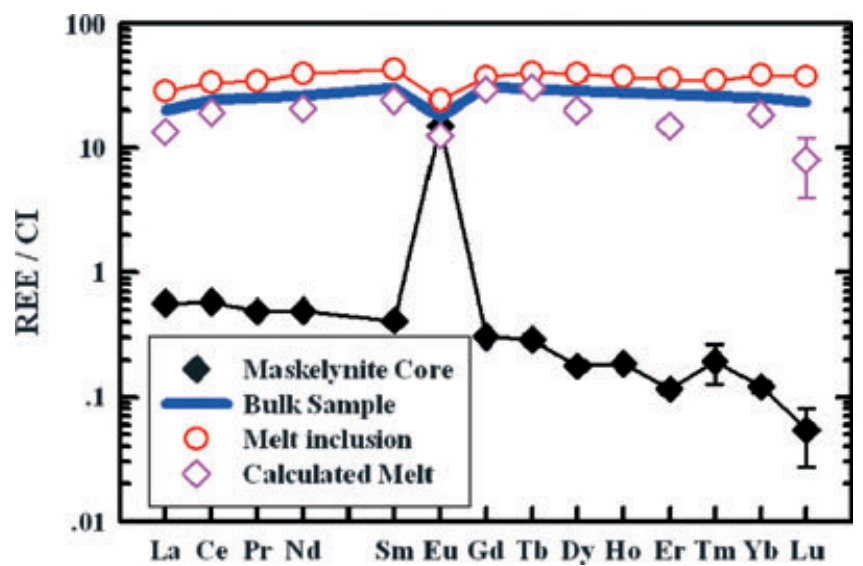

5288

NONDESTRUCTIVE THREE-DIMENSIONAL IMAGING OF AN INTERPLANETARY DUST PARTICLE AT THE NANOSCALE

Z. W. $\mathrm{Hu}^{1}$ and R. Winarski ${ }^{2}$. ${ }^{1} \mathrm{XNano}$ Sciences Inc., P. O. Box 12852, Huntsville, AL 35812, USA. E-mail: zwhu@xnano.org. ${ }^{2}$ Center for Nanoscale Materials, Argonne National Laboratory, Argonne, IL 60439, USA.

Interplanetary dust particles (IDPs) collected from the stratosphere [1], perhaps dominated by comet fragments as implied by the latest dynamical model of zodiacal cloud [2], represent the most primitive extraterrestrial materials currently available for laboratory study [3-5]. Chondritic porous (CP) IDPs and cluster IDPs in general exhibit properties consistent with a cometary origin, and they are fragile, porous aggregates of mostly submicron-sized surviving presolar grains and nebular condensates. Much knowledge about early solar system processes has been gained from the laboratory analysis of the mineralogy and compositions of microtomed thin IDP sections ( $\leq 100 \mathrm{~nm}$ thick). Yet, subtle, fine structures indigenous to interstellar grains and parent bodies preserved in IDPs may be altered by destructive processing. A question arises then: Is it possible to peer into delicately-bound-aggregate IDPs noninvasively to map their unaltered structures in three-dimensional (3D) nanoscale detail? Such information is crucial to our understanding of presolar and early solar nebular processes and the origin and evolution of comets and the solar system at a fundamental level. We have been tackling this issue using a nondestructive $3 \mathrm{D}$ nanoimaging approach that exploits the high penetrating power of hard X-rays and the high sensitivity of phase contrast imaging. Results from the initial experiment will be presented, along with the discussion of implications for understanding the structure and formation of interstellar grains, ice and dust agglomeration in the early solar nebula, and the origin and evolution of comets. New opportunities will also be discussed to investigate both presolar grains in situ in host IDPs and cometary structure and evolution at the fundamental level.

Acknowledgment: ZWH wishes to thank G. J. Flynn, D. E. Brownlee, M. E. Zolensky, L. R. Nittler, B. Lai, and J. Masor for valuable discussions, V. Rose for technical assistance, and NASA for allocating IDPs for this research. Use of the Center for Nanoscale Materials and the Advanced Photon Source at Argonne National Laboratory was supported by DOE, Office of Science, Office of Basic Energy Sciences, under Contract No. DE-AC02-06CH11357

References: [1] Brownlee D. E. 1985. Annual Review of Earth and Planetary Sciences 13:147-173. [2] Nesvorný D. 2010. The Astrophysical Journal 713:816-836. [3] Ishii H. A. et al. 2008. Science 319:447-450. [4] Busemann H. 2009. Earth and Planetary Science Letters 288:44-57. [5] Messenger S. 2000. Nature 404:968-971. 
5382

\section{THE IMPORTANCE OF COSMIC RAY EFFECTS FOR PALLADIUM-SILVER CHRONOMETRY IN IRON METEORITES: AN EVALUATION USING NOBLE GASES}

L. Huber ${ }^{1}$, M. Schönbächler ${ }^{2}$, I. Leya ${ }^{3}$, R. Wieler ${ }^{1}$, K. J. Theis ${ }^{2}$, M. Rehkämper ${ }^{4}$ and G. K. Benedix ${ }^{5}$. ${ }^{1}$ Institute for Geochemistry and Petrology ETH Zürich, CH-8092 Zürich, Switzerland. E-mail: liliane.huber@erdw.ethz.ch. ${ }^{2}$ SEAES, The University of Manchester, M13 9PL, UK. ${ }^{3}$ Space Research and Planetary Sciences, University of Bern, Switzerland. ${ }^{4}$ IARC and Dept. of Earth Science and Engineering, Imperial College London, SW7 2AZ, UK. ${ }^{5}$ IARC and Department of Mineralogy, The Natural History Museum, Cromwell Road, London, SW7 5BD, UK.

Introduction: The short-lived ${ }^{107} \mathrm{Pd}-{ }^{107} \mathrm{Ag}$ decay system (halflife $=6.5 \mathrm{Myr})$ is a powerful tool to date core crystallization of asteroids [1]. During the travel as meteoroids from the asteroid parent body to the earth galactic cosmic-ray (GCR) particles can produce cosmogenic Ag isotopes through interactions with $\mathrm{Pd}$ (e.g., ${ }^{106} \mathrm{Pd}(n, \gamma)$ ) [2]. This production of $\mathrm{Ag}$ can potentially compromise the $\mathrm{Pd}-\mathrm{Ag}$ dating system. It is therefore essential to determine the concentration of cosmogenic $\mathrm{Ag}$ in these meteorites. Reedy [2] modeled the cosmogenic production of $\mathrm{Ag}$ isotopes and obtained an upper limit of the ${ }^{107} \mathrm{Ag}_{\cos } /{ }^{108} \mathrm{Pd}$ ratio of $1.4 \times 10^{-7}$ for an exposure age of $1 \mathrm{Ga}$ at a position of maximum GCR particle flux within meteoroids. This would be negligible for Pd-Ag age determinations, because the measured ratios are in the range of approximately $1 \times 10^{-5}[1,2]$.

This study aims to reassess these early calculations and utilizes noble gas concentrations as a measure for the fluence of cosmic-rays. This in turn can be used to estimate the magnitude of the GCR effects that may influence the Pd-Ag dating system.

One way to exclude that the Pd-Ag dating system is compromised by cosmogenic $\mathrm{Ag}$ is to compare deviations from $\mathrm{Pd}-\mathrm{Ag}$ isochrones (which are defined by several iron meteorites [3]) with the amount of produced noble gases. If samples with very low noble gas concentrations fall with the same probability on the isochrone as those with high contents the influence of the GCR induced effects can be neglected.

Samples and Methods: The noble gases will be measured in samples adjacent to those which were used for Pd-Ag analyses [4]. New cosmic-ray exposure ages will be determined for Coahuila (IIAB), Boxhole (IIIA), and Goose Lake (IAB), which have not been analyzed for light noble gases previously. Further analyzed will be Toluca, Thunda, Canyon Diablo, Odessa, Henbury, Sikhote-Alin, and North Chile and for these samples the reported exposure ages will be verified based on the newly obtained noble gas data and recent physical model calculations [4].

For the noble gas analyses, the samples were cut into $80-150 \mathrm{mg}$ pieces. Following cleaning with ethanol they are loaded into the sample storage arms of a high-T furnace, where they are heated in vacuum for two days at $80^{\circ} \mathrm{C}$ to desorb atmospheric noble gases. The gas extraction occurs at $1700{ }^{\circ} \mathrm{C}$ using a RF-heating system. We will present the light noble gas data $(\mathrm{He}, \mathrm{Ne}, \mathrm{Ar})$ and the corresponding cosmic ray exposure ages of the meteorites.

References: [1] Schönbächler M. et al. 2008. Geochimica et Cosmochimica Acta 72:5330-5341. [2] Reedy R. C. 1980. 11th Lunar and Planetary Science Conference. pp. 1169-1178. [3] Theis et al. 2011 Meteoritics \& Planetary Science, this volume. [4] Ammon K. et al. 2009. Meteoritics \& Planetary Science 44:485-503.

\section{7}

COMPONENTS OF DISTAL SUDBURY EJECTA

M. S. Huber ${ }^{1}$ and C. Koeberl ${ }^{1,2}$. ${ }^{1}$ Department of Lithospheric Research, University of Vienna, Althanstrasse 14, A-1090 Vienna, Austria. E-mail: matthew.huber@univie.ac.at. ${ }^{2}$ Natural History Museum, Burgring 7, A1090 Vienna, Austria.

Introduction: The $1850 \mathrm{Ma}$ [1] Sudbury impact is one of the largest meteorite impact events recorded in Earth history. The economic mineral deposits within the Sudbury crater that are related to the vast differentiated melt sheet are well studied, but the ejecta have not been thoroughly characterized. Impact ejecta have been found from approximately $500 \mathrm{~km}$ to $950 \mathrm{~km}$ from the crater center [2, 3, 4]. The most distal ejecta are located in the "Colerain" region of Minnesota, about $950 \mathrm{~km}$ from the crater center. Near the US-Canada border in Ontario, a drill core through the ejecta layer was taken near the Pine River ("PR") at a distance of approximately $650 \mathrm{~km}$ from the crater center. The nearest ejecta deposits are found south of Lake Superior in the Upper Peninsula of Michigan at the "Connors Creek" location, about $500 \mathrm{~km}$ from the crater center.

Different locations have varying thicknesses and compositions of ejecta. The deposits are located in slightly differing geological settings, but are generally similar near-shore continental shelf deposits with mostly thin bedded deposits both stratigraphically above and below the ejecta deposits [3]. Few surface exposures of the ejecta deposits have been found, but the Connors Creek location provides a small outcrop. Although part of the upper portions of the ejected material has been truncated by erosion, about two meters of ejecta are preserved, with glasses and accretionary lapilli as the main impact derived phases. The Colerain and PR samples preserve some of the fine textures lost to alteration and erosion at the Connors Creek location.

Samples have been characterized by petrographic and geochemical analyses. Samples from all sites have similar mineral content, including carbonates, shocked and unshocked quartz, various micas that appear to represent devitrified glass, pyrite, chalcopyrite, sphalerite, and some spherules of organic carbon (possibly locally derived, or possibly of similar origin to organic carbon observed in [5]). Samples are primarily silicates, with up to 23 wt. $\% \mathrm{Al}_{2} \mathrm{O}_{3}$, and 3-4 wt. $\% \mathrm{MgO}$ and $\mathrm{Fe}_{2} \mathrm{O}_{3}$. In addition, the trace elements $\mathrm{V}, \mathrm{Cr}, \mathrm{Rb}$, and $\mathrm{Zr}$ are enriched.

From near to far, the main differences between the various deposits are (1) Accretionary lapilli are not present at the most distal locations; (2) Evolution of glass particles from angular toward rounded (spheroidal) shapes; (3) Decrease in the abundance of channel deposits; and (4) Decrease in locally derived detritus mixed with glasses (from approximately $20 \mathrm{vol} \%$ to $<5 \mathrm{vol} \%$ ). These features are consistent with decreasing energy at greater distance from the crater. Thicknesses of deposits are not consistent with the theoretical calculations of [6], likely a result of reworking and wave action following initial deposition. There is a relationship between underlying strata and local expressions of the ejecta layer.

Acknowledgments: Special thanks to Klaus Schulz, Bill Addison, Greg Brumpton, Bill Cannon, and Mark Severson.

References: [1] Krogh et al. 2004. Ontario Geological Survey Special Volume 1:431-446. [2] Addison W. A. et al. 2005. Geology 33:193-196. [3] Cannon W. F. et al. 2010. GSA Bulletin 122:50-75. [4] Addison W. A. et al. 2010. GSA Special Paper 465:245-268. [5] Wright A. J. et al. 2010. Precambrian Research 177:23-38. [6] Melosh H. J. 1989. Oxford Monographs on Geology and Geophysics 11:87-111. 
5136

PONTLYFNI, THE INSIDE STORY: DISTRIBUTION OF METAL AND SULFIDE REVEALED BY MICRO-COMPUTED TOMOGRAPHY

A. C. Hunt ${ }^{1}$, G. K. Benedix ${ }^{1,2}$ and L. Howard ${ }^{3}$. ${ }^{1}$ Impacts and Astromaterials Research Centre (IARC), The Natural History Museum, Department of Mineralogy, Cromwell Road, London, SW7 5BD, UK, E-mail: alison.hunt@nhm.ac.uk. ${ }^{2}$ Department of Earth Science and Engineering, IARC, Imperial College London, South Kensington Campus, London SW7 2AZ, UK. ${ }^{3}$ Department of Mineralogy, The Natural History Museum, Cromwell Road, London, SW7 5BD, UK.

Introduction: Winonaites are primitive achondrites with roughly chondritic mineralogy [1]. They are related to silicate inclusions in IAB iron meteorites via their oxygen isotope compositions [2]. Pontlyfni is the only fall among the winonaites. Previous studies have shown that Pontlyfni is probably the most reduced and finest-grained of the winonaites, with textures and mineralogy that vary widely $[1,3]$. In particular, Fe-Ni metal and troilite are heterogeneously distributed, with metal occurring as veins up to $6 \mathrm{~mm}$ in length and as particles up to $1 \mathrm{~mm}$ in dimension [1]. Here, we aim to elucidate the structures and abundances of the metal- and troilite-rich regions using micro-computed tomography (CT). Micro-CT is particularly suited to studying meteorites such as the winonaites, as they contain minerals with significantly different $\mathrm{x}$-ray attenuation coefficients, giving rise to clearly defined variations in the greyscale values of the resultant $\mathrm{x}$-ray images, and allowing easy separation of mineralogical phases.

Method: We scanned an $\sim 18 \mathrm{~g}$ piece of Pontlyfni (BM 1975, M6), which measures $3 \mathrm{~cm}$ by $2 \mathrm{~cm}$ by $2 \mathrm{~cm}$. Micro-CT scanning was carried out at the NHM using a Metris X-Tek HMXST 225 CT scanner. The specimen was scanned using a W target, at $225 \mathrm{kV}$ and $175 \mu \mathrm{A}$, with a $1.5 \mathrm{~mm}$ copper filter and $500 \mathrm{~ms}$ exposure at a voxel size of $\sim 17 \mu \mathrm{m}$. Data were reconstructed with a cone beam algorithm using CT-PRO. Analysis of the resulting data was carried out using VG Studio Max 2.1.

Discussion: In hand specimen and thin section, Pontlyfni comprises silicate material with variably weathered metal and troilite, typical of the winonaites. Our new data reveal the highly brecciated nature of this meteorite. Angular and rounded silicate clasts measuring up to $4.5 \mathrm{~mm}$ in dimension are distinguished from a metal- and sulfide-rich matrix. Veins of metal and sulfide also cross-cut these clasts. The CT images reveal that metal and sulfide are more abundant in Pontlyfni than previously determined. Mineral modes calculated from combined elemental maps of Pontlyfni thin sections indicate an abundance of $\mathrm{Fe}-\mathrm{Ni}$ metal + troilite of $30-39 \%$, similar to published abundances of $30-37 \% \quad[1,3]$. By comparison, new micro-CT data show a much higher volume of metal + sulfide $(51.7 \%)$ and a lower volume of silicate phases $(48.3 \%)$. Angular, chondritic silicate inclusions have been described in some IAB irons, with an abundance of up to $40 \%$ [4]. The combination of silicaterich clasts set in a metal- and sulfide-rich matrix indicates that Pontlyfni more closely resembles a IAB iron meteorite, similar to some parts of Campo del Cielo or Caddo County, than a typical winonaite.

References: [1] Benedix G. K. et al. 1998 Geochimica et Cosmochimica Acta 62:2535-2553. [2] Clayton R. N. and. Mayeda T. K. 1996. Geochimica et Cosmochimica Acta 60:1999-2017. [3] Floss C. et al. 2008. Meteoritics \& Planetary Science 43:657-674. [4] Benedix G. K. et al. 2000. Meteoritics \& Planetary Science 35:1127-1141.

\section{4}

OXYGEN ISOTOPIC COMPOSITION OF A STARDUST CHONDRULE FRAGMENT NAMED IRIS

G. R. Huss ${ }^{1}$, R. C. Ogliore ${ }^{1}$ K. Nagashima ${ }^{1}$, A. L. Butterworth ${ }^{2}$, Z. Gainsforth ${ }^{2}$, J. Stodolna ${ }^{2}$ and A. J. Westphal ${ }^{2}$. ${ }^{1}$ Univ. of Hawaii at Mãnoa, USA. E-mail: ghuss@ @igp.hawaii.edu. ${ }^{2}$ University of California, Berkeley, USA.

Introduction: Oxygen isotopic composition is key to understanding the origins of and relationships among solar system materials. Measuring the $\mathrm{O}$ isotopic composition of Stardust samples is challenging because of the small grain sizes. The first $\mathrm{O}$ measurements were done on a CAI fragment [1]. A few measurements of relatively large fragments of lessrefractory material have also been made [1-4]. Here we report data for Iris, a complex fragment with characteristics of a type II chondrule $[5,6]$.

Methods: Iris is a $23 \times 15 \times 5 \mu \mathrm{m}$ terminal particle from Stardust track C2052,2,74. We mounted the Iris potted butt, without modification, in a specially designed holder [7]. The sample is in the middle of a oneinch $\mathrm{Al}$ disk behind a $500-\mathrm{nm}$-thick $\mathrm{Si}_{3} \mathrm{~N}_{4}$ window with a $300-\mu \mathrm{m}$ central hole. Appropriate polished standards are mounted with epoxy in a ring of holes on a cover plate that holds the $\mathrm{Si}_{3} \mathrm{~N}_{4}$ window [7]. The sample was documented by SEM, and analysis spots were marked by putting a focused electron beam on the sample for several minutes to produce a spot visible as a depleted $\mathrm{O}$ signal in scanning ${ }^{16} \mathrm{O}^{-}$ion image. Oxygenisotope data were collected using the UH ims 1280 ion microprobe in multicollection mode. A $25-30 \mathrm{pA} \mathrm{Cs}^{+}$primary ion beam focused to $\sim 2 \mu \mathrm{m}$ was used. The mass resolving power for ${ }^{17} \mathrm{O}^{-}$was $\sim 5500(\mathrm{M} / \Delta \mathrm{M})$. Oxygen-16 was measured on the L1 Faraday cup, ${ }^{17} \mathrm{O}$ on the monocollector electron multiplier (EM), and ${ }^{18} \mathrm{O}$ on the $\mathrm{H} 1 \mathrm{EM}$. The potential contribution from interfering ${ }^{16} \mathrm{OH}^{-}$signal to ${ }^{17} \mathrm{O}^{-}$was evaluated by checking ${ }^{16} \mathrm{OH}^{-}$after each measurement. Data were corrected for Faraday cup background, EM deadtime, detector yield, and ${ }^{16} \mathrm{OH}^{-}$contribution to ${ }^{17} \mathrm{O}$ (typically $<0.2 \%$ ). There is a systematic offset of $\sim 2.7 \%$ in $\delta^{18} \mathrm{O}$ between samples behind the $\mathrm{Si}_{3} \mathrm{~N}_{4}$ window and those mounted on the cover plate. We quantified this offset using San Carlos olivine. Uncertainties in $\delta^{18} \mathrm{O}$ include the measurement uncertainty and the uncertainty in the calibration of the offset. Those for $\Delta^{17} \mathrm{O}$ include the measurement uncertainty and the standard deviation of $\Delta^{17} \mathrm{O}$ from a group of standards.

Results and Discussion: We measured the $\mathrm{O}$ isotopic compositions of olivine, mesostasis, and chromite in Iris. The results are in close agreement and indicate that Iris is isotopically homogeneous. Mean $\Delta \Delta^{17} \mathrm{O}$ of Iris is $\sim-0.3 \% \pm \sim 2 \%$ o $(2 \sigma)$, indistinguishable from terrestrial $\mathrm{O}$. Iris thus appears to show relatively high $\Delta^{17} \mathrm{O}$ compared to other chondrulelike material from comet Wild 2 [2-4]. Mean $\delta^{18} \mathrm{O}$ for Iris is $\sim+8 \% \pm$ $\sim 2 \%$ o $(2 \sigma)$ and plots near the intersection of the terrestrial fractionation line and carbonaceous chondrite anhydrous mineral line on an $\mathrm{O}$ threeisotope plot. Iris thus appears to have a higher $\delta^{18} \mathrm{O}$ than most anhydrous chondritic materials [cf. 8].

References: [1] McKeegan K. D. 2006. Science 314:1724-1728. [2] Nakamura T. et al. 2008. Science 321:1664-1667. [3] Nakashima D. et al. 2011. Abstract \#1240. 42nd Lunar and Planetary Science Conference. [4] Nakamura T. et al. 2009. Meteoritics and Planetary Science 44:A153. [5] Gainsforth Z. et al. 2010. Meteoritics \& Planetary Science 46:A60. [6] Gainsforth Z. et al. 2011. Meteoritics \& Planetary Science 46: this issue. [7] Westphal A. J. et al. 2011. Meteoritics \& Planetary Science 46: this issue. [8] Yurimoto H. et al. 2008. Reviews in Mineralogy and Geochemistry vol. 68. pp. 41-186. 
5091

NEW DENSE COLLECTION AREA IN THE ATACAMA DESERT, CHILE: PRELIMINARY RESULTS

A. Hutzler ${ }^{1}$, J. Gattacceca ${ }^{1}$, P. Rochette ${ }^{1}$, T. Jull ${ }^{2}$, M. Valenzuela ${ }^{3}$, C. Cournede $^{1}$, M. Uehara ${ }^{1}$, N. Laridhi-Ouazaa ${ }^{4}$, M. Gounelle ${ }^{5}$, R. Braucher ${ }^{1}$. ${ }^{1}$ CEREGE, UMR6635 CNRS; Aix-Marseille Université, Aix-en-Provence, France. ${ }^{2}$ NSF Arizona Accelerator Mass Spectrometer Facility, The University of Arizona, Tucson, AZ 85721, USA. ${ }^{3}$ Dpto. Geologia, Universidad de Chile, Chile Plaza Ercilla 803, Santiago, Chile. ${ }^{4}$ University of Tunis El Manar, Faculty of Sciences, Department of Geology, Crystalline Petrology and Sedimentary Unit, Campus 2092 Manar-II, Tunis, TUNISIE ${ }^{5}$ LMCM, Museum national d'Histoire naturelle, CP52, 61 rue Buffon, 75005 Paris, France.

The Atacama Desert (Chile) is the driest and one of the oldest deserts on Earth [1]. It thus offers good possibilities for meteorite recovery [2][3]. We describe here the main characteristics of a new dense collection area (DCA) located in the Central Depression of the Atacama Desert. We propose to name it Paposo, after the LL6 ordinary chondrite Paposo, found close by [4]. During a joint CEREGE-University of Chile expedition in 2010, we recovered 62 meteorites, all ordinary chondrites except for one $\mathrm{CO} 3$. Meteorites were collected searching on foot on a hilly deflation surface, and recognized because of their chocolate-brown color that contrasts with the darker local magmatic rock. Petrographic observations, electron microprobe analyses, and magnetic susceptibility were used for pairing. The 62 meteorites represent at least 44 different falls. The Paposo area thus qualifies as a new DCA.

Surface ages were determined using in situ produced cosmogenic ${ }^{10} \mathrm{Be}$ concentrations measured in quartz pebbles collected on the surface. Assuming no denudation, Paposo area exposure ages are of several Myr. Regarding weathering, $75 \%$ of the meteorites have a weathering grade of W2 or W3. They are thus more weathered than in the other close-by chilean DCA of San Juan [3], but still less weathered compared to other hot desert DCAs in the world. Terrestrial ages are being determined with in-situ 14C and will be discussed [5].

Meteorites masses are between $1 \mathrm{~g}$ and $582 \mathrm{~g}$, with $95 \%$ under $280 \mathrm{~g}$. The collection shows an overabundance of small meteorites, and a lack of large ones, compared to other hot desert DCAs. The abundance of small stones indicates that only searching on foot allows efficient recovery of small meteorites. This mass distribution might also reflect a fragmentation of meteorites during weathering, although this not strongly supported by the low pairing rate.

$\mathrm{H}$ and $\mathrm{L}$ chondrites abundances are in agreement with Antarctic and hot deserts collections, with the same abundance of $\mathrm{L}$ and $\mathrm{H}$. This tendency has to be confirmed with future search that will allow increasing meteorite density.

References: [1] Dunai et al. 2005. Geology 33:321-324. [2] Muñoz C., Guerra N., Martinez-Fris J., Lunar C., and Cerda J. 2007. Journal of Arid Environments 71:188-200. [3] Gattacceca et al. Description of the densest meteorite collection area in hot deserts: The San Juan meteorite field (Atacama Desert, Chile). Meteoritics \& Planetary Science, in revision. [4] Meteoritical Bulletin, no. 89, 2005. Meteoritics \& Planetary Science 40:A201-A263. [5] Jull A. J. T., Donahue D. J., and Linick T. W. 1989. Geochimica et Cosmochimica Acta 53:2095-2100.
5522

\section{ASTEROID ITOKAWA (LL CHONDRITE) REGOLITH SIZE FREQUENCY CHARACTERISTICS AND COMPARISON TO DISAGGREGATED TAGISH LAKE (C2 UNGROUPED) METEORITE FRAGMENTS}

M. I. Ibrahim and A. R. Hildebrand. University of Calgary. E-mail: emiibrah@ucalgary.ca.

Introduction: Asteroid compositional diversity is indicated by spectral and albedo variation and the lithologic range of recovered meteorites. Asteroid regoliths have proven to be unexpectedly ubiquitous and have been imaged with varying resolution on asteroids Gaspra, Ida, Mathilde, Eros, Itokawa, Stein, and Lutetia, and the analogous Martian moons, Deimos and Phobos. The characteristics of asteroid regolith, and the processes that produce it, are of increasing interest as asteroid sampling missions have begun and asteroid dynamic responses to orbit diversion techniques are considered. In this study, we begin by comparing Itokawa [1] regolith fragments' size-frequency and shape distributions between three different terranes. We then determine the size-frequency and shape distributions of fragments of disaggregating Tagish Lake meteorite [2] to compare fragmentation in the much weaker dark asteroid lithologies.

Data Analysis and Results: Image "st_2532629277_v.fits" from the Descent and touchdown sequence of Hayabusa [3] was used to calculate the size frequency distribution of fragments on four equal areas. The image resolution of $10 \mathrm{~cm}$ pixel $^{-1}$ allowed four areas of $\sim 170 \mathrm{~m}^{2}$ to be picked on the typical rough terrane and two smooth areas (within Komaba Crater and Muses-C). The cumulative size frequency distributions of fragments on the rough terrains yielded a slope of -1.63 and -1.32 while Komaba Crater and Muses-C had a slope of -3.33 and -3.07 , respectively. The mean $\mathrm{b} / \mathrm{a}$ axial ratios of the fragments were found to be $0.73 \pm 0.03$ and $0.75 \pm 0.03$ within Komaba Crater and the Muses-C region, respectively.

A total of 195 fragments of Tagish Lake meteorite were measured with a digital caliber and produced a cumulative size distribution slope of -5.7 and their calculated axial ratios $\mathrm{b} / \mathrm{a}$ and $\mathrm{c} / \mathrm{a}$ had mean values of $0.76 \pm 0.01$ and $0.53 \pm 0.01$, respectively. Note that working with fragments allows measuring of three axes; imagery data allows measurement of only two and an assumption of flat-lying fragments is required to conclude that the measured ratio corresponds to $\mathrm{b} / \mathrm{a}$.

Discussion: The size frequency within Komaba, a 30 m-diameter crater, had a steeper slope than those of the rough terrane (and even steeper than that of the Muses- $\mathrm{C}$ region where abundant fine material collected), indicating that impact-induced fracturing and intra-cratering flows are very effective in eliminating larger fragments and resulting in a more uniform size distribution consistent with observed terrestrial erosion processes.

Tagish Lake meteorite fragment samples show a significantly steeper slope for the cumulative size distribution compared to Itokawa terranes. The steeper slope of the Tagish Lake lithologies is interpreted to reflect the lower strength of these hydrated phyllosilicate lithologies (typical of other similar meteorite lithologies such as $\mathrm{CI}$ and $\mathrm{CM}$ ). This implies that the easier fracturing of Tagish Lake-like lithologies will result in enhanced regolith generation on their parent bodies. However, the b/a fragment axial ratio remain the same for the lithologies of contrasting strengths.

References: [1] Fujiwara et al. 2006. Science 312:1330-1334. [2] Hildebrand A. R. et al. 2006. Meteoritics \& Planetary Science 41:407-431. [3] http://darts.isas.jaxa.jp/planet/project/hayabusa/amica. 
5259

\section{THREE-DIMENSIONAL MICRO-STRUCTURES OF DUST AGGREGATE BY RANDOM BALLISTIC DEPOSITION USING MICRO-TOMOGRAPHY}

Y. Imai ${ }^{1}$, A. Tsuchiyama ${ }^{1}$, J. Blum ${ }^{2}$, K. Uesugi ${ }^{3}$, A. Takeuchi ${ }^{3}$, T. Nakano $^{4}$ and M. Uesugi ${ }^{5}$. ${ }^{1}$ Department Earth \& Space Sci., Osaka Univ. E-mail: imai@astroboy.ess.sci.osaka-u.ac.jp. ${ }^{2} \mathrm{TU}$ Braunschweig. ${ }^{3}$ JASRI/SPring-8. ${ }^{4}$ GSJ/AIST. ${ }^{5}$ JAXA.

Introduction: In a formation scenario of planetary system, it is widely accepted that planetesimals form due to collisions and aggregation of sub-micron solid particles (dust). In recent years, several studies have been made on the dust aggregation process. Blum et al. (2006) [1] describe the dust aggregation by annalistic theoretical approach. Wada et al. (2008) [2] determined an equation of state of dust aggregates by three dimensional numerical simulations of dust aggregate collisions. In terms of experimental studies, Blum and Wurm (2008) [3] reviewed a series of their studies of experiments about dust aggregation, and clarified macroscopic picture of dust aggregation. However, three dimensional (3D) structure of the dust aggregate has not been determined experimentally yet. In order to reveal dust aggregation in protoplanetary disk, it is necessary to clarify 3D structure of the dust aggregates experimentally.

Experiments: Dust aggregate analogs were made in a laboratory as simulation of random ballistic deposition process in the early solar system. Single silica sphere grains of 1.5 microns in diameter were dropped down by cogwheel [3] with a feasible low-velocity in a vacuum chamber evacuated at $100 \mathrm{~Pa}$. During the falling, the grains were aggregated with each other, and finally deposited on a glass fiber of 40 microns in diameter. 3-D structures of the deposited aggregates were obtained by nano-tomography using phase contrast at beamline BL47XU in SPring- 8 with monochromatic X-ray beams of $8 \mathrm{keV}$. Successional slice images with the voxel size of $48 \times 48 \times 48 \mathrm{~nm}$ were reconstructed.

Results and Discussion: We succeed to recognize individual gains of the aggregates, and found that the aggregates are mainly composed of chains of grains with some branching. Positions of the center of gravity of individual grains were obtained using image analysis after binarization of the grains. The coordination numbers of the grains vary mainly from 2 to 3 , and the fractal dimension of the aggregates ranges from 1.8 to 2.4 . The numerical simulation of dust aggregation [2] showed that the fractal dimension of the aggregates does not exceed 2.5 in ballistic cluster-cluster aggregation process. The result of the numerical simulation is consistent with the result of the present study. However, some aggregates of the experiment have higher coordination numbers than those of the numerical simulation, although the fractal dimension of the experimental and numerical simulation aggregates are almost the same. We found that angles between neighboring grains in the experimental aggregates concentrate in 60 degree. In contrast, the aggregates of the numerical simulation do not have such feature. This suggests that there is difference of 3-D structure between the aggregates of experimental and numerical simulations, and the aggregates may have different physical properties such as strength and collisional sticking probability.

References: [1] Blum J. et al. 2006. The Astrophysical Journal 652:1768. [2] Wada et al. 2008. The Astrophysical Journal 677:1296-1308. [3] Blum J. and Wurm G. 2008. Annual Review of Astronomy and Astrophysics $46: 21-56$
5236

SULFUR ISOTOPE COMPOSITIONS OF TROILITE INCLUSIONS FROM THE MURCHISON CARBONACEOUS CHONDRITE

T. R. Ireland, P. Holden. Research School of Earth Sciences, The Australian National University, Canberra, ACT0200, Australia. E-mail: trevor.ireland@anu.edu.au.

Introduction: As one of the first projects undertaken on the new SHRIMP SI, we have developed a technique for rapid measurement of the four isotopes of sulfur. In this work we have analyzed troilite inclusions separated from the Murchison carbonaceous chondrite. The inclusions occur typically as rounded objects and are up to several hundred micrometers across. Most of the grains appear to be recovered from isolated sulfide grains in the matrix, although some fragments with adhering silicate suggest a chondrule origin. The grains were mounted in epoxy along with a pyrite reference.

Methods: In situ isotopic analysis was performed using sensitive high-mass resolution ion microprobe-stable isotope (SHRIMP-SI). A $6 \mathrm{nA}$ primary $\mathrm{Cs}^{+}$ion beam $(13 \mathrm{keV})$ was focused to approximately 20 microns with $\mathrm{S}^{-}$secondary ions extracted at $10 \mathrm{keV}$. No electron gun was required for charge compensation. The sulfur isotopes were collected in multiple collection mode: ${ }^{32} \mathrm{~S}$ with $400 \mu \mathrm{m}$ collector slit and $10^{10} \Omega$ current mode resistor, ${ }^{33} \mathrm{~S}$ with $150 \mu \mathrm{m}$ c.s. and $10^{12} \Omega,{ }^{34} \mathrm{~S} 200 \mu \mathrm{m}$ c.s. and $10^{11} \Omega$, and ${ }^{36} \mathrm{~S}$ with $200 \mu \mathrm{m}$ c.s. and $10^{12} \Omega$. In this mode of acquisition, SHRIMP SI is capable of measuring $\delta^{34} \mathrm{~S}$ to around $0.2 \%$ (2 SE) $\Delta^{33} \mathrm{~S}$ to around $0.3 \%(2 \mathrm{SE})$ and $\Delta^{36} \mathrm{~S}$ to $0.5 \%$ (2 SE).

Results: The Murchison troilite inclusions are uniform within each inclusion but show differences between inclusions of up to several permil in terms of mass dependent fractionation. No nonlinear effects have been detected at this stage in either $\Delta^{33} \mathrm{~S}$ or $\Delta^{36} \mathrm{~S}$.

Discussion: SHRIMP analysis allows analysis of four-isotope $\mathrm{S}$ compositions with only a few nanograms of material. The external precision obtained for $\Delta^{36} \mathrm{~S}$ is similar to that attained by conventional gas source mass spectrometry. Mass dependent fractionation in the Murchison inclusions is consistent with evaporation/condensation in the solar nebula. 
5231

NORTHWEST AFRICA 6704: A UNIQUE CUMULATE PERMAFIC ACHONDRITE CONTAINING SODIC FELDSPAR, AWARUITE AND "FLUID" INCLUSIONS, WITH AN OXYGEN ISOTOPIC COMPOSITION IN THE ACAPULCOITE-LODRANITE FIELD

A. J. Irving ${ }^{1}$, R. Tanaka ${ }^{2}$, A. Steele ${ }^{3}$, S. M. Kuehner ${ }^{1}$, T. E. Bunch ${ }^{4}$, J. H. Wittke ${ }^{4}$ and G. M. Hupé. ${ }^{1}$ Department of Earth \& Space Sciences, University of Washington, Seattle, WA 98195, USA. E-mail: irving@ess. washington.edu ${ }^{2}$ Institute for Study of Earth's Interior, Okayama University, Misasa, Japan. ${ }^{3}$ Geophysical Laboratory, Carnegie Institution, Washington, DC, USA. ${ }^{4}$ Geology Program, SESES, Northern Arizona University, Flagstaff, AZ, USA.

Petrography: A very fresh, dense, yellowish-green stone with rounded, shiny exterior surfaces and small patches of remnant black fusion crust found in Algeria is a unique, medium grained (gs 0.3 $1.5 \mathrm{~mm}$ ), unshocked achondrite with an igneous cumulate texture. Smaller grains of olivine $\left(\mathrm{Fa}_{51.6-53.2} ; \mathrm{FeO} / \mathrm{MnO}=120-140 ; \mathrm{NiO}=0.9-1.0 \mathrm{wt} \%\right)$ and chromite $[\mathrm{Cr} /(\mathrm{Cr}+\mathrm{Al})=89.0)$ are enclosed within large (up to $1.5 \mathrm{~mm})$ orthopyroxene oikocrysts $\left(\mathrm{Fs}_{41.6-42.4} \mathrm{Wo}_{2.8-3.6}, \mathrm{FeO} / \mathrm{MnO}=81-\right.$ 106), which are surrounded by intercumulus indistinctly-twinned, very sodic plagioclase $\left(\mathrm{Ab}_{92} \mathrm{An}_{4} \mathrm{Or}_{4}\right)$ and tiny grains of awaruite $(\mathrm{Ni}=75$ $\mathrm{wt} \%)$. Orthopyroxene grains contain curvilinear trains of tiny rounded to irregularly-shaped, clear to pinkish inclusions $(<2-30 \mu \mathrm{m})$, which appear on polished surfaces to be empty bubbles with smooth rounded walls. Confocal Raman spectroscopy of inclusions below thin section surfaces did not reveal any fluid species.

Oxygen Isotopes: Replicate analyses of acid-washed bulk material by laser fluorination gave: $\delta^{17} \mathrm{O} 1.015,0.880 ; \delta^{18} \mathrm{O} 3.922,3.613 ; \Delta^{17} \mathrm{O}$ $-1.048,-1.020$ per mil, respectively. These results plot in the field for acapulcoites and lodranites $[1,2]$.

Discussion: This specimen is unlike any other known achondrite in its combined mineralogical features: mafic silicate minerals with elevated $\mathrm{FeO} / \mathrm{MnO}$ ratios, extremely sodic plagioclase, trapped bubble trains and very $\mathrm{Ni}$-rich metal. Despite its oxygen isotopic composition, NWA 6704 bears no mineralogical resemblance to members of the acapulcoitelodranite clan. It appears to be a cumulate igneous rock related to an unknown "planetary" parent body, which evidently was capable of interior melting and crystal accumulation. The relatively ferroan silicates, very sodic plagioclase and now empty (?) fluid inclusions (see below) suggest a somewhat "Earth-like," differentiated parent body.

Bubble trains and cleavages in orthopyroxene; WOF $0.6 \mathrm{~mm}$

References: [1] Clayton R. and Mayeda T. 1996. Geochimica et Cosmochimica Acta 60:1999-2017; [2] Irving A. et al. 2007. Abstract \#1338. 38th Lunar and Planetary Science Conference.

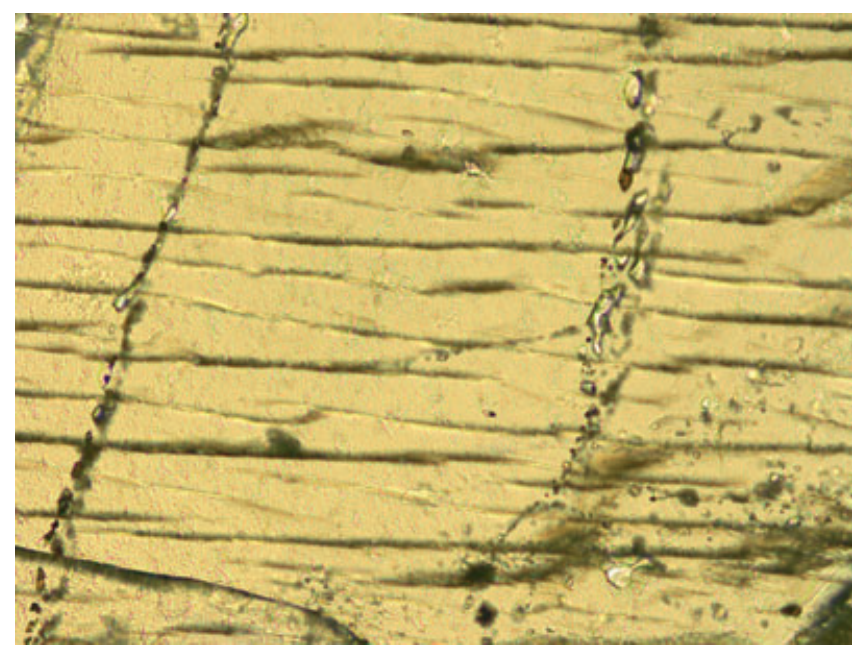

5232

PAIRED FINE GRAINED, PERMAFIC OLIVINE-PHYRIC SHERGOTTITES NORTHWEST AFRICA 2990/5960/6234/ 6710: TRACE ELEMENT EVIDENCE FOR A NEW TYPE OF MARTIAN MANTLE SOURCE OR COMPLEX LITHOSPHERIC ASSIMILATION PROCESSES

A. J. Irving ${ }^{1}$, C. D. K. Herd ${ }^{2}$, M. Gellissen ${ }^{3}$, S. M. Kuehner ${ }^{1}$ and T. E. Bunch ${ }^{4}$. ${ }^{1}$ Earth \& Space Sciences, University of Washington, Seattle, WA 98195, USA, E-mail: irving@ess.washington.edu. ${ }^{2}$ Earth \& Atmos. Sciences, University of Alberta, Edmonton, Canada. ${ }^{3}$ Institute of Geosciences, Universität zu Kiel, Germany. ${ }^{4}$ Geology, SESES, Northern Arizona University, Flagstaff, AZ 86011, USA.

Background: NWA 2990 was the first of at least five fresh crusted stones allegedly found in Mali representing an unusual type of Martian igneous lithology. Our initial studies [1] established that NWA 2990 is an olivine-phyric shergottite with unusually fine grainsize, and based upon its REE abundances we suggested that it had affinities to "enriched" shergottites. Bulk major and trace element compositions were measured on a very small fragment of the stone; however, subsequent analyses on more representative powders of paired stones now lead us to present revised compositional data, which are much more consistent with the observed mineralogy and have significant implications.

Paired Specimens: NWA 5960, NWA 6234 and NWA 6710 all share with NWA 2990 the same microporphyritic texture, mineralogy and mineral compositions. Bulk XRF analyses of NWA 5960 and NWA 6234 in the same laboratory establish that these are all permafic shergottites [2] with $\mathrm{CaO}=6.5 \mathrm{wt} \%, \mathrm{MgO}=16.6 \mathrm{wt} \%$ and $\mathrm{Mg} /(\mathrm{Mg}+\mathrm{Fe})=0.572$. The major element composition reported previously [1] evidently was on powder that undersampled the olivine and chromite microphenocrysts, yet bulk abundances of incompatible elements (residing in groundmass phases) were accurate. REE data for NWA 6234 by ICPMS show good agreement for common elements with INAA data for NWA 2990, but new data for $\operatorname{Pr}$ and $\mathrm{Nd}$ require that the affinity of these specimens be revised from "enriched" to intermediate.

Discussion: The more complete REE pattern implies either that NWA 2990 and pairings are related to a Martian mantle source distinct from the depleted, intermediate and "enriched" sources already known [3], or else that assimilative interaction of an "enriched" Martian magma with lithospheric materials (possibly hydrothermally altered?) changed its $\mathrm{Sm} / \mathrm{Nd}$ ratio.

References: [1] Bunch T. et al. 2009. Abstract \#2274. 40th Lunar and Planetary Science Conference. [2] Irving A. et al. 2011. Abstract \#1612. 42nd Lunar and Planetary Science Conference. [3] Borg L. and Draper D. 2003. Meteoritics \& Planetary Science 38:1713-1731. [4] Lapen T. et al. 2010. Abstract \#2448. 41st Lunar and Planetary Science Conference.

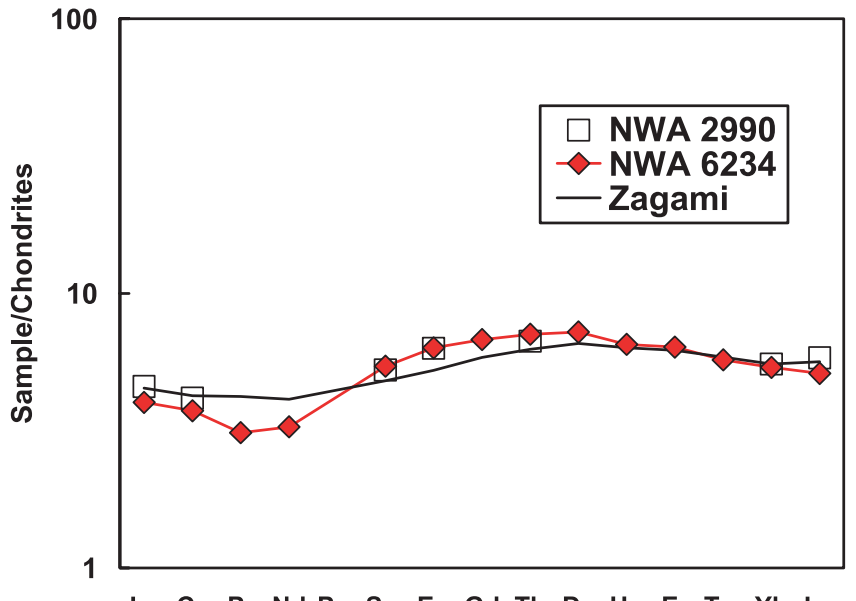




\section{0}

\section{OXIDATION IN R CHONDRITES}

Junko Isa, Alan E. Rubin, and John T. Wasson. Institute of Geophysics, University of California, Los Angeles, CA 90095-1567, USA. E-mail: jisa@ucla.edu.

$\mathrm{R}$ chondrites are among the most oxidized chondrite groups, characterized by the rarity or absence of metallic Fe-Ni [1-5], high Ni contents in the remaining metal (mainly awaruite) [2-5], abundant NiObearing olivine ( $\mathrm{Fa35}-42$ ) [5], the presence of $\mathrm{Fe}^{3+}$-bearing oxides and, in one sample (R5 LAP 04840), major hornblende and accessory biotite [6]. These mineralogical features reflect variations in local conditions on the $\mathrm{R}$ chondrite parent body. To understand such parent-body processes, we studied the bulk chemistry and petrology of $14 \mathrm{R}$ chondrites. Our INAA data show only minor chemical variations in $\mathrm{R}$ chondrites $[5,7,8]$. Nevertheless, we observed diverse compositions among oxide phases in equilibrated $\mathrm{R}$ chondrites [7]. The amount of $\mathrm{Fe}^{3+}$ in magnetite-chromite solid solutions increases with the amount of $\mathrm{NiO}$ in olivine. In addition, the sample with the most $\mathrm{Fe}^{3+}$-enriched oxides (A-881988 and LAP 031156) are associated with the lowest olivine Fa ( 35 mole\%), implying that these rocks are the most oxidized samples. These trends are not a function of petrologic type; this indicates that the degree of oxidation is independent of the temperature history and instead, probably reflects local conditions. Variations in redox state caused variations in the distribution of $\mathrm{Fe}$ and $\mathrm{Ni}$ among sulfides and oxides. For example, $\mathrm{Fe}$ occurs in metal $\left(\mathrm{Fe}^{0}\right)$, is bivalent in silicates, sulfides and partly in oxides, and is trivalent in some oxides.

We used the optical microscope and point-counter to determine modal abundances and petrologic type; we also used EPMA for phase analysis. The respective abundances of silicate, sulfide and oxide are: R6 Y-980702, Fa38 (94 vol\%, 5 vol\%, 0.6 vol\%), R4 A-881988, Fa35 (92 vol\%, 5 vol $\%, 3$ vol\%), R4 LAP 031156, Fa35 (89 vol\%, 7 vol\%, 4 vol\%), R4 NWA 2198, Fa39 (91 vol\%, 8 vol\%, 1 vol\%), R4-clast in Bencubbin, Fa38 (94 vol\%, 6 vol $\%, 0.7$ vol $\%)$. The sulfide modal abundances (5-8 vol\%) are not correlated with the phase compositions and have been affected by terrestrial weathering to different extents [9]. On the other hand, the oxide/silicate volume-ratios (0.007-0.04) are correlated with the olivine and magnetite compositions. The modal abundance of oxides increases with the amount of $\mathrm{Fe}^{3+}$ in oxide, and decreases with the olivine $\mathrm{Fa}$ value. The observed trends (the chief oxidant) probably result from the inhomogeneous distribution of water in the $\mathrm{R}$ chondrite parent body.

References: [1] Rubin A. E. and Kallemeyn G. W. 1989. Geochimica et Cosmochimica Acta 53:3035-3044. [2] Schulze H. et al. 1994. Meteoritics 29:275-286. [3] Rubin A. E. and Kallemeyn G. W. 1994. Meteoritics 29:255-264. [4] Bischoff A. et al. 1994. Meteoritics 29:264-274. [5] Kallemeyn G. W. et al. 1996. Geochimica et Cosmochimica Acta 60:2243-2256. [6] McCanta M. C. et al. 2008. Geochimica et Cosmochimica Acta 72:5757-5780. [7] Isa et al. 2010. Abstract \#5403. Meteoritics \& Planetary Science. [8] Greenwood et al. 2000. Geochimica et Cosmochimica Acta 64:3897-3911. [9] Rubin A. E. and Huber H. 2005. Meteoritics \& Planetary Science 40:1123-1130.
5399

QUE 97186 AND RBT 04143: TWO EXTREME VARIETIES OF CV3 CARBONACEOUS CHONDRITES

H. Ishida, T. Nakamura, H. Miura, and Y. Kakazu. Department of Earth and Planetary Material Sciences, Tohoku University, Miyagi 9808578 Japan. E-mail: h-ishida@s.tohoku.ac.jp.

CV3 meteorites of reduced type are known to retain the most primitive mineralogical characteristcs of $\mathrm{CV} 3$ chondrites, because it has undergone the least degrees of aqueous alteration and thermal metamorphism [e.g., 1]. At the beginning of the present study, we have carried out petrologic study on six CV3 samples, RBT 04143, QUE 97186, GRA 06101, LAP 04843, LAP 02206, and LAP 02228 in order to identify reduced type CV3s. The results showed that RBT 04143 and QUE 97186 are classified to reduced CVs and further mineralogical and isotope study was carried out on the two meteorites to uncover their formation and evolution process.

RBT 04143 has a very porous matrix and rounded undeformed chondrules. Submicron to several-micron size silicates of mostly olivine and minor amounts of low- and high-Ca pyroxene are major phases in matrix. Individual matrix olivine were analyzed by using FE-EPMA and showed a wide range of $\mathrm{Fe} /(\mathrm{Mg}+\mathrm{Fe})$ ratios from $\mathrm{Fa} 0$ to 80 . The wide $\mathrm{Fa}$ variation indicates that olivine dust in the solar nebula had an extreme wide range of $\mathrm{Fe} / \mathrm{Mg}$ ratios and $\mathrm{RBT} 04143$ has undergone the least degrees of aqueous alteration and thermal metamorphism after accretion of parent body.

On the other hand, the matrix of QUE 97186 is highly compacted with porosity much lower than RBT 04143 and chondrules are flattened to high aspect ratios and show a preferred orientation. The texture strongly suggests that the meteorite has experienced shock impact on the meteorite parent body. Olivine and pyroxene phenocrysts in QUE 97186 chondrules show undulatory extinctions and planar deformation fractures. This result indicates that the meteorite experienced shock pressure around $20 \mathrm{GPa}$ according to the results of previous shock recovery experiments [2] and shock-stage classification scheme [3].

Shock-induced heating resulted in homogenizing matrix olivine composition: QUE 97186 matrix olivine showed $\mathrm{Fa}$ range from Fa45 to 60, which is much narrower than that in RBT 04143. Low melting temperature material is also subject to heating: troilite grains in the matrix are totally or partially melted, suggesting that peak shock temperature exceeded $\mathrm{Fe}-\mathrm{FeS}$ eutectic melting temperature of $1000{ }^{\circ} \mathrm{C}$. So as to estimate the cooling rate from peak residual temperature $(800$ approximately $1300{ }^{\circ} \mathrm{C}$ based on internal energy increase derived from hugoniot calculation [2] and mineralogical observation), we simulated homogeneization of $\mathrm{Fa}$ with time by considering size distribution of matrix olivine and Fe-Mg diffusion length by both grain-boundary and lattice diffusion [4-6]. The results of simulation indicate that the cooling rates are in the range from $10^{-5}-10^{-1} \mathrm{~K} \mathrm{~s}^{-1}$. This corresponds to the heating region smaller than $10 \mathrm{~m}$ on the QUE 97186 parent body.

References: [1] McSween. 1977. Geochimica et Cosmochimica Acta 41:1777-1790. [2] Nakamura et al. 2000. Icarus 146:289-300. [3] Stoffler et al. 1991 Geochimica et Cosmochimica Acta 55:3845-3867. [4] Yurimoto and Wasson. 2002 Geochimica et Cosmochimica Acta 66:1777-1790. [5] Mishin and Herzig. 1999 Material Science and Engineering 260:55-71. [6] Dohmen and Chakrabory. 2007. Physics and Chemistry of Minerals 34:409-430. 
5213

THE QUESTION OF GEMS IN COMET 81P/WILD 2: STARDUST ANALOG IMPACTS OF FINE-GRAINED MINERAL AGGREGATES H. A. Ishii ${ }^{1}$, P. J. Wozniakiewicz ${ }^{1}$, A. T. Kearsley ${ }^{2}$, M. J. Burchell ${ }^{3}$, J. P. Bradley $^{1}$, N. Teslich ${ }^{1}$, M. C. Price ${ }^{3}$ and M. J. Cole ${ }^{3}$. ${ }^{1}$ Institute of Geophysics and Planetary Physics, Lawrence Livermore National Laboratory, 7000 East Avenue, Livermore, CA 94550, USA E-mail: hope.ishii@1lnl.gov. ${ }^{2}$ Impacts \& Astromaterials Research Centre, Department of Mineralogy, Natural History Museum, London SW7 5BD, UK. ${ }^{3}$ Centre for Astrophysics and Planetary Science, School of Physical Sciences, University of Kent, Canterbury, Kent CT2 7NH, UK.

Introduction: During the Preliminary Examination phase for comet $81 \mathrm{P} /$ Wild two samples returned by NASA's Stardust mission, GEMS-like objects similar in texture to submicron amorphous silicate glass embedded with metal and sulfides (GEMS) found in cometary chondritic porous interplanetary dust particles [1] were found in hypervelocity impact tracks generated by capture of comet dust in silica aerogel at approximately $6.1 \mathrm{~km} \mathrm{~s}^{-1}$. We showed that sulfide particles fired into aerogel resulted in the same texture as Stardust GEMS-like objects (sulfide-rimmed metal beads embedded in glass) [2]. We now explore results of hypervelocity impact of fine-grained mineral aggregate particles like those inferred to have impacted Stardust $\mathrm{Al}$ foil collectors [3, 4]. We ask if such aggregates can explain the heterogeneous glass chemistry seen in GEMS-like objects in impact tracks in Stardust aerogel as well as the mixtures of quenched melt and residual crystals seen in impact residues in Stardust foils.

Materials and Methods: Fine-grained artificial mineral aggregates were generated from $<5 \mu \mathrm{m}$, relatively monodisperse, size-separated powders of olivine, diopside and pyrrhotite cemented together with an acrylate adhesive and fired by light gas gun (LGG) at flight spare Al foil and silica aerogel at Stardust encounter speed. SEM with EDX was used to locate impact craters with multi-mineral residue in foils for focused ion beam (FIB) thin section preparation. Impact tracks in aerogel were extracted by ultrasonic blades [5], compressed and embedded in epoxy for ultramicromed thin section preparation. Thin sections were analyzed using LLNL's Titan aberration-corrected (scanning) transmission electron microscope ((S)TEM).

Results and Discussion: In foil, impacts of relatively-monodisperse aggregates result in complete melting, loss of $\mathrm{S}$, incorporation of $\mathrm{Al}$ and segregation of Fe-rich, Al-bearing crystalline beads. The glass composition is intermediate in $\mathrm{Mg}$ and $\mathrm{Ca}$ and depleted in Fe relative to olivine and diopside precursors. In aerogel, aggregate impacts result in bulbous tracks. Results of aggregate impacts in the aerogel will also be presented as a function of track depth and compared with prior single component projectile shots. We will discuss implications of aggregate particles on localized heating and chemical inhomogeneity in impacts.

Acknowledgments: We thank NASA for Al foils and STFC for LGG support. This work was supported by NNH07AG46I and NNH11AQ79I to HAI and LDRD 09-ERI-004 to JPB. Portions of this work were performed under the auspices of US DOE by LLNL under Contract DE-AC52-07NA27344.

References: [1] Keller L. P. et al. 2006. Science 314:1728-1731. [2] Ishii H. A. et al. 2008. Science 319:447-450. [3] Price M. C. et al. 2010. Meteoritics \& Planetary Science 45:1409-1428. [4] Leroux H. et al. 2008. Meteoritics \& Planetary Science 43:143-160. [5] Ishii H. A. and Bradley J. P. 2006. Meteoritics \& Planetary Science 41:233-236.
5072

FORMATION OF REFRACTORY INCLUSIONS FROM ${ }^{16} \mathrm{O}$-RICH AND ${ }^{16}$ O-POOR SOLIDS: EVIDENCE FROM COMPLEX CAIS IN CV CHONDRITES

M. A. Ivanova ${ }^{1,2}$, A. N. Krot ${ }^{3}, \quad$ K. Nagashima ${ }^{3}$ and G. J. MacPherson ${ }^{2} .{ }^{1}$ Vernadsky Institute of Geochemistry, Moscow 119991, Russia. E-mail: meteorite2000@mail.ru. ${ }^{2}$ Smithsonian Institution, Washington, DC. ${ }^{3}$ University of Hawai'i at Mãnoa, Honolulu, HI 96822, USA.

Introduction: $\mathrm{CAIs}$ enriched in $\mathrm{Zr}$, $\mathrm{Sc}$ and $\mathrm{Y}$ provide important records of the highest temperature processes in the early solar nebula [14]. We recently reported mineralogy, petrography, and O-isotope compositions of two complex refractory inclusions consisting of normal (Type B, CTA, or FTA) CAIs and Zr-rich CAIs (which probably are ultrarefractory) composed of $\mathrm{Zr}$,Sc-rich pyroxenes, $\mathrm{Zr}$-Y-Sc oxides, and Y-rich perovskites, $3 N-24$ from NWA 3118 and 33E-1 from Efremovka [5]. In both $\mathrm{Zr}$-rich CAIs, the $\mathrm{Zr}$, Sc-rich pyroxenes, the Y-rich perovskites and the $\mathrm{Zr}$-Y-Sc-oxides are depleted in ${ }^{16} \mathrm{O}\left(\Delta^{17} \mathrm{O} \sim-3\right.$ $\pm 2 \%$ ), whereas the co-occurring $\mathrm{Al}$,Ti-diopside and spinel are ${ }^{16} \mathrm{O}$-rich $\left(\Delta{ }^{17} \mathrm{O} \sim-23 \pm 2 \%\right)$ [5]. In this regard $3 N-24$ and $33 E-1$ differ from an ultrarefractory CAI "Romulus" from the CM chondrite Murchison [4] that is uniformly ${ }^{16} \mathrm{O}$-rich $\left(\Delta^{17} \mathrm{O} \sim-20 \%\right)$. In order to better understand this enigmatic dichotomy of O-isotope compositions in $3 N-24$ and $33 E-1$, we performed detailed spot analyses of the $\mathrm{Zr}$, Sc-rich pyroxenes in $3 N-24$ along a profile outward from the $\mathrm{Zr}$-Y-Sc-oxides into the enclosing $\mathrm{Al}$, Ti-diopside to explore potential correlations between pyroxene isotopic and mineral chemical compositions.

Results and Discussion: In $3 N-24$, the $\mathrm{Zr}$, Sc-rich pyroxenes form the halo around the $\mathrm{Zr}$-Y-Sc-rich oxides intergrown with $\mathrm{Y}$-rich perovskite. The pyroxenes have positive $\mathrm{Zr}-\mathrm{Sc}, \mathrm{Zr}-\mathrm{Y}$, and $\mathrm{Zr}$ - $\mathrm{Hf}$ correlations, and show a systematic change in $\Delta^{17} \mathrm{O}$ value from ${ }^{16} \mathrm{O}$-poor to ${ }^{16} \mathrm{O}$-rich and decreasing $\mathrm{Zr}$, Sc, and $\mathrm{Y}$ contents with increasing distance from the $\mathrm{Zr}-\mathrm{Y}$ $\mathrm{Sc}$ oxides. However, chemical and O-isotope compositions of the pyroxenes appear to be decoupled. There is a sharp $(\sim 5 \mu \mathrm{m})$ boundary between the ${ }^{16} \mathrm{O}$-poor $\mathrm{Zr}$, Sc-rich pyroxenes and the surrounding ${ }^{16} \mathrm{O}$-rich Al, Ti-diopside. Both types of pyroxenes enclose small $(1-3 \mu \mathrm{m})$ spinel grains which are similarly ${ }^{16} \mathrm{O}$-rich $\left(\Delta^{17} \mathrm{O} \sim-22 \pm 3 \%\right)$.

In $33 E-1$, pyroxene in the core is highly enriched in $\mathrm{Sc}, \mathrm{Zr}, \mathrm{Y}$ and $\mathrm{Hf}$, but correlations between these elements are not as clear as in $3 N-24$. Al-diopside from the rim of $33 E-1$ is more enriched in $\mathrm{Zr}, \mathrm{Sc}, \mathrm{Y}$ and $\mathrm{Hf}$ than that of $3 N-24$. As in $3 N-24$, we observed no correlation between O-isotope compositions of the $\mathrm{Zr}$, Sc-rich pyroxene and their chemical compositions, although again the $\mathrm{Zr}$, Sc-pyroxene in the CAI core is ${ }^{16} \mathrm{O}$-poor and the Al-diopside in the rim is ${ }^{16} \mathrm{O}$-rich.

We infer that the complex CAIs from Efremovka and NWA 3118 recorded the multistage formation of refractory inclusions and the coexistence of ${ }^{16} \mathrm{O}$-poor (Zr-rich oxides, perovskite and $\mathrm{Zr}$, Sc-rich pyroxenes) and ${ }^{16} \mathrm{O}$-rich (Al-diopside, spinel) solids in the early solar system [6]. The $\mathrm{Zr}$, Y, and Sc-rich ${ }^{16} \mathrm{O}$-poor mineral assemblages in $3 N-24$ and $33 E-1$ appear to be relict; they were partially melted and mixed with material formed in an ${ }^{16} \mathrm{O}$-rich environment. Oxygen-isotope exchange between the ${ }^{16} \mathrm{O}$-rich and ${ }^{16} \mathrm{O}$-poor materials during this melting was very limited.

References: [1] Davis A. M. 1991. Journal of Geophysical Research 90:1151-1154. [2] Simon S. B. et al. 1996. Meteoritics \& Planetary Science 31:106-115. [3] El Goresy A. et al. 2002. Geochimica et Cosmochimica Acta 66:1459-1491. [4] Ushikubo T. et al. 2004. Meteoritics \& Planetary Science 39:\#5111. [5] Ivanova M. A. et al. 2011. Abstract \#1728. 42nd Lunar and Planetary Science Conference. [6] Krot A. N. et al. 2010. The Astrophysical Journal 713:1159-1166. 
5503

THE TIMING OF AQUEOUS ALTERATION IN UNEQUILIBRATED ORDINARY CHONDRITES

B. Jacobsen ${ }^{1}$, J. Matzel ${ }^{1}$, A. N. Krot ${ }^{2}$, I. D. Hutcheon ${ }^{1}$, M. Telus ${ }^{2}$ and K. Nagashima ${ }^{2}$. ${ }^{1}$ Lawrence Livermore National Laboratory. ${ }^{2}$ University of Hawai'i at Mānoa, USA.

Introduction: The time scale of secondary mineral formation in chondrites is important for distinguishing between nebular and asteroidal alteration processes. Although most chondrites are affected by varying degrees and types of alteration, the time frame for different episodes of fluid-assisted thermal metamorphism is poorly constrained. Aqueous activity occurred nearly simultaneously on the parent bodies of at least three classes of unequilibrated carbonaceous chondrites-CI, CM and $\mathrm{CV}$-suggesting water played an important role in the chemical evolution of primitive solar system bodies [1]. Whereas the timing of aqueous alteration in $\mathrm{CV}$ chondrites is well constrained, the timing of formation of secondary minerals in unequilibrated ordinary chondrites (UOCs) is not. In order to constrain the timing of aqueous alteration on the UOC parent body, we studied the ${ }^{53} \mathrm{Mn}-{ }^{53} \mathrm{Cr}$ systematics of fayalitic olivine in the UOCs, Ngawi (LL3.0-3.6) and EET 90161 (L3.05).

Results and Discussion: Fayalitic olivine $\left(\mathrm{Fa}_{50-100}\right)$ is a major constituent in the matrices of many UOCs and carbonaceous $(\mathrm{CV}, \mathrm{CK}$, $\mathrm{CO})$ chondrites and both nebular and asteroidal models have been proposed to explain its origin [2]. Most recent models, however, favor fayalite formation during fluid-assisted thermal metamorphism on the chondrite parent bodies $[3,4]$. In this regard, the ${ }^{53} \mathrm{Mn}-{ }^{53} \mathrm{Cr}$ systematics of fayalite can be used to constrain the timing of aqueous alteration on chondrite parent bodies. The $\mathrm{Cr}$ isotopic composition of fayalite in two UOCs, Ngawi and EET 90161, was measured with the NanoSIMS 50 at the Lawrence Livermore National Laboratory using a ${ }^{16} \mathrm{O}^{-}$primary beam focused to $\sim 300 \mathrm{~nm}$ spatial resolution and a mass resolving power of $\sim 3500$. Fayalite in Ngawi, with ${ }^{55} \mathrm{Mn} /{ }^{52} \mathrm{Cr}$ ratios ranging between 1100 and 1950 , shows no excess ${ }^{53} \mathrm{Cr}$ beyond analytical uncertainty. Fayalite grains in EET 90161 exhibit a much wider range in ${ }^{55} \mathrm{Mn} /{ }^{52} \mathrm{Cr}$ ratios of 430-20,000 and two fayalites with ${ }^{53} \mathrm{Mn} /{ }^{52} \mathrm{Cr}$ of $\sim 19,000$ show large ${ }^{53} \mathrm{Cr}$ excesses $\left(\delta^{53} \mathrm{Cr} \sim 375 \%\right.$ ) resolved at the $2 \sigma$ level. A regression through the $\mathrm{Mn}-\mathrm{Cr}$ data for EET 90161 fayalite defines a best fit line with a slope corresponding to $\left({ }^{53} \mathrm{Mn} /{ }^{55} \mathrm{Mn}\right)_{0}=(2.6 \pm 2.2) \times 10^{-6}$. The inferred initial ${ }^{53} \mathrm{Mn} /{ }^{55} \mathrm{Mn}$ ratio cannot be resolved yet from that in ordinary chondrite chondrules reported by [5], $(5.1 \pm 1.6) \times 10^{-6}$.

These data provide the first evidence for radiogenic ${ }^{53} \mathrm{Cr}$ in secondary phases in UOCs and demonstrate that the formation of fayalitic olivine during aqueous alteration occurred simultaneously, about $6 \mathrm{Ma}$ after CAI formation, on at least three carbonaceous and one ordinary chondrite parent body. The time scale is consistent with heating of asteroids by ${ }^{26} \mathrm{Al}$, and suggests that water played an important role in the chemical evolution of primitive solar system bodies.

References: [1] Hutcheon I. D. et al. 1998. Science 282:1865-1867. [2] Lauretta D. et al. 2003. Geochimica et Cosmochimica Acta 65:13371353. [3] Krot A. et al. 2004. Proceeding of National Institute of Polar Research 17:154-172. [4] Zolotov M. Yu. et al. 2006. Meteoritics \& Planetary Science 41:1775-1796. [5] Yin Q.-Z. et al. 2007. The Astrophysical Journal 662:L43-L46.
5043

TRANSPORT OF CHONDRITE COMPONENTS IN THE PROTOPLANETARY DISK: INTERPRETING THE CARBONACEOUS/ NON-CARBONACEOUS CHONDRITE DICHOTOMY

E. Jacquet ${ }^{1}$, S. Fromang ${ }^{2}$ and M. Gounelle ${ }^{1}{ }^{1}$ LMCM, UMR 7202, CNRS \& MNHN, CP 52, 57 rue Cuvier, 75005 Paris, France. E-mail: ejacquet@mnhn.fr. ${ }^{2}$ CEA, Irfu, SAp, Centre de Saclay F-91191 Gif-surYvette. ${ }^{3}$ UMR AIM, CEA-CNRS-Univ. Paris VII, Centre de Saclay, F-91191, Gif-sur-Yvette, France.

Introduction: A fundamental observation in cosmochemistry is the first-order distinction between carbonaceous ("CCs") and noncarbonaceous chondrites (Enstatite, Ordinary, Rumuruti chondrites, "EORs"). How this dichotomy arose in the protoplanetary disc and how it is to be understood chronologically and/or spatially [1] is inseparable from the long-standing debates on the chemical, isotopic and petrographic fractionations in the different chondrite groups (e.g. [2],[3]). In particular, it is important to investigate fractionations of petrographic components of chondrites during their several Ma-long [4] journey as free-floating objects in the disc until their final agglomeration.

Approach: We have revisited via simplified analytic calculations the radial dynamics of mm- and sub-mm-sized solids [5]. In order to draw conclusions independently of the details of the disk structure, we have based our reasonings on as generic a disk model as possible and compared our inferences to calculations for specific disc models published in the literature (e.g. [7-9]).

Results and Discussion: We find that the dynamics of solids are essentially controlled by a gas-solid "decoupling" parameter called S first introduced by [6]: If $S<1$, solids are essentially tied to the gas and no (whole-rock) fractionation other than exchange with the gas phase occurs. If $\mathrm{S}>1$, solids tend to settle toward the disk midplane and drift toward the Sun faster than the gas. In many disk models, $\mathrm{S}$ should be an increasing function of time and heliocentric distance.

Based on (1) abundance of refractory inclusions (2) proportion of matrix (3) lithophile element abundances and (4) oxygen isotopic composition of chondrules, we find that the properties of CCs are most consistent with $\mathrm{S}<1$ when they accreted while those of EORs suggest $\mathrm{S}>1$. For example, complementarity in CCs ([10-11]) would have been maintained until accretion because differential drift between chondrules and dust grains was negligible. Given how $\mathrm{S}$ varies in disk models, this suggests that $\mathrm{CC}$ components agglomerated (but not necessarily formed) earlier on average than those of EORs (as suggested by [12]). Thus, refractory inclusions formed in the early phases of disk history [13], and found in considerable abundance in CCs, would have been depleted in the disk by the time EORs accreted because of drift toward the Sun.

If our first-order conjecture regarding the $\mathrm{CC} / \mathrm{EOR}$ duality were to prove correct, this would open the way to considerable progress in the understanding of chondrite diversity in the accretion disk context.

References: [1] Wood J. A. 2005. Ch. and the p. p. d., 953-971. [2] Wasson J. T. and Chou C.-L. 1974. Meteoritics 9:69-84. [3] Anders E. 1964. Space Science Review 3:583-714. [4] Villeneuve J. et al. 2009. Science 325:985-988. [5] Jacquet E. et al. (to be submitted to Icarus). [6] Cuzzi J. N. et al. 1996, in Ch. and the p. p. d., 35-43. [7] Clarke C. J. and Pringle J. E. 1988. MNRAS 235:365-373. [8] Ciesla F. J. 2009. Icarus 200:655-671. [9] Hughes A. L. H. and Armitage P. J. 2010. The Astrophysical Journal 719:1633-1653. [10] Bland P. et al. 2005. Proceedings of the National Academy of Sciences 102:13755-13760. [11] Hezel D. C. and Palme H. 2010. Earth and Planetary Science Letters 294:85-93. [12] Chambers J. 2006. in MESS2 487-497. [13] Jacquet E. et al. Astronomy \& Astrophysics 526:L8. 


\section{0}

CARBON, NITROGEN, AND SILICON ISOTOPIC COMPOSITIONS OF PRESOLAR SILICON CARBIDE GRAINS FROM THE LL3.1 CHONDRITE BISHUNPUR

M. Jadhav ${ }^{1}$, K. Nagashima ${ }^{1}$ and G. R. Huss ${ }^{1}{ }^{1}$ Hawai'i Institute of Geophysics and Planetology, University of Hawai'i at Mānoa, Honolulu, HI 96822. E-mail: manavi@higp.hawaii.edu.

Introduction: Several studies have indicated that the $\mathrm{N}$ isotopic compositions of presolar $\mathrm{SiC}$ grains vary between different meteorites and among different size ranges in single meteorites [1-5]. The $\mathrm{N}$ compositions do not correlate with the cosmic ray exposure ages of the host meteorites, and terrestrial contamination is unlikely in most cases [3, 4]. We know that abundances and other characteristics of presolar grains correlate with the bulk compositions of the host meteorites [6, 7]. This suggests the possibility that $\mathrm{N}$ isotopes carry a record of the pre-accretionary radiation history of the material making up each chondrite. To test this hypothesis, we have begun an investigation of presolar $\mathrm{SiC}$ grains from various chondrites. Here, we report preliminary results of $\mathrm{C}, \mathrm{N}$, and $\mathrm{Si}$ isotopic measurements on presolar $\mathrm{SiC}$ grains from the Bishunpur (LL3.1) chondrite.

Method: A portion of the SiC-spinel separate prepared by [6] was deposited on gold foil mounts. Thirty-five $\mathrm{SiC}$ grains were identified by mapping the mounts for $\mathrm{C}, \mathrm{Si}, \mathrm{Al}, \mathrm{Mg}$, and $\mathrm{Cr}$ using wavelength dispersive spectrometry on the UH JXA-8500F field-emission electron microprobe. Most grains were $<1.5 \mu \mathrm{m}$ in size. Isotopic analysis of $\mathrm{C}, \mathrm{N}$, and Si were performed using the UH ims-1280 ion microprobe. A 3-5 pA $\mathrm{Cs}^{+}$primary beam focused to $\sim 0.5 \mu \mathrm{m}$ was rastered over $5 \times 5 \mu \mathrm{m}^{2}$ regions and scanning isotope images were collected. Carbon and $\mathrm{Si}$ isotopes were measured first, followed by $\mathrm{C}$ and $\mathrm{N}$ (measured as $\mathrm{CN}^{-}$). Isotopic ratios were calculated from regions of interest using L'image software.

Results and Discussion: Thirty-three of the grains are mainstream grains with ${ }^{12} \mathrm{C} /{ }^{13} \mathrm{C}$ ratios ranging from 20 to 100 and $\delta^{29} \mathrm{Si}$ and $\delta^{30} \mathrm{Si}$ ranging from $0 \%$ to $+200 \%$ relative to solar silicon. Two grains have ${ }^{12} \mathrm{C} /{ }^{13} \mathrm{C}$ ratios $<10$ and can be classified as $\mathrm{A}+\mathrm{B}$ grains. Nitrogen in most of the Bishunpur grains is highly enriched in ${ }^{14} \mathrm{~N}$, with ${ }^{14} \mathrm{~N} /{ }^{15} \mathrm{~N}$ up to 12 times the solar value. Some grains appear to be less enriched in ${ }^{14} \mathrm{~N}$ than similar-sized SiC grains from Murchison [1], Orgueil [3], Colony [4], and ALHA77307 [5]. We are cautious to interpret this result because the less ${ }^{14} \mathrm{~N}$-rich $\mathrm{N}$ compositions could be due to contamination by terrestrial $\mathrm{N}$. While measuring $\mathrm{C}$ and $\mathrm{Si}$ isotopes, the $\mathrm{C} / \mathrm{Si}$ ratio of a majority of the grains decreased over the measurement period. The ion images showed a large $\mathrm{C}$ signal around the grains that sputtered away during the course of the measurement. This indicates that the grains were covered by a C-rich contaminant. It is possible that $\mathrm{N}$ from what was left of this $\mathrm{C}$-rich material affected the $\mathrm{N}$ compositions. We intend to check these measurements by preparing new grain mounts, taking special precautions to remove the C-rich material, as described in [3], and measuring more presolar $\mathrm{SiC}$ grains from Bishunpur. (GRH).

Acknowledgments: Supported by NASA grant NNX08AB58G

References: [1] Hoppe P. et al. 1994. The Astrophysical Journal 430:870-890. [2] Hoppe P. et al. 1996. Geochimica et Cosmochimica Acta 60:883-907. [3] Huss G. R. et al. 1997. Geochimica et Cosmochimica Acta 23:5117-5148. [4] Smith J. B. and Huss G. R. 2003. Meteoritics \& Planetary Science 38:A118. [5] Smith J. B. et al. 2004. Abstract \#2006. 35th Lunar and Planetary Science Conference. [6] Huss G. R. and Lewis R. S. 1995. Geochimica et Cosmochimica Acta 59:115-160. [7] Huss G. R. et al. 2003. Geochimica et Cosmochimica Acta 67:4823-4848.

\section{9}

RAMAN SPECTROSCOPY INVESTIGATIONS OF TAGISH LAKE NANODIAMONDS

T. Jakubowski ${ }^{1}$, M. Kozanecki ${ }^{2}$, U. Ott ${ }^{3}$ and A. Karczemska ${ }^{4}$. ${ }^{1}$ Balonowa 5/2 Wroclaw, Poland. E-mail: illaenus@gmail.com. ${ }^{2}$ Department of Molecular Physics, Technical University of Łódź, Łódź, Poland. ${ }^{3}$ Department of Geochemistry, Max Planck Institute for Chemistry, Mainz, Germany. ${ }^{4}$ Institute of Turbomachinery, Technical University of Lodź, Łodź, Poland.

Introduction: Tagish Lake, a carbonaceous chondrite $\mathrm{C} 2$, fell on January 18, 2000, in Canada. According to [1], the meteorite contains about $3650-4330 \mathrm{ppm}$ of nanodiamonds, while inferred from measurements of xenon [2] the abundance is $1800-2500 \mathrm{ppm}$. This is the highest nanodiamond abundance reported in chondrites. Raman spectroscopic signatures of separated nanodiamonds have been studied in the work presented here.

Samples and Experiments: Nanodiamonds were separated following established procedures [3] Raman spectra have been recorded using the confocal Raman micro-spectrometer T-64000 (Jobin-Yvon) with argon line $\lambda=514.5 \mathrm{~nm}$.

Results: The spectra show a diamond peak at $1329 \mathrm{~cm}^{-1}$ (FWHM of about $50 \mathrm{~cm}^{-1}$ ) and a broad peak around $1600 \mathrm{~cm}^{-1}$ (Fig. 1) [4]. Previous investigations of Allende meteorite presolar nanodiamonds showed peaks at 1326 and $1590 \mathrm{~cm}^{-1}$ [5] what is in agreement of laboratory manufactured nanodiamonds Raman investigations [6].

Shifts of diamond peak positions (monocrystalline cubic diamond has Raman peak at $1332 \mathrm{~cm}^{-1}$ ) occur because of the nanometer crystal sizes [6] or because of different polytypes [7] of diamond occurring in the sample.

References: [1] Grady M. M. et al. 2002. Meteoritics \& Planetary Science 37:713-735. [2] Jakubowski T. et al. 2011. Abstract \#2944. Goldschmidt 2011 Conference. [3] Amari S. et al. 1994. Geochimica et Cosmochimica Acta 58:459-470. [4] Jakubowski T. 2011. Ph.D. thesis, Technical University of Lodz. [5] Gucsik A. et al. 2008. Proceedings of the IAU Symposium 251 "Organic Matter in Space":335-339. [6] Osswald S. et al. 2009. Physical Review B 80:075419. [7] Phelps A. W. 1999. Abstract \#1749. Lunar and Planetary Science Conference.

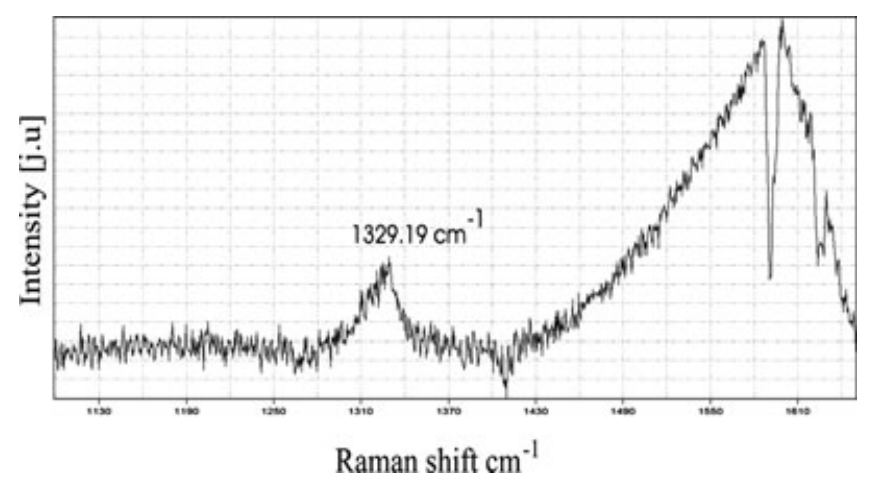

Fig.1. Raman spectra of nanodiamonds from Tagish Lake carbonaceous chondrite. The diamond peak is detected at the position $1329 \mathrm{~cm}^{-1}$. 
5167

EVIDENCE FOR RHÖNITE IN ANGRITES D'ORBIGNY AND SAHARA 99555

A. Jambon and O. Boudouma. CNRS UMR 7193, UPMC-PARIS 6, ISTEP 4 place Jussieu, 75005 Paris, France. E-mail: albert.jambon@ upmc.fr.

Introduction: Angrites are rare achondrites with a specific and odd mineralogy: anorthite, calcic olivine and Al-Ti-diopside-hedenbergite are the major phases [1]. Magmatic angrites: D’Orbigny, SAH 99555 and NWA 1296 share textural and mineralogical similarities, with a decreasing grain size indicating an increasing cooling rate. An enigmatic Al-Tisilicate was described in D'Orbigny [1]. It was later suggested that it could be rhönite [2]. More recently this mineral was discovered in another angrite, NWA 4590 of the plutonic type [3].

Analytical Techniques: We investigated three polished sections, two of D’Orbigny (NHM Wien; 1176-1179) and one of SAH 99555. We performed SEM imaging (Zeiss Ultra 55VP), EDS identification and EMP analyses at $15 \mathrm{kV}, 10 \mathrm{nA}$ (Cameca SX100). Raman spectra were obtained for D'Orbigny with the SEM built-in spectrometer

Results: Rhönite is present in both samples in close relationship with final crystallization phases. Its size is typically $20-100 \mu \mathrm{m}$. The structural formula is (20 O and 14 cations): $\left(\mathrm{Si}_{3.95} \mathrm{Al}_{1.73} \mathrm{Ti}_{0.32}\right) \mathrm{Fe}_{5}\left(\mathrm{Fe}_{0.17}\right.$, $\left.\mathrm{Ti}_{0.75}\right) \mathrm{Ca}_{2.03} \mathrm{O}_{20}$. Notice that the tetrahedral site is significantly deficient in Al. Since $\mathrm{Fe}^{3+}$ is absent, excess Ti was assigned to the tetrahedral site. Rhönite is $\mathrm{Mg}$-free like all other mafic phases during the final stages of crystallization of D'Orbigny and SAH 99555. Comparisons with NWA 4590 rhönite indicates a slightly different chemistry: in particular the absence of $\mathrm{Fe}^{3+}$ and $\mathrm{Cr}^{3+}$, and the $\mathrm{Si}, \mathrm{Al}$ deficiency in the tetrahedral site.

Interpretation and Conclusions: Rhönite is a rare mineral found in terrestrial alkalic rocks and CAIs. It was also found in a Luna 4 sample [4]. It is characteristic of a low silica activity in $\mathrm{Ca}$ and Ti-rich melts. It may be present in other angrites as well even though not described until now and appears another oddity of angrites. As already suggested, it points to some similarity between angrites and terrestrial alkalic rocks [2].

References: [1] Mittlefehldt et al. 2002. Meteoritics \& Planetary Science 37:345-369. [2] Jambon A. et al. 2005. Meteoritics \& Planetary Science 40:361-375. [3] Kuehner S. M. and Irving A. J. 2007. EOS Transactions of the American Geophysical Union 88: Abstract P41A0219. [4] Treiman A. 2008. American Mineralogist 93:488-491.

\section{9}

CHLORINE IN THE PARIS CM: A PRELIMINARY STUDY

A. Jambon ${ }^{1}$, O. Boudouma ${ }^{1}$, B. Zanda ${ }^{2}$, and F. Couffignal ${ }^{3}$. ${ }^{1}$ UPMCPARIS 6, ISTEP 4 place Jussieu, CNRS UMR 7193, 75005 Paris, France. E-mail: albert.jambon@upmc.fr. ${ }^{2}$ LMCM-DHT- CP52, Museum National d'Histoire Naturelle and CNRS UMR 7202, 61 rue Buffon, 75005 Paris, France. ${ }^{3}$ CAMPARIS 4 place Jussieu, 75005 Paris, France.

Introduction: Paris is a recently discovered $\mathrm{CM}$ chondrite with moderate aqueous alteration compared to other CM2 [1]. This specimen is presently studied by a consortium and we were allocated section 2010-6 by MNHN Paris. Here we focus on the abundance and distribution of chlorine in this sample compared to Mighei (MNHN section 2008-42) and Cold Bokkeveld (MNHN section 2010-16). The abundance of chlorine in $\mathrm{CM}$ chondrites is highly variable and poorly understood. This work is part of a more comprehensive survey of halogens in CMs.

Analytical Techniques: In this first approach we performed SEM imaging and EMP analyses. In addition, chemical maps by EDS and WDS allowed characterizing the chlorine distribution.

Results: EMP analyses in raster mode over areas of $100 \times 100 \mu \mathrm{ms}$ yields the following average abundances: $1160( \pm 240), 1620( \pm 230)$ and $830( \pm 180) \mathrm{ppm}( \pm$ variability for 10 points) for Paris, Mighei and Cold Bokkeveld respectively. EDS chemical mapping is consistent with EMP results. For Paris, it indicates that olivine, troilite and kamacite are chlorine free, while there is a low chlorine background of about $1200( \pm 600=2$ sigma $) \mathrm{ppm}$ in the fine matrix and a few hotspots with several $\mathrm{wt} \% \mathrm{Cl}$ are. These hot spots correspond to tochilinite patches surrounded by cronstedtite.

This distribution contrasts with that observed in both Mighei and Cold Bokkeveld in which a chlorine background is present at the level of $1600 \mathrm{ppm} \mathrm{Cl}$ and $800 \mathrm{ppm} \mathrm{Cl}$, respectively. In Mighei hotpsots are absent but in Cold Bokkeveld, rare halite crystals of a few micrometers can be found. We did not find the Cl-tochilinite which appears to be specific of Paris.

Spot analyses of the Paris tochilinite indicate a variable $\mathrm{Cl}$ concentration from 1.0 to $3.7 \mathrm{wt} \%$. When tochilinite is more closely associated with cronstedtite, the $\mathrm{Cl}$ content falls to an average of $5000 \mathrm{ppm}$ and can be as low as $1000 \mathrm{ppm}$.

Conclusions: In situ analyses yield high chlorine values compared to all previous values obtained from bulk chemical analyses. For instance mean $\mathrm{CM}$ composition is given at $160 \mathrm{ppm} \mathrm{Cl}$ [2]. The presence of significant amounts of chlorine in tochilinite is, as far as we know of, specific of Paris and may explain its high chlorine abundance. The low level from bulk analysis may be due to high chlorine mobility in aqueous fluids.

References: [1] The Meteoritical Bulletin \#97. [2] Wasson J. T. and Kallemeyn G. W. 1988. Philosophical Transactions of the Royal Society of London A 325:535. 


\section{7}

\section{NEW INSIGHTS ON HYDROUS PHASES IN R CHONDRITES NWA 6491 AND 6492}

N. S. Jamsja ${ }^{1}$, A. M. Ruzicka ${ }^{1}$, M. Fries ${ }^{2} .{ }^{1}$ Cascadia Meteorite Laboratory, Portland State University, 17 Cramer Hall, 1721 SW Broadway, Portland OR 97207, USA. E-mail: niinaj@pdx.edu. ${ }^{2}$ Planetary Science Institute, 1700 E. Fort Lowell Road, Suite 106, Tucson, AZ 85719, USA.

Introduction: $\mathrm{R}$ chondrites are among the most oxidized meteorites and show evidence of hydrous alteration represented by phases such as biotite and hornblende [1-3]. We recently reported two hydrous phases (HP1 and HP2) that were believed to be related to laihunite and cronstedtite in the NWA 6491 and 6492 R chondrites, genomict breccias containing type 3-5 and 3-6 material [4]. In this abstract we summarize new results from both meteorites that provide additional information about these hydrous and other phases.

Results: Additional study of electron microprobe (EMPA) and SEM data from both meteorites, and Raman spectroscopy data from NWA 6492, show that the HP2 phase, believed to be related to laihunite [4], is indeed an intermixture of laihunite and olivine. Two-component mixing models based on EMPA data imply olivine with approximately $\mathrm{Fa}_{60-70}$, whereas preliminary Raman data suggest olivine with approximately $\mathrm{Fa}_{88}$. Both $\mathrm{Fa}$ values are significantly higher than typical for equilibrated $\mathrm{R}$ chondrites $\left(\mathrm{Fa}_{37-41}\right)$ [5]. EMPA data also imply that the olivine and laihunite intermixture may contain water.

Additional analysis of EMPA data suggests that the HP1 phase, earlier suggested to be cronstedtite [4], is in fact goethite that is intermixed with one or more other phases. Raman microscopy conducted on an atypical goethite-bearing assemblage in NWA 6492 suggests a complex intermixture of jarosite and anhydrite in the core, and goethite and hematite in a rim and in veins that cross-cut the core.

Conclusions: We infer that laihunite, goethite, anhydrite and jarosite are of pre-terrestrial origin. We base our conclusion on: (1) the overall low weathering grade of NWA 6491 and 6492; (2) the lack of terrestrial cross-cutting weathering veins; (3) the systematic change of goethite texture and grain size from petrographic type 3 to type 5 and 6 clasts, which implies that it was present at the time of metamorphism in the $\mathrm{R}$ chondrite parent body; (4) the apparent broken edges of certain laihunite grains in mineral and lithic clasts which implies that laihunite was present prior to brecciation; and (5) veining structures, which imply formation of sulfate prior to goethite.

Laihunite, goethite, and jarosite probably formed by aqueous alteration under hydrous and oxidizing conditions during open system processes. Laihunite likely formed by the breakdown of ferrous olivine.

References: [1] McCanta M. C. et al. 2008. Geochimica et Cosmochimica Acta 72:5757-5780. [2] McCanta M. C. et al. 2007. Abstract \#2149. 38th Lunar and Planetary Science Conference. [3] Mikouchi T. et al. 2007. Abstract \#1928. 38th Lunar and Planetary Science Conference. [4] Jamsja N. and Ruzicka A. 2011. Abstract \#2324. 42nd Lunar and Planetary Science Conference. [5] Hutchison R. 2004. Meteorites: A petrological, chemical and isotopic synthesis.

\section{9}

GERZEH, A PREHISTORIC EGYPTIAN METEORITE

D. Johnson ${ }^{1}$, M. M. Grady ${ }^{1,2}$, and J. Tyldesley ${ }^{3}$. ${ }^{1}$ PSSRI, Open University, Walton Hall, Milton Keynes, MK76AA, UK, E-mail: D.Johnson@open.ac.uk. ${ }^{2}$ Natural History Museum, Cromwell Road, London, UK. ${ }^{3} \mathrm{KNH}$ Centre for Biomedical Egyptology, Manchester University, Oxford Road, Manchester, M139PT, UK.

Iron from meteorites has been utilized in artefacts by peoples from different cultures, e.g., the Inuit of Greenland, who fashioned knives and weapons from the Cape York meteorite [1]. One of the most ancient examples of worked iron meteorites comes from a prehistoric Egyptian cemetery. The site of El Gerzeh, 40 miles south of Cairo, was excavated in 1911-1912, and 301 graves dating from around 3300 BCE were discovered [2]. A few of the graves contained rare and precious materials such as gold and carnelian. Two graves, Tombs 67 and 133, were also found to contain iron beads; at the time of excavation these examples of Egyptian pre-dynastic culture were considered to be the earliest specimens of worked iron. Subsequent analysis revealed the iron to contain significant levels of nickel, leading to identification of their meteoritic origin [3]. Iron meteorites were also used to make other items later in Egyptian history, including a dagger blade from Tutankhamen's tomb (18th dynasty, 1340-1320 BCE), but no artifacts have been found to be made from meteorite iron after this date.

The most recently published analysis of the Gerzeh iron beads was in 1929 [3]. Our study is intended as an illustration of the non-destructive analysis of an intact artifact, in order to allow a better understanding of this historically rare and precious sample. Optical imaging, electron microscopy and EDS were used to both define and analyze the bead. A thick weathering layer of oxidized iron was easily seen; this incorporates rounded quartz grains that originated in the grave, which was filled with sand. Patches of the oxidized areas have degraded and partially fallen away, probably post-excavation, allowing us to examine underlying regions in which small areas of metal were observed. The metal has an average composition of $9 \mathrm{wt} \% \mathrm{Ni}$. The tubular bead structure interior was also found to be filled with ferric oxides and sand, at one end of the bead plant fibres of the strand used to string the beads together were also observed.

This study explores one of the earliest examples of the use of meteoritic iron in artifacts. It is impossible to estimate the original mass of the meteorite: nine beads were discovered in the two tombs, but because of the rarity of iron in early Egypt, it is assumed all beads were produced from the same meteorite. The meteorite must have fallen prior to approximately $3300 \mathrm{BCE}$, as this is the accepted date of the tombs. It is also possible that it fell beyond Egyptian borders as some of the other materials found in the tombs are believed to have arrived in Egypt through foreign trade routes.

Acknowledgments: We would like to thank Dr Karen Exell, Curator of Egypt and the Sudan, at the Manchester Museum, for the loan of a Gerzeh bead, accession number: 5303.

References: [1] Buchwald V. F. 1975. Handbook of iron meteorites, University of California Press, Berkeley, pp. 1418. [2] Wainwright G. A. 1912. Revue Archeologique 19:255-259. [3] Desch C. H. 1929. Reports on the metallurgical examination of specimens for the Sumerian Committee of the British Association. Reports of the British Association for the Advancement of Science. 
5404

METEORITES, ORGANICS AND FISCHER-TROPSCH TYPE REACTIONS: PRODUCTION AND DESTRUCTION N. M. Johnson ${ }^{1}$, A. S. Burton ${ }^{1,2}$ and J. A. Nuth III $^{3}$. ${ }^{1}$ NASA Goddard Space Flight Center, Astrochemistry Laboratory, Code 691, Greenbelt, MD 20771, USA. ${ }^{2}$ NASA Postdoctoral Program, CA, USA. ${ }^{3}$ NASA GSFC, solar systems Exploration Division, Code 690, Greenbelt, MD 20771, USA. E-mail: natasha.m.johnson@nasa.gov.

Background: There has been an ongoing debate about the relative importance about the various chemical reactions that formed organics in the early solar system. One proposed method that has long been recognized as a potential source of organics is Fischer-Tropsch type (FTT) synthesis [e.g., 1, 2]. This process is commonly used in industry to produce fuels (i.e., complex hydrocarbons) by catalytic hydrogenation of carbon monoxide [3]. Hill and Nuth [4] were the first to publish results of FTT experiments that also included Haber-Bosch (HB) processes (hydrogenation of nitrogen. Their findings included the production of nitrile-bearing compounds as well as trace amounts of methyl amine. Previous experience with these reactions revealed that the organic coating deposited on the grains is also an efficient catalyst [5] and that the coating is composed of insoluble organic matter (IOM) and could be reminiscent of the organic matrix found in some meteorites [6]. This current set of FTT-styled experiments tracks the evolution of a set of organics, amino acids, in detail.

Experimental Procedure: The FTT/HB reactions are conducted in a closed gas circulating system that monitors the evolved gases in real time using Fourier transform infrared spectroscopy. The experiment reported here was heated to $550{ }^{\circ} \mathrm{C}$ and used a catalyst of iron-silicate amorphous grains. The gas mixture combines 75 torr of $\mathrm{CO}, 75$ torr of $\mathrm{N}_{2}$ and 550 torr of $\mathrm{H}_{2}$. Individual reactions proceed until approximately $90 \%$ of the $\mathrm{CO}$ is depleted. The reactants are then pumped away, the bulb is refilled with the same gas mixture and another reaction using the same catalyst is carried out. Fifteen such runs were done at the specified temperature and results in coated iron silicate grains approximately $10 \%$ by mass carbon and $0.2 \%$ nitrogen. A complete description of the experiment methodology can be found in previous publications $[4,5]$. The coated catalyst/grain sample was analyzed at different time steps to follow the evolution of the amino acids. The amino acids were analyzed using derivatization and liquid chromatography-fluorescence detection/time-offlight mass spectrometry.

Results: We will present a time sequence for a select set of amino acids and their abundance over the course of the reaction. The data show that the amino acids are produced early in the reaction cycle and then are either destroyed or are incorporated into the IOM on the catalyst/grain surface. We will also report on the generation of a rare, nonbiogenic amino acid.

References: [1] Kress M. E. and Tielens A. G. G. M. 2001. Meteoritics \& Planetary Science 36:75-91. [2] Hayatsu R. and Anders E. 1981. In Cosmo and geochemistry, edited by Bosche. pp. 1-39. [3] Hindermann J. P. et al. 1993. Catalysis Reviews-Science and Engineering 35:1-127. [4] Hill and Nuth J. A. 2003. Astrobiology 3:291-304. [5] Nuth J. A. et al. 2008. The Astrophysical Journal 673:L225-L228. [6] Johnson N. M. et al. 2004. Abstract \#1876. 35th Lunar and Planetary Science Conference.
5475

EXSOLUTION IN FELDSPAR IN THE TUXTUAC (LL5) CHONDRITE: A NEW PERSPECTIVE ON COOLING RATES FOR METAMORPHOSED CHONDRITES

R. H. Jones and A. J. Brearley. Department of Earth and Planetary Sciences, University of New Mexico, NM, USA. E-mail: rjones@unm. edu.

Introduction: Petrologic type 4-6 ordinary chondrites have undergone metamorphism on their parent bodies. There are currently few means to obtain quantitative estimates of peak temperatures and cooling rates for this process. We recently observed possible exsolution of $\mathrm{K}$ feldspar in albitic plagioclase in the LL5 chondrite Tuxtuacs [1], and in L5 Roy (1933) [2]. Since exsolution microstructures can potentially be used as cooling rate indicators, we have studied this feature further using transmission electron microscopy (TEM).

Methods: We obtained quantitative WDS analyses of feldspar using EPMA. A TEM sample was extracted from a feldspar grain in Tuxtuac using focused ion beam (FIB) extraction techniques, and was characterized on a JEOL $2010200 \mathrm{kV}$ TEM.

Results: Exsolution in albitic feldspar occurs in several relict chondrules in a thin section of Tuxtuac [1]. Textures vary from grains that are predominantly albitic with very fine-scale (sub- $\mu \mathrm{m})$ K-rich (sub)parallel lamellae, to regions of grains that contain approximately equal amounts of coarser (up to $10 \mu \mathrm{m}$ ) intergrown albite and $\mathrm{K}$-feldspar. EPMA analyses give compositions of $\mathrm{An}_{9} \mathrm{Ab}_{89} \mathrm{Or}_{2}$ and $\mathrm{An}_{3} \mathrm{Ab}_{18} \mathrm{Or}_{79}$ for the two phases, measured in coarse-grained areas with a $5 \mu \mathrm{m}$ electron beam [1].

We selected a grain with fine-scale (sub- $\mu \mathrm{m}$ ) K-rich lamellae, and cut a FIB section perpendicular to the length of the lamellae. The section shows an exsolution texture of K-feldspar lenses in host albite: albite has albite twinning with individual twin widths of $100-160 \mathrm{~nm}$. The section intersects several $\mathrm{K}$-feldspar lenses, which have maximum widths of $90 \mathrm{~nm}$ up to $\sim 0.5 \mu \mathrm{m}$. K-feldspar lamellae also contain albite twins. In addition, some local regions within the albite have very fine-scale $\mathrm{K}$ feldspar lamellae that are just a few nanometers in thickness and occur on a wavelength of $60-90 \mathrm{~nm}$. The interface between K-feldspar and albite exhibits a zigzag structure and significant strain at the interface. This zigzag interface is caused by the evolution of ordering and development of albite twins in the K-feldspar.

Discussion: Our study shows conclusively that albitic plagioclase in Tuxtuac has an exsolution microstructure. The microstructure is very comparable to that seen in plagioclase of similar compositions in a terrestrial syenite intrusion, that probably cooled through the exsolution interval of around 600 to $500{ }^{\circ} \mathrm{C}$ over a time scale of $10^{4}$ years $[3,4]$. This cooling rate is significantly faster than metallographic cooling rates calculated for LL5 and LL6 chondrites of tens of degrees per million years, or less [5,6], over a similar temperature range. There is no obvious explanation for this large difference in cooling rates. To date, we have not observed exsolution in any type 6 chondrites, which would be predicted to cool more slowly than type 5, according to an onion-shell model.

References: [1] Kovach H. A. and Jones R. H. 2010. Meteoritics \& Planetary Science 45:246-264. [2] Gallegos J. and Jones R. H. 2011. This conference. [3] Brown W. L. and Parsons I. 1988. Contributions to Mineralogy and Petrology 98:444-454. [4] Brown W. L. et al. 1983. Contributions to Mineralogy and Petrology 82:13-25. [5] Taylor G. J. et al. 1987. Icarus 69:1-13. [6] Willis J. and Goldstein J. I. 1981. Proceedings of Lunar and Planetary Science 12B:1135-1143. 


\section{6}

\section{SELENIUM ISOTOPES IN SOME CHONDRITES}

R. Jotter and U. Ott. Max-Planck-Institut für Chemie, Postfach 3060, D-55020 Mainz, Germany. E-mail: uli.ott@mpic.de.

Introduction: Among heavy elements, selenium is exceptional with respect to the extent of isotopic mass fractionation that may occur in nature (e.g., [1]). It has six stable isotopes $\left({ }^{74} \mathrm{Se}\right.$ to $\left.{ }^{82} \mathrm{Se}\right)$, can occur in five difference valence states (-II to $+\mathrm{VI}$ ) and is one of the most redoxsensitive elements. Equilibrium isotope fractionation (in terms of ${ }^{82} \mathrm{Se} /{ }^{76} \mathrm{Se}$ ) at low temperature may range up to several percent [2]. Chemically and in chondritic meteorites Se largely follows the $\sim 2500 \times$ more abundant sulfur [3].

Selenium isotopes in meteorites have mostly been investigated with regard to nucleosynthetic anomalies, e.g., by stepwise dissolution $[4,5]$. However, in this work due internal normalization, any information on mass dependent effects has been lost. To our knowledge, only [6] have reported useful isotopic data without internal normalization, but these are restricted to a few troilite samples from iron meteorites. Their Se isotopic composition turned out to be indistinguishable from that of the NIST SRM 3149 standard $\left( \pm 0.39 \%\right.$ in ${ }^{82} \mathrm{Se} /{ }^{76} \mathrm{Se}$; [7] $)$. We have set out to obtain a wider overview of $\mathrm{Se}$ isotopic composition in meteorites, beginning with bulk chondrites.

Experimental: Typically $\sim 100-200 \mathrm{mg}$ of meteorite are dissolved by $\mathrm{HF} / \mathrm{HNO}_{3}$ digestion. Selenium is separated using a microcolumn containing activated alumina [8] and purified using ascorbic acid as described in [9]. Mass spectrometric measurements are by N-TIMS using multiple collection on a Finnigan MAT 262 for isotopes ${ }^{76} \mathrm{Se}$ to ${ }^{80} \mathrm{Se}$, with the extreme isotopes ${ }^{74} \mathrm{Se}$ and ${ }^{82} \mathrm{Se}$ measured on the central Faraday cup. Mass fractionation $\left({ }^{76} \mathrm{Se} /{ }^{80} \mathrm{Se}\right)$ is determined using a ${ }^{74} \mathrm{Se} /{ }^{82} \mathrm{Se}(\sim 0.27: 1)$ double spike.

First Results: At this time, data have been obtained for the carbonaceous chondrites Allende and Murchison as well as the L3.4 chondrite ALH 81032. Selenium abundances of Murchison and Allende agree well with literature values [3], while the abundance in ALH 81032 is close to the average concentration for $\mathrm{L}$ chondrites [10]. Isotopic data need to be considered preliminary, with an uncertainty in $\delta{ }^{76} \mathrm{Se} /{ }^{80} \mathrm{Se}$ close to $1 \%$. For a precise assessment (including analytical uncertainties) we will have to refine the composition of our double spike and assess the external reproducibility of standard measurements. Relative to the NRM 3149 standard both Allende and ALH 81032 may be somewhat light ( $\delta$ ${ }^{76} \mathrm{Se} /{ }^{80} \mathrm{Se} \sim 2$ and $\sim 1 \%$, resp.), while Se in Murchison is indistinguishable from the reference.

References: [1] Johnson T. M. 2004. Chemical Geology 204:201-214. [2] Li X. and Liu Y. 2011. Earth and Planetary Science Letters 304:113120. [3] Dreibus G. et al. 1995. Meteoritics 30:439-445. [4] Plagge M. et al. 2003. Abstract \#1217. 34th Lunar and Planetary Science Conference. [5] Dauphas N. et al. 2003. Abstract \#1807. 34th Lunar and Planetary Science Conference. [6] Rouxel O. et al. 2002. Geochimica et Cosmochimica Acta 66:3191-3199. [7] Carignan J. and Wen H. 2007. Chemical Geology 242:347-350. [8] Pyrzynska K. et al. 1998. Analytica Chimica Acta 363:141-146. [9] Tanzer D. and Heumann K. G. 1991. Analytical Chemistry 63:1984-1989. [10] Wasson J. T. and Kallemeyn G. W. 1988. Philosophical Transactions of the Royal Society London A $325: 535-544$

\section{0}

PETROGRAPHY OF LUNAR METEORITES DHOFAR 925 AND 961 K. H. Joy ${ }^{1,2,3}$, D. A. Kring ${ }^{1,2}$ and M. E. Zolensky ${ }^{3}$. ${ }^{1}$ CLSE, LPI/USRA, 3600 Bay Area Blvd., Houston, TX 77058, USA. E-mail: joy@, lpi.usra.edu. ${ }^{2}$ NASA Lunar Science Institute. ${ }^{3}$ ARES, NASA Johnson Space Center, Houston, TX 77058, USA.

Introduction: Lunar meteorites Dhofar (Dho) 925 and 961 are brecciated lunar meteorites of intermediate $\mathrm{Fe}$ composition $(7-17 \mathrm{FeO}$ wt\%) [1-3]. They are reputedly paired with each other, and are also grouped with the Dho 960 and Sayh al Uhaymir (SaU) 449 stones [1-3]. As Dho 961 is compositionally similar to regoliths in the South PoleAitkin (SPA) basin, it has been hypothesized that the group may have been launched from SPA on the farside of the Moon [3-5]. As such, they may contain chronological evidence to date the oldest lunar basin, helping to constrain the early impact history of the Moon.

Methods: One thick section from each meteorite was carbon coated and analyzed using the NASA JSC JEOL-7600F field-emission SEM and Cameca SX100 electron microprobe (EMP).

Results: Dho 925 and 961 are petrographically distinct [1, 5], but contain lithics with similar textures and mineral chemistries:

Dho 925: The sample $(\sim 7 \times 6 \mathrm{~mm})$ is composed of a clast-bearing dark-grey impact melt breccia. Clasts range from small $(<10 \mu \mathrm{m})$ mineral and glass fragments up to $2 \mathrm{~mm}$ lithics (equilibrated magnesian anorthositic plutonics (plagioclase typically $>60 \%$ by area, $\mathrm{An}_{94-97}$; mafics typically $<40 \%, \mathrm{Mg} \# 68-80$ [see also 1, 5]); very-low $\mathrm{Ti}$ (VLT) and low-Ti basalts (quenched variolitic and ophitic texture); impact melts and melt breccias; fragmental breccias; and rare Si-K-feldspar assemblages).

Dho 961: The sample $(\sim 22 \times 12 \mathrm{~mm})$ is a fragmental breccia with a notable large $(\sim 4 \times 2 \mathrm{~mm})$ dark-grey, clast-bearing, impact melt breccia clast and a large $(\sim 5 \times 3 \mathrm{~mm})$ cream-coloured ophitic basalt clast (similar to the KREEPy igneous rocks described by [1]). Dho 961 contains a more diverse textural range of clasts than seen in Dho 925. For example, plutonic igneous fragments are typically more mafic (20-60\% mafics), and VLT and low-Ti basalts are texturally diverse (variolitic, subophitic, ophitic textures). A $300 \mu \mathrm{m}$ thick melt vein in Dho 961 has a composition similar to the Dho 025 bulk rock [3].

Impact Melts: Impact melts and clast-bearing melt breccias (of size range $4 \mathrm{~mm}$ to $300 \mu \mathrm{m}$ ) in Dho 925 and 961 were analyzed using a $20 \mu \mathrm{m}$ defocused EMP beam. Most are normative anorthositic gabbro, with compositions similar to the Dho 025 bulk rock [3]. A few clasts are normative anorthositic troctolite and troctolitic anorthosite. All are alkali poor $\left(<0.6 \mathrm{Na}_{2} \mathrm{O}+\mathrm{K}_{2} \mathrm{O}\right.$ wt\%), similar to KREEP-poor feldspathic lunar meteorites, but are comparatively less aluminous: their Al-contents are more akin to Apollo VHA-impact melts (e.g., 20-25 $\mathrm{Al}_{2} \mathrm{O}_{3}$ [6]). We found no clasts in either stone of the olivine-bearing, ITE-rich, mafic impact melt breccia $\left(\mathrm{Al}_{2} \mathrm{O}_{3} \sim 15.4 \mathrm{wt} \%\right)$ reported to be a dominant component of Dho 961 [4-5] and used as supporting evidence for an SPA basin origin [4].

References: [1] Demidova S. I. et al. 2005. Abstract \#1607. 36th Lunar and Planetary Science Conference. [2] Demidova S. I. et al. 2007. Petrology 15:386-407. [3] Korotev R. L. et al. 2010. Abstract \#2126. 41st Lunar and Planetary Science Conference. [4] Jolliff B. A. et al. 2008. Abstract \#2519. 39th Lunar and Planetary Science Conference. [5] Zeigler R. A. et al. 2010. Abstract \#1985. 41st Lunar and Planetary Science Conference. [6] Spudis P. D. and Davis P. A. 1986. Journal of Geophysical Research 91:E84-E90. 
5456

FIRST DESCRIPTIONS OF TWO NEW BOREHOLE PROFILES FROM THE RIES IMPACT CRATER, GERMANY

D. Jung ${ }^{1}$, S. Sturm ${ }^{2}$, G. Arp ${ }^{3}$, J. Pohl ${ }^{4}$ and T. Kenkmann ${ }^{2}$. ${ }^{1}$ Bayerisches Landesamt für Umwelt LfU, ${ }^{2}$ Institut für Geowissenschaften, AlbertLudwigs-Universität Freiburg, Germany. ${ }^{3}$ Universität Göttingen, ${ }^{4}$ LMUMünchen. E-mail: Sebastian.Sturm@geologie.uni-freiburg.de.

Introduction: The Ries impact crater with a diameter of $\sim 25 \mathrm{~km}$, located in southern Germany, is a complex crater structure that is famous for its largely preserved ejecta blanket inside and outside the crater $[1,2]$. The principal outline of the crater interior consists of (i) a central crater basin filled with suevite and lake deposits, (ii) an inner crystalline ring with a diameter of $12 \mathrm{~km}$, that consists of uplifted brecciated basement units, (iii) the megablock zone displaying a hummocky relief and containing parautochthonous and allochthonous megablocks, and (iv) the tectonic crater rim [3,4]. Here we present borehole profiles of two new drilling sites at the Ries impact crater in southern Germany that were drilled by the Geological Survey of Bavaria (LfU). The first drilling location was selected to investigate a post-impact hydrothermal spring site and its basement, which is part of the crystalline ring. The second drilling location was aimed at constraining the occurrence and volume of impact melt rock in the Ries crater.

Drilling Sites: The first drilling site is located south of the city of Nördlingen and north of Reimlingen on the southern crystalline ring. (R4390848/H5411481). The $65 \mathrm{~m}$ deep borehole profile can be subdivided from base to top: (1) 65.0-63.9 m: altered, brecciated gneisses embedded in reddish clay material (bunte breccia?), (2) 63.9-56.2 m: altered $\mathrm{mm}$ to $\mathrm{cm}$-sized gneiss and amphibolite fragments embedded in a grayish, finegrained matrix, calcite veins and melt veins? present, (3) 56.2-36.7: grayish breccia, partly clast supported, with abundant biotite-rich gneiss and amphibolite fragments, (4) 36.7-23.0 m: intact gneisses with foliation dipping consistently $45^{\circ}$, (5) 23.0-22.5: grayish fine-grained matrix including cm-sized granitic clasts, (6) 22.5-0.4 m: Travertin limestone and Ries lake deposits, (7) $0.4-0.0 \mathrm{~m}$ : topsoil.

The second drilling site is located a few meters south of the outcrop Polsingen, in which impact melt rock is exposed (R4405236/H5420826). The $35 \mathrm{~m}$ deep borehole profile is subdivided from base to top: (1) 35.0 34.0: iron-rich, ocher sandstone (Dogger), (2) 34.0-27.5: brecciated limestone (Malmian) (3) 27.5-23.8: grayish marly limestone, quartzite at top, (4) 23.8-20.5 m: iron-rich, ocher sandstone (Dogger), (5) 20.5-5.8 m: heavily brecciated and sand-sized crystalline basement material (especially granitic fragments), (6) $5.8-3.0 \mathrm{~m}: \mathrm{mm}$ to cm-sized crystalline clasts (especially granites) embebbed in a dense reddish to grayish impact melt matrix. (7) $3.0-0.1 \mathrm{~m}$ : strongly brecciated reddish impact melt rock with $\mathrm{mm}$ to cm-size crystalline clasts (8) $0.1-0.0 \mathrm{~m}$ : topsoil.

References: [1] Pohl J. et al. 1977. Impact and explosion cratering, Flagstaff, Arizona. [2] Kenkmann T. and Ivanov B. 2006. Earth and Planetary Science Letters 252:15-29. [3] Stöffler D. et al. 2001. Meteoritics \& Planetary Science 36(9). [4] Stöffler D. 1997. Geologica Bavarica 75:163189.
5415

\section{JOINT BELGIUM-JAPAN SEARCH FOR ANTARCTIC METEORITES IN THE 2010-2011 FIELD SEASON}

H. Kaiden ${ }^{1,2}$, S. Goderis ${ }^{3}$, V. Debaille ${ }^{4}$ H. Kojima ${ }^{1,2}$ and $\mathrm{Ph}$. Claeys ${ }^{3}$. ${ }^{1}$ National Institute of Polar Research, Tachikawa, Tokyo 1908518, Japan. E-mail: kaiden@nipr.ac.jp. ${ }^{2}$ The Graduate University for Advanced Studies, Tachikawa, Tokyo 190-8518, Japan. ${ }^{3}$ Vrije Universiteit Brussel, Pleinlaan 2, B-1050 Brussels, Belgium. ${ }^{4}$ Université Libre de Bruxelles, 50, Av. F.D. Roosevelt, CP 160/02, B-1050 Brussels, Belgium.

Since the 2009-2010 field season in Antarctica, Belgium and Japan have jointly conducted searches for meteorites in the Sør Rondane Mountains (SRM), East Antarctica [1]. A total of 635 meteorite specimens were found in the Mt. Balchen area (approximately $72.0^{\circ} \mathrm{S}$, $27.5^{\circ} \mathrm{E}$ ), which is located at the eastern end of the SRM, in the 20092010 field season [2]. Here we report the search for meteorites hosted by the Belgian Antarctic Research Expedition (BELARE) in the Nansen Ice field (approximately $72.7^{\circ} \mathrm{S}, 24.2^{\circ} \mathrm{E}$ ) that is located on the south of the SRM in the 2010-2011 field season.

The 29th Japanese Antarctic Research Expedition (JARE) conducted systematic searches for meteorites on the Nansen Ice field and found about 1400 meteorite specimens in the 1988-1989 field season [3]. The meteorites were termed Asuka- 88 meteorites, which include rare types such as the Asuka-881371 angritic achondrite and the Asuka-881757 gabbroic mare basalt. A meteorite search team consisting of three Belgians and two Japanese performed systematic meteorite searches in the Nansen Ice Field, where the meteorite search had not been carried out since 1989, from January 1 to 23, 2011, known as the SAMBA (Search for Antarctic Meteorites: Belgian Contribution) mission [4]. A total of 218 meteorites and meteorite fragments were collected during this period. Most of the collected meteorites are ordinary chondrites, but at least two achondrites were recognized in the field. The meteorites were individually put in clean polyethylene bags in the field and kept frozen until they were put in a freezer at the National Institute of Polar Research (NIPR), Japan. The position of each meteorite was recorded by GPS. The initial processing (weighing, measuring, photography, naming and brief description) and classification will be carried out at the NIPR. The meteorites collected by the joint Belgium-Japan team in the 2010-2011 field season will be termed Asuka 10 meteorites. Only a third of the Nansen Ice field was surveyed in 2010/2011, thus JARE and BELARE are planning to cover the rest of the area in 2012/2013.

The meteorite search was performed with the support of the Belgian Science Policy (BELSPO), the International Polar Foundation (IPF) and the Belgian Defense.

References: [1] Kaiden H. et al. 2010. Meteoritics \& Planetary Science 45:A100. [2] Kaiden H. et al. 2010. 33rd Antarctic Meteorites:34 35. [3] Naraoka H. et al. 1990. Antarctic Records 34:216-224. [4] Claeys Ph. et al. 33rd Antarctic Meteorites:5-6. 


\section{6}

MICRO RAMAN SPECTROSCOPY AND CATHODOLUMINESCENCE INVESTGATIONS OF DIAMONDS IN UREILITES

A. Karczemska ${ }^{1}$ and T. Jakubowski ${ }^{2}$. ${ }^{1}$ Technical University of Łódź, Institute of Turbomachinery, Łódź, Poland. ${ }^{2}$ Balonowa 5/2 Wroclaw, Poland.

Introduction: Raman mapping and scanning electron microscopy with cathodoluminescence are the powerful tools for investigations the diversity of carbon in meteorites [1-7]. They allow to show the distribution of diamonds in the samples. Diamonds in ureilites are of different sizes, from nanometers up to micrometers [4]. There are still questions about their origin. New results show wide diversity of diamonds types basis on Raman shifts and on cathodoluminescence spectra investigations.

Samples and Experiments: Study are based on several ureilites (DaG 868, Dhofar 1303, NWA 3140, JaH 054, Sahara 98505, Almahata Sitta). Raman mapping studies have been done with use of WITec alpha RA instrument equipped with an one grating $\left(600 \mathrm{~g} \mathrm{~mm}^{-1}, \mathrm{BLZ}=500 \mathrm{~nm}\right)$ UHTS 300 spectrometer, Newton-CCD camera $(1024 \times 127$ pixels $)$ and Nikon $100 \times(\mathrm{NA}=0.95)$ objective. Frequency doubled NdYAG laser (532 nm line) was used for sample excitation. Scanning electron microscope LEO, type 1430 with microprobe EDS-ISIS 300 and cathodoluminescence device type VIS-View with spectrometer have been used for cathodoluminescence investigations.

Results: Monocrystalline cubic diamond has Raman peak at $1332 \mathrm{~cm}^{-1}$. The Raman spectra in ureilites show the diamond peak shifts from 1297 to $1340 \mathrm{~cm}^{-1}$ and the wide range of FWHM parameter. It is possibly because of the nanometer crystals sizes, or internal stresses in crystals, or because of different polytypes of diamond occurring in the sample. Even unshocked ureilites contain diamonds, it means that probably some of ureilitic diamonds have not shock origin.

References: [1] Karczemska A. 2010. JAMME 45:114-119. [2] Le Guillou C. et al. 2010. Geochimica et Cosmochimica Acta 74:4167-4185. [3] Bischoff A. et al. 1999. Abstract \#1100. Lunar and Planetary Science Conference. [4] Grund T. and Bischoff A. 1999. Abstract \#5074. 62nd Annual Meteoritical Society Meeting. [5] Karczemska A. et al. 2009. JAMME 37/2:292-297. [6] Jakubowski T. et al. 2009. Abstract \#1382. 40th Lunar and Planetary Science Conference. [7] Karczemska A. Jakubowski T., and Kozanecki M. 2009. Abstract \#5382. 72nd Annual Meteoritical Society Meeting.
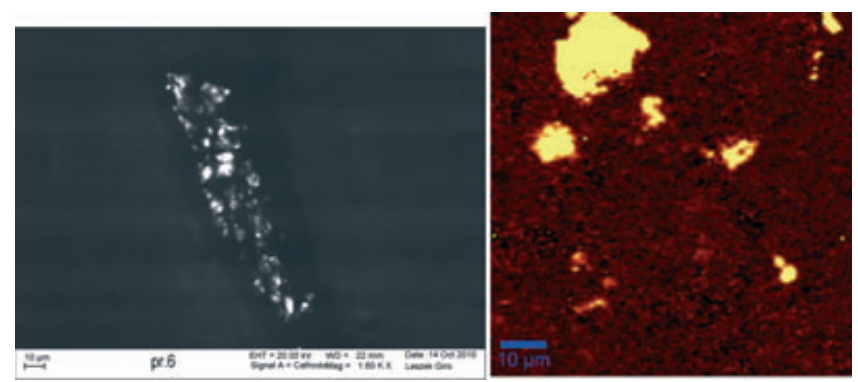

Fig.1. CL image of DaG 868 and Raman mapping of $\mathrm{JaH} \mathrm{054,}$ ureilite diamonds are bright spots.
5336

A NEW METEORITE FALL AT SOTMANY, POLAND

Ł. Karwowski ${ }^{1}$, A. S. Pilski ${ }^{2}$, T. A. Przylibski ${ }^{3}$, J. Gattacceca ${ }^{4}$, P. Rochette $^{4}$, K. Luszczek $^{3}$, R. Kryza ${ }^{5}$, B. Woźniak ${ }^{6}$, M. Woźniak ${ }^{6}$. ${ }^{1}$ University of Silesia, Faculty of Earth Sciences, ul. Będzińska 60, 41-200 Sosnowiec, Poland. ${ }^{2}$ Nicolaus Copernicus Museum, ul. Katedralna 8, 14-530 Frombork, Poland. ${ }^{3}$ Wrocław University of Technology, Faculty of Geoengineering, Mining and Geology, Wybrzeze S. Wyspiańskiego 27, 50-370 Wrocław, Poland. ${ }^{4}$ CEREGE, Aix-Marseille Université, Aix-en-Provence, France. ${ }^{5}$ Wrocław University, Institute of Geological Sciences, ul. Cybulskiego 30, 50-205 Wrocław, Poland. ${ }^{6}$ University of Warsaw, Department of Biology, ul. Miecznikowa 1, 02-096 Warsaw, Poland.

Introduction: On April 30, 2011, at 6:03 CEST, in a farm at the village of Sołtmany, NE Poland, a stone penetrated the edge of a roof and crushed onto a concrete step below. It was immediately found by the owners of the farm, alarmed by loud noise. Mr. Roman Rzepka from the nearby town of Giżycko, recognized the stone as meteoritic, and informed the authors. On May 2, fragments of the stone were purchased and distributed among laboratories for examination. Here are some preliminary results.

Samples and Macroscopic Examination: The meteorite was broken by impact into pieces: $813 \mathrm{~g}, 155 \mathrm{~g}$ and many smaller ones. TKW is at least $1066 \mathrm{~g}$. Nearly all examined pieces have the fusion crust of about $1-1.5 \mathrm{~mm}$ thick. The crust is of the same thickness all around the meteorite, which suggests that the stone was a single fall. The hole in the roof and the trace of impact onto the step below proved that the fall was nearly vertical. Macroscopic examination showed that the stone was extremely fresh (Wo), nearly white in color, with clearly visible troilite and FeNi grains, up to $2-3 \mathrm{~mm}$ in size, dispersed uniformly through entire meteorite. Only few chondrules were distinguished macroscopically.

Mineralogy and Petrology: The mean compositions of olivine $\left(\mathrm{Fa}_{25.5}\right)$ and low-Ca pyroxene $\left(\mathrm{Fs}_{22}\right)$ indicate that the meteorite can be classified as an L chondrite [1]. This is confirmed by magnetic susceptibility $\log \chi=4.71 \pm 0.04$, typical of the lower range of $\mathrm{L}$ chondrites [2]. The texture examined both macroscopically and in thin section, the homogeneous compositions of olivines and pyroxenes, and the large grain-size of plagioclase, all indicate type 6 chondrite. There is no difference in composition of olivines and pyroxenes in chondrules and out of them. Low-Ca pyroxene is usually accompanied by $\mathrm{Ca}$ pyroxene (Wo: $\sim 45 \%$; Fs: $\sim 46.5 \%$ ). In larger grains of feldspar (An: $\sim 10 \%$; Or: $\sim 5 \%$ ), albitic twinning is visible. Sparse chondrules are well crystallized; no glass is visible. $\mathrm{F}-\mathrm{Cl}$ apatite is an accessory. Opaque minerals are represented by troilite, kamacite, taenite and rare chromite. Taenite shows an unusual texture, with areas resembling plessite. In taenite grains, tiny inclusions of metallic copper are visible. The undulatory extinction in olivine and pyroxene indicates that the chondrite is weakly shocked (S2). This is confirmed by low anisotropy of magnetic susceptibility [3].

Acknowledgments: The authors are grateful to the finder of the meteorite, Mrs. Alfreda Lewandowska.

References: [1] Weisberg M. K. et al. 2006. In Meteorites and the early solar system II pp. 19-52. [2] Rochette P. et al. 2003. Meteoritics \& Planetary Science 38:251-268. [3] Gattacceca J. et al. 2005. Earth and Planetary Science Letters 234:351-368. 
5263

IMPACT DISRUPTION OF IRON METEORITE PARENT BODIES UNDER WARM CONDITIONS

T. Katsura ${ }^{1}$, A. M. Nakamura ${ }^{1}$, S. Hasegawa ${ }^{2}$, A. Takabe ${ }^{1}$, M. Setoh ${ }^{1}$, and K. Sangen ${ }^{1} .{ }^{1}$ Earth and Planetary Science, Kobe University, 1-1 Rokkodai-cho, Nada-ku, Kobe, 657-8501 Japan. E-mail: amnakamu@kobe-u.ac.jp. ${ }^{2}$ ISAS/JAXA, 3-1-1 Yoshinodai, Chuo-ku, Sagamihara, Kanagawa 252-5210, Japan.

Introduction: Between the differentiation of the parent bodies of iron meteorites and the last collision events that thrust the population of iron meteorites into interplanetary space, the parent bodies, iron cores, and fragments must have experienced collisional events over a range of mass scales and impact velocities. The parent bodies of iron meteorites may have formed in the terrestrial planet region. Afterward, they or their fragments were scattered into the main belt [1]. As the ductile-brittle transition temperature of an iron meteorite on impact is around $200 \mathrm{~K}$ [2], the impact strength of iron samples [3] at room temperature is of interest.

Impact Experiments: We performed impact experiments on steel and iron meteorites at room temperature in two different velocity regimes. High-velocity impact experiments (velocities of $1.4-4.8 \mathrm{~km} \mathrm{~s}^{-1}$ ) were performed using a two-stage light-gas gun at the Institute of Space and Astronautical Science (ISAS). The projectiles were disks of steel and Mundrabilla and Campo del Cielo iron meteorites a few millimeters in size. The targets were serpentinite and dunite blocks. Low-velocity impact experiments (velocities up to $1 \mathrm{~km} \mathrm{~s}^{-1}$ ) were performed using a singlestage vertical powder gun at Kobe University. Copper cylinders $15 \mathrm{~mm}$ in diameter and $15 \mathrm{~mm}$ in height were used as projectiles on steel and Campo del Cielo cylinders as targets. After each impact experiment, we looked for fragments of steel and iron meteorites using a magnet. We measured the mass of the recovered fragments of iron material after impact.

Results: After the high-velocity impacts, all iron projectiles were thinned and deformed. Some projectiles were disrupted into several fragments with torn surfaces. The surface on the impact side lost metallic luster and was rough. The mass fraction of the largest fragments ranged from 0.07 to 0.99 of the originally projectile mass. After the low-velocity impacts, the mass fraction of the largest iron fragment ranged from 0.11 to 1.0. The iron targets were disrupted by a large crack penetrating parallel to the impact direction. Given larger-input kinetic-energy densities, radial disruption occurred. We estimated the initial peak pressure using a planer impact approximation. We found that the initial peak pressure required to disrupt catastrophically the smaller (millimeter size) specimens of iron materials was two to three times larger than the peak pressure required for the larger (centimeter size) specimens.

Summary: Impact disruption experiments of small (millimeter) and larger (centimeter) specimens of iron material indicated that the impact strength of iron meteorite parent bodies depends on the scale of the collision, even at temperatures above the ductile-brittle transition temperature.

References: [1] Bottke W. F., Nesvorný D., Grimm R. E., Morbidelli A. and O'Brien D. P. 2006. Nature 439:821-824. [2] Remo J. L. and Johnson A. A. 1975. Journal of Geophysical Research 80:37443748. [3] Ryan E. V. and Davis D. R. 1994. DPS Meeting \#26, \#31.10, BAAS, vol. 26, p. 1180 .

\section{5}

CATHODOLUMINESCENCE CHARACTERIZATION OF HIGHPRESSURE SILICA AND FELDSPAR MINERALS

M. Kayama ${ }^{1}$, H. Nishido ${ }^{1}$, T. Sekine ${ }^{2}$ and K. Ninagawa ${ }^{3} .{ }^{1}$ Research Institute of Natural Sciences, Okayama University of Science, Japan. E-mail: kayama@rins.ous.ac.jp. ${ }^{2}$ Department of Earth and Planetary Systems Science, Graduate School of Science, Hiroshima University, Japan. ${ }^{3}$ Department of Applied Physics, Okayama University of Science, Japan.

Introduction: High-pressure minerals in various meteorites have been investigated in planetary sciences for a clarification of shock metamorphic processes. Although recent studies apply cathodoluminescence (CL) of stishovite and high-pressure glass to identify such high-pressure silica minerals, CL mechanisms of high-pressure silica minerals have not been clarified in detail. Furthermore, high-pressure feldspar minerals such as $\mathrm{KAlSi}_{3} \mathrm{O}_{8}$-hollandite and maskelynite have not been studied from the perspective of CL spectroscopy. In this study, natural and synthetic highpressure silica and feldspar minerals have been characterized by $\mathrm{CL}$ spectroscopy to clarify their emission mechanisms.

Samples and Methods: Synthesized stishovite, coesite, $\mathrm{KAlSi}_{3} \mathrm{O}_{8}-$ hollandite, and experimentally shock-induced sanidine and quartz at pressure of $40 \mathrm{GPa}$ by a propellant gun were selected for CL measurements. A scanning electron microscopy-cathodoluminescence (SEM-CL) was used to obtain CL spectra of these samples.

Results and Discussion: CL spectrum of synthetic stishovite has a pronounced emission band at $\sim 390 \mathrm{~nm}$. CL intensity of the blue emission decreases with an increase in electron irradiation time. Similar blue emission was observed in CL spectra of silica minerals in Martian meteorites of NWA 2975 and Shergotty. On the other hand, synthetic coesite exhibits dull UV emission peaked below $300 \mathrm{~nm}$ and blue emission at $\sim 380 \mathrm{~nm}$. CL spectrum of experimentally shocked quartz at $40 \mathrm{GPa}$ shows blue emissions at $460 \mathrm{~nm}$, which have not been recognized in CL of natural quartz up to now. CL spectral patterns and features of these high-pressure silica minerals are quite different from other silica minerals such as quartz, tridymite and cristobalite.

$\mathrm{CL}$ spectrum of $\mathrm{KAlSi}_{3} \mathrm{O}_{8}$-hollandite consists of emission bands at 330 and $380 \mathrm{~nm}$ in UV-blue region. Similar UV-blue emission bands were detected in experimentally shocked sanidine at $40 \mathrm{GPa}$. Alkali feldspar glasses in NWA 2975 and Shergotty also exhibit UV-blue emissions at 330 and $380 \mathrm{~nm}$, which might be assigned to defect centers in $\mathrm{Al}$ and $\mathrm{Si}$ octahedra produced under high pressure process. $\mathrm{KAlSi}_{3} \mathrm{O}_{8}$-hollandite has higher emission intensities than shocked samples at $40 \mathrm{GPa}$ and the glasses in shergottite. It might be responsible for completely octahedral coordination of $\mathrm{Si}$ and $\mathrm{Al}$ in $\mathrm{KAlSi}_{3} \mathrm{O}_{8}$-hollandite and partially one in diaplectic glass. CL spectrum of $\mathrm{KAlSi}_{3} \mathrm{O}_{8}$-hollandite also shows emissions at 550, 580, 660, 710 and $730 \mathrm{~nm}$, which were undetectable in shocked sanidine at $40 \mathrm{GPa}$ and the glasses in shergottite. These emission bands are characteristics of CL signals derived from $\mathrm{KAlSi}_{3} \mathrm{O}_{8}$-hollandite.

These results indicate that $\mathrm{CL}$ signals can be applicable to identify high-pressure silica and feldspar minerals and to observe their distributions in meteorites with high spatial resolution $(\sim 1 \mu \mathrm{m})$. CL and infrared-CL (from 800 to $1800 \mathrm{~nm}$ ) spectroscopy at room and liquid nitrogen temperature for these synthetic and experimentally shocked samples will be discussed here for further clarification of their CL mechanism. 
5380

COMETARY DUST RESIDUE IN LARGE STARDUST FOIL CRATERS: HOW MUCH SURVIVES, AND HOW TO SAFELY EXTRACT IT FOR ANALYSIS

A. T. Kearsley ${ }^{1}$, M. J. Burchell ${ }^{2}$, M. C Price ${ }^{2}$, M. J. Cole ${ }^{2}$, P. J. Wozniakiewicz ${ }^{3}$, H. A. Ishii ${ }^{3}$, N. Teslich ${ }^{3}$, J. P. Bradley ${ }^{3}$ and T. Salge $^{4} .{ }^{1}$ IARC, Department of Mineralogy, Natural History Museum, London SW7 5BD, UK. E-mail: antk@nhm.ac.uk. ${ }^{2}$ School of Physical Sciences, University of Kent, Canterbury, Kent CT2 7NH, UK. ${ }^{3}$ IGPP, Lawrence Livermore National Laboratory, 7000 East Avenue, Livermore, CA 94550, USA. ${ }^{4}$ Bruker Nano GmbH, Schwarzschildstrasse 12, 12489 Berlin, Germany.

Introduction: Craters on Stardust foils show impacts by Wild 2 dust of a wide size range [1]. Sections from focussed ion beam (FIB) instruments reveal composition and structural preservation of dust in smaller craters [2]. There has been reluctance to attempt such preparations for larger craters (up to $>200 \mu \mathrm{m}$ ), as their size and shape make widespread surface contamination by re-deposition of FIB-ablated material probable, and the extraction angle is too steep for an in-chamber micromanipulator. Before any preparation, it is important to know where residue is present. Auger mapping has been applied very successfully [3] but is slow to document large areas. Although energy-dispersive X-ray (EDX) mapping can find residue on walls and lips of craters [4], occasionally ideal targets for NanoSIMS [5], deeper parts are not seen by typical inclined EDX detectors. Here we explain methods to locate residue across the whole crater surface, quantify the amount, and change crater shapes for safe extraction of material.

Methods: Light gas gun shots were performed at Canterbury, using powders of lead sulfide, Bjurbole and Allende meteorites, and poly methylmethacrylate (PMMA), fired onto $100 \mu \mathrm{m}$ thick All100 foil at approximately $6 \mathrm{~km} \mathrm{~s}^{-1}$. A Zeiss EVO $15 \mathrm{LS}$ scanning electron microscope (SEM) was used for stereo imagery of $\mathrm{PbS}$ craters, to measure $\mathrm{C} / \mathrm{Al}$ count ratios in PMMA craters for comparison to carbon thin-film calibration, and to map Bjurbole impact residue. Silicon drift detector (SDD) take-off angle was increased by long sample working distance, allowing examination of over $97 \%$ of the crater area, but this was relatively slow (16 h). An annular four-channel Bruker XFlash 5060F SDD, placed beneath the pole-piece of a Zeiss Supra 55 field emission SEM, gave hyperspectral X-ray maps of a whole Allende crater in $<45 \mathrm{~min}$. Digital elevation models (DEM) were created for the $\mathrm{PbS}$ craters, prior to and after deformation with a carefully controlled, shaped, steel needle.

Results and Conclusions: The mapped area and estimated thickness of Bjurbole residue suggest that approximately $55 \%$ of the impacting particle was preserved, but measurement of carbon film thickness revealed that $<3 \%$ of each PMMA particle survived. The DEM showed that craters on Stardust foil can be made much broader and shallower, bringing all residue to locations for safe extraction by proven methods. It is now possible to locate, measure and extract residues from larger Stardust craters. This will release another important repository of cometary dust from Wild 2.

References: [1] Price M. C. et al. 2010. Meteoritics \& Planetary Science 45:1409-1428. [2] Leroux H. et al. 2008. Meteoritics \& Planetary Science 43:143-160. [3] Stadermann F. J. et al. 2009. Abstract \#2120. 40th Lunar and Planetary Science Conference. [4] Kearsley A. T. et al. 2008. Meteoritics \& Planetary Science 43:41-73. [5] Stadermann F. J. et al 2008. Meteoritics \& Planetary Science 43:299-313.
5280

METEORITE POLISHED SECTIONS: X-RAY MAP IMAGERY FOR DOCUMENTATION, CURATION AND 'VIRTUAL-LOAN' OF IRREPLACEABLE MATERIALS

A. T. Kearsley ${ }^{1}$, C. L. Smith ${ }^{1}$, J. Spratt ${ }^{1}$, G. K. Benedix ${ }^{1}$, A. Hunt ${ }^{1}$, S. S. Russell $^{1}$, K. H. Joy ${ }^{1,2,3}$ and M. Gounelle ${ }^{4}{ }^{1}$ IARC, Department of Mineralogy, Natural History Museum, London, SW7 5BD, UK. E-mail: antk@nhm.ac.uk. ${ }^{2}$ CLSE, LPI-USRA, 3600 Bay Area. Blvd. Houston, Tx-77058, USA. ${ }^{3}$ NASA Lunar Science Institute. ${ }^{4}$ LMCM, CNRS \& MNHN, UMR 7202, CP52, 57 rue Cuvier, 75005 Paris, France.

Introduction: Polished meteorite surfaces are primary research materials and each may reveal unique textural relationships between phases of diagnostic composition and equilibration level. Many meteorites are represented by only a low total mass. They are challenging materials to prepare, with soft or reactive components that may alter in prolonged contact with water and air. Informed choice of abrasive compounds for lapping and polishing is important to avoid later confusion in searches for interesting grains (such as $\mathrm{SiC}, \mathrm{Al}_{2} \mathrm{O}_{3}$ and diamond). Each section is therefore a substantial investment in precious sample and preparation time. As the significance of features may only be realized much later, with development of new analytical techniques, careful curation, storage and informed access is essential to use these resources responsibly. Fortunately, digital storage of diverse analytical data from a sample may now aid this process.

Compositional Imagery: Analytical scanning electron microscopy (SEM) is widely applied in meteorite research. Many textures are revealed by backscattered electron images (BEI), shown in almost all petrographic publications. Unfortunately, similarity of BE coefficients between diverse compositions often prevents distinction by gray tones in these images. Maps of element distribution may overcome this limitation, yielding such images as the three-color combined X-ray maps used to document calcium- and aluminum-rich inclusions so effectively [e.g., 1]. However, mapping by wavelength dispersive X-ray spectrometers [e.g., 2] requires long collection time, limiting the area examined in an acceptable length of instrument use. Recent improvements in energy dispersive (EDX) collection efficiency (especially large area silicon drift detectors (SDD), faster pulse processing, classifying many tens of thousands of X-rays per second), combined with sophisticated methods of automated beam and stage movement, now allow collection of a full EDX spectrum from each micrometer-scale pixel across areas of multiple square centimeters in instrument sessions of a few hours [e.g., 3]. A sample holder can be loaded at the end of a day of instrument use, map areas defined, and the instrument left to acquire data overnight, automatically saving results which are downloaded to a separate server the next morning, for processing and extraction of selected element X-ray maps. An entire collection of meteorite sections can be documented in this way, with copies of compositional image files then available as a 'virtual-loan,' allowing on-line selection of appropriate samples for research, without the need to transport precious samples whose suitability for a specific investigation would otherwise be unknown in advance.

References: [1] Krot A. N. and Keil K. 2002. Meteoritics \& Planetary Science 37:91-111. [2] Ivanova M. A. et al. 2008. Meteoritics \& Planetary Science 43:915-940. [3] Bland P. A. et al. 2007. Meteoritics \& Planetary Science 42:1417-1427. 


\section{9}

ORGANIC ANALYSIS OF XENOLITHIC CLASTS IN METEORITES

Y. Kebukawa ${ }^{1 *}$, M. E. Zolensky ${ }^{2}$, M. D. Fries ${ }^{3}$, A. Steele ${ }^{1}$, A. L. D. Kilcoyne $^{4}$ and G. D. Cody ${ }^{1}$. ${ }^{1}$ Geophysical Laboratory, Carnegie Institution of Washington, Washington, DC, USA. ${ }^{2}$ NASA Johnson Space Center, Houston, TX, USA. ${ }^{3}$ Planetary Science Institute, San Diego, CA, USA. ${ }^{4}$ Advanced Light Source, Lawrence Berkeley National Laboratory, Berkeley, CA, USA. *E-mail: ykebukawa@ciw.edu.

Introduction: Primitive xenolithic clasts have been found in regolithbearing meteorites [1]. They are most commonly similar to type 1-3 chondrites, but significant differences usually exist. Some of these clasts should derive from Kuiper Belt Objects (KBOs) trapped in the inner asteroid belt by scattering by giant planets in later period of planetary formation, in the so-called Nice model $[2,3]$. Here we present organic analyses of several of these clasts by various spectroscopic methods, e.g., carbon X-ray absorption near edge structure (C-XANES), Fourier transform infrared micro-spectroscopy (microFTIR), and Raman spectroscopy.

Results: We have analyzed clasts from four meteorites; Kapoeta (Howardite), Zag (H5), Sharps (H3.4) and Cold Bokkeveld (CM2). CXANES and FTIR spectra show mostly aromatic nature with slightly aliphatics. The carbonyl features obtained by C-XANES might have been caused by focused ion beam (FIB) used in sample preparation. C-XANES spectra of all clasts did not show significant $1 \mathrm{~s}-\sigma^{*}$ exiton features, suggesting that these clasts have not experienced high temperature, probably $<200{ }^{\circ} \mathrm{C}$ [4], although some of their parent meteorites has subjected strong thermal processing. However, the Raman G-band center and full width at half maximum (FWHM) of the Zag clast show similarities to thermally metamorphosed CVs.

Most interestingly, the clasts from Sharps are significantly organic carbon rich and contain less silicates and carbonates, compare to the clasts from other three meteorites. G-band parameters obtained by Raman spectra of two distinct Sharps clasts both show similarity with CV and $\mathrm{CO}$ chondrites. However, these would be two different starting materials that have been metamorphosed to the type 3.4 petrographic class together. One clast contains what are probably vapor-grown graphite grains, some of which have been disrupted by shock. The other clast appears to have had a $\mathrm{O}, \mathrm{N}$-rich starting material. Both show signs of slight thermal metamorphismalmost certainly while entrained in the Sharps H3.4 parent body. These Raman spectral features are consistent with FTIR spectra which show less aliphatics and carbonyls.

Conclusions: These primitive xenolithic clasts have organic features that are distinct from their parent meteorites and preserve their history from before accretion on single parent bodies. However, they may also have been affected by secondary process on their parent bodies, which results in very complex histories..

References: [1] Zolensky M. Z. et al. 2009. Abstract \#2162. 40th Lunar and Planetary Science Conference. [2] Tsiganis K. et al. 2005. Nature 435:459-461. [3] Morbidelli A. et al. 2005. Nature 435:462-465. [4] Cody G. D. et al. 2008. Earth and Planetary Science Letters 272:446-455.

\section{5}

IRRADIATION OF FeS: RELATIVE SPUTTERING RATES OF TROILITE AND Mg SILICATES

L. P. Keller ${ }^{1}$ and Z. Rahman ${ }^{2}$. ${ }^{1}$ Robert M. Walker Laboratory for Space Science, ARES, NASA/JSC, Houston, TX 77058, USA. E-mail: Lindsay.P.Keller@nasa.gov. ${ }^{2}$ ESCG/Jacobs Technology, TX 77058, USA.

Introduction: Astronomical measurements of $\mathrm{S}$ abundances in the diffuse interstellar medium (ISM) indicate ionized $\mathrm{S}$ is the dominant species with little $(<5 \%) \mathrm{S}$ residing in grains [e.g., 1]. This is an enigmatic result, given that abundant $\mathrm{Fe}$-sulfide grains are observed in dust around pre- and post-main sequence stars [2,3] and are major components of primitive meteoritic and cometary samples. These disparate observations suggest that the lifetime of Fe-sulfides in the ISM is short because of destruction processes. Our previous work has shown that FeS retains its crystallinity and does not amorphize during radiation processing, whereas enstatite and forsterite are readily amorphized [4,5]. Here, we have extended this study to measure the relative sputtering rates of $\mathrm{FeS}$ compared to silicates.

Experimental: We used a FEI Quanta 600 3D dual-beam focused ion beam (FIB) instrument to sputter sharp grain boundaries between troilite and enstatite or forsterite in chondrules within polished sections of the Vigarano $\mathrm{CV} 3$ chondrite. A $7 \times 10 \mu \mathrm{m}$ rastered region extending across the grain boundary was sputtered at normal incidence using either $30 \mathrm{kV}, 7 \mathrm{nA} \mathrm{Ga}^{+}$for $1 \mathrm{~min}$, or $5 \mathrm{kV}, 1 \mathrm{nA}, \mathrm{Ga}^{+}$for $7 \mathrm{~min}$. For the $30 \mathrm{kV}$ experiments, we measured the depth of the sputtered pit directly in secondary electron images. For the $5 \mathrm{kV}$ experiments, we extracted a FIB lift-out section from two of the pits and measured the pit depth and microstructure in the transmission electron microscope (TEM).

Results and Discussion: The $30 \mathrm{kV}$ experiments produced pits in troilite that were $\sim 4 \times$ deeper than the adjacent pits in enstatite or forsterite. More precise measurements were obtained from TEM examination of the $5 \mathrm{kV}$ experiment FIB cross sections. The FeS/enstatite pit depth ratios for the two FIB sections were 4.2 and 3.9. The depth ratio variation is likely related to crystallographic orientation differences. Irradiation of $\mathrm{FeS}$ with $5 \mathrm{kV} \mathrm{Ga}^{+}$resulted in preferential sputtering of $\mathrm{S}$ and the formation of a 5-8 nm thick surface layer of nanophase Fe metal. $\mathrm{X}$-ray mapping shows that the zone of $\mathrm{S}$ sputtering extends to a depth of nearly $20 \mathrm{~nm}$, but there is no evidence for FeS amorphization, consistent with our previous study [4].

The dominant grain destruction mechanism in the ISM is sputtering from passage of supernova-generated shock waves [6]. Our results indicate that FeS grains produced in evolved stars and injected into the ISM will be destroyed more rapidly than crystalline silicates. Despite their expected shorter interstellar lifetime, circumstellar Fe-sulfides should be preserved in meteoritic materials, but are as yet, undetected. Floss et al. [7] measured an abundance of presolar silicates in isotopically primitive interplanetary dust particles (IDPs) of $810 \mathrm{ppm}$. Given the $\mathrm{S} / \mathrm{Si}$ ratio of $\sim 0.5$ in the diffuse ISM [1] and a destruction rate $4 \times$ faster than silicates, we estimate an abundance of presolar sulfides of $\sim 100 \mathrm{ppm}$ in isotopically primitive IDPs.

References: [1] Jenkins E. B. 2009. The Astrophysical Journal 700:1299. [2] Keller L. P. et al. 2002. Nature 417:148. [3] Hony S. et al. 2002. Astronomy \& Astrophysics 393:L103. [4] Keller L. P. et al. 2010. Abstract \#1172. 41st Lunar and Planetary Science Conference. [5] Christoffersen R. et al. 2011. Meteoritics \& Planetary Science, in press. [6] Jones A. P. and Nuth J. A. 2011. Astronomy \& Astrophysics 530:A44. [7] Floss, C. et al. 2006. Geochimica et Cosmochimica Acta 70:2371. 


\section{8}

\section{A FIB-TEM STUDY OF Ca- AND Fe-RICH PYROXENES IN THE MATRICES OF OXIDIZED $R$ AND CV3 CARBONACEOUS CHONDRITES}

L. P. Keller ${ }^{1}$ and Z. Rahman ${ }^{2}$. ${ }^{1}$ Robert M. Walker Laboratory for Space Science, ARES, NASA/JSC, Houston, TX 77058, USA. ${ }^{2}$ ESCG/ Jacobs Technology, TX 77058, USA. E-mail: Lindsay.P.Keller@nasa.gov.

Introduction: $\mathrm{Ca}-\mathrm{Fe}-$ rich pyroxenes are ubiquitous components in the matrices of the oxidized subgroup of the CV3 chondrites and are inferred to have formed during low grade, fluid-assisted metamorphism on their parent bodies [1,2]. Oxygen isotopic measurements of individual hedenbergite (Hd)-rich inclusions in Allende matrix are isotopically heavy, consistent with interaction with a ${ }^{16} \mathrm{O}$-depleted reservoir [2]. The precursors of the Hd-bearing assemblages are unknown, although [1] has suggested the precursors were similar to the anhydrous matrix materials present in the reduced $\mathrm{CV} 3$ chondrites. We are using electron microscopy to investigate the nm-scale mineralogy and petrography of the $\mathrm{Hd}$-rich inclusions in the matrix of a type 3 clast in the PRE $95404 \mathrm{R}$ chondrite and in ALH 85006 CV3 chondrite matrix.

Experimental: X-ray mapping and SEM imaging show that the Hdrich inclusions are typically $10-50 \mu \mathrm{m}$ rounded to amoeboid objects with a central void. We prepared a cross section of one of these objects from PRE 95404 using a FEI Quanta 600 3D dual-beam focused ion beam (FIB) instrument. Transmission electron microscopy (TEM) and quantitative $\mathrm{x}$-ray mapping data were obtained from the FIB section using the JEOL 2500SE field-emission STEM equipped with a thin window, energy-dispersive x-ray (EDX) spectrometer.

Results and Discussion: The Hd-rich inclusion is highly porous and consists of $\mu \mathrm{m}$-sized grains of Ca-rich pyroxene with minor pentlandite. EDX analyses show that the pyroxenes are either Hd100 or Di90Hd10 that share sharp compositional boundaries at the sub- $\mu \mathrm{m}$ scale. The crystal size coarsens toward the central void and many of the crystals show euhedral terminations into the void. The Hd grains typically contain nanophase inclusions of pentlandite, while the Di-rich grains only contain numerous sub- $\mu \mathrm{m}$ voids throughout their volume. X-ray mapping reveals that the Di-rich domains are elongate and lath-shaped with high aspect ratios. Some of the Hd domains are also lath-shaped, but most are larger, more rounded and equant.

The abundant voids within the Di-rich domains in these inclusions are consistent with volatile $\left(\mathrm{H}_{2} \mathrm{O}\right)$ loss during their formation from a $\mathrm{Mg}$ rich precursor, probably phyllosilicates. The Hd precursor may also have been phyllosilicates or more likely Fe-sulfides. In the latter case, the pentlandite inclusions in $\mathrm{Hd}$ are remnants of originally low-Ni Fe sulfides. The Ca source is less clear. Krot et al. [1] suggested that Ca was lost from chondrules and $\mathrm{Ca}$ - and Al-rich inclusions and re-precipitated in matrix during metasomatic alteration. Alternatively, $\mathrm{CaCO}_{3}$ may have been part of the precursor assemblage in which case some of the porosity and void space in the inclusions resulted from decarbonation reactions.

The precursors to the Hd-rich inclusions in the oxidized CV3 and R chondrites were hydrated phases formed at low temperatures and subsequently metamorphosed on the parent body.

References: [1] Krot A. N. et al. 1998. Meteoritics \& Planetary Science 33:623. [2] Cosarinsky M. et al. 2003. Abstract \#1043. 31st Lunar and Planetary Science Conference.

\section{5}

COLLAPSE OF A TRANSIENT CRATER CAVITY: FIRST RESULTS FROM ANALOG EXPERIMENTS

T. Kenkmann and P. Burgert. Institute of Geosciences-Geology, AlbertLudwigs-University Freiburg, Germany. E-mail: thomas.kenkmann@ geologie.uni-freiburg.de.

Introduction: Except for very small impact craters, the shape of almost all craters is modified by gravity driven mass movements. In simple craters the shape of the transient cavity that forms at the end of the excavation stage [1] is moderately modified, in complex craters the mass movements are substantial. To understand the kinematics of crater wall collapse in simple and complex craters we conducted analogue experiments with a variety of materials (sand, sand + flour mixtures, glass beads) and recorded the particle displacements by means of particle image strainometry (PIS) [2].

Experiments: The experimental setup consisted of a $50 \mathrm{~cm}$ box filled with the analogue material. A paraboloidal cavity mimicking a transient crater was created either with a replica that was removed after filling of analogue material was completed or by a partly buried balloon. The gravitational instability of the cavity was induced by a piston installed beneath the cavity moving downward at constant velocity [2]. In case of the balloon experiments, the cavity walls instantaneously collapsed when the balloon was punctured. Two cameras, installed at high angles to the target surface, recorded the collapse of the cavity. Pulsed LED flashes illuminated the setup. We used the Strain Master 3D software package by La Vision to record changes in the position of material points. PIS provides an accurate measure of the instantaneous displacement field of laboratory flow and is adapted for strain monitoring in analogue sandbox experiments [3]. Scaling of material cohesion and density between experiment and nature resulted in a scaling factor of approximately $10^{4}$, thus the $10 \mathrm{~cm}$ cavities formed in the experiments roughly correspond to $1 \mathrm{~km}$ cavities in nature.

Results: In the first set of experiments (using the replica), numerous slumps developed at the cavity rim and reached the cavity center. A slump often triggered the onset of subsequent adjacent flows. Slumps created lobate headscarps and superimposed onto each other at the bottom of the cavity, thereby filling the cavity. Additionally a system of circumferential tensile fractures formed outside the cavity. These concentric fissures allowed for slow creeping of the entire mass inside the concentric fissures into the cavity. Their occurrence resembles the concentric fissures around the $108 \mathrm{~m}$ Snowball crater [4].

The second set of experiments (exploding balloon, sand + flour) led to the simultaneous initiation of cohesive slumps along the steep rim. While moving inward these slumps subsequently disintegrated into granular flows due to oblique and frontal collision with other flows. The central portion of the crater floor is characterized by a rather turbulent and chaotic flow that resulted in a flattish morphology.

References: [1] Grieve R. A. F. 1991. Meteoritics 26:175-194. [2] Kenkmann T. and Burgert P. 2011. Abstract \#1511. 42nd Lunar and Planetary Science Conference. [3] Adam J. et al. 2005. Journal of Structural Geology 27:283-301. [4] Jones G. H. S. 1977. In: Roddy D. J., ed. Impact and Explosion cratering. pp. 163-183. 
5468

\section{A TWO-STAGE APPROACH FOR PREDICTING COMPOSITION FROM MICROSCOPIC IMAGES: EXAMPLES FROM THE MARS EXPLORATION ROVERS SPIRIT AND OPPORTUNITY}

K. Kiatpanichagij ${ }^{1}$, J. G. Spray ${ }^{1}$ and T. Trabucchi ${ }^{1}$. ${ }^{1}$ Planetary and Space Science Centre, University of New Brunswick, Fredericton, NB E3B 5A3, Canada. E-mail: kirkkiat@unb.ca.

Introduction: Rovers employed in planetary exploration missions are typically equipped with multiple measurement and observational instruments, e.g., an alpha particle X-ray spectrometer (APXS) for determining the chemical composition of target sites, and cameras for capturing target images at the geologist's hand-lens scale. Measurement via an APXS is a time-consuming process (the complete analysis of one sample has typically required at least three hours during MER operations). Conversely, cameras can capture images at much faster rates. Since there is a relationship between the area of chemical analysis and a complementary (coincident) image, a triage system can be designed that facilitates the use of images to limit the need to perform compositional analysis. Here we describe how such a system has been constructed and tested.

Procedure: The system consists of three building blocks: (1) an image segmentation block, (2) the first artificial neural network (ANN1), and (3) the second artificial neural network (ANN2). The image segmentation block groups similar pixels into meaningful regions. ANN1 mimics the ability of a geologist to classify the image regions into predefined rock or surface types, e.g., blueberries, basalts, dark soils, bright dusts. Finally, ANN2 predicts the chemical composition of the captured image from the areas of these surface types. ANN1 thus performs a classification task, and ANN2 performs a prediction task.

Results: Initially, our primary triage system employed pixel-based segmentation in the first building block. A low classification accuracy of the ANN1 was observed. However, with a low accuracy ANN1, the prediction error of ANN2, which is considered a system error, was bounded and the predicted values of chemical compositions were fairly close to the target values. To improve the system accuracy, a state-of-theart multi-resolution segmentation approach was deployed in the first building block, derived from commercially available eCognition software (a technique usually used for analyzing remotely sensed images and medical images). It was in-house implemented and used in the developed triage system. Additionally, to comply with the increasing use of multicore processors, a parallel version of the algorithm was developed and tested.

Conclusions: Approximately 30 compositional analyses and matching images are required to facilitate image-only compositional estimations given a common family of materials (e.g., blueberries set in sedimentary host). Ultimately, the goal is to enable the rover to perform autonomous decisions in real time. Currently, due to limitations in processor performance, the aim is to assist mission operations geologists in analyzing images of target sites, for which chemical compositions are not available.
5283

FINE-GRAINED REFRACTORY INCLUSIONS FROM THE REDUCED CV3 CHONDRITE THIEL MOUNTAINS 07007

H. Kim and B.-G. Choi. Earth Science Education, Seoul National University, Seoul, 151-748, Korea; E-mail: bchoi@snu.ac.kr.

The Thiel Mountains (TIL) 07007 was found by the 2nd Korea Expedition for Antarctic Meteorites; it has been classified as CV3 chondrite based on the bulk oxygen isotopic composition $\left(\Delta{ }^{17} \mathrm{O}=-4.35 \%\right)$ and petrological characteristics $[1,2]$.

A thin section of the meteorite (SNU-T068) having the area of $\sim 266 \mathrm{~mm}^{2}$ was studied for Petrological characteristics with optical microscope, scanning electron microscope and electron microprobe analyzer. Oxygen isotopic compositions of individual mineral phases are being measured using the NanoSIMS 50 at Korea Basic Science Institute and the data will be presented at the meeting.

TIL 07007 consists of relatively large chondrules, fine-grained refractory inclusions, amoeboid olivine inclusions and fine-grained matrix. Chondrules are mostly metal- and sulfide-rich type I porphyritic olivine or porphyritic olivine pyroxene chondrules: type II chondrules are relatively rare. Average area occupied by a single chondrule in the thin section is $\sim 0.75 \mathrm{~mm}^{2}$, which is somewhat larger than that of Allende we measured $\left(\sim 0.50 \mathrm{~mm}^{2}\right)$. Possibly due to impact compaction, chondrules and inclusions are all elongated to the same direction. Numerous micro-faults also probably due to impact exist in chondrules, inclusions and matrix. Average major and minor axes of chondrules are 1.13 and $0.67 \mathrm{~mm}$, respectively, giving the average eccentricity of $\sim 0.75$ (aspect ratio of 1.7). TIL 07007 has no magnetite, its sulfide grains are nearly Ni-free and modal matrix/chondrule ratio is 0.50 ; these characteristics are similar with the other reduced CV3 chondrites, especially with Efremovka and Leoville $[3,4]$.

Nineteen refractory inclusions larger than $80 \mu \mathrm{m}$ have been found in the thin section. Two of them are completely or partially surrounded by amoeboid olivine inclusions. Similar to chondrules, the inclusions are also elongated: one extreme is $2040 \times 140 \mu \mathrm{m}$. All of them are fine-grained: no coarse-grained $\mathrm{Ca}$-Al-rich inclusions of igneous texture are found. The mineral assemblage and texture of the fine-grained refractory inclusions are very similar to those in Leoville and Efremovka [4]. Mineralogy varies but typically is spinel, pyroxene (Al-diopside and Al-Ti diopside), anorthite and melilite as major minerals. Perovskite is also found in most of them. Three inclusions have minor or trace amount of forsterite. Hibonite is absent or rare. Some inclusions have a few $\mathrm{Fe}-\mathrm{Ni}$ metal and sulfide grains. Secondary minerals are absent in most inclusions but found in two inclusions. Some inclusions show a zoned structure in mineralogy.

Seven inclusions are relatively spinel-rich and very similar to the fined-grained spinel-rich inclusions in Leoville and Efremovka [4]: two of the spinel-rich inclusions are melilite-free. Another seven inclusions are melilite-rich $\left(\mathrm{Ak}_{0-20}\right)$ : melilite occupies most area of the inclusions and has fine-grained irregular shaped spinel \pm hibonite \pm perovskite and anorthite \pm diopside aggregates in it. The other three inclusions are rich in Al-diopside.

References: [1] Weisberg M. K. et al. 2010. Meteoritics \& Planetary Science 45:1530-1551. [2] Choi B.-G. et al. 2009. Journal of the Geological Society of Korea 45:593-605. [3] Krot A. N. et al. 1995. Meteoritics \& Planetary Science 30:748-775. [4] Krot A. N. et al. 2004. Meteoritics \& Planetary Science 39:1517-1553. 
5037

\section{OPAQUE MINERALS IN CH CHONDRITES: INDICATORS OF FORMATION CONDITIONS}

M. Kimura ${ }^{1}$, T. Karube ${ }^{1}$, M. K. Weisberg ${ }^{2,3}$, T. Mikouchi ${ }^{4}$ and T. Noguchi ${ }^{1}$. ${ }^{1}$ Ibaraki University, Japan. E-mail: makotoki@ mx.ibaraki.ac.jp. ${ }^{2}$ Kingsborough College of the City University of New York, USA. ${ }^{3}$ American Museum of Natural History, USA. ${ }^{4}$ University of Tokyo, Japan.

Introduction: $\mathrm{CH}$ chondrites are characterized by remarkable features, such as $\mathrm{Fe}-\mathrm{Ni}$ metal with solar $\mathrm{Co} / \mathrm{Ni}$ ratio, small chondrule size distribution, and refractory inclusions with kushiroite and grossite [1,2]. Here we report our analytical results on opaque minerals in two $\mathrm{CH}$ chondrites, SaU 290 and Asuka 881020, in order to explore the formation conditions of $\mathrm{CH}$ chondrites. The opaque minerals are good indicators of formation conditions, especially the redox states.

Petrology and Mineralogy: We found Si-free and -enriched $(<13$ $\mathrm{wt} \%$ ) $\mathrm{Fe}-\mathrm{Ni}$ metals, suessite, perryite, $\mathrm{FeSi}$ (fersilicite), barringerite, andreyivanovite, troilite, daubreelite, pyrrhotite, pentlandite, and magnetite in two $\mathrm{CH}$ chondrites. Some troilite contains $\mathrm{Cr}(<3 \mathrm{wt} \%)$. These minerals generally occur as isolated grains, among tiny chondrules, $80 \mu \mathrm{m}$ in average diameter, and isolated silicates. Most of these minerals have not been previously found in $\mathrm{CH}$ chondrites, and we are using EBSD for phase indentification. Some of these opaques occur as intimate intergrowths; perryite, barringerite, and daubreelite are present in close association with Si-rich Fe-Ni metal, whereas pentlandite, pyrrhotite, and magnetite occur only in dark clasts.

Discussion: Some of the opaque minerals in $\mathrm{CH}$ chondrites, such as Si-bearing $\mathrm{Fe}-\mathrm{Ni}$ metal, perryite, Cr-bearing troilite, and daubreelite, are typical in $\mathrm{E}$ chondrites. However, some Fe-Ni metals contain higher $\mathrm{Si}$ concentrations than those in any $\mathrm{E}$ chondrite. Some typical minerals in $\mathrm{E}$ chondrites, such as niningerite and oldhamite, are not found in $\mathrm{CH}$ chondrites. On the other hand, the occurrences of Si-rich Fe-Ni metal and suessite are similar to some mineral assemblages in ureilites. These assemblages formed under more reducing conditions than opaque minerals in the E chondrites [3]. Magnetite, pentlandite and pyrrhotite, in close association with hydrous silicates, in dark clasts, formed under highly oxidizing conditions. Si-free $\mathrm{Fe}-\mathrm{Ni}$ metal and $\mathrm{Cr}$-free troilite are commonly encountered in ordinary chondrites and others.

We conclude that $\mathrm{CH}$ chondrites are highly unequilibrated mixtures of components that originated under highly reducing to highly oxidizing conditions. It is unlikely that these components formed from a single reservoir. This may be consistent with the highly heterogeneous oxygen isotopic compositions of $\mathrm{CH}$ chondrules which suggests that not all of the chondrules formed from the same reservoir [4].

References: [1] Weisberg M. K. et al. 1988. Earth and Planetary Science Letters 91:19-32. [2] Kimura M. et al. 2009. American Mineralogist 94:1479-1482. [3] Ikeda Y. 2007. Polar Science 1:45-53. [4] Nakashima D. et al. 2010. Meteoritics \& Planetary Science 45:A148.
5389

AGREEMENT OF EXPERIMENT AND THEORY IN HOMOGENEOUS NUCLEATION

Y. Kimura ${ }^{1 *}$, K. K. Tanaka ${ }^{2}$, H. Miura ${ }^{1}$ and K. Tsukamoto ${ }^{1}{ }^{1}$ Tohoku University, Tohoku, Japan. E-mail: ykimura@m.tains.tohoku.ac.jp. ${ }^{2}$ Hokkaido University, Hokkaido, Japan.

Introduction: Classical nucleation theory has been widely used to describe dust formation in ejecta gas from evolved stars, supernovae and possibly in a plume after energetic shock in primitive solar nebula, whereas the classical theory always has large gaps with experiments. For example, homogeneous nucleation rates of droplets were measured as a function of temperature and supersaturation using ethanol and nonane [7, 8]. They calculated temperature and supersaturation ratio in the chamber from the initial temperature and pressure, and homogeneous nucleation rate was determined after the drops have grown to visible size to count the number after taken by camera. The nucleation rates were different in several orders of magnitude from the classical nucleation theory. Recently, it has been shown that semiphenomenological nucleation theory, which was proposed by Dillmann and Meier (1991) [3], can explain the result of molecular dynamic simulation of nucleation at relatively low temperature for liquid and solid $[4,5]$. We made a new chamber with interferometer to measure the homogeneous nucleation environment and compared actual number of formed particles with that calculated by semiphenomenological nucleation theory.

Experiments: The newly constructed smoke chamber can produce nanometer sized particles via homogeneous nucleation after vaporization of a starting material. Mach-Zehnder type interferometer is also prepared together with the chamber and it can detect the changing of temperature and gas density as a difference of an optical path length. The interferometer is able to detect a small difference of refractive index as small as $10^{-6}$. Using the noncontact method, homogeneous condensation temperature and supersaturation of smoke particles are directly determined. The size and number of produced particles were observed by transmission electron microscope and compared with the calculated values.

Results: Preliminary experiment using manganese demonstrated that semiphenomenological nucleation model shows good agreement with the experiment for the nucleation temperature, size and number of formed particle, when we give initial temperature, pressure and time scale of supersaturation increase for nucleation from the experiment. Accordingly, we conclude the semi-phenomenological nucleation theory is useful for describe nucleation events even at such relatively higher temperature environments. Sticking coefficient and the surface free energy at the temperature of super cooling, i.e., lower than equilibrium temperature, can be obtained from the experiment and calculation. Those values are applicable to dust formation.

References: [1] Schmitt J. L., Adams G. W. and Zalabsky R. A. 1982. The Journal of Chemical Physics 77:2089-2097. [2] Adams G. W. Schmitt J. L. and Zalabsky R. A. 1984. The Journal of Chemical Physics 81:5074-5078. [3] Dillmann A. and Meier G. E. A. 1991. The Journal of Chemical Physics 94:3872. [4] Tanaka K. K. et al. 2005 The Journal of Chemical Physics 122:184514. [5] Tanaka K. K., Tanaka H., Yamamoto T., and Kawamura K. 2011. The Journal of Chemical Physics 134, in press. 
5499

DETERMINING TRACE ELEMENT ABUNDANCES IN SINGLE PRESOLAR SiC GRAINS

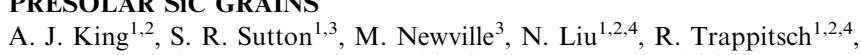
P. R. Heck ${ }^{2,5}$ A. M. Davis ${ }^{1,2,5,6}$ M. J. Pellin ${ }^{1,2,4,6}$ and T. Stephan ${ }^{1,2,4,5}$. ${ }^{1}$ Department of the Geophysical Sciences. ${ }^{2}$ Chicago Center for Cosmochemistry, University of Chicago, Chicago, IL, USA. E-mail: ajking@uchicago.edu. ${ }^{3}$ Consortium for Advanced Radiation Sources, Univ. of Chicago, Chicago, IL, USA. ${ }^{4}$ Materials Science Division, Argonne National Laboratory, Argonne, IL, USA. ${ }^{5}$ Robert A. Pritzker Center for Meteoritics and Polar Studies, The Field Museum, Chicago, IL, USA. ${ }^{6}$ Enrico Fermi Institute, University of Chicago, Chicago, IL, USA.

Motivation: Presolar $\mathrm{SiC}$ grains have been well characterized according to their isotopic compositions, providing details of the nucleosynthetic processes that take place in carbon-rich stars and supernovae. In addition, the abundances of trace elements in presolar $\mathrm{SiC}$ grains are also likely to be directly related to both nucleosynthesis and the condensation environment of the parent stars. Most studies of presolar $\mathrm{SiC}$ grains have been performed using secondary ion mass spectrometry (SIMS). However, this technique is destructive and suffers from matrix effects and a lack of suitable $\mathrm{SiC}$ standards, such that there have been few studies combining isotopic compositions and trace element abundances within individual presolar $\mathrm{SiC}$ grains [1].

Synchrotron X-ray fluorescence (SXRF), for which matrix effects are well understood and easily corrected for, has been successfully used to detect a wide range of trace elements in presolar $\mathrm{SiC}$ grains [2, 3]. Also, as SXRF is nondestructive, the same grains can then be investigated with other analytical techniques. However, in the earlier SXRF studies, the isotopic compositions of the grains were never measured due to technical difficulties in the design of the sample mount. We have now begun a combined trace element and isotopic study of individual presolar $\mathrm{SiC}$ grains.

Experimental: Presolar $\mathrm{SiC}$ grains from the KJG size fraction (1.5$3 \mu \mathrm{m}$ ) [4] will be deposited upon CVD diamond films. Previously, Kapton and $\mathrm{SiN}$ have been used as the mounting substrate but these were found to be fragile, made locating grains difficult, and in the latter case, prevented the use of $\mathrm{Si}$ as the reference element $[2,3]$. The diamond films will initially be examined using a scanning electron microscope (SEM) in order to identify and map the $\mathrm{SiC}$ grains, with the same grains relocated at the Advanced Photon Source (APS) using a reflected light microscope. SXRF analyses will then be made using the GeoSoilEnviroCARS beamline 13-IDE. This beam-line can provide a spot size of $\sim 2 \mu \mathrm{m}$, suitable for measurements of individual $\mathrm{SiC}$ grains. A beam energy of $\sim 22.5 \mathrm{keV}$ should be sufficient for fluorescence of many elements known to be present at ppm levels. Spectra will be calibrated using two NIST reference materials and background corrections made by measuring the substrate.

Summary: We will report trace element abundance data for $\sim 15$ presolar $\mathrm{SiC}$ grains. The sample mount is designed so that the isotopic compositions of the same grains can then be analyzed using SIMS and newly developed instruments such as CHILI [5].

References: [1] Amari S. et al. 1995. Meteoritics 30:679-693. [2] Kashiv Y. et al. 2010. The Astrophysical Journal 713:212-219. [3] Knight K. et al. 2008. Abstract \#2135. 39th Lunar and Planetary Science Conference. [4] Amari S. et al. 1994. Geochimica et Cosmochimica Acta 58:459-470. [5] Stephan T. et al. 2011. Meteoritics \& Planetary Science, this volume.

\section{4}

HIGH PRECISION OXYGEN ISOTOPE SYSTEMATICS OF A TYPE B1 CAI FROM LEOVILLE (CV3)

N. T. Kita ${ }^{1}$, T. Ushikubo ${ }^{1}$, K. B. Knight ${ }^{2,3}$, R. A. Mendybaev ${ }^{2}$, A. M. Davis $^{2}$, F. M. Richter ${ }^{2}$, D. Nakashima ${ }^{1}$, M. J. Spicuzza ${ }^{1}$ and J. W. Valley ${ }^{1}$. ${ }^{1}$ WiscSIMS, University of Wisconsin, Madison, WI 53706, USA. E-mail: noriko@geology.wisc.edu. ${ }^{2}$ University of Chicago, Chicago, IL 60637, USA. ${ }^{3}$ Lawrence Livermore National Laboratory, Livermore, CA 94550, USA.

Introduction: Oxygen three-isotope ratios of $\mathrm{Ca}$-, Al-rich inclusions (CAIs) often show a large range of heterogeneity among different mineral phases in a single inclusion [e.g., 1, 2], which may reflect the complex history of CAIs in both the solar nebula and the parent asteroids. Here, we report new highly precise and accurate Secondary Ion Mass Spectrometer (SIMS) analyses of a type B1 CAI from Leoville (3535-1), which was previously studied for $\mathrm{Mg}$ and $\mathrm{Si}$ isotope fractionation and ${ }^{26} \mathrm{Al}-{ }^{26} \mathrm{Mg}$ chronology [3-5]. This is a large pristine CAI $(6 \times 8 \mathrm{~mm})$ with a 0.5 -mm-thick melilite mantle. Secondary minerals, such as nepheline, are absent in this CAI.

SIMS Analysis: An IMS-1280 at WiscSIMS was used for analysis, which yields analytical uncertainties of a single $15 \mu \mathrm{m}$ spot of $0.3-0.4 \%$ (2SD) for $\delta^{18} \mathrm{O}$ and $\delta^{17} \mathrm{O}[6]$. To obtain accurate $\delta^{18} \mathrm{O}$ and $\delta^{17} \mathrm{O}$ values from minerals in the CAI, we used natural and synthetic mineral standards for spinel, melilite and anorthite. For fassaite, synthetic glass standards with major element compositions similar to fassaite in the type B CAIs were synthesized. Homogeneity of oxygen isotope ratios and SIMS matrix correction factors were carefully evaluated for individual standards.

Results: Oxygen isotope ratios of spinel, fassaite and anorthite plot near the lower end of the CCAM line [1] with $\delta^{18} \mathrm{O}$ values of $-44 \%$ to $-40 \%$. The $\Delta^{17} \mathrm{O}$ values of spinel, fassaite and anorthite are $-23.6 \%$, from $-23.8 \%$ to $-21.5 \%$ and $-22.9 \%$, respectively. The highest $\delta^{18} \mathrm{O}$ and $\Delta^{17} \mathrm{O}$ values were obtained from fassaite within the melilite mantle. These data plot along the slope 1.0 line in $\delta^{18} \mathrm{O}$ vs. $\delta^{17} \mathrm{O}$ diagram, but are displaced to the right side of CCAM line. Melilite data plot at $\delta^{18} \mathrm{O}$ and $\delta^{17} \mathrm{O}$ of $\sim+11 \%$ and $\sim+4 \%$, respectively, correponding to a $\Delta^{17} \mathrm{O}$ value of $-1.5 \%$. Melilite data are also significantly higher in $\delta^{18} \mathrm{O}$ relative to the CCAM.

Discussion: Slightly elevated $\Delta^{17} \mathrm{O}$ values of anorthite and some fassaite compared to spinel could be caused by limited oxygen isotopic exchange with solar nebular gas when the CAI was reheated within 0.1 $\mathrm{Ma}$ of the earliest recorded events of the solar system [5]. Oxygen isotope ratios of melilite are not in equilibrium with other minerals and show high $\delta^{18} \mathrm{O}$ values, suggesting isotopic exchange at low temperature, possibly with parent body fluid or with nebular gas. However, simple diffusive isotopic exchange between CAI minerals and nebula gas or parent body fluid may not explain ${ }^{16} \mathrm{O}$-poor melilite in the entire CAI, while preserving the ${ }^{16} \mathrm{O}$-rich signature in anorthite $[2,7]$.

References: [1] Clayton R. N. et al. 1977. Earth and Planetary Science Letters 34:209-224. [2] Fagan T. J. et al. 2004. Meteoritics \& Planetary Science 39:1257-1272. [3] Richter F. M. et al. 2007. Abstract \#2303. 38th Lunar and Planetary Science Conference. [4] Knight K. B. et al. 2009. Abstract \#2360. 40th Lunar and Planetary Science Conference. [5] Kita N. T. et al. 2010. Abstract \#2154. 41st Lunar and Planetary Science Conference. [6] Kita N. T. et al. 2010. Geochimica et Cosmochimica Acta 74:6610-6635. [7] Nagashima K. et al. 2010. Abstract \#2255. 41th Lunar and Planetary Science Conference. 


\section{1}

\section{A PRELIMINARY MICRO-SPECTROSCOPIC ANALYSIS OF THE CARBONACEOUS MATTER IN THE PARTICLES RECOVERED BY THE HAYABUSA MISSION}

F. Kitajima ${ }^{1}$, M. Kotsugi ${ }^{2}$, T. Ohkochi ${ }^{2}$, H. Naraoka ${ }^{1}$, Y. Ishibashi ${ }^{3}$, M. $\mathrm{Abe}^{3}$, A. Fujimura ${ }^{3}$, R. Okazaki ${ }^{1}$, T. Yada ${ }^{3}$, T. Nakamura ${ }^{4}$, T. Noguchi ${ }^{5}$,

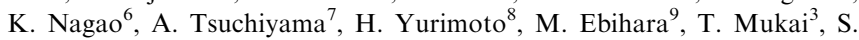

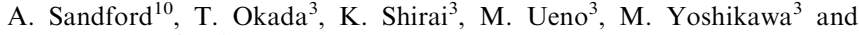
J. Kawaguchi ${ }^{3}$. ${ }^{1}$ Department of Earth \& Planet Sciences, Kyushu University, E-mail: kitajima@geo.kyushu-u.ac.jp. ${ }^{2}$ SPring-8/JASRI ${ }^{3}$ ISAS/JAXA ${ }^{4}$ Department of Earth Planet \& Material Sciences, Tohoku University. ${ }^{5}$ Coll. Sci., Ibaraki University. ${ }^{6}$ Geochem. Res. Center, Univ. Tokyo. ${ }^{7}$ Department of Earth Space Sciences, Osaka University. ${ }^{8}$ Natl Hist. Sci., Hokkaido University. ${ }^{9}$ Grad. School Sci. Engineer. Tokyo Metropol. University. ${ }^{10}$ NASA/ARC.

Introduction: Insoluble organic matter (IOM) is the major fraction of the chondritic carbonaceous matter [1]. Its disordered structure converts to ordered during thermal metamorphism, and it suggests to what extent thermal metamorphism has proceeded. [2]. Several spectroscopic techniques such as Raman [3, 4], infrared (IR) [5], solidstate ${ }^{13} \mathrm{C}$ NMR and carbon X-ray absorption near edge structure (C-XANES) [4] are useful tools to evaluate the maturity level of IOM. The asteroid 25143 Itokawa belongs to S-type and its surface has been considered to have an olivine-rich mineral assemblage similar to that of LL5 or LL6 chondrites [6]. However, it is a rubble-pile object [7], suggesting possible carbonaceous fragments. Such fragments can be a clue to the thermal process involved in the formation history of Itokawa.

Samples and methods: Five particles (RA-QD02-0017, 0033, 0044, 0049, and 0064) were analyzed by non-destructive methods; micro-Raman and IR techniques. Because we hope to analyze the particles as intact as possible without using organic resin, newly developed a sample holder made from diamond [8]. Raman spectra were obtained using an excitation wavelength of $514.5 \mathrm{~nm}$ on an argon ion laser. The laser beam was focused by a microscope equipped with a $50 \times$ objective leading to a spot diameter of 2 micrometer. Mid-infrared measurements were performed using an aperture of which size was 50 micrometer $\times 50$ micrometer.

Results and Discussion: All Raman spectra from the five particles show principal bands at around 821 and $851 \mathrm{~cm}^{-1}$. These bands can be assigned to olivine, and it seems the major mineral. A few Raman spectra occasionally show Raman bands from the other minerals; pyroxene from the particle 0033, for example. However, all the particles show neither D- nor G-bands, which can be usually observed from chondritic IOM. All IR spectra show $\mathrm{Si}-\mathrm{O}$ absorption at around $1000 \mathrm{~cm}^{-1}$, and a broad absorption from about 2200 to $1650 \mathrm{~cm}^{-1}$, which can also be assigned to olivine. However, the spectra lack in absorptions relating to carbonaceous matter. Although the particles may contain trace amount of low molecular weight organic compounds, there is no evidence for chondritic IOM.

References: [1] Sephton M. A. 2002. Natural Product Reports 19:292-311. [2] Kitajima F. et al. 2002. Geochimica et Cosmochimica Acta 66:163-172. [3] Sandford S. A. et al. 2006. Science 314:1720-1724. [4] Cody G. D. et al. 2008. Earth and Planetary Science Letters 272:446-455. [5] Kebukawa Y. et al. 2010. Meteoritics \& Planetary Science 45:99-113. [6] Abe M. et al. 2006. Science 312:1334-1338. [7] Fujiwara A. et al. 2006. Science 312:1330-1334. [8] Kitajima F. et al. 2011. Abstract \#1855. 42nd Lunar and Planetary Science Conference.

\section{0}

LIMITS FOR ASTEROID $2008 \mathrm{TC}_{3}$ SIZE AND MASS BASED ON DENSITIES OF ALMAHATA SITTA METEORITES

R. Kiuru ${ }^{1,2}$, T. Kohout ${ }^{1,3}$, M. Montonen ${ }^{1}$, D. Britt ${ }^{4}$, R. Macke ${ }^{4}$, P. Scheirich $^{5}$ and G. Consolmagno ${ }^{6}$. ${ }^{1}$ Department of Physics, University of Helsinki, Helsinki, Finland. E-mail: risto.kiuru@helsinki.fi. ${ }^{2}$ Geological Survey of Finland, Espoo, Finland. ${ }^{3}$ Institute of Geology, Academy of Sciences of the Czech Republic, Prague, Czech Republic. ${ }^{4}$ Department of Physics, University of Central Florida, Orlando, USA. ${ }^{5}$ Astronomical Institute, Academy of Sciences of the Czech Republic, Ondřejov, Czech Republic. ${ }^{6}$ Vatican Observatory, Vatican City State.

Introduction: On October 6, 2008 a small asteroid designated $2008 \mathrm{TC}_{3}$ was discovered in space $20 \mathrm{~h}$ prior to impact on Earth [1]. So far over 600 fragments of the corresponding Almahata Sitta meteorites have been recovered at impact site in Nubian Desert, Sudan. We characterized these meteorites through measurement of their bulk and grain densities and porosities [2]. Most Almahata Sitta meteorite fragments were classified as ureilites [1], but recently a significant amount of various chondritic compositions has been found [3-6].

Density of the Almahata Sitta Meteorite Fragments: Our measurements on the Almahata Sitta ureilites reveal bulk densities between $2.9-3.3 \mathrm{~g} \mathrm{~cm}^{-3}$, the average being $3.1 \mathrm{~g} \mathrm{~cm}^{-3}$. These seem to be generally higher than those reported by [1], but closer to previous data from other ureilites by [7] and [8].

Implications on Size and Mass of Asteroid 2008 TC $_{3}$ : Based on the densities of Almahata Sitta ureilites as the dominant lithological fraction of the asteroid, we can say that its bulk density should not exceed that of the meteorites, $3.1 \mathrm{~g} \mathrm{~cm}^{-3}$ (this includes the microporosity of the meteorite fragments). This value used together with albedo estimates of $0.09-0.2$ by [9] and shape model by [10] was used to estimate the asteroid's mass. Since the asteroid broke down during atmospheric passage, we consider presence of significant macroporosity likely, and this will lower the mass estimate. For macroporosities of $20-50 \%$ we get mass estimates between 4 and $23 \mathrm{t}$. This is lower than those reported by [1] and $[11]$.

Acknowledgments: We would like to thank the Grant Agency of the Czech Academy of Sciences of the Czech Republic (grant no. KJB300130903), Väisälän Foundation and University of Helsinki for research and travel funding, students and staff of University of Khartoum for their hospitality, help and support, and Peter Jenniskens and Muawia Shaddad for providing us with access to the Almahata Sitta meteorite material.

References: [1] Jenniskens P. et al. 2009. Nature 458:485-488. [2] Kohout T. et al. 2011. Icarus 212:697-700. [3] Bischoff A. et al. 2010. Meteoritics \& Planetary Science 45:1638-1656. [4] Kohout T. et al. 2010. Meteoritics \& Planetary Science 45:1778-1788. [5] Shaddad M. et al. 2010. Meteoritics \& Planetary Science 45:1557-1589. [6] Zolensky et al. 2010. Meteoritics \& Planetary Science 45:1618-1637. [7] Consolmagno G. et al. 2008. Chemie der Erde 68:1-29. [8] Macke R. et al. 2011. Meteoritics \& Planetary Science 46:311-326. [9] Hiroi T. et al. 2010. Abstract \#1148. 41st Lunar and Planetary Science Conrefence. [10] Scheirich P. et al. 2010. Meteoritics \& Planetary Science 45:1804-1811. [11] Borovička J. et al. 2009. Astronomy \& Astrophysics 507:1015-1022. 
5255

THE MG ISOTOPE COMPOSITION OF PRESOLAR SILICATE GRAINS FROM AGB STARS: CONSTRAINTS ON THE Mg ISOTOPE INVENTORY OF THE LOCAL INTERSTELLAR MEDIUM AT SOLAR BIRTH

J. Kodolányi and P. Hoppe. Max Planck Institute for Chemistry, 55128 Mainz, Germany. E-mail: j.kodolanyi@mpic.de.

Introduction: The ${ }^{25} \mathrm{Mg} /{ }^{24} \mathrm{Mg}$ and ${ }^{26} \mathrm{Mg} /{ }^{24} \mathrm{Mg}$ ratios of the Sun (and the solar system) are at the lower end of ratios observed for stars with solar metallicity $\left(Z_{\text {solar }}\right)$ and are substantially lower than those predicted by galactic chemical evolution (GCE) models for stars with $Z_{\text {solar }}$ in the solar neighbourhood [1,2]. An evaluation of the $\mathrm{Mg}$ isotope composition of the material from which the solar system formed could help understand this discrepancy. As more than half of the $\mathrm{Mg}$ in the interstellar medium (ISM) is hosted by oxide and silicate dust produced by evolved stars [3], the Mg isotope inventory of the local ISM at solar birth could be constrained partially by the $\mathrm{Mg}$ isotope composition of presolar silicate and oxide grains, which represent interstellar dust at the time of solar system formation. Magnesium isotope data on presolar silicate grains $[4,5]$ reveal that silicate grains from asymptotic giant branch (AGB) stars, the most prolific sources of presolar silicate grains [e.g., 6], have ${ }^{25,26} \mathrm{Mg} /{ }^{24} \mathrm{Mg}$ close to or slightly higher than solar $[4,5]$. Here we provide additional $\mathrm{Mg}$ isotope data for presolar silicate grains from AGB stars.

Experimental: Presolar silicate grains were identified in the matrix of Acfer 094 (an ungrouped carbonaceous chondrite), based on their $\mathrm{O}$ isotope compositions and $\mathrm{Si}-\mathrm{Al}-\mathrm{O}$ systematics. These were determined by the NanoSIMS, as were the grains' $\mathrm{Mg}$ isotope compositions (for analytical conditions see [4]).

Results and Discussion: Based on their O isotope composition (all grains belong to Group 1 of [7]), the identified 26 presolar silicate grains were produced in the winds of AGB stars with initial masses of 1.15-2.2 $\mathrm{M}_{\text {solar }}$ and $\mathrm{Z} \sim \mathrm{Z}_{\text {solar }}$ [8]. The five grains analyzed successfully so far for $\mathrm{Mg}$ isotopes have $\delta^{25} \mathrm{Mg}$ between $-10( \pm 17 ; 1 \sigma$ uncertainty $)$ and 64 $( \pm 26) \%$ and $\delta^{26} \mathrm{Mg}$ between $-22( \pm 41)$ and $46( \pm 25) \%$, i.e., roughly comparable to the solar composition, but, on average, slightly higher than that. These compositions are similar to the composition of presolar oxide grains from low-mass AGB stars [e.g., 9 without grains with significant contribution to ${ }^{26} \mathrm{Mg}$ from ${ }^{26} \mathrm{Al}$ decay]. Our data probably reflect GCE, since stellar evolution models do not predict significant increase in ${ }^{25,26} \mathrm{Mg} /{ }^{24} \mathrm{Mg}$ in the envelopes of low-mass AGB stars with $\sim Z_{\text {solar }}$, when compared to initial ratios [e.g., 10]. Furthermore, the measured $\delta^{25,26} \mathrm{Mg}$ values plot along a slope $\sim 1$ line (within error) in a $\delta^{25} \mathrm{Mg}$ vs. $\delta^{26} \mathrm{Mg}$ representation, which suggests only a small contribution (if any) to ${ }^{26} \mathrm{Mg}$ from the decay of ${ }^{26} \mathrm{Al}$. Thus the silicate component of the local ISM probably had a slightly heavier $\mathrm{Mg}$ isotope composition on average than our Sun, at the time of solar system formation.

References: [1] Yong D. et al. 2003. The Astrophysical Journal 599:1357-1371. [2] Fenner Y. et al. 2003. Publications of the Astronomical Society of Australia 20:340-344. [3] Fitzpatrick E. L. 1997. The Astrophysical Journal 482:L199-L202. [4] Kodolányi J. and Hoppe P. 2011. Proceeding Science (NIC XI) 142. [5] Kodolányi J. and Hoppe P. 2011. Abstract \#1094. 42nd Lunar and Planetary Science Conference. [6] Vollmer C. et al. 2009. Geochimica et Cosmochimica Acta 73:7127-7149. [7] Nittler L. R. et al. 1997. The Astrophysical Journal 483:475-495. [8] Nittler L. R. 2009. Publications of the Astronomical Society of Australia 26:271-277. [9] Zinner E. et al. 2005. Geochimica et Cosmochimica Acta 69:4149-4165. [10] Palmerini S. et al. 2011. The Astrophysical Journal 729.

\section{9}

\section{BERYLLIUM-10 IN AUSTRALASIAN MICROTEKTITES}

C. Koeberl ${ }^{1}$, K. Nishiizumi ${ }^{2}$, M. W. Caffee ${ }^{3}$ and B. P. Glass ${ }^{4}{ }^{1}$ University of Vienna, Althanstrasse 14, 1090 Vienna, Austria, and Natural History Museum, 1010 Vienna, Austria. E-mail: christian. koeberl@univie.ac.at. ${ }^{2}$ Space Science Laboratory, University of California, Berkeley, CA 94720, USA. ${ }^{3}$ Purdue University, West Lafayette, IN 47907, USA. ${ }^{4}$ University of Delaware, Newark, DE 19716, USA.

Previously, Australasian tektites have been measured for the concentrations of the cosmogenic radionuclide ${ }^{10} \mathrm{Be}$, which forms by interaction of cosmic rays with nitrogen and oxygen in the atmosphere and is concentrated in the top of any sediment column. These data help to constrain the location and characteristics of the source material. The ${ }^{10} \mathrm{Be}$ half-life is $1.36 \mathrm{Myr}$; thus, it can only be studied in relatively young impact structures and glasses. The average value of ${ }^{10} \mathrm{Be}$ in Australasian tektites is about $100 \times 10^{6}$ atoms $\mathrm{g}^{-1}$ [1]. This is comparable to ${ }^{10} \mathrm{Be}$ contents of near-surface source materials, e.g., soils or sediments (marine and land). Also, within the Australasian strewn field there is a correlation between the tektite type and the ${ }^{10} \mathrm{Be}$ concentration [1]. Aerodynamically shaped tektites (Australites) found farther from the presumed impact site in southeast Asia have higher contents (on average about $136 \times 10^{6}$ atoms $\mathrm{g}^{-1}$ ) compared to layered (Muong Nong-type) tektites, which have not been transported as far (on average about $59 \times 10^{6}$ atoms $\mathrm{g}^{-1}$ ). This has obvious implications on the formation mechanism of tektites. The Ivory Coast tektites are regular (splash-form) tektites related to the Bosumtwi crater formed at $1.07 \mathrm{Ma}$, and if these tektites also formed from surficial materials as did the Australasian tektites, then they are expected to contain similar concentrations of ${ }^{10} \mathrm{Be}$. Measurements by [2] show that ${ }^{10} \mathrm{Be}$ concentrations of Ivory Coast tektites are consistent with formation from mostly near-surface sediments or soils. Traces of ${ }^{10} \mathrm{Be}$ were also found by [2] in a moldavite (Central European tektite). Thus all tektites in which ${ }^{10} \mathrm{Be}$ can be measured show the presence of that nuclide, indicating that tektites in general are produced from near-surface target rocks.

One open question is if microtektites also contain Be-10, and how much? Due to the small sizes of microtektites, such measurements were difficult to impossible until now. As a first attempt, we selected three microtektites and a fragment from Site 1144A in the South China Sea, three microtektites from V29-39 and two from V29-40 from the Central Indian Ocean, and two from site V16-70 (which is father from the Indochina area). The compositions were measured by microprobe, and afterwards the microtektites were released from the epoxy, cleaned, and pooled, giving a total mass of $620.5 \mu \mathrm{g}$. Initially we hoped to measure the three main locations separately but the weight was insufficient. The content of Be-10 was measured by accelerator mass spectrometry at Purdue University. The microtektite composite contains $260 \pm 60 \times 10^{6}$ atoms ${ }^{10} \mathrm{Be} / \mathrm{g}$. This concentration is notable for at least two reasons: first, this is about two times higher than that for the splash-form (ablated) australites, which otherwise have the highest ${ }^{10} \mathrm{Be}$ content in the strewn field; second, as this is an average for microtektites recovered at various distances from the inferred source area, some microtektites could contain even higher contents of ${ }^{10} \mathrm{Be}$. The immediate conclusion is that microtektites are derived from the immediate surface of the target and that the form even before the "normal" tektites.

References: [1] Ma P. et al. 2004. Geochimica et Cosmochimica Acta 68:3883-3896. [2] Serefiddin F. et al. 2007. Geochimica et Cosmochimica Acta 71:1574-1582. 
5195

\section{MAGNETIC SUSCEPTIBILITY AS A TOOL FOR CHARACTERIZATION OF AN ASTEROID REGOLITH AND SAMPLE RETURN}

T. Kohout ${ }^{1,2}$ and D. Britt ${ }^{3}$. ${ }^{1}$ Department of Physics, University of Helsinki, Helsinki, Finland. E-mail: tomas.kohout@ helsinki.fi. ${ }^{2}$ Institute of Geology, Academy of Sciences, Prague, Czech Republic. ${ }^{3}$ Department of Physics, University of Central Florida, Orlando, FL, USA.

Introduction: Magnetic susceptibility of a rock is dependent on concentration of ferromagnetic minerals. It proved to be reliable nondestructive tool in distinguishing various types of extraterrestrial materials $[1,2,3]$, and in searching for compositional variations of meteorite falls $[4,5]$. Magnetic susceptibility of an asteroid regolith can be compared to database of known meteorites in order to diagnose its mineralogy, homogeneity and maturity.

In-situ Susceptibility Measurement of the Asteroid Regolith: A simple susceptibility measurement coil can be incorporated into lander or surface drop probe. Its design can be similar to commercially available handheld susceptibility meters or to one described in [6] or [7]. The regolith homogeneity can be tested by spot measurements in various locations around asteroid.

Regolith on airless bodies is subject to space weathering often associated with production of iron nanoparticles. These particles are often in superparamagnetic state exhibiting high frequency dependence of magnetic susceptibility. Thus degree of susceptibility frequency dependence is quantitatively related to regolith maturity [8].

Susceptibility Measurement of the Sample Return: The magnetic susceptibility measurements of a sample return will provide similar information as in-situ measurements, but laboratory instrumentation provide higher precision. Additionally magnetic susceptibility results can be directly compared with mineralogical and chemical analysis. However, any sample return is likely to be a rather small, localized quantity.

Conclusions: Magnetic susceptibility can provide diagnostic parameters to determine regolith mineralogy and meteorite analog. Additionally regolith maturity can be estimated from frequency dependence of regolith susceptibility.

References: [1] Rochette P. et al. 2003. Meteoritics \& Planetary Science 38:251-268. [2] Rochette P. et al. 2008. Meteoritics \& Planetary Science 43:959-980. [3] Rochette P. et al. 2009. Meteoritics \& Planetary Science 44:405-427. [4] Consolmagno G. J. et al. 2003. Meteoritics \& Planetary Science 41:331-342. [5] Kohout et al. 2010. Meteoritics \& Planetary Science 45:1778-1788. [6] Rochette P. et al. 2004. Planetary and Space Science 52:987-995. [7] Bentley M. S. 2009. Planetary and Space Science 57:1491-1499. [8] Bentley M. S. 2011 Planetary \& Space Science 59:79-91.

\section{4}

SHOCK ORIGIN INFERRED FOR UNUSUAL TEXTURES IN EH3 CHONDRITE PCA 82518

M. Komatsu ${ }^{1}$, T. Fagan ${ }^{2}$, T. Mikouchi ${ }^{3}$ and M. Miyamoto ${ }^{3}$. ${ }^{1}$ Institute for Advanced Study, Waseda University, Tokyo, Japan. E-mail: komatsu@aoni.waseda.jp. ${ }^{2}$ Department of Earth Sciences, Waseda University. ${ }^{3}$ Department of Earth and Planetary Science, University of Tokyo.

Introduction: Enstatite chondrites are the most reduced group among chondrites. Like the other types of chondrites, the enstatite chondrites experienced various degrees of metamorphism and shock [e.g., 1]. We have examined the mineralogy and chemistry of EH3 chondrites in order to understand low temperature metamorphic reactions on the EH parent body. Here we describe the mineralogy, chemistry and matrix textures that distinguish EH3 chondrite PCA 82518, from several other EH3 chondrites (ALHA81189, Y-691, ALH 84170, and Sahara 97096).

Results: This meteorite is mainly composed of porphyritic to granular pyroxene chondrules, and irregularly shaped metal-sulfide clumps. Euhedral silica is commonly observed near chondrule rims and in pyroxene fragments. The presence of olivine and well-defined chondrules suggest that it belongs to type 3 .

Many of the low-Ca pyroxene crystals in PCA contain numerous blebs of sub-micron sized Ni-poor Fe metal and troilite. These pyroxenes appear opaque in transmitted light and tend to be mantled by a thin layer of pure enstatite [2].

The matrix in PCA 82518 is different from that of other EH3s. The matrices of most EH3s are characterized by silicate sulfide and metal clasts ranging in size from one to several $\mu \mathrm{m}$-across ("clastic matrix" of [3]). The fragmental, clastic textures of these grains can be seen easily in back-scattered electron images. In contrast, much of the PCA matrix is so fine that individual grains are not observed; this matrix may be glassy. The matrix contains kamacite crystals with bulbous shapes (convex crystal margins with matrix). In addition, elongate enstatite crystals extend from chondrules into matrix.

Pyroxene in PCA shows a wide range of Fs content similar to other $\mathrm{EH} 3$ s; however, the $\mathrm{Cr}_{2} \mathrm{O}_{3}$ content in olivine is lower. In type $3 \mathrm{OC}$ and $\mathrm{CO}$ chondrites, $\mathrm{Cr}_{2} \mathrm{O}_{3}$ apparently diffuses out of olivine during metamorphism [4].

Discussion: Well-defined chondrules and the presence of olivine indicate that PCA 82518 is a type 3 chondrite. However, the very fine, non-clastic matrix and bulbous metal crystals distinguish PCA from other EH3s. The bulbous metal texture indicates in situ crystallization or annealing, not clastic transport. This texture and elongate pyroxene extending into matrix may have resulted from a shock event, similar to that inferred for RKPA80259 [5]. The lower $\mathrm{Cr}_{2} \mathrm{O}_{3}$ of olivine compared to other EH3s is consistent with metamorphism at higher temperature [4]

PCA 82518 is classified by [1] as shock stage S3, based primarily on lamellar twinning and undulatory extinction in coarse enstatite. However, actual shock energy might have been higher than suggested by this classification if enstatite crystals were annealed. In any case, PCA 82518 experienced more extensive heating, probably due to shock, than the other EH3 chondrites in our study.

References: [1] Rubin A. et al. 1997. Geochimica et Cosmochimica Acta 60:847-858. [2] Lusby D. et al. 1987. Geochimica et Cosmochimica Acta 30:E679-E695. [3] Rubin A. et al. 2009. Meteoritics \& Planetary Science 44:589-601. [4] Grossman J. N. and Blearley A. J. 2005. Meteoritics \& Planetary Science 40:87-122. [5] Fagan T. J. et al. 2000. Meteoritics \& Planetary Science 35:31-329. 
5426

FIRST ${ }^{40} \mathrm{Ar}-{ }^{39} \mathrm{Ar}$ DATA OF THREE RECENTLY DISCOVERED ACHONDRITES: SAU 402, DH 312, AND DH 500

E. V. Korochantseva ${ }^{1,2}$, A. I. Buikin ${ }^{1,2}$, J. Hopp ${ }^{1}$, A. V. Korochantsev ${ }^{2}$ and M. Trieloff ${ }^{1}$. ${ }^{1}$ Institut für Geowissenschaften, Universität Heidelberg, Heidelberg, Germany. E-mail: Mario.Trieloff@geow.uniheidelberg.de. ${ }^{2}$ Vernadsky Institute of Russian Academy of Sciences, Moscow, Russia.

Introduction: A relative scarcity of chronological data on primitive achondrites and current great interest in enstatite meteorites prompted us to undertake this high-resolution ${ }^{40} \mathrm{Ar}-{ }^{39} \mathrm{Ar}$ study. Here we report first ${ }^{40} \mathrm{Ar}-{ }^{39} \mathrm{Ar}$ data for three unrelated achondrites (1) SaU 402-ungrouped enstatite that could be an impact melt rock [1]; (2) Dh 312-a primitive achondrite, classified as an acapulcoite with low shock stage and weathering grade W3 [2]; (3) Dh 500 - an unusual ungrouped brecciated primitive achondrite (S4, W4) possibly having some links to winonaites and silicate inclusions of the IAB/IIICD irons [3].

Results and Discussion: The age spectrum of $\mathrm{SaU} 402$ yields an age plateau with an age of $4275 \pm 17 \mathrm{Ma}$ over approximately $50 \%$ of ${ }^{39} \mathrm{Ar}$ release $(1 \sigma$ error here and further). Most probably this age reflects the formation time of this impact melt rock. However, it may also date a later postformation event, similar to another enstatite impact melt rock Ilafegh 009 where the Ar-Ar age was significantly younger than the I-Xe age. The last total reset of both Dhofar meteorites occurred approximately $4.45 \mathrm{Ga}$ ago. The age spectrum of Dh 312 shows a partial plateau with an age of $4450 \pm 24 \mathrm{Ma}\left(66-100 \%\right.$ of ${ }^{39} \mathrm{Ar}$ release). Within uncertainty this age is the same as Ar-Ar ages of other acapulcoites and lodranites constraining the time of thermal metamorphism [5-7]. According to [3], the polymict breccia Dh 500 experienced intensive thermal and impact metamorphism which is consistent with the complex history inferred from this Ar-Ar study. The age spectrum of Dh 500 shows at least three events. It gives two partial plateaux with ages of $4307 \pm 11 \mathrm{Ma}\left(17-39 \%\right.$ of $\left.{ }^{39} \mathrm{Ar}\right)$ and $4454 \pm 11 \mathrm{Ma}\left(53-94 \%\right.$ of $\left.{ }^{39} \mathrm{Ar}\right)$. These ages are in the range of Ar-Ar ages of winonaites and silicate from IAB irons as well as unshocked ordinary chondrites [8-10]. Secondary events causing partial ${ }^{40} \mathrm{Ar}$ loss $\leq 3.2 \mathrm{Ga}$ ago are indicated by age spectra of all samples. Primordial ${ }^{36} \mathrm{Ar}$ is detected in all samples and is released at temperatures $>1100{ }^{\circ} \mathrm{C}$ from primitive meteorites and in the temperature range of $800-1140{ }^{\circ} \mathrm{C}$ from SaU 402. Thus, all three meteorites carry valuable information on the processes in the early solar system.

References: [1] Connolly H. C., Jr. et al. 2006. Meteoritics \& Planetary Science 41:1383-1418. [2] Grossman J. N. and Zipfel J. 2001. Meteoritics \& Planetary Science 36:A293-A322. [3] Lorenz C. A. et al. 2003. Abstract \#5045. Meteoritics \& Planetary Science 38. [4] McCoy T. J. et al. 1995. Geochimica et Cosmochimica Acta 59:161-175. [5] Pellas P. et al. 1997. Geochimica et Cosmochimica Acta 61:3477-3501. [6] McCoy T. J. et al. 1997. Geochimica et Cosmochimica Acta 61:623-637. [7] Mittlefehldt D. W. et al. 1996. Geochimica et Cosmochimica Acta 60:867882. [8] Benedix G. K. et al. 1998. Geochimica et Cosmochimica Acta 62:2535-2553. [9] Bogard D. D. et al. 2005. Meteoritics \& Planetary Science 40:207-224. [10] Trieloff M. et al. 2003. Nature 422:502-506.

\section{3}

LUNAR METEORITES FROM ANTARCTICA AND OMAN

R. L. Korotev. Dept. of Earth \& Planetary Sciences, Washington Univ., St. Louis, Missouri, U.S.A. E-mail: korotev@wustl.edu.

Thirty-three named lunar meteorite stones representing 19-21 meteorites have been found in Antarctica, all by government-sponsored expeditions (Japan and U.S.). (It has not yet been established whether the 4 MIL 0900XX lunar stones represent 1, 2, or 3 falls [1].) The total mass of lunar material recovered from Antarctica is $5.43 \mathrm{~kg}$, and $0.12 \%$ (by stone name, excluding provisional names) of the meteorites from Antarctica currently listed in the Meteoritical Bulletin Database are from the Moon.

In comparison, 57 named lunar meteorites stones representing 16-22 meteorites have been found in Oman, all but one (SaU 169) by private collectors. Many of these are not well characterized, although we have analyzed samples at least one stone of all Omani lunaites by INAA (39 stones). The mass of lunar material from Oman, a country about the size of Poland, is $9.75 \mathrm{~kg}$. In the area of the main concentration of meteorites (those with names Dhofar, Jiddat al Harasis, and Shişr), the concentration of lunar meteorite finds is a remarkable $0.9 \mathrm{~g} \mathrm{~km}^{-2}$. This value is $3.8 \times$ that calculated by Nazarov et al. [2] because of 10 more years of new finds, including $5.5-\mathrm{kg}$ Shişr 162 . The fraction of lunar meteorites among named stones from Oman, 2.35\% (by stone name), is $2000 \times$ that for Antarctica presumably because of collection bias, i.e., many ordinary chondrites from Oman never receive names.

Assuming 21 Antarctic lunaites, 13 are feldspathic breccias (3-9\% $\mathrm{FeO})$, four are mare basalts $(21-23 \% \mathrm{FeO})$, and 4 are breccias containing both highlands and mare material $(12-18 \%$ FeO). None has a large proportion of KREEP (MIL 090036 and Yamato 983885 have about 2 $\mu \mathrm{g} / \mathrm{g}$ Th). Assuming 21 Omani lunaites (author's preference), 17 are feldspathic breccias, 1 (Dhofar 287) is a mare basalt, 1 (Dhofar 1180) is a mare-highlands breccia, and 2 are KREEP-rich breccias (SaU 169 with two lithologies having 9 and $30 \mu \mathrm{g} / \mathrm{g}$ Th and Dhofar 1442, a regolith breccia, having $14 \mu \mathrm{g} / \mathrm{g} \mathrm{Th}$ ). Thus, the distribution of lunar rock types differs considerably between the two regions.

Among the Antarctic lunaites, several cases of launch pairings are established or suspected, leading to 13-17 lunar craters represented. Because of lack of cosmic-ray exposure data for most Omani lunaites, launch pairings among the Omanian meteorites are not established. On the basis of similar composition and texture, SaU 449 is likely launchpaired with Dhofar 925/960/961 (340 km separation) [3] and JaH 348 may be launch paired with paired stones Dhofar 1436 and Dhofar 1443 (143 km separation). Although texturally different, Shişr 166 is compositionally identical to Dhofar 026 found $33 \mathrm{~km}$ away. These stones may represent yet another launch pairing.

On the basis of similar texture and compositions, possible AntarcticOman launch pairings include Dhofar 1428 and MAC 88104/5. Dhofar $1436 / 1443$, an impact-melt breccia, is compositionally identical to MIL 07006, a regolith breccia.

Omani lunaites are contaminated, to varying degrees, with $\mathrm{K}, \mathrm{Sr}$, $\mathrm{Cs}, \mathrm{Ba}, \mathrm{As}, \mathrm{Se}, \mathrm{Br}, \mathrm{Sb}$, and $\mathrm{U}$ compared to their Antarctic counterparts and Apollo samples.

References: [1] Korotev R. L. et al. 2011. Abstract \#1999. 42nd Lunar and Planetary Science Conference. [2] Nazarov M. A. et al. 2004. Solar System Research 38:49-58. [3] Korotev R. L. et al. 2009. Meteoritics \& Planetary Science 44:1287-1322. 


\section{2}

\section{A PROTOPLANETESIMAL DUST COLLISION MODEL BASED ON EXPERIMENTS}

S. Kothe ${ }^{1}$, C. Güttler ${ }^{1}$, J. Blum ${ }^{1}$, E.Beitz ${ }^{1}$ and R. Weidling ${ }^{1} .{ }^{1}$ Institut für Geophysik und extraterrestrische Physik, TU Braunschweig, E-mail: s.kothe@tu-bs.de.

Introduction: The formation of solid bodies in the early solar system, like e.g. planets, meteorites or asteroids started with the growth of small aggregates out of (sub-) micrometer sized dust grains. At distances of $1 \mathrm{AU}$ from the young Sun, most of the particles are silicates. Collisions between those dust grains have initially low velocities and lead to sticking and hence to the growth of larger, at first fractal, aggregates. Further impacts are supposed to compact these agglomerates to non-fractal but still highly porous particles. Due to the interaction with the surrounding gas, these agglomerates collide at higher velocities. Therefore these collisions do not necessarily lead to sticking.

However, none of these processes is directly accessible by observations so that we depend on theories, simulations and experiments to develop a picture of these processes.

The collision behavior of two dust agglomerates has been studied in different experiments (e.g., reviewed in [1]) with typically micrometer sized dust particles and aggregates thereof. To reach low collision velocities, which are realistic in a protoplanetary disk, most of the experiments were performed under microgravity conditions on parabolic flights or in the drop tower in Bremen.

The experiments showed a variety of outcomes: while small particles at low collision velocities are still likely to stick, an increase in the impact energy leads to rebound of the particles, or, for even higher velocities of the order of $1 \mathrm{~m} \mathrm{~s}^{-1}$, even to fragmentation. Both of these processes can restrain the growth of larger bodies.

Collision Model: To make the diversity of possible outcomes in these experiments accessible for theoretical approaches, we compiled them in a dust collision model (c.f. [2]). Depending on the particle masses, velocities, and porosities, the model predicts the outcome of collisions and distinguishes between different types of collisional outcomes, which are based on our experiments and extrapolations of these. For each type of collision, the model returns the particles porosities and size distribution after the collision.

Improvements: Since the original publication, several new experiments were conducted to improve the model. We found that there is a transition between the sticking and the bouncing regime and growth is still possible at velocities where we expect rebound [3]. Moreover, under certain circumstances fragmentation, which at first appears to constrain further growth, can actually lead to growth due to a small mass transfer $[4,5]$.

This talk will give an introduction to our model and new experiments which were used to improve it.

References: [1] Blum J. and Wurm G. 2008. Annual Review of Astronomy and Astrophysics 46:21-56. [2] Güttler C. et al. 2010. Astronomy \& Astrophysics 513:A56. [3] Weidling et al. arXiv 1105:3909. [4] Beitz et al. 2011. arXiv 1102:4441. [5] Kothe et al. 2010. The Astrophysical Journal 725:1242-1251.
5501

CALCULATING IMPACT MELT VOLUMES PRODUCED BY NONVERTICAL IMPACTS ON THE EARTH, MOON, AND MARS

D. A. Kring ${ }^{1}$, O. Abramov ${ }^{1}$ and S. M. Wong ${ }^{2} .{ }^{1}$ Center for Lunar Science and Exploration, USRA-Lunar and Planetary Institute, 3600 Bay Area Blvd., Houston Texas 77058, USA. E-mail: kring@lpi.usra.edu. ${ }^{2}$ Lunar and Planetary Laboratory, University of Arizona, Tucson, Arizona 85721, USA.

Introduction: Previous studies have shown that the volume of impact melt generated and its volume relative to fragmented rock increases with crater diameter [1-3]. In a comparison of the parameters affecting impacts on different planetary surfaces, Grieve and Cintala [4] also suggested the melt volume associated with similar-sized transient craters is nearly six times greater on the Earth and Venus than on the Moon. Comparisons of this type were derived from analytical expressions that assume a vertical impact trajectory and, thus, are limited to only a small proportion of the impacts that occur on a planet. The most probable trajectories have impact angles of $45^{\circ}$ [5] and impact events with even shallower inclinations are possible. Recently computer codes have been developed that allow three-dimensional simulations of impact events. One numerical study [6] used hydrocode modeling to determine the amount of melt produced by impacts at various impact angles on Earth and showed that it decreases with impact angle. We use that type of result to render an easy-to-use analytical expression for calculating impact melt volumes.

Results: Our analytical expression is derived for melt volumes produced by oblique impacts as a function of gravity, impact velocity, impact angle, and density of impact projectiles and target surfaces on the Earth, Moon, and Mars. The most probable oblique impact $\left(45^{\circ}\right)$ produces $\sim 2$ times less melt volume than a vertical impact, and $\sim 2-7$ times more melt than impacts with $30^{\circ}$ and $15^{\circ}$ trajectories, respectively. The melt volume for a particular crater diameter increases with gravity and impact velocity, so a crater on Earth will have more melt than similar-size craters on Mars and the Moon. For example, the formation of a transient crater with a particular diameter on Earth generates $\sim 3$ times more melt than on Mars and $\sim 5$ times more melt than on the Moon. However, the melt volume for a particular projectile diameter does not depend on gravity, but has a strong dependence on impact velocity, so the melt generated by a given projectile on the Moon is $\sim 2$ times greater than that on Mars. Collectively, these results imply thinner central melt sheets and smaller proportion of melt particles in impact breccias on the Moon and Mars than on Earth.

References: [1] Ahrens T. J. and O'Keefe J. D. 1977. In Impact and explosion cratering. pp. 639-656. [2] Grieve R. A. F. and Cintala M. J. 1992. Meteoritics 27:526-538. [3] Pierazzo E. et al. 1997. Journal of Geophysical Research 103:28,607-28,625. [4] Grieve R. A. F. and Cintala M. J. 1997. Advances in Space Research 20:1551-1560. [5] Shoemaker E. M. 1962. In Physics and astronomy of the Moon. 283-359. [6] Pierazzo E. and Melosh H. J. 2000. Icarus 145:252-261. 
5412

ORIGIN OF FERROAN OLIVINE IN MATRICES OF UNEQUILIBRATED CHONDRITES

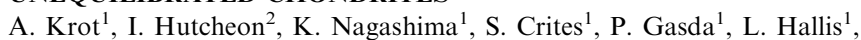
C. Jilly ${ }^{1}, \quad$ M. Petaev ${ }^{3}, \quad$ K. Robertson ${ }^{1}$, G. Taylor ${ }^{1}$ and M. Telus ${ }^{1}$ ' ${ }^{1}$ University of Hawai'i at Mãnoa, Honolulu, HI, USA. ${ }^{2}$ Lawrence Livermore National Laboratory, Livermore, CA, USA. ${ }^{3}$ Harvard University, Cambridge, MA, USA.

Ferroan olivine $\left(f a, \mathrm{Fa}_{50-100}\right)$ is one of the major minerals in matrices of unequilibrated ordinary (UOCs) and carbonaceous (CV, CK, $\mathrm{CO})$ chondrites. Two classes of models, nebular and asteroidal, have been proposed to explain its origin. According to the nebular models, matrix $f a$ formed by gas-solid condensation under highly-oxidizing conditions resulted from evaporation of dust \pm ice-enriched nebular regions during chondrule formation [1]. Recent thermodynamic calculations, however, do not support these models [2]. According to the asteroidal models, matrix $\mathrm{fa}$ formed during fluid-assisted thermal metamorphism on the chondrite parent asteroids [3, 4]. These models are consistent with the mineralogical observations and $\mathrm{O}-$ and ${ }^{53} \mathrm{Mn}-{ }^{53} \mathrm{Cr}$ isotope systematics of $f a$ in $\mathrm{CVs}$ and MAC 88107 (ungr.) [3-7]. Here we report on the mineralogy and Oisotope compositions of $f a$ in matrices of UOCs; ${ }^{53} \mathrm{Mn}-{ }^{53} \mathrm{Cr}$ measurements are in progress.

Ferroan olivine $\left(\mathrm{Fa}_{60-99}\right)$ is found in several textural occurrences in Ngawi (1) 1-10 $\mu \mathrm{m}$-sized grains in fine-grained rims around type I chondrules containing abundant opaque nodules composed of magnetite $(m g t), \mathrm{Fe}-\mathrm{Ni}$ sulfide $(s f)$, Ni-rich metal, and $\mathrm{Fe}-\mathrm{Ni}$ carbides in their peripheries; (2) $f a \pm m g t \pm s f$ veins crosscutting fine-grained rims around chondrules; (3) $f a$ replacing opaque nodules in chondrule peripheries; (4) $\mathrm{fa}+$ phyllosilicates replacing chondrule mesostasis; and (5) isolated coarse $(10-30 \mu \mathrm{m})$ grains and grains overgrowing opaque nodules in the matrix. Coarse $f a$ grains commonly contain inclusions of $s f$ and $m g t$ and show the inverse chemical zoning: fayalite content decreases toward the edges of the grains, suggesting $\mathrm{Fe}-\mathrm{Mg}$ interdiffusion during thermal metamorphism. The similar textural occurrences of $f a$ are found in finegrained rims and matrices of QUE 97008, EET 90161 (L3.05), MET 96503 (L3.1), and MET 00452 (H3.5). The $\Delta^{17} \mathrm{O}$ value of Ngawi $f a$, approximately $5 \%$, measured in situ by the UH Cameca ims-1280 is similar to those of coarse $m g t$ grains in Ngawi and Semarkona [7].

These and previously published observations of the mineralogy and isotopic compositions of $f a$ in matrices of metamorphosed ordinary and carbonaceous chondrites [3-7], and the nearly complete absence of $f a$ in matrices of unmetamorphosed chondrites and cometary IDPs, support origin of matrix $f a$ during fluid-assisted thermal metamorphism. ${ }^{53} \mathrm{Mn}-{ }^{53} \mathrm{Cr}$ and O-isotope studies of matrix $f a$ in UOCs and CCs can be used to constrain time of aqueous alteration and, in combination with $\mathrm{D} /$ $\mathrm{H}$ measurements of organics and phyllosilicates, sources of water ice (inner versusouter disk) that accreted into their parent asteroids [8].

References: [1] Lauretta D. et al. 2003. Geochimica et Cosmochimica Acta 65:1337-1353. [2] Grossman L. 2010. Meteoritics \& Planetary Science 45:7-20. [3] Krot A. et al. 2004. Proceedings of the National Institute of Polar Research 17:154-172. [4] Zolotov M. et al. 2006. Meteoritics \& Planetary Science 41:1775-1796. [5] Dobrică E. and Brearley A. 2011. Abstract \#2092. 42nd Lunar and Planetary Science Conference. [6] Choi B.-G. et al. 2000. Meteoritics \& Planetary Science 35:1239-1249. [7] Choi B.-G. et al. 1998. Nature 35:1365-1387. [8] Alexander C. et al. 2010. Geochimica et Cosmochimica Acta 74:4417-4437.
5294

VOLATILE ELEMENT DEPLETION AND NEUTRON CAPTURE EFFECTS IN IRON METEORITES: NEW CONSTRAINTS FROM CADMIUM

T. S. Kruijer ${ }^{1}$, M. Zbinden ${ }^{1}$, P. Sprung ${ }^{1}$, T. Kleine ${ }^{2}, \quad$ R. Wieler ${ }^{1}$. ${ }^{1}$ Institute of Geochemistry and Petrology, ETH Zurich. Correspondence: thomas.kruijer@erdw.ethz.ch. ${ }^{2}$ Institut für Planetologie, Westfälische Wilhelms-Universität Münster.

Introduction: Cosmic-ray effects can hamper chronological applications to extraterrestrial materials [e.g., 1], e.g. obtaining accurate core formation ages for iron meteorites using the Hf-W system [e.g., 1, 2]. Some lunar rocks show cosmic-ray-induced ${ }^{113} \mathrm{Cd}$ anomalies [3], but yet no study investigated $\mathrm{Cd}$ isotopes in iron meteorites. Cadmium, a chalcophile and volatile element, is expected to occur in very low, but measurable concentrations in iron meteorites. We here present new isotope dilution $\mathrm{Cd}$ data for metal and troilite samples from different groups of iron meteorites, and for the first time report a cosmic-ray induced Cd isotope anomaly in an iron meteorite.

Methods: Troilite and metal samples were dissolved in concentrated $\mathrm{Hcl}$ or Aqua Regia. $\mathrm{A}^{106} \mathrm{Cd}$-enriched spike was added to $200-700 \mathrm{mg}$ aliquots for concentration determinations. Cadmium was separated from the matrix using a multi-stage ion exchange chromatography modified after [4]. Cadmium concentrations and isotope compositions were measured using a $\mathrm{Nu}$ Plasma MC-ICPMS at ETH Zurich. External uncertainties on concentration data for troilite are estimated to be $3 \%$ (2SD), while the uncertainties for metal samples are considerably higher due to significant blank corrections. Total procedural blanks varied between 4 and $12 \mathrm{pg} \mathrm{Cd}$.

Results and Discussion: Cadmium abundances in troilite inclusions range from 0.4 to $5 \mathrm{ppb}$, while concentrations in the metal phase are all below $30 \mathrm{ppt}$. Cadmium abundances in the metal are 2-3 orders of magnitude lower than previously reported [5,6], but consistent with a more recent study [7]. Although average moderately volatile element (Ga and $\mathrm{Ge}$ ) contents for different iron groups [8] generally correlate with the $\mathrm{Cd}$ contents presented here, $\mathrm{Cd}$ abundances vary between different troilite nodules from single meteorites. One troilite nodule separated from Toluca (IAB) has $\varepsilon^{113 / 111} \mathrm{Cd}=-7.3 \pm 3.3$ and $\varepsilon^{114 / 111} \mathrm{Cd}=4.1 \pm 4.7$ (2SD) (i.e., $10^{4}$ deviation relative to the terrestrial value). The $\mathrm{Cd}$ isotope anomalies presented here are consistent with expected neutron capture effects for $\mathrm{Cd}$ isotopes, and in good agreement with correlated $\varepsilon^{114} \mathrm{Cd}$ vs $\varepsilon^{113} \mathrm{Cd}$ variations reported for irradiated lunar samples [4].

Outlook: The very low $\mathrm{Cd}$ concentrations and their systematic variation reflect the strong volatile element depleted nature of magmatic iron meteorites. Albeit analytically challenging, the first cosmic-ray induced $\mathrm{Cd}$ isotope anomaly reported here appears promising for the use of ${ }^{113} \mathrm{Cd}$ as neutron capture dose monitor in iron meteorites.

References: [1] Leya I. et al. 2000. Earth and Planetary Science Letters 175:1-12. [2] Kleine T. et al. 2009. Geochimica et Cosmochimica Acta 73:5150-5188. [3] Sands D. G. et al. 2001. Earth and Planetary Science Letters 186:335-346. [4] Wombacher F. 2003 Geochimica et Cosmochimica Acta 67:4639-4654. [5] Schmitt R. A. et al. 1963. Geochimica et Cosmochimica Acta 27:1077-1088. [6] Rosman K. J. R. and De Laeter J. R. 1974. Geochimica et Cosmochimica Acta 58:1665-1677. [7] Chen J. H. and Wasserburg G. J. 1983. 14th Lunar and Planetary Science Conference 1052. [8] Haack, H. and McCoy T. J. 2003. Treatise on Geochemistry. 
5143

PSEUDOTACHYLITIC MELTING AND UNMIXING OF SILICATE, CARBONATE AND SULFIDE MELTS IN THE PUŁTUSK H-CHONDRITE

A. Krzesińska ${ }^{1}$, A. Żelaźniewicz ${ }^{1}$ and R. Orłowski ${ }^{2} .{ }^{1}$ Institute of Geological Sciences, Pol. Acad. Sc., Res. Centre in Wrocław, Podwale 75, PL-50449 Wrocław, Poland. ${ }^{2}$ Res. Centre in Warsaw, Twarda 51/55, PL-00818, E-mail: agatakrz@twarda.pan.pl.

Introduction: The Pultusk meteorite is H4-5 S3 chondrite with silicates of typical, equilibrated composition [1-3]. It was significantly deformed due to shock-related shearing at high strain-rate (without high shock pressures) [3, 4]. In most of the specimens cataclastic, darkened zones occur. The conducted EDS measurements indicate that in such darkened zones, glassy blebs composed of iron-depleted silicate-, ironnickel sulfide- and carbonate-domains are present.

Iron-pure silicates and iron-nickel sulfides in cataclased zones: In matrix of cataclased parts of chondrite, $\sim 20-120 \mu \mathrm{m}$ large, irregular blebs occur. They are generally composed of amorphous phases; however, some microcrystallites may also be detected. Such blebs never occur in the light, host part of the chondrite. Modal composition of blebs differs from the host rock's composition as blebs are either completely free of metal and sulfides or contain many laths of iron-nickel sulfides. Silicate glasses in blebs have generally composition of enstatite $\left(\mathrm{En}_{94.1} \pm 2.6 \quad \mathrm{Fs}_{5.0} \pm 2.9\right.$ $\left.\mathrm{Wo}_{0.9} \pm 0.3\right)$ and iron-pure diopside $\left(\mathrm{En}_{52.9} \pm 7.4 \mathrm{Fs}_{4.2} \pm 2.5 \mathrm{Wo}_{42.9} \pm 8.0\right)$ and in some cases of forsterite $\left(\mathrm{Fo}_{98.6} \pm 0.5 \mathrm{Fa}_{1.4} \pm 0.5\right)$.

The composition of the silicates in the vicinity of all of these objects is typical for $\mathrm{H}$ chondrite $\left(\mathrm{En}_{80.5} \mathrm{Fs}_{17.5 \pm 1.6} \mathrm{Wo}_{2.0 \pm 1.5}\right.$ and $\mathrm{Fo}_{80.7} \mathrm{Fa}_{19.3 \pm 1.2}$ ). No transition zone is observed.

Carbonates inside of blebs: In one of blebs, $\mathrm{CaO}$-rich microcrystallites occur. Their composition is: $\mathrm{SiO}_{2}-0.22 ; \mathrm{FeO}-0.85$; $\mathrm{MgO}-0.05$ and $\mathrm{CaO}-54.79 \mathrm{wt} \%$. They are most likely calcitic because of carbon contents.

Discussion and Conclusion: Blebs of such iron-pure silicate composition are hardly expected in a thermally equilibrated chondrite and must postdate nebular and accretional processes. The composition of blebs cannot be related to enstatite chondrite projectile as sulfides in blebs do not contain normally litophile elements. The origin of calcite seems to be the most mysterious problem. Origin as weathering product [5] is excluded as specimens are fresh finds and blebs occur inside them

Since blebs occur only inside zones with the obvious evidence of cataclasis, they may be related to pseudotachylitic melting and unmixing due to high strain-rate impact event. It seems that three immiscible melts then unmixed: magnesium-silicate, iron sulfide and carbonate. The origin of carbon is however unknown. Moreover, pseudotachylitic melting is likely as such unmixing of silicate and sulfide/carbonate melts occur in terrestrial impact structures [6,7] and iron-depleted glasses have been formed in $\mathrm{H}$ chondrite deformed at high strain-rate [8].

Acknowledgments: This work was supported by MNiSW grant no. N N307 474838.

References: [1] Binns R. A. 1968 Geochimica et Cosmochimica Acta 32:299-317. [2] Stöffler D. et al. 1991. Geochimica et Cosmochimica Acta 55:3845-3867. [3] Krzesinska A. 2010. Abstract \#1140. 41st Lunar and Planetary Science Conference. [4] Krzesińska A. 2010. vol. 5. EPSC2010499. [5] Rubin A. E. 1997. Meteoritics \& Planetary Science 32:231-247. [6] Osinski and Spray. 2010. EPSL 194:17-29. [7] Deutsch A. et al. 1998. Abstract \# 1386. 29th Lunar and Planetary Science Conference. [8] Van der Bogert C. H. et al. 2003. Meteoritics \& Planetary Science 38:15211531 .
5257

YOUNG ${ }^{26} \mathrm{Al}^{26} \mathrm{Mg}$ AGES OF CHONDRULES IN THE LL3.1 CHONDRITE BISHUNPUR: EVIDENCE FOR THERMAL PROCESSING ON ASTEROIDAL BODY?

E. Kurahashi ${ }^{1,2}$ K. Nagashima ${ }^{3}$, A. N. Krot ${ }^{3}$ and S. S. Russell ${ }^{2}$. ${ }^{1}$ Institut für Planetologie, Westfälische Wilhelms-Universität Münster, Germany. E-mail: e.kurahashi@uni-muenster.de. ${ }^{2}$ Natural History Museum, London, UK. ${ }^{3} \mathrm{HIGP} / \mathrm{SOEST}$, University of Hawai'i at Mãnoa, USA.

Introduction: An increasing amount of data on protoplanetary solids using precise isotope measurements provides important constraints on the formation ages of solids in the early solar system [e.g., 1-5]. Here, we report new results of the $\mathrm{Al}-\mathrm{Mg}$ isotope system of the Bishunpur (LL3.1) chondrules.

Mineralogy and Petrology: A type I chondrule (\#25, 450 $\mu \mathrm{m}$ in diameter) consists of elongated orthopyroxene $\left(\mathrm{Fs}_{5} \mathrm{Wo}_{1}\right)$, high-Ca pyroxene $\left(\mathrm{Fs}_{11} \mathrm{~W}_{37}\right)$ and glassy mesostasis containing fine grains of metal and high-Ca pyroxene. A type II chondrule $(\# 24,520 \times 260 \mu \mathrm{m})$ consists of olivine $\left(\mathrm{Fo}_{\sim 81}\right)$ and glassy mesostsasis including high-Ca pyroxene crystallites $\left(\mathrm{Fs}_{19} \mathrm{Wo}_{41}\right)$. There are no secondary minerals in either chondrule. ${ }^{26} \mathrm{Al}-{ }^{26} \mathrm{Mg}$ isotope systematics: $\mathrm{Mg}$-isotope measurements were performed with the UH Cameca ims-1280. Four points in glassy mesostasis $(\mathrm{Al} / \mathrm{Mg}=23-69)$ in $\# 25$ and 3 points in glassy mesostasis $(\mathrm{Al} /$ $\mathrm{Mg}=26-37)$ in \#24 were measured with a $\sim 5 \mu \mathrm{m}$ beam spot. Both chondrules show no clear evidence of ${ }^{26} \mathrm{Mg}$-excesses and provide only upper limits of the initial ${ }^{26} \mathrm{Al} /{ }^{27} \mathrm{Al}$ ratios of $9.9 \times 10^{-7}$ and $4.2 \times 10^{-6}$, respectively. Assuming a homogeneous distribution of ${ }^{26} \mathrm{Al}$ in the early solar system with the inferred initial ${ }^{26} \mathrm{Al} /{ }^{27} \mathrm{Al}$ ratio of $5 \times 10^{-5}$ [6], these data imply relative chondrule formation ages of $>4.1 \mathrm{Ma}$ for \#25 and $>2.6 \mathrm{Ma}$ for \#24 after Ca, Al-rich inclusions (CAIs).

Discussion: Despite the pristine mineralogy of the chondrules studied, the inferred Al-Mg ages are clearly younger than the previously reported chondrule ages from Bishunpur, ranging from 0.8 to $2.5 \mathrm{Ma}$ after CAIs [1, 2]. To evaluate a possibility of instrumental artefact, a chondrule from CR2 Elephant Moraine (EET) 92174, which has been previously measured for ${ }^{26} \mathrm{Al}-{ }^{26} \mathrm{Mg}$ systematics [5], was analyzed under the same conditions. The results obtained are in excellent agreement with the data reported by Nagashima et al. [5]. Based on the petrological observations of metal-troilite-silicate inclusions in matrix and fine-grained rims around chondrules in Bishunpur, Kojima et al. [7] concluded that this meteorite is a breccia that experienced impact process(es) prior to the final accretion. Both chondrules studied here are partly surrounded by fine-grained rims and metal-sulfide-silicate inclusions occur next to the chondrules. We infer that there is a possibility of redistributions of radiogenic ${ }^{26} \mathrm{Mg}$ in chondrules during re-accretion of Bishunpur parent body by impact event(s).

References: [1] Kita N. et al. 2005. In Chondrites and the protoplanetary disk. pp. 558-587. [2] Mostefaoui S. et al. 2002. Meteoritics \& Planetary Science 37:421-438. [3] Kunihiro T. et al. 2004. Geochimica et Cosmochimica Acta 68:2947-2957. [4] Kurahashi E. et al. 2008. Geochimica et Cosmochimica Acta 72: 3865-3882. [5] Nagashima K. et al. 2007. Abstract \#5291. Meteoritics \& Planetary Science 42. [6] MacPherson G. J. et al. 1995. Meteoritics 30:365-386. [7] Kojima T. et al. 2003. Geochimica et Cosmochimica Acta 67:3065-3078. 
5005

EVIDENCES OF A SHOCK METAMORPHISM IN THE CHONDRITE GRUZ'KE (H4)

N. V. Kychan. Institute of environmental geochemistry NAS of Ukraine, 34a Palladin av., Kyiv, Ukraine. E-mail: Nkychan@i.ua.

The chondrite Gruz'ke was found on 2007 March next to the village Gruz'ke in the Kirovograd region, Kirovograd district, Ukraine $\left(48^{\circ} 32^{\prime} \mathrm{N}\right.$, $\left.32^{\circ} 03^{\prime} \mathrm{E}\right)$. According to previous study [2], meteorite Gruz'ke was classified as an $\mathrm{H} 4$ ordinary chondrite. Presence of the numerous evidences of a shock metamorphism allowed us to classify the chondrite as a shock stage S4, based on the scheme of Stöffler et al. [3]. On the basis of the weathering classification of Wlotzka [4], the Gruz'ke chondrite belongs to the stage W2.

Here we present the additional data on a scanning electron microscope (SEM) and energy dispersive spectroscopy (EDS) study of inclusions in the metal and troilite particles [2] and shock effects in polished sections of the Gruz'ke chondrite. One of the polished sections was etched by $5-\%$ nital during $10 \mathrm{~s}$. The ordinary for chondrites shock effects are presented in minerals of the meteorite by the structures of deformation (planar structures in olivine, Neumann lines in kamacite and lamellas in troilite), shock heating (mosaicism of olivine, polycrystalline structure of kamacite and troilite, partial or complete transformation of martensite to coarse plessite within a zone taenite, secondary troilite) and melting (veins of troilite and pockets of silicates). Some shocked minerals have evidences of additional fragile and plastic deformations, such as shear structures of lamellas and monocrystals in troilite, distortion of the Neumann lines in kamacite and grains of zone taenite. At the same time, the presence within the chondrite of melted regions composed of maskelenite $\left(\mathrm{Ab}_{78.9} \mathrm{An}_{13.2} \mathrm{Or}_{6.89}\right)$ with numerous regular or irregular microcrystals of chromite and amoeboid coarse grains of kamacite and troilite with a high content of large inclusions (a pallasite-like structure) are the main features of the chondrite. Due to the high content of inclusions, the structure of the kamacite is similar to that of the Galkiv chondrite (H4) [1]. EDS-data indicate to the following average composition of the studied inclusions in the metal and troilite (in $\mathrm{wt}_{\mathrm{o}}$ ): olivine $\quad\left(\mathrm{Fa}_{18.6}\right), \quad$ pyroxene $\quad\left(\mathrm{Fs}_{16.7} \mathrm{En}_{82.2} \mathrm{Wo}_{1.41}\right)$, plagioclase $\left(\mathrm{Ab}_{80.6} \mathrm{An}_{11.7} \mathrm{Or}_{7.78}\right)$, chromite $\left(\mathrm{Cr}_{2} \mathrm{O}_{3}-55.7 ; \mathrm{FeO}-29.3 ; \mathrm{Al}_{2} \mathrm{O}_{3}-7.05\right.$; $\left.\mathrm{MgO}-3.98 ; \quad \mathrm{TiO}_{2}-2.22 ; \quad \mathrm{MnO}-0.80 ; \quad \mathrm{V}_{2} \mathrm{O}_{5}-0.73\right)$, merrillite $\left(\mathrm{P}_{2} \mathrm{O}_{5}-48.7 ; \mathrm{CaO}-43.4 ; \mathrm{MgO}-3.37 ; \mathrm{Na}_{2} \mathrm{O}-2.63 ; \mathrm{FeO}-1.87\right)$, native cupper $(\mathrm{Cu}-94.1 ; \mathrm{Fe}-3.93 ; \mathrm{Ni}-2.01)$ and silica $\left(\mathrm{SiO}_{2}-98.5\right.$; $\mathrm{FeO}-1.47)$.

The presence of a lot of structures of shock metamorphism and the character of their distribution within the chondrite point to as minimum two shocks in the cosmic history of the meteorite parent body. The first one resulted in the main shock alteration of the chondrite due to a shock pressure in the range of $30-35 \mathrm{GPa}$, which was accompanied by a local increase of temperature up to $988^{\circ} \mathrm{C}$ and post-shock one $\leq 500{ }^{\circ} \mathrm{C}$. The large sizes of inclusions, which were produced in result of solid-state diffusion during shock metamorphism, testify to a relatively slow cooling of the meteorite parent body from the post-shock temperature. The last shock was no more than $8 \mathrm{GPa}$ and produced of only fragile and plastic deformation within the minerals.

References: [1] V. P. Semenenko and A. L. Girich. 1998. Meteoritics \& Planetary Science 33:A193-A196. [2] Semenenko et al. 2010. Mineralogical Review 60:59-69 (in Ukrainian). [3] D. Stöffler et al. 1991. Geochimica et Cosmochimica Acta 55:3845-3867. [4] F. Wlotzka. 1993. Meteoritics 28:460.
5384

BASAL SUEVITE AND BASAL IMPACT MELT ROCKS, A SINGLE IMPACTITE UNIT MARKING THE BOTTOM OF ROCHECHOUART TRANSIENT CRATER

P. Lambert ${ }^{1}$, G. R. Osinski ${ }^{2}$, and H. M. Sapers ${ }^{2}$. ${ }^{1}$ Sciences \& Applications, 218 Bd Albert 1 er, F33800 Bordeaux, France. E-mail: lambertbdx@numericable.fr. ${ }^{2}$ Department Earth Sciences, University of Western Ontario, London, ON, Canada.

Early studies suggested a continuum between impact melt-bearing breccia ("suevite") and impact melt rocks at Rochechouart, with an intermediate population where lithic matrix and melt matrix domains coexists at the hand specimen scale (D-E population in [1]). More recently "upper" and "basal" suevite have been distinguished within the Rochechouart impact deposits [2]; and Montoume breccias have been interpreted as particulate impact melt rocks, transitional between a melt rock and a melt-rich impact breccia [3]. Notably, a large proportion of impact melt rocks currently exposed at Rochechouart bears a significant proportion of lithic clasts $[1,2]$. All the impact melt rocks exposed stand in direct contact with bedrock $[1,2]$ and can be referred as to "basal impact melt rocks."

Field, petrographic and geochemical data indicate that basal suevite and basal impact melts rocks at Rochechouart are members of a single impactite unit (basal impact melt-bearing unit) where the proportion of melt is more significant than its setting (in the matrix or as clasts), the latter varying locally and depending on the scale of observation. The unit spreads over the SW portion of the preserved crater deposits. It is characterized by (1) systematic setting in direct contact with the autochtonous-parautochtonous bedrock; (2) limited mixing and transportation deduced from petrographic and/or geochemical data indicating a local origin of a significant proportion of both melts and lithic fractions [2]; (3) complex textures including local diffuse contacts between lithic matrix and melt matrix fractions, "melts in melts," schlieren melt matrix breccia clasts, [2]; and (4) depletion or absence of homogeneous melts.

The Rochechouart basal impact melt-bearing unit is interpreted as the result of combined mechanisms associated with the collapse of the transient cavity. These mechanisms involve mechanical fragmentation of the shocked bedrock in the floor of the transient cavity related to uplift and pressure release, superimposed heating due to structural uplift known to cause elevated transient local geotherms at terrestrial impact craters above $20 \mathrm{~km}$ diameter [4], and further "intrusion" and mixing with the crater fill deposits. This hypothesis is consistent with the relative inefficiency in homogenizing and mixing of melt at Rochechouart noted by [2]. More generally it could explain the lack of coherent impact melt sheet at Rochechouart. Field and petrological data indicate that significant melting occurs beyond readjustment during the slow thermal re-equilibration process within the heterogeneous mixture of lithic debris and melts.

Beyond impactite nomenclature and related issues, recognition of basal suevite and basal impact melt rocks bear important geological applications. They may constrain the position of the center of the original crater (and its size to a lesser extend) at deeply eroded and/or reprocessed impact structures.

References: [1] Lambert P. 1977. Earth and Planetary Science Letters 35:258-268. [2] Lambert P. 2010. Geological Society of America Special Paper 465:509-541. [3] Sapers H. M. et al. 2009. Abstract \#1284. 40th Lunar Prospector Spacecraft. [4] Naumov M. V. 2005. Geofluids 5:165184 


\section{7}

MICROSTRUCTURAL CHARACTERISTICS OF SHOCKED RUTILE FROM THE CAPE CHARLES DRILL HOLE, CHESAPEAKE BAY IMPACT STRUCTURE, VIRGINIA, USA

F. Langenhorst ${ }^{1}$, J. W. Horton Jr. ${ }^{2}$, J. C. Jackson ${ }^{2}$, H. E. Belkin ${ }^{2}$ and I.-M. Chou ${ }^{2}$. ${ }^{1}$ Institute of Geoscience, Friedrich-Schiller University Jena, Burgweg 11, D-07749 Jena, Germany. E-mail: Falko.Langenhorst@ uni-jena.de. ${ }^{2}$ U. S. Geological Survey, Reston, VA 20192, USA.

Introduction: The late Eocene Chesapeake Bay impact structure is buried at shallow to moderate depths beneath continental-margin sediments in southeastern Virginia, USA. In preparation for the international Chesapeake Bay Impact Structure Deep Drilling Project, the United States Geological Survey drilled an $823 \mathrm{~m}$ test hole into the central uplift of the crater near Cape Charles, Virginia [1]. A combined $\mathrm{X}$-ray diffraction and Raman study on monomict gneissic impact breccias from this test hole revealed the existence of a high-pressure polymorph of $\mathrm{TiO}_{2}$ with the $\alpha-\mathrm{PbO}_{2}$ structure $\left(\mathrm{TiO}_{2}\right.$ II) [2]. After the Ries impact structure, Chesapeake Bay is the second crater where this polymorph has been found. Here, we have undertaken a transmission electron microscopic study to understand the mechanism of the phase transformation, which was fast enough to operate under the very short time scale of shock compression.

Methods: Brecciated gneisses from the Cape Charles drill hole were prepared into thin sections [2] and then ion-milled using a conventional Gatan Duomill as well as a Quanta 3D DualBeam. Microstructural observations were made using a $200 \mathrm{kV}$ LEO922 transmission electron microscope (TEM).

Results and Discussion: The $\mathrm{TiO}_{2}$ grains studied so far are the mixtures of rutile and the $\alpha-\mathrm{PbO}_{2}$-structured high-pressure polymorph that were originally described in [2]. TEM inspection of these grains shows that the initial $\mathrm{TiO}_{2}$ phase, rutile, is very defect-rich, containing numerous stacking faults and microtwins. These planar faults occur on multiple, symmetrically equivalent $\{011\}$ planes, forming an interconnected network. The local symmetry of the faults corresponds to the $\alpha-\mathrm{PbO}_{2}$ structure, but larger areas of the defect-free high-pressure polymorph have not yet been detected.

This overall microstructure is compatible with a martensitic (shear) transformation from rutile to the $\alpha-\mathrm{PbO}_{2}$-structured high-pressure polymorph, which has been proposed by Hyde and Andersson [3]. According to Hyde and Andersson [3] the rutile structure can be regarded as hexagonal close packed oxygen atoms with titanium atoms occupying half the close packed rows of octahedral interstices. Shear on oxygen planes of the rutile structure pushes the titanium atoms into new positions and results in the formation of the $\alpha-\mathrm{PbO}_{2}$-structured high-pressure polymorph. Since the shock duration was very short, there was not enough time for a complete realignment of atoms, explaining the defectrich nature of the $\mathrm{TiO}_{2}$ grains.

Acknowledgments: We are grateful for financial support provided by the Deutsche Forschungsgemeinschaft (LA 830 13-1 and 14-1).

References: [1] Horton J. W. Jr., Gohn G. S., Powars D. S. and Edwards L. E. 2008. Geological Society of America Special Paper 437:7397. [2] Jackson J. C., Horton J. W. Jr., Chou I.-M. and Belkin H. E. 2006. American Mineralogist 91:604-608. [3] B. G. Hyde and Andersson S. 1988. Inorganic Crystal Structures. pp. 67-72.
5352

SILICA SPECIATION: COUPLING SEM RAMAN AND CATHODOLUMINESCENCE

A. Laroussi ${ }^{1}$, A. Jambon ${ }^{2}$, O. Boudouma ${ }^{2}$ and H. Chennaoui Aoudjehane ${ }^{1,2}$. ${ }^{1}$ Laboratoire GAIA, Département de Géologie, Faculté des Sciences, Université Hassan II, BP 5366 Maârif, Casablanca Maroc (E-mail: a.laroussi@fsac.ac.ma). ${ }^{2}$ UPMC -Paris6, ISTeP, Case 110, 4 place Jussieu, 75252 Paris, France.

Seifertite, stishovite, cristobalite, tridymite, quartz and high and low pressure glass, are silica phases found in Martian meteorites, eucrites, enstatite chondrites and lunar meteorites. Crystalline state of silica in meteorites and terrestrial rocks essentially depend on the intensity of the shock on the parent body and the chemical composition of the original silica.

Identification of silica phase is important, it allow having information on (P-T) conditions of its formation, especially the intensity of the shock. Cathodoluminescence CL spectroscopy on SEM, allowed to identify most silica phases and to valid the technique, that is simple, rapid, efficient and non destructive. Reference spectra have been collected $[1-4]$.

We perform a Raman spectrometer prototype coupling to SEM and to $\mathrm{CL}$ spectrometer. Collecting spectra from a same minerals permit by using both techniques to intercalibrate the system. The aim is to be able to identify minerals and phases by using one or the other technique or both of them in the same equipment. It is especially important to be able to do it for minerals that are instable and can be destroy by one or the other technique, under the laser or electrons beam.

Raman spectra have been recorded on previously identified phases by $\mathrm{CL}$ of silica, plagioclase, maskelynite and pyroxenes; in order to valid this approach by comparing results.

We present here analyses on the lunar meteorite NWA 4734 [5-6], exceptional because the presence of high an low pressure silica phases: stishovite, seifertite, tridymite and cristobalite, and plagioclase and maskelynite $[5,7]$.

We began by the location of interesting phases by SEM images, CL spectra have been collected and the same points have been used to collect Raman spectra on VP Laser 532 mode, $0.2 \mathrm{mV}$ with different counting timing.

Comparing results shows that this new approach seems to be interesting, at least for some minerals, and it could be used for meteorites and terrestrial rocks.

References: [1] Chennaoui Aoudjehane H. et al. 2005. Meteoritics \& Planetary Science 40:967-979. [2] Chennaoui Aoudjehane H. et al. 2006. Abstract \#1036. 37th Lunar Prospector Spacecraft. [3] Chennaoui Aoudjehane H. et al. 2006. Abstract \#1037. 37th Lunar Prospector Spacecraft. [4] Chennaoui Aoudjehane H. et al. 2007. Abstract \#1714. 37th Lunar Prospector Spacecraft. [5] Jambon A. et al. (soumis). [6] Connolly H. C. et al. 2008. Meteoritics \& Planetary Science 43:571-632. [7] Chennaoui Aoudjehane et al. 2008. Meteoritics \& Planetary Science. Abstract 5058 . 


\section{2}

A COMPARATIVE STUDY OF TRACE-ELEMENT DISTRIBUTIONS IN R AND H CHONDRITIC SULFIDES: QUANTITATIVE ASSESSMENT OF ENHANCED SULFUR FUGACITY IN THE EARLY SOLAR SYSTEM

D. S. Lauretta ${ }^{1}$, A. V. Andronikov ${ }^{1}$, K. M. Jackson ${ }^{1}$ and L. Ojha ${ }^{1}$. ${ }^{1}$ Lunar and Planetary Laboratory, The University of Arizona, Tucson, AZ, USA. E-mail: lauretta@lpl.arizona.edu.

Introduction: The $\mathrm{R}$ chondrites are a well-established chondrite group that contains chondrules, mineral fragments, sulfides, CAIs, and fine-grained matrix [1-5]. R chondrites have high $\mathrm{A}^{17} \mathrm{O}(+2.9)[1,6,7]$. They are oxidized as indicated by their high $\mathrm{Fe}\left(\mathrm{Fa}_{37-40}\right)$ and $\mathrm{Ni}$ (up to $0.25 \mathrm{wt} \%$ ) contents in olivine and lack of metal $[1,6-8]$. They are also highly sulfidized, containing abundant pyrrhotite $\left(\mathrm{Fe}_{1-\mathrm{x}} \mathrm{S}\right)$ and pentlandite $\left[(\mathrm{Fe}, \mathrm{Ni})_{9} \mathrm{~S}_{8}\right]$ as well as other rare phases including chalcopyrite $\left(\mathrm{FeCuS}_{2}\right)$ and reports of pyrite $\left(\mathrm{FeS}_{2}\right)[1,2,3,7,9,10]$. The $\mathrm{R}$ chondrites record one of the most sulfidizing environments in the early solar system.

Using pyrrhotite-magnetite equilibrium, [9] determined the sulfur fugacity of LAP 04840 (R6) to be iron-troilite (IT) +2 . We used Cu-Fechalcopyrite equilibrium and the presence of $\mathrm{Ni}$ in the pyrrhotite to constrain sulfur fugacity in three $\mathrm{R}$ chondrites to IT +1.6 to 2.8 [10]. Here, we use trace-element abundances in sulfide minerals to constrain the conditions of their formation. Since the H-chondrites are buffered at IT, they serve as a baseline for comparing the elemental abundances in $\mathrm{R}$ chondrite sulfides.

Analytical Techniques: We analyzed nine assemblages from three $\mathrm{R}$ chondrites (LAP031135, LAP04840, and PRE95411). In addition, we analyzed sixteen troilite grains from four $\mathrm{H}$ chondrites (Djati-Pengilon, Zag, Oum Dreyga, and Djoumine). Chemical analyses of trace element abundances in situ of metals and sulfides are carried out using LA-ICPMS. The LA system at LPL consists of CETAC LSX-213 Nd:YAG 213 nm laser coupled with the Element 2 ICP-MS. We use the Hoba IVB iron meteorite sample [11] and NIST SRM-1250 as standards for analyzing metals, and MASS-1 USGS sulfide standard for sulfides [12].

Results: Compared to H-chondrite troilite, pyrrhotite in $\mathrm{R}$ chondrites is enriched in platinum group elements by factors ranging from 100-1000×. Trace element abundances in R-chondrite pyrrhotites combined with thermodynamic analysis indicate formation at IT $+2.3( \pm 0.8)$ and $1050 \mathrm{~K}$. If these conditions are representative of the nebular environment in which the $\mathrm{R}$ chondrites formed, then the determined sulfur fugacity is consistent with an $\mathrm{H}_{2} \mathrm{~S} / \mathrm{H}_{2}$ ratio of this region that is enhanced by a factor of $\sim 670 \times$ over solar. Detailed characterization of these phases will provide better constraints on their formation.

Acknowledgments: This work was funded by NASA Grant NNX10AH50G (DSL PI).

References: [1] Kallemeyn et al. 1996. Geochimica et Cosmochimica Acta 60:2243-2256. [2] Rubin and Kallemeyn. 1989. Geochimica et Cosmochimica Acta 53:3035-3044. [3] Weisberg et al. 1991. Geochimica et Cosmochimica Acta 55:2657-2669. [4] Bischoff and Srinivasa. 2003. Meteoritics \& Planetary Science 38:5-12. [5] Rout and Bischoff. 2008. Meteoritics \& Planetary Science 43:1439-1464. [6] Bischoff et al. 1994. Meteoritics 29:264-275. [7] Schulze et al. 1994. Meteoritics 29:275-286. [8] Righter and Neff. 2007. Polar Science 1:25-44. [9] McCanta et al. 2008. Geochimica et Cosmochimica Acta 72:5757-5780. [10] Jackson and Lauretta. 2010. Meteoritics \& Planetary Science Supplement \#5164. [11] Walker et al. 2008. Geochimica et Cosmochimica Acta 72:2198-2216. [12] Danyushevsky et al. 2011. Geochemistry: Exploration, Environment, Analysis 11:51-60.
5133

SPECTRAL INDICES FOR IDENTIFYING TERRAINS ON VESTA WITH DAWN FRAMING CAMERA FILTERS

L. Le Corre ${ }^{1}$, V. Reddy ${ }^{1,2}$, and A. Nathues ${ }^{1}$. ${ }^{1}$ Max-Planck-Institut für Sonnensystemforschung, Katlenburg-Lindau, Germany, E-mail: lecorre@ mps.mpg.de. ${ }^{2}$ Department of Space Studies, University of North Dakota, Grand Forks, USA.

Introduction: Vesta is the 3rd largest asteroid in the main belt [1] and the first target of NASA's Dawn mission which will begin its mapping phase in survey orbit around Vesta in August 2011. The Framing Camera (FC), one of three scientific instruments onboard the spacecraft [2], contains a set of seven color filters ranging from 0.44 to 0.97 microns in addition to a clear filter. The latter will be used mainly for mapping the morphology of Vesta's surface whereas the color filters will provide hints about surface composition either through the creation of color composite images allowing identifying spectrally distinct units, or through the analysis of the color spectra in combination with laboratory spectra of well-known howardite-eucrite-diogenite (HEDs) and non-HEDs samples.

Goals: Our study aims to find out what filter ratios and spectral criteria are best to characterize terrains on Vesta using the data acquired with the FC filters in the visible-near infrared. Obviously, our limitation is that we do not have ground truth measurements from the surface of this asteroid therefore we are going to assume that HEDs [3] and potentially other related types such as mesosiderites, pallasites and minerals like olivine-orthopyroxene (OPX) mixtures are analogous to terrains that might be observed on Vesta [4].

Spectral Criteria: Several spectral criteria such as reflectance ratios, spectral slopes and pseudo Band I minimum were plotted as a function of ratios of FC color filters to look for the best color parameters that can be used to extract compositional information. Band I minimum is used as a proxy for band center as FC cannot measure the whole 1-micron absorption band that is essential to study the mineralogy of HED terrains. The slope between 0.75 and 0.92 microns is also used to characterize the left wing of the pyroxene Band I. Spectral parameters have been computed using publicly available laboratory spectra of HEDs, pallasites, mesosiderites and olivine-OPX mixtures after resampling to FC filters.

Classification: In order to be able to distinguish the putative meteorite analogs on the surface of Vesta we selected the most relevant plots showing clusters of data points in distinctive areas or linear trends for each type. For each of them, four or five plots were attributed and will be used to identify surface composition in FC data. According to our study, it is possible to distinguish between eucrites and diogenites by using specific FC filters. Other non-HEDs types are recognizable as well. If none of the spectral criteria are fulfilled, we may assume it corresponds to howardite-like terrains or alternatively to another type of mineralogy that one can add to our spectral library after identification. Further testing of our method will involve effects of calibration, phase angle, and temperature on the selection of plots and cross-calibration with laboratory samples.

References: [1] Thomas P. et al. 1997. Science 277:1492-1495. [2] Sierks H. et al. 2011. Space Science Reviews online. [3] McSween H. et al. 2010. Space Science Reviews online. [4] Gaffey M. 1997. Icarus 127:130157. 
5351

\section{CHARACTERIZATION AND POSSIBLE ORIGIN OF A CARBONACEOUS COATING ON AN UNUSUAL NANOCRYSTALLINE SILICATE AGGREGATE IN THE MATRIX OF THE MET 00426 CR3 CHONDRITE}

C. Le Guillou ${ }^{1}$, A. J. Brearley ${ }^{1}$ and S. Bernard ${ }^{2}$. ${ }^{1}$ Department of Earth and Planetary Sciences, MSC03-2040, University of New Mexico, New Mexico, USA. E-mail: corentin.san@gmail.com. ${ }^{2}$ LMCM-MNHN, Paris, France.

Introduction: Pristine chondrites are the best samples to study the nebular dust from which asteroidal parent bodies accreted. MET 00426 (CR3.00) is known to have preserved one of the highest concentrations of presolar silicates [1], as well as potential nebular condensates, as a result of minimal parent body aqueous alteration and metamorphism. Its matrix contains amorphous hydrated silicate, phyllosilicates, sulfides and organic matter particles [2,3]. During FIB-TEM studies of MET 00426 matrix, we encountered an unusual particle coated with organic material, embedded within the normal fine-grained matrix. Here we report the results of TEM and STXM studies of this particle and discuss the possible formation mechanism of the carbonaceous material.

Results: The aggregate (500 nm in size) is composed of nanocrystalline $\mathrm{Mg}-\mathrm{Fe}-\mathrm{Ca}$ silicates $(20-40 \mathrm{~nm}$, probably diopside or augite) associated with sulfides and organic matter. The silicates are more Mg-rich $(\mathrm{Mg} \#>80$ atom\%) than the surrounding matrix and some individual domains are richer in Ca. Energy filtered TEM shows a continuous coating (approximately $20 \mathrm{~nm}$ in thickness) of carbonaceous material on the aggregate. High resolution TEM indicates that the carbonaceous material is slightly ordered, with small $(1-5 \mathrm{~nm})$ graphene layers stacked together and oriented parallel to the surface of the silicates. This degree of structural ordering is different from the more disordered organics found elsewhere in the matrix [3]. STXM mapping confirms the dominant aromaticity of this carbon coating and the absence of oxidized functional groups usually present in CR organics [3].

Discussion: This intriguing aggregate shares several microstructural similarities with presolar grains, such as grain size, high- $\mathrm{Mg}$ and $\mathrm{Ca}$ content, chemical variation at the nanometer scale and nanocrystalline features [4]. However, an attempt to measure the O-isotopic composition by NanoSIMS was unsuccessful. Nevertheless, we interpret this particle as having a nebular or presolar origin. Therefore, the occurrence of the carbon-coating provides information about organic formation prior to parent body accretion. This kind of coating has also been described around silicates in CP-IDPs [5], but the silicate mineralogy is different. Two possible synthesis mechanisms for the formation of this coating were suggested by [5]; Fischer-Tropsch processes [6] and polymerization of simple organic molecules by UV irradiation within an icy mantle on silicate grains that can occur in the interstellar medium [7]. In both cases, these observations demonstrate that organic synthesis reactions on the surface of silicate grains did contribute to the structural and chemical diversity of carbonaceous material in chondrites.

References: [1] Floss C. and Stadermann F. 2009. The Astrophysical Journal 697:1242-1255. [2] Abreu N. M. and Brearley A. J. 2010. Geochimica et Cosmochimica Acta 74:1146-1171. [3] Le Guillou C. et al. 2011. Abstract \#1296. 42nd Lunar and Planetary Science Conference. [4] Vollmer C. et al. 2009. The Astrophysical Journal 700:774-782. [5] Flynn G. J. et al. 2010. Abstract \#1079. 40th Lunar and Planetary Science Conference. [6] Nuth J. A. et al. 2008. Organic Matter in Space, Proceedings of the International Astronomical Union, IAU Symposium, 251 pp. 403-408. [7] Bernstein M. P. et al. 1999. Science 283:1135-1138.

\section{5}

MATRIX MINERAL CHEMISTRY OF TIL 08007 CM CHONDRITE: IMPLICATIONS FOR INTERMEDIATE ALTERATION

H. W. Lee ${ }^{1,2}$, S. T. Kwon' ${ }^{1}$ J. I. Lee' , I. S. $\mathrm{Ahn}^{2}$, T. Nakamura ${ }^{3}$, A. Nakato ${ }^{3}$. ${ }^{1}$ Yonsei University. E-mail: 1hw615@yonsei.ac.kr. ${ }^{2}$ Korea Polar Research Institute. ${ }^{3}$ Tohoku University, Tohoku, Japan.

Introduction: $\mathrm{CM}$ chondrites contain tochilinite, cronstedtite, serpentine, carbonate, and sulfide in their matrix. These secondary minerals in matrix are well known as sensitive indicators for aqueous alteration on their parent bodies [1]. Tochilinite and cronstedtite are generally observed in PCP (poorly characterized phase) and serpentine replaced olivine or pyroxene. Carbonate and sulfide are associated with PCP or occur in matrix. As alteration advances, chemistry of secondary minerals of matrix is changed from Fe-rich (cronstedtite-like) to Mg-rich (serpentine-like) composition [2]. In addition, $\mathrm{Mg}, \mathrm{Fe}, \mathrm{Mn}$-bearing carbonates occur in heavily altered CM chondrites. Modal abundance of sulfide increases during progressive alteration. In this study, we report mineral chemistry of matrix in TIL 08007 recovered by the $3 \mathrm{rd}$ KOREAMET and classified as CM chondrite [3], and discuss its implications associated with aqueous alteration.

Analytical Methods: We examined a polished thin section (KOPRIT018) by scanning electron microscopy (SEM), and electron probe micro analyzer (EPMA). Through SEM observation, we qualitatively identified PCP-like phases, carbonates, and sulfides in matrix. In order to analyze quantitatively matrix components, we carried out EPMA analysis with both focused and broad beams. Four areas containing PCP-like phases of a separated slice from TIL 08007 were selected for synchrotron radiation X-ray diffraction (SR-XRD) analyses.

Results and Discussions: In SEM-BSE, tochilinite-like phase and phyllosilicates are primarily observed within PCP-like phase $(8.5 \mathrm{vol} \%)$. Some of them include $\mathrm{Ca}$-carbonate grains. Tochilinite-like minerals in TIL 08007 are characterized by lower S content (by 10 approximately 15 wt\%) and slightly higher $\mathrm{Mg} / \mathrm{Fe}$ ratio compared to other typical $\mathrm{CM}$ chondrites (Murchison and Murray) [4]. Modal abundance of sulfide is 10 vol\% ranging between less altered CMs (Murchison and Murray) and extensive altered ones (LAP 02277 and MET 01070) [5]. Phyllosilicates appear to be Fe-bearing serpentine. Based on SR-XRD analysis, major components of PCP-like phase are serpentine (7.2 $\AA$ ), and tochilinite and serpentine mixed-layer mineral $(6.0 \AA)$. Weak peak of $5.4 \AA$ probably indicates minor presence of tochilinite. From these results, lower $\mathrm{S}$ content in tochilinte-like phase may indicate that $\mathrm{S}$ was consumed to make sulfides and brucite layer of tochilinite formed mixed-layer mineral. Also, it is apparent that Fe-bearing serpentine was converted from cronstedtite by uptake of $\mathrm{Mg}$ and $\mathrm{Si}$ released from olivine and pyroxene during aqueous alteration. Additionally, the co-occurrence of both tochilinite-like phase and serpentine in PCP-like phase means that they are presumably on the transitional pathway during progressive aqueous alteration. The simple occurrence of Ca-carbonate supports that TIL 08007 did not reach extensive alteration. Therefore, the mineral chemistry of matrix implies that TIL 08007 is an intermediately altered CM2.3 chondrite.

References: [1] Tomeoka and Buseck. 1985. Geochimica et Cosmochimica Acta 49:2149-2163. [2] McSween. 1979a. Geochimica et Cosmochimica Acta 43:1761-1770. [3] Choi et al. 2008. Journal of the Geological Society of Korea 45:593-605. [4] Palmer. 2009. Ph.D. thesis. The University of Arizona. [5] Rubin et al. 2007. Geochimica et Cosmochimica Acta 71:2361-2382. 
5272

INVESTIGATING THE PRESOLAR GRAIN INVENTORY OF CH CHONDRITES: A NANOSIMS STUDY OF ACFER 182

J. Leitner ${ }^{1}$, P. Hoppe ${ }^{1}$ and J. Zipfel ${ }^{2}{ }^{1}$ Max Planck Institute for Chemistry, 55128 Mainz, Germany. E-mail: jan.leitner@mpic.de. ${ }^{2}$ Forschungsinstitut und Naturmuseum Senckenberg, 60325 Frankfurt, Germany.

Introduction: Primitive solar system materials contain varying amounts of presolar dust grains that formed in the winds of evolved stars or in the ejecta of stellar explosions. Presolar silicates and oxides are among the most abundant types of these grains [e.g., 1, 2]. The CR chondrite clan, consisting of the $\mathrm{CR}, \mathrm{CH}$, and $\mathrm{CB}$ chondrite groups, contains some of the most primitive meteorites, sharing certain mineralogical and chemical similarities [3,4]. High abundances of presolar silicates and carbonaceous grains were recently reported in individual $\mathrm{CR}$ chondrites [5-7], and a first presolar silicate grain was observed in the $\mathrm{CH} / \mathrm{CB}$ chondrite Isheyevo [8]. Noble gas data imply the presence of carbonaceous presolar grains also in $\mathrm{CH}$ chondrites [9], but no presolar $\mathrm{O}$-anomalous grains have been detected so far. In this ongoing study, we conduct a search for O-anomalous presolar silicate and oxide grains in fine-grained material located in lithic clasts of the $\mathrm{CH} 3$ chondrite Acfer 182. Investigating the occurrence and content of presolar matter in $\mathrm{CH}$ chondrites can shed light on the formation history of their parent body.

Samples and Experimental: We performed ion imaging of $10 \times 10 \mu \mathrm{m}^{2}$-sized matrix areas in a thin section of the $\mathrm{CH} 3$ chondrite Acfer 182 (paired with Acfer 207 and Acfer 214 [10]) with the NanoSIMS 50 ion probe in Mainz. ${ }^{16} \mathrm{O}^{-},{ }^{17} \mathrm{O}^{-},{ }^{18} \mathrm{O}^{-},{ }^{28} \mathrm{Si}^{-}$, and ${ }^{27} \mathrm{Al}^{16} \mathrm{O}^{-}$were measured in multi-collection to identify presolar silicate and oxide grains.

Results and Discussion: $4,700 \mu \mathrm{m}^{2}$ of fine-grained material in matrix clasts have been analyzed for their O-isotopic composition. No presolar grains have been identified so far; based on the average presolar silicate grain size of $\sim 300 \mathrm{~nm}$, we estimate an upper limit of $\sim 15 \mathrm{ppm}$ for Acfer 182. This is comparable to the presolar silicate abundance of $\sim 10 \mathrm{ppm}$ we found for Isheyevo. The formation scenario for the $\mathrm{CH}$ chondrites is still under debate. According to a popular model, $\mathrm{CH}$ chondrites may represent a mixture of material produced by a giant impact between planetary bodies and nebular components that formed by thermal processing of dust in the protoplanetary disk [11, and references therein]. Detection of presolar grains in these meteorites would proof that at least one of the components contained interstellar matter that survived the impact. This would give support to the idea that surviving molecular cloud material might be one of the carriers of the large ${ }^{15} \mathrm{~N}$ enrichments of $\mathrm{CH}$ chondrites. 1385.

Acknowledgments: We acknowledge support by DFG through SPP

References: [1] Nguyen A. et al. 2007. The Astrophysical Journal 656:1223-1240. [2] Hoppe P. 2008. Space Science Reviews 138:43-57. [3] Weisberg M. K. et al. 1995. Proceedings of the NIPR Symposium on Antarctic Meteorites 8:11-32. [4] Krot A. N. et al. 2002. Meteoritics \& Planetary Science 37:1451-1490. [5] Floss C. and Stadermann F. J. 2009. Geochimica et Cosmochimica Acta 73:2415-2440. [6] Leitner J. et al. 2011. The Astrophysical Journal, submitted. [7] Nguyen A. N. et al. 2010. The Astrophysical Journal 719:166-189. [8] Leitner et al. 2010. Meteoritics \& Planetary Science 45:A166. [9] Huss G. R. et al. 2003. Geochimica et Cosmochimica Acta 67:4823-4848. [10] Bischoff A. et al. 1993. Geochimica et Cosmochimica Acta 57:2631-2648. [11] Bonal L. et al. 2010. Geochimica et Cosmochimica Acta 74:6590-6609.

\section{8}

\section{PHOSPHATES IN ORDINARY CHONDRITES}

L. Leschner ${ }^{1}$, J. Roszjar ${ }^{1}$, T. John ${ }^{2}$, and A. Bischoff ${ }^{1}$. ${ }^{1}$ Institut fuer Planetologie. Wilhelm-Klemm-Str. 10, 48149 Muenster, Germany, E-mail: 1_lesc01@uni-muenster.de. ${ }^{2}$ Institut fuer Mineralogie. Corrensstr. 24, 48149 Muenster, Germany.

Introduction: Ca-phosphates, including both, apatite and merrillite are common accessory phases in most ordinary chondrites and as major hosts for $\mathrm{U}, \mathrm{Pb}$, and REE $[1,2]$ important mineral phases. They typically occur in type 4-6 ordinary chondrites and are less abundant in unequilibrated rocks. We have studied the grain sizes, and mineral chemistry of apatites and merrillites found in six ordinary chondrites of different type.

Analytical Techniques: Quantitative analyses were obtained using a JEOL JXA 8900 Superprobe electron probe micro analyzer operated at an acceleration voltage and beam current of $15 \mathrm{kV}$ and $15 \mathrm{nA}$ for major and minor elements, and $15 \mathrm{kV}$ and $4 \mathrm{nA}$ for halogens, respectively, using a defocused beam of $10 \mu \mathrm{m} . \mathrm{H}_{2} \mathrm{O}$ values for apatites were calculated based on stoichiometry.

Results: Mineralogy: Both, apatite and merrillite are typically intergrown with pyroxene, plagioclase, chromite, and troilite. Their grain size is roughly increasing from type 4 (typical sizes: $40-120 \mu \mathrm{m}$ ) to type 6 ordinary chondrites (typical sizes: $200-300 \mu \mathrm{m}$ ). Merrillite is generally larger than apatite. Importantly, the relative abundances of both phases vary significantly, e.g., merrillite is strongly dominating in Allegan, whereas in Bruderheim and Barwell apatite is by far more abundant.

Chemical composition: We found that both Ca-phosphates have very similar chemical compositions among the $\mathrm{H}, \mathrm{L}$, and LL chondrites. This is obvious for $\mathrm{CaO}$ and $\mathrm{P}_{2} \mathrm{O}_{5}$, but also valid for the more minor components $\mathrm{Na}_{2} \mathrm{O}$ and $\mathrm{MgO}$ (Table 1). Slight variations exist for FeO-, $\mathrm{SiO}_{2}-$, and $\mathrm{Ce}_{2} \mathrm{O}_{3}$-concentrations among the minerals from different meteorites. Merrillite represents an important additional REE host. In contrast to terrestrial apatite, which is dominantly F-rich, all analyzed apatites are $\mathrm{Cl}$-rich, which clearly indicates formation under $\mathrm{F}$ and $\mathrm{OH}$ poor conditions, as the latter both do have the more favorable ionic size to be incorporated into the apatite structure. Barwell apatite has the highest F-, Adzhi-Bogdo apatite the highest $\mathrm{Cl}$ concentrations.

References: [1] Brearley A. J. and Jones R. H. 1998. Planetary materials (ed. Papike J.) Reviews in Mineralogy, vol. 36. pp. 3-1 - 3-396. [2] Crozaz G. et al. 1989. Earth and Planetary Science Letters 93:157-169. [3] Bischoff A. et al. 1993 Meteoritics 28:570-578. [4] Terada K. and Bischoff A. 2009. The Astrophysical Journal 699:L68-L71.

Table 1. Phosphates in ordinary chondrites. Results in $\mathrm{wt} \%$.

\begin{tabular}{|c|c|c|c|c|c|c|c|c|c|c|}
\hline & \multicolumn{5}{|l|}{ Apatite } & \multicolumn{5}{|c|}{ Merrillite } \\
\hline & $\mathrm{Y}(3)$ & PV(4) & $\mathrm{AB}(3)$ & $\mathrm{BH}(9)$ & $\mathrm{B}(4)$ & $\mathrm{Y}(2)$ & $\mathrm{A}(9)$ & $\mathrm{PV}(10)$ & $\mathrm{AB}(2)$ & $\mathrm{BH}(4)$ \\
\hline $\mathrm{CaO}$ & 52.2 & 53.1 & 53.1 & 53.3 & 53.0 & 45.5 & 46.7 & 46.7 & 45.1 & 45.2 \\
\hline $\mathrm{P}_{2} \mathrm{O}_{5}$ & 39.7 & 41.7 & 41.2 & 41.1 & 40.9 & 46.3 & 46.9 & 46.8 & 47.0 & 46.7 \\
\hline $\mathrm{Na}_{2} \mathrm{O}$ & 0.30 & 0.46 & 0.26 & 0.37 & 0.38 & 2.72 & 2.75 & 2.75 & 2.90 & 2.74 \\
\hline $\mathrm{K}_{2} \mathrm{O}$ & 0.02 & n.d. & 0.03 & 0.02 & n.d. & 0.07 & 0.05 & 0.06 & 0.06 & 0.05 \\
\hline $\mathrm{MgO}$ & 0.07 & 0.03 & 0.09 & 0.04 & n.d. & 3.6 & 3.6 & 3.6 & 3.5 & 3.6 \\
\hline $\mathrm{MnO}$ & 0.06 & 0.07 & $<0.01$ & 0.04 & 0.04 & 0.05 & 0.04 & 0.04 & 0.04 & $<0.01$ \\
\hline $\mathrm{SiO}_{2}$ & 0.29 & n.d. & 0.24 & 0.13 & 0.06 & 0.23 & 0.02 & n.d. & 0.05 & 0.05 \\
\hline $\mathrm{FeO}$ & 0.65 & 0.38 & 0.65 & 0.14 & 0.22 & 0.68 & 0.32 & 0.47 & 0.99 & 0.51 \\
\hline $\mathrm{Al}_{2} \mathrm{O}_{3}$ & 0.04 & $<0.01$ & 0.03 & 0.03 & $<0.02$ & 0.06 & $<0.01$ & 0.03 & $<0.01$ & n.d. \\
\hline $\mathrm{Cl}$ & 5.0 & 5.6 & 5.7 & 5.5 & 5.0 & n.a. & n.a. & n.a. & n.a. & n.a. \\
\hline $\mathrm{F}$ & 0.46 & 0.30 & 0.30 & 0.46 & 0.63 & n.a. & n.a. & n.a. & n.a. & n.a. \\
\hline $\mathrm{H}_{2} \mathrm{O}$ & 0.22 & 0.19 & 0.14 & 0.13 & 0.17 & n.c. & n.c. & n.c. & n.c. & n.c. \\
\hline Total & 99.01 & 101.83 & 101.74 & 101.26 & 100.40 & 99.21 & 100.38 & 100.45 & 99.64 & 98.85 \\
\hline \multicolumn{11}{|c|}{$\begin{array}{l}\text { Y, Ybbsitz (H4); A, Allegan (H5); PV, Portales Valley (H6); AB, Adzhi Bogdo (LL3-6); BH, } \\
\text { Bruderheim (L6); B, Barwell (L6); Sr, S and Ti always below the detection limit; Ce was } \\
\text { always at or below detection limit; n.a., not analyzed; n.d., not detected; n.c., not } \\
\text { calculated; <, below detection limit; no. of grains in brackets; Phosphates from Adzhi } \\
\text { Bogdo include grains from granite-like clasts }[3,4] \text {. }\end{array}$} \\
\hline
\end{tabular}


5235

SMALL PRESOLAR Si-RICH GRAINS FROM THE QINGZHEN (EH3) METEORITE

Y. Lin ${ }^{1}$, P. Hoppe ${ }^{2}$, J. Huth ${ }^{2}$, J. Zhang ${ }^{1}$ and X. Zhao ${ }^{1}{ }^{1}$ Key Laboratory of the Earth's Deep Interior, Institute of Geology and Geophysics, Chinese Academy of Science, Beijing, China. Email: LinYT@mail.iggcas.ac.cn. ${ }^{2}$ Max Planck Institute for Chemistry, Mainz, Germany.

Introduction: In a previous study, an acid residue of the Qingzhen (EH3) meteorite, consisting mainly of $\mathrm{SiC}, \mathrm{Si}_{3} \mathrm{~N}_{4}$ and spinel, was separated into eight size fractions, ranging from $0.05 \mu \mathrm{m}$ up to $>10 \mu \mathrm{m}$. Only two of them (QZR4: $0.4-0.8 \mu \mathrm{m}$, QZR5: $0.8-2.0 \mu \mathrm{m}$ ) had been studied previously $[1,2]$. In this work, we analyzed grains from two other size fractions (QZR2: $0.1-0.2 \mu \mathrm{m}$, QZR3: $0.2-0.4 \mu \mathrm{m}$ ) with the NanoSIMS. We compared the results with those for the larger grain size fractions from the same meteorite and those from the Murchison carbonaceous chondrite [3], in order to explore the distribution of various types of presolar Si-rich grains among size fractions from different chemical groups of chondrites.

Results and Discussion: $1501 \mathrm{Si}$-rich grains from a mount of the 0.1$0.2 \mu \mathrm{m}$ fraction (labeled as QZR2A) and 1490 Si-rich grains from a mount of the $0.2-0.4 \mu \mathrm{m}$ fraction (labeled as QZR3A) were analyzed for ${ }^{12} \mathrm{C},{ }^{13} \mathrm{C},{ }^{28} \mathrm{Si},{ }^{29} \mathrm{Si}$ and ${ }^{30} \mathrm{Si}$ by automated ion imaging with the NanoSIMS at MPI for Chemistry [4]. Abundance ratios of $\mathrm{SiC} / \mathrm{Si}_{3} \mathrm{~N}_{4}$ are 4.71:1 for QZR2A and 1.35:1 for QZR3A, slightly different from the SEM observation (3.19:1 for QZR2A, 2.03:1 for QZR3A). Twenty supernova (SN) X grains from QZR2A and $19 \mathrm{X}$ grains from QZR3A were identified, representing abundances of $2 \%$ and $1.6 \%$ of all $\mathrm{SiC}$ grains, respectively. Five grains from QZR2A with large ${ }^{29} \mathrm{Si}$-enrichment, which have a SN origin as well [3], and another four from QZR3A were discovered, which gives total $\mathrm{SN}$ grain abundances of $2.5 \%$ and $2.0 \%$, respectively. These abundances are similar to those inferred for small presolar SiC grains from Murchison [3], but approximately 8 times as high as for the larger grains from Qingzhen [1]. AB grains are more abundant in both QZR2A (6.1\%) and QZR3A $(6.7 \%)$ in comparison with those from Murchison (3.1-3.7\%) [3]. Three $\mathrm{Si}_{3} \mathrm{~N}_{4}$ grains with ${ }^{12} \mathrm{C} /{ }^{13} \mathrm{C}<10$ (AB type) and $10 \mathrm{Si}_{3} \mathrm{~N}_{4}$ grains with ${ }^{12} \mathrm{C} /{ }^{13} \mathrm{C}$ of ${ }^{15-24}$ were identified from QZR3A by ion imaging, whereas only one $\mathrm{Si}_{3} \mathrm{~N}_{4}$ grain with significant ${ }^{13} \mathrm{C}$ excess $\left({ }^{12} \mathrm{C} /{ }^{13} \mathrm{C}\right.$ of 17) from QZR2A was found. These $\mathrm{Si}_{3} \mathrm{~N}_{4}$ grains were relocated in the SEM, and at least for two of them no adjacent $\mathrm{SiC}$ grains were evident. Re-analysis of two of the $\mathrm{AB}-\mathrm{Si}_{3} \mathrm{~N}_{4}$ grains with the NanoSIMS 50L at IGGCAS showed normal $\mathrm{N}$ isotopes, and only slight ${ }^{13} \mathrm{C}$ excesses $\left({ }^{12} \mathrm{C} /{ }^{13} \mathrm{C}\right.$ of $\left.34 \pm 14,25 \pm 3\right)$, suggesting that the first measurements were probably affected by adjacent $\mathrm{SiC} \mathrm{AB}$ grains. For grains with $\mathrm{X}$ signature, the abundance ratio of $\mathrm{Si}_{3} \mathrm{~N}_{4} / \mathrm{SiC}$ is between $1: 9$ in this work and 1:4 in the large size fractions [1]. In contrast, AGB $\mathrm{Si}_{3} \mathrm{~N}_{4}$ grains are comparatively rare compared to $\mathrm{SiC}$. This is consistent with a previous report of only one candidate $\mathrm{AGB} \mathrm{Si}_{3} \mathrm{~N}_{4}$ grain from Indarch (EH4) [5].

Acknowledgment: This study was supported by Natural Science Foundation of China (40830421).

References: [1] Lin Y. et al. 2002. The Astrophysical Journal 575:257-263. [2] Lin Y. et al. 2010. The Astrophysical Journal 709:11571173. [3] Hoppe P. et al. 2010. The Astrophysical Journal 719:1370-1384. [4] Gröner E. and Hoppe P. 2006. Applied Surface Science 252:7148-7151. [5] Zinner E. et al. 2007. Geochimica et Cosmochimica Acta 71:4786-4813.
5237

A PETROGRAPHY-SIMS COMBINED STUDY OF ${ }^{60} \mathrm{Fe}^{-60} \mathrm{Ni}$ AND ${ }^{53} \mathrm{Mn}-{ }^{53} \mathrm{Cr}$ IN SULFIDES FROM EH3 CHONDRITES

Y. Lin, J. Guo, S. Hu, W. Shen and Y. Liu. Key Laboratory of the Earth's Deep Interior, Institute of Geology and Geophysics, Chinese Academy of Science. Email: LinYT@mail.iggcas.ac.cn.

Introduction: Presence of ${ }^{60} \mathrm{Fe}$ was confirmed in troilite $[1,2]$ and $\mathrm{FeO}$-rich chondrules [3] from primitive ordinary chondrites. Recent study of unequilibrated enstatite chondrites showed disturbance of ${ }^{60} \mathrm{Fe}-{ }^{60} \mathrm{Ni}$ and ${ }^{53} \mathrm{Mn}-{ }^{53} \mathrm{Cr}$ in sulfides [4]. Our previous study of Qingzhen revealed various formations and thermal histories of sulfides [5]. In this study, we conducted SIMS analyses on sulfides with detailed petrographic settings from the Qingzhen and Sahara 97159 (EH3) meteorites, in order to explore ${ }^{60} \mathrm{Fe}-{ }^{60} \mathrm{Ni}$ and ${ }^{53} \mathrm{Mn}-{ }^{53} \mathrm{Cr}$ systems.

Results and Discussion: Troilite and sphalerite grains with schreibersite spherules and/or isolated in silicates from Qingzhen and Sahara 97159 were analyzed for isotopes of $\mathrm{Fe}, \mathrm{Ni}, \mathrm{Mn}, \mathrm{Cr}$, and $\mathrm{Zn}$ with the CAMECA ims 1280 at IGGCAS. These sulfides are Ni-free for EPMA [5], confirmed by SIMS with $\mathrm{Fe} / \mathrm{Ni}$ ratios up to $2.4 \times 10^{5} .{ }^{60} \mathrm{Ni}$ excesses were detected in all 10 sulfide assemblages (five from each meteorite). Each of them defines an ${ }^{60} \mathrm{Ni} /{ }^{61} \mathrm{Ni}-{ }^{56} \mathrm{Fe} /{ }^{61} \mathrm{Ni}$ "isochron" line, except for analyses with $\mathrm{Fe} / \mathrm{Ni}$ ratios $>10^{5}$ observed only in sphalerite. The high $\mathrm{Fe} / \mathrm{Ni}$ analyses plot on the right to the "isochron" line, indicative of $\mathrm{Fe}-\mathrm{Ni}$ redistribution. This is consistent with a previous report of lower $\left({ }^{60} \mathrm{Fe} /{ }^{56} \mathrm{Fe}\right)_{0}$ values for higher $\mathrm{Fe} / \mathrm{Ni}$ grains [4]. Sphalerite in Qingzhen is usually normally zoned, with $\mathrm{Fe}$ decreasing towards troilite [5]. This would increase the $\mathrm{Fe} / \mathrm{Ni}$ ratios because $\mathrm{Ni}$ diffusing faster than $\mathrm{Fe}[6]$. This is confirmed by a negative relationship between the $\mathrm{Fe} / \mathrm{Ni}$ and $\mathrm{Fe} / \mathrm{Zn}$ ratios. Seven of the 10 sulfide assemblages (three from Sahara 97159, four from Qingzhen) have $\left({ }^{60} \mathrm{Fe} /{ }^{56} \mathrm{Fe}\right)_{0}$ of $(0.93-1.6) \times 10^{-6}$, and three others from $0.76 \times 10^{-6}$ to $0.5 \times 10^{-6}$. The high $\left({ }^{60} \mathrm{Fe} /{ }^{56} \mathrm{Fe}\right)_{0}$ ratios are consistent with the estimation of $(0.5-1) \times 10^{-6}$ from chondrules [3], in the view of fast condensation of the solar nebula [7]. In case of ${ }^{53} \mathrm{Mn}-{ }^{53} \mathrm{Cr}$ system, only a sphalerite from Qingzhen shows ${ }^{53} \mathrm{Cr}$ excess, with an inferred $\left({ }^{53} \mathrm{Mn} /{ }^{55} \mathrm{Mn}\right)_{0}$ of $6.3 \times 10^{-8}$. Absence of ${ }^{53} \mathrm{Cr}$ excesses is likely due to $\mathrm{Cr}$ diffusion during thermal metamorphism in the parent body, as indicated by exsolution of tiny daubreelite in all troilite grains coexisting with Cr-sulfides [5].

Conclusions: Ni-free of schreibersite-bearing sulfides is confirmed by the SIMS analysis, supplying with robust evidence for condensation. Most of the sulfide assemblages have $\left({ }^{60} \mathrm{Fe} /{ }^{56} \mathrm{Fe}\right)_{0}$ of $(0.93-1.6) \times 10^{-6}$, probably representative of the solar value. The partial $\mathrm{Fe}-\mathrm{Ni}$ redistribution can be explained by diffusion during cooling. In contrast, $\mathrm{Mn}-\mathrm{Cr}$ system was more severely affected by secondary events, consistent with the petrographic observation [5].

Acknowledgment: This study was supported by the Natural Science Foundation of China (40830421).

References: [1] Tachibana S. and Huss G. R. 2003. Abstract \#1737. Lunar and Planetary Science Conference. [2] Mostefaoui S. et al. 2005. The Astrophysical Journal 625:271-277. [3] Tachibana S. et al. 2006. The Astrophysical Journal 639:L87-L90. [4] Guan Y. et al. 2007. Geochimica et Cosmochimica Acta 71:4082-4091. [5] Lin Y. and El Goresy A. 2002. Meteoritics \& Planetary Science 37:577-599. [6] Lauretta D. S. 2005. Oxidation of Metals 64:1-22. [7] Wang G. et al. 2007. Meteoritics \& Planetary Science 42:1281-1289. 
5304

CHARACTERISTICS AND GEOMETRY OF IMPACT-INDUCED CALCITE TWINNING

P. Lindgren ${ }^{1}$, M. R. Lee ${ }^{1}$ and M. J. Burchell ${ }^{2}$. ${ }^{1}$ School of Geographical and Earth Sciences, University of Glasgow. ${ }^{2}$ School of Physical Sciences, University of Kent. E-mail: paula.lindgren@glasgow.ac.uk.

Introduction: Calcite twinning can be used to infer stress magnitude and stress orientation, and is therefore widely applied as a palaeopiezometer in various terrestrial geological settings [1]. Also, the calcite microstructure in carbonaceous chondrite meteorites has been proven to be a useful tool for reconstructing the parent body deformation history during and/or after aqueous alteration [2]. In carbonaceous chondrites, calcite twinning is thought to be a result of deformation via impact gardening on the parent body (ies). However, the characteristics and geometry of calcite twinning during impact are not well-known, as the methods for calcite twin stress analyses are generally applied to calcite that was deformed at lower strain rates during tectonism [1]. To better understand the characteristics and geometry of calcite twinning in response to impacts and to test the methods of stress analyses, we have here carried out two sets of physical impact experiments on calcite using a light-gas gun [4]. We are currently analyzing the results using SEM imaging (2D and 3D) and EBSD analyses. Calcite speleothems were chosen for the experiments, as these are composed of pure calcite and are tectonically undeformed.

Results and Discussion: The first set of experiments used $1.5 \times 1.5 \mathrm{~mm}$ sized cubes of calcite as projectiles, which were fired into a target of water at a speed of $1.068 \mathrm{~km} \mathrm{~s}^{-1}$. These conditions gave a maximum peak pressure of ca $1 \mathrm{GPa}$ [5]. SEM and EBSD analyses of the recovered calcite fragments show that they are heavily ruptured along cleavage planes, and have developed twins. The twins occur on the $\{\overline{\mathrm{I}} 018\}$ planes, and hence are calcite e-twins. This set of experiments shows that calcite twins can form by impact at shock pressures of ca $1 \mathrm{GPa}$, which are more realistic of impact gardening on asteroids than previous impact experiments on calcite at $85 \mathrm{GPa}$ [6]. The second set of experiments used $0.8 \mathrm{~mm}$ diameter stainless steel bullets which were fired into two calcite targets at 1.068 and $1.098 \mathrm{~km} \mathrm{~s}^{-1}$. In the first target the projectile was fired normal to the calcite c-axes, and in the second target the projectile was fired parallel to the calcite c-axes. Craters formed in both targets with maximum diameters of $7.4 \mathrm{~mm}$ and $5.9 \mathrm{~mm}$, and volumes of ca $11 \mathrm{~mm}^{3}$ and $8.5 \mathrm{~mm}^{3}$, respectively. Both craters had similar depths of about $1.6 \mathrm{~mm}$. The first target produced a highly irregular circumference and internal topography, while the second target produced a more bowl shaped crater. Calcite in the interiors of the craters is highly fractured and twinned. Melting occur at the base of both craters. Preliminary EBSD analyses of the top surface of the target, immediately outside the craters, show that twinning is absent here. Future EBSD analyses will investigate the characteristics and geometry of the calcite twins in cross sections of the interiors of the craters.

References: [1] González-Casado J. M. et al. 2006. Journal of Structural Geology 28: 1084-1092. [2] Lindgren P. et al. 2011. Earth and Planetary Science Letters, in press. [3] Brearley A. et al. 1999. Abstract \#1301. 30th Lunar and Planetary Science Conference. [4] Burchell M. J. et al. 1999. Measurement Science and Technology 10: 41-50. [5] Melosh H. J. 1989. Impact Cratering: A Geologic Process, Oxford University Press, Oxford. [6] Langenhorst F. et al. 2003. High Pressure Compression of Solids V: Shock chemistry with applications to meteorite impacts.
5305

3D MICROSTRUCTURE AND CALCITE TWIN STRESS ANALYSES IN THE MURCHISON (CM2) METEORITE

P. Lindgren and M. R. Lee. School of Geographical and Earth Sciences, University of Glasgow. E-mail: paula.lindgren@glasgow.ac.uk.

Introduction: The internal microstructure of a meteorite can provide important insights into its regional deformation history and the parent body environment within which it has resided [1, 2]. The CM2 carbonaceous chondrites contain a few volume percent carbonates, mainly calcite, which formed by parent body aqueous alteration [3], and may also be used to explore the deformation. Calcite is widely used in terrestrial structural geology since it deforms to produce twins whose orientations are geometrically related to the c axes of the host calcite and to the principal axes of stress [4]. The samples used in terrestrial studies contain abundant calcite and the twins are formed by low strain rates during tectonism. However, only small samples of carbonaceous chondrites are available for analysis, the calcite is rare, and the twins are likely to be impact induced $[1,5]$. To test the applicability of calcite twin stress analyses to carbonaceous chondrites we carried out a 3D microstructural investigation on a fragment of Murchison (CM2). The fragment was embedded in resin and polished in to a block $8 \times 7 \times 5 \mathrm{~mm}$ in size. The orientation of calcite twins and the principal axes of stress were determined via SEM imaging and electron backscatter diffraction (EBSD) analyses on three sides of the block oriented normal to each other.

Results and Discussion: SEM imaging of the three sides of the block shows that the internal microfabric, i.e. the alignment of deformed chondrules and the foliation of the matrix components, links up in 3D as expected. EBSD analyses show that aragonite found on one side is oriented with its $\mathrm{c}$ axis in the same plane as the microfabric, implying that the aragonite and the microfabric formed under the same uniaxial stress regime. The calcite grains on the three sides have a weak crystallographic preferred orientation, but here the data are limited with a broad scatter. These calcite grains also have $\{\overline{1018}\}$ e-twins and preliminary results from two sides are somewhat scattered, but still suggest that the calcite twin stress analyses are consistent, i.e. the principal axes of stress (compression and extension) are connected in $3 \mathrm{D}$. Results also reveal that multiple phases of twinning are present in at least one of the calcite grains, where one set of twins is offset and cross-cut by a second set of twins, indicating at least two deformation events. Some of the calcite grains exhibit a posttwinning subgrain microstructure with low/high angle boundaries (all $<20^{\circ}$ misorientation). Other calcite grains are aggregates of individual calcite crystals with high-angle grain boundaries, which grew in different orientations prior to twinning. Twins in these aggregate grains yield clustered principal axes of stress, which confirms that calcite twin stress analyses are a robust method to analyze deformation of carbonaceous chondrites and other calcite-bearing meteorites. Further work on the microstructures is to be complimented with 3D X-ray tomography.

References: [1] Lindgren P. et al. 2011. Earth and Planetary Science Letters, in press. [2] Trigo-Rodríguez J. M. 2006. Geochimica et Cosmochimica Acta 70: 1271-1290. [3] Brearley A. J. 2006. Meteoritics and the early solar system II. The University of Arizona Press, Tucson. [4] Turner F. J. 1953. American Journal of Science 251: 276-298. [5] Brearley A. et al. 1999. Abstract \#1301. 30th Lunar and Planetary Science Conference. 


\section{6}

Cu AND Fe ISOTOPE ABUNDANCES IN LOW Ti LUNAR BASALTS F. N. Lindsay ${ }^{1}$, G. F. Herzog ${ }^{1}$ and F. Albarède ${ }^{2}$. ${ }^{1}$ Department of Chemistry \& Biological Chemistry, Rutgers University, New Brunswick, NJ 08854, USA. E-mail: flindsay@rci.rutgers.edu ${ }^{2}$ Ecole Normale Supérieure (LST) 69364 Lyon cedex 7, France.

Introduction: Enrichment in heavy isotopes of the moderately volatile elements $\mathrm{Cu}$ and $\mathrm{Zn}$ has been reported in high Ti lunar basalts [1, 2] relative to terrestrial counterparts for reasons not yet clear. Isotope measurements of low Ti basalts [3] hinted at similar enrichments on average. Here, we report $\mathrm{Cu}$ and $\mathrm{Fe}$ isotope abundances for six low $\mathrm{Ti}$ basalts as well as elemental abundances.

Experimental Methods: Powdered samples were treated as in [3]. Recoveries of elemental $\mathrm{Cu}$ and $\mathrm{Zn}$ were measured by high-resolution ICPMS) and of Fe with a Varian ICP-optical emission spectrometer (OES). Concentrations were calculated using a standard solution calibration. Isotopic analysis was done at ENS, Lyon using a Nu Plasma multicollector mass spectrometer (MC- ICP-MS) [1]. Isotope ratios are expressed as $\delta$ values relative to: ${ }^{63} \mathrm{Cu}$ and NIST 976 for $\mathrm{Cu} ;{ }^{54} \mathrm{Fe}$ and IRMM-14 for $\mathrm{Fe}$.

Results and Discussion: $\mathrm{Fe}$ isotope abundances (Table 1) are compatible with those reported by $\left[5 ; \quad\left(\delta^{56} \mathrm{Fe} 0.07 \pm 0.03 \%, \quad \delta^{57} \mathrm{Fe}\right.\right.$ $0.11 \pm 0.04 \% \mathrm{o})]$ and show less fractionation than samples with high Ti $[5$; $\left.\left(\delta^{56} \mathrm{Fe} 0.19 \pm 0.03 \%, \delta^{57} \mathrm{Fe} 0.22 \pm 0.05 \%\right)\right]$. High Ti basalts also show more fractionation in $\mathrm{Zn}\left(\delta^{66} \mathrm{Zn} 1.2 \pm 0.02 \%\right.$ ) than low Ti basalts $\left(\delta^{66} \mathrm{Zn}\right.$ $0.27 \pm 0.06 \%$ ) [2, 3]. In contrast, $\mathrm{Cu}$ isotopes of low Ti basalts are more fractionated than high Ti samples: (high $\mathrm{Ti} \delta^{65} \mathrm{Cu} 0.5 \pm 0.1 \%$ [2], low Ti $\delta^{65} \mathrm{Cu} 2.0 \pm 0.2 \%$ ). This surprising observation and the fact that $\delta^{65} \mathrm{Cu}$ versus $\mathrm{Cu}(\mathrm{ppm})$ does not show a trend compatible with Rayleigh distillation (Fig. 1) suggests that the isotopic fractionation neither is controlled by volatilization, nor occurs during evaporation; preliminary $\mathrm{Zn}$ results [1] show a similar trend. We think it more likely that the fractionation is regulated by mineral chemistry and/or diffusion.

References: [1] Moynier F. et al. 2006. Geochimica et Cosmochimica Acta 70:6103-6117. [2] Herzog G. F. et al. 2009. Geochimica et Cosmochimica Acta 73:5884-5904. [3] Lindsay F. et al. 2011. Abstract \#1907. 42nd Lunar and Planetary Science Conference. [4] Klemme S. et al. 2006. Chemical Geology 234:251-263. [5] Liu Y. et al. 2010. Geochimica et Cosmochimica Acta 74:6249-6262.

Table 1. Comparison of measured samples with literature values shown in grayed rows. Error is $1 \sigma$.

\begin{tabular}{|c|c|c|c|c|c|}
\hline & ${ }^{56 / 54} \mathrm{Fe}$ & ${ }^{57 / 54} \mathrm{Fe}$ & Fe wt $\%$ & $\delta^{65} \mathrm{Cu}$ & $\mathrm{Cu}(\mathrm{ppm})$ \\
\hline \multicolumn{6}{|l|}{ Lunar Basalts } \\
\hline 12053,284 & $0.02 \pm 0.04$ & $-0.08 \pm 0.07$ & $\begin{array}{l}14.0 \pm 0.7 \\
14.3-15.6\end{array}$ & $1.59 \pm 0.2$ & $\begin{array}{l}5.4 \pm 0.37 \\
7.1-26\end{array}$ \\
\hline 12056,20 & $\begin{array}{l}-0.08 \pm 0.03 \\
-0.04\end{array}$ & $\begin{array}{l}-0.18 \pm 0.04 \\
-0.10\end{array}$ & $\begin{array}{l}20.3 \pm 0.5 \\
16.79\end{array}$ & $2.02 \pm 0.2$ & $6.07 \pm 0.30$ \\
\hline 12063,329 & $\begin{array}{r}-0.03 \pm 0.05 \\
0.07\end{array}$ & $\begin{array}{r}-0.05 \\
0.12\end{array}$ & $\begin{array}{l}15.0 \pm 0.4 \\
16.5-16.7\end{array}$ & $1.60 \pm 0.2$ & $\begin{array}{l}4.56 \pm 0.33 \\
5.0-12.9\end{array}$ \\
\hline 12021,597 & $\begin{array}{c}-0.03 \pm 0.05 \\
0.01-0.06\end{array}$ & $\begin{array}{l}0.00 \pm 0.07 \\
0.05-0.08\end{array}$ & $\begin{array}{l}15.8 \pm 0.4 \\
14.9-15.6\end{array}$ & $1.90 \pm 0.2$ & $\begin{array}{l}3.73 \pm 0.42 \\
8.1-14\end{array}$ \\
\hline 12022,297 & $0.00 \pm 0.06$ & $0.04 \pm 0.08$ & $\begin{array}{l}16.9 \pm 0.4 \\
16.7-17.2\end{array}$ & $2.13 \pm 0.2$ & $\begin{array}{l}3.74 \pm 0.31 \\
5.0-17.6\end{array}$ \\
\hline 12075,90 & $-0.07 \pm 0.04$ & $-0.07 \pm 0.04$ & $\begin{array}{l}15.5 \pm 0.2 \\
15.7-16.8\end{array}$ & $1.68 \pm 0.2$ & $\begin{array}{l}3.81 \pm 0.26 \\
6.0-8.5\end{array}$ \\
\hline
\end{tabular}

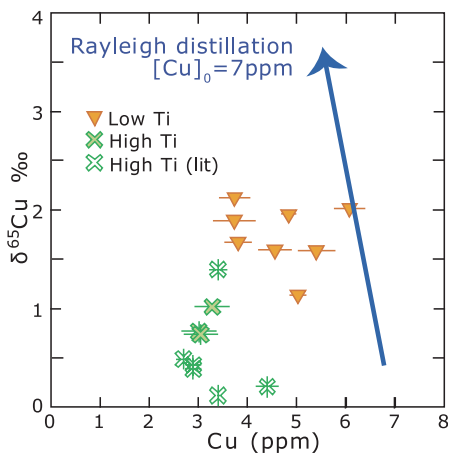

5184

SINGLE CRYSTAL ${ }^{40} \mathrm{Ar} /{ }^{39} \mathrm{Ar}$ AGES FROM GRA 06128

F. N. Lindsay ${ }^{1,3}$, B. Turrin ${ }^{2,3}$, G. F. Herzog ${ }^{1,3}$ and C. C. Swisher $\mathrm{III}^{2,3}$. ${ }^{1}$ Chemistry and Chemical Biology ${ }^{2}$ Earth Planetary Science ${ }^{3}$ Rutgers University, Piscataway, NJ 08854, USA.

Introduction: Lindsay et al. [1] reported the feasibility of dating single feldspar grains from the unusual, brachinite-like achondrite GRA 06129. An age difference of $100 \mathrm{My}$ between GRA 06128 (4.34 $\pm 0.01 \mathrm{Ga}$, [2] and GRA 06129 (4.42 $\pm 0.03 \mathrm{Ga}$, [1]) (hereafter GRA8 and GRA9) was noted $(4.34 \pm 0.01 \mathrm{Ga}$, [2] versus $4.42 \pm 0.03 \mathrm{Ga}$, [1], respectively); this discrepancy prompted us to date GRA8 using our single grain method. We now report an age for GRA8 of $4.34 \pm 0.02 \mathrm{Ga}$, which confirms the results of [2] and strengthens the arguments for a long, complicated thermal history on the parent body of these paired achondrites [3-9].

Experimental Methods: We separated 30 feldspar grains $(0.004<$ volume $\left[\mu^{3}\right]<0.112 ; 1<$ mass $\left.[\mu \mathrm{g}]<30\right)$ from GRA8 by handpicking techniques. Eight feldspar grains (endmember composition $\mathrm{An}_{25} \mathrm{Ab}_{72} \mathrm{Or}_{3^{-}}$ $\mathrm{An}_{24} \mathrm{Ab}_{73} \mathrm{Or}_{3}$, which is slightly less albitic than reported by $\left.[4,9,10]\right)$ were irradiated (with $\mathrm{Cd}$ shielding) for $80 \mathrm{~h}$ at the USGS Triga reactor along with reference minerals FC-2 sanidine (28.02 Ma) and $\mathrm{Hb} 3 \mathrm{Gr}$ amphibole (1073.6 Ma). Single grains were heated in 8-10 steps with a $\mathrm{CO}_{2}$ laser. The Ar isotopes were analyzed using a MAP 215-50 spectrometer operated in pulse-counting mode. For grains of 3-29 $\mu \mathrm{g}$ with $0.16-0.29 \mathrm{~K}$ wt $\%$, the total gas released $\left(10^{-12} \mathrm{~mol} \mathrm{~g}{ }^{-1}\right):{ }^{40} \mathrm{Ar}=3493-10826 ;{ }^{39} \mathrm{Ar}=6.54-18.15$; typical system blank $\left(10^{-18} \mathrm{~mol}\right)$ is: ${ }^{40} \mathrm{Ar}=748 \pm 24 ;{ }^{39} \mathrm{Ar}=7.7 \pm 1.0$; ${ }^{38} \mathrm{Ar}=0.6 \pm 0.2 ;{ }^{37} \mathrm{Ar}=24 \pm 0.2 ;{ }^{36} \mathrm{Ar}=2.4 \pm 0.14$. With a few exceptions, each heating increment yielded $1 / 6$ th to $1 / 8$ th of the total amount of gas.

Results and Discussion: Our single grain ages for GRA8 and GRA9 are comparable to published whole rock dates for GRA9 $(4.46 \pm 0.02$ [9], $4.39 \pm 0.04$ [11]) and a plagioclase separate date for GRA8 $(4.35 \pm 0.02$ [2]). Our method allows us to use substantially less sample (30 $\mu \mathrm{g}$ versus $3-13 \mathrm{mg}$ ) and yields spectra that are simple to interpret. Figure 1 shows the range of ages of 28 grains; we believe this range points to fine scaled heterogeneities between oligoclase grains, which may show differential amounts of resetting due to metamorphism and/or metasomatism over a span of $100 \mathrm{My}$.

References: [1] Lindsay F. et al. 2010. Abstract \#5322. Meteoritics \& Planetary Science 45. [2] Park J. et al. 2010. Abstract \#1365. 41st Lunar and Planetary Science Conference. [3] Usui T. et al. 2010. ibid. Abstract \#1186. [4] Arai T. et al. 2008. Abstract \#2465. 39th Lunar and Planetary Science Conference. [5] Zeigler R. A. et al. 2008 ibid. Abstract \#2456. [6] Mikouchi T. and Miyamoto M. 2008 ibid. Abstract \#2297. [7] Nyquist L. E. et al. 2009. Abstract \#1290. 40th Lunar and Planetary Science Conference. [8] Day J. M. D. et al. 2009. Nature 457:179-182. [9] Shearer C. K. et al. 2010 Geochimica et Cosmochimica Acta 74:1172-1199. [10] Trieman A. H. et al. 2008. Abstract \#2215. 39th Lunar and Planetary Science Conference. [11] Bajo T. et al. 2010. Meteoritics \& Planetary Science 45. Abstract \#5089.

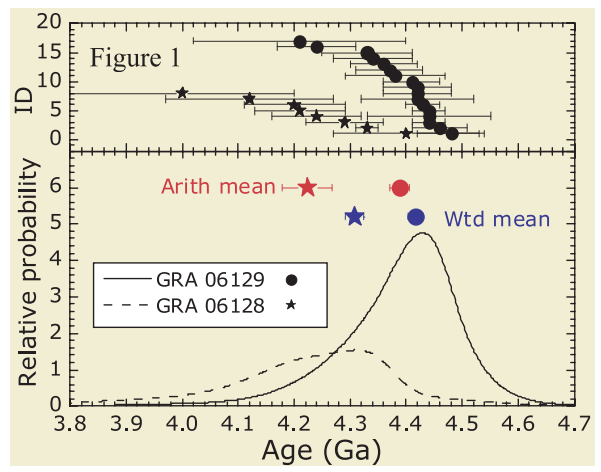


5278

\section{A HETEROGENEOUS SOLAR NEBULA AS SAMPLED BY CM HIBONITES}

Ming-Chang Liu ${ }^{1,2}$, Marc Chaussidon ${ }^{1}$, Christa Göpel ${ }^{3}$ and Typhoon Lee $^{2}$. ${ }^{1}$ CNRS-CRPG, Nancy, France. E-mail: mcliu@crpg.cnrs-nancy.fr. ${ }^{2}$ ASIAA, Taipei, Taiwan. ${ }^{3}$ IPGP, Paris, France.

Introduction: The degree of uniformity of isotope distribution in the solar nebula has long intrigued cosmochemists, as it is crucial for the use of short-lived radionuclides for chronometry. Model simulations showed that in a marginally gravitationally unstable disk, initial isotope variations, if any, could be well mixed to $\sim 10 \%$ level spatial heterogeneity within a few thousand years [1]. From this view, the formation time of refractory inclusions plays a key role in understanding the observed isotopic compositions.

Hibonite $\left(\mathrm{CaAl}_{12} \mathrm{O}_{19}\right)$, primarily found in $\mathrm{CM}$ meteorites as 20 $80 \mu \mathrm{m}$ individual inclusions in the matrix, is believed to be one of the oldest solar system solids [2]. The inferred ${ }^{26} \mathrm{Al}$ abundances split $\mathrm{CM}$ hibonite grains into two distinct groups [e.g., 3]. ${ }^{26} \mathrm{Al} /{ }^{27} \mathrm{Al}$ in individual Spinel-HIBonite spherules (SHIBs) is consistent with $\sim 5 \times 10^{-5}$ within large analytical errors. In contrast, PLAty hibonite Crystals (PLACs) display a range of $\Delta^{26} \mathrm{Mg}^{*}$ (deviation from a mass dependent fractionation line) from $+2 \%$ to $-4 \%$. Negative $\Delta^{26} \mathrm{Mg}$ in PLACs indicates that these grains must have formed prior to the presence of ${ }^{26} \mathrm{Al}$ in the solar nebula [4]. However, the chronology for SHIBs is still not clear, primarily due to large analytical uncertainties. Here we report new high precision $\mathrm{Mg}$ data of hibonite, in hopes of better constraining the formation time of SHIBs.

Experimental: Forty-three new hibonite samples (9 PLACs and 34 SHIBs) studied here were hand-picked from a Murchison acid residue (Courtesy of A. Davis), and a high density fraction of Paris meteorite, and are on average $\sim 40 \mu \mathrm{m}$ in size. High precision $\mathrm{Mg}$ isotopic analysis was performed on the Cameca 1280HR2 at CRPG-CNRS. Polished, epoxy-mounted hibonite grains were sputtered with a $20 \mathrm{nA}{ }^{16} \mathrm{O}^{-}$beam (? $\sim 30-40 \mu \mathrm{m})$. Secondary ions were collected simultaneously with four faraday cups. Instrumental mass fractionation and relative sensitivity factor were determined and corrected for by using Madagascar hibonite. The inferred ${ }^{26} \mathrm{Al} /{ }^{27} \mathrm{Al}$ for each grain was calculated by connecting the data point to the origin $\left(\Delta^{26} \mathrm{Mg} *={ }^{27} \mathrm{Al} /{ }^{24} \mathrm{Mg}=0\right)$.

Results and Discussion: The $\mathrm{Mg}$ isotopic compositions of Murchison and Paris hibonite are consistent with previous discoveries, but the higher precision enables us to resolve an ${ }^{26} \mathrm{Al} /{ }^{27} \mathrm{Al}$ scatter from 1 to $6 \times 10^{-5}$ in SHIBs, although analytical errors for grains with $6 \times 10^{-5}$ would also allow for overlapping with $5.2 \times 10^{-5}$, the "canonical ratio" defined in CV CAIs [5]. Such a variation could result from early formation of SHIBs before ${ }^{26} \mathrm{Al}$ was homogenized or contemporaneous formation of SHIBs and CAIs followed by subsequent disturbance in SHIBs. However, each measurement here is analogous to "bulk" analysis, which should be insensitive to internal re-equilibration, unless the disturbance was an open-system behavior. Furthermore, if the grains were indeed subject to loss of radiogenic ${ }^{26} \mathrm{Mg}$, one should expect to see some SHIBs without any ${ }^{26} \mathrm{Al}$. Therefore, we explain the observed ${ }^{26} \mathrm{Al} /{ }^{27} \mathrm{Al}$ variation in SHIBs as recording ${ }^{26} \mathrm{Al}$ heterogeneity in the disk, possibly prior to the CAI formation.

References: [1] Boss A. P. 2008. Earth and Planetary Science Letters 268:102-109. [2] Grossman L. 1972. Geochimica et Cosmochimica Acta 36:597-619. [3] Ireland T. R. 1988. Geochimica et Cosmochimica Acta 52:2827-2839. [4] Liu M.-C. et al. 2009. Geochimica et Cosmochimica Acta 73:5051-5079. [5] Jacobsen B. et al. 2008. Earth and Planetary Science Letters 272:353-364.

\section{7}

\section{NEW STORY OF SHERGOTTITE EETA79001}

Yang Liu ${ }^{1}$, Cyrena. A. Goodrich ${ }^{2}$ and Lawrence A. Taylor ${ }^{1}$. ${ }^{1}$ Planetary Geosciences Institute, Department of Earth and Planetary Science, University of Tennessee, Knoxville, TN 37996, USA. E-mail: yangl@utk.edu. ${ }^{2}$ Planetary Science Institute, Tucson, AZ 85719, USA.

Introduction: Elephant Moraine (EET) A79001 is a unique shergottite, containing two juxtaposing lithologies: A and B [1]. The origin of Lithology A has been a point of debate since its collection (e.g., [1-5]). Here we report new observations suggesting assimilation/mixing at high $\mathrm{P}$ and followed by crystallization in a closed system.

Mineral Inclusions in Lithology A: We observed round olivine and orthopyroxene (opx) inclusions in olivine (ol) megacrysts. The round opx inclusion is $\mathrm{Mg}$-rich in the interior $\left(\mathrm{Mg} \# 79.6, \mathrm{En}_{78} \mathrm{Wo}_{2}\right)$ and more $\mathrm{Fe}$ rich at the rim near the interface $\left(\mathrm{Mg} \# 76.5, \mathrm{En}_{77} \mathrm{Wo}_{2}\right)$, both of which are NOT in equilibrium with the host olivine ( $\mathrm{Mg \#} \mathrm{67).} \mathrm{This} \mathrm{is} \mathrm{opposite} \mathrm{to}$ what is expected for crystallization at low $\mathrm{P}$, where ol would be enclosed by opx. A subhedral ol $\left(\mathrm{Fo}_{68}\right)$ is also enclosed in a megacrystic ol $\left(\mathrm{Fo}_{68}\right)$. The similar composition between ol inclusion and host can be explained by re-equilibration as the $\mathrm{Fe}-\mathrm{Mg}$ diffusion is fast in olivines.

The round shape of the mineral inclusions indicates resorption event(s) due to interaction with melt. Two possible scenarios can cause dissolution (1) mixing with melt of a different composition; (2) decrease in pressure so that opx becomes unstable. If (2) is the sole process, we would expect more Mg-rich ol $(\mathrm{Mg} \#>70)$ than Mg-rich opx. This is not consistent with the available literature data $[1,6]$, where there is comparable modal abundances of Mg-rich olivine and orthopyroxene. Furthermore, occurrence of $\mathrm{Mg}$-rich ol and opx as individual megacrysts in other sections $[1,4,5]$ indicates both $\mathrm{Mg}$-rich ol and opx are stable liquidus phases, a feature typical for crystallization at high P [7].

"Melt" Inclusions in Olivine: We measured bulk trace element abundances of "melt" (now devitrified) inclusions in olivines using LAICP-MS. Melt inclusions in Mg-rich olivines $\left(\mathrm{Fo}_{75-73}\right)$ suggest that these olivines crystallized from incompatible-element depleted melt ([La/ $\mathrm{Yb}]_{\mathrm{N}}=0.04-0.15, \mathrm{~N}$ denotes $\mathrm{CI}$ chondrite normalized), similar to shergottites with depleted incompatible elements (e.g., Yamato-980459, [8]). Melt inclusions from more Fe-rich olivines is similar to whole-rock data of EETA79001B.

Implications: Based on the above observations, we suggest that EETA79001A was formed by: (1) crystallization of Mg-rich ol + opx at high $\mathrm{P}$; (2) mixing at high $\mathrm{P}$ with a magma containing slightly more enriched incompatible elements (not enriched basaltic shergottites!) that assimilated some ol + opx; (3) crystallization of megacrystic ol and opx at high P, (4) eruption/ascent to lower P. Different from previous models, we emphasize that (1)-(3) occurred at pressure $>0.7 \mathrm{GPa}(>60 \mathrm{~km})$ (inferred from [7]).

References: [1] McSween H. Y. and Jarosewich E. 1983. Geochimica et Cosmochimica Acta 47:1501-1513. [2] Wadhwa M. et al. 1994. Geochimica et Cosmochimica Acta 58:4213-4229. [3] Mittlefehldt D. W. et al. Meteoritics \& Planetary Science 34:357-367. [4] Steele I. M. and Smith J. V. Proceedings, 13th Lunar and Planetary Science Conference, JGR, 87, supplement, A375-384. [5] Goodrich C. A. 2003. Geochimica et Cosmochimica Acta 67:3735-3771. [6] Melin M.2008. Masters thesis. [7] Filiberto J. and Treiman A. H. 2009. Chemical Geology 263:60-68. [8] Shirai N. and Ebihara M. 2004. Abstract \#1511. 35th Lunar and Planetary Science Conference. 
5260

\section{CALCULATIONS ON PHOTOPHORETIC MOTION OF CHONDRULES IN THE EARLY SOLAR SYSTEM AND TEMPERATURE DISTRIBUTIONS IN ILLUMINATED CHONDRULES}

C. Loesche ${ }^{1}$, J. M. Friedrich ${ }^{2}$, J. Teiser ${ }^{1}$ and G. Wurm ${ }^{1}$. ${ }^{1}$ Faculty for Physics, University Duisburg-Essen, AG Wurm. E-mail: christoph. loesche@uni-due.de. ${ }^{2}$ Department of Chemistry, Fordham University, Bronx, New York.

Chondrules play an important role in the formation process of planets [1]. We study if the effect of photophoresis might transport particles to the asteroid belt. Photophoresis has been proposed as an efficient mechanism for radial material transport. However, these models are based on idealized assumptions concerning the particle geometry and material properties $[2,3]$. We present calculations/simulations in order to complement ongoing lab experiments on photophoresis and to develop more realistic transport models.

First, tomographic data from chondrules of the L/LL4 chondrite Bjurböle are used to extract the geometric shape and to define material functions using Mathematica-Code. After that, a finite elements method (FEM) is used to calculate the temperature distribution inside the tomographed chondrules resulting from solar irradiation. With these results it is possible to improve the understanding of chondrule motion and sorting in the early solar system as a result from the photophoretic force.

Currently first preliminary results are available. Comparison with experimental data by Hesse et al. (in prep.) shows that the numerical simulations give realistic data, including effects of rotating chondrules under irradiation. Further goals are to get information about torques influencing chondrule rotation and to improve materials equations of state.

References: [1] Scott E. R. D. 2007. Annual Review of Earth and Planetary Science 35:577-620. [2] Wurm G. and Krauss O. 2006. Icarus 180:487-495. [3] Wurm G. and Haack H. Meteoritics \& Planetary Science 44:689-699.

Additional Information: If you have any questions regarding the preparation of your abstract, call the LPI at 281-486-2142 or -2188 (or send an e-mail message to publish@lpi.usra.edu).

\section{6}

SPATIAL ANALYSIS OF PLANAR DEFORMATION FEATURES

A. Losiak ${ }^{1}$, I. Golebiowska ${ }^{2}$ and C. Koeberl ${ }^{1,3}$. ${ }^{1}$ Dept. Lithospheric Research, Univ. Vienna, 1090 Vienna, Austria. E-mail: anna.losiak @ univie.ac.at. ${ }^{2}$ Univ. Warsaw, Faculty of Geography and Regional Studies, Poland. ${ }^{3}$ Natural History Museum, 1010 Vienna, Austria.

Introduction: Planar deformation features (PDFs) are multiple sets of parallel planes of amorphous material in minerals, $\leq 2 \mu \mathrm{m}$ thick and spaced $2-10 \mu \mathrm{m}$ apart, which form due to shock in specific crystallographic orientations [e.g., 1, 2]. PDFs are usually measured on randomly selected grains from a thin section, thus microtextural context information is lost. We attempt to measure PDF orientations of all quartz grains within the field of view in a thin section to check if any spatial relations exist between grains containing PDFs. This project will help the understanding of PDF formation mechanisms and shock wave propagation on the single grain scale.

Methods: Planar deformation features are measured using a U-stage and indexed using the program of [3]. Care has been taken to keep a constant orientation of the thin section in relation to the U-stage, ensuring that all measured azimuths have the same reference system. All measured grains were referenced on a map of a thin section based on a microphotograph. A database with information about the orientation of c-axes and PDFs as well as the number and type of PDFs present in each grain was referenced to a thin section photo-map using ArcGIS software. Spatial associations between grains in relation to their various characteristics (e.g., number of PDFs present) were analyzed.

The studied test sample is a meta-graywacke from the Bosumtwi crater (Ghana) from $271.4 \mathrm{~m}$ depth in the LB-08A drill core [e.g., 4]. It consists mostly of large multigrain aggregates of quartz $(\leq 1 \mathrm{~mm})$ and large feldspar grains $(\leq 1.5 \mathrm{~mm})$ set in a fine-grained crystalline matrix.

Results: We measured 102 quartz grains within a total area of $9.62 \mathrm{~mm}^{2}$; the average number of PDFs per grain was 1.76 (including 15 grains with no PDFs). The following observations were made: (1) quartz grains with no PDFs are located on the external part of the quartz aggregates, while ones with a strongly distorted crystallographic lattice are located in the center of quartz aggregates; (2) grains sharing boundaries often contain similar numbers of PDFs; (3) grains sharing borders and having similar orientations of their c-axes can have different numbers of PDFs; (4) grains with the same PDF indices tend to occur close to each other.

Discussion: Firstly, clustering of PDFs of specific types suggest that, on a micro-scale, the formation of PDFs within the grain is dependent on the surroundings of the grain. Secondly, the angle between the c-axis of a quartz grain and the shock wave propagation direction is not a main factor influencing development of PDFs. However, it is also possible that the observed differences in a number of PDFs are due to differences in the c-axis orientation (Dauphiné twining). Thirdly, stress within quartz aggregates is not distributed evenly.

References: [1] Stöffler D. and Langenhorst F. 1994. Meteoritics \& Planetary Science 29:155-181. [2] Ferrière L. et al. 2009. Meteoritics \& Planetary Science 44:925-940. [3] Losiak A. et al. 2011. Abstract \#1286. 42nd Lunar and Planetary Science Conference. [4] Ferrière L. et al. 2008. Science 322:1678-1681. 


\section{4}

ISOTOPIC CONSTRAINTS OF MODERATELY VOLATILE AND SIDEROPHILE ELEMENTS DURING CORE FORMATION

B. Luais. CRPG-CNRS, Nancy-Université, BP 20, 54501 Vandoeuvre-lèsNancy Cedex, France. E-mail: luais@crpg.cnrs-nancy.fr.

Introduction: Scenarios of core-mantle formation and subsequent processes of planetary accretion leading to the observed elemental composition of the Earth upper mantle are based on experimental metalsilicate partitioning of siderophile elements. Recent models require homogeneous accretion at high $P \quad(\approx 30-40 \mathrm{GPa}), \quad T \quad\left(\approx 3000^{\circ} \mathrm{K}\right)$ conditions with an increase with time of oxygen fugacity to account for the budget (depletion) of moderately siderophile elements in the mantle $[1,2]$. However, these models differ on the $\left(P, T, f \mathrm{O}_{2}\right)$ conditions relevant to the depletion of the moderately volatile siderophile element (e.g., $\mathrm{Ge}$ ) in the Earth upper mantle. These elements provide additional constraints on the possible loss of volatile elements during impact processes, which are linked to the main metal-silicate differentiation stage. Specifically, isotopes of these elements can be strongly fractionated during evaporation processes, which makes isotopic composition an appropriate tool for testing the role of volatility processes.

Results and Discussion: New germanium isotopic data $\left(\delta^{74} \mathrm{Ge}\right.$ relative to JMC standard, $2 \sigma \pm 0.2 \%$ ) were obtained on Earth silicate mantle (peridotites, basalts) and carbonaceous chondrites (CC), and then compared with Ge isotopic composition of iron meteorites [3]. Earth silicate mantle samples have $\delta^{74} \mathrm{Ge}=+0.892 \pm 0.062 \%$, which is significantly lower than the mean value for magmatic iron meteorites $\left(\delta^{74} \mathrm{Ge}=+1.77 \pm 0.22 \%\right.$ o $)$. First $\delta^{74} \mathrm{Ge}$ data of some carbonaceous chondrites indicate light isotopic composition.

The heavy Ge isotopic composition of Fe-meteorites and Earth silicates with respect to $\mathrm{CC}$ would indicate a major loss of $\mathrm{Ge}$ during core formation, most probably due to evaporation induced by a Moonforming impact. This isotopic effect of evaporation leads to a strong isotopic fractionation which would hide the small if detectable kinetic isotopic fractionation associated to diffusion of Ge from the silicate to the metal phase under the reducing conditions of metal-silicate segregation [4]. The light Ge isotopic composition of the Earth silicate mantle cannot represent the resulting silicate phase (Bulk Silicate Earth) after core segregation, this silicate depleted reservoir $(\approx 1 \mathrm{ppm} \mathrm{Ge})$ would have in this case heavier $\mathrm{Ge}$ isotopic composition than the core. It is suggested that the $\delta^{74} \mathrm{Ge}$ value of $+0.892 \%$ is representative of the Earth Upper Mantle. The Ge isotopic composition of the silicate phase at the $P, T, f \mathrm{O}_{2}$ conditions of core segregation would reflect the change in oxidation state and valence of Ge associated with pressure [1,2].

References: [1] Siebert J. et al. 2011. Geochimica et Cosmochimica Acta 62:1631-1642. [2] Righter K. 2011. Earth and Planetary Science Letters 304:379-388. [3] Luais B. 2007. Earth and Planetary Science Letters 262:21-36. [4] Luais et al. 2007. Eos Trans. AGU, 88(52), Fall Meet. Suppl. Abstract \# V51E-0833.
5520

PHOTOCHEMISTRY AND THE OBSERVED ENRICHMENT OF O, C, N, AND H ISOTOPES IN METEORITE IOM

J. R. Lyons ${ }^{1}$. ${ }^{1}$ Institute of Geophysics \& Planetary Physics, UCLA, Los Angeles, CA 90095-1567, USA. E-mail: jimlyons@ucla.edu.

Introduction: The remarkable $\mathrm{O}$ MIF signatures seen in acidinsoluble organic matter (IOM) from a CR2 meteorite [1] show some correlation with enhanced $\delta^{13} \mathrm{C}$ values, suggesting that both enrichments may be a result of $\mathrm{CO}$ photochemistry. ${ }^{2} \mathrm{H}$ and ${ }^{15} \mathrm{~N}$ enrichments in the same IOM do not correlate with ${ }^{17} \mathrm{O}$ and ${ }^{18} \mathrm{O}$ and $/$ or ${ }^{13} \mathrm{C}$ enrichments, but do show some correlation with each other [1]. Both $\mathrm{N}$ and $\mathrm{H}$ isotopes have been shown to be strongly fractionated by ion-molecule reactions in molecular clouds $[2,3]$. Here, the question I address is whether these results, particularly those for meteorite IOM [1], are consistent with expected photochemical and ion-molecule processes.

Photochemical Models of Clouds and the Solar Nebula: It is well established that in model calculations $\mathrm{CO}$ self-shielding produces large enrichment in product ${ }^{17} \mathrm{O}$ and ${ }^{18} \mathrm{O}$, which are then stored in $\mathrm{H}_{2} \mathrm{O}$. Product $\mathrm{C}$ is similarly enriched in ${ }^{13} \mathrm{C}$, although by a factor of $\sim 2$ less than the enrichment in ${ }^{17} \mathrm{O}$ and ${ }^{18} \mathrm{O}$ [4]. Self-shielding enrichment of ${ }^{13} \mathrm{C}$ is predicted by photochemical models of disks [5], but $\mathrm{CO}$ exchange with $\mathrm{C}^{+}$and $\mathrm{CO}$ condensation [6, 7], are also important. Disk model calculations, including ${ }^{13} \mathrm{C}$ at temperatures well above $\mathrm{CO}$ condensation ( $>20 \mathrm{~K}$ ), are in progress using the oxygen isotope code of [8]. Shielding functions from [4] are used for $\mathrm{C}^{13} \mathrm{O}$. As with $\mathrm{CO}, \mathrm{N}_{2}$ also undergoes selfshielding, producing enriched $\mathrm{N}$ and $\mathrm{N}\left({ }^{2} \mathrm{D}\right)$, with the $\mathrm{N}$ stored in $\mathrm{HCN}$. The $\mathrm{N}\left({ }^{2} \mathrm{D}\right)$ rapidly forms $\mathrm{NH}$, leading to reformation of $\mathrm{N}_{2}$ by reaction of $\mathrm{N}$ and $\mathrm{NH}$, and thus erasing much of the photo-induced fractionation. Large initial ${ }^{15} \mathrm{~N}$ enrichments are possible by $\mathrm{N}_{2}$ self-shielding, but the total amount of enriched material produced is small [9]. Finally, $\mathrm{H}_{2}$ also has a line-type absorption spectrum, and so also undergoes self-shielding at the edges of clouds and high surface of disks. At greater depths into these objects, where $\mathrm{CO}$ and $\mathrm{N}_{2}$ self-shielding become important, $\mathrm{H}_{2}$ lines are highly broadened and will shield HD lines, thus reducing D enrichment. These results are consistent with a photochemical origin for $\mathrm{O}$ and (possibly) $\mathrm{C}$ isotopes signatures in IOM, and ion-molecule origin for $\mathrm{H}$ and $\mathrm{N}$ isotopes signatures in IOM.

References: [1] Hashizume K. et al. 2011. Nature Geoscience 4:165. [2] Rodgers S. D. and Charnley S. B. 2008. MNRAS 385:L48. [3] Aleon J. and Robert F. 2004. Icarus 167: 424. [4] Visser R. et al. 2009. Astronomy \& Astrophysics 503: 323. [5] Woods P. and Willacy K. 2009. The Astrophysical Journal 693: 1360. [6] Smith R. et al. 2011. Abstract \#1281. 42nd Lunar and Planetary Science Conference. [7] Young E. and Schauble E. 2011. Abstract \#1323. 42nd Lunar and Planetary Science Conference. [8] Lyons J. R. and Young E. D. 2005. Nature 435:317-320. [9] Lyons J. R. 2010. Abstract \#5424. 73rd MetSoc. 
5169

DISCOVERY OF METEORITIC LAKARGIITE $\left(\mathrm{CaZrO}_{3}\right)$, A NEW ULTRAREFRCTORY MINERAL FROM THE ACFER 094 CARBONACEOUS CHONDRITE

Chi Ma. Division of Geological and Planetary Sciences, California Institute of Technology, Pasadena, CA 91125, USA. E-mail: chi@gps.caltech.edu

Introduction: During a nano-mineralogy investigation of the Acfer 094 carbonaceous chondrite, lakargiite $\left(\mathrm{CaZrO}_{3}\right)$ was identified as submicrometer inclusions in an isolated hibonite grain in section USNM 7233-1. The hibonite grain is described in Simon and Grossman [1]. Lakargiite is a recently found perovskite-group mineral from hightemperature skarns in ignimbrites of the Upper-Chegem volcanic structure, the North Caucasus, Russia [2]. Reported here is the first extraterrestrial occurrence of lakargiite, as a new ultrarefractory mineral in a primitive meteorite, among the first solids formed in the solar system. Field-emission SEM with EDS and electron back-scatter diffraction (EBSD) was used to characterize its composition and structure and associated phases.

Occurrence, Chemistry, and Crystallography: Lakargiite $\left[\mathrm{Ca}_{0.95}\left(\mathrm{Zr}_{0.87}\right.\right.$ $\left.\left.\mathrm{Ti}_{0.16}\right) \mathrm{O}_{3}\right]$ occurs along with tazheranite $\left[\left(\mathrm{Zr}_{0.52} \mathrm{Ti}_{0.18} \mathrm{Ca}_{0.16} \mathrm{Y}_{0.06} \mathrm{Fe}_{0.05}\right.\right.$ $\left.\mathrm{Sc}_{0.03}\right) \mathrm{O}_{1.75}$, Zr-bearing perovskite $\left[\mathrm{Ca}_{0.94}\left(\mathrm{Ti}_{0.98} \mathrm{Zr}_{0.06}\right) \mathrm{O}_{3}\right]$ and Os-W-alloy $\left[\mathrm{Os}_{0.81} \mathrm{~W}_{0.13} \mathrm{Fe}_{0.06}\right]$ as fine-inclusions scattered in the central area of the hibonite $\left[\mathrm{Ca}\left(\mathrm{Al}_{11.70} \mathrm{Ti}_{0.14} \mathrm{Fe}_{0.10} \mathrm{Mg}_{0.06}\right) \mathrm{O}_{19}\right]$ grain (Fig. 1). Lakargiite appears as irregular or subhedral lath-shaped grains, $300-900 \mathrm{~nm}$ in size, sometimes in contact with Os-W or perovskite. Associated tazheranite is a Ca-stabilized cubic zirconia (CSZ), likely being the first reported meteoritic CSZ. EBSD analysis revealed that the lakargiite has a perovskite Pbnm structure, identical to that of synthetic $\mathrm{CaZrO}_{3}$ [3], showing $a=5.591 \AA, b=5.762 \AA, c=8.017 \AA, V=258.3 \AA^{3}, Z=4$.

Origin and Significance: Lakargiite is a new Zr-dominant ultrarefractory mineral, joining the Zr-rich refractory minerals allendeite $\left(\mathrm{Sc}_{4} \mathrm{Zr}_{3} \mathrm{O}_{12}\right)$ [4], tazheranite (Sc- or $\mathrm{Y}$ - stabilized cubic zirconia) $[4,5]$ and panguite [6]. Texturally, lakargiite, tazheranite, $\mathrm{Zr}$-bearing perovskite and Os-W formed before host hibonite. Lakargiite is likely an early condensate. Further work is under way to determine its place in the nebula.

References: [1] Simon S. B. and Grossman L. 2011. Meteoritics \& Planetary Science, in press. [2] Galuskin E. V. et al. 2008. American Mineralogist 93:1903-1910. [3] Koopmanns H. J. A. et al. 1983. Acta Crystallographica Section C 39:1323-1325. [4] Ma C. et al. 2009. Abstract \#1402. 40th Lunar and Planetary Science Conference. [5] Ma C. and Rossman G. R. 2008. Geochimica et Cosmochimica Acta 72:12S, A577. [6] Ma C. et al. 2011. Abstract \#1276. 42nd Lunar and Planetary Science Conference.

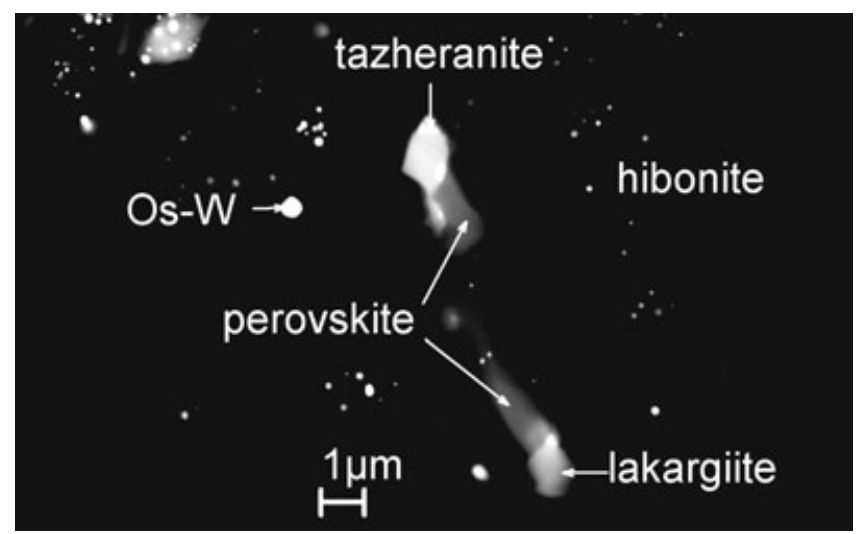

Fig 1. BSE image showing lakargiite in Acfer 094.

\section{1}

THORTVEITITE $\left(\mathrm{Sc}_{2} \mathrm{Si}_{2} \mathrm{O}_{7}\right)$, THE FIRST SOLAR SILICATE?

Chi Ma ${ }^{1}$, John R. Beckett ${ }^{1}$, Oliver Tschauner $^{1,2}$ and George R. Rossman ${ }^{1}$. ${ }^{1}$ Division of Geological and Planetary Sciences, California Institute of Technology, Pasadena, CA 91125, USA. ${ }^{2}$ High Pressure Science and Engineering Center and Department of Geoscience, University of Nevada, Las Vegas, NV 89154, USA. E-mail: chi@gps.caltech.edu.

Introduction: In condensation calculations for a cooling gas of solar composition, or dust enriched variants, melilite is invariably the earliest condensing silicate except at very high dust-to-gas ratios [e.g., 1]. The possibility that silicates of the highly refractory lithophiles, such as Sc and $\mathrm{Zr}$, actually form first is not tested because of a lack of thermodynamic data. Meteoritic occurrences may, therefore, provide the best clues to the earliest evolution of $\mathrm{Si}$ in nebular solids. During a nano-mineralogy investigation of the Murchison CM2 carbonaceous chondrite, we identified thortveitite $\left(\mathrm{Sc}_{2} \mathrm{Si}_{2} \mathrm{O}_{7}\right)$, along with davisite $\left(\mathrm{CaScAlSiO}{ }_{6}\right)$, panguite $\left[(\mathrm{Ti}, \mathrm{Sc}, \mathrm{Al}, \mathrm{Mg})_{1.8} \mathrm{O}_{3}\right]$, spinel, and $\mathrm{Sc}$-rich diopside, in the Sc-enriched ultrarefractory inclusion MUR1. This is the first meteoritic occurrence of thortveitite and the second natural occurrence of panguite, a Ti-rich oxide recently discovered in Allende [2]. Field-emission SEM with EDS, electron back-scatter diffraction, and electron microprobe were used to characterize the compositions and structures of these two minerals and associated phases.

Occurrence, Chemistry, and Crystallography: Thortveitite $\left[\left(\mathrm{Sc}_{1.60}\right.\right.$ $\left.\left.\mathrm{Zr}_{0.13} \mathrm{Ti}_{0.08} \mathrm{Mg}_{0.06} \mathrm{Y}_{0.04} \mathrm{Fe}_{0.04} \mathrm{Ca}_{0.02}\right)\left(\mathrm{Si}_{1.91} \mathrm{Ti}_{0.08} \mathrm{Al}_{0.01}\right) \mathrm{O}_{7}\right]$ occurs with davisite (15 wt $\left.\% \quad \mathrm{Sc}_{2} \mathrm{O}_{3}\right)$, panguite $\left[\left(\mathrm{Ti}_{0.71} \mathrm{Sc}_{0.40} \mathrm{Al}_{0.24} \quad \mathrm{Mg}_{0.14} \mathrm{Si}_{0.08} \mathrm{Fe}_{0.06}\right.\right.$ $\left.\left.\mathrm{Zr}_{0.05} \mathrm{Cr}_{0.05} \mathrm{Ca}_{0.05} \mathrm{Y}_{0.03}\right) \quad \sum_{1.82} \mathrm{O}_{3}\right]$, and $\mathrm{MgAl}$-spinel. These phases form clots that are in 2-D mostly or entirely enclosed in Sc-bearing diopside (3-8 wt $\% \quad \mathrm{Sc}_{2} \mathrm{O}_{3}$ ). There is a step function in composition between davisites and the diopside where in contact. Panguite appears as irregular grains, $0.5-2 \mu \mathrm{m}$ in size, along with fine-grained spinel within davisite or serpentinized regions, presumably after davisite. The thortveitite is subhedral, $1.5 \times 3.5 \mu \mathrm{m}$ to $6.5 \times 9.0 \mu \mathrm{m}$, and is in contact with davisite/ alteration or diopside. Where in contact with davisite, thortveitite often appears embayed, suggestive of a reaction relationship. Thortveitite appears to be inclusion free.

Origin and Significance: With the discovery of thortveitite, the Sc-, $\mathrm{Zr}$-rich menagerie in carbonaceous chondrites now includes thortveitite, panguite, davisite, allendeite $\left(\mathrm{Sc}_{4} \mathrm{Zr}_{3} \mathrm{O}_{12}\right)$ [3], tazheranite (Sc- and/or Ystabilized cubic zirconia) [3, 4], and lakargiite $\left(\mathrm{CaZrO}_{3}\right)$ [5]. MUR1 contains three of these Sc-enriched phases, thortveitite, panguite and davisite. Texturally, thortveitite formed first in MUR1, possibly with later partial reaction with vapor (?) to form davisite + spinel + panguite. Molar Sc/Zr of MUR1 thortveitite (approximately 12) is intermediate between davisite (approximately 15) and panguite (approximately 9), consistent with conservation of $\mathrm{Sc} / \mathrm{Zr}$ in a reaction between thortveitite and vapor (source of $\mathrm{Ti}, \mathrm{Ca}, \mathrm{Mg}$, and $\mathrm{Al}$ ) to produce davisite + panguite + spinel. Since $\mathrm{Sc} / \mathrm{Zr}$ in all of these Sc-, Zr-bearing phases are much higher than solar (1.2), prior fractionation of a separate $\mathrm{Zr}$-rich phase may be indicated. Further work is required to determine MUR1's place in the nebular framework but, if the thorveitite is an early condensate, it may well be the first silicate and it certainly is more refractory than melilite. Indeed, davisite, which appears to be a reaction product of thortveitite in MUR1, may also predate melilite.

References: [1] Ebel D. S. and Grossman L. 2000. Geochimica et Cosmochimica Acta 64:339-366. [2] Ma C. et al. 2011. Abstract \#1276. 42nd Lunar and Planetary Science Conference. [3] Ma C. et al. 2009. Abstract \#1402. 40th Lunar and Planetary Science Conference. [4] Ma C. and Rossman G. R. 2008. Geochimica et Cosmochimica Acta 72:12S, A577. [5] Ma C. 2011. 74th Annual Meeeting of the Meteoritical Society. Abstract \#5169. 
5093

POROSITY, MAGNETIC SUSCEPTIBILITY, AND DENSITY OF LUNAR METEORITES

R. J. Macke ${ }^{1}$, D. T. Britt ${ }^{1}$, W. S. Kiefer ${ }^{2}$, A. J. Irving ${ }^{3}$ and G. J. Consolmagno ${ }^{4}{ }^{1}$ Department of Physics, University of Central Florida. E-mail: macke@jesuits.net. ${ }^{2}$ Lunar and Planetary Institute. ${ }^{3}$ Department of Earth and Space Sciences, University of Washington Seattle. ${ }^{4}$ Specola Vaticana.

Introduction: The interpretation of gravimetric and topographical data from lunar missions to model the Moon's interior requires a comprehensive database of lunar rock densities and porosities. To date we have measured porosity, density and magnetic susceptibility for five Apollo lunar samples and 50 stones from 44 of the 74 known lunar meteorites, representing a wide range of lithologies and lunar regions. Our measurement methods as outlined in Consolmagno [1] are fast, nondestructive and non-contaminating. Grain density is measured by helium ideal-gas pycnometry, bulk density by the glass bead method developed by Consolmagno and Britt [2]. Porosity is calculated directly from bulk and grain densities. We also measure magnetic susceptibility, using an SM-30 magnetic susceptibility meter [3].

Basalts: The low- $\mathrm{Ti}\left(1-5 \mathrm{wt} \% \mathrm{TiO}_{2}\right)$ basalts measured here group tightly in grain density (avg. $3.30 \pm 0.06 \mathrm{~g} \mathrm{~cm}^{-3}$ ) and magnetic susceptibility $(\log \chi=2.86 \pm 0.11)$. Porosities range from zero to $10 \%$, averaging $5.6 \%$. There is no discernible difference between the two Apollo basalts (12051 and 15555) and the six basaltic meteorites taken as a whole.

Feldspathic Breccias: Grain densities averaged $2.85 \pm 0.11 \mathrm{~g} \mathrm{~cm}^{-3}$. Magnetic susceptibilities varied over a wide range, from $\log \chi=2.1$ to 4.5. Two distinct populations are visible in porosity, with most below $8 \%$ porous, but a few (most notably all stones of the pairing NWA 2995/ $2996 / 4503 / 5151 / 6252$ ) have a porosity in excess of $10 \%$. These samples represent a mix of regolith breccias, fragmental breccias, and impact-melt breccias.

Other Breccias: Diverse lithologies (including KREEP-rich breccias) occupy a different range of density and magnetic susceptibility than the other two groups. The meteorites in this group have low porosities (like the felsdpathic breccias and basalts), but in contrast Apollo breccias have porosities above $\sim 15 \%$.

Discussion: The data presented here are early results of an ongoing study. Much work still remains to be done, including further measurements already in progress of both meteorites and Apollo samples. Expanding the database of Apollo samples will help address issues about the porosity of in-situ breccias as opposed to meteorite breccias. It will also fill in missing data with the inclusion of thus-far unrepresented highTi $\left(9-14 \mathrm{wt} \% \mathrm{TiO}_{2}\right)$ basalts as well as expanding the representation of low-Ti basalts. In addition, the Apollo samples have known geologic contexts and include lithologies that have not been recognized in the lunar meteorite collection.

Acknowledgments: CAPTEM, Caroline Smith (NHM), Carl Agee (UNM), Kevin Righter (NASA-JSC), Ted Bunch (NAU), Randy Korotev (WUSTL), Ian Nicklin (ROM), Philip Mani, Gregory Hupé, Don Edwards, and Andrew Abraham. NASA Planetary Geology and Geophysics Program grant NNX09AD91G.

References: [1] Consolmagno G. J. et al. 2008. Chemie der Erde-Geochemistry 68:1-29. [2] Consolmagno G. J. and Britt D. T. 1998. Meteoritics \& Planetary Science 33:1231-1241. [3] Gattacceca J. et al. 2004. Geophysical Journal International 158:42-49.
5159

\section{SCHREIBERSITES IN MINCY AND VACA MUERTA MESOSIDERITES}

D. E. Magnelli ${ }^{1}$, M. E. Varela ${ }^{1}$ and F. Brandstätter ${ }^{2} .{ }^{1}$ ICATECONICET, San Juan, Argentina. ${ }^{2}$ Naturhistorisches Museum, Vienna, Austria.

Introduction: The iron-nickel phosphide schreibersite is a common accessory phase in many iron and iron-rich meteorites [1]. In mesosiderites, it is found as disseminated crystals characterized by a high $\mathrm{Ni}$ content (approximately $50 \mathrm{wt} \%$ ) [2]. This contribution is a report on petrographic features and chemical compositions of schreibersites from Mincy (nine objects) and Vaca Muerta (four objects) mesosiderites.

Samples and Methods: The studied PTS are Vaca Muerta K and Mincy L from NHMV. Electron microprobe measurements were performed with an ARL-SEMQ at NHMV.

Results: Phosphide $(\mathrm{Ph})$ crystals are totally or partially (e.g., metal + silicate) enveloped by metal. The schreibersite/silicate interfaces are irregular with schreibersite copying silicates edges. Conversely, schreibersite shows regular - occasionally euhedrally outlined-faces when it is enveloped by metal (kamacite $[\alpha \mathrm{Fe}]$ and/or taenite $[\gamma \mathrm{Fe}]$ ). The grain size is quite variable and ranges from $20 \mu \mathrm{m}$ up to $300 \mu \mathrm{m}$. Nickel profiles performed across $\mathrm{Ph}+\alpha \mathrm{Fe}$ on Mincy (Fig.) are in agreement with those previously measured on Emery [3]. We also show the distribution of $\mathrm{Ni}$ when schreibersite is in contact with taenite $(\gamma \mathrm{Fe})$.

Acknowledgment: The present research was supported by Agencia PICT 212.

References: [1] Skála R. and Císarová I. 2005. Physics and Chemistry of minerals 31:721-732. [2] Powell B. N. 1971. Geochimica et Cosmochimica Acta 35:5-34. [3] Kulpecz A. A. and Hewins R. H. 1978. Geochimica et Cosmochimica Acta 42:1495-1500.

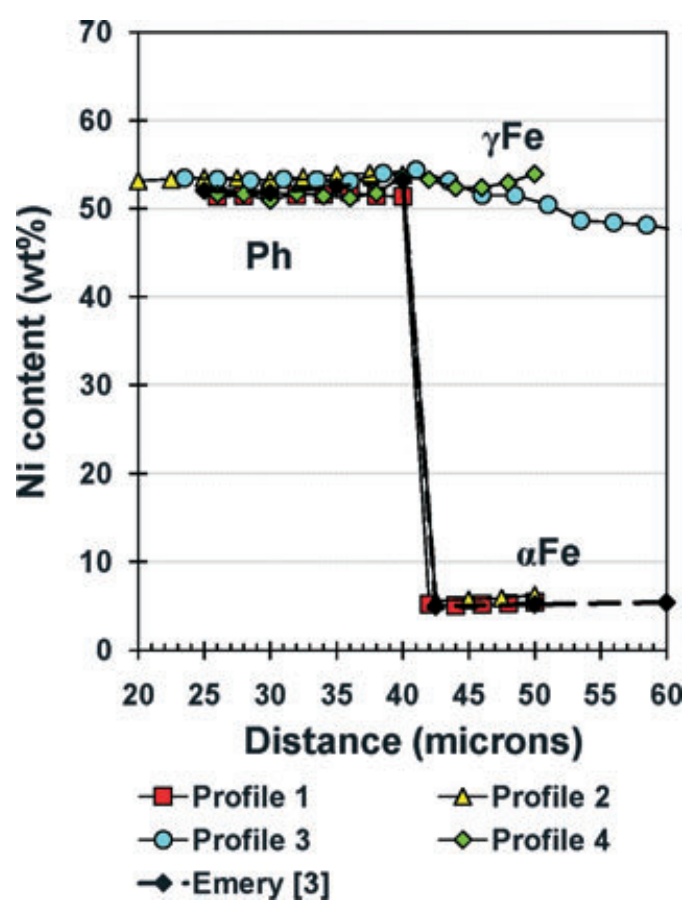


5157

EQUILIBRIUM TEMPERATURE ESTIMATIONS ON COEXISTING PHASES IN MESOSIDERITES

D. E. Magnelli ${ }^{1}$, M. E. Varela ${ }^{1}$, F. Brandstätter $^{2}$ and R. B. Scorzelli ${ }^{3} .{ }^{1}$ ICATE-CONICET, San Juan, Argentina. E-mail: dmagnelli@ icate-conicet.gob.ar. ${ }^{2}$ Naturhistorisches Museum, Vienna, Austria. ${ }^{3}$ Centro Brasileiro de Pesquisas Físicas, RJ, Brazil.

Introduction: The thermal history of mesosiderites is not totally understood yet. Materials with different physical and chemical properties (e.g., silicates, oxides, sulfides, and metal) make up the whole rock, showing a heterogeneous mineral distribution with variable degrees of recrystallization. Here we report on new equilibrium temperature calculations made on the silicate pairs high-Ca/low-Ca pyroxene and low$\mathrm{Ca}$ pyroxene/olivine. We took into account only clinopyroxene and orthopyroxene exsolutions present as lamellae and/or blebs.

Methods: To assess equilibria among pyroxene-pyroxene and low$\mathrm{Ca}$ pyroxene-olivine pairs, we have used the oxythermobarometry program QUILF [1]. The program was run using 1 bar as pressure condition since mesosiderites silicates have been considered to be part of shallow crustal levels [e.g., 2, 3].

Samples: The studied PTS are Vaca Muerta K, Vaca Muerta O, Crab Orchard L3771, Mincy L, Estherville M2072 (from NHMV) and ALHA77219,74-10, Asuka-87106.51.3 and Asuka-882023.51-3 (from NIPR).

Results and Discussion: For thermometric purposes, the molar compositions of all involved phases were recalculated into the scheme $\mathrm{Mg}-\mathrm{Fe}-\mathrm{Ca}$ according to QUILF projection. Almost all compositions corresponding to both, high- and low-Ca pyroxenes nearly fit the equilibrium model in $\mathrm{Fe}-\mathrm{Mg}$ exchange. The equilibrium temperatures fall in the range from $872{ }^{\circ} \mathrm{C} \pm 49{ }^{\circ} \mathrm{C}$ to $1057{ }^{\circ} \mathrm{C} \pm 28^{\circ} \mathrm{C}$. This temperature range is in agreement with that previously estimated for Budulan and Mincy [4]. For Crab Orchard, QUILF gives temperatures with relatively large uncertainty values $\left(1018{ }^{\circ} \mathrm{C} \pm 75^{\circ} \mathrm{C}\right.$ to $\left.1025^{\circ} \mathrm{C} \pm 121{ }^{\circ} \mathrm{C}\right)$ suggesting that equilibrium conditions were not established.

If olivines and orthopyroxene rims are taking into account the estimated temperatures give similar results. Concerning $\mathrm{Fe}-\mathrm{Mg}$ exchange, the highest temperature corresponds to Vaca Muerta $\mathrm{K} 1038{ }^{\circ} \mathrm{C} \pm 0{ }^{\circ} \mathrm{C}$ and the lowest to Mincy $\mathrm{L}\left(848{ }^{\circ} \mathrm{C} \pm 0{ }^{\circ} \mathrm{C}\right)$ with a mean value placed at $950.5^{\circ} \mathrm{C}$. In all olivine-orthopyroxene pairs an exact solution $\left( \pm 0{ }^{\circ} \mathrm{C}\right.$ uncertainty) was verified indicating that $\mathrm{Fe}-\mathrm{Mg}$ partitioning has achieved a near equilibrium state. However, if $\mathrm{Fe}-\mathrm{Mg}-\mathrm{Ca}$ is taken into account, the measured amount of calcio-olivine component is too low to reach equilibrium conditions. Did olivine lose Ca during cooling?

Acknowledgment: The authors are greatly indebted to Dr. Donald Lindsley for the helpful discussions. This work was supported by Agencia PICT 212.

References: [1] Andersen D. J. et al. 1993. Computer \& Geosciences 19:1333-1390. [2] Powell B. N. 1971. Geochimica et Cosmochimica Acta 35:5-34. [3] Ganguly J. et al. 1994. Geochimica et Cosmochimica Acta 58:2711-2723. [4] Hewins R. H. and Harriot T. A. 1986. Proceedings, 16th Lunar and Planetary Science Conference. pp. D365-D372.

\section{3}

\section{Li Be B IN REFRACTORY HIBONITE OBJECT}

K. K. Marhas and Y. Kadlag. Physical Research Laboratory, Navrangpura, Ahmedabad, Gujarat, India 380009. E-mail: kkmarhas@prl.res.in.

Presence of ${ }^{10} \mathrm{Be}\left(\mathrm{t}_{1 / 2} \sim 1.5 \mathrm{Ma}\right)$ in the early solar system has been confirmed with initial ${ }^{10} \mathrm{Be} /{ }^{9} \mathrm{Be}$ ranging from $3 \times 10^{-4}-18 \times 10^{-4}[1-5]$ in various early solar system objects. Potential source to the origin of this short-lived nuclide in the early solar system has been argued between irradiation of gas and dust in the solar nebula by solar energetic particles $[6,7]$ and ${ }^{10} \mathrm{Be}-\mathrm{GCR}$ trapped within the proto-solar molecular cloud [8]. Possible presence of ${ }^{7} \mathrm{Be}\left(\mathrm{t}_{1 / 2} \sim 53\right.$ days) in the Allende CAI [9] favors the energetic particle interaction as the source of these $\left({ }^{7} \mathrm{Be},{ }^{10} \mathrm{Be}\right)$ short-lived nuclides whereas the absence of ${ }^{7} \mathrm{Be}$ in platelet hibonite gives an additional outlook on mixing of ${ }^{7} \mathrm{Li}$ produced from spallation and the chondritic material [5]. Platelet hibonites with the absence of ${ }^{26} \mathrm{Al}$ and ${ }^{41} \mathrm{Ca}$ are considered as the first forming solids in the early solar system [10] and hence should have seen the early irradiation in turn producing ${ }^{7} \mathrm{Be}$ along with ${ }^{10} \mathrm{Be}$ within the grain. We have initiated Li Be B study of platelet type hibonites with absence of ${ }^{26} \mathrm{Al}$ and ${ }^{41} \mathrm{Ca}$ in order to check on the possible presence of ${ }^{7} \mathrm{Be}$ and to understand the irradiation scenario in the early solar system.

SH-7 is a $2 \mathrm{~mm}$-sized hibonite rich refractory object found on a broken surface of the Murchison meteorite [11]. It is devoid of short-lived nuclide $\quad{ }^{26} \mathrm{Al} \quad\left[\left({ }^{26} \mathrm{Al} \quad{ }^{27} \mathrm{Al}\right)_{\mathrm{i}}<1.5 \times 10^{-6}\right] \quad$ and $\quad{ }^{41} \mathrm{Ca} \quad\left[\left({ }^{41} \mathrm{Ca} /{ }^{40} \mathrm{Ca}\right)_{\mathrm{i}}\right.$ $<3.3 \times 10^{-9}$ ] and indicates presence of ${ }^{10} \mathrm{Be}$ with $\left({ }^{10} \mathrm{Be} /{ }^{9} \mathrm{Be}\right)_{\mathrm{i}} \sim 4 \times 10^{-4}$ $[10,3] .{ }^{16} \mathrm{O}$ and group III REE pattern [1] imply object to be refractory with negligible effect of secondary processes after its formation.

Utilizing the high spatial resolution capability of NanoSIMS, PRL nanoSIMS was used in multicollection mode $\left({ }^{6} \mathrm{Li}^{+},{ }^{7} \mathrm{Li}^{+},{ }^{9} \mathrm{Be}^{+},{ }^{10} \mathrm{~B}^{+}\right.$, ${ }^{11} \mathrm{~B}^{+}$) with an $\mathrm{O}^{-}$primary beam of approximately $2 \mathrm{nA}$ rastering the sample for more than $1.5 \mathrm{~h}$ in order to achieve better counting statistics. Eight different areas of the refractory object $(\mathrm{SH} 7)$ were analyzed with varying ${ }^{9} \mathrm{Be} /{ }^{6} \mathrm{Li}$ values (ranging from 90 to 280). Concentration of Be for these analyzed areas varied from 140 to $500 \mathrm{ppb} .\left({ }^{10} \mathrm{Be} /{ }^{9} \mathrm{Be}\right)_{\mathrm{i}}$ values obtained by PRL nanoSIMS are reproducible (within errors) compared to the earlier SIMS measurements on the same sample [3].

No definite excess in ${ }^{7} \mathrm{Li}$ has been observed for $\mathrm{SH} 7$ hibonite. Lower value of the ${ }^{7} \mathrm{Li} /{ }^{6} \mathrm{Li}$ (minimum approximately $-100 \%$ ) as compared to chondritic value $\left({ }^{7} \mathrm{Li} /{ }^{6} \mathrm{Li}=12.02\right)$ in most of the analyses probably indicates the mixing of the spallation product $\left({ }^{7} \mathrm{Li} /{ }^{6} \mathrm{Li}=0.8\right)$ with the chondritic material $\left({ }^{7} \mathrm{Li} /{ }^{6} \mathrm{Li}=12.02\right)$. Further understanding definitely requires a detailed study of diffusion processes of $\mathrm{Li}$ in hibonite grains with different conditions.

References: [1] McKeegan K. D. et al. 2000. Science 289: 1334-1337. [2] Sugiura, N. et al. 2001. Meteoritics \& Planetary Science 36: 1397-1408. [3] Marhas K. K. et al. 2002. Science 298:2182-2185. [4] MacPherson, G. J. et al. 2003. Geochimica et Cosmochimica Acta 67: 3165-3179. [5] Liu M. -C. et al. 2010. The Astrophysical Journal Letters 719: L99-L103. [6] Goswami J. N. et al. 2001. The Astrophysical Journal 549:1151-1159. [7] Gounelle M. et al. 2001. The Astrophysical Journal 548:1051-1070. [8] Desch S. J. et al. 2003. The Astrophysical Journal 602: 528-542. [9] Chaussidon M. et al. 2006. Geochimica et Cosmochimica Acta 70:224-245. [10] Sahijpal S. et al. 1998. Nature 391:559-561. [11] Hashimoto A. et al. 1986. 17th Lunar and Planetary Science Conference. pp. 317-318. 


\section{3}

\section{Si ISOTOPIC COMPOSITION OF EFREMOVKA E101.1 CAI}

J. Marin-Carbonne ${ }^{1}$, J. Aléon ${ }^{2}$, K. D. McKeegan ${ }^{1}$ and A. El Goresy ${ }^{3}$. ${ }^{1}$ Department of Earth and Space Sciences, University of California, Los Angeles, CA 90095-1567, USA. ${ }^{2}$ CSNSM, CNRS/IN2P3/ Univ. Paris Sud, Bat 104, 91405 Orsay Campus, France. ${ }^{3}$ Bayerisches Geoinstitut, Bayreuth, Germany. E-mail: jmarin@ess.ucla.edu, mckeegan@ess.ucla.edu

Inclusion E101.1 from the CV3 carbonaceous chondrite Efremovka is a compact type A CAI, highly enriched in ultrarefractory oxides [1]. This inclusion consists of several different lithological subunits with a complex petrography. The core contains ultrarefractory fassaitic clinopyroxene, enriched in $\mathrm{Sc}$ and $\mathrm{Zr}$, enclosed in large melilite crystals [1]. This core also contains some impact glass [1,2]. Surrounding the inclusion is a well-defined Wark-Lowering rim (WLR). This inclusion has been extensively studied for $\mathrm{O}$ [2] and $\mathrm{Mg}$ isotopes [3].

Here, we investigate the $\mathrm{Si}$ isotopic composition of the different subunits of this inclusion. Our goals are to better understand the conditions of formation of the CAI and the relationship between the different lithological units. We performed $\mathrm{Si}$ isotope analyses using the Cameca ims 1270 at UCLA in multicollection mode. San Carlos olivine, pyroxene and melilite reference materials were used to correct for instrumental mass fractionation. The reproducibility obtained on these reference materials is better than $0.4 \%$ at one sigma.

One profile of $200 \mu \mathrm{m}$ from the rim to the core of the inclusion was performed. Melilite close to the WLR is fairly homogeneous, around$4.8 \pm 1.6 \%$, in contrast to the melilite from the center of the core. Only two analyses were performed on the center of the core and we will perform more analyses to investigate this discrepancy. The impact glass is isotopically lighter than the melilite with a mean value of $-7.7 \%$. The $\mathrm{WR}$ is more fractionated than the core of the inclusion with values between $+7.3 \%$ and $+14.7 \%$.

No correlation is apparent between $\mathrm{Mg}$ and $\mathrm{Si}$ isotopic compositions in the few data that we have. As shown by the $\mathrm{O}$ [2] and the $\mathrm{Mg}$ [3] studies, this inclusion could have experienced some evaporation event. Further $\mathrm{Si}$ isotope analyses are currently ongoing to investigate in detail the relationship between the sub-units and the nature of the evaporation event(s) forming the inclusion rim.

References: [1] El Goresy et al. 2002. Geochimica et Cosmochimica Acta 66:1459-1491. [2] Aléon et al. 2010. Meteoritics \& Planetary Science 45:A7. [3] Aléon et al. 2011.
5383

Mg AND Si ISOTOPIC COMPOSITIONS OF VIGARANO FUN INCLUSION 1623-5

J. Marin-Carbonne ${ }^{1}$, K. D. McKeegan ${ }^{1}$, A. M Davis ${ }^{2}$, G. J. MacPherson $^{3}$, R. A. Mendybaev ${ }^{2}$, and F. M. Richter ${ }^{2}$. ${ }^{1}$ Department of Earth and Space Sciences, University of California, Los Angeles, CA 90095-1567, USA. (jmarin@ess.ucla.edu). ${ }^{2}$ Department of the Geophysical Sciences and Enrico Fermi Institute, University of Chicago, IL 60637-1433, USA. ${ }^{3}$ Department of Mineral Sciences, National Museum of Natural History, Smithsonian Institution, Washington DC 20560-0119, USA.

Among the CAIs, some inclusions have extreme isotopic properties, with large mass-dependant isotopic fractionation of major elements $(\mathrm{Mg}$, $\mathrm{Si}, \mathrm{O})$ accompanied by large nonradiogenic nuclear anomalies in various minor and trace elements [1]. The origin of these fractionations and the composition of the precursors of these so-called FUN inclusions are still unknown. Vigarano 1623-5 has strong isotopic affinities to the Allende FUN inclusion $\mathrm{Cl}$ and is the only "classical" FUN inclusion that still exists in thin section and so can be studied by in situ methods [2]. This object, which shows the first unequivocal correlated petrologic and isotopic evidence for volatilization [2,3], is composed of two chemically distinct parts, separated by a melilite-rich mantle and a hibonite-bearing vesicle. One part is pyroxene-rich and the other is a melilite-rich. A discontinuous $200 \mu \mathrm{m}$ thick melilite mantle surrounds these cores [2]. Here, we investigate in situ isotopic compositions of $\mathrm{Mg}$ and $\mathrm{Si}$ of the different parts of this unique inclusion. Our goal is to better understand the relationship between the mantle and the core of this inclusion to constrain its origin and environment of formation.

The analyses were done on a Cameca ims-1270 at UCLA at high mass resolution in monocollection and multicollection modes. Olivine, pyroxene, and melilite standards were used to correct for instrumental mass fractionation. The matrix effect due to variation of Ak content [4] was also investigated by analyzing synthetic zoned melilite.

Individual minerals show a large range of mass fractionation in $\delta^{30} \mathrm{Si}$ and $\delta^{25} \mathrm{Mg}$, forsterite from +6.9 to $+23.2 \%$ and +23.1 to $+29.0 \%$, respectively, and mantle melilite from +6.8 to $+26.2 \%$ and from +30.6 to $+32.9 \%$, respectively. Olivines show less mass-dependent fractionation for $\mathrm{Mg}$ than do pyroxene and melilite, but the same range of fractionation for $\mathrm{Si}$ is present in all of these minerals. There is no apparent relationship between the magnitude of fractionation and spatial distribution in either core of the inclusion. Olivines show a correlation between $\mathrm{Mg}$ and $\mathrm{Si}$ isotopes, which is slightly different from the relationship defined by the free evaporation of $\mathrm{Mg}_{2} \mathrm{SiO}_{4}$ residues [5]. The relationship between the degree of evaporation and the isotopic mass fractionation can be modeled using the Rayleigh equation. Our data imply an evaporation fraction (taking $\alpha=0.99009$, [6]) of approximately $72 \%$ for the pyroxene-rich core and approximately $84 \%$ for the mantle. The precursor of the core of $1623-5$ was at least partially evaporated as it was crystallizing.

References: [1] Loss, R. D. et al. 1994. The Astrophysical Journal. 436:193-196. [2] Davis A. M. et al. 1991. Geochimica et Cosmochimica Acta, 55, 621-637. [3] McKeegan K. D. et al. 2005. Abstract \#2077. 36th Lunar and Planetary Science Conference. [4] Knight K. B. et al. 2009. Geochimica et Cosmochimica Acta, 73:6390-6401. [5] Davis A. M. et al. 1990. Nature, 347:655-657. [6] Mendybaev et al. 2009. Abstract \#2461. 40th Lunar and Planetary Science Conference. 


\section{8}

\section{ORIGIN OF XENON ISOTOPIC COMPOSITION OF PHASE $Q$}

Y. Marrocchi ${ }^{1}$, M. Kuga ${ }^{1}$, B. Marty ${ }^{1}$ and P. Reinhardt ${ }^{2} .{ }^{1}$ CRPGCNRS, BP20, 54501 Vandoeuvre les Nancy, France. ${ }^{2}$ Laboratoire de Chimie Théorique, UPMC-Paris VI, 75252 Paris, E-mail: yvesm@ crpg.cnrs-nancy.fr.

Introduction: Noble gases trapped in primitive meteorites are isotopically mass-dependant fractionated with respect to solar noble gases. They are quantitatively hosted by a poorly defined, organic phase [1], labeled phase Q. Xenon, which is the least prone to isotopic fractionation due to its lowest relative mass difference between isotopes, is enriched in heavy isotopes by $+1.26 \pm 0.05 \%$ per atomic mass unit in the carbonaceous chondrite Orgueil relative to Solar. In order to understand the origin of phase $\mathrm{Q}$ and its xenon isotopic composition, we have performed an experimental study coupled with theoretical calculation.

Experimental Method and Theoretical Calculation: We performed adsorption experiments $(<1 \mathrm{eV})$ using $\mathrm{Xe}$ atoms and ions, ionized in a radiofrequency plasma. Within the reaction vessel, anthracite was heated in a furnace and the resulting organic particles were deposited onto the walls of the vessel, resulting in carbon-rich films. Xe was trapped either as ions in the ionization zone of the vessel, or as neutral atoms outside this zone.

Using the finite nucleus model [2], we determined the first ionization potential (FIP) by solving the relativistic Schrödinger-Dirac equation for each $\mathrm{Xe}$ isotopes for neutral atoms and for ions.

Results: Xe trapped from ionized $\mathrm{Xe}$ is tightly bound and is enriched by $+1.36 \pm 0.06 \% \mathrm{amu}^{-1}(2 \sigma)$ in heavy isotopes, reproducing the isotopic fractionation of Xe trapped in chondrites relative to Solar. Neutral Xe is more loosely trapped, is much lower in abundance, and is not isotopically fractionated. Ionized conditions allow the monotonic Xe isotopic composition observed in meteorites during stepwise heating release to be reproduced.

Discussion: Xe was not implanted into the film, because the energies of the incident $\mathrm{Xe}$ atoms and ions are far too low $(<1 \mathrm{eV})$. From the difference of behavior between ionic and neutral forms, we propose that $\mathrm{Xe}$ ions were trapped via chemical bonding at the surface of the newly created C-rich film. The observed mass-dependant fractionation of Xe is unlikely to have occurred in the gas phase as the difference in FIPs correspond to $\approx 1 \times 10^{-4} \mathrm{eV}$ between ${ }^{124} \mathrm{Xe}$ and ${ }^{136} \mathrm{Xe}$. As the mean experimental first ionization energy of xenon is $12.13 \mathrm{eV}$, the isotopic effect makes only $\approx 0.00008 \%$ difference in ionization energy between two $\mathrm{Xe}$ isotopes. $\mathrm{Xe}$ isotopic fractionation is more probably related to variations in chemical bonding strengths of $\mathrm{Xe}$ isotopes as chemical bonds involving heavy $\mathrm{Xe}$ isotopes are more stable than those involving light ones. The extent of fractionation could have been kinetically enhanced as a result of competition between film growth and Xe dissociation rate. The Q-noble gas fractionation and trapping characteristics are likely related to UV irradiation (from nearby young stars) of the surface of growing organic grains in the outer part of the solar system. For young stars, photons emitted in the far UV energy range $(<100 \mathrm{~nm})$ that are capable of ionizing noble gases were orders of magnitude more abundant than for the Present-day Sun, allowing efficient ionization of gaseous species

References: [1] Marrocchi et al. 2005. Earth and Planetary Science Letters 236:569-578. [2] Dyall K.G. et al. 1989 Comp. Phys. Comm. $55: 425-446$.
5335

A Fe-RICH PHASE ENRICHED IN REE AND HFSE: NEW CLUES ON CHONDRITE HISTORY?

Céline Martin ${ }^{1}$, Vinciane Debaille ${ }^{2}$, Philippe Claeys ${ }^{1}$. ${ }^{1}$ Earth System Sciences, Vrije Universiteit Brussel, Belgium. ${ }^{2}$ Département des Sciences de la Terre et de l'Environnement, Université Libre de Bruxelles, Belgium.

Chondrites are among the most pristine objects of the solar system and their average chemical composition estimates the initial composition of the Earth. Particularly, the Sm-Nd and Lu-Hf isotopic systems are used together as tracers of early planetary processes because they are not fractionated either by condensation/volatilization processes in the early solar system or by metal/silicate separation during core formation in terrestrial planets. The CHUR (CHondritic Uniform Reservoir) serves as the established reference value for these two systems. It is clearly demonstrated that $\mathrm{Sm}-\mathrm{Nd}$ values are well constrained and homogeneous in chondrites. However, recent data shows that this is not the case for Lu-Hf system that displays large $\mathrm{Lu} / \mathrm{Hf}$ ratio heterogeneities (up to $28 \%$ ), hence restraining its use in cosmo/geochemistry. A better knowledge of these meteorites and the geological processes they underwent will ensure a better understanding of the first million years of the solar system, including the early evolution of terrestrial planets and more importantly the Earth.

The study of accessory phases provides significant information on the PT conditions of their formation as well as on their alteration state. Moreover, such minerals are assumed to contain a large part of rare earth elements (REE) and high field strength elements (HFSE). Among those accessory phases, an undescribed $\mathrm{Fe}( \pm \mathrm{Ni})$-rich phase, founded as single grain as well as rim of sulfide or as veins has been found almost ubiquitously in chondrites (H3, H4, L6). The interest of this kind of interstitial phase will be discussed in order to better constrain Sm-Nd and Lu-Hf systematics, as it contains significant amount of LREE (up to $75 \mathrm{ppm}$ of $\mathrm{Nd}$ ) and $\mathrm{Hf}$ (up to $40 \mathrm{ppm}$ ). 
5421

\section{THREE-DIMENSIONAL OBSERVATION AND IMAGE ANALYSIS OF ORGANIC NANOGLOBULES IN CARBONACEOUS CHONDRITES USING X-RAY MICROTOMOGRAPHY}

T. Matsumoto ${ }^{1}$, A. Tsuchiyama ${ }^{1}$, K. Nakamura-Messenger ${ }^{2}$, M. E. Zolensky $^{2}$, T. Nakano ${ }^{3}$, and K. Uesugi ${ }^{4}$. ${ }^{1}$ Department of Earth and Space Science, Osaka University, Osaka, Japan. E-mail: matsumoto@ as-troboy.ess.sci.osaka-u.ac.jp. ${ }^{2}$ NASA JSC, Houston, TX 77058, USA. ${ }^{3}$ GSJ/AIST. ${ }^{4}$ JASRI/SPring-8.

Introduction: In carbonaceous chondrites, IDPs, and dust from comet $81 \mathrm{P} /$ Wild 2, spherical organic objects of a few hundred nanometers, called organic nanoglobules were found [1-3]. They have usually hollow structures, and it has been proposed that they were formed from composite particles of organic materials and ice in molecular clouds or aqueous alteration of organic materials in asteroids [4].In the both origins, hollow regions of the globules might be filled with $\mathrm{H}_{2} \mathrm{O}$-rich fluids. However, any fluids have not been detected due to destructive analysis, such as by TEM, in the previous studies. In order to determine the existence of such fluids, we tried to observe organic nanoglobules nondestructively using synchrotron radiation-based X-ray microtomography.

Experiments: The imaging experiments were done at BL47XU of SPring-8, JAPAN. The samples were pieces of Tagish Lake meteorite of $60 \mu \mathrm{m}$ in size. Their 3-D structures were obtained by the tomography system with the spatial resolution of about $250 \mathrm{~nm}$. Then, the samples were microtomed and observed under a transmission electron microscope (TEM) to compare with the CT images.

Results and Discussions: Comparison between the CT and TEM images showed that nanoglobules were identified by the $\mathrm{CT}$ images. However, CT images of nanoglobules are not exactly the same as TEM images probably due to X-ray refraction in the sample. In order to evaluate CT images of nanoglobules and to determine the presence of fluids in the hollows, simulation of CT images was made by considering $\mathrm{X}$-ray absorption and refraction for nanoglobules with or without water in the hollows. The simulation suggests that at least the observed nanoglobule do not seem to contain any water in the hollow. The simulation also revealed that the external shapes of nanoglobules can be identified although internal structures are hardly recognized by CT images. Based on the simulation, three-dimensional shapes of nanoglobules in the Targish Lake samples were examined. Three-axial ellipsoids approximation of the nanoglobule shapes revealed that they are almost spherical (the ratio of the longest and shortest axis is no less than approximately 0.7 ). If nanoglobules have molecular clouds origin based on $\mathrm{N}$ and $\mathrm{H}$ isotopic features [4], icy dust in the molecular clouds should be spherical.

References: [1] Nakamura K. et al. 2002. International Journal of Astrobiology 1:179. [2] Messenger S. et al. 2008. Abstract \#2391. 34th Lunar and Planetary Science Conference. [3] De Gregorio B. T. 2009. Abstract \#1130. 40th Lunar and Planetary Science Conference. [4] Nakamura-Messenger K. et al. 2006. Science 314:1439-1442.
5287

SILICATE-BEARING SULFIDE-METAL ASSEMBLAGES IN PRIMITIVE ORDINARY CHONDRITES

M. Matthes ${ }^{1}$ and A. Bischoff ${ }^{1}$. ${ }^{1}$ Institut für Planetologie, WilhelmKlemm-Str. 10, 48149 Münster, Germany. E-mail: max.matthes@ uni-muenster.de.

Introduction: The ordinary chondrites Adrar 003 (L/LL3.10), Krymka (LL3.2), and Semarkona (LL3.00) belong to the most primitive chondrites known [1]. These rocks were found to contain different types of silicate-bearing sulfide-metal assemblages in terms of texture, size, and relative abundance of silicates and opaques (sulfides, metal). We have studied 53 aggregates within the three chondrites and found that troilite is the most abundant phase within the assemblages. As major silicates olivine and pyroxene occur.

Mineralogy: The assemblages were identified and documented in thin section by optical microscopy in reflected and transmitted light. Most of the assemblages occur as individual objects between chondrules. In several cases these aggregates form broad chondrule rims presumably indicating a close genetic relationship. The mineral sizes within the aggregates vary extremely as well as the relative abundances of silicates and opaques. Besides troilite, pentlandite is of minor abundance and the metal grains analyzed so far are primarily kamacite; taenite only occurs sporadically.

341 olivines and 157 low-Ca pyroxenes were analyzed within these sulfide-metal assemblages by SEM-EDX. Although the range and average composition of pyroxene within the aggregates are similar to those of the bulk chondrites, significant differences were obtained for olivine. Considering all three samples, olivine provides a much broader range in Fa-content in the assemblages than in the bulk rocks. In addition, the average olivine composition of the grains within the aggregates of all three samples $\left(\sim \mathrm{Fa}_{30}\right)$ is significantly different from the average composition of olivine in the three bulk rocks $\left(\sim \mathrm{Fa}_{12}\right.$; Table 1$)$.

Discussion: The different textural and mineralogical characteristics of the silicate-bearing sulfide-metal aggregates suggest different formation conditions. The differences in olivine compositions (bulk chondrites vs. aggregates) will be considered in detail in ongoing studies.

Reference: [1] Grossman J. N. and Brearley A. J. 2005. Meteoritics \& Planetary Science 40:87-122.

Table 1. Compositions of olivine (Ol) and low-Ca pyroxene (Px) in the studied bulk chondrites and within sulfide-metal aggregates (agg.); $\mathrm{n}=$ number of analyzed grains; data in $\mathrm{mol} \%$.

\begin{tabular}{|c|c|c|c|c|}
\hline & $\mathrm{Ol}(\mathrm{Fa})$ & $n$ & Px (Fs) & $n$ \\
\hline \multicolumn{5}{|l|}{ Adrar 003} \\
\hline Bulk chondrite (range) & $0.4-35.8$ & 98 & $0.6-40.3$ & 74 \\
\hline Average & $13.2 \pm 8.1$ & & $10.4 \pm 8.0$ & \\
\hline Sulfide-metal agg. (range) & $0.3-60.0$ & 155 & $1.4-27.4$ & 69 \\
\hline Average & $32.4 \pm 15.1$ & & $10.1 \pm 7.0$ & \\
\hline \multicolumn{5}{|l|}{ Krymka } \\
\hline Bulk chondrite (range) & $0.4-47.1$ & 92 & $1.0-32.5$ & 54 \\
\hline Average & $12.0 \pm 8.5$ & & $8.4 \pm 7.2$ & \\
\hline Sulfide-metal agg. (range) & $0.6-60.5$ & 128 & $0.6-63.2$ & 65 \\
\hline Average & $29.8 \pm 16.0$ & & $10.0 \pm 10.9$ & \\
\hline \multicolumn{5}{|l|}{ Semarkona } \\
\hline Bulk chondrite (range) & $0.3-26.3$ & 65 & $0.6-32.3$ & 46 \\
\hline Average & $10.8 \pm 6.9$ & & $8.9 \pm 7.4$ & \\
\hline Sulfide-metal agg. (range) & $1.1-37.3$ & 58 & $2.6-23.4$ & 23 \\
\hline Average & $26.3 \pm 8.1$ & & $9.9 \pm 7.2$ & \\
\hline
\end{tabular}


5528

\section{$\mathrm{Fe}^{2+}$-Mg PARTITIONING BETWEEN OLIVINE AND MARTIAN BASALTIC MELTS}

A. K. Matzen, J. R. Beckett, M. B. Baker and E. M. Stolper. California Institute of Technology, MC 170-25, Pasadena, CA 91125, USA. E-mail: amatzen@caltech.edu.

Introduction: The partitioning of elements between olivine and liquid is key to understanding igneous processes in mafic and ultramafic systems [1]. The landmark study of Roeder and Emslie [2] concluded that the olivine (ol)-liquid (liq) exchange coefficient, $K_{\mathrm{D}, \mathrm{Fe}^{2+}-\mathrm{Mg}}=(\mathrm{FeO} / \mathrm{MgO})^{\mathrm{ol}} /$ $(\mathrm{FeO} / \mathrm{MgO})^{\text {liq }}$ (by weight), is $0.30 \pm 0.03$, independent of temperature $(T)$ and liquid composition. Subsequent work [3] has shown a dependence on liquid composition, but the canonical value of 0.30 is still widely used in both terrestrial and Martian applications [e.g., 4, 5]. Here, we use previously published experiments on bulk compositions relevant to Martian magmas to determine the appropriate $K_{\mathrm{D}, \mathrm{Fe}^{2+}-\mathrm{Mg}}(\mathrm{s})$ for Martian systems; we then use this value to test whether any of the ol-phyric shergottites represent liquids.

Experiments on Martian Compositions: One-atm experiments on model Martian compositions provide the tightest constraint on $K_{\mathrm{D}, \mathrm{Fe}^{2+}-\mathrm{Mg}}$ because $T$ and oxygen fugacity $\left(f \mathrm{O}_{2}\right)$ are well known, allowing us to use [6] to predict the amount of ferrous iron present in each liquid. The median of 17 published 1 -atm experiments yields $K_{\mathrm{D}, \mathrm{Fe}^{2+}-\mathrm{Mg}}=$ $0.354 \pm 0.008$ (error is one mean absolute deviation, MAD). There are higher pressure $(P)$ experiments on model Martian compositions, but their $f \mathrm{O}_{2} \mathrm{~S}$ are less well constrained. High- $P$ experiments in graphite capsules are reducing [7], but $\mathrm{Fe}^{3+}$ still materially affects estimates of $K_{\mathrm{D}, \mathrm{Fe}^{2+}-\mathrm{Mg}}$; correcting for the $\mathrm{Fe}^{3+}$ using $[6,7]$ increases $K_{\mathrm{D}, \mathrm{Fe}^{2+}-\mathrm{Mg}}$, on average, by 0.016. We see no strong correlations of $K_{\mathrm{D}, \mathrm{Fe}^{2+}-\mathrm{Mg}}$ with liquid or ol composition for the high or low- $P$ experiments and a weak increase in $K_{\mathrm{D}, \mathrm{Fe}^{2+}-\mathrm{Mg}}$ with increasing $P$. A marked increase in $K_{\mathrm{D}, \mathrm{Fe}^{2+}-\mathrm{Mg}}$ for high- $P$ experiments at $T<\sim 1150{ }^{\circ} \mathrm{C}$ is likely due to lack of equilibrium. Accepting high- $P$ experiments with $T>1150{ }^{\circ} \mathrm{C}$ leads to a median $K_{\mathrm{D}, \mathrm{Fe}^{2+}-\mathrm{Mg}}$ of $0.369 \pm 0.024$ (MAD), higher than the 1 -atm experiments but notably higher than the canonical value of 0.30 [2]. Combining 1-atm. and high- $P$, high- $T$ experiments yields a median $K_{\mathrm{D}, \mathrm{Fe}^{2+}-\mathrm{Mg}}$ of $0.360 \pm 0.013$ (MAD), close to the recently reported value of $0.35 \pm 0.01$ [8], which was obtained without correcting for $\mathrm{Fe}^{3+}$ in the liquid.

Olivine-Phyric Shergottites: Using $K_{\mathrm{D}, \mathrm{Fe}^{2+}-\mathrm{Mg}}=0.30$ leads to the result that none of the ol cores in ol-phyric shergottites is in equilibrium with liquids equivalent to their bulk. Applying our $K_{\mathrm{D}, \mathrm{Fe}^{2+}-\mathrm{Mg}}$ to these shergottites leads to the possibility that Y980459, NWA 5789 and 2990 are liquid compositions (others are not), identical to the results of Filiberto and Dasgupta [8]. Accounting for $\mathrm{Fe}^{3+}$ in the liquid also allows us to constrain plausible $\mathrm{O}_{2}$ s during cooling when independent measures are unavailable or not yet determined; e.g., if the olivines in NWA 2990 are in equilibrium with a liquid whose composition is that of the bulk meteorite, crystallization must have occurred under reducing conditions where the $\mathrm{Fe}^{3+} / \sum \mathrm{Fe}$ is small, $\sim \mathrm{IW}+0.5$ at 1 atm or $\mathrm{IW}+1$ at $1 \mathrm{GPa}$.

References: [1] Basaltic Volcanism Study Project. 1981. [2] Roeder P. L. and Emslie R. F. 1970. Contributions to Mineralogy and Petrology 29:275-289. [3] Sack R. O. et al. 1987. Contributions to Mineralogy and Petrology 96:1-23. [4] Falloon T. J. et al. 2007. Chemical Geology 241:207-233. [5] Peslier A. H. et al. 2010. Geochemistry Geophysics Geosystems 74:4543-4576. [6] O'Neill et al. 2006. American Mineralogist 91:404-412. [7] Médard E. et al. 2008. American Mineralogist 93:18381844. [8] Filiberto J. and Dasgupta R. 2011. Earth and Planetary Science Letters 304:527-537.
5173

EXPLORING XRF AS A NEW TECHNIQUE FOR BASIC METEORITE CLASSIFICATIONS

R. G. Mayne ${ }^{1}$, A. J. Ehlmann ${ }^{1}$ and K. C. Daviau ${ }^{2} .{ }^{1}$ Monnig Meteorite Collection, School of Geology, Energy, and the Environment, Texas Christian University, TCU Box 298830, Fort Worth, TX 76129, USA. E-mail: r.g.mayne@tcu.edu. ${ }^{2}$ Physics Department, Bard College, PO Box 5000, Annandale-on-Hudson, NY 12504, USA.

Current Classification Methods: In the 2010/2011 season, the Antarctic Search for Meteorites (ANSMET) collected around 1200 meteorites. These will all be classified over the next 2 years, according to the U.S. Federal Regulation on Antarctic Meteorites [1], by the curatorial staff at the Smithsonian Institution's National Museum of Natural History. The vast majority of the meteorites collected in Antarctica are equilibrated ordinary chondrites and the current method employed for their identification is that of visual examination combined with oil immersion of olivine grains. This technique requires that a small amount of each meteorite be powdered, sieved, and then examined in refractive index oils under a petrographic microscope. This is a timely process and the Smithsonian's main goal is to be able to "separate the approximately $10 \%$ of meteorites that deserve further scientific study" [1].

XRF: The Monnig Meteorite Collection deals with many requests from the general public for meteorite identification. The vast majority of these samples are industrial slag. The newly purchased Bruker handheld XRF will be used to analyze these samples to confirm that their composition is not meteoritic.

The goal of this study is to explore XRF as a new technique for meteorite identification. In this preliminary study we will present data on the first two parts of this study (1) estimating the Ni-content of iron meteorites (2) examining previously classified ordinary chondrites to establish a calibration technique for unknowns. The eventual aim is to see if the XRF technique outlined here can be used to distinguish LL, L, and $\mathrm{H}$ chondrites from one another, to provide an alternative streamlined process to oil immersion.

Methodology: All meteorites will be analyzed on the Bruker Tracer III-SD handheld XRF system at Texas Christian University. For part (1) only meteorites from the Monnig Meteorite Collection with a published $\mathrm{Ni}$ content were selected for analysis. All Ni contents were taken from [2]. A minimum of three analyses were taken for each sample. The number of counts for $\mathrm{Ni} \mathrm{K} \alpha$ were tabulated each time and a calibration curve was constructed. Previously classified ordinary chondrites were selected from the Monnig Collection for part (2) of the study. Twenty meteorites of each ordinary chondrite group $(\mathrm{H}, \mathrm{L}, \mathrm{LL})$ will be measured.

Preliminary Results: Initial results relating $\mathrm{Ni} \mathrm{K} \alpha$ counts to $\mathrm{Ni}$ content of iron meteorites show that these two components do appear to be directly related and no further reduction of the data (into percentage $\mathrm{Ni}$ ) is required. The calibration curve constructed from the preliminary data has an $R^{2}$ value of 0.94 .

At the time of writing, only five $\mathrm{H}, \mathrm{L}$, and LL meteorites have been analyzed on the XRF. While there appear to be some differences between the ordinary chondrite groups, there is currently not enough data for statistical analysis to see if these differences are significant enough to allow their identification.

References: [1] Corrigan C. M. et al. 2008. Meteoritics \& Planetary Science 43:A180. [2] Wasson J. T. 2010. Geochimica et Cosmochimica Acta $75: 1757-1772$. 
5179

THE 100th ANNIVERSARY OF THE FALL OF NAKHLA: THE SUBDIVISION OF BM1913,25

K. M. McBride ${ }^{1}$ and K. Righter ${ }^{2}$. ${ }^{1}$ ESCG Jacobs Technology, NASA Johnson Space Center, 2101 Nasa Pkwy, Houston, TX 77058, USA. E-mail: kathleen.mcbride-1@nasa.gov. ${ }^{2}$ NASA-JSC, 2101 Nasa Pkwy, Houston, TX 77058, USA.

This year marks the 100th anniversary of the fall of Nakhla, a cumulate clinopyroxenite of Martian origin that fell near Alexandria, Egypt, in 1911. Multiple fragments of the meteorite were seen to fall over an area of $4.5 \mathrm{~km}$ in diameter. Approximately 40 stones were recovered with a combined weight of about $10 \mathrm{~kg}$ [1]. Most of the larger specimens found their way to museums and meteorite collections in Cairo, Paris, Berlin, and the Smithsonian, to name a few.

In 1998, the British Museum sent a $641 \mathrm{~g}$ (BM1913,25), fully fusion crusted stone of Nakhla to the Johnson Space Center (JSC) for processing in the Antarctic Meteorite Lab in order to allocate samples to the scientific community. The stone was split in half in a dry nitrogen glove box. One half of the stone was sent back to the museum and the other half (346 g) was used for sample allocations. From 1998-2001, 37 scientists requested 65 separate samples of Nakhla, including 2 thin sections.

This set of allocations was especially important in that all of the sample splits are from the same piece of Nakhla and it had a known history since it was acquired by the museum in 1913. With the multiple fragments of Nakhla, it is not known from what pieces the main bulk of research has been done, what variation may exist between all the pieces and to what contaminants the fragments may have been exposed, (i.e., water, solvents or cutting fluids, etc.). All of the allocations prepared at JSC were processed in a nitrogen cabinet using only stainless steel, aluminum, and Teflon tools and containers to reduce the chance of introducing any new contaminants.

The focused effort to subdivide and distribute samples of Nakhla to the meteorite community resulted in enhanced understanding of Nakhla and nakhlites in general: organic geochemistry [2, 3], weathering [4], sulfur isotopes [5], radiometric age [6], and magmatic history [7, 8].

There are 13 Nakhlites that have been recovered to date: Nakhla, Lafayette, Governador Valdares, three from NW Africa, three from Yamato and four from Miller Range regions in Antarctica. The Yamato Nakhlites are paired [9] as are the Miller Range samples [10].

References: [1] Meyer C. 2004. Mars Meteorite Compendium II1II14. [2] Jull A. J. T. et al. 2000. Geochimica et Cosmochimica Acta 64:3763-3772. [3] Glavin D. et al. 1999. Proceedings of the National Academy of Sciences 96:8835-8838. [4] Bridges J. and Grady M. M. 2000. Earth and Planetary Science Letters 176:267-279. [5] Farquhar J. 2000. Nature 404:50-54. [6] Shih C.-Y. et al. 2010. Abstract \#1367. 41st Lunar and Planetary Science Conference. [7] Stockstill K. et al. 2005. Meteoritics \& Planetary Science 40:377-396. [8] Neal C. R. et al. 2001. Abstract \#1682. 32nd Lunar and Planetary Science Conference. [9] Imae N. et al. 2005. Meteoritics \& Planetary Science 40:1581-1598. [10] Antarctic Meteorite Newsletter 33, no. 2, 2010.

\section{0}

METEORITE MOVEMENT IN ANTARCTICA AND CLIMATE CHANGE

G. J. H. McCall. Honorary Associate Western Australian Museum (retired); 44 Robert Franklin Way, South Cerney, Glos., UK, GL7 5UD E-mail: joemccall@tiscali.co.uk.

Introduction: Large ice sheets lose ice in two ways; slow movement of ice by processes of crystal deformation [1]; and summersurface melting and base flow [2, 3]. The Japanese made the first meteorite finds in the Yamato Mountains, Antarctica, in 1969. Antarctic meteorites can be shown by cosmic ray studies to have fallen thousands, even millions of years ago and are flowing at a very slow rate. Haack et al. [4], who failed to find meteorites on blue (deoxygenated) ice surfaces in Greenland, suggested that the meteorites were possibly washed down by the melting. Greenland has a higher average temperature than Antarctica. The meteorites may be removed from the surface by the summer melt before they can enter the deep ice flow system; or they may have entered the deep ice as the snow built up and been carried in the blue ice, as in Antarctica, but are not found on the surface. Katabatic winds that are believed to bring meteorites to the surface of blue ice in Antarctica [5], after the very slow flow is impeded by nunataks etc, may not operate in Greenland's climate. The presence or absence of arresting barriers such as nunataks and the amount of snowfall forming ice inland are other possible. Where the blue ice is other possible exposed, impeded and slowed up by nunataks, it is isoclinally folded, and this may also bring meteorites to the surface: also the Greenland ice sheet may have been almost entirely lost in the Eemian interglacial [100,000-70,000 years ago] - the East Antarctic Ice sheet is millions of years old. West Antarctica is losing ice, in East Antarctica there is equilibrium between loss and increment from snowfall inland. The slow movement of meteorites is not affected by the fact that the ice sheet overlies kilometer deep basins, because of the positive topography of the ice surface, which builds up faster than the weight of the ice pushes the land surface down, and in both Greenland and Antarctica the land surface originally ( 7 and 34 million years ago respectively) had positive topography before the ice formed. Slow movement of the meteorites has no bearing on the arguments about global warming, but surface melt and basal flow does. Because of deep depression of the ice base, rapid surface melt and basal flow can only operate at the coastal margins of the sheet (as is the case in Greenland). The difference in climate between coastal regions and centre of the ice sheet is an important factor also in the distribution of ice loss from surface melting and base flow. Summer melt and basal flow may be insignificant except at the coastal margins in E. Antarctica. The absence in West Antarctica is likely due to climatic or topographic causes.

References: [1] Ollier C. 2010. Geoscientist 20:16-21. [2] Hambrey M., Bamber J., Christofferson P., Glasser N., Hubbard A., and Hubbard B. 2010. Geoscientist 20:18-23. [3] Jenkins M. 2010. National Geographic Magazine 217:34-47. [4] Haack H., Schutt J., Meibom A., and Harvey R. 2007. Meteoritics \& Planetary Science 42:1727-1733. [5] Zolensky M. 1998. In: Meteorites: Flux with time and impact effects. Edited by Grady M. M., Hutchison R., McCall G. J. H., and Rothery D. A. Geological Society, London, Special Publication 140:93-104. [6] Naish T. et al. 2009. Nature 458:322-328. 
5041

\section{ARE WE ALONE? THE EVOLUTION FACTOR IN THE CONTEXT OF THE EXOPLANET-PROLIFERATED UNIVERSE}

G. J. H. McCall. Honorary Associate Western Australian, Museum (Retired) 44 Robert Franklin Way, South Cerney, GL7 5UD, UK. E-mail: joemccall@ticali.co.uk

Introduction: Recent discussions of the question "Are we alone in the universe" heavily emphasized the astronomical and physical arguments, but seemed largely to ignore the arguments related to the long history of evolution on Earth, and the likelihood or otherwise on exoplanets of any repetition or "mirror image"[1] of the path of evolution, as we know it on Earth, in the habitable zone in the Milky Way galaxy or galaxies more distant. The discovery of about $\sim 500$ exoplanets in our galaxy is a scientific revolution of great importance, and these are only "the tip of the iceberg." Myriads more must exist. Yet such is the nature of the variables relating to evolution, the possibility of it taking numerous pathways - even the possibility of different patterns of evolution on other planets in the habitable zone-that it is concluded that there is no chance at all of a repetition of our own planet's evolutionary pathway being repeated on any exoplanet in the habitable zone [2]. Evolution is subject to innumerable constraints, internally within the development of life through the ages, externally, through different geological histories of the host planet and interaction with different coexistent life populations at any point in geological time; and on exoplanets through differences in the parent star, arrangement of the planetary system, and the physical conditions that these impose on the planet: in addition the Earth has the peculiarity of shields such as the magnetosphere and the ozone layer, and the peculiarity of surface liquids in the form of seas (within which much of evolutionary history took place) and a peculiar secondary oxygenic atmosphere, a product of early photosynthetic unicellular life itself. Plate tectonics allows the escape of the Earth's internal heat, and absorbs excess carbon dioxide [3], preventing a Venus-like runaway greenhouse effect, in which advanced life is impossible: Plate tectonics is unique in the solar system, and it may well have appeared mid-way through Earth's life history [4], and allowed eukaryote development? [5-8] It may be unique in the galaxy? The conclusion is reached that, without the likelihood of repetition of the Earth's evolutionary pathways (which may be unique, despite the myriads of exoplanets out there), there will surely be no sufficiently similar intelligent beings out there, who can communicate back to us? Probably the evolutionary pathway on any exoplanet possessed of life is unique. Evolution is essentially a random process. As R. A. Fortey has concluded "There is nothing inexorable about the course of Evolution: rather it is a pas de deux between the changing environment and the capacity of organisms to respond to these changes" [9].

References: [1] Gramling C. 2009. Earth 54:36-43. [2] Marquardt M. 2010. Earth 55:50. [3] Baker D, Ratcliff T. 2010. The 50 most extreme places in the Solar System. Belknap Press of Harvard University Press, London England and Cambridge, Massachusetts 151-155. [4] McCall G. J. H. 2010. Australian Journal of Earth Sciences 57:349-355. [5] Katz L., Bhatacharya, D. 2006. Genomics and the evolution of the microbial eukaryotes. Oxford University Press, 243. [6] Margolis L. 1970. Origin of Eukaryotic Cells. Yale University Press, Newhaven. [7] Keeling P. J. et al. 2006. TRENDS in ecology and evolution, 20:670-676. [8] Albani A. et al. 2010. Nature 466:100-104. [9] Fortey R. A. (Geoscientist, in press).

\section{0 \\ THE RELATIONSHIP BETWEEN IIE IRONS AND H CHONDRITES: PETROLOGIC AND OXYGEN ISOTOPE CONSTRAINTS}

K. H. McDermott ${ }^{1}$, R. C. Greenwood ${ }^{1}$, I. A. Franchi ${ }^{1}$, M. Anand ${ }^{1}$ and E. R. D. Scott ${ }^{2}$. ${ }^{1}$ CEPSAR, The Open University, Milton Keynes MK7 6AA, UK. E-mail: k.h.mcdermott@open.ac.uk. ${ }^{2}$ HIGP, University of Hawai'i, Honolulu, HI 96822, USA.

Introduction: Silicate inclusions have been observed in about half of all currently identified IIE irons (21 specimens) [1]. Based mainly on their similar oxygen isotope compositions, a genetic relationship between the IIE irons and $\mathrm{H}$ chondrites had been proposed [2, 3]. However, this relationship was subsequently questioned due to the limited overlap between laser fluorination data for equilibrated $\mathrm{H}$ chondrites and the earlier IIE silicate data $[4,5]$. We are undertaking a detailed oxygen isotope and geochemical study of both the IIE silicate inclusions and $\mathrm{H}$ chondrites, with the aim of investigating further the relationship between the two groups.

Analytical Techniques: Oxygen isotope analysis of both mineral separates and bulk silicate inclusions from 9 IIE irons, as well as a suite of $12 \mathrm{H}$ chondrite samples, was undertaken by infrared laser-assisted fluorination [6]. Textural and quantitative analysis of IIE silicate inclusions was carried out using a Cameca SX-100 Electron Microprobe and a FEI Quanta 200 FIB-ASEM.

Petrographical Results: Detailed petrographic study of the chondritic IIE-an Netschaevo, $\left(\mathrm{Fa}_{14}\right.$, excluding $\mathrm{Fa}_{25}$ secondary olivines) reveals that it not only contains well-developed chondrule relicts, but also extensive evidence of partial melting. The more primitive IIE silicates have olivine compositions that fall within the $\mathrm{H}$ chondrite range, i.e., Techado $\left(\mathrm{Fa}_{16}\right)$ and Watson $001\left(\mathrm{Fa}_{20}\right)$, both of which have also experienced melting.

Oxygen Isotopic Compositions: Oxygen isotopes in the IIEs show a range of $\Delta^{17} \mathrm{O}$ values from $0.58 \%$ to $0.90 \%$, with a mean of $0.72 \pm 0.11 \%$ o $(2 \sigma) . \quad \delta^{18} \mathrm{O}$ for the 9 samples studied also show a considerable range from $3.06 \%$ to $6.25 \%$, the mean $\delta^{18} \mathrm{O}$ value is $4.69 \pm 0.59 \%$ oo $(2 \sigma)$. The wide range in $\delta^{18} \mathrm{O}$ values reflects the fact that some of the IIE silicate inclusions are highly differentiated (Kodaikanal and Colomera), as discussed by [5]. For comparison, the oxygen isotope analyses of the 12 equilibrated $\mathrm{H}$ chondrites examined gave a mean $\delta^{18} \mathrm{O}$ value of $4.11 \pm 0.56 \%(2 \sigma)$ and $\Delta^{17} \mathrm{O}$ value of $0.71 \pm 0.12 \%(2 \sigma)$.

Discussion: The presence of relict chondrules in Netschaevo suggests that the group as a whole may be derived from a chondritic parent body. The nearly identical mean $\Delta^{17} \mathrm{O}$ values for the IIE irons and $\mathrm{H}$ chondrites support the possibility of a genetic link between these two groups. In addition, the substantial variation in $\Delta^{17} \mathrm{O}$ seen in both groups suggests that they were derived from parent bodies with similar levels of primary isotopic heterogeneity. The wide variation in $\delta^{18} \mathrm{O}$ values seen in some IIE silicate indicates that they underwent significant degrees of differentiation. In conclusion, our new laser fluorination data show that the $\mathrm{H}$ chondrites and IIEs could both have originated on the same unequilibrated $\mathrm{H}$ chondrite parent body.

References: [1] Mittlefehldt D. W. et al. 1998. Reviews in Mineralogy 36. [2] Goldstein J. I. et al. 2009. Chemie der Erde 69:293-325. [3] Clayton R. N. and Mayeda T. K. 1996. Geochimica et Cosmochimica Acta 60:1999-2018. [4] Folco L. et al. 2004. Geochimica et Cosmochimica Acta 68:2379-2397. [5] Franchi I. A. 2008. Reviews in Mineralogy and Geochemistry, vol. 68. pp. 345-397. [6] Miller M. F. et al. 1999. Rapid Communications in Mass Spectrometry 13:1211-1217. 
5139

LASER ABLATION MAPPING OF SIDEROPHILE ELEMENTS IN THE LATE ARCHAEAN REIVILO IMPACT SPHERULE LAYER, SOUTH AFRICA

McDonald $^{1}$, B. Simonson ${ }^{2}$, C. Koeberl ${ }^{3}$ and W. U. Reimold ${ }^{4}$. ${ }^{1}$ School of Earth \& Ocean Sciences, Cardiff University, Cardiff CF10 3YE, UK, E-mail: mcdonaldi1@cf.ac.uk. ${ }^{2}$ Oberlin College, Oberlin, OH 44074-1052, USA. ${ }^{3}$ University of Vienna, Althanstrasse 14, A-1090 Vienna, Austria. ${ }^{4}$ Museum für Naturkunde-Leibniz Institute at Humboldt University Berlin, Invalidenstrasse 43, 10115 Berlin, Germany.

Introduction: Layers of spherules formed during late Archaean to early Proterozoic impacts have been recognized in the coeval Hamersley Basin of western Australia and the Griqualand West basin of South Africa [1]. Three spherule layers in the Hamersley Basin (the Jeerinah, Paraburdoo and Dales Gorge layers) can be correlated with equivalents (Monteville, Reivilo and Kuruman) in the Griqualand West Basin [2]. The Paraburdoo-Reivilo layer is remarkable in that it contains an extremely high (up to $40 \%$ ) extraterrestrial component (ETC), represented by platinum-group element (PGE) contents, similar to some early Archaean spherule layers [3, 4] from Barberton. The high ETC present in the layer allows in-situ trace element mapping of the distribution of the extraterrestrial components within and around impact spherules in order to constrain the processes by which they formed and the manner by which projectile material became incorporated.

Methods: Individual spherules (or clusters of spherules) and surrounding matrix from the Reivilo layer were mapped by time resolved laser ablation ICP-MS using a 12 micron laser beam and similar procedures to those described by [5]. The isotopes used for analysis were ${ }^{24} \mathrm{Mg},{ }^{27} \mathrm{Al},{ }^{29} \mathrm{Si},{ }^{34} \mathrm{~S},{ }^{39} \mathrm{~K},{ }^{44} \mathrm{Ca},{ }^{47} \mathrm{Ti},{ }^{51} \mathrm{~V},{ }^{52} \mathrm{Cr},{ }^{55} \mathrm{Mn},{ }^{57} \mathrm{Fe},{ }^{59} \mathrm{Co},{ }^{61} \mathrm{Ni}$, ${ }^{65} \mathrm{Cu},{ }^{66} \mathrm{Zn},{ }^{75} \mathrm{As},{ }^{95} \mathrm{Mo},{ }^{99} \mathrm{Ru},{ }^{103} \mathrm{Rh},{ }^{106} \mathrm{Pd},{ }^{107} \mathrm{Ag},{ }^{121} \mathrm{Sb},{ }^{125} \mathrm{Te},{ }^{140} \mathrm{Ce}$, ${ }^{185} \mathrm{Re},{ }^{189} \mathrm{Os},{ }^{193} \mathrm{Ir},{ }^{195} \mathrm{Pt},{ }^{197} \mathrm{Au}$ and ${ }^{209} \mathrm{Bi}$. NIST610 was used as a reference standard and for repeat analyses to monitor drift over the analytical run.

Results: Element maps produced to date suggest that Os, Ir, Ru, $\mathrm{Rh}$, and $\mathrm{Pt}, \mathrm{Ni}$ and $\mathrm{Co}$ are confined to the interiors of spherules but also that some spherules are free of siderophiles. The PGE occur most notably as microparticles (nuggets?) that occur close to spherule rims and not evenly through the spherule. Palladium and $\mathrm{Au}$ are present at low concentrations and concentrated in the matrix between spherules. Some spherules have been infilled or partially replaced by sulfides (millerite, pyrite, chalcopyrite). These have elevated $\mathrm{Ni}, \mathrm{Cu}$, and $\mathrm{Co}$ contents, but the sulfides are free of PGE and PGE peaks elsewhere never coincide with elevated $\mathrm{S}$. Other elements, such as $\mathrm{Mg}, \mathrm{Mn}$, and $\mathrm{Fe}$, are depleted in the spherules and concentrated in the matrix.

Discussion: The presence of spherules with high temperature PGE nuggets and also siderophile-free spherules is significant. If these findings are confirmed by further work, it suggests the spherules formed from a mix of projectile-rich and projectile-free impact melts, not a homogenized cloud of rock vapor [1].

References: [1] Simonson B. et al. 2009. Precambrian Research 169:100-111. [2] Hassler S. W. et al. 2011. Geology 39:307-310. [3] Simonson B. et al. 2009. Precambrian Research 175:51-76. [4] Kyte F. et al. 1992. Geochimica et Cosmochimica Acta 56:1365-1372. [5] Ulrich T. et al. 2009. Canadian Mineralogist 47:1001-1012.
5141

GEOCHEMISTRY OF POST-KINEMATIC MAFIC INTRUSIONS AT MANIITSOQ, WEST GREENLAND: CONTAMINATED MANTLE MELTS OR IMPACT MELTS?

McDonald $^{1}$ and A. A. Garde ${ }^{2}$. ${ }^{1}$ School of Earth \& Ocean Sciences, Cardiff University, Cardiff CF10 3YE, U.K., E-mail: mcdonaldi1@cf.ac.uk. ${ }^{2}$ Geological Survey of Denmark and Greenland, Øster Voldgade 10, 1350 Copenhagen K, Denmark.

Introduction: The $\sim 2.975 \mathrm{Ga}$ Maniitsoq structure in West Greenland may be a Mesoarchaean impact structure [1]. It is centred on a $\sim 200 \mathrm{~km}$ wide aeromagnetic anomaly and contains a near-circular, $>40 \mathrm{~km}$ wide homogenised granitoid core with a diffused boundary, surrounded by a still wider belt of shattered and brecciated country rock with variably deformed and downgraded quartz PDFs, dykes of fluidised microbreccia, abundant evidence of partial melting, and massive hydrothermal alteration. A $70 \mathrm{~km}$ long contemporaneous curvilinear belt of unshocked norite-diorite sills and diorite plugs with proto-orbicular textures, indicative of rapid cooling of superheated melt, occurs east of the core. The norites and diorites are geochemically diverse [2], with zones of sulfide mineralization and the aim of this study has been to test whether any could be downward injections of impact melt below the crater in the manner of offset dykes at Sudbury [3].

Results: Analyses for major elements, trace elements and platinumgroup elements (PGE) were performed by ICP and ICP-MS. The noritesdiorites display an enormous range of $\mathrm{MgO}(5.9-23.7 \mathrm{wt} . \%)$ and $\mathrm{Cr}(120-$ $3600 \mathrm{ppm}$ ) over a limited range of $\mathrm{SiO}_{2}(47-59 \mathrm{wt} . \%)$. On Harker plots the intrusions fall on a general array, between primitive mantle melts and local gneisses/amphibolites, that is more extensive, dispersed and shows a stronger mantle component than typically found with impact melts [3]. Unmineralized high-Mg norites contain low concentrations of PGE $(\mathrm{Ir}=0.03-0.2 \mathrm{ppb})$. Mineralized norites may carry up to $10 \mathrm{ppb}$ Ir but sulfide metal tenors are low and high $\mathrm{Cu} / \mathrm{Pd}$ ratios $\left(10^{4}-10^{6}\right)$ indicate removal of PGE by immiscible sulphides before final emplacement. Norites and diorites typically display strongly suprachondritic $\mathrm{Ru} / \mathrm{Ir}, \mathrm{Rh} /$ $\mathrm{Ir}, \mathrm{Ru} / \mathrm{Rh}$ and $\mathrm{Pt} / \mathrm{Ir}$ typical of mantle melts. However, one low-Mg diorite (283761) south of the structure core plots off the main compositional array and contains the highest concentration of Ir ( $0.59 \mathrm{ppb}$ ), along with chondritic $\mathrm{Ru} / \mathrm{Ir}, \mathrm{Rh} / \mathrm{Ir}$ and $\mathrm{Ru} / \mathrm{Rh}$ ratios.

Discussion: The evidence suggests that most of the 2.975 Ga noritediorite intrusions are not impact melts but represent contaminated mafic magma, intruding upwards from the mantle below the structure, possibly in response to post-impact decompression. However one intrusion sampled to date may represent impact melt injected downwards from a former overlying melt sheet.

References: [1] Garde A. A. 2010. Abstracts and Proceedings of the Geological Society of Norway 1:57-58. [2] Garde A. A. 1997. Geological Survey of Greenland Bulletin 177:115. [3] Wood C. R. and Spray J. G. 1998. Meteoritics \& Planetary Science 33:337-347. 
5362

\section{DOES VESTA HAVE MOONS?}

L. A. McFadden ${ }^{1}$, M. Sykes ${ }^{2}$, S. Joy ${ }^{3}$, P. Tricarico ${ }^{2}$, D. O'Brien ${ }^{2}$, J. Y. $\mathrm{Li}^{4}$, M. Mutchler ${ }^{5}$, Nargess Memarsadeghi ${ }^{1}$, H. Safavi ${ }^{1}$, P. GutierrezMarques $^{6}$, A. Nathues ${ }^{6}$, S. Mottola ${ }^{7}$, H. Sierks ${ }^{6}$, S. Schroder ${ }^{6}$, C. Polansky ${ }^{8}$, R. Jacobson ${ }^{8}$, C. T. Russell ${ }^{3}$, C. A. Raymond ${ }^{8}$, M. Rayman $^{8}$, S. Weinstein-Weiss ${ }^{8}$, E. Palmer ${ }^{2} .{ }^{1}$ NASA, Goddard Space Flight Center. E-mail: lucy.mcfadden@nasa.gov. ${ }^{2}$ Planetary Science Institute, Tucson, USA. ${ }^{3}$ IGPP, UCLA. ${ }^{4}$ U. Maryland. ${ }^{5}$ Space Telescope Science Institute. ${ }^{6}$ Max-Planck-Institut für Sonnensystemforschung, Lindau, Germany. ${ }^{8}$ California Inst. Technology, Jet Propulsion Laboratory.

Previous searches for moons around Vesta have found nothing to an upper limit of 22.5 magnitude, that corresponds to $44 \pm 4 \mathrm{~m}$ diameter assuming the same albedo as Vesta. The Dawn mission's approach phase has dedicated satellite search observations consisting of two mosaic sequences bracketing the first observations of a complete rotation of Vesta scheduled for early July, 2011. In addition, we use the approach optical navigation image sequences for initial satellite searches. We will report any findings from these observations, and upper limits of magnitude and size.
5051

NAKHLA: A MARTIAN METEORITE WITH INDIGENOUS ORGANIC CARBONACEOUS FEATURES

D. S. McKay ${ }^{1}$, E. K. Gibson ${ }^{1}$, K. L. Thomas-Keprta ${ }^{2}$, S. J. Clemett ${ }^{2}$, L. $\mathrm{Le}^{2}$, Z. Rahman ${ }^{2}$ and S. J. Wentworth ${ }^{2}$. ${ }^{1}$ ARES, NASA/JSC, Mail Code KA, Houston, TX 77058, USA. ${ }^{2}$ ESCG at NASA/JSC, Mail Code JE23, Houston, TX 77058, USA. E-mail: david.s.mckay@nasa.gov.

The Nakhla meteorite possesses discrete, well defined, structurally coherent morphologies of carbonaceous phases present within iddingsite alteration zones. Based upon both isotopic measurements and analysis of organic phases the presence of pre-terrestrial organics is now recognized. Within the microcrystalline layers of Nakhla's iddingsite, discrete clusters of salt crystals are present. These salts are predominantly halite $(\mathrm{NaCl})$ with minor $\mathrm{MgCl}_{2}$ crystals. Some $\mathrm{CaSO}_{4}$, likely gypsum, appears to be partially intergrown with some of the halite. EDX mapping shows discrete $\mathrm{C}$-rich features are interspersed among these crystals.

A hollow semi-spherical 'bowl' structure $(\sim 3 \mu \mathrm{m})$ has been identified and analyzed after using a focused ion beam (FIB) to cut a transverse TEM thin section of the feature and the underlying iddingsite. TEM/ EDX analysis reveals that the feature is primarily carbonaceous containing $\mathrm{C}$ with lesser amounts of $\mathrm{Si}, \mathrm{S}, \mathrm{Ca}, \mathrm{Cl}, \mathrm{F}, \mathrm{Na}$, and minor Mnand Fe; additionally a small peak consistent with $\mathrm{N}$, which has been previously seen in Nakhla carbonaceous matter, is also present. Selected area electron diffraction (SAED) shows that this C-rich material is amorphous (lacking any long-range crystallographic order) and is not graphite or carbonate. Micro-Raman spectra acquired from the same surface from which the FIB section was extracted demonstrate a typical kerogen-like $\mathrm{D}$ and $\mathrm{G}$ band structure with a weak absorption peak at 1350 and a stronger peak at $1600 \mathrm{~cm}^{-1}$. The C-rich feature is intimately associated with both the surrounding halite and underlying iddingsite matrix.

Both iddingsite and salts are interpreted as having formed as evaporate assemblages from progressive evaporation of water bodies on Mars. This assemblage, sans the carbonaceous moieties, closely resembles iddingsite alteration features previously described which were interpreted as indigenous Martian assemblages. These distinctive macromolecular carbonaceous structures in Nakhla may represent one of the sources of the high molecular weight organic material previously identified in Nakhla.

While we do not speculate on the origin of these unique carbonaceous structures, we note that the significance of such observations is that it may allow us to construct a C-cycle for Mars based on the chemistry of the Martian meteorites with obvious implications for astrobiology and the prebiotic evolution of Mars. In any case, our observations strongly suggest that organic $\mathrm{C}$ exists as micrometer-size, discrete structures on Mars.

Acknowledgment: Receipt of the Nakhla samples from Caroline Smith and the British Museum Natural History is acknowledged. 
5113

Mn-Cr SYSTEMATICS OF PALLASITE OLIVINE BY SIMS: CONTRASTING Cr IN BRAHIN AND BRENHAM

S. J. McKibbin, T. R. Ireland, Y. Amelin, P. Holden and P. Lanc. Research School of Earth Sciences, Australian National University, Australia. E-mail: seann.mckibbin@anu.edu.au.

Introduction: Pallasite meteorites are mixtures of $\mathrm{Fe}-\mathrm{Ni}$ metal and olivine and would appear to be good candidates for ${ }^{53} \mathrm{Mn}-{ }^{53} \mathrm{Cr}$ dating $\left(\mathrm{t}_{1 / 2} 3.7 \mathrm{Ma}\right)$. However, conflicting ion-probe results have been reported for these rocks, with some workers finding elevated ${ }^{53} \mathrm{Cr} /{ }^{52} \mathrm{Cr}$ correlated with ${ }^{55} \mathrm{Mn} /{ }^{52} \mathrm{Cr}[1,2]$ while others do not [3]. Recently, it has been suggested that a statistical effect at low count rates arising from the reduction of data as a series of ratios could introduce positive bias into isotope measurements for some applications [4], leading [5] to retract earlier findings of elevated ${ }^{53} \mathrm{Cr} /{ }^{52} \mathrm{Cr}$ in pallasite olivine $[1,2]$. To investigate whether in-situ accumulated radiogenic ${ }^{53} \mathrm{Cr}$ exists in these meteorites, we have re-analyzed olivine from two petrographically contrasting pallasites: Brahin (which has a fragmental texture), and Brenham (with rounded olivine).

Methods: In situ isotopic analysis was performed using sensitive high-mass resolution ion microprobe reverse geometry (SHRIMP-RG) on olivine and chromite from Brahin and olivine from Brenham. The primary sputtering beam was run at high current (17-66 nA as a mixture of $\mathrm{O}^{-}$and $\mathrm{O}_{2}^{-}$) and focused to a $\sim 30$ micron diameter spot, giving strong $\mathrm{Cr}$ secondary ion beams and hence minimizing the effect described by [4]. ${ }^{52} \mathrm{Cr}^{+},{ }^{53} \mathrm{Cr}^{+}$and ${ }^{55} \mathrm{Mn}^{+}$were measured with an electron multiplier, and San Carlos olivine was used as an external standard to correct for instrumental fractionation on ${ }^{53} \mathrm{Cr}^{+} /{ }^{52} \mathrm{Cr}^{+}$and ${ }^{55} \mathrm{Mn}^{+} /{ }^{52} \mathrm{Cr}^{+}$.

Results: We find that olivine and chromite in Brahin has normal $\mathrm{Cr}$ isotopic composition within analytical error $\left(\begin{array}{lll}\sim & 1 \%\end{array}\right)$. In contrast, Brenham olivine shows $\mathrm{Cr}$-isotope excesses (up to $6 \pm 1 \% 1 \sigma$ ) within a few 10's of microns of olivine rims. These are well correlated with ${ }^{55} \mathrm{Mn} /{ }^{52} \mathrm{Cr}$ and imply an initial ${ }^{53} \mathrm{Mn} /{ }^{55} \mathrm{Mn}$ of $2.55( \pm 0.62) \times 10^{-5}$ at the time of isotopic closure of $\mathrm{Cr}$ in Brenham olivine rims.

Discussion: Our finding of isotopically normal $\mathrm{Cr}$ in Brahin is in agreement with [3], while our initial ${ }^{53} \mathrm{Mn} /{ }^{55} \mathrm{Mn}$ for Brenham is even higher than originally reported by $[1,2]$. Previous ion-probe studies had 30-250 counts per cycle for ${ }^{52} \mathrm{Cr}^{+}$over 150 cycles [5], but with higher beam currents and improved ion transmission we have achieved $1-4 \times 10^{4}$ counts per cycle, with $80-200$ cycles for each analysis. We estimate bias arising from the effect highlighted by [4] to be $\sim 0.1 \%$ for our measurements.

The contrasting Mn-Cr systematics found here is good evidence for localised $\mathrm{Cr}$-isotope variation in some, but not all, pallasites. The initial ${ }^{53} \mathrm{Mn} /{ }^{55} \mathrm{Mn}$ found for Brenham is not compatible with current understanding of extinct nuclide chronology (with solar system initial ${ }^{53} \mathrm{Mn} /{ }^{55} \mathrm{Mn}$ of $\left.9.1 \quad( \pm 1.7) \times 10^{-6} ; \quad[6]\right)$ and probably represents concentration of radiogenic ${ }^{53} \mathrm{Cr}$ at olivine rims. This appears to be related to the arrangement of olivine, with the interconnected networks in Brenham [7] exhibiting anomalous $\mathrm{Cr}$ while isolated olivine fragments in Brahin do not.

References: [1] Hsu et al. 1997. Abstract \#1783. 28th Lunar and Planetary Science Conference. [2] Hsu W. 2005. Geochemical Journal 39:311. [3] Tomiyama T. et al. 2007. Abstract \#2007. 38th Lunar and Planetary Science Conference. [4] Ogliore et al. 2011. Abstract \#1592. 42nd Lunar and Planetary Science Conference. [5] Huss et al. 2011. Abstract \#2608. 42nd Lunar and Planetary Science Conference. [6] Nyquist et al. 2009. Geochimica et Cosmochimica Acta 73:5115-5136. [7] Spinsby et al. 2008. Computers \& Geosciences 34:1-7.
5114

SIMS Mn-Cr SYSTEMATICS OF OLIVINE FROM THE ANGRITE
METEORITE D'ORBIGNY: AN INTER-LABORATORY
COMPARISON

S. J. McKibbin ${ }^{1}$, T. R. Ireland ${ }^{1}$, Y. Amelin ${ }^{1}$, N. Sugiura ${ }^{2}$, P. Holden ${ }^{1}$ and P. Lanc ${ }^{1}$. ${ }^{1}$ Research School of Earth Sciences, Australian National University, Australia. E-mail: seann.mckibbin@gmail.com. ${ }^{2}$ Department of Earth and Planetary Science, University of Tokyo, Tokyo, Japan.

Introduction: In recent years, the angrite meteorites have proven suitable for dating by the ${ }^{53} \mathrm{Mn}-{ }^{53} \mathrm{Cr}$ extinct nuclide system using in situ techniques [1-3]. However, analyses of the D'Orbigny angrite using two secondary ion mass spectrometers (SIMS) [1,2] revealed a significant interlaboratory bias. The initial ${ }^{53} \mathrm{Mn} /{ }^{55} \mathrm{Mn}$ for this meteorite is reported as $2.84( \pm 0.24) \times 10^{-6}$ by [1] and $\sim 3.5 \times 10^{-6}$ [2]. To test whether heterogeneous $\mathrm{Mn}-\mathrm{Cr}$ systematics or differences in methodology caused this bias, we have re-analyzed the piece of D'Orbigny from [1] using the method of [2].

Methods: The D'Orbigny sample was mounted inside a circular standard holder containing reference materials to monitor instrumental mass and inter-element fractionation. We have previously developed a method for in situ measurement of $\mathrm{Mn}-\mathrm{Cr}$ systematics in olivine using Sensitive High-mass Resolution Ion Micro Probe (SHRIMP; models RG and II) which we apply here. $\mathrm{Mn}-\mathrm{Cr}$ relative sensitivity was obtained from synthetic $\mathrm{Fe}$ - and $\mathrm{Ca}$-rich olivines [2]. Our correction for relative sensitivity is in the range of $0.54-0.80$ depending on the matrix.

Results: As previously reported, D'Orbigny olivine is strongly zoned in major and trace elements, and we find a good correlation between ${ }^{55} \mathrm{Mn}^{+} /{ }^{52} \mathrm{Cr}^{+}$and ${ }^{53} \mathrm{Cr}^{+} / 52 \mathrm{Cr}^{+}$. After correction for inter-element sensitivity (using a three-component mixing model to account for the continuously varying major-element matrix) and mass fractionation of $\mathrm{Cr}$ isotopes, the gradient of our regression gives an initial ${ }^{53} \mathrm{Mn} /{ }^{55} \mathrm{Mn}$ of 3.61 $( \pm 0.39) \times 10^{-6}$.

Discussion: Our new result agrees with our previous finding for another piece of D'Orbigny [2], but disagrees with previous work on this chip at the University of Tokyo [1]. This indicates that the discrepancy is not caused by partial resetting of the $\mathrm{Mn}-\mathrm{Cr}$ system or presence of cosmogenic isotopes. The $\mathrm{Mn}-\mathrm{Cr}$ relative sensitivity factors of $0.54-0.80$, determined in this study, do not seem to be responsible either, since they are similar to that of $0.617 \pm 0.046$ reported in [1]. Potential biases arising during data reduction of low count rate measurements [4,5] could influence $\mathrm{Cr}$-isotope ratios of low-Cr olivine in angrites, but rejection of analyses with the lowest $\mathrm{Cr}$ concentrations (and hence highest $\mathrm{Mn} / \mathrm{Cr}$ ) does not change the gradient of our regression to systematically lower values (e.g., for analyses with ${ }^{55} \mathrm{Mn} /{ }^{52} \mathrm{Cr}<10^{5}$, the same range as in [1], we find initial ${ }^{53} \mathrm{Mn} /{ }^{55} \mathrm{Mn}$ of $\left.3.63( \pm 0.47) \times 10^{-6}\right)$. We are currently investigating the effects of different data reduction methods. The discrepancy might also be related to differing styles of instrumental mass fractionation in CAMECA and SHRIMP ion probes, in which case the two-isotope external correction method may not accurately account for Cr-isotope fractionation in one or both of these instruments.

References: [1] Sugiura N. et al. 2005 Earth Planets and Space 57: e13-e16. [2] McKibbin S. J. et al. 2008. Abstract \#5132. 71st MetSoc. [3] McKibbin S. J. et al. 2010. Abstract \#5172. 73rd Metsoc. [4] Ogliore R. C. et al. 2011. Abstract \#1592. 42nd Lunar and Planetary Science Conference. [5] Huss G. R. et al. 2011. Abstract \#2608. 42nd Lunar and Planetary Science Conference. 


\section{1}

\section{DAWN ARRIVES AT VESTA}

H. Y. McSween ${ }^{1}$, C. T. Russell ${ }^{2}$ and C. A. Raymond ${ }^{3}$. ${ }^{1}$ Department of Earth and Planetary Sciences, University of Tennessee, Knoxville, TN 37996-1410, USA. ${ }^{2}$ Institute of Geophysics and Planetary Physics, University of California, Los Angeles, CA 90095-1567, USA. ${ }^{3}$ Jet Propulsion Laboratory, Pasadena, CA 91109, USA.

The Dawn spacecraft, launched in 2007 and fueled by ion engines, will perform a rendezvous with asteroid 4 Vesta, a differentiated body thought to be the source of the HED meteorites. Dawn will spend roughly a year in Vesta orbit studying its surface and gravity field to determine its geologic history and to establish its relationship with the HEDs. The Framing Camera (FC) will obtain image mosaics in multiple color filters at $<100 \mathrm{~m} \mathrm{px}^{-1}$ spatial resolution, and partial coverage at $25 \mathrm{~m} \mathrm{px}^{-1}$ resolution, to map the geology, geomorphology, and tectonic fabric, and to characterize space weathering. Multi-angle clear filter images will be used to construct a topographic model to $100 \mathrm{~m}$ spatial resolution and $10 \mathrm{~m}$ height accuracy. The Visible and InfraRed Spectrometer (VIR) maps spectral variations between 0.25 and $5 \mu \mathrm{m}$ to resolve mineralogy, geologic units, weathering effects and thermal brightness variations. The elemental composition of the surface is resolved to 10 spatial pixels by the Gamma Ray and Neutron Detector (GRaND). Together with the spherical harmonic gravity field derived from navigation, these observations will provide a comprehensive data set to investigate Vesta's differentiation, magmatic and tectonic history, effects of impacts, and weathering of the surface. During approach, rotational characterizations will be performed to update Vesta's spin pole, finalize instrument calibrations, and tune exposure times for imaging instruments.

At the time of the 2011 METSOC meeting, Dawn will have just entered its Survey orbit, observing Vesta for seven orbits over 21 days at a mean radius of $3000 \mathrm{~km}$, while the Sun is at its highest southern latitudes. After achieving global color and multispectral maps with FC and VIR, the spacecraft transfers to the High-Altitude Mapping Orbit (HAMO, mean radius $950 \mathrm{~km}$ ). In HAMO FC performs detailed global mapping in multiple color filters, while VIR collects high-resolution multispectral data in the southern hemisphere. After 30 days in HAMO, Dawn transfers to the Low-Altitude Mapping Orbit (LAMO, mean radius $460 \mathrm{~km}$ ), where GRaND maps Vesta's surface for nominally 70 days, while daily tracking passes map the gravity field. Following LAMO, Dawn spirals out and stops at HAMO altitude to map the newly illuminated terrain in the northern polar region. Departure for Ceres is scheduled for July 2012 to meet an arrival in February 2015.

\section{7}

\section{ANALYSIS OF ALMAHATA SITTA METEORITE}

H. T. Meadni ${ }^{1}$ and G. M. Taha ${ }^{2}{ }^{1}$ Student Department of Physics. E-mail: haboyaasl@yahoo.com. ${ }^{2}$ Student/department of Chemistry. E-mail: ghadamhmd11@yahoo.com.

Fragments of Almahata Sitta meteorite was analyzed by a team of undergraduate students at the departments of Chemistry and Physics at University of Khartoum, as an attempt to obtain chemical information to classify the meteorite chemically. Sample \#27 was analyzed using Atomic Absorption spectroscopy for the elemental analysis ${ }^{2}$, the results shows various amounts of cobalt, calcium, magnesium, zinc, manganese, and high percentage of iron up to $(44.6 \%)$. And for the identification of amino acids the high performance liquid chromatography is used to detect trace amounts of six different amino acids.

Also a fragment sample\#43 was analyzed using X-ray diffraction technique in order to obtain structural information and the XRD data was studied using WINFIT and POWDERCELL software. The estimated crystallite size of different phases of the sample was found to range between $20 \mathrm{~nm}$ to $60 \mathrm{~nm}$.

The plasma formation technique is used to detect the light elements in Almahata Sitta meteorite. This was investigated by using high voltage pluses which produce high pluses current. The electrons beam was focused by external magnetic field to defuse electron on target surface geometry with pulse 177 ns. Brightness of plasma emission and spectroscopic analysis show different content of some meteorite samples. The spectroscopic absorption pattern indicates the existence of some light elements in Almahata Sitta meteorite such as $(\mathrm{Cu}, \mathrm{N}, \mathrm{F}, \mathrm{I}, \mathrm{Kr})$ in addition to $\mathrm{Ne}$ and $\mathrm{Pm}$.

References: [1] SHADDAD M. H. et al. 2010. Meteoritics \& Planetary Science 45:1553-1556. [2] Skoog D. A. and West D. M. 1980. Principles of instrumental analysis, second edition. 
5039

MAKING THE MOON FROM THE EARTH'S MANTLE-THE GEOCHEMICAL PERSPECTIVE

M. M. M. Meier ${ }^{1}$, A. Reufer ${ }^{2}$, W. Benz ${ }^{2}$ and R. Wieler ${ }^{1} .{ }^{1}$ Earth Sciences, ETH Zurich, Switzerland. E-mail: meier@erdw.ethz.ch. ${ }^{2}$ Physics Department, University of Berne, Switzerland.

Introduction: The formation of the Moon from a circum-terrestrial disk ejected in a collision between the proto-Earth and a roughly Marssized impactor (a.k.a. the giant impact or GI hypothesis [1]) is widely accepted today. However, the close geochemical similarity of lunar rocks and Earth's mantle has posed a serious problem for the hypothesis, as simulations of the GI consistently [1] had the Moon forming predominantly $(\sim 80 \%)$ from impactor material. In view of large isotopic and elemental inhomogeneities in different solar system objects, e.g., in $\mathrm{O}$, $\mathrm{Si}, \mathrm{Cr}$ and $\mathrm{W}$ isotopic ratios, or $\mathrm{Mg} / \mathrm{Cr}$ and $\mathrm{Hf} / \mathrm{W}$ elemental ratios, it seems unlikely that the impactor had a composition closely matching that of the Earth. Similarity in $\mathrm{W}$ isotopes and $\mathrm{Hf} / \mathrm{W}$ ratios further implies that proto-Earth and impactor must have formed their cores at the same time. A post-impact re-equilibration model has been proposed to account for the similarity in $\mathrm{O}$ isotopes [2], but so far, no complete, quantitative model of this exists [3]. It therefore remains unclear at this stage whether this re-equilibration model can satisfy all geochemical constraints. Recently, a modified GI scenario with new parameters was presented, where a steep, high-velocity collision yields an iron-depleted disk mainly derived from terrestrial (or "target") material and a reasonable EarthMoon angular momentum [4]. Here, we compare the predictions from this model with constraints from the above-mentioned geochemical systems.

Results and Discussion: The geochemical similarity of Earth and Moon can be expressed in a factor $\delta \mathrm{f}_{\mathrm{T}}=\left(\mathrm{M}_{\text {target }} / \mathrm{M}_{\text {total }}\right)_{\text {disk }} /\left(\mathrm{M}_{\text {target }} /\right.$ $\left.\mathrm{M}_{\text {total }}\right)_{\text {post-impact Earth }}-1$, reflecting the depletion of terrestrial material in the Moon-forming disk. Since both Earth and Moon incorporate significant amounts of impactor material, it is this difference in relative contributions that has to approach zero to satisfy geochemical constraints. The exact value of $\delta \mathrm{f}_{\mathrm{T}}$ depends on the composition of protoEarth and impactor, but also on their initial mass and the mass lost from the system. Typical values for $\delta \mathrm{f}_{\mathrm{T}}$ in the "canonical" model [1] do not exceed $-66 \%$, incompatible with geochemical constraints. For an impactor that resembles Mars in $\mathrm{O}, \mathrm{Si}$ and $\mathrm{Cr}$ isotopic composition, $\delta \mathrm{f}_{\mathrm{T}}$ should exceed $-5 \%,-59 \%$ and $-45 \%$, respectively. A typical outcome of the new high-velocity GI model is a $\delta \mathrm{f}_{\mathrm{T}}$ of $-34 \%$, compatible with these constraints except for $\mathrm{O}$. However, $\mathrm{O}$ may be a special case, as there are at least three different solar system materials (Earth, E- and CIchondrites) that plot on the terrestrial fractionation line, possibly because they all sample the same reservoir, from which also the impactor could have been derived. Alternatively, post-impact re-equilibration as proposed by [2][3] may have erased any remaining isotopic differences.

Conclusions: The high-velocity GI model [4] can satisfy the known geochemical constraints. A possible exception is $\mathrm{O}$, if the impactor is as different in $\mathrm{O}$ isotope composition as Mars. In this case, post-impact reequilibration [2] is still needed, for which our model constitutes a new starting point, as the high-velocity GI model also predicts a much hotter early Earth. This may have implications for geophysics and the formation of the atmosphere.

References: [1] Canup et al. 2001. Nature 412:708. [2] Pahlevan and Stevenson. 2007. Earth and Planetary Science Letters 262:438. [3] Pahlevan et al. 2011. Earth and Planetary Science Letters 301:433. [4] Reufer et al. 2011. Abstract \#1136. 42nd Lunar and Planetary Science Conference.

\section{3}

THERMAL HISTORY OF VIG1623-5 FUN CAI: EXPERIMENTAL MODELING

R. A. Mendybaev and F. M. Richter. University of Chicago, IL, USA. E-mail: ramendyb@uchicago.edu.

Introduction: Vig 1623-5 is a FUN CAI composed mainly of 30$170 \mu \mathrm{m}$ grains of forsterite (Fo) enclosed within pyroxene (Px) and/or åkermanitic $\left(\AA \mathrm{K}_{89}\right)$ melilite (Mel) and surrounded by incomplete gehlenitic $\left(\AA \mathrm{k}_{16-60}\right)$ melilite mantle [1]. The inclusion is characterized by extremely large mass-dependent fractionations of magnesium, silicon and oxygen isotopes [1, 2]. Earlier we showed [3] that isothermal evaporation of FUN1 melt $\left(\mathrm{Mg}_{2} \mathrm{SiO}_{4}\right.$ plus several wt $\%$ of $\mathrm{CaO}$ and $\left.\mathrm{Al}_{2} \mathrm{O}_{3}\right)$ at $1900{ }^{\circ} \mathrm{C}$ results in residues with chemical and isotopic compositions of $\mathrm{Mg}, \mathrm{Si}$ and $\mathrm{O}$ close to those in Vig1623-5. In this paper we present results of cooling experiments aimed at reproducing the texture of 1623-5.

Experiments: Most experiments have been conducted in a vacuum furnace using FUN1 and FUN2 melt compositions; the latter is a product of evaporation of FUN1 and has a composition close to 1623-5 (see [3] and references therein). A one-atmosphere furnace was used in experiments when FUN2 melt was cooled from temperatures below $1600{ }^{\circ} \mathrm{C}$ in $\mathrm{H}_{2}$.

Results: Cooling FUN1 from $1850{ }^{\circ} \mathrm{C}$ in vacuum at $30-120{ }^{\circ} \mathrm{C} \mathrm{h}^{-1}$ results in residues composed of Fo and Px. Contrary to the crystallization in a closed system, fast cooling $\left(\sim 120^{\circ} \mathrm{C} \mathrm{h}^{-1}\right)$ leads in formation of massive Fo (up to $1 \mathrm{~mm}$ in size) while slow cooling $\left(\sim 30^{\circ} \mathrm{C} \mathrm{h}^{-1}\right)$ results in "quench-like" textures. This is due to the fact that as $\mathrm{Mg}$ and $\mathrm{Si}$ evaporate, the liquidus of the melt decreases, but when the evaporation rate becomes sufficiently slow, instant nucleation and crystallization of Fo begins. These experiments show that although evaporation of $\mathrm{Mg}$ and $\mathrm{Si}$ from FUN1 melt results in residues appropriately enriched in heavy $\mathrm{Mg}$ and $\mathrm{Si}$ isotopes [3], the texture and mineral compositions of the residues are distinctly different from that of Vig1623-5.

Cooling of FUN2 melt in vacuum from $\sim 1650{ }^{\circ} \mathrm{C}$ at $30-60{ }^{\circ} \mathrm{C} \mathrm{h}^{-1}$ results in Fo (up to $200-300 \mu \mathrm{m}$ ) embedded in Px and Mel. Small Sp grains and some An are also observed in the residues. The compositions of forsterite (up to $1.5 \mathrm{wt} \% \mathrm{CaO}$ ) and the $\mathrm{Ca}-$, Al-rich pyroxene in the residues are close to that of Vig1623-5 [1]. Melilite from these experiments, however, is more gehlenitic $\left(\sim \AA \mathrm{k}_{75}\right)$ than the $\AA \mathrm{k}_{89}$ found in the main mass of Vig1623-5.

We showed that when Type B melt is exposed to $\mathrm{H}_{2}$ melilite mantle forms [4]. Similar experiments are underway using FUN2 melt. Run products from the vacuum experiments described above were reheated into one atm $\mathrm{H}_{2}$ and cooled from $\mathrm{T} \leq 1600^{\circ} \mathrm{C}$. We have not yet succeed in forming a melilite mantle, but cooling from $1425^{\circ} \mathrm{C}$ at 10 and $25^{\circ} \mathrm{C} \mathrm{h}^{-}$ ${ }^{1}$ results in $10-50 \mu \mathrm{m}$ thick forsterite-free mantle $(5-10 \mu \mathrm{m} \mathrm{Sp}$ grains are enclosed into Mel and Px). The expectation based on the phase diagram is that slower cooling (or slightly higher starting temperatures) will evaporate sufficient $\mathrm{Mg}$ and $\mathrm{Si}$ so that $\mathrm{Px}$ would convert into $\mathrm{Mel}$ followed by dissolution of $\mathrm{Sp}$ in the gehlenitic Mel. The last step in this process was observed in evaporation experiments with Type B-like compositions [4].

Conclusions: Formation of texture similar to Vig1623-5 FUN CAI seems to require multiple heating and cooling events.

References: [1] Davis et al. 1991. Geochimica et Cosmochimica Acta 55:621-637. [2] Marin-Carbonne et al. 2011. 74th Meteoritics Society Meeting. [3] Mendybaev et al. 2010. Abstract \#2725. 41st Lunar and Planetary Science Conference. [4] Mendybaev et al. 2006. Geochimica et Cosmochimica Acta 70:2622-2642. 
5145

$\mu$-FTIR, $\mu$-RAMAN SPECTROSCOPIES AND SEM-EDX ANALYSES OF GRAINS OF THE PARIS METEORITE

S. Merouane ${ }^{1}$, Z. Djouadi ${ }^{1}$, R. Brunetto ${ }^{1}$, J. Borg ${ }^{1}$ and P. Dumas ${ }^{2}$. ${ }^{1}$ IAS, UMR 8617 Orsay, France. ${ }^{2}$ Synchrotron SOLEIL, SaintAubin, France. E-mail: sihane.merouane@ias.u-psud.fr.

Introduction: We investigated a few fragments from the Paris meteorite through mid- and far-infrared micro-spectroscopy $(2.5-100 \mu \mathrm{m})$, Raman spectroscopy and SEM-EDX analyses to get information on their mineralogy and composition (organics and elementary). These data allow us to compare Paris (at least these fragments if representative of the meteorite) with other carbonaceous chondrites in particular, and, more generally, with other primitive extraterrestrial objects present in our collections (e.g., IDPs for Interplanetary Dust Particles).

Experiments: Few micron-sized fragments among the $13 \mathrm{mg}$ received from the MNHN (Paris, France) were crushed into a Diamond compression cell [1]. Infrared spectra were obtained on the SMIS2 beamline of the SOLEIL synchrotron (France) with a NicPlan microscope attached to a Fourier transform infrared spectrometer (FTIR). Raman spectra were acquired with a spectrometer DXR from Thermo Fisher with a $532 \mathrm{~nm}$ laser and EDX analyses were performed with a SEM Hitachi $3600 \mathrm{~N}$ and an EDX spectrometer ThermoNoran System SIX at IEF (Orsay, France).

Results and Discussion: Mineralogy Infrared spectra are dominated by phyllosilicates and carbonates, implying a significant aqueous alteration, most probably on the parent body. Far-infrared and Raman spectra reveal the presence of magnetite and traces of sulfides, possibly troilite. Only one inclusion of crystalline anhydrous silicate (mixture of magnesium-rich olivine and pyroxene) was found in the analyzed fragments.

Organic Matter The study by IR spectroscopy of the aliphatic organic matter present in the meteorite leads to a $\mathrm{CH}_{2} / \mathrm{CH}_{3}$ ratio of $2.3 \pm 0.2$, which is in good agreement with the reported values in Orgueil (CI) and Murchison (CM) [2]. The aromatic carbon (detected by Raman spectroscopy) has a quite ordered structure indicating that these grains have suffered a weak thermal alteration and seem to be less primitive than IDPs [3].

Elementary Composition Some elementary ratios were extracted from the EDX data analyses. The obtained values $\mathrm{Mg} / \mathrm{Si}=0.88, \mathrm{Fe} / \mathrm{Si}=0.95$, $\mathrm{S} / \mathrm{Si}=0.42, \quad \mathrm{Al} / \mathrm{Si}=0.091, \quad$ compatible with the chondritic values reported by Anders and Grevesse [4], in the range of CI and CM elementary ratios $[5,6]$. However, $\mathrm{Ni} / \mathrm{Si}=0.078$ and $\mathrm{Ca} / \mathrm{Si}=0.25$ are higher than for the $\mathrm{CI}$ and $\mathrm{CM}$ chondrites. Our analytical techniques show an excellent correlation between the spatial distribution of the carbonates (found by IR spectroscopy) and element $\mathrm{Ca}$ (as shown by EDX mapping) implying that major part of the $\mathrm{Ca}$ is incorporated in the carbonates.

Conclusion: The combination of different analytical techniques enables both qualitative and quantitative studies of the samples. Microspectroscopy is also a useful tool to determine the distribution of the different components in micron-sized grains.

The different analyses of the few fragments indicate that they have undergone a significant aqueous alteration and a weak thermal alteration. Both their mineralogical and organic composition, as well as their aromatic component, classify these grains of the Paris meteorite close to the CI or CM meteorite-type.

References: [1] Grossemy F. 2008. Ph.D. thesis [2] Matrajt G. et al. 2005. Astronomy \& Astrophysics 433:979-995 [3] Raynal P. I. 2003. Ph.D. thesis. [4] Anders E. and Grevesse N. 1989. Geochimica et Cosmochimica Acta 53:197-214. [5] Brownlee D. E. 1978. IAU Colloq. 52:134-150 [6] Hudson G. J. et al. 1981. Science 211:383-386.
5146

QUANTITATIVE STUDY OF THE $10 \mu M$ SILICATE BAND IN INTERPLANETARY DUST PARTICLES

S. Merouane ${ }^{1}$, Z. Djouadi ${ }^{1}$, R. Brunetto ${ }^{1}$, J. Borg ${ }^{1}$ and $P$. Dumas ${ }^{2} .{ }^{1}$ IAS, UMR 8617, Orsay, France. ${ }^{2}$ Synchrotron SOLEIL, Saint-Aubin, France, E-mail: sihane.merouane@ias.u-psud.fr.

Introduction: Interplanetary dust particles (IDPs) are particles originating from an asteroidal or cometary parent body. Their mineralogical composition can give clues on their parent bodies' characteristics and thus on the formation of the solar system [1].

In this study we analyze a few IDPs of the NASA collection with complementary analytical techniques (Infrared (IR) micro-spectroscopy, Raman spectroscopy and SEM-EDX analyses) to get information on their mineralogy, their organics and their elementary composition.

Experiment: The studied IDPs were crushed into a Diamond'EXPress compression cell following the protocol of $[2,3]$. IR micro-spectroscopy from mid to far $(2.5-100 \mu \mathrm{m})$ infrared is performed on the SMIS2 beamline of the SOLEIL synchrotron (France) using a NicPlan microscope attached to a Fourier Transform infrared spectrometer (FTIR). Raman spectra were acquired at SOLEIL with a spectrometer DXR from Thermo Fisher with a $532 \mathrm{~nm}$ laser and SEMEDX analyses were performed with a SEM Hitachi $3600 \mathrm{~N}$ and an EDX spectrometer ThermoNoran System SIX at "l'Institut d'Electronique Fondamentale" (IEF, Orsay, France).

Procedure: The present work focuses on the mineralogical composition of the grains, and more particularly on the silicate signature, given in IR by the $10 \mu \mathrm{m}$ feature, corresponding to the well-known stretching mode of the Si-O bonds. We are developing an analytical procedure consisting on a "deconvolution" of this band in order to quantify its different contributors (olivine, pyroxene, as well as other possible species, either crystalline or amorphous). These contributors are identified by combining the obtained results with the different analytical techniques (IR, Raman and SEM-EDX).

Silicate-type spectra were first acquired for standard minerals (olivine, pyroxene). We used crystalline minerals crushed in a diamond cell (as the same manner as the IDPs) and amorphous thin films synthesized by electron beam evaporation from the crystalline silicates [4] on a $\mathrm{KBr}$ or CsI substrate. Using a linear combination of the standard spectra (amorphous and crystalline), we are able to fit the $10 \mu \mathrm{m}$ band of the IDPs.

We will present the first results obtained on the validation of our analytical procedure on synthetic samples and its application on IDPs. This work is still under improvement but this technique seems promising to infer the quantitative mineralogical composition of IDPs and thus give clues on the processes undergone by their parent bodies.

References: [1] Sandford S. A. and Bradley J. P. 1989. Icarus 82:146-166. [2] Grossemy F. 2008. Ph.D. thesis, France. [3] Brunetto R. et al. 2011. Icarus 212:896-910. [4] Djouadi Z. et al. 2005. Astronomy \& Astrophysics 440:179-184. 
5471

FIRST ANALYSIS OF ALL XENON ISOTOPES IN GENESIS SOLAR WIND AloS COLLECTOR

A. Meshik ${ }^{1}$, O. Pravdivtseva ${ }^{1}$, C. M. Hohenberg ${ }^{1}$, J. H. Allton ${ }^{2}$ and D. S. Burnett ${ }^{3}$. ${ }^{1}$ Washington University, Physics CB1105, Saint Louis, MO 63130. E-mail: am@physics.wustl.edu. ${ }^{2}$ NASA, Johnson Space Center, Houston. ${ }^{3}$ Geology 100-23, CalTech, Pasadena.

Introduction: Recently we made successful measurements of all solar wind (SW) krypton isotopes in Genesis Al-collectors [1]. Here we report results of 20 independent analysis of Xe extracted from Aluminum on Sapphire (AloS) under four different experimental conditions.

Experimental: Separation of the solar wind heavy noble gases, captured in Genesis collectors, from surface and possible interface contaminations is not trivial. One way of dealing with these contaminations is depth profiling. But due to low SW-Xe abundance it requires an extremely sensitive mass spectrometer, such as RELAX [2]. In this work we developed a different approach, extracting total $\mathrm{Xe}$ and $\mathrm{Kr}$ and making a blank correction on the basis of measured $\mathrm{Kr} / \mathrm{Xe}$ ratios which are different in the SW and the blank. The later turned out to be not atmospheric and was determined by minimizing the spread of calculated SW-Xe fluencies between different extracted areas.

Results: SW fluence and preliminary isotopic composition of Xe extracted from AloS at four different conditions are shown in the table below, where $\alpha$-angle between incident laser beam and the plane normal to the surface of Al-film; $\mathrm{n}$ - number of independent laser extractions.

Conclusion: Our preliminary Genesis Xe results are generally agree with SW-Xe obtained from the lunar soil $[3,4]$. Some subtle differences need to be further investigated. We plan to verify our data treatment and possibly develop some rejection criteria since in several cases the data scatter exceed statistically expected deviations.

Acknowledgment: Supported by NASA grant NNX07AM76G.

References: [1] Meshik A. P. et al. 2011. Abstract \#2703. 42nd Lunar and Planetary Science Conference. [2] Crowther S. A. and Gilmour J. D. 2011. Abstract \#1969. 42nd Lunar and Planetary Science Conference. [3] Wieler R. and Baur H. 1994. Meteoritics 29:570-580. [4] Pepin R. O. et al. 1995. Geochimica et Cosmochimica Acta 59:4997-5022.

\begin{tabular}{cccccc}
\hline & $\begin{array}{l}\alpha=\pi / 8 \\
\mathrm{n}=4\end{array}$ & $\begin{array}{l}\alpha=\pi / 4 \\
\mathrm{n}=6\end{array}$ & $\begin{array}{l}\alpha=\pi / 2 \\
\mathrm{n}=6\end{array}$ & $\begin{array}{l}\alpha=\pi / 4 \\
\mathrm{n}=4\end{array}$ & $\begin{array}{l}71501 \\
\text { lunar soil }[3,4]\end{array}$ \\
\hline $\begin{array}{l}{ }^{132} \mathrm{Xe} \mathrm{Sw}_{\mathrm{Sw}} \\
{ }^{10^{6} \mathrm{at} \mathrm{cm}}{ }^{-2}\end{array}$ & 1.23 & 1.01 & 1.16 & 1.18 & \\
${ }^{136} \mathrm{Xe} /{ }^{132} \mathrm{Xe}$ & $.2966 \pm .0028$ & $.2987 \pm .0025$ & $.3002 \pm .0015$ & $.3013 \pm .0007$ & $.2985 \pm .0009$ \\
${ }^{134} \mathrm{Xe} / 132 \mathrm{Xe}$ & $.3713 \pm .0029$ & $.3699 \pm .0028$ & $.3749 \pm .0018$ & $.3724 \pm .0008$ & $.3666 \pm .0015$ \\
${ }^{131} \mathrm{Xe} /{ }^{132} \mathrm{Xe}$ & $.8135 \pm .0083$ & $.8297 \pm .0047$ & $.8190 \pm .0026$ & $.8302 \pm .0016$ & $.8272 \pm .0028$ \\
${ }^{130} \mathrm{Xe} /{ }^{132} \mathrm{Xe}$ & $.1613 \pm .0020$ & $.1641 \pm .0017$ & $.1614 \pm .0010$ & $.1612 \pm .0005$ & $.1661 \pm .0009$ \\
${ }^{129} \mathrm{Xe} / 132 \mathrm{Xe}$ & $1.0413 \pm .0051$ & $1.0473 \pm .0025$ & $1.0408 \pm .0014$ & $1.0358 \pm .0015$ & $1.0420 \pm .0070$ \\
${ }^{128} \mathrm{Xe} /{ }^{132} \mathrm{Xe}$ & $.0829 \pm .0014$ & $.0833 \pm .0012$ & $.0818 \pm .0007$ & $.0846 \pm .0003$ & $.0848 \pm .0009$ \\
${ }^{126} \mathrm{Xe} /{ }^{132} \mathrm{Xe}$ & $.00405 \pm .00029$ & $.00389 \pm .00027$ & $.00371 \pm .00017$ & $.00438 \pm .00013$ & $.00423 \pm .00014$ \\
${ }^{124} \mathrm{Xe} /{ }^{132} \mathrm{Xe}$ & $.00520 \pm .00036$ & $.00510 \pm .00030$ & $.00460 \pm .00018$ & $.00484 \pm .00009$ & $.00488 \pm .00012$ \\
\hline
\end{tabular}


5457

PRESOLAR SILICON CARBIDE X GRAINS, EXPLOSIVE HYDROGEN BURNING, AND THE EVOLUTION OF MASSIVE STARS

B. S. Meyer ${ }^{1}$, M. J. Bojazi ${ }^{1}$, L.-S. The ${ }^{1}$ and M. F. El Eid ${ }^{2} .{ }^{1}$ Department $^{2}$ of Physics and Astronomy, Clemson University, Clemson, SC 29634-0978, USA. ${ }^{2}$ Department of Physics, American University of Beirut, Beirut, Lebanon.

Introduction: The nitrogen-15 abundance in presolar silicon carbide (SiC) X grains remains a puzzle. These grains presumably formed in the outflows from exploding massive stars (supernovae). Detailed supernova yields [1] fail to explain the correlation between aluminum and nitrogen isotopic signatures in the presolar $\mathrm{SiC} \mathrm{X}$ grains [2].

Nitrogen-15 Production: To help understand the nitrogen isotopic abundances in presolar $\mathrm{SiC} X$ grains, we explore ${ }^{15} \mathrm{~N}$ nucleosynthesis in massive stars. Nitrogen-15 is produced as the supernova shock passes through the helium-rich layers [3] and heats them up. Nitrogen-14 captures an alpha particle to make ${ }^{18} \mathrm{~F}$. The ${ }^{18} \mathrm{~F}$ can then undergo a $(\mathrm{n}, \alpha)$ reaction to produce ${ }^{15} \mathrm{~N}$. It can also be produced by explosive hydrogen burning when the shock first encounters the hydrogen-rich outer layers of the star. In these shock-heated layers, abundant ${ }^{14} \mathrm{~N}$ captures a proton to become ${ }^{15} \mathrm{O}$. After the shocked layers expand and cool, the ${ }^{15} \mathrm{O}$ decays to ${ }^{15} \mathrm{~N}$. The amount of ${ }^{15} \mathrm{~N}$ produced depends on the post-shock temperature.

Presupernova Stellar Evolution: Most supernova models do not show evidence of strong explosive hydrogen burning; however, the 25 solar mass model of [1] does show a strong spike in the ${ }^{15} \mathrm{~N}$ abundance due to this burning. The carbon and nitrogen signatures in presolar SiC $\mathrm{X}$ grains can be explained if material from the ${ }^{15} \mathrm{~N}$ spike is included in mixing models to account for the grains' origins [2]. The 25 solar mass model in [1] shows an ${ }^{15} \mathrm{~N}$ spike whereas, for example, the 15 solar mass models does not because the pre-shock temperature at the inner edge of the hydrogen shell in the 25 solar model is roughly $5 \times 10^{7} \mathrm{~K}$ while it is about $2 \times 10^{7} \mathrm{~K}$ for the 15 solar mass model. Since the post shock temperature is a multiple of the pre-shock temperature (about 2 for a Mach 2 shock and 4 for a Mach 3 shock), the 25 solar mass model can achieve a higher post-shock temperature than the 15 solar mass model for the same speed shock.

Our presupernova models of a 25 solar mass star show significant differences in the pre-shock temperature at the inner H-shell depending on the treatment of convection and mass loss [4]. If we include mass loss and use the Schwarzschild convection criterion, the temperature at the inner edge of the hydrogen shell is $2.5 \times 10^{7} \mathrm{~K}$. If we include semi-convection using the Ledoux criterion, the inner-H shell temperature is $\sim 3.5 \times 10^{7} \mathrm{~K}$. If we use the Schwarzschild convection criterion and do not include mass loss, the temperature is $\sim 2 \times 10^{7} \mathrm{~K}$. These differences mean that, if indeed ${ }^{15} \mathrm{~N}$-spike material is needed to explain the nitrogen composition of $\mathrm{SiC} \mathrm{X}$ grains, presolar $\mathrm{SiC} \mathrm{X}$ grains may help constrain mixing and mass loss in presupernova stellar evolution.

References: [1] Rauscher T. et al. 2002. The Astrophysical Journal 576:323-348. [2] Lin Y. et al. 2010. The Astrophysical Journal 709:11571173. [3] Meyer B. S. and Bojazi M. J. 2011. Abstract \#2376. 42nd Lunar and Planetary Science Conference. [4] El Eid M. F. et al. 2009. Space Science Reviews 147:1-29.

\section{9}

PROCESS OF THE SUEVITE GENESIS OF THE RIES CRATER, GERMANY

C. Meyer and M. Jébrak. Université du Québec à Montréal, Canada. E-mail: cornelia.meyer@utweiler.de.

Introduction: The processes of formation and transport of particles in suevite during impact crater formation remain poorly understood and were investigated at the 15 -Ma-old, $25 \mathrm{~km}$ wide Ries crater, Germany. The suevite of the Ries crater occurs in three different geological settings: (1) Crater suevite in the central crater cavity inside the inner ring; (2) Outer suevite on top of the continuous ejecta blanket; and (3) Dikes in the crater basement and in displaced megablocks. For suevite genesis, the following processes have been discussed to-date in literature: (1) Fall-back of material into the crater and its periphery upon collapse of an ejecta plume [1], and (2) Horizontal transport of ejected material, akin to (a) an impact melt flow [2], (b) a pyroclastic flow [3], (c) initiated by phreatomagmatic explosion [4].

Methods: In order to differentiate between these emplacement modes we analyzed at different locations inside and outside the crater: (1) The three-dimensional shape and orientation of the suevite particles, (2) The stereometric parameters of melt particles and lithic clasts (grain shape, grain size distribution, and content of particles), and (3) The chemical composition of the melt particles compared to their corresponding matrix.

Results: The suevite can be distinguished in the following subunits: (1) Suevite with similar melt and matrix composition, which can be considered as original impact melt; (2) Strongly altered suevite, with similar melt and matrix composition; (3) Suevite containing K-poor and K-rich melt with either silicate matrix similar in chemistry to the melt, or non filled pore spaces; and (4) Suevite with different melt and matrix composition.

Discussion: Five stages can be distinguished for the formation and deposition of the Ries suevite: (1) An early ejecta plume, mainly composed of projectile and sediments, is formed during the excavation stage and is deposited ( $<2 \mathrm{~m}$ thick) inside and outside the crater on top of the Bunte Breccia after crater formation, (2) After a hiatus, the interaction of water with the impact melt lake induce a phreatomagmatic explosion of the upper melt lake, (3) A basal, non erosive pyroclastic surge, initiated by the explosion, moves radially outward and is deposited inside and outside the crater, (4) At the same time an ejection column rises. After its collapse, a density stratified pyroclastic flow is initiated which propagates to the crater margin and partially covers the first deposited suevite layer, (5) The collapse of the ejection column creates contemporaneously an upward rising ejecta plume, where accretionary lapilli are formed. Finally, these lapilli, together with the residual plume material, are deposited in the inner crater (a) into an existing water layer, or (b) reworked by incoming water in the crater at a later time.

Acknowledgment: This work was prepared at the Museum für Naturkunde, Berlin, and was supported by the Deutsche Forschungsgemeinschaft (RE 528/4-1).

References: [1] Stöffler D. 1977. Geologica Bavaria 75:443-458. [2] Osinski G. R. et al. 2004. Meteoritics \& Planetary Science 39:1655-1683. [3] Newsom H. E. 1986. Journal of Geophysical Research 91:E239-E251. [4] Artemieva N. A. et al. 2009. Abstract \#1526. 40th Lunar and Planetary Science Conference. 


\section{9}

ELECTRON BACK-SCATTER DIFFRACTION STUDY OF IRON METAL IN ALMAHATA SITTA UREILITE

T. Mikouchi ${ }^{1}$, C. A. Goodrich ${ }^{2}$, V. H. Hoffmann ${ }^{3,4}$, M. E. Zolensky ${ }^{5}$ and K. Sugiyama ${ }^{6}{ }^{1}$ Department of Earth and Planetary Science, University of Tokyo, Tokyo 113-0033, Japan. ${ }^{2}$ Planetary Science Institute, Tucson, AZ 85719, USA. ${ }^{3}$ Department of Geoscience, University of Tuebingen, 72076 Tuebingen, Germany. ${ }^{4}$ Department of Geography-Environmental Science, University of Munich, 80333 Muenchen, Germany. ${ }^{5}$ NASA-JSC, Houston, TX 77058, USA. ${ }^{6}$ Institute of Materials Research, Tohoku University, Miyagi 980-0812, Japan. E-mail: mikouchi@eps.s.u-tokyo. ac.jp.

Almahata Sitta (AS) is a polymict breccia mainly composed of ureilite lithologies showing variable mineralogy [1]. These ureilite lithologies can be texturally divided into two types. One is a compact and coarse-grained, and composed of large olivine and pigeonite (reaching mm-size) with graphite and metal, and is similar to typical ureilites. The other is porous and consists of fine-grained (10-20 $\mu \mathrm{m}$ in size) olivine and pyroxene with small amounts of metal and graphite. Fe metal is commonly observed in both types and its unique mineralogy can offer important clues to understand the ureilite formation history [2, 3]. Here we report electron back-scatter diffraction (EBSD) analysis of Fe metal and associated opaque phases in three ureilite samples of AS.

The sample \#1 (AS\#1) is fine-grained similar to the sample \#7 (AS\#7) that was first described after the fall [1]. We observed both large vein and small scattered $\mathrm{Fe}$ metal grains in these two samples by EBSD. Almost all $\mathrm{Fe}$ metal grains are $b c c$ kamacite $(\alpha-\mathrm{Fe})$ although they show variable grain sizes $(5-100 \mu \mathrm{m})$. The sample \#44 (AS\#44) is a typical coarse-grained sample and contains vein metal (approximately $500 \mu \mathrm{m}$ long) at grain boundaries between silicates [2]. These areas show a large diversity of phase assemblages and textures [2, 4]. One common assemblage is a crystalline intergrowth of two phases with apparent contrast in BEI in spite of no obvious compositional difference by EMPA [2]. The sample prepared by colloidal-silica polishing for EBSD work shows clear relief surface in these areas corresponding to the BEI contrast. In other intergrowths, elongated laths (up to $30 \mu \mathrm{m}$ long) are present and are inferred from BEI and EMPA to be cohenite $\left(\mathrm{Fe}_{3} \mathrm{C}\right)$. EBSD analysis showed that the obtained Kikuchi bands match with the cohenite structure. In these intergrowths, $\mathrm{Fe}$ metal is homogeneous in BEI, but EBSD shows that small amounts of $\gamma$-Fe $(f c c)$ were present as well as kamacite.

The coexistence of two Fe metal phases ( $\alpha$ and $\gamma$ ) and cohenite in one of the AS\#44 metal intergrowths is important and similar occurrence can be found in commercial iron alloy [5]. $\gamma$-Fe ("austenite") is stable at high temperature and upon cooling it decomposes into $\alpha$-Fe ("ferrite") and $\mathrm{Fe}_{3} \mathrm{C}$ ("cementite") at low temperature [5]. The small amount of $\gamma$-Fe in AS\#44 may be relict because of fast cooling by shock melting [2]. Alternatively, shock remelting of pre-existing metal + graphite formed a eutectic-looking texture. Because the carbon content of these areas is far too high to have been dissolved in austenite at high temperature, we prefer the latter hypothesis. In contrast, Fe metal in AS\#1 and \#7 does not contain cohenite and $\gamma$-iron. This is probably due to their different thermal history and lower $\mathrm{C}$ content.

References: [1] Zolensky M. E. et al. 2010. Meteoritics \& Planetary Science 45:1618-1637. [2] Goodrich C. A. et al. 2010. Meteoritics \& Planetary Science 45:A66. [3] Hoffmann V. H. et al. 2011. Meteoritics \& Planetary Science 46: in press. [4] Ross A. et al. 2011. Abstract \#2720. 42nd Lunar and Planetary Science Conference. [5] Smith W. F. and Hashemi J. 2006. Foundations of materials science and engineering, McGraw-Hill. pp. 1056.

\section{7}

HYDROCODE SIMULATIONS OF BINARY ASTEROID IMPACTS

K. Miljkovic, S. Mannick, G. S. Collins and P. A. Bland. Earth Science \& Engineering, Imperial College London, UK. E-mail: k.miljkovic@ impreial.ac.uk.

Introduction: Observational data [1, 2] and numerical modeling [3, 4] show that $15 \%$ of the near-Earth asteroid (NEA) population are binary. However, the observed crater populations on Earth [5] and Venus [6] indicate that the number of doublet impact craters is much less than $15 \%$. This discrepancy could originate from the assumption that may imply that not all binary asteroids create a clearly separated doublet crater, many could form a single or a complex shaped crater. In this work we use numerical modeling to quantify the threshold for the formation of distinct doublet craters on Earth and compare the complex crater morphology formed by a closely separated binary asteroid impact with that formed by oblique single asteroid impact.

Background: Identification of craters formed by binary asteroid impact on Earth is not simple because the craters are obscured by erosion and the presence of oceans. Conversely, on less active planetary bodies identifying doublet craters is hampered by a saturation of craters [6]. A rare example of a terrestrial doublet crater is Clearwater East and West that is estimated to have formed $290 \pm 20 \mathrm{Myr}$ ago [7]. The low probability of two craters forming so close together in space and time suggests they were formed at the same time by a binary asteroid. Clearwater East and West craters are $32 \mathrm{~km}$ and $22 \mathrm{~km}$ in diameter, respectively. Applying impact scaling laws [8] to the size of these craters we estimate that the impacting binary asteroid comprised a $3.3 \mathrm{~km}$ diameter primary and $2.1 \mathrm{~km}$ diameter secondary. The two craters are separated by $28.5 \mathrm{~km}$, which corresponds to 11 times the primary asteroid diameter (Dp).

Method: The iSALE-3D hydrocode [9] was used to simulate binary asteroid impacts on Earth, using a granite material model as a representative for Earth's surface and the asteroids. In the simulations, the asteroid diameters were: (i) $\mathrm{Dp}=1200 \mathrm{~m}$ for the primary and $\mathrm{Ds}=600 \mathrm{~m}$ for the secondary asteroid and (ii) $\mathrm{Dp}=\mathrm{Ds}=600 \mathrm{~m}$. The separation between the binary components (L) was varied from $1.25 \mathrm{Dp}$ to $10 \mathrm{Dp}$. These values are representative of $\mathrm{Ds} / \mathrm{Dp}$ and $\mathrm{L}$ for binary asteroids in NEA region, based on current observations [1].

Results: Preliminary results show that impact of a closely separated binary asteroid forms an elongated crater shape. For case (i) a single crater forms with a tear drop shape for $\mathrm{L}<8 \mathrm{Dp}$; a doublet crater is formed if $\mathrm{L}>8 \mathrm{Dp}$. For case (ii) a single, elliptical crater is formed for $\mathrm{L}<6 \mathrm{Dp}$, if $6<\mathrm{L}<8 \mathrm{Dp}$ the elliptical crater starts to show two offcentre peaks and only for $\mathrm{L}>10 \mathrm{Dp}$ does the impact form a doublet.

References: [1] Pravec P. et al. 2006. Icarus 181:63-93. [2] Margot J. L. et al. 2002. Science 296:1445-1448. [3] Richardson D. C. et al. 1998. Icarus 134:47-76. [4] Bottke W. F. and Melosh H. J. 1996. Icarus 124:327-391. [5] Melosh H. J. and Stansberry J. A. 1991. Icarus 94:171179. [6] Cook C. M. et al. 2003 Icarus 165:90-100. [7] Earth Impact database (http://www.passc.net/EarthImpactDatabase/index.html). [8] Schmidt R. M. and Housen K. R. 1987. International Journal of Impact Engineering 5:543-560. [9] Elbeshausen D. et al. 2009 Icarus 204:716-731. 
5484

$\mathrm{Mg}$ AND $\mathrm{Cr}$ ISOTOPIC COMPOSITIONS OF INDIVIDUAL CHONDRULES FROM PRIMITIVE CHONDRITES

C. A. Miller, R. W. Carlson and C. M. O'D. Alexander. ${ }^{1}$ Department of Terrestrial Magnetism, Carnegie Institution of Washington, 5241 Broad Branch Road NW, Washington, D.C. 20015, USA. E-mail: cmiller@dtm.ciw.edu.

Temporal constraints on the evolution of the early solar system are provided by high-precision long-lived (absolute; $\mathrm{Pb}-\mathrm{Pb}$; [1]) and shortlived (relative; e.g., Al-Mg, Mn-Cr; [2, 3]) radiochronometers. Short-lived chronometers are particularly informative as their geochemical variety permits evaluation of a variety of processes.

A series of high-precision $\mathrm{Pb}-\mathrm{Pb}$ and $\mathrm{Al}-\mathrm{Mg}$ ages indicate that, with the exception of the $\mathrm{CBs}$, chondrules formed over an interval of 1.8 Myr [2, 4]. A correlation between older chondrule ages and lower concentrations of volatile elements such as $\mathrm{Si}$ has also been suggested [2]. Such an extensive period of chondrule formation is difficult to reconcile with models for a turbulent protoplanetary disk that is thoroughly mixed on $10^{4}-10^{6}$ year time scales [5]. If the Al-Mg age spread is an accurate indicator of chondrule formation ages, then the conditions under which chondrules formed must be revisited; if not, then the variability may be due to mixing of older-refractory and younger-volatile reservoirs within chondrules, or of Al-Mg "resetting."

Evaluating individual chondrules for both $\mathrm{Mg}$ and $\mathrm{Cr}$ isotope composition is a way of evaluating these hypotheses. Like Al-Mg, the $\mathrm{Mn}-\mathrm{Cr}$ chronometer is active during the interval of chondrule formation. Relative age differences between chondrules that are consistent across both chronometers would support a lengthy interval of chondrule formation. Also, while the parent element is more refractory than the daughter within the Al-Mg system, the situation is reversed for $\mathrm{Mn}-\mathrm{Cr}$ [6]. Therefore, if chondrule age variability results from the mixing of refractory and volatile reservoirs, individual chondrules with relatively old $\mathrm{Al}-\mathrm{Mg}$ ages should have relatively younger $\mathrm{Mn}-\mathrm{Cr}$ ages.

Techniques for the purification of $\mathrm{Mg}$ and $\mathrm{Cr}$ have been developed and isotope data from will be presented for individual chondrules from primitive carbonaceous and ordinary chondrites.

References: [1] Amelin Y. et al. 2002. Science 297:1678-1683. [2] Mostefaoui S. et al. 2002. Meteoritics \& Planetary Science 37:421-438. [3] Shukolyukov A. and Lugmair G. W. 2006. Earth and Planetary Science Letters 250:200-213. [4] Kurahashi E. et al. 2008. Geochimica et Cosmochimica Acta 72:3865-3882. [5] Alexander C. M. O'D. 2005. Chondrites and the protoplanetary disk pp. 972-1002. [6] Lodders K. 2003. The Astrophysical Journal 591:1220-1247.
5481

${ }^{26} \mathrm{Al}^{-26} \mathrm{Mg}$ CHRONOMETRY OF CAIS FROM CV3 CHONDRITES

R. K. Mishra and M. Chaussidon. Centre de Recherches Pétrographiques et Géochimiques, CRPG - Nancy Université-CNRS, UPR 2300, 15 rue Notre Dame des Pauvres, BP 20, 54501Vandoeuvre les Nancy, France. E-mail: ritesh@crpg.cnrs-nancy.fr.

Introduction: The advancements in analytical capabilities for meteoritic studies have enabled greater precision and detection of smaller isotopic variations for understanding phenomena, processes and their time scales hitherto unexplored. These improvements in analytical techniques open the possibility of utilizing the long-trusted work horse of ${ }^{26} \mathrm{Al}-{ }^{26} \mathrm{Mg}$ systematics to test a few critical assumptions [1-3]. Calcium, aluminumrich inclusions (CAIs) represent the first formed solids of Solar system and have $\mathrm{Pb}-\mathrm{Pb}$ absolute ages of $4568 \mathrm{Ma}[4,5]$. High Precision $\mathrm{Al}-\mathrm{Mg}$ systematics of CAIs can allow to constrain the physico-chemical conditions and their evolution during the earliest stage of birth of solar system [6, 7]. CAIs of different types in Vigarano and Efremovka (CV 3.1-3.4) were analyzed in the present study to: (1) Estimate the spatiotemporal scale of homogeneity (heterogeneity) of isotopic compositions, (2) Elucidate existence (or non existence) of a particular epoch leading to formation of a particular (or all) type of CAIs, (3) Existence of epochs or continuum of high temperature events leading to constraint on their causal's.

Results from ${ }^{26} \mathrm{Al}-{ }^{26} \mathrm{Mg}$ analysis of three CAIs and one AOA from Efremovka and nine CAIs from Vigarano are presented here. The analyzed CAIs include typical type Bs, fine-grained spinel rich, and igneous ones. Their $\mathrm{Mg}$ isotopes show that they formed as early as $70 \mathrm{kyr}$ to as late as $2.5 \mathrm{Myr}$ after the birth of solar system (Fig. 1). Their isotopic studies along with bulk $\mathrm{Al}-\mathrm{Mg}$ ratio can be used to decipher their complex evolution from condensation to melting and re-melting.

References: [1] Villeneuve J. et al. 2009. Science 325:985-988. [2] Jacobsen B. et al. 2008. Earth and Planetary Science Letters 272:353-364. [3] Davis et al. 2010. Abstract \#2496. 41st Lunar and Planetary Science Conference. [4] Bouvier A. and Wadhwa M. 2010. Nature geosciences 3:637-41. [5] Amelin Y. et al. 2010. Earth and Planetary Science Letters 300:343-350. [6] MacPherson G. J. et al. 2010. The Astrophysical Journal 711:L117-12. [7] Kita et al. 2010. Abstract \#2154. 41st Lunar and Planetary Science Conference.

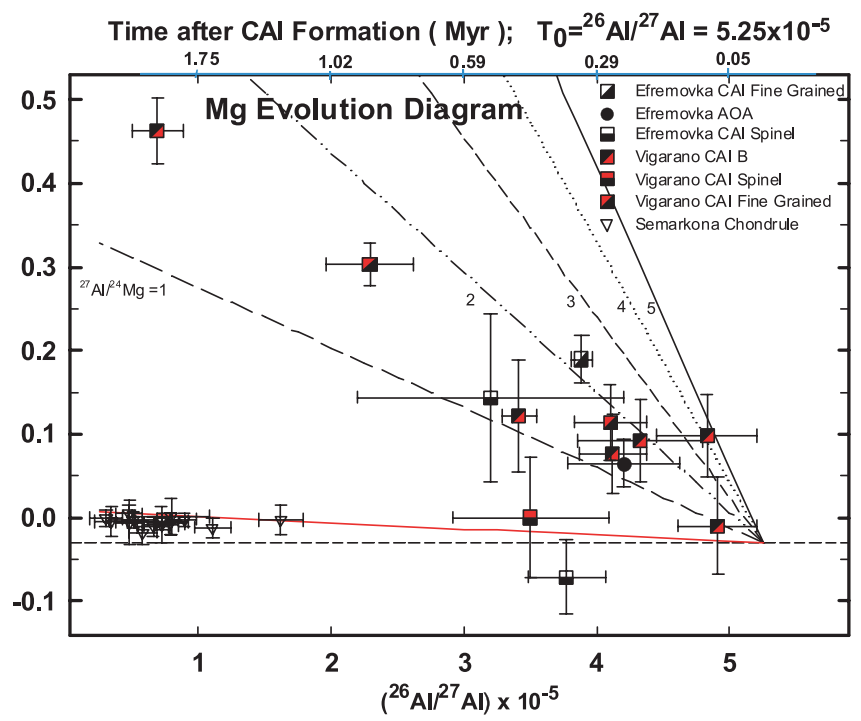

Fig. 1. $\delta{ }^{26} \mathrm{Mg}_{0} \mathrm{~V}_{\mathrm{s}}{ }^{26} \mathrm{Al} /{ }^{27} \mathrm{Al}$ plot of early solar system solids. 
5420

TOWARD A REGOLITH MATURITY INDEX FOR HOWARDITES

D. W. Mittlefehldt ${ }^{1}$, J. A. Cartwright ${ }^{2}$, J. S. Herrin ${ }^{3}$ and K. N. Johnson ${ }^{4}$. ${ }^{1} \mathrm{NASA} /$ Johnson Space Center, Houston, TX, USA. E-mail: david.w.mittlefehldt@nasa.gov. ${ }^{2}$ Max-Planck-Institut für Chemie, Mainz, Germany. ${ }^{3}$ ESGC/Johnson Space Center, Houston, TX, USA. ${ }^{4}$ Arizona State University, Tempe, AZ, USA.

The Dawn spacecraft has just arrived at asteroid 4 Vesta, parent of the howardite, eucrite and diogenite (HED) meteorites [1], to begin a yearlong surface study from orbit [2]. As Dawn will view a debris-covered surface, understanding the formation and mixing processes for the debris layer will strongly aid surface data interpretations. Howardites are polymict breccias mainly composed of clasts derived from basaltic (eucritic) and orthopyroxenitic (diogenitic) parent materials [3]. Some howardites are poorly reworked (fragmental howardites) whilst others have been extensively gardened in an active regolith (regolithic howardites) [4]. The latter may represent an ancient, well-mixed regolith, whilst the former may be from more recent ejecta deposits [4]. Due to environmental differences, regolith development on Vesta differs in detail from that on the Moon [4-6].

We have been developing petrological criteria to apply to howardite thin sections to determine their relative regolithic maturity, which we are fine-tuning with comparison to noble gas data $[7,8]$. Whilst we previously emphasized the abundance of "reworked" clasts (fragmental and impactmelt breccia clasts), this is an imperfect criterion: one howardite with abundant reworked clasts (EET 99408) shows no evidence of solar wind $\mathrm{Ne}$ (SW-Ne), yet, two of our alleged fragmental howardites have clear SW-Ne signatures (LEW 85313, MET 00423) [7, 8].

We are now investigating the diversity in minor and trace element contents of low-Ca pyroxene clasts in howardites as a measure of regolith grade, and will begin analyses of such grains within reworked clasts. Our hypothesis is that regolithic howardites (and the breccia clasts they contain) will show greater diversity because they sampled more diverse diogenitic plutons than fragmental howardites, which formed from ejecta from only a few impacts [e.g., 4]. Our initial LA-ICP-MS work showed ranges in trace element diversity in low-Ca pyroxenes (estimated from the standard error of the mean of analyses), where those howardites considered of medium to high regolithic grade showed greater diversity [9]. Our EMPA results (from a larger howardite suite) show an overall greater diversity in our putative medium to high regolithic grade howardites, though there are exceptions. The greatest diversity is found for paired howardites GRO 95574 and GRO 95581, which were not considered regolithic in our initial study. We will continue investigating avenues to determine regolith maturity in thin section, factoring in bulk rock compositional data, and will coordinate these studies with noble gas results.

References: [1] Drake M. J. 2001. Meteoritics \& Planetary Science 36:501-513. [2] Russell C. T. et al. 2006. Advances in Space Research 38:2043-2048. [3] Mittlefehldt D. W. et al. 1998. Reviews in Mineralogy vol. 36, chapter 4. [4] Warren P. H. et al. 2009. Geochimica et Cosmochimica Acta 73:5918-5943. [5] Housen K. R. and Wilkening L. L. 1982. Annual Review of Earth and Planetary Sciences 10:355-376. [6] Mittlefehldt D. W. et al. 2011. Abstract \#2569. 42nd Lunar and Planetary Science Conference. [7] Cartwright J. A. et al. 2011. Abstract \#2655. 42nd Lunar and Planetary Science Conference. [8] Cartwright J. A. et al. 2011. Abstract \#5042. This Conference. [9] Johnson K. N. et al. 2011. Abstract \#2073. 42nd Lunar and Planetary Science Conference.
5422

IN SITU TRACE ELEMENT MEASUREMENTS ON RODA AND THE ORIGIN OF DIOGENITES

D. W. Mittlefehldt ${ }^{1}$ and J. S. Herrin ${ }^{2} .{ }^{1}$ NASA/Johnson Space Center, Houston, TX, USA. E-mail: david.w.mittlefehldt@nasa.gov. ${ }^{2} E S G C /$ Johnson Space Center, Houston, TX, USA.

The origin of diogenites remains poorly understood. A recent model interprets many diogenites to have been formed from melts that were derived by remelting initial magma ocean cumulates, and these penultimate parent melts were then contaminated by melts derived from remelting of the basaltic (eucritic) crust to form the ultimate diogenite parent melts [1] (hereafter the remelting model). This is a very complicated petrogenesis that has profound implications for the geological evolution of 4 Vesta if correct. This model was developed based on trace element analyses of bulk rock samples that had been leached in acids to remove phosphates; the compositions of the residues were interpreted to be close to those of cumulus orthopyroxenes \pm plagioclase, chromite and olivine [1]. In situ measurements of phases in diogenites can be used to test this model. We have begun a campaign of laser ablation ICP-MS of orthopyroxene grains in diogenites for this purpose. Here we report our first results on one diogenite, Roda.

We have determined a suite of trace lithophile elements on nine, $\mathrm{mm}$-sized pyroxene grains separated from Roda that have previously been studied $[2,3]$. A key observation supporting the remelting model is the very low $\mathrm{Eu} / \mathrm{Eu}^{*}$ of leached residues; values too low to represent orthopyroxene that crystallized from melts with chondritic $\mathrm{Sm} / \mathrm{Eu}$ and $\mathrm{Gd} / \mathrm{Eu}[1] .\left(\mathrm{Eu}^{*}=\mathrm{Eu}\right.$ interpolated from REE diagrams.) Crustal remelts have low $\mathrm{Sm} / \mathrm{Eu}$ and $\mathrm{Gd} / \mathrm{Eu}$, and orthopyroxenes that crystallized from parent melts contaminated by them would have very low $\mathrm{Eu} / \mathrm{Eu}^{*}[1]$.

Roda grains have $\mathrm{Eu} / \mathrm{Eu}^{*}$ of 0.243 to 0.026 ; the latter a value lower than any measured on bulk diogenite leached residues (0.041) [1]. There is a general negative correlation between $\mathrm{Eu} / \mathrm{Eu}^{*}$ and some incompatible elements $(\mathrm{Zr}, \mathrm{Nb}, \mathrm{Hf})$, but not others (LREE). This appears inconsistent with the remelting model as it would suggest an evolving parent melt with $\mathrm{La}$ decreasing as $\mathrm{Zr}$ increased and $\mathrm{Eu} / \mathrm{Eu}^{*}$ decreased. Grain $\mathrm{R}-15$ includes trace element-rich trapped melt phases $[2,3]$. This grain has the highest $\mathrm{Eu} / \mathrm{Eu}^{*}$ and LREE contents, indicating that the trapped melt had a high $\mathrm{Eu} / \mathrm{Eu}^{*}$. Thus, our first data on one diogenite do not provide support for the remelting model [1].

Roda is unusual in that its orthopyroxene grains show wide ranges in trace element contents [4]. Previous in situ REE analyses of grain R-15 did not reveal evidence for subsolidus equilibration with trace elementrich trapped melt phases, and led to the suggestion that Roda may be polymict, with different grains representing different lithologies of diverse compositions [3]. Thus, based on our results on Roda, it is perhaps premature to abandon the remelting model. In situ measurements on a suite of diogenites is planned to further address this issue.

References: [1] Barrat J.-A. et al. 2010. Geochimica et Cosmochimica Acta 74:6218-6231. [2] Mittlefehldt D. W. 1994. Geochimica et Cosmochimica Acta 58:1537-1552. [3] Fowler G. W. et al. 1997. Abstract \#1494. 28th Lunar and Planetary Science Conference. [4] Fowler G. W. et al. 1995. Geochimica et Cosmochimica Acta 59:3071-3084. 


\section{3}

CARBON-BEARING IMPACT-RELATED MATERIALS OF BURIED CRATER BROKEN AT TAKAMATSU, JAPAN

Y. Miura. Yamaguchi University and Al. I. Cuza University. E-mail: moonyas@hotmail.com.

Introduction: Impact-related evidences at buried and broken impact crater structure in Takamatsu, Shikoku, Japan are reported mainly by gravity anomaly and shocked-related materials on the Cretaceous Ryoke granite [1-10]. Together with impact-related materials on the granitic basement rock, anomalous materials with carbon-bearing materials which are not usually included in the granitic rocks are found at drilled materials. Main purposes of the paper are to elucidate carbon and calcium-bearing nano-particles at the Takamatsu, Shikoku, Japan $[9,10]$.

Anomalous Carbon and Calcium Contents: The Ryoke granitic rock at Takamatsu has few carbon and calcium contents in bulk XRF analyses. However, drilled sample at $950 \mathrm{~m}$ in depth contains nano-grains with high carbon $(0.001-0.01 \mathrm{~mm})$ and $\mathrm{CaSiCO}_{3}$ in composition (in $100 \mathrm{~nm}$ size). Carbon-rich grains in Takamatsu show very weak nanodiamond-like peak in the Raman spectra.

Formation Process of Carbon-Bearing Grains: Main source of carbon and calcium cannot be found in the granitic rock as follows:

1) Carbon sources mainly found at crater bottom (ca. $950 \mathrm{~m}$ in depth) are considered to be foreign rocks of sedimentary limestone (from sea-sediments) or carbon dioxides gas during impact reaction probably on sea-water impact.

2) Calcium sources to form nano-grains of $\mathrm{CaSiCO}_{3}$ in composition are also considered to be sedimentary limestone stored in the underground due to similar origins of carbon sources.

Summary: The present study is summarized as follows:

1) Very high carbon contents at Takamatsu in Japan are obtained in drilled sample of $950 \mathrm{~m}$ in depth, which indicates the sources from sedimentary sea-limestone and/or carbon dioxides gas during impact reaction on sea-impact.

2) Calcium sources with similar origin of carbon to form nanograins of $\mathrm{CaSiCO}_{3}$ in composition are considered to be sedimentary limestone existed in the underground.

References: [1] Kono Y. et al. 1994. Report of Kaken B (Japan) 36 pp. [2] Miura et al. 1995. Meteoritics (USA) 30:552. [3] Miura et al. 1999. Abstract \#1127. 30th Lunar and Planetary Science Conference. [4] Miura et al. 2000. Abstract \#3152. 31st Lunar and Planetary Science Conference. [5] Miura Y. et al. 2000. Abstract \#4132. 31st Lunar and Planetary Science Conference. [6] Miura Y. et al. 2001. Abstract \#1981. 32nd Lunar and Planetary Science Conference. [7] Miura Y. 2002. Abstract \#1231. 33rd Lunar and Planetary Science Conference. [8] Miura Y. et al. 2004. Abstract \#2094. 35th Lunar and Planetary Science Conference. [9] Miura Y. 2005. Abstract \#2394. 34th Lunar and Planetary Science Conference. [10] Miura Y. et al. 2006. Abstract \#1239. 37th Lunar and Planetary Science Conference. [11] Miura Y. 2009. Abstract \#2565. 40th Lunar and Planetary Science Conference.
5047

FIRST EVIDENCE FOR NATURAL DISSOCIATION OF OLIVINE TO SILICATE-PEROVSKITE AND MAGNESIOWÜSTITE IN A SHOCKED MARTIAN METEORITE DAG 735

M. Miyahara ${ }^{1}$, E. Ohtani ${ }^{1}$, S. Ozawa ${ }^{1}$, M. Kimura ${ }^{2}$, A. El Goresy ${ }^{3}$, T. Sakai $^{1}$, T. Nagase ${ }^{1}$, K. Hiraga ${ }^{4}$, N. Hirao ${ }^{5}$ and Y. Ohishi ${ }^{5} .{ }^{1}$ Tohoku University. E-mail: miyahara@m.tohoku.ac.jp. ${ }^{2}$ Ibaraki University. ${ }^{3}$ Universität Bayreuth. ${ }^{4}$ IMR. ${ }^{5}$ SPring-8.

Introduction: High-pressure and -temperature experiments expect that olivine dissociates to silicate-perovskite $(\mathrm{Pv})+$ magnesiowüstite (Mw) at $24 \mathrm{GPa}$ (e.g., [1]). However, the dissociation of olivine at highpressure and -temperature condition has never been reported from any natural samples so far. Therefore, the dissociation mechanism of olivine in natural samples is still unclear. Olivine is one of major constituents of the Martian meteorite. Some Martian meteorites record high-pressure conditions of $\sim 24 \mathrm{GPa}$ or even inferred higher pressures during natural dynamic events (e.g, [2]). It is likely that olivine in such Martian meteorites dissociates into $\mathrm{Pv}+\mathrm{Mw}$ because the pressure conditions are beyond the stable pressure field of olivine, wadsleyite and ringwoodite. Accordingly, we carefully scanned olivine grains in the Martian meteorite, Dar al Gani (DaG) 735 with FE-SEM and FIB-TEM techniques, and here, we report the first evidence for olivine dissociation at high-pressure and -temperature conditions.

Results and Discussion: DaG 735 is an olivine-phyric shergottite and contains a shock-melt vein. The dissociation texture of olivine $\left(\mathrm{Fa}_{34-41}\right)$ was observed in olivine grains adjacent to the shock-melt vein. FE-SEM images show that the dissociation textures can be divided into two settings; 1) fine-grained granular ( $<\sim 100 \mathrm{~nm}$ ) part and 2) coarse-grained $(<\sim 1.0 \mu \mathrm{m})$ granular part. Fine-grained granular textures exist always adjacent to the shock-melt vein. The abundance of coarse-grained granular texture increases in dominance with increasing a distance from the shock-melt vein wall to the interior of the olivine grains. Raman spectra corresponding to pyroxene-glass were obtained from both dissociation settings. FIB-TEM studies show that the fine-grained granular parts consist of equigranular $\mathrm{Mw}$ and vitrified-Pv. The coarsegrained granular parts include fragments with alternating lamellar intergrowth. The alternating lamellae consist of $\mathrm{Mw}$ and vitrified-Pv.

The dissociation mechanisms of olivine or ringwoodite to $\mathrm{Mw}+\mathrm{Pv}$ were previously studied using high-pressure generating devices (e.g., [3]). Micro-textural evolution from lamellar into equigranular, similar to the textural sequence encountered here, was previously observed with increasing temperature but at the same pressure conditions. The microtextural evolution observed in DaG 735 probably resulted from the decreasing thermal gradient but at the same pressure magnitude into the olivine grain during a dynamic event. Olivine dissociation requires the pressure condition of $\sim 24 \mathrm{GPa}$ or more. In addition, a kinetic barrier exists around $700^{\circ} \mathrm{C}$ for the dissociation of olivine [4]; i.e., the dissociation does not take place below $\sim 700^{\circ} \mathrm{C}$ even at higher overstepped pressure. Accordingly, pressure condition recorded in the shock-melt vein of DaG 735 would be beyond $\sim 24 \mathrm{GPa}$, and temperature condition should be $>700^{\circ} \mathrm{C}$ at least.

References: [1] Ito E. and Takahashi E. 1989. Journal of Geophysical Research 94:10637-10646. [2] Stöffler D. et al. 1986. Geochimica et Cosmochimica Acta 50:889-903. [3] Poirier J. P. et al. 1986. Nature 321:603-605. [4] Wang Y. et al. 1997. Science 275:510-513. 
5477

DAG 1054: A NEW ANOMALOUS UREILITE FROM DAR AL GANI V. Moggi-Cecchi ${ }^{1}$, S. Caporali ${ }^{1,3}$ and G. Pratesi ${ }^{2}$. ${ }^{1}$ Museo di Scienze Planetarie, Provincia di Prato, Via Galcianese, 20/h, I-59100 Prato, Italy E-mail: v.moggi@pratoricerche.it. ${ }^{2}$ Dipartimento di Scienze della Terra, Università di Firenze, Via La Pira, 4, I-50121, Florence, Italy. ${ }^{3}$ Dipartimento Chimica, Università di Firenze, Via della Lastruccia, 3, I-50019, Sesto Fiorentino, Italy.

Introduction: $\mathrm{DaG} 1054$ is a new meteorite found in 1999 by Romano Serra during a scientific expedition in the Dar al Gani rocky desert area of Libya. The meteorite consists of a single stone weighing $28.4 \mathrm{~g}$, with no traces of fusion crust. A cut surface on one side reveals an achondritic texture. The type specimen $(8 \mathrm{~g})$, a thick section and a polished thin sections are on deposit at Museo di Scienze Planetarie, Prato, Italy (inv. $\mathrm{N}^{\circ}$ 5138). The Museo del Cielo e della Terra (OAM), Bologna, Italy holds the main mass [1].

Description: The sample has a very fine-grained, homogeneous unbrecciated and mosaicized texture mainly consisting of very small (up to $30 \mu \mathrm{m}$ ) rounded and non-zoned olivine crystals and rare orthopyroxene (Fig. 1a). Carbon-rich material, including very small diamond grains, occurs at grain boundaries but no distinct aggregates of carbonaceous material are visible. Opaque phases are formed almost exclusively by $\mathrm{Fe}-$ silicides of non-stoichometric suessitic composition, dispersed in small to very small (up to $100 \mu \mathrm{m}$ across) grains or sub parallel veins, frequently altered to oxides (Fig. 1b). Very rare sulfide grains (mainly troilite) can be occasionally found. EMPA analyses performed on olivine and pyroxene provided the following results: Low-Ca pyroxene: $\mathrm{Fs}_{12,2-20.9} \mathrm{Wo}_{0.7-3.0}$, $\mathrm{Cr}_{2} \mathrm{O}_{3}=0.85-0.92 \mathrm{wt} \%$; Olivine $\left(\mathrm{Fa}_{11.0-17.1}, \mathrm{CaO}=0.29-0.31 \mathrm{wt} \%\right.$, $\mathrm{Cr}_{2} \mathrm{O}_{3}=0.80-0.85 \mathrm{wt} \%$ ). SEM-EDS analyses performed on the suessitic phase proved it to have a variable, non-stoichometric composition, with 2 main clusters at $\mathrm{Fe}_{78} \mathrm{Si}_{21}$ at $\%(\mathrm{Cr}=0.68, \mathrm{Ni}=0.49)$ and at $\mathrm{Fe}_{74} \mathrm{Si}_{23}$ at. \% $(\mathrm{Cr}=1.34, \mathrm{Ni}=1.54)$. Troilite contains up to $4.3 \mathrm{wt} \% \mathrm{Cr}$. The moisaicized structure suggests a high shock stage; weathering grade is low.

Conclusions: Although several features like the fine grained and mosaicized texture are similar to those displayed by polymict ureilites like Haverö, Y-74154, ALH 81101, and Almahata Sitta, the thin section contains no coarse-grained clasts and appears homogeneous and unbrecciated. The lack of zoning of olivine and the presence of suessite of variable composition, also reported by [2-5] for other ureilites, suggest a closer relationship with both NWA 1241 and the fine-grained clast MS161 of Almahata Sitta [6]. These anomalous features indicate that this meteorite could be a fine-grained monomict clast of a larger polymict body.

References: [1] Weisberg M. K. et al. 2011. Meteriotics \& Planetary Science 46:3, in press. [2] Ikeda Y. 2007. Polar Science 1:45. [3] Herrin J. S. et al. 2007. Abstract \#2404. 38th Lunar and Planetary Science Conference. [4] Ross A. J. et al. 2010. Abstract \#2361. 41st Lunar and Planetary Science Conference. [5] Smith C. L. et al. 2010. Meteriotics \& Planetary Science 45:Suppl., A192. [6] Bishoff A. et al. 2010. Meteriotics \& Planetary Science 45:10-11, 1638.

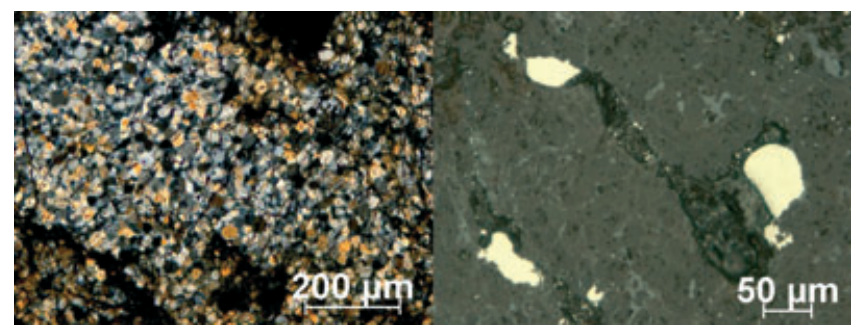

5011

COMETARY NOBLE GASES TRAPPED BY THE STARDUST AEROGEL

R. K. Mohapatra ${ }^{1}$, U. Ott ${ }^{2}$ and I. D. Clark ${ }^{1}{ }^{1}$ University of Ottawa, Ottawa K1N 6N5, Canada. ${ }^{2}$ Max-Planck-Institut für Chemie, D-55020 Mainz, Germany. E-mail: uli.ott@mpic.de.

Introduction: The Stardust mission used monolithic silica aerogel for collecting particles from comet Wild 2 [1] because of its remarkable porosity. During its flight through the comet's coma, Stardust encountered also cometary gas, however. We report here preliminary observations from laboratory experiments and modeling of cometary gas flux at the Stardust aerogel.

Adsorption and Desorption: Results from our experiments on a sample of "pre-flight" aerogel indicate efficient gas adsorption and desorption properties. An aerogel cold trap, presumably cleaner in terms of hydrocarbon background, may be ideal for processing gas samples in simultaneous nitrogen and noble gas mass spectrometry.

Implantation of Cometary Gas: We have also explored the possibility of sampling cometary gas introduced into the Stardust aerogel by implantation. The encounter velocity of $6.1 \mathrm{~km} \mathrm{sec}^{-1}$ [2] corresponds to implantation energy of roughly $0.2 \mathrm{eV}$ per nucleon, which does not support trapping of the light noble gases. We have used the TRIM code [3] to investigate trapping efficiency by implantation for the heavy noble gases- $\mathrm{Ar}, \mathrm{Kr}$, and $\mathrm{Xe}$. Incident cometary argon at the Stardust aerogel was modeled by assuming $\mathrm{Ar} / \mathrm{O}$ as measured in Hale-Bopp [4] and oxygen inferred from water production by Wild $2[2,5]$. Abundances of $\mathrm{Kr}$ and $\mathrm{Xe}$ were derived by assuming solar like $\mathrm{Ar} / \mathrm{Kr} / \mathrm{Xe}$. Results are compiled in Fig. 1. For an aerogel density of $0.066 \mathrm{~g} \mathrm{~cm}^{-3}$, determined on our pre-flight aerogel, implanted $\mathrm{Ar}, \mathrm{Kr}$, and $\mathrm{Xe}$ may reside in the upper $4.5-32 \mathrm{~nm}$ region of the flown aerogel; it would be sited accordingly deeper for a density $<0.01 \mathrm{~g} \mathrm{~cm}^{-3}$ characteristic for the impact surface [1].

Conclusions: Preliminary results from laboratory experiments and modeling of cometary gas suggest near-surface samples of the Stardust aerogel should be searched for possibly implanted cometary gases.

Acknowledgments: We thank M. Zolensky for discussions and the Stardust Allocation committee for providing aerogel samples for our tests.

References: [1] Brownlee et al. 2006. Science 314:1711-1716. [2] http://stardust.jpl.nasa.gov/science/wild2.html. [3] Ziegler J. F. et al. 2010. Nuclear Instruments and Methods in Physics Research B 268:1818-1823. [4] Stern S. A. et al. 2000. The Astrophysical Journal Letters 544:L169L172. [5] de Val-Borro M. et al. 2010. Astronomy \& Astrophysics 521:L50.

\section{$\square$ Avail. Implantation space $\mathbf{0}$ Incident Gas Blanks}

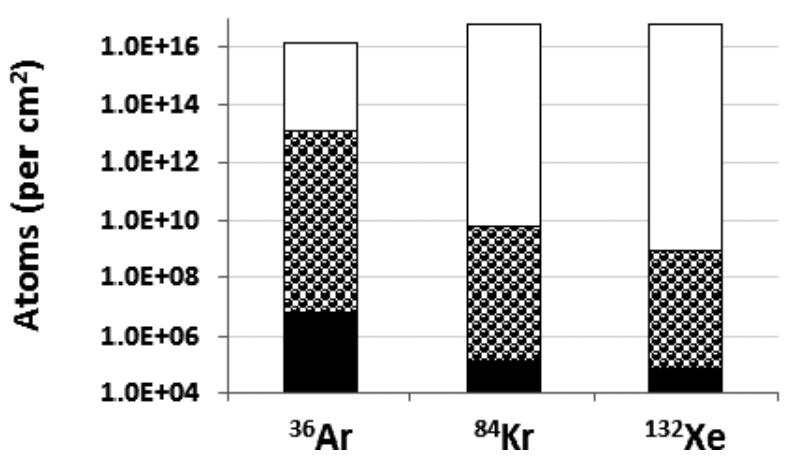

Fig.1. Model assessment of expected cometary gas in aerogel. 


\section{3}

\section{REVISITING FIRES AT THE K-Pg BOUNDARY}

J. Morgan ${ }^{1}$, N. Artemieva ${ }^{2,3}$, C. Belcher ${ }^{4}$, T. Goldin ${ }^{5}$ and Elisabetta Pierazzo ${ }^{2}$. ${ }^{1}$ Imperial College London, London, UK. E-mail: j.morgan@imperial.ac.uk. ${ }^{2}$ Planetary Science Institute, Tucson, AZ, USA. ${ }^{3}$ Institute for Dynamics of Geospheres, Moscow, Russia. ${ }^{4}$ University of Vienna, Vienna, Austria. ${ }^{5}$ University of Edinburgh, Edinburgh, UK.

Introduction: The discovery of large amounts of soot in K-Pg boundary clays led to the hypothesis that there were major wildfires at the K-Pg boundary $[1,2]$. Impact generated fires could be ignited in two ways: through the fireball for distances of up to $3000 \mathrm{~km} \mathrm{[3]} \mathrm{and} \mathrm{globally}$ through the re-entry of hypervelocity ejecta [4]. Models of the latter have assumed a particular velocity and mass flow for the re-entering ejecta $[4$, 5]. In our new simulations we model the entire process, starting with the ejection of material from Chicxulub and ending with these ejecta re-entering the Earth's atmosphere. We have also reviewed the evidence that has been used to argue against the global wildfire hypothesis [6, 7], including the lack of charcoal and relative abundance of uncharred material in K-Pg boundary sites in North America.

Method: We simulate the Chicxulub impact with the 3D hydrocode SOVA [8] complemented by the ANEOS equation of state for geological materials [9]. With SOVA, we model (1) the impact and initial ejection of material and (2) the ballistic continuation of ejecta on a spherical earth until the ejecta arrives at the top of the atmosphere [10]. We then use the 2D two-phase fluid flow code KFIX-LPL to model the atmospheric reentry of ejecta [5] and the thermal pulse delivered to the Earth's surface. We have modeled the ejection of material for two impact angles, $45^{\circ}$ and $60^{\circ}$, and calculated the associated thermal pulse at $2000-2500 \mathrm{~km}$ and $7000-8000 \mathrm{~km}$ from Chicxulub. These distances correspond to the southern USA and Europe-locations where there is a wealth of observational data.

Results: When sedimentation rates and dilution of charcoal with other ejecta material are taken into account, the K-Pg boundary record in North America may be consistent with local wildfires. Some fires at, or shortly after, impact appear to be required to explain the large mass of soot in the global K-Pg layer. The thermal pulse delivered to the surface depends on radiating temperature of the ejecta and the opacity distribution of the atmosphere and thus is strongly dependent on re-entry velocity, spherule size and mass flow of ejecta, and these change with angle of impact and direction (downrange, uprange, etc.). The thermal pulse $\left(>20 \mathrm{~kW} \mathrm{~m}^{-2}\right)$ in the downrange direction at sites up to a few thousand kilometers from Chicxulub may be sufficient to ignite fires, but is less severe $\left(<20 \mathrm{~kW} \mathrm{~m}^{-2}\right)$ for distal sites and in other directions. Our results suggest that ejecta re-entry ignited some wildfires, but did not instantaneously ignite fires globally.

References: [1] Wolbach W. et al. 1985. Science 230:167-170. [2] Wolbach W. et al. 1990. Geological Society of America Special Paper 247:391-400. [3] Shuvalov V. and Artemieva N. 2002. Geological Society of America Special Paper 356. pp. 695-703. [4] Melosh H. et al. 1990. Nature 343:251-254. [5] Goldin T. and Melosh H. 2009. Geology 37:11351138. [6] Belcher C. et al. 2005. Geological Society of London 162:591-602. [7] Belcher C. et al. 2009. Proceedings of the National Academy of Sciences 106:4112-4117. [8] Shuvalov V. 1999. Shock Waves 9:381-390. [9] Thompson S. L. and Lauson H. S. 1972. SC-RR-61 0714. Sandia Nat. Lab. 119 p. [10] Artemieva N. and Morgan J. 2009. Icarus 201:768-780.
5346

SIMULATION OF GAS-GRAIN COLLISIONS: A MECHANISM FOR CHONDRULE FORMATION

A. Morlok ${ }^{1}$, Y. C. Sutton ${ }^{2}$, M. Patel ${ }^{1}$, N. St. J. Braithwaite ${ }^{2}$ and M. M. Grady ${ }^{1,3}$. ${ }^{1}$ PSSRI, The Open University, Walton Hall, Milton Keynes, MK7 6AA, UK. E-mail: a.morlok@open.ac.uk. ${ }^{2}$ Department of Physics \& Astronomy, The Open University, Walton Hall, Milton Keynes, MK7 6AA, UK. ${ }^{3}$ The Natural History Museum, Cromwell Road, SW7 5BD, London, UK.

Introduction: The exact process of chondrule formation is still not known. The most common scenarios are formation in a protoplanetary nebular environment: e.g., X-wind model [1] or nebular shock fronts, where precursor grains are heated by collisions with gas [2]. Most chondrules probably have been heated to $1500-1600{ }^{\circ} \mathrm{C}$, followed by a cooling rate of usually between 10 and $1000{ }^{\circ} \mathrm{C} \mathrm{h}^{-1}$ [3]. In this part of our study about the formation of chondrules by shock processes, we try to simulate the formation of chondrules by gas-grain collisions in a nebular environment.

Earlier, related studies usually did not result in chondrule-like objects [4]. In a first series of experiments using plasma arcs, we produced successfully spherical silicate droplets in a simple set-up under atmospheric conditions. These chondrule-like objects already show similarities to actual chondrules [5]. In a second series, the experiments take place in an environment closer to the earlier solar nebula, at low pressure and a reducing environment..

Techniques: A plasma arc is generated at atmospheric pressure using a low voltage solid state switching circuit to drive a Tesla coil (natural frequency $\sim 325 \mathrm{kHz}$ ). The gas temperature was determined in an earlier experiment through fitting of modeled spectra to observed emission spectra: At $25 \mathrm{~W}$, the gas temperature is in the region of $>2100^{\circ} \mathrm{K}$.

The plasma generator is placed in an environmental chamber at the PSSRI/Open University. With the help of a remote controlled dispenser, starting material (mixture of olivine, feldspar, metal, and carbon) is dropped through the arc) under varying atmospheres (air and N2) at changing pressures of $5 \times 10-1,1 \times 10-1,4.8 \times 10-2,5 \times 10-3 \mathrm{bar}$.

The run products are recovered below the arc and analyzed using SEM/EDX for their structure and chemical composition.

First Results: The first run did show interaction of the material with the plasma arc. However, preliminary screening of the run products with SEM/EDX indicates only few melt droplets, which are difficult to identify in the original material [5]. Here we will present the results of a new series of experiments with varying starting compositions.

References: [1] Shu et al. 2001 The Astrophysical Journal 548:10291050. [2] Desch and Connolly Jr. 2002. Meteoritics \& Planetary Science 37:183-208. [3] Zanda B. 2004. Earth and Planetary Science Letters 224:124. [4] Güttler et al. 2008. Icarus 195:5094-5510. [5] Morlok et al. 2011. Abstract \#1081. 42nd Lunar and Planetary Science conference. 
5202

\section{THERMAL HISTORIES OF CHONDRULES: AN ASSESMENT OF THE EFFECT OF A SIZE DISTRIBUTION OF PRECURSOR PARTICLES}

M. A. Morris ${ }^{1}$ and S. J. Desch ${ }^{1}$. ${ }^{1}$ School of Earth and Space Exploration, Arizona State University, AZ, USA. E-mail: melissa.a. morris@asu.edu.

Introduction: Chondrules represent one of the best probes of the physical conditions and processes acting in the early solar nebula. Chondrule formation models are assessed on their ability to match the meteoritic evidence, especially constraints on their thermal histories. The model most consistent with chondrule thermal histories appears to be passage through shock waves in the solar nebula $[1,2]$. These models meet all known constraints on thermal histories during chondrule formation, except for the restriction on the amount of time chondrules remain at $>1300 \mathrm{~K}$ prior to achieving peak temperature, which [3] estimate is $<30 \mathrm{~min}$, based on the lack of isotopic fractionation of sulfur. In the shock model of [4], chondrules are "preheated" to temperatures above $1300 \mathrm{~K}$ in the preshock region, remaining there for over $4 \mathrm{~h}$. Heating in the preshock region is controlled mainly by opacity, which is greatly affected by the sizes of solids, with smaller particles contributing more. Previous models have typically included chondrule precursors of only one size [4-7]. We feel it is now necessary to include a simple size distribution of precursor particles in the shock model in order to assess the effects on the thermal histories of chondrules, especially in the preshock region.

Methods: We used the shock code of [4] to carry out our calculations of chondrule thermal histories with the addition of "microchondrules" of radius, $a=10 \mu \mathrm{m}$, mixed with $300 \mu \mathrm{m}$ chondrule precursors and submicron-sized dust. This improves on the models of $[4,5]$, which assumed a simple monodispersion of $300 \mu \mathrm{m}$ precursors, as well as micron-sized dust. The dust, considered coupled to the gas, evaporates completely and nearly instantaneously at $1500 \mathrm{~K}$ [4]. Chondrules and microchondrules evaporate at identical, energy-limited rates when their temperatures exceed their liquidus temperature of approximately $1820 \mathrm{~K}$. Vapor evaporated from the chondrule precursors is added back to the gas.

Results and Discussion: We considered two cases: one in which all large solids are in chondrules, and one in which half of the mass of chondrules is replaced by microchondrules. A key result from these studies is that microchondrules are present in the preshock region, where they significantly enhance the opacity (by a factor of 15), but they evaporate in the postshock region, and therefore do not contribute to the opacity or cooling rate there. Cooling rates through the crystallization range are raised only slightly over the canonical case of [4], from approximately $10-20$ to $20-40 \mathrm{~K} \mathrm{~h}^{-1}$, still well within the range consistent with the meteoritic constraints on thermal histories. The time spent at $T>1300 \mathrm{~K}$ before the shock, however, is reduced to $<30 \mathrm{~min}$.

Conclusion: The inclusion of a simple size distribution of chondrules, containing "microchondrules" of $\leq 10 \mu \mathrm{m}$ in radius, leads the shock model to match the last unmet constraint on chondrule formation.

References: [1] Desch S. J., Morris M. A., Connolly H. C., Jr., and Boss A. P. 2011. Meteoritics \& Planetary Science, submitted. [2] Desch S. J., Morris M. A., Connolly H. C., Jr., and Boss A. P. 2010. The Astrophysical Journal 725:692. [3] Tachibana S. and Huss G. R. 2005. Geochimica et Comochimica Acta 69:3075. [4] Morris M. A. and Desch S. J. 2010. The Astrophysical Journal 722:1474. [5] Desch and Connolly, 2002. Meteoritics \& Planetary Science 37:183. [6] Ciesla F. J. and Hood L. L. 2002. Icarus 158:281. [7] Iida A., Nakamoto T., Susa H., and Nakagawa Y. 2001. Icarus 153:430.
5270

\section{LABORATORY EXPERIMENTS OF DEPENDANT CATASTROPHIC DISRUPTION \\ ROTATIONALLY} A. J. W. Morris, M. J. Cole, M. C. Price and M. J. Burchell. School of Physical Science, University of Kent, Canterbury, CT2 7NH, UK. E-mail: am661@kent.ac.uk.

Introduction: Catastrophic disruption of minor bodies is a major evolutionary process for rocky bodies in the asteroid belt, e.g., see [1] for a general review and [2] for a typical recent paper (concerning the Themis family). Two tools are available for its study, hydrocode simulations and laboratory experimentation. Both offer advantages. In the latter case it is possible to control key parameters and see how they influence the outcome of impact events (albeit at laboratory, i.e., cm scale). Previous studies have paid attention to areas concerning gravity or impact related disruption [2] and the use of hydrocode modeling to ascertain internal faults and their effects on disruption [3] thresholds to mention a few variables usually investigated. But although spin is a feature of asteroids, it is rarely considered in impact experiments. Here we use laboratory impact experiments to study the influence of target spin on the outcome of the impact event. Accordingly, we examine the effects governing hypervelocity impacts on solid cement spheres.

Method: The target spheres were made in-house and had (on average) a diameter and mass of $75 \mathrm{~mm}$ and $367 \mathrm{~g}$ respectively. A rotating target holder was used which had an average rotation rate of $3.44 \mathrm{revs}^{-1}$. Two datasets were obtained for stationary $\left(0 \mathrm{rev}^{-1}\right)$ and rotating shots $\left(3.44 \mathrm{revs}^{-1}\right)$ with 22 impacts in total. Impacts were obtained using a two stage light gas gun [4] firing stainless steel spheres (dia. $1-3 \mathrm{~mm}$ ) at speeds between 1 and $7.5 \mathrm{~km} \mathrm{~s}^{-1}$. This gave impact energy densities $Q$ (kinetic energy/original target mass) in the range $7-2600 \mathrm{~J} \mathrm{~kg}^{-1}$. This spanned the regime of cratering to disruption defined by $\mathrm{m}_{\mathrm{f}} / \mathrm{m}_{\mathrm{i}}$ (mass largest fragment/original target mass) being greater than or less than 0.5 respectively, with $Q^{*}$ being the energy density at $\mathrm{m}_{\mathrm{f}}$ / $\mathrm{m}_{\mathrm{i}}=0.5$. The $\mathrm{m}_{\mathrm{f}} / \mathrm{m}_{\mathrm{i}}$ results ranged from 0.99 to 0.04 in each of the data sets. For stationary targets it was found that $Q^{*}=1500 \mathrm{~J} \mathrm{~kg}^{-1}$, and we noted that $\mathrm{m}_{\mathrm{f}} / \mathrm{m}_{\mathrm{i}}$ fell below 0.8 at $Q=750 \mathrm{~J} \mathrm{~kg}^{-1}$. For the rotating targets we found that whilst $Q^{*}=1450 \mathrm{~J} \mathrm{~kg}^{-1}, \mathrm{~m}_{\mathrm{f}} / \mathrm{m}_{\mathrm{i}}$ fell below 0.8 at $Q=450 \mathrm{~J} \mathrm{~kg}^{-1}$.

Discussion: From the present data we found that the stationary and rotating targets had similar $Q^{*}$ values and thus the level of rotation in these experiments is insufficient to affect the overall disruption threshold. There may however be a difference in behavior at low $Q$ values with rotating targets removing more spall material from craters - this is being investigated further.

References: [1] Ryan E. V. 2000. Annual Review of Earth and Planetary Science 28:367-389. [2] Leliwa-Kopystynski J. et al. 2009. Meteoritics \& Planetary Science 44:1929-1935. [3] Michel P. et al. 2004. Icarus 168:420-432. [4] Stewart S. T. et al. 2009. The Astrophysical Journal 691:L133-L137. [5] Burchell M. J. et al. 1999. Measurement Science \& Technology 10:41-50. 


\section{0}

ESA LUNAR LANDER'S SEARCH FOR VOLATILES

A. D. Morse ${ }^{1}$, S. J. Barber ${ }^{1}$, J. M. Pillinger ${ }^{1}$, S. Sheridan ${ }^{1}$, I. P. Wright ${ }^{1}$, E. K. Gibson ${ }^{2}$, J. A. Merrifield ${ }^{3}$, N. R. Waltham ${ }^{4}$, L. J.Waugh ${ }^{5}$ and C. T. Pillinger ${ }^{1}$. ${ }^{1}$ Planetary \& Space Sciences Research Institute, Walton Hall, The Open University, Milton Keynes. MK7 6AA, UK. E-mail: a.d.morse@open.ac.uk. ${ }^{2}$ Astromaterials Research Office, NASA Johnson Space Center, Houston, TX 77058, USA. ${ }^{3}$ Fluid Gravity Engineering, 1 West Street, Emsworth, PO10 7DX, UK. ${ }^{4}$ RAL Space, Didcot, Oxfordshire, OX11 0QX, UK. ${ }^{5}$ EADS Astrium Ltd., Gunnels Wood Rd., Stevenage SG1 2AS, UK.

Introduction: Following the Apollo era the moon was considered a volatile poor body. Samples collected from the Apollo missions contained only ppm levels of water formed by the interaction of the solar wind with the lunar regolith [1]. However more recent orbiter observations have indicated that water may exist as water ice in cold polar regions buried within craters at concentrations of a few $w t \%$ [2]. Infrared images from M3 on Chandrayaan-1 have been interpreted as showing the presence of hydrated surface minerals with the ongoing hydroxyl/water process feeding cold polar traps. This has been supported by observation of ephemeral features termed "space dew" [3]. Meanwhile laboratory studies indicate that water could be present in appreciable quantities in lunar rocks [4] and could also have a cometary source [5].

Lunar Lander: The European Space Agency (ESA's Directorate of Human Spaceflight and Operations) have provisionally scheduled a robotic mission to demonstrate key technologies to enable later human exploration. Planned for launch in 2018, the primary aim is for precise automated landing, with hazard avoidance, in zones which are almost constantly illuminated (e.g., at the edge of the Shackleton crater at the lunar south pole). The Lander design requirement for constant solar illumination restricts the landing location, however these sunlit areas are close to permanently shadowed volatile rich regions and the analysis of volatiles is a major science objective of the mission.

Lunar Volatile and Resources Analysis Package: The authors have been commissioned by ESA to conduct an evaluation of possible technologies to be included in L-VRAP which aims to demonstrate volatile extraction and determine the volatile inventory of the moon with a view for future In Situ Resource Utilization (ISRU). Surface samples will be collected by a robotic arm with the possibility of a rover to collect more distant samples. The concentration, chemical and accurate isotopic ratios $\left(\mathrm{D} / \mathrm{H},{ }^{12} \mathrm{C} /{ }^{13} \mathrm{C},{ }^{15} \mathrm{~N} /{ }^{14} \mathrm{~N},{ }^{18} \mathrm{O} /{ }^{16} \mathrm{O}\right.$ and noble gases) of liberated volatiles will be determined, possibly using similar technology to the Philae comet lander of the Rosetta mission [6]. In addition the chemical and isotopic composition of the transient lunar atmosphere will be monitored [7]. Modeling the effects of contamination from the Lander is an essential part of this study so that these can be recognized and minimized.

References: [1] Pillinger C. T. 1979. Report Progress Physics 42:897961. [2] Mitrofanov I. G. et al. 2010. Science 330:483-486. [3] McCord T. B. et al. 2010. Journal Geophysical Research, submitted. [4] Boyce J. W. et al. 2010. Nature 466:466-469. [5] Greenwood J. P. et al. 2011. Nature Geoscience 4:79-82. [6] Morse A. D. et al. 2009. Rosetta: ESA's mission to the origin of the solar system, 19.6:669-686. [7] Hofmann J. H. 1975. Lunar Atmospheric Composition Experiment, NASA report.
5505

SIZE OF THE GROUP IVA IRON METEORITE CORE: CONSTRAINTS FROM THE AGE AND COMPOSITION OF MUONIONALUSTA

N. A. Moskovitz ${ }^{1}$ and R. Walker ${ }^{2}$. ${ }^{1}$ Carnegie Institution of Washington, DTM, DC, USA. E-mail: nmoskovitz@dtm.ciw.edu. ${ }^{2}$ University of Maryland, MD, USA.

Introduction: The group IVA fractionally crystallized iron meteorites display a diverse range of metallographic cooling rates, ranging from 100 to $6600 \mathrm{~K} \mathrm{Myr}^{-1}[1,2]$. These have been attributed to their formation in a metallic core, $\sim 150 \mathrm{~km}$ in radius that cooled to crystallization without any appreciable insulating mantle. Such an exposed core may have resulted from a hit-and-run collision [3] between two large $\left(\sim 10^{3} \mathrm{~km}\right)$ proto-planetary bodies. Here we build upon this formation scenario by incorporating several new constraints. These include (1) a recent U-Pb radiometric closure age of $4565.3 \mathrm{Mya}(<2.5 \mathrm{Myr}$ after CAIs) for the group IVA iron Muonionalusta [4], (2) new measurements and modeling of highly siderophile element compositions for a suite of IVAs, and (3) consideration of the thermal effects of heating by the decay of the shortlived radionuclide ${ }^{60} \mathrm{Fe}$.

IVA Fractional Crystallization Model: We model the fractional crystallization sequence of the IVA system using an approach similar to [5]. With initial S, P, Re, and Os compositions of 3\%, 0.1\%, $295 \mathrm{ppb}$, and $3250 \mathrm{ppb}$ respectively we find that compositions similar to Muonionalusta are produced after $\sim 60 \%$ fractional crystallization. For an inwardly crystallizing core [6] this suggests formation at $70 \%$ the radius, $\mathrm{R}$, of the body.

Thermal Conduction Model: To match the wide range of IVA cooling rates we follow [1] by assuming an exposed core that cools without any insulating silicate mantle. We solve the 1D thermal conduction equation [7] for cores with variable amounts of live ${ }^{60} \mathrm{Fe}$, ranging from zero [8] up to a plausible maximum of ${ }^{60} \mathrm{Fe} /{ }^{56} \mathrm{Fe}=4 \times 10^{-7}$ [9]. With core size and ${ }^{60} \mathrm{Fe}$ abundance as the primary variables in this model, we fit both the range of IVA cooling rates and the crystallization of Muonionalusta at $4565.3 \mathrm{Myr}$ at a radius of $0.7 \mathrm{R}$.

Results: Our calculations suggest that the IVA core was $50-110 \mathrm{~km}$ in radius after the hit-and-run collision. This range is primarily due to uncertainties in the initial abundance of live ${ }^{60} \mathrm{Fe}$ incorporated into the IVA core. This emphasizes the need to better constrain initial ${ }^{60} \mathrm{Fe}$ abundances for the IVAs and other iron meteorite parent bodies that may have crystallized within a few half-lives of ${ }^{60} \mathrm{Fe}$ after CAIs $\left(\tau_{1 / 2}=\right.$ 2.62 Myr). Candidates for such early formation include the IIAB, IIIAB and IVB groups.Lastly, our models define a relationship between cooling rate and closure age. For example, IVAs with the fastest cooling rates $\left(>1000 \mathrm{~K} \mathrm{Myr}^{-1}\right)$ may have absolute ages separated by as little as a few times $10^{5}$ years after CAI formation. The details of this relationship can be firmly established if old U-Pb ages are confirmed/measured for Muonionalusta and other IVAs.

References: [1] Yang J., Goldstein J. I., and Scott E. R. D. 2007. Nature 446:888-891. [2] Yang J., Goldstein J. I., and Scott E. R. D. 2008. Geochimica et Cosmochimica Acta 72:3042-3061. [3] Asphaug E. et al. 2006. Nature 439:155-160. [4] Blichert-Toft J. et al. 2010. Earth and Planetary Science Letters 296:469-480. [5] Walker R. J. et al. 2008. Geochimica et Cosmochimica Acta 73:5189-5201. [6] Ruzicka A. and Hutson M. 2006. Meteoritics \& Planetary Science 41:1959-1987. [7] Moskovitz N. and Gaidos E. 2011. Meteoritics \& Planetary Science, in press. [8] Quitte G. et al. 2010. The Astrophysical Journal 720:12151224. [9] Mishra R. K. et al. 2010. The Astrophysical Journal Letters 714:L217-L221. 
5170

\section{THE SEARCH FOR PRESOLAR OXIDES IN PARIS}

S. Mostefaoui. Muséum National d'Histoire Naturelle, LMCM UMR CNRS - 7202, Case 52, 57 Rue Cuvier 75321 Paris Cedex 05, France. E-mail: smail@mnhn.fr.

Introduction: Paris is considered the less aqueously altered among the CM chondrites [1]. Its material is assumed to be better preserved and therefore represents the best $\mathrm{CM}$ candidate for the detection of presolar oxides in the matrix. In this study I present preliminary NanoSIMS results of the O-isotopography of the Paris matrix. The estimation of presolar oxide abundance in comparison with other chondrites will allow to better understanding the alteration and thermal histories of this meteorite group.

Experiment: A polished thick section of Paris was observed with optical microscope. Three areas of the fine matrix largely distant from each other and reflecting the alteration lithologies of the meteorite were meticulously selected excluding zones of large grains and/or chondrule fragments. The areas were screened for the presence of presolar O-rich dust using the NanoSIMS. A focussed $\mathrm{Cs}^{+}$ion beam $(<100 \mathrm{~nm})$ of $<1 \mathrm{pA}$ was rastered over $9 \times 9 \mu^{2}$ areas. Negative secondary ions of the three O-isotopes, ${ }^{28} \mathrm{Si}$, and ${ }^{27} \mathrm{Al}^{16} \mathrm{O}$ were simultaneously measured in multicollection and $256 \times 256$ pixel image sequences (total integration time of approximately $1 \mathrm{~h}$ per image set) were acquired on different locations.

Results: Seventy-six NanoSIMS $9 \times 9 \mu \mathrm{m}^{2}$ images, covering a total matrix area of $6156 \mu \mathrm{m}^{2}$, were acquired for the search of presolar oxides in Paris. None of the measured areas show a sign of an anomalous O-isotopic hotspot suggesting the absence (at least to the level allowed by the NanoSIMS spatial resolution) of presolar oxides in these areas. This testifies the rarity of such grains in Paris. Their absence prevents determining the precise abundance of presolar oxides in this meteorite. But, assuming the finding of a presolar oxide in the subsequent $9 \times 9 \mu \mathrm{m}^{2}$ area of the matrix with a size typical of presolar oxide grains in chondrites one can estimate an upper limit of the matrix-normalized abundance in Paris not to exceed approximately $10 \mathrm{ppm}$.

Discussion: The present results show that the abundance of presolar oxides in Paris is low. It is definitely lower than the abundances of presolar oxides in the CRs QUE 99177 and MET 00426 (220 and $160 \mathrm{ppm}$, respectively) [2] and in the Acfer 094 carbonaceous chondrite for which an equivalent matrix area was scanned for 12 presolar oxides identified [3]. In Murchison, which is a CM like Paris, the abundance of presolar oxides (all silicates) was estimated to approximately $3 \mathrm{ppm} \mathrm{[4].}$ Although Paris is predictable to have a higher abundance than Murchison, one need to explore more than $2 \times$ the area I explored here in order to confirm it. Despite the Paris material is the less suffering among $\mathrm{CM}$ chondrites from aqueous alteration either thermal metamorphism [5] or still the aqueous alteration [4] were responsible for destruction of oxide presolar isotopic signatures in Paris. Alternatively, a preaccretionary selective destruction [6] leading to heterogeneous distributions of presolar oxide grains within carbonaceous chondrites formation environment cannot be excluded. Continuing NanoSIMS measurements are in progress in order to better estimate the abundance of presolar oxide in this chondrite.

References: [1] Zanda B. et al. 2010. Meteoritics \& Planetary Science \#5312. [2] Floss C. and Stadermann F. J. 2009. Geochimica et Cosmochimica Acta 73:2415. [3] Mostefaoui S. et al. 2004. The Astrophysical Journal 613:L149. [4] Nagashima K. et al. 2005. Abstract \#1671. 36th Lunar and Planetary Science Conference. [5] Huss G. R. and Lewis R. S. 1995. Geochimica et Cosmochimica Acta 59:115. [6] Huss G. R. 2004. Antarctic Meteorite Research 17:132.
5028

SYNCHROTRON X-RAY DIFFRACTION AND MÖSSBAUER SPECTROSCOPY STUDIES OF WEATHERING EFFECTS ON ORDINARY CHONDRITES FROM THE ATACAMA DESERT, CHILE

P. Munayco, J. Munayco, R. R. Avillez, M. Valenzuela, J. Gattacceca, P. Rochette and R. B. Scorzelli.

This work reports the results obtained by ${ }^{57} \mathrm{Fe}$ Mössbauer spectroscopy (MS) and synchrotron radiation X-ray diffraction (SRXRD) on 15 meteorite samples of weathered ordinary chondrites (OC), collected in the San Juan area of the Atacama Desert, northern Chile, that continues previous work done [1] with samples of this densest collection area.

MS is a powerful tool to determine the differences in alteration rate between Fe-bearing phases in a weathered meteorite. The analyses of the spectral area of the primary phases in relation to the total amount of oxidation allow the determination of which phases are most susceptible to weathering. Since the abundance of ferric iron is directly related to the level of terrestrial weathering, the decrease in the spectral area of a primary mineral, should be followed by an increase in a ferric component. In this work the MS results are complemented by SR-XRD experiments performed in the D10A-XRD2 line of the National Synchrotron Light Laboratory (LNLS, Brazil). The use of synchrotron radiation amplifies the use of XRD since it provides very intense and sharp lines for very crystalline phases. SR-XRD is particularly suited to weathered meteorites which usually have small amounts of many corrosion phases, together with the primary phases. Further, XRD is not restricted to iron contained phases.

The Mössbauer spectra at RT exhibit two $\mathrm{Fe}^{2+}$ doublets, associated to olivine and pyroxene, a third $\mathrm{Fe}^{3+}$ doublet, corresponding to superparamagnetic oxides and/or oxyhydroxides, and additionally, four sextets due to magnetically ordered phases - troilite, magnetite, kamacite/ taenite and large particle goethite. At low temperature $(4.2 \mathrm{~K})$ the spectra exhibit the same components observed at RT and additionally, a magnetic component (not seen at RT) identified as akaganéite. An increase in the relative areas of goethite and magnetite is also observed and in some samples hematite/maghemite was also detected. The presence of akaganéite and hematite as well as the increase in relative areas, is due to the magnetic splitting at $4.2 \mathrm{~K}$ of the superparamagnetic $\mathrm{Fe}^{3+}$ doublet observed at RT.

The fit of the SR-XRD results was done using the Bruker TOPAS $4.2^{\odot}$ program with the fundamental parameters approach. A standard silicon powder was used to optimize the fundamental parameters. Up to 18 different phases were considered during the Rietveld modeling, Iron was allowed to substitute the magnesium sites of the pyroxene and olivine phases. All the other phases were considered stoichiometric or with constant composition. The goethite amount seems to provide a clear indication for meteoritic weathering. Further the total amount of weathering related phases correlates very well with its Mossbauer equivalent.

References: [1] J. Munayco, P. Munayco, E. M. Valenzuela, J. Gattacceca, P. Rochette, R. B. Scorzelli. 2010. Abstract \#5035. 73rd Meteoritical Society Meeting, New York, USA. 
5191

\section{ELECTRONIC STRUCTURE CALCULATIONS OF WATER ADSORPTION ON FORSTERITE GRAINS: IMPLICATIONS FOR PLANETARY WATER}

Krishna Muralidharan ${ }^{1}$ and Michael J. Drake ${ }^{2} .{ }^{1}$ Material Science and Engineering, The University of Arizona, Tucson, AZ, 85721, USA. ${ }^{2}$ Lunar and Planetary Laboratory, The University of Arizona, Tucson, AZ, 85721, USA.

Introduction: Recent computational investigations have clearly indicated that adsorption occurring during the early stages of planetary accretion is a viable delivery source of planetary water $[1,2]$. In this study we use rigorous electronic structure and atomistic simulations to examine and accurately characterize the mechanisms of water adsorption on to forsterite grains-a primary planetary building block. Specifically, we focus on examining dissociative adsorption of water. Previous investigations had focused on associative molecular adsorption, which serves as a precursor to the ultimate dissociative adsorption of water.

Results and Discussion: Using density functional theory (DFT), the dissociation energy-landscapes of three surface orientations $(\{100\},\{110\}$, $\{010\})$ of forsterite were studied. Of particular interest is the $\{100\}$ surface, as it contains multiple adsorption sites. The unhydrated $\{100\}$ surface, which is a primary cleavage plane of olivine, contains three possible dissociation sites (S2, S3, and S4), each characterized by the presence of undercoordinated oxygen and magnesium atoms, thereby lending themselves well to the dissociation of a water molecule. The S2 site is coordinated to three oxygen atoms, while $\mathrm{S} 3$ is nested between two surface tetrahedra and is bonded to four oxygen atoms. S4 is also fourcoordinated, with three oxygen atoms lying on the surface. In order to calculate the dissociative adsorption energies of the three sites, plane wave-DFT utilizing the Troullier Martin pseudopotential [3] to represent the electron-nuclear interaction, in conjunction with the local density approximation [4] (LDA) for exchange-correlation was used. Amongst the 3 sites, S2 yields the highest dissociation energy, corresponding to $420 \mathrm{KJ} \mathrm{mol}^{-1}$, which can be attributed to the fact that it is the least coordinated cation as well as to the fact that it is located close to the surface and is extremely susceptible to hydroxylation. S3 and S4 also yield very high dissociation energy values of $288 \mathrm{~kJ} \mathrm{~mol}^{-1}$ and $180 \mathrm{~kJ} \mathrm{~mol}^{-1}$, respectively. Note that molecular water can associatively bind to the $\{100\}$ surface very strongly, with energies ranging between $100-150 \mathrm{~kJ} \mathrm{~mol}^{-1}$. Thus from a computational standpoint, one can assume that on $\{100\}$ both associative and dissociative adsorption can occur, though based on energetics dissociation is preferred.

Conclusion: Using electronic-structure calculations, we have shown that adsorption of water on to forsterite grains is energetically stable at thermodynamic conditions pertaining to the accretion disk. Further, we identify that water dissociation is the dominant adsorption mechanism and conclude that adsorption can be a significant source of terrestrial planetary water. The conclusions of Muralidharan et al. [2] that Earth could acquire many oceans of water by adsorption remain robust. Terrestrial planetary systems around other stars should also contain water.

References: [1] King M. E. et. al. 2010. Earth and Planetary Science Letters 300:11. [2] Muralidharan K. et al. 2008. Icarus 198:400. [3] Troullier N. and Martin J. L. 1991. Physical Review B 43:1993. [4] Ceperley D. M. and Alder B. J. 1980. Physical Review Letters 45:566.
5367

CONDENSATION KINETICS AND CHEMICAL FRACTIONATION OF SILICATE AND METAL IN A PROTOPLANETARY DISC

H. Nagahara ${ }^{1}$ and K. Ozawa ${ }^{2}$. ${ }^{1}$ Department Earth Planetary Science, The University Tokyo, Tokyo, Japan. E-mail: hiroko@eps.s.u-tokyo. ac.jp. ${ }^{2}$ Department Earth Planetary Science, The University Tokyo, Tokyo, Japan.

Introduction: Condensation is the first step of planet formation. Although recent finding of CAIs in cometary samples $[1,2]$ have been thought to represent large scale material transportation from the inner edge to outer region of the disc, it is not clear whether CAIs were formed at the inner edge of the disc or by transient heating in the outer region. Rather, the presence of a large variety of chondrites prefers mixing of dusts in a limited scale. Time scales of condensation and physical evolution of dusts in a range of total pressure, gas cooling time scale, and dust/gas separation conditions are evaluated by using a kinetic condensation model including homogeneous and heterogeneous condensation cases.

Model: The model is based on kinetic condensation theory in cooling gas [3-5] with solar composition $(\mathrm{H}-\mathrm{He}-\mathrm{C}-\mathrm{N}-\mathrm{O}-\mathrm{Mg}-\mathrm{Al}-\mathrm{Si}-\mathrm{Fe})$ developed by our group is applied. The model calculates the changes of dust size, number density, chemical composition, occurrence of different phases with time, where cooling time scale and critical size for dust separation from the residual gas are free parameters. We use experimentally determined kinetic parameters for condensation and evaporation by our group.

Results and Discussion: If condensation proceeds without dust separation, the condensed dusts have a very narrow size range and the system finally does not show chemical fractionation, where the grain size of dusts is exclusively controlled by cooling time scale of the system. The grain size distribution and bulk composition of both residual system and separated dusts showed a wide range when dust are separated from the residual gas when they reached a critical size, which is due to repeated homogeneous nucleation. If metal grains with size as small as those we find in GEMS (tens of nanometer in size), the cooling condition is fairly well constrained, which is comparable to the chondrule cooling time scale. Thus, the critical size for dust/gas separation plays an important role for chemical fractionation and dust size distribution. The mode of nucleation (homogeneous or heterogeneous) is found to be the most crucial parameter for chemical fractionation: totally heterogeneous condensation does not explain $\mathrm{Mg} / \mathrm{Si} / \mathrm{Al}$ fractionation among chondrites, suggesting $\mathrm{A} 12 \mathrm{O} 3$ separation in the early stage of condensation in order to explain compositions of chondrites.

References: [1] Brownlee D. 2006. Science 314:1711-1716. [2] McKeegan K. D. et al. 2006. Science 314:1724-1728. [3] Yamamoto T. and Hasegawa H. 1977. Progress of Theoretical Physics 58:816. [4] Drain B. T. and Salpeter E. E. 1977. The Journal of Chemical Physics 67:2230. [5] Kozasa and Hasegawa. 1989. Progress of Theoretical Physics 77:1402. 
5238

SOLAR NOBLE GASES IN ITOKAWA REGOLITH MATERIALS RETURNED BY THE HAYABUSA MISSION

K. Nagao 1 , R. Okazaki ${ }^{2}$, T. Nakamura ${ }^{3}$, Y. N. Miura ${ }^{4}$, T. Osawa ${ }^{5}$, K. Bajo ${ }^{1}$, S. Matsuda ${ }^{1}$, M. Ebihara ${ }^{6}$, T. R. Ireland ${ }^{7}$, F. Kitajima ${ }^{2}$, H. Naraoka $^{2}$, T. Noguchi ${ }^{8}$, A. Tsuchiyama ${ }^{9}$, M. Uesugi ${ }^{12}$, H. Yurimoto ${ }^{10}$, M. E. Zolensky ${ }^{11}$, K. Shirai ${ }^{12}$, M. Abe ${ }^{12}$, T. Yada ${ }^{12}$, Y. Ishibashi ${ }^{12}$, A. Fujimura ${ }^{12}$, T. Mukai ${ }^{12}$, M. Ueno ${ }^{12}$, T. Okada ${ }^{12}$, M. Yoshikawa ${ }^{12}$ and J. Kawaguchi ${ }^{12}$. ${ }^{1}$ Geochemical Research Center, University Tokyo, Tokyo, Japan. E-mail: nagao@eqchem.s.u-tokyo.ac.jp. ${ }^{2}$ Department Earth Planetary Science, Kyushu University, Kyushu, Japan. ${ }^{3}$ Department Earth Planetary Mater. Science, Tohoku University, Tohoku, Japan. ${ }^{4}$ Earthquake Research Institute, University Tokyo, Tokyo, Japan. ${ }^{5}$ Japan Atomic Energy Agency. ${ }^{6}$ Graduate School Science Engineering, Tokyo Metropolitan University, Tokyo, Japan. ${ }^{7}$ Research School Earth Science, Australian National University. ${ }^{8}$ College Science, Ibaraki University, Ibaraki, Japan. ${ }^{9}$ Department Earth Space Science, Osaka University, Osaka, Japan. ${ }^{10}$ National Hist. Science, Hokkaido University, Hokkaido, Japan. ${ }^{11}$ NASA JSC. ${ }^{12}$ JAXA-ISAS.

Introduction: The Hayabusa spacecraft successfully returned regolith materials from the surface of S-type asteroid 25143 Itokawa [1]. The samples were undamaged by the collection on the Itokawa surface, and handled in the clean chambers of the JAXA curation facility under vacuum and nitrogen gas [2], which is essentially different from other extraterrestrial materials. We report noble gas elemental and isotopic compositions of three grains RA-QD02-0015 (\#0015), RA-QD02-0053 (\#0053), and RA-QD02-0065 (\#0065).

Experimental Procedures: The samples are blocky and translucent olivine grains with dimensions of approximately 40, 40, and $60 \mu \mathrm{m}$ [3], and their estimated masses are 0.06, 0.06, and 0.21 $\mu \mathrm{g}$ for \#0015, \#0053, and $\# 0065$, respectively. Noble gases were extracted from each grain by means of stepwise heating at approximately 200 and $300^{\circ} \mathrm{C}$, and finally melted for complete extraction using a Nd-YAG CW laser. The extracted noble gases were measured with a modified VG5400/MS-III at the Geochemical Research Center, University of Tokyo.

Results and Implications: High concentrations of $\mathrm{He}, \mathrm{Ne}$, and $\mathrm{Ar}$, as high as those in lunar soils and IDPs, are released at variable temperatures from each sample. Their isotopic compositions are essentially identical to those of solar wind (SW). This clearly confirms that the grains resided on the surface of Itokawa. Only $\mathrm{He}$ is depleted compared with the relative abundances of $\mathrm{He}, \mathrm{Ne}$, and $\mathrm{Ar}$ in $\mathrm{SW}$. These noble gas characteristics can be explained by repeated implantation and preferential loss of solar He by removal of weathered He-rich rim on the grain surface [4] through friction among regolith grains on Itokawa. Duration of SW implantation to the measured samples is estimated as several hundreds of years. Concentrations of cosmogenic noble gases produced by galactic cosmic rays (GCR) were so low that they could not be detected in excess to the SW isotopic ratios beyond experimental errors. An upper limit of GCR exposure ages for the Hayabusa samples based on the $\mathrm{Ne}$ isotopic compositions is approximately $10 \mathrm{Myr}$. The short exposure age suggests that the regolith materials have been continuously escaping from Itokawa's surface at the relatively high rate of several tens of $\mathrm{cm} \mathrm{Myr}^{-1}$. The weak gravitational force at Itokawa's surface (escape velocity approximately $0.2 \mathrm{~m} \mathrm{~s}^{-1}$ ) [5] should be a main reason for the short residence time of the Itokawa regolith grains.

References: [1] Yano H. et al. 2006. Science 312:1350-1353. [2] Okazaki R. et al. 2011. Abstract \#1653. 42nd Lunar and Planetary Science Conference. [3] Nakamura T. et al. 2011. Science (submitted). [4] Noguchi T. et al. 2011. Science (submitted). [5] Miyamoto H. et al. 2007. Science 316:1011-1014.

\section{9}

GLOBAL DISTRIBUTION OF SILICON BY KAGUYA GAMMA-RAY SPECTROMETER

H. Nagaoka ${ }^{1}$, N. Hasebe ${ }^{1}$, K. J. Kim², Y. Karouji ${ }^{3}$, S. Kobayashi ${ }^{3}$, M. Hareyama $^{3}$, N. Yamashita ${ }^{4}$, O. Gasnault ${ }^{4}$, O. Forni ${ }^{4}$, C. d'Uston ${ }^{4}$, R. C. Reedy $^{5}$, E. Shibamura ${ }^{6}$, M. N. Kobayashi ${ }^{7}$, H. Takeda ${ }^{8}$, and the Kaguya Gamma Ray Spectrometer team. ${ }^{1}$ Waseda University, Waseda, Japan. E-mail: hiroshi-nagaoka@asagi.waseda.jp. ${ }^{2}$ KIGAM. ${ }^{3}$ JAXA. ${ }^{4}$ IRAP. ${ }^{5}$ PSI. ${ }^{6}$ Saitama Pref. University, Saitama, Japan. ${ }^{7}$ Chiba Institute Tech, Chiba, Japan. ${ }^{8}$ University of Tokyo, Tokyo, Japan.

Introduction: The distribution of major and important trace elements on the lunar surface is essential to understand the origin and evolution of the lunar crust. Gamma-ray spectrometers (GRS) carried on previous lunar and planetary missions have played important roles to determine the chemical element abundance of the surface of planetary body [e.g., 1]. A high precision GRS was carried on the first Japan's large-scaled lunar explorer, Kaguya which observed from December 2007 to June 2009 [2]. The GRS mounted on Kaguya (KGRS), which was the first to use a germanium detector for a lunar mission [3], has measured gamma rays emitted from the Moon with the high energy resolution. The distribution maps of three radioactive elements were reported, based on KGRS data analysis (K [4], Th, and U [5]). Preliminary results for Fe, Ti, $\mathrm{Ca}$, and $\mathrm{Al}$ have been reported [6-8]. In this work, we present preliminary results of analysis for a thermal neutron capture reaction peak from $\mathrm{Si}$ using KGRS data obtained during the low altitude observation.

Data Processing: KGRS data obtained in the lower altitude observation (approximately $50 \mathrm{~km}$ from February to June, 2009) in the late portion of its mission were used. First, the lunar surface was pixelated by $600 \times 600 \mathrm{~km}$ equal areas, gamma-ray counts were accumulated in each pixel, and 106 spectra were made. Noises in resultant spectra were filtered out. The $\mathrm{Si}$ distribution was surveyed using $3539.5 \mathrm{keV}$ peak, which is well resolved by KGRS spectra. The net counting rates of this peak were determined on the basis of the total peak area method [9]. Neutron correction was applied to the net counting rates using Lunar Prospector (LP) thermal neutron data $[10,11]$.

Results: The $3539.5 \mathrm{keV}$ neutron-capture peak from $\mathrm{Si}$ was used to map $\mathrm{Si}$ over the entire lunar surface. The counting rates of $\mathrm{Si}$ in mare region (Procellarum, Imbrium, Tranquillitatis) seem to be lower, as compared with highlands. The counting rates of $\mathrm{Si}$ in the farside highland regions near Hertzsprung were slightly high, as compared with their surrounding highland regions. Ample studies of this result are required in future.

References: [1] Prettyman T. H. et al. 2006. Journal of Geophysical Research 111:E12007. [2] Kato M. et al. 2010. Space Science Review 154:3-19. [3] Hasebe N. et al. 2008. Earth Planets, and Space 60:299-312. [4] Kobayashi S. et al. 2010. Space Science Review 154:193-218. [5] Yamashita N. et al. 2010. Geophysics Research Letter 37:L10201. [6] Gasnault O. et al. 2009. Abstract \#2253. 40th Lunar and Planetary Science Conference. [7] Karouji Y. et al. 2010. Abstract \#5166. 73rd Annual Meteoritical Society Meeting. [8] Yamashita N. et al. 2011. Abstract \#2093. 42nd Planetary Science Conference. [9] Gilmore G. and Hemingway J. D. 1995. Practical Gamma-ray Spectrometry. Wiley, Chichester. [10] The Planetary Data System, http://pds.nasa.gov/ [11] Maurice S. et al. 2004. Journal of Geophysical Research 109:E07S04. 
5461

OXYGEN-ISOTOPE COMPOSITIONS OF CHONDRULES AND MATRIX GRAINS IN KAKANGARI CHONDRITE

K. Nagashima ${ }^{1}$, A. N. Krot ${ }^{1}$ and G. R. Huss ${ }^{1}{ }^{1}$ HIGP/SOEST, University of Hawai'i at Mãnoa, USA. E-mail: kazu@higp.hawaii.edu.

Introduction: The relationship between chondrules and matrix in primitive chondrites is not well understood. Some workers see matrix as raw material for the chondrules that did not experience chondruleforming process [e.g., 1], while others see matrix as complementary to chondrules in the sense that material that evaporated from the chondrules during chondrule melting events re-condensed in the matrix [e.g., 2]. Oxygen isotopes can potentially provide important constraints on the relationship between chondrules and matrix and on thermal processing of matrix in the solar nebula. Here we report on an in situ O-isotope study of chondrules and matrix grains in the Kakangari chondrite. The Kakangari chondrules and matrix have similar chemical compositions but slightly different $\mathrm{O}$-isotope compositions: matrix is ${ }^{16} \mathrm{O}$-enriched by $2.6 \%$ compared to the bulk chondrules which plot along the terrestrial fractionation (TF) line [3-5].

Methods: Oxygen-isotope compositions were measured with the UH Cameca ims-1280 SIMS. For chondrules, we used $\sim 1 \mathrm{nA}$ primary $\mathrm{Cs}^{+}$ beam with $7 \mu \mathrm{m}$ raster and 2 Faraday cups $(\mathrm{FCs})$ for ${ }^{16} \mathrm{O}$ and ${ }^{18} \mathrm{O}$, and an electron multiplier (EM) for ${ }^{17} \mathrm{O}$. Matrix grains were measured with $\sim 15 \mathrm{pA}$ beam focused to $\sim 1 \mu \mathrm{m}$ using $\mathrm{FC}-\mathrm{EM}-\mathrm{EM}$ for ${ }^{16} \mathrm{O},{ }^{17} \mathrm{O}$, and ${ }^{18} \mathrm{O}$, respectively. Analysis spots were marked by focusing the electron beam on the points of interest to produce a spot that is visible as depleted oxygen signal in scanning ${ }^{16} \mathrm{O}^{-}$ion image, allowing us to identify exact locations of small grains in matrix.

Results and Discussion: Oxygen-isotope compositions of olivine $\left(\mathrm{FO}_{\sim 97}\right)$ and low-Ca pyroxene $\left(\mathrm{En}_{\sim 95-97}\right)$ in five chondrules (Type I PO and POP) plot near the intersection of the TF line and the Young and Russell line [6] with mean $\Delta^{17} \mathrm{O}$ values of $+0.2 \pm 0.6 \%(2 \sigma)$ and $-0.1 \pm 0.7 \%$, respectively. There are no significant systematic differences among chondrules. The $\Delta^{17} \mathrm{O}$ values of chondrule phenocrysts are in good agreement with $\mathrm{O}$-isotope compositions of bulk chondrules, but the $\delta^{18} \mathrm{O}$ values are slightly lower than those reported by [3]. One olivine grain in a coarse-grained chondrule rim is ${ }^{16} \mathrm{O}$-rich $\left(\Delta^{17} \mathrm{O} \sim-23 \%\right.$ ). Oxygen-isotope compositions of matrix olivine $\left(\mathrm{Fo}_{\sim 96-97}\right)$ and low-Ca pyroxene $\left(\mathrm{En}_{\sim 94-96}\right)$ grains of $\sim 5-20 \mu \mathrm{m}$ in size have a bimodal distribution: 12 out of 13 olivine and 4 out of 17 pyroxene grains are similarly ${ }^{16} \mathrm{O}$-rich $\left(\Delta^{17} \mathrm{O} \sim\right.$ $-23.5 \pm 2.9 \%$ ), others are similarly ${ }^{16} \mathrm{O}$-poor $\left(\Delta^{17} \mathrm{O} \sim-0.1 \pm 1.7 \%\right.$ ) These results indicate the ${ }^{16} \mathrm{O}$-enriched bulk matrix composition compared to bulk chondrules [3] could be explained by ubiquitous presence of ${ }^{16} \mathrm{O}$ rich grains in matrix. Nearly identical O-isotope compositions of chondrules and ${ }^{16} \mathrm{O}$-poor matrix grains may imply that both components formed in the same nebular region. ${ }^{16} \mathrm{O}$-rich matrix olivine and pyroxene might be related to AOAs and perhaps formed at a different time and place from Kakangari chondrules, suggesting those grains escaped thermal processing during chondrule formation and were mixed with Kakangari ${ }^{16} \mathrm{O}$-poor matrix grains later.

References: [1] Huss G. R. 2003. Geochimica et Cosmochimica Acta 67:4823-4848. [2] Klerner S. and Palme H. 1999. Meteoritics \& Planetary Science 34:A64. [3] Prinz M. et al. 1989. 20th Lunar and Planetary Science Conference, pp. 870-871. [4] Brearley A. J. 1989. Geochimica et Cosmochimica Acta 53:2395-2411. [5] Weisberg M. K. et al. 1996. Geochimica et Cosmochimica Acta 60:4253-4263. [6] Young E. D. and Russell S. S. 1998. Science 282:452-455.
5379

MINERALOGY AND THERMAL HISTROY OF ITOKAWA SURFACE PARTICLES RECOVERED BY HAYABUSA MISSION

T. Nakamura ${ }^{1}$, T. Noguchi ${ }^{2}$, M. Tanaka ${ }^{3}$, M. E. Zolensky ${ }^{4}$, M. Kimura ${ }^{2}$, A. Tshuchiyama ${ }^{5}$, A. Nakato ${ }^{1}$, T. Ogami ${ }^{1}$, H. Ishida ${ }^{1}$, M. Uesugi ${ }^{6}$, T. Yada $^{6}$, K. Shirai ${ }^{6}$, A. Fujimura ${ }^{6}$, R. Okazaki ${ }^{8}$, S. Sandford ${ }^{7}$, S. Wakita ${ }^{1}$, Y. Ishibashi ${ }^{6}$, M. Abe ${ }^{6}$, T. Okada ${ }^{6}$, M. Ueno ${ }^{6}$, T. Mukai ${ }^{6}$, M. Yoshikawa $^{6}$ and J. Kawaguchi ${ }^{6}$. ${ }^{1}$ Department Earth \& Planet. Mat. Sci., Tohoku University, Tohoku, Japan. E-mail: tomoki@m.tohoku.ac.jp. ${ }^{2}$ The College of Science at Ibaraki University, Ibaraki, Japan. ${ }^{3}$ WEBRAM,SPring-8, National Institute for Maths Science. ${ }^{4}$ NASA JSC. ${ }^{5}$ Department Earth \& Space Science, Osaka University, Osaka, Japan. ${ }^{6}$ JAXA-ISAS. ${ }^{7}$ NASA ARC. ${ }^{8}$ Department Earth \& Planetary Science, Kyushu University, Kyushu, Japan.

Introduction: Surface dust particles of asteroid Itokawa were successfully collected by spacecraft Hayabusa [1], but are very small similar in size range to the particles recovered by Stardust mission. Analysis of these tiny particles required multidiscipline edge-of-the-art techniques. We have developed a sequential experimental procedure consisting of synchrotron-based analysis, scanning and transmission electron microscopy, and secondary ion mass spectrometry [2].

Experiments: Approximately forty particles of about $30-180 \mu \mathrm{m}$ in size were attached on the top of thin carbon fibers. Layers of epoxy resin with several-micron thick covered entire surface of the particle to minimize exposure to atmosphere. The particles were analyzed at beamline $3 \mathrm{~A}$ of KEK and BL15XU of SPring-8 for X-ray diffraction. The particles were embedded in epoxy resin and processed by ultramicrotomy and/or polishing. Polished sections of the particles were analyzed by scanning electron microscopes for chemical composition and EBSD. Areas of interest in some particles were lifted up and thinned by FIB for observation by a transmission electron microscope.

Results and Discussion: Most particles are composed mainly of relatively coarse (typically from 10 to $50 \mu \mathrm{m}$ in size) and well-crystalline silicates such as olivine, low- and high-Ca pyroxene, and plagioclase. Some particles contain chromite, chlorapatite, merrillite, and troilite up to $20 \mu \mathrm{m}$ in size. Small inclusions (up to $10 \mu \mathrm{m}$ ) of taenite, kamacite, troilite, and chromite occur within coarse silicates in many particles. Compositions of silicates are relatively homogeneous. The common occurrence of triple junctions suggests that most particles have been thermally annealed sufficiently long for the silicates to have recrystallized. Olivine approximately $\mathrm{Fa}_{29}$, low-Ca pyroxene approximately $\mathrm{Fs}_{22}$, and kamacite Co (approximately $10 \mathrm{wt} \%$ ) and $\mathrm{Ni}$ (approximately $4 \mathrm{wt} \%$ ) concentrations of Itokawa particles are within the range of LL chondrites [3]. The presence of coarsened plagioclase (typically $20-50 \mu \mathrm{m}$ in size) and diopside (typically $10-30 \mu \mathrm{m}$ ) suggests that most of the particles have experienced high-temperature metamorphism with a metamorphic grade from 5 to 6. Metamorphic temperatures were estimated based on twopyroxene geothermometry to be approximately $800{ }^{\circ} \mathrm{C}$, which is slightly lower than LL6 temperatures [4].

References: [1] Nakamura T. et al. 2011. Abstract \#1776. 42nd Lunar and Planetary Science Conference. [2] Tsuchiyama A. et al. 2011. Abstract \#1777. 42nd Lunar and Planetary Science Conference. [3] Rubin A. E. Geochimica et Cosmochimica Acta 54:1217-1232. [4] Nakamuta Y. and Motomura.Y. 1999. Meteoritics \& Planetary Science 34:763-772. 
5515

STABLE CHLORINE ISOTOPE STUDY OF MARTIAN SHERGOTTITES AND NAKHLITES; WHOLE ROCK AND ACID LEACHATES AND RESIDUES

N. Nakamura ${ }^{1,6}$, L. E. Nyquist ${ }^{1}$, Y. Reese ${ }^{2}$, C.-Y. Shih ${ }^{3}$, T. Fujitani ${ }^{4}$ and O. Okano ${ }^{5}$. ${ }^{1}$ NASA Johnson Space Center, ARES Mail Code KR, 2101 NASA Pkwy, Houston, TX 77058, USA. E-mail: noboru. nakamura@nasa.gov. ${ }^{2}$ Mail Code JE-23, ESCG/Muniz Engineering, Houston, TX 77058, USA. ${ }^{3}$ ESCG Jacobs-Sverdrup, Houston, TX 77058,USA. ${ }^{4}$ Marine Tech. College, Ashiya 659, Japan. ${ }^{5}$ Okayama University, Okayama 700,Japan. ${ }^{6}$ Kobe University, Nada, Kobe 657, Japan. E-mail: nakamuransjp@yahoo.co.jp.

Introduction: We have established a precise analytical technique for stable chlorine isotope measurements of tiny planetary materials by TIMS (thermal ionization mass spectrometry) [1], for which the results are basically consistent with the IRMS technique (gas source mass spectrometry) [2-4]. We present here results for Martian shergottites and nakhlites; whole rocks, $\mathrm{HNO}_{3}$-leachates and residues, and discuss the chlorine isotope evolution of planetary Mars.

Experimental: Whole rocks of Zagami (shergottite, basalt), Nakhla (nakhlite, basalt) and MIL03346 (nakhlite, basalt) and $1 \mathrm{~N}-\mathrm{HNO}_{3}$ leachates and residues of Zagami and MIL samples were examined for chlorine contents by ion chromatography and isotopic composition by TIMS in this work. The $1 \mathrm{~N}-\mathrm{HNO}_{3}$ leaching was carried out at room temperature for $15-20 \mathrm{~h}$. Bulk and residue samples were analyzed for chlorine isotopes after treatment by $\mathrm{HF}$ leaching, $\mathrm{AgCl}$ precipitation and Cs-form resin. Isotopic analyses of leachates were also carried out by a similar method without HF-treatment.

Results and Discussion: Leaching effect- The second Zagami sample subjected to leaching experiments shows higher total $\mathrm{Cl}$ abundance $(160 \mathrm{ppm})$ compared to the 1st sample (114 ppm) reported at 42th LPSC [1], but rather similar abundance to that of Dreibus et al. [5] (145 ppm). About $60 \%$ of total chlorine in the bulk sample was leached out in the $1 \mathrm{~N}-\mathrm{HNO}_{3}$ fraction, indicating that major parts of chlorine exist in soluble phases (for example, chloroapatite) in Zagami. On the other hand, only $20 \%$ of bulk MIL chlorine $(221 \mathrm{ppm})$ was leached out in the acid fraction.

Chlorine Isotopic Composition: Our Orgueil (CI) results show significantly smaller $\delta^{37} \mathrm{Cl}_{\text {SMOC }}(-0.5 \%)$ compared to that of Sharp et al. $\left(\delta^{37} \mathrm{Cl}_{\mathrm{SMOC}}=1.2 \%\right.$ \% $[4]$ but still within the range of $\delta^{37} \mathrm{Cl}_{\mathrm{SMOC}}$ values reported by the New Mexico Univ. group [6]. Bulk Zagami, shergottite, shows $\delta^{37} \mathrm{Cl}_{\mathrm{SMOC}}$ almostthe same as seawater. On the other hand, Zagami leachate and two bulk nakhlites (Nakhla and MIL03346) show relatively similar $\delta^{37} \mathrm{Cl}_{\text {SMOC }}$ values $(1.5 \pm 0.5 \%)$. In Marrtian meteorites, the acid leachates were considered to be derived from more leachable components such as fluids, suggesting that they might represent Martian crustal components [7].

References: [1] Nakamura N. et al. 2011. Abstract \#2513. 42nd Lunar and Planetary Science Conference. [2] Bonifacie M. et al. 2007. Chemical Geology 242:187-201. [3] Musashi M. et al. 1998. Analytical Chemistry 363:261-269. [4] Sharp D. Z. et al. 2007. Nature 446:10621064. [5] Dreibus G. et al. 1979. Origin and Distribution of the Elements. pp. 33-38. [6] Mercer J. A. et al. 2011. Abstract \#2463. 42nd Lunar and Planetary Science Conference. [7] Sautter V. et al. 2006. Earth and Planetary Science Letters 252:45-55.
5448

NANO-SCALE ANATOMY OF Stardust COMETARY TRACKS CONTINUED: BULBOUS TRACKS 147 AND 168

K. Nakamura-Messenger ${ }^{1,2}$, L. P. Keller ${ }^{1}$, S. Messenger ${ }^{1}$, S. J. Clemett ${ }^{1,3}$, A. N. Nguyen ${ }^{1,2}$ and D. Frank ${ }^{4}{ }^{1}$ Robert $M$ Walker Laboratory for Space Science, ARES, NASA/JSC, Houston, TX, USA. ${ }^{2}$ ESCG/Jacobs Technology, Houston, TX, USA. ${ }^{3}$ ESCG/ERC Inc., Houston, TX, USA. ${ }^{4}$ ESCG/Hamilton Sundstrand, Houston, TX, USA. E-mail: keiko. nakamura-1@nasa.gov.

Introduction: Comet Wild 2 dust grains captured by the Stardust spacecraft show a wide range of chemical compositions, both among and within individual particle tracks [1]. This sample diversity complicates answering basic questions such as the bulk composition of the comet and modal mineralogy. We have developed new sample preparation and analytical techniques tailored for analysis of entire Wild 2 aerogel tracks, and successfully ultramicrotomed a "carrot" type A track along its axis while preserving its original shape [2]. This innovation allowed us to examine the distribution of fragments along the entire track from the entrance hole all the way to the terminal particle. This report focuses on our systematic disassembly and coordinated analysis of two Stardust bulbous type tracks from the $\mathrm{mm}$ to $\mathrm{nm}$-scale.

Experimental: The walls of tracks $\# 147(4600 \mu \mathrm{m}$ long with 7 terminal particles, TPs) and \#168 (2640 $\mu \mathrm{m}$ long, 3 TPs $)$ were photodocumented using an extended depth-of-field image processing technique that generates a single infocus image from a series of photographs. The TPs were removed, embedded in epoxy, and ultramicrotomed separately. Scanning TEM was used for elemental and detailed mineralogy characterization. This approach also enabled spatially resolving the target sample from fine-scale mixtures of compressed aerogel and melt.

Results and Discussion: We obtained preliminary mineralogical analyses of 3 TPs and particles recovered from a crack near the entrance area $(30 \mu \mathrm{m})$ from Track \#147, and 2 TPs from T168. T147,TP2 $(18 \mu \mathrm{m})$ entirely consists of $\mathrm{Fe}-\mathrm{Ni}$ alloy $(5$ atom $\%$ Ni). T147,TP3 $(19 \mu \mathrm{m})$ contains $\mathrm{Fa} 28$ with partial olivine-pyroxene intergrowth and minor albite. T147,TP4 $(12 \mu \mathrm{m})$ contains pentlandite, Fe-olivine, albite and high $\mathrm{Ca}$ pyroxene with $\mathrm{Na}$ and $\mathrm{Cr}$ (kosmochlor component). The $\mathrm{Fe}$ olivine + albite + Ca-pyroxene assemblage is common in other Wild 2 samples as well [3]. No surviving crystalline cometary materials were observed from track walls yet, but only mixed and melted materials of aerogel and cometary constituents. T168,TP1 $(10 \mu \mathrm{m})$ contains Fe-olivine, albite and pentlandite. T168,TP2 $(8 \mu \mathrm{m})$ has concentric texture with a core of olivine grains $(100 \mathrm{~nm}$ in size) with coexisting indigenous amorphous $\mathrm{SiO}_{2}$ surrounded by a carbon mantle (800 nm thickness), which in turn is surrounded by a layer of compressed aerogel. A nebular origin as possible precursors to type II chondrules in ordinary chondrites has been proposed for the Fe-olivine + albite + Ca-pyroxene assemblages in Wild 2 [3]. However, in T147, this assemblage coexists with pentlandite which would not have been a stable phase in the nebula [4]. In future work, NanoSIMS will be used for isotopic analyses, and ultrafast two-step laser mass spectrometry (ultra $\mathrm{L}^{2} \mathrm{MS}$ ) to investigate the nature and distribution of organic phases. The albite in T168,TP1 may be large enough to measure $\mathrm{Mg} / \mathrm{Al}$ system using NanoSIMS. We also plan to measure H, N, C isotopes of the carbon shell of T168,TP2 and O isotopes of the silicates inside.

References: [1] Brownlee D. E. et al. 2006. Science 314:1711. [2] Nakamura-Messenger K. et al. 2011. Meteoritics \& Planetary Science, in press. [3] Joswiak D. et al. 2009. Meteoritics \& Planetary Science 44:1561. [4] Brearley A. J. 1999. Science 285:1380. 


\section{5}

STUDY OF CHONDRULES IN CH CHONDRITES - III: OXYGEN ISOTOPE RATIOS OF SILICA-BEARING, METAL-BEARING, AND ${ }^{16} \mathrm{O}$-RICH CHONDRULES

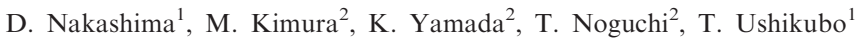
and N. T. Kita ${ }^{1} \cdot{ }^{1}$ University of Wisconsin-Madison, USA. E-mail: naka@geology.wisc.edu. ${ }^{2}$ Ibaraki University, Japan.

Introduction: From a systematic survey of chondrule oxygen isotope ratios in Asuka-881020 $(\mathrm{CH})$, we reported that magnesian and ferroan cryptocrystalline (CC) chondrules were derived from two distinct isotope reservoirs with $\Delta^{17} \mathrm{O}$ values of $-2 \%$ and $+1.5 \%$, respectively [1]. $\mathrm{CH}$ chondrites also contain ferroan nonporphyritic chondrules such as silicabearing ( $\mathrm{SB}$ ) and FeNi metal-bearing (MB) chondrules [2], which may be related to ferroan $\mathrm{CC}$ chondrules according to their similar sizes $(<100 \mu \mathrm{m})$ and $\mathrm{FeO}$-rich chemistry. Exceptionally ${ }^{16} \mathrm{O}$-rich oxygen isotope ratios were reported from a glassy chondrule with olivine rim by [3] in Acfer $214(\mathrm{CH})$. Here we report additional oxygen three-isotope analyses of several minor types of chondrules in Asuka-881020.

Results: IMS-1280 at UW-Madison was used to obtain oxygen isotope ratios using $2 \times 4 \mu \mathrm{m}$ spots, which yield analytical precisions of $1-2 \%$ ( $2 \mathrm{SD}$ ). All data plot along a slope 1 line on a three-isotope oxygen diagram $\left(\delta^{17} \mathrm{O}\right.$ vs. $\left.\delta^{18} \mathrm{O}\right)$. Isotope ratios of ferrous pyroxene and silica in SB chondrules are consistent within the analytical uncertainties. Nine SB chondrules with spherical shapes have nearly identical $\Delta^{17} \mathrm{O}$ values with the average $+1.2 \pm 1.4 \%$ ( $2 \mathrm{SD}$ ), while five $\mathrm{SB}$ chondrules with irregular shapes and fine-grained textures have variable $\Delta^{17} \mathrm{O}$ values from -6 to $+5 \%$. Three ferroan $\mathrm{MB}$ chondrules have $\Delta^{17} \mathrm{O}$ values from 0 to $+2 \%$. Two olivine-rimmed Al-rich chondrules show $\Delta \Delta^{17} \mathrm{O}$ values of $-12 \%$ and $-6 \%$, respectively. We analyzed one magnesian MB chondrule that shows the $\Delta^{17} \mathrm{O}$ value $(-21 \%)$ as low as CAIs, though it has no olivine rim.

Discussion: Nearly identical oxygen isotope ratios of ferrous pyroxene and silica in SB chondrules suggest formation from common isotope reservoirs. Spherical SB and ferroan MB chondrules have similar $\Delta{ }^{17} \mathrm{O}$ values to ferroan $\mathrm{CC}$ chondrules, and are depleted in refractory and volatile elements like ferroan $\mathrm{CC}$ chondrules. Thus, these three types of ferroan chondrules might have formed via fractional condensation from an isotopically uniform gaseous reservoir. $\Delta^{17} \mathrm{O}$ range of irregular-shaped SB chondrules overlaps with that of type I porphyritic chondrules in Asuka-881020 [1], implying that the irregular-shaped SB chondrules formed in the solar nebula like the porphyritic chondrules [4].

Al-rich chondrules with olivine rim show intermediate $\Delta{ }^{17} \mathrm{O}$ values between ${ }^{16} \mathrm{O}$-rich $\mathrm{CAIs}$ [5] and ferromagnesian chondrules [1] in $\mathrm{CH}$ chondrites, indicating mixing of $\mathrm{CAI}$-like materials and ferromagnesian chondrules as their precursors. The lowest $\Delta^{17} \mathrm{O}$ value from this work was obtained from the magnesian MB chondrule, which is not enriched in refractory elements unlike CAIs. The origin of the magnesian MB chondrule would be related to that of olivine-rimmed glassy chondrule in [3]. They may have formed from a ${ }^{16} \mathrm{O}$-rich gaseous reservoir or preserved the ${ }^{16} \mathrm{O}$-rich composition after melting [3].

References: [1] Nakashima D. et al. 2010. Meteoritics \& Planetary Science 45:A148. [2] Krot A. N. et al. 2000. Meteoritics \& Planetary Science 35:1249-1258. [3] Kobayashi K. et al. 2003. Geochemical Journal 37:663-669. [4] Krot A. N. et al. 2010. Geochimica et Cosmochimica Acta 74:2190-2211. [5] Sahijpal S. et al. 1999. Meteoritics \& Planetary Science 45:A101.
5076

\section{HIGH-PRECISION OXYGEN ISOTOPE ANALYSIS OF EQUILIBRATED ORDINARY CHONDRITES: FOR COMPARISON WITH THE ITOKAWA PARTICLES}

D. Nakashima, T. Ushikubo and N. T. Kita. University of WisconsinMadison, WI, USA. E-mail: naka@geology.wisc.edu.

Introduction: X-ray fluorescence and near-infrared measurements by the Hayabusa spacecraft suggested that the surface materials of the asteroid Itokawa were similar to LL5 or LL6 chondrites [1, 2], which was confirmed by preliminary examination of particles recovered from the asteroid Itokawa [e.g., 3, 4]. Oxygen isotope ratios of equilibrated ordinary chondrites (EOCs) by ion microprobe are important for comparison with the Itokawa particles, but are scarce. Here we report oxygen isotope ratios of four phases (olivine, low-Ca and high-Ca pyroxene, and plagioclase) in LL5 (Siena), LL6 (St. Séverin), and H6 (Guareña).

Results: IMS-1280 at UW-Madison was used to obtain oxygen isotope ratios using $10 \times 15 \mu \mathrm{m}$ spots, which yield analytical precisions of $\sim 0.4 \%$ for $\delta^{18} \mathrm{O}, \delta^{17} \mathrm{O}$, and $\Delta^{17} \mathrm{O}$ (2 SD). Olivine, low-Ca and high-Ca pyroxene, and plagioclase in respective meteorites have indistinguishable $\Delta{ }^{17} \mathrm{O}$ values and show averages of $+1.2 \pm 0.1 \%$ o $(n=29$; Siena $)$, $+1.3 \pm 0.1 \% \quad(n=32 ; \quad$ St. Séverin $)$, and $+0.9 \pm 0.1 \% \quad(\mathrm{n}=32$; Guareña) (2SE). The $\delta^{18} \mathrm{O}$ values range from +4.7 to $+6.3 \%$ (Siena), from +4.5 to $+5.8 \%$ (St. Séverin), and from +3.7 to $+5.2 \%$ (Guareña). The four mineral phases in all three meteorites show systematic changes in $\delta^{18} \mathrm{O}$ values, in the order of high-Ca pyroxene, olivine, low-Ca pyroxene, and plagioclase. Morphology of chondrules remains in Siena (LL5), and we analyzed several chondrules in Siena. The average $\Delta^{17} \mathrm{O}$ values are variable among chondrules $(+0.4$ to $+1.5 \%$ ). One POP chondrule with the average $\Delta \Delta^{17} \mathrm{O}$ of $\sim+1.5 \%$ contains an olivine grain with $\Delta^{17} \mathrm{O}$ of $\sim-2.0 \%$, which may be a relict olivine.

Discussion: Siena and St. Séverin (LL) have higher $\Delta^{17} \mathrm{O}$ values than Guareña $(\mathrm{H})$, which is consistent with bulk chondrite data [5]. The $\delta^{18} \mathrm{O}$ values of olivine in Siena are systematically higher than those in St. Séverin by $0.5 \%$, consistent with the negative correlation between $\delta^{18} \mathrm{O}$ values and petrologic types observed in bulk chondrites [5].

Oxygen isotope equilibrium temperatures were calculated from plagioclase and high-Ca pyroxene pairs in matrices. The differences in $\delta^{18} \mathrm{O}$ between two minerals were $1.6 \pm 0.4 \%$ (Siena), $1.3 \pm 0.2 \%$ o (St. Séverin), and $1.6 \pm 0.1 \%$ o (Guareña), which correspond to temperatures of $720 \pm 140{ }^{\circ} \mathrm{C}$ (Siena), $870 \pm 120^{\circ} \mathrm{C}$ (St. Séverin), and $750 \pm 50{ }^{\circ} \mathrm{C}$ (Guareña). These temperatures are consistent with those for oxygen thermometry in EOCs by [6] as well as olivine-spinel thermometry [7].

The relict olivine grain with $\Delta^{17} \mathrm{O}$ of $-2.0 \%$ in the POP chondrule has $\mathrm{Fa}_{24.7}$, which is within the range in Siena $\left(\mathrm{Fa}_{24} .8 \pm 0.2 ; n=16\right)$. Our data suggests that oxygen isotope heterogeneity originally in primitive chondrules was survived even in type 5 EOCs. Such a heterogeneous grain could exist among Itokawa particles.

References: [1] Okada T. et al. 2006. Science 312:1338-1341. [2] Abe M. et al. 2006. Science 312:1334-1338. [3] Nakamura T. et al. 2011. Abstract \#1766. 42nd Lunar and Planetary Science Conference. [4] Yurimoto H. et al. 2011. Abstract \#1755. 42nd Lunar and Planetary Science Conference. [5] Clayton R. N. et al. 1991 Geochimica et Cosmochimica Acta 55:2317-2337. [6] Clayton R. N. 1993. Annual Review of Earth and Planetary Sciences 21:115-149. [7] Kessel R. et al. 2007. Geochimica et Cosmochimica Acta 71:1855-1881. 
5416

THE VARIETY OF THERMAL EVOLUTION OF PRIMITIVE HYDROUS ASTEROIDS RECORDED IN DEHYDRATED CARBONACEOUS CHONDRITES

A. Nakato ${ }^{1}$, T. Nakamura ${ }^{1}$, T. Noguchi ${ }^{2}$, I. $\mathrm{Ahn}^{3}$ and J. I. Lee ${ }^{3}{ }^{1}$ Tohoku University, Miyagi 980-8578, Japan. E-mail: aiko@s. tohoku.ac.jp. ${ }^{2}$ Ibaraki University, Ibaraki 310-8512, Japan. ${ }^{3}$ Korean Polar Research Institute, Incheon 406-840, Korea.

Diversities of thermal evolution, starting from low-temperature aqueous alteration and subsequent high-temperature thermal dehydration, in primitive hydrous asteroids can be evaluated based on mineralogical and chemical variation in dehydrated carbonaceous chondrites [e.g., 1]. Synchrotron XRD analysis indicates that matrix phyllosilicates in Belgica (B-) 7904, Dhofar 735, PCA 91084, PCA 91008, Yamato-86695, EET 87522, WIS 91600, LAR 06318, ALH 84033, DOM 03183, and MIL 05152 are decomposed by heating [2]. Most of samples, excepting B-7904 and Dhofar 735, experienced weak thermal metamorphism. To know the degree of heating based on evidence from organics, we analyzed matrices by using micro-Raman spectroscopy. As expected, most of weak heated samples, except for PCA 91008 that underwent heavy weathering at Antarctica, showed a broad D1-band suggestive of low maturation grade of organics. The results suggest that those samples suffered heating weaker than Murchison samples that were experimentally heated at $600{ }^{\circ} \mathrm{C}$ for $1 \mathrm{~h} \mathrm{[3].}$

LAR 06318 and ALH 84033 show unique oxygen isotopic compositions. Although most weakly heated samples that we analyzed are plotted on CM-CO-Tagish Lake line, only LAR 06318 and ALH 84033 are plotted on or close to CCAM line. Because partially decomposed phyllosilicates are most dominant phases in these two samples, their oxygen compositions are close to those of the decomposed phases. Based on low totals of FE-EPMA analysis, dehydration of phyllosilicates was not so much progress. Therefore, oxygen composition of the two meteorites were established during aqueous alteration and not severely affected by heating. Phyllosilicates having various oxygen isotopic compositions can be formed under following conditions during aqueous alteration (1) original solution had various oxygen isotopic ratios. (2) water/rock ratio varies considerably.

Our results suggest that B-7904 and Dhofar 735 suffered heating at the highest temperatures among 11 samples. This interpretation is consistent with previous studies [e.g., 4, 5]. Based on micro-Raman analysis, their maturation grade of organics is higher than heated Murchison at $900{ }^{\circ} \mathrm{C}$. But narrow $\mathrm{Fe}-\mathrm{Mg}$ zoning at outer edges of chondrule olivines in B-7904 and Dhofar735 implies that duration of the heating is much shorter than those expected for internal heating by shortlived radionuclide, like ${ }^{26} \mathrm{Al}$. As shown in [5], oxygen isotope compositions of the two samples determined in our study also show smaller $A \hat{A}{ }^{17} \mathrm{O}$ than those of the other samples, and matrices have Si-rich chemical composition, suggesting that the conditions of thermal evolution are different from those in typical $\mathrm{CM}$ and dehydrated $\mathrm{CM}$ chondrite parent bodies.

References: [1] Hiroi T. et al. 1993. Science 261:1016-1018. [2] Nakato A. et al. Abstract \#1725. 42nd Lunar and Planetary Science Conference. [3] Nakato A. et al. 2008. Earth, Planets and Space 60:855854. [4] Tomeoka K. 1990. Proceedings of the NIPR Symposium on Antarctic Meteorites 3:40-54. [5] Ivanova M. A. et al. 2010. Meteoritics \& Planetary Science 45:1108-1123.
5207

EXAMINATION OF ORGANIC COMPOUNDS IN THE HAYABUSA SAMPLES FROM THE ASTEROID ITOKAWA

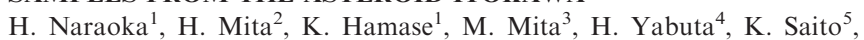
K. Fukushima ${ }^{5}$, F. Kitajima ${ }^{1}$, S. A. Sandford ${ }^{6}$, T. Nakamura ${ }^{7}$, T. Noguchi $^{8}$, R. Okazaki ${ }^{1}$, K. Nagao ${ }^{9}$, M. Ebihara ${ }^{10}$, H. Yurimoto ${ }^{11}$, A. Tsuchiyama $^{4}$, M. Abe ${ }^{12}$, K. Shirai ${ }^{12}$, M. Ueno ${ }^{12}, \mathrm{~T} . \mathrm{Yada}^{12}, \mathrm{Y}$. Ishibashi $^{12}$, T. Okada ${ }^{12}$, A. Fujimura ${ }^{12}$, T. Mukai ${ }^{12}$, M. Yoshikawa ${ }^{12}$ and J. Kawaguchi ${ }^{12}$. ${ }^{1}$ Kyushu University, Kyushu, Japan. E-mail: naraoka@geo.kyushu-u.ac.jp. ${ }^{2}$ Fukuoka Institute of Technology, Fukuoka, Japan. ${ }^{3}$ Shiseido Co. Ltd. ${ }^{4}$ Osaka University, Osaka, Japan. ${ }^{5}$ Nagoya University, Nagoya, Japan. ${ }^{6}$ NASA/ARC ${ }^{7}$ Tohoku University, Tohoku, Japan. ${ }^{8}$ Ibaraki University, Ibaraki, Japan. ${ }^{9}$ University of Tokyo, Tokyo, Japan. ${ }^{10}$ Tokyo Metropolitan University, Tokyo, Japan. ${ }^{11}$ Hokkaido University, Hokkaido, Japan. ${ }^{12}$ ISAS/JAXA.

Introduction: So far, two sample return missions have provided samples for organic analyses; lunar soils by the Apollo Program [1] and cometary dust particles by the Stardust mission [2]. The Hayabusa mission has provided the first opportunity to examine the occurrence of organic matter directly on the surface of an asteroid. Asteroid Itokawa is an S-type asteroid, a parent body of LL5-6 ordinary chondrites [3]. Although it seems to be depleted in organic matter due to its high formation temperature, the organic matter may be delivered from comets or carbonaceous meteorites, and/or organic precursors may be implanted by solar wind [4]. In this study, organic compound analyses were performed for the solvent-extract of the Hayabusa particles.

Materials and Methods: Five particles (approximately 40-180 $\mu \mathrm{m}$ ), which consist mainly of olivine and pyroxene in mineral composition, were rinsed with small amount of organic solvents in a diamond holder. After acid hydrolysis the amino acids with enantiomeric separation was analyzed by two-dimensional high performance liquid chromatography with highly sensitive fluorescence detection (detection limit; approximately sub femto mole). Other organic compound analysis including a search for PAHs was performed using ToF-SIMS. Carbonaceous grains (approximately $50 \mu \mathrm{m}$ ) of Y791191 (CM2) were also analyzed as a reference.

Results and Discussion: The glycine (Gly) and DL-alanine (Ala) were observed from the particle extracts only at a procedural blank level. L-Ala was more enriched relative to D-Ala, indicating a terrestrial signature rather than asteroidal signatures. On the other hand, Gly, Ala and $\alpha$-aminoisobutyric acid (approximately $100 \mathrm{ppm}$ ) were detected in the $\mathrm{HCl}$ extract of $\mathrm{Y} 791191$, where Ala was present as a nearly racemic mixture. As to ToF-SIMS analysis, no substantial difference was observed in positive and negative ions between the Hayabusa sample and procedural blank. The concentration of indigenous organic compounds is less than approximately ppm, if present.

References: [1] Harada K. et al. 1971. Science 173:433-435. [2] Sandford S. et al. 2006. Science 314:1720-1724. [3] Abe M. et al. 2006. Science 312:1334-1338. [4] Fox S. W. et al. 1976. Geochimica et Cosmochimica Acta 40:1069-1071. 
5130

DAWN AT VESTA: FIRST RESULTS FROM THE FRAMING CAMERAS

Nathues $^{1}$, C. T. Russell ${ }^{2}$, C. Raymond ${ }^{3}$, U. Christensen ${ }^{1}$, H. Sierks ${ }^{1}$, R. Jaumann $^{4}$, H. U. Keller ${ }^{5}$, S. Mottola ${ }^{4}$, G. Neukum ${ }^{6}$, M. Hoffmann ${ }^{1}$, L. Le Corre ${ }^{1}$, V. Reddy ${ }^{1}$, S. E. Schröder ${ }^{1}$, J. -Y. $\mathrm{Li}^{7}$, B. J. Buratti ${ }^{3}$, P. Gutiérrez Marqués $^{1}$, I. Buettner ${ }^{1}$, I. Hall ${ }^{1}$, T. Maue ${ }^{1}$, M. Richards ${ }^{1}$ and the Dawn Science Team. ${ }^{1}$ MPI for Solar System Research, KatlenburgLindau, Germany. E-mail: nathues@mps.mpg.de. ${ }^{2}$ UCLA. ${ }^{3}$ JPL. ${ }^{4}$ DLR. ${ }^{5}$ TUB. ${ }^{6}$ FUB. ${ }^{7}$ UMD.

Introduction: Vesta is the largest intact differentiated asteroid [1] that is considered to be a model for the initial stages of planetary differentiation [2]. NASA's Dawn mission is expected to rendezvous with Vesta in July 2011 for a yearlong global characterization mission. The Framing Cameras (FC) onboard the Dawn spacecraft will image the asteroid in clear and seven narrow band filters $(0.4-1.0 \mu \mathrm{m})$ [3].

We plan to present the first results from the Dawn FC observations of Vesta obtained during approach. Our aim is to answer three basic questions by comparing Dawn FC data with our current understanding of Vesta from HST and ground-based studies. These questions are: (1) Is the hemispherical albedo dichotomy on Vesta confirmed? (2) Is there color variation on Vesta, if so how does the color variation correlate with HST color and ground-based spectral maps? (3) Is the large topographic feature on Vesta's South Pole confirmed?

Scientific Question 1: Albedo dichotomy: Low resolution resolved color data can be helpful in answering several key questions about the nature and origin of Vesta's surface. From HST observations [4] the hemispheric albedo dichotomy on Vesta is well known. The eastern hemisphere of Vesta has higher albedo than the western hemisphere; this is consistent with ground-based lightcurve observations [2]. Such global albedo variations can be detected and confirmed from observations in approach.

Scientific Question 2: Color variegation: Spectral [2] and color variations [4] on Vesta have been attributed to compositional heterogeneity of distinct surface units. Low-resolution global color ratio maps from FC data can identify and confirm these features reported by HST [4] and ground-based telescopes [5]. Average color spectra of progressive rotational phases from the rotational characterizations can be directly compared with similar spectra from HST and ground-based telescopes.

Scientific Question 3: Global topographic feature: The largest topographic feature on Vesta detected from HST images by [6] is the South Pole crater with an estimated diameter $\sim 460 \mathrm{~km}$ and a depth of $\sim 20 \mathrm{~km}$ [1]. Current shape models of Vesta indicate a large central peak within this crater. Images at resolution greater than HST $\left(38 \mathrm{~km} \mathrm{pix}^{-1}\right)$ should confirm the presence of this feature and help define its origin.

References: [1] Thomas et al. 1997. Icarus 128. [2] Gaffey. 1997. Icarus 127. [3] Sierks et al. 2011. Space Science Reviews, in press. [4] Binzel et al. 1997. Icarus 128. [5] Reddy et al. 2010. Icarus 210. [6] Thomas et al. 1997. Science 277.
5514

ORIGIN AND COMPOSITION OF CARBONATES, SULFATES, AND HALITE ON MARS

A. W. Needham ${ }^{1,2}$, N. A. Starkey ${ }^{1}$, D. Johnson ${ }^{1}$, T. Tomkinson ${ }^{1,3}$, C. Guillermier $^{1,4}$, R. L. Abel ${ }^{2,3}$, I. A. Franchi ${ }^{1}$ and M. M. Grady ${ }^{1,2}$. ${ }^{1}$ PSSRI, The Open University, Walton Hall, Milton Keynes, MK7 6AA, UK. E-mail: A.W.Needham@open.ac.uk. ${ }^{2}$ Department of Mineralogy, The Natural History Museum, London, SW7 5BD, UK. ${ }^{3}$ SUERC ${ }^{4}$ Harvard Medical School ${ }^{5}$ Imperial College, London.

Introduction: The nakhlite meteorites, widely accepted to originate on Mars, preserve complex mixtures of silicate alteration, carbonates, sulfates, halides, oxides, and iron oxy-hydroxides [1-3]. These phases may have formed at different times, under different fluid flow regimes (groundwater, hydrothermal, crater lake), experiencing a range of waterrock ratios, evaporative histories, and varying degrees of interaction with the atmosphere and bedrock $[2,3]$.

Using information gathered from our 3D investigations of Nakhla's fluid pathways [4] we have undertaken a range of in-situ geochemical and isotopic analyses of carbonate, sulphate and halite in the Nakhla meteorite.

Samples and Methods: The 3D mineralogy of a small section of Nakhla was analyzed by computed tomography (CT) [4]. Using a Metris X-Tek HMX ST 225 System a resolution of $\sim 5 \mu \mathrm{m}$ per voxel was acheived, producing several thousand CT "slices," subsequently reconstructed to provide 3D representations of the sample. Several sites of interest were identified in the CT data and the section of Nakhla was physically cut to reveal these sites for in situ studies. High-resolution backscatter electron and false-color EDS images were obtained using a FEI Quanta 200 dual-beam system; the focused ion beam (FIB) capabilities of this instrument have been used to extract FIB sections ranging from electron-transparent $(\sim 100 \mathrm{~nm})$ to $\sim 5 \mu \mathrm{m}$ thick sections for Raman spectroscopy, electron microprobe, and NanoSIMS analyses. We have previously demonstrated the capabilities of the NanoSIMS $50 \mathrm{~L}$ for analyzing the range of carbon and oxygen isotope compositions in the Martian meteorite ALH 84001 [5]. Continuing improvements in the precision of oxygen isotope analyses (presented by [6]), especially including ${ }^{17} \mathrm{O}$ measurements, are essential for analyses of samples with smaller isotopic differences, such as for our analyses of Nakhla secondary minerals, e.g., carbonates with $<10 \% \delta^{18} \mathrm{O}$ variations $[7,8]$ in order to understand the nature of the fluids and formation conditions.

Summary: Combining 3D mineralogy with subsequent highprecision and high-spatial resolution 2D mineralogical, chemical, and isotopic analyses, provides detailed information on the formation and alteration of secondary minerals on Mars. Understanding all aspects of these minerals is essential to reconstructing the true history of water on Mars, and the inherent implications for past climate and potential habitability.

References: [1] Gooding J. L. et al. 1991. Meteoritics 26:135-143. [2] Bridges. J. C. and Grady. M. M. 2000. Earth and Planetary Science Letters 176: 267-279. [3] Grady M. M. et al. 2007. Abstract \#1826. 38th Lunar and Planetary Science Conference. [4] Needham A. W. et al. 2011. Abstract \#5440. 72nd Annual Meeting of the Meteoritical Society. [5] Tomkinson T. et al. 2010. Abstract \#5368. 72nd Annual Meeting of the Meteoritical Society. [6] Starkey et al. 2011. 73rd Annual Meeting of the Meteoritical Society. [7] Vicenzi and Eiler 1998. Meteoritics \& Planetary Science 33. [8] Saxton J. M. et al. 2000. Geochemistry Geophysics Geosystems 64:1299-1309. 


\section{9}

MICROSTRUCTURES OF RARE SILICATE Stardust FROM NOVA AND SUPERNOVAE

A. N. Nguyen ${ }^{1,2}, \quad$ L. P. Keller ${ }^{1}, \quad$ Z. Rahman ${ }^{1,2}$ and S. Messenger ${ }^{1}$. ${ }^{1}$ Robert M. Walker Laboratory for Space Science, ARES, NASA JSC, Houston TX, USA. ${ }^{2}$ ESCG/Jacobs Technology, Houston, TX, USA. E-mail: lan-anh.n.nguyen@nasa.gov.

Introduction: Most silicate stardust analyzed in the laboratory and observed around stellar environments derives from O-rich red giant and AGB stars [1,2]. Supernova (SN) silicates and oxides are comparatively rare, and fewer than 10 grains from nova or binary star systems have been identified to date. Very little is known about dust formation in these stellar environments. Mineralogical studies of only three O-rich SN [3-5] and no nova grains have been performed. Here we report the microstructure and chemical makeup of two SN silicates and one nova grain.

Experimental: Silicate grains having exotic O isotopic compositions were identified amongst matrix grains of the Acfer 094 meteorite by NanoSIMS $\mathrm{O}$ and $\mathrm{Si}$ isotopic imaging. Grains having unusual isotopic signatures were then measured for $\mathrm{Mg}$, and in some cases $\mathrm{Fe}$, isotopes after removal of surrounding grains by focused ion beam milling [6]. Cross sections were then produced for three of these presolar silicates and their microstructures were determined by transmission electron microscopy (TEM).

Results and Discussion: Supernova silicates. Two silicates had $\mathrm{O}$ and $\mathrm{Mg}$ isotopic ratios consistent with condensation in 15M SNe. Grain 6_16 is enriched in ${ }^{18} \mathrm{O}(1.5 \times$ solar $)$ and slightly depleted in ${ }^{25} \mathrm{Mg}$. Si and $\mathrm{Fe}$ isotopic ratios are solar within error. This grain is amorphous and has a chemical composition consistent with $\mathrm{Mg}$-rich olivine (Fo95). Grain 2 33b is anomalous in both $\mathrm{O}$ and $\mathrm{Mg}\left(\delta^{17} \mathrm{O}=355 \%, \delta^{18} \mathrm{O}=700 \%\right.$, $\delta^{25} \mathrm{Mg}=-250 \%, \quad \delta^{26} \mathrm{Mg}=130 \%$ ), but not Si. Little grain material remained after the NanoSIMS analyses, but TEM analysis indicated an amorphous $\mathrm{Fe}$-rich silicate with minor $\mathrm{Mg}$ and $\mathrm{Ca}$ and nanophase $\mathrm{FeNi}$ metal inclusions. This structure is reminiscent of glass with embedded metal and sulfides (GEMS) [7], though beam damage cannot be excluded. While presolar GEMS have been identified in interplanetary dust particles [8], conclusive identification of these grains in meteorite samples has not been made.

The mineralogies of two other SN silicates have previously been determined: $\mathrm{B} 10 \mathrm{~A}$ is an aggregate of crystalline olivine (Fo83) [3] and 2_4 is amorphous $\mathrm{MgSiO}_{3}$ [5]. The chemical compositions of $\mathrm{B} 10 \mathrm{~A}, 24$ and 6 6_16 suggest equilibrium condensation. In contrast to other $\mathrm{SN}$ dust phases, the SN silicates have varied mineralogies and are mostly amorphous, though alteration from an initially crystalline state cannot be ruled out.

Nova silicate. Silicate $4 \_2$ was found to be highly ${ }^{17} \mathrm{O}$-rich and also anomalous in ${ }^{18} \mathrm{O}$ and $\mathrm{Mg}$ isotopes $\left(\delta^{17} \mathrm{O}=32410 \%, \delta^{18} \mathrm{O}=-130 \%\right.$, $\delta^{25} \mathrm{Mg}=1025 \%, \delta^{26} \mathrm{Mg}=90 \%$ ), suggestive of a nova source (see [6]). An extremely limited amount of material remained for TEM analysis, but the silicate contains $\mathrm{Al}(\mathrm{Al} / \mathrm{Si}=0.3)$ and some $\mathrm{Fe}$, likely contamination from an underlying $\mathrm{Fe}$ oxide grain. Nova grains are exceptionally rare and no other microstructural studies of O-rich nova dust exist to date. Identification of additional nova silicates is highly desirable.

References: [1] Nguyen A. N. et al. 2010. The Astrophysical Journal 719:166. [2] Waters L. B. F. M. et al. 1996. Astronomy \& Astrophysics 315:L361. [3] Messenger S. et al. 2005. Science 309:737. [4] Stroud R. M. et al. 2008. Abstract \#1778. 39th Lunar and Planetary Science Conference. [5] Nguyen A. N. et al. 2010. Meteoritics \& Planetary Science 45:A5423. [6] Nguyen A. N. et al. 2011. Abstract \#2711. 42nd Lunar and Planetary Science Conference. [7] Bradley J. P. and Dai Z. R. 2004. The Astrophysical Journal 617:650. [8] Keller L. P. and Messenger S. 2011. Geochimica et Cosmochimica Acta, in revision.

\section{5}

\section{LOVINA: IS THIS A METEORITE?}

K. Nishiizumi ${ }^{1}$ and M. W. Caffee ${ }^{2}$. ${ }^{1}$ Space Sciences Laboratory, University of California, Berkeley, CA 94720-7450, USA. E-mail: kuni@ ssl.berkeley.edu. ${ }^{2}$ Department of Physics, Purdue University, West Lafayette, IN 47907-1396, USA.

Introduction: Lovina $(8.2 \mathrm{~kg}$ ) was found on Lovina beach in Bali, Indonesia, on January 1981. It is a strikingly oblong, weathered iron with $\mathrm{cm}$-scale pyramids projecting from its upper surface (orientation as found on beach) and $\mathrm{cm}$-sized vugs in the lower surface [1]. Based on petrographic, mineralogical, and geochemical analysis [2], Lovina is classified as an ungrouped iron meteorite (ataxite). The bulk chemical composition, including trace elements, suggests Lovina is a high $\mathrm{Ni}$ iron meteorite, although its unusual appearance casts some doubt on this assertion. Since all recovered extraterrestrial materials contain cosmogenic nuclides produced in space we have measured the concentrations of cosmogenic nuclides ${ }^{10} \mathrm{Be},{ }^{26} \mathrm{Al}$, and ${ }^{36} \mathrm{Cl}$ to verify its extraterrestrial origin.

Results and Discussion: A piece of Lovina was cleaned by etching with $5 \% \mathrm{HNO}_{3} /$ ethanol solution using an ultrasonic bath for $\sim 30 \mathrm{~min}$. $249 \mathrm{mg}$ of the cleaned sample was then dissolved in $4 \mathrm{~N} \mathrm{HNO}_{3}$ along with the carrier solutions. The chemical composition of our sample is $57.8 \% \mathrm{Fe}, 0.94 \% \mathrm{Co}$, and $36.4 \% \mathrm{Ni}$. After chemical separation and purification the cosmogenic radionuclides were measured by AMS at Purdue University. Lovina contains $0.0055 \pm 0.0005 \mathrm{dpm}{ }^{10} \mathrm{Be} \mathrm{kg}^{-1}$, $0.0029 \pm 0.0021 \mathrm{dpm}{ }^{26} \mathrm{Al} \mathrm{kg}^{-1}$, and $0.0025 \pm 0.0008 \mathrm{dpm}{ }^{36} \mathrm{Cl} \mathrm{kg}^{-1}$. These activities are 3 to 4 orders of magnitude lower than those of typical iron meteorites, and the lowest among all iron meteorites except interior pieces from the Nantan or Gibeon meteorites. If the cosmogenic nuclides in Lovina were produced in space, the recovered sample of Lovina was near the center of an object more than $\sim 3 \mathrm{~m}$ in diameter, or it resided at $\sim 1.5 \mathrm{~m}$ depth in a large object in space. In addition, the ${ }^{10} \mathrm{Be}$ and ${ }^{36} \mathrm{Cl}$ concentrations require a $\sim 1.4 \mathrm{Myr}$ terrestrial age. Alternatively, this concentration of ${ }^{36} \mathrm{Cl}$ can be produced on the Earth if Lovina were exposed at the surface in Indonesia for $\sim 0.1 \mathrm{Myr}$. Although the observed ${ }^{10} \mathrm{Be}$ is slightly higher than would be expected with a $0.1 \mathrm{Myr}$ terrestrial exposure, the ${ }^{10} \mathrm{Be}$ could be elevated from exposure of Lovina to terrestrial contamination. Although we etched the sample, the concentration of ${ }^{10} \mathrm{Be}, \sim 5 \times 10^{5}$ atom $\mathrm{g}^{-1}$, could be absorbed from rain or seawater. It is perhaps noteworthy that Lovina has many pits in its surface. Tektites from Southeast Asia contain more than 2 orders of magnitude higher ${ }^{10} \mathrm{Be}$ than found in Lovina. Based on cosmogenic radionuclide concentrations, it is our opinion that Lovina is unlikely to be of extraterrestrial origin. We will remeasure the cosmogenic radionuclides, as well as noble gases in a larger piece to verify these conclusions.

Acknowledgments: We thank Daryl Pitt of Macovich Collection for providing sample.

References: [1] Meteoritical Bulletin, No. 93, 2008 March. [2] Flemming R. L. et al. 2008. Abstract \#2412. 39th Lunar and Planetary Science Conference. 
5361

\section{X-RAY ABSORPTION NEAR-EDGE SPECTROSCOPY OF SUPERNOVA CARBONACEOUS GRAINS}

L. R. Nittler ${ }^{1}$, E. Groopman ${ }^{2}$, T. J. Bernatowicz ${ }^{2}$, T. K. Croat ${ }^{2}$ and E. K. Zinner $^{2}$. ${ }^{1}$ Department of Terrestrial Magnetism, Carnegie Institution of Washington, Washington, DC 20015, USA. E-mail: Inittler@ciw.edu. ${ }^{2}$ Laboratory for Space Sciences and Department of Physics, Washington University, St. Louis, MO 63130, USA.

Introduction: Transmission electron microscopy and Raman spectroscopy have been previously used with great success to characterize presolar carbonaceous grains and infer useful astrophysical information about their formation conditions [1-3]. Here, we report the first application of synchrotron transmission X-ray microscopic (STXM) techniques to meteoritic graphitic grains with isotopic compositions indicating a presolar formation in type II supernovae.

Samples and Methods: We analyzed ultramicrotome slices of two presolar graphites: KE3-e6, from Murchison [2], and OR1d5m-G15 (ORG15), from Orgueil. STXM measurements were performed at beamline 5.3.2.2 of the Advanced Light Source (Lawrence Berkeley National Lab). X-ray absorption near-edge spectroscopic (XANES) analyses were made in "image stack" mode, wherein the sample is moved in a raster pattern under the $<50 \mathrm{~nm}$ X-ray beam and a complete absorption spectrum is acquired at each pixel. XANES stacks were acquired at the $\mathrm{C}, \mathrm{N}$, and $\mathrm{O} \mathrm{K}$-edges and the $\mathrm{Ti}_{2,3}$ edge.

Results and Discussion: Isotopic analyses of the two graphite grains indicate a SN origin, e.g., ${ }^{18} \mathrm{O},{ }^{15} \mathrm{~N}$, and ${ }^{28} \mathrm{Si}$ excesses. TEM analysis of the analyzed slice of KE3-e6 indicates a turbostratic graphite morphology and several Ti-rich subgrains. The C-edge XANES spectrum for this grain shows typical graphite features at 285.2 and approximately $291 \mathrm{eV}$, due to $1 \mathrm{~s} \pi^{*}$ and $\sigma^{*}$ transitions, respectively, as well as a small feature at $288.6 \mathrm{eV}$. This is probably due to $\mathrm{C}=\mathrm{O}$ bonding (also seen in the $\mathrm{O} \mathrm{K}$ edge spectrum), suggesting that the observed $\mathrm{O}$ is bonded to the graphene layers as a lactone. N-XANES spectra reveal significant $\mathrm{N}$ contents and an absorption feature at approximately $401 \mathrm{eV}$, possibly indicating amidetype bonding. Ti- and O-XANES spectra of five Ti-rich sub-grains indicate that one is most likely $\mathrm{TiO}_{2}$, and the rest $\mathrm{TiC}$. However, the spectra suggest the presence of some $\mathrm{O}$ (and $\mathrm{N}$ ) even in the TiC grains, as previously inferred from SIMS measurements of other supernova grains [4]. The STXM data for grain ORG15 indicate a "cauliflower-like" morphology and a C-XANES spectrum slightly different from that of KE3-e6. Rather than a single sharp $1 \mathrm{~s} \pi^{*}$ feature at $285.2 \mathrm{eV}$, ORG15 shows features at 284.8, 285.4 and to a lesser extent at $287.5 \mathrm{eV}$. Most likely, this grain consists of a composite of graphitic $\mathrm{C}$ with some smaller, well-organized PAH-like material. In contrast, the N-edge spectrum for ORG15 is similar to that of KE3-e6. Our preliminary results demonstrate the utility of XANES analysis of graphitic presolar grains. In future studies, we will probe the chemistry of Fe-rich sub-grains and the $\mathrm{C}$-edge structure of grains for which Raman spectra indicate a strongly disordered nature [3].

Acknowledgments: We thank David Kilcoyne for help with the STXM and George Cody for useful discussions.

References: [1] Bernatowicz T. J. et al. 1996. The Astrophysical Journal 472:760-782. [2] Croat T. K. et al. 2003. Geochimica et Cosmochimica Acta 67:4705-4725. [3] Zinner E. et al. 1995. Meteoritics 30:209-226. [4] Stadermann F. J. et al. 2005. Geochimica et Cosmochimica Acta 69:177-188.
5117

FIRST DIRECT EVIDENCE OF ASTEROIDAL SPACE WEATHERING FOUND ON THE SURFACE OF ITOKAWA PARTICLES

T. Noguchi ${ }^{1}$, T. Nakamura ${ }^{2}$, M. Kimura ${ }^{1}$, M. E. Zolensky ${ }^{3}$, M. Tanaka ${ }^{4}$, T. Hashimoto ${ }^{5}$, M. Konno ${ }^{5}$, A. Nakato ${ }^{2}$, T. Ogami ${ }^{2}$, A. Fujimura ${ }^{6}$, M.

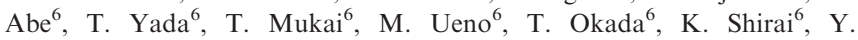
Ishibashi $^{6}$ and R. Okazaki ${ }^{7}$. ${ }^{1}$ Ibaraki University. E-mail: tngc@mx. ibaraki.ac.jp. ${ }^{2}$ Tohoku University. ${ }^{3}$ NASA/JSC. ${ }^{4}$ National Institute for Materials Science. ${ }^{5}$ Hitachi High-Technologies Corporation. ${ }^{6}$ ISAS/ JAXA. ${ }^{7}$ Kyushu University.

Introduction: Surfaces of airless bodies exposed to interplanetary space gradually have their structures, optical properties, chemical compositions, and mineralogy changed by solar wind implantation and sputtering, irradiation of galactic and solar rays, and micrometeorite bombardment. These alteration processes and the resultant optical changes are called "space weathering" [1-3]. It has been already proved by transmission electron microscopy (TEM) that metallic iron nanoparticles deposited on the surfaces of lunar soils result from space weathering [4]. TEM observation suggested that both micrometeorite bombardment and solar wind implantation and sputtering played important roles in the formation of the nanoparticles. Asteroidal space weathering has been advocated based on ground-based and in situ observation of asteroids [5-7]. Initial investigation of fine-grained particles retrieved from the asteroid Itokawa by the Hayabusa spacecraft revealed that the S-type asteroid Itokawa is surfaced by space weathered LL chondrite-like materials [8]. Here we describe the surface modification of the Itokawa particles.

Materials and Methods: We analyzed 10 Itokawa particles. We prepared ultrathin sections of the particles. Four particles were prepared in a $\mathrm{N}_{2}$ purged environment and six were in the earth's atmosphere. In both cases, we used dehydrated ethylene glycol instead of distilled water as the trough liquid to prevent contact with water. Ultrathin sections were observed by spherical aberration corrected scanning TEM.

Results and Discussion: Five out of 10 particles we examined have 30-60-nm-thick rims containing Fe-enriched nanoparticles often accompanied with minor sulfur. Sulfur-bearing Fe-rich nanoparticles exist on the top of the surface ( 10-nm thick). Except for the very surface, materials that contain the nanoparticles considerably preserve the crystal structures of their substrate minerals. This fact shows a stark contrast to the amorphous nature of the host of the metallic Fe nanoparticle-bearing rims in lunar soils [4]. Lattice fringes of most nanoparticles were difficult to observe, with a few exceptions. Textures and chemical compositions of the nanoparticle-bearing rims are dependent on the substrate minerals. We think that the main agent of space weathering of Itokawa is sputtering by solar wind rather than micrometeorite bombardment.

References: [1] Hapke B. 2001. Journal of Geophysical Research 106:10039-10073. [2] Clark B. E. et al. 2002. Asteroids III. Tucson: University of Arizona Press. [3] Chapman C. R. 2004. Annual Review of Earth and Planetary Science 32:539-567. [4] Keller L. P. and McKay D. S. 1997. Geochimica et Cosmochimica Acta 61:2331-2341. [5] Binzel R. P. et al. 2002. Asteroids III. Tucson: The University of Arizona Press. [6] Clark et al. 2001. Meteoritics \& Planetary Science 36:1617-1637. [7] Hiroi T. et al. 2007. Nature 443:56-58. [8] Kerr R. A. Science 332:302. 


\section{2}

\section{HYDROTHERMAL ALTERATION EXPERIMENTS OF AMORPHOUS SILICATES: EFFECT OF ORGANIC-INORGANIC INTERACTION}

R. Noguchi ${ }^{1}$, A. Tsuichiyama ${ }^{1}$, H. Yabuta ${ }^{1}$ and S. Ichikawa ${ }^{2} . \quad{ }^{1}$ Department of Earth Space Science, Osaka University, Osaka, Japan. E-mail: noguchi@astroboy.ess.sci.osaka-u.ac.jp. ${ }^{2}$ Institute for Nano Science Design, Osaka University, Osaka, Japan.

Introduction: Most carbonaceous chondrites with primitive chemical compositions have experienced aqueous alteration in their parent bodies. In order to understand the formation of minerals during the aqueous alteration process, we have previously carried out hydrothermal alteration experiments using $\mathrm{Fe}$-free amorphous silicates with $\mathrm{CI}$ chondritic composition [1]. The previous study showed that saponite, serpentine, calcite, and a relatively small amount of aragonite are formed from amorphous silicates and deionized water. This study focuses on the organic-inorganic interaction upon the aqueous alteration process of chondrites, since carbonaceous chondrites contain large amounts of organic compounds (approximately $2 \%$ ) [2] that could have been involved to formation of some minerals, such as carbonates.

Experiments: The glass of the system, $\mathrm{SiO}_{2}-\mathrm{MgO}-\mathrm{Al}_{2} \mathrm{O}_{3}-\mathrm{CaO}$ $\mathrm{Na}_{2} \mathrm{O}$, with the CI chondrite chemical composition was used. The glass sample was ground to make micron-scale powders. Two kinds of amino acids, glycine and alanine, were used as the analog of whole organic matter in the carbonaceous chondrites. The ratio of alanine to glycine is 2.56:1.00, based on that in the GRA 95229 which is weakly altered CR chondrite [3]. Silicates $(100 \mathrm{mg})$, amino acids $(23 \mathrm{mg})$ and deionized water ( 0.1 or $0.5 \mathrm{~mL}$ ) were placed in Teflon vessels so that water to rock mass ratios were 1.0 and 5.0, sealed with stainless jacket, and were heated in an electric furnace at $150^{\circ} \mathrm{C}$ for $1,2,4$, and 8 weeks under water saturated vapor pressures (approximately 3.0 bars). After heating experiments, vessels were rapidly cooled to room temperature in water. Solid products were recovered from solutions and dried in vacuum at room temperature. Parts (approximately $80 \mathrm{mg}$ ) of solid materials were extracted with hot Milli-Q water for $24 \mathrm{~h}$. The water extracts were analyzed with highperformance liquid chromatography (HPLC) to quantify the concentrations of amino acids. Solid products were analyzed with powder X-ray diffractmeter (XRD) and field emission scanning electron microscopy (FE-SEM) to identify the formed mineral pahse.

Results and Discussion: The XRD analysis and the FE-SEM observations of all the samples obtained from the hydrothermal experiments showed that serpentine and saponite, which are major phase in the chondrites, were formed. Aragonite, minor phase in CM chondrite, was also formed in all the experiments. However, calcite was not formed. The concentrations of alanine and glycine decreased with increasing time of heating, which is consistent with the trend of amino acid concentrations in aqueous-altered carbonaceous chondrites. On the other hand, the ratio of alanine to glycine decreased with increasing time of heating. This is a reverse trend to that of carbonaceous chondrites.

References: [1] Noguchi R. et al. 2011. Abstract \#1789. 42nd Lunar and Planetary Science Conference. [2] Pizzarello S. et al. 2006. Meteorites and the early solar system II:625-651. [3] Martins Z. et al. 2007. Meteoritics \& Planetary Science 42:2125-2136.
5438

OXYGEN ISOTOPIC ANALYSES OF WATER IN BJURBÖLE MATRIX AND CHONDRULES

M. Nunn and M. Thiemens. Department of Chemistry and Biochemistry, University of California, San Diego, La Jolla CA, USA. E-mail: mnunn@ucsd.edu.

Introduction: Past oxygen isotopic analyses of Bjurböle have been limited to whole rock and chondrule studies. We present here the first oxygen isotopic measurements of water contained in the matrix and chondrules of the L4 equilibrated ordinary chondrite Bjurböle.

Method: Water was extracted by vacuum pyrolysis from a $1 \mathrm{~g}$ sample of matrix and a $1.6 \mathrm{~g}$ sample of chondrules from the Bjurböle meteorite. A new, low volume, ultra low blank system was built specifically for these measurements. Each fraction was pumped overnight on a vacuum line to remove adsorbed terrestrial water before heating step-wise to $150,350,600$, and $1000{ }^{\circ} \mathrm{C}$. While heating, evaporated volatiles were collected in a liquid nitrogen cold trap. Water was quantitatively converted to molecular oxygen with bromine pentafluoride. Isotopic abundances were measured on a double-collecting isotope ratio mass spectrometer.

Results and Discussion: Direct fluorination of Bjurböle chondrules and whole rock and UV laser probe analyses of individual Bjurböle chondrules have shown the $\Delta^{17} \mathrm{O}$ value to cluster around $1[1,2]$. Compared to these data, water extracted from the Bjurböle matrix and chondrules is isotopically light $\left(\Delta^{17} \mathrm{O} \leq 0.8\right)$, presumably reflecting different equilibration histories of water and oxygen-bearing minerals. Additionally, the proximity of $\Delta^{17} \mathrm{O}$ values of water extracted at lower temperatures to that of terrestrial water indicates low-temperature heating is necessary to remove adsorbed terrestrial water and yield more accurate results.

References: [1] Clayton et al. 1991. Geochimica et Cosmochimica Acta 55:2317. [2] Ash et al. 1997. Abstract \#5282. 60th Annual Meteoritical Society Meeting. 


\section{5}

A QUANTITATIVE, TIME-DEPENDENT MODEL OF OXYGEN ISOTOPES IN THE SOLAR NEBULA: STEP 1

J. A. Nuth ${ }^{1}$, J. A. Paquette ${ }^{2,3}$ A. Farquhar ${ }^{2,4}$ and N. M. Johnson $^{2}$. ${ }^{1}$ Solar System Exploration Division, Code 690, NASA's Goddard Space Flight Center, Greenbelt MD 20771, USA. E-mail: joseph. a.nuth@nasa.gov. ${ }^{2}$ Astrochemistry Laboratory, Code 691, NASA GSFC. ${ }^{3}$ NASA Postdoctoral Fellow. ${ }^{4}$ USRP Student Intern.

Introduction: The remarkable discovery that oxygen isotopes in primitive meteorites were fractionated along a line of slope 1 rather than along the typical slope 0.52 terrestrial fractionation line occurred almost 40 years ago [1]. However, a satisfactory, quantitative explanation for this observation has yet to be found, though many different explanations have been proposed. The first of these explanations proposed that the observed line represented the final product produced by mixing molecular cloud dust with a nucleosynthetic component, rich in ${ }^{16} \mathrm{O}$, possibly resulting from a nearby supernova explosion. Donald Clayton [2] suggested that galactic chemical evolution would gradually change the oxygen isotopic composition of the interstellar grain population by steadily producing ${ }^{16} \mathrm{O}$ in supernovae, then producing the heavier isotopes as secondary products in lower mass stars. Thiemens and collaborators proposed a chemical mechanism that relied on the availability of additional active rotational and vibrational states in otherwise-symmetric molecules, such as $\mathrm{CO}_{2}, \mathrm{O}_{3}$ or $\mathrm{SiO}_{2}$, containing two different oxygen isotopes [3, 4] and a second, photochemical process that suggested that differential photochemical dissociation processes could fractionate oxygen [5]. This second line of research has been pursued by several groups $[6,7]$, though none of the current models is quantitative.

Lightning as the Processing Agent: Based on experiments [8] done at GSFC that produced nonmass-dependently fractionated solid oxides from plasma, we have previously suggested [9] that dust processed via nebular lightning could explain the observed oxygen isotopic distribution in the solar system. Necessary conditions for nebular lightning have been previously discussed [10-12] and we will present preliminary work suggesting that lightning is a major component of protostellar nebulae.

We will also present preliminary results of the time dependent evolution of oxygen isotopes in nebular dust and the largest uncertainties that remain in the model.

References: [1] Clayton R., Grossman L., and Mayeda T. 1973. Science 182:485. [2] Clayton D. 1988. The Astrophysical Journal 334:191195. [3] Thiemens M. H. and Jackson T. 1987. Geophysical Research Letters 6:624. [4] Thiemens M. H. 1996. In Chondrules and the protoplanetary disk, edited by Hewins R. H., Jones R. H., and Scott E. R. D. Boston: Cambridge University Press. pp. 107-118. [5] Thiemens M. H. and Heidenreich J. E. 1983. Science 219:1073. [6] Clayton R. N. 2002. Nature 415:860-861. [7] Lyons J. R. and Young E. D. 2005. Nature 435:317-320. [8] Kimura Y., Nuth J. A., Chakraborty S., and Thiemens M. H. 2007. Meteoritics \& Planetary Science 42:1429-1439. [9] A Simple Mechanism for Fractionating Oxygen Isotopes in the Solar System Joseph A. Nuth III, Annual Meeting of the Meteoritical Society, Nancy France, July 2009. Abstract\#. [10] Gibbard S. G., Levy E. H., and Morfill G. E. 1997. Icarus 130:517-533. [11] Love S. G., Keil K., and Scott E. R. D. 1995. Icarus 115:97-108. [12] Desch S. J., and Cuzzi J. N. 2000. Icarus 143:87-105.

\section{5}

Rb-Sr AND Sm-Nd STUDY OF ASUKA-881394: EVIDENCE OF "LATE" METAMORPHISM

L. E. Nyquist ${ }^{1}$, C.-Y. Shih ${ }^{2}$, Y. Reese ${ }^{3}$ and H. Takeda ${ }^{4}{ }^{1}$ KR, NASA Johnson Space Center, Houston, TX 77058, USA. E-mail: laurence.e.nyquist@nasa.gov. ${ }^{2}$ ESCG Jacobs-Sverdrup, Houston, TX 77058, USA. ${ }^{3}$ Mail Code JE-23, ESCG/Muniz Engineering, Houston, TX, 77058, USA. ${ }^{4}$ Chiba Inst. of Tech., 2-17-1 Tsudanuma, Narashino City, Chiba 275-0016, Japan.

Introduction: The Asuka-881394 achondrite contains fossil ${ }^{26} \mathrm{Al}$ and ${ }^{53} \mathrm{Mn}$ [1-3] and has a ${ }^{207} \mathrm{~Pb} /{ }^{206} \mathrm{~Pb}$ age of $4566.5 \pm 0.2 \mathrm{Ma}$ [3], the oldest for an achondrite. Preliminary results showed initial ${ }^{146} \mathrm{Sm} /{ }^{144} \mathrm{Sm}=(7.4 \pm 1.2) \times 10^{-3}$, indicative of an ancient age, but ${ }^{87} \mathrm{Rb}-{ }^{87} \mathrm{Sr}$ and ${ }^{147} \mathrm{Sm}^{-143} \mathrm{Nd}$ ages of $4370 \pm 60$ and $4490 \pm 20 \mathrm{Ma}$, respectively [1], were younger than expected from the presence of shortlived nuclides. We revisit the Rb-Sr and Sm-Nd chronology of A-881394 in an attempt to establish whether "late" metamorphism led to inconsistency in its apparent ages.

Min-Pet: The granulitic texture of A-881394 led Wadhwa et al. [4] to suggest that it had experienced an extensive thermal event in which it lost $\mathrm{Na}$ and other volatiles, accounting for its very calcic $\mathrm{An}_{98}$ plagioclase. Prior to discovery of its fossil short-lived nuclides, very calcic plagioclase and unusually magnesian pyroxene were considered to be its most important features [2, 4]. It was suggested that $\mathrm{Na}$ may have been lost via catastrophic bombardment into a magma ocean during late-stage accretion, resulting in increased anorthite, magnesian pyroxene, and silica at the expense of albite [2].

Rb-Sr Data: Combined data from investigations in 2001 [1] and 2010 give a "disturbed isochron" corresponding to $4.50 \pm 0.18 \mathrm{Ga}$. Evidence for early crystallization is preserved in low ${ }^{87} \mathrm{Sr} /{ }^{86} \mathrm{Sr}$ of plagioclase separates. ${ }^{87} \mathrm{Rb} /{ }^{86} \mathrm{Sr}$ is lower than for cumulate eucrites $\mathrm{Y}$ 980318/433, and comparable to values for angrites. Initial ${ }^{87} \mathrm{Sr} /{ }^{86} \mathrm{Sr}=0.698992 \pm 19$, within uncertainty of $0.698972 \pm 8$ for angrite LEW 86010 [5], but not clearly resolved from values for eucrites. Handpicked tridymite (2001) and a mineral separate leachate (2010) determine the isochron slope, and when combined with one pyroxene separate and its residue after leaching give an apparent secondary isochron age of $4.0 \pm 0.8 \mathrm{Ga}$.

Sm-Nd Data: New data (2010) give an isochron age of $4.524 \pm 0.063 \mathrm{Ga}$, and all thirteen data from both investigations give $4.511 \pm 0.033 \mathrm{Ga}$. It is likely that the $\mathrm{Sm}-\mathrm{Nd}$ system also has been disturbed. Handpicked tridymite (2001) plus combined leachates (2010) define an apparent age with pyroxenes of $4.4 \pm 0.2 \mathrm{Ga}$. Removing these "outliers" (tridymite and two leachates) leads to a revised age of $4.516 \pm 0.037 \mathrm{Ga}$. Combined data from both investigations give revised initial ${ }^{146} \mathrm{Sm} /{ }^{144} \mathrm{Sm}=(9.1 \pm 1.4) \times 10^{-3}$ and $T_{\mathrm{LEW}}=4.584 \pm 0.023 \mathrm{Ga}$ relative to $4558 \mathrm{Ma}[6]$ and ${ }^{146} \mathrm{Sm} /{ }^{144} \mathrm{Sm}=7.6 \times 10^{-3}$ for LEW 86010 [5].

Conclusion: Disturbed and partially reset ${ }^{87} \mathrm{Rb}-{ }^{87} \mathrm{Sr}$ and ${ }^{147} \mathrm{Sm}-{ }^{143} \mathrm{Nd}$ ages appear to be consistent with disturbance of these isotopic systems by some catastrophic or metamorphic events on the A881394 parent asteroid. These events followed an earlier catastrophic bombardment as envisioned by the Na-loss model leading to $\mathrm{An}_{98}$ plagioclase [2]. The occurrence of such events probably continued to times less than approximately $4.4 \mathrm{Ga}$ ago.

References: [1] Nyquist L. E. et al. 2001. Meteoritics \& Planetary Science 36:A151. [2] Nyquist L. E. et al. 2003. Earth and Planetary Science Letters 214:11-25. [3] Wadhwa M. et al. 2009. Geochimica et Cosmochimica Acta 73:5189-5201. [4] Takeda H. et al. 1997. Antarctic Meteorite Research 10:401-413. [5] Nyquist L. E. et al. 1994. Meteoritics 29:872-885. [6] Lugmair G. W. and Galer S. J. G. 1992. Geochimica et Cosmochimica Acta 56:1673-1694. 


\section{5}

MEASUREMENTS OF ${ }^{26}$ Al IN THE Stardust CHONDRULE FRAGMENT "IRIS"

R. C. Ogliore ${ }^{1}$, A. L. Butterworth ${ }^{2}$, Z. Gainsforth ${ }^{2}$, G. R. Huss ${ }^{1}$, K. Nagashima $^{1}$, J. Stodolna ${ }^{2}$ and A. J. Westphal ${ }^{2}$. ${ }^{1}$ University of Hawai'i at Mānoa, Honolulu, HI, USA. E-mail: ogliore@higp.hawaii.edu. ${ }^{2}$ University of California, Berkeley, CA, USA.

Introduction: The former presence of radioactive ${ }^{26} \mathrm{Al}\left(t_{1 / 2}=\right.$ $0.73 \mathrm{Myr}$ ) has been identified in chondrules that have experienced little metamorphism [e.g., 1]. The lack of radiogenic ${ }^{26} \mathrm{Mg}$ in other chondrules can be interpreted as a disturbance of the ${ }^{26} \mathrm{Al}-{ }^{26} \mathrm{Mg}$ isotope system due to thermal or aqueous alteration, inhomogeneity of ${ }^{26} \mathrm{Al}$ in the solar nebula, or a few Myr time delay between the formation of these objects and the earliest solar system objects, CAIs (from which the canonical ${ }^{26} \mathrm{Al} /{ }^{27} \mathrm{Al} \approx 5 \times 10^{-5}$ is deduced) [1]. Jupiter-family comets appear to be made of a mixture of solar system materials with different histories [2], including chondrule fragments [3]. We report measurements of the ${ }^{26} \mathrm{Al}-{ }^{26} \mathrm{Mg}$ system in a type II chondrule fragment from comet Wild-2, returned to Earth by NASA's Stardust mission.

Methods: We extracted a $23 \times 15 \times 5 \mu \mathrm{m}$ terminal particle from Stardust track C2052,2,74 ("Iris") [4]. This particle has characteristics of a type II chondrule fragment [5]. We identified fragments of oligoclase $\left(\mathrm{An}_{\sim 20}\right)$ on four approximately $100 \mathrm{~nm}$ microtomed slices on a TEM grid. Since the Al-rich phases needed for useful measurements of the ${ }^{26} \mathrm{Al}-{ }^{26} \mathrm{Mg}$ system were surrounded by Mg-rich phases, we covered the potentially interfering neighboring phases with a Pt shield deposited by FIB. We then glued the grid to a bullet, and measured ${ }^{24} \mathrm{Mg},{ }^{25} \mathrm{Mg},{ }^{26} \mathrm{Mg},{ }^{27} \mathrm{Al}$ using the UH Cameca ims-1280 (primary ${ }^{16} \mathrm{O}^{-}$beam at approximately $10 \mathrm{pA}$, approximately $2 \mu \mathrm{m}$ spot size, simultaneous measurement of $\mathrm{Mg}$ isotopes followed by peak-jumping to ${ }^{27} \mathrm{Al}$, approximately 4800 mass-resolving power). We acquired approximately 200 cycles at each spot with $15 \mathrm{~s}$ for the $\mathrm{Mg}$ isotopes and $1 \mathrm{~s}$ for ${ }^{27} \mathrm{Al}$ per cycle, until the sample was nearly consumed. On each slice we measured one or two spots in the Stardust oligoclase. We used Miyakejima anorthite, prepared and mounted similarly to the Stardust sample, as the standard.

Analysis: For each cycle, we assume the dead-time corrected counts of ${ }^{26} \mathrm{Mg}$ in the Stardust chondrule fragment follow a Poisson distribution, with expectation value equal to the sum of two sources: (1) the natural ${ }^{26} \mathrm{Mg}$ abundance (deduced from the plagioclase standard) proportional to the ${ }^{24} \mathrm{Mg}-$ (also, ${ }^{25} \mathrm{Mg}$-) corrected counts for that cycle, and (2) a radiogenic contribution proportional to the corrected, time-interpolated ${ }^{27} \mathrm{Al}$ counts. The constant of proportionality of (2) is the initial ${ }^{26} \mathrm{Al} /{ }^{27} \mathrm{Al}$, and is easily derived by the maximum likelihood method. We determined a robust confidence interval for the initial ${ }^{26} \mathrm{Al} /{ }^{27} \mathrm{Al}$ by using a Monte Carlo simulation that accounts for statistical uncertainty and instrumental mass fractionation.

Results: We found no evidence for extinct ${ }^{26} \mathrm{Al}$ in the Stardust chondrule fragment; the one-sided $2 \sigma$ upper bound for initial ${ }^{26} \mathrm{Al} /{ }^{27} \mathrm{Al}$ is approximately $3 \times 10^{-6}$. The Stardust fragment either formed or was heated in an asteroidal parent body at least $3 \mathrm{Myr}$ after CAIs formation. Incorporation into the comet must have occurred after that. Our result constrains the formation of cometary nuclei.

References: [1] Kita N. T. et al. 2005. In Chondrites and the protoplanetary disk, ASP 341:558-587. [2] Brownlee D. E. et al. 2006. Science 314:1711-1716. [3] Joswiak D. J. et al. 2010. Abstract \#2119. 41st Lunar and Planetary Science Conference. [4] Butterworth A. L. et al. 2010. Abstract \#2446. 41st Lunar and Planetary Science Conference. [5] Gainsforth Z. et al. 2011. Meteoritics \& Planetary Science. This conference.
5451

THE STAC FADA "IMPACT EJECTA" LAYER: NOT WHAT IT SEEMS

G. R. Osinski ${ }^{1}$, L. Preston ${ }^{1}$, L. Ferrière ${ }^{1,2}$, T. Prave $^{3}$, J. Parnell ${ }^{4}$, A. Singleton $^{1}$ and A. E. Pickersgill ${ }^{1}$. ${ }^{1}$ Department Earth Sciences, University of Western Ontario, London, ON, Canada. E-mail: gosinski@uwo.ca. ${ }^{2}$ Natural History Museum, Burgring 7, A-1010 Vienna, Austria. ${ }^{3}$ Department Earth Sciences, University of St. Andrews, St. Andrews, UK. ${ }^{4}$ Department Geology \& Petroleum Geology, University of Aberdeen, Aberdeen, UK.

Introduction: The Stac Fada Member (SFM) forms part of the Stoer Group of the Torridonian of NW Scotland. The SFM is unique in the Torridonian, being characterized by the presence of greenish altered glass clasts. Its origin has been debated for decades with several hypotheses being proposed but all invoking some connection with volcanic activity in the region (e.g., [1]). More recently, Amor et al. [2] suggested that the SFM represents "a chord section through the continuous ejecta blanket surrounding an impact crater." Here, we confirm the presence of shocked material within the SFM and then discuss its origin.

Methods: Fieldwork was carried out in August 2008 and June 2009. We performed optical microscopy on 26 polished thin sections using an optical microscope and a four-axis universal stage. Quantitative analyses and investigation of textures were carried out on a JEOL JXA-8900 L electron microprobe.

Confirmation of Shocked Material within the SFM: The investigated breccia samples display a large heterogeneity of shock effects in quartz grains; including grains with PFs and/or PDFs (mostly 1-2 sets; up to 4 sets per grain). It is notable that some samples contain no shocked quartz grains. The crystallographic orientations of $90 \mathrm{PF}$ and PDF sets in 59 quartz grains were measured by U-stage. A large proportion of the measured PDFs show orientations parallel to $\omega\{\}$ and $\sim 12 \%$ of the measured PDFs are parallel to the $\pi\{\}$ orientation. Amor et al. [2] identified possible PDFs in 25 quartz grains from 9 thin sections. Our observations confirm the presence of PDFs in quartz in the SFM; although several discrepancies are notable.

Impact Origin? We have confirmed that the SFM contains shocked material. However, based on a review of all terrestrial impact ejecta deposits and considerations of impact ejecta emplacement [3] we find several observations and properties of the SFM that are not consistent with it being a "continuous ejecta blanket" as proposed by [2]. Most importantly, it contains an order of magnitude less shocked material than other proximal impact melt-bearing ejecta layers (e.g., "suevite" from the Ries impact structure Germany [4]); and it is very well sorted compared to other impact ejecta deposits. In this latter respect, it shares many similarities to the Onaping Formation of the Sudbury impact structure, Canada, which has been recently reinterpreted as hot impact melt that interacted with seawater [5]. As such, we propose that the SFM is not of primary impact origin but is more akin to volcaniclastic rocks, which represent volcanic materials that have been transported and reworked by wind or water. This has implications for the recognition of impact ejecta deposits and their emplacement in $\mathrm{H}_{2} \mathrm{O}$-rich environments.

Acknowledgments: This work was funded by the Canadian Space Agency CARN Program and NSERC.

References: [1] Young G. M. 2002. Transactions Earth Sciences 93:116. [2] Amor K. et al. 2008. Geology 36:303-306. [3] Osinski G. R. et al. 2011. Earth and Planetary Science Letters, in press. [4] Engelhardt W.v. 1997. Meteoritics \& Planetary Science 32:545-554. [5] Grieve R. A. F. et al. 2010. Meteoritics \& Planetary Science 45:759-782. 
5221

U-Pb and Pb-Pb DATINGS AND D/H RATIOS OF PHOSPHATES IN THE MARTIAN METEORITES

Y. Ota ${ }^{1,2}$, N. Takahata ${ }^{2}$, Y. Sano ${ }^{2}$ and N. Sugiura ${ }^{1}$. ${ }^{1}$ School of Science, University of Tokyo, Tokyo, Japan. E-mail: y_ohta@aori.u-tokyo.ac.jp. ${ }^{2}$ Atmosphere and Ocean Research Institute.

Introduction: There are many studies that reported $\mathrm{U}-\mathrm{Pb}$ and $\mathrm{Pb}-\mathrm{Pb}$ dating in phosphates in the Martian meteorites. The ages of Shergottites are reported about 4 billion years [1] and $200 \mathrm{Myr}$ [2]. These Martian meteorite ages are not precisely determined and this age is very important for the discussion about variation of $\mathrm{D} / \mathrm{H}$ ratio in Mars. Here we show the $\mathrm{U}-\mathrm{Pb}$ and $\mathrm{Pb}-\mathrm{Pb}$ ages and $\mathrm{D} / \mathrm{H}$ ratio in Martian Meteorites at the same phosphate grains.

Samples and Methods: We use the ALH 84001, Zagami, DaG476 and some Martian meteorites. Phosphate minerals in a thin section were detected by SEM-EDS and it was coated with gold films before the NanoSIMS measurement. For $\mathrm{U}-\mathrm{Pb}$ and $\mathrm{Pb}-\mathrm{Pb}$ dating, primary $\mathrm{O}$ - ions with a beam intensity of $10 \mathrm{nA}$ were used in a spot diameter of about 10 $20 \mu \mathrm{m}$, and for $\mathrm{D} / \mathrm{H}$ ratios, with a beam intensity of $1 \mathrm{nA}$ in a spot diameter of about a few micrometers. The apatite derived from Prairie Lake called PRAP whose age was known [3] was used as a standard, and the apatite collected from Emilchil Errachidia in Morroco whose water abundance was measured was used as a standard of $\mathrm{D} / \mathrm{H}$ ratio and water abundance.

Result: The phosphate mineral in ALH 84001 crystallized about 4 billion years ago according to the U-Pb, $\mathrm{Pb}-\mathrm{Pb}$ system. The age obtained in this study is consistent with those of the previous studies [4] within the experimental error. $\mathrm{D} / \mathrm{H}$ ratios in $\mathrm{ALH} 84001$ are up to 3000 approximately $3500 \%$. There is a negative correlation between the $\delta \mathrm{D}$ and amount of water. The U-Pb ages of phosphate minerals in Zagami are almost zero, so it had reset because of some metamorphism. But the $\mathrm{Pb}-\mathrm{Pb}$ isochron age at the same spot of $\mathrm{U}-\mathrm{Pb}$ dating is about 4 Gyr. This age is defined by two grains. $\mathrm{D} / \mathrm{H}$ ratios in Zagami and DaG476 are about 0 approximately $1000 \%$. Our data show Zagami crystallized at $4 \mathrm{Gyr}$ ago by the $\mathrm{Pb}-\mathrm{Pb}$ age, and it has experienced some metamorphism so $\mathrm{U}-\mathrm{Pb}$ age is almost zero.

Discussion: The crystallization ages of phosphates in ALH 84001 and Zagami are probably about $4 \mathrm{Ga}$, but $\mathrm{U}-\mathrm{Pb}$ ages of phosphates in Zagami had reset recently. It seems that $\mathrm{D} / \mathrm{H}$ ratios of phosphates in ALH 84001 keep the value 4 billion years ago and this value is higher than previous studies [5]. However $\mathrm{D} / \mathrm{H}$ ratios of phosphates in Zagami are almost terrestrial values or mixed values with terrestrial and Martian water because of resetting age. There are many data of phosphate in Shergottites that $\mathrm{U}-\mathrm{Pb}$ ages are about $200 \mathrm{Myr}$ and $\mathrm{D} / \mathrm{H}$ ratios are over $4000 \%$ [6]. Our data support the scenario that these Martian meteorites crystallize about $4 \mathrm{Gyr}$ ago, and metamorphose resetting $\mathrm{U}-\mathrm{Pb}$ ages and replacing $\mathrm{D} / \mathrm{H}$ ratio about $200 \mathrm{Myr}$ ago.

References: [1] Bouvier A. et al. 2009. Earth and Planetary Science Letters 280:285-295. [2] Nyquist et al. 1998. Journal of Geophysical Research 103:31445-31455. [3] Sano Y. et al. 1999. Chemical Geology 153:171-179. [4] Terada K. et al. 2003. Meteoritics \& Planetary Science 38:1697-1703. [5] Greenwood et al. 2008. Geophysical Research Letter 35:L05203. [6] Watson et al. 1994. Science 265:86-90.
5012

COSMOGENIC NOBLE GASES OF THE GEBEL KAMIL IRON METEORITE

U. Ott ${ }^{1}$, S. Merchel ${ }^{2}$, L. Folco ${ }^{3}$ and M. D’Orazio ${ }^{4}$. ${ }^{1}$ MPI für Chemie, Postfach 3060, D-55020 Mainz, Germany. E-mail: uli.ott@mpic.de. ${ }^{2}$ HZDR, D-01328 Dresden, Germany. ${ }^{3}$ MNA, Siena, Italy. ${ }^{4}$ DST Pisa, Italy.

Introduction: The Kamil Crater in southern Egypt was first identified during a Google Earth survey. A closer inspection during a geophysical expedition in February 2010 not only revealed details of its structure but also identified several thousand iron meteorite specimens with a total weight of $\sim 1.7$ tons $[1,2]$. The meteorite is classified as an ungrouped $\mathrm{Ni}$-rich ataxite [3]. $\mathrm{He}, \mathrm{Ne}$ and $\mathrm{Ar}$ measurements have been performed on samples from the only regmaglypted $83 \mathrm{~kg}$ individual as well as from a piece of the shrapnel produced during the impact $[1,2]$.

Experimental: We measured two samples each from the individual and the shrapnel, separated by a distance of $\sim 1 \mathrm{~cm}$ in each case. Material from in between is being used for accelerator mass spectrometry of longlived cosmogenic radionuclides. Because of instabilities in the gain of the electron multiplier, the noble gas abundances were not determined by the (standard) peak height method, but rather by isotope dilution. For that, about $1 / 3$ of the sample gas was "spiked" with a gas mixture $\left({ }^{3} \mathrm{He} /{ }^{4} \mathrm{He} /{ }^{22} \mathrm{Ne} /{ }^{36} \mathrm{Ar} \sim 1.2 \times 10^{-8} / 1.1 \times 10^{-6} / 1.0 \times 10^{-10} / 3.8 \times 10^{-9} \mathrm{cc}, \mathrm{Ne}\right.$ and $\mathrm{Ar}$ isotopic compositions atmospheric).

Results: As normal for iron meteorites, noble gases are purely spallogenic. Relevant results are listed in Table 1 (concentrations in $10^{-8} \mathrm{cc} \mathrm{g}^{-1}$ units; uncertainties in the last digits in parentheses; $\mathrm{I}=$ individual, $\mathrm{S}=$ shrapnel).

Discussion: Using the model calculations of [4], we can derive bounds on the pre-atmospheric size of the object and can define a possible range of cosmic ray exposure ages. Most useful is the ${ }^{4} \mathrm{He} /{ }^{38} \mathrm{Ar}$ ratio (cf. Fig. 13 in [4]). Based on the maximum ratio of $\sim 90$ (S-C), a minimum radius for the meteoroid is $\sim 85 \mathrm{~cm}$. This implies a preatmospheric mass of $>20,000 \mathrm{~kg}$, in excellent agreement with the estimate of [1]. Furthermore, the shrapnel samples must originate from further inside the meteoroid than the individual $(35-45 \mathrm{~cm}$ vs $15-25 \mathrm{~cm}$ ). To reach agreement between ${ }^{4} \mathrm{He} /{ }^{38} \mathrm{Ar}$ and ${ }^{4} \mathrm{He} /{ }^{21} \mathrm{Ne}$, a sulfur/ phosphorus content in the range $0.4-0.8$ wt $\%$ contributing to ${ }^{21} \mathrm{Ne}$ production is required (4, 5; cf. Fig. 11 in [4]). Conflicting cosmic ray exposure ages, however, follow from the relation between ${ }^{4} \mathrm{He} /{ }^{38} \mathrm{Ar}$ ratio and ${ }^{38} \mathrm{Ar}$ production rate according to [4]. While for the individual an age on the order of $400-500 \mathrm{Ma}$ is indicated, estimates for the shrapnel samples are lower in the 200-300 Ma range. The discrepancy may be solved by the radionuclide measurements, which are in progress.

References: [1] Folco L. et al. 2010. Science 329:804. [2] Folco L. et al. 2011. Geology 39:179-182. [3] Weisberg M. K. et al. 2010. Meteoritics \& Planetary Science 45:1530-1551. [4] Ammon K. et al. 2009. Meteoritics \& Planetary Science 44:485-503; in detail at: http:// noblegas.unibe.ch/index.php?content $=$ noblegas/data. [5] Ammon K. et al. 2008. Meteoritics \& Planetary Science 43:685-699.

\begin{tabular}{lllll}
\hline Sample & ${ }^{3} \mathrm{He}$ & ${ }^{4} \mathrm{He}$ & ${ }^{21} \mathrm{Ne}$ & ${ }^{38} \mathrm{Ar}$ \\
\hline I-C & $147(10)$ & $622(18)$ & $1.59(4)$ & $9.47(29)$ \\
I-g & $138(9)$ & $604(20)$ & $1.58(4)$ & $8.20(34)$ \\
S-C & $44(5)$ & $214(16)$ & $0.42(1)$ & $2.39(10)$ \\
S-f & $41(5)$ & $193(18)$ & $0.35(1)$ & $2.31(11)$ \\
\hline
\end{tabular}


5518

EXTREME CADMIUM AND THALLIUM ISOTOPE FRACTIONATIONS IN ENSTATITE CHONDRITES

C. S. Palk ${ }^{1,2}$, M. Rehkämper ${ }^{1,2}$, R. Andreasen ${ }^{1,2}$ and A. Stunt ${ }^{1} .{ }^{1}$ IARC, Department of Earth Science \& Engineering, Imperial College, London SW7 2AZ, UK. E-mail: c.palk09@imperial.ac.uk. ${ }^{2}$ Natural History Museum, London SW7 5BD, UK.

Introduction: The extinct ${ }^{205} \mathrm{~Pb}^{205} \mathrm{Tl}$ decay system $\left(t_{1 / 2}=15 \mathrm{Myr}\right)$ features two volatile elements and may hence be useful for studying volatile loss in the early solar system. Analyses of the $\mathrm{Pb}-\mathrm{Tl}$ system are best combined with measurements of $\mathrm{Cd}$ isotope compositions to monitor stable isotope fractionations from evaporation and condensation [1, 2]. In this study, we measure $\mathrm{Pb}, \mathrm{Tl}$ and $\mathrm{Cd}$ abundances and isotopic compositions in bulk samples of enstatite chondrites. To date, results have been obtained for Indarch (EH4), Abee (EH4), St. Mark's (EH5), MAC 02839 (EL3), PCA 91020 (EL3), and Khairpur (EL6). Data for additional samples will be available at the meeting.

Results: $\mathrm{The} \mathrm{Pb}, \mathrm{Tl}$ and $\mathrm{Cd}$ abundances of the samples display considerable and systematic variability. In particular, the EL3 and EH4 enstatite chondrites have much higher volatile element contents (of about 40-100 ppb Tl, 300-900 ppb Cd) than the EH5 and EL6 samples, which record more intense thermal metamorphism and feature $\sim 5 \mathrm{ppb} \mathrm{Tl}$ and $\sim 10-20 \mathrm{ppb} \mathrm{Cd}$. The $\mathrm{Pb}$ abundances also show a similar offset between samples of petrologic types 3 and 4 vs. 5 and 6 , but two of the meteorites (Indarch, St. Mark's) have $\mathrm{Pb}$ isotope compositions, which are indicative of pervasive contamination by terrestrial $\mathrm{Pb}$.

The most extreme stable isotope fractionations were found in two EL chondrites (PCA 91020, Khairpur), which are depleted in light Cd isotopes and display $\varepsilon^{114 / 110} \mathrm{Cd}$ values of about +70 (relative to a bulk silicate Earth with $\varepsilon^{114 / 110} \mathrm{Cd} \approx 0$ ). Moderate $\mathrm{Cd}$ stable isotope effects with $\varepsilon^{114 / 110} \mathrm{Cd}$ values of about +8 were furthermore identified for MAC 02839 and St. Mark's. Only Indarch and Abee feature essentially unfractionated $\mathrm{Cd}$, relative to carbonaceous chondrites, with $\varepsilon^{114 / 110} \mathrm{Cd} \approx$ +3. In contrast, only a single sample (PCA 91020) displays a clearly fractionated $\mathrm{Tl}$ isotope composition characterized by $\varepsilon^{205} \mathrm{Tl} \approx+22$, whereas all other meteorites have $\varepsilon^{205} \mathrm{Tl}$ of between -2.9 and +0.8 , similar or identical to the bulk silicate Earth value of $-2.0 \pm 0.5$.

Discussion: The data obtained so far indicate that stable isotope fractionations are commonplace for $\mathrm{Cd}$ in enstatite chondrites. This is in accord with the results of earlier work [3], which found that Cd isotopes are an excellent monitor of parent body processes that effect the abundances of volatile elements. The only meteorite to display both large $\mathrm{Cd}$ and $\mathrm{Tl}$ stable isotope effects is the highly shocked (S5) sample PCA 91020 [4], possibly suggesting that $\mathrm{Tl}$ may be readily mobilized during shock metamorphism. Due to the presence of stable isotope fractionation for $\mathrm{Cd}$ and, at least in some samples, $\mathrm{Tl}$, together with the terrestrial $\mathrm{Pb}$ contamination of two meteorites, it will be challenging to construct a ${ }^{205} \mathrm{~Pb}^{205} \mathrm{Tl}$ parent body isochron for enstatite chondrites.

References: [1] Baker R. G. A. et al. 2010. Earth and Planetary Science Letters 291:39-47. [2] Andreasen R. et al. 2009. Abstract \#A43. Geochimica et Cosmochimica Acta 73:13. [3] Wombacher F. et al. 2008. Geochimica et Cosmochimica Acta 72:646-667. [4] Rubin A. E. et al. 1997. Geochimica et Cosmochimica Acta 61:847-858.
5302

FE/MG COMPLEMENTARITY IN CHONDRULES AND MATRIX OF CARBONACEOUS CHONDRITES

H. Palme ${ }^{1}$ and D.C. Hezel ${ }^{2}{ }^{1}$ Forschungsinstitut und Naturmuseum Senckenberg, Senckenberganlage 25, D-60325 Frankfurt am Main, Germany. E-mail: herbert.palme@uni-koeln.de. ${ }^{2}$ Institut für Geologie und Mineralogie, Universität zu Köln, Zülpicherstrasse 49b, D-50674 Köln.

Introduction: We have recently shown that matrices of carbonaceous chondrites (CC) are consistently high in $\mathrm{SiO}_{2}$ and low in $\mathrm{MgO}$ while chondrules have the opposite signature [1]. The bulk CI-ratio of CC requires the formation of both components from a single reservoir [1, 2]. The distribution of $\mathrm{Mg}$ and $\mathrm{Si}$ between chondrules and matrix must have been established in the solar nebula. We show here that the same reasoning applies to $\mathrm{Fe}$.

We have complemented recent chemical data by INAA on Mokoia (CV3) chondrules [2] by a large number of new matrix analyses. Fig. 1 indicates high $\mathrm{Fe}$ and low $\mathrm{Mg}$ for matrix and low $\mathrm{Fe}$ and high $\mathrm{Mg}$ for chondrules. Metal is absent in Mokoia. CC bulk Fe and Mg contents [3] plot between chondrules and matrix, although $\mathrm{Fe}$ is slightly variable, which is attributed to bulk reservoir fractionation [4]. Overall there is a clear complementary relationship between chondrules and matrix. Both thermal metamorphism and aqueous alteration would transport $\mathrm{Fe}$ from matrix into chondrules and thus erase the differences. Despite significant aqueous alteration of the Mokoia matrix the chondrules were not or only slightly affected [3].

A compilation of CC matrix data by [5] shows the same spread in matrix $\mathrm{Fe}$ and $\mathrm{Mg}$ as in Fig. 1. Chondrules should thus have a similar compositional range in $\mathrm{Fe}$ and $\mathrm{Mg}$ as in Fig. 1.

This suggests forsteritic olivine as a major chondrule component in CC. Since $\mathrm{Fe}, \mathrm{Ni}$ and $\mathrm{Co}$ occur in chondritic ratios in Mokoia and Allende chondrules $[3,6,7] \mathrm{Fe}$ must have entered chondrules as $\mathrm{Fe} \mathrm{Ni-}$ metal and was then oxidized during chondrule melting.

References: [1] Hezel D. C. and Palme H. 2010. Earth and Planetary Science Letters 280:85-93. [2] Hezel D. C. and Palme H. 2008. Earth and Planetary Science Letters 265:716-725. [3] Jones R. H. and Schilk A. J. 2009. Geochimica et Cosmochimica Acta 73:5854-5883. [4] Wolf D. and Palme H. 2001. Meteoritics \& Planetary Science 36: 559-571. [4] Palme H. and Lodders K. 2009. Abstract \#5243. Annual Meteoritical Society Meeting. [5] Huss G. R. et al. 2005. In: Chondrites and the protoplanetary disk (ASP Conference series, vol. 341), pp. 701-731. [6] Rubin A. E. and Wasson J. T. 1982. Geochimica et Cosmochimica Acta 51:1923-1937. [7] Palme H. and Spettel B. Unpublished data.

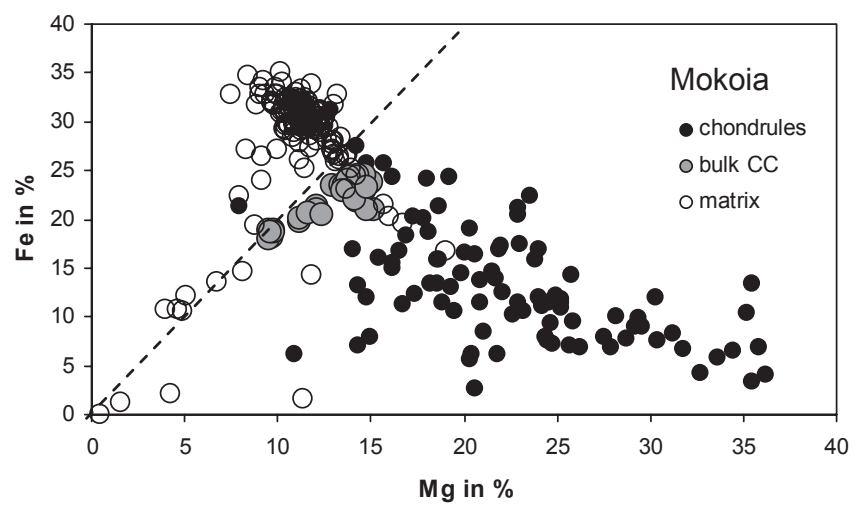

Fig. 1. Fe vs. $\mathrm{Mg}$ in matrix and chondrules of Mokoia. 
5164

DISK DISPERSAL AND THE FORMATION OF PLANETARY SYSTEMS

I. Pascucci. Lunar and Planetary Laboratory, The University of Arizona, AZ, USA. E-mail: pascucci@lpl.arizona.edu.

Gas-rich dust disks around young stars (hereafter, protoplanetary disks) provide the raw material to build up planets. Thus, the time scale over which they disperse and the physical mechanisms contributing to their dispersal are key in understanding what type of planets can form and on what time scale. Significant progress has been made in the past few years in measuring the dispersal time scale of protoplanetary disks. Surveys of nearby star-forming regions and associations have established that by age $10 \mathrm{Myr}$ only a few percent of premain sequence sun-like stars still retain a gas-rich dust disk [1-3]. Still much debate exists on the disk dispersal mechanisms and on their efficiency.

In this talk, I will discuss the theoretical mechanisms proposed to explain the dispersal of protoplanetary disks with special emphasis on viscous accretion, planet formation, and star-driven photoevaporation. I will seek constraints from observations of protoplanetary disks and from the Solar System to evaluate the role of these mechanisms in dispersing primordial gas and dust. I will also present newly discovered gas lines that can be used to trace star-driven photoevaporative flows and discuss how to measure the rate at which disks lose their mass via photoevaporation [4-6]. Finally, I will show how these disk dispersal mechanisms impact the final architecture of planetary systems.

References: [1] Pascucci I. et al. 2006. The Astrophysical Journal 651:1177. [2] Hernandez J. et al. 2008. The Astrophysical Journal 686:1195. [3] Pascucci I. and Tachibana S. 2010. In Protoplanetary dust: Astrophysical and cosmochemical perspectives, edited by Apai D. and Lauretta D. Cambridge University Press. pp. 263-298. [4] Pascucci I. and Sterzik M. 2009. The Astrophysical Journal 702:724. [5] Pascucci I. et al. 2011. The Astrophysical Journal, in press (arXiv:1105.0045). [6] Sacco G., Flaccomio E., and Pascucci I. et al. In preparation.
5174

FIRST REPORT OF SUEVITE FROM DHALA IMPACT STRUCTURE, INDIA

J. K. Pati. Department of Earth and Planetary Sciences, Nehru Science Centre, University of Allahabad, Allahabad-211 002, India. E-mail: jkpati@yahoo.co.in.

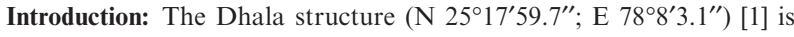
a confirmed impact structure [2] of Paleoproterozoic age [3] in the western part of the Archean Bundelkhand craton, India. The target rocks $(2.5 \mathrm{Ga}$ Archean Bundelkhand granitoids [3]) are covered by a thick $(5-150 \mathrm{~m})$ melt breccia sheet. This is, in turn, overlain by postimpact sediments (Dhala Group [4]) of unknown age followed by the Vindhyan Supergroup of rocks of Proterozoic age. In addition to impact melt breccia, the two other breccia types observed earlier include monomict granitoid breccia and another type of apparently lithic breccia.

Dhala Suevite: This latter, dark greenish gray to greenish brown, apparently lithic breccia contains altered microscopic melt clasts in a matrix dominated by clastic material. It occurs above the impact melt breccia with a gradational contact to the footwall. The sediments belonging to the Dhala Group occur with a sharp unconformable/ erosional (?) contact above lithic breccia. The thickness of the melt clastbearing breccia unit is about $1.5 \mathrm{~m}$ in the central elevated area of the impact structure. This rock comprises lithic clasts $(20-30 \mathrm{vol} \%)$, mineral clasts $(20-40 \mathrm{vol} \%)$, and melt clasts (1-5 vol\%), besides martix, with a general clast to matrix ratio of 40:60. Sizes of mineral and lithic clasts range from $<1$ to $60 \mathrm{~mm}$. The mineral clasts are angular to subrounded, whereas the lithic clasts are angular to subangular. The mineral clasts include quartz, feldspar, biotite, apatite, zircon, and opaques. The dominant shock features observed in quartz and feldspar grains are planar deformation features (PDF): one to four sets of PDF in quartz clasts and one or two sets of PDF in feldspar grains. Zircon grains (up to $100 \mu \mathrm{m}$ in size) show fractures cross-cutting the oscillatory zoning. A few zircon grains show granular texture. The melt clasts are pale to dark green, pale to dark brown, dark gray and reddish brown. They vary in size from $<5 \mathrm{~mm}$ to $>40 \mathrm{~mm}$, and in shape from circular, elliptical, bomb-like, globular, spindle-shape, and fishing-hook to barrel shapes. They are only partially devitrified and remain largely isotropic. Some of the reddish brown melt clasts contain unmelted mineral and lithic clasts that are mostly rounded in shape and show reaction rims with the surrounding melt with microlites. These microclasts show deformation in the form of fractures, bent twin lamelle and shock deformation features (like PDFs). The pale brown colored melt clasts contain randomly distributed and oriented pale green microlites. Many melt clasts show fluidal texture.

Conclusion: This is the first report of a melt clast-bearing breccia containing diagnostic shock metamorphic evidence from the Dhala structure, India. The characteristics of this breccia define it as suevite. The oldest suevite $(1.85 \mathrm{Ga})$ previously reported derives from the Sudbury structure, Canada [5]. Once the age of the Dhala impact event will be better constrained, the associated suevite could possibly become the oldest occurrence of this important type of impact breccia.

Acknowledgments: J. K. P. thanks Uwe Reimold, Germany, for his continued help and support. PLANEX is also thanked for their research support.

References: [1] Pati J. K. 2005. Meteoritics \& Planetary Science 40:A121. [2] Pati J. K. et al. 2008. Meteoritics \& Planetary Science 43:13831398. [3] Pati J. K. et al. 2010. Geological Society of America Special Paper 465. pp. 571-591. [4] Jain S. C. et al. 2001. Geological Survey of India Special Publication 64:289-297. [5] French B. M. 1998. Lunar and Planetary Institute, Houston, TX, Contribution CB-954. 120 p. 
5476

IN SITU SYNCHROTRON X-RAY TRANSMISSION MICROSCOPY OF CR CHONDRITES QUE 99177 AND GRA 95229

Z. Peeters ${ }^{1}$, C. M. O'D. Alexander ${ }^{1}$, H. Changela ${ }^{2}$, L. R. Nittler ${ }^{1}$, S. Price $^{1}$ and R. M. Stroud ${ }^{2}$. ${ }^{1}$ Carnegie Institution of Washington, Washington, DC 20015, USA. E-mail: zpeeters@dtm.ciw.edu. ${ }^{2}$ Naval Research Laboratory, Code 6s366, 4555 Overlook Ave. SW, Washington, DC 20375-532, USA.

Introduction: As part of a coordinated, multiple-analysis approach to understanding the structure and origin of the insoluble organic matter (IOM) in primitive meteorites, we have performed X-ray absorption nearedge spectroscopy (XANES) of matrix materials from two CR chondrites, QUE 99177 and GRA 95229. QUE 99177 is a very primitive CR3 chondrite with little evidence for parent-body alteration [1], whereas GRA 95229 is a more aqueous altered CR2. Previous nanoSIMS isotopic mapping of sections of QUE 99177 [2,3] revealed carbon to be largely distributed throughout the matrix as sub-?m sized particles with a wide range of $\mathrm{H}, \mathrm{C}$ and $\mathrm{N}$ isotopic compositions. However, during mapping, an unusually large $(8.4 \times 2.8 \mu \mathrm{m})$ carbon-rich vein was discovered. The vein was targeted for focussed ion-beam (FIB) lift-out with the Carnegie Zeiss Auriga FIB-SEM. For comparison, FIB lift-out sections were also extracted from a different (random) part of the QUE 99177 matrix and from a polished section of GRA95229. The FIB sections were taken to the scanning transmission X-ray microscope (STXM) on beamline 5.3.2.2 at the Advanced Light Source in Berkeley for XANES mapping.

Results and Discussion: The carbon-rich vein in QUE 99177, as measured by nanoSIMS from the top of the inclusion (i.e., before lift-out) was enriched in $\mathrm{D}(\delta \mathrm{D}=+6000 \%)$ and in ${ }^{15} \mathrm{~N}\left(\delta^{15} \mathrm{~N}=+500 \%\right)$, significantly higher than the bulk IOM composition for this meteorite $\left(\delta \mathrm{D}=+3139 \%, \delta^{15} \mathrm{~N}=+187 \%\right.$. [4] $)$. The FIB section showed that the carbon-rich vein extended $5.5 \mu \mathrm{m}$ into the meteorite. C-edge XANES mapping of the carbon-rich vein revealed three bands (aromatic or vinylic carbon bonds, carbonyl bonds, and carboxyl bonds) commonly seen in chondritic IOM, plus carbonate. Compared to other carbon in the FIB sections of QUE 99177 and GRA 95229, the C-XANES spectrum of the C-rich vein appears slightly different, both in the positions and ratios of the observed absorption features. Similarly, the N-edge XANES spectra of the vein differs from the other studied materials in showing significantly more absorption due to imidyl and nitrile bonds. Planned TEM and nanoSIMS isotopic analysis of the FIB sections will allow detailed sub-?m correlation of chemical, mineralogical and isotopic data to better understand the nature of the organic matter and its relation to inorganic matrix materials.

References: [1] Abreu N. M. and Brearley A. J. 2010. Geochimica et Cosmochimica Acta 74:1146-1171. [2] Nguyen A. N. et al. 2008. Meteoritics \& Planetary Science 43:A5277. [3] Floss C. and Stadermann F. J. 2009. The Astrophysical Journal 697:1242-1255. [4] Alexander C. M. O’D. et al. 2007. Geochimica et Cosmochimica Acta 71:4380-4403.
5353

ABUNDANCES OF RADIOACTIVE ELEMENTS ON THE SURFACE OF MERCURY: FIRST RESULTS FROM THE MESSENGER GAMMA-RAY SPECTROMETER

P. N. Peplowski ${ }^{1}$, D. K. Hamara ${ }^{2}$, D. J. Lawrence ${ }^{1}$, T. J. McCoy ${ }^{3}$, W. V. Boynton $^{2}$, L. G. Evans ${ }^{4}$, E. A. Rhodes ${ }^{1}$, S. C. Solomon ${ }^{5}$, L. R. Nittler ${ }^{5}$ and A. L. Sprague ${ }^{2}$. ${ }^{1}$ Johns Hopkins University, Applied Physics Laboratory, Laurel, MD 20723, USA. ${ }^{2}$ University of Arizona, Tucson, AZ 85721, USA. ${ }^{3}$ Smithsonian Institution, Washington, DC 20560-0119, USA. ${ }^{4}$ Computer Sciences Corporation, Lanham-Seabrook, MD 20706, USA. ${ }^{5}$ Carnegie Institution of Washington, Washington, DC 20015, USA.

Introduction: On 18 March 2011, MESSENGER became the first spacecraft to enter orbit around Mercury, inaugurating a one-year campaign of orbital operations [1]. This investigation includes mapping the surface composition from orbit using the MESSENGER Gamma-Ray Spectrometer (GRS) [2]. Data from the GRS can be used to determine the surface composition to depths of tens of centimeters by measuring gamma rays originating from both radioactive elements $(\mathrm{K}$, Th, and $\mathrm{U})$ and excitation of stable elements (e.g., $\mathrm{Si}, \mathrm{Fe}, \mathrm{Ti}, \mathrm{O}$ ) by interactions with incident galactic cosmic rays. This work details the measurement of $\mathrm{K}$ and Th abundances on the surface, the results of which have important implications for our understanding of the formation and thermal history of Mercury [3].

Preliminary Results: GRS data acquired during the first month of orbital operations have been used to determine the average abundances of $\mathrm{K}(1078 \pm 49 \mathrm{ppm})$ and $\mathrm{Th}(0.173 \pm 0.088 \mathrm{ppm})$ in the northern hemisphere of Mercury. The $\mathrm{K} / \mathrm{Th}$ ratio in this region is $6230 \pm 3180$, which is within the range of values for the other terrestrial planets but is significantly higher than the average lunar value of 360 [4].

Discussion: Ratios of the volatile incompatible $\mathrm{K}$ to the refractory incompatible Th provide insights into volatile element depletions in planetary bodies. The $\mathrm{K} / \mathrm{Th}$ ratio for the surface of Mercury is similar to that for Mars (4000-7000 over $95 \%$ of the surface [5]). The $\mathrm{K} / \mathrm{Th}$ ratio for Mercury is also similar to that for Martian meteorites. Absolute surface abundances for Mercury are a factor of three to four lower than for Mars, however, probably reflecting the enhancement of $\mathrm{K}$ and Th abundances at the Martian surface by aqueous processes. The $\mathrm{K} / \mathrm{Th}$ ratio of Mercury, particularly when coupled with the high S abundance of Mercury [6], suggests that evaporation or refractory condensation models [3] for the formation of Mercury are not viable. These models should produce volatile-poor compositions, counter to the observations presented here. Other models are less constrained by these data, although chondritic melting models appear to predict higher Th abundances (approximately $1 \mathrm{ppm}$ ) [4] than observed. Future determination of $U$ abundances from GRS observations should provide additional constraints on these models.

References: [1] Solomon S. C. et al. 2007. Space Science Review 131:3-39. [2] Goldsten J. O. et al. 2007. Space Science Review 131:339391. [3] Taylor G. J. and Scott E. R. D. 2003. Treatise on Geochemistry, vol. 1, pp. 477-485. [4] Taylor G. J. et al. 2006. Journal of Geophysical Research 111, E03S06-E03S10. [5] Taylor G. J. et al. 2006. Journal of Geophysical Research 111, E03S06. [6] Weider S. Z. et al. This conference. 
5306

\section{A COMPARATIVE STUDY OF TROILITE IN VARIOUS METEORITES USING MÖSSBAUER SPECTROSCOPY WITH A HIGH VELOCITY RESOLUTION}

E. V. Petrova ${ }^{1}$, V. I. Grokhovsky ${ }^{1}$, M. I. Oshtrakh ${ }^{1}$, R. N. Kolunin ${ }^{1}$ and V. A. Semionkin ${ }^{1,2}$. ${ }^{1}$ Faculty of Physical Techniques and Devices for Quality Control, Ural Federal University, Ekaterinburg, 620002, Russian Federation. E-mail: grokh47@mail.ru. ${ }^{2}$ Faculty of Experimental Physics, Ural Federal University, Ekaterinburg, 620002, Russian Federation.

Introduction: Troilite $\mathrm{FeS}$ is the main sulfur mineral in the extraterrestrial matter. FeS was found in various meteorites in various forms (inclusions in iron meteorites or component of ordinary chondrites). Therefore, troilite may vary in different meteorites. To study meteoritic troilite Mössbauer spectroscopy with a high velocity resolution was used. This technique is useful for analysis of iron hyperfine parameters with high accuracy.

Experimental: Samples of various ordinary chondrites (Farmington L5, Tsarev L5, Vengerovo H6, Richardton H5, Saratov L4, Zvonkov H6) and FeS extracted from iron meteorite Sikhote-Alin IIAB were prepared as powder. Mössbauer spectra of these samples were measured using automated precision Mössbauer spectrometric system with a high velocity resolution [1] at room temperature in 4096 channels.

Results and Discussion: Mössbauer spectra of ordinary chondrites demonstrated usual complicated pattern with three magnetic sextets related to $\mathrm{Fe}-\mathrm{Ni}$ alloy and $\mathrm{FeS}$ and five quadrupole doublets related to olivine, pyroxene and iron oxide. Mössbauer spectrum of troilite extracted from Sikhote-Alin consists of one magnetic sextet. It was interesting to observe variation of the values of magnetic hyperfine field for troilite in studied meteorites. This variation was in the range of approximately 308 $323 \mathrm{kOe}$. The values of magnetic hyperfine field for troilite in ordinary chondrites may be divided in two groups with small differences of the field outside the instrumental error. The largest value of magnetic hyperfine field for troilite in ordinary chondrites was approximately $314 \mathrm{kOe}$ for Zvonkov H6 while the smallest one was of approximately $308 \mathrm{kOe}$ for Farmington L5. In contrast, the value of magnetic hyperfine field for troilite from Sikhote-Alin meteorite was approximately $323 \mathrm{kOe}$. These differences may be a result of small structural variations and deviations in stoichiometry in troilite from different meteorites. It is possible that small structural and composition variations reflect differences in mineral formation in the studied meteorites. Therefore, accurate Mössbauer hyperfine parameters of troilite may provide additional information for analysis of meteorites formation and evolution.

Acknowledgments: This work was supported in part by the Federal Grant-in-Aid Program "Human Capital for Science and Education in Innovative Russia" (Governmental Contracts No. P1154 and No 14.740.11.1006).

Reference: [1] Semionkin V. A. et al. 2010. Bulletin of the Russian Academy of Sciences: Physics 74:416-420.
5150

IN SITU NANOSIMS MEASUREMENT OF D/H RATIO AND H EMISSION IN MATRIX ANALOGUES

L. Piani ${ }^{1}$, F. Robert ${ }^{1}$ and L. Remusat ${ }^{1}$. ${ }^{1}$ LMCM, CNRS-MNHN, UMR7202, Case 52, 57 Rue Cuvier, 75005 Paris, France, E-mail: lpiani@mnhn.fr.

Introduction: The nature and consequences of the possible interactions between water/clay minerals and insoluble organic matter (IOM) on chondrites is still debated $[1,2]$. The in situ comparison of their respective $\mathrm{D} / \mathrm{H}$ ratio should shed new light on the processes that occurred during the hydrothermal alteration on chondrites parent bodies. A decade ago, SIMS measurements using negative primary oxygen beam were performed to assess the $\mathrm{H}$ isotopic composition of ordinary chondrites (OC) matrices [3]. Under these analytical conditions, $\mathrm{H}$ emission for $\mathrm{H}$-bearing silicates is higher than that for IOM: up to $92 \%$ of secondary $\mathrm{H}^{+}$extracted from the matrix comes from hydroxylated minerals. Thus, the $\mathrm{D} / \mathrm{H}$ ratios of the $\mathrm{H}$-bearing silicates in matrix could be measured in situ with this method but the $\mathrm{D} / \mathrm{H}$ ratio of the associated IOM has to be determined. An attempt has been tested by [4] with a $\mathrm{Cs}^{+}$primary beam on the ims 1280 ion microprobe. However, such a measurement is challenging: in their analytical conditions (1) the $\mathrm{H}^{-}$emission between IOM and phyllosilicates are not markedly different (higher for the phyllosilicates by only a factor 3 to 10) and (2) "matrix effects" modify the $\mathrm{D} / \mathrm{H}$ ratio. In this context, we have investigated the $\mathrm{H}^{-}$emission and the instrumental $\mathrm{H}$ isotopic fractionation on laboratory prepared mixtures of phyllosilicates and IOM with NanoSIMS.

Experimental: Terrestrial montmorillonite (hereafter referred to as Mtm) having $\mathrm{D} / \mathrm{H}=143 \times 10^{-6}$, terrestrial type III kerogen (D/ $\left.\mathrm{H}=141 \times 10^{-6}\right)$ and the IOM isolated from the OC GRO $95502(\mathrm{D} /$ $\mathrm{H}=669 \times 10^{-6}$ [2]) were mixed. The Mtm was mixed with either the kerogen or the IOM in variable proportions. Analyses were performed using $\mathrm{Cs}^{+}$and $\mathrm{O}^{-}$primary beams. The Hemission (and $\mathrm{C}^{-}, \mathrm{OH}^{-}, \mathrm{OD}^{-}$, $\mathrm{Si}^{-}$for $\mathrm{Cs}^{+}$) and the $\mathrm{D} / \mathrm{H}$ ratio for different laboratory prepared mixtures were determined.

Results: I. $\mathrm{O}^{-}$Primary Ion Beam. The emission yields of $\mathrm{H}$ for the IOM and Mtm are similar: 46 and $45 \mathrm{cps} / \mathrm{pA}$, respectively. Consequently, the $\mathrm{D} / \mathrm{H}$ ratio of the mixings should only depend on the proportion of each end-member: the measured $\mathrm{D} / \mathrm{H}$ ratios of the mixtures are consistent with mass balance predictions.

II. $\mathrm{Cs}^{+}$Primary Ion Beam. $\mathrm{H}$ and $\mathrm{OH}$ emission yields for the IOM are respectively 20 and 2 times higher than those for the Mtm. Therefore, in mixtures (natural or not), the cross contributions of IOM and Mtm on both the $\mathrm{D} / \mathrm{H}$ and the $\mathrm{OD} / \mathrm{OH}$ ratios cannot be neglected. Furthermore, the $\mathrm{H}$ and $\mathrm{OH}$ emission yields for the 50/50 (wt\%) IOM and Mtm mixture are enhanced by a factor of 1.6 and 4.0, respectively, compared with those measured for pure IOM. Measured $\mathrm{D} / \mathrm{H}$ ratios in this IOM and Mtm mixture is lower than expected by calculation using the emission yields of the pure end-members: $404 \pm 22 \times 10^{-6}$ instead of $454 \times 10^{-6}$. Since the mixture $\mathrm{OH} / \mathrm{H}$ ratio is closer to $\mathrm{Mtm}$ than expected, this isotopic effect likely results from an enhanced contribution of the Mtm on $\mathrm{H}$ and $\mathrm{OH}$ due to the addition of the carbonaceous compound (IOM). Further experiments are in progress to better constrain this matrix effect.

References: [1] Remusat L. et al. 2010. The Astrophysical Journal 713:1048-1058. [2] Alexander C. M. O'D. et al. 2007. Geochimica et Cosmochimica Acta 71:4380-4403. [3] Deloule E and Robert F. 1995. Geochimica et Cosmochimica Acta 59:4695-4706. [4] Bonal L. et al. 2010. Geochimica et Cosmochimica Acta 74:6590-6609. 


\section{7}

APPLICATION OF LICHENOMETRY TO METEORITE STUDIES

J. M. Pillinger ${ }^{1}$, C. T. Pillinger ${ }^{1}$, M. Powell ${ }^{2}$ and M. Butler ${ }^{3}$. ${ }^{1}$ Planetary and Space Sciences Research Institute, The Open University, Milton Keynes MK7 6AA, UK. ${ }^{2} 15$, Rotten Row, Riseley MK44 1EJ, UK. ${ }^{3} 96$, Townfield Road, Flitwick MK45 1JG, UK. E-mail: j.m.pillinger@ open.ac.uk.

Introduction: The lichen colonisation of a large, weathered, ordinary chondrite that had been surface exposed in Wiltshire, UK, for numerous decades, was studied. As the terrestrial provenance of the meteorite was unclear, it was proposed that an examination of the lichens present on the surface may offer confirmation of the geographical location at which it was reported, at least at the continent level. Additionally using the technique of lichenometry [1] information about the time of exposure at the location was sought. Lichen growths on meteorites exposed to the natural environment are not uncommon; they are considered by some to adversely affect the appearance of the specimen. A study of the lichens on the Ella Island (Greenland) meteorite demonstrated early colonisation of a fall [2]. Unfortunately, the meteorite studied in the work reported here remained in storage for 20 years after removal from the exposed location. Nevertheless the lichens were in sufficiently good state of preservation to allow identification. The community is described and discussed.

Results: Eight species of lichen have been named on the meteorite with some degree of certainty. These include the most conspicuous species and were determined by simple inspection as Diploicia canescens (rare), Lecanora albescens (rare), L. campestris (occassional), L. dispersa (occasional), L. muralis (occasional), Phaeophyscia orbicularis (occasional), Physcia adscendens (rare) and Verrucaria fuscella (rare). One small thallus was present that strongly resembles Caloplaca arcis (rare). Large colonies of a fertile Caloplaca species (frequent) was provisionally identified as Caloplaca holocarpa sens. str as was Amandinea punctata (occasional). Large areas of sterile crust, forming a background to the more distinctive lichens were present in some quantity on the meteorite and included Lecania erysibe (abundant) and possibly Caloplaca chlorina (abundant). A tentative identification was of Verrucaria muralis (rare). Two thalli of Lecanora campestris measured $6.0 \times 3.2 \mathrm{~cm}$ and $4.3 \times 3.5 \mathrm{~cm}$ respectively. Two thalli of L. muralis measured $3.6 \times 2.0 \mathrm{~cm}$ and $2.2 \times 1.5 \mathrm{~cm}$. A single thallus of Diploicia canescens measured $1.7 \mathrm{~cm}$ in diameter.

Discussion: The meteorite had not acquired any of the specialist lichens associated with the sarsen stones of Wiltshire, nor specialized iron species. The meteorite flora is reminiscent of that of ironstones and ironrich sandstones (gravestones) and some types of brick: no species associated with a non-UK origin were identified. The community is consistent with exposure at a poorly eutrophic site where nutrients from bird droppings is low. All species were considered pioneers of bare rock thus a time of decades of environmental exposure at the Wiltshire location is not inconsistent by comparison to similar colonies on dated gravestones. Study of further meteorite finds exhibiting lichen colonisation may produce a useful dataset allowing conclusions to be reached about exposure time on Earth, location and climatic conditions endured.

References: [1] Winchester V. 1998. Botanical Journal of the Linnean Society 96:57-68. [2] Hansen E. S. and Graff-Petersen P. 1986. Lichenologist 18:71-78.
5317

EVALUATION OF VARIOUS HYPOTHESES RELATING TO THE LOCATION OF THE FALL AND THE SUBSEQUENT HISTORY OF A LARGE METEORITE LATELY RECOVERED IN WILTSHIRE, UK C. T. Pillinger and J. M. Pillinger. Planetary and Space Sciences Research Institute, The Open University, Milton Keynes, MK7 67AA, UK. E-mail: c.t.pillinger@open.ac.uk.

Introduction: A large, $60 \mathrm{~kg}$, weathered meteorite was reported to have been in the UK for at least 60 years when it was taken to the Natural History Museum, London, twenty years ago. Previous to 1991, eye witness and photographic evidence demonstrate that it rested for many years near to the front door of an imposing country house in Wiltshire. It was reputed to have been either brought to the UK or otherwise collected locally by Lt Col Frederick George Glyn Bailey in the early twentieth century. The descendents of FGG Bailey have made the specimen available and encouraged a study to ascertain more information about the meteorite before arranging for it to be on permanent local public display. The meteorite is described in an accompanying abstract.

The presence of a meteorite of unknown terrestrial provenance offers a considerable challenge. While a scientific examination [1] can offer some information, much depends on an exhaustive examination of contemporary documents. Some while ago we were able to piece together a detailed account of the circumstances surrounding the fall of the Wold Cottage meteorite to show it should not be considered as just any old ordinary chondrite [2].

Results: The life and times of Lt Col FGG Bailey have been researched thoroughly in order to highlight any possible links he may have had to a meteorite find either in the UK or the rest of the World. The meteorite is inextricably linked to the house where it was located for so many years: Lake House. Thus the history of the Lake House Estate, the house, its previous occupants and their associates, including their interests and scientific aptitude, have also been studied in an attempt to establish the earthly provenance of the specimen.

Several hypotheses have been considered to account for the presence of the meteorite in Wiltshire. Necessarily these hypotheses revolve round the people who could conceivably have had an involvement in the meteorite's history on Earth. Questions regarding whether they might have had the motivation, opportunity and capability to recognize a meteorite have been considered. Even if they did, they would have needed the resources to move such a large stone and have the desire to collect and display it.

A document trail has been initiated which provides new and verifiable information about the terrestrial provenance of the meteorite associated with Lake House. The latest findings will be presented together with an up-to-date probability/possibility assessment of the place of first collection and hence the site of the fall. The work reported here may offer some pointers to others searching archive material for reports connected to meteorite related events.

References: [1] Pillinger C. T. et al. This conference. [2] Pillinger C. T. and Pillinger J. M. 1996. Meteoritics \& Planetary Science 31:589-605. 
5321

\section{THE DANEBURY IRON AGE METEORITE}

C. T. Pillinger ${ }^{1}$, J. M. Pillinger ${ }^{1}$, R. C. Greenwood ${ }^{1}$, D. Johnson ${ }^{1}$, A. G. Tindle $^{2}$, A. J. T. Jull ${ }^{3}$ and D. W. H. Allen ${ }^{4}$. ${ }^{1}$ PSSRI ${ }^{2}$ EES, Open University, Milton Keynes MK7 6AA, UK. ${ }^{3}$ University of Arizona, Tucson AZ85721, USA. ${ }^{4}$ Hampshire County Museums Service, Winchester UK. E-mail: c.t.pillinger@open.ac.uk.

Introduction: The Danebury meteorite's existence was first reported in the Meteoritical Bulletin in 1991 [1] following an effort to characterize metal containing artifacts recovered in 1974 from an archaeological dig conducted at the site of an Iron Age hillfort, Danebury Ring, in the county of Hampshire, England. After that initial report the object was "mislaid" and no further details appeared. In 2008 we initiated a search for the specimen and it has been recovered, sadly less a central slice removed to make a probe mount. We are now able to provide a better petrological description using a PTS of an interior cut of the $20 \mathrm{~g}$ surviving mass ( $\mathrm{H} 5$, low weathering grade $\mathrm{W} 1 / 2)$ and more information about the circumstances under which the meteorite was found.

The Anatomy of a Hill Fort: The Iron Age is a period of prehistory which in Britain extends from around $800 \mathrm{BC}$ until the Roman occupation of the mid first century AD. Southern Britain abounds with sites affording evidence of a turbulent period when inhabited locations are identified by the emphasis placed on defence fortifications. Danebury Ring can be regarded as a classic Iron Age hill fort site. It has been studied throughout a major campaign of annual excavations undertaken between 1969 and 1988 [2]. Systematic study of the 5 ha enclosure revealed evidence for scores of buildings. Hundreds of thousands of pottery shards, bone fragments as well as numerous objects of stone and metal were located with $10 \mathrm{~cm}$ accuracy before being recovered from some 2500 pits.

Iron Age Grain Pits: The Iron Age populace of Britain were essentially farmers. The pits excavated on Danebury Hill were dug originally to store the summer's grain harvest over the winter while the crop to be consumed immediately was kept above ground in granaries. Some budding Iron Age scientist had discovered that whilst burying grain might sound counter-productive, grain placed in a chalk pit capped with clay begins to ferment at the margins creating an anaerobic environment where the rest of the material survives for later use. The thousands of grain pits excavated at Danebury attest the efficacy of the science.

The Terrestrial Residence History of the Meteorite: The Danebury meteorite was recovered was excavated from the back-filled grain pit \#706. Its stratigraphic location revealed that the meteorite went into the hole during a second episode of the back-filling process. Pits no longer in use were initially refilled with the spoil from newly dug pits. After subsidence (2nd generation filling) "pot-holes" were flattened off with spoil from even later pits. The location of the meteorite in the 2 nd level implies it could have been where it was found because that is the place it actually fell. We have measured ${ }^{14} \mathrm{C}$ weathering age of the meteorite and obtained a value of $2350 \pm 120 \mathrm{BP}$. This date coincides with a period identified when the fort was highly active i.e. concurrent with construction of its most sophisticated fortifications.

References: [1] Meteoritical Bulletin 70. 1991. Meteoritics 26:68-69. [2] Cunliffe B. et al. 1984. Council for British Archeology Research Report 52.
5326

THE METEORITE FROM LAKE HOUSE

C. T. Pillinger ${ }^{1}$, J. M. Pillinger ${ }^{1}$, R. C. Greenwood ${ }^{1}$, D. Johnson ${ }^{1}$, A. G. Tindle ${ }^{2}$, A. J. T. Jull ${ }^{3}$ and M. Ashcroft ${ }^{4}$. ${ }^{1}$ PSSRI. ${ }^{2}$ EES Open University, Milton Keynes MK7 6AA, UK. ${ }^{3}$ AMF, University of Arizona, Tucson AZ85721, USA. ${ }^{4}$ Magna Parva Ltd., Bardon Hill, LE67 1UB, UK. E-mail: c.t.pillinger@open.ac.uk.

Introduction: In 1991 a very big meteorite (>60 kg, dimensions: diameter $50 \mathrm{~cm} \times$ height $40 \mathrm{~cm}$ ) was brought to the Natural History Museum by the then occupier of Lake House, a country mansion in Wiltshire, UK, associated with a large estate of the same name. The circumstances concerning the likely origins of this "find" are dealt with in a companion abstract [1]. Our attention was attracted to it because of its proximity to Danebury Hill (20 km east), where the only British meteorite "find", collected under controlled circumstances, was located in 1974 [2]. If the large meteorite from Lake House turned out to be paired with the much smaller Danebury find, then the mystery surrounding its origin would be instantly solved.

The sample from Lake House was confirmed as a meteorite by Robert Hutchison [3] and subsequently returned to its owners. Our electron microprobe data from a PTS made from a chip taken at the OU suggest it should be classified as type H5 similar to our assignment for Danebury.

Petrography: The sample is a heavily weathered (W5) [4], moderately shocked (S4) [5], equilibrated ordinary chondrite (H5). Distinct chondrules are present, but these tend to have poorly defined boundaries. Porphyritic types predominate, but barred olivine and radial pyroxene textured chondrules are also common. Chondrule mesostasis is recrystallized, with grain sizes generally below $50 \mu \mathrm{m}$. The sample is cut by a network of veins, up to $2 \mathrm{~mm}$ thick, filled with secondary weathering products.

Discussion: In respect of weathering characteristics, the two specimens are entirely different - areas in our Danebury PTS are grade $\mathrm{W} 1$ and the whole specimen is no worse than W2, whereas the meteorite from Lake House is W5. The thin section of the latter was made from a near surface chip of the highly corroded sample; the Danebury analyses were performed using a fresh interior portion. Not wishing to have what appeared to be a very delicate specimen disintegrate, we attempted to extract an interior core from the larger meteorite using an experimental drill being developed for robotic space missions. Much to our surprise prolonged drilling was unable to penetrate more than a millimeter into a location where the crust appeared to be absent. In earlier performance tests the drill had no difficulty in obtaining $1.5 \mathrm{~cm}$ long cores from basalt and concrete. We conclude that the interior of the meteorite from Lake House may not be as weathered as it appears from the outside.

Because we had obtained a very precise terrestrial weathering age for Danebury, samples of the meteorite from Lake House were removed for AcceleratorMass Spectrometry terrestrial residence dating. The first sample studied has a ${ }^{14} \mathrm{C}$ weathering age of $10,600 \pm 1800$ years BP. A second run with a better specimen gave $9500 \pm 560$ years. The large meteorite is clearly not related to the small one authenticated from the archeological site a few kilometers away. Given that it is a single stone, the meteorite represents an unusually large ordinary chondrite find.

References: [1] Pillinger C. T. and Pillinger J. M. This conference. [2] Pillinger C. T. et al. This conference. [3] Personal communication. [4] Wlotzka F. 1993. Meteoritics 28:460. [5] Stöffler D. et al. 1991. Geochimica et Cosmochimica Acta 55:3845-3867. 
5080

MELT ON THE STRIATED SURFACE OF SHATTER CONES IN BASALT

L. Pittarello ${ }^{1}$, C. Koeberl ${ }^{1,2}$ and A. P. Crósta ${ }^{3}$. ${ }^{1}$ Department of Lithospheric Research, University of Vienna, A-1090 Vienna. E-mail: lidia.pittarello@univie.ac.at. ${ }^{2}$ Natural History Museum, A-1010 Vienna. ${ }^{3}$ Department of Geology \& Natural Resources, Institute of Geosciences, Campinas, SP, Brazil.

Introduction: Shatter cones are one of the most reliable types of macroscopic evidence of shock metamorphism [1]. The local occurrence of melt on the striated surfaces of shatter cones, in the form of glassy siliceous spherules decorating the topographic lows of the striations, has been the described from large impact structures, such as Vredefort [e.g., 2, 3]. Different models [4-6] were proposed for melt formation on the surface of shatter cones, but none of them can explain all the observations [7]. Furthermore, a recent study on shock deformation distribution within shatter cones has demonstrated that the deformation in not localized exclusively on the surface and that the recorded shock peak is higher than how previously assumed [8]. Here we describe a homogeneous melt layer decorating the striated surface of shatter cones formed in fine-grained basalts, from the Vista Alegre impact structure, a $9.5 \mathrm{~km}$ diameter structure recently confirmed as being of impact origin, excavated in the basalts of the Paraná Basin, Brazil [9].

Results: A cross section of the cm-sized shatter cones from the Vista Alegre reveals several fractures parallel to the striated surface. Such fractures are generally filled by an ultracataclasite (grain size $<1 \mu \mathrm{m}$ ), where some clasts of pyroxene are preserved. Along the striated surface a $50-\mu \mathrm{m}$-thick homogeneous film of melt is locally preserved. The melt is locally quenched, forming dendritic microlites $4-5 \mu \mathrm{m}$ in size, with a mixed composition between plagioclase and augitic pyroxene. Raman micro-spectroscopy has confirmed the occurrence of amorphous material, but its exact nature still needs to be confirmed. No shatter cones have been reported from the other impact craters in basalts (Lonar, India and Logancha, Russia), and those from the Vargeão impact structure, Brazil, have not yet been studied in detail. One possible explanation for melt occurrence in basaltic shatter cones is the lower melting temperature of basalt compared to silica-rich rocks; another that the lack of evidence for friction could suggest a shock origin for the melt.

References: [1] French B. and Koeberl C. 2010. Earth Science Reviews 98:123-170. [2] Gay N. C. et al. 1978. Earth and Planetary Science Letters 41:372-380. [3] Nicolaysen L. O. and Reimold W. U. 1999. Journal of Geophysical Research 104:4911-4930. [4] Gibson H. M and Spray J. G. 1998. Meteoritics \& Planetary Science 33:329-336. [5] Sagy A. et al. 2002. Nature 418:310-313. [6] Baratoux D. and Melosh H. J. 2003. Earth and Planetary Science Letters 216:43-54. [7] Wieland F. et al. 2006. Meteoritics \& Planeteray Science 41:1737-1759. [8] Ferrière L. and Osinski G. R. 2010. Abstract \#5273. Meteoritics \& Planetary Science 45:A53. [9] Crósta A. P. et al. 2010. Meteoritics \& Planetary Science 45:181-194.

Back-scattered FE-SEM image of a microlitic aggregate.

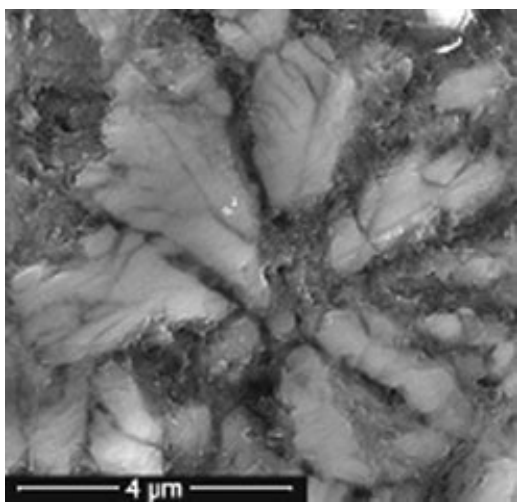

5081

PETRO-CHEMICAL CHARACTERIZATION OF THE UNIQUE IMPACT CRATER ON EARTH IN SILICEOUS VOLCANIC ROCKS: THE EL'GYGYTGYN DRILL CORE

L. Pittarello ${ }^{1}$ C. Koeberl ${ }^{1,2}$ and the El'gygytgyn Scientific Party. ${ }^{1}$ Department of Lithospheric Research, University of Vienna, A1090 Vienna. E-mail: lidia.pittarello@univie.ac.at. ${ }^{2}$ Natural History Museum, A-1010 Vienna.

Introduction: The El'gygytgyn impact structure, 3.6 Ma old and $18 \mathrm{~km}$ in diameter, was excavated in Late Cretaceous siliceous volcanic rocks of the central Chukotka, northeastern Russia [1]. It is the only known terrestrial impact structure formed in siliceous volcanic target and thus enables to investigate shock metamorphism in such lithologies. The impact structure, filled by a lake $12 \mathrm{~km}$ in diameter, was drilled in 2009 during an ICDP drilling project [2]. The drill core, penetrating through post-impact sediments, impactites and the fractured igneous basement, was sampled and studied through petrographic (optical and electron microscope, Raman micro-spectroscopy) and chemical analyses (XRF and INAA), from $316.08 \mathrm{~m}$ to $517.30 \mathrm{~m}$ in depth.

Results:

Core Stratigraphy: The main rock types of the crater basement are ignimbrite, tuff, and lava of rhyolitic composition; rarely from andesite and dacite [3]. The simplified stratigraphy of the core is: (1) $316-385 \mathrm{~m}$ impact lithic breccia including volcanic-like and melt clasts, locally with shocked minerals, in a fine-grained clastic matrix; (2) $385-427 \mathrm{~m}$ transition zone characterized by a reddish breccia alternating with dark veins of basalt-like melt; (3) 427-517 m greenish ignimbrite-like rock, with abundant melt particles, quartz-free and elongated parallel to flattening direction. This latter formation is crosscut by abundant fractures locally filled by carbonate, silicate and clay veins.

Shock Features: The abundance of shock features decreases rapidly from the top to the bottom of the studied core section, being almost absent in the brecciated layer (3). Specifically, in the layer (1) clasts of volcanic-like rocks include shocked phenocrysts. Locally, quite fresh PDFs occur in quartz grains, with up to 3 sets per grain. In the matrix, quartz grains are commonly either completely transformed into diaplectic glass or unshocked. Some $\mu \mathrm{m}$-sized grains of coesite occur in quartz grains. Some feldpars contain PDFs and evidence of melting. The mafic minerals (biotite, amphibole) are rare and generally highly altered locally kinked or partially melted, maybe for volcanic or tectonic processes. In layer (3) no or very few shock features occur, with rare incipient basal PDFs in quartz.

Geochemistry: The bulk analyses of layers (1) and (3) are consistent with a trachy-rhyolite composition. Clasts in the breccia (1), separately analyzed, cover the full range of composition but are mainly rhyolites. On the other hand, the melt particles included in (3) show a mafic to intermediate composition. In (2) some basalt-like samples, with high loss of ignition, contains anomalous content of $\mathrm{Co}, \mathrm{Cr}$, and $\mathrm{Ni}$, and will further investigated to relate such contamination to hydrothermal alteration or a possible meteoritic component.

Acknowledgment: This work was supported by the Austrian Science Foundation (FWF) project P21821-N19.

References: [1] Gurov E. P. et al. 1979. Abstract. 10th Lunar and Planetary Science Conference. pp. 479-481. [2] Melles M. et al. 2011 Scientific Drilling 11:29-40. [3] Gurov E. P. et al. 2005. Geochimica et Cosmochimica Acta Special Paper 384:391-412. 
5369

IMPACT MELT IN SMALL SIMPLE LUNAR HIGHLANDS CRATERS

J. B. Plescia ${ }^{1}$ and M. J. Cintala ${ }^{2}$. ${ }^{1}$ Applied Physics Laboratory, The Johns Hopkins University, Laurel, MD 20723, USA. E-mail: jeffrey. plescia@jhuapl.edu. ${ }^{2}$ Code KR, NASA, Johnson Space Center, Houston, TX 77058, USA.

Introduction: Impact melt is a typical characteristic of large complex impact craters on all of the terrestrial planets; it also occurs in some larger simple craters. The melt forms pools on the crater floor, flows beyond the rim, and drapes the inner crater wall. Melt deposits have been assumed to only be associated with larger simple craters and complex craters [1-6] with the smallest recognized crater with melt being approximately $750 \mathrm{~m}$ in diameter. Theoretical models of impact melt generation suggested that only small volumes would be produced [7-8] and most of that would be ejected from the crater interior.

Observations: LRO LROC images show that impact-melt deposits are recognized associated with simple highlands craters down to diameters of approximately $200 \mathrm{~m}$. The melt forms pools on the crater floor, veneer on the crater walls, flows on the crater and ejecta outside the crater. Melt also forms stringers of dark material extending over the rim and across the surface. Such melt deposits are relatively rare, and can be recognized only in some fresh craters. These observations indicate that identifiable quantities of impact melt can be produced in small impacts and the presence of such deposits shows that the material can be aggregated into recognizable deposits. Estimates of the amount of melt present in craters with melt pools on the floor $\left[10^{-3}-10^{-5} \mathrm{~km}^{3}\right]$ are of the same order of magnitude as that estimated from the models of melt generation [8].

Interpretations: While target properties can influence the amount of melt produced during an impact, the physical properties of the highland megaregolith are more or less uniform in the context of impact crater formation. Similarly, variations in the properties of the projectile do not significantly change the amount of melt produced. Thus, some other aspect of the cratering process must be responsible. It is concluded that these craters represent vertical impacts rather than the more common oblique events. For a vertical impact, [10-11] the shock pressures can be larger and they are focused at the center of the crater. In such cases more melt is produced and less is ejected than in an oblique event.

Conclusions: Impact melt is observed in fresh simple lunar highlands craters as small as approximately $200 \mathrm{~m}$ diameter. Such deposits are rare. Small craters with recognizable impact melt are interpreted to represent vertical, rather than oblique, impacts.

References: [1] Dence M. 1971. Journal of Geophysical Research 76:5552-5565. [2] Moore H. 1972. Apollo 16 Preliminary Science Report, NASA SP315:29-45-29-51. [3] Howard K. and Wilshire H. 1975. Journal Research U.S.G.S. 3:237-251. [4] Hawke B. and Head J. 1977. 8th Lunar Prospector Spacecraft Conference, 415-417. [5] Hawke B. and Head J. 1977. Impact and Explosion Cratering, pp. 815-841. [6] Hawke B. and Head J. 1979. 10th Lunar Prospector Spacecraft Conference, pp. 510-512. [7] Schmidt R. and Housen K. 1987. International Journal Impact Engineering 5:543-560. [8] Cintala M. and Grieve R. 1998. Meteoritics \& Planetary Sciences 33:889-912. [9] Robinson M. et al. 2010. Space Science Review 150:81-124. [10] Pierazzo E. et al. 1997. Icarus 127:408-423. [11] Pierazzo E. and Melosh H. 2000. Icarus 145:252-261.

\section{0 \\ FEATHER FEATURES IN QUARTZ: STRUCTURAL AND TEXTURAL INSIGHTS FROM FIRST TEM AND EBSD MEASUREMENTS}

M. H. Poelchau ${ }^{1}$, G. Trullenque ${ }^{1}$ and T. Kenkmann ${ }^{1}$. ${ }^{1}$ Institut für Geowissenschaften, Universität Freiburg, Germany. E-mail: michael. poelchau@geologie.uni-freiburg.de.

Introduction: Feather features (FFs) are a recently discovered [1], deformational feature in quartz that has been found in 26 confirmed impact craters so far [2]. They are suggested to form under low shock pressures [2] and potentially can be added to the list of shock-induced microstructures in quartz, along with PDFs and planar fractures.

FFs consist of a main planar fracture (PF) that has been sheared during shock compression and from which short, parallel to subparallel lamellae emanate in one direction, typically at an angle of $50-60^{\circ}$. The orientation of these lamellae is always consistent with the sense of shear along the fracture. The crystallographic orientation of the fracture and lamellae show a degree of crystallographic control, with (0001), $\{10 \overline{1} ; 1\}$ and $\{11 \overline{2} ; 2\}$ as the most common rational low index crystallographic planes.

Generation of FFs: Analysis of multiple sets of FFs in natural samples shows that all FF lamellae within a sample trend in roughly the same direction, while the sheared PFs form conjugate sets at $\sim 90^{\circ}$ angles. The principal axis of stress of the shock wave is therefore assumed to lie at a $45^{\circ}$ angle to the conjugate sets and parallel to the FF lamellae. Thus FFs can be used as indicators for the orientation of differential stress within the shock wave.

Although the generation of FFs as a shear-induced structure is clear in principle, details of their formation are not yet completely understood. Specifically, the quartz grain's lattice is commonly distorted near the shear fracture, visible as an undulatory extinction pattern under the microscope. In conventional structural geology, the orientation of this lattice distortion forms as the result of "dragging" of material along the shear plane. Surprisingly, FFs show the exact opposite orientation.

Results and Implications: EBSD (electron backscatter diffraction) measurements are currently being performed on FFs to analyze lattice deformation at a high resolution. Preliminary EBSD results confirm the lattice deformation determined by microscope analyses. Based on these initial results, we suggest that lattice deformation along the PF is caused by elastic lattice strain accumulated during shearing in the shock wave, which is then rapidly released during unloading, resulting in the opening of the FF lamellae as extensional fractures (mode I) while rotating the lattice between two individual lamellae, causing additional mode II in-plane shear along the lamellae and resulting in a distortion pattern visible in EBSD measurements. Preliminary TEM analyses show that the junctions of the FF lamellae with the PF are filled with vesicular amorphous material.

Further EBSD and TEM work is planned to give more detailed insights into the kinematics of dynamic fracturing and shearing. A deeper understanding of this process can potentially be used to constrain and differentiate fracturing mechanisms in shock waves (and their visible expressions in quartz grains) from fracturing in lower-dynamic, tectonic regimes.

References: [1] French B. M. et al. 2004. GSA Bulletin 116:200-218. [2] Poelchau M. H. and Kenkmann T. 2011. Journal of Geophysical Research 116:B02201. 
5462

IMPACTS INTO SANDSTONE: POROSITY EFFECTS IN THE STRENGTH REGIME

M. H. Poelchau, A. Dufresne, T. Kenkmann and the MEMIN Team. Instut für Geowissenschaften, Universität Freiburg, Germany. E-mail: michael.poelchau@geologie.uni-freiburg.de.

Introduction: The MEMIN (Multidisciplinary Experimental and Modeling Impact Research Network) is currently focused on experimental impact cratering into sandstone. One of the main goals of these experiments is determining the role porosity has on the impact process and on the resulting crater morphology. So far, four sets of impact experiments have been performed at the two-stage light gas gun facilities of the Ernst-Mach-Institut, Freiburg, Germany. Aluminum, steel and iron meteorite projectiles were accelerated to velocities between $2.5-7.8 \mathrm{~km} \mathrm{~s}^{-1}$, resulting in impact energies ranging from $0.7-58.4 \mathrm{~kJ}$. Targets were dry Seeberger sandstone blocks with a grain size of $\sim 70 \mu \mathrm{m}$, a porosity of 20 $25 \%$, and a uniaxial compressive strength (UCS) of 35-40 $\mathrm{MPa}[1,2]$.

Cratering Results and Discussion of Porosity Effects: All crater dimensions were measured with a 3D laser scanner. Crater depth to diameter ratios range from 0.14 to 0.25 . Craters formed by steel or meteoritic iron projectiles are slightly deeper $(0.20)$ than the average craters formed in crystalline rocks with similar high-density projectiles $(0.18)[3,4]$, indicating that the sandstone's porosity results in slightly increased penetration depth. The sandstone craters formed by aluminum projectiles, on the other hand, are much shallower (0.15), and thus reflect a stronger dependency of penetration depth on projectile density than on porosity, at least for porosity values of $\sim 25 \%$.

The effect of porosity becomes more apparent when crater volumes of the sandstone and non-porous crystalline rocks [3-5] are compared. For the same impact energy and roughly similar impact conditions (projectile mass, density and speed) the same crater volumes result, in spite of a difference of target crushing strength of nearly one order of magnitude (UCS of crystalline rocks is assumed at $300 \mathrm{MPa}$ ). This is in agreement with numerical modeling results [6] that show a dampening of the shock wave through porosity. Thus, the amount of energy available to work against the target's strength to form a crater is reduced by the work required to close pore space. Although density effects of the projectile on crater volume should be expected, no major differences were detected.

The cratering efficiency (the ratio of excavated target mass to projectile mass) in sandstone is also greatly reduced compared to strength scaled cratering efficiency values of crystalline rocks [3-5] by almost one order of magnitude. Based on the limited number of data, it is currently difficult to determine the exact effects of porosity on strength-scaled size parameters beyond a general reduction of cratering efficiency. Impact experiments into more highly porous target rocks are planned to better quantify the effects porosity has on cratering in strength dominated regimes.

References: [1] Kenkmann T. et al. 2011. Meteoritics \& Planetary Science, in press. [2] Poelchau M. H. et al. 2011. Abstract \#1824. 42nd Lunar and Planetary Science Conference. [3] Polanskey C. A. and Ahrens T. J. 1990. Icarus 84:140-155. [4] Burchell M. J. and Whitehorn L. 2003. Monthly Notices of the Royal Astronomical Society 341:192-198. [5] Smrekar S. et al. 1986. Journal of Geophysical Research 13:745-748. [6] Güldemeister N. et al. 2011. Abstract \#1104. 42nd Lunar and Planetary Science Conference.

\section{7}

HIGH FIDELITY STUDIES OF INTERSTELLAR DUST ANALOG IMPACTS IN Stardust AEROGEL AND FOILS

F. Postberg, C. Allen, S. Bajt, H. A. Bechtel, J. Borg, F. E. Brenker, J. Bridges, D. E. Brownlee, M. Burchell, M. Burghammer, A. L. Butterworth, P. Cloetens, A. M. Davis, R. Doll, C. Floss, G. J. Flynn, D. Frank, Z. Gainsforth, E. Grün, P. R. Heck, J. K. Hillier, P. Hoppe, L. Howard, G. R. Huss, J. Huth, A. Kearsley, A. J. King, B. Lai, J. Leitner, L. Lemelle, A. Leonard, H. Leroux, L. R. Nittler, R. C. Ogliore, W. J. Ong, M. C. Price, S. A. Sandford, J. A. Sans Tresseras, S. Schmitz, T. Schoonjans, K. Schreiber, G. Silversmit, A. Simionovici, R. Srama, T. Stephan, J. Stodolna, R. M. Stroud, S. R. Sutton, R. Toucoulou, M. Trieloff, P. Tsou, A. Tsuchiyama, T. Tyliczszak, B. Vekemans, L. Vincze, A. J. Westphal, M. E. Zolensky and >29,000 Stardust@home dusters. ISPE author affiliations are at http://www.ssl.berkeley.edu/ $\sim$ westphal/ISPE/.

In 2000 and 2002 the Stardust Mission exposed aerogel collector panels for a total of about 200 days to the stream of interstellar grains sweeping through the solar system. The material was brought back to Earth in 2006.

We present laboratory calibration of the collection process by shooting high speed [3-30 $\mathrm{km} \mathrm{s}^{-1}$ ] interstellar dust (ISD) analogs onto Stardust aerogel and foil flight spares. Particle impact speeds up to $50 \mathrm{~km} \mathrm{~s}^{-1}$ can only be achieved by a Van de Graaff accelerator such as operated at the MPI für Kernphysik (Heidelberg) [1], and requires coating of nonconductive particles with thin films of either platinum [2] or polypyrrole [3].

Particle impact tracks in aerogel were identified by an optical microscope. Track diameters and depths were measured optically at high magnification using an encoded stage with $0.5 \mu \mathrm{m}$ precision. Subsequently tracks in picokeystones were extracted and analyzed with Scanning Transmission X-ray Microscopy (STXM). This enabled an investigation into both the morphology of impact tracks as well as structural and chemical modification of projectile and collector material. Major campaigns were completed in spring 2011 with the goal to characterize tracks of interstellar grains with respect to the projectile speed, size, and density. Three different materials (orthopyroxene, iron, polystyrene) were therefore shot within several narrow speed and size windows (e.g., 14 $\left.16 \mathrm{~km} \mathrm{~s}^{-1}, 0.37-0.43 \mu \mathrm{m}\right)$. For each set of parameters, about 50 particles were collected.

First results show a bulbous track shape at $15 \mathrm{~km} \mathrm{~s}^{-1}$ similar to type A Stardust tracks with a terminal particle. For the first time it could be shown experimentally that cores of submicron minerals survive aerogel capture at speeds well above $10 \mathrm{~km} \mathrm{~s}^{-1}$. The calibration is not only important for the systematic search and analyses of foils and aerogel [4, 5], but also allows recalculation of the ISD flux [6] for the Stardust collection period.

References: [1] Stübig M. et al. 2001. Planetary and Space Science 49:853. [2] Hillier J. K. et al. 2009. Planetary and Space Science 57:2081. [3] Armes S. et al. 1991. Polymer 32:2325. [4] Floss C. et al. 2011. This conference. [5] Stroud R. M. et al. 2011. This conference. [6] Landgraf M., Müller M., and Grün E. 1999. Planetary and Space Science 47:1029. 
5116

THE FIRST DOCUMENTED GEORGIA-AREA TEKTITE FOUND IN BIBB COUNTY, GEORGIA

H. Povenmire. Florida Institute of Technology, 215 Osage Drive, Indian Harbour Beach, FL 32937, USA. E-mail: katiehal1@yahoo.com.

About 1999, a black glassy rock was found approximately in the middle of Bibb County just south of Macon, GA. This was identified as a Georgia tektite on May 19, 2011. This $18.42 \mathrm{~g}$ tektite is a flattened tear drop shape and is $47 \times 22 \times 15 \mathrm{~mm}$ in size. It had been in running water for a long time and the surface was smoothed. There are no bubbles or inclusions. The find location was on the Fall Line and the Piedmont Plateau. The two western adjoining counties, Crawford and Peach have similar geology and will probably be confirmed with further field work. The approximate find location was lat. $32 \mathrm{~d} 42^{\prime} \mathrm{N}$ and long. $83 \mathrm{~d} 44^{\prime} \mathrm{W}$.

In 2010, a spectacular Georgia-area tektite was found in Aiken County, South Carolina. In 2011, a second tektite was found in Allendale County, South Carolina. This is a small disk- shaped tektite and was found in an Indian midden overlooking the Savannah River. It was not found in situ and further field work is in progress for more specimens.

About ten percent of Georgia tektites have shown indications of being flaked by early Indians. However, complete artifacts are extremely rare. In early 2011, a second Georgia tektite which had been flaked into a projectile point was reported. This specimen was found in Dodge county and artifact specialists have suggested that it was of archaic age and the aborigines flaking it were likely of the Pickwick or Wacissa Cultures. This $7.1 \mathrm{~g}$ tektite was flaked, then damanged and then reworked several times.

In 2010, the third largest Georgia splash form tektite was re-ported from Bleckley County. It has a weight of approximately $65 \mathrm{~g}$. Precise data on it is not available but it is of significant importance because it fills a statistical gap in the size distribution of the large Georgia tektites.

In the last year, several museum quality tektites have been found. These have come from Bleckley, Wilcox and Houston counties. Since these specimens have come from previously con-firmed counties, they are good scientific specimens but do not add to our knowledge of distribution.

There have now been approximately 2700 Georgia-area tektites found in 26 counties. The total area of the Georgia-area strewn field is now approximately 10,000 square miles and this is larger than the Texas bediasite strewn field.

Acknowledgments: I want to thank the help of Mike Stokes, Thomas Thurman, Sharon Veal and Kalen Icard.

References: [1] Povenmire H. 2003. Tektites: A Cosmic Enigma Blue Note Publishing, Cocoa Beach, FL. [2] Povenmire H. and Lambert J. 2010. The First Georgia-Area Tektite found in Aiken County, South Carolina, Meteoritics \& Planetary Science, 73rd Annual Meeting Abstract, New York.
5509

FEASIBILITY OF IODINE AND BROMINE ANALYSIS IN GENESIS SOLAR WIND COLLECTORS

O. Pravdivtseva ${ }^{1}$, A. Meshik ${ }^{1}$, C. M. Hohenberg ${ }^{1}$ and D. S. Burnett ${ }^{2}$. ${ }^{1}$ McDonnell Center for the Space Sciences and Physics Department, Washington University, St. Louis, MO 63130, USA. E-mail: olga@physics.wustl.edu. ${ }^{2}$ Geology 100-23, CalTech, Pasadena, CA, USA.

Comparison of elemental abundances in sun, meteorites and earth provides understanding of the formation and evolution of the solar system. Yet, the majority of the solar system abundances are based on meteoritic values [1-6]. Here we report an attempt to estimate a feasibility of direct measurements of iodine and bromine in the GENESIS solar wind Aluminum on Sapphire collector (AloS) using neutron induced conversions: ${ }^{127} \mathrm{I}(\mathrm{n}, \gamma \beta){ }^{128} \mathrm{Xe},{ }^{79} \mathrm{Br}(\mathrm{n}, \gamma \beta){ }^{80} \mathrm{Kr}$ and ${ }^{81} \mathrm{Br}(\mathrm{n}, \gamma \beta)^{82} \mathrm{Kr}$.

To estimate the extent of terrestrial halogen contamination in GENESIS collectors, several flown fragments of AloS were submerged in methanol (for $1 \mathrm{~h}$ and for $48 \mathrm{~h}$ ), rinsed in methanol, dried, sealed under vacuum in fused quartz ampoules and irradiated at the Missouri University Research Reactor receiving fluence $\sim 2 \times 10^{19}$ thermal neutrons $\mathrm{cm}^{-2}$.

We analyzed two areas $\left(0.07\right.$ and $\left.0.7 \mathrm{~cm}^{2}\right)$ of AloS the solar wind collector washed for $48 \mathrm{~h}$, and one area $\left(0.13 \mathrm{~cm}^{2}\right)$ of the collector washed for $1 \mathrm{~h}$. Xenon extracted from the largest area had a clear signature of solar wind as indicated by ${ }^{129} \mathrm{Xe} /{ }^{132} \mathrm{Xe}=1.045 \pm 0.005$ and other major isotopes, while ${ }^{128} \mathrm{Xe} /{ }^{132} \mathrm{Xe}=1.01 \pm 0.03$ had a 12 -fold excess compared to the solar value. Longer washing apparently reduces iodine contamination 4 times, implying that it is surface correlated. Evidently, to separate ${ }^{128} \mathrm{Xe}$, derived from solar iodine, and the solar wind ${ }^{128} \mathrm{Xe}$, the iodine contamination should be reduced further, at least 10 times. It may be achieved by combination of a longer and more aggressive cleaning procedure (i.e., ozone cleaning followed by washing in non-polar solvents at elevated temperatures) and depth-profiling laser extraction.

Krypton analyses of the irradiated AloS collectors demonstrates solar wind signature with $17 \%$ excess in ${ }^{82} \mathrm{Kr}$ and 3 -fold excess in ${ }^{80} \mathrm{Kr}$. Apparently, cleaning in methanol is much more efficient for $\mathrm{Br}$ that for $\mathrm{I}$, since a longer 48-hour washing reduced bromine contamination 50 times.

Our first "quick and dirty" experiment suggests that determination of solar iodine and bromine is potentially feasible, especially considering availability of cleaner Si-based Genesis collectors and the possibility of analyzing larger collector areas irradiated with tripled neutron fluence.

Acknowledgment: This work is supported by NASA grant NNX07AM76G.

References: [1] Anders E. and Ebihara M. 1982. Geochimica et Cosmochimica Acta 46:2363-2380. [2] Anders E. and Grevesse N. 1989. Geochimica et Cosmochimica Acta 53:197-214. [3] Cameron A. G. W. 1968. In Origin and distribution of the elements, Ahrens L. H., ed. Pergamon, Oxford. pp. 125-143. [4] Cameron A. G. W. 1973. Space Science Reviews 15:121-146. [5] Suess H. E. and Urey H. C. 1956. Reviews of Modern Physics 28:53-74. [6] Lodders K. 2010. Principles and Perspectives in Cosmochemistry 379-417. 
5356

HYDROCODE MODELING OF MICROMETER SCALE IMPACTS INTO AI FOIL: IMPLICATIONS FOR ANALYSES OF Stardust ISPE CRATERS

M. C. Price ${ }^{1}$, A. T. Kearsley ${ }^{2}$, M. J. Burchell ${ }^{1}$, S. P. Armes ${ }^{3}$, M. J. Cole ${ }^{1}$, J. K. Hillier ${ }^{4}$, F. Postberg ${ }^{5}$ and N. Starkey ${ }^{4}$. ${ }^{1}$ School of Physical Sciences, University of Kent, Canterbury, CT2 7NH, UK. E-mail: mcp2@star.kent.ac.uk. ${ }^{2}$ Impacts \& Astromaterials Research Centre, Natural History Museum, London SW7 5BD, UK. ${ }^{3}$ Department of Chemistry, University of Sheffield, Sheffield, S3 7HF, UK. ${ }^{4}$ PSSRI, Open University, Milton Keynes, MK7 6AA, UK. ${ }^{5}$ Institute for Geosciences, Heidelberg University, Heidelberg, Germany.

Introduction: This work provides a validated hydrocode model to help interpret impact velocity and angle (and thus impactor origin) from nondestructive measurements of the dimensions of craters found on the Stardust interstellar collector foil. We have used a modified CowperSymonds [1] strength model for Al-1100 within AUTODYN, incorporating strain rate (and strain) hardening and pressure dependent melting [2] Our model has now been validated against experimental data for silica and sodalime glass (slg) projectiles fired onto Stardust flight spare foil at velocities of $6.1 \mathrm{~km} \mathrm{~s}^{-1}$ [3, 4] and sub-micron iron particles accelerated in a Van de Graaff (VdG) accelerator $[5,6]$ at velocities up to $27 \mathrm{~km} \mathrm{~s}^{-1}$. Results from recent experiments using the VdG accelerator in Heidelberg [7] are also being used to extend the validation.

VdG Analog Foil Shots: Two campaigns used Stardust flight spare foil as targets: March 2010: Platinum coated orthopyroxene (OPX) with particle diameter approximately $0.4 \mu \mathrm{m}$, accelerated in four runs to 6 (twice), 15 and $20 \mathrm{~km} \mathrm{~s}^{-1}$. Data from these four foils are described in [2]. April-May 2011 (1) Polypyrrole coated olivine projectiles of approximately $0.25 \mu \mathrm{m}$ diameter, accelerated to: 3, 6, 10, 15, 20, $70 \mathrm{~km} \mathrm{~s}^{-1}$. (2) Polypyrrole coated polystyrene (approximately $0.4 \mu \mathrm{m}$ diameter) accelerated to $3,6,10 \mathrm{~km} \mathrm{~s}^{-1}$. (3) Pt coated OPX (approximately 0.25 and $0.4 \mu \mathrm{m}$ diameter): accelerated to $3,6,10$, $15 \mathrm{~km} \mathrm{~s}^{-1}$.

Results: The hydrocode model reproduces the experimentally derived crater dimensions to within $10 \%$ for the majority of the data and, at worst, to within $25 \%$ for the highest speed and smallest projectile. This validation covers a range of projectile sizes from $150 \mathrm{~nm}$ to $4 \mu \mathrm{m}$, impact velocity $6-27 \mathrm{~km} \mathrm{~s}^{-1}$ and projectile density from 2.4 to $7.8 \mathrm{~g} \mathrm{~cm}^{-3}$.

Discussion: Recent analyses [8,9] of two sub- $\mu \mathrm{m}$ diameter craters on the Stardust interstellar collector foil show they were almost certainly made by secondary ejecta from the $\mathrm{Zn}$ - and Ce-bearing solar panel cover glass. Secondary glass particles are likely to have been ejected with a wide range of speeds, up to a few tens of $\mathrm{km} \mathrm{s}^{-1}$, to impact on the collector at oblique angles [10]. 3D numerical modeling of glass impactors is ongoing to determine the effect that solid impactor shape, impact angle and velocity may have on crater shape and if any measureable crater metric could be used to help determine an impactor's trajectory and velocity.

References: [1] Cowper G. R and Symonds P. S. 1957. Technical Report \#28, Brown University. [2] Price M. C. et al. 2011. Meteoritics \& Planetary Science (sub.). [3] Price M. C. et al. 2010. Meteoritics \& Planetary Science 45:1409. [4] Kearsley A. T. et al. 2006. Meteoritics \& Planetary Science 41:167. [5] Davidson R. F. and Walsh M. L. 1995. Proceedings of the SCCM. [6] Stradling G. L. et al. 1992. Proceedings of the HVIS. [7] Mocker A. et al. 2011. Review of Science Instruments (sub.). [8] Stroud et al. 2011. This issue. [9] Floss et al. 2011. This issue. [10] Westphal A. et al. 2008. Abstract \#1855. 39th Lunar Prospector Spacecraft.
5198

NEW DATA ON GRAPHITE FROM THE ZAKŁODZIE ENSTATITE ACHONDRITE

T. A. Przylibski ${ }^{1}$, T. Jakubowski ${ }^{2}$, L. Karwowski ${ }^{3}$, R. Kryza ${ }^{4}$ and K. Uszczek ${ }^{1}{ }^{1}$ Faculty of Geoengineering, Wrocław University of Technology, Mining and Geology, Wybrzeże S. Wyspiańskiego 27, 50-370 Wrocław, Poland. ${ }^{2}$ Balonowa 5/2, 54-129 Wrocław, Poland. ${ }^{3}$ Faculty of Earth Sciences, University of Silesia, ul. Będzińska 60, 41-200 Sosnowiec, Poland. ${ }^{4}$ University of Wrocław, Institute of Geological Sciences, ul. Cybulskiego 30, 50-205 Wrocław, Poland.

Introduction: Zakłodzie is one of the ungrouped enstatite achondrites. It was classified by some authors $[1,2]$ as an impact-melt rock, but others (T. A. Przylibski and research team) have suggested that a new meteorite group of primitive enstatite achondrites can be defined. They proposed to include into that group, apart from Zakłodzie, several other enstatite meteorites, e.g., Happy Canyon, Ilafegh 009, QUE 94204 and Yamato-8404 [3-5]. Zakłodzie has an extraordinary mineral composition, comprising e.g., keilite [5] and, in the innermost part, spherical nodules of graphite and metal, surrounded by enstatite crystals. Both, inclusions of graphite in metal grains and metal grains in graphite are found. These kinds of inclusions are important for establishing the temperatures of metamorphism leading to chondrule borders effaceing and recrystallization of plagioclase II [4]. Further constraints on the crystallization temperature can be inferred from determination of the graphite structure.

Samples and Experiments: Analyses were made on a fragment of the innermost part of Zakłodzie, a few $\mathrm{cm}^{2}$ in size. Element mapping was done on SEM JEOL JSM-5500LV. Raman spectra were obtained on confocal microspectrometer T-64000 (Jobin-Yvon) equipped with microscope Olympus BX-40; the $514.5 \mathrm{~nm}$ Ar line was used for sample excitation. X-ray powder diffraction analyses were performed on X'Pert Philips PW 3710 diffractometer (Co K $\alpha$ ).

Results: Element mapping shows that carbon nodules are set in iron or, locally, enclosed in silicate minerals. The diameters of the nodules are between 0.1 and $4 \mathrm{~mm}$ (exceptionally, up to $9 \mathrm{~mm}$ ). Graphite was observed in association with all other mineral components of the meteorite, i.e., sinoite, schreibersite, troilite, enstatite, and FeNi phases. The Raman spectra show graphite lines at $1580 \mathrm{~cm}^{-1}$ for $\mathrm{G}$ band, and $1355 \mathrm{~cm}^{-1}$ for $\mathrm{D}$ band; they also show broad peaks at $2700 \mathrm{~cm}^{-1}$. According to Lespade method [6], the graphite from Zakłodzie is determined as semiordered to ordered. X-ray diffraction results confirm the hexagonal structure of alphagraphite. Further investigations are in progress to establish more detailed structural characteristics of the Zakłodzie graphite.

References: [1] Burbine T. H. et al. 2000. Meteoritics \& Planetary Science 35:A36. [2] Keil K. and Bischoff A. 2008. Meteoritics \& Planetary Science 43:1233-1240. [3] Pilski A. S. 2004. 3rd Meteorite Conference in Poznań. pp. 41-46. [4] Przylibski T. A. et al. 2005. Meteoritics \& Planetary Science 40:A185-A200. [5] Karwowski Ł. et al. 2007. American Mineralogist 92:204-209. [6] Lespade P. et al. 1982. Carbon 20:427-430. 


\section{9}

CORRELATED NUCLEOSYNTHETIC ISOTOPE VARIABILITY IN $\mathrm{Cr}, \mathrm{Sr}, \mathrm{Ba}, \mathrm{Sm}, \mathrm{Nd}$, AND Hf IN MURCHISON AND QUE 97008 L. Qin ${ }^{1,2}$, R. W. Carlson', and C. M. O'D. Alexander ${ }^{1} .{ }^{1} \mathrm{DTM}$, Carnegie Institution of Washington, Washington, DC 20015, USA. ${ }^{2}$ Center for Isotope Geochemistry, Lawrence Berkeley National Laboratory, Berkeley, CA 94720, USA. E-mail: lqin@1bl.gov.

Introduction: Step-wise acid digestion is a very useful means of increasing the magnitude of nucleosynthetic isotope anomalies and probing the nature of the carriers of isotope anomalies in chondrites. We performed step-wise dissolution of two primitive meteorites, the CM2 chondrite Murchison and the L3.05 chondrite QUE 97008. The isotopic compositions of $\mathrm{Cr}, \mathrm{Sr}, \mathrm{Ba}, \mathrm{Nd}, \mathrm{Sm}$ and $\mathrm{Hf}$ were characterized. We also examined the consequences of nucleosynthetically-induced isotope variations for the interpretation of the variations of radiogenic isotope abundances.

Methods: Approximately $5 \mathrm{~g}$ of each meteorite was crushed to $<60 \mu \mathrm{m}$, and sequentially leached with (i) $8.5 \mathrm{~N}$ acetic acid (at room temperature for $24 \mathrm{~h}$ ), (ii) $6 \mathrm{~N} \mathrm{HNO}_{3}$ (at room temperature for $5 \mathrm{~d}$ ), (ii) $6 \mathrm{~N} \mathrm{HCl}$ (at $75{ }^{\circ} \mathrm{C}$ for $24 \mathrm{~h}$ ), and the residue was dissolved with an HF$\mathrm{HNO}_{3}$ mixtureusing a Parr Bomb (at $170{ }^{\circ} \mathrm{C}$ for $1 \mathrm{w}$ ). From each leach and residue, $\mathrm{Cr}, \mathrm{Sr}, \mathrm{Ba}, \mathrm{Nd}, \mathrm{Sm}$, and $\mathrm{Hf}$ were extracted and purified using conventional ion-exchange techniques. The isotopic compositions for all elements except Hf were measured on a Triton thermal ionization mass spectrometer. Hf isotopic compositions were determined on a $\mathrm{Nu}$ plasma multi-collector ICP-MS.

Results: Isotopic anomalies of nucleosynthetic origin are observed in all elements in all leaches and the residue of Murchison. In general, where resolved, the nucleosynthetic isotopic anomalies are 2-5 fold smaller in QUE 97008 than in Murchison. The difference in the magnitude of anomalies is similar to the difference in their matrix abundances consistent with the suggestion that matrix is the main carrier of anomalous material. The anomalies in all but $\mathrm{Cr}$ are best explained by variable additions of pure $s$-process nuclides to a background nebular composition slightly enriched in $r$-process isotopes compared to average Solar System material. Leaching leaves a residue in Murchison that is strongly enriched in $s$-process nuclides with depletions of over $1 \%$ in ${ }^{135} \mathrm{Ba}$ and $7 \varepsilon$ in ${ }^{84} \mathrm{Sr}$. Combined with concentration data, this indicates that the isotopic compositions of $\mathrm{Ba}$ and $\mathrm{Sr}$ in the Murchison residue are strongly influenced by $s$-process-rich presolar $\mathrm{SiC}$. Neodymium, Sm, and $\mathrm{Hf}$ display variable $s^{-}, r$-process nuclide abundances as do $\mathrm{Ba}$ and $\mathrm{Sr}$, but the anomalies are much smaller. Samarium shows variable relative abundances of the $p$-process isotope ${ }^{144} \mathrm{Sm}$ that do not correlate with ${ }^{142} \mathrm{Nd}$, suggesting that the direct $p$-process contribution to ${ }^{142} \mathrm{Nd}$ is small. Correcting for nucleosynthetic variability in $\mathrm{Nd}$ explains the range in ${ }^{142} \mathrm{Nd} /{ }^{144} \mathrm{Nd}$ seen between $\mathrm{C}$-, and O-, E-chondrites, but not the difference between chondrites and all modern Earth rocks, leaving decay of ${ }^{146} \mathrm{Sm}$ and a superchondritic $\mathrm{Sm} / \mathrm{Nd}$ ratio as the likely explanation for Earth's high ${ }^{142} \mathrm{Nd} /{ }^{144} \mathrm{Nd}$. The $s$-, $r$ - variations observed in Hf caution relying on isotopically anomalous refractory inclusions for the initial abundance of the short-lived ${ }^{182} \mathrm{Hf}$, a $r$-process nuclide, in the Solar System.
5129

ORIGIN OF THE CENTRAL MAGNETIC ANOMALY AT HAUGHTON IMPACT STRUCTURE, CANADA

Y. Quesnel ${ }^{1}$, J. Gattacceca ${ }^{1}$, G. R. Osinski ${ }^{2}$ and P. Rochette ${ }^{1}$. ${ }^{1}$ CEREGE, CNRS, Aix-Marseille University, Aix-enProvence, France, E-mail: quesnel@cerege.fr. ${ }^{2}$ Department of Earth Sciences, University of Western Ontario, London, Ontario, Canada.

Context: Located on Devon Island, Nunavut, Canada, the 23-km diameter Haughton impact structure is one of the best-preserved mediumsize complex impact structures. The impact occurred $\sim 39 \mathrm{Ma}$ ago in to a target formation composed of a 2-km thick sequence of Lower Paleozoic sedimentary rocks of the Arctic Platform overlying Precambrian metamorphic basement of the Canadian Shield [1]. Impact melt breccias filled the crater, while impact-related hydrothermal activity took place, but since then no significant geological event affected the area [2].

In the 1980s, ground magnetic and gravity measurements were carried out within the central part of the crater [3]. A significant anomaly was discovered and coarsely modeled by a source body of simple geometry. A recent airborne magnetic survey delivered additional data that covered the whole crater but no modeling was done [4].

Method: During Summer 2010, we performed a new ground magnetic survey to better characterize the central magnetic anomaly. A Geometrics G858 caesium vapor magnetometer was used. After correct removal of the external and core magnetic fields, the resulting dataset allowed us to produce a local magnetic field map of high quality at ground level, whereas [3] only used profiles. We precisely modeled the anomaly to constrain the geometry and magnetization of its source, also using results from laboratory measurements of magnetic properties (remanent magnetization and magnetic susceptibility) of all target lithologies.

Results: The amplitude of the central magnetic anomaly reaches $\sim 900 \mathrm{nT}$ over a distance of $1.5 \mathrm{~km}$. A local sharp transition of the gradient was identified, probably indicating a very shallow top of the magnetized source. Modeling indicates that a $0.7 \mathrm{~km}^{3}$ magnetized body with possible local superficial branches could be the source of this anomaly. Among the local lithologies, all Paleozoic sedimentary rocks are too weakly magnetic to account for the magnetic anomaly. Even the highest total (i.e. induced + remanent) magnetization intensities measured on basement samples are about $1 \mathrm{~A} \mathrm{~m}^{-1}$, slightly weaker than those resulting from source modeling $\left(1.5-2.5 \mathrm{~A} \mathrm{~m}^{-1}\right)$.

Discussion: The geometry of the modeled source body corresponds to the one expected with a central uplift. Magnetization property investigations exclude Paleozoic sedimentary rocks from comprising this inner part of the central uplift. Because the pre-impact magnetization intensities of basement formations cannot account for the magnetic anomaly too, an additional impact-related process is required. Postimpact hydrothermal alteration could have affected this uplifted basement, since evidence of such alteration is common at Haughton [2]. This geological process will enhance both remanent magnetization and magnetic susceptibility. In addition, the modeled magnetization directions correspond to the expected normal polarity regional directions of the main magnetic field when the impact occurred [5].

References: [1] Osinski G. R. et al. 2005. Meteoritics \& Planetary Science 40:1759-1776. [2] Osinski G. R. et al. 2005. Meteoritics \& Planetary Science 40:1859-1877. [3] Pohl J. et al. 1988. Meteoritics 23:235-238. [4] Glass B. J. et al. 2005. Abstract \#2398. 36th Lunar and Planetary Science Conference. [5] Gattacceca J. et al. 2011. This meeting. 
5026

A REAPPRAISAL OF THE METAMORPHIC HISTORY OF EH3 AND EL3 ENSTATITE CHONDRITES

E. Quirico ${ }^{1}$, M. Bourot-Denise ${ }^{2}$, C. Robin ${ }^{1}$, G. Montagnac ${ }^{3}$ and P. Beck $^{1}$. ${ }^{1}$ IPAG CNRS/University Grenoble 138041 Grenoble France. E-mail: eric.quirico@obs.ujf-grenoble.fr. ${ }^{2}$ LEME Museum National Histoire naturelle Paris France. ${ }^{3}$ Laboratoire de Géologie ENS Lyon France.

The thermal history of a series of EH3 and EL3 chondrites has been investigated by studying the degree of structural order of the organic matter (OM) located and characterized in matrix areas by Raman microspectroscopy [1]. By comparison with unequilibrated ordinary chondrites (UOCs) and $\mathrm{CO}$ and $\mathrm{CV}$ carbonaceous chondrites, the following petrologic types have been assigned to various E chondrites: Sahara 97096 and Alan Hills 84206: 3.1-3.4; Alan Hills 85170 and Parsa: 3.5; Alan Hills 85119: 3.7; Qingzhen, MacAlpine Hills 88136 and MacAlpine Hills 88184: 3.6-3.7. The petrologic type of Qingzhen is consistent with the abundance of the P3 noble gas component, a sensitive tracer of the grade of thermal metamorphism [2]. The petrologic types are qualitatively consistent with the abundance of fine-grained matrix for the whole series. No significant effects of shock processes on the structure of OM were observed. However such processes certainly compete with thermal metamorphism and the possibility of an effect cannot be fully discarded, in particular in the less metamorphosed objects. The OM precursors accreted by the EH3 and EL3 parent bodies appear to be fairly similar to those of UOCs and $\mathrm{CO}$ and $\mathrm{CV}$ carbonaceous chondrites. Raman data however show some slight structural differences that could be partly accounted for by shock processes. The metamorphic history of EH3 and EL3 chondrites has often been described as complex, in particular regarding the combined action of shock and thermal metamorphism $[3,4]$. Because OM maturity is mostly controlled by the temperature of peak metamorphism, it is possible to distinguish between the contributions of long duration thermal processes and that of shock processes. Comparison of the petrologic types with the closure temperatures previously derived from opaque mineral assemblages [5] has revealed that the thermal history of EH3 and EL3 chondrites is consistent with a simple asteroidal onion shell model. Thermal metamorphism in enstatite chondrites appears to be fairly similar to that which takes place in other chondrite classes. The complex features recorded by mineralogy and petrology and widely reported in the literature appear to be mostly controlled by shock processes.

References: [1] Quirico E. et al. 2011. Geochimica et Cosmochimica Acta 75:3088-3102. [2] Huss G. R. and Lewis R. S. 1994. Meteoritics 29:811-829. [3] Zhang Y. et al. 1995. Journal of Geophysical Research 100:9417-9438. [4] Kimura et al. 2005. Meteoritics \& Planetary Science 40:855-868. [5] Zhang Y. and Sears D. W. G. 1996. Meteoritics \& Planetary Science 31:647-655.
5098

NON-UNIFORM DISTRIBUTION OF THE MARTIAN REGOLITH COMPONENT IN SHERGOTTITES

M. N. Rao ${ }^{1}$, L. E. Nyquist ${ }^{2}$, D. K. Ross ${ }^{1}$ and J. Park ${ }^{3}$. ${ }^{1}$ Jacobs-ESCG, Johnson Space Center, Houston. TX. USA. E-mail: nageswara.rao@ nasa.gov. ${ }^{2}$ ARES, NASA Johnson Space Center, Houston. TX. USA. ${ }^{3}$ Chemistry Department, Rutgers University, Piscataway, NJ, USA.

Introduction: Shergottite samples that show evidence for thermal neutron irradiated $(\mathrm{n}, \gamma)$ products such as ${ }^{80} \mathrm{Kr}$ excesses and ${ }^{149} \mathrm{Sm}$ isotopic deficits necessarily contain a Martian regolith component because the $(\mathrm{n}, \gamma)$ products are produced only in the top few meters of the Martian surface $[1,2]$. The ${ }^{80} \mathrm{Kr}$ excesses from $\mathrm{Br}$ and the ${ }^{149} \mathrm{Sm}$ deficits determined in GRIM (gas-rich impact-melt) glasses, 27,8 and 104 from EET 79001 Lith A and B and in Shergotty-B yielded an integrated neutron fluence of $\sim 10^{15} \mathrm{n} / \mathrm{cm}^{2}$ to which the glass constituents were exposed near Mars surface [1,2]. Some of these glasses (8 and104) further show evidence for fast neutron $\left(\mathrm{N}\right.$, xp yn) products such ${ }^{83} \mathrm{Kr}$ and ${ }^{84} \mathrm{Kr}$ excesses from $\mathrm{Sr}$ and $\mathrm{Rb}$ yielding an integrated fast neutron $(\mathrm{N})$ fluence of $\sim 10^{16} \mathrm{~N} / \mathrm{cm}^{2}$ providing definitive evidence for a Martian regolith component in these glasses [2].

Results: We determined the ${ }^{149} \mathrm{Sm}$ isotopic deficit in the whole rock EET 79001, Lith B, amounting to a $\varepsilon{ }^{149} \mathrm{Sm}$ value of $-0.21 \pm 0.16$. This value corresponds to a thermal neutron fluence of $(0.52 \pm 0.39) \times 10^{15} \mathrm{n} / \mathrm{cm}^{2}$ to which this Lith B sample was exposed on Mars. This value is in agreement (within errors) with the fluence of $(1.33 \pm 0.75) \times 10^{15} \mathrm{n} / \mathrm{cm}^{2}$ determined using isotopic shifts in ${ }^{149} \mathrm{Sm}$ and ${ }^{157} \mathrm{Gd}$ isotopes by [3] in another Lith B (bulk) sample EET 79001,69. Because of this high fluence in bulk Lith B, we expected to find large pockets of secondary sulfides similar to those studied by us in, 104 (a GRIM) sample belonging also to Lth B [4]. We therefore studied a thin section EET 79001, 69 (Lith B) by EMPA/SEM techniques. However, we found very little immiscible sulfide melt blebs in this case.

In the case of EET 79001, Lith A bulk sample, we reported earlier [1] a thermal neutron fluence of $(0.24 \pm 0.31) \times 10^{15} \mathrm{n} / \mathrm{cm}^{2}$ which overlaps zero value. Further, this result is consistent with the observation that no ${ }^{80} \mathrm{Kr}$ excess was observed in the case of Lith A sample (EET 79001, 34) studied by [5] for $\mathrm{Kr}$ isotopic composition. However, in the case of GRIM glasses from Lith A, gypsum laths and S-rich aluminosiliactes were detected by [6] and positive elemental correlations between " $\mathrm{SO}_{3}$ ", and $\mathrm{CaO}$ and " $\mathrm{SO}_{3}$ " and $\mathrm{Al}_{2} \mathrm{O}_{3}$ were found by [7].

In the lherzolite Y00027 (bulk), $\mathrm{Kr}$ isotopic composition was determined by [8] and no evidence for the neutron capture ${ }^{80} \mathrm{Kr}$ on $\mathrm{Br}$ was found. But, in a melt-vein glass (GRIM) from the same lherzolite, they found evidence for the occurrence of ${ }^{80} \mathrm{Kr}$ excess from $\mathrm{Br}$ indicative of a regolith component.

These results suggest that the S-rich Martian regolith component is not uniformly distributed in shergottite GRIM glasses and bulk samples.

References: [1] Rao M. N. et al. 2011. Journal of Geophysical Research-Planets, in press. [2] Rao M. N. et al. 2002. Icarus 156:352-372. [3] Hidaka H. et al. 2009. Earth and Planetary Science Letters 288:564571. [4] Sutton S. et al. 2008. Abstract \#1961. 39th Lunar and Planetary Science Conference. [5] Becker R. H. and Pepin R. O. 1984. Earth and Planetary Science Letters 69:225-242. [6] Gooding J. L. and Muenow D. W. 1986. Geochimica et Cosmochimica Acta 50:1049-1059. [7] Rao M. N. et al. 2004. Abstract \#1501. 35th Lunar and Planetary Science Conference. [8] Nagao K. et al. 2008. Polar Science 2:195-214. 
5126

TEMPERATURE AND SPECTRAL PHASE EFFECTS ON HED METEORITES: IMPLICATIONS FOR DAWN AT VESTA

V. Reddy ${ }^{1,2}$, E. A. Cloutis ${ }^{3}$, A. Nathues ${ }^{1}$, J. P. Mann ${ }^{3}$, M. J. Gaffey ${ }^{2}$ and U. Schade ${ }^{4}{ }^{1}$ Max-Planck Institute for Solar System Research, Germany. E-mail: reddy@mps.mpg.de. ${ }^{2}$ University of North Dakota, USA. ${ }^{3}$ University of Winnipeg, Canada. ${ }^{4}$ Helmholtz Zentrum Berlin, BESSY II, Germany.

Introduction: Asteroid 4 Vesta is the first target of NASA's Dawn mission, which is expected to begin its yearlong orbital mission in July 2011. The Framing Camera (FC), one of the three scientific instruments onboard the spacecraft [1], contains a set of seven filters ranging from 0.44 to 0.97 microns in addition to a clear filter. While the VIR spectrometer on Dawn has larger spectral coverage (0.25-5 microns) and spectral resolution (2-10 nm), Dawn FC has higher spatial resolution (up to $\sim 20 \mathrm{~m})$ than VIR $(\sim 50 \mathrm{~m})$.

Spectral phase effects on Vesta have been already detected and approximately quantified by [2]. Gaffey [2] noted a change in the intensity of Vesta's 1-micron band with increasing phase angle. Hence we expect to see a wavelength dependent phase effect, which will alter the reflectance values of FC data depending on the band pass of the filter and viewing geometry (mainly phase angle). Beside the viewing geometry we expect also to observe spectral changes due to surface temperature, which will likely be present in the spectrometer data. Temperature uncertainties arise when spacecraft spectral data, obtained at much colder surface temperatures $(80-300 \mathrm{~K})$, is interpreted using laboratory calibrations developed at room temperatures $(\sim 300 \mathrm{~K})$. In an effort to pre-quantify these phase angle-, and temperature-induced spectral effects, we conducted a series of laboratory studies of HED meteorites.

Methodology: Laboratory spectral measurements (0.3-25 microns) of Moama eucrite and orthopyroxene PYX032 (analog for a diogenite) at different phase angles $\left(18-120^{\circ}\right)$ were measured at the University of Winnipeg Planetary Spectrophotometer Facility. Data of temperature spectral series were obtained from two published sources [3,4], and reanalyzed after resampling to Dawn FC wavelength ranges. Spectral band parameters like Band I and II centers, Band Area Ratio (BAR) and band depths were measured using methods described in [5].

Results: Using laboratory spectral measurements of HED meteorites at different phase angles and temperatures we have developed a set of equations to correct Dawn FC color images and VIR cubes for temperature and phase angle effects. We noted a general increase in band depths and BAR at the shortest phase angle $\left(13^{\circ}\right)$ and a shallow decline between $30^{\circ}$ and $90^{\circ}$ and a dramatic drop between $90^{\circ}$ and $120^{\circ}$ for Moama eucrite. Eucrites and diogenites show maximum shift in Band II center with increase in temperature. The equations will correct the spectral parameters to standard geometry $\left(0^{\circ}\right)$ for phase angle data and room temperature $(300 \mathrm{~K})$.

References: [1] Sierks et al. 2011. Space Science Reviews. [2] Gaffey. 1997. Icarus 127. [3] Hinrichs et al. 2002. Icarus 155. [4] Schade and Wäsch. 1999. Meteoritics \& Planetary Science 34. [5] Reddy. 2009. PhD dissertation, UND.
5006

PSEUDOTACHYLITIC BRECCIA AND IMPACT MELT ROCK FROM VREDEFORT: CHEMICAL AND ISOTOPIC SYSTEMATICS W. U. Reimold ${ }^{1}$, M. Thirlwall ${ }^{2}$ and T. Mohr-Westheide ${ }^{1}$. ${ }^{1}$ Museum for Natural History, Leibniz Institute at Humboldt University Berlin, Invalidenstrasse 43, 10115 Berlin, Germany E-mail: uwe.reimold@ mfn-berlin.de; tanja.mohr@mfn-berlin.de. ${ }^{2}$ Department of Earth Sciences, Royal Holloway University of London, Egham, Surrey TW20 0EX, UK. E-mail: m.thirlwall@gl.rhul.ac.uk.

Pseudotachylitic breccias (PTB) are frequently reported as characteristic melt breccia occurrences in impact structures. In contrast, notable occurrences are severely restricted, and massive ones occur only in the crater floors to the Vredefort Dome, South Africa, the Sudbury impact structure, Canada, and at Araguainha, Brazil. In recent years, particularly the Vredefort PTB have been studied extensively for structural, mineralogical and chemical evidence of the processes leading to the formation of these enigmatic rocks and for criteria that could be used to distinguish them from similar melt breccias. Extensive chemical analysis of PTB ranging from $<1 \mathrm{~mm}$ veinlets to many meter sized, massive occurrences have made a strong case for either local generation through shock compression melting (thin veinlets - akin to shock veins known from chondritic meteorites) or decompression melting ( $\mathrm{cm}$ to massive melt breccias) in the course of rapid formation of central uplifts [1]. The role of friction melting is, at best, limited-as evidenced by field and thin section observations. Nevertheless, these extensive chemical data are still challenged by a small group of workers who persist that massive PTB represent impact melt that intruded from the crater into the crater floor and thereby assimilated a lot of basement material. To further test this hypothesis - that, in any case, is not supported by the chemical data available to date, $\mathrm{Rb}-\mathrm{Sr}, \mathrm{Sm}-\mathrm{Nd}$ and $\mathrm{U}-\mathrm{Pb}$ isotopic analyses have been carried out by us on several Vredefort PTB samples from massive occurrences in granite and amphibolite, their respective host rocks, and-for comparison-several Vredefort Granophyre (impact melt rock) samples. The results demonstrate that PTB and their host rocks have significantly different isotopic systematics (recalculated to the impact age of $2.02 \mathrm{Ga}$ ) from that of the Granophyre. Furthermore, analyses of PTB/ HR from different locations yielded locally different isotopic results. This further supports the chemical results that PTB melt was formed from local reservoirs (the respective host rocks), which are different from the reservoir of impact melt (mixture of the full crustal sequence affected by the impact). With respect to the $\varepsilon N d$ values calculated for an age of 3.15 $\mathrm{Ga}$, the approximate age for the Archean granitoids that represent the host rock to PTB and the major precursor component of Granophyre, a significant variation between granitoid-hosted PTB and Granophyre is observed. In conclusion, not only the chemical data base but also these isotopic results do not support the "PTB =impact melt + assimilated" floor rock hypothesis - also in keeping with an isotopic study of Sudbury PTB and host rocks [2].

References: [1] Mohr-Westheide T. and Reimold W. U. 2010. GSA Special Paper 465. pp. 619-645. [2] Lafrance B. and Kamber B. 2010. Precambrian Research 180:237-250. 
5281

\section{THE POSSIBLE ORIGIN OF THE NON MASS-DEPENDENT ISOTOPE FRACTIONATION IN OZONE AND ITS EXTENSION TO COSMOCHEMISTRY}

P. Reinhardt ${ }^{1}$ and F. Robert ${ }^{2}$. ${ }^{1}$ Lab. Chimie Théorique. CNRS - UPMC UMR 7616 - 75252 Paris. E-mail: reinh@lct.jussieu.fr. ${ }^{2}$ LMCM, CNRSMNHN, UMR7202, Case 52, 61 Rue Buffon, 75005 Paris, France. E-mail: robert@mnhn.fr.

Introduction: A non-mass-dependent oxygen isotopic compositions was reproduced through the synthesis of ozone [1]. Numerous theoretical models [2-3] have been proposed to account for the almost equal enrichment in ${ }^{17} \mathrm{O}$ and ${ }^{18} \mathrm{O}$ relative to ${ }^{16} \mathrm{O}$-referred in the literature to as "the non-mass dependent isotopic fractionation." The generally accepted theory is based on a modification of the standard RRKM model of unimolecular dissociation [2].

Here we follow a different approach. Ozone is formed via the stabilization of the complex $\mathrm{O}_{3} *\left(\mathrm{O}_{3} *+\mathrm{M} \rightarrow \mathrm{O}_{3}+\mathrm{M}\right)$ where $\mathrm{O}_{3} *$ results from $\mathrm{O}+\mathrm{O}_{2} \rightarrow \mathrm{O}_{3}^{*}$. We propose that the lifetime of $\mathrm{O}_{3} *$ is different if the isotopes involved in the reaction are dis- or indistinguishable; for example: ${ }^{16} \mathrm{O}+{ }^{17} \mathrm{OO} \rightarrow{ }^{16} \mathrm{O}^{17} \mathrm{OO}^{*}$ or ${ }^{16} \mathrm{O}+{ }^{16} \mathrm{OO} \rightarrow{ }^{16} \mathrm{O}^{16} \mathrm{OO}^{*}$, respectively (the isotopically non labeled $\mathrm{O}$ atom of the molecule has no role in this process). In our calculations, in order to emphasize this idea, the masses of the oxygen isotopes were numerically labeled but their mass differences were ignored (all calculations were actually performed for $16 \mathrm{amu})$.

Principle: In our numerical simulation, $\mathrm{O}_{3} *$ is produced via the collision between $\mathrm{O}$ and $\mathrm{O}_{2}$ but the third body $\mathrm{M}$ is absent. Therefore $\mathrm{O}_{3}$ * always disintegrate. However, we assume (as in most chemical models) that for a lifetime $\tau$ longer than a critical value $\tau_{\text {Crit. }}$ the complex can be stabilized. Therefore, without an explicit model for ozone formation, we extrapolate our simulations to a real situation. We note $\tau$ the average lifetime of the complex in a range of $\tau$ values where $\mathrm{O}_{3}{ }^{*}$ can be stabilized as $\mathrm{O}_{3}$ and $\mathrm{P}$ the probability to find it; the subscripts $\mathrm{R}$ and NR stand for isotope exchange (Reactive) and non-exchange (NonReactive) reactions, respectively. The effect arises from the fact that the partition $w$ of the average lifetime is: $w=\tau_{\mathrm{R}} \mathrm{P}_{\mathrm{R}}+\tau_{\mathrm{NR}} \mathrm{P}_{\mathrm{NR}}$ when isotopes are distinguishable but $w=\left(\tau_{\mathrm{R}}+\tau_{\mathrm{NR}}\right) 1 / 2\left[\mathrm{P}_{\mathrm{R}}+\mathrm{P}_{\mathrm{NR}}\right]$ when they are indistinguishable. Lifetimes can be derived from these partitions.

Results: The scattering is carried out within classical trajectory simulations, in an ab-initio potential of three oxygen atoms in a singlet ground state. This energy surface was calculated by the group of $\mathrm{R}$. Schinke [4] with up-to-date quantum chemical means. The calculated isotopic fractionation factor $\alpha$ reproduces (1) the experimental results (for ${ }^{16} \mathrm{O}^{17} \mathrm{O}^{18} \mathrm{O}$ the calculated $\alpha$ is 1.26 compared with 1.21 for the measured value) and (2) the observed variations with $\mathrm{P}$ and $\mathrm{T}[5,6]$.

Cosmochemical Consequences: Since the present interpretation does not depend on the interaction potential involved in the formation of the complex, the M. Thiemens' proposal [7] according to which the ozone effect is relevant to Cosmochemistry is supported by the present treatment.

References: [1] Thiemens M. H. and Heidenreich III J. E. 1983. Science 219:1073. [2] Gao Y. Q. and Marcus R. M. 2001. Science 293:259. [3] Babikov D. et al. 2003. The Journal of Chemical Physics 119:2577. [4] Schinke R. and Fleurat-Lessart P. 2004. The Journal of Chemical Physics 121:5789. [5] Morton J. et al. 1990. Journal of Geophysical Research 95:901. [6] Janssen C. and Tuzson B. A. 2010. The Journal of Chemical Physics 114:9709. [7] Thiemens M. H. 2006. Annual Review of Earth and Planetary Sciences 34:217.
5327

PARENT BODY INFLUENCE ON ORGANIC MATTER: THE MESSAGE FROM PARIS

L. Remusat ${ }^{1}$, S. Bernard ${ }^{1}$, C. Le Guillou ${ }^{2}$ and A. Brearley ${ }^{2}$. ${ }^{1}$ LMCM, CNRS-MNHN, UMR7202, Paris, France. ${ }^{2}$ Department of Earth and Planetary Sciences, UNM, Albuquerque, USA. E-mail: remusat@mnhn.fr.

Introduction: Among the CM carbonaceous chondrites, Paris appears to be the less altered and has experienced mild thermal metamorphism [1]. Like other CM, Paris exhibits variations in alteration state within the same sample. This unique object among the CM class should shed new light on the parent body influence on the isotopic and molecular structure of the organic matter in carbonaceous chondrite. The effects of fluid circulation on chondritic organics remain unclear and have implications on the interpretations of the isotopic and molecular signatures of the insoluble organic matter (IOM) in chondrites [2, 3].

Recently, the analysis of organic matter in several pieces of Tagish Lake (ungrouped $\mathrm{C} 2$ chondrite intermediate between $\mathrm{CI}$ and $\mathrm{CM}$ ) showing variable degrees of alteration has revealed chemical and isotopic heterogeneities for IOM and soluble compounds [4, 5]. These observations have lead to the conclusion that aqueous alteration has induced modifications of organic matter. It was also concluded that isotopic exchange has altered the $\mathrm{D} / \mathrm{H}$ signature of the IOM in Tagish Lake. This conclusion is in contrast with the in situ NanoSIMS observation that the isotopic signature of organic particles is poorly affected by hydrothermal alteration [3]. The aim of this study is to compare the molecular properties and isotopic signature (measured in situ) of organic particles in the two lithologies found in Paris meteorite.

Experimental: A polished section of Paris meteorite was prepared without epoxy and was studied by NanoSIMS. Several areas on the least and most altered regions were rastered by a focused $\mathrm{Cs}^{+}$beam to generate secondary ion images of $\mathrm{H}^{-}, \mathrm{D}^{-},{ }^{12} \mathrm{C}^{-},{ }^{16} \mathrm{O}^{-},{ }^{26} \mathrm{CN}^{-},{ }^{28} \mathrm{Si}^{-}$and ${ }^{32} \mathrm{~S}^{-}$. The spatial resolution is estimated to be $230 \mathrm{~nm}$. Several FIB (focused ion beam) sections were prepared from the same areas for STXM (Scanning Transmission X-ray Microscopy) experiment to identify the functional groups and TEM (Transmission Electron Microscopy) study of the textural associations between organics and minerals.

Results and Discussion: Like in other CM, Paris exhibits micron size organic particles distributed randomly in the matrix. However, some areas showing a high signal in $\mathrm{S}$ and $\mathrm{H}$ lack organic particles. TEM should allow the characterization of these phases. Our NanoSIMS data indicate that the $\mathrm{D} / \mathrm{H}$ ratio of organic particles in the heavily and poorly altered matrices is undistinguishable. Most of the organic particles exhibit $0<\delta \mathrm{D}<2000 \%$; these data are consistent with other CM matrices [3]. A few D-rich hot spots are also observed, with a maximum at $\delta \mathrm{D}=7950 \pm 330 \%$. $\mathrm{C} / \mathrm{H}$ and $\mathrm{N} / \mathrm{C}$ are also similar in the two lithologies, though $\mathrm{C} / \mathrm{H}$ in Paris appears significantly lower than their counterparts in other CM.

Our data thus indicate that aqueous alteration on CM parent body does not induce a significant modification of the composition of organic particles. Further STXM and TEM observations will be presented and will be used to check these interpretations based on NanoSIMS imaging.

References: [1] Zanda B. et al. 2010. Abstract \#5312. 73rd MetSoc Meeting. [2] Alexander C. M. O'D. et al. 2007. Geochimica et Cosmochimica Acta 74:4417-4437. [3] Remusat L. et al. 2010. The Astrophysical Journal 713:1048-1058. [4] Glavin D. P. et al. 2010. Abstract \#5131. 73rd MetSoc Meeting. [5] De Gregorio B. T. et al. 2010. Abstract \#5398. 73rd MetSoc Meeting. 
5212

D/H RATIOS OF HIGHLY EVOLVED LUNAR ROCKS

K. L. Robinson ${ }^{1}$, G. J. Taylor ${ }^{1}$, E. Hellebrand ${ }^{1}$ and $\mathrm{K}$. Nagashima ${ }^{1}$. ${ }^{1}$ HIGP, UHNAI, University of Hawai'i at Mãnoa. E-mail: krobinson@higp.hawaii.edu.

Introduction: The felsites are highly evolved lunar rocks, typically containing approximately $70 \mathrm{wt} \% \mathrm{SiO}_{2}$. Most have igneous textures that feature graphic intergrowths of K-feldspar and quartz [1]. Sampled felsites occur as clasts in breccias, sometimes coexisting with Fe-rich, low-Si clasts. This pairing of Fe-rich and Si-rich compositions suggests that the felsites could have formed through silicate liquid immiscibility from a magma with KREEP basalt composition in small intrusive bodies [1].

Recently, water has been detected in lunar pyroclastic glasses [2] and apatites $[3,4]$. Apatite $\left[\mathrm{Ca}_{5}\left(\mathrm{PO}_{4}\right)_{3}(\mathrm{~F}, \mathrm{Cl}, \mathrm{OH})\right]$ incorporates water as $\mathrm{OH}$ into its crystal structure. At low abundances, water acts like an incompatible element and will become enriched in the melt. Thus, KREEP-rich magmas should be enriched in water when apatite begins to crystallize. Apatites in felsites could contain water sufficient to allow the $\mathrm{D} / \mathrm{H}$ isotopic composition of the water to be determined, which is important for determining the source of the Moon's water and the extent of water loss during magmatic processing [e.g., 4, 5].

We identified four apatite grains large enough $(>20 \mu \mathrm{m})$ for SIMS analysis in two lunar rocks. Sample 14321,1047 is a quartz-K-feldspar felsite, while sample 77538,16 consists of felsite and associated Fe-rich phases; the apatite was within the Fe-rich phase.

Methods: Apatites were analyzed for $\mathrm{P}, \mathrm{Ca}, \mathrm{Si}, \mathrm{Na}, \mathrm{Fe}, \mathrm{Ce}, \mathrm{F}, \mathrm{Cl}$, $\mathrm{Y}$, and $\mathrm{La}$ with the UH JXA-8500F electron microprobe. Hydrogen isotopic composition and water content were analyzed in situ with the UH ims 1280 ion microprobe with a $\mathrm{Cs}^{+}$primary beam. $\mathrm{H}_{2} \mathrm{O}$ content of apatite samples was estimated from a calibration curve on $\mathrm{H}_{2} \mathrm{O}(\mathrm{wt} \%)$ versus ${ }^{1} \mathrm{H} /{ }^{18} \mathrm{O}$ determined using four apatite standards with different $\mathrm{H}_{2} \mathrm{O}$ contents (provided by F. McCubbin). The detection limit for $\mathrm{H}_{2} \mathrm{O}$ was approximately $100 \mathrm{ppm}$. Uncertainties on $\delta \mathrm{D}$ and water content are approximately $75 \%$ and $30 \%(2 \sigma)$, respectively.

Results: We analyzed a total of five points among four apatite grains in two samples. The single large apatite in 14321,1047 had $\mathrm{H}_{2} \mathrm{O}$ below our detection limit in both points analyzed. Two apatite grains were measured in 77538,16 . They have $\mathrm{H}_{2} \mathrm{O}$ contents of approximately $180 \mathrm{ppm}$. $\delta \mathrm{D}$ values for these points are $+300-+400 \%$.

Discussion: We found very little water in the apatites measured. Apatites in both samples contain significant (wt \% level) amounts of REEs, indicating that these highly-evolved, KREEP-rich magmas contained little water. In turn, this suggests that the lunar magma ocean (and the bulk Moon) had a low initial water content, as argued by [5]. The $\delta \mathrm{D}$ values in apatite in 77538 with detectable water are elevated, which agrees with findings in [4]. However, since the measured $\mathrm{H}_{2} \mathrm{O}$ is barely above detection limit, we cannot say with absolute certainty that the $\delta \mathrm{D}$ of lunar water in this magmatically evolved lunar rock is elevated with respect to Earth.

References: [1] Robinson K. L. and Taylor G. J. 2011. Abstract \#1257. 42nd Lunar and Planetary Science Conference. [2] Saal A. E. et al. 2008. Nature 454:192-195. [3] McCubbin F. M. et al. 2010. PNAS 27:11223-11228. [4] Greenwood J. P. et al. 2011. Nature Geoscience 4:7982. [5] Elkins-Tanton L. et al. 2011. Earth and Planetary Science Letters, in press.
5479

WESTON: THE RECOVERY AND DISPERSION OF A HISTORICALLY SIGNIFICANT METEORITE FALL

M. C. Robson. John J. McCarthy Observatory, New Milford, C 06776, USA. E-mail: Monty.Robson@mccarthyobservatory.org.

Introduction: Weston, the first scientifically documented meteorite fall in the New World, occurred in the decade in which most scientists finally agreed that stones do fall from the sky. It fell during morning astronomical twilight on 14 December 1807. Seven fragments were recovered from an elliptical strewn field in central Fairfield County, Connecticut, USA. This $12 \mathrm{~km}$ by $4 \mathrm{~km}$ ellipse is orientated north-south and centered on $\mathrm{N} 41^{\circ} 16^{\prime}$ and $\mathrm{W} 73^{\circ} 16^{\prime}$ [1].

The first fragments were recovered within about half an hour of the fall and the last of the seven within three weeks. The consensus of modern literature is that $150 \mathrm{~kg}$ of the meteorite was recovered. But meteoritic scholars from Wülfing [2] to Koblitz [3] only account for about $20 \mathrm{~kg}$. In the first edition of the Catalogue of meteorites, G. T. Prior [4] added a note for Weston which has been included in all subsequent editions of the work: "Comparatively little has been preserved." Reasons for this apparent mass discrepancy will be discussed.

In an age when the fastest communication and transportation were limited to horse speed it seems remarkable how rapidly the mass of Weston was dispersed around the world. Unfortunately this took place without good record keeping so the history of only one Weston fragment is easily traced from recovery to today. Ongoing research now indicates two more fragments may have a completely known history.

Discussion: Two teams investigated the fall sites of Weston. Dr. Isaac Bronson and Rev. Dr. Horace Holley conducted the first investigation on December 19, 1807. The better-known on-site expedition to the area was performed by Yale College professors Benjamin Silliman and James Kingsley starting two days later. A careful reading of both the reports generated from these expeditions $[5,6]$ is necessary to understand the recovery of Weston. Together these reports allow a recovery chronology to be developed that includes when, where, and by whom the fragments were recovered. The reports also describe the fall circumstances and give mass estimates for each fragment. This valuable information shows why so little of the meteorite can be accounted for today.

Acknowledgments: Sincere thanks go to these researchers for professional courtesy during visits to their collections: Dr. Timothy McCoy, National Meteorite Collection, Smithsonian Institution, Washington D.C.; Dr. Caroline Smith, Natural History Museum, London; Dr. Laurence Garvie, Center for Meteorite Studies, Tempe. Thanks also to Dr. Franz Brandstätter, of the Naturhistorisches Museum, Vienna, for his valuable correspondence.

References: [1] Robson M. and Pagliaro F. 2009. Meteoritics \& Planetary Science 44:1343-1354. [2] Wülfing E. A. 1897. Die meteoriten in sammlungen und ihre literature. Tübingen: H. Laupp'schen. 460 p. [3] Koblitz, Journal MetBase 7.1. CD-ROM version 7.1. [4] Prior G. T. 1923. Catalogue of meteorites with special reference to those represented in the collection of the British Museum (Natural History). London: British Museum. ix +196 p. [5] Bronson I. 1807. Letter to the editor. New-York Commercial Advertiser. December 28, 1807. [6] Silliman B. and Kingsley J. 1807. Letter to the editor. New Haven, Connecticut, Herald December 29, 1807. 
5038

COSMIC SPHERULE DENSITY IN SOIL AND SURFACE EXPOSURE AGE IN THE ATACAMA DESERT, CHILE

P. Rochette, A. Hutzler, A. Nyindong, S. Spitale, D. Bourles, R. Braucher, P. Chaurand and J. Gattacceca. CEREGE, CNRS/Université Aix-Marseille, Aix-en-Provence, France.

We have sampled various surfaces in the Atacama Desert during our meteorite search expeditions [1] and determined cosmic spherule (CS) density within these surfaces. Soil was sampled down to $10 \mathrm{~cm}$ depth on a surface varying from 0.1 to $1 \mathrm{~m}^{2}$. Raw soil was sieved in the $200-800 \mu \mathrm{m}$ fraction and subjected to magnetic extraction either on site (for larger samples) or back to the laboratory. CS larger than $200 \mu \mathrm{m}$ were picked under the binocular and their extraterrestrial nature confirmed by SEM imaging and micro-chemical analysis using $\mu$ XRF. CS $>200 \mu \mathrm{m}$ density varies in the eight sites sampled from 100 to $2500 \mathrm{CS} \mathrm{m}^{-2}$. Repeated sampling on the same surface for three sites (within a $\mathrm{km}$ distance) yield consistent CS densities. For the richest samples logarithmic cumulative size distribution slope around -5 indicates minor size distortion with respect to Antarctic collections, suggesting little horizontal (by wind or water) or vertical (by percolation below the sampled surface) transport. In those sites the proportion of Iron CS (ICS) is about 4\%, again in agreement with Antarctic collections, taking into account the lack of glass CS due to magnetic extraction. However, in less rich samples, the size distribution slope (up to -3 ) and the ICS\% (from 15 to $60 \%$ ) indicate some density and size sorting. Assuming that ICS $>200 \mu \mathrm{m}$ is the least mobile fraction of the CS flux, due to size, resistance to weathering and density, we propose that their density may be a proxy of surface age.

To test this hypothesis, surface exposure ages have been determined using in situ produced ${ }^{10} \mathrm{Be}$ content in large quartz pebbles sampled on the same surfaces. Measurements were performed on ASTER, the 5MV AMS located at CEREGE [2]. Surface exposure ages calculated assuming no erosion vary from 0.15 to $6 \mathrm{Myr}$. Correlation between ICS density and exposure ages appears good in all sites except one, from a different climatic zone. Silicate CS density is much less well correlated with exposure age, comforting our hypothesis of higher reliability of ICS density. Drawback is that larger samples are needed to recover a significant ICS number.

References: [1] Gattacceca et al. 2009. Abstract \#5083. Meteotrical Society Meeting. [2] Arnold M. et al. 2010. Nuclear Instruments and Methods in Physics Research B 268:1954-1959. Doi:10.1016/ j.nimb.2010.02.107.
5193

PETROGRAPHY AND MODAL ABUNDANCE OF METAL IN UREILITES: A COMBINED 2D AND 3D STUDY

A. J. Ross ${ }^{1,2}$, D. C. Hezel ${ }^{1}$, L. E. Howard ${ }^{1}$, C. L. Smith ${ }^{1}$, H. Downes ${ }^{2}$, J. S. Herrin, P. Jenniskens ${ }^{3}$ and M. Shaddad ${ }^{4}$. ${ }^{1}$ Mineralogy Department, Natural History Museum, London, UK. E-mail: aidan.ross@ucl.ac.uk. ${ }^{2}$ Centre for Planetary Sciences at UCL/Birkbeck, London, UK. ${ }^{3}$ SETI Institute, Mountain View, CA, USA. ${ }^{4}$ Physics \& Astronomy Department, University of Khartoum, Khartoum, Sudan.

Introduction: Most unbrecciated ureilites are believed to represent intact samples of the ureilite parent body (UPB) mantle, consisting predominantly of olivine and pyroxenes with accessory carbon, metal and sulfide [1]. Although much of the original metal has been lost [2], what remains records partial melting and differentiation processes on the UPB. Knowing the modal abundances of metals within ureilite samples helps with the interpretation of bulk siderophile elements $[2,3]$ and of magnetic susceptibility [4]. X-ray computed tomography (CT) scanning allows nondestructive 3D imaging of samples, visualizing 3D spatial relationships which are not apparent in traditional petrographic studies of individual $2 \mathrm{D}$ thin sections.

We have analyzed a range of ureilite samples in $2 \mathrm{D}$ and $3 \mathrm{D}$, including several samples of the anomalous ureilite Almahata Sitta (AS) [5]. As a quickly recovered fall, AS samples do not show oxidation of metal as seen in most other ureilites.

Methods: Samples were scanned using the Metris X-Tek HMX ST 225 CT system at the NHM and reconstructed using CT-PRO 2.0. Analysis of the scans was performed using VG Studio Max 2.0, using greyscale thresholding to separate phases. Thin sections or probe blocks were examined using the LEO 1455 VP SEM at the NHM. Whole section EDS X-ray chemical maps were acquired using Oxford Instruments INCA software and combined to differentiate minerals. These were processed by pixel counting in Photoshop to obtain modal mineralogies.

Results and Discussion: Metal in Almahata Sitta shows a large variation in modal percent and appearance between samples. For example, from SEM data AS\#22 has approximately $1.8 \%$ metal with grain boundary metal not exceeding approximately $35 \mu \mathrm{m}$ in width, whereas AS\#44 shares many features with the anomalous ureilite LAR 04315 and is particularly metal-rich, with approximately $3.4 \%$ metal and some unusually large grains $>250 \mu \mathrm{m}$ in size. These percentages include siderophile-bearing metal in veins and primary inclusions as well as siderophile-free secondary reduction metal. All metal affects magnetic susceptibility, whereas bulk siderophile concentrations are not affected by reduction metal. Reduction metal makes up a greater percentage of the metal content of AS\#22 than AS\#44. Preliminary data analysis suggests that whilst it is easier to differentiate metal and sulfide using the SEM, carbon is harder to quantify in 2D as SEM samples are carbon-coated. The main sources of error in extrapolating the data are likely to be small sample size and sample heterogeneity.

Acknowledgments: We would like to thank R. Abel, A. T. Kearsley and A. Ball for equipment assistance. We thank ANSMET and the NHM for comparative ureilite samples.

References: [1] Mittlefehldt D. W. et al. 1998. In Planetary materials. Reviews in Mineralogy 36. [2] Rankenburg K. et al. 2008. Geochimica et Cosmochimica Acta 72:4642-4659. [3] Friedrich J. M. et al. 2010. Meteoritics \& Planetary Science 45:1718-1727. [4] Kohout T. et al. 2010. Meteoritics \& Planetary Science 45:1778-1788. [5] Jenniskens P. et al. 2009. Nature 458:485-488. 
5402

\section{ANALYSIS OF SECTIONED AEROGEL TRACKS OF COMET WILD 2 PARTICLES WITH C60-TOF-SIMS}

D. Rost, T. Henkel and I. Lyon. The University of Manchester, Manchester M13 9PL, UK. E-mail: detlef.rost@manchester.ac.uk.

Introduction: The Stardust mission returned material from comet Wild 2 captured in aerogel. The analysis of these samples is a challenging task, especially for organic compounds. Driven by the impact explosion, some if not most of the surviving organics are found in the neighboring aerogel $[1,2]$. There is intrinsic contamination in the aerogel $[2,3]$ and further terrestrial contamination should be avoided by analyzing samples as unaltered as possible, i.e., not pressed or embedded. Preferably, the spatial resolution should be on the scale of the (sub)micrometer sized surviving cometary particles. Finally, minimal sample consumption allows comprehensive examination with additional methods. Meeting all these needs is very challenging (e.g., two-step laser mass spectrometry is limited to spatial resolution $>10 \mu \mathrm{m}[1,2])$.

Method: Recent developments in time-of-flight secondary ion mass spectrometry (ToF-SIMS) offer a viable new approach. Using cluster ions as the primary beam enables a gentle and efficient desorption of large molecules from the sample surface while a spatial resolution of down to $1 \mu \mathrm{m}$ or less can be achieved. $\mathrm{C}_{60}$ clusters have been shown to be most efficient for detecting organic compounds [e.g., 4], but also many major and some minor and trace elements can be measured at the same time with high accuracy [5]. For this study we use one of our ToF-SIMS instruments equipped with a $40 \mathrm{kV} \mathrm{C}_{60}$ primary ion gun. We have previously demonstrated that some organics, including polycyclic aromatic hydrocarbons (PAHs), can be easily detected as intact molecules with only minor fragmentation [6]. We have also measured aerogel surface exposed to the cometary particle flux [7] and found indications for an enrichment in organics relative to unexposed blank flight aerogel.

Results: We analyzed a longitudinal section of track 15 (tile C2012) including adjacent aerogel. The untreated sample is held in place by a covering grid. We found organic compounds at constant background levels throughout the aerogel with no apparent enrichment toward the cometary track. This includes the characteristic PAHs naphthalene, phenanthrene and pyrene. Several micrometer sized enrichments of $\mathrm{Na}$, $\mathrm{Mg}, \mathrm{Al}$, and $\mathrm{K}$ indicate inorganic cometary material stuck in the track walls.

Discussion: While organics dispersed into the surrounding aerogel have been identified in more than half of the tracks examined for this feature [1, 8], not all tracks show them [8]. Since bulbous tracks indicate a violent explosion upon particle entry their impactors are thought of being rich in volatile and organic components. Our analyzed track, however, is of carrot shape and at only $250 \mu \mathrm{m}$ in length rather short. Therefore, it is reasonable that the impacting cometary particle contained only very few volatile and organic components, in accordance with our results.

Further optimizations to our instrument are planned to improve our detection threshold for organics. We plan to analyze more tracks, especially of the bulbous type.

References: [1] Sandford S. A. et al. 2006. Science 314:1720-1724. [2] Clemett S. J. et al. 2010. Meteoritics \& Planetary Science 45:701-722. [3] Sandford S. A. et al. 2010. Meteoritics \& Planetary Science 45:406433. [4] Fletcher J. S. et al. 2006. Applied Surface Science 252:6513-6516. [5] Henkel T. et al. 2009. Rapid Communications in Mass Spectrometry 23:3355-3360. [6] Rost D. et al. 2010. Abstract \#1973. 41st Lunar and Planetary Science Conference. [7] Rost D. et al. 2009. Abstract \#2480. 40th Lunar and Planetary Science Conference. [8] Bajt S. et al. 2009. Meteoritics \& Planetary Science 44:471-484.
5502

SOLAR SYSTEM INITIAL ${ }^{176} \mathrm{Hf} /{ }^{177} \mathrm{Hf}$ INFERRED FROM THE EUCRITE NORTHWEST AFRICA 5073

J. Roszjar ${ }^{1}$, E. E. Scherer ${ }^{2}$, K. Mezger ${ }^{3}$ and A. Bischoff ${ }^{1}{ }^{1}{ }^{1}$ Institut für Planetologie, WWU Münster, Germany. E-mail: j_rosz01@uni-muenster.de. ${ }^{2}$ Institut für Mineralogie, WWU Münster, Germany. ${ }^{3}$ Institute of Geological Sciences, University of Bern, Switzerland.

Introduction: Northwest Africa (NWA) 5073 is a new Stannerntrend eucrite with the coarsest texture ever found among eucrite samples [1]. It is mainly composed of pyroxene and plagioclase and comprises abundant mesostasis. The mesostasis primarily consists of tridymite, Ni-poor metallic Fe, troilite, plagioclase, ilmenite, chromite, Caphosphate, zircon and baddeleyite. Zr-bearing phases are abundant and up to $25 \mu \mathrm{m}$ in diameter [1]. Previous Lu-Hf analyses on this sample showed that the whole rock plotted on the external isochron for other basaltic eucrites, but that complex internal Lu-Hf systematics, yielded two ages, $4.68 \pm 0.14 \mathrm{Ga}$ and $4.31 \pm 0.14 \mathrm{Ga}$, and two apparent initial values $\left({ }^{176} \mathrm{Hf} /{ }^{177} \mathrm{Hf}_{\mathrm{i}}\right), 0.27976 \pm 13(2 \sigma)$ and $0.28020 \pm 8(2 \sigma)[2]$. These results correlate with the petrographic observation that this sample had a complex thermal history [1]. To better define the internal isochron for this rock and to better constrain the Solar System initial ${ }^{176} \mathrm{Hf} /{ }^{177} \mathrm{Hfvalue}$, we separated unradiogenic ilmenite and zircon from the sample.

Methods: Zircon and ilmenite were separated from about $6.7 \mathrm{~g}$ of cleaned and crushed sample. These minerals were concentrated with a selective acid digestion procedure using $\mathrm{HF}-\mathrm{HNO}_{3}-\mathrm{HCl}$. Via handpicking, a pure ilmenite and an ilmenite-zircon-mixture mineral fraction were separated. Chemical separation and isotope analysis procedures were those of [3] and [4]. Samples were analyzed for their Lu-Hf isotopic composition using an IsoProbeMC-ICP-MS. The accuracy and 2 s.d. external precision of $\mathrm{Hf}$ isotope analyses as a function of $\mathrm{Hf}$ concentration were evaluated by repeated measurement of standard solutions at different intensities [5]. Ages were calculated using a decay constant of $1.867 \times 10^{-11}$ year $^{-1}$ for ${ }^{176} \mathrm{Lu}$ (e.g., $\left.[3,6]\right)$.

Results and Conclusion: The ilmenite fraction lies above the older, $4.68 \mathrm{Ga}$ isochron at low $\mathrm{Lu} / \mathrm{Hf}$. The ilmenite + zircon fraction, together with the whole rock and three pyroxene fractions lie on a $4.61 \pm 0.11 \mathrm{Ga}$ trend. Although this is within error of the expected age of the sample, the initial ${ }^{176} \mathrm{Hf} /{ }^{177} \mathrm{Hf}$ of 0.279832 (77) is higher than that of some other eucrite isochrons. This may suggest that ilmenite in the ilmenite + zircon fraction either partially re-equilibrated isotopically during a heating event or it lost some Lu during the selective digestion procedure. In either case, it seems that a pure zircon fraction, if recovered, might yield a somewhat lower initial ${ }^{176} \mathrm{Hf} /{ }^{177} \mathrm{Hf}$ than the value reported here.

References: [1] Roszjar J., Metzler K., Bischoff A., Barrat J.-A., Geisler T., Greenwood R. C., Franchi I. A., and Klemme S. 2011. Meteoritics \& Planetary Science, submitted. [2] Roszjar J. and Scherer. E. E. 2010. Meteoritics \& Planetary Science 45:A175. [3] Scherer E. E., Münker C., Mezger K. 2001. Science 293:683-686. [4] Münker C., Weyer S., Scherer E., and Mezger K. 2001. Geochemistry Geophysics Geosystems 2:1064, doi: 10.1029/2001GC000183. [5] Bizzarro M., Baker J. A., Haack H., Ulfbeck D., and Rosing M. 2003. Nature 421:931-933. [6] Söderlund U., Patchett P. J., Vervoort J. D., and Isachsen C. E. 2004. Earth and Planetary Science Letters 219:311-324. 
5376

A POSSIBLE PAIRED FALL OF NICOLÁS LEVALLE FROM ARGENTINA AND ITS P IN CHONDRULES

R. Rönick ${ }^{1}$, T. Ntaflos ${ }^{1}$ and H. Palme ${ }^{2}{ }^{1}$ University of Vienna, Althanstrasse 14, A-1090, Vienna. (roman.roenick@univie.ac.at). ${ }^{2}$ Senckenberg Forschung, Senckenberganlage 25, D- 60325, Frankfurt am Main.

Introduction: Recently a meteorite from Argentina was classified as L5 and named Nicolás Levalle (Meteoritical Bulletin Database, in press). We examined a meteorite which is presumably paired to this possible fall considering the date and location which was reported to us from locals (Mr. Blanco). We classified our sample as LL4. In addition, we studied this meteorite focusing on the abundance and distribution of $\mathrm{P}$ in chondrules.

Petrology and Mineral Chemistry: Modal abundances are $78 \%$ chondrules (PO, BO, RP), 14\% matrix, 5\% troilite, $1 \%$ taenite, $1 \%$ chromite and $1 \%$ minor phases (e.g., apatite). The $\mathrm{Fa}$ content of the olivines is $26.00 \pm 0.33$ mole $\%, n=67$ and the orthopyroxene composition is Fs $=22.12 \pm 0.52 \mathrm{~mole} \%, \mathrm{En}=76.60 \pm 0.44 \mathrm{~mole} \%$, $n=26$. Still, the PO chondrules studied contain a clear to turbid glassy mesostasis. Therefore, we suggest to classify the sample as LL4. Undulatory extinctions of the olivines accompanied by rare planar fractures are indicative of a shock stage S3 [1]. The Meteoritical Bulletin lists a lower $\mathrm{Fa}$ content of $24.9 \pm 0.20 \mathrm{~mole} \% \quad(n=6)$ possibly indicating a brecciated meteorite.

P-zoned Olivines in Particular Chondrules: Some type IIA chondrules show P-zonation patterns within the olivines similar to those investigated in H-chondrites by [2], [3] and Semarkona LL3 by [4]. While divalent cations like $\mathrm{Fe}$ and $\mathrm{Mg}$ are completely equilibrated during thermal metamorphism, $\mathrm{P}$ retains the magmatic zonation patterns of the olivines. The incorporation of $\mathrm{P}$ during olivine crystallization is indicative of rapid crystallization and high $\mathrm{P}$ contents in the melt [5]. The content of $\mathrm{P}_{2} \mathrm{O}_{5}$ of the mesostasis of five investigated chondrules varies within a factor of about 5 (from 0.67 to $0.12 \mathrm{wt} \%$ ) as $\mathrm{P}$ is locked up in phosphate whereas the content of $\mathrm{SiO}_{2}, \mathrm{MgO}, \mathrm{FeO}, \mathrm{CaO}, \mathrm{Al}_{2} \mathrm{O}_{3}$ and $\mathrm{Cr}_{2} \mathrm{O}_{3}$ remains relatively constant due to diffusion of the elements across the chondrule rims. At the interface between dendritic clinopyroxene and glass within the mesostasis of chondrules with high $\mathrm{P}_{2} \mathrm{O}_{5}$ contents tiny phases $(<1 \mu \mathrm{m})$ rich in $\mathrm{Ca}$ and $\mathrm{P}$ occur which we consider as microcrystals of apatite formed during thermal metamorphism. The glass is free of $\mathrm{P}_{2} \mathrm{O}_{5}$. This may allow us to distinguish different types of chondrules whose original chemistry is erased during thermal metamorphism. Other type IIA PO chondrules have lower contents of $\mathrm{P}_{2} \mathrm{O}_{5}$ within the mesostasis. No P-zonation features of the olivines were found in the latter. [6] also found a positive correlation between $\mathrm{P}$ content in olivine and the mesostasis. Further studies of the zoning of $\mathrm{P}$ and other trace elements in UOCs and EOCs are planned.

References: [1] Stöffler D. et al. 1991. Geochimica et Cosmochimica Acta 55:3845-3867. [2] McCanta M. C. et al. 2008. Abstract \#1807. 39th Lunar and Planetary Science Conference. [3] McCanta M. C. et al. 2009. Abstract \#2048. 40th Lunar and Planetary Science Conference. [4] Hewins R. H. 2009. 40th Lunar and Planetary Science Conference. [5] Boesenberg J. S. et al. Abstract \#1366. 35th Lunar and Planetary Science Conference. [6] Jones R. H. 1990 Geochimica et Cosmochimica Acta 54:1785-1802.
5189

ORIGIN AND ALTERATION OF AMOEBOID OLIVINE INCLUSIONS IN CK3 AND CV3 CHONDRITES

Alan E. Rubin. Institute of Geophysics, University of California, Los Angeles, CA 90095-1567, USA. E-mail: aerubin@ucla.edu.

Amoeboid olivine inclusions (AOIs) are composed of major olivine, minor Ca-pyx, anorthite, and in some cases, spinel \pm low-Ca pyx \pm melilite [1]. Some AOIs enclose small CAIs with Wark-Lovering rims [1, 2]. Although rare, melilite occurs in a few AOIs from minimally altered carbonaceous chondrites [1]. AOIs vary in size among chondrite groups: OC, 0.04-0.35 mm [3]; CO, 0.1-0.5 mm [4]; CR, <0.5 mm [5]; $\mathrm{CV}, 0.1-10 \mathrm{~mm}[6,7]$. I report here the occurrence of a $0.54 \times 1.31 \mathrm{~mm}$ AOI in CK3 NWA 1559; I also studied 9 AOIs from CV3 Allende. CK and CV chondrites are closely related groups that likely formed in the same region of the nebula [8, 9]; their AOIs probably have similar histories.

The CK3 AOI interior contains irregular 3-15 $\mu \mathrm{m}$-size Ca- pyx grains $\left(\mathrm{Fs}_{1.6} \mathrm{Wo}_{48.8}\right)$ with $2.2 \mathrm{wt} \% \mathrm{Al}_{2} \mathrm{O}_{3} \quad$ (approximately $70 \mathrm{vol} \%$ ), 2-20 $\mu \mathrm{m}$-size pores (approximately $30 \mathrm{vol} \%$ ) and accessory plagioclase. The 75-160 $\mu$ m-thick rim of the AOI contains ferroan, 20-130 $\mu$ m-size olivine grains $(\mathrm{Fa} 34.3 \pm 0.4)$ with faceted $\left(120^{\circ}\right.$ triple junctures) and fragmental shapes. Coarse $(25-50 \mu \mathrm{m}$-size $)$ patches of plagioclase $\left(\mathrm{Ab}_{61.8}\right.$ $\left.\mathrm{Or}_{1.1}\right)$ with $2-18 \mu \mathrm{m}$-thick Ca-pyx rinds occur in several places just beneath the olivine rim. (The CK3 AOI mineral compositions were all modified by parent-body processes.)

Nearly $80 \%$ of Allende AOIs have rims composed of $10-30 \mu \mathrm{m}$-size olivine grains, many with $120^{\circ}$ triple junctures [10]. Rim olivine consists of low-FeO cores surrounded by ferroan overgrowths produced by parent-body processes. The rims are $10-80 \mu \mathrm{m}$ thick and enclose porous patches of Ca-pyx, plagioclase \pm spinel. They may be related to accretionary rims with $20-40 \mu \mathrm{m}$ anhedral forsterite grains [10] that surround many CV CAIs [11].

On average, olivine constitutes (in vol $\%$ ) approximately $50 \%$ of OC, $\mathrm{CO}$, and CR AOIs [3-5], 50\% of the CK3 AOI, and approximately $80 \%$ of AOIs in reduced $\mathrm{CV}$ chondrites [2]. CV AOIs may have acquired additional forsterite in the $\mathrm{CV}-\mathrm{CK}$ region of the nebula (previously inferred to contain abundant olivine-rich dust [9]). This may account for the large sizes of CV AOIs. Although not particularly olivine-rich, the CK3 AOI probably formed in a similar manner.

Amoeboid olivine inclusions were sintered to partly melted in the nebula, plausibly by the same process that formed chondrules; many AOIs preserved some of their initial porosity. In more-extensively-melted AOIs, olivine crystallized first, followed by melilite, spinel, Ca-pyx and small amounts of anorthite. Much of the melilite and spinel was altered and replaced by Ca-pyx and anorthite [1]. Hydrothermal experiments show melilite to be very susceptible to parent-body alteration [12], consistent with its paucity in CM CAIs [13] and its corrosion in Allende CAIs [14]. Some of the pores in AOIs may have been formed by the corrosion of melilite.

References: [1] Krot A. et al. 2004. Chemie der Erde 64:185-239. [2] Komatsu M. 2003. Ph.D. thesis, University of Tokyo. [3] Itoh S. et al. 2007. Meteoritics \& Planetary Science 42:1241-1247. [4] Chizmadia L. et al. 2002. Meteoritics \& Planetary Science 37:1781-1796. [5] Aléon J. et al. 2002. Meteoritics \& Planetary Science 37:1729-1755. [6] Grossman L. and Steele I. 1976. Geochimica et Cosmochimica Acta 40:149-155. [7] Komatsu M. et al. 2001. Meteoritics \& Planetary Science 36:629-641. [8] Greenwood R. et al. 2010. Geochimica et Cosmochimica Acta 74:16841705. [9] Rubin A. 2010. Geochimica et Cosmochimica Acta 74:4807-4828. [10] Kornacki A. and Wood J. 1984. 14th Lunar and Planetary Science Conference. pp. B573-B587. [11] MacPherson G. et al. 1985. Geochimica et Cosmochimica Acta 49:2267-2279. [12] Nomura K. and Miyamoto M. 1998. Geochimica et Cosmochimica Acta 62:3575-3588. [13] Rubin A. 2007. Meteoritics \& Planetary Science 42:1711-1726. [14] MacPherson G. and Grossman L. 1984. Geochimica et Cosmochimica Acta 48:29-46. 


\section{8}

A SHOCKING TALE: TEM OBSERVATIONS OF DEFORMED OLIVINE IN ORDINARY CHONDRITES

A. Ruzicka ${ }^{1,2}$ and R. Hugo ${ }^{2}$. ${ }^{1}$ Cascadia Meteorite Laboratory and Portland State University, Portland, OR 97207, U.S.A. ${ }^{2}$ Department of Geology, Portland State University, Portland, OR 97207 U.S.A. E-mail: ruzickaa@pdx.edu.

Introduction: Previous transmission electron microscopy (TEM) work suggested that Portales Valley [H7 (S1)] and MIL 99301 [LL6 (S1)] experienced syn-metamorphic shock deformation at an early stage $[1,2]$. To better interpret the microstructures of these and other chondrites, we used TEM to study two L6 chondrites with a nominally simple shock history, including Park (S1) and Leedey (S4, revised from a literature designation of S3 based on our optical microscopy data). Our expectation was that these $\mathrm{L}$ chondrites could have been shocked to various degrees from a cold starting temperature at a late stage, possibly even in the same, major collisional event that affected the $\mathrm{L}$ parent body approximately $0.5 \mathrm{Ga}$ ago [e.g., 3].

Results: Olivine microstructures found for Leedey include (1) moderately high dislocation densities $\left(10^{9}-10^{10} \mathrm{~cm}^{-1}\right)$; (2) predominantly $\mathbf{b}=[001]$ dislocations with straight segments oriented along two to three distinct line vectors, with only occasional bowing of dislocations, suggesting low temperature deformation; (3) rare low angle grain boundaries indicating limited recovery; (4) small (10 s of nanometers) $\mathrm{b}=[001]$ dislocation loops.

Specific microstructures found for olivine in Park include (1) a moderate dislocation density $\left(10^{8} \mathrm{~cm}^{-1}\right)$; (2) a prevalence of $\mathbf{b}=[001]$ dislocations, predominantly bowed, with some $\mathbf{b}=[100]$ dislocations, indicative of deformation at an elevated temperature; (3) occasional tangles formed by intersections of $\mathbf{b}=[001]$ dislocations with $\mathbf{b}=[100]$ dislocations; (4) no low angle grain boundaries (subgrain boundaries), indicating limited recovery; and (5) small (10 s of nanometers) $\mathbf{b}=[001]$ dislocation loops, suggestive of dislocation climb.

Discussion: Altogether, Leedey appears to be a good example of an S4 chondrite that was relatively strongly deformed from a cold starting temperature. This conforms to the hypothesis that this meteorite could have been affected by the relatively late shock event that affected the L chondrite parent body. Using the data for Leedey as a guide, we suggest that Portales Valley could have been deformed to an S3 shock stage with subsequent annealing to obtain S1 characteristics. In contrast to Leedey, Park appears to have been subjected to low-to-moderate shock pressures at elevated temperatures. There is no evidence that Park was significantly more deformed than it is now, so the low shock stage probably cannot be attributed to an annealing (recovery) process. Instead, we suggest that Park largely escaped the late, strong shock event that affected most L chondrites and instead records one or more, early, relatively weak shock events that occurred while the $\mathrm{L}$ chondrite parent body was being metamorphosed. This history of weak shock at elevated temperature is similar to that experienced by MIL 99301 [2]. We now have TEM evidence for early collisions affecting each of the H, L, and LL parent bodies.

References: [1] Hutson M. et al. 2007. Abstract \#5072. 42nd Meteoritics and Planetary Science. [2] Hutson M. et al. 2009. Abstract \#1081. 40th Lunar and Planetary Science Conference. [3] Bogard D. D. 1995. Meteoritics 30:244-268.

\section{6}

MACRO-, TO MICRO- TO NANOSCALE ADVANCED SEM/EDS ANALYSIS USING STAGE CONTROL AND AN ANNULAR FOURCHANNEL SILICON DRIFT DETECTOR: APPLICATIONS IN IMPACT STUDIES

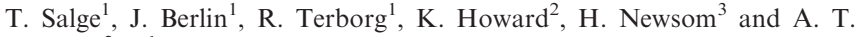
Kearsley ${ }^{2}$. ${ }^{1}$ Bruker Nano GmbH, Schwarzschildstr. 12, 12489 Berlin, Germany. E-mail: tobias.salge@bruker-nano.de. ${ }^{2}$ Department of Mineralogy, Natural History Museum, London SW7 5BD, UK. ${ }^{3}$ Institute of Meteoritics, Department of Earth and Planetary Sciences, University of New Mexico, Albuquerque, NM 87131, USA.

Introduction: Imaging of ever smaller structures, in situ within large samples, requires low electron beam energy to enhance spatial resolution, and therefore also use of low energy X-ray lines for elemental analysis. To separate significant overlapping peaks e.g., N-K $(392 \mathrm{eV})$ and Ti- $\mathrm{L}_{1}$ $(395 \mathrm{eV})$ [1], the incorporation of line deconvolution algorithms [2] in energy dispersive X-ray (EDX) software is of crucial importance. In this paper we describe applications of a new system to impact studies.

Methods: Without adequate X-ray count statistics, deconvolution is unlikely to be effective. We therefore used an annular Silicon Drift Detector (SDD), the Bruker XFlash 5060F. This has parallel processing of data from four channels, giving an output count rate of $1.1 \mathrm{Mcps}$. It is placed between the pole piece and sample, covering a very large solid angle of X-ray collection, rapidly acquiring sufficient data even on beam sensitive samples. High take-off angle and collection of X-rays from four different directions allow data collection across samples with substantial surface topography. Automated stage control and spectrum imaging (an entire EDS spectrum is collected at each pixel) allow large data sets to be acquired within a short time.

Examples of Applications: 1. Large area, high resolution mapping (with tiling or stitching of neighboring areas) is useful for understanding processes in the formation of tektites [3], revealing flow textures and layering, without destructive section preparation. Coalescence textures formed during the transition from melt to solid are seen, with surface pitting produced by microimpact collisions in the impact plume, and surface etching by chemical attack in the impact plume, or later weathering.

2. Large area, high resolution spectrum imaging of the matrix at impact melt breccia of the Chicxulub impact crater (Yax-1 borehole, Unit $5861.72 \mathrm{~m}$ ) [3] reveals the distribution of unusual, resorbed andradite garnets. Analysis at low accelerating voltage reveals secondary mineral formation, such as $<500 \mathrm{~nm} \mathrm{NaCl}$ and $<150 \mathrm{~nm}$ Fe-Ti-oxides associated with garnet resorption, indicating a complex history of matrix genesis during the fluid evolution of high temperature seawater-derived brine.

3. Complex topographic surfaces, such as the interior of experimental Stardust foil craters, can be imaged very rapidly and in detail in order to locate projectile residues [4] without loss of data due to "shadowing" of any area at depth.

References: [1] Salge et al. 2010. Abstract \#I6.4. 17th International Microscopy Congress. [2] Aßmann A. and Wendt M. 2003. SpectrochimicaActa 58:711-716. [3] Howard K. T. 2011. Geological Society of London 573-591. [3] Salge T. and Newsom H. 2010. Abstract \#5337. 73rd Annual Meeting of the Meteoritical Society. [4] Kearsley et al. 2011. Submitted to 74th Annual Meeting of the Meteoritical Society. 
5122

TRACE ELEMENT GEOCHEMISTRY OF THE NAKHLITE NORTHWEST AFRICA 5790

M. E. Sanborn ${ }^{1}$, M. Wadhwa ${ }^{1}$, J. B. Balta ${ }^{2}$, R. Mayne $^{3}$ and H. Y. McSween $\mathrm{Jr}^{2}$. ${ }^{1}$ SESE, Arizona State University, Tempe, AZ, 852871404. E-mail: Matthew.Sanborn@asu.edu. ${ }^{2}$ Dept. of Earth and Planetary Sciences, University of Tennessee, Knoxville, TN. ${ }^{3}$ Dept. of Geology, Texas Christian University, Fort Worth, TX.

Introduction: Northwest Africa (NWA) 5790 is a recently recovered hot desert nakhlite that brings the total number of samples of this SNC subgroup to 13. Petrographically similar to the other known nakhlites [1], NWA 5790 contains phenocrysts of augite and olivine, embedded in a fine-grained mesostasis. The mesostasis is composed primarily of feldspar, phosphate, olivine, pyroxene, and oxides. An initial investigation of NWA 5790 noted that the whole-rock rare earth element (REE) abundances were elevated in comparison to the other known nakhlites [2]. Here, we report REE and selected trace and minor element abundances measured in situ in individual minerals of NWA 5790 with the goal of constraining its petrologic history and understanding its relationship to the other nakhlites.

Results and Discussion: The concentrations of REE and other selected trace elements were measured in augite, olivine, mesostasis, and phosphate (apatite) of NWA 5790 using the Cameca IMS-6f ion microprobe at Arizona State University. The augite has a concavedownward REE pattern that is LREE-depleted $\left([\mathrm{La} / \mathrm{Sm}]_{\mathrm{CI}} \sim 0.4\right)$, with a small negative Eu anomaly $\left(\mathrm{Eu} / \mathrm{Eu}^{*} \sim 0.9\right)$ and a slight decrease from the middle- to the HREE $\left([\mathrm{Gd} / \mathrm{Lu}]_{\mathrm{CI}} \sim 1.5\right)$. This mineral exhibits a moderate range of REE abundances ( $\mathrm{La} \sim 1.5-3.2 \times \mathrm{CI}$ ). The REE as well as Ti, Y, and $\mathrm{Zr}$ abundances in the augite cores of the NWA 5790 nakhlite are higher than those in the other nakhlites (e.g., Nakhla and Lafayette) with the exception of MIL 03346 augite cores (which exhibit similar abundances to those of NWA 5790) [3,4]. The olivine in NWA 5790 is HREE enriched $\left([\mathrm{Lu} / \mathrm{Gd}]_{\mathrm{CI}} \sim 12\right)$ with low REE abundances $(\mathrm{Lu} \sim 1.2 \times \mathrm{CI})$. The fine-grained mesostasis was analyzed with a beam spot large enough to obtain a "bulk" analysis. It has a LREE-enriched pattern ( $\mathrm{La} \sim 60-95 \mathrm{xCI}$; $\left.[\mathrm{La} / \mathrm{Lu}]_{\mathrm{CI}} \sim 5-6\right)$ that parallels that of the NWA 5790 whole-rock. Apatite usually occurs as very small grains in the mesostasis; however, at least one apatite grain was large enough to obtain a clean ion microprobe analysis. The apatite in NWA 5790 is significantly LREE-enriched $\left([\mathrm{La} / \mathrm{Lu}]_{\mathrm{CI}} \sim 50\right)$, and has the highest REE abundances of all the analyzed phases $(\mathrm{La} \sim 1100 \times \mathrm{CI})$. This mineral also exhibits a significant negative $\mathrm{Eu}$ anomaly $\left(\mathrm{Eu} / \mathrm{Eu}^{*} \sim 0.29\right)$ similar to that in other nakhlite apatites (e.g., [3]).

Conclusions: The REE patterns of the various mineral of NWA 5790 are similar to those observed in the other known nakhlites indicating that NWA 5790 crystallized from a parent melt that originated from the same geochemical source reservoir as the other nakhlites. However, the elevated whole-rock REE concentrations [2] coupled with the relatively higher REE, Ti, Y, and $\mathrm{Zr}$ abundances observed in the augite cores suggest that the NWA 5790 parent melt was more evolved than those of most previously studied nakhlites (e.g., Nakhla [3]).

References: [1] Treiman A. 2005. Chemie der Erde 65:203-270. [2] Jambon A. et al. 2010. Abstract \#1696. 41st Lunar and Planetary Science Conference. [3] Wadhwa M. and Crozaz G. 1995. Geochimica et Cosmochimica Acta 59:3629-3645. [4] Day J. et al. 2006. Meteoritics \& Planetary Science 41:581-606.

\section{8}

\section{CHONDRULES: CLUES TO EARLY PLANETESIMALS}

I. S. Sanders ${ }^{1}$ and E. R. D. Scott ${ }^{2} .{ }^{1}$ Trinity College Dublin, Ireland. E-mail: isanders@tcd.ie. ${ }^{2}$ University of Hawaii, USA.

Introduction: Most chondrules were evidently made in huge batches of closely spaced droplets [1] about $2 \mathrm{Myr}$ after the solar system began. Modeling by [2] suggests that at that time enormous fan-shaped plumes of chondrule droplets, like 'spray from a hose-pipe nozzle', would have emerged downstream from the overshooting fraction of molten impactors following oblique low-velocity collisions and partial planetesimal mergers. If chondrules originated in this way, then what can they tell us about their planetesimal antecedents?

Planetesimal Oxidation State: Two main kinds of molten planetesimal probably existed - reduced and oxidized - to account for Type I (FeO-poor) and Type II (FeO-bearing) chondrules respectively. Reduced planetesimals perhaps accreted from partially condensed matter in the young, hot inner nebula, as proposed by [3], and their cores in some cases had evidently formed in the first $0.5 \mathrm{Myr}$ [4]. The timing of disruption of one particularly large molten planetesimal, which left the IVA iron parent body in its wake, suitably coincides with the time of chondrule formation [5,6]. Oxidized planetesimals, on the other hand, perhaps accreted in a cold part of the disk, possibly later or further from the sun than the reduced ones, and incorporated grains of water ice. As the planetesimals became heated, hydrated and eventually melted, the $\mathrm{H}_{2} \mathrm{O}$ plausibly oxidized the metal, and supplied the ${ }^{16} \mathrm{O}$-poor oxygen whose abundance in chondrules in $\mathrm{E}, \mathrm{H}, \mathrm{L}, \mathrm{LL}$ and $\mathrm{R}$ chondrites correlates so well with $\mathrm{FeO} /(\mathrm{FeO}+\mathrm{MgO})$. To make Type II chondrules in conventional ways is, incidentally, beset with problems [7]

Planetesimal Anatomy: A typical molten planetesimal perhaps had a coating of cold loose regolith passing downwards with increasing temperature into sintered rigid 'crust', through a zone of incipient melting into the molten silicate interior from which the metal core was separating. Hence the overshooting plume would have carried a diverse suite of materials. The spray would probably have sampled much more mantle than core, thus explaining iron depletion in all chondrite groups relative to CIs. Phenocrysts in the melt would explain chondrules with mm-sized single crystals. With olivine phenocrysts settling through time, laterformed chondrules would have become increasingly pyroxene-rich, as is observed [8]. Incipiently melted fragments of the crust would account for chondrules packed full of unmelted 'relict' grains [9]. Small solid objects, including fragments of sintered crust [10], perhaps became wetted and engulfed by droplets they encountered in flight, accounting for the presence of these objects within chondrules.

Reservoirs of Ingredients: Chondrules and other debris launched by collisions presumably accreted to new or existing planetesimals before having time to become homogenized in the disk by radial mixing and turbulence. Thus each chondrite parent body (chondrite group?) was dominated by ingredients supplied by recent impacts in its 'feeding zone' and so acquired its own distinctive petrographic and chemical characteristics [11].

References: [1] Alexander C. M. O'D. et al. 2008. Science 320:16171619. [2] Asphaug E. 2011. Abstract \#1647. 42nd Lunar and Planetary Science Conference. [3] Bland P. A. and Ciesla F. J. 2010. Abstract \#1871. 41st Lunar and Planetary Science Conference. [4] Burkhardt C. et al. 2008. Geochimica et Cosmochimica Acta 72:6177-6197. [5] Yang J. et al. 2007. Nature 446:888-891. [6] Blichert-Toft et al. 2010. Earth and Planetary Science Letters 296:469-480. [7] Fedkin A. V. and Grossman L. 2010. Abstract \#1448. 41st Lunar and Planetary Science Conference. [8] Kita N. T. et al. 2005. Abstract \#1750. 36th Lunar and Planetary Science Conference. [9] Ruzicka A. et al. 2010. Abstract \#1956. 41st Lunar and Planetary Science Conference. [10] Libourel G. and Krot A. N. 2007. Earth and Planetary Science Letters 251:1-8. [11] Jones R. H. 2010. Workshop on chondrules, New York. 
5162

XANES ANALYSIS OF ORGANIC RESIDUES FROM THE IRRADIATION OF ASTROPHYSICAL ICE ANALOGS AND COMPARISON WITH Stardust SAMPLES

S. A. Sandford ${ }^{1}$, M. Nuevo', S. N. Milam ${ }^{2}$, G. D. Cody $^{3}$, A. L. D. Kilcoyne $^{4}$, B. T. De Gregorio ${ }^{5}$ and R. M. Stroud ${ }^{6}{ }^{1}$ NASA-Ames Research Center, Moffett Field, CA, USA. E-mail: Scott.A. Sandford@nasa.gov. ${ }^{2}$ NASA Goddard Space Flight Center, Greenbelt, MD, USA. ${ }^{3}$ Carnegie Institution of Washington, Washington, DC, USA. ${ }^{4}$ Advanced Light Source, Berkeley, CA, USA. ${ }^{5}$ NASA Johnson Space Center, Houston, TX, USA. ${ }^{6}$ Naval Research Laboratory, Washington, DC, USA.

Introduction: The NASA Stardust mission successfully collected authentic cometary grains from Comet $81 \mathrm{P} /$ Wild 2 [1], including organicrich materials [2]. X-ray absorption near-edge structure (XANES) analysis of these samples indicates that these grains contain O-rich and N-rich organic materials having a broad variety of functional groups: carbonyls, $\mathrm{C}=\mathrm{C}$ bonds, aliphatic chains, amines, amides, etc. [2, 3]. Stardust samples were also recently shown to contain the amino acid glycine [4]. Some of the organic materials found in Stardust samples contain very little aromatic carbon and resemble, at least superficially, the organic residues produced by the UV irradiation of interstellar/cometary ice analogs. Organic residues produced from the irradiation of astrophysical ice analogs are known to contain a large suite of prebiotic molecules including amino acids [5-7], and amphiphilic compounds (fatty acids) [8]. In the work reported here, residues were produced in the laboratory from the UV irradiation of mixtures of ices containing $\mathrm{H}_{2} \mathrm{O}, \mathrm{CH}_{3} \mathrm{OH}, \mathrm{CO}$, and $\mathrm{NH}_{3}$ in relative proportions 100:50:1:1 at $7 \mathrm{~K}$. Additional residues were produced from mixtures with no $\mathrm{NH}_{3}$, and mixtures containing alkanes or naphthalene $\left(\mathrm{C}_{10} \mathrm{H}_{8}\right)$. C-, N-, and O-XANES spectra of these residues were measured using Beamline 5.3.2 at the Advanced Light Source in Berkely. These XANES spectra were used to assess the samples' organic functional group chemistry and overall atomic composition, as well as their $\mathrm{N} / \mathrm{C}$ and $\mathrm{O} / \mathrm{C}$ ratios. The first results of this study indicate the presence of a number of chemical bonds and functions, namely, carbonyls, $\mathrm{C}=\mathrm{C}$ bonds, alcohols, amides, amines, and nitrile groups. The relative proportions of these functional groups can be compared with XANES measurements of Stardust samples [9]. The samples were also found to be modified by accumulated X-ray exposure, the principal change being the conversion of some aliphatic carbon into aromatic carbon.

References: [1] Brownlee D. E. et al. 2006. Science 314:1711. [2] Sandford S. A. et al. 2006. Science 314:1720. [3] Cody G. D. et al. 2008. Meteoritics \& Planetary Science 43:353. [4] Elsila J. E. et al. 2009. Meteoritics \& Planetary Science 44:1323. [5] Bernstein M. P. et al. 2002. Nature 416:401. [6] Muñoz Caro G. M. et al. 2002. Nature 416:403. [7] Nuevo M. et al. 2008. Origins of Life and Evolution of the Biosphere 38:37. [8] Dworkin J. P. et al. 2001. Proceedings of the National Academy of Sciences 98:815. [9] Nuevo M. et al. 2011. Advances in Space Research in press.

\section{3}

APATITE AS A PROBE OF VOLATILE COMPOSITION IN HED MAGMA

A. R. Sarafian, M. F. Roden and A. E. Patiño-Douce. University of Georgia. E-mail: povault@uga.edu.

Introduction: The HED parent body is a well-sampled planetary body, with nearly 1000 known meteorites. Although this parent body is thought to be volatile-depleted [1], apatite, a volatile-bearing $(\mathrm{F}, \mathrm{Cl}, \mathrm{OH}$, S) mineral, is a minor constituent of eucrites, along with a volatile-free phosphate, merrillite. Representative basaltic eucrites (Stannern, Nuevo Laredo, Pasamonte, Juvinas, LEW 88010, LEW 88009, EET 87542, and GRA 98098) were selected for study on the basis of bulk rock $\mathrm{TiO}_{2}$ and $\mathrm{Mg} \#$ [2]. Four cumulate eucrites, Moore County, Serra de Magé, ALH 85001, and EET 87548 were also selected.

Results: Apatite compositions were measured using the JEOL 8600 electron microprobe at the University of Georgia; operating conditions and procedures followed those of Patiño-Douce et al. [3]. We assume $\mathrm{X}_{\mathrm{Cl}}+\mathrm{X}_{\mathrm{F}}+\mathrm{X}_{\mathrm{OH}}=1$ and calculate $\mathrm{OH}$ by difference. A typical apatite from basaltic eucrites has $40.7 \mathrm{P}_{2} \mathrm{O}_{5}, 55.7 \mathrm{CaO}, 0.2 \mathrm{Cl}, 3.6 \mathrm{~F}$ (all values in wt $\%$ ), and no detectable $\mathrm{SrO}, \mathrm{Na}_{2} \mathrm{O}, \mathrm{Ce}_{2} \mathrm{O}_{3}, \mathrm{FeO}, \mathrm{MgO}, \mathrm{SO}_{3}, \mathrm{Y}_{2} \mathrm{O}_{3}$, $\mathrm{AsO}_{4}$, or $\mathrm{SiO}_{2}$. A typical apatite from cumulate eucrites has $41.8 \mathrm{P}_{2} \mathrm{O}_{5}$, $56.2 \mathrm{CaO}, 3.0 \mathrm{~F}$ (all values in wt\%), and no detectable $\mathrm{Cl}, \mathrm{SrO}, \mathrm{Na}_{2} \mathrm{O}$, $\mathrm{Ce}_{2} \mathrm{O}_{3}, \mathrm{FeO}, \mathrm{MgO}, \mathrm{SO}_{3}, \mathrm{Y}_{2} \mathrm{O}_{3}, \mathrm{AsO}_{4}$, or $\mathrm{SiO}_{2}$. The $\mathrm{F}: \mathrm{Cl}: \mathrm{OH}$ ratio for apatite in basaltic eucrites and cumulate eucrites are 10.5:0.53:1 and 11.8:0:1 respectively.

Discussion: The F-rich nature of eucrite apatites indicate either an intrinsically volatile-poor parent body or the eucritic magmas were devolatilized and lost $\mathrm{H}_{2} \mathrm{O}$ and $\mathrm{Cl}$. However, we found a measurable vacancy in the anion site $(\sim 15 \%$ in cumulate eucrites and up to $25 \%$ in basaltic eucrites), which we inferred to be occupied with $\mathrm{OH}$. Chlorine concentration increases slightly $(\sim 0.2 \mathrm{wt} \%)$ as $\mathrm{Mg} \#$ decreases. Although partition coefficients are poorly constrained for the halogens between apatite and melt, a first approximation for melt composition can be obtained from partition coefficients for $\mathrm{F}$ and $\mathrm{Cl}$ between a fluidsaturated basaltic melt and apatite [4]; we estimate the $\mathrm{Cl} / \mathrm{F}$ weight ratio in the melt to be $\sim 0.25$. Patiño-Douce et al. [3] described a thermodynamic model suggesting that a rock with both merrillite and apatite reflects relatively low $\mathrm{H}_{2} \mathrm{O}$ content, high halogen content (in this case $\mathrm{F}$ ), and high $\mathrm{P}$ content compared to typical terrestrial samples containing apatite only. The last stages of the volatile-depleted magma on the HED parent body must have concentrated enough $\mathrm{F}$ (and possibly $\mathrm{H}_{2} \mathrm{O}$ ) to crystallize apatite. The HED parent body differs from the volatile-rich parent body of GRA 06128/9 where the late stage fluid was $\mathrm{Cl}$ rich, and chlorapatite replaced merrillite [5].

Acknowledgments: We would like to thank Joe Boesenberg at the American Museum of Natural History and Kevin Righter at the Johnson Space Center for facilitating the loan of thin sections.

References: [1] Papike J. J. 2008. In Reviews in Mineralogy, vol. 36. Planetary Materials. Mineralogical Society of America: Washington, D.C. pp. 7-01-7-10. [2] BVSP, Basaltic volcanism on the terrestrial planets 1981. New York: Pergam on Press Inc. 1286 p. [3] Patiño-Douce A. E. et al. 2011. Chemical Geology, submitted. [4] Mathez E. A. and J. D. Webster. 2005. Geochimica et Cosmochimica Acta. 69:1275-1286. [5] Shearer C. et al. 2010. Geochimica et Cosmochimica Acta. 74:1172-1199. 
5513

HANDLING HEAVENLY JEWELS-35 YEARS OF ANTARCTIC METEORITE PROCESSING AT JOHNSON SPACE CENTER C. E. Satterwhite ${ }^{1}$, K. M. McBride ${ }^{1}$, R. Harrington ${ }^{1}$, C. M. Schwarz ${ }^{1}$ and K. Righter ${ }^{2}$. ${ }^{1}$ ESCG Jacobs Technology, NASA Johnson Space Center, 2101 Nasa Pkwy, Houston, TX 77058, USA. E-mail: cecilia.e. satterwhite@nasa.gov. ${ }^{2}$ NASA-JSC, 2101 Nasa Pkwy, Houston, TX 77058, USA.

Introduction: The ANSMET program began in 1976, and since that time more than 18,000 meteorites have been processed in the Meteorite Processing Lab at Johnson Space Center in Houston, TX [1]. The meteorites are collected and returned to JSC on a freezer truck and remain frozen until they are initially processed.

Initial Processing of Meteorites: Initial processing involves drying the meteorites in a nitrogen glove box for $24-48 \mathrm{~h}$, photographing, measuring, weighing and writing a description of the interior and exterior. The meteorite is broken and a representative sample is sent to the Smithsonian Institution for classification.

Newsletter and Requests: Once initial processing has been complete and the meteorites have been classified, the information is published in the Antarctic Meteorite Newsletter [2,3]. The newsletter is published twice yearly and is sent electronically to researchers around the world and is also available on line. Researchers are asked to fill out a request form and submit it to the Meteorite Working Group secretary. All sample requests will be reviewed by either the meteorite curator or the Meteorite Working Group depending on the type of meteorite and the research being conducted.

Processing for Sample Requests: In the meteorite processing lab, meteorite samples are prepared several different ways. Most samples are prepared as chips obtained by use of stainless steel chisels in a chipping bowl or rock splitter. In special situations where a researcher needs a slab the meteorite samples can be bandsawed in a dry nitrogen glove box with a diamond blade, no liquids are ever introduced into the cabinet. The last type of sample preparation is thin/thick sections. The meteorite thin section lab at JSC can prepare standard 30-micron thin sections, thick sections of variable thickness (100-200 microns), or demountable sections using superglue.

Information for Researchers: It is important that researchers fill the sample request form completely, in order to make sure the meteorite is processed correctly [4]. Researchers should list any special requirements on the form, i.e., packaging of samples (poly vs. stainless), thick sections and thickness needed, superglue needed, interior chips, exterior chips, fusion crust, contamination issues, all concerns should be listed so processing can be done accurately and any concerns the researcher has can be addressed before the meteorites are broken.

References: [1] http://curator.jsc.nasa.gov/antmet/index.cfm. [2] http://curator.jsc.nasa.gov/antmet/classdb.cfm. [3] http://curator.jsc.nasa. gov/antmet/amn/amn.cfm. [4] http://curator.jsc.nasa.gov/antmet/request dates.cfm.
5034

MEASUREMENT OF LOW-TEMPERATURE HEAT CAPACITY OF METEORITES

M. W. Schaefer ${ }^{1}$, G. J. Consolmagno ${ }^{2}$, D. Britt ${ }^{3}$ and B. E. Schaefer ${ }^{1} .{ }^{1}$ Louisiana State University. E-mail: mws@1su.edu. ${ }^{2}$ Vatican Observatory. ${ }^{3}$ University of Central Florida.

Introduction: The heat capacity of a meteorite is a mass-weighted sum of the heat capacities of its component minerals, unlike thermal conductivity or porosity, which are functions of the fabric of the meteorite. As such it is an independent constraint on the mineral composition of a meteorite. It has been shown previously [1] that many meteorite groups can be categorized by their grain density and magnetic susceptibility; the measurement of heat capacity might provide yet another, independent, way of determining the bulk composition of a whole meteorite. One can look for systematic differences between various meteorite groups; systematic differences between falls and finds; and systematic differences between weathered and unweathered samples.

Meteorite heat capacity is also an essential parameter in a number of modeling techniques of interest to planetary scientists, including thermal models of asteroids and icy bodies expected to have a significant rocky component similar to meteorites; models of the evolution of small body orbits as a function of thermal inertia via the Yarkovsky and YORP effects; the modeling of meteor brightness as a function of the meteor initial size and speed; and parameters used in the modeling of the response of planetary surfaces to impact cratering.

To perform such measurements on a suitably large, representative number of samples across meteorite types requires a quick and nondestructive way of measuring the heat capacity of whole rocks. Because heat capacity is itself a strong function of temperature, it is also desirable to measure it over a range of temperatures.

Experimental Procedure: We are developing a new, non-destructive procedure for measuring the heat capacity of minerals and rocks, based on the technique of [2]. An insulated dewar of liquid nitrogen is placed on a balance, and its decrease in mass from evaporation is measured over time. A small (several grams) sample is dropped into the dewar, and the increased amount of nitrogen evaporated by the heat input from the sample is determined. This mass of nitrogen is then used to calculate the average heat capacity over the temperature range between room temperature and the boiling temperature of liquid nitrogen. By having the sample at different starting temperatures, it will be possible to derive the temperature dependence of the heat capacity as well.

Preliminary Results: Since last year, we have experimented with a number of different combinations of containers and starting conditions. We have developed a mathematical model for the flow of heat from the system, in order to suggest ways to optimize the shape and size of the liquid nitrogen container. During this development phase, measurements of quartz are underway for comparison to published values. The average heat capacity over this temperature range derived for the samples measured to date is consistent with literature values. The technique appears to hold good promise of providing rapid, non-destructive measurements of this important thermal parameter.

References: [1] Consolmagno G. J. et al. 2008. Chemie der ErdeGeochemistry 68:1-29. [2] Koch A. et al. 2010. Abstract \#5163. Meteoritics \& Planetary Science 45. 


\section{0}

\section{DIFFERENTIATION OF Fe-Ni SPHERULES IN IMPACT GLASS}

M. Schaffer, T. Kasama, R. E. Dunin-Borkowsky, A. N. MacDonald and C. Bender Koch. University of Copenhagen, Denmark

Metallic, micron sized Fe-Ni spherules are frequently reported from glasses formed during impact processes. They have a high preservation potential and are a valid indicator of impactor type.

However, the details of their formation remain obscure and their potential as specific indicator of impact details unexploited.

In this work on the impact glass from Wabar we report on of the relation between the simple spherules themselves and the hosting glass using SEM, and a detailed study of the spherules using a combination of FIB/SEM reconstruction and TEM.

Overall, we identified a late stage differential contraction of the spherules in the hosting glass, facilitating mechanical separation of the spherules from the host. The structure of the spherules is consistent with entry of a small nugget of the meteorite and some associated air into the molten glass. The spherical shape is acquired as the molten glass induces melting of the metal and its minor components. While the metal is molten a density related differentiation of the light components like iron sulfide and voids results in the accumulation of these at or close to the surface of the spheres.

With these principal processes being established the huge variation in the detailed sphere morphology can be rationalized.

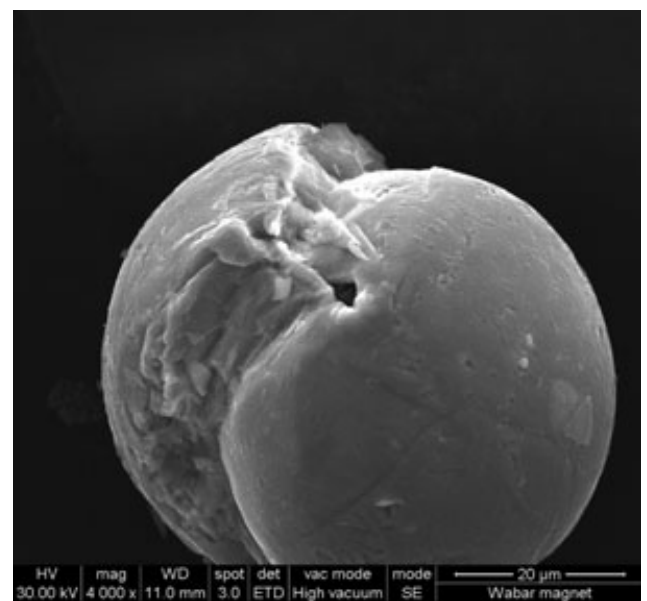

Fig. 1. Composite Fe-Ni spherule from Wabar impactite glass.
5059

NALBACH (SAARLAND, GERMANY) AND WABAR (SAUDI ARABIA) GLASS-TWO OF A KIND?

M Schmieder ${ }^{1}$, W. Müller ${ }^{2}$ and E. Buchner ${ }^{1,3}$. ${ }^{1}$ Institut für Planetologie, Universität Stuttgart, Herdweg 51, 70174 Stuttgart, Germany. E-mail: martin.schmieder@geologie.uni-stuttgart.de. ${ }^{2}$ Diefflerstr. 217, 66809 Nalbach/Saar, Germany. ${ }^{3}$ Rieskrater-Museum Nördlingen, EugeneShoemaker-Platz 1, 86720 Nördlingen, Germany.

Introduction: The recent discovery of enigmatic glasses near Nalbach (Saarland, Germany) by one of the authors (W. M.) [1] poses the major question whether these glasses were produced by an impact event [2]. Although no shocked quartz grains have been detected so far, the Nalbach glass shows a number of features typical for terrestrial impact glasses, such as those found at, e.g., Monturaqui (Chile), Henbury (Australia), or the Libyan Desert Glass. In particular, the Nalbach glass exhibits a striking similarity to the Wabar (Saudi Arabia) impact glass [3, 4].

Nalbach and Wabar Glass-Comparison and Interpretation: The Nalbach and Wabar glasses both exhibit bluish to greenish varieties with a fluidal and schlieren-rich, vesicular, $\mathrm{SiO}_{2}$-rich glassy groundmass (Fig. 1A, B). Both glass types contain abundant ballen $\alpha$-cristobalite as a high-temperature $\left(>1000{ }^{\circ} \mathrm{C}\right)$ modification of silica [2]. Microscopic $\mathrm{Fe}-$ $\mathrm{Ni}$ spheres considered as droplets of iron meteoritic matter [2-4] are distributed in a very similar way in both glass types. The Nalbach and Wabar glasses show microstructural evidence for a dynamic liquid interaction between the silicatic melt, incorporated Fe-Ni droplets, and gas (vesicles), which is expressed by the occurrence of flattened $\mathrm{Fe}-\mathrm{Ni}$ spheres (Fig. 1C, D) and microspheres adherent to the inner surfaces of vesicles (Fig. 1E, F). Our preliminary observations are consistent with the formation of both glasses as an "emulsion" of an iron meteoritic melt in a hot, degassing target melt [4] and, like for Wabar, favor an impact origin for the Nalbach glass. Further analyses are ongoing.

References: [1] Müller W. 2011. Prims: A possible Holocene meteorite impact in the Saarland region, West Germany. Scribd document no. 51477759 (available online). [2] Buchner et al. 2011. This volume. [3] Hörz F. et al. 1989. Proceedings, 19th Lunar and Planetary Science Conference. pp. 697-709. [4] Schmieder M. and Buchner E. 2009. Abstract volume, $1^{\text {st }}$ AICAC Meeting, Amman, Jordan, CD-ROM.

Fig. 1. Nalbach and Wabar glass. A: Uncut specimen, Nalbach (from [1]). B: Polished section, Wabar. C: Backscattered electron image (BEI) of vesicular glassy melt matrix (gray) and flattened $\mathrm{Fe}-\mathrm{Ni}$ sphere (white) at the melt/vesicle interface, Nalbach. D: Similar to C but from Wabar (BEI). E: Fe-Ni microspheres adherent to the inner surface of a vesicle, Nalbach (BEI). F: Similar to E, with larger Fe-Ni sphere, Wabar (BEI).

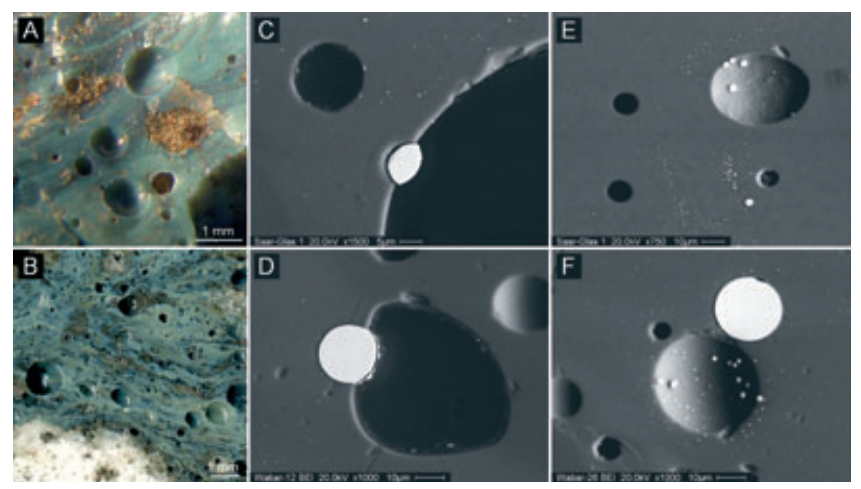


5060

\section{IMPACTITES AND RELATED LITHOLOGIES} GERMANY-CURRENT STATE OF KNOWLEDGE

M Schmieder ${ }^{1}$, W. Müller ${ }^{2}$, L. Förster ${ }^{3}$ and E. Buchner ${ }^{1,4}$. ${ }^{1}$ Institut für Planetologie, Universität Stuttgart, Herdweg 51, 70174 Stuttgart, Germany. E-mail: martin.schmieder@geologie.uni-stuttgart.de. ${ }^{2}$ Diefflerstr. 217, 66809 Nalbach/Saar, Germany. ${ }^{3}$ Eichkamp 35, 23714 Bad Malente. ${ }^{4}$ Rieskratermuseum, Eugene-Shoemaker-Platz 1, 86720 Nördlingen, Germany.

Background: Germany, as the country of the Nördlinger Ries and pioneer impact studies [1], is of particular significance to impact research. The approximate $24 \mathrm{~km}$ and approximate $14.6 \mathrm{Ma}$ Ries crater and the approximate $3.8 \mathrm{~km}$ Steinheim Basin in S Germany, both associated with a full suite of proximal impact ejecta (i.e., lithic impact breccias, suevites, and impact melt rocks), count among the best preserved impact structures on Earth $[2,3]$. Distal Ries ejecta comprise the Central European tektite ("moldavite") strewn field, parts of which extend into Lusatia/Saxony [4], as well as the "Brock-horizont" layer in Bavaria and Baden-Württemberg [5]. Apart from the Ries and Steinheim craters, some enigmatic structures have been proposed to be of impact origin [6-10], however, all of these geologic features currently lack evidence for shock metamorphism and/or meteoritic matter as proof for impact.

New Discoveries: Fluidal, schlieren-rich, and vesicular silicatic glass containing abundant $\mathrm{Fe}-\mathrm{Ni}$ spheres and ballen-textured $\alpha$-cristobalite was recently discovered (W.M.) near Nalbach in the Saarland. The petrography and geochemistry of the glass revealed a striking resemblance to the Wabar impact glass found in Saudi Arabia and support a likely impact origin of the Nalbach glass [11, 12].

Erratic boulders and cobbles of Fennoscandian impact melt rocks were found (L. F.) in Pleistocene glacial till near Sieversdorf and Braak in Schleswig-Holstein, as well as from the Baltic Sea coast near Sassnitz on Rügen Island, Mecklenburg-Western Pomerania. Shock petrographic investigations suggest that these rocks were glacially transported over distances of up to approximately $800 \mathrm{~km}$ from the approximately $19 \mathrm{~km}$ Dellen and approximately $9 \mathrm{~km}$ Mien impact structures, Sweden, as their source craters $[13,14]$.

Magnetic $\mathrm{Fe}-\mathrm{Ni}$ and silicatic spherules were reported from Neogene (Miocene) cave sediments of the Swabian Alb, Baden-Württemberg [15], which might suggest a link to the Ries-Steinheim event; similar magnetic spherules were found in Eocene-Oligocene clays in Hesse [16]. It will be worthwhile undertaking a first focused search for distal ejecta produced by large Paleozoic to Cenozoic terrestrial impacts in the German sedimentary record.

References: [1] Shoemaker E. M. and Chao E. C. T. 1961. Journal of Geophysical Research 66:3371-3378. [2] Buchner E. et al. 2010. Meteoritics \& Planetary Science 45:662-674. [3] Buchner E. and Schmieder M. 2010. Meteoritics \& Planetary Science 45:1093-1107. [4] Lange J. M. 1995. Proceedings, 26th Lunar and Planetary Science Conference. pp. 827-828. [5] Buchner E. et al. 2003. Icarus 191:360-370. [6] Rutte E. 1974. Obberrh. geol. Abh. 23:66-105. [7] Ernstson K. et al. 2010. Journal of Siberian Federal University Engineering and Technologies 1:72-103. [8] Fehr K. T. et al. 2005. Meteoritics \& Planetary Science 40:187-194. [9] Auth R. 2010. Buchenblätter 14:44-46. [10] Harting M. et al. 2003. Abstract \#5201. 66th MetSoc. [11] Buchner E. et al. 2011. This conference. [12] Schmieder M. et al. 2011. This volume. [13] Förster L. et al. 2010. Abstract \#5026. 73rd MetSoc. [14] Meyer K.-D. 1987. Der Geschiebesammler 20:125-146. [15] Schmieder M. et al. 2008. Abstract \#55. Paneth, 15-16. [16] Schidlowski M. and Ritzkowski S. 1972. Neues Jahrbuch für Mineralogie, Geologie und Paläontologie. 3:170-182.
5316

RELICT STRUCTURE OF A HYDROUS MINERAL IDENTIFIED IN WILD 2 DUST

S. Schmitz and F. E. Brenker. Geoscience Dept., Goethe University, Altenhoefer Allee 1, D-60438 Frankfurt / Main, Germany. E-mail: schmitz@em.uni-frankfurt.de.

Introduction: In January 2006, the Stardust spacecraft returned to Earth two unprecedented and independent types of extraterrestrial samples: bonafide samples of a comet, and the first grains of contemporary interstellar dust. Over a thousand dust particles from comet Wild 2 that have been collected by the Stardust spacecraft have been extensively investigated [1-5]. None of them ever yield compelling evidence for a hydrous phase. Here we present for the first time TEM data from a terminal particle (TP) (track 32) indicating the former existence of the phyllosilicate sepiolite or a Na-poor saponite.

Technique: A TEM grid from the terminal particle (TP) of Track 32 (C2027, 3, 32, 3, 8) was investigated in great detail using transmission electron microscopy. We performed high-resolution (HRTEM), bright field-(BF) imaging and selected area electron diffraction (SAED) to characterize the crystal structures. Additional energy dispersive X-ray (EDX) analyses were performed for determining the chemical composition. D-spacings were obtained from lattice fringe images and SAED patterns respectively.

Results: The complex TP of track 32 shows two clearly distinct mineral phases. The first, enstatite $(\mathrm{Mg} / \mathrm{Si} 0.919 ; \mathrm{Fe} / \mathrm{Si} \mathrm{0.026;} \mathrm{Ca} / \mathrm{Si} \mathrm{0.011;}$ $\mathrm{Al} / \mathrm{Si} 0.010 ; \mathrm{Cr} / \mathrm{Si} 0.011)$ shows high stacking disorder parallel (100) which includes alternating clinoenstatite (CLEN) and orthoenstatite (OREN) lamellae, (100) twins and pronounced stacking disorder. Attached and partly enclosed into the enstatite a structurally separated second phase $(\mathrm{Mg} / \mathrm{Si} 0.694 ; \mathrm{Fe} / \mathrm{Si} 0.037 ; \mathrm{Ca} / \mathrm{Si} 0.026 ; \mathrm{Al} / \mathrm{Si} 0.032 ; \mathrm{Cr} / \mathrm{Si}$ $0.015 ; \mathrm{Mn} / \mathrm{Si} 0.015 ; \mathrm{Ti} / \mathrm{Si} 0.013$ ) was discovered showing significantly different compositional and textural behavior. Chemical characterization is consistent with sepiolite or Na-poor saponite, both are phyllosilicates. Detailed TEM studies reveal sigmoidal fractures in this second phase which are typically observed as the result of dehydration in smectites or similar hydrous phases under vacuum. Microstructural data (HRTEM, SAED) are partly consistent with a high temperature triclinic clinoenstatite structure. The structural deviation is most likely due to the lower $\mathrm{Mg} / \mathrm{Si}$ ratio of the new phase. Collected EELS spectra didn't show $\mathrm{OH}-$ related pre peaks indicating complete dehydration of the sample. Our data suggest that sepiolite or low $\mathrm{Na}$ saponite are the most likely candidates for the missing hydrous phase of comet Wild 2 samples, which transform to high temperature triclinic enstatite due to impact heating during capture into Aerogel.

References: [1] Brownlee et al. 2006. Science 314:1711-1716. [2] Flynn et al. 2006. Science 314:1731-1735. [3] Zolensky et al. 2006. Science 314:1735-1739. [4] Schmitz S. and Brenker F. E. 2008. The Astrophysical Journal Letters 681:L105-108. [5] Schmitz et al. 2009. Geochimica et Cosmochimica Acta 73:5483-5492. 
5343

RELATIONSHIP BETWEEN FEO CONTENT AND ÁÂA $\hat{A}^{17}$ IN CHONDRULES FROM CR CHONDRITES: LINKING OXYGEN FUGACITY AND O-ISOTOPE EVOLUTION

D. L. Schrader ${ }^{1}$, H. C. ConnollyJr. ${ }^{1,2,3}$, D. S. Lauretta ${ }^{1}$, K. Nagashima ${ }^{4}$ and G. R. Huss ${ }^{4}$. ${ }^{1}$ LPL, University of Arizona, Tucson, AZ 85721, USA. ${ }^{2}$ Department of Physical Sciences, Kingsborough Community College of CUNY, Brooklyn, NY 11235 and Earth and Environmental Sciences, The Graduate Center of CUNY, New York, NY 10024, USA. ${ }^{3}$ Department of Earth and Planetary Sciences, AMNH, NY 110024, USA. ${ }^{4}$ HIGP, University of Hawai'i at Mānoa, Honolulu, HI 96822, USA, E-mail: schrader@1pl.arizona.edu.

Introduction: In situ O-isotope measurements of chondrules provide clues about their solid precursors, melting history, and exchange with a gas reservoir [1, 2]. We present observations which constrain the formation environment of ferromagnesian chondrules from CR chondrites, part of an ongoing study to understand the formation conditions of type-II chondrules [2, 3].

Methods: We investigated chondrules from CR chondrites QUE 99177, GRA 95229, and GRA 06100. We analyzed element abundances of the olivines in 8 type-I and 12 type-II chondrules with the Cameca SX50 electron microprobe at LPL. These same grains were analyzed with the UH Cameca ims 1280 ion microprobe for their O-isotope composition [2].

Results: O-isotope composition of olivine in type-II $\left(>\mathrm{Fa}_{10}\right)$ chondrules varies with the abundance of FeO-poor relict grains. Most type-II chondrules without relict grains have olivine phenocrysts with $\mathrm{O}$ compositions that cluster slightly above the TF line $\left(\Delta^{17} \mathrm{O}=0.2 \pm 1.0 \%\right.$, $2 \sigma$ ). A second population (from MAC 87320 [1,2] and GRA 95229) of relict-free type-II chondrules has phenocrysts with compositions that plot below the TF line near the CCAM line $\left(\Delta^{17} \mathrm{O}=-1.6 \pm 0.5 \%\right)$. Relict grains within type-II chondrules have $\Delta^{17} \mathrm{O}=-4.4 \pm 1.1 \%$, similar to olivine in type-I chondrules $\left(\Delta^{17} \mathrm{O}=-3.2 \pm 0.9 \%\right.$ [ [2]. Type-II chondrules with $\mathrm{FeO}$-poor relict grains contain both ${ }^{16} \mathrm{O}$-rich $\left(\Delta^{17} \mathrm{O}=\right.$ $-2.5 \pm 1.1 \%$ ) and ${ }^{16} \mathrm{O}$-poor $\left(\Delta^{17} \mathrm{O}=0.2 \pm 0.7 \%\right)$ FeO-rich olivine.

Discussion: The $\mathrm{FeO}$ content of olivine and degree of melting experienced by chondrules are both related to $\dot{A} \hat{A}^{17} \mathrm{O}$. In general $\mathrm{FeO}$ poor olivine has low $\Delta^{17} \mathrm{O}$, and FeO-rich olivine has high $\Delta^{17} \mathrm{O}$. Type-I chondrule olivine has the lowest $\Delta^{17} \mathrm{O}$ and $\mathrm{FeO}$ content. FeO-rich phenocrysts in type-II chondrules without relict grains have the highest $\Delta{ }^{17} \mathrm{O}$. Type-II chondrules with relicts contain olivine with both low $\Delta^{17} \mathrm{O}$ and high $\Delta^{17} \mathrm{O}$.

These data suggest that $\mathrm{FeO}$ and $\Delta^{17} \mathrm{O}$ of olivine increased concurrently during type-II chondrule formation. FeO-poor, ${ }^{16} \mathrm{O}$-rich precursors contributed to the final isotopic composition of type-II chondrules [2]. The oxidation states and isotopic compositions of type-II chondrule olivines may have been established by: (1) the composition of their silicate precursors, a mixture of ${ }^{16} \mathrm{O}$-rich FeO-poor type-I chondrule fragments and ${ }^{16} \mathrm{O}$-poor FeO-rich material; (2) exchange between the chondrule melt and an ${ }^{16} \mathrm{O}$-poor, oxidizing gas reservoir; or (3) chondrule precursor materials such as $\mathrm{H}_{2} \mathrm{O}$ ice or reduced carbon $[2,4]$. Components of these hypotheses are not mutually exclusive, and a combination of processes is plausible.

Acknowledgments: This work was supported by the DDF of the NAI (DSL, PI) and NASA Cosmochemistry (HCC, PI).

References: [1] Krot A. N. et al. 2006. Geochimica et Cosmochimica Acta 70:767-779. [2] Connolly H. C. Jr. and Huss G. R. 2010. Geochimica et Cosmochimica Acta 74:2473-2483. [3] Schrader D. L. 2008. Geochimica et Cosmochimica Acta 72:6124-6140. [4] Connolly H. C. Jr. et al. 1994. Nature 371:136-139.
5013

FORMATION OF REFRACTORY METAL ALLOYS: THE FIRST CONDENSATES OF THE SOLAR SYSTEM?

D. Schwander ${ }^{1,2}$, T. Berg ${ }^{2}$ H. Palme ${ }^{3}$ U. Ott ${ }^{1}$ and G. Schönhense ${ }^{2}$. ${ }^{1}$ Max-Planck-Institut für Chemie, Joh.-Joachim-BecherWeg 27, D-55128 Mainz, Germany. E-mail: d.schwander@mpic.de. ${ }^{2}$ Institut für Physik, Johannes Gutenberg-Universität, Staudingerweg 7, D-55128 Mainz, Germany. ${ }^{3}$ Forschungsinstitut und Naturmuseum Senckenberg, Senckenberganlage 25, D-60325 Frankfurt am Main, Germany.

Sub-micrometer sized refractory metal nuggets (RMN) separated from acid resistant residues of the Murchison meteorite have concentrations of the refractory metals Os, W, Ru, Mo, Ir that match calculated high temperature condensates having equilibrated with a solar composition gas at $1400-1600 \mathrm{~K}$ at $10^{-4}$ bar. The presence of the proper amounts of the easily oxidizable elements $\mathrm{Mo}$ and $\mathrm{W}$ in the alloy makes alteration in the meteoritic environment or during chemical processing very unlikely and thus supports a condensation origin [1]. A TEM study of a subset of these grains indicates a hexagonal close-packed crystal structure in all cases, supporting single phase condensation [2].

The most important objective in further studies is the in situ identification of the RMN: In polished sections of Allende and Murchison we have begun a systematic search for submicron refractory metal particles in order to see if the morphology and chemistry of these particles is identical to the particles recovered from acid resistant residues.

First results indicate that the RMN are very rare and are probably restricted to certain areas of the meteorites. It is presently unclear if the RMN are associated with CAI or occur as isolated grains in matrix, or both.

At the same time we have produced additional acid residues from Murchison. In one of these residues another population of RMN was identified. By the time of the conference we will present the compositions of these RMN. It is of interest to see if this new population of RMN covers exactly the same range in condensation temperatures as the particles of the population described by [1]. Furthermore we have started to produce acid residues from Allende to see if there are particles which could be similar or identical to those from Murchison.

References: [1] Berg T. et al. 2009. The Astrophysical Journal 702:L172-L176. [2] Harries D. et al. 2011. Abstract \#1837. 42nd Lunar and Planetary Science Conference. 
5127

ALHA77005: MONITOR FOR TERRESTRIAL INFLUENCE ON NOBLE GASES AND ANALOGUE FOR MARS

S. P. Schwenzer ${ }^{1,2}$, J. Schwanethal ${ }^{1}$, U. Ott ${ }^{2}$, A. G. Tindle ${ }^{1}$, M. Anand ${ }^{1}$ and S. P. Kelley ${ }^{1}$. ${ }^{1}$ The Open University, Walton Hall, Milton Keynes, MK7 6AA, UK. E-mail: s.p.schwenzer@open.ac.uk. ${ }^{2}$ Max-Planck Institut für Chemie, J.-J. Becher Weg, 55128 Mainz, Germany.

Introduction: The noble gases in Martian meteorites are derived from the Martian interior and atmosphere, and cosmogenic nuclides [1], but quantifying the components is complicated by terrestrial cold desert storage which can cause serious contamination, partially obscuring the extraterrestrial heavy noble gas signatures [2, and references therein] upon which key observations have been made (e.g., Martian atmosphere in EETA 79001, fractionated or ancient Martian atmosphere in ALHA77005 [see 1 for references]). This study investigates the rim and interior of ALHA77005 to characterize terrestrial air contamination, and derive potential correction procedures.

Sample and Methods: We obtained rim and interior samples of ALHA77005 (NASA, split ALH 77005, 225, 227, thin section 54). Each split was gently crushed, $\sim 20$ grains were handpicked, irradiated at McMaster reactor (Canada), and measured by melting grains individually by laser heating for Ar isotope analysis (MAP215-50). Microscopy, virtual microscope documentation, and electron microprobe (Cameca SX 100) were done at the OU.

Results and Discussion: Analysis of the rim of the sample revealed open fractures, which are lined by alteration minerals up to $\sim 0.5 \mathrm{~mm}$ into the meteorite, but appear alteration free deeper into the sample.

$\mathrm{Ar}-\mathrm{Ar}$ analysis reveals clear differences between rim and interior samples. Interior samples yield a range of ${ }^{36} \mathrm{Ar} /{ }^{40} \mathrm{Ar}$ of 0.001 to 0.003 but little correlation with ${ }^{39} \mathrm{Ar} /{ }^{40} \mathrm{Ar}$. In contrast, exterior sample grains fall into two groups, one group displaying variable ${ }^{39} \mathrm{Ar} /{ }^{40} \mathrm{Ar}$ with a clear trend towards terrestrial atmospheric ${ }^{36} \mathrm{Ar} /{ }^{40} \mathrm{Ar}$, but some analyses with low ${ }^{36} \mathrm{Ar}$ are associated with high ${ }^{40} \mathrm{Ar} /{ }^{36} \mathrm{Ar}$. Average ${ }^{40} \mathrm{Ar} /{ }^{36} \mathrm{Ar}$ in these samples is lower than the values measured at Mainz (MZ) by the fusion of bulk-sample $(50 \mathrm{mg})$ and by spot fusion measurement of the sample at OU $\left[{ }^{40} \mathrm{Ar}{ }^{36} \mathrm{Ar} 1229(\mathrm{MZ})\right.$ and $\left.887(\mathrm{OU})\right]$. If relative probability plots of ${ }^{40} \mathrm{Ar} /{ }^{36} \mathrm{Ar}_{\text {tr }}$ (cosm. corr. with $\left({ }^{38} \mathrm{Ar} /{ }^{36} \mathrm{Ar}\right)_{\text {tr }}=0.244$, [3] $)$ are investigated, both populations show two peaks with the exterior sample being considerably closer to terrestrial atmosphere $\left({ }^{40} \mathrm{Ar}^{36}{ }^{36} \mathrm{Ar}_{\text {tr }}\right.$ of $\sim 350$ and 800 exterior; $\sim 750$ and 1400 interior). The second maximum of the interior sample is identical with ${ }^{40} \mathrm{Ar} /{ }^{36} \mathrm{Ar}_{\text {tr }}$ measured in ol and pyx separates of ALHA77005 [4].

Conclusions: Our Ar-Ar study reveals significant air contamination even in the inner parts of the ALHA77005 sample. Future work will add heavy noble gas and trace element data in order to evaluate correction procedures for terrestrial contamination. The goal is to be able to see through terrestrial contamination even if only a small and weathered sample is available for study. Moreover, this will serve as an analogue to enhance our understanding of gas incorporation processes in cold deserts, which will help measuring Ar and noble gases on Mars [5,6].

References: [1] Swindle T. D. 2002. Martian noble gases. Reviews in Mineralogy and Geochemistry, vol. 47. pp. 171-190. [2] Schwenzer S. P. et al. 2009. Polar Science, doi:10.1016/j.polar.2009.06.00. [3] Wiens R. C. 1988. Earth and Planetary Science Letters 91:55-65. [4] Schwenzer S. P. et al. 2006. Meteoritics \& Planetary Science 41:A161. [5] Bogard D. D. 2009. Meteoritics \& Planetary Science 44:3-14. [6] Talboys D. L. et al. 2009. Planetary and Space Science 57:1237-1245.
5276

THE SECONDARY MINERAL-FORMING FLUID IN THE NAKHLITES

S. P. Schwenzer ${ }^{1}$ and J. C. Bridges ${ }^{2}$. ${ }^{1}$ Dept. of Earth Sciences, CEPSAR, The Open University, Milton Keynes MK7 6AA, UK; s.p.schwenzer@open.ac.uk. ${ }^{2}$ Space Research Centre, Dept. of Physics \& Astronomy, University of Leicester LE1 7RH, UK; E-mail: j.bridges@le.ac.uk.

Introduction: The nakhlite secondary minerals are mainly a siderite/ smectite and serpentine/silicate gel/salt assemblage which varies in composition between the nakhlites [1, 2]. This increasingly accurate understanding of the secondary mineralogy now allows us to model the associated fluid. Fluid modeling also helps test our hypothesis that the alteration occurred in the aftermath of an impact into ice-bearing Martian crust [2]. We utilize experience on thermochemical modeling of Martian impact hydrothermal systems [3], with the presence of $\mathrm{CO}_{2}$ [4] to derive likely formation conditions of the nakhlite alteration assemblage.

Methods: We use CHILLER [5] to model the fluid. Lafayette bulk chemistry [6], olivine [6], mesostasis compositions, and combinations of them are used as dissolving host rock chemistry. The initial fluid is a diluted brine with $\mathrm{Fe}, \mathrm{Ca}$ and $\mathrm{Mg}$ in the $10^{-2} \mathrm{~mol} \mathrm{~L}^{-1}$ range, charge balanced by $\mathrm{Cl}^{-}$. We modeled $\mathrm{H}_{2} \mathrm{CO}_{3}$ concentrations between $0.1 \times 10^{-4} \mathrm{~mol} \mathrm{~L}^{-1} \mathrm{H}_{2} \mathrm{CO}_{3}$ and $5 \mathrm{~mol} \mathrm{~L}^{-1}$. Each analysis has data for water to rock ratios (W/R) between 1 and 100,000 . W/R is defined as the amount of rock that completely dissolved in the given amount of initial fluid. Model temperatures (at 10 bar) are 10 and $100{ }^{\circ} \mathrm{C}$.

Results and Conclusions: At both temperatures the nature of the mineral assemblages can be grouped e.g. high $\mathrm{W} / \mathrm{R}$, where $\mathrm{Fe}$ and $\mathrm{Al}$ mainly form minerals such as hematite (at $100{ }^{\circ} \mathrm{C}$ ) or goethite (at $10{ }^{\circ} \mathrm{C}$ ), but silicates - such as are seen in the nakhlites-are not formed. At intermediate $\mathrm{W} / \mathrm{R}$ phyllosilicates like some of those observed [2] are predicted: nontronite, chlorite, talc, serpentine, depending on $\mathrm{T}$ and composition of the host rock, occur together with hematite/goethite. At low $\mathrm{W} / \mathrm{R}$ smectite (nontronite) is not formed from most host rock chemistries, but the assemblages include serpentine, chlorite and other minerals. In the presence of $\mathrm{CO}_{2}$, carbonates precipitate replacing any oxide and forming $\mathrm{Mg}-(\mathrm{Ca})$-siderite at low $\mathrm{W} / \mathrm{R}$. At $10{ }^{\circ} \mathrm{C}$ and $\mathrm{W} / \mathrm{R}$ of $1.5-8$ the model carbonate contains $\sim 45 \%$ sd, $32 \%$ cc, $23 \% \mathrm{mg}$, which matches some of the compositions in Nakhla [1]. From our observation of Lafayette with carbonate being precipitated first followed by smectite and serpentine [2], we assume that $\mathrm{CO}_{2}$ was exhausted by carbonate precipitation and was followed by phyllosilicate. During initial carbonate formation the $\mathrm{pH}$ is buffered by the presence of dissolved $\mathrm{H}_{2} \mathrm{CO}_{3}$. Smectite (nontronite $43 \%$ ) with serpentine (antigorite 36\%) and traces of chlorite plus goethite, apatite, pyrite precipitated in our model from Lafayette bulk rock, $\mathrm{W} / \mathrm{R} \sim 380$ and $\mathrm{pH} 8$. Our results are consistent with the nakhlite mineral assemblages and $\mathrm{W} / \mathrm{R}$ ratios decreasing from Lafayette to Nakhla and low fluid temperatures

References: [1] Bridges J. C. et al. Space Science Reviews 2001. 96:365-392. [2] Changela H. G. and Bridges J. C. 2010. Meteoritics \& Planetary Science 45:1847-1867. [3] Schwenzer S. P. and Kring D. A. 2009. Geology 37:1091-1094. [4] Schwenzer S. P. and Kring D. A. 2009. Meteoritics \& Planetary Science 44:A188. [5] Reed M. H. and Spycher N. F. 2006. Users Guide for CHILLER: A Program for Computing WaterRock Reactions, Boiling, Mixing and Other Reaction Processes in Aqueous-Mineral-Gas systems and Minplot Guide.U. of Oregon. [6] Treiman A. H. 2005. Chemie der Erde 65:203-270. 


\section{6}

THERMAL AND IMPACT HISTORY OF H CHONDRITES: WAS THE ONION SHELL STRUCTURE PUNCTURED BY IMPACTS DURING METAMORPHISM?

Edward R. D. Scott ${ }^{1}$, Tatiana V. Krot ${ }^{1}$, Joseph I. Goldstein ${ }^{2}$ and G. Jeffrey Taylor ${ }^{1} .{ }^{1}$ HIGP, University of Hawaii, Honolulu, HI, USA. E-mail: escott@hawaii.edu. ${ }^{2}$ Dept. of Mechanical and Industrial Engineering, University of Massachusetts, Amherst, MA. USA.

Introduction: The thermal and impact histories of ordinary chondrites provide important clues to the formation and impact histories of asteroids. Bodies that were heated by ${ }^{26} \mathrm{Al}$ and were undisturbed by impacts developed an onion-shell structure with the least metamorphosed type 3 material on the outside and types 4-6 arranged in concentric shells in an onion-shell structure. Trieloff et al. [1] argued from their Ar-Ar and $\mathrm{Pb}-\mathrm{Pb}$ ages and $\mathrm{Pu}$ fission track data for $9 \mathrm{H} 4-6$ chondrites that the onion-shell structure was undisturbed during cooling and that the metallographic cooling rates, which showed little or no correlation between type and cooling rate [2], were flawed or compromised by later impact reheating. Harrison and Grimm [3] discarded fast metallographic cooling rates and inferred that only a very small fraction of the age and cooling rate data for $\mathrm{H}$ chondrites were inconsistent with an onion-shell model.

Methods: We have studied taenite grains and shock levels in 13 H4-6 chondrites which are largely shock stage S1/S2. Metallographic cooling rates were determined from central $\mathrm{Ni}$ contents of taenite grains using the Wood technique to constrain thermal histories during kamacite growth at $500-600{ }^{\circ} \mathrm{C}$. Cloudy taenite particles, which formed below $350{ }^{\circ} \mathrm{C}$, were measured using a FEI field emission SEM with a resolution of $1-2 \mathrm{~nm}$.

Results: Zones of cloudy taenite are clearly visible in all chondrites except for three fast cooled $\mathrm{H} 4$ chondrites. The $0.1 \mu \mathrm{m}$ scale of these intergrowths precludes any diffusion during reheating that could modify the $\mathrm{Ni}$ zoning in taenite on scales of micrometers. Thus the metallographic cooling rates could not have been modified by impact reheating. Cloudy zone dimensions are inversely correlated with metallographic cooling rate and are consistent with iron meteorite data [4]. Our results and published data [2] for $11 \mathrm{H} 4$ chondrites give cooling rates of $5-100{ }^{\circ} \mathrm{C} \mathrm{Myr}^{-1}$ except for three that cooled at $>5000{ }^{\circ} \mathrm{C} \mathrm{Myr}^{-1}$ and which anchor the H4-6 sequence of Trieloff et al. [1]. Our cooling rates for these $\mathrm{H} 4 \mathrm{~s}$ are consistent with the Trieloff ages but are far too high for chondrites that were heated by ${ }^{26} \mathrm{Al}$. These H4s could not have cooled undisturbed near the surface and were probably excavated $\sim 3 \mathrm{Myr}$ after the $\mathrm{H}$ body accreted so that they quickly cooled near the surface. Early impacts like this one could have shock melted sulfides in low-shock type 5 and 6 material $[5,6]$. We predict that most H4s will have $\mathrm{Ar}-\mathrm{Ar}$ ages that are younger than the Trieloff samples. Similarly, H5 and H6 chondrites both show wide ranges of cooling rates that are incompatible with undisturbed cooling. Butsura, for example, cooled about five times faster than four other H6 chondrites including Estacado and Guarena. We infer that several impacts punctured the $\mathrm{H}$ chondrite body to type 6 depths while it was cooling causing disturbances in the thermal histories of many $\mathrm{H}$ chondrites.

References: [1] Trieloff M. et al. 2003. Nature 422:502-506. [2] Taylor G. J. et al. 1987. Icarus 69:1-13. [3] Harrison K. P. and Grimm R. E. 2010. Geochimica et Cosmochimica Acta 74:5410-5423. [4] Yang J. et al. 2010. Geochimica et Cosmochimica Acta 74:4471-4492. [5] Rubin A. E. 2004. Geochimica et Cosmochimica Acta 68:673-689. [6] Scott E. R. D. et al. 2010. Meteoritics \& Planetary Science 45:A184.

\section{3}

TWO TYPES OF GRAPHITE CRYSTALS WITHIN THE KRYMKA CHONDRITE (LL3.1)

V. P. Semenenko and A. L. Girich. Institute of Environmental Geochemistry, NAS of Ukraine, Palladina 34a, Kyiv-142, 03180, Ukraine. E-mail: virasem@i.com.ua.

Previous studies [4, 5] showed that carbonaceous xenoliths in Krymka contain uniformly dispersed regular crystals of graphite, which were probably formed as a result of metamorphism from primary organic compounds. Recently, we found similar crystals of graphite $(\leq 4 \times 0.2 \mu \mathrm{m}$ in size) within a primitive, fine-grained rim around a polyzone, olivinepyroxenes macrochondrule $(6 \times 4.3 \mathrm{~mm}$ in size). The rim contains rare grains of $\mathrm{Ca}, \mathrm{Al}$-rich minerals (hibonite, anorthite, diopside and spinel), and its $\mathrm{SiO}_{2} / \mathrm{MgO}$ and $\mathrm{FeO} /(\mathrm{FeO}+\mathrm{MgO})$ ratios are higher (3.11 and 0.86, respectively) than those of the opaque matrix [1].

The macrochondrule consists of several concentric zones: the silicate core is surrounded by two metal-troilite-rich zones, which are separated from each other by a silicate zone. This whole assemblage is enclosed by the fine-grained rim. The metal-troilite mantles contain rounded or irregular graphite grains with a fine, polycrystalline structure. The grains are larger (up to $100 \mu \mathrm{m}$ in size) than metal-associated carbon inclusions in primitive chondrites [3]. By these mineralogical features this unique macrochondrule differs from other known chondrules and macrochondrules [2].

The following speculations about the nature of the two types of graphites found within the studied object can be made. Their presence points to an enrichment in carbon of the precursor material of the macrochondrule and its primitive rim. The existence of two metal-troilite mantles in the macrochondrule attests to its multistage formation. A finegrained, silicate precursor of the macrochondrule was probably mantled by a metal-troilite dust in a mineralogically heterogeneous dusty environment at least twice, like those of accretionary, fine-grained spherules within the Krymka xenolith BK 13 [6]. The following flash melting of the macrochondrule precursor, probably with partial evaporation of its surface led to the separation of immiscible metaltroilite-silicate liquids and the preservation of the melted metal-troilite mantles. Crystallization of graphite during the formation of macrochondrule must be connected with the presence of C-bearing material, for example C-containing metal or organic compounds in the precursor. The primitive, fine-grained rim of the macrochondrule was formed due to deposition of dusty particles onto its surface from a dusty environment before or during accretion of the Krymka parent body. Regular graphite crystals within the fine-grained rim were probably formed from primary organic compounds due to shock metamorphism of the Krymka parent body. Thus, the two types of graphite crystals, which differ in size, shape and structure, have different nature, i.e. metamorphic within the rim and "igneous" within the macrochondrule, due to distinct processing of the primary C-containing material.

Acknowledgments: We are sincerely grateful to Claude Perron for helpful discussion and correction of our English.

References: [1] Huss G. R. et al. 1981. Geochimica et Cosmochimica Acta 45:33-51. [2] Hutchison R. and Bridges J. C. 1995. Meteoritics 30:523-524. [3] Mostefaoui et al. 2000. Geochimica et Cosmochimica Acta 64:1945-1964. [4] Semenenko V. P. et al. 2004. Geochimica et Cosmochimica Acta 68:455-475. [5] Semenenko V. P. et al. 2005. Geochimica et Cosmochimica Acta 69:2165-2182. [6] Semenenko V. P. et al. 2001. Meteoritics \& Planetary Science 36:1067-1085. 


\section{2}

THE END TO END MARS SAMPLE RETURN CAMPAIGN

M. A. Sephton ${ }^{1}$, C. D. K. Herd ${ }^{2}$ and the MEPAG E2E-iSAG team. ${ }^{1}$ Earth Science and Engineering, Imperial College London, South Kensington, United Kingdom, SW7 2AZ. E-mail: m.a.sephton@ imperial.ac.uk ${ }^{2}$ Earth and Atmospheric Sciences, University of Alberta, Edmonton, Canada, T6G 2E3.

Introduction: Mars Sample Return (MSR) is a top priority for the next decade of space exploration [1]. Returned samples would allow their detailed investigation by the most advanced instruments on Earth providing new insights into the history of Mars. The success of MSR would require multiple flight missions and a subsequent non-flight project, a scenario now termed the "MSR Campaign." The campaign objectives need to be understood in detail at an early stage in order to plan the role of each component mission. The End to End International Science Analysis Group (E2E-iSAG) was chartered by the Mars Exploration Program Analysis Group (MEPAG) to undertake this task by building on efforts of previous MEPAG-chartered groups. Since August 2010 the E2E-iSAG has been (1) defining potential objectives, (2) defining candidate criteria for sampling and landing sites definition (3) defining reference landing sites (4) developing a first draft of a returned sample measurement plan, and (5) initial inputs to technology planning for the overall sample return campaign (from the first mission to the analysis of samples on Earth), and not just one segment of this program.

Results: The scientific objectives proposed by E2E-iSAG are, in priority order (1) Critically assess any evidence for past life or its chemical precursors, and place detailed constraints on the past habitability and the potential for preservation of the signs of life, (2) Quantitatively constrain the age, context and processes of accretion, early differentiation and magmatic and magnetic history of Mars, (3) Reconstruct the history of surface and near-surface processes involving water, (4) Constrain the magnitude, nature, timing, and origin of past planet-wide climate change, (5) Assess potential environmental hazards to future human exploration, (6) Assess the history and significance of surface modifying processes, including, but not limited to: impact, photochemical, volcanic, and Aeolian, (7) Constrain the origin and evolution of the Martian atmosphere, accounting for its elemental and isotopic composition with all inert species, (8) Evaluate potential critical resources for future human explorers. With an additional obvious but unprioritised objective-determine if the surface and near-surface materials contain evidence of extant life.

The findings of the E2E-iSAG most relevant to the meteoritical community are those involving sample selection, sample sizing and sample distribution once back on Earth. Sample types of highest priority to the objectives listed above are: (1) Lacustrine or hydrothermal deposits, (2) Hydrothermally altered rocks, (3) Low-T fluid-altered rocks, (4) Unaltered Igneous rocks, (5) Regolith, (6) Airfall dust, and (7) Atmosphere, rocks with trapped atmosphere. Subsurface rocks made accessible by either drilling or natural excavation would be desirable. Sample suites would be preferable for some sample types. The total proposed returned sample mass would be about $500 \mathrm{~g}$ with a proposed subdivision of this amount between sample numbers and types detailed in the E2E-iSAG report. With decades of experience involving curation and analysis of extraterrestrial samples, the meteoritical community may have a new opportunity to apply their skills to Mars samples returned by a campaign of dedicated space missions.

Reference: [1] Squyres S. W. et al. 2011. Washington D.C.: The National Academies Press.
5344

CATALYTIC HYDROGENATION OF THE MACROMOLECULAR MATERIAL IN METEORITES

M. A. Sephton ${ }^{1}$, W. Meredith ${ }^{2}$, A. Verchovsky ${ }^{3}$, J. S. Watson ${ }^{3}$ and C. E. Snape $^{2} .{ }^{1}$ Earth Science and Engineering, Imperial College London, South Kensington, SW7 2AZ, UK, E-mail: m.a.sephton@imperial.ac.uk. ${ }^{2}$ School of Chemical, Environmental and Mining Engineering, University of Nottingham, Nottingham, NG7 2RD, UK. ${ }^{3}$ Planetary and Space Sciences Research Institute, Open University, Milton Keynes, MK7 6AA, UK.

Introduction: By far the major organic component in meteorites is a large and relatively complex organic network or macromolecule [1]. The macromolecule is insoluble in common organic solvents and is generally assumed to be completely indigenous owing to its high molecular weight and immobility. However, it is this attribute which creates analytical difficulties and repulses straightforward investigations.

In recent years non-degradative techniques such as nuclear magnetic resonance spectroscopy $[2,3]$ and degradative methods such as hydrous pyrolysis $[4,5]$ have provided insights into the make up the macromolecular material. One additional and particularly effective method involves the catalytic addition of hydrogen to the macromolecule to liberate its constituent units [6]. The products of catalytic hydrogenation are highly amenable to analysis by mass spectrometric techniques. Catalytic hydrogenation is highly efficient and has released more bound carbon from the macromolecule than any other thermal degradation technique. This approach has also produced the largest ever aromatic units from the thermal degradation of meteorites and has partly reconciled the disharmony between observations of large interstellar molecules and their smaller meteoritic counterparts [6].

To date, this method has only been applied to the type II Murchison meteorite. New experiments have extended this work to other meteorites of differing petrologic type (types I and III), revealing the potential influence of differing organic source materials and subsequent effects of parent body alteration. Moreover, the previous off-line method has been supplemented by an on-line approach that could provide the opportunity for the analysis of a wider range of meteorites only available in small amounts.

Preliminary data suggest the presence of occluded units within the macromolecular material that may reveal which organic compounds formed first during macromolecule construction before being swamped and immobilized by the forming organic network. Contaminant organic compounds are also occasionally present reflecting the propensity of the macromolecule to retain terrestrial compounds despite previous extraction with organic solvents.

References: [1] Sephton. 2002. Natural Product Reports 19:292-311. [2] Gardinier et al. 2000. Earth and Planetary Science Letters 184:9-21. [3] Cody G. D. et al. 2002. Geochimica et Cosmochimica Acta 66:1851-1865. [4] Sephton M. A. et al. 1998. Geochimica et Cosmochimica Acta 62:18211828. [5] Sephton M. A. et al. 2000. Geochimica et Cosmochimica Acta 64:321-328. [6] Sephton M. A. et al. 2004. Geochimica et Cosmochimica Acta 68:1385-1393. 
5209

EXPERIMENTAL TEST OF THE CARBON MONOXIDE SELFSHIELDING MODEL FOR THE EARLY SOLAR SYSTEM'S OXYGEN ISOTOPE EVOLUTION

X. Y. Shi ${ }^{1}$, Q.-Z. Yin ${ }^{2}$, Y.-C. Chang ${ }^{1}$, Z. Luo ${ }^{1}$, H. Huang ${ }^{1}$, C.-Y. $\mathrm{Ng}^{1}$. ${ }^{1}$ Department of Chemistry, University of California, Davis, CA 95616, USA. E-mail: xyshi@ucdavis.edu. ${ }^{2}$ Department of Geology, University of California, Davis, CA 95616, USA. E-mail: qyin@ ucdavis.edu.

We designed a laboratory experiment to test the carbon monoxide self-shielding (COSS) model advanced to explain the oxygen isotope distribution in the early Solar System materials [1-3]. Our unique windowless vacuum ultraviolet (VUV) ultra high resolution laser photodissociation and photoionization mass spectrometry is designed to mimic the solar nebular photochemistry environment and experimentally verify if the CO photodissociation at VUV wavelengths (90-110 nm) would produce the expected mass independent oxygen isotope fractionation as predicted in the recently revived self-shelding model [1-3]. This model has been invoked to explain the peculiar oxygen isotope distribution observed in early solar system materials and has a specific prediction for the Sun's oxygen isotope composition [4], a top science priority of NASA's GENESIS mission.

However, the first experimental test of COSS model by [5] using the Advanced Light Source (ALS) at Lawrence Berkeley National Laboratory (LBNL), poses a serious question on the COSS model, although the validity of [5] were immediately challenged by [6-8]. The significance of COSS model for the early solar system warrants a second opinion with closer experimental scrutiny. If the COSS model stands the test of experimental verification, it has major implications for the origin and transport of water in the Solar System that governs the ultimate habitablility of planets.

Our VUV laser system can generate four to five orders of magnutide higher optical resolution, with brightness more than 6 orders of magnitude greater than the broadband synchrontron light source at ALS at LBNL. This will ensure higher signal-to-noise ratios when studying weak transitions or transitions of less abundant isotopologues, such as ${ }^{13} \mathrm{C}^{17} \mathrm{O}$. Our initial experimental results support a "slope- 1 " ${ }^{16} \mathrm{O}$ depletion line as the COSS model postulates and observed in the early solar system material and now observed in the Sun [9].

References: 1] Clayton R. N. 2002. Nature 415:860. [2] Yurimoto and Kuramoto 2004. Science 305:1763. [3] Lyons and Young 2005. Nature 435:317. [4] Yin Q.-Z. 2004. Science 305:1729. [5] Chakraborty et al. 2008. Science 321:1328. [6] Lyons et al. 2009. Science 324:1516a. [7] Federmann and Young 2009. Science 324:1516b. [8] Yin Q.-Z. et al. 2009. Science 324:1516c. [9] McKeegan et al. 2011. Science, in press.
5004

FINE MORPHOLOGICAL FEATURES OF MINERAL GRAINS IN THE NORTON COUNTY AUBRITE

K.O. Shkurenko, V.P. Semenenko, T.M. Gorovenko and V. M. Slyvinsky. Institute of Environmental Geochemistry, NAS of Ukraine, Palladina 34a, Kyiv-142, 03180, Ukraine. E-mail: koshkurenko@ gmail.com.

Previous scanning electron microscopy (SEM) study of chondrites $[1,4]$ and pallasites $[2,5]$ indicates the presence of a primary and secondary sculptures on the surface of mineral grains. Most of the sculptures are thought to be produced during thermal and shock effects connected with a complex, multistage formation history of the meteorites. Here we present the data on a SEM and energy dispersive spectroscopy (EDS) examination of sculptural features and chemical composition of a surface of grains separated from a fine-grained fraction of the Norton County achondrite [6]. The following minerals and their intergrowths are detected as separate grains: enstatite, forsterite, troilite, kamacite, brezinaite, ferroalabandite, daubreelite, and oldhamite. Iron hydroxides, regular crystals of calcite and gypsum, and fine-grained films of manganite are detected as weathering products. As we know, this is the first time the brezinaite is found in the Norton County achondrite. It was revealed as a friable grain of green-grayish colour with perfect-developed cleavage [6]. The EDS data indicate to the following composition $\left(\mathrm{wt}^{\mathrm{o}} \mathrm{)}\right)$ : 48.2 Cr, 41.2 S, 6.78 Ca, 1.72 Mg, and admixture of Mn, Fe, Ti, Ni, Mg, and $\mathrm{Si}$. The presence of $\mathrm{Ca}, \mathrm{Ni}, \mathrm{Mg}, \mathrm{Mn}$, and $\mathrm{Si}$ is probably due to analytical contamination by surrounding minerals. The presence of refractory elements such as $\mathrm{Ti}, \mathrm{V}$, and $\mathrm{Cr}$ is the main chemical feature of the sulfides that indicates to a high temperature formation of the aubrite. For example, troilite contains (wt\%) up to $2.91 \mathrm{Ti}, 1.04 \mathrm{Cr}$ and $0.27 \mathrm{~V}$, daubreelite does up to $2.04 \mathrm{Ti}$ and $0.24 \mathrm{~V}$, and brezinaite - up to $0.79 \mathrm{Ti}$.

The surface of the examined mineral grains and their fractures is characterized by the following sculptures: (1) elongated voids (rivulet-like voids of [3]) which are parallel to the axis c of enstatite crystals; (2) inclusions of troilite on enstatite, $\mathrm{Cu}-\mathrm{Fe}$-Ti-alloy on olivine, and some unidentified minerals which result in their preferable crystallization on a mineral phase boundary during slow cooling; (3) submicron pores on enstatite and olivine grains that could indicate possibly some gas inclusions; (4) well-developed cleavage along (110) for enstatite, and lamellar deformations on enstatite, olivine, troilite and kamacite formed as a result of shock; (5) sticked drops, emulsions and streams of melt on enstatite, olivine and troilite grains produced by shock heating; (6) thin growth steps on a face of a troilite crystal that testify to their slightly unequilibrated formation probably as result of a post-shock heating.

Thus, the SEM and EDS-study of a small portion of the material allows to get additional data about a mineralogical composition and a fine structure of the achondrite. The morphological and chemical features support the previous petrographic results [3] on important role of multistage shock metamorphism in the cosmic history of the Norton County parent body.

References: [1] Cristophe Michel-Levy M. 1981. Earth and Planetary Science Letters 54:67-80. [2] Kvasnytsya V. and Semenenko V. P. 2003. Meteoritics \& Planetary Science 7:A10. [3] Okada A. et al. 1988. Meteoritics 23:59-74. [4] Semenenko V. P. and Tertichnaya B. V. 1994. 25th Lunar and Planetary Science Conference. pp. 1261-1264. [5] Semenenko V. P. et al. 2008. Proceedings of Ukranian Minerologic Society 5:68-74 (in Ukrainian). [6] Semenenko V. P. et al. 2010. Proceedings of Ukranian Minerologic Society 7:28-35 (in Ukrainian). 
5140

\section{A STUDY OF EVAPORATION OF MURCHISON CHONDRITE AND CAI INCLUSIONS IN EFREMOVKA USING THE KNUDSEN EFFUSION CELL}

S. I. Shornikov and O. I. Yakovlev. RAS Vernadsky Institute of Geochemistry \& Analytical Chemistry, Kosygin st., 19, Moscow, 119991, Russia. E-mail: sergey.shornikov@gmail.com.

Introduction: Vaporization (condensation) is among the key physicochemical processes that form the meteorite substance composition. These processes played an especially important role at formation of the substance of white inclusions in carbonaceous chondrites (CAI). The problems of the origin and physicochemical conditions for CAI formation remain the subject of continuous discussions [1]. A weak side of various genetic models of CAI is their construction on a purely theoretical basis without due reliance on experimental data. Therefore, of special interest are the results of the study on evaporation of CAI substance from the Efremovka meteorite [2].

Experiment: The experiments were performed by the mass spectrometric method at evaporation from a Knudsen effusion tungsten cell of CAI inclusions in Efremovka and Murchison sample. To reduce the interaction of cell material the samples were placed in a rhenium boat on the bottom of a tungsten cell. It did not fully rule out the reducing action of tungsten. The temperature dependence of changes in the studied melts was determined at temperatures up to $2900 \mathrm{~K}$. Experiments were spent in the conditions of step temperature lifting with step of $50 \mathrm{~K}$ at the constant temperature about $15 \mathrm{~min}$. The chosen conditions of step heating allowed to keep a molecular mode of the expiration of steam at the given weight of the sample and optimum to reduce the experiment duration.

Results and Discussion: We identified the gas phase composition (monoatomic forms: $\mathrm{K}, \mathrm{Na}, \mathrm{Fe}, \mathrm{Mg}, \mathrm{Ca}, \mathrm{Al}$; oxide forms: $\mathrm{FeO}, \mathrm{SiO}$, TiO, $\mathrm{TiO}_{2}, \mathrm{CaO}, \mathrm{AlO}, \mathrm{Al}_{2} \mathrm{O}$ ) and found the partial pressure values of vapor species.

Results of experiments on the Murchison chondrites have shown that the temperature sequence of evaporation components melt to a gas phase is almost identical to the known data on evaporation of the lunar basalt [3]. The maximum concentration of silicon dioxide in melt is observed to temperature $2000 \mathrm{~K}$. At high temperature its concentration sharply falls at increasing of concentrations $\mathrm{CaO}$ and $\mathrm{Al}_{2} \mathrm{O}_{3}$ (to 60 $65 \mathrm{wt} \% \mathrm{Al}_{2} \mathrm{O}_{3}$ ).

The CAI inclusions in Efremovka evaporation characterized of silicon monoxide presence in the gas phase with the high partial pressure $\left(10^{-6} \mathrm{~atm}\right)$ at the temperature range $2400-2700 \mathrm{~K}$. The partial pressure values of $p(\mathrm{SiO})$ are closed to the same of $p(\mathrm{Ca})$ and $p(\mathrm{Al})$. It is obviously the such unusual values of $p(\mathrm{SiO})$ are due to the high values of $p\left(\mathrm{O}_{2}\right)$ in comparison to typical for evaporation of Murchison chondrite and lunar basalt. For example, at temperature $2350 \mathrm{~K}$ the value of $p\left(\mathrm{O}_{2}\right)=1.0 \times 10^{-6} \mathrm{~atm}$ for CAI inclusions in Efremovka, and the value of $p\left(\mathrm{O}_{2}\right)=2.0 \times 10^{-7}$ atm for Murchison chondrite.

The obtained result has a genetic importance which showed of the special conditions of formation of CAI inclusions in Efremovka. The CAI inclusions in Efremovka feature is in their formation in the regions with high oxygen concentration.

References: [1] Shornikov S. I. and Yakovlev O. I. 2010. Abstract \#1408. 41st Lunar and Planetary Science Conference. [2] Yakovlev O. I. et al. 1984. 15th Lunar and Planetary Science Conference. pp. 945-946. [3] Markova O. M. et al. 1986. Geokhimiya 11: 1559-1569 (in Russian).
5517

\section{SYNCHROTRON X-RAY IRRADIATION OF Stardust INTERSTELLAR CANDIDATES: FROM "NO" TO "LOW" DAMAGE EFFECTS}

A. Simionovici ${ }^{1}$, C. Allen, S. Bajt, R. Bastien, H. Bechtel, J. Borg, F. E. Brenker, J. C. Bridges, D. E. Brownlee, M. J. Burchell, M. Burghammer, A. Butterworth, P. Cloetens, A. M. Davis, C. Floss, G. Flynn, D. Frank, Z. Gainsforth, E. Grün, P. R. Heck, J. Hillier, P. Hoppe, L. Howard, G. R. Huss, J. Huth, A. T. Kearsley, A. J. King, B. Lai, J. Leitner, L. Lemelle, H. Leroux, R. Lettieri, W. Marchant, L. Nittler, R. Ogliore, F. Postberg, S. Sandford, J.A. Sans Tresseras, T. Schoonjans, S. Schmitz, G. Silversmit, V.A. Solé, R. Srama, T. Stephan, J. Stodolna, R. M. Stroud, S. Sutton, M. Trieloff, P. Tsou, A. Tsuchiyama, T. Tyliczs-zak, B. Vekemans, L. Vincze, A. J. Westphal, D. Zevin, M. E. Zolensky and $>29,000$ Stardust@home dusters ${ }^{2}$. ${ }^{1}$ ISTerre, Observatory of Sciences of the Universe of Grenoble, BP 53, 38041 Grenoble, FRANCE. E-mail: alexandre.simionovici@ujf-grenoble.fr. ${ }^{2}$ ISPE team affiliations are listed at http://www.ssl.berkeley.edu/ ${ }^{\sim}$ westphal/ISPE.

Introduction: Although synchrotron radiation X-Ray fluorescence (SR-XRF) is among the least destructive analysis methods applied to rare extraterrestrial grains, we have observed radiation damage effects following high flux synchrotron analyses. Track 30 of the IS collector of the Stardust mission [1], containing 2 candidates dubbed Orion and Sirius [2] was analyzed at ESRF, France, on beamlines ID13 and ID22NI by nano-XRF/XRD scanning methods. Beam damage effects were noticed on both samples and a quantitative analysis of their irradiation history was established [3], allowing us to propose new experimental protocols as well as fluence limits, minimizing such effects in the future. The purpose of this study is to present these facts, analyze potential damage mechanisms and offer alternatives.

Cosmic Irradiation Limit: The cosmic X-rays background in the 3$300 \mathrm{keV}$ energy range, most likely produced by active galactic nuclei components impacting our IS samples was fitted by a simple analytical expression by Gruber et al. [4] and a limit of cosmic irradiation of $3 \cdot 10^{19}$ $\mathrm{ph} \mathrm{cm}^{-2}$, dubbed astrophysical limit (AL) was established. X-ray scanning of IS samples at fluences (flux - time) exceeding the AL are potentially damaging, except in specific cases, known in biochemistry as "recovery effects" appear, where multiple shorter exposures are preferable to long ones [7].

Damage Mechanisms: Main particle impact damage include electron or proton/alpha irradiation-induced heating, ion displacement and charging $[6,7]$ of microscopic samples, particularly material in an insulator matrix.

References: [1] Brownlee et al. 2006. Science 314:1711-1715. [2] Westphal et al. 2011. Abstract \#2083. 40th Lunar and Planetary Science Conference. [3] Simionovici et al. 2011. Abstract \#2812. 42nd Lunar and Planetary Science Conference. [4] Gruber D. E. et al. 1999. The Astrophysical Journal 520:124-129. [5] Egerton R. F. et al. 2004. Micron 35:399-409. [6] Lemelle L. et al. 2003. Geochimica et Cosmochimica Acta 67:1901-1910. [7] Oger P. et al. 2008. Spectrochimica Acta Part B 63:512517. 


\section{4}

\section{WHERE ARE ALL THE TERRESTRIAL METEORITES?}

Michael J. Simms. National Museums Northern Ireland, Cultra, BT18 0EU, Northern Ireland. E-mail: michael.simms@nmni.com.

Meteorites clearly can reach Earth from the Moon and from Mars, launched by larger impacts. This raises the inevitable question: can solid debris be ejected from Earth by large impacts, to return subsequently, survive re-entry, and be recognized as a meteorite?

Recognition: Meteorites launched from Earth will not be recognizable from lithology or geochemistry. The only diagnostic feature would be a fusion crust formed during re-entry, but this will not develop on many sedimentary rocks [1]. The Antarctic collection programmes are the best prospect for recognizing a fusion-crusted terrestrial meteorite but no candidate has yet been reported. Might this absence be significant and not merely chance?

Residence Times in Space and on Earth: CRE ages for Lunar meteorites [2] are typically $<<1 \mathrm{Ma}$, which suggests that ejected terrestrial debris will have comparably short space residence times. In geological terms return may be almost synchronous with the impact launch event. Maximum terrestrial residence times of achondrites are $<1 \mathrm{Ma}$ [3]. Combined space and terrestrial residence times of a terrestrial meteorite, probably $<1 \mathrm{Ma}$, require a launching impact within the last few hundred ka for there to be any prospect of such a meteorite being recognized at the surface.

Launch Conditions: Minimum crater diameters necessary to achieve significant dispersal of Lunar and Martian material into space are $0.4 \mathrm{~km}$ and $3 \mathrm{~km}$ respectively. Scaling-up to Earth-based parameters suggests a minimum crater diameter of $\sim 25 \mathrm{~km}$, even before the effects of atmospheric drag on Earth-launched projectiles is considered. Spallation may launch solid material at high velocities from a narrow and very shallow zone around the impact site [4], with vaporization of volatiles in the target rock perhaps providing further acceleration. To escape Earth's gravity requires launch velocities perhaps $75 \%$ of impact velocity, which exceeds most published estimates [5] and questions the potential existence of terrestrial meteorites. For the high shock pressures associated with a suitably large Earth impact, any potential meteoroids will be small,

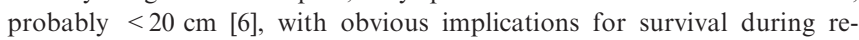
entry.The shallow location of the spallation zone has significant implications for recognizing 'terrestrial' meteorites. The target lithologies for many large impacts were sedimentary (e.g., limestone for the Chicxulub, Manicouagan, and Ries sites). Fusion crusts would not form on such lithologies and hence it would be effectively impossible to identify them as having re-entered the atmosphere as a meteorite.

Conclusions: A fusion crust is the only feature by which a terrestrially derived meteorite might be recognized. None have yet been reported. Minimum crater diameter of $\sim 25 \mathrm{~km}$ and combined space and terrestrial residence times of $<1 \mathrm{Ma}$ for potential Earth-derived meteorites precludes the existence of any at the surface today; a crater of such size and recency is unlikely to have been overlooked by impact hunters scouring Google Earth. Ancient terrestrial meteorites might be sought in strata that slightly post-date major impacts in the geologic past.

References: [1] Brandstätter F. et al. 2008. Planetary and Space Science 56:976-984. [2] Eugster O. et al. 2006. Meteorites and the Early Solar System II. pp. 829-851. [3] Jull A. J. T. 2006. Meteorites and the Early Solar System II. pp. 889-905. [4] Melosh H. J. 1984. Icarus 59:234 260. [5] Wells L. E. et al. 2003. Icarus 162:38-46. [6] Nyquist L. E. 2001. Space Science Reviews 96:105-164.
5151

A STUDY OF GLASSES IN HOWARDITES: IMPACT MELT CLASTS VERSUS PYROCLASTS

S. A. Singerling and H. Y. McSween. Department of Earth \& Planetary Sciences, University of Tennessee, Knoxville, TN 37996, USA. E-mail: ssingerl@utk.edu.

Introduction: Theoretical calculations predict that explosive eruptions ought to have occurred on volatile-bearing asteroids of sufficient size $[1,2]$. The resulting deposits, pyroclastics, should then be preserved in samples derived from these asteroids. The large asteroid 4 Vesta is one candidate that might have experienced explosive volcanism, but pyroclastic material has not yet been definitively identified in meteorite samples (HEDs) from this body [3]. Preliminary work by David Mittlefehldt has identified what may be pyroclasts in several howardite samples (personal communication). The purpose of this research is to identify, describe, and analyze glasses in howardites and distinguish their origin: impact melt-derived or pyroclastic.

The following table outlines expected differences between the two glass types: impact melt clasts and pyroclasts.

Significance: Impact melt clasts act as representative samples of surface lithologies, the study of which will further constrain the range of compositions that occur on Vesta. Pyroclasts, if recognized as such in HEDs samples, could yield information on eruptive conditions operating in/on Vesta and could indicate a new role for volatile elements on Vesta.

Expected Results: This work will focus on compositional results obtained from electron microprobe analysis. Variation diagrams will help distinguish the two compositionally (i.e., $\mathrm{CaO}$ versus $\mathrm{MgO}, \mathrm{FeO} / \mathrm{MgO}$ versus $\mathrm{Al}_{2} \mathrm{O}_{3}$ ) and in regard to volatile elements (i.e., $\mathrm{Na}_{2} \mathrm{O} / \mathrm{CaO}$ versus $\mathrm{Fe} \#$ and $\mathrm{K}_{2} \mathrm{O} / \mathrm{CaO}$ versus $\mathrm{Fe} \#$ ). For an example of such diagrams relating to HEDs, see [4]. Impact melt clasts should plot near a howardite composition on such diagrams since they represent pre-existing material on the surface that was impacted. Pyroclasts should plot near a primitive basaltic eucrite composition since they represent the initial melt. Any xenocrysts in the impact melts clasts should have evolved compositions. Phenocrysts in pyroclasts should have primitive compositions. We expect to present chemical results at the meeting.

References: [1] Wilson L. and Keil K. 1997. Meteoritics \& Planetary Science 32:813-823. [2] Wilson L. et al. 2010. Meteoritics \& Planetary Science 45:1284-301. [3] Keil K. 2002. In Asteroids III pp. 573-85. [4] Mittlefehldt D. W. et al. 1998. In Planetary materials. pp. 4-122.

\begin{tabular}{lll}
\hline & Impact melt clasts & Pyroclasts \\
\hline Composition & $\begin{array}{c}\text { Howardite-like and } \\
\text { mineral-like } \\
\text { Evolved melt }\end{array}$ & $\begin{array}{c}\text { Eucritic-like } \\
\text { Initial melt }\end{array}$ \\
& Heterogeneous & Homogeneous \\
$\begin{array}{c}\text { Volatile-element } \\
\text { content }\end{array}$ & Low & High \\
$\begin{array}{c}\text { Incompatiable-element } \\
\text { content }\end{array}$ & Low & High \\
$\begin{array}{c}\text { Texture } \\
\text { Vitric }\end{array}$ & Vitric \\
& Vitrophyric & Vitrophyric \\
& Dendritic & Dendritic \\
& Partly resorbed clasts & \\
\hline & Schlerin & \\
\hline
\end{tabular}


5142

EFFECTS OF SHOCK METAMORPHISM ON ZIRCONS FROM THE HAUGHTON IMPACT STRUCTURE

C. Singleton ${ }^{1}$, G. R. Osinski ${ }^{1,2}$, and D. Moser ${ }^{1}$. ${ }^{1}$ Department of Earth Sciences, University of Western Ontario, ON N6A 5B7, Canada E-mail: asingle2@uwo.ca. ${ }^{2}$ Department of Physics and Astronomy, University of Western Ontario, London, ON N6A 5B7, Canada.

Introduction: The extreme pressure and thermal conditions attained during shock metamorphism at sometimes very limited spatial and temporal scales [1] result in unique microscopic features that remain to be fully explored in some common rock-forming minerals, including zircon. The type of shock effect(s) created in a given sample depends upon the pressures and temperatures involved as well as the composition, density and material's location in the target. This has prompted our analysis of zircon grains in variably shocked and exceptionally preserved crystalline basement assemblages of the Haughton impact structure, Canada. The purpose of this study is to identify and describe various effects in zircons grains caused by the impact event.

Results: Our results provide valuable new information regarding the effects of shock in zircon.

Shock Effects in Quartz and Shock Levels. In this sample suite it was found that quartz shows the full range of shock features including fracturing, PDFs, toasting, decrease in birefringence, diaplectic melting, full melting with loss of grain boundaries and finally flow banding.Based on these and other shock features shock levels from 1 to 7 were assigned to 52 samples of shocked crystalline material from the Haughton impact structure.

Shock Effects in Zircon. A number of shock effects were identified in the zircon grains. The first of these are a variety of fractures, which range from non-linear to linear. The non-linear fractures are also present in some un-shocked samples so these features do not always indicate shock. Other effects include less defined zoning and micro-faulting. Zircons that have been highly shocked show a distinct granular texture that is proposed to be due to recrystallization of the zircon.

Raman Spectroscopy. Raman spectroscopy investigations were carried out on eight zircons from various shock levels. Level 0 shows peaks at approximately $225,292,354,413,435,819,973$ and 1003. Level 2 shows peaks at approximately 204, 223, 355, 438, 813, 973 and 999. Level 3 shows peaks at approximately 201, 222, 351, 434, 818, 973 and 1000. Level 5 shows peaks at approximately 201, 223, 355, 439, 973 and 1007. Level 6 shows peaks at approximately 201, 222, 353, 438, 972 and 1005 . These results indicate mainly zircon type structures with some evidence for the scheelite-type phase and transition to reidite [2].

Summary: The shock effects identified in these samples range from shock level 1-7 indicating pressures ranging from 2 to $80 \mathrm{GPa}$ which agrees with previously published estimates for Haughton samples [3]. The progression of shock effects seen in quartz is in keeping with other studies. A number of shock effects have been identified in zircon grains of various shock levels and raman spectroscopy investigations suggest evidence of zircon and scheelite-type phase and possible transition to reidite. [add some more text here to fill in the space]

References: [1] Langenhorst F. 2002. Bulletin of the Czech Geological Survey 77:265-282. [2] Gucsik A. 2007. Workshop on Impact Cratering II. [3] Stöffler D. 1966. Contributions to Mineralogy and Petrology 12:1524.
5373

DISTRIBUTION AND ORIGIN OF MAFIC IMPACT EJECTA FROM THE LOCKNE CRATER, SWEDEN

A. S. L. Sjöqvist ${ }^{1}$, P. Lindgren ${ }^{2}$, E. F. F. Sturkell ${ }^{1}$, and M. R. Lee $^{2}$. ${ }^{1}$ Department of Earth Sciences, University of Gothenburg. ${ }^{2}$ School of Geographical and Earth Sciences, University of Glasgow, E-mail: axel@student.gu.se.

Introduction: The Lockne crater formed approximately $455 \mathrm{Myr}$ ago in a target composed of a crystalline Proterozoic basement, overlain by early Palaeozoic sedimentary rocks, and seawater. The inner crater has a diameter of approximately $7 \mathrm{~km}$, with an up to $2.5 \mathrm{~km}$ wide brim of ejected, crushed crystalline basement spread around it [1]. Large ejecta blocks within this rim range from a few meters to approximately $100 \mathrm{~m}$ in diameter are found as far $15 \mathrm{~km}$ from the edge of the crater. The largest ejecta blocks have created smaller, secondary craters around Lockne with diameters of 100-200 m (i.e., Målingen, Karsåtjärnen, and Kloxåsen) [2]. The field distribution of the Kloxåsen ejecta blocks is unknown but determining their relation to the rest of the impact structure is important for a fuller understanding of the formation of Lockne and other similarly sized craters in mixed (crystalline and sedimentary) targets. Here, we have mapped the distribution of ejecta around Kloxåsen. As the chemical and mineralogical composition of the Kloxåsen ejecta blocks is unknown we have also carried out a mineralogical and chemical analysis of this material to establish the source rock of the mafic ejecta blocks, to characterize the extent of hydrothermal alteration, and to determine the levels of shock in the ejecta.

Results and Discussion: Mapping of the Kloxåsen area showed that the mafic ejecta breccia is much more widely distributed than previously thought, although the granitic breccia dominates. Optical petrography, SEM-EDS, and Raman spectroscopy revealed extensive and curious hydrothermal alteration in the mafic clasts. Olivine has been completely replaced by serpentine and chlorite that in turn are wholly or partly replaced by calcite (and translucent sphalerite). This strongly depletes the clasts in $\mathrm{Fe}$ and $\mathrm{Mg}$, and enriches them in $\mathrm{Ca}$. Titanaugite has been replaced by quartz, chlorite, calcite, and anatase, although unaffected titanaugite grains still occur. Some biotites appear "split open" and are replaced by quartz and anatase, but no kinkbanding has been observed. Titanaugite affected by mechanical twinning suggests shock pressure of at least $5 \mathrm{GPa}$ [3]. Fluid-inclusion analysis in calcite revealed that the hydrothermal system in Kloxåsen is the same as in the rest of the crater [2]. Sector-zoned titanaugite oikocrysts, which are only known to occur in CSDG (Central Scandinavian Dolerite Group) rocks [4], and whole-rock geochemistry conducted in this study, strongly suggest the Åsby dolerite as a protolith for the observed mafic impact ejecta at Kloxåsen.

Acknowledgments: The Royal Astronomical Society is thanked for funding this project.

References: [1] Lindström M. et al. 2005. Impact tectonics, Berlin: Springer. pp. 357-388. [2] Sturkell E. F. F. 1998. Geologische Rundschau 87:253-267. [3] Melosh H. J. 1989. Impact cratering: A geologic process. New York, NY: Oxford University Press. [4] Claeson D. T. et al. 2007. Journal of Petrology 48:711-728. 


\section{9}

USING PETROLOGY AND MINERALOGY TO UNDERSTAND THE SURFACE OF VESTA: A COLLECTION OF FINE-GRAINED EUCRITES

S. E. Smith ${ }^{1}$, R. G. Mayne ${ }^{1}$ and C. M. Corrigan ${ }^{2} .{ }^{1}$ Monnig Meteorite Collection, School of Geology, Energy, and the Environment, TCU Box 298830, Texas Christian University, Fort Worth, TX 76109, USA. E-mail: samantha.e.smith@tcu.edu. ${ }^{2}$ Smithsonian Institution, National Museum of Natural History, 10th \& Constitution NW, Washington, D.C. 205600119, USA.

Introduction: Recent work has suggested that fine-grained eucrites could be key to understanding the petrogenesis of Vesta [1]. These eucrites represent original surface or near-surface material that may give us information about Vesta's geologic past. It is unlikely that such units will be visible with the Dawn spacecraft, however, because of the brecciation on the surface of Vesta.

Previous studies, such as [1-3] indicate that individual fine-grained eucrites are not common; however, howardites are the most abundant member of the HEDs family and can contain unique eucrite clasts not found in eucrite-only samples. Therefore, the textural variety of eucrite clast types within howardites is far greater.

By studying these fine-grained eucrite clasts, we can better understand the different units found on the surface of Vesta, and what processes might have produced them.

Methodology: In this study, we have examined all howardites in the National Meteorite Collection at the Smithsonian for the presence of finegrained eucrite clasts. These clasts were mapped using the FEI NovaSEM 600 scanning electron microscope in the Mineral Sciences Department at the Smithsonian. The resulting X-ray maps and BSE images have been used to analyze minerals present, modal abundances, and textures. The mineral chemistries of pyroxene and plagioclase within each of the clasts have been analyzed using the JEOL $9800 \mathrm{R} / 5$ electron microprobe, also at the Smithsonian. So far eight fine-grained eucrite clasts have been identified in five howardites.

Results and Discussion: After obtaining SEM and microprobe data we determined that there is a variety of textural and compositional features within the fine-grained eucrite clasts. Texurally, we found several clasts that exhibited ophitic textures and granular pyroxene and plagioclase. Some clasts were found to have a quenched, glassy texture and others contained skeletal grains. Compositionally, the fine-grained eucrites encompass both equilibrated and unequilibrated pyroxene compositions suggesting that they experienced a range of metamorphic conditions. Minor elements in pyroxene suggest that they formed from both early primitive and late stage, more evolved melts. There are also a large range of plagioclase compositions such as slilica-enriched plagioclase composition glass to crystalline plagioclase with a compositional range of $\mathrm{An}_{62-95}$. Most samples have unequilibrated plagioclase of $\mathrm{An}_{\sim 10}$.

SEM and electron microprobe data show a large variety of textures and compositions within the data set. Such diversity has given us insight into the diverse geologic setting in which these eucrites were formed on Vesta.

References: [1] Mayne R. G., Sunshine J. M., McSween H.Y., McCoy T. J., Corrigan C. M. and Gale A. 2010. Meteoritics \& Planetary Science, in press. [2] Stolper E. 1977. Geochimica Cosmochimica Acta 41:587-611. [3] Russell C. T. et al. 2004. Planetary and Space Science 52:465-489.
5406

OBSERVATIONS OF ${ }^{12} \mathrm{CO}-{ }^{13} \mathrm{CO}$ PARTITIONING TOWARD SOLAR-TYPE PROTOSTARS AS PROXIES FOR SOLAR SYSTEM CHEMICAL EVOLUTION

R. L. Smith ${ }^{1}$, K. M. Pontoppidan 2 , E. D. Young ${ }^{1,3}$ and M. R. Morris ${ }^{4}$. ${ }^{1}$ Department of Earth and Space Sciences, University of California Los Angeles (UCLA), Los Angeles, CA, USA. E-mail: rsmith@ess.ucla.edu. ${ }^{2}$ Space Telescope Science Institute, Baltimore, MD, USA. ${ }^{3}$ Institute of Geophysics and Planetary Physics, UCLA, Los Angeles, CA, USA. ${ }^{4}$ Division of Astronomy and Astrophysics, Department of Physics and Astronomy, UCLA, Los Angeles, CA, USA.

Introduction: The solar system $\left[{ }^{12} \mathrm{C}\right] /\left[{ }^{13} \mathrm{C}\right]$ ratio is approximately $86-$ 89 [1-4], values which are peculiar as compared to local interstellar clouds [approximately 62-69; 4-6]. Explanations for this discrepancy in carbon isotopes have invoked Galactic chemical evolution (GCE), and assume that the solar system isotopically represents the local interstellar medium approximately $4.6 \mathrm{Gyr}$ ago, and that presently-evolving protostars should be chemically similar to their parent clouds [7]. This study utilizes highresolution near-infrared observations of $\mathrm{CO}$ toward local young stellar objects and molecular clouds as a probe of protostellar chemical evolution.

Observations: Near-infrared spectra of the CO rovibrational bands toward 12 local star-forming regions were observed using the CRIRES instrument at very high resolution $(R=95,000)$. These data trace several foreground clouds and protostellar envelopes, and two circumstellar disks, thereby representing varying stages of protostellar evolution. Precise CO isotopologue ratios were derived from the observed line profiles; a subset of these observations has been reported [8-10].

Results: Derived $\left[{ }^{12} \mathrm{CO}\right] /\left[{ }^{13} \mathrm{CO}\right]$ ratios reveal a large spread (approximately 84-158) as compared to the solar system and local interstellar medium, indicating that protostars may vary significantly in $\left[{ }^{12} \mathrm{C}\right] /\left[{ }^{13} \mathrm{C}\right]$ with respect to their parent clouds. Further, comparisons with the $\mathrm{CO}$ ice column densities derived from published optical depths [11] and upper limits for these objects reveal a general trend which suggests that ${ }^{12} \mathrm{CO}-{ }^{13} \mathrm{CO}$ partitioning between the ice and gas may influence the carbon isotopic composition in protostellar reservoirs. It is also apparent that complex chemistry, possibly including isotopic exchange with graphitic, hydrocarbon and/or other carbon reservoirs, likely contributes to the chemical evolution of these systems, and simple ice-gas fractionation is insufficient for explaining the observations to date.

Conclusions: A robust set of observations of $\left[{ }^{12} \mathrm{CO}\right] /\left[{ }^{13} \mathrm{CO}\right]$ toward local star-forming regions suggests that protostars are not necessarily similar to their parent clouds in carbon isotopes, violating GCE assumptions for describing early solar system $\left[{ }^{12} \mathrm{C}\right] /\left[{ }^{13} \mathrm{C}\right]$ evolution. Ice-gas partitioning in ${ }^{12} \mathrm{CO}_{-}{ }^{13} \mathrm{CO}$ may play a role in determining the isotopic evolution of protostars, and may have significant implications for understanding the carbon isotopic budget in the early solar system.

References: [1] Scott P. C. et al. 2006. Astronomy \& Astrophysics 456:675-688. [2] Coplen T. B. et al. 2002. Pure and Applied Chemistry 74:1987-2017. [3] Clayton D. D. and Nittler L. R. 2004. Annual Review of Astronomy and Astrophysics 42:39-78. [4] Wilson T. L. 1999. Reports on Progress in Physics 62:143-185. [5] Langer W. D. and Penzias A. A. 1993. The Astrophysical Journal 408:539-547. [6] Milam S. N. et al. 2005. The Astrophysical Journal 634:1126-1132. [7] Prantzos N. et al. 1996. Astronomy \& Astrophysics 309:760-774. [8] Smith R. L. et al. 2009. The Astrophysical Journal 701:163-175. [9] Smith R. L. et al. 2010. Meteoritics \& Planetary Science 45:5381. [10] Smith R. L. et al. 2011. Abstract \#1281. 42nd Lunar and Planetary Science Conference. [11] Pontoppidan K. M. et al. 2011. Astronomy \& Astrophysics 408:981-1007. 
5226

OXYGEN ISOTOPE MEASUREMENTS OF SIMULATED WILD 2 IMPACT CRATER RESIDUES

C. J. Snead ${ }^{1}$, K.D. McKeegan ${ }^{1}$, A.T. Kearsley ${ }^{2}$ and M.J. Burchell ${ }^{3}$. ${ }^{1}$ Department of Earth and Space Sciences, University of California, Los Angeles, Los Angeles, CA 90095-1567, USA. E-mail: stardust2006@ucla.edu. ${ }^{2}$ Dept. of Mineralogy, The Natural History Museum, London SW7 5BD, UK. ${ }^{3}$ School of Physical Science, University of Kent, Canterbury, Kent CT2 7NH UK.

Introduction: Oxygen isotope measurements of samples from Comet $81 \mathrm{P} /$ Wild 2 collected by NASA's Stardust mission have provided surprising similarities to minerals in carbonaceous chondrites which have been interpreted as evidence for large-scale radial migration of dust components from the inner solar nebula to the accretion regions of Jupiter-family comets [1, 2]. If this interpretation is correct, what the studies have so far not achieved is a measurement of the average oxygen isotope composition of the dust component of a known comet parent body. The reason is that the analyses have so far been restricted to large terminal particles because the fine-grained component of impacting particles suffered from intimate mixing with oxygen-rich aerogel. However, the aluminum foil substrates on the Stardust collector provide an opportunity for a low background alternative to the aerogel collectors for measuring the O-isotopic composition of both the coarse and the finegrained components of impacting dust particles. We have been developing the experimental approaches necessary to assess potential fractionation effects of capture by measuring impact craters produced by projectiles of known composition. Here we present results of oxygen isotope measurements of San Carlos olivine residues shot into aluminum foil targets at Stardust encounter velocities.

Results: Simulated impacts were produced by firing San Carlos Olivine into foil targets at approximately $6 \mathrm{~km} \mathrm{~s}^{-1}$ using a light gas gun at the University of Kent in Canterbury [3]. Craters ranging from 20-50 microns in diameter were cut from the foil target, flattened and pressed into Indium. The samples were then mounted with a San Carlos Olivine standard for ion probe analysis by the UCLA IMS-1270. Measurements were made using a $10 \mathrm{keV}, 1-2 \mathrm{nA}$ Cs primary beam; spot size was approximately $20 \mu \mathrm{m}$. The detectors were configured for 2-oxygen isotope composition measurements; a Faraday cup was used to measure the ${ }^{16} \mathrm{O}$ signal, while the ${ }^{18} \mathrm{O}$ signal was measured using an electron multiplier. Four measured craters contained enough residue to sustain a ${ }^{16} \mathrm{O}$ count rate $>10^{6}$ counts $\mathrm{s}^{-1} ; \delta^{18} \mathrm{O}$ values for these craters, normalized to the San Carlos Olivine standard were $3.1 \pm 0.2 \%, 2.6 \pm 0.4 \%, 2.0 \pm 0.8 \%$, $0.9 \pm 1.2 \%$, and $4.2 \pm 0.7 \%$. Quoted uncertainties are $1 \mathrm{SD}$.

Discussion: Crater measurements with ${ }^{16} \mathrm{O}$ count rates $>10^{6}$ counts $\mathrm{s}^{-1}$ reproduced the standard value of San Carlos olivine to within $4 \%$. The most accurate measurements are correlated with higher ${ }^{16} \mathrm{O}$ count rates. Sample preparation of impact craters has a strong effect on the level of precision that can be achieved; craters must be flattened in order avoid distortion of the accelerating electric field and inaccuracies in the instrumental mass fractionation correction.

References: [1] McKeegan K. D. et al. 2006. Science 314:1724-1728. [2] Nakamura T. et al. 2008. Science 321:1664-1667. [3] Kearsley A. T. et al. 2006. Meteoritics \& Planetary Science 41:167-180.
5250

ARAGONITE IN THE CM CARBONACEOUS CHONDRITES: A PROXY FOR THE MAGNITUDE OF AQUEOUS ALTERATION

M. R. Sofe, M. R. Lee and P. Lindgren. School of Geographical and Earth Sciences, University of Glasgow, Glasgow G128QQ, UK. E-mail: msofe@glasgow.ac.uk.

Introduction: The degree of aqueous alteration of CM carbonaceous chondrites can be tracked by changes in properties including in the volume of fine-grained matrix, the volume and chemical composition of its constituent phyllosilicates, and the extent of alteration of chondrules [e.g., 1-3]. The carbonate minerals aragonite, calcite and dolomite were also formed during alteration and whereas calcite is seen in all CMs, dolomite has been recorded only from the more highly altered meteorites [e.g., 4] and little is known about the occurrence of aragonite. Here we have sought to understand better the significance of the correspondence between carbonate mineralogy and degree of alteration by undertaking a systematic study of intermeteorite differences in the abundance and properties of aragonite.

Methods: Six CM2s were studied, namely Pollen, Murray, Mighei, LON 94101, Nogoya, and QUE 93005 (listed in the approximate order of increasing degree of alteration), together with the CM1 SCO 06043. Dolomite was identified by SEM imaging and X-ray microanalysis, and aragonite was distinguished from calcite using electron backscatter diffraction and Raman spectroscopy.

Results: Aragonite occurs in the matrices of five of the meteorites studied and is absent from the most highly altered samples (i.e., QUE 93005 and SCO 06043). Only Nogoya contains both aragonite and dolomite. The approximate abundance of aragonite, expressed as grains $\mathrm{mm}^{-2}$, is: Pollen (0.20), Murray (0.30), Mighei (0.23), LON 94101 (0.10), Nogoya (0.17). The mean sizes of these aragonite grains are also similar: Pollen $(21 \mu \mathrm{m})$, Murray $(22 \mu \mathrm{m})$, Mighei $(33 \mu \mathrm{m})$, LON 94101 $(25 \mu \mathrm{m})$, Nogoya $(14 \mu \mathrm{m})$. The largest grain $(101 \mu \mathrm{m})$ was found in Mighei. Unlike calcite, the aragonite grains usually lack serpentinetochilinite rims, although many of them have been partially replaced by matrix phyllosilicates. Most of the crystals occur in loose clusters and their $c$ axes have a common alignment.

Discussion: As aragonite crystals are embayed by phyllosilicates they formed prior to the end of aqueous activity, and their common alignment suggests crystallization under uniaxial lithostatic pressure at depth within the parent body. Given that aragonite occurs only in the less altered meteorites and dolomite in the more highly processed CMs, with the crossover at Nogoya (classified as CM2.2 by [2]), these minerals track the degree of aqueous alteration by their presence or absence. We suggest that crystallization of aragonite rather than calcite is favored in solutions with $\mathrm{Mg} / \mathrm{Ca}>$ approximately 1 , and significantly higher $\mathrm{Mg} / \mathrm{Ca}$ ratios will yield dolomite. If aragonite and dolomite crystallized after calcite, then the $\mathrm{Mg} / \mathrm{Ca}$ of bicarbonate rich solutions must have increased with time, and reached significantly greater values in those meteorites that were more highly altered overall.

Acknowledgments: We thank the Natural History Museum (London) and NASA-JSC for loan of the samples studied.

References: [1] McSween H. Y. 1979. Geochimica et Cosmochimica Acta 43:1761-1770. [2] Rubin A. E. et al. 2007. Geochimica et Cosmochimica Acta 71:2361-2382. [3] Howard K. T. et al. 2009. Geochimica et Cosmochimica Acta 73:4576-4589. [4] Johnson C. A. and Prinz M. 1993. Geochimica et Cosmochimica Acta 57:2843-2852. 
5392

\section{ZONING OF CALCITE IN CM CARBONACEOUS CHONDRITES AND ITS RELATIONSHIP TO DEGREE OF AQUEOUS ALTERATION}

M. Sofe ${ }^{1}$, M. R. Lee ${ }^{1}$, P. Lindgren ${ }^{1}$ and C. L. Smith ${ }^{2}$. ${ }^{1}$ School of Geographical \& Earth Sciences, University of Glasgow, Glasgow G12 8QQ, UK. ${ }^{2}$ Department of Mineralogy, Natural History Museum, London, SW7 5BD, U.K. E-mail: Mahmood.Sofe@ges.gla.ac.uk.

Introduction: Carbonates occur in CM2 carbonaceous chondrites in a variety of phases including: Ca-carbonate (aragonite and calcite), dolomite and breunnerite [e.g., 1, 2]. Petrographic work has revealed multiple generations of Ca-carbonates including aragonite, calcite replacing olivine/pyroxene, calcite rimmed by tochilinite and partially or completely replaced by phyllosilicates, and calcite grains that are free of inclusions and rims. In this study we have sought to understand the conditions of Ca-carbonate crystallization within the $\mathrm{CM}$ parent body and how they may have varied between meteorites that show different magnitudes of aqueous alteration.

Methods: This study used the following CM2 falls: Murchison, Murray, Pollen, Mighei, and Nogoya. Over 300 grains were located and their textures and microstructures characterized using backscattered electron (BSE) and cathodoluminescence (CL) microscopy. Work by laser Raman spectroscopy and electron probe microanalysis (EPMA) was also undertaken for mineral identification and chemical analysis respectively.

Results and Discussion: Based on the aqueous alteration index of $\mathrm{CM}$ chondrites of [3], we related CL intensity and patterns of zoning in the Ca-carbonates into degree of alteration. The least altered meteorites studied (Murchison, Murray, Pollen and Mighei) contain 1.3-2.0 vol\%. of Ca-carbonate (both aragonite and calcite) that have a variety of $\mathrm{CL}$ characteristics. All calcite grains that have replaced olivine/pyroxene and many grains of aragonite and non-rimmed calcite have a low CL intensity and are unzoned, and this can be related to the stability of fluid composition during crystal growth. Some aragonite and non-rimmed calcite grains, and all of calcites rimmed with tochilinite, display one of three styles of $\mathrm{CL}$ emission. (1) Concentric zoning with a triangle-shaped seed crystal in one part, subsequent zoning of hexagonal habit, and other bands of zoning to fill the pore space. Growth of this type was controlled by the change in fluid composition and delivery of ions to the growing crystal face. (2) Grains that have only a high CL intensity throughout as a consequence of compositional stability of the fluid during the crystal growth; however this fluid was different in chemical composition to that which crystallized grains with a low CL intensity. (3) Some grains have patchy low and high CL intensity which possibly reflects a complex growth history as a result of a changeable fluid chemical compositions. Ca-carbonate grains in Nogoya, the more highly aqueously altered CM2, show no $\mathrm{CL}$ variation and a low emission intensity; this implies that they crystallized from fluids that were stable in chemical composition. This correspondence between CL properties and degree of alteration agrees very well with the finding by [4] that concentrations of manganese and iron in the $\mathrm{CM} 2$ carbonates decrease with increasing alteration of their host rock.

References: [1] Lee M. et al. 2010. Meteoritics \& Planetary Science 45:A115. [2] Brearley A. J. 1998. Abstract \#1246. 29th Lunar and Planetary Science Conference. [3] Rubin E. et al. 2007. Geochimica et Cosmochimica Acta 71:2361-2382. [4] De Leuw S. et al. 2010. Meteoritics \& Planetary Science 45:513-530.

\section{2}

\section{${ }^{60} \mathrm{Fe}^{60} \mathrm{Ni}$ SYSTEMATICS IN THE ANGRITES}

L. J. Spivak-Birndorf, M. Wadhwa and P. E. Janney. School of Earth and Space Exploration, Arizona State University, Tempe, AZ 85287-1404, USA. E-mail: Lev.Spivak-Birndorf@asu.edu.

Introduction: The abundance of ${ }^{60} \mathrm{Fe}\left(t_{1 / 2}=2.6 \mathrm{Ma}\right)$ in the early Solar System is not well constrained. Previously reported estimates of the initial ${ }^{60} \mathrm{Fe} /{ }^{56} \mathrm{Fe}$ ratio vary considerably depending on the samples and techniques used (e.g., [1-3]). We have begun an investigation of the ${ }^{60} \mathrm{Fe}-{ }^{60} \mathrm{Ni}$ systematics in angrites to constrain the abundance and distribution of ${ }^{60} \mathrm{Fe}$ in the early Solar System, preliminary results of which were presented earlier [4]. Here we report Fe-Ni data for additional angrite samples.

Materials and Methods: Nickel isotopes and $\mathrm{Fe} / \mathrm{Ni}$ ratios were measured by ICP-MS using methods described by [4]. The study includes two bulk samples and two mineral separates (olivine and pyroxene) from the quenched angrite D'Orbigny, as well as bulk samples of the plutonic angrites NWA 4590, NWA 4801, and NWA 6291 (paired with NWA 2999).

Results: The three plutonic angrite samples span a wide range of ${ }^{56} \mathrm{Fe} /{ }^{58} \mathrm{Ni}$ ratios (approximately $70-10,000$ ), and all have $\mathrm{Ni}$ isotopic compositions identical to the terrestrial value within analytical errors. In contrast, while bulk samples and mineral separates of D'Orbigny also span a wide range of ${ }^{56} \mathrm{Fe} /{ }^{58} \mathrm{Ni}$ ratios (approximately $4600-11,000$ ), all have excesses in ${ }^{60} \mathrm{Ni}$ that correlate with their $\mathrm{Fe} / \mathrm{Ni}$ ratios. The D'Orbigny samples define an internal ${ }^{60} \mathrm{Fe}^{60} \mathrm{Ni}$ isochron corresponding to a ${ }^{60} \mathrm{Fe} /{ }^{56} \mathrm{Fe}=(3.4 \pm 1.5) \times 10^{-9}$ and $\varepsilon^{60} \mathrm{Ni}_{0}=-0.18 \pm 0.29$ at the time of the last equilibration of $\mathrm{Ni}$ isotopes in this meteorite.

Discussion: A recent investigation of ${ }^{60} \mathrm{Fe}^{60} \mathrm{Ni}$ systematics in angrites showed that $\varepsilon^{60} \mathrm{Ni}^{*}$ is correlated with $\mathrm{Fe} / \mathrm{Ni}$ ratios in bulk samples of D'Orbigny, Sahara 99555 (SAH 99555), and NWA 2999 [3]. The authors of that study interpreted this relationship as a whole-rock (WR) isochron corresponding to the timing of widespread $\mathrm{Fe} / \mathrm{Ni}$ fractionation during differentiation of the angrite parent body. They further argue that the source reservoirs of the various angrites formed contemporaneously and have remained closed systems with respect to their ${ }^{60} \mathrm{Fe}^{-60} \mathrm{Ni}$ systematics. However, D'Orbigny and SAH 99555 crystallized at the same time $[5,6]$ and the ${ }^{60} \mathrm{Fe}-{ }^{60} \mathrm{Ni}$ systematics of NWA 2999 are very similar to that of chondrites, making it possible that the angrite WR isochron reported by [3] actually corresponds to the time of formation of D'Orbigny and SAH 99555 from a chondritic source. Bulk samples of plutonic angrites NWA 4590 and NWA 4801 have ${ }^{56} \mathrm{Fe} /{ }^{58} \mathrm{Ni}$ ratios as high as approximately 10,000 and should have excess ${ }^{60} \mathrm{Ni}$ if their sources formed contemporaneously with those of the quenched angrites, as suggested by [3]. However, we find no ${ }^{60} \mathrm{Ni}$ excesses in these samples indicating that ${ }^{60} \mathrm{Fe}$ was extinct by the time the source reservoirs of these meteorites formed. Assuming that NWA 4590 and NWA 4801 formed at the same time, we determine a ${ }^{60} \mathrm{Fe} /{ }^{56} \mathrm{Fe} \leq 9 \times 10^{-10}$ when this event occurred. This corresponds to an age of $\leq 4558.3 \mathrm{Ma}$ relative to D'Orbigny, which is consistent with $\mathrm{Pb}-\mathrm{Pb}$ ages reported for the plutonic angrites [5]. Finally, the ${ }^{60} \mathrm{Fe}-{ }^{60} \mathrm{Ni}$ systematics of D'Orbigny and the age difference between CAIs and this angrite [7] allows us to estimate an initial Solar System ${ }^{60} \mathrm{Fe} /{ }^{56} \mathrm{Fe}$ of $(1.2 \pm 0.6) \times 10^{-8}$.

References: [1] Chen J. H. et al. 2009. Geochimica et Cosmochimica Acta 73:1461-1471. [2] Tachibana S. et al. 2006. The Astrophysical Journal 639:L87-L90. [3] Quitté G. et al. 2010. The Astrophysical Journal 720:1215-1224. [4] Spivak-Birndorf L. J. et al. 2011. Abstract \#2281. 42nd Lunar and Planetary Science Conference. [5] Amelin Y. 2008. Geochimica et Cosmochimica Acta 72:221-232. [6] Amelin Y. 2008. Geochimica et Cosmochimica Acta 72:4874-4885. [7] Bouvier A. and Wadhwa M. 2010. Nature Geoscience 3:637-641. 


\section{1}

\section{SHOCK-INDUCED MELT POCKET FORMATION IN NWA 4468: EVIDENCE FOR PHASE TRANSFORATION INDUCED IMPLOSION}

J. G. Spray ${ }^{1}$ and S. Boonsue ${ }^{1}$. ${ }^{1}$ Planetary and Space Science Centre, University of New Brunswick, Fredericton, NB E3B 5A3, Canada. E-mail: jgs@unb.ca.

Northwest Africa (NWA) 4468 is 675 g Martian meteorite recovered from the Western Sahara in 2006 [1]. It is an olivine basaltic shergottite, comprising clinopyroxene, olivine, maskelynite, chromite, ilmenite, Caphosphates, troilite and pyrrhotite. There have been several studies of NWA 4468, including its geochronology, petrology and bulk composition, but aspects of its shock history are less well constrained. The meteorite exhibits both bulk (maskelynite formation) and localized shock effects, the latter in the form of shock veins and melt pockets that contain various high pressure and/or high temperature polymorphs, glasses and neocrystallites. This work presents new results following extensive melt pocket characterization based on analytical field emission scanning electron microscopy and micro-Raman spectroscopy. The melt pockets are typically $2-5 \mathrm{~mm}$ in diameter and subspherical. Three components are identified in the melt pockets (1) fragmented/fractured mineral phases inherited from the bulk sample, (2) inherited mineral phases that have undergone solid-state phase transformation (e.g., olivine to ringwoodite), and (3) glasses and neocrystalline phases that originated via fusion. Metal phases (mainly Fe-Ti-Cr oxides) have undergone melting to yield disseminated clouds of immiscible spheres set within a silica-rich glass matrix. Three Ca-phosphate polymorphs have been recognized [2, 3]: $\alpha-, \beta$ - and $\gamma$ - apatite (chlorapatite, merrilite and tuite, respectively), with their structures confirmed by Raman spectroscopy. Ca-hollandite and stishovite are also present. Evidence for a possible new high-pressure polymorph of low-Ca clinopyroxene is also presented and discussed. Ringwoodite is developed at the rim of the pockets, which, overall, suggest an increasing pressure gradient from periphery to core. Most if not all melt pockets are characterized by the presence of Ca-phosphate phases in their core regions. We propose that the relatively low pressure transformation of apatite to merrillite, which took place during the bulk shock event, may have provided the necessary energy for the thermalpressure spike as a result of concomitant volume reduction and associated implosion.

References: [1] Irving A. J. et al. 2007. Abstract \#1526. 38th Lunar Prospector Spacecraft. [2] Beck P. et al. 2007. Geophysical Research Letters 34:L01203, doi:10.1029/2006GL028141. [3] Xie X. et al. 2002. Geochimica et Cosmochimica Acta 66:2439-2444.
5519

CARBON RAMAN SPECTROSCOPY: NATURAL AND EXPERIMENTALLY-INDUCED VARIATIONS IN IDPS

N. H. Spring and H. Busemann. SEAES, The University of Manchester, UK. E-mail: nicole.spring-2@postgrad.manchester.ac.uk.

Introduction: Carbon Raman spectroscopy has been shown to complement other techniques in identifying levels of thermal alteration in meteorites. As it has high spatial resolution and is relatively nondestructive, it can also be applied to micron-sized IDPs. Many IDPs are thought to originate from comets rather than asteroids [1], and so are expected to show lesser degrees of parent body thermal alteration than meteorites. Indeed, the majority of IDPs contain organic matter (OM) that is similar to or more disordered than the most primitive insoluble organic matter (IOM) separated from meteorites [2, 3]. In particular, some of the IDPs collected to coincide with the passing though comet Grigg-Skjellerup's dust stream (GSC) show Raman characteristics which are among the most primitive to date, complementing NanoSIMS isotope data on $\mathrm{H}$ and $\mathrm{N}$ which conclude the same [4]. However, average parameters for GSC-IDPs, normal IDPs and the most unaltered IOM are similar, suggesting asteroids and comets acquired their $\mathrm{OM}$ from a common reservoir.

We have applied this technique to 27 IDPs to determine the degree of order of their OM, and identify the most primitive and C-rich particles for a detailed NanoSIMS study. Fragments of these IDPs have also been analyzed with FTIR spectroscopy [5]. Ultimately they will be examined in RELAX, a time of flight mass analyzer for Xe [6]. The samples are cluster particles of varying textures and compositions from eight different collections.

Method: We used a Renishaw inVia Raman microscope with a $514 \mathrm{~nm}$ laser, based at Manchester Metropolitan University to determine $\mathrm{D}$ and $\mathrm{G}$ band widths $\Gamma$ and positions $\omega$. Meteoritic IOM separates from several meteorite types were used as standards. The IDPs were unpressed and positioned on gold foil covered SEM stubs. The laser was focused using $\times 50$ magnification. The spot size was $\sim 2 \mu \mathrm{m}$ and the laser power was kept $<30 \mu \mathrm{W}$.

Results: Of the 20 IDPs yielding Raman parameters with low errors, all but three plot alongside the most primitive IOM (EET 92042, Semarkona, Murchison), agreeing with other studies that show IDPs have the most disordered $\mathrm{C}$, and therefore least thermal alteration, adding to the likelihood that they are cometary material. The OM of the GSC particle is the most amorphous from the $\mathrm{G}$ band data, possibly indicative of irradiation in space.

Our data are comparable with literature trends (taken with $532 \mathrm{~nm}$ excitation [2]) if shifts in $\mathrm{D}$ and to a much lesser extent $\mathrm{G}$ band are accounted for. These shifts can be explained by (i) different laser excitation, whereby $\mathrm{D}$ and $\mathrm{G} \omega$ decrease with increasing excitation wavelength [7]. (ii) Shifts due to laser power, whereby we observe differences of up to $100 \mathrm{~cm}^{-1}$ in D band width between 25 and $125 \mu \mathrm{W}$ for most disordered IOM. Given the smaller beam in the previous study [2], the power density they used was significantly greater than $125 \mu \mathrm{W}$. Adjusted accordingly, trends for IDPs and IOM agree with previous studies.

Acknowledgments: We thank V. Vishnyakov at the MMU DRIAM facility funded by EPSRC grant EP/F056117/1.

References: [1] Nesvorný D. et al. 2010. The Astrophysical Journal 713:816-836. [2] Busemann H. et al. 2011. Abstract \#4086. Corals II. [3] Starkey N. A. and Franchi I. A. 2011. Abstract \#1938. 42nd Lunar and Planetary Science Conference. [4] Busemann H. et al. 2009. Earth and Planetary Science Letters 288:44-57. [5] Busemann H. et al. 2011. Meteoritics \& Planetary Science 46. This conference. [6] Busemann H. et al. 2010. Abstract \#1947. 41st Lunar and Planetary Science Conference. [7] Ferrari A. C. and Robertson J. 2001. Physical Review B 64:075414. 
5527

THE SUSCEPTIBILITY OF PHASE Q TO PYRIDINE: ARE CI CHONDRITES UNIQUE?

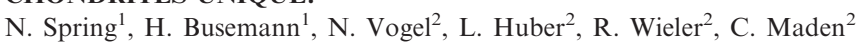
and C.M.O'D. Alexander ${ }^{3}$. ${ }^{1}$ SEAES, University of Manchester, UK. E-mail: nicole.spring-2@postgrad.manchester.ac.uk. ${ }^{2}$ ETH Zurich, Switzerland. ${ }^{3}$ Carnegie Institution of Washington, USA.

Introduction: The origin of the primordially trapped noble gases in the elusive phase Q is an unresolved question in meteoritics [1]. Acidresistant residues, enriched in insoluble organic matter (IOM) in all meteorite classes contain Q-gases of remarkably similar composition, that are strongly fractionated relative to solar gases [2,3]. Q-gas signatures in achondrites and comet Wild 2 dust $[4,5]$ show that phase $\mathrm{Q}$ was ubiquitously incorporated during accretion in the inner and outer solar system. Pyridine applied to the IOM of CI Orgueil released large amounts of Q-Xe due to swelling [6, 7]. In contrast, pyridine did not affect phase Q in LL, CV, CR and CM chondrites [7-9]. This may suggest that different types of phase Q exist. Either phase Q was modified due to particular conditions on the CI parent body (e.g., severe aqueous alteration) or processes on all other parent bodies (e.g., mild thermal alteration) modified a pyridine-susceptible fraction. Here we present noble gas data on IOM from pristine meteorites. In particular, we show that phase Q in CIs Ivuna and Orgueil are not affected by pyridine.

Experimental: We used an IR laser to extract noble gases in IOM $(<1-5 \mathrm{mg})$ of CIs Orgueil and Ivuna, CV3 Allende, L3.05 QUE 97008, $\mathrm{C} 2$ Tagish Lake. IOM aliquots before and after treatment with pyridine were compared [see 8 for details]. Nb tubes were used to avoid sample loss during heating. As quantitative gas release has not been reached for all samples yet, we compare isotope and element ratios, which can readily distinguish the release of Q-gases $[8,9]$, whilst avoiding ambiguity due to sample loss during preparation and extraction.

Results and Discussion: All residues contain large amounts of Q noble gases, in agreement with their primitive character. All isotope and elemental ratios are in the range expected for IOM [2, 3]. As demonstrated earlier [8], the reported $60 \%$ loss of phase Q [6] would be visible in the elemental ratios (favoring the lighter element) and also Xe isotopes (Xe-HL would be more dominant). However, even the most suitable Xe isotopic ratios are typically identical (within 3\%) in treated and untreated IOM. Similarly, the element ratios vary by typically 10 $30 \%$, however, without a systematic trend, hence not supporting the idea of phase Q loss due to pyridine treatment. To the contrary, more ratios point to a depletion of presolar phases relative to phase Q, which hence may simply reflect sample heterogeneity. Most importantly, treated and untreated CI Ivuna IOM shows similar ratios and we do not observe a significant loss of Q-gases in Orgueil. The CI parent body acquired and still carries the same phase Q and its noble gases as all other groups, despite different alteration processes.

References: [1] Wieler R. et al. 2006. In Meteorites and the early solar system II: 499-521. [2] Busemann H. et al. 2000. Meteoritics \& Planetary Science 35:949-973. [3] Huss G. R. et al. 1996. Geochemistry Geophysics Geosystems 60:3311-3340. [4] Busemann H. and Eugster O. 2002. Meteoritics \& Planetary Science 37:1865-1891. [5] Marty B. et al. 2008. Science 319:75-78. [6] Marrocchi Y. et al. 2005. Earth and Planetary Science Letters 236: 569-578. [7] Matsuda J.-I. et al. 2010. Meteoritics \& Planetary Science 45:1191-1205. [8] Spring N. et al. 2010. Abstract \#2640. 41st Lunar and Planetary Science Conference. [9] Busemann H. et al. 2008. Abstract \#1777. 39th Lunar and Planetary Science Conference.
5101

THE MASON GULLY METEORITE FALL IN SW AUSTRALIA: FIREBALL TRAJECTORY AND ORBIT FROM PHOTOGRAPHIC RECORDS

P. Spurný ${ }^{1}$, P. A. Bland ${ }^{2,3}$, L. Shrbený ${ }^{1}$, M. C. Towner ${ }^{2}$, J. Borovička ${ }^{1}$, A. W. R. Bevan ${ }^{4}$ and D. Vaughan ${ }^{5}$. ${ }^{1}$ Astronomical Institute of the Academy of Sciences, Fričova 298, CZ-251 65 Ondřejov Observatory, Czech Republic. E-mail: spurny@asu.cas.cz. ${ }^{2}$ IARC, Department of Earth Sci. \& Eng., Imperial College London, SW7 2AZ, UK. ${ }^{3}$ Dept. Applied Geol., Curtin University of Technology, GPO Box U1987, Perth WA 6845, Australia. ${ }^{4}$ Dept. Earth and Planet. Sci., Western Australian Museum, Locked Bag 49 Welshpool DC, WA 6986, Australia. ${ }^{5}$ PO BOX 187, Nedlands, Perth, WA 6909, Australia.

Introduction: We report a new instrumentally recorded meteorite fall, named Mason Gully, which was recorded by the Desert Fireball Network (DFN), an ambitious project dedicated to mapping of fireballs over the remote area of the Nullarbor Region of SW Australia, a very suitable place for meteorite recoveries [1]. This is the second case of such successful recovery after Bunburra Rockhole, the major milestone of this project [2].

Results and Discussion: The reported meteorite fall occurred over SW Australia on April 13th, 2010 at $10^{\mathrm{h}} 36^{\mathrm{m}} 12.7^{\mathrm{s}} \pm 0.1^{\mathrm{s}}$ UT (maximum brightness). The fireball designated DN130410 was recorded photographically and photoelectrically by two eastern stations of the DFN. Its luminous trajectory started at an altitude of $83.46 \mathrm{~km}$ and after a $73.8 \mathrm{~km}$ long flight terminated at an altitude of $23.84 \mathrm{~km}$. The angle of the atmospheric trajectory to the Earth's surface was $53.9^{\circ}$. The object, with initial mass of about $40 \mathrm{~kg}$, entered the atmosphere with a very low speed of $14.53 \mathrm{~km} \mathrm{~s}^{-1}$, and during a 6.0 -s long flight decelerated to the terminal speed of $4.1 \mathrm{~km} \mathrm{~s}^{-1}$, when the fireball brightness decreased below the sensitivity limit of both DFOs. The maximum absolute $(100-\mathrm{km}$ distance) brightness of -9.4 magnitude was reached in a short flare at an altitude of $35.8 \mathrm{~km}$. The initial meteoroid before its collision with Earth orbited the Sun on a very low inclined orbit defined by the following parameters: semimajor axis (AU): $2.470 \pm 0.004$; eccentricity: $0.6023 \pm 0.0007$; perihelion distance (AU): $0.98240 \pm 0.00007$; aphelion distance (AU): $3.958 \pm 0.008$; argument of perihelion $\left(^{\circ}\right)$ : $18.95 \pm 0.03$; longitude of ascending node $\left({ }^{\circ}\right)$ : 203.2112; inclination $\left(^{\circ}\right)$ : $0.832 \pm 0.013$; period (years): $3.882 \pm 0.009$. All angular elements are given in J2000 equinox. This is one of the most precise orbits ever determined for a meteorite. The computation of the darkflight, and determination of impact position was complicated by very strong lateral stratospheric winds reaching $45 \mathrm{~m} \mathrm{~s}^{-1}$, shifting the resulting impact position for the meteorite of the recovered size $(25 \mathrm{~g})$ by about $6 \mathrm{~km}$ aside! In spite of this unfavorable fact the meteorite was found up to $150 \mathrm{~m}$ from the predicted site (for more details see [3]). This case along with the Bunburra Rockhole meteorite clearly show that this project in the remote and hostile environment of the Australian desert is meaningful, and that these observations can bring data of fundamental value to solar system research. We also proved the correctness of our observational and computational methods and models.

References: [1] Bland P. A. et al. 2006. Meteoritics \& Planetary Science 41:5197. [2] Bland P. A. et al. 2009. Science 325:1525. [3] Towner M. C. et al. 2011. Meteoritics \& Planetary Science 46, This conference. 
5411

\section{NANOSIMS OXYGEN ISOTOPE ANALYSES ON GRIGG- SKJELLERUP COLLECTION IDPS}

N. A. Starkey ${ }^{1}$, I. A. Franchi ${ }^{1}$ and J. Davidson ${ }^{2} .{ }^{1}$ PSSRI, Open University, Milton Keynes, MK7 6AA, UK. E-mail: n.starkey@ open.ac.uk. ${ }^{2}$ Lunar \& Planetary Laboratory, University of Arizona, Tucson, AZ 85721-0092, USA.

Introduction: The oxygen isotope composition of meteorites has revealed much about the origin and history of inner solar system materials and processes operating across a large volume of the protoplanetary disk. Our knowledge of oxygen isotope signatures beyond the asteroid belt is limited to a few analyses of particles from comet Wild 2 [1-4] and anhydrous CP-IDPs [4-7]. Almost all such analyses studied large, ferromagnesian silicate crystals with the predominant finegrained material largely overlooked. While the fine grain size of this material prohibits isotopic measurements on a grain by grain basis, the signature and variability of the bulk isotopic composition of this material offers insight into early solar system oxygen isotopic reservoirs. Here we present NanoSIMS 50L high precision three-oxygen isotope spot analyses on a set of Grigg-Skjellerup collection IDPs from a larger sample set previously characterized by SEM, Raman and NanoSIMS (C,N,O isotope mapping) [8]. All the IDPs are anhydrous fine-grained particles containing presolar grains emphasizing the primitive nature of the samples [8].

Method: Particles were pressed into gold foil and $\mathrm{a} \approx 50 \mathrm{pA}$ probe was rastered over $5 \times 5 \mu \mathrm{m}^{2}$ areas for analyses with ${ }^{16} \mathrm{O}$ measured on a Faraday cup and ${ }^{17} \mathrm{O},{ }^{18} \mathrm{O}$ and ${ }^{24} \mathrm{Mg}^{16} \mathrm{O}$ on electron multipliers. The mass resolution was set to $>10,000$ (Cameca-definition). Isotope ratios $\left(\delta^{17} \mathrm{O}\right.$ and $\delta^{18} \mathrm{O}$ ) were normalized to SMOW using analyses of San Carlos olivine. The mixed mineralogy of the fine-grained samples precludes any matrix effect correction, with variations between the olivine standard and the main phases in the IDPs thought to be negligible. Combined $2 \sigma$ precision from sets of five analyses on Eagle Station yield $\pm 0.8 \%$ for $\delta^{18} \mathrm{O}$ and $\pm 1.2 \%$ for $\delta^{17} \mathrm{O}$, and mean values within $2 \sigma$ of true.

Results: The IDPs display a wide range in $\delta^{18} \mathrm{O}$ values from approximately $0 \%$ to $<-30 \%$, plotting close to, or above the CCAM line. The offset from the CCAM line appears to be related to ${ }^{16} \mathrm{OH}$ interference revealed by a strong linear relationship with ${ }^{16} \mathrm{OH}$ abundances. The reason ${ }^{16} \mathrm{OH}$ is impacting the IDP results remains unclear as no such effect is observed for the olivine standard or Acfer094 matrix pressed into gold (despite similarly high ${ }^{16} \mathrm{OH}$ ). $\delta^{18} \mathrm{O}$ are far less susceptible to interference and therefore reflect the true values of the IDPs, most likely falling along the CCAM line. With two samples having $\delta^{18} \mathrm{O}$ values of -25 and $-31 \%$, these results extend the limited range of oxygen isotopes previously reported for IDPs. While the mineralogical composition of each sputtered area is unknown, it may contain many thousands of small grains and it is therefore unlikely that such large variations are the result of random sampling of refractory ${ }^{16} \mathrm{O}$-rich material. These results indicate a much larger range in bulk compositions of cometary materials compared to that from asteroids, recording more primitive signatures of reservoirs in the early solar system.

References: [1] McKeegan K. D. et al. 2006. Science 314:1724-1728. [2] Nakamura T. et al. 2008. Science 321:1664-1667. [3] Joswiak D. J. et al. 2010. Abstract \#2119. 41st Lunar and Planetary Science Conference. [4] Nakashima D. et al. 2011. Abstract \#1240. 42nd Lunar and Planetary Science Conference. [5] Messenger et al. 2003. Science 300:105-108. [6] Aleon J. et al. 2009. Geochimica et Cosmochimica Acta 73:4558-4575. [7] Floss C. et al. 2011. Meteoritics \& Planetary Science 45:1889-1905. [8] Davidson J. 2009. Ph.D. thesis, Open University.
5419

OXYGEN ISOTOPE ANALYSIS OF A CHONDRULE-LIKE WILD 2 TERMINAL PARTICLE USING NANOSIMS

N. A. Starkey ${ }^{1}$, I. A. Franchi ${ }^{1}$, J. C. Bridges ${ }^{2}$, H. G. Changela ${ }^{2,3}$ and L. J. Hicks ${ }^{2}$. ${ }^{1}$ PSSRI, Open University, Milton Keynes MK7 6AA, UK. E-mail: n.starkey@open.ac.uk. ${ }^{2}$ SRC, University of Leicester, Leicester LE1 7RH, UK. ${ }^{3} \mathrm{NRL}, 4555$ Overlook Ave. SW, Washington, D.C. 20375, USA.

Introduction: Oxygen isotopes provide a key tool in determining origins and links of diverse solar system materials. SIMS analyses of terminal particles returned from comet Wild 2 with sufficient analytical precision for useful comparison to meteorites are very limited [1-3]. Generally, analyses of grains have used potted butts or single grains pressed into gold in order to provide sufficient sample thickness to analyse with high current probes. At most, only one potted butt can exist for any characterized terminal particle and in many cases it is required for other analytical techniques or no longer contains cometary material. Here we utilize the more readily available microtomed sections as a means of extracting useful oxygen isotopic information. NanoSIMS 50L isotope imaging mode allows the material available for analysis from these very thin sections $(\approx 70 \mathrm{~nm})$ to be maximized, although analytical uncertainty reported by others using this technique is large [4].

Sample: The microtomed TEM section is from Stardust terminal particle \#2, Track $154(\mathrm{C} 2063,1,154,1,15)$. This approximately $4 \times 1.5 \mu \mathrm{m}^{2}$ particle has previously been characterized by TEM [5] and is composed of Al-rich diopside and pigeonite with minor enstatite and forsterite, and is thought to be a fragment of an Al-rich chondrule rather than a CAIs [5].

Analyses: The back of the TEM grid containing the sections was reinforced with a $200 \mathrm{~nm}$ gold coat and a further $20 \mathrm{~nm}$ gold coat was deposited on the top of the sections to facilitate charge dissipation. A $2 \mathrm{pA}$ probe was rastered over an $8 \times 8 \mu^{2}$ area for analysis. The Cameca-defined mass resolution was $>10,000$ with ${ }^{16,17,18} \mathrm{O},{ }^{28} \mathrm{Si}$ and ${ }^{24} \mathrm{Mg}^{16} \mathrm{O}$ measured on electron multipliers. $\delta^{17} \mathrm{O}$ and $\delta^{18} \mathrm{O}$ were normalized to SMOW from San Carlos olivine measurements. Results were processed using L'image software and corrected for position drift, QSA effects and detector aging. The difference in matrix effect between the low-FeO pyroxene and the olivine standard are thought to be negligible compared to the precision and a correction is not applied. Reproducibility determined from analyses on comparable areas of San Carlos olivine was $0.9 \%$ for $\delta^{18} \mathrm{O}$ and $4 \%$ for $\delta^{17} \mathrm{O}$ and analyses of Eagle Station gave correct values within error of true value.

Results: The particle was surrounded by aerogel with the sample area being defined on the basis of ${ }^{24} \mathrm{Mg}^{16} \mathrm{O}$ and ${ }^{16} \mathrm{O}$ counts. The isotopic composition of the particle was determined as: $\delta^{18} \mathrm{O}=-8.2 \pm 2.3 \%$ and $\delta^{17} \mathrm{O}=-5.0 \pm 5.4 \%$ ( $1 \sigma$ errors $)$. On a three-isotope diagram the sample plots just above (but well within $2 \sigma$ ) of the CCAM line. The isotopic composition is indistinguishable from that of Al-rich chondrules found in carbonaceous chondrites, in agreement with its mineralogy [5], and clearly distinct from unaltered CAIs-like materials. The chondrule-like compositions are similar to those reported previously [1-3] and support a common origin for chondrules in asteroids and comets.

References: [1] McKeegan K. D. et al. 2006. Science 314:1724-1728. [2] Nakamura T. et al. 2008. Science 321:1664-1667. [3] Nakashima D. et al. 2011. Abstract \#1240. 42nd Lunar and Planetary Science Conference. [4] Joswiak D. J. et al. 2010. Abstract \#2119. 41st Lunar and Planetary Science Conference. [5] Bridges J. C. and Changela H. G. 2010. Abstract \#2058. 41st Lunar and Planetary Science Conference. 
5423

RAMAN VARIATION OF ORGANIC MATTER IN LARGE CLUSTER IDPS AND METEORITIC IOM

N. A. Starkey ${ }^{1}$, I. A. Franchi ${ }^{1}$ and C. M. O'D. Alexander ${ }^{2}$. ${ }^{1}$ PSSRI, Open University, Milton Keynes MK7 6AA, UK. E-mail: n.starkey@ open.ac.uk. ${ }^{2}$ DTM, Carnegie Institution of Washington, 5241 Broad Branch Road, Washington, D.C. 20015, USA.

Introduction: CP-IDPs display a range of primitive features indicative of a cometary origin. The organic material $(\mathrm{OM})$ present in IDPs may be the result of formation processes that occurred across a large volume of the protoplanetary disk to small-scale on the cometary body itself. Raman microscopy offers a nondestructive approach for determining general characteristics of the OM in IDPs. Compared to the large number of potential parent bodies or the huge volume of the protoplanetary disk that may have contributed to the formation of comets, the number of IDPs analyzed to date remains relatively small. Raman measurements were performed on nine IDPs (from five large cluster particles; collectors L2005 and L2006) offering the opportunity to investigate the variability of the $\mathrm{OM}$ on a number of scales. Previous Raman analyses of IDPs and IOM from meteorites have used a number of different wavelengths, predominantly green. While it has been shown that $\mathrm{D}$ band position $(\omega \mathrm{D})$ is influenced by excitation $\lambda[1]$, it is rarely quantified. Interestingly, the wavelength dependent shift in the $\omega \mathrm{G}$ is known to vary with degree of ordering of OM. Multiwavelength analyses made here offer an independent measure of organic maturity.

Results: Meteoritic IOM (samples as in [2]) and IDPs mapped using $514 \mathrm{~nm}$ and $473 \mathrm{~nm}$ lasers showed a reproducible downshift of 10$13 \mathrm{~cm}^{-1}$ in $\omega \mathrm{D}$. For $\omega \mathrm{G}$, the IDPs displayed a larger downshift (approximately $4 \mathrm{~cm}^{-1}$ ) than the IOM (negligible). The range of $\omega \mathrm{D}$ and $\omega \mathrm{G}$ in any one IDP cluster is limited, with values comparable to meteoritic IOM. For particles within individual clusters, $\omega D$ varies by $<6 \mathrm{~cm}^{-1}$ and $\omega \mathrm{G}$ by $<3 \mathrm{~cm}^{-1}$. Each cluster IDP displays distinctive internal peak parameter covariation.

Discussion: The large $\Gamma \mathrm{D}$ and $\Gamma \mathrm{G}$, high $\omega \mathrm{D}$, low $\omega \mathrm{G}$ and $\mathrm{ID} / \mathrm{IG}$ ratios of $0.9-1.3$ indicate that the IDPs are all primitive. The samples plot near to the $\mathrm{CI}, \mathrm{CM}$ and $\mathrm{CR}$ field. The dispersion in $\omega \mathrm{G}$ with excitation $\lambda$ is related to the degree of disordering of the carbon [1], and therefore the greater $\omega \mathrm{G}$ dispersion observed for the IDPs suggests that the OM in the IDPs may be more disordered than meteoritic IOM, in contrast to their similar peak parameters. The IDPs selected for this study do not appear to be as primitive as Grigg-Skjellerup Collection (GSC) particles [3], but are comparable to other non-GSC IDPs [4].

While great isotopic and structural variability exists within individual particles at the sub- $\mu \mathrm{m}$ scale (e.g., [3]), these results indicate that the OM within IDPs is reasonably homogeneous at the few to tens of $\mu \mathrm{m}$-scale, and that there is greater variability between particles than within. The variability between particles most likely reflects accretion from different reservoirs, potentially on the scale of individual comets given the distinctive signatures from GSC-IDPs [3]. Secondary processes on the comet, in interplanetary space or during atmospheric entry may also play a role despite low peak metamorphic temperatures $\left(<200{ }^{\circ} \mathrm{C}\right.$ (after [5])).

References: [1] Ferrari A. C. and Robertson J. 2001. Physical Review B 63:075414. [2] Alexander C. M. O'D. et al. 2007. Geochimica et Cosmochimica Acta 71:4380-4403. [3] Busemann H. et al. 2009. Earth and Planetary Science Letters 288:44-57. [4] Davidson J. 2009. Ph.D. thesis, Open University. [5] Busemann H. et al. 2007. Meteoritics \& Planetary Science 42:1387-1416.
5192

CHILI-APPROACHING THE FINAL FRONTIERS IN LATERAL RESOLUTION AND SENSITIVITY FOR ISOTOPIC AND CHEMICAL ANALYSIS

T. Stephan ${ }^{1,2,3}$, A. M. Davis ${ }^{1,2,4}$, M. J. Pellin ${ }^{1,2,3,4}$, M. R. Savina ${ }^{1,3}$ I. V. Veryovkin $^{1,3}$, A. J. King ${ }^{1,2}$, N. Liu ${ }^{1,2,3}$, R. Trappitsch ${ }^{1,2,3}$ and R. Yokochi ${ }^{1,2} .{ }^{1}$ Chicago Center for Cosmochemistry. E-mail: tstephan@ uchicago.edu. ${ }^{2}$ Department of the Geophysical Sciences, University of Chicago, Chicago, IL 60637, USA. ${ }^{3}$ Materials Science Division, Argonne National Laboratory, Argonne, IL 60439, USA. ${ }^{4}$ Enrico Fermi Inst., University of Chicago, Chicago, IL 60637, USA.

Introduction: Cometary, asteroidal, and interstellar dust collected by recent sample return missions Stardust and Hayabusa, by high-flying aircraft in the stratosphere, or extracted from meteorites all require analytical techniques with high lateral resolution and high sensitivity. In secondary ion mass spectrometry (SIMS), lateral resolution has reached approximately $50 \mathrm{~nm}$ and the useful yield - the ratio of detected to consumed atoms from a sample-only rarely exceeds $1 \%$. The final frontiers, however, to determine the isotopic nature and the location of every single atom in a given sample seem to be no longer beyond imagination. The final limiting factors are the number of atoms in an analyzed volume and how fast they can be detected.

CHILI (Chicago Instrument for Laser Ionization), a new resonance ionization mass spectrometry (RIMS) instrument, which is about to be completed at the University of Chicago, is a major step toward these frontiers [1]. CHILI is designed for isotopic and elemental analysis at a few nanometers spatial resolution and a useful yield of $40-50 \%$.

Major Components: CHILI is equipped with a COBRA-FIB highresolution liquid metal ion gun, which can be focused to $2.5 \mathrm{~nm}$, and an $\mathrm{e}^{-}$CLIPSE Plus field emission electron gun (both from Orsay Physics) with a beam diameter of $<4 \mathrm{~nm}$. The electron gun supports the built-in diffraction-limited (approximately $0.5 \mu \mathrm{m}$ ) optical microscope for sample imaging at high resolution. A piezoelectric driven stage for nanometerscale motions and a sample holder that accepts a wide variety of samples are operational.

The flight tube for the time-of-flight mass spectrometer will be mounted vertically above the sample chamber, which resides in the center of an $\mathrm{H}$-shaped laser table supported by active vibration cancelation devices.

For resonance ionization, six tunable Ti:sapphire solid state lasers pumped with three $40 \mathrm{~W}$ Nd:YLF lasers will be used. This will allow at least three elements to be analyzed simultaneously.

Counting statistics limit the dynamic range and the precision of isotopic ratios in existing RIMS instruments [2,3] due to dead time effects [4] of the single anode microchannel plate detectors. CHILI will initially be equipped with such a detector, but a new detector that will allow much higher count rates is presently under development, in collaboration with the Large-Area Picosecond Photo-Detectors Project (http://psec.uchicago.edu).

Conclusions: CHILI reflects many recent developments in instrument design, approaching their physical limits. CHILI will be applied to a multitude of cosmochemical problems where high lateral resolution and high sensitivity are indispensable.

References: [1] Stephan T. et al. 2011. Abstract \#1995. 42nd Lunar and Planetary Science Conference. [2] Savina M. R. et al. 2003. Geochimica et Cosmochimica Acta 67:3215-3225. [3] Veryovkin I. V. et al. 2004. Nuclear Instruments and Methods in Physics Research B 219220:473-479. [4] Stephan T. et al. 1994. Journal of Vacuum Science \& Technology A 12:405-410. 
5064

EXTRACTING PYROXENE MINERAL SPECTRA FROM MARTIAN METEORITES

N. R. Stephen ${ }^{1,2}$, G. K. Benedix ${ }^{1,2}$ and P. A. Bland ${ }^{1,2,3}$. ${ }^{1}$ Dept. of Mineralogy, The Natural History Museum, Cromwell Road, London, SW7 5BD, UK. E-mail: n.stephen@nhm.ac.uk. ${ }^{2}$ Impacts \& Astromaterials Research Centre, (IARC) and Dept. of Earth Science and Engineering, Imperial College London, South Kensington Campus, London, SW7 2AZ, UK. ${ }^{3}$ Dept. Applied Geol., Curtin University of Technology, GPO Box U1987, Perth WA 6845, Australia.

Introduction: Without Martian sample return, Martian meteorites provide the best in situ opportunity to study Martian material [e.g. 1] on Earth and to compare to data collected via remote sensing techniques. Thermal emission spectroscopy is one method that has been used to produce several maps of the surface geology and mineralogy of Mars [2-4]. This method uses a spectral library of mostly terrestrial minerals to deconvolve whole rock spectra of the surface of Mars into its constituent minerals. The terrestrial minerals in the database cover a wide range of compositions. However, minerals in Martian meteorites have compositions still not represented in the spectral library and getting mineral separates from hand samples is difficult. One method that can be used is micro-FTIR [5-9]. In this study we are expanding ours and others previous studies [8-10] to measure spectra for the range of pyroxene compositions found in Martian meteorites and compare these to bulk rock spectra.

Samples and Analytical Techniques: Spectra of pyroxenes were measured in thin sections of Los Angeles (BM2000, M12) and Zagami (BM1966, 54 and MfN) using a Perkin Elmer SpectrumOne FTIR with an AutoIMAGE microscope.

Results and Discussion: Compositional differences of pyroxenes in Martian meteorites are reflected in their spectra. Pyroxene (augite and pigeonite) in the Martian meteorites have a much larger range of $\mathrm{Mg}$ and $\mathrm{Fe}$ in pigeonite and lower $\mathrm{Ca}$ contents in augite as compared to pyroxenes in the spectral library. There are two ways to extract pyroxene spectra using the microscope FTIR system (1) Individually measure each mineral grain or (2) create a full spectrum map of an entire thin section. The first method can be hampered by grain orientation, which is known to effect IR spectra. To test the usefulness of the microscope-derived spectra on deconvolutions, we will create a theoretical "bulk" rock by combining individual mineral spectra together according to modal mineralogy analysis of thin sections and bulk powders. This 'bulk' will also be compared to the measured spectra of the bulk thin section.

References: [1] McSween H. Y. 2003. In Meteorites, Comets, and Planets (ed. A. M. Davis), Vol 1 Treatise on Geochemistry (eds H. D. Holland and K. K. Turekian). [2] Christensen P. et al. 2001. Journal of Geophysical Research 106:23823-23871. [3] Christensen P. et al. 2004. Space Science Review 110:85-130. [4] Christensen P. et al. 2004. Science 306:1733-1739. [5] Palomba et al 2006. Icarus 182:68-79. [6] Klima R. and Pieters C. 2006. Journal of Geophysical Research 111:E01005. [7] Morlok A. et al. 2006. Meteoritics \& Planetary Science 41:773-784. [8] Benedix G. and Hamilton V. 2007. Abstract \#1805. 38th Lunar and Planetary Science Conference. [9] Benedix G. K. and Hamilton V. E. 2009. Abstract \#5082. 72nd Annual Meteoritical Society Meeting. [10] Hamilton V. E. 2000. Journal of Geophysical Research 105:9701-9716.
5297

MODELING INTERSTELLAR DUST DYNAMICS IN THE SOLAR SYSTEM: APPLICATION TO Stardust

V. J. Sterken ${ }^{1,2}$, N. Altobelli ${ }^{3}$, S. Kempf ${ }^{2,4}$, F. Postberg ${ }^{1,5}$, G. Schwehm ${ }^{3}$, R. Srama ${ }^{1,6}$, and E. Grün ${ }^{1,4} \cdot{ }^{1}$ MPIK, Heidelberg, Germany E-mail: Veerle.Sterken@mpi-hd.mpg.de. ${ }^{2}$ IGEP, TU Braunschweig, Germany. ${ }^{3}$ ESA, Madrid, Spain. ${ }^{4}$ LASP, University of Colorado, Boulder, CO, USA. ${ }^{5}$ Institut für Geowissenschaften, Universität Heidelberg, Germany. ${ }^{6}$ IRS, TU Stuttgart, Germany.

Introduction: Interstellar dust (ISD) has been detected in the solar system with space missions like Helios [1], Ulysses [2-5], Galileo [3, 6], and Cassini [7], using onboard dust detectors [8]. In addition, in 2000 and 2002, the Stardust mission has exposed its aerogel collectors to the interstellar dust stream for sample return of interstellar dust. Up to now, a few ISD candidates are found in the aerogel, and efforts are on-going in the frame of the Inter Stellar Preliminary Examination (ISPE) [9]. For better understanding the results of the Stardust ISD capture, we calculated the relative densities and fluxes of ISD during the mission collection phase. Also an estimate of the absolute number of particles to be expected in the aerogel is made.

Method: Simulations of interstellar dust trajectories through the solar system are made, taking into account gravity, solar radiation pressure and Lorentz forces due to the interaction of the charged dust with the interplanetary magnetic field. The latter leads to time-variable fluxes. From the simulations, densities and fluxes are derived at the location of Stardust with respect to the undisturbed flow of ISD. An estimate is made for the total amount of particles hitting the collector, by starting with the MRN distribution of dust [10] derived from astronomical observations, and applying the particle filtering at the Heliospheric boundary [11] and in the inner solar system [4]. The method is tested against Ulysses data and modeling.

Results: The proposed method of estimating total interstellar dust numbers is evaluated and future work is defined.

References: [1] Altobelli N. et al. 2006. Astronomy \& Astrophysics 448:243. [2] Grün E. et al. 1993. Nature 362:428. [3] Baguhl M. et al. 1996. Space Science Reviews 78:165-172. [4] Landgraf M. et al. 2000. Journal of Geophysical Research 105:10303. [5] Krüger H. et al. 2007. Space Science Reviews 130:401. [6] Altobelli N. et al. 2005. Journal of Geophysical Research 110:7102. [7] Altobelli N. et al. 2003. Journal of Geophysical Research 108:8032. [8] Srama R. et al. 2004. Space Science Reviews 114:465. [9] Westphal A. J. et al. 2011. Abstract \#2083. 42nd Lunar and Planetary Science Conference. [10] Mathis J. S. 1977. The Astrophysical Journal Part 1 217:425-433. [11] Slavin J. D. et al. 2010. 12th International Solar Wind Conference 1216:497-501. 
5482

INVESTIGATING PRESSURE MAGNITUDES AT DEPTH FOR OBLIQUE IMPACTS INTO LAYERED TARGETS: APPLICATIONS TO IMPACTS INTO SEDIMENTARY TARGETS

A. M. Stickle and P. H. Schultz. Brown University Geological Sciences, Providence RI, USA, 02912. E-mail: angela_stickle@brown.edu.

Introduction: Experimental and numerical models reveal the effects of low-impedance layers on top of competent substrates, including reduced shock pressure at depth in the substrate. This becomes increasingly important for the terrestrial bodies where low-impedance layers typically cover the surface (i.e., ice, sediments or mega-regolith). The earth, specifically, is $75-80 \%$ covered in sediments; therefore, understanding the effects of layered targets is important for our understanding of resultant crater structures seen on the surface and the shock effects recorded in the rock record. One useful case study is the Rock Elm impact structure in southwest Wisconsin, USA, which is a deeply eroded crater found in a region of otherwise virtually undeformed sedimentary rocks lying on top of a Precambrian crystalline basement. Field studies [i.e., 1] identified shock features within quartz grains within the structure; however, these features were formed at very low shock pressures (5-10 GPa) and other diagnostic shock effects such as shatter cones were absent.

Approach: A suite of experiments were performed at the NASA Ames Vertical Gun Range at the NASA Ames Research Center into planar PMMA targets with surface layers of varying materials and thicknesses. Direct comparisons were made using 3D CTH calculations to interpret the processes observed in high-speed imaging. Large-scale simulations, then, allow application to planetary impacts. For large-scale impacts into sedimentary targets with thicknesses on the order of one- to two-projectile diameters, pressure magnitudes at depth are determined and compared to field observations at the Rock Elm impact structure.

Results: Experiments show that damage structures and crater size in the competent substrate are significantly reduced or non-existent for impacts into a layered target. 3D CTH simulations reveal that this is a manifestation of the impact energy being absorbed/scattered in the surface layer and the layer acting as a wave guide for very oblique impacts $\left(15-30^{\circ}\right)$. For laboratory scale impacts, the peak pressure in the competent substrate drops by $\sim 80 \%$ for a thin (1 projectile diameter) surficial sedimentary layer. In contrast, for surface ice layers, the peak pressure drops by only $\sim 35 \%$ because of the lower impedance contrast. At larger scales, a $90 \%$ lower impedance (sedimentary layer) results in a $70 \%$ drop in peak pressure near the surface of the underlying bedrock [2].

The Rock Elm impact structure provides an application of these results. This crater formed in $500-600 \mathrm{~m}$ thick sediments covering a crystalline basement rock [1]. The finding that quartz in the central uplift is only weakly shocked may be a result of the impact into a soft, overlying sedimentary layer where the upper layers of sediments absorb much of the impact energy. Large-scale CTH models, calibrated by experiments, can be used to constrain pressure magnitudes at depth within such a target lithology.

References: [1] French B. M., Cordua W. S., and Plescia J. B. 2004. Geological Society of America Bulletin 116:188-200. [2] Stickle A. M. and Schultz P. H. 2010. Abstract U51B-0043. American Geophysical Union Fall Meeting.

\section{5}

SHOCK CLASSIFICATIONS OF PLANETARY SILICATE ROCKS REVISITED: UPDATE OF THE 1991 GCA AND 2007 IUGS PROPOSALS

D. Stöffler. Museum für Naturkunde-Leibniz Institute at the Humboldt University Berlin, D-10115 Berlin, Germany. E-mail: dieter. stoeffler@mfn-berlin.de.

Introduction: A wide variety of rocks from all planetary bodies explored so far display shock effects attributed to hyper-velocity impacts acting on the these bodies since their formation. The present record of shocked rocks includes terrestrial, lunar, and Martian crustal rocks and fragments from different asteroidal bodies (stony and iron meteorites). Since the first shock recovery experiments became available for calibration of shock effects around 1960 various classification systems have been proposed until a unified system was developed in the scope of the IUGS Systematics of Metamorphic Rocks [1].

Current Classification Systems: Shock classifications are based on the principle of "progressive shock metamorphism" defined first in 1966 $[2,3]$. Subsequently, classification systems were proposed focusing on the rocks' parent bodies, i.e. Earth, Moon, Mars, and asteroids [4-7]. However, the systematics of shocked rocks is largely independent of the parent bodies but rather depends on the type of rock, its physical properties (e.g., constituent minerals and their shock impedance, porosity), and the ambient temperature during shock. Moreover, shock classifi-cations of different rocks were published in different, sometimes hardly accessible journals and books $[1,7]$, and a coherent summarizing review of the current state focusing on all major rock types independent of their parent body is missing as the IUGS systematics deals primarily with the classification of impactites in general [1].

Recommended Shock Classifications of Planetary Rocks: A set of shock classifications is proposed for different "basic" rock types based on pressure calibrated "equilibrium" shock effects of the main constituent minerals (quartz, plagioclase, olivine, pyro-xene), and on "disequilibrium" effects such as high pressure polymorphs and intergranular melt veins and pockets. The shock classified rock types comprise: (1) igneous and metamorphic crystalline rocks such as felsic (quartzo-feldspathic) rocks, mafic rocks, and ultramafic rocks, (2) consolidated sedimentary rocks such as sandstone, and (3) unconsolidated sediments such as sand and (planetary) regolith. These subgroups include shock classifi-cations for the following specific rocks: (1) granitoids and gneisses, quartzite; basalt, gabbro, norite, troctolite, anorthosite, basaltic achondrites; dunite, peridotite, pyroxenite, chondrites, chassignite, ureilite, aubrite, diogenite, (2) sandstone, and (3) sand, lunar regolith, asteroidal regolith. For these rocks 5-7 stages of shock metamorphism are defined on the basis of shock effects calibrated by shock recovery experiments on the major minerals (see references in [1, 7]).

Outlook: More shock recovery experiments on plagioclase, olivine, and pyroxene at low ambient temperatures $(\sim 100-250 \mathrm{~K})$ are desirable for improving classification of extraterrestrial rocks.

References: [1] Fettes D. and Desmons J. 2007. Meta-morphic rocks. Cambridge University Press. [2] Stöffler D. 1966. Contributions to Mineralogy and Petrology 12:15-24. [3] Chao E. C. T. 1967. Science 156:192-202. [4] Dodd R. T. and Jarosewich E. J. 1979. Earth and Planetary Science Letters 44:335-340. [5] Van Schmus W. R. and Ribbe P. H. Geochimica et Cosmochimica Acta 31:747-765. [6] Stöffler D. et al. 1991. Geochimica et Cosmochimica Acta 55:3845-3887. [7] Joliff B. L. et al. 2006. Reviews in Mineralogy \& Geochemistry, vol. 60. pp. 1-81 and 519-596. 
5355

COMBINED NANOSIMS AND TEM IN SITU ANALYSIS OF PRISTINE MATRIX MATERIAL OF ALHA77307 AND ACFER 094 A. N. Stojic ${ }^{1}$, F. E. Brenker ${ }^{1}$ and P. Hoppe ${ }^{2} .{ }^{1}$ Goethe Universitaet, Institut fuer Geowissenschaften, 60438 Frankfurt/Main, Germany. ${ }^{2}$ MaxPlanck Institute for Chemistry, P.O. Box 3060, 55020 Mainz, Germany E-mail: stojic@em.uni-frankfurt.de.

Only a few structural studies on presolar silicates and oxides exist [1]. Commonly the NanoSIMS mapping technique is used to identify presolar grains in the meteoritic matrix from a polished thin section. To extract these presolar grains for further TEM analysis, a complex and risky lift out procedure by FIB is needed [1], reducing the availability of such grains for structural studies. In contrast, argon ion slicing (ArIS), a recently introduced TEM sample preparation technique in geosciences, yields super large electron transparent areas of up to $40,000 \mu \mathrm{m}^{2}$ [2]. The availability of extremely large thin foils makes a substantial change in the sequence of procedure steps possible, i.e., preparation of a large thin foil first, followed by the NanoSIMS scan on the previously obtained thin foil (Fig. 1). No additional sample preparation for TEM is required once the grains are identified by NanoSIMS.

Pieces of Acfer 094 and ALHA77307 were ArIS treated until large electron transparent areas were obtained. These TEM thin foils were scanned for oxygen isotope ratios with a Cameca NanoSIMS 50. Based on large O-isotopic anomalies we detected two presolar grains (both silicates) out of eight ion images $\left(10 \times 10 \mu \mathrm{m}^{2}\right)$ in ALHA77307, and 7 presolar grains (six silicates and one oxide) out of 22 ion images (same size) from Acfer 094.

References: [1] Vollmer et al. 2009. The Astrophysical Journal 700:774-782. [2] Stojic and Brenker. 2010. European Journal of Mineralogy 22:17-21.

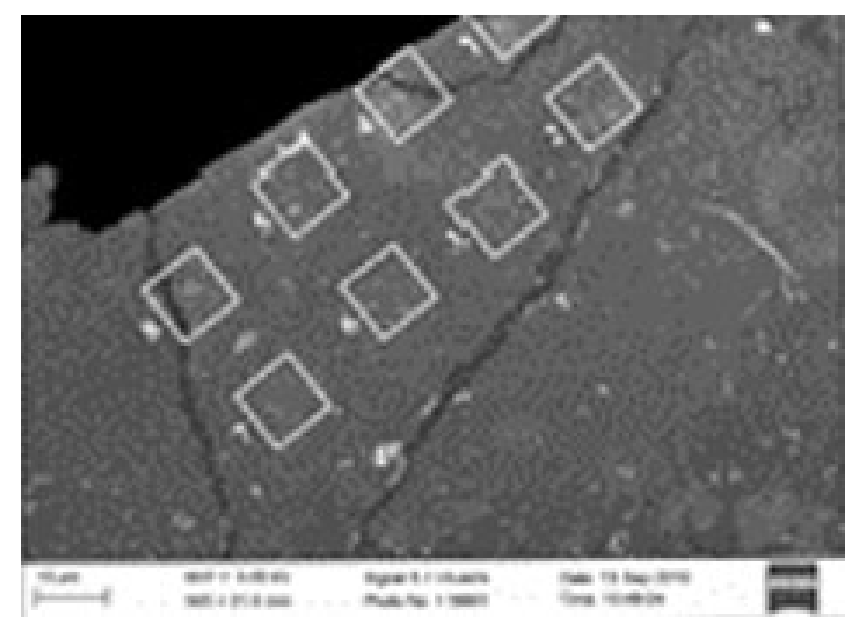

Fig. 1. Thin foil section of ALHA77307 with NanoSIMS scan windows indicated.
5439

\section{A COMPARISON OF POROSITY USING DIFFERENT MEASUREMENT TECHNIQUES \\ M. M. Strait. Alma College, Alma, MI 48801, USA. E-mail: straitm@alma.edu.}

Introduction: Porosity is one of several physical properties of meteorites that are being explored by the planetary science community. Knowledge of porosity will affect the modeling of a variety of physical phenomenon, such as impact disruption. Porosity is being measured in a variety of ways by different laboratories, including a combination of helium pycnometry and glass beads to measure whole rock porosity [1], SEM/BSE imaging and computer processing to measure thin sections [2] and X-ray tomography on whole rock samples [3]. There are advantages and disadvantages to each of these techniques, but they each provide important information about porosity.

A related three-dimensional X-ray technique using Micro CT scanning has been reported [4]. This technique was applied by this laboratory to a suite of rocks that have also been measured using pycnometry and thin section imaging. The first report on this comparison looked at its use as a visualization technique [5]. Here we report on a comparison of porosity as measured by the three methods.

Results and Discussion: A set of five terrestrial samples that had previously been measured using pycnometry and imaging [6] were measured using the GE eXplore specCZT scanner at the University of Western Ontario. The set of samples chosen were low in porosity, and, as a result, the size of the pores are on the same size scale as the resolution of the Micro CT, limiting the usefulness of the technique to measure porosity. Other problems encountered were the variations in the color range from slice to slice in the three-dimensional image stack and the overlap of the color range in the center of the sample with the pore space further out into the sample. More work is being done to resolve color overlap problems, but the resolution problem cannot be overcome.

In general, we find that samples with low porosity $(<5 \%)$ do not agree well between the three techniques, but the nature of the porosity is not well understood in these samples. There is better agreement with more highly porous samples $(>10 \%)$, but again, there is some variation between the methods used.

Overall, we conclude that CT imaging is best used to get a basic view of the appearance of the porosity in a hand sample. The technique is easy to use, fast, and there is no sample preparation needed. Pycnometry and point counting produce better numerical measures for porosity, especially at smaller values. Pycnometry is the best technique for quick, reliable whole rock measurements, although it gives no visualization of the porosity in the sample. In conclusion, porosity needs to continue to be evaluated using multiple methods to get a better feel for both the numerical range of porosity values as well as the physical appearance of the pore space.

References: [1] Britt D. T. and Consolmagno G. J. 2003. Meteoritics \& Planetary Science 38:1161-1180. [2] Strait M. M. and Consolmagno G. J. 2002. Meteoritics \& Planetary Science 37:A137. [3] Friedrich J. M. et al. 2008. Planetary and Space Science 56:895-900. [4] McCausland P. J. A. et al. 2010. Abstract \#2584. 41st Lunar and Planetary Science Conference. [5] Strait M. M. et al. 2011. Abstract \#2052. 42nd Lunar and Planetary Science Conference. [6] Strait M. M. et al. 2007. Meteoritics \& Planetary Science 42:A146. 
5350

ULTRASENSITIVE DETERMINATION OF ${ }^{81} \mathrm{Kr}-\mathrm{Kr}$ COSMIC RAY EXPOSURE AGES OF EUCRITES AND BUNBURRA ROCKHOLE I. Strashnov ${ }^{1}$, P. A. Bland ${ }^{2}$, P. Spurný ${ }^{3}$, M. C. Towner ${ }^{2}$ and J. D. Gilmour ${ }^{1}$. ${ }^{1}$ SEAES, University of Manchester, Manchester, E-mail: Ilya.Strashnov@manchester.ac.uk. ${ }^{2}$ IARC, Department of Earth Science and Engineering, Imperial College London, SW7 2AZ, UK. ${ }^{3}$ Astronomical Institute of the Academy of Sciences, Fričova 298, CZ-251 65 Ondřejov Observatory, Czech Republic.

Introduction: Cosmic ray exposure ages (CRE ages) measure the length of time a meteoroid was exposed to cosmic rays as a small body between separation from its parent asteroid or planet and arrival at the Earth. They can be determined by comparing the equilibrium concentration of radioactive ${ }^{81} \mathrm{Kr}$ to the concentrations of stable cosmogenic $\mathrm{Kr}$ isotopes. Among the various types of methods ${ }^{81} \mathrm{Kr}-\mathrm{Kr}$ CRE ages appear the most reliable for eucrites because no adjustment is needed for shielding and sample composition [1]. However, low ${ }^{81} \mathrm{Kr}$ concentrations (few thousand atoms $\mathrm{mg}^{-1}$ [2]) and low ${ }^{81} \mathrm{Kr} / \mathrm{Kr}$ ratios have been constraints on the use of the method since bulk (up to $1 \mathrm{~g}$ ) samples have been required. Here we report ${ }^{81} \mathrm{Kr}-\mathrm{Kr}$ ages acquired by RIMSKI-Resonance Ionization Mass Spectrometer for Krypton Isotopes [3, 4].

Results and Discussions: Typical samples were grains of $1-3 \mathrm{mg}$, each containing a few thousand atoms of ${ }^{81} \mathrm{Kr}$ and $\sim 10^{5}-10^{6}$ atoms of stable krypton isotopes. Between 3-6 replicate analyses have been made for each meteorite in this study. Gas was extracted by laser heating. Isotope ratios were corrected for mass discrimination using interspersed analyses of krypton sourced from the terrestrial atmosphere. For 7 eucrites our data are consistent with previously reported literature data [4] once account is taken of the recent revision of the ${ }^{81} \mathrm{Kr}$ half-life to $2.29 \times 10^{5}$ yr [5].

We have also analyzed two samples for which ${ }^{81} \mathrm{Kr}-\mathrm{Kr}$ ages have not been previously reported. Our preliminary age for the eucrite Lakangaon is $24.59 \pm 0.87 \mathrm{Myr}$, while that for the anomalous eucrite Bunburra Rockhole is $26.71 \pm 1.05 \mathrm{Myr}$.

Eugster and Michel [1] assign known eucrites to five CRE age clusters corresponding to presumed impacts on the parent body. Our data suggest that Lakangaon belongs to a cluster with Millbillillie, for which we obtain an ${ }^{81} \mathrm{Kr}-\mathrm{Kr}$ age of $23.57 \pm 1.87 \mathrm{Myr}$ (compare to the literature age of $22.38 \pm 0.73 \mathrm{Myr}$ [2]) and Bereba 26.29 $\pm 1.65 \mathrm{Myr}$ $(27.17 \pm 2.29$ Myr [2]).

Bunburra Rockhole [6] was recovered by The Desert Fireball Network in Western and has a calculated pre-atmospheric orbit suggesting its parent body is a member of the Aten group of earthcrossing asteroids. While our data suggest an ${ }^{81} \mathrm{Kr}-\mathrm{Kr}$ age similar to that of Millbillillie, Bereba and Lakangaon, oxygen isotope and other data imply that it did not originate on the eucrite parent body [6]. Our ${ }^{81} \mathrm{Kr}$ age is distinctly different from those of the other anomalous eucrite falls, Pasamonte (7.7 $\pm 0.52 \mathrm{Myr}$ [2]) and Ibitira (12.5 $\pm 0.52 \mathrm{Myr}$ [2]), suggesting they sample three impacts in contrast to the five impacts on the Eucrite parent body that have produced 26 falls with measured ${ }^{81} \mathrm{Kr}-\mathrm{Kr}$ ages to date. Eucrite samples were provided by Natural History Museum. This work was funded by the STFC, grant number ST/ G003068/1.

References: [1] Eugster O. and Michel Th. 1995. Geochimica et Cosmochimica Acta 59:177-199. [2] Shukolyukov A. and Begeman F. 1996. Meteoritics \& Planetary Science 31:60-72. [3] Strashnov I. et al. 2011. Journal of Analytical Atomic Spectrometry, doi:10.1039/ C1JA10051C, in press. [4] Strashnov et al. This conference. [5] Baglin C. M. 2008. Nuclear Data Sheets 109:2257-2437. [6] Bland P. A. et al. 2009. Science 325:1525-1527.
5273

RIMSKI-A RESONANCE IONIZATION MASS SPECTROMETER FOR DETERMINATION OF KRYPTON ISOTOPE RATIOS OF EXTRATERRESTRIAL SAMPLES

Strashnov I. and J. D. Gilmour. SEAES, University of Manchester, Manchester. E-mail: Ilya.Strashnov@manchester.ac.uk.

RIMSKI [1]-A Resonance Ionization Mass Spectrometer for Krypton Isotopes - is a newly developed ultrasensitive instrument with a cryogenic sample concentrator and a laser ion source designed for isotopic analysis of extraterrestrial material. Laser heating is used for extraction of krypton from samples into the mass spectrometer volume. Atoms continuously condense on a cold spot in the ion source, and are repeatedly released by laser heating. They are ionized in the evaporation plume using three tunable dye lasers and one frequency tripled Qswitched Nd:YAG laser. Two resonant transitions are employed (at $116.5 \mathrm{~nm}$, produced by four-wave frequency mixing in $\mathrm{Xe}$, and $558.1 \mathrm{~nm}$ ) and are followed by single photon ionization at $1064 \mathrm{~nm}$.

Multiple analyses of air aliquots and $<5 \mathrm{mg}$ samples of meteorites, both containing $\sim 10^{6}$ total krypton atoms, show precision $\sim 1 \%$ for major isotope ratios. They are reproducible to this level through a day's analyses, allowing calibration for linear and non-linear mass discrimination by sample-standard bracketing. The mass spectrometer blank corresponds to $\sim 5800$ krypton atoms $\left(\sim 3300\right.$ atoms of ${ }^{84} \mathrm{Kr}$ ). The lifetime against detection of krypton atoms is $7 \mathrm{~min}$.

A further test of the instrument is provided by $\mathrm{Kr}$ isotope ratio determinations of eucrites with known isotope compositions. Cosmogenic ${ }^{81} \mathrm{Kr}$ can be reliably detected at the few thousand atom level, corresponding to determination of cosmic ray exposure ages - the length of time a meteoroid has spent as a small body between separation from its parent asteroid or planet and arrival at the Earth-from samples of a few milligrams. Determined ages and literature values (in brackets [2]) of-Bereba: $26.29 \pm 1.65$ My $(27.17 \pm 2.29 \mathrm{My})$, Bouvante: $6.93 \pm$ $0.33 \mathrm{My}(6.99 \pm 0.40 \mathrm{My})$, Pasamonte: $7.71 \pm 0.49 \mathrm{My}(7.70 \pm 0.52$ My), Padvarnikai: $14.32 \pm 0.79$ My (15.2 \pm 2.08 My), Juvinas: $11.14 \pm$ $0.45 \mathrm{My} \quad(11.04 \pm 0.42 \mathrm{My})$, Millbillillie: $23.57 \pm 1.87 \mathrm{My} \quad(22.38 \pm$ $0.73 \mathrm{My})$, Stannern: $36.37 \pm 2.08 \mathrm{My}(37.10 \pm 0.94 \mathrm{My})-$ agrees within experimental error once account is taken of the recent revision of the ${ }^{81} \mathrm{Kr}$ half-life to $2.29 \times 10^{5}$ years [3].

Further instrument development includes design of a new ion source and implementation of the Xe resonance ionization scheme similar to RELAX [4] that would allow simultaneous $\mathrm{Xe} / \mathrm{Kr}$ analysis. Among planned measurements are primitive meteorites, presolar $\mathrm{SiC}$ grains as well as material from sample return messions e.g., Stardust or Genesis. Determination of elemental $\mathrm{Kr} / \mathrm{Xe}$ ratios will allow aqueous and igneous processes to be traced. method applicability forcing use of bulk (up to $1 \mathrm{~g})$ samples.

Acknowledgment: Eucrite samples were provided by Natural History Museum. This work was funded by the STFC, grant number ST/ G00306811.

References: [1] Strashnov I. et al. 2011. Journal of Analytical Atomic Spectrometry, doi:10.1039/C1JA10051C, in press. [2] Shukolyukov A. and Begeman F. 1996. Meteoritics \& Planetary Science 31:60-72. [3] Baglin C. M. 2008. Nuclear Data Sheets 109:2257-2437. [4] Crowther S. A. et al. 2008. Journal of Analytical Atomic Spectrometry 23:938-947. 
5118

ELEMENTAL ANALYSIS OF IMPACT RESIDUES IN CRATERS ON THE Stardust INTERSTELLAR FOILS

R. M. Stroud, C. Allen, A. Ansari, S. Bajt, N. Bassim, H. A. Bechtel, J. Borg, F. Brenker, J. Bridges, D. E. Brownlee, M. Burchell, M. Burghammer, A. L. Butterworth, P. Cloetens, A. M. Davis, R. Doll, C. Floss, G. J. Flynn, D. Frank, Z. Gainsforth, E. Grün, P. R. Heck, J. K. Hillier, P. Hoppe, L. Howard, G. R. Huss, J. Huth, B. Hvide, A. Kearsley, A. J. King, P. G. Kotula, B. Lai, J. Leitner, L. Lemelle, A. Leonard, H. Leroux, L. R. Nittler, R. C. Ogliore, W. J. Ong, F. Postberg, M. C. Price, S. A. Sandford, J. A. Sans Tresseras, S. Schmitz, T. Schoonjans, K. Schreiber, G. Silversmit, A. Simionovici, R. Srama, T. Stephan, J. Stodolna, S. R. Sutton, R. Toucoulou, M. Trieloff, P. Tsou, A. Tsuchiyama, T. Tyliczszak, B. Vekemans, L. Vincze, A. J. Westphal, M. E. Zolensky and $>29,000$ Stardust@home dusters. ISPE author affiliations are at http://www.ssl.berkeley.edu/ westphal/ISPE/.

Introduction: Preliminary imaging studies show the abundance of craters on the interstellar foils is 50 to 100 times the expected value for interstellar particle impacts [1]. Thus, the majority of the craters are likely due to other sources, such as secondary impacts from interplanetary dust particles that impacted the solar panels, or direct impact of high velocity nanoparticles.

In order to distinguish amongst the possible origins for the craters, elemental analyses of any retained impact residue, and/or crater morphology measurements, are necessary. Solar cell cover glass is primarily silica, but contains wt. \% levels of $\mathrm{Ce}$ and $\mathrm{Zn}$, which can be used as an indicator of spacecraft origin. The specific features of craters from the impact of high velocity nanoparticles, i.e., $10-50 \mathrm{~nm}$ particles traveling at $100-400 \mathrm{~km} \mathrm{~s}^{-1}$, are unknown, however, lack of detectable residue and/or high depth-to-diameter and narrow-rimmed craters are expected to be diagnostic. Results from analog shot studies suggest that interstellar particle impact craters are likely to contain residue detectable by Auger spectroscopy, and have crater morphologies consistent with impact velocities of $5-25 \mathrm{~km} \mathrm{~s}^{-1}$.

Experimental: Elemental analysis of six craters was performed, including those with symmetric and asymmetric geometries, and both larger and smaller than $1 \mu \mathrm{m}$. Auger measurements were done with the PHI 700 Auger Nanoprobe at Washington University [2]. EDX measurements were performed at the MPI for Chemistry in Mainz using a FEG SEM with a conventional EDX system, and at Sandia National Lab, NM, using a FEG SEM with a custom Bruker on-axis silicon driftdetector EDX system. FIB-TEM studies were carried out at the Naval Research Lab, with a FEI Nova 600 FIB-SEM and JEOL 2200FS TEM.

Results and Discussion: All six craters contained residues with levels of $\mathrm{Si}$ and $\mathrm{O}$ dectectable by Auger or $\mathrm{EDX}$, but not $\mathrm{Ce}$, or $\mathrm{Zn}$. In addition, $\mathrm{Fe}$ was detected in four craters, $\mathrm{Mg}$ in two, and $\mathrm{Na}$ in two. Subsequent TEM analysis of FIB sections from two craters first analyzed by EDX revealed residues $\sim 10$ to $50-\mathrm{nm}$ thick, with major silica and minor levels of $\mathrm{Ti}, \mathrm{Ce}$ and $\mathrm{Zn}$. These measurements are consistent with analysis of tracks in aerogel from the IS collector, which revealed $\sim 80 \%$ of tracks originate from secondary impacts. Additional elemental analysis of craters is planned to identify bona fide interstellar particle impacts.

References: [1] Stroud R. M. et al. 2011. Abstract \#1753. 42nd Lunar and Planetary Science Conference. [2] Floss C. et al. 2011. This conference.

\section{4}

ORDINARY CHONDRITE-RELATED GIANT COSMIC SPHERULES C. Suavet ${ }^{1,2}$, C. Cordier $^{1}$, P. Rochette ${ }^{3}$, L. Folco ${ }^{1}$, J. Gattacceca ${ }^{3}$, C. Sonzogni ${ }^{3}$, D. Damphoffer ${ }^{3}$. ${ }^{1}$ MNA, Siena, Italy. ${ }^{2}$ MIT, Cambridge, MA, USA. E-mail: csuavet@mit.edu. ${ }^{3}$ CEREGE, Aix-en-Provence, France.

Micrometeorites are extraterrestrial particles smaller than approximately $2 \mathrm{~mm}$ collected on the Earth's surface [1]. Most of them melt during atmospheric entry; they are called cosmic spherules. A previous study [2] found that all three spherules with porphyritic olivine (PO) and cryptocrystalline (CC) textures were related to ordinary chondrites based on their oxygen isotopic signature. Here we report the oxygen isotope compositions of 15 giant $(>800 \mu \mathrm{m})$ cosmic spherules from the Transantarctic Mountains, Antarctica [3], including six PO, eight $\mathrm{CC}$ and one barred olivine (BO) textural types [4].

Eleven spherules (approximately $70 \%$ of them: $4 / 6$ PO and $6 / 8 \mathrm{CC}$, and the BO spherule) are related to ordinary chondrites based on oxygen isotopic compositions. In ordinary chondrite-related PO spherules olivines have $\mathrm{Fa}_{8.5-11.8}$ composition, they are Ni-poor to Ni-rich (0.04-1.12 wt\%), and $\mathrm{CaO}$-rich $(0.10-0.17 \mathrm{wt} \%)$. Ordinary chondrite-related spherules also have high magnetite contents (approximately $2-12 \mathrm{wt} \%$ ). One PO and one $\mathrm{CC}$ spherules are related to previously identified ${ }^{17} \mathrm{O}$-enriched cosmic spherules for which the parent body is unknown [2]. One CC spherule has an oxygen isotopic signature relating it with $\mathrm{CM} / \mathrm{CR}$ carbonaceous chondrites.

These results show that the texture of cosmic spherules is not only controlled by atmospheric entry heating conditions but also depends on the parent body, whether be it through orbital parameters (entry angle and velocity), or chemistry, mineralogy, or grain size of the precursor.

Acknowledgments: This work was supported by the PNRA and by the EU-funded ORIGINS Marie Curie Action.

References: [1] Rubin A. E. and Grossmann J. N. 2010. Meteoritics \& Planetary Science 45:114-122. [2] Suavet C., Alexandre A., Franchi I. A., Gattacceca J., and Sonzogni C. et al. 2010. Earth and Planetary Science Letters 293:313-320. [3] Rochette P. et al. 2008. Proceedings of the National Academy of Sciences 105:18206-18211. [4] Genge et al. 2008. Meteoritics \& Planetary Science 43:497-515. 


\section{5}

CORRELATION BETWEEN ${ }^{54}$ CR ANOMALIES AND ACCRETION AGES OF METEORITE PARENT BODIES: IMPLICATIONS FOR THE EVOLUTION OF THE SOLAR NEBULA

N. Sugiura and W. Fujiya. Department of Earth and Planetary Science, University of Tokyo, Tokyo, Japan. E-mail: Sugiura@eps.s.u-tokyo.ac.jp.

Introduction: A fair correlation between ${ }^{54} \mathrm{Cr}$ anomalies and accretion ages is observed among meteorites, including iron meteorites, a pallasite, mesosiderites, an aubrite, HED meteorites, an angrite, NWA 011, ureilites and chondrites (including $\mathrm{E}, \mathrm{O}, \mathrm{R}, \mathrm{CK}, \mathrm{CO}, \mathrm{CV}$, $\mathrm{CH}-\mathrm{CB}, \mathrm{CR}, \mathrm{CM}$, and $\mathrm{CI}$ ). The ${ }^{54} \mathrm{Cr}$ data are taken from [1-8], whereas the accretion ages were estimated in [9]. Data on the Eagle Station pallasite are not included because spallogenic $\mathrm{Cr}$ may contribute significantly to the ${ }^{54} \mathrm{Cr}$ anomaly [10].

The ${ }^{54} \mathrm{Cr}$ anomalies range from $-0.9 \varepsilon$ in differentiated meteorites to $1.6 \varepsilon$ in primitive chondrites. The accretion ages range from $<0.6 \mathrm{Ma}$ after CAI for differentiated meteorites to $3.5 \mathrm{Ma}$ after CAI for primitive chondrites. NWA 011 is an outlier of the trend that is defined by the other meteorites. It is an achondrite that formed early [11] but the $\varepsilon^{54} \mathrm{Cr}$ is as high as those of $\mathrm{C}$ chondrites [1]. An explanation could be devised for the high $\varepsilon^{54} \mathrm{Cr}$. Hence, the correlation between ${ }^{54} \mathrm{Cr}$ anomalies and accretion ages is considered significant in spite of the presence of an outlier. The carriers of the ${ }^{54} \mathrm{Cr}$ anomalies were recently identified as tiny presolar oxide grains $[12,13]$. Hence, we could elucidate the evolution of the solar nebula by investigating how the presolar grains were mixed and distributed in the solar nebula.

Results: We made one-dimensional numerical simulations to find out whether the correlation between ${ }^{54} \mathrm{Cr}$ anomalies and accretion ages can be explained by advection-diffusion of presolar grains injected into the solar nebula. The nebula is assumed to be in a steady state, moving inward at a constant velocity. The injection is assumed to have happened at the time of CAI formation. Adjustable parameters are the turbulence diffusion parameter $(\alpha)$ and the location of the injection. Accretion locations of parent bodies are assumed to shift gradually with time from $1 \mathrm{AU}$ (differentiated meteorites) to $4 \mathrm{AU}$ (primitive $\mathrm{C}$ chondrites). Results of the numerical simulations suggest that the correlation observed among meteorites can be reproduced if the presolar grains were injected at an appropriate distance that is proportional to $\alpha$. For $\alpha=10^{-3}$ that is often assumed for the solar nebula, the injection location is $\sim 100 \mathrm{AU}$.

References: [1] Bogdanovski O. and Lugmair G.W. 2004. Abstract \#1715. 30th Lunar and Planetary Science Conference. [2] Shukolyukov A. and Lugmair G.W. 2006. Abstract \#1478. 37th Lunar and Planetary Science Conference. [3] Shukolyukov A. and Lugmair G.W. 2006. Earth and Planetary Science Letters 250:200. [4] Trinquier A. et al. 2007. The Astrophysical Journal 655:1179. [5] Qin L. et al. 2010. Meteoritics \& Planetary Science 45:1771. [6] Qin L. et al. 2010. Geochimica et Cosmochimica Acta 74:1122. [7] Yamakawa A. et al. 2010. The Astrophysical Journal 720:150. [8] Yamashita K. et al. 2010. The Astrophysical Journal 723:20. [9] Sugiura N. and Fujiya W. 2011. Meteoritical Society Meeting Abstract. This conference. [10] Papanastassiou D. A. and Chen J. H., 2011. Abstract \#2195. 42nd Lunar and Planetary Science Conference. [11] Sugiura N. and Yamaguchi A. 2007. Abstract \#1431. 38th Lunar and Planetary Science Conference. [12] Dauphas N. et al. 2010. The Astrophysical Journal 720:1577. [13] Qin L. et al. 2011. Geochimica et Cosmochimica Acta 75:629.

\section{6}

\section{ACCRETION AGES OF METEORITE PARENT BODIES}

N. Sugiura and W. Fijiya. Department of Earth and Planetary Science, University of Tokyo, Tokyo, Japan. E-mail: Sugiura@eps.s.u-tokyo.ac.jp.

Introduction: Accretion of parent bodies is the first stage of the solar system evolution. Mainly based on $\mathrm{W}$ isotopic information [1] and heat source consideration [2], it is now generally accepted that iron meteorite and achondrite parent bodies accreted earlier than parent bodies of chondrites. However, details of the accretion ages are not well established for many meteorite parent bodies yet. Here we compile accretion ages (Tacc calculated from CAI formation) if available and if not available, we estimate Tacc based on choronological data, heat source consideration and thermal histories. Magmatic irons, HED achondrites, angrites, aubrites, NWA 2976, pallasites, mesosiderites, ureilites and chondrites (E, $\mathrm{O}, \mathrm{R}, \mathrm{CK}, \mathrm{CO}, \mathrm{CV}, \mathrm{CH}-\mathrm{CB}, \mathrm{CR}, \mathrm{CM}$ and $\mathrm{CI}$ ) are included in this study. Instantaneous accretion of parent bodies is assumed for simplicity.

Methods and Results: Tacc of magmatic irons is estimated to be $<0.6 \mathrm{Ma}$ based on the $\mathrm{W}$ isotope [2]. Tacc of HED achondrites is estimated to be $\sim 1.3 \mathrm{Ma}$, based on the core formation age at $\sim 2.5 \mathrm{Ma}$ after CAI [3]. Tacc of angrites is estimated to be $1.6 \mathrm{Ma}$, based on the model core formation age of $\sim 4 \mathrm{Ma}$ after CAI [4]. Tacc of aubrites is not constrained well and is estimated to be $<1.8 \mathrm{Ma}$, according to the requirement of melting of chondritic materials due to ${ }^{26} \mathrm{Al}$ heating. Tacc of NWA 2976 (paired with NWA 011) is estimated to be $\sim 1.5$ Ma, based on the model Al-Mg age of $3.5 \mathrm{Ma}$ after CAI [5]. W isotopic compositions of metal in pallasites are similar to those in magmatic irons [6]. Hence Tacc of pallasite is estimated to be $<0.6 \mathrm{Ma}$. Although W isotopes in many mesosiderites appear to be affected by slow cooling, the least radiogenic composition is similar to those in magmatic irons [6]. Hence Tacc of mesosiderites is estimated to be $<0.6 \mathrm{Ma}$. Estimate of Tacc of ureilites is complicated because of the possible loss of ${ }^{26} \mathrm{Al}$ by explosive volcanism. We favor Tacc $1.1 \mathrm{Ma}$ though we cannot explain the details here.

Tacc of chondrites (E, O, R, CK, CO, CV, CH-CB and CR) are estimated based on the abundances of $\mathrm{Al}$ and the peak metamorphic temperatures. They are 1.8, 2.1, 2.4, 2.6, 2.8, 3.0, 3.3 and $3.5 \mathrm{Ma}$. The ambient temperatures for the $\mathrm{C}$ chondrites (except for $\mathrm{CR}$ ) were assumed to be $\sim-50 \mathrm{C}$. In the case of $\mathrm{CR}$, presence of water ice and heat of hydration were taken into consideration and the ambient temperature is assumed to be $\sim-100 \mathrm{C}$. The accretion ages of $\mathrm{O}, \mathrm{CO}$ and $\mathrm{CR}$ reported here are similar to those reported by [1].

Tacc of CM and CI are estimated to be $\sim 3.5 \mathrm{Ma}$, based on the Mn$\mathrm{Cr}$ ages of carbonates [7, 8] and the thermal histories of the parent bodies. Here again, presence of water ice, heat of hydration and ambient temperature of $\sim-100 \mathrm{C}$ are taken into account.

References: [1] Kleine T. et al. 2009. Geochimica et Cosmochimica Acta 73:5150. [2] Hevey P. J. and Sanders I. S. 2006. Meteoritics \& Planetary Science 41:95. [3] Touboul M. et al. 2008. Abstract \#2336. 39th Lunar and Planetary Science Conference. [4] Markowski A. 2007. Earth and Planetary Science Letters 262:214. [5] SchillerM. et al. 2010. Geochimica et Cosmochimica Acta 74:4844. [6] Quitte G. et al. 2005. Geochimica et Cosmochimica Acta 69:1321. [7] Fujiya W. et al. 2010. Abstract \#1644. 41st Lunar and Planetary Science Conference. [8] Fujiya W. et al., 2011. Abstract \#1397. 42nd Lunar and Planetary Science Conference. 


\section{8}

\section{METEORITES IN SLOVAKIA-SUMMARY}

J. Svoren ${ }^{1}$, V. Porubcan ${ }^{1,2}$, M. Husarik ${ }^{1}$. ${ }^{1}$ Astronomical Institute of SAS, SK-05960 Tatranska Lomnica, Slovak Republic, E-mail: astrsven@ta3.sk. ${ }^{2}$ Department of Astronomy, Comenius University, Mlynska dolina, SK-84248 Bratislava, Slovak Republic.

In the contribution an overview of Slovak meteorites from the viewpoint of the fall/find and location of the fragments at present, is presented. In the territory of the Slovak Republic (an area of $49035 \mathrm{~km}^{2}$ ) three falls and three meteorite findings over two centuries have been registered. Additional details are in [1].

The first known meteorite listed in Hungarian transcript as Lenarto is a find of an iron meteorite found in October 1814 in the vicinity of the eastern-Slovak village of Lenartov, west of Bardejov. The meteorite is classified as a medium octahedrite, a transitional member of group IIIA-IIIB (2). Contains large amounts of occlusions of gases, mainly hydrogen, $90.4 \%$ of iron and $8.8 \%$ of nickel. The main mass $(73.6 \mathrm{~kg})$ is deposited in the museum of Budapest. Additional larger fragments are in Tubingen $(3.3 \mathrm{~kg})$, Vienna $(3.2 \mathrm{~kg})$ and Budapest $(2.9 \mathrm{~kg})$.

The second meteorite known as Magura is also a finding of an iron meteorite in around 1835 in the vicinity of the northern-Slovak village of Slanica near the town of Namestovo. A large number of boulders weighing from 1 to $46 \mathrm{~kg}$ were found. Approximately $1600 \mathrm{~kg}$ of the meteoric material was melted. There has never been found a crater. Magura is a heavily shocked, coarse octahedrite with $92.6 \%$ of iron and $6.7 \%$ of nickel. Iron from Magura is located in a number of museums around the world, with the largest pieces in Tubingen $(45.5 \mathrm{~kg})$, Vienna (30.2 kg), Budapest (18.5 kg), Berlin (10.1 kg) and London (9.0 kg) [2].

The first meteorite fall recorded in Slovakia is dated on July 24, 1837. The stone meteorite listed in Hungarian transcript as Gross-Divina fell at noon in the vicinity of village Divina near the town of Zilina. Classified as a chondrite. It is very rare in museum collections. The main mass $(9.9 \mathrm{~kg})$ is deposited in the Budapest museum and smaller fragments are in Prague $(0.26 \mathrm{~kg})$ and Wien $(0.06 \mathrm{~kg})$.

The second fall is a stone listed in Hungarian transcript as NagyBorove which fell in the vicinity of the village of Velke Borove near the town of Liptovsky Mikulas on May 9, 1985. Classified as a chondrite with well-defined glassy chondrules. It is very rare in museum collections. The largest piece $(5.9 \mathrm{~kg})$ is exposed in the museum in Budapest and additional smaller fragments are in Chicago $(0.18 \mathrm{~kg})$ and London $(0.05 \mathrm{~kg})$.

The last find is from August 1994, where a stone meteorite of the weight of $4.3 \mathrm{~kg}$ was found, during harvest in the vicinity of the village of Rumanova near the town of Sered. Classified as an H5 chondrite with $21.2 \%$ of iron, $18.2 \%$ of silicon and $13.2 \%$ of magnesium. Two largest fragments are deposited in the Slovak national museum in Bratislava $(2.5 \mathrm{~kg})$.

The last one is the fall of an $\mathrm{H} 5$ chondrite near the town of Kosice on February 28, 2010 [3].

Acknowledgment: This work has been supported by the APVV project No. 0516-10.

References: [1] Tucek K. 1981. Meteorites and their occurrences in Czechoslovakia (in Czech). Prague: Academia. [2] Buchwald V. F. 1975. Handbook of iron meteorites. Berkeley: University California Press. [3] Toth J. and Svoren J. 2010. Meteorit Košice (in Slovak). In Meteor news of the slovak astronomical society 31:65-69.

\section{7 \\ ${ }^{40} \mathrm{Ar}-{ }^{39} \mathrm{Ar}$ DATING OF LARKMAN NUNATAK 06299: COMPARISON TO PAIRED SAMPLE LAR 06298 AND TO OTHER LL CHONDRITES}

T. D. Swindle ${ }^{1,2}$, J. R. Weirich ${ }^{1}$, C. E. Isachsen ${ }^{2}$, A. Wittmann ${ }^{3}$ and D. A. Kring ${ }^{3} \cdot{ }^{1}$ Lunar and Planetary Laboratory, University of Arizona, Tucson, AZ, USA. E-mail: tswindle@email.arizona.edu. ${ }^{2}$ Department of Geosciences, University of Arizona, Tucson, AZ, USA. ${ }^{3}$ Lunar and Planetary Institute, Houston, TX, USA.

Larkman Nunatak (LAR) 06298 and 06299 are paired LL chondrite impact melt breccias. We previously analyzed four splits of LAR 06298 using ${ }^{40} \mathrm{Ar}-{ }^{39} \mathrm{Ar}$ dating [1], and determined that all showed signs of partial degassing at $\sim 1000 \mathrm{Ma}$ or later, in some cases $\sim 500 \mathrm{Ma}$ or later. We were unable to obtain a more precise age because no two of the four splits had anything resembling concordant plateaus. Since LAR 06299 is paired with LAR 06298, we analyzed three splits of LAR 06299 to shed more light on the situation. Two of the splits look much like two of the LAR 06298 splits, with minimum ages of $\sim 1000 \mathrm{Ma}$, but no good plateaus. The third split, however, yielded several extractions giving much younger ages, of $\sim 400 \mathrm{Ma}$ or less. Since minimum apparent ages are likely to be maximum ages of partial degassing, we interpret this as indicating a partial resetting event no more than $400 \mathrm{Ma}$ ago. This split has a significant amount of ${ }^{36} \mathrm{Ar}$, indicating the presence of trapped $\mathrm{Ar}$, and does not yield an isochron, so various assumptions about trapped $\mathrm{Ar}$ yield ages anywhere from $400 \mathrm{Ma}$ to near zero. Since none of the seven splits has yielded a good plateau at an age that could represent a resetting event, further Ar-Ar analysis of LAR 06298 or LAR 06299 is unlikely to be worthwhile, but even an upper limit of $\sim 400 \mathrm{Ma}$ is significant.

First, this is the youngest degassing event recorded in an LL chondrite. In fact, of $>20$ LL chondrites for which shock ages have been determined, only one other (Oberlin, $420 \pm 30 \mathrm{Ma}$ [2]) is < $500 \mathrm{Ma}$. The impact melt textures of these two chondrites are macroscopically similar to that of Cat Mountain [3], which implies this degassing occurred during a major impact event (perhaps in the presence of a significant gravitational field [4]). Alternatively, the impact melt textures could have been established during an earlier event, and a later event, not yet detected petrographically, could have caused the degassing.

Second, with the return of samples of LL chondrite composition from asteroid 25143 Itokawa by Hayabusa [5], the history of shocked LL chondrites can potentially be linked to specific events and objects in the asteroid belt. For example, the $\leq 400 \mathrm{Ma}$ degassing event may be related to the impact disruption of an LL-chondrite asteroid and reassembly of Itokawa, consistent with the calculated $75-1000 \mathrm{Ma}$ age of the surface of Itokawa [6]. Unfortunately, we were unable to determine a precise degassing age for LAR 06298/06299, and its cosmic ray exposure age (20$25 \mathrm{Ma}$ ) is less than the calculated surface age of Itokawa, so we would need to find an LL chondrite with a better-preserved thermal history to fully make the connection. However, the LAR 06298/06299 results suggest that such a task may be possible.

References: [1] Weirich J. R. et al. 2009. Meteoritics \& Planetary Science 44:A215. [2] Bogard D. D. et al. 1976. Journal of Geological Research 81:5664-5678. [3] Kring D. A. et al. 1996. Journal of Geological Research 101:29,329-29,371. [4] Rubin A. E. and Moore W. B. 2011. Meteoritics \& Planetary Science 46: 737-747. [5] Nakamura T. et al. 2011. Abstract \#1766. 42nd Lunar and Planetary Science Conference. [6] Michel P. et al. 2009. Icarus 200:503-513. 


\section{4}

\section{THERMAL CONDUCTIVITY OF METEORITES}

M. Szurgot. Center of Mathematics and Physics, Technical University of Łódź, Al. Politechniki 11, 90924 Łódź, Poland. E-mail: mszurgot@, p.lodz.pl.

Thermal conductivity of meteorites has been studied by a number of researchers [1-4]. The aim of the paper was to determine and analyze thermal conductivity of various meteorites at room temperature.

Thermal conductivity $K$ of meteorites was determined using the wellknown relationship

$K=D d \mathrm{Cp}$,

where $D$ is thermal diffusivity, $d$ is bulk density and $\mathrm{Cp}$ is specific heat capacity.

According to our data, thermal conductivity of chondrites is in the range of $(1.5-4.5) \mathrm{W} \mathrm{m}^{-1} \mathrm{~K}^{-1}$, stony-irons $(2.5-17) \mathrm{W} \mathrm{m}^{-1} \mathrm{~K}^{-1}$, and octahedrites (23-67) $\mathrm{W} \mathrm{m}^{-1} \mathrm{~K}^{-1}$. Exemplary values of $K$ are: NWA 4039 (eucrite, $1.1 \mathrm{~W} \mathrm{~m}^{-1} \mathrm{~K}^{-1}$ ), $\mathrm{HaH} 286$ (eucrite, $1.9 \mathrm{~W} \mathrm{~m}^{-1} \mathrm{~K}^{-1}$ ), Allende (CV3, $\left.1.4 \mathrm{~W} \mathrm{~m}^{-1} \mathrm{~K}^{-1}\right)$, DaG $610\left(\mathrm{H} 4,2.5 \mathrm{~W} \mathrm{~m}^{-1} \mathrm{~K}^{-1}\right)$, Gold Basin (L4, $\left.3.7 \mathrm{~W} \mathrm{~m}^{-1} \mathrm{~K}^{-1}\right)$, El Hammami $\left(\mathrm{H} 5,4.5 \mathrm{~W} \mathrm{~m}^{-1} \mathrm{~K}^{-1}\right)$, Vaca Muerta (mesosiderite-A1, $2.5 \mathrm{~W} \mathrm{~m}^{-1} \mathrm{~K}^{-1}$ ), Brahin (pallasite PMG, $\quad(5.4$ 16.5) $\mathrm{W} \mathrm{m}^{-1} \mathrm{~K}^{-1}$ ), Morasko (IAB-MG, $(25-67) \mathrm{W} \mathrm{m}^{-1} \mathrm{~K}^{-1}$ ), Odessa (IAB-MG, $45 \mathrm{~W} \mathrm{~m}^{-1} \mathrm{~K}^{-1}$ ), Campo del Cielo (IAB-MG, $52 \mathrm{~W} \mathrm{~m}^{-1} \mathrm{~K}^{-1}$ ), and Gibeon (IVA, (23-25) $\mathrm{W} \mathrm{m}^{-1} \mathrm{~K}^{-1}$ ).

The relationship between thermal conductivity and bulk density of meteorites has been established. Thermal conductivity can be expressed as a function of bulk density by various empirical equations. Power, linear, and quadratic fit are satisfactory.

According to the power function fit, we have

$K=K_{0} \cdot\left(d / d_{0}\right)^{\mathrm{n}}$,

where $K$ is thermal conductivity $\left(\mathrm{W} \mathrm{m}^{-1} \mathrm{~K}^{-1}\right), d$ is bulk density $\left(\mathrm{kg} \mathrm{m}^{-3}\right)$, and $d_{0}=1000 \mathrm{~kg} \mathrm{~m}^{-3}$. Coefficients $K_{0}$ and $\mathrm{n}$ are constant for a given temperature $\left(K_{0}=(0.044 \pm 0.050) \mathrm{W} \mathrm{m}^{-1} \mathrm{~K}^{-1}, \quad\right.$ and $n=3.4 \pm 0.6$, $\mathrm{R}^{2}=0.90$, at $\left.300 \mathrm{~K}\right)$.

Linear fit is given by the formula

$K=\mathrm{A} \cdot \mathrm{d}+\mathrm{B}$,

where $K$ is thermal conductivity $\left(\mathrm{W} \mathrm{m}^{-1} \mathrm{~K}^{-1}\right), d\left(\mathrm{~kg} \mathrm{~m}^{-3}\right)$ is bulk density of meteorites, and coefficients $A$ and $B$ are constant for a given temperature $\left(A=(8.81 \pm 0.61) \bullet 10^{-3} \mathrm{~W} \mathrm{~m}^{2} \mathrm{~kg}^{-1}, \quad B=-26.7 \pm 2.8 \mathrm{~W} \mathrm{~m}^{-1} \mathrm{~K}^{-1} \quad\right.$ at $\left.298 \mathrm{~K}, \mathrm{R}^{2}=0.89\right)$.

$K$ depend on $d$ since $D, C p$, and thermal capacity $C p \bullet d$ depend on density of meteorites [5,6]. Literature data [2,4] confirm validity of equations (2) and (3). Using values of grain density $d_{g}$ and $K$ measured by Opeil and coworkers [2] enable one to determine $A$ and $B$ values for various temperatures:

$$
\begin{aligned}
& A=2.99 \bullet 10^{-3} \mathrm{~W} \mathrm{~m}^{2} \mathrm{~kg}^{-1}, B=-5.84 \mathrm{~W} \mathrm{~m}^{-1} \mathrm{~K}^{-1} \text { at } 100 \mathrm{~K}, \\
& A=3.92 \bullet 10^{-3} \mathrm{~W} \mathrm{~m}^{2} \mathrm{~kg}^{-1}, B=-8.50 \mathrm{~W} \mathrm{~m}^{-1} \mathrm{~K}^{-1} \text { at } 200 \mathrm{~K}, \\
& A=4.84 \bullet 10^{-3} \mathrm{~W} \mathrm{~m}^{2} \mathrm{~kg}^{-1}, B=-10.9 \mathrm{~W} \mathrm{~m}^{-1} \mathrm{~K}^{-1} \text { at } 300 \mathrm{~K}, \mathrm{R}^{2}=
\end{aligned}
$$
0.97 .

Conclusions: Relationship between thermal conductivity and density of meteorites has been established that can be applied for evaluation of thermal conductivities of extraterrestrial matter at various temperatures.

References: [1] Szurgot M. et al. 2008. Crystal Research and Technology 43:921-930. [2] Opeil C. P. et al. 2010. Icarus 208:449-454. [3] Beach M. et al. 2009. Planetary and Space Science 57:764-770. [4] Yomogida K. and Matsui T. 1983. Journal of Geophysical Research 88:9513-9533. [5] Szurgot M. and Wojtatowicz T. W. 2011. Abstract \#5036. 74th Annual Meteoritical Society Meeting. [6] Szurgot M. 2011. Abstract \#1150. 42nd Lunar and Planetary Science Conference.

\section{6}

\section{THERMAL DIFFUSIVITY OF METEORITES}

M. Szurgot ${ }^{1}$ and T. W. Wojtatowicz ${ }^{2}$. ${ }^{1}$ Center of Mathematics and Physics, Technical University of Łódź, Al. Politechniki 11, 90924 Łódź. E-mail: mszurgot@p.lodz.pl. ${ }^{2}$ Institute of Physics, Technical University of Łódź, Wolczanska 219, 93005 Łódź, Poland.

Introduction: Determination of thermal properties of meteorites is important to describe the evolution of asteroids and planets. Thermal diffusivity of selected meteorites has been studied by a number of researchers [1-6]. The aim of the paper was to determine and analyze thermal diffusivity of various meteorites at room temperature.

Methods: Thermal diffusivity D of meteorites was measured by the laser flash method at ambient conditions, at $298 \mathrm{~K}$ in one atmosphere air. Bulk density of the samples was determined by the Archimedean method. Relative errors of measurements of diffusivity are about $1-6 \%$, and density about $1 \%$.

Results and Discussion: Thermal diffusivity of achondrites is in the range of $(0.4-2.4) \times 10^{-6} \mathrm{~m}^{2} \mathrm{~s}^{-1}$, chondrites $(0.5-2.0) \times 10^{-6} \mathrm{~m}^{2} \mathrm{~s}^{-1}$, stonyirons $(1.3-7.0) \times 10^{-6} \mathrm{~m}^{2} \mathrm{~s}^{-1}$, and octahedrites $(7-19) \times 10^{-6} \mathrm{~m}^{2} \mathrm{~s}^{-1}$. Exemplary values of D are: NWA 4039 (eucrite, $0.41 \times 10^{-6} \mathrm{~m}^{2} \mathrm{~s}^{-1}$ ), HaH 286 (eucrite, $0.93 \times 10^{-6} \mathrm{~m}^{2} \mathrm{~s}^{-1}$ ), Zaklodzie (enstatite achond., $\left.1.04 \times 10^{-6} \mathrm{~m}^{2} \mathrm{~s}^{-1}\right), \quad$ Allende (CV3, $\left.0.56 \times 10^{-6} \mathrm{~m}^{2} \mathrm{~s}^{-1}\right), \quad$ NWA 4967 $\left(\mathrm{CO} 3.2,1.00 \times 10^{-6} \mathrm{~m}^{2} \mathrm{~s}^{-1}\right)$, NWA $1609\left(\mathrm{H} 4-6,1.08 \times 10^{-6} \mathrm{~m}^{2} \mathrm{~s}^{-1}\right)$, El Hammami $\left(\mathrm{H} 5,1.57 \times 10^{-6} \mathrm{~m}^{2} \mathrm{~s}^{-1}\right), \mathrm{DaG} 610\left(\mathrm{H} 4,1.64 \times 10^{-6} \mathrm{~m}^{2} \mathrm{~s}^{-1}\right)$, Thuathe $\left(\mathrm{H} 4 / 5,2.01 \times 10^{-6} \mathrm{~m}^{2} \mathrm{~s}^{-1}\right)$, NWA869 (L4-6, $\left.1.03 \times 10^{-6} \mathrm{~m}^{2} \mathrm{~s}^{-1}\right)$, Gold Basin (L4, $1.32 \times 10^{-6} \mathrm{~m}^{2} \mathrm{~s}^{-1}$ ), Vaca Muerta (mesosiderite-A1, $1.34 \times 10^{-6} \mathrm{~m}^{2} \mathrm{~s}^{-1}$ ), Brahin (pallasite PMG, $(2.1-6.9) \times 10^{-6} \mathrm{~m}^{2} \mathrm{~s}^{-1}$ ), Campo del Cielo (IAB-MG, $15.8 \times 10^{-} 6 \mathrm{~m}^{2} \mathrm{~s}^{-1}$ ), Odessa (IAB-MG, $12.4 \times 10^{-6} \mathrm{~m}^{2} \mathrm{~s}^{-1}$ ), Morasko (IAB-MG, $(7-19) \times 10^{-6} \mathrm{~m}^{2} \mathrm{~s}^{-1}$ ), Henbury (IIIAB, $10.3 \times 10^{-6} \mathrm{~m}^{2} \mathrm{~s}^{-1}$ ), and Gibeon (IVA, $7.2 \times 10^{-6} \mathrm{~m}^{2} \mathrm{~s}^{-1}$ ).

Measurements of 20 meteorites revealed relationship between thermal diffusivity and bulk density of meteorites. Thermal diffusivity is a linear function of bulk density which is expressed by the empirical equation

$D=A \cdot d+B$,

where $\mathrm{D}$ is thermal diffusivity $\left(10^{-6} \mathrm{~m}^{2} \mathrm{~s} \times^{-1}\right), d\left(\mathrm{~kg} \mathrm{~m}^{-3}\right)$ is bulk density of meteorites, and coefficients $A$ and $B$ are constant for a given temperature $\left(A=2.49 \times 10^{-9} \mathrm{~m}^{5} \mathrm{~kg}^{-1} \mathrm{~s}^{-1}, \quad B=-7.11 \times 10^{-6} \mathrm{~m}^{2} \mathrm{~s}^{-1}\right.$ at 298 K). Not only our but also literature data confirm validity of eq. (1). Coefficients $A$ and $B$ depend on temperature and express effect of temperature on thermal diffusivity. Using values of $D$ and $\mathrm{d}$ measured for five meteorites (four chondrites and one octahedrite) by Osako [6] enabled us to determine $A$ and $B$ values for various temperatures:

$$
\begin{aligned}
& A=2.37 \times 10^{-9} \mathrm{~m}^{5} \mathrm{~kg}^{-1} \mathrm{~s}^{-1}, B=-6.19 \times 10^{-6} \mathrm{~m}^{2} \mathrm{~s}^{-1} \text { at } 100 \mathrm{~K}, \\
& A=2.11 \times 10^{-9} \mathrm{~m}^{5} \mathrm{~kg}^{-1} \mathrm{~s}^{-1}, B=-6.24 \times 10^{-6} \mathrm{~m}^{2} \mathrm{~s}^{-1} \text { at } 200 \mathrm{~K}, \\
& A=2.10 \times 10^{-9} \mathrm{~m}^{5} \mathrm{~kg}^{-1} \mathrm{~s}^{-1}, B=-6.52 \times 10^{-6} \mathrm{~m}^{2} \mathrm{~s}^{-1} \text { at } 300 \mathrm{~K} .
\end{aligned}
$$

Conclusions: Relationship between thermal diffusivity and bulk density of meteorites has been established that can be applied for evaluation of thermal diffusivities of extraterrestrial matter at various temperatures.

References: [1] Szurgot M. et al. 2008. Crystal Research and Technology 43:921-930. [2] Opeil C. P. et al. 2010. Icarus 208:449-454. [3] Beach M. et al. 2009. Planetary and Space Science 57:764-770. [4] Yomogida K. and Matsui T. 1983. Journal of Geophysical Research 88:9513-9533. [5] Matsui T. and Osako M. 1979. Memories of the National Institute of Polar Research 15:243-252 (Special Issue). [6] Osako M. 1981. Bulletin of the National Science Museum Series E, 4:1-8. 
5027

\section{DEGREE OF AQUEOUS ALTERATION IN SIX CM/CI CARBONACEOUS CHONDRITES}

D. Takir and H. Y. McSween Jr. Department of Earth \& Planetary Sciences and Planetary Geosciences Institute, University of Tennessee, Knoxville, TN 37996-1410. E-mail: dtakir@utk.edu.

Introduction: We have begun a project to quantify the degree of aqueous alteration in $\mathrm{CM} / \mathrm{CI}$ carbonaceous chondrites, obtain spectra of these chondrites, and measure spectra of possibly related outer Main Belt asteroids in order to explore the nature of aqueous alteration on these asteroids $[1,2]$. Here, we present petrograhic and geochemical analyses for six CM/CI carbonaceous chondrites (LAP 02277, MIL 07700, QUE 99770, Cold Bokkeveld, Bells, and Ivuna). We applied and compared the alteration scales of Browning et al. [3] and Rubin et al. [4], which include detailed petrographic observations and electron microprobe analyses.

Petrology and Geochemistry of $\mathbf{C M} / \mathbf{C I}$ Chondrites: [3] proposed a scale to track the degree of aqueous alteration of $\mathrm{CM}$ chondrites. This scale is based on the relative progress of coupled substitutions in the progressive alteration of cronstedtite to $\mathrm{Mg}$-serpentine, which increases as alteration proceeds, the so-called the mineralogic alteration index (MAI). [3] proposed two additional indicators of aqueous alteration, the abundance of isolated anhydrous mineral fragments (derived from disaggregation of chondrules) in the matrix and the relative percentage of chondrule alteration. Applying this scale to the chondrites analyzed thus far, we found a good correlation between the MAI and the volume percent of chondrule alteration. [4] proposed an alteration scale that ranges downward from moderately altered chondrites (petrologic subtype 2.6) to highly altered chondrites (petrologic subtype 2.0). [4] used petrologic properties that result from early and intermediate alteration processes, which include hydration of matrix and formation of phyllosilicates and production of large clumps of serpentine-tochilinite intergrowths (PCP). [4] also used petrologic properties that reflect progressive alteration processes and include oxidation of metallic $\mathrm{Fe}-\mathrm{Ni}$, alteration of mafic silicate phenocrysts in chondrules, changes in PCP composition (increase in the phyllosilicate/sulfide ratio), and changes in carbonate mineralogy. Applying this scale to our chondrites, we also found a good correlation between the petrologic type and the "FeO" $/ \mathrm{SiO}_{2}$ ratio.

Implications: The two scales correlate for the meteorites analyzed thus far except for Bells, which is thought to be extensively brecciated and altered [5]. Once we analyze additional meteorites, we will utilize the most quantifiable or significant features in both scales. High-quality reflectance spectra of the analyzed chondrites are being taken in order to correlate the $3-\mu \mathrm{m}$ band spectral properties (e.g., shape, depth, width, band center) with the petrological/geochemical parameters. When coupled with telescopic spectra, this investigation will allow a better understanding of the nature of aqueous alteration of outer Main Belt asteroids spanning the $2.5<\mathrm{a}<4.0 \mathrm{AU}$ region, and will lead to crucial constrains on how, when, and where this alteration occurred.

References: [1] Takir D. et al. 2011. Abstract \#1182. 42nd Lunar and Planetary Science Conference. [2] Takir D. and Emery J. P. 2010. Abstract \#53.09. 42nd American Astronomical Society, Division for Planetary Science. [3] Browning L. B. et al. 1996. Geochimica et Cosmochimica Acta 60:2621-2633. [4] Rubin et al. 2007. Geochimica et Cosmochimica Acta 71:2361-2382. [5] Brearley A. J. 1995. Geochimica et Cosmochimica Acta 59:2291-2317.
5339

ATMOSPHERIC OXYGEN EXCHANGE IN MICROMETEORITES S. Taylor ${ }^{1}$, Y. Guan ${ }^{2}$ and G. Matrajt ${ }^{3}$. ${ }^{1}$ CRREL, 72 Lyme Road, Hanover, NH 03755, USA. ${ }^{2}$ Geol. \& Planet. Sci, Caltech Pasadena, CA 91125, USA. ${ }^{3}$ Department of Astronomy, University of Washington, Seattle WA 98105, USA.

Introduction: Micrometeorites (MM), $30 \mu \mathrm{m}$ to $2 \mathrm{~mm}$ extraterrestrial particles, are heated to varying degrees entering the Earth's atmosphere. Heating, melting and volatilization can change their chemical and isotopic composition; mass-dependent isotopic fractionation is seen in severely heated MMs [1].

Single minerals of olivine and pyroxene partially melt or are affected by sub-solidus reactions [2] entering the Earth's atmosphere. We think this altered zone is demarcated by the presence of magnetite crystallites whose number and size decrease toward the center of the particle (Fig. 1a). The thickness of this zone is quite uniform for a given MM but varies among single mineral MMs probably due to the duration and temperature of heating and on the composition of the mineral.

Sample Description and Analysis: We measured ${ }^{18} \mathrm{O}$ and ${ }^{16} \mathrm{O}$ in the core and near the magnetite edge of four single mineral MMs of forsteritic composition to determine whether oxygen exchange during atmosheric entry altered their isotopic compositions. Analyses were made using the Cameca ims 7f-Geo at CalTech.

Discussion: Results show that all the forsterite MMs are isotopcally light (Fig. 1b) similar to relict grains in carbonaceous chondrites [3]. Isotope analyses made near the rim (adjacent to the magnetite zone) and in the center of the MM show no gradient in isotopic composition (Fig. 1b). The one analyses made within the magnetite rim, Ol-7-8, plots in the same region as terrestrial olivines and is isotopically heavier that its core.

These data support the idea that oxygen exchange with the Earth's atmosphere produces a visible magnetite zone in iron containing minerals. The lack of an isotopic gradient in the homogeneous looking interior of these MMs suggests that their centers have not been altered during atmospheric heating and thus can be compared to values from other solar system materials.

References: [1] Alexander et al. 2002. Geochimica et Cosmochimica Acta 66:173-183. [2] Toppani et al. 2001. Meteoritics \& Planetary Science 36:1377-1396. [3] Leshin et al. 1997. Geochimica et Cosmochimica Acta 61:835-845.
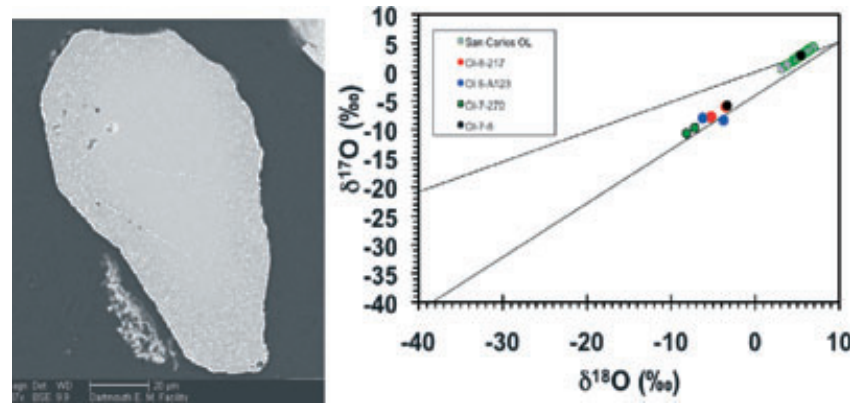

Fig. 1(a) Single mineral olivine MM Ol 5-A123; (b) a three-oxygen isotope plot showing analyses for the center and edge of four single mineral olivine MMs. 


\section{8}

EVOLUTION AND PROPERTIES OF DECIMETER BODIES IN THE SOLAR NEBULA

J. Teiser, I. Engelhardt, and G. Wurm. Faculty of Physics, University Duisburg-Essen. E-mail: jens.teiser@uni-due.de.

Introduction: The first step in planet formation is the coagulation of micron sized dust particles to macroscopic dust agglomerates at low collision velocities. With increasing particle sizes the collision velocities also increase. Centimeter sized dust agglomerates settle towards the midplane of protoplanetary disks and drift towards the central star. On their way, they can sweep up small $(100 \mu \mathrm{m})$ particles at collision velocities between $1 \mathrm{~m} / \mathrm{s}$ and $10 \mathrm{~m} / \mathrm{s}$, as small particles are well coupled to the gas $[1,2]$.

Experimental Aspects: Here, we present an experimental study on the evolution and the macroscopic mechanical properties of decimeter dust agglomerates in a self-consistent growth scenario. A beam of small dust agglomerates (approximately $100 \mu \mathrm{m}$ ) is generated using a sieve with a mesh width of $250 \mu \mathrm{m}$. The generated particles are accelerated by gravity and hit the targets at collision velocities between 1.5 and $7 \mathrm{~m} \mathrm{~s}^{-1}$ depending on the drop height. The dust agglomerates consist of quartz dust with irregularly shaped grains in the size range between 0.1 and $10 \mu \mathrm{m}$ and $80 \%$ of the mass between 1 and $5 \mu \mathrm{m}$, as considered relevant for protoplanetary disks. Different target sizes are used in the range between $3 \mathrm{~mm}$ and $3.5 \mathrm{~cm}$ in diameter.

Results: In the experiments targets grow by a combination of direct sticking and re-accretion of ejecta due to gravity. This is consistent with bodies in protoplanetary disks, as they move through the gas and impact ejecta are exposed to a head wind, which accelerates them back to the target surface. Under certain conditions, this headwind is of the same order as gravity in the laboratory experiments. However, in all experiments targets grow with a homogeneous structure, independent of the impact velocities and of the target sizes. The ratio between reaccretion and direct sticking becomes larger for increasing target sizes. As the target porosities and shapes do not depend on the target size, this leads to the conclusion, that the mechanisms leading to growth do not influence the properties of the resulting targets.

For the development of coagulation models the mechanical strength of dust aggregates is very important, which is mainly determined by the volume filling of the agglomerates. The volume filling of the forming targets increases with increasing impact velocity, as the dynamic pressure scales with $v^{2}$. However, for impact velocities larger than $5 \mathrm{~m} / \mathrm{s}$ the volume filling reaches a saturation at a volume filling of $32 \%$ and does not increase further. This value is also consistent with the maximum volume filling feasible by local compression as already described by [3].

Conclusions: The experiments lead to the conclusion that the properties of growing dust aggregates do not change whether they grow by direct sticking or by re-accretion, but only on the impact velocities they are exposed to. However, once a volume filling of $32 \%$ is reached, further collisions do not lead to further compaction. For decimeter bodies only the maximum volume filling feasible by local compression has to be considered. The growth history is erased by numerous impacts and the resulting bodies have a homogeneous structure.

References: [1] Weidenschilling S. J. and Cuzzi J. N. 1993. Protostars and Planets III 1031ff. [2] Weidenschilling S. J. 1997. Icarus 127:290. [3] Wurm G. et al. 2005. Icarus 178:253.
5489

${ }^{60} \mathrm{Fe}-{ }^{60} \mathrm{Ni}$ ANALYSIS OF PORPHYRITIC CHONDRULES FROM PRIMITIVE ORDINARY CHONDRITES

M. Telus, G. R. Huss, K. Nagashima and R. C. Ogliore. University of Hawai'i at Mānoa, Honolulu, HI 96822. E-mail: telus@higp.hawaii.edu.

Introduction: The ${ }^{60} \mathrm{Fe}-{ }^{60} \mathrm{Ni}$ system $\left(\mathrm{t}_{1 / 2}=2.6 \mathrm{Myr}\right)$ provides the resolution necessary to date a variety of early solar system processes. However, the initial solar system abundance of ${ }^{60} \mathrm{Fe}$ is poorly constrained. Ion probe measurements provide some of the best estimates of the initial ${ }^{60} \mathrm{Fe}$ abundance in the solar system. But the existing data have several problems: (1) data reduced using the mean of measured ratios is subject to a systematic bias that overestimates the ${ }^{60} \mathrm{Fe}$ abundance [1,2]; (2) most detections of ${ }^{60} \mathrm{Fe}$ come from radiating pyroxene chondrules, some of which may not have remained closed systems since formation [3]; (3) Fe/ $\mathrm{Ni}$ ratios in large phenocrysts of porphyritic chondrules, which likely have remained isotopically closed, are lower than in radiating chondrules, making measurements difficult. Here we report data gathered with an improved multi-collection protocol on porphyritic chondrules from type 3.0-3.1 ordinary chondrites.

Methods: We have added some new chondrites to our study. QUE 97008 is classified as L3.05 [4]. Raman spectroscopic measurements made using the protocol of [5] give results consistent with this classification. Chondrules were selected for high $\mathrm{Fe}$ content and low Ni content using SEM-EDS and EMPA.

$\mathrm{Fe}$ and $\mathrm{Ni}$ isotopes were measured in multicollection mode at the University of Hawai'i using the Cameca ims 1280 ion microprobe. Nickel$60,{ }^{61} \mathrm{Ni}$, and ${ }^{62} \mathrm{Ni}$, were measured on electron multipliers (EMs) and ${ }^{56} \mathrm{Fe}$ was measured on a Faraday cup. We used a $6-7 \mathrm{nA}{ }^{16} \mathrm{O}^{-}$primary beam. Each spot was presputtered using a $25 \mu \mathrm{m}$ raster, which was reduced to $15 \mu \mathrm{m}$ for the measurements. A mass resolving power of $\sim 5500$ was achieved using multicollector exit slits of $250 \mu \mathrm{m}$. Major molecular interferences at the masses of the $\mathrm{Ni}$ isotopes were checked at the end of each measurement. EM gains were monitored by repeated measurements of standards and corrections for detector drift were applied to the data. Isotope ratios were calculated from time-interpolated total counts, following [1], and data were corrected for detector background, deadtime, and tails of interference peaks. Internal mass-fractionation corrections were performed for each point.

Results: We measured five porphyritic chondrules, two from QUE 97008 and three from Bishunpur (LL3.1). Four are porphyritic olivine or olivine-pyroxene chondrules and one is a barred olivine chondrule. The $\mathrm{Fe} / \mathrm{Ni}$ ratios in these chondrules are factors of 5-10 lower than in radiating pyroxene chondrules, and we were not able to resolve clear evidence for ${ }^{60} \mathrm{Fe}$ in any of them. However, the upper limits for the initial ${ }^{60} \mathrm{Fe} /{ }^{56} \mathrm{Fe}$ ratios for these chondrules $\left(1-2 \times 10^{-7}\right)$ are broadly consistent with previously reported detections [e.g., 3, 6]. We will continue our efforts to measure radiating pyroxene and porphyritic chondrules to refine estimates of the initial ${ }^{60} \mathrm{Fe} /{ }^{56} \mathrm{Fe}$ ratio in the early solar system.

References: [1] Ogliore R. C. et al. 2011. Nuclear Instruments and Methods in Physics Research Section B, doi: 10.1016/j.nimb.2011.04.120. [2] Huss G. R. et al. 2011. Abstract \#2608. 42nd Lunar and Planetary Science Conference. [3] Telus M. T. et al. 2011. Abstract \#2559. 42nd Lunar and Planetary Science Conference. [4] Grossman J. N. and Brearley A. J. 2005. Meteoritics \& Planetary Science 40:87-122. [5] Bonal L. et al. 2006. Geochimica et Cosmochimica Acta 70:1849-1863. [6] Tachibana et al. 2006. The Astrophysical Journal 639:L87-L90. 


\section{6}

OXYGEN ISOTOPE RATIOS IN CHONDRULES FROM PRIMITIVE CR CHONDRITE MET 00426: A CORRELATION WITH CHONDRULE Mg\#

T. J. Tenner ${ }^{1}$, D. Nakashima ${ }^{1}$, T. Ushikubo ${ }^{1}$, N. T. Kita ${ }^{1}$, and M. K. Weisberg ${ }^{2,3}$. ${ }^{1}$ University of Wisconsin-Madison, USA. E-mail: tenner@wisc.edu. ${ }^{2}$ Kingsborough Community College, CUNY, USA. ${ }^{3}$ American Museum Natural History, NY, USA.

Introduction: Meteorite Hills (MET) 00426 is classified as a CR 3.00 chondrite [1]. Its chondrules are prevalently large $(300 \mu \mathrm{m}$ to $2 \mathrm{~mm}$ dia.), with olivine and pyroxene $\mathrm{Mg \#}>95$ (where $\mathrm{Mg \#}=$ mole $\%[\mathrm{MgO}]$ $[\mathrm{MgO}+\mathrm{FeO}])$. A tiny volume percentage consists of smaller type II chondrules. Previous in situ O-isotope analyses of chondrules in CR chondrites [2-4] exhibit O-isotope ratios similar to those in carbonaceous [3-6] and ordinary [7] chondrites; i.e., $\Delta^{17} \mathrm{O}: ? 8.0$ to $+1 \%$ (where $\left.\Delta^{17} \mathrm{O}=\delta^{17} \mathrm{O}-0.52 \times \delta^{18} \mathrm{O}\right)$. Recent studies of chondrules in Y81020 (CO3) [5] and Acfer 094 (ungrouped-C) [6] show correlations between O-isotope ratios and $\mathrm{Mg \#}$; i.e., $\mathrm{Mg \#} \mathrm{>} 95$ and $\mathrm{Mg \#}<95$ chondrules cluster at $\Delta^{17} \mathrm{O}=-5 \%$ and $-2 \%$, respectively. However, the relationship between $\mathrm{Mg} \#$ and $\mathrm{O}$-isotope ratios in chondrules has yet to be investigated for CR chondrites. Here we present O-isotope ratios of 29 chondrules in MET 00426, measured with the WiscSIMS IMS-1280, using a $10 \times 15 \mu \mathrm{m} \mathrm{Cs}+$ primary beam [7], and discuss their relationship with corresponding $\mathrm{Mg \#}$.

Results: All individual spot data from chondrules in MET 00426 plot along a slope-1 line in the oxygen three-isotope diagram. $\Delta^{17} \mathrm{O}$ values of multiple spots (olivine and/or pyroxene) within a given chondrule are indistinguishable $( \pm 0.5 \%$ 2 SD). Type II (Mg\# 53-62) chondrule fragments $(n=3)$ have $\Delta^{17} \mathrm{O}$ values of $0.4 \pm 0.2 \%$. Type I chondrules $(n=26)$ have $\Delta^{17} \mathrm{O}$ values that increase from $-6 \%$ to $-1 \%$ as $\mathrm{Mg \#}$ decreases from 99 to 94 . Only one of 29 chondrules has a relict grain (relict olivine $\Delta^{17} \mathrm{O}:-3.2 \%$; host olivine/pyroxene $\Delta^{17} \mathrm{O}:-1.6 \%$; Mg\# $\#_{\text {relict. host }}$ : 97.7).

Discussion: Our observations indicate that chondrules in MET 00426 formed in a variable environment where an increase in oxidation state (which lowers chondrule $\mathrm{Mg \#} \mathrm{[8])} \mathrm{is} \mathrm{correlated} \mathrm{with} \mathrm{increasing} \Delta^{17} \mathrm{O}$ values. Two factors, (1) addition of $\mathrm{H}_{2} \mathrm{O}$, and (2) an increase in dust enrichment (relative to solar system) potentially contributed to more oxidizing conditions. A $5 \%$ increase in $\Delta^{17} \mathrm{O}$ over a narrow range in chondrule Mg\# (99-95) could be from oxidation of precursor material by addition of $\mathrm{H}_{2} \mathrm{O}$ with positive $\Delta^{17} \mathrm{O}$, due to cooling of the local disk, under relatively low dust enrichment $(<200 \times$ solar system [8]). The inferred positive $\Delta^{17} \mathrm{O}$ of the $\mathrm{H}_{2} \mathrm{O}$ added to precursor material may have been similar to that of $\mathrm{H}_{2} \mathrm{O}$ that accreted into the chondrite [9]. Increased dust enrichment $(>1000 \times$ solar system [8]) would be necessary to form type II chondrules (Mg\# approximately 55), along with a slightly higher $\mathrm{H}_{2} \mathrm{O}$ contribution to precursor material (relative to type I chondrules) to yield their approximately $0.4 \% \Delta^{17} \mathrm{O}$ values. Such variability in the chondrule-forming environment could have occurred spatially and/or through time.

References: [1] Abreu N. M. and Brearley A. J. 2010. Geochimica et Cosmochimica Acta 74:1146-1171. [2] Connolly Jr. H. C. and Huss G. R. 2010. Geochimica et Cosmochimica Acta 74:2473-2483. [3] Chaussidon M. et al. 2008. Geochimica et Cosmochimica Acta 72:1924-1938. [4] Libourel G. and Chaussidon M. 2011. Earth and Planetary Science Letters 301:921. [5] Tenner T. J. et al. 2011. Abstract \#1426. 42nd Lunar Prospector Spacecraft. [6] Ushikubo T. et al. 2011. Abstract \#1183. 42nd Lunar Prospector Spacecraft. [7] Kita N. T. et al. 2010. Geochimica et Cosmochimica Acta 74:6610-6635. [8] Ebel D. S. and Grossman L. 2000. Geochimica et Cosmochimica Acta 64:339-366. [9] Clayton R. N. and Mayeda T. K. 1999. Geochimica et Cosmochimica Acta 63:2089-2104.
5286 SIDEROPHILE ELEMENTS IN THE ELGA (IIE) METAL

S. N. Teplyakova ${ }^{1}$, M. Humayun ${ }^{2}$. ${ }^{1}$ Vernadsky Institute of Geochemistry and Analytical Chemistry, Russian Academy of Sciences, Moscow, Kosygina st.19, 119991 (E-mail: elga.meteorite@gmail.com) ${ }^{2}$ National High Magnetic Field Laboratory and Department of Earth, Ocean \& Atmospheric Science, Florida State University, 1800 E. Paul Dirac Drive, Tallahassee, FL 32310, USA.

Introduction: The IIE irons belong to a non-magmatic group of iron meteorites because magmatic Ga-Ni, Ir-Ni trends are poorly defined [1]. It was suggested that IIE metal like IAB metal formed as a pool of impact-generated melt in a chondritic body [1]. Based on oxygen isotopes, silicate inclusions in IIE irons resemble $\mathrm{H}$ chondrites [2]. The IIE iron Elga meteorite is the finest octahedrite containing silicate inclusions (SIs) [3]. Based on concentrations of $\mathrm{Ni}, \mathrm{Ga}, \mathrm{Ge}$ in the metal the Elga meteorite was classified as IIE iron $[4,5]$. Here, we report results on mineralogy and LA-ICP-MS siderophile element analyses of the metal in the Elga IIE and discuss the possible genetic relations between this meteorite and chondritic precursor material.

Results and Discussion: Elga consists of Fe-Ni metal with silicate (18 vol\%) and troilite (less than $1 \mathrm{vol} \%$ ) inclusions. The metal consists of taenite (taenite, plessite, martensite) $-9.5 \mathrm{vol} \%$, kamacite $-85 \mathrm{vol} \%$, and schreibersite $-4.5 \mathrm{vol} \%$.

A long laser track $(1.2 \mathrm{~cm})$ was analyzed in metal in octahedritic structure and aureole for 36 elements including 22 siderophiles. Bulk major and trace element abundances are (ppm) and RSD [\%]: $\mathrm{Fe} 911825.1 \pm 2, \mathrm{Ni} 75682.0 \pm 17, \quad \mathrm{Co} 4623 \pm 7, \quad \mathrm{P} 4880 \pm 168$, $\mathrm{S} 2136 \pm 97, \mathrm{Si} 520 \pm 231, \mathrm{Cr} 17 \pm 120, \mathrm{Cu} 145 \pm 17, \mathrm{Ga} 11 \pm 11$, Ge $75.4 \pm 11$, As $11.4 \pm 15$, Mo $12.1 \pm 11$, Ru $8.3 \pm 0.1$, Rh $1.4 \pm 11$, Pd $3.7 \pm 14, \mathrm{~W} 1.1 \pm 13$, Re $0.4 \pm 16$, Os $5.03 \pm 12$, Ir $4.8 \pm 11$, Pt $11.2 \pm 11$, Au $1.2 \pm 18$.

The elemental abundances of $\mathrm{Co}, \mathrm{Cu}, \mathrm{Ge}, \mathrm{Ga}$ and $\mathrm{Ir}$, are similar to those previously reported for Elga [1, 4], while the abundances of $\mathrm{W}$ are lower and As, Au are higher. The metal exhibits a flat refractory siderophile pattern relative to $\mathrm{CI}$ and $\mathrm{Ni}$. The metal is enriched $(1-1.7 \mathrm{xCI}$ ) in siderophile elements (W, Re, Os, Ir, Ru, Pt, Mo), and depleted in $\mathrm{Sb}, \mathrm{Cu}, \mathrm{Ga}, \mathrm{Ge}$, and $\mathrm{Sn}$ relative to $\mathrm{CI}$ and $\mathrm{Ni}$. Our model calculations of partial melting of $\mathrm{H}$ chondrite metal showed depletion in refractory siderophiles of partial melts and enrichment in solids that are in equilibrium with these liquids. Elga metal exhibits a pattern similar to solid metal that is in equilibrium with a $40 \%$ partial melt of bulk $\mathrm{H}$ chondrite metal ( $\sim 6 \%$ partial melt of $\mathrm{H}$ chondrite), for most elements except $\mathrm{Sb}$. Therefore siderophile pattern in Elga metal is consistent with fine-grained unfractionated metal particles of $\mathrm{H}$-chondrite or solid metal crystallized from H-chondrite precursor.

References: [1] Wasson J. T. and Wang J. 1986. Geochimica et Cosmochimica Acta 50:725-732. [2] Clayton R. N. and Mayeda T. K. 1996. Geochimica et Cosmochimica Acta 60:1999-2017. [3] Plyashkevich L. N. 1962. Meteoritika 22:51-60. [4] Wasson J. T. 1970. Geochimica et Cosmochimica Acta 34:957-964. [5] Dyakonova M. I. 1979. Nauka 48. [6] Humayun M. et al. 2007. Geochimica et Cosmochimica Acta 71:46094627. [7] Kong P. et al. 1998. Meteoritics \& Planetary Science 33:993-998. 
5332

DATING CORE CRYSTALLIZATION OF IIAB AND IIIAB IRON METEORITES USING THE PALLADIUM-SILVER DECAY SYSTEM K. J. Theis ${ }^{1}$, M. Schönbächler ${ }^{1}$, G. K. Benedix ${ }^{2,3}$, M. Rehkämper ${ }^{3}$ and R. Andreasen ${ }^{3}$. ${ }^{1}$ SEAES, University of Manchester, M13 9PL, UK, E-mail: karen.theis@postgrad.manchester.ac.uk. ${ }^{2}$ IARC/Department of Mineralogy, The Natural History Museum, Cromwell Road, London, UK SW7 5BD. ${ }^{3}$ IARC and Dept. of Earth Science and Engineering, Imperial College London, SW7 2AZ, UK.

Introduction: The IIAB and IIIAB iron meteorites exhibit distinct fractional crystallization trends, which indicate that they formed by the crystallization of a metallic liquid during the segregation of an iron core in an asteroidal parent body. Core segregation for the IIAB parent body is estimated to have occurred around 1.2 Ma after the formation of Allende CAIs based on the Hf-W decay system [1] and Pt-Re-Os dating of core crystallization yielded an age of $4546 \pm 46 \mathrm{Ma}$ [2]. The ${ }^{107} \mathrm{Pd}-{ }^{107} \mathrm{Ag}$ decay system can also be used to date these iron meteorites and it has the advantage that it yields a higher degree of precision due to the relatively short half-life of ${ }^{107} \mathrm{Pd}(6.5 \mathrm{Ma})$. Previous data show [3, 4] that IIAB iron meteorites may define two isochrons with ages of (1) 8.9 Ma after solar system formation for IIABs with high Ir contents and (2) $10.9 \mathrm{Ma}$ for IIABs, which are inferred to have formed later in the crystallization sequence based on their low content of the compatible element Ir. Likewise, the IIIAB iron meteorites also display fractional crystallization trends, but with relatively high scatter in the Ir versus $\mathrm{Au}$ diagram. The scatter is thought to be the result of parental melt that was trapped within the crystallizing metal [5]. As these conclusions are based on only a few data, this study aims to further constrain the $\mathrm{Pd}-\mathrm{Ag}$ isochrons for both IIAB and IIIAB iron meteorites.

Analytical Techniques: Ion exchange chemistry [6] is used to separate $\mathrm{Pd}$ and Ag from four IIAB (Sikhote-Alin (x2), North Chile and Coahuila) and three IIIAB (Thunda, Boxhole and Henbury) iron meteorites. Silver isotopes are analyzed on the $\mathrm{Nu}$ Plasma MC-ICPMS at Manchester relative to the NIST SRM978a Ag standard. Palladium and $\mathrm{Ag}$ concentration are acquired by isotope dilution.

Results and Discussion: The results for North Chile are in good agreement with those of previous studies [3,4] and fall on the same linear correlation in the $\mathrm{Pd}-\mathrm{Ag}$ isochron diagram, corresponding to an age of 10.9 Ma after CAI formation [3]. Coahuila falls between the two IIAB isochrons [3] indicating that the sample analyzed here may be disturbed or formed slightly later (by $\sim 1 \mathrm{Ma}$ ). Sikhote-Alin, which crystallizes late in the sequence according to elemental data [7], plots well below both isochrons. This suggests very late crystallization or later resetting. Our analyzed sample displays an unusually high $\mathrm{Ag}$ content (4.77 ppb) and a duplicate will be analyzed to confirm the data. The IIIABs will also be analyzed and the results presented at the conference.

References: [1] Markowski A. et al. 2006. Earth \& Planetary Science Letters 250:104-115. [2] Cook D. L. et al. 2004. Geochimica et Cosmochimica Acta 68:1413-1431. [3] Schönbächler M. et al. 2010 Meteoritics \& Planetary Science 45:A183. [4] Chen J. H. et al. 2002. Geochimica et Cosmochimica Acta 66:3793-3810. [5] Wasson J. 1999. Geochimica et Cosmochimica Acta 63:2875-2889. [6] Schönbächler M. et al. 2007. International Journal of Mass Spectrometry 261:183-191. [7] Wasson J. T. et al. 2007. Geochimica et Cosmochimica Acta 71:760-781.
5095

SCALE-DEPENDENT METHODS FOR NITROGEN ELEMENTAL QUANTIFICATION IN INSOLUBLE ORGANIC MATTER BY IMAGING WITH THE NANOSIMS

A. Thomen and F. Robert. Laboratoire de Minéralogie et de Cosmochimie du Muséum. E-mail : athomen@mnhn.fr.

Introduction: Correlated microscopic variations between elemental and isotopic compositions of $\mathrm{N}$ in insoluble organic matter (IOM) of carbonaceous chondrites were tentatively search in recent studies [1,2]. At a micrometer scale, i.e., the typical size of a hotspot in the IOM, this topic is addressed by quantitative imaging with a NanoSIMS. We proposed here two methods of $\mathrm{N}$ elemental quantification with "improper" samples, (i.e., rough sample surface) the surfaces of which often mimics those of natural samples (IPDs or IOM).

Materials and Method: The calibration of the $\mathrm{N}$ concentrations was done with: a charcoal, a type 3 kerogen, a type 1 kerogen and Murchison IOM, with increasing $\mathrm{N} / \mathrm{C}$ from $2.2 \times 10^{-3}$ to $3.27 \times 10^{-2}$, respectively. Several $10 \times 10 \mu \mathrm{m}^{2}$ images of $\mathrm{CN}^{-}, \mathrm{C}^{-}$and $\mathrm{C}_{2}^{-}$of $128^{2}$ pixels of the standards were acquired by rastering the $\mathrm{Cs}^{+}$primary beam with $\sim 2 \mathrm{pA}$ and $200 \mathrm{~nm}$ diameter. Ion images are sequentially reconstructed. Steadystate equilibirum is achieved by implantation of $\mathrm{a} \mathrm{Cs}^{+}$dose of $2 \times 10^{17} \mathrm{~cm}^{-2}$ prior to the analysis. Image files are processed with L'IMAGE software (L.Nittler copyright). The $\mathrm{CN}^{-} / \mathrm{C}^{-}$and $\mathrm{CN}^{-} / \mathrm{C}_{2}^{-}$ ratios are extracted at two different spatial scales with the whole image defined by (i) one ROI and by (ii) $\sim 300$ ROIs (that is $6^{2}$ pixels for each ROI). Each scale has its own drawbacks: (i) at the image scale, the artifacts of the chemical ion ratios linked to the topographic state of the samples, have a tendency to vanish, (ii) at a $6^{2}$ pixels scale $\left(0.2 \mu \mathrm{m}^{2}\right)$, our calibration accounts for topographic effects and the possible chemical heterogeneities of the standard. On ideal standards, i.e., polished and chemically homogeneous standards, the two approaches should yield identical results.

Results: Whatever the scale, the precision and detection limit for the $\mathrm{N}$ content in IOM is better determined by the $\mathrm{CN}^{-} / \mathrm{C}_{2}^{-}$than by the $\mathrm{CN}^{-} /$ $\mathrm{C}^{-}$ratios. At the scale (i) inversion of the $95 \%$ confidence interval for the mean $\mathrm{CN}^{-} / \mathrm{C}^{-}$ratios corresponds to a relative uncertainty of $\pm 60 \%$ of the atomic $\mathrm{N} / \mathrm{C}$ ratio. In the same way for $\mathrm{CN}^{-} / \mathrm{C}_{2}^{-}$, the relative uncertainty is $\pm 22 \%$. At the scale (ii) we observe for the $\mathrm{CN}^{-} / \mathrm{C}^{-}$and $\mathrm{CN}^{-} / \mathrm{C}_{2}^{-}$distributions of each standards, pronounced tails with clear departures from Gaussian distribution. These non-Gaussian distribution strongly affect the correlation coefficient of least-square regression and thus the precisions of the calibrations. When transformed in log-normal distributions, the variance of the residues is nearly constant, illustrating the improvement of the regression quality.

At the scale (ii) prediction interval [3] is used to quantify the uncertainties on the $\mathrm{N}$ content measured at a micrometer scale: inversion of $95 \%$ level gives a relative uncertainty of $\pm 33 \%$ for the calibration based on the $\mathrm{CN}^{-} / \mathrm{C}_{2}^{-}$but $\pm 100 \%$ for $\mathrm{CN}^{-} / \mathrm{C}^{-}$(that is covering the whole range of natural variations in kerogens or IOM!). We will show several applications of these methods for IOM of chondrites at the conference.

References: [1] Busemann H. et al. 2006. Science 312:728-730. [2] De Gregorio B. T. et al. 2011. Geochimica et Cosmochimica Acta 15:4454-4470. [3] Draper N. and Smith H. 2008. Applied Regression Analysis. 
5463

BASAL BRECCIA AT MANICOUAGAN: FORMATION MECHANISMS, THE SUEVITE CONNECTION AND LUNARMARTIAN COMPARISONS

L. M. Thompson. Planetary and Space Science Centre, University of New Brunswick, 2 Bailey Drive, Fredericton, New Brunswick E3B 5A3 Canada. E-mail: 1thompso@unb.ca.

Introduction: The term suevite has been used to describe impactderived, polymict breccias containing a mixture of shocked and unshocked, lithic and melt fragments and generally considered to possess clastic matrices [1]. Recent re-interpretation of the type suevites of the Ries crater as clast-rich impact melts [2], has sparked a renewed interest in the nature of these impactite lithologies. This work concerns suevites associated with the $\sim 80 \mathrm{~km}$ rim diameter, $214 \mathrm{Ma}$ Manicouagan impact structure of Quebec, Canada.

Suevite at Manicouagan: Suevites are well exposed at the outer edge of the central island and occur as weakly consolidated, friable layers and lenses immediately underlying, and in contrast to, the more coherent, columnar jointed, typically vesiculated, basal, clast-laden impact melt (IM) sheet. They exhibit fine-grained, clastic matrices enclosing angular to rounded lithic and mineral fragments, and blebs, schlieren, stringers and larger masses of melt. Lithic fragments are polymict, range from $<\mathrm{mm}$ to tens of $\mathrm{m}$ in size and exhibit evidence of various degrees of deformation and shock prior to incorporation into the suevite. This indicates derivation from disparate sources and extensive mixing. Melt inclusions range in size from fine, $\mathrm{cm}$-scale stringers and blebs to larger, $\mathrm{m}$-size masses, which are typically clast laden and may be vesiculated, resembling the basal, clast laden IM that is exposed in the vicinity, indicating derivation from this source. Contacts of the larger melt masses and meltrimmed clasts are typically irregular, the melt appearing to be disaggregated and strung out, grading into finer melt schlieren and stringers. Flow textures within IM immediately adjacent to suevite and fluidal, elongate melt blebs within the suevite indicate flow parallel to contacts and interaction of melt that was still relatively hot and plastic. Striated surfaces of some clasts attest to the dynamic and erosive nature of the suevite. In places, a gradation is observed from clastic- to meltdominated suevite immediately adjacent to basal IM. As well, facies within the basal IM, which exhibit a high proportion of fine, clastic material, indicate a continuum between allochthonous, clastic breccias, through suevite to basal, clast laden IM.

Conclusion: Suevite exposed at the base of the IM sheet at Manicouagan is not a distinct impactite facies. It represents the dynamic interface between the basal IM and underlying clastic breccias and can be modeled by the Kelvin-Helmholtz phenomenon. The melt and clastic breccia represent two fluids moving at different velocities relative to one another, whereby perturbations are amplified and mixing occurs resulting in suevite. This work provides new insights into the formation mechanism of this little-studied variant of suevite and has implications for the formation of suevites in other settings, as well as possible links to lunar and Martian breccias, e.g., Martian megabreccia.

References: [1] von Engelhardt W. and Graup G. 1984. Geologische Rundschau 73:2:447-481. [2] Osinski G. R., Grieve R. A. F., and Spray J. G. 2004. Meteoritics \& Planetary Science 39:10:1655-1684.
5279

EBSD STUDY OF LATTICE PREFERRED ORIENTATION (LPO) OF THE HARZBURGITE NWA 5480

B. J. Tkalcec and F. E. Brenker. Goethe University, Geoscience Institute, 60438 Frankfurt, Germany. E-mail: tkalcec@em.uni-frankfurt.de.

Introduction: Classified as belonging to the Howardite-EucriteDiogenite (HED) group of achondrite, Northwest Africa 5480, like other olivine-rich diogenites, is thought to represent an ultramafic cumulate formed within a magma chamber in the upper mantle of the differentiated asteroid 4 Vesta, or a Vesta-like body [1]. Dominated by olivine $(57 \mathrm{vol} \%$ ) and orthopyroxene (42 vol\%) NWA 5480 has further been classified a harzburgite [2], whereby the distribution of olivine and orthopyroxene is very heterogeneous, with some areas displaying up to $90 \%$ of either of the two minerals. In this study, structural analysis was performed on the olivine grains of NWA 5480 using electron backscatter diffraction (EBSD), which allows us to measure and visualize the crystallographic orientation of the crystal axes to discover any lattice preferred orientation (LPO) [3].

NWA 5480 Analysis: The sample was categorized into two regions for targeted analysis: (a) Zone A, dominated by coarse-grained olivine, and (b) Zone B, dominated by orthopyroxene-olivine schlieren. A total of 1361 EBSD crystallographic orientation measurements of coarse-grained olivine were recorded from 58 sites (each site covering $1 \mathrm{~mm}^{2}$ ) within Zone A and a total of 148 measurements of finer-grained olivine were recorded from 20 sites within Zone B. Only EBSD measurements with a mean angular deviation (MAD) of $<1$ were accepted and recorded. The EBSD results of Zones $\mathrm{A}$ and $\mathrm{B}$ display pronounced yet distinctly differing LPOs, suggesting two separate deformation processes and/or events. A comparison with olivine LPO in terrestrial cumulates and deformed mantle peridotites illustrates the unlikeliness that the olivine LPO from Zone A of NWA 5480 was formed through cumulation or compaction processes. In contrast, a distinct similarity to the olivine LPO formed by pencil glide ((0kl) [100] glide system), typical of plastic deformation in the terrestrial mantle, causes us to consider alternative formation processes for NWA 5480, including the feasibility of convection within the Vestan mantle.

References: [1] McSween H. Y. Jr. et al. 2010. Space Science Reviews doi:10.1007/s11214-010-9637-z. [2] Beck A. W. and McSween H. Y. Jr. 2010. Meteoritics \& Planetary Science 45:850-872. [3] Prior D. J. et al. 1999. American Mineralogist 84:1741-1759. 


\section{5}

\section{A HYDRATED CLAST IN THE MOKOIA CV3 CARBONACEOUS CHONDRITE: EVIDENCE FOR INTENSIVE AQUEOUS ALTERATION IN THE CV PARENT BODY}

K. Tomeoka ${ }^{1}$ and I. Ohnishi ${ }^{1,2}$. ${ }^{1}$ Department of Earth and Planetary Sciences, Faculty of Science, Kobe University, Nada, Kobe 657-8501, Japan. E-mail: tomeoka@kobe-u.ac.jp. ${ }^{2} J E O L$ Ltd., 3-1-2 Musashino, Akishima, Tokyo 196-8558, Japan.

We report the discovery of a highly hydrated clast in the Mokoia CV3 carbonaceous chondrite that contains eight chondrules and one amoeboid olivine inclusion (AOI), embedded in a matrix dominated by hydrous phyllosilicates. Anhydrous silicates in the chondrules and AOI were extensively replaced by phyllosilicates (mostly saponite with minor phlogopite). The mineralogy and texture indicate that the clast resulted from intensive aqueous alteration of a precursor equivalent to the host Mokoia meteorite, and it can be regarded as the CV2-type lithology.

This clast reveals abundant evidence of progressive and dynamic aqueous alteration processes. The chondrules were replaced inward from their margins, and the alteration products were disaggregated and mixed into the matrix; as a result, the chondrules increasingly became smaller in size and irregular in shape. Some of the chondrules were separated into pieces. The results indicate that a major proportion of the matrix materials were produced by the alteration of chondrules and inclusions and that there was a process in which the alteration products and the original matrix material were extensively and uniformly mixed together.

The matrix of the clast has a composition intermediate between saponite and serpentine and contains abundant framboidal magnetite, being similar to the matrix of the CI chondrites. The results suggest that more advancing alteration of the clast leads to a CI1-like lithology and raise the possibility that the $\mathrm{CI} 1$ chondrites could have resulted from aqueous alteration of a precursor that had a type 3 lithology (which consists of chondrules and inclusions set in a matrix) under conditions similar to those for the alteration of the Mokoia clast.

The results indicate that there was a highly wet region within the Mokoia parent body, where intensive aqueous alteration occurred, and that this clast escaped subsequent thermal metamorphism. The past studies of CV dark clasts [e.g., 1-3] and the present study of the Mokoia clast suggest that the $\mathrm{CV}$ parent body had a heterogeneous distribution of water and that various degrees of aqueous alteration, thermal metamorphism, and shock metamorphism took place in different regions. These inferences imply that the CV parent body was dynamic in terms of the physical and chemical processes.

The presence of the clast supports the previously proposed model [4], which suggests that the aqueous alteration in the chondrules/rims in Mokoia occurred in a wet region (or regions) different from where the host meteorite was finally lithified and that these aqueously altered chondrules/rims were afterward mixed with anhydrous matrix grains by brecciation on the parent body.

References: [1] Kojima T. and Tomeoka K. 1996. Geochimica et Cosmochimica Acta 60:2651-2666. [2] Krot A. N. et al. 1997. Meteoritics \& Planetary Science 32:31-49. [3] Ohnishi I. and Tomeoka K. 2002. Meteoritics \& Planetary Science 37:1843-1856. [4] Tomeoka K. and Ohnishi I. 2010. Geochimica et Cosmochimica Acta 74:4438-4453.
5464

"DOGGED" SEARCH OF FRESH NAKHLA SURFACES REVEALS NEW ALTERATION TEXTURES

T. Tomkinson ${ }^{1}$ M. R. Lee ${ }^{2}$ D. F. Mark ${ }^{1}$ and F. Stuart ${ }^{1}$. ${ }^{1}$ SUERC, East Kilbride, Glasgow, U.K. ${ }^{2}$ GES, Glasgow University, UK. E-mail: tim.tomkinson@glasgow.ac.uk.

Introduction: Nakhla is the only nakhlite fall and hence is the best sample available for investigating products of aqueous alteration on Mars. Alteration products including clays, salts, carbonates and Fesilicate gels have been previously observed filling fractures within this meteorite [1-3]. These materials have been interpreted as remnants of an ancient aqueous alteration event related to an impact-induced hydrothermal system or sustainable subsurface brine [3,4]. However, the poor crystallinity of the Fe-silicates suggests a short formation time scale on Mars. Given their microstructural, crystallographic and chemical complexity these alteration products are still not fully understood. Hitesh and Bridges [3] have examined the fine-scale structure of the Fe-silicate gels and clays by transmission electron microscope (TEM) study of foils cut from veins exposed in polished thin sections using the focused ion beam (FIB) technique. Here we have chosen to examine alteration product-coated grain surfaces in freshly broken samples of Nakhla, and we have also extracted layers of alteration products using a micromanipulator for high-resolution chemical and microstructural characterization.

Methods: The sample studied (BM1913, 25) was kindly loaned by the Natural History Museum (London). It is a fresh interior surface fragment (1.6 g) prepared by Dr. Caroline Smith and has not been carbon or gold coated or washed. The zones of weakness along which the sample has broken expose large areas of alteration products. These samples were characterized by field-emission SEM, low-voltage scanning transmission electron microscopy, and by TEM.

Results: We have observed a variety of alteration products that have not been previously described. The Fe-silicate gels are composed of multiple thin sheets, some of which are concentrically zoned with respect to $\mathrm{Si}, \mathrm{Cl}, \mathrm{Ca}, \mathrm{Mn}$ and $\mathrm{Fe}$ and over scales of $\sim 0.1 \mathrm{~mm}$. The gels are also cross-cut by veins of a $\mathrm{Cl}$-rich material that have a nanoscale fibrous structure and are decorated by tens of nanometre sized euhedral calcium sulphate crystals. Additionally we have observed radial growth structures emanating from halite grains, and sites displaying stages of nucleation and growth of the gel.

The features that we have observed suggest that the Fe-silicate gel formed during fluctuations in fluid compositions, and the presence of $\mathrm{Cl}-$ rich alteration products cross-cutting the gel indicates subsequent injection of a saline fluid. The sharp contacts of this later material with the gel suggest that this brine percolated through Nakhla at very low temperatures [5] thus not remobilizing and equilibrating with the surrounding material. One important message of this study is that caution is required when samples are being prepared. The nanoscale features that we have found would undoubtedly be lost in conventional thin section manufacture and may explain why they have not been observed previously.

References: [1] Bunch T. E. and Reid A. M. 1975. Meteoritics 10:303-324. [2] Gooding J. L. et al. 1991. Meteoritics 26:135-143. [3] Changela H. G. and Bridges J. C. 2011. Meteoritics \& Planetary Science 45:1847-1867. [4] Rao M. N. et al. 2006. Abstract \#1969. 37th Lunar and Planetary Science Conference. [5] Hausrath E. M. and Brantley S. L. 2010. Journal of Geophysical Research 115. 
5466

CRYSTALLOGRAPHY AND ORIGINS OF ETCH PITS IN NAKHLA OLIVINE

T. Tomkinson ${ }^{1}$, M. R. Lee ${ }^{2}$, D. F Mark ${ }^{1}$, F. Stuart ${ }^{1}$ and P. Lindgren ${ }^{2}$. ${ }^{1}$ SUERC, East Kilbride, Glasgow, UK. ${ }^{2}$ GES, Glasgow University, UK. E-mail: tim.tomkinson@glasgow.ac.uk.

Introduction: Etch pitted olivine grains have been observed within the nakhlite meteorite MIL 03346 [1] but have rarely been described from Nakhla [2]. Velbel et al. in 2010 [1] concluded that the pits in MIL 03346 olivine were a result of terrestrial weathering in Antarctica as they occur only within the outermost few hundred microns of the meteorite. However, the pitted olivines that we have found in Nakhla are on freshly exposed pieces of the meteorite that were taken from the interior of this fall ( $\sim 5 \mathrm{~cm}$ into the sample). As these etch pits occur only on some olivines and the majority are filled with alteration products of Martian origin, they must have formed on Mars.

Results: The Nakhla etch pits range from diamond to wedge to circular in shape, reflecting the crystallographic orientation of the grain surface on which they have formed. The largest of the two pitted regions found has an array of 80 by 100 round pits with a preferred orientation. The smaller region is a mix of small arrays and single lines of diamond shaped pits. The largest pit observed is $\sim 30 \mu \mathrm{m}$ and the smallest is submicron in diameter. Some of the pits are empty or filled with a hydrous Fe-silicate that has been interpreted by [3] to be a gel, while others contain a $\mathrm{Cr}$-rich material that was exposed during sample preparation. If this $\mathrm{Cr}$ enriched material occurs beneath the amorphous gel it is likely to predate the gel formation.

Implications: These etch pits can be used to obtain information on the duration of exposure to aqueous solutions. For these calculations pit volumes are required, and they will be obtained by imaging their profiles using SEM and making digital elevation models of the largest pits ( $\sim 30 \mu \mathrm{m}$ diameter) using Alicona MEX software, and by excavating cross sections through them using the focused ion beam (FIB) technique. These data will yield the minimum duration of exposure to the etching fluid [4]. As the pits are observed in only two of the 14 olivine grains so far examined, we will investigate the crystal orientation of the etched olivine grains in order to assess whether there is a preferred etching orientation. Alternatively the etching could predate the main phase of aqueous activity when the fluid was exposed to a limited surface area of the sample when it was less densely fractured.

Acknowledgments: The Nakhla sample (BM1913_25) used for this study was from the U.K. Natural History Museum and kindly allocated by Dr Caroline Smith.

References: [1] Velbel M. A. et al. 2010. Abstract \#2223. 41st Lunar and Planetary Science Conference. [2] Velbel M. A. 2010. AGU Fall Meeting. Abstract \#P52B-07. [3] Changela H. G. and Bridges J. C. 2011. Meteoritics \& Planetary Science 45:1847-1867. [4] Olsen A. A. 2007. American Mineralogist 92:598-602.
5472

STEPS TOWARD PRECISE Ar/Ar CHRONOLOGIES FOR FLUIDROCK INTERACTION THROUGHOUT THE SOLAR SYSTEM

T. Tomkinson ${ }^{1}$, D. F. Mark', M. R. Lee ${ }^{2}$ and F. Stuart ${ }^{1}$. ${ }^{1}$ SUERC, East Kilbride, UK. ${ }^{2}$ GES, Glasgow University, UK. E-mail: t.tomkinson@glasgow.ac.uk.

Introduction: Determination of ages for pre-terrestrial fluid-rock interaction has only been carried out on two of the twelve known nakhlites (Lafayette and Yamato 000593). The only K-Ar ages for the Kbearing "iddingisite" range from 0-670 $\pm 91 \mathrm{Ma}$ [1]. Within the nakhlites fine-grained iddingsite is present in discrete veins and as grain coatings. The low K-content and fine-grain size of the iddingsite hinder the $\mathrm{Ar} / \mathrm{Ar}$ approach as: (1) only small amounts of material are available for isotope extraction, and (2) potential recoil of ${ }^{39} \mathrm{Ar}$ and ${ }^{37} \mathrm{Ar}$ from the fine-grained clays during irradiation. This contribution will focus on novel approaches for $\mathrm{Ar} / \mathrm{Ar}$ dating of fine-grain K-bearing clay in meteorites using cutting edge mass spectrometer technology (high-sensitivity high-resolution HELIX Split Flight Tube noble gas mass spectrometer), a range of irradiation protocols (e.g., in vacuo encapsulation, D-D fusion neutrons and irradiation of thin sections), and a variety of Ar extraction tools (e.g., $\mathrm{CO}_{2}$ and UV lasers, bulb furnace). Analog materials from the Isle of Arran (Scotland) have been collected to aid initial investigations. After testing of our approaches we will target K-bearing clay in both the nakhlites (e.g., Nakhla) and carbonaceous chondrites (e.g., Murchison).

Arran Analogs: Samples for a "proof of concept" study were sourced from the east coast of Arran, where a highly altered Carboniferous basaltic lava flow is exposed. The flow consists of grains of plagioclase and olivine that show similarities to olivine crystals from the nakhlites. Veins and patches of iddingsite are evident. These alteration products are been utilized to test and refine sample preparation methods, detection limits and achievable spatial resolution prior to analysis of the meteorites. This analogue material is especially useful as its age (c. $330 \mathrm{Ma}$ ) falls mid-way between the estimates for alteration of Lafayette (i.e., 0-670 Ma), its $\mathrm{K}$-content is comparable to that of the nakhlite iddingsite, and veins of alteration products are $2-20 \mu \mathrm{m}$ wide, also similar to the average size of veins in the nakhlites (4-16 $\mu \mathrm{m}$ [2]).

Analytical Methodology: SUERC has taken delivery of the first two Thermo Fisher HELIX SFT noble gas mass spectrometers. We have built a custom designed low volume extraction line, which is dedicated to $\mathrm{Ar} /$ Ar dating of extraterrestrial materials. Attached to this system is a New Wave UP-213 UV laser that enables in situ analysis of material with a spatial resolution (spot size) of $4 \mu \mathrm{m}$. The HELIX SFT is able resolve isobaric interferences at mass 36 allowing for the first time a true ${ }^{36} \mathrm{Ar}$ measurement and accurate atmospheric correction. Ion counting electron multipliers and high-sensitivity resistor Faradays will allow for precise measurement of small ion beams, facilitating determination of precise $\mathrm{Ar} /$ Ar ages.

References: [1] Swindle T. D. et al. 2000. Meteoritics \& Planetary Science 35:107-115. [2] Changela H. G. and Bridges J. C. 2011. Meteoritics \& Planetary Science 45:1847-1867. 
5124

MASON GULLY: THE SECOND METEORITE RECOVERED BY THE DESERT FIREBALL NETWORK

M. C. Towner ${ }^{1}$, P. A. Bland ${ }^{1}$, P. Spurný ${ }^{2}$, G. K. Benedix ${ }^{3}$, K. Dyl ${ }^{1}$, R. C. Greenwood $^{4}$, J. Gibson ${ }^{4}$, I. A. Franchi ${ }^{4}$, L. Shrbený ${ }^{2}$, A. W. R. Bevan ${ }^{5}$ and D. Vaughan ${ }^{6}$. ${ }^{1}$ IARC, Dept Earth Sci. \& Eng., Imperial College London, SW7 2AZ, UK. E-mail: m.towner@imperial.ac.uk. ${ }^{2}$ Astronomical Institute of the Academy of Sciences, Fričova 298, CZ-251 65 Ondřejov Observatory, Czech Republic. ${ }^{3}$ IARC, Department of Mineralogy, Natural History Museum, London SW7 5BD, UK. ${ }^{4}$ PSSRI, Open University, Walton Hall, Milton Keynes MK7 6AA, UK. ${ }^{5}$ Department of Earth and Planetary Sciences, Western Australian Museum, Locked Bag 49 Welshpool DC, WA 6986, Australia. ${ }^{6}$ PO BOX 187, Nedlands, Perth, WA 6909, Australia.

Introduction: Mason Gully (MG) is the second meteorite recovered using data obtained by the Desert Fireball Network (DFN) project $[1,2]$. MG has been classified as an $\mathrm{H} 5$ ordinary chondrite, with minimal weathering, due to its short terrestrial exposure age in an area of low precipitation.

Recovery: Analysis of the camera images gave orbital information [3], and a prediction of the fall position to an area of $2-3 \mathrm{~km}^{2}$. Searching was carried out on foot, by a team of six people, over 12 days in November 2010. The meteorite was recovered within $150 \mathrm{~m}$ of the predicted fall site. We have now undertaken 4 field campaigns, and recovered meteorites on 2 of them: a $50 \%$ success rate, validating the concept of a network based in the Australian outback.

Physical Characteristics: MG has a mass of $24.54 \mathrm{~g}$. The stone is $\sim 3 \mathrm{~cm}$ in long dimension, and $50 \%$ fusion crusted. Individual pyroxene, olivine, and feldspar crystals are visible on the broken surface, with no evidence of alteration of silicates. Some rust patches around metal grains on original broken surfaces. Although the meteorite appears to have high porosity (based on observations of sawn surfaces), metal in the interior shows no sign of alteration. Fusion crust is black and fresh. We compared alteration of $\mathrm{MG}$ crust, broken surfaces, and interior, to a recent $\mathrm{H}$ chondrite fall (recovered $<3$ weeks later with no rain between fall and recovery). In contrast, $\mathrm{MG}$ experienced $23 \mathrm{~cm}$ of rain between fall and recovery. MG was less weathered. The overall level of alteration was consistent with a 7 month residence time in the desert [4].

Petrography: The meteorite has a typical petrologic type 5 texture, with discernible, but not distinct chondrules. Chondrule types include barred olivine and radiating pyroxene. Minerals are heterogeneously distributed. Modal mineralogy (in vol\%) is: olivine (33\%); orthopyroxene $(38 \%)$; clinopyroxene $(5 \%)$; plagioclase $(8 \%)$; metal $(11 \%)$; sulfide $(5 \%)$; minor components around $1 \%$.

Mineral Compositions and Geochemistry: Mineral compositions as determined by EMP: olivine, Fa19.2 \pm 0.6 ; low-Ca pyroxene, Fs16.4 \pm $0.4 \mathrm{Wol} .4 \pm 0.3 ; \quad$ chromite $\quad(\mathrm{Fe} / \mathrm{Fe}+\mathrm{Mg}=0.84 ; \quad \mathrm{Cr} / \mathrm{Cr}+\mathrm{Al}=0.86)$. Oxygen isotopes: $\delta 17 \mathrm{O}=3.04 ; \delta 18 \mathrm{O}=4.42 ; \Delta 17 \mathrm{O}=0.74$ (all in per mil).

References: [1] Bland P. A. et al. 2009. Abstract \#1664. 40th Lunar and Planetary Science Conference, www.desertfireballnetwork.org. [2] Spurný P. et al. 2009. Abstract \#1498. 40th Lunar and Planetary Science Conference. [3] Spurný P. et al. 2011. Meteoritics and Planetary Science 46 (this conference). [4] Bland P. A., Berry F. J., and Pillinger C. T. 1998. Meteoritics \& Planetary Science 33:127-129.
5523

HOW BIG IS THE MOROKWENG IMPACT STRUCTURE, SOUTH AFRICA? A REAPPRAISAL OF ITS PRESENT SIZE USING REMOTE SENSING, GEOPHYSICS AND GIS

C. Townsend ${ }^{1}$, M. A. G. Andreoli ${ }^{2,3}$, G. Cooper ${ }^{2}$, S. Misra ${ }^{3}$ and I. Stengel ${ }^{4,5}$. ${ }^{1} 30$, Arnold Road, DL1 1JG, U.K. E-mail: skymapper639@ impactcraters.info, skymapper639@impactcraters.info. ${ }^{2}$ School of Geoscience, University of the Witwatersrand, Johannesburg 2050, South Africa. ${ }^{3}$ SGS, University of KwaZulu-Natal, Durban-4000, South Africa. ${ }^{4}$ NamibGeoVista Geoconsult \& Imaging, Windhoek, Namibia. ${ }^{5}$ Department of Geography, University of Pretoria, Pretoria, South Africa.

Introduction: The Morokweng impact structure in South Africa, formed close to the Jurassic-Cretaceous boundary (approximately $145 \pm 2 \mathrm{Ma}$ ) by the impact of an LL- 6 chondrite body [ 1 and references therein], could be one of the largest known terrestrial craters if the issue surrounding its final diameter can be resolved. Morokweng, presently buried under cover of Cenozoic Kalahari continental deposits, is described to have a diameter between approximately $70 \mathrm{~km} \mathrm{[2,3]} \mathrm{and}$ $340 \mathrm{~km}$ [4]. The present integrated study, involving the use of Landsat 7 imagery, SRTM-DEM data, and the results of a magnetic and gravity survey, coupled with field observations and borehole data, suggest the minimum diameter of this structure is likely to be approximately $160 \mathrm{~km}$.

Techniques of Study: The Landsat 7 imagery and DEM were investigated for arcuate and impact related topographical features; these datasets were merged with magnetic and gravity data and analysed spatially using a Geographical Information System (GIS).

Observations: The Morokweng structure is characterized by a large, elliptically shaped gravity low, approximately $155 \times 125 \mathrm{~km}$ in diameter, centered on the melt sheet. When correlated with the SRTM-DEM and Landsat 7 imagery, it appears sharply truncated to the North, West and Southwest by the Kuruman Hills. Enhancement of the gravity data has revealed a smaller elliptical anomaly some $55 \times 68 \mathrm{~km}$ in diameter, the center of which reveals a small low at $23.5^{\circ} \mathrm{E} 26.5^{\circ} \mathrm{S}$, being the approximate center of the melt sheet.

Conclusions: We suggest that the Morokweng structure has a minimum diameter of approximately $160 \mathrm{~km}$, with a probable final diameter of approximately $200 \mathrm{~km}$ [cf. 5, 6], making it one of the largest known terrestrial impact structures.

References: [1] Misra S. et al. 2011. Abstract \#1102. 42nd Lunar and Planetary Science Conference. [2] Henkel H. et al. 2002. Journal of Applied Geophysics 49:129-147. [3] Reimold W. U. et al. 2002. Earth and Planetary Science Letters 201:221-232. [4] Corner B. et al. 1997. Earth and Planetary Science Letters 146:351-364. [5] Baker T. 2001. A Remote Sensing Investigation of the Morokweng Impact Structure, South Africa. University of Greenwich, UK. B.Sc. thesis (Unpublished). [6] Andreoli M. A. G. et al. 2008. Abstract \#1236. 39th Lunar and Planetary Science Conference. 
5473

ANGRA DOS REIS: RECONCILIATION OF COMPOSITION AND TEXTURE, INVOKING IMPACT

A. H. Treiman. Lunar and Planetary Institute, 3600 Bay Area Boulevard, Houston TX 77058 E-mail: treiman\#lpi.usra.edu.

Introduction: The angrite meteorites are ancient basaltic rocks from a highly differentiated body [1-5]. Angra dos Reis itself (AdoR) is unique in being a pyroxenite, not a basalt. By mineralogy and chemistry, AdoR has been interpreted as an igneous cumulate [1,6], but its texture (Fig. 1, [7]) suggests a more complex history.

AdoR Texture: AdoR consists of $93 \%$ fassaite pyroxene, with olivine, Mg-Fe spinel, merrillite, etc. [6]. The fassaite occurs in two textural patterns: poikilitic megacrysts up to $3 \mathrm{~mm}$ across, seen in thin section as disjoint grains in optical continuity; and equant 'groundmass' grains of $\sim 100 \mu \mathrm{m}$ across (Fig. [7]). Originally [6], groundmass grains were interpreted as cumulus and the megacrysts as intercumulus infill. Alternatively, [7] suggested that the megacrysts were porous phenocrysts, and that the groundmass represented devitrified magma. The former idea is physically implausible [7], and the latter chemically difficult [1].

Yet Another Idea: By analogy with the 'granular bands' in the ALH 84001 meteorite [8], the 'groundmass' fassaite grains in AdoR could be interpreted as annealed [9] remnants of shock-deformed (or melted) material. The poikilitic megacrysts would be unrecrystallized remnants of original cumulus grains. This explanation reconciles AdoR's bulk composition (pyroxene cumulate) with its texture, at the expense of requiring a multi-stage history. The relatively young age of AdoR, 4557.6 versus 4564.4 Ma for quenched angrites [4,10], would then represent shock metamorphic equilibration. This evidence for strong shock deformation and extended cooling duration is consistent with the concept of an angrite planetesimal or dwarf planet [5].

References: [1] Mittlefehldt D. W. and Lindstrom M. M. 1990. Geochimica et Cosmochimica Acta 54:3209-3218. [2] Mittlefehldt D. W. et al. 1998. Non-chondritic meteorites from asteroidal bodies. Ch. 4 in Planetary materials (ed. J. J. Papike), Reviews in Mineralogy 36. [3] Mittlefehldt D. W. et al. 2002. Meteoritics \& Planetary Science 37:345-369. [4] Amelin Y. 2008. 1997. Geochimica et Cosmochimica Acta 72:221-232. [5] Weiss B. P. 2008. Science 322:713-716. [6] Prinz M. et al. 1977. Earth and Planetary Science Letters 35:317-330. [7] Treiman A. H. 1989. 19th Lunar and Planetary Science Conference. pp. 443-450. [8] Treiman A. H. 1998. Meteoritics \& Planetary Science 33:753-764. [9] Fittipaldo M. A. et al. 2005. Meteoritics \& Planetary Science 42:573-589. [10] Markowski A. et al. 2007. Earth and Planetary Science Letters 262:216-229.

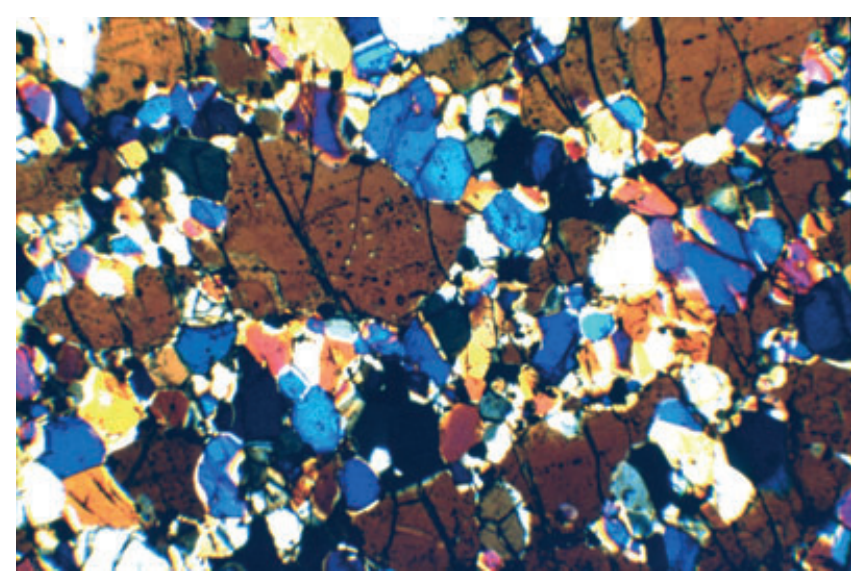

Fig. 1. Angra dos Reis thin section, crossed polars, $2.1 \mathrm{~mm}$ across. All fassaite pyroxene. Megacryst of grains in optical continuity (brown), crossed by bands of smaller grains in random orientations.
5247

THE ROLE OF SYNCHROTRON RADIATION-BASED X-RAY MICROTOMOGRAPHY IN EXAMINATION OF SMALL EXTRATERRESTRIAL SAMPLES RECOVERED BY SPACECRAFT IN THE Stardust AND HAYABUSA MISSIONS

A. Tsuchiyama ${ }^{1}$, T. Nakamura ${ }^{2}$, M. Uesugi ${ }^{3}$, K. Uesugi ${ }^{4}$, T. Nakano ${ }^{5}$, A. Takeuchi $^{4}$, Y. Suzuki ${ }^{4}$, Y. Iida ${ }^{1}$, R. Niimi ${ }^{1}$, R. Noguchi ${ }^{1}$, T. Matsumoto ${ }^{1}$, J. Matsuno ${ }^{1}$, T. Nagano ${ }^{1}$, Y. Imai ${ }^{1}$, T. Ogami ${ }^{2}$, T. Noguchi ${ }^{6}$, M. E. Zolensky $^{7}, \quad$ T. Mukai ${ }^{3}, \quad$ M. $\mathrm{Abe}^{3}, \quad$ T. $\mathrm{Yada}^{3}$ and A. Fujimura $^{3}$. ${ }^{1}$ Department of Earth \& Space Science, Osaka University, Osaka, Japan. E-mail: akira@ess.sci.osaka-u.ac.jp. ${ }^{2}$ Department of Earth \& Planetary Mat. Science, Tohoku University. ${ }^{3}$ JAXA. ${ }^{4}$ JASRI/SPring-8. ${ }^{5}$ GSJ/AIST. ${ }^{6}$ The Coll. of Sci. at Ibaraki University. ${ }^{7}$ NASA JSC.

Introduction: Coma dust particles of comet $81 \mathrm{P} /$ Wild 2 coma were returned to Earth by NASA's Stardust mission in January 2006 and have been examined in laboratories [1]. Regolith particles of asteroid 25143 Itokawa were returned by JAXA's Hayabusa mission in June 2010 and preliminary examination has started [2,3]. Particle sizes of both the samples are small (<approximately $100 \mu \mathrm{m})$ and their total amounts are very small compared with Apollo lunar samples. Nondestructive microtomography gives unique 3-D data and also plays an important role in later destructive analyses in order to optimize the information obtained from these small, precious particles. Our research group has used a synchrotron radiation (SR) based microtomography system for these samples. An overview of these results will be presented here.

SR-Based Microtomography: Tomography using X-ray absorption is a conventional method to give spatial distribution of X-ray linear attenuation coefficients (LACs) as a digital image (CT image). SR X-ray beams with high flux density and high coherence gives CT images of high spatial resolution (nano-scale resolution is possible by X-ray microscope optics using a FZP) and high $\mathrm{S} / \mathrm{N}$ ratio. High flux $\mathrm{X}$-ray beams are easily monochromated, and this gives quantitative LAC values in CT images, which can be used for identification of minerals and their rough chemical compositions. We used microtomography systems at BL20B2 and BL47XU of SPring-8 for the sample examination.

Stardust Samples: Comet coma dust was captured using silica aerogel at a relative velocity of $6.1 \mathrm{~km} \mathrm{~s}^{-1}$ by the Stardust spacecraft. Dust particles were disrupted during the capture and impact tracks were formed. Relatively coarse crystalline grains, harvested from impact tracks, were examined by microtomography, which determined how to best cut the sample particles for later oxygen isotope analysis [4]. 3-D morphologies of impact tracks were also examined, and densities of impacted dust particles were estimated based on these data [5].

Hayabusa Samples: A quantitative 3-D mineral distribution was obtained for each particle by imaging at 7 and $8 \mathrm{keV}$. These 3 -D mineral maps were essential for planning later destructive analysis in the preliminary examination analysis flow. External 3-D shape features of these particles were also examined to reveal details of regolith formation and evolution on Itokawa [3].

References: [1] Brownlee D. E. et al. 2006. Science 314:1711-1716. [2] Nakamura T. et al. 2011. This issue. [3] Tsuchiyama A. et al. 2011. This issue. [4] Nakamura T. et al. 2008. Science 321:1664-1667. [5] Iida Y. et al. Meteoritics \& Planetary Science 45:1302-1319. 


\section{3}

ORIGIN AND EVOLUTION OF ITOKAWA REGOLITH PARTICLES BASED ON THREE-DIMENSIONAL SHAPES AND SIZES OF HAYABUSA SAMPLES

A. Tsuchiyama ${ }^{1}$, M. Uesugi ${ }^{2}$, T. Matsushima ${ }^{3}$, T. Michikami ${ }^{4}$, T. Kadono $^{5}$, T. Nakamura ${ }^{6}$, K. Uesugi ${ }^{7}$, T. Nakano ${ }^{8}$, S. A. Sandford ${ }^{9}$, R. Noguchi $^{1}$, T. Matsumoto ${ }^{1}$, J. Matsuno ${ }^{1}$, T. Nagano ${ }^{1}$, Y. Imai ${ }^{1}, A$. Takeuchi $^{7}$, Y. Suzuki ${ }^{7}$, T. Ogami ${ }^{6}$, J. Katagiri ${ }^{3}$, M. Ebihara ${ }^{10}$, T. R. Ireland $^{11}$, F. Kitajima ${ }^{12}$, K. Nagao ${ }^{13}, \mathrm{H}$. Naraoka ${ }^{12}$, T. Noguchi ${ }^{14}$, R. Okazaki $^{12}$, H. Yurimoto ${ }^{15}$, M. E. Zolensky ${ }^{16}$, T. Mukai $^{2}$, M. Abe ${ }^{2}$, T. Yada $^{2}$, A. Fujimura ${ }^{2}$, M. Yoshikawa ${ }^{2}$ and J. Kawaguchi ${ }^{2} .{ }^{1}$ Department Earth \& Space Science, Osaka University, Osaka, Japan. E-mail: akira@ ess.sci.osaka-u.ac.jp. ${ }^{2}$ JAXA. ${ }^{3}$ Department Engineering Mechanical \& Energy, University of Tsukuba, Tsukuba, Japan. ${ }^{4}$ Fukushima National College of Technology. ${ }^{5}$ ILE, Osaka University, Osaka, Japan. ${ }^{6}$ Department Earth \& Planetary Mat. Science, Tohoku University, Tohoku, Japan. ${ }^{7}$ JASRI/SPring-8. ${ }^{8}$ GSJ/AIST. ${ }^{9}$ NASA ARC. ${ }^{10}$ Graduate School of Science \& Engineering, Tokyo Metropolitan University, Tokyo, Japan. ${ }^{11}$ Research School of Earth Science, ANU. ${ }^{12}$ Department Earth \& Planetary Science, Kyushu University, Kyushu, Japan. ${ }^{13}$ Geochemical Research Center, The University of Tokyo, Tokyo, Japan. ${ }^{14}$ The College of Science at Ibaraki University, Ibaraki, Japan. ${ }^{15}$ National History Science, Hokkaido University, Hokkaido, Japan. ${ }^{16}$ NASA JSC.

Introduction: Particles of S-type asteroid 25143 Itokawa were successfully recovered by the Hayabusa mission, and at least 1500 recovered particles have been identified as having an Itokawa origin $[1,2]$. This is the first sample recovered from an asteroid and returned to Earth, and the second extraterrestrial regolith to have been sampled, the first being the Moon, which was sampled by the Apollo and Luna missions. We obtained three-dimensional (3-D) structures of Itokawa particles using X-ray microtomography in the Hayabusa sample preliminary examination in order to understand their textures in comparison with meteorites and the 3-D shape features in connection with the regolith formation and evolution on the asteroid [2].

Experiments: Forty particles of approximately $30-180 \mu \mathrm{m}$ in size were imaged at BL47XU of SPring-8 with effective spatial resolutions of approximately 200 or $500 \mathrm{~nm}$. Imaging at two X-ray energies of 7 and $8 \mathrm{keV}$ made identification of minerals in CT images possible. A successive set of 3-D CT images, which shows quantitative 3-D mineral distribution, was obtained for each particle.

Results and Discussion: Modal mineral abundances of the whole sample and the bulk density $\left(3.4 \mathrm{~g} \mathrm{~cm}^{-3}\right)$ calculated form the modal abundance and average chemical compositions of the minerals [1] are similar to those of LL chondrites. 3-D structures of the particles having textural variations indicate a mixture of equilibrated and less-equilibrated chondrite materials. No particles showing melting were observed, indicating low impact velocities similar to typical relative impact velocities among asteroids (approximately $5 \mathrm{~km} \mathrm{~s}^{-1}$ ). 3-D shape features of the particles (size and shape distributions together with presence of particles with rounded edges) suggest that they were formed by meteoroid impacts on the asteroid surface, transported to the smooth terrain, and eroded in situ there by granular processes, which include major resurfacing processes in small asteroids [3]. The above discussion indicates that the returned sample roughly represents the general surface materials on Itokawa.

References: [1] Nakamura T. et al. 2011. Abstract \#1776. 42nd Lunar and Planetary Science Conference. [2] Tsuchiyama A. et al. 2011. Abstract \#1777. 42nd Lunar and Planetary Science Conference. [3] Miyamoto H. et al. 2007. Science 316:1011-1014.

\section{3}

\section{EVIDENCE FOR EXTINCT ${ }^{36} \mathrm{Cl}$ FROM EXCESS ${ }^{36} \mathrm{Ar}$ IN ALLENDE SODALITE}

G. Turner ${ }^{1}$, R. Burgess ${ }^{1}$, S. P. Kelley ${ }^{2}$ and G. J. Wasserburg ${ }^{3}$. ${ }^{1}$ SEAES, University of Manchester, UK, E-mail: Grenville.turner@manchester. ac.uk. ${ }^{2}$ Dept. of Earth and Environmental Science, Open University, UK. ${ }^{3}$ Division of Geological and Planetary Science, California Institute of Technology, USA.

Introduction: Excesses of ${ }^{36} \mathrm{~S}$ have previously been reported in sodalite $\left(\mathrm{Na}_{8} \mathrm{Al}_{6} \mathrm{Si}_{6} \mathrm{O}_{24} \mathrm{Cl}_{2}\right)$ and wadalite $\left(\mathrm{Ca}_{6} \mathrm{Al}_{5} \mathrm{Si}_{2} \mathrm{O}_{16} \mathrm{Cl}_{3}\right)$ in CAI and chondrules from the Allende and Ningqiang meteorites and used to infer the presence of ${ }^{36} \mathrm{Cl}$ (half-life $3 \times 10^{5}$ a) in the early solar system [1-3]. In spite of the fact that ${ }^{36} \mathrm{Cl}$ decays predominantly $(98 \%)$ to ${ }^{36} \mathrm{Ar}$, all attempts to locate the very large amounts of ${ }^{36} \mathrm{Ar}$ implied by these ${ }^{36} \mathrm{~S}$ excesses have been negative. Here we present results of a new method to identify ${ }^{36} \mathrm{Ar}$ from ${ }^{36} \mathrm{Cl}$ decay, based on low fluence fast neutron activation.

Method: We have irradiated two batches of Allende sodalite (Pink Angel) with different fluencies of fast neutrons in the Cd-shielded CLICIT facility of the Oregon State TRIGA reactor. In the absence of trapped argon, the upper intercept of a plot of ${ }^{36} \mathrm{Ar} /{ }^{38} \mathrm{Ar}$ against ${ }^{37} \mathrm{Ar} /{ }^{38} \mathrm{Ar}$ defines a relationship between ${ }^{36} \mathrm{Ar}$ and ${ }^{38} \mathrm{Ar}$ from the action of cosmic rayinduced secondary neutrons and mono-isotopic ${ }^{36} \mathrm{Ar}$ from ${ }^{36} \mathrm{Cl}$ decay. By combining data from two irradiations, the two contributions can be unambiguously distinguished. Figure 1 shows allowed combinations of ${ }^{36} \mathrm{Cl} /{ }^{35} \mathrm{Cl}$ and the product of cosmogenic secondary neutron fluence and ${ }^{37} \mathrm{Cl}$ cross section for each series. The intersection corresponds to ${ }^{36} \mathrm{Cl} /{ }^{35} \mathrm{Cl}=(1.9 \pm 0.5) \times 10^{-8}$ and $\left\langle\sigma_{37} . \Phi>=(1.1 \pm 0.8) \times 10^{-10}\right.$.

The latter is in accord with the neutron fluence observed in CAIs in Allende [4]. The ${ }^{36} \mathrm{Cl} /{ }^{35} \mathrm{Cl}$ ratio is 3 orders of magnitude lower than the highest value inferred from ${ }^{36} \mathrm{~S}$ excesses. If interpreted chronologically, i.e., as a closure age, it would correspond to a period of $3 \mathrm{Ma}$ or so of activity involving the precipitation of sodalite, which is consistent with I-Xe data [5]. Alternatively, the low ratio may represent ${ }^{36} \mathrm{Ar}$ loss in line with the low activation energy for Ar diffusion in sodalite. The presence of excess ${ }^{36} \mathrm{Ar}$ together with ${ }^{36} \mathrm{~S}$ supports the idea that live ${ }^{36} \mathrm{Cl}$ was present in the sodalite, as opposed to the ${ }^{36} \mathrm{~S}$ being inherited. The mechanism for producing ${ }^{36} \mathrm{Cl}$ several million years after the formation of the solar system is still unknown.

References: [1] Lin Y. et al. 2005. Proceedings of the National Academy of Sciences 102:1306-1311. [2] Hsu W. et al. 2006. The Astrophysical Journal 640:525-529. [3] Jacobsen B. et al. 2009. Abstract \#2553. 40th Lunar and Planetary Science Conference. [4] Wasserburg G. J. et al. 2011. Geochimica et Cosmochimica Acta, in press. [5] Turner G. et al. Meteoritics \& Planetary Science 44:A5255.

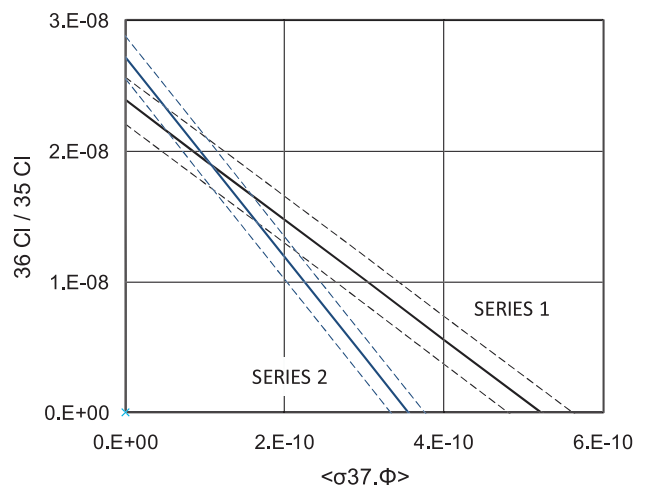


5446

DECIPHERING THE HISTORY OF CARBONATES AND ASSOCIATED MINERALS DURING SECONDARY ALTERATION WITHIN CR1 GRO 95577

M. A. Tyra, C. Le Guillou and A. J. Brearley. Department of Earth \& Planetary Sciences, MSC03-2040, University of New Mexico, Albuquerque, NM 87131, USA. E-mail: matyra@unm.edu.

Introduction: Secondary carbonates in CR chondrites are commonly pure $\mathrm{CaCO}_{3}$ but can contain up to $7 \mathrm{wt} \% \mathrm{FeO}, 14 \mathrm{wt} \% \mathrm{MgO}$, and $2 \mathrm{wt} \% \mathrm{MnO}$ [1]. The highly altered CR1 chondrite GRO 95577, however, is an exception; Ca-carbonate, dolomite, and Fe-rich carbonate have all been identified and carbonate oxygen isotope compositions suggest that Ca-carbonate formation predated Fe-rich carbonate [2]. Carbonates occur in the matrix with other alteration phases. We have studied carbonate mineral relationships in GRO 95577 using SEM and TEM to understand their chemical heterogeneity and formation sequence.

Results: Carbonates often occur spatially correlated with pyrrhotite and some magnetite. Pyrrhotite ranges from euhedral-to-abraded, and magnetite, individually and within framboids, is euhedral. Some Fe-rich carbonate encrusts calcite and pyrrhotite. Calcite, Fe-rich carbonate, and pyrrhotite show dissolution features. Fibrous and porous phyllosilicate rims are systematically found rimming all other secondary phases.

We extracted a FIB section $(27 \mu \mathrm{m}$ across $)$ that transects matrix, Ferich carbonate with associated fibrous phyllosilicate, pyrrhotite, heterogeneous carbonate, and a lamellar pentlandite-phyllosilicate intergrowth. Three distinct carbonate compositions occur (1) nearly-pure Ca-carbonate (some $\mathrm{Mn}$ ), (2) an intermediate Ca-rich carbonate with highly variable $\mathrm{Mg}, \mathrm{Mn}$, and $\mathrm{Fe}$, and (3) a carbonate high in $\mathrm{Fe}, \mathrm{Mg}$, and $\mathrm{Mn}$, and almost free of $\mathrm{Ca}$. The Fe-rich carbonate is located within $500 \mathrm{~nm}$ of the pyrrhotite, but is separated by a layer of Ca-carbonate and intermediate carbonate grains from the pyrrhotite. Intermediate carbonate contains four chemically-zoned areas within $1 \mu \mathrm{m}$ with $\mathrm{Ca} / \mathrm{Mg}$ ranging from 1 to 2 . These zones are parallel to a pyrrhotite crystal face. Finegrained matrix phyllosilicates and fibrous grains have compositions and $d$ spacings consistent with serpentine $(15<\mathrm{Mg \#}<30$ atom\%).

Discussion: Pyrrhotite is almost always associated with $\mathrm{Ca}$ carbonate, which may have formed early in the precipitation sequence together with magnetite. The zoned intermediate carbonate precipitated after pyrrhotite, as it grew parallel to a pyrrhotite crystal face. Intermediate carbonate is also richer in Fe next to the pyrrhotite, which may indicate that local chemical heterogeneity of the fluid could be controlled by pyrrhotite alteration. Fe-rich carbonate always surrounds both Ca-carbonate and pyrrhotite, and is a later stage phase. Lastly, Fe-rich carbonate dissolution and replacement by phyllosilicates is evident both on the SEM and TEM scale where $100 \mathrm{~nm}$-wide serpentine fibers grow parallel to Fe-rich carbonate grain surfaces. These serpentines have a larger grain size than matrix, are also found intergrown with pentlandite, and may result from late stage precipitation in voids at grain boundaries. This interplay between sulfides, magnetite, carbonates, and phyllosilicates may result from heterogeneous and changing nano-scale chemical environments.

Acknowledgments: This work was supported by NASA grant NNG06GG37G (A. J. Brearley).

References: [1] Weisberg et al. 1993. Geochimica et Cosmochimica Acta 57:1567-1586. [2] Tyra M. A. et al. 2011. Abstract \#1639. 42nd Lunar and Planetary Science Conference.
5218

DEVELOPMENT OF A METHOD FOR QUANTITATIVE ANALYSIS BY SYNCHROTRON RADIATION X-RAY CT, TOWARD HAYABUSA2 MISSION

M. Uesugi ${ }^{1}$ and $\mathrm{K}$. Uesugi ${ }^{2}$. ${ }^{1}$ Osaka University, JAXA/JSPEC, 3-1-1 Yoshinodai Sagamihara, Kanagawa 252-5210, Japan. E-mail: uesugi@ planeta.sci.isas.jaxa.jp. ${ }^{2}$ JASRI / SPring-8 1-1-1, Kouto, Sayo-cho, Sayogun, Hyogo 679-5198 Japan.

Introduction: Hayabusa2, subsequent mission of Hayabusa, targets 1999 JU3, a C-type asteroid which thought as a parent body of carbonaceous chondrite. Synchrotron radiation X-ray CT is one of the important tools of the initial analysis of those retuned samples, because it enables us not only to observe samples three-dimensionally but also to analyze materials inside the meteorites quantitatively by estimating X-ray absorption of materials [1-4]. By comparing calculated values of linear attenuation coefficient (LAC) and observed LAC values those expressed as contrasts of the slice images, we can determine materials inside the samples [2-4]. In this paper, we observed several carbonaceous chondrites by SR-X-Ray CT. Uesugi et al. [4] showed that histograms of the LAC are useful for rough determination of meteorite class. Using obtained histograms, we discuss the method for the quantitative analysis of Hayabusa2 samples.

Results and Discussions: Experiments were carried out at BL20B2 in SPring-8, Japan. X-ray CT data were obtained by optimized environment for the CT observation of meteorites [4]. Figure shows the histograms of carbonaceous chondrites obtained by $30 \mathrm{keV}$ X-ray.

Corresponding materials of each peak in histograms are determined by thresholding the slice images around the peak. Peaks of chondrules appear at $3.1,4.3$ and $4.3 \mathrm{~cm}^{-1}$ and peaks of matrix appear at $6.2,8.0$, and $9.2 \mathrm{~cm}^{-1}$, for Murchison, Allende, and Y-81020, respectively. The peak values of chondrule are equivalent to Fo\# 97, 91, and 91 of olivine, and En\# 96, 87, and 87 of pyroxene, for Murchison, Allende, and Y-81020, respectively. These values depend on the chemical compositions of each component, and enable us to determine the meteorite class of the samples, though we should increase the number of data to increase accuracy.

References: [1] Tsuchiyama A. et al. 2005. American Mineralogist 90:132-142. [2] Nakamura T. et al. 2008. Science 321:1664-1667. [3] Tsuchiyama A. et al. 2011. Abstract \#1777. 42nd Lunar and Planetary Science Conference. [4] Uesugi M. et al. 2010. Earth and Planetary Science Letters 299:359-367.

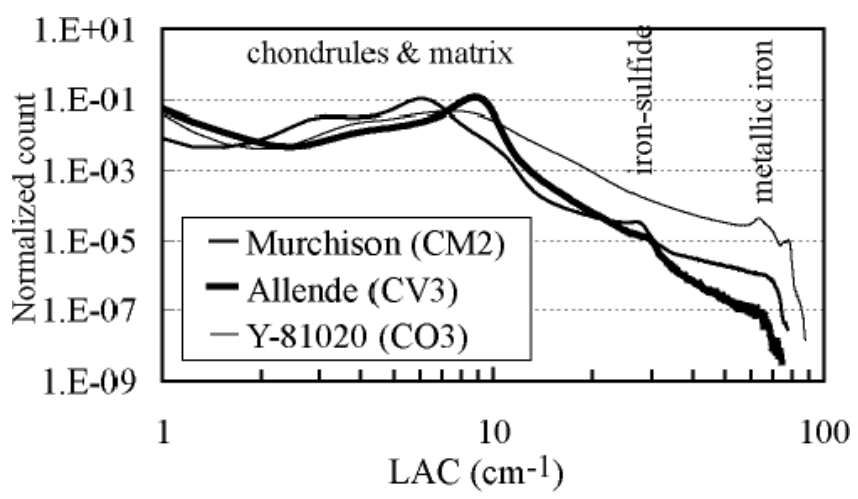


5296

THE PARENT BODY OF THE CA. 480 KYR-OLD TUNGUSKA-LIKE IMPACT OVER ANTARCTICA

M. van Ginneken ${ }^{1}$, C. Suavet ${ }^{2,3}$, C. Cordier $^{1}$, L. Folco ${ }^{1}$, P. Rochette ${ }^{3}$ and C. Sonzogni ${ }^{3}$. ${ }^{1}$ Museo Nazionale dell'Antartide, Università di Siena, Italy. E-mail: vanginneken@unisi.it. ${ }^{2}$ Massachusetts Institute of Technology, USA. ${ }^{3}$ CEREGE, Université Aix-Marseille III, France.

Introduction: Meteoritic ablation spheres (MAS) recently found on top of the Victoria Land Transantarctic Mountains (TAM) and in the EPICA Dome-C and Dome-Fuji ice core document the impact of a $10^{8} \mathrm{~kg}$ (or larger) cosmic body in the Antarctic region ca. $480 \mathrm{kyr}$ ago [1-3]. The exact nature of the impactor is unknown, and whether the impactor struck the Antarctic ice sheet or exploded in the atmosphere is matter of debate. In order to address these questions, we discuss highprecision oxygen isotope compositions of two aggregates of MAS from the TAM.

Methods: Two MAS aggregates, namely \#20c.25 and \#20c.351, from micrometeorite trap \#20c, Miller Butte, Antarctica, were selected with a Scanning Electron Microscope (SEM) for oxygen isotope analysis. Two samples of host granite detritus, of which one was cleaned of weathering products, were also selected to study the effect of weathering and bedrock contamination. Oxygen isotope compositions were determined using the IR-laser fluorination coupled with mass spectrometry (IRMS) technique adapted for the study of extraterrestrial materials following [4].

Results: SEM observations show a larger amount of weathering products in \#20c.351 compared to \#20c.25. Oxygen isotope compositions are $\delta^{18} \mathrm{O}=2.98$ and $\Delta^{17} \mathrm{O}=-3.26$ for $\# 20 \mathrm{c} .25, \quad \delta^{18} \mathrm{O}=6.50$ and $\Delta^{17} \mathrm{O}=-0.54$ for $\# 20 \mathrm{c} .351, \delta^{18} \mathrm{O}=13.65$ and $\Delta^{17} \mathrm{O}=0.03$ for cleaned host granite, and $\delta^{18} \mathrm{O}=4.97$ and $\Delta^{17} \mathrm{O}=0.09$ for host granite with weathering products (analytical uncertainties are $\pm 0.27 \%$ for $\delta^{18} \mathrm{O}$ and $\pm 0.07 \%$ for $\left.\Delta^{17} \mathrm{O}\right)$.

Discussion: Comparison of oxygen isotope compositions of MAS aggregates and host granites indicate that alteration broadly shifts the MAS aggregate composition toward those of host granite, with maximum alteration on the Terrestrial fractionation line. Pristine bulk composition of the MAS, derived from modal composition of sample \#20c.25 [1], is $\Delta{ }^{17} \mathrm{O} \approx-3.86 \%$, thus similar to those of $\mathrm{CV}, \mathrm{CO}$, and $\mathrm{CK}$ chondrites and of anhydrous silicates in short period comet $81 \mathrm{P} /$ Wild 2. Mixing models indicate that during the formation of MAS, interaction with oxygen from the Antarctic ice was likely minor, therefore ruling out direct impact on the ice sheet and favouring a Tunguska-like airburst in the low-atmosphere. This conclusion is consistent with the continental distribution of the paired MAS [1].

Conclusion: MAS from the TAM and the EPICA Dome-C and Dome-Fuji ice cores resulted from a Tunguska-like airburst over Antarctica ca. $480 \mathrm{kyr}$ ago, implying an object several tens of meters in size or larger, either an asteroid of $\mathrm{CV}, \mathrm{CO}$ or $\mathrm{CK}$ composition or a short-period comet similar to $81 \mathrm{P} /$ Wild 2 .

Acknowledgments: This work was supported by the PNRA and by the EU-funded ORIGINS project.

References: [1] van Ginneken et al. 2010. Earth and Planetary Science Letters 293:104-113. [2] Narcisi et al. 2007. Geophysical Research Letters 34:L15502. [3] Misawa et al. 2010. Earth and Planetary Science Letters 289:287-297. [4] Suavet et al. 2010. Earth and Planetary Science Letters 293:313-320.
5330

MONT DIEU A IIE NON-MAGMATIC IRON METEORITE WITH CHONDRULES

N. Van Roosbroek ${ }^{1,2}$, S. Goderis ${ }^{1}$, V. Debaille ${ }^{3}$, G. Hublet ${ }^{3}, \mathrm{Ph}$ Claeys ${ }^{1}{ }^{1}$ Earth System Sciences, Vrije Universiteit Brussels, B-1050 Brussels, E-mail: nadia.vanroosbroek@student.kuleuven.be. ${ }^{2}$ Geoinstitute, Katholieke Universiteit Leuven, B-3001 Leuven. ${ }^{3}$ Département des Sciences de la Terre et de l'Environnement, Université Libre de Bruxelles, B-1050 Brussels, Belgium.

The $\sim 450 \mathrm{~kg}$ fragment of the Mont Dieu iron meteorites at the Museum of the Royal Belgian Institute of Natural Sciences has further been characterized, with special focus on the silicate inclusions. As the original fragments in the collection of the Musée National d'Histoire Naturelle in Paris show much more rust damage [1, 2], the good state of preservation of this large fragment provides a unique way to study the Mont Dieu meteorites. Recently, Mont Dieu II was classified as a fine octahedrite IIE iron meteorite [3]. The metal phase shows a clear widmanstätten texture, composed essentially of kamacite, with fine lines of Ni-rich taenite, and locally troilite associated with schreibersite [3].

The presented study focuses on the abundant large, rounded, greenish silicate inclusions present in Mont Dieu. These inclusions are mainly composed of olivine $\left(\sim \mathrm{SiO}_{2}: 39.7 \mathrm{wt} \%, \mathrm{FeO}: 15.8 \mathrm{wt} \%, \mathrm{MgO}\right.$ : $43.2 \mathrm{wt} \%$, MnO: $\left.0.4 \mathrm{wt}^{\%} \%\right)$, pyroxene $\left(\sim \mathrm{SiO}_{2}: 60.0 \mathrm{wt} \%, \mathrm{FeO}: 9.3 \mathrm{wt} \%\right.$, MgO: 31.2 wt $\%, \mathrm{MnO}: 0.4$ wt $\%, \mathrm{CaO}: 0.9 \mathrm{wt} \%, \mathrm{Al}_{2} \mathrm{O}_{3}: 0.2 \mathrm{wt} \%, \mathrm{TiO}_{2}$ : $0.1 \mathrm{wt} \%)$ and plagioclase $\left(\sim \mathrm{SiO}_{2}: 64.6 \mathrm{wt} \%, \mathrm{Al}_{2} \mathrm{O}_{3}: 21.1 \mathrm{wt} \%, \mathrm{Na}_{2} \mathrm{O}: 9.3\right.$ wt $\%, \mathrm{~K}_{2} \mathrm{O}: 0.9$ wt $\%$, FeO: 0.8 wt $\%, \mathrm{CaO}: 2.7$ wt $\%$ ), with chromite, troilite, schreibersite, (chlor)apatite and metal as minor mineral phases. The bulk composition has also been determined with ICP-OES and ICPMS. Large chondrules $(0.8-1.5 \mathrm{~mm})$ are present in the examined inclusions, of which two are barred olivine chondrules. This feature appears unique in non-magmatic iron meteorites. The inner part of the chondrules consists mainly of olivine $\left(\sim \mathrm{SiO}_{2}: 41.3 \mathrm{wt} \%, \mathrm{Fe}_{2} \mathrm{O}_{3}: 16.3 \mathrm{wt} \%\right.$, MgO: $40.7 \mathrm{wt} \%, \mathrm{MnO}: 0.26 \mathrm{wt} \%)$, pyroxene $\left(\sim \mathrm{SiO}_{2}: 58.3 \mathrm{wt} \%, \mathrm{Fe}_{2} \mathrm{O}_{3}\right.$ : $9.7 \mathrm{wt} \%, \mathrm{MgO}: 30.6 \mathrm{wt} \%, \mathrm{MnO}: 0.21 \mathrm{wt} \%, \mathrm{CaO}: 0.79 \mathrm{wt} \%, \mathrm{Al}_{2} \mathrm{O}_{3}: 0.04$ wt $\left.\%, \mathrm{TiO}_{2}: 0.03 \mathrm{wt} \%\right)$, plagioclase $\left(\sim \mathrm{SiO}_{2}: 65.9 \mathrm{wt} \%, \mathrm{Al}_{2} \mathrm{O}_{3}: 20.7 \mathrm{wt} \%\right.$, $\mathrm{Na}_{2} \mathrm{O}: 8.2 \mathrm{wt} \%, \mathrm{~K}_{2} \mathrm{O}: 0.88 \mathrm{wt} \%, \mathrm{Fe}_{2} \mathrm{O}_{3}: 1.6 \mathrm{wt} \%, \mathrm{CaO}: 2.4 \mathrm{wt} \%$ ) and troilite, crossed with metal veins. The chondrules are surrounded by troilite, (chlor) apatite, metal, chromite and schreibersite. The fayalite and ferrosilite molar contents of the seven chondrules are similar to those observed in H-type ordinary chondrite that have been linked to IIE NMI based on their oxygen isotopic compositions [4]. Due to the presence of obvious chondrules in the silicate inclusions, Mont Dieu can be considered as part of the Netschaëvo class within the IIE group. Based on these characteristics, the Mont Dieu II meteorite promises to provide more insight in the further understanding of the IIE NMI formation process.

References: [1] Grossman. 1997. Meteoritics \& Planetary Science 31 (Suppl.): A159-166. [2] Desrousseaux et al. 1996. Meteoritics \& Planetary Science 31:A36. [3] Van Den Borre et al. 2007. Meteoritics \& Planetary Science 42:A153. [4] Olsen et al. 1994. Meteoritics 200-213. 
5153

CHONDRULES IN THE TIESCHITZ CHONDRITE: A CHEMICALPETROGRAPHIC APPROACH

M. E. Varela ${ }^{1}$, E. Zinner ${ }^{2}$ and G. Kurat ${ }^{3}$. ${ }^{1}$ ICATE/CONICET, San Juan, Argentina. E-mail: evarela@icate-conicet.gob.ar. ${ }^{2}$ Physics Department, Washington University, St. Louis, MO, USA. ${ }^{3}$ Department Lithospheric Science, University of Vienna, Vienna, Austria (deceased).

Introduction: Although large amounts of petrological and geochemical data on chondrules exist, there is still no consensus, neither on the different processes that were active during and after chondrule formation, nor on the nature of the processes that give rise to liquid droplets [e.g., 1]. However, most of the scientists working on chondrules today believe that crystals in chondrules are precipitation products of the liquid (e.g., melt) and that mesostasis represents residual liquid of the bulk melt. Here we compare the texture of three chondrules from the UOC Tieschitz L/H3.6 (PTS, Tieschitz L 3441-3443, NHMV) with the chemical composition of their clear glassy mesostasis (residual melt?) in order to contribute to our knowledge of the chondrule forming mechanism.

Results: Tieschitz 3-(2) is a PO chondrule (glass: $13 \%$, euhedral Ol [Fo98]: $87 \%$ ), $2.3 \mathrm{~mm}$ in apparent diameter and containing several metal and sulfide belts and diffusion controlled aggregation at the surface (lobate surface). Tieschitz L(A) is a POP chondrule (diameter: $680 \mu \mathrm{m}$ ) with a rim of low-Ca pyroxene (glass: $3.6 \%, \mathrm{Ol}\left[\mathrm{Fo}_{98}\right]$ : $51 \%$, Px $\left[\mathrm{En}_{97.6}\right.$, $\mathrm{Fs}_{1.4}$ ]: $45 \%$ ). Tieschitz XII is a glass-rich (glass: 64\%) chondrule that contains olivine plates $(16.5 \%)$, Ca-rich pyroxene $(2.5 \%)$, sulfide dendrites, and a large spinel that is surrounded by a cryptocrystalline matrix $(16.5 \%)$ [2]. Normalized REE abundance patterns of the glassy mesostasis are shown in the figure.

The studied objects have almost identical elemental abundance patterns but different mineralogy and texture. Trace element abundances and patterns appear not to correlate with the textures and/or the mineralogy of the objects, as was previously observed in some chondrules from UOCs [3]. Glasses show no sign of geochemical fractionation but instead a clear sign of vapor fractionation. Unraveling the role of liquids in crystal growth is crucial for our understanding of chondrule formation processes.

References: [1] Zanda 2004. Earth and Planetary Science Letters 224:1-17. [2] Kurat et al. 2010. Abstract \#5315. Lunar and Planetary Science Conference. [3] Engler et al. 2007. Icarus 192:286.

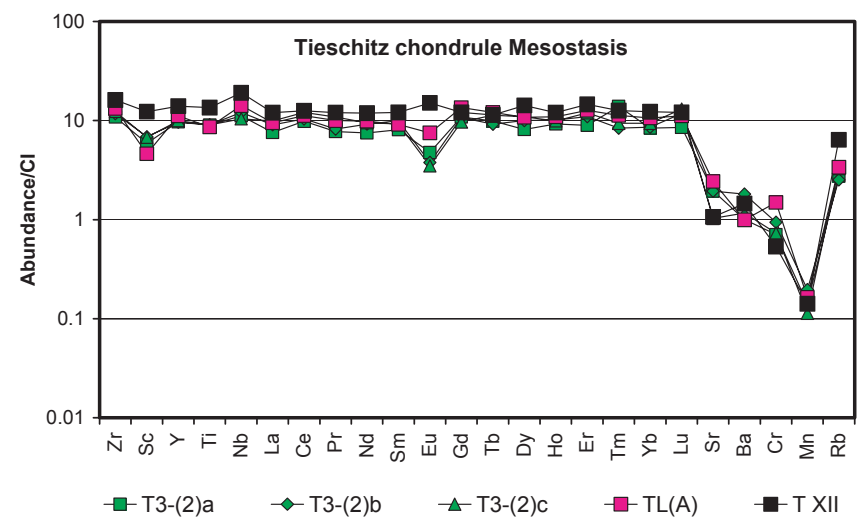

5044

AEROGEPHYSICAL ANOMALIES OVER SERRA DA CANGALHA (SdC) IMPACT STRUCTURE, BRAZIL

M. A. R. Vasconcelos and A. P. Crósta. Institute of Geosciences, University of Campinas, Campinas, SP, Brazil. E-mail: vasconcelos@ ige.unicamp.br; alvaro@ige.unicamp.br.

Introduction: $\mathrm{SdC}$ is a complex impact structure $\sim 13 \mathrm{~km}$ in diameter with a central uplift of $\sim 5.8 \mathrm{~km}$ in diameter [1]. It was formed in undisturbed Phanerozoic sedimentary rocks of the Parnaíba Basin, northeastern Brazil. Inside SdC there are four stratigraphic units, namely the Longá, Piauí, Poti and Pedra de Fogo formations, from the lowermost to the uppermost unit. The Longá Fm. comprises dark shales of the Devonian/Lower Carboniferous representing the oldest rocks exposed in the central uplift. The other three units comprise basically by sandstones.

Data and Results: We used relatively high-resolution aeromagnetic and aerogammaspectrometric data, acquired using a flight line spacing of $500 \mathrm{~m}$ and flight height of $100 \mathrm{~m}$. This portion of the Parnaíba Basin exhibits a conspicuous NE-SW magnetic anomaly related to crystalline basement. The regional magnetic field the same directional trend and it was partially removed by filtering the data, revealing a $15 \mathrm{nT}$ residual magnetic anomaly over the crater still oriented towards NE-SW. We then applied the analytic signal method in order to position the anomaly over the source. The result revealed an anomaly coinciding with the area of occurrence of the the Longá Fm. in the middle of the central uplift, together with other anomalies that can be related to the NW boundary of the central uplift. The Euler deconvolution method [2] was applied to the analytical signal results and showed $1000-1500 \mathrm{~m}$ deep sources under the central uplift possibly related to basement rocks. Gammaspectrometric data provided interesting results related to lithologic/mineralogic characteristics of exposed rocks. The $\mathrm{K}$ channel marked anomalies with high values $(0.5 \%)$ related to all vertical ridges within the central uplift, as well as the boundary and center of the central uplift, whereas the Th channel marked the topographic contrast that defines the annular basin $(4 \mathrm{ppm})$ and the rim $(20 \mathrm{ppm})$. The $\mathrm{U}$ channel shows very well defined boundaries related to the central uplift and rim ( $3 \mathrm{ppm})$. A ternary map is presented in the Fig. 1.

Conclusions: An uncharacteristic high concentration of radioelements is observed mainly into the central uplift. This high concentration might be explained by the absorption of the radioelements by clay minerals and/or their combination with iron oxides, increasing the concentration on the surface. Indeed, high amounts of iron oxides are observed in thin-sections of rocks from the collar and in breccias from the central depression. These conspicuous anomalies suggest that the radioelements were remobilized and combined with oxides and clays, possibly as a result of the circulation of hydrothermal fluids in later stages of the cratering process.

References: [1] Kenkmann T. et al. 2010. Abstract \#1237. 41st Lunar and Planetary Science Conference. [2] Thompson D. T. 1982. Geophysics 47:31-37.

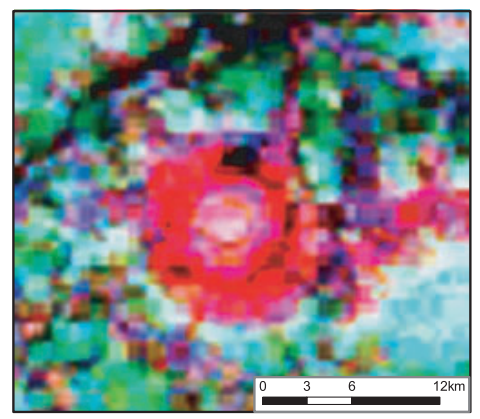

Fig. 1. Ternary aerogammaspectrometric map $(\mathrm{RGB}=\mathrm{K}, \mathrm{Th}, \mathrm{U}$ channels, respectively). 


\section{3}

ACCRETION DISK ORIGIN OF EARTH'S WATER: LABORATORY EXPERIMENTS

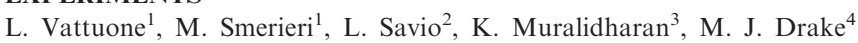
and M. Rocca ${ }^{1}$. ${ }^{1}$ Dipartimento di Fisica dell'Università di Genova and IMEM-CNR Unità operativa di Genova, Via Dodecaneso 33, 16146 Genova, Italy. ${ }^{2}$ IMEM-CNR Unità operativa di Genova, Via Dodecaneso 33, 16146 Genova, Italy. ${ }^{3}$ Department of Materials Sciences and Engineering, University of Arizona, Tucson, AZ 85721, USA. ${ }^{4}$ Lunar and Planetary Laboratory, University of Arizona, Tucson, AZ 85721, USA.

The presence of water on the Earth is an enigma. It is generally agreed that it was too hot at $1 \mathrm{AU}$ for hydrous minerals to be stable in the accretion disk. Thus, Earth's water is conventionally believed to be delivered by comets or wet asteroids after Earth formed. However, wet asteroids and comets have elemental and isotopic properties that are inconsistent with those of Earth [1] limiting the amount of water derived from comets and wet asteroids. Drake [1] proposed that water was introduced during planet growth in the accretion disk in a form stable under high temperature conditions. This hypothesis is supported by the presence of water in the disks around young stars [2] and by numerical simulations of water adsorption on silicate grains under conditions corresponding to those in the accretion disk [3-5] which showed that molecular chemisorption of water on forsterite (a main constituent of the dust grains) might account for the formation of several Earth oceans [4]. Here we show by laboratory experiments (see Figure) that water adsorbs dissociatively on the olivine $\{100\}$ surface at the temperature (approximately $500-1500 \mathrm{~K}$ ) and water pressure (approximately $10^{-8}$ bar) expected for the accretion disk, leaving an $\mathrm{OH}$ adlayer that is stable at least up to $900 \mathrm{~K}$. This high temperature stability may result in the accretion of many Earth oceans to Earth and other terrestrial planets, provided that a viable mechanism to produce water from hydroxyl exists.

References: [1] Drake M. J. 2005. Meteoritics \& Planetary Science 40:519-527. [2] Bethell T. and Bergin E. 2009. Science 326:1675-1677. [3] Stimpfl M., Walker A. M., Drake M. J., de Leeuw N. H., and Deymier P. 2006. Journal of Crystal Growth 294:83-95. [4] Muralidharan K., Deymier P., Stimpfl M., de Leeuw N. H., and Drake M. J. 2008. Icarus 198:400-407. [5] Muralidharan K. and Drake M. J., 2011. Meteoritics \& Planetary Science. This conference.

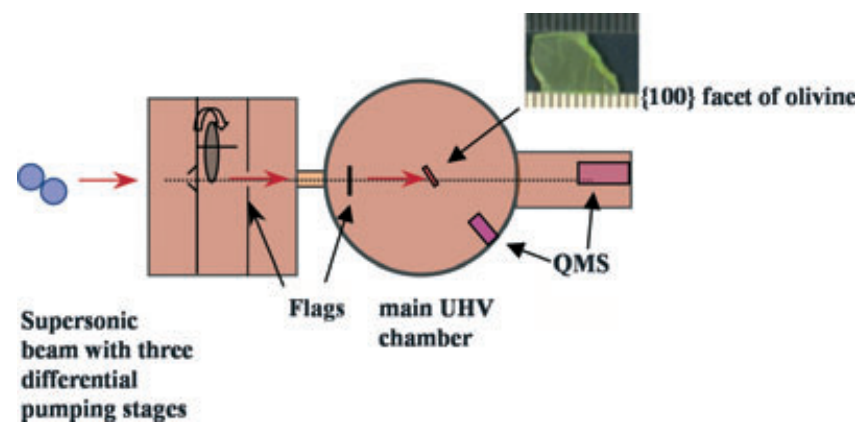

Fig. 1. Schematic drawing of the ultra high vacuum chamber (UHV) and quadrupole mass spectrometer (QMS). Blue filled circles represent water molecules. Green is olivine crystal.

\section{8}

Q NOBLE GASES IN THE ORGUEIL Hf/HCI RESIDUE: A HIGH PRESSURE EXPERIMENT

A. B. Verchovsky ${ }^{1}$, W. Montgomery ${ }^{2}$ and M. A. Sephton ${ }^{2}$. ${ }^{1}$ Open University, Milton Keynes, UK. ${ }^{2}$ Earth Science and Engineering, Imperial College London, South Kensington, SW7 2AZ, UK.

Introduction: The origin of the planetary noble gases (PNG) carrier (Q) in meteorites remains enigmatic. All attempts to separate Q have been unsuccessful in spite of a number of characteristic distinctive features. It is carbonaceous and accounts for a small (approximately 5\%) fraction of the total carbonl in meteorites [1]. Solvation experiments with pyridine [2] suggest that it has an organic structure that can be partially destroyed by the reagent, resulting in a partial loss of PNG. Q is destroyed by oxidizing reagents such as $\mathrm{HNO}_{3}, \mathrm{H}_{2} \mathrm{O}_{2}, \mathrm{HClO}_{4}$ and oxidized in pure oxygen atmosphere at low temperatures $\left(400-600^{\circ} \mathrm{C}\right)$. It is more readily transformed into diamond by shock metamorphism than the other organics [3]. On the other hand it is less susceptible to parent body metamorphism than most of the macromolecular material [4]. In the present study we decided to examine how $\mathrm{Q}$ reacts to high static pressures. For this purpose a $\mathrm{HF} / \mathrm{HCl}$ residue from Orgueil has been taken to high pressure and modest temperature in a diamond-anvil cell (DAC). The treated and original samples then were analyzed for $\mathrm{C}, \mathrm{N}, \mathrm{Ar}$ and $\mathrm{Xe}$ and by micro-Raman.

Experimental: A small aliquot (approximately $10 \mathrm{ng}$ ) of the $\mathrm{HF} / \mathrm{HCl}$ residue was loaded into a membrane-type DAC containing type II diamonds with $500 \mu \mathrm{m}$ culets. A rhenium gasket with a $200 \mu \mathrm{m}$ diameter sample chamber and a preindented thickness of approximately $50 \mu \mathrm{m}$ depth was placed between the diamonds to contain the sample. The sample was compressed to $9.7 \mathrm{GPa}$ and heated at $250-300{ }^{\circ} \mathrm{C}$ for $7 \mathrm{~h}$, then allowed to cool. Pressure was monitored continuously using the ruby florescence method. [5]

$\mathrm{C}, \mathrm{N}$, and noble gases have been analyzed using mass spectrometric multi-element analyzer Finesse at the Open University. The gases were extracted in three combustion steps- 300,700 , and $1000^{\circ} \mathrm{C}$. In the first step most of surface contamination has been removed; at $700{ }^{\circ} \mathrm{C}$ most of the gases from $\mathrm{Q}$ are released, and the last step is required just to confirm that no more gases can be released. Raman spectra have been obtained using the mapping method for areas approximately $20 \times 20 \mu \mathrm{m}$ with resolution of $1 \mu \mathrm{m}$.

Results and Discussion: The Raman spectra of the original and treated sample are similar. They show usual broad D and $G$ bands characteristics of macromolecular material. No diamond feature is seen in the treated sample, which does not necessarily mean that diamond has not been formed under high pressure, but rather that it is small in size and has low abundance.

The noble gas results indicate that ${ }^{36} \mathrm{Ar}$ and ${ }^{132} \mathrm{Xe}$ have been lost during the high pressure expose: $70 \%$ of $\mathrm{Ar}$ and $40 \%$ of Xe. Since at $300{ }^{\circ} \mathrm{C}$ no PNG losses are expected from Q the result suggests that it is the pressure that resulted in the noble gas release.

Conclusions: Static high pressure affects the Q resulting in its structural transformation and loss of noble gases. We plan to treat the samples with variable pressure and more detailed investigations of the pressure-treated material in order to understand the observed effects.

References: [1] Lewis R. S. et al. 1975. Science 190:1251-1262. [2] Marrocchi Y. et al. 2005. Earth and Planetary Science Letters 236:569578. [3] Gobel R. et al. 1978. Journal of Geophysical Research 83:855-867. [4] Verchovsky A. B. et al. 2002. Earth and Planetary Science Letters 199:243-255. [5] Mao H. K., Xu J., and Bell P. M. 1986. Journal of Geophysical Research Solid Earth Planets 91:4673-4676. 


\section{7}

CONCORDANT EARLY SOLAR SYSTEM TIME SCALES FROM Pb-Pb AND EXTINCT CHRONOMETERS

M. Wadhwa, A. Bouvier and G. A. Brennecka. ${ }^{1}$ Arizona State University, Tempe, AZ 85287, USA. E-mail: wadhwa@asu.edu.

In recent years, high-resolution chronometers based on long- and short-lived radionuclides have been applied extensively to toward determining the time scales, with sub-Ma precision, of events that occurred in the early solar system (ESS). However, these chronometers have often yielded discordant ages (e.g., $[1,2])$. Our recent work focuses on highprecision $\mathrm{Pb}-\mathrm{Pb}$ and $\mathrm{Al}-\mathrm{Mg}$ systematics of several meteorites and their components (e.g., [1-4]). We also showed that ${ }^{238} \mathrm{U}^{235} \mathrm{U}$ in meteoritic materials can be variable $[5,6]$. We recently presented a $\mathrm{Pb}-\mathrm{Pb}$ age of $4568.2_{-0.4}^{+0.2}$ Ma for a CAIs from the NWA 2364 CV3 meteorite [3]; although its $\mathrm{U}$ isotopic composition was not measured, the uncertainty in its reported $\mathrm{Pb}-\mathrm{Pb}$ age incorporates possible deviation of its ${ }^{238} \mathrm{U} /{ }^{235} \mathrm{U}$ ratio from the previously assumed value $(=137.88)$. We find that the $\mathrm{Al}-\mathrm{Mg}$ ages using the NWA 2364 CAIs as the anchor agree with those obtained using D'Orbigny as the anchor (Fig. 1), implying that the relatively old age for this CAIs is robust and ${ }^{26} \mathrm{Al}$ was uniformly distributed in the ESS. These $\mathrm{Al}-\mathrm{Mg}$ ages also agree with the $\mathrm{Mn}-\mathrm{Cr}$ and $\mathrm{Pb}-\mathrm{Pb}$ ages (Fig. 1). One exception is Asuka-881394, the $\mathrm{Pb}-\mathrm{Pb}$ age of which is approximately $1 \mathrm{Ma}$ older than its $\mathrm{Al}-\mathrm{Mg}$ and $\mathrm{Mn}-\mathrm{Cr}$ ages. Its $\mathrm{Pb}-\mathrm{Pb}$ age was calculated assuming that its ${ }^{238} \mathrm{U} /{ }^{235} \mathrm{U}$ has the same value as that which we measured in other achondrites [4, 7], which may not be a valid assumption. Nevertheless, Fig. 1 shows that our recent high-precision chronological investigations yield consistent time scales for ESS events such as planetesimal differentiation.

References: [1] Wadhwa M. et al. 2007. Workshop on Chronology of Meteorites and the ESS. Abstract \#4053. [2] Wadhwa M. et al. 2009. Geochimica et Cosmochimica Acta 73:5189-5201. [3] Bouvier A. and Wadhwa M. 2010. Nature Geoscience 3:637-641. [4] Bouvier A. et al. 2011. Geochimica et Cosmochimica Acta (submitted). [5] Bouvier A. et al. 2011. Goldschmidt Conference (submitted). [6] Brennecka G. et al. 2010. Science 327:449-451. [7] Brennecka G. and Wadhwa M. 2011. Goldschmidt Conference (submitted). [8] Sugiura N. and Yamaguchi A. 2007. Abstract \#1431. 38th Lunar and Planetary Science Conference. [9] Shukolyukov A. and Lugmair G. 2008. Abstract \#2094. 39th Lunar and Planetary Science Conference. [10] Shukolyukov A. et al. 2009. Abstract \#1381. 40th Lunar and Planetary Science Conference. [11] McKibbin S. et al. 2010. Abstract \#5172. 73rd Meteoritical Society Meeting.

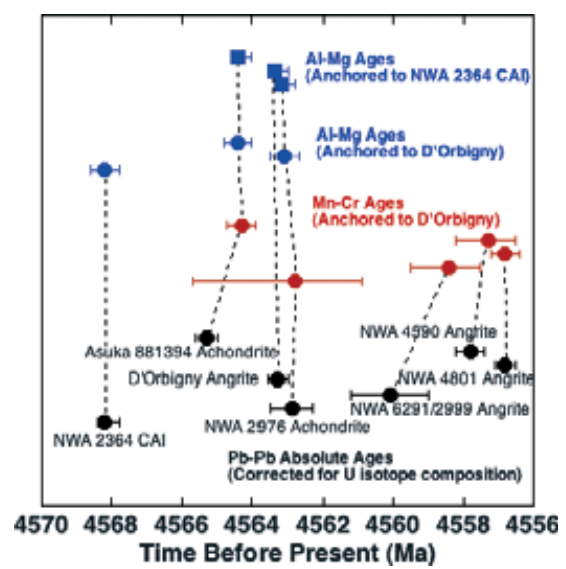

Fig. 1. $\mathrm{Pb}-\mathrm{Pb}$ ages (calculated using measured ${ }^{238} \mathrm{U} /{ }^{235} \mathrm{U}$ ratios for NWA 2976 and the angrites [4, 7], and inferred values for NWA 2364 CAIs and Asuka-881394) compared to $\mathrm{Al}-\mathrm{Mg}$ and $\mathrm{Mn}-\mathrm{Cr}$ ages. $\mathrm{Pb}-\mathrm{Pb}$ and $\mathrm{Al}-\mathrm{Mg}$ data from [2-7] and references therein; $\mathrm{Mn}-\mathrm{Cr}$ data from [2, 8-11].
5324

A LASER PROBE ${ }^{40} \mathrm{Ar} /{ }^{39} \mathrm{Ar}$ INVESTIGATION OF SHERGOTTITE NORTHWEST AFRICA 4797: IMPLICATIONS FOR SHOCK METAMORPHISM PRIOR TO EJECTION

E. L. Walton ${ }^{1,2}$, S. P. Kelley ${ }^{3}$, C. D. K. Herd ${ }^{2}$, A. J. Irving ${ }^{4}$ and T. E. Bunch ${ }^{5}{ }^{1}$ Dept. Phys. Sci., Grant MacEwan University, Canada ${ }^{2}$ Dept. Earth \& Atm. Sci., University of Alberta, Canada. ${ }^{3}$ Dept. Earth Sci., The Open University, UK. ${ }^{4}$ Dept. of Earth \& Space Sci., University of Washington, USA. ${ }^{5}$ Dept. Geol., Northern Arizona University, USA. E-mail: ewalton@ualberta.ca.

Introduction: We report laser probe argon isotope analyses of "lherzolitic" shergottite Northwest Africa (NWA) 4797. The results are discussed in the context of deciphering shock history. NWA 4797 is texturally complex, with strong shock overprinting primary igneous features [1]. Shock effects in olivine and pyroxene include strong mosaicism, grain boundary melting, reduced birefringence, shock blackening, and pervasive fracturing. Maskelynite has not been observed in NWA 4797; rather, precursor igneous plagioclase has been completely melted, vesiculated and recrystallized by shock to form plagioclase microlites in glass. A vein (approximately $1 \mathrm{~mm} ; 10.8 \mathrm{vol} \%$ ) of quenched melt transects the sample, from which skeletal olivine $\left(\mathrm{Fo}_{69-79}\right)$, pyroxene $\left(\mathrm{En}_{44-51} \mathrm{Fs}_{14-18}\right.$, and chromite have crystallized in alkali-rich glass containing spheres of iron sulfide.

Samples, Methods, and Results: A total of 37 extractions were obtained on recrystallized plagioclase, the shock melt vein and igneous groundmass using a New Wave $193 \mathrm{~nm}$ eximer laser ablation system (approximately $70 \mu \mathrm{m}$ diameter melt pits). This system prevents heating outside laser pits, precisely targeting plagioclase and the vein. Corrections for mass spectrometer discrimination, irradiation interferences and cosmogenic ${ }^{36} \mathrm{Ar}$ concentrations have been applied to all argon data following extraction.

Groundmass. Extractions yielded a wide range of ages from $690 \pm 30 \mathrm{Ma}$ to several apparent ages $>4.5 \mathrm{Ga}$. All groundmass analyses yielded ages older than the conventional range for shergottites (approximately 200-600), indicating the presence of excess argon.

Recrystallized Plagioclase. Several young ages were obtained, ranging from $50 \pm 20 \mathrm{Ma}$ to $150 \pm 15 \mathrm{Ma}$. However, many of the plagioclase analyses were contaminated by groundmass, yielding ages up to $2760 \pm 40 \mathrm{Ma}$. These older ages are correlated with the $\mathrm{Ca} / \mathrm{K}$ ratio of the analysis.

Shock melt vein. A transect of laser pits crossing the melt vein showed on average lower ${ }^{40} \mathrm{Ar} /{ }^{36} \mathrm{Ar}$ ratios within the vein in contrast to other shergottites. This is reflected in the mean age of the vein (800 Ma) compared with the groundmass (2600 Ma).

Cosmic Ray Exposure (CRE) ages. A CRE age of $2.2 \pm 0.4 \mathrm{Ma}$ was measured using spallogenic ${ }^{36} \mathrm{Ar}$ and ${ }^{38} \mathrm{Ar}$ production.

Discussion: The CRE age of $2.2 \pm 0.4 \mathrm{Ma}$ approximates the time of impact ejection. We interpret young ages from recrystallized plagioclase to indicate the timing of argon loss during an impact event on Mars. While the youngest age is significantly older than the CRE age, this may reflect partial loss during the impact event, but clearly the ages are younger than conventional crystallization ages for shergottites. The combined lack of a Martian atmospheric signature and significantly younger ages in recrystallized plagioclase is attributed to longer cooling times for this melt. Argon extractions from the shock melt vein show lower ${ }^{40} \mathrm{Ar} /{ }^{36} \mathrm{Ar}$ ratios compared to shock melts in other shergottites. We favor a formation mechanism by injection rather than in situ pore collapse for the shock melt vein. These observations make NWA 4797 a unique shergottite worthy of further study.

Reference: [1] Walton E. L. et al. 2009. Abstract \#1464. 40th Lunar and Planetary Science Conference. 
5234

\section{STABLE ISOTOPES: A NONCARBONACEOUS PEDIGREE FOR UREILITES, IN COMMON WITH ALMOST ALL DIFFERENTIATED PLANETARY MATERIALS}

Paul H. Warren. Institute of Geophysics, UCLA, Los Angeles, CA 90095-1567, USA; E-mail: pwarren@ucla.edu.

The abundant, diverse ureilite meteorites are peridotitic asteroidal mantle restites that have remarkably high bulk carbon contents (average 3 $\mathrm{wt}^{\%} \%$ ) and have long been linked to the so-called carbonaceous chondrites. This term is "somewhat of a misnomer" [1], because the high petrologic type carbonaceous chondrites are, if anything, C-poor compared to ordinary chondrites. But it has long been customary to describe as "carbonaceous" a distinctive set of chondrites that show kinship in various ways without necessarily being carbon-rich. Here, the word carbonaceous is used in that strict, meteoritics-customary sense.

Ureilite oxygen isotopic compositions, i.e., diversely negative $\Delta^{17} \mathrm{O}$, viewed in isolation, have long been viewed as confirming the carbonaceous-chondritic pedigree hypothesis. However, a very different picture emerges through analysis of a compilation of recently published high-precision isotopic data for $\mathrm{Cr}$ [2-8; especially 8], $\mathrm{Ti}$ [9-10] and $\mathrm{Ni}$ [11-13] for ureilites and various other planetary materials (earlier publications by same first authors not cited). Ureilites have lower $\varepsilon^{62} \mathrm{Ni}$ and far lower $\varepsilon^{50} \mathrm{Ti}$ and $\varepsilon^{54} \mathrm{Cr}$ than any known variety of carbonaceous chondrite. On a plot of $\varepsilon^{50} \mathrm{Ti}$ versus $\varepsilon^{54} \mathrm{Cr}$, and similarly $\Delta^{17} \mathrm{O}$ versus $\varepsilon^{54} \mathrm{Cr}$, ureilite compositions cluster far from and in a direction approximately orthogonal to a trend internal to the carbonaceous chondrites, and the carbonaceous chondrites are separated by a wide margin from all other planetary materials. Thus, notwithstanding the impressive resemblance to carbonaceous chondrites in terms of diversely negative $\Delta^{17} \mathrm{O}$, the ureilite precursors accreted from preponderantly noncarbonaceous (sensu stricto) materials.

Despite total depletion of basaltic matter, ureilites retain moderate py (三 pyroxene/[olivine + pyroxene]) ratios, averaging $30 \mathrm{vol} \%$; a result expected from simple partial melting of moderate- $\mathrm{SiO}_{2} /(\mathrm{FeO}+\mathrm{MgO})$ noncarbonaceous chondritic matter, but implying an additional process of major reduction of $\mathrm{FeO}$ if the precursor material were carbonaceouschondritic. The ureilites' diversity in $p y$, silicate-core $m g$ and $\Delta^{17} \mathrm{O}$ implies the parent asteroid accreted a diverse mélange of noncarbonaceous materials.

The striking bimodality of planetary materials on the $\varepsilon^{50} \mathrm{Ti}$ versus $\varepsilon^{54} \mathrm{Cr}$ and $\Delta^{17} \mathrm{O}$ versus $\varepsilon^{54} \mathrm{Cr}$ diagrams may be an extreme manifestation of the effects of episodic accretion of early solids in the protoplanetary nebula. However, an alternative, admittedly speculative, explanation is that the bimodality corresponds to a division between materials that originally accreted in the outer solar system (carbonaceous) and materials that accreted in the inner solar system (noncarbonaceous, including the ureilites).

References: [1] Krot A. N. et al. 2004. In Meteonities, Comets, and Plants, edited by Davis A. M. Treatise on Geochemistry, vol. 1. pp. 83128. [2] Yamashita K. et al. 2005. NIPR Symposium Antarctica Meteorite 100. [3] Shukolyukov A. et al. 2011. Abstract \#1527. 42nd Lunar and Planetary Science Conference. [4] Ueda T. et al. 2006. NIPR Symposium Antarctica Meteorite 117. [5] Trinquier A. et al. 2008. Geochimica et Cosmochimica Acta 72:5146. [6] de Leuw S. et al. 2010. Abstract \#2703. 41st Lunar and Planetary Science Conference. [7] Qin L. et al. 2010. Meteoritics \& Planetary Science 45:1771. [8] Yamakawa A. et al. 2010. The Astrophysical Journal 720:150. [9] Leya I. et al. 2008. Earth and Planetary Science Letters 266:233. [10] Trinquier A. et al. 2009. Science 324:374. [11] Regelous M. et al. 2008. Earth and Planetary Science Letters 272:330. [12] Dauphas N. et al. 2008. The Astrophysical Journal 686:560.

[13] Quitte G. et al. 2010. The Astrophysical Journal 720:1215.
5230

NORTHWEST AFRICA 6693, A NEAR-ULTRAMAFIC ACHONDRITE WITH LOW (0.6 $\times$ CHONDRITIC) $\mathrm{Mg} / \mathrm{Si}, \quad \mathrm{Na}-$ FELDSPAR, Ni-RICH Fo50 OLIVINE AND LOW $\Delta^{17} \mathrm{O}$

P. H. Warren ${ }^{1}$, A. E. Rubin ${ }^{1}$, J. Isa ${ }^{1}$, E. Thompson ${ }^{2}$, I. Ahn ${ }^{3}$ and B-G. Choi ${ }^{4}$. ${ }^{1}$ Institute of Geophysics, UCLA, Los Angeles, CA, USA; E-mail: pwarren@ucla.edu 22Lake Oswego, OR, USA. ${ }^{3}$ Korea Polar Research Institute, Korea, Japan. ${ }^{4}$ Earth Science Education, Seoul National University, Korea, Japan.

The NWA 6693 meteorite was found as a single $5.1 \mathrm{~kg}$ stone. Much of its surface is weathered, but the many scattered metal grains in the interior are rust-free. This may be partly a consequence of the metal composition, which clusters tightly at (by weight) $\mathrm{Ni}_{81} \mathrm{Fe}_{19} \mathrm{Co}_{0.7}$. A mode shows (in vol\%) pyroxene 70, olivine 16, feldspar 13, Cr-spinel 0.6, metal 0.4 , with traces of Ni-rich sulfides and merrillite. Pyroxene composition clusters tightly at $\mathrm{En}_{57} \mathrm{WO}_{3.3}$ and $\mathrm{FeO} / \mathrm{MnO}=90$; with scattered $\mathrm{En}_{40} \mathrm{WO}_{40}$ lamellae rarely (where blebby) large enough for EPMA. Olivine clusters tightly at $\mathrm{Fo}_{50}$, with $0.77 \mathrm{wt} \% \mathrm{NiO}$ (approximately $10 \%$ of the bulk rock's $\mathrm{Ni}$ occurs as $\mathrm{NiO}$ in olivine). The feldspar is $\mathrm{Ab}_{92} \mathrm{Or}_{2.5}$, except for rare grains of $\mathrm{Ab}_{57} \mathrm{Or}_{42}$. The spinel composition (in $\mathrm{wt} \%$ ) is 57.8 $\mathrm{Cr}_{2} \mathrm{O}_{3}, 32.0 \mathrm{FeO}$, approximately $2.1 \mathrm{Fe}_{2} \mathrm{O}_{3}, 2.8 \mathrm{TiO}_{2}, 1.7 \mathrm{MgO}$.

The igneous (no relict chondrules) texture is hard to categorize. The dominant pyroxene includes an oikocryst $5 \mathrm{~mm}$ across, enclosing chadacrysts of both olivine and feldspar. However, most pyroxenes are nonpoikilitic and $<2 \mathrm{~mm}$. Most olivines are blocky and $0.5-1 \mathrm{~mm}$; some grains have embayed shapes and a few grains exhibit a mantle of pyroxene. The feldspar appears interstitial, yet separated outcrops exhibit optical continuity over distances as great as $2.5 \mathrm{~mm}$. In places the feldspar wraps around small mafic silicates; cf. "pincer" textures observed [1] in impact-melted but essentially undifferentiated chondrites.

The bulk oxygen isotopic composition, determined by replicate laser-fluorination at KOPRI, averages $\delta^{18} \mathrm{O}=4.32 \%, \delta{ }^{17} \mathrm{O}=1.19 \%$ o and $\Delta^{17} \mathrm{O}=-1.08 \%$. These data are in principle consistent with the acapulcoite-lodranite clan [2], but in those meteorites the mafic silicates are highly magnesian, and feldspars have lower $\mathrm{Na} / \mathrm{Ca}$. The ungrouped LEW 88763, with $\Delta^{17} \mathrm{O}=-1.2 \%$ o $[2,3]$, is a closer precedent to NWA 6693, but LEW 88763 consists mainly of olivine, its mafic silicates have higher $m g$ (e.g., $\mathrm{Fo}_{65}$ ) and its feldspars range to $\mathrm{Ab}_{55} \mathrm{An}_{44}$. Several $\mathrm{CH} /$ CB chondrites are also similar in O-isotopes to NWA 6693 [e.g., 4].

The bulk composition is in most respects close to chondritic. Two approximately $360 \mathrm{mg}$ chips were analyzed by INAA and FB-EPMA, representing (A) fresh interior and (B) material including some rusty brown alteration. Highly siderophile elements (Os, Ir and $\mathrm{Au}$ ) and $\mathrm{Ni}$ are essentially chondritic in (A); in (B) they are $0.3 \times$ chondritic, except $\mathrm{Ni}$, $0.5 \times$. The REE pattern of $(\mathrm{A})$ is sloped from $0.8 \times \mathrm{CI}$ at $\mathrm{La}-\mathrm{Sm}$, to $0.4 \times \mathrm{CI}$ at $\mathrm{Lu}$, with a $(+) \mathrm{Eu}$ anomaly $(1.5 \times \mathrm{CI})$. Ga/Al $(w t)=0.00016$. The numerically greatest divergence from typical chondritic composition is shown by highly volatile elements; e.g., $\mathrm{Zn}$ in (A) is $<0.02 \times \mathrm{CI}$. The most significant divergence, however, is that the mafic silicates are so ferroan, bulk $\mathrm{Mg} / \mathrm{Si}(0.48$, by wt.) is only about $0.6 \times$ chondritic; and no juggling of the olivine/pyroxene ratio (to model vagaries of crystal accumulation) can shift $\mathrm{Mg} / \mathrm{Si}$ to $>0.8 \times$ chondritic. Thus, in contrast with "primitive achondrites," $\mathrm{Mg} / \mathrm{Si}$ indicates NWA 6693 is no restite. The origin of this unique material is enigmatic.

References: [1] Rubin A. E. and Jones R. H. 2003. Meteoritics \& Planetary Science 38:1507-1520. [2] Greenwood R. C. et al. 2007. Abstract \#2163. 38th Lunar Prospector Spacecraft. [3] Swindle T. D. et al. 1998. Meteoritics \& Planetary Science 33:31-48. [4] Ivanova M. A. et al. 2008. Meteoritics \& Planetary Science 43:915-940. 


\section{9}

A TALE OF TWO CRATERS: (U-Th)/He DATING AND MODELING OF AGE RESETTING OF TWO SMALL/LOW ENERGY IMPACT STRUCTURES

J-A. Wartho ${ }^{1}$, M. C. van Soest ${ }^{1}$, I. Ukstins Peate ${ }^{2}$, D. T. King Jr. ${ }^{3}$, L. W. Petruny $^{3}$ and K. V. Hodges ${ }^{1}$. ${ }^{1}$ School of Earth and Space Exploration, Arizona State University, PO Box 871404, Tempe, AZ 85287, USA. ${ }^{2}$ Department of Geoscience, University of Iowa, Iowa City, IA 52242, USA. ${ }^{3}$ Department of Geology and Geography, Auburn University, Box 870338, Auburn, AL 36849, USA.

Introduction: (U-Th)/He single apatite and zircon crystal dating has been successfully undertaken on two small/low-energy impact structures: Wetumpka (a $\sim 5 \mathrm{~km}$ diameter shallow-marine structure [1]) and Monturaqui (a $350 \mathrm{~m}$ diameter structure [2]). A range of ages was obtained, but the youngest cluster of apatite/zircon ages yielded impact ages of $84.4 \pm 1.4(2 \sigma)$ for Wetumpka [3] and $663 \pm 90 \mathrm{Ka}(95 \%$ confidence) for Monturaqui [2]. These (U-Th)/He ages are in agreement with previous stratigraphic [1] and cosmogenic/palaeomagnetic ages [4-5].

However, the target rocks of these two small size/low energy craters experienced heterogeneous and relatively low shock metamorphism conditions of 5-15 GPa (Wetumpka [6]) and < 10-65 GPa (Monturaqui [7]) that relate to temperatures of $100-150{ }^{\circ} \mathrm{C}$ and $100->1500{ }^{\circ} \mathrm{C}$, respectively [8]. Therefore, how did the radiogenic ${ }^{4} \mathrm{He}$ in $\sim 10 \%$ of the apatite and zircon crystals we have analyzed become reset during these short-lived impact events?

Mathematical modeling of He diffusion in apatite and zircon has been undertaken to better understand how these grains can become reset during impact events, and how the (U-Th)/He technique can be successfully applied to the dating of small impact structures.

Results: Mathematical modeling of the \% of He diffusion loss from apatite and zircon crystals, has shown that (a) short, but high temperature fireball [e.g., 9], (b) very localized short high shock metamorphism events [e.g., 10], (c) longer duration but lower temperature hydrothermal events [e.g., 11] or (d) a combination of all three processes $(\mathrm{a}-\mathrm{c})$ are all capable of resetting (U-Th)/He ages in apatite and zircon grains during the formation of low energy or small impact structures.

Conclusions: As the He diffusion modeling shows, the unique fast diffusion properties of $\mathrm{He}$ in U-bearing minerals (e.g., apatite and zircon) make the low-temperature (U-Th)/He geochromometer a powerful tool for dating impact structures, especially low energy and small impact structures.

References: [1] King D. T. Jr. et al. 2006. Meteoritics \& Planetary Science 41:1625-1631. [2] Ukstins Peate I. et al. 2010. Abstract \#2161. 41st Lunar and Planetary Science Conference. [3] Wartho J.-A. et al. 2011. Abstract \#1524. 42nd Lunar and Planetary Science Conference. [4] Valenzuela M. 2008. Jaresbericht Annual Report 27. [5] Valenzuela M. 2008. Abstract \#5185. 72nd Meteoritical Society. [6] Neathery T. L. et al. 1976. Geological Society of America Bulletin 87:567-573. [7] Bunch T. E. and Cassidy W. A. 1972. Contributions to Mineralogy \& Petrology 36:95112. [8] French, B. M. 1998. Traces of Catastrophe. Lunar and Planetary Institute. 120 p. [9] Collins G. S. et al. 2005. Meteoritics \& Planetary Science 40:817-840. [10] Biren M. B. et al. 2010. Abstract \#2314. 41st Lunar and Planetary Science Conference. [11] Jõeleht A. et al. 2005. Meteoritics \& Planetary Science 40:21-33.

\section{1}

\section{COOLING RATES OF IVA AND IIIAB METEORITES}

J. T. Wasson and P. Hoppe. ${ }^{1}$ University of California, Los Angeles, CA, USA. E-mail: jtwasson@ucla.edu. ${ }^{2}$ Max-Planck Institut f. Chemie, Mainz, Germany.

Introduction: A major puzzle in iron meteorite research is why metallographic cooling rates (MCR) show wide ranges (factor of 60 in group IVA [1], factor of 6 in group IIIAB [2]) within fractionally crystallized (magmatic) iron-meteorite groups, and why MCR correlate with compositions. Possible answers are that essentially all the insulating silicates had been stripped from the metallic cores [3] or that there are effects associated with composition or impact histories that are not properly treated in the conversion of phase-composition data to MCR [4]. In an attempt to better assess relative cooling rates within these groups we developed a new method based on $\mathrm{Co} / \mathrm{Ni}$ ratios in kamacite and taenite; our preliminary results indicate comparatively little variation in cooling rates within IVA and IIIAB.

Method: Our approach is based on the variation with temperature of the double ratio $(\mathrm{Co} / \mathrm{Ni}) \alpha /(\mathrm{Co} / \mathrm{Ni}) \gamma[5]$. We call this ratio $\mathrm{R} \alpha \gamma$; it ranges from approximately 3 at $1000 \mathrm{~K}$ to approximately 30 at $720 \mathrm{~K}$. Thus, the lower the cooling rate, the higher the value of Ray.

Experimental: We used the MPIC NanoSIMS to determine Co in kamacite and taenite; the spatial resolution was about $0.5 \mu \mathrm{m}$ and the sampling depth $<0.1 \mu \mathrm{m}$. By measuring the ratio near the interface we focused on the low-temperature record and minimized the effects of factors that may have affected the systems at high temperatures in the $\alpha+\gamma$ field. Our pilot study consisted of IVA irons Bishop Canyon and Duchesne with MCR differing by a factor of 25 and IIIAB irons Haig and Cumpas with MCR differing by a factor of 4.5 .

The IVA irons have similar R $\alpha \gamma$ values of 22-23 implying that they cooled at rates that differ by less than a factor of $3(2 \sigma)$. The R $\alpha \gamma$ in IIIAB Cumpas was similar (approximately 21), but that in Haig was approximately 29 indicating that it cooled several times slower, in contrast to the Haig MCR which is $4.5 \times$ higher than that for Cumpas [2].

Discussion: To help assess the reason for the contrasting IIIAB cooling rates we reexamined the MCR results [2]; to help eliminate possible high-temperature effects we limited the comparison to narrow taenite lamellae (halfwidths between 4 and $10 \mu \mathrm{m}$ ). We discovered that the Haig data distribution is anomalous and Bella Roca partly anomalous and that the central $\mathrm{Ni}$ contents in the other 11 irons were unresolvable; irons differing in MCR by a factor of 6 showed the same central $\mathrm{Ni}$ contents implying identical cooling rates at low temperatures. We infer that the anomalous behavior of Haig in both studies reflects shock effects.

Summary: Our preliminary study using a new cooling rate method indicates no difference in cooling rates between two IVA irons with MCR differing by a factor of 25 . Our results imply that the Haig IIIAB iron cooled more slowly than Cumpas, in contrast to MCR data showing Haig to have cooled $4 \times$ faster. We suggest that this reflects shock damage in Haig that created tiny fractures in the taenite. These results cast doubt on published cooling rates showing large variations in groups IVA and IIIAB.

References: [1] Yang J. et al. 2008. Geochimica et Cosmochimica Acta 72:3043. [2] Yang J. and Goldstein J. 2006. Geochimica et Cosmochimica Acta 70:3197. [3] Yang J. et al. 2007. Nature 446:888. [4] Wasson J., Matsunami Y., and Rubin A. 2006. Geochimica et Cosmochimica Acta 70:3149. [5] Widge S. and Goldstein J. 1977. Metallurgical and Materials Transactions A 8:309. 
5529

ANALYSES OF TWO MARTIAN METEORITES FOR THE RAMAN LASER SPECTROMETER ONBOARD EXO MARS

I. Weber ${ }^{1}$, U. Böttger ${ }^{2}$, E.K. Jessberger ${ }^{1}$ and H. W. Hübers ${ }^{2,3}$. ${ }^{1}$ Institute of Planetology, Muenster, Germany. E-mail: sonderm@uni-muenster.de. ${ }^{2}$ Department of Experimental Planetary Physics, Institute of Planetary Research, German Aerospace Center, Berlin, Germany. ${ }^{3}$ Institut für Optik und Atomare Physik, Technische Universität Berlin, Berlin, Germany.

Introduction: Exo Mars is the first mission in ESA's Aurora Exploration Programme, which will start 2018. The Raman Laser Spectrometer (RLS) onboard Exo Mars will perform measurements on Mars to identify mineral products and organic compounds. In preparation for the data from Mars we investigate Martian meteorites as well as analogue materials with a Raman instrument working under "real" conditions. For a first complete mineralogical identification the material is investigated by light microscopy and SEM. Afterwards Raman analyses at room temperature and in Martian-like conditions were made. Here we present the results of these measurements on two Martian meteorites.

Measurements and Experimental Setup: The Raman measurements are performed with a confocal Raman microscope Witec alpha300 R system at room temperature under air at ambient pressure. The Raman laser excitation wavelength is $532 \mathrm{~nm}$. The spectral resolution of the spectrometer is $4-5 \mathrm{~cm}^{-1}$. A Nikon $10 \times$ objective is used. The spot size on the sample in focus is about $1.5 \mu \mathrm{m}$. The laser power of $1 \mathrm{~mW}$ on the sample is chosen to represent the RLS instrument on Exo Mars.

Samples: The two Martian meteorites DaG 670 [1] and SAU 051 [2] are basaltic shergottites consisting of pyroxenes, maskelynite and relict plagioclase as well as of olivines. They were chosen for the investigations because the basaltic shergottites are the most likely candidate rocks to be found on the surface on Mars.

Results: Sections of both meteorites were scanned with light microsopy and eight regions of interest each are selected for detailed SEM and Raman investigations. The areas are marked with an $100 \times 100 \mu \mathrm{m}$ "L" using laser ablation. All regions were mapped with a JEOL JSM6610LV SEM working in low-vacuum to avoid artefacts in the Raman spectra from carbon coating. The Raman measurements are in progress and the results will be presented.

References: [1] Wadhwa M. et al. 2001. Meteoritics \& Planetary Science 36:195-208. [2] Gnos E. et al. 2002. Meteoritics \& Planetary Science 37:835-854.
5357

MAJOR-ELEMENT ABUNDANCES OF SURFACE MATERIALS ON MERCURY: FIRST RESULTS FROM THE MESSENGER X-RAY SPECTROMETER

S. Z. Weider ${ }^{1}$, L. R. Nittler ${ }^{1}$, R. D. Starr ${ }^{2}$, W. V. Boynton ${ }^{3}$, L. G. Evans $^{4}$, J. O. Goldsten ${ }^{5}$, T. J. McCoy ${ }^{6}$, R. L. McNutt Jr. ${ }^{5}$, C. E. Schlemm II ${ }^{5}$, S. C. Solomon ${ }^{1}$ and A. L. Sprague ${ }^{3}$. ${ }^{1}$ Carnegie Institution of Washington, Washington, DC 20015, USA. E-mail: sweider@ ciw.edu. ${ }^{2}$ Catholic University, Washington, DC 20064, USA. ${ }^{3}$ University of Arizona, Tucson, AZ 85721, USA. ${ }^{4}$ Computer Sciences Corporation, Lanham-Seabrook, MD 20706, USA. ${ }^{5}$ Johns Hopkins University, Applied Physics Laboratory, Laurel, MD 20723, USA. ${ }^{6}$ Smithsonian Institution, Washington, DC 20013, USA.

Introduction: MESSENGER became the first spacecraft to orbit Mercury on 18 March 2011. One of the mission's major scientific objectives is to characterize the chemical composition of Mercury's surface in order to address key questions on the formation and geologic history of the least-explored, innermost planet [1]. The X-Ray Spectrometer (XRS) on MESSENGER [2] employs the technique of planetary X-ray fluorescence (XRF) to estimate quantitatively the abundances of major rock-forming elements (e.g., $\mathrm{Mg}, \mathrm{Al}, \mathrm{Si}, \mathrm{S}, \mathrm{Ca}, \mathrm{Ti}$, and $\mathrm{Fe}$ ) on Mercury's surface. The instrument measures characteristic Xrays that are excited by the incident (and variable) flux of solar X-rays from within the top approximately $100 \mu \mathrm{m}$ of the regolith.

Preliminary Results: Preliminary analysis of XRS data acquired during solar flare events indicates that Mercury's crust is substantially richer in $\mathrm{Mg}$ and poorer in $\mathrm{Al}$ and $\mathrm{Ca}$ than both lunar crustal rocks and terrestrial basalts. The derived $\mathrm{Mg} / \mathrm{Si}, \mathrm{Al} / \mathrm{Si}$, and $\mathrm{Ca} / \mathrm{Si}$ ratios lie within the range of terrestrial ultramafic rocks. Analysis of "quiet Sun" data gives $\mathrm{Mg}, \mathrm{Al}$, and $\mathrm{Si}$ abundances consistent with the flare data. The flare spectra also indicate low total concentrations of $\mathrm{Ti}(<1 \mathrm{wt} \%)$ and $\mathrm{Fe}$ (< approximately $5 \mathrm{wt} \%$ ) and a surprisingly high $\mathrm{S}$ abundance $(\mathrm{S} / \mathrm{Si}$ approximately 0.1 ) in surface materials on Mercury.

Discussion: In order to explain Mercury's high density and metal/ silicate ratio [3], it has been suggested that a primary crust and much of the original mantle may have been stripped away, e.g., by a giant impact event $[4,5]$. The $\mathrm{Mg} / \mathrm{Si}, \mathrm{Al} / \mathrm{Si}$, and $\mathrm{Ca} / \mathrm{Si}$ data reported here indicate a relatively low abundance of plagioclase feldspar, perhaps consistent with this model. However, models [e.g., 6] whereby Mercury originally formed from highly reduced (e.g., enstatite chondrite) material may also explain our elemental data. Melting of such precursory material produces basaltic and metal-rich ( $\mathrm{Fe}, \mathrm{Ni}-\mathrm{FeS})$ melts, leaving an ultramafic (mainly enstatite) residuum. Melting experiments [6] on material from the EH-chondrite Indarch have been used to suggest a "synthetic Mercurian basalt" composition whose $\mathrm{Mg} / \mathrm{Si}, \mathrm{Al} / \mathrm{Si}, \mathrm{Ca} / \mathrm{Si}$, and $\mathrm{S} / \mathrm{Si}$ ratios lie within the range seen by MESSENGER, although even the relatively small amount of $\mathrm{Fe}$ seen is higher than predicted by the melting experiments. The relatively low apparent $\mathrm{Fe}$ and $\mathrm{Ti}$ abundances reported here indicate that these elements are not responsible for the thermal neutron absorption observed during MESSENGER's Mercury flybys [7].

References: [1] Solomon S. C. et al. 2001. Planetary and Space Science 49:1445-1465. [2] Schlemm C. E. et al. 2007. Space Science Reviews 131:393-415. [3] Lewis J. S. 1972. Earth and Planetary Science Letters 15:286-290. [4] Wetherill G. W. 1988. In: Mercury, University Arizona Press. pp. 670-691. [5] Benz W. et al. 1988. Icarus 74:516-528. [6] Burbine T. H. et al. 2002. Meteoritics \& Planetary Science 37:1233-1244. [7] Lawrence, D. J. et al. 2010. Icarus 209:195-209. 
5483

COSMOGENIC RADIONUCLIDES IN ANTARCTIC HOWARDITES: TERRESTRIAL AGES AND PAIRING

K. C. Welten ${ }^{1}$, K. Nishiizumi ${ }^{1}$ and M. W. Caffee ${ }^{2}$. ${ }^{1}$ Space Sciences Laboratory, University of California, Berkeley, CA 94720, USA. E-mail: kcwelten@ssl.berkeley.edu. ${ }^{2}$ Department of Physics, Purdue University, West Lafayette, IN 47907, USA.

Introduction: In anticipation of the upcoming visit of the Dawn spacecraft to asteroid 4 Vesta, there is renewed interest in howardites, which are believed to dominate the surface of 4 Vesta. The Antarctic meteorite collection contains more than 100 howardites, including several showers, such as the Pecora Escarpment (PCA) 02009 pairing group [1, 2]. The identification of paired howardites is important to understand their heterogeneity on a $\mathrm{cm}$ to $\mathrm{m}$ scale. We have previously shown that cosmogenic radionuclides can help to identify paired HED meteorites, even when the individual samples show a wide range in composition [2]. In this work we present concentrations of cosmogenic ${ }^{10} \mathrm{Be}$ (halflife $=1.36 \mathrm{Myr}), \quad{ }^{26} \mathrm{Al} \quad(0.705 \mathrm{Myr})$, and ${ }^{36} \mathrm{Cl} \quad(0.301 \mathrm{Myr})$ in 24 howardites and 3 brecciated eucrites from the Antarctic meteorite collection.

Experimental Procedures: We dissolved howardite samples of 50 $150 \mathrm{mg}$ in $\mathrm{HF} / \mathrm{HNO}_{3}$. After dissolution, small aliquots of the dissolved samples were taken for chemical analysis. We separated $\mathrm{Be}, \mathrm{Al}$, and $\mathrm{Cl}$ and measured the radionuclide concentrations by accelerator mass spectrometry at Purdue's PRIME Lab.

Results and Discussion: Chemical analysis of the samples shows a wide range in bulk compositions with $\mathrm{Al}$ ranging from 1 to $7 \mathrm{wt} \%, \mathrm{Ca}$ from 2 to $8 \mathrm{wt} \%$, and $\mathrm{Mg}$ from 4 to $15 \mathrm{wt} \%$. The bulk composition of Mt. Pratt (PRA) 04401 is consistent with a large contribution of CM-type material $[3,4]$.

Concentrations of cosmogenic ${ }^{10} \mathrm{Be}\left(15-25 \mathrm{dpm} \mathrm{kg}^{-1}\right)$ and ${ }^{26} \mathrm{Al}(52-$ $109 \mathrm{dpm} \mathrm{kg}{ }^{-1}$ ) indicate that all howardites were exposed in space long enough ( $>5 \mathrm{Myr}$ ) to reach $>90 \%$ of ${ }^{10} \mathrm{Be}$ saturation level. The variations in ${ }^{10} \mathrm{Be}$ and ${ }^{26} \mathrm{Al}$ are mainly due to differences in shielding, although the high ${ }^{26} \mathrm{Al}$ concentration $\left(109 \mathrm{dpm} \mathrm{kg}^{-1}\right)$ in MET 96500 may be due to production of ${ }^{26} \mathrm{Al}$ by solar cosmic rays. Concentrations of cosmogenic ${ }^{36} \mathrm{Cl}$ range from 4 to $12 \mathrm{dpm} \mathrm{kg}^{-1}$, with the exception of PRA 04401, with shows a value of $24 \mathrm{dpm} \mathrm{kg}$. The high ${ }^{36} \mathrm{Cl}$ concentration in PRA indicates a large contribution from neutron-capture ${ }^{36} \mathrm{Cl}$, most likely in the $\mathrm{Cl}$-containing $\mathrm{CM}$ clasts. The remaining variations in ${ }^{36} \mathrm{Cl}$ are due to a combination of differences in (i) the concentration of $\mathrm{Ca}$, a major target element for the production of ${ }^{36} \mathrm{Cl}$, (ii) shielding effects as a function of size and depth, and (iii) the terrestrial age. Using the relationship between the ${ }^{36} \mathrm{Cl}$ production rate from $\mathrm{Ca}$ and the ${ }^{26} \mathrm{Al}$ production rate [5], we derive terrestrial ages up to $\sim 250 \mathrm{kyr}$ for the Antarctic howardites. Based on the radionuclide results, we identified one pairing group at Miller Range (MIL) and one at Queen Alexandra Range (QUE), the latter including both howardites and diogenites. The radionuclide results can also be used to constrain the CRE histories of howardites when combined with recent cosmogenic noble gas results [6].

Acknowledgments: We thank the Meteorite Working Group for providing the meteorite samples.

References: [1] Beck A. et al. 2007. Meteoritics \& Planetary Science 42:A17. [2] Welten K. et al. 2009. Meteoritics \& Planetary Science 44:A216. [3] Mittlefehldt D. et al. 2011. Abstract \#2569. 42nd Lunar and Planetary Science Conference. [4] Herrin J. et al. 2011. Abstract \#2806. 42nd Lunar and Planetary Science Conference. [5] Leya I. and Masarik J. 2009. Meteoritics \& Planetary Science 44:1061-1086. [6] Cartwright J. et al. 2011. Abstract \#2655. 42nd Lunar and Planetary Science Conference.
5274

REVERSIBLE SAMPLE PREPARATION OF SMALL PARTICLES IN POTTED BUTTS FOR SIMS ANALYSES

A. J. Westphal ${ }^{1}$, A. Butterworth ${ }^{1}$, Z. Gainsforth ${ }^{1}$, G. R. Huss ${ }^{2}$, K. Nakashima $^{2}$, R. C. Ogliore ${ }^{2}$ and J. Stodolna ${ }^{1}{ }^{1}$ Space Sciences Laboratory, U. C. Berkeley, Berkeley, CA 94720, USA. E-mail: westphal@ssl.berkeley.edu. ${ }^{2}$ Hawai'i Institute of Geophysics and Planetology, University of Hawaii at Mãnoa, 1680 East-West Road, POST 504, Honolulu, HI 96822, USA.

Introduction: With the return of Stardust samples the cosmochemistry community faced the novel challenge of conducting coordinated analyses on a suite of extraterrestrial samples that are simultaneously extremely tiny (nanogram to picogram), extremely limited, and extremely complex and fine-grained. This has required the development of novel techniques [1]. Current techniques generally make efficient use of the samples [2], but isotopic analyses have required a sample preparation technique that requires contact with the sample and modification of the potted butt. Here we describe an easily reversible sample preparation technique for SIMS measurements that facilitates multiple rounds of TEM, STXM and isotopic analyses, thus making full use of rare particles. We anticipate that this technique will be appropriate for Hayabusa samples from 25143 Itokawa, which share the same challenging properties as Stardust samples.

Silicon Nitride Window Mounting Protocols for SIMS: The most common sample-preparation protocol for Stardust samples is to embed particles in epoxy and to use an ultramicrotome to produce multiple $100 \mathrm{~nm}$-thick sections for TEM or STXM, and a potted butt that usually contains the remaining $70-90 \%$ of the original particle. Further rounds of ultramicrotomy can be done on the potted butt if it is preserved as a cylindrical "bullet." To achieve sufficiently high precision to place samples on the solar-system three-isotope oxygen plot, measurements must be made in the bulk grain in the potted butt. We developed a reversible SIMS sample preparation technique as part of our coordinated analysis of the particle Iris [3-5]. X-ray fluorescence microprobe analysis showed that Iris is a complex particle with several phases not uncovered in the first rounds of ultramicrotomy. This technique involves no physical contact with the sample or modification of the potted butt. The mount consists of two components: a Cameca ims 1280-compatible Al round which holds the potted butt at a fixed altitude, and a "buckler," which simultaneously surrounds the particle with a flat conductive plane, matching the altitude of the particle, and integrates a ring of 12 standards that closely match the known phases in the particle and that surround the particle. The buckler consists of a thin Al disk with embedded standards, polished to a mirror finish, with a central $3 \mathrm{~mm}$ hole. We mounted a Aucoated, $500 \mathrm{~nm}$-thick $\mathrm{Si}_{3} \mathrm{~N}_{4}$ membrane in which we had previously drilled a $300 \mu \mathrm{m}$ diameter hole using an ion mill, over the hole in Al disk. We used a motorized, encoded micromanipulator with $200 \mathrm{~nm}$ resolution (Sutter MP-285) to integrate the complete assembly, since $\sim 1-\mu \mathrm{m}$ precision is required in the three-dimensional alignment of the $\mathrm{Si}_{3} \mathrm{~N}_{4}$ window with the particle. Using San Carlos olivine standards in potted butts, we have shown that $\mathrm{O}$-isotopes are measured with $\sim 2 \%$ precision $(2 \sigma)$ in $\Delta^{17} \mathrm{O}$ using this technique[5].

References: [1] Westphal A. J. et al. 2004. Meteoritics \& Planetary Science 39:1375. [2] Zolensky M. et al. 2000. Meteoritics \& Planetary Science 35:9. [3] Gainsforth Z. et al. 2011. Meteoritics \& Planetary Science 46. This conference. [4] Ogliore R. et al. 2011. Meteoritics \& Planetary Science 46. This conference. [5] Huss G. R. et al. 2011 Meteoritics \& Planetary Science 46. This conference. 


\section{4}

ANALYSIS OF TITANIUM ISOTOPE RATIOS IN REFRACTORY INCLUSIONS BY LA-MC-ICP-MS

C. D. Williams ${ }^{1}$, M. Wadhwa ${ }^{1}$, P. E. Janney ${ }^{1}$, R. R. Hines ${ }^{1}$, E. S. Bullock $^{2}$ and G. J. MacPherson ${ }^{2} .{ }^{1}$ School of Earth and Space Exploration, Arizona State University, Tempe, AZ, USA. E-mail: Curtis.Williams@asu.edu. ${ }^{2}$ US National Museum of Natural History, Smithsonian Institution, Washington, DC, USA.

Introduction: Calcium-aluminum-rich inclusions (CAIs) with fractionation and unknown nuclear (FUN) effects preserve a record of large isotopic heterogeneities at small spatial scales in the early solar system (ESS). Constraining the origin of these isotopic heterogeneities and their temporal and spatial distribution is critical for understanding stellar inputs into the solar nebula and the conditions that prevailed during the earliest epoch of Solar System formation. Thus far, no absolute dates have been determined for any FUN CAIs and there is little to no material remaining from previously studied ones for chronological investigations. As such, it is important to locate new FUN CAIs so that their distinctive isotopic signatures can be placed in temporal context in the ESS.

The FUN CAIs preserve large anomalies in several neutron-rich isotopes including ${ }^{50} \mathrm{Ti}$ (e.g., $\left.[1,2]\right)$. Therefore, the titanium isotope system is well suited for the identification of these objects. Furthermore, laser ablation multicollector inductively coupled plasma mass spectrometry (LA-MC-ICP-MS) allows precise, yet rapid analysis of $\mathrm{Ti}$ isotopic compositions, making this technique suitable for analyzing a large number of CAIs to search for ones with FUN effects. Here we report preliminary results of titanium isotope ratio measurements of two coarsegrained Allende CAIs by LA-MC-ICP-MS.

Analytical Technique: Titanium isotope ratios were measured with the Thermo Finnigan Neptune MC-ICP-MS at ASU. The isotope ratios were measured in two peak jumping steps: ${ }^{44} \mathrm{Ca},{ }^{46} \mathrm{Ti},{ }^{47} \mathrm{Ti},{ }^{48} \mathrm{Ti},{ }^{49} \mathrm{Ti},{ }^{50} \mathrm{Ti}$ (step 1) and ${ }^{49} \mathrm{Ti},{ }^{51} \mathrm{~V},{ }^{53} \mathrm{Cr}$ (step 2). All analyses were made in mediumresolution with count times of $8.4 \mathrm{~s}$ in step 1 and $4.2 \mathrm{~s}$ in step 2. Each run consisted of 20 integrations, resulting in a approximately 5 min analysis time. Gas blanks were measured at least four times per analytical session. In situ analyses of minerals in polished sections were made using a Photon Machines Analyte 193 excimer laser ablation system connected to the MC-ICP-MS using $\mathrm{He}$ as the carrier gas. Typical laser operating conditions included a $150 \mu \mathrm{m}$ spot size and $4 \mathrm{~Hz}$ repeat rate at full power (7 mJ per pulse). Instrumental mass bias was corrected using the samplestandard bracketing technique (with a San Carlos augite megacryst as the bracketing standard) and internal normalization to a ${ }^{49} \mathrm{Ti} /{ }^{47} \mathrm{Ti}$ ratio of 0.749766 [3]. Typical ${ }^{48} \mathrm{Ti}$ intensity measured on the San Carlos augite (approximately $1.5 \mathrm{wt} \% \mathrm{TiO}_{2}$ ) was $1.6 \times 10^{-11} \mathrm{~A}$.

Results: Several runs (10-15) were made on titanium-rich phases (predominantly fassaite) in each of two coarse-grained Allende CAIs (19B65 and CPM2-1). The average values for the Ti isotopic ratios and 2SD uncertainties for each CAIs were as follows: For 19B65, $\varepsilon^{46} \mathrm{Ti} /{ }^{47} \mathrm{Ti}=4.38 \pm 6.68, \quad \varepsilon^{48} \mathrm{Ti} /{ }^{47} \mathrm{Ti}=0.98 \pm 1.96 \quad$ and $\quad \varepsilon^{50} \mathrm{Ti} /{ }^{47} \mathrm{Ti}=$ $10.50 \pm 4.35 ; \quad$ For $\quad$ CPM2- $1, \quad \varepsilon^{46} \mathrm{Ti} /{ }^{47} \mathrm{Ti}=6.22 \pm 4.52, \quad \varepsilon^{48} \mathrm{Ti} /{ }^{47} \mathrm{Ti}=$ $2.87 \pm 2.58$ and $\varepsilon^{50} \mathrm{Ti} /{ }^{47} \mathrm{Ti}=0.67 \pm 7.74$. These values are within the reported range for normal, non-FUN CAIs (e.g., [1-3]). Analyses of additional CAIs are currently in progress.

References: [1] Niederer F. R. et al. 1980. The Astrophysical Journal 240:L73-L77. [2] Niemeyer S. and Lugmair G. W. 1981. Earth and Planetary Science Letters 53:211-225. [3] Niederer F. R. et al. 1985. Geochimica et Cosmochimica Acta 49:835-851.
5253

POTENTIAL RELATIONSHIP BETWEEN TITANIUM AND ZIRCONIUM ISOTOPE HETEROGENEITIES WITHIN SOLAR SYSTEM MATERIALS

N. H. Williams, W. M. Akram and M. Schönbächler. SEAES, The University of Manchester, M13 9PL, UK. E-mail: Niel.Williams-2@ postgrad.manchester.ac.uk.

Introduction: Isotopic heterogeneities in the neutron-rich isotopes ${ }^{96} \mathrm{Zr}$ and ${ }^{50} \mathrm{Ti}$ between bulk carbonaceous chondrites and the Earth-Moon system are now well established [1-3]. Previous work indicated that ${ }^{96} \mathrm{Zr}$ and ${ }^{50} \mathrm{Ti}$ anomalies in calcium-aluminum rich inclusions (CAI) might be correlated $[4,5]$. This raises the question, whether $\mathrm{Ti}$ and $\mathrm{Zr}$ anomalies in bulk solar system materials are linked to the same carrier phase (i.e., CAI), which was heterogeneously distributed within the solar system. A potential ${ }^{96} \mathrm{Zr}-{ }^{50} \mathrm{Ti}$ correlation may help to identify (i) a common nucleosynthetic site, in which these anomalies were generated and/or (ii) the processes experienced by the carrier phase(s) that allowed the specific nucleosynthetic signature to be preserved. A recent study revealed a linear relationship between ${ }^{46} \mathrm{Ti}$ and ${ }^{50} \mathrm{Ti}$ isotope anomalies within CAIs, bulk meteorites and the Earth, when normalizing to a fixed ${ }^{49} \mathrm{Ti} /{ }^{47} \mathrm{Ti}$ ratio [3]. The isotopes ${ }^{46} \mathrm{Ti}$ and ${ }^{50} \mathrm{Ti}$ have a different nucleosynthetic production site [6], which indicates that most likely processes within the solar system lead to the observed ${ }^{46} \mathrm{Ti}-{ }^{50} \mathrm{Ti}$ correlation.

In order to better constrain the potential relationship of $\mathrm{Zr}$ and $\mathrm{Ti}$ isotope anomalies in our solar system, we will obtain high precision $\mathrm{Ti}$ isotope analyses on a wide variety of solar system materials, which were previously analyzed for $\mathrm{Zr}$ isotopes [1].

Analytical Techniques: Titanium is separated via a two-step ion exchange procedure previously described in [7, 8]. The $\mathrm{Zr}$ data is reported elsewhere [1]. Samples are further purified using an additional separation step involving AG50-X8 cation resin. The resin is cleaned with $4 \mathrm{M}$ $\mathrm{HNO}_{3}$, then preconditioned and the samples are loaded onto the column in $0.25 \mathrm{M} \mathrm{H}_{2} \mathrm{SO}_{4}-1 \% \mathrm{H}_{2} \mathrm{O}_{2}$. Titanium is separated from $\mathrm{Ca}, \mathrm{Cr}$ and $\mathrm{V}$ and other matrix elements in $4 \mathrm{M} \mathrm{HNO}_{3}$.

All five $\mathrm{Ti}$ isotopes are analyzed simultaneously on a $\mathrm{HR} \mathrm{Nu}$ plasma MC-ICPMS at high resolution. The samples are bracketed by an Alfa Aesar Ti standard solution. Instrumental mass fractionation is corrected using the exponential law relative to ${ }^{49} \mathrm{Ti} /{ }^{47} \mathrm{Ti}$. Calcium, $\mathrm{V}$ and $\mathrm{Cr}$ corrections are applied to account for isobaric interferences on ${ }^{48} \mathrm{Ti}(\mathrm{Ca})$ and ${ }^{50} \mathrm{Ti}\left(\mathrm{V}\right.$ and $\mathrm{Cr}$ ). Samples are introduced in $0.5 \mathrm{M} \mathrm{HNO}_{3}-0.005 \mathrm{M}$ HF mixtures by means of desolvating nebulizer.

The samples to be analyzed include solar system material from various heliocentric distances: the geostandards $\mathrm{BHVO} 2$ and Cody Shale, four carbonaceous chondrites (Allende, Murchison, Colony, and Orgueil), two ordinary chondrites (Richardton and Forest Vale), two eucrites (Pasamonte and Sioux County), CAIs from Allende and lunar basalts.

References: [1] Akram W. M. et al. 2011. Abstract \#1908. 42nd Lunar and Planetary Science Conference. [2] Leya I. et al. 2008. Earth and Planetary Science Letters 266:233-244. [3] Trinquier A. et al. 2009. Science 324:374-376. [4] Leya I. et al. 2009. The Astrophysical Journal 702:1118-1126. [5] Harper C. L. et al. 1991. Abstract \#1257. 22nd Lunar and Planetary Science Conference. [6] Clayton D. 2003. Handbook of Isotopes in the Cosmos: Hydrogen to Gallium. Cambridge University Press. [7] Schönbächler M. et al. 2004. Analyst 129:32-37. [8] Leya I. et al. 2007. International Journal of Mass Spectrometry 262:247-255. 
5187

THE SPACE ACADEMY: A SUCCESSFUL STEM PARTNERSHIP

R. C. Wilson ${ }^{1}$, J. J. Remedios ${ }^{1}$, M. Barstow ${ }^{2}$, S. Roberts ${ }^{3}$, T. Moore ${ }^{3}$, A. $\mathrm{Ojha}^{4}$ and S. Bull ${ }^{5}$. ${ }^{1} \mathrm{EOS}$, Department of Physics and Astronomy, University of Leicester, Leicester, UK. E-mail: rcw15@1e.ac.uk. ${ }^{2}$ College of Science and Engineering, University of Leicester, Leicester, UK. ${ }^{3}$ IESSG, Nottingham Geospatial Building, University of Nottingham, Nottingham, UK. ${ }^{4}$ Space Academy, National Space Center, Leicester, UK. ${ }^{5}$ Science Learning Center East Midlands, School of Education, University of Leicester, Leicester, UK.

Introduction: Launched in October 2008, the Space Academy is a collaboration between the University of Leicester, the University of Nottingham, the National Space Center, the Science Learning Center East Midlands, STEMNET and the East Midlands Development Agency. The project has an Earth/planetary research scientist at each university, dedicated full-time staff and outstanding teachers seconded as lead educators to the project. The Space Academy offers curriculum focused full-day programmes in biology, chemistry, physics, astronomy, earth sciences and applied sciences for students from Key Stages 3 to 5, tailormade to the curriculum focus of the participating school. In addition, the Space Academy hosts an annual teacher CPD conference and INSET sessions for science educators.

Methodology: A suite of materials using space sciences and climate change as the context for learning and engagement in KS3-5 curriculum themes have been developed in collaboration with internationally recognized experts in space science, education and industry. Researchers and teachers work together to ensure that masterclass content is curriculum-focussed and include current discoveries and scientific information. The program is delivered by both research scientists experienced in public engagement and teachers (seconded from their schools) that are recognized as outstanding educators. An example Space Academy program includes an A Level/Cambridge Pre-U masterclass that uses "an away day to Mars" as the context for learning topics such as Newton's law and forces, gravitational potential and field strength, EM spectrum and spectroscopy through the investigation of physical properties/environment of Mars and its habitability. Similarly, a GCSE/A Level chemistry masterclass uses "Comets: harbingers of doom or agents of life?" as the context for learning low-temperature physics, simulating a comet nucleus, kinetics and mass spectrometry.

Evaluation: The Space Academy methodology has received international recognition by the science education community, enabling the project to contribute on an international-scale including (1) hosting the largest session at the NASA Space Exploration Educators Conference, (2) leading a session at the ESA human space-flight teachers workshop and (3) leading a session at the European Space Camp at the Andoya Rocket Range in Northern Norway. The Space Academy masterclasses and teacher CPD sessions have been externally evaluated by both the University of Leicester and an OFSTED inspector in terms of lasting student understanding and teacher effectiveness. There is evidence that the Space Academy program increases attainment in national examinations.

Acknowledgments: We acknowledge funding from the East Midlands Development Agency for this project 2008-2011.
5222

NORTHWEST AFRICA 5131: ANOTHER TAFASSASSET-LIKE METACHONDRITE RELATED TO THE CR CHONDRITE PARENT BODY

J. H. Wittke ${ }^{1}$, T. E. Bunch ${ }^{1,5}$, A. J. Irving ${ }^{2,5}$, D. Rumble III ${ }^{3}$ and P. P. Sipiera ${ }^{4,5}$. ${ }^{1}$ Geology Program, SESES, Northern Arizona University, Flagstaff, AZ 86011, USA, E-mail: james.wittke@nau.edu. ${ }^{2}$ Earth \& Space Sciences, University of Washington, Seattle, WA, USA. ${ }^{3}$ Geophysical Laboratory, Carnegie Institution, Washington, DC, USA. ${ }^{4}$ Field Museum of Natural History, Chicago, IL, USA. ${ }^{5}$ Planetary Studies Foundation, Galena, IL, USA.

Petrography: NWA 5131 is a $533 \mathrm{~g}$ achondrite previously thought to have affinities with LL chondrites (based largely on its olivine composition); however, new oxygen isotopic data clearly indicate that it is instead related to CR chondrites, and is in many ways quite similar to Tafassasset. The specimen is an inequigranular metamorphic aggregate of olivine (62 vol\%, $\left.\mathrm{Fa}_{29.3}, \mathrm{FeO} / \mathrm{MnO}=68\right)$, orthopyroxene (28 vol\%, $\left.\mathrm{Fs}_{24.7} \mathrm{Wo}_{2.1}\right)$, and metal (5 vol\%) with accessory clinopyroxene, intermediate plagioclase, chromite ( $c r \# 82)$ and troilite. Finer grained portions consist of polygonal grains meeting at $120^{\circ}$ junctions, but there also are regions with poikiloblastic texture. Orthopyroxene poikiloblasts up to $4 \mathrm{~mm}$ across enclose smaller chadacrysts of olivine and metal. No chondrules can be distinguished, but the poikiloblastic texture may represent former chondrule regions that have been highly recrystallized.

Oxygen Isotopes: Results obtained by laser fluorination of acidwashed material $\left(\delta^{18} \mathrm{O}=2.12,2.10 ; \delta^{17} \mathrm{O}=0.42,-0.45 ; \Delta^{17} \mathrm{O}=-1.536\right.$, $-1.555 \%$, respectively) plot well below the TFL along the trend for CR chondrites.

Discussion: NWA 5131 provides additional supporting evidence for the existence of at least some regions of extensive thermal metamorphism within the $\mathrm{CR}$ chondrite parent body. Although the vast majority of $\mathrm{CR}$ chondrites are hydrated and of petrologic type 2, there are two known highly equilibrated CR6 chondrites (NWA 2994, NWA 3100) [1]. Tafassasset (like NWA 5131) is best termed a CR metachondrite, since it is completely lacking in chondrules. As we have already argued [1, 2], such thermally metamorphosed specimens, as well as the paired, very ancient igneous-textured achondrites NWA 011/2400/2976/4587/ 4901, with very similar oxygen isotopic compositions [3], imply much more complexity within the $\mathrm{CR}$ parent body than originally envisioned. Like the CV-CK chondrite parent body, it may well have a metallic core surrounded by a metamorphic mantle (perhaps containing plutonic igneous bodies) and a chondrule-rich regolithic crust. It remains to be seen whether NWA 5131 also exhibits the fractionated, nonchondritic compositional characteristics found in Tafassasset, NWA 2994 and NWA 3100 [1]. Hypotheses about the size and/or thermal history of the CR chondrite parent body may need to be reconsidered in light of discoveries of highly equilibrated specimens like NWA 5131.

References: [1] Bunch T. et al. 2008. Abstract \#1991. 39th Lunar and Planetary Science Science. [2] Bunch T. et al. 2005. Abstract \#2308. 36th Lunar and Planetary Science Conference. [3] Yamaguchi A. et al. 2002. Science 296:334-336. [4] Floss C. et al. 2005. Meteoritics \& Planetary Science 40:343-360. [5] Spivak-Birndorf L. et al. 2009. 72nd Met. Soc Mtg., \#5390; Bouvier A. and Wadhwa M. 2010. Abstract \#489. 41st Lunar and Planetary Science Conference. 
5223

\section{A NOMENCLATURE SYSTEM FOR DIOGENITES CONSISTENT WITH THE IUGS SYSTEM FOR NAMING TERRESTRIAL ULTRAMAFIC ROCKS}

J. H. Wittke ${ }^{1}$ A. J. Irving ${ }^{2}$, T. E. Bunch ${ }^{1}$ and S. M. Kuehner ${ }^{2}$. ${ }^{1}$ Geology Program, SESES, Northern Arizona University, Flagstaff, AZ 86011, USA. E-mail: james.wittke@nau.edu. ${ }^{2}$ Earth \& Space Sciences, University of Washington Seattle, WA 98195, USA.

Background: Traditionally diogenites have been considered to be extraterrestrial ultramafic rocks dominated by orthopyroxene and having igneous (presumed to be cumulate) textures. Many diogenites have been shocked, and exhibit cataclastic (crushed) textures, although few are true breccias (requiring rotation of clasts). With the discovery of many more specimens in both hot and cold deserts, the range of orthopyroxene compositions found in diogenites has expanded considerably [e.g., 1], and now spans a range from $\mathrm{Fs}_{13}$ to $\mathrm{Fs}_{34}$. Furthermore the modal mineralogy of diogenitic rocks now includes not only chromite (and minor Ni-poor metal) but variable amounts of olivine and calcic plagioclase (yet very little clinopyroxene).

Recently it has been suggested [2] that the name diogenite be expanded to include rocks containing $>40 \mathrm{vol} \%$ olivine (previously termed olivine diogenites by us [3]), and even to related rocks composed of more than $90 \mathrm{vol} \%$ olivine (of which there are at least five known examples [4]).

A Logical Nomenclature System: The new terms harzburgitic diogenite and dunitic diogenite are very reasonable descriptors for those examples with $40-90 \mathrm{vol} \%$ olivine and $\geq 90 \mathrm{vol} \%$ olivine, respectively. However, in keeping with the established IUGS nomenclature for terrestrial ultramafic rocks [5], we suggest that the additional terms olivine-orthopyroxenitic diogenite and noritic diogenite be used to describe the few specimens with $10-40 \mathrm{vol} \%$ olivine and with $>10$ vol $\%$ plagioclase, respectively. The diagram below is modified from Figure 2.6 of [5], and on it are plotted the modes for 21 Northwest African and Arabian Peninsula diogenitic specimens that we have studied in detail. Note that diogenites can contain up to $10 \mathrm{vol} \%$ plagioclase before becoming noritic, and such examples could be termed plagioclase-bearing diogenites (like their terrestrial peridotite counterparts).

References: [1] Bunch T. et al. 2007. 70th Meteorite Society Mtg., \#5133. [2] Beck A. and McSween H. 2010. Meteoritics \& Planetary Science 45:850-872. [3] Irving A. et al. 2005. Lunar and Planetary Science Abstract \#2188. [4] Bunch T. et al. 2010. 73rd Meteorite Society Mtg., \#5315. [5] LeMaitre et al. 2002. Igneous Rocks: A Classification and glossary of terms, 2nd ed. Cambridge University Press.

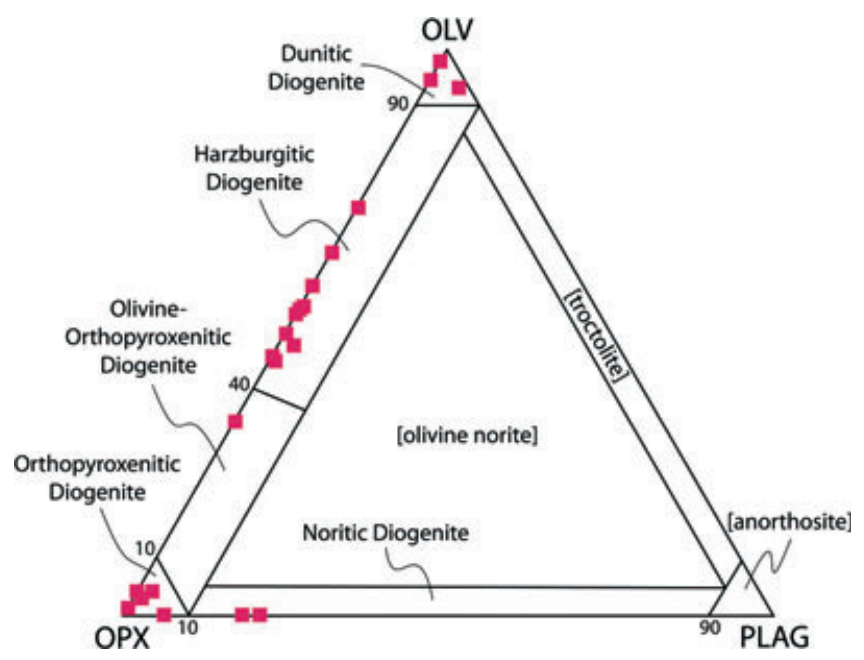

\section{2}

ORGUEIL LOW-DENSITY PRESOLAR CARBON AIN'T GRAPHITE BUT GLASSY CARBON

B. Wopenka ${ }^{1}$, E. Groopman ${ }^{2}$ and E. Zinner ${ }^{2}$. ${ }^{1}$ Earth and Planetary Sciences and ${ }^{2}$ Physics Department, Washington University, St. Louis, MO 63130, USA. E-mail: bwopenka@levee.wustl.edu.

Presolar carbonaceous grains from Orgueil traditionally have been called "graphite" [1]. Graphite is a crystalline solid with a well defined 3-D structure. A recent Raman study [2] of grains from the Orgueil highdensity fraction OR 1f $\left(2.02-2.04 \mathrm{~g} \mathrm{~cm}^{-1}\right)$ confirmed that the majority of them $(86 \%)$ were indeed graphitic. However, this is not the case for grains from Orgueil's low-density separate OR1d $\left(1.75-1.92 \mathrm{~g} \mathrm{~cm}^{-3}\right)$. The majority of low-density carbon grains (11 of 20) that were analyzed by Raman spectroscopy followed by NanoSIMS analysis [3] are not graphite, but rather glassy carbon, an amorphous solid. Only 3 of 20 grains were graphitic, and the remainder (6 of 20) had a "kerogen-type" Raman signature, i.e., they also were non-crystalline $\mathrm{sp}^{2}$-bonded carbon. The physical properties of glassy carbon are well known to materials scientists. It has a unique structure with a random combination of small-sized (nanocrystalline) basal planes and edge planes with many large void spaces in between. The latter explains its low density (listed as $1.42 \mathrm{~g} \mathrm{~cm}^{-3}$ ) compared to the one for graphite (listed as 2.09$\left.2.23 \mathrm{~g} \mathrm{~cm}^{-3}\right)$.

Based on their first and second-order Raman spectra, the $\mathrm{sp}^{2}$ bonded carbon materials in Orgueil grains can be grouped into three different types [see also 2]. (1) Graphitic grains with more or less structural disorder have very narrow 1st- order peaks (D and G) and very strong second-order peaks; their $\mathrm{D} / \mathrm{G}$ intensity ratios can be used to infer the graphitic in-plane crystallite size; (2) Allende-type grains have wider first-order peaks than graphitic grains, but with a distinctive feature: their $\mathrm{D}$ band is more intense than their $\mathrm{G}$ band; in addition they have a very weak (or no) second-order spectrum; and (3) kerogen-type grains have extremely wide first-order and no second-order peaks. From the Raman spectroscopic perspective, only grains of type 1 deserve to be called "graphite," whereas grains of types 2 and 3 are amorphous. The very characteristic and unusual Raman signature of Allende-type grains is typical for glassy carbon and is not known to occur in any other form of $\mathrm{sp}^{2}$-bonded carbon. Thus, we propose that the carbon in both Allende and the majority of the low-density presolar carbon grains in Orgueil are a form of glassy carbon, rather than crystalline graphite. This finding not only explains the low density of this so-called "graphite" separate, but it is also consistent with the hypothesis, based on isotopic data [4], that grains from the low- and high-density fractions come from different stellar sources. The presolar graphite probably comes from AGB stars (and dominates the high-density separate), whereas the presolar glassy carbon comes from supernovae (and dominates the low-density separate). Indeed, all grains with Allende-type (i.e., glassy carbon) spectra of this study have isotopic signatures (such as ${ }^{14} \mathrm{~N},{ }^{18} \mathrm{O},{ }^{28} \mathrm{Si}$ excesses and high ${ }^{26} \mathrm{Al} /{ }^{27} \mathrm{Al}$ ratios) indicative of a supernovae origin [3]. One possible explanation for the presence of glass (rather than crystals) in carbon material from supernovae could be the relatively rapid cooling of their ejecta.

References: [1] Jadhav M. et al. 2006. New Astronomical Review 50:591-595. [2] Wopenka B. et al. 2011. Abstract \#1162. 42nd Lunar and Planetary Science Conference. [3] Groopman E. et al. This conference. [4] Zinner E. et al. 2006. Proceeding of Science (NIC-IX) 019. 
5205

INVESTIGATING CARBONATE SURVIVAL IN Stardust ALUMINUM FOILS

P. J. Wozniakiewicz ${ }^{1}$, H. A. Ishii ${ }^{1}$, A. T. Kearsley ${ }^{2}$, M. J. Burchell ${ }^{3}$, J. P. Bradley $^{1}$, N. Teslich ${ }^{1}$ and M. J. Cole ${ }^{3}$. ${ }^{1}$ Institute of Geophysics and Planetary Physics, Lawrence Livermore National Laboratory (LLNL), 7000 East Avenue, Livermore, CA 94550, USA (wozniakiewic1@1lnl.gov). ${ }^{2}$ Impacts \& Astromaterials Research Centre (IARC), Department of Mineralogy, Natural History Museum (NHM), London SW7 5BD, UK. ${ }^{3}$ Centre for Astrophysics and Planetary Science, School of Physical Sciences, University of Kent, Canterbury, Kent CT2 7NH, UK.

Introduction: Hydrous meteorites and interplanetary dust particles commonly contain carbonates believed to result from parent body aqueous alteration. Such alteration requires the prolonged presence of liquid water, and comets, generally believed to have remained frozen since their accretion, were not expected to contain these minerals. Intriguingly, the presence of carbonates in cometary nuclei was inferred from VEGA 1 and 2 mass spectrometry data obtained from comet $1 \mathrm{P} / \mathrm{Halley}$ [1] and Spitzer space telescope spectra obtained from comet 9P/Temple 1 [2]. Recently, carbonates were positively identified in samples of comet $81 \mathrm{P} /$ Wild 2 collected in Stardust aerogel [e.g., 3, 4]. However, the authors recognized concerns over aerogel premission purity and postmission contamination. Confirmation of cometary carbonates may be provided by analyses of Stardust Al foils. However, collection by impact at $6 \mathrm{~km} \mathrm{~s}^{-1}$ likely caused alteration requiring impact analog investigations to assess the effects.

Method: Powdered calcite was fired in light gas gun shots at flight spare Al foils under Stardust encounter conditions. Scanning electron microscopy with energy dispersive X-ray analysis (SEM EDX) was used to locate regions of residue. Focused ion beam (FIB) microscopy was used to prepare sections from preimpact projectile and postimpact crater residues for analysis and comparison by transmission electron microscopy (TEM).

Results and Discussion: TEM analyses show calcite residues in craters $>50 \mu \mathrm{m}$ are a mixture of crystalline and amorphous material. Crystalline materials exhibit close to preimpact projectile compositions and polycrystalline structure. This material is interpreted as surviving impactor. Amorphous material is vesiculated and contains $\mathrm{Al}$ suggesting a melt origin and loss of volatiles. Although calcite decomposes releasing $\mathrm{CO}_{2}$ at high pressures [e.g., 5], the amorphous residue chemistry is depleted in $\mathrm{C}$ but not $\mathrm{O}$ relative to the original projectile, possibly as a result of incorporating and oxidizing $\mathrm{Al}$ as observed in previous studies of phyllosilicate residues [6]. We conclude that where surviving material is not found, carbonates may be identified by their amorphous vesiculated residues which retain original cation to $\mathrm{O}$ ratios but have incorporated $\mathrm{Al}$. The $\mathrm{Al}$ foils therefore represent a valuable sample set for investigation of cometary carbonates.

Acknowledgments: We thank NASA for providing $\mathrm{Al}$ foils, and STFC for support of the LGG. Parts of this work were performed under the auspices of U.S. DOE by LLNL under Contract DE-AC5207NA27344. This work was supported by grants: NASA NNH07AG46I to HAI \& LDRD 09-ERI-004 to JPB.

References: [1] M. N. Fomenkov et al. 1992. Science 258:266. [2] C. M. Lisse et al. 2006. Science 313:635. [3] S. Wirick et al. 2007. Abstract \#1534. 38th Lunar Prospector Spacecraft. [4] G. J. Flynn et al. 2008. Abstract \#1979. 39th Lunar Prospector Spacecraft. [5] Lange M. A. and Ahrens T. J. 1983. 14th Lunar Prospector Spacecraft p. 419. [6] Wozniakiewicz et al. Abstract \#2357. 39th Lunar Prospector Spacecraft.
5206

KWAJALEIN ATOLL: A NEW COLLECTION SITE FOR MICROMETEORITES

P. J. Wozniakiewicz ${ }^{1}$, J. P. Bradley ${ }^{1}$, M. E. Zolensky ${ }^{2}$, D. E. Brownlee ${ }^{3}$ and H. A. Ishii ${ }^{1}$. ${ }^{1}$ Institute of Geophysics \& Planetary Physics, Lawrence Livermore National Laboratory (LLNL), 7000 East Avenue, Livermore, CA 94550, USA (wozniakiewic1@1lnl.gov). ${ }^{2}$ NASA Johnson Space Center, Houston, TX 77058, USA. ${ }^{3}$ Department of Astronomy, University of Washington, Seattle, WA 98195, USA.

Introduction: Although micrometeorites are constantly arriving at the Earth's surface, they are quickly hidden amongst vast amounts of natural and anthropogenic terrestrial dust. Using magnets, metal-bearing micrometeorites have been successfully separated from deepsea sediments [e.g., 1, 2] and dissolved prehistoric (preindustry) limestones [e.g., 3] and salts [e.g., 4]. However, these samples do not fully represent the incident population of micrometeorites. More complete collections are obtained where the terrestrial dust flux is so low that micrometeorites represent the major dust component and additional separation techniques are not required, for example, from the upper atmosphere by high altitude planes [e.g., 5] or from polar ice, snow and well water [e.g., 6-8]. These samples are proving invaluable to understanding interplanetary dust. However, atmospheric collections are captured in silicone oil requiring removal by solvents, which may be problematic to some analyses (e.g., organics), and polar samples have been exposed to water ice [9].

We are evaluating collection of micrometeorites on Kwajalein atoll in the Pacific Ocean, more than 1000 miles from the nearest continent. From mid-December to mid-May, trade winds blow continuously from the northeast at 15-20 knots providing a continuous stream of ocean air to sample. The main sources of nonextraterrestrial dust are sea spray and coral, both of which are easily distinguishable from micrometeorites. We report the findings of our preliminary background samples.

Method: A high volume air sampler was installed at Kwajalein High School and fitted with a polycarbonate membrane filter with $5 \mu \mathrm{m}$ perforations. The flow rate was set to sample approximately $100 \mathrm{~m}^{3}$ of air per hour. Filters were returned to LLNL for survey by optical microscopy. A selection of micrometeorite candidates were picked and analyzed by scanning electron microscopy.

Findings and Future Work: The sampler was intended to run for 7 days, but was stopped after only three due to clogging of the filters by salt. Despite the short exposure the filter contains "particles of interest" in addition to background aerosol and analysis results will be presented. NASA funding has been awarded to purchase, modify and install a new air sampler at Kwajalein airport and to design and implement several alternative collectors. These plans will also be presented.

Acknowledgments: We thank S. Yakuma, B. Barnett, D. Dearborn and T. Jones (LLNL) for use of their samplers and help throughout the project and Paula Fluhrer and her students at Kwajalein High School for running the experiment. Parts of this work were performed under the auspices of U.S. DOE by LLNL under Contract DE-AC52-07NA27344.

References: [1] Fredriksson K. 1956. Nature 177:32. [2] Pettersson H. and Fredriksson K. 1958. Pacific Science 12:71. [3] Dredge et al. 2010. Scottish Journal of Geology 46:7. [4] Davidson J. et al. 2007. Abstract \#1545. 38th Lunar Prospector Spacecraft. [5] Brownlee et al. 1977. Proceedings, 8th Lunar Science Conference. p. 149. [6] Maurette et al. 1991. Nature 351:44. [7] Duprat et al. 2007. Advances in Space Research 39:605. [8] Taylor et al. 1998. Nature 392:899. [9] Bradley et al. 2011. Abstract \#1320. 42nd Lunar Prospector Spacecraft. 
5208

TAKING PLANETARY SCIENCE AND ASTRONOMY TO STUDENTS IN THE MIDDLE OF THE PACIFIC OCEAN

P. J. Wozniakiewicz ${ }^{1}$, J. P. Bradley ${ }^{1}$, M. E. Zolensky ${ }^{2}$, D. E. Brownlee ${ }^{3}$, H. A. Ishii ${ }^{1}$. ${ }^{1}$ Institute of Geophysics and Planetary Physics, Lawrence Livermore National Laboratory (LLNL), 7000 East Avenue, Livermore, CA 94550, USA (wozniakiewic1@1lnl.gov). ${ }^{2}$ NASA Johnson Space Center, Houston, TX 77058, USA. ${ }^{3}$ Dept. of Astronomy, University of Washington, Seattle, WA 98195, USA.

Introduction: Kwajalein Island is the southernmost island in the Kwajalein atoll, one of the Micronesian nation of atolls and islands in the middle of the Pacific Ocean. The remote location means that approximately 300 children living on the island generally miss out on some learning opportunities in science taken for granted at most mainland schools; e.g., take your children to work days, talks and presentations by scientific visitors, and opportunities to get involved in current research. Such experiences can be the inspiration behind successful scientific and medical careers and without them potential scientists may not be aware of all career paths available to them. Working with Kwajalein Junior/Senior High School science teacher, Paula Fluhrer, we have initiated an outreach project that aims to provide the students at Kwajalein High School with inclass and extracurricular opportunities for hands-on participation in astronomy and planetary science.

The Outreach Project: For this outreach project we have identified two key tasks. First, we are introducing students to the field of planetary science and astronomy through a series of presentations and after school activities. The presentations are written to compliment their curriculumbased classes in Chemistry, Physics and Earth Sciences and are to be given by visiting scientists from LLNL and JSC in November 2011. The private donation of a Celestron Ultima 2000 telescope has allowed us to initiate an after school astronomy club to inspire potential astronomers and planetary scientists of tomorrow. Second, we are giving students the opportunity to gain some hands-on research experience through participation in the research collaboration between LLNL, JSC and Univ. of Washington that was recently awarded NASA funding to evaluate the collection of micrometeorites on Kwajalein [1]. Kwajalein is an ideal location to test micrometeorite collection because it is $>1000$ miles from the nearest continent, and the primary sources of nonextraterrestrial dust are sea salt and coral, both of which are easily distinguished from micrometeorites. Also, Kwajalein micrometeorites will not have been subjected to ice, snow, well water or silicone oil and solvents like other collections. Students will participate by running the air sampler to collect dust and by performing some searches by optical microscope (to be donated) to identify micrometeorite candidates. Follow-up analyses will be carried out at LLNL, and data collected on particles identified by students and its relevance will be presented to the students to keep them involved and excited about their role in the unfolding science.

Acknowledgments: We thank S. Yakuma, B. Barnett, D. Dearborn, and T. Jones (LLNL) for use of their samplers and help throughout the project. We thank Paula Fluhrer, a dedicated teacher at Kwajalein High School, for her enthusiastic collaboration in this project, and we thank her students for running the experiment. Parts of this work were performed under the auspices of U.S. DOE by LLNL under Contract DE-AC52-07NA27344.

Reference: [1] Wozniakiewicz P. J. et al. Abstract \#5206. This conference.
5261

ASTEROIDAL ATMOSPHERES: A NEW SUBJECT AREA

I. P. Wright, S. J. Barber, S. Sheridan, G. H. Morgan, D. J. Andrews, C. T. Pillinger and A. D. Morse. Planetary \& Space Sciences, Open University, UK.

Introduction: A few years ago the Moon was widely thought to be a dry, barren place. Today, with results from both spacecraft observations [e.g., 1] and laboratory analyses [e.g., 2], the regolith of the Moon is considered to contain a number of different forms of water at various levels of concentrations (up to \% levels). Indeed, it is now commonplace to talk about a lunar hydrosphere and its associated water cycle. One component of this arises from a substance that is seen to come and go [3], known as "space dew" [4], which is most likely to be the result of an interaction between the solar wind $(\mathrm{H})$ and the lunar surface $(\mathrm{O})$. It is a short step to postulate that an equivalent effect would also be associated with the surfaces of asteroidal bodies. And, by extension, these would also have atmospheres (perhaps more accurately considered as exospheres, although if cycling of dew at the surface of the Moon is a reality then perhaps "exosphere" is semantically incorrect). This would be on top of the large volumes of water that are already considered to exist within the upper layers of large bodies like Ceres, and as prominent surface deposits on asteroids such as 24 Themis.

Lutetia: On July 10, 2010, the Rosetta spacecraft (ESA's mission to rendezvous with, and land on, a cometary nucleus) flew past the asteroid 21 Lutetia. This is by far the largest such body thus far visited by a space mission and some spectacular images were obtained [5]. Amongst the investigations carried out during the flyby was a campaign mounted by various gas analysis instruments to search for evidence of an atmosphere around the asteroid. Included in this was the Ptolemy instrument, which is located on the Lander (Philae) element of the mission [6].

Discussion: Although there is not space here to describe the results from all of the Rosetta instruments it is clear that at closest approach (ca. $15,000 \mathrm{~km}$, at the sub-solar point) the gas analysis instruments detected increases in signals from volatiles such as $\mathrm{H}_{2} \mathrm{O}$ and $\mathrm{CO}_{2}$. The question is: were these the result of the spacecraft traversing an asteroidal atmosphere, or were they somehow a result of spacecraft outgassing during the various manoeuvers that were necessary for tracking purposes during the flyby? At face value the results from Ptolemy would suggest asteroidal outgassing rates of about $5 \times 10^{3} \mathrm{~kg} \mathrm{~s}^{-1}$ (i.e., considering the entire surface of the body). In principle this is the sort of level that would be expected from an active cometary nucleus and clearly seems unlikely. However, if Rosetta had flown through a collimated outgassing feature, the globally normalized rates would appear to be much lower. Now, it could be argued that such a coincidence is unlikely, but we should point out that some asteroids, like 596 Sheila, are known to have tails (whilst certain comets have "tails" that point towards the Sun). Furthermore, recent results regarding the presence of water on the Moon should act to remind us of how quickly and completely a paradigm can change.

References: [1] Colaprete A. et al. 2010. Science 330:463-468. [2] Greenwood J. P. et al. 2011. Nature Geoscience 4:79-82. [3] Pieters C. M. et al. 2009. Science 326:568-572. [4] McCord T. B. et al. 2011. OH/ $\mathrm{H}_{2} \mathrm{O}$ in the Lunar Soil discovered by M3: Possible Space Dew? Journal of Geophysical Research, in review. [5] See, for instance http://www.esa.int/ esaMI/Rosetta/ [6] Andrews D. J. et al. 2011. Ptolemy operations and results during the Lutetia flyby, Planetary and Space Science, submitted. 
5526

SHOCK METAMORPHISM OF FRESH AND ALTERED BASALT PROTOLITHS FROM LONAR CRATER, INDIA: WHY ARE SNCS ALWAYS "FRESH" AND CLASS 2?

S.P. Wright. Institute of Meteoritics, University of New Mexico, Albuquerque, NM 87131, USA. E-mail: spwright@unm.edu.

Introduction: The study of shock metamorphism on basaltic minerals [1-3] is of much interest given that our only $\sim 55$ samples of Mars are shocked basalts. Identifying the source craters/regions of SNC meteorites [4] is also of interest as we have no sample return and hence no geologic context to compare to the increasing amount of remote data being returned from orbiters. This is a quandary as all $\sim 40$ shergottites have been shocked $\sim 25-45 \mathrm{GPa}$, yet all remote data is of pixels of primarily unshocked basalt. Further, the finding of alteration minerals on Mars [5-6] has implications for water in the geologic history of Mars, and thus upcoming missions such as MSL [7]. Deccan basalt has been called an excellent analog for Surface Type 1 (basalt) found in the equatorial regions of Mars [8]. Comparisons to and implications for shergottites and data from Mars orbiters and Rovers are noted. For this study, from a previous classification [9], Class 1 ( $<20 \mathrm{PGa})$, Class 2 (20-40 GPa), Class 3 (40-60 GPa), Class 4 (60-80 GPa), and Class 5 (>80 GPa) shocked basalt, or "impact melts" of both fresh and altered basalt, along with one soil protolith, have been found, and petrographic and BSE images will be shown.

Deccan and Lonar Background: Prior to impact, Deccan basalts primarily composed of labradorite and augite erupted $\sim 65 \mathrm{Ma}$. Aqueous alteration that we attribute to groundwater in the lower 3 of 6 flows resulted in chlorite, serpentine, celadonite, hematite, zeolite, and silica being deposited in the basalts. Impact $\sim 570 \mathrm{ka}$ [10] produced impactites from a wide range of shock pressures [9] of both fresh and altered protoliths.

Petrography and BSE Images: Class 2 shocked basalt show intense shattering and fracturing of clinopyroxene grains, and labradorite has been converted to maskelynite $[2,9]$. These data are comparable to the petrography of shergottites [e.g., 11]. Petrographic images of impact melts exhibit schlieren and flow features similar to lechatelierite from Meteor Crater and other terrestrial impact melts. Classes 3 and 4 show flowing and vesiculated plagioclase glass, respectively (NOT maskelynite). New finds related to Mars alteration include 1.) melt glass "encasing" the alteration minerals listed above to preserve their pre-impact (groundwater) alteration now accessible as breccia clasts in the ejecta, and 2.) only Class 2 rocks with altered protoliths acquire "decompression cracks", as there was no melting to accommodate the change in volume as seen in higher classes.

Implications: We receive a biased sampling as only fresh, Class 2 shocked basalts are ejected off Mars. The melting of higher shock classes and/or the "decompression cracking" of altered basalts must decrease the particle velocity term in the Huguenot equations, and hence the Martian escape velocity of $5.0 \mathrm{~km} \mathrm{~s}^{-1}$ is not reached. These materials likely remain on Mars to be found by future rovers.

References: [1] Stöffler. 1971. Journal Of Geophysical Research 76:5541-5551. [2] Stöffler. 1972. Fortschritte der Mineralogie 49:50-113. [3] Stöffler 1974. Fortschritte der Mineralogie 51:256-289. [4] Hamilton et al. 2003. Meteoritics \& Planetary Science 38:871-885. [5] Ehlmann et al. 2010. Geophysical Research Letters [6] Glotch et al. 2010. Geophysical Research Letters. [7] Mahaffey. 2008 ISSI 25:255-268. [8] Bandfield et al. 2000. Science 287:1626-1630. [9] Kieffer. et al. 1976. 7th Lunar and Planetary Science Conference. 1391-1412. [10] Jourdan et al. 2011. Geology, in press. [11] McCoy et al. 1999. Geochemistry Geophysics Geosystems 63:1249-1262.
5292

INDICATORS FOR AN IMPACT DIRECTION IN THE CENTRAL PIT OF AN UNNAMED MARTIAN CRATER

G. Wulf, M. H. Poelchau and T. Kenkmann. ${ }^{1}$ Institute of Geosciences Geology, Albert-Ludwigs-University Freiburg, Germany. E-mail: gerwin.wulf@geologie.uni-freiburg.de.

Introduction: The ejecta blanket is the most distinctive indicator for the impact direction in oblique impacts, showing the formation of "forbidden" zones and "butterfly" patterns $[1,2]$. The position of the central uplift relative to the crater center was proposed as another diagnostic feature [3], but could not be verified statistically [4] and might be caused by heterogeneous target structures [5]. A further promising indicator for obliquity is provided by the internal structure of central uplifts. Some terrestrial craters show a preferred stacking of layered bedrock in the central uplift $[6,7]$ with bedding strike perpendicular to the long axis of the crater ellipse [7]. Further analysis of Martian craters $[8,9]$ provide evidence that preferred strike orientation in the central uplift could be indicative for an impact direction. Here we present new results of the mapping of an unnamed central pit crater that confirm preferred strike orientation within central structures as a possible indicator for an impact direction.

Methods: An unnamed central pit crater south of Valles Marineris $\left(15.8^{\circ} \mathrm{S} 63.7^{\circ} \mathrm{W}, 54.5 \mathrm{~km}\right.$ crater rim diameter, $13 \mathrm{~km}$ pit diameter) was mapped with emphasis on structural trends of the exposed layered units in the central pit. We used high-resolution image data (HRSC, CTX, HiRISE) to determine the apparent strike values and additionally used an available HiRISE DTM of the western part of the central pit to get true strike and dip values using the Layertools extension for ArcGIS [10].

Results: The central pit crater can be characterized as an oblique impact crater using THEMIS infrared images showing a "forbidden" zone of the ejecta blanket which indicates an impact direction from 290 $300^{\circ}$. Apparent and true strike orientation shows a very distinct preferred trend of $30-40^{\circ}$ over the majority of the central pit. Strata predominantly dip WNW.

Discussion: An oblique impact and the impact direction from 290$300^{\circ}$ could clearly be determined based on the ejecta pattern. The consistent $30-40^{\circ}$ strike and westward dip of the analyzed stack of layers surrounding the central pit indicate material motion and shortening along $300-310^{\circ}$ and a shear component top-to-the-SSE. This direction shows only a minute deviation from the impact direction inferred from the ejecta blanket. Shortening and stacking in the central crater floor is explained by the remnant horizontal momentum transferred from the impacting projectile to the target during the oblique impact [7]. The yet unsolved processes of central pit formation will also be considered and current models of pit formation will be cross-checked with the constraints given by the structural data gathered in this work. For instance, the orientation of central pit strata appear to be unaffected by the pit formation.

References: [1] Gault D. E. and Wedekind J. A. 1978. Proceedings, 9th Lunar and Planetary Science Conference. pp. 3843-3875. [2] Anderson J. L. B. et al. 2004. Meteoritics \& Planetary Science 39:303-320. [3] Schultz P. H. and Anderson R. R. 1996. Geological Society of American Special Paper 302:397-417. [4] Ekholm G. A. and Melosh H. J. 2001. Geophysical Research Letters 28:623-626. [5] Collins G. S. et al. 2008. Earth and Planetary Science Letters 270:221-230. [6] Scherler D. et al. 2006. Earth and Planetary Science Letters 248:43-53. [7] Kenkmann T. and Poelchau M. H. 2009. Geology 37:459-462. [8] Wulf G. et al. 2011. Abstract \#1440. 41st Lunar and Planetary Science Conference. [9] Poelchau M. H. et al. 2009. Abstract \#1796. 40th Lunar and Planetary Science Conference. [10] Kneissl T. et al. 2010. Abstract \#1640. 41st Lunar and Planetary Science Conference. 


\section{7}

SHOCK-PRODUCED COESITE IN THE XIUYAN CRATER, CHINA

W Xiao, M. Chen and X. Xie. Guangzhou Inst. Geochem., Chinese Academy of Sciences, Guangzhou 510640, China. E-mail: wsxiao@gig.ac.cn.

Introduction: The Xiuyan crater is located in the Liaodong Peninsula of northeastern China. It is a simple bowl-shaped crater with a diameter of $1.8 \mathrm{~km}$. The crystalline basement around the crater is made up of Proterozoic metamorphic rock complex composed of granulite, hornblendite, gneisses tremolite marble, and marble [1]. A drilling positioning at the center of the crater reveals a crater-fill breccia unit of $188 \mathrm{~m}$ in thickness lain under lacustrine sediments [2]. Below the breccia layers are fractured bedrock of tremolite marble or still the breccia units at the crater bottom. A layer of impact breccia composed of clasts of granulite, gneiss, hornblendite and fragments of glass as well as clastic matrix, occurs at the lower part of the breccia units in the depth interval from 260 to $295 \mathrm{~m}$. We have examined abundant coesite from the impact breccia.

Results and Discussion: Clasts of gneiss in the impact breccia have been investigated in petrology and mineralogy. The gneiss is composed of quartz, feldspar and small amount of amphibole. Feldspar was completely molten and transform to vesicular glass. Quartz in gneiss was transformed into silica glass. Amphibole was mostly transformed to iron-magnesium oxides, which commonly occur as irregular patches surrounding silica glasses. Coesite occurs within silica glass and commonly appears as polycrystalline aggregates of stringers and irregular patches, as well as granular, needle-like and dendritic crystals. The composition of coesite is identical to the silica glass. The occurrence of coesite is indicative of crystallization from a silica liquid [3]. The silica glass contains coesite up to $80 \%$ by volume. Raman spectrum of coesite displays the strongest peak at $521 \mathrm{~cm}^{-1}$ and other weak peaks at 1146, 1038, 815, 785, 468, 427, 356, 326, 269 and $205 \mathrm{~cm}^{-1}$, and all these peaks can be attributed to coesite [4]. The coesite associating with silica glass is considered to be a diagnostic feature for shock metamorphism of target rocks [5]. The occurrence of coesite provides a unambiguous evidence for an impact origin of the Xiuyan crater.

Acknowledgments: This research was supported by CAS (Kzcx2-yw140), NNSF of China (40872041) and President Foundation of CAS.

References: [1] Chen M. 2008. Chinese Science Bulletin 53:392-395. [2] Chen M. et al. 2010. Chinese Science Bulletin 55:1777-1781. [3] Chen M. et al. 2010. Earth and Planetary Science Letters 297:306-314. [4] Boyer H. et al. 1985. Physics and Chemistry of Minerals. 12:45-48. [5] Chao E. C. T. et al. 1960. Science 132:220-222.

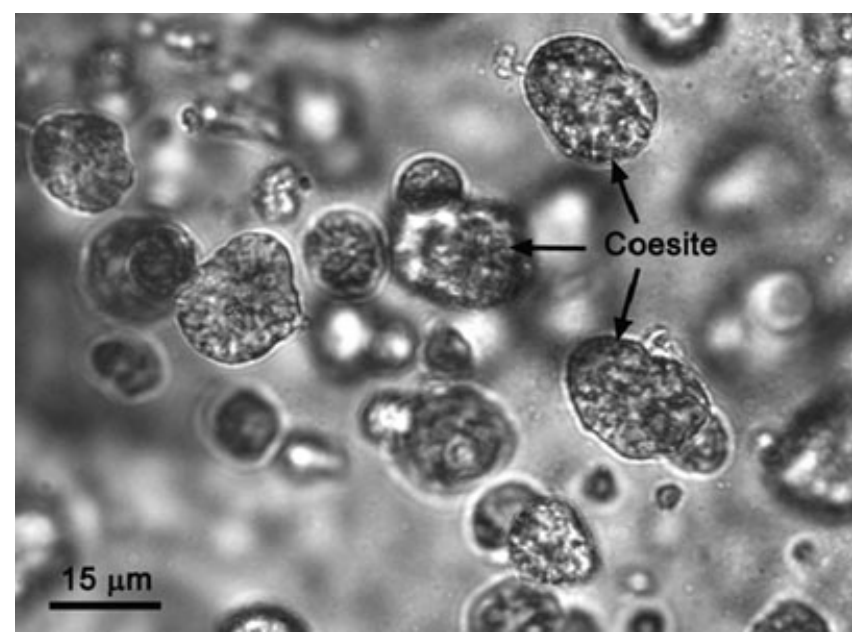

5387

LA-ICP-MS STUDY OF METALLIC Fe-Ni FROM THREE MESOSIDERITES NEWLY FOUND IN GROVE MOUNTAINS, ANTARCTICA

L. $\mathrm{Xu}^{1,2}, \quad$ Y. $\mathrm{Lin}^{2}$, Y. Zhang ${ }^{2}$, C. $\mathrm{Li}^{1} \cdot{ }^{1}$ National Astronomical Observatories, Chinese Academy of Sciences, Beijing, China. E-mail: xul@nao.cas.cn. ${ }^{2}$ Key Laboratory of the Earth’s Deep Interior, Institute of Geology and Geophysics, Chinese Academy of Sciences, Beijing, China.

Introduction: Mesosiderites consist of subequal amounts of metal and basaltic-pyroxenitic silicates. Previous analyses by neutron activation show possible genetic links of the metal to IIIAB iron meteorites, but with distinct differences in detail $[1,2]$. In this study, we analysed in situ highly siderophile elements (HSE) and other trace elements in metallic Fe$\mathrm{Ni}$ from three mesosiderites found in Grove Mountains, Antarctica, in order to clarify genetic relationship of the metallic $\mathrm{Fe}-\mathrm{Ni}$ with other groups of meteorites.

Results and Discussion: Of the three mesosiderites, GRV 055364 is mild weathered. The silicate portion shows coarse-grained textures, and olivine clasts have coronas decorated by fine-grained chromite. GRV 050212 and GRV 021553 are significant altered, and highly brecciated. Their silicate portions are very heterogeneous and commonly zoned. Exsolution of low-Ca and augite are well developed, especially in GRV 050212.

HSE (Re, Os, Ir, Ru, Pt, Rh, Pd and $\mathrm{Au}$ ), $\mathrm{Ni}, \mathrm{Co}$ and other trace elements (W, Mo, As, $\mathrm{Cu}, \mathrm{Sb}, \mathrm{Ga}, \mathrm{Ge}$ ) of metal clasts from these meteorites were analyzed in situ by LA-ICP-MS. Metal grains from GRV 055364 show a wide range of compositions. There seems a correlation between the concentrations and abundances patterns of HSE. As the concentrations of HSE decrease, their CI-, Ni-normalized abundance patterns vary from refractory-rich $(\mathrm{Re} / \mathrm{Ni}: 3.7 \times \mathrm{CI})$ to flat patterns, and to slightly refractory-depleted $(\mathrm{Re} / \mathrm{Ni}: 0.28 \times \mathrm{CI})$. All of them have negative Pd-anomalies, and are depleted in the moderately volatile elements, including $\mathrm{As}, \mathrm{Cu}, \mathrm{Sb}, \mathrm{Ga}$ and Ge. Analyses of GRV 021553 exhibit a relatively narrow range of compositions, with the HSE patterns similar to the refractory grains from GRV 055364. Those from GRV 050212 are the most HSE-enriched, and six out of nine analyses have Ninormalized abundances of $\mathrm{Re}$ of 3.9-5.0 × CI. The most HSE-enriched grains show a steep slope of the abundance patterns toward Re.

Except for the HSE-depleted grains from GRV 055364, the HSE patterns of all other analyses from the three meteorites are very similar to those of metallic Fe-Ni from equilibrated H-group chondrites [3, 4]. Our analyses confirm a close genetic relationship of metallic $\mathrm{Fe}-\mathrm{Ni}$ between mesosiderites and H-group chondrites. In contrast, HSE-rich IIIAB irons show no negative Pd-anomaly with lower Re concentrations relative to other HSE [5], different from those of metal from the mesosiderites. The wide variation of HSE of metal Fe-Ni from GRV 055364 is probably due to fractional crystallization of molten metal.

Acknowledgments: This work was supported by the Knowledge Innovation Program of Chinese Academy of Sciences (KZCX2-YW-Q08) and NSF of China (41073053).

References: [1] Hassanzadeh J. et al. 1990. Geochimica et Cosmochimica Acta 54:3197-3208. [2] Wasson J. T. et al. 1974. Geochimica et Cosmochimica Acta 38:135-149. [3] Horan M. F. et al. 2003. Chemical Geology 196:27-42. [4] Fischer-Goedde M. et al. 2010. Geochimica et Cosmochimica Acta 74:356-379. [5] Mullane E. et al. 2004. Chemical Geology 208:5-28. 
5386

HAYABUSA SAMPLE CURATION AT PLANETARY MATERIAL SAMPLE CURATION FACILITY IN JAXA

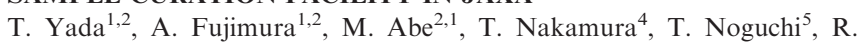
Okazaki $^{6}$, T. Okada ${ }^{2,1}$, Y. Ishibashi ${ }^{1}$, K. Shirai ${ }^{2}$, M.E. Zolensky ${ }^{7}$, S. Sandford $^{8}$, M. Uesugi ${ }^{1}$, Y. Karouji ${ }^{1}$, M. Ueno ${ }^{2,1}$, T. Mukai ${ }^{3}$, M. Yoshikawa $^{2,1}$, and J. Kawaguchi ${ }^{1,2}$. ${ }^{1}$ JAXA's Space Exploration Center. ${ }^{2}$ Institute Space Astronautical Science. ${ }^{3}$ Japan Aerospace Exploration Agency, Sagamihara, Kanagawa 252-5210, JAPAN, E-mail: yada@planeta.sci.isas.jaxa.jp. ${ }^{4}$ Department Earth Science, Graduate School Science, Tohoku University, Sendai, Miyagi 980-8578 JAPAN. ${ }^{5}$ College Sci., Ibaraki University, Mito, Ibaraki 310-8512, JAPAN. ${ }^{6}$ Department Earth Planet. Science, Graduate School Science, Kyushu University, Fukuoka 812-8581, JAPAN. ${ }^{7}$ NASA Johnson Space Center, Mail Code KT, Houston, TX 77058, USA. ${ }^{8}$ NASA Ames Research Center, M/S 245-6, Moffett Field, CA 94035-1000, USA.

Introduction: A Japanese asteroid explorer Hayabusa has successfully returned its reentry capsule on June 13th, 2010. As detailed previously [1], a series of processes have been held at the Planetary Material Sample Curation Facility in JAXA. The sample container was extracted from the reentry capsule and then introduced into clean chambers to be opened in there. After opening, the sample catcher, which consists mainly of two rooms (Room-A and -B) and a rotational cylinder, was extracted from the container. Then the particles inside the catcher were recovered with three different sampling methods by (1) direct pickup from the catcher, (2) Teflon spatula scooping and (3) compulsory fall onto a silica glass disk, followed by analyses by FESEM-EDS. We eventually concluded that the rocky particles must have originated from S-type Asteroid 25143 Itokawa based on their mineralogy and elemental ratios relative to remote sensing results of the asteroid surface [2].

Sample Curation for Initial Analyses: There are more than a thousand particles of $>10 \mu \mathrm{m}$ in size recognised on the silica glass disk which gathered those fallen compulsory from the catcher Room-A. Among them, more than a hundred particles have been removed and more than fifty were distributed for the initial analyses thus far. Their preliminary results are presented in [2-10].

Future Schedule for Further Sample Distribution: The initial analyses are still in progress, and we will distribute some of particles recovered from the catcher Room-B. Then some part of the particles will be distributed to NASA, based on the Memorandum of Understanding (MOU) between Japan and USA for the Hayabusa mission. Finally, in the near future an international Announcement of Opportunity (AO) for sample analyses will be open to any interested party.

References: [1] Fujimura A. et al. 2011. Abstract \#1829. 42nd Lunar and Planetary Science Conference. [2] Nakamura T. et al. 2011. Abstract \#1766. 42nd Lunar and Planetary Science Conference. [3] Tsuchiyama A. et al. 2011. Abstract \#1766. 42nd Lunar and Planetary Science Conference. [4] Ebihara M. et al. 2011. Abstract \#1902. 42nd Lunar and Planetary Science Conference. [5] Yurimoto H. et al. 2011. Abstract \#1755. 42nd Lunar and Planetary Science Conference. [6] Noguchi T. et al. 2011. Abstract \#1596. 42nd Lunar and Planetary Science Conference. [7] Okazaki R. et al. 2011. Abstract \#1653. 42nd Lunar and Planetary Science Conference. [8] Nagao K. et al. 2011. Abstract \#2119. 42nd Lunar and Planetary Science Conference. [9] Kitajima F. et al. 2011. Abstract \#1855. 42nd Lunar and Planetary Science Conference. [10] Naraoka H. et al. 2011. Abstract \#1672. 42nd Lunar and Planetary Science Conference.
5300

AB INITIO CALCULATION ON PHOTODISSOCIATION OF CO MOLECULES TO TEST THE SELF-SHIELDING MODEL

A. Yamada ${ }^{1}$, S. Nanbu ${ }^{2}$ and M. Ozima ${ }^{1}{ }^{1}$ Department of Earth and Planetary Science, University of Tokyo, Tokyo 113-0033, Japan. E-mail: yamada@eps.s.u-tokyo.ac.jp. ${ }^{2}$ Sophia University.

The mass-independently fractionated oxygen isotope in CAI of primitive meteorites is commonly attributed to self-shielding effect of $\mathrm{CO}$ [e.g., 1]. Besides its unusual implication that CAI oxygen must be the same as the solar oxygen, the self-shielding hypothesis is based on several dubious premises including no isotopic effect of photo-dissociation of CO. Here, we show from a quantum mechanical calculation that photodissociation of $\mathrm{CO}$ seems to give rise to yield substantial isotopic effect on the photo-dissociation.

$\mathrm{Ab}$ initio reaction dynamics simulations were performed to compute the photolysis rate for the $\mathrm{E}^{1} \angle$ ? $\mathrm{X}^{1} \Sigma^{+}$electronic transition with nonadiabatic transition to $\mathrm{k}^{3} \Pi$ and dissociative channel. This transition is considered one of the important transition for a $\mathrm{CO}$ self-shielding model. We used the Born-Oppenheimer approximation; in the first step the time independent Schrödinger equation was solved only for the electronmotion, and in the second step we performed the time dependent wavepacket dynamics for the nuclei-motion in the potential energy curves. The theoretical photo-dissociation cross section as a function of wavelength of excitation light was estimated by the Fourier transform of the autocorrelation function, $<\phi(0) \mid \phi(\mathrm{t})>[2]$. Assuming the Boltzmann distribution for vibrational and rotational state, we summed up to get the total cross section. Using photo-dissociation cross secions calculated for respective isotopes ( $i=16,17,18)$, we will disscuss photo-dissociation rate $J_{i}=\int I(z, \lambda) \phi_{i}(\lambda) \sigma_{i}(\lambda) \mathrm{d} \lambda$, where $i$ is for an isotope, $I$ is light intensity, $\phi$ is the quantum yield assumed unity, $\sigma$ is the cross secion.

References: [1] Lyons et al. 2009. Geochimica et Cosmochimica Acta 73:4998-5017. [2] Heller 1978. Journal of Chemical Physics 68:2066-2075. 


\section{3}

ALKALI-RICH FRAGMENTS IN LL-CHONDRITIC BRECCIAS

T. Yokoyama ${ }^{1}, \mathrm{~K}$. Misawa ${ }^{1,2}$, and O. Okano ${ }^{3}$. ${ }^{1}$ Graduate University for Advanced Studies, Tachikawa, Tokyo 190-8518, Japan. E-mail: yokoyama.tatsunori@nipr.ac.jp. ${ }^{2}$ Antarctic Meteorite Research Center, NIPR, Tachikawa, Tokyo 190-8518, Japan. ${ }^{3}$ Faculty of Science, Okayama University, Okayama 700-8530, Japan.

Introduction: Alkali-rich igneous fragments were identified in the brecciated LL chondrites, Krähenberg (LL5) [1], Bhola (LL3-6) [2], and Yamato (Y)-74442 (LL4) [3-5], and show characteristic fractionation patterns of alkaline elements (e.g., $\mathrm{Na}$ approximately $0.5 \times \mathrm{CI}, \mathrm{K}$ approximately $12 \times \mathrm{CI}, \mathrm{Rb}$ approximately $45 \times \mathrm{CI}$, and $\mathrm{Cs}$ approximately $70 \times$ CI [6]). The alkali-rich fragments in Krähenberg, Bhola, and Y74442 are very similar in mineralogy, petrography, and mineral chemistry, implying that they could have formed from related precursor materials [5]. In order to understand origin of these alkali-rich fragments as well as to constrain timing of their formation, we have undertaken $\mathrm{Rb}-\mathrm{Sr}$ isotopic studies on alkali-rich fragments in Bhola and Y-74442.

Results and Discussion: Abundances of rubidium in the whole-rock samples of Bhola and Y-74442 (50-100 mg in weight) are 2-10 times of those of ordinary chondrites, indicating a contribution of alkaline-rich fragments. Alkali-rich fragments separated from Bhola and Y-74442 show extreme enrichments of rubidium (10-70 $\times$ chondrites). Model ages of Bhola and Y-74442 whole-rock samples are calculated to be 4.67 and $4.63 \mathrm{Ga}$, respectively, when LL-chondrite ${ }^{87} \mathrm{Sr} /{ }^{86} \mathrm{Sr}$ initial ratio of 0.69900 [7] (using $\lambda_{87 \mathrm{Rb}}=1.402 \times 10^{-11} \mathrm{a}^{-1}$; adjusted NBS-987 ${ }^{87} \mathrm{Sr} /{ }^{86} \mathrm{Sr}$ to 0.710250 ) is used. Bhola and Y-74442 (including three data points of [8]) yield a whole-rock Rb-Sr age of $4596 \pm 96 \mathrm{Ma}$ with an initial ${ }^{87} \mathrm{Sr} /{ }^{86} \mathrm{Sr}$ of $0.7000 \pm 0.0046$. Relatively young ${ }^{39} \mathrm{Ar}-{ }^{40} \mathrm{Ar}$ ages of approximately $4200 \mathrm{Ma}$ were reported for alkali-rich fragments in Krähenberg and Bhola [9], and the whole-rock sample of Y-74442 [10], which suggests that they suffered impact event(s) with the partial degassing of Ar.

Potassium, rubidium, and cesium are also classified as large ion lithophiles and are partitioned into residual phases during crystallization. The alkali-rich fragments in Krähenberg and Bhola possess flat REE patterns, which is different from the alkali-rich early planetary objects, Graves Nunatak (GRA) 06128/06129, showing LREE/HREE fractionations [11]. Geochemistry (i.e., solid/liquid fractionation process) could not be responsible for the enrichments of heavier alkalis in the Krähenberg, Bhola, and Y-74442 fragments. Taking into account for the lack of detectable potassium isotopic fractionation in the Krähenberg fragment [12] along with the old formation ages of approximately $4.56 \mathrm{Ga}$ for the Krähenberg and Y-74442 fragments [1, 13], an alkali-rich component could have formed during an early stage of solar system evolution and incorporated into precursor materials of fragments prior to a final melting event.

References: [1] Kempe W. and Müller O. 1969. Meteorite Research, pp. 418. [2] Noonan A. F. et al. 1978. Geological Survey Open File Report 78-701, 311. [3] Yanai K. et al. 1978. Memoirs of the National Institute Polar Research Special Issue 8:110. [4] Ikeda Y. and Takeda H. 1979. Memoirs of the National Institute Polar Research Special Issue 15:123. [5] Yokoyama T. et al. 2011. Abstract \#1941. 42nd Lunar Prospector Spacecraft. [6] Wlotzka F. et al. 1983. Geochimica et Cosmochimica Acta 47:743. [7] Minster J. F. and Allègre C. J. 1981. Earth Planetary Sciences 56:89. [8] Okano O. et al. 1990. Geochimica et Cosmochimica Acta 54:3509. [9] Trieloff M. et al. 1994. Meteoritics 29:541. [10] Kaneoka I. and Nagao K. 1993. Proceedings of NIPR Symposium Antarctica Meteorites 6:88. [11] Day J. M. D. et al. 2009. Nature 457:179. [12] Humayun M. and Clayton R. N. 1995. Geochimica et Cosmochimica Acta 59:2131. [13] Nishiya N. et al. 1995. Okayama University of Earth Science Report 2:91.

\section{9}

HYDROUS MINERALOGY OF CARBONACEOUS CHONDRITES FROM THERMOGRAVIMETRY ANALYSIS

Z. Yoldi-Martinez ${ }^{1}$, P. Beck ${ }^{1}$, G. Montes-Hernandez ${ }^{2}$, R. Chiriac ${ }^{3}$, E. Quirico $^{1}$, L. Bonal ${ }^{1}$, B. Schmitt ${ }^{1}$ and F. Moynier ${ }^{4}$. ${ }^{1}$ UJF-Grenoble 1/ CNRS-INSU, Institut de Planetologie et d'Astrophysique de Grenoble (IPAG), France, E-mail: pierre.beck@obs.ujf-grenoble.fr. ${ }^{2}$ Institut des Sciences de la Terre (IsTERRE), UJF-Grenoble 1, France. ${ }^{3}$ Université de Lyon, Université Lyon 1, Laboratoire des Multimatériaux et Interfaces UMR CNRS 5615.

Introduction: Carbonaceous chondrites are considered as amongst the most primitive Solar System samples available. One of their primitive characteristics is their enrichment in volatile elements. This includes hydrogen, which is present in hydrated and hydroxylated minerals. More precisely, the mineralogy is expected to be dominated by phyllosilicates in the case of CM chondrites, and by Montmorillonite type clays in the case of CI. Here, in order to characterize and quantify the abundance of lowtemperature minerals in carbonaceous chondrites, we performed thermogravimetric analysis of matrix fragments of Tagish Lake, Murchison and Orgueil.

Methods: Chunks of the matrix of Murchison, Tagish Lake, and Orgueil were extracted for TGA analysis following [1]. TGA experiments were performed with TGA/SDTA $851^{\mathrm{e}}$ Mettler Toledo under the following conditions: sample mass of about $10 \mathrm{mg}$, platinum crucible of $150 \mu \mathrm{L}$ with a pinhole, heating rate of $10^{\circ} \mathrm{C} \mathrm{min}^{-1}$, and inert $\mathrm{N}_{2}$ atmosphere of $50 \mathrm{~mL} \mathrm{m^{-1 }}$. Sample mass loss and associated thermal effects were obtained by TGA/SDTA. In order to integrate the different mass loss steps, the TGA first derivative (mass loss rate) was used. TGA apparatus was calibrated in terms of mass and temperature. A total of about 15 reference minerals were analyzed for comparison.

Exchangeable Water: The three samples studied show significant endothermic mass loss below $200{ }^{\circ} \mathrm{C}$. Two distinct peaks are present on the first derivative curve in the case of Murchison and Orgueil, corresponding to adsorbed $\mathrm{H}_{2} \mathrm{O}$ (peak centered at $70^{\circ} \mathrm{C}$ ) and water molecules trapped in mesopores (peak centered at $130{ }^{\circ} \mathrm{C}$ ). Tagish Lake shows the presence of adsorbed $\mathrm{H}_{2} \mathrm{O}$ only. The total amount of this "exchangeable" water is 2.8, 6 and $7.8 \mathrm{wt} \%$ for Murchison, Tagish Lake and Orgueil respectively.

-OH bearing mineral phases: In the case of Orgueil, a significant amount of mass loss is centered at $250{ }^{\circ} \mathrm{C}$, likely related to the decomposition of ferrihydrite, confirming previous studies [2]. In addition, mass releases are observed at $570{ }^{\circ} \mathrm{C}$ and $730{ }^{\circ} \mathrm{C}$, which can be attributed to serpentine and $\mathrm{Mg}$-rich phyllosilicate. In the case of Murchison, the occurrence of a Mg-rich serpentine is suggested by the presence of a mass loss at $730{ }^{\circ} \mathrm{C}$, together with cronstedtite (mass loss at $380^{\circ} \mathrm{C}$ and $545^{\circ} \mathrm{C}$ ). Tagish Lake shows a thermal behavior distinct from that of Murchison and Orgueil since (1) Mg-serpentine appear to be absent (2) the first derative curve shows that the mass loss occurs at $570{ }^{\circ} \mathrm{C}$ with a shoulder at $520{ }^{\circ} \mathrm{C}$.

Conclusions: This study shows that TGA enables to quantify the amount of "exchangeable water" for a given meteorite sample. This water might play a dynamic role on the early water cycle of hydrous asteroids. In addition, TGA are of great help for estimating the $\mathrm{H}$ budget in various chondrite groups and understanding the radial transfer of volatiles in the Solar System.

References: [1] Montes-Hernandez et al. submitted. [2] Tomeoka K and Buseck P.R. 1988. Geochimica et Cosmochimica Acta 52:1627-1640. 
5470

EARLY FORMATION OF DIFFERENTIATED PARENT BODIES AND LATE FORMATION OF CHONDRITE PARENT BODIES BEYOND THE ASTEROID BELT

E. D. Young. Department of Earth \& Space Sciences, University of California Los Angeles, 595 Charles E. Young Drive East, Los Angeles, CA 90095. E-mail: eyoung@ess.ucla.edu.

Introduction: Our understanding of the chronology of planetesimal formation has changed significantly in the past several years. Highprecision chronometers (e.g., ${ }^{182} \mathrm{Hf} /{ }^{182} \mathrm{~W},{ }^{26} \mathrm{Al} /{ }^{26} \mathrm{Mg}$ ) reveal that differentiated bodies formed very rapidly, often with metal-silicate separation ages nearly indistinguishable from CAI formation (i.e., time zero). These same constraints require that chondrite parent bodies generally formed several million years after CAIs. In retrospect, thermal models for planetesimal formation and evolution could have been used to reach these same conclusions. Although thermal models are often employed to simulate the evolution of individual bodies for detailed comparisons with meteoritical data [1], the spectrum of possible evolution histories provides robust constraints on feasible combinations of accretion times and peak temperatures (e.g., differentiated vs. undifferentiated; [2]).

Method: A spherically symmetric finite difference code that simulates the thermal evolution of planetesimals, taking into account enthalpies of both water and rock phase transitions and convective enhancement of heat flow, was constructed. The base state assumes $30 \%$ by volume water. The heat sources in these calculations are the shortlived nuclides. The code was applied to a wide range of initial conditions that map out possible and impossible combinations of time and peak temperature.

Results: Mapping results in terms of accretion time (post-CAI) and radii of the bodies shows clearly that silicate-metal segregation dated by the $\mathrm{Hf} / \mathrm{W}$ system requires accretion of these parent bodies prior to $1.5 \mathrm{Myr}$ post-CAI. The result is robust and is consistent with recent dating of igneous meteorites [3]. A minimum radius of $\sim 30 \mathrm{~km}$ applies for formation of bodies destined for metal-silicate segregation at $1.5 \mathrm{Myr}$, with smaller radii possible at $<1.5 \mathrm{Myr}$. Chondrite parent bodies in which temperatures did not exceed ca. 1,000 K must have formed $>1.5 \mathrm{Myr}$ post-CAI for all radii $>15 \mathrm{~km}$, consistent with the emerging new chronological constraints for chondrules and their host rocks. For bodies that never suffered temperatures greater than $500 \mathrm{~K}$ (e.g., some carbonaceous chondrites), accretion times are limited to $>2.2 \mathrm{Myr}$ for radii $>15 \mathrm{~km}$. Thermal models require early $(<1.5 \mathrm{Myr})$ formation of differentiated bodies and late $(>2 \mathrm{Myr})$ formation of carbonaceous chondrite parent bodies.

Discussion: Models for the dynamics of the early solar system linked to giant planet migration raise significant questions about the provenance of bodies populating the asteroid belt. The constraints on accretion times provided by thermal models, in apparent agreement with emerging chronological constraints, suggest that carbonaceous chondrites may represent objects accreted at heliocentric distances well beyond the asteroid belt (e.g., if accretion times for bodies tens of kilometers in diameter varied with distance $R$ from the nascent Sun according to $\tau(\mathrm{yrs})$ $\left.\sim 2000(R / 1 \mathrm{AU})^{3 / 2}[4]\right)$.

References: [1] Young E. D. et al. 1999. Science 286:1331-1335. [2] Ghosh A. et al. 2006. Meteorites and the early solar system II, 555-556. [3] Kleine T. et al. 2009. Geochimica et Cosmochimica Acta 73:5150-5188. [4] Weidenschilling S. J. 2000. Space Science Reviews 92:295-310.

\section{3}

EVOLUTION TO WEAK NUCLEAR STATISTICAL EQUILIBRIUM AND THE NEUTRON-RICH IRON GROUP ISOTOPES

T. $\mathrm{Yu}$ and B. S. Meyer. Department of Physics and Astronomy, Clemson University, Clemson, SC 29634-0978, USA.

Introduction: FUN CAIs and hibonite grains show roughly correlated excesses and deficits in the neutron-rich iron-group isotopes such as ${ }^{48} \mathrm{Ca}$ and ${ }^{50} \mathrm{Ti}$ (e.g., [1] and references therein). Such effects seem to call for correlation of these isotopes in the precursor dust of the Solar System. From a nucleosynthesis point of view, this makes sense because the neutron-rich iron-group isotopes are copiously co-produced in lowentropy expansions of neutron-rich matter [2], which presumably occur in rare Type Ia (thermonuclear) supernovae. The production of these isotopes in such expansions, however, depends on how neutron-rich the material becomes, which in turn depends on the complicated nuclear dynamics of hot, dense, neutron-rich matter.

Weak Interaction Rates: To explore the production of the neutronrich iron-group nuclei, we have developed tools [3] to follow the evolution of nuclear reaction networks into weak nuclear statistical equilibrium (WSE). Weak interaction rate compilations have been calculated before (e.g., $[4,5])$. However these compilations do not guarantee a network evolves to a weak nuclear statistical equilibrium (WSE) that is consistent with the other nuclear data in the problem because the compilations treat forward and reverse rates separately. We have developed tools to compute the reverse weak rates from detailed balance. This enables us to compare network and equilibrium calculations consistently.

Results: With our tools, we calculated the nucleosynthesis in matter at fixed high temperature $\left(6 \times 10^{9} \mathrm{~K}\right)$ and density $\left(9 \times 10^{9} \mathrm{~g} \mathrm{cc}^{-1}\right)$ and found that $Y_{\mathrm{e}}$ (number of net electrons per nucleon) evolves to 0.397 , the WSE value. If we instead do not insist on weak detailed balance, $Y_{\mathrm{e}}$ evolves to 0.419 with rates from reference [3]. This value is inconsistent with WSE. The resulting ${ }^{48} \mathrm{Ca}$ abundance differs significantly in the two calculations. Another interesting result is that the system evolves into WSE via a quasi-equilibrium, not a nuclear statistical equilibrium (NSE). NSE is a state in which all nuclei are in equilibrium under strong and electromagnetic, but not necessarily weak, reactions. The reason for this is that, as dense, hot matter is undergoing the electron captures to reduce $Y_{\mathrm{e}}$, the number of heavy nuclei $Y_{\mathrm{h}}$ must also change. The tight balance between forward and reverse strong and electromagnetic reactions causes the effective timescale for $Y_{\mathrm{h}}$ to evolve to become comparable to that for $Y_{\mathrm{e}}$ to change.

Conclusions: A proper treatment of forward and reverse weakinteraction rates is important for calculations of the yields of neutron-rich isotopes in low-entropy freeze-outs. We also have found that in such lowentropy environments, the nuclear populations can evolve to WSE through a quasi-equilibrium instead of a regular NSE. This result will be important for those computing effective neutronization rates in dense Type Ia supernovae.

References: [1] Meyer B. S. and Zinner E. In Meteorites and the early solar system II (Tucson: University of Arizona Press), pp. 69-108. [2] Meyer B. S. et al. 1996. The Astrophysical Journal 462:825-838. [3] See https://sourceforge.net/projects/nucnettools/ [4] Fuller G. M. et al. 1985. The Astrophysical Journal 293:1-16. [5] Langanke K. and MartínezPinedo G. 2000. Nuclear Physics A 673:481-508. 
5320

OXYGEN AND MAGNESIUM ISOTOPIC COMPOSITIONS OF ASTEROID 25143 ITOKAWA RETURNED BY THE HAYABUSA MISSION

H. Yurimoto ${ }^{1}$, K. Abe ${ }^{1}$, M. Abe $^{2}$, M. Ebihara $^{3}$, A. Fujimura ${ }^{2}$, M.

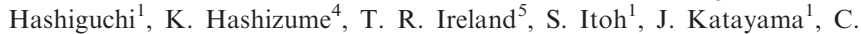
Kato $^{1}$, J. Kawaguchi ${ }^{2}$, N. Kawasaki ${ }^{1}$, F. Kitajima ${ }^{6}$, S. Kobayashi ${ }^{1}$, T. Meike $^{1}$, T. Mukai ${ }^{2}$, K. Nagao ${ }^{7}$, T. Nakamura ${ }^{8}$, H. Naraoka ${ }^{6}$, T.

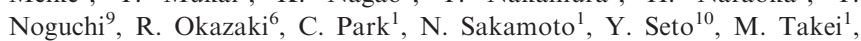
A. Tsuchiyama ${ }^{4}$, M. Uesugi ${ }^{2}, \mathrm{~S}$. Wakaki ${ }^{1}, \mathrm{~T}_{\text {. Yada }}{ }^{2}, \mathrm{~K}$. Yamamoto ${ }^{1}, \mathrm{M}$. Yoshikawa $^{2}$ and M. E. Zolensky ${ }^{11}$. ${ }^{1}$ Hokkaido University. E-mail: yuri@ep.sci.hokudai.ac.jp. ${ }^{2}$ JAXA-ISAS. ${ }^{3}$ Tokyo Metropolitan University. ${ }^{4}$ Osaka University. ${ }^{5}$ The Australian National University. ${ }^{6}$ Kyushu University. ${ }^{7}$ The University of Tokyo. ${ }^{8}$ Tohoku University. ${ }^{9}$ Ibaraki University. ${ }^{10}$ Kobe University. ${ }^{11}$ NASA Johnson Space Center.

Introduction: The asteroid Itokawa is classified as an S-type asteroid and inferred, based upon reflectance spectrometry, to consist of materials similar to primitive achondrites or ordinary chondrites $[1,2]$. In order to unequivocally establish the connection between asteroids and meteorites, determination of oxygen isotopic composition of asteroidal material is absolutely imperative. For example, primitive achondrites and ordinary chondrites are clearly distinguishable by their oxygen isotopic compositions [3]. This is an updated report of the oxygen isotopic composition from the preliminary report [4] and the first report of the magnesium isotopic composition of Itokawa.

Experiments: Each grain was mounted at the center of an epoxy disk and the surface was polished under the processes established for the preliminary examination. The samples were gold-coated to a thickness of $60 \mathrm{~nm}$.

Oxygen and magnesium isotope compositions have been investigated by the Hokudai isotope microscope system [5]. $\mathrm{A} \mathrm{Cs}^{+}$primary beam of $20 \mathrm{keV}$ and $\mathrm{O}^{-}$of $23 \mathrm{keV}$ was focused to a spot of approximately $10 \mu \mathrm{m}$ on the surface of the sample for $\mathrm{O}$ and $\mathrm{Mg}$ isotope analyses, respectively. Secondary ions were collected simultaneously in Faraday cups or in electron multipliers corresponding to the secondary ion intensities using a Cameca multi-collection system. Terrestrial standards of the corresponding minerals were used for normalization.

Results and Discussion: All oxygen isotopic compositions of the minerals from Itokawa plot on the upper side of terrestrial materials on an oxygen three-isotope diagram and are distributed parallel to the terrestrial mass fractionation line. The $\Delta \Delta^{17} \mathrm{O}_{\text {SMOw }}$ for Itokawa is $1.46 \% \pm 0.41(2 \sigma)$ for olivine, $1.57 \% \pm 0.62(2 \sigma)$ for orthopyroxene, and $1.15 \% \pm 0.51(2 \sigma)$ for plagioclase. The mean $\Delta \Delta^{17} \mathrm{O}_{\text {SMOW }}$ for minerals from Itokawa, $1.39 \% \pm 0.36(2 \sigma)$, coincide with those of LL or $\mathrm{L}$ chondrites, but not with those of $\mathrm{H}$ chondrites.

We have observed no $\delta^{26} \mathrm{Mg}$-excesses for olivine, orthopyroxene and plagioclase from Itokawa in $2 \sigma$ confidence of the analytical precisions. The initial ${ }^{26} \mathrm{Al} /{ }^{27} \mathrm{Al}$ ratio is calculated to be $3 \pm 8(2 \sigma) \times 10^{-8}$ and the initial $\delta^{26} \mathrm{Mg}$ is to be $0.01 \pm 0.02 \%$, which coincide with those of equilibrated chondrites.

References: [1] Abell P. A. et al. 2007. Meteoritics \& Planetary Science 42:2165-2177. [2] Abe M. et al. 2006. Science 312:1334-1338. [3] Clayton, R. N. 2005. In Meteorites, Comets, and Planets, Treatise on Geochemistry, vol. 1. Amsterdam: Elsevier. pp. 129-142. [4] Yurimoto H. et al. 2011. Abstract \#1755. 42nd Lunar and Planetary Science Conference. [5] Yurimoto H. et al. 2003. Applied Surface Science 203-204, 793-797.

\section{8}

CHEMISTRY OF CARBONACEOUS CHONDRITE MATRICES: PARENT-BODY ALTERATION AND CHONDRULE-MATRIX COMPLEMENTARITY

B. Zanda ${ }^{1,2}$, M. Humayun ${ }^{3}$, J.-A. Barrat ${ }^{4}$, M. Bourot-Denise ${ }^{1}$ and R. H. Hewins ${ }^{1,2} .{ }^{1}$ MNHN \& CNRS, 61 rue Buffon, 75005 Paris, France (zanda@mnhn.fr). ${ }^{2}$ Department of Earth \& Planet. Sci., Rutgers University, Piscataway, NJ, USA. ${ }^{3}$ Department of Earth, Ocean \& Atmospheric Science and National High Magnetic Field Laboratory, FSU, Tallahassee, FL 32310, USA. ${ }^{4}$ Université Européenne de Bretagne, CNRS UMR 6538, U.B.O-I.U.E.M., Place Copernic, 29280 Plouzané, France.

Introduction: The contribution of chondrite matrices to the volatile element budget and the $\mathrm{Mg} / \mathrm{Si}$ of the bulk rocks is fundamental. Bland et al. [1] showed LA-ICP-MS volatile element abundances of CC matrices to be related to those of the bulk rocks, suggesting a "complementarity" with their adjoining high $\mathrm{T}$ fractions. [2] also advocates "complementarity" based on the $\mathrm{Mg} / \mathrm{Si}$ of these components determined by EMP. However, [3, 4] argued that these relationships were due to parent body alteration. Here we present new data to support this conclusion.

Method: We selected a suite of carbonaceous chondrites with varying degrees of alteration [5,6], including CI chondrites [7] and the Paris CM breccia, which contains significantly different lithologies [4]. Bulk chemical analyses were conducted both at UBO by ICP-MS and ICP-AES after wet chemistry, and at NHMFL by an in-situ rastering with LA-ICP-MS. In-situ analyses of matrices were performed both by EMP and LA-ICP-MS.

Results and Discussion: Bulk analyses conducted by the two methods are reproducible and consistent [7]. In particular, they show the bulk compositions of the less altered and more altered zones of Paris to be indistinguishable. On the other hand, in-situ analyses of the matrix differ significantly: matrix in the less altered zones has a CI composition, while that of the more altered zones has lost $\mathrm{S}$ and chalcophiles and gained $\mathrm{Fe}$ and siderophiles [4]. EMP data for other CM matrices indicate that their $\mathrm{S} / \mathrm{Si}$ decreases with the extent of parent-body alteration, in agreement with the above results. Matrix compositions thus vary with alteration although the bulk rocks remain isochemical. This suggests local exchange between matrices and high temperature fractions, and a CI composition for the matrices at the time of accretion.

Conclusion: These results contradict pre-accretion complementarity between matrix and the high $\mathrm{T}$ fraction as advocated by $[1,2]$ and support the two-component model of Anders [8]. They allow these components to be formed independently and accrete in varying proportions to generate the range of bulk $\mathrm{CC}$ compositions. It is thus possible that high $\mathrm{T}$ components formed near the Sun were transported over large distances and mixed with matrix in colder regions of the disk, which would explain their presence in cometary materials.

References: [1] Bland P. et al. 2005. Proceedings of National Academic Sciences 102:13755-13760. [2] Hezel D. and Palme H. 2010. Earth and Planetary Science Letters 294:85-93. [3] Zanda B. et al. 2009. Abstract \#5280. Meteoritics \& Planetary Science, vol. 44, suppl. [4] Zanda et al. 2011. Abstract \#2040. Lunar and Planetary Sciences. [5] Browning et al. 1996. Geochimica et Cosmochimica Acta 60:2621-2633. [6] Rubin et al. 2007. Geochimica et Cosmochimica Acta 71:2361-2382. [7] Barrat et al. 2011. This conference. [8] Anders E. 1964. Space Science Review 3:583-714. 
5265

THE Stardust INVESTIGATION INTO THE CR2 CHONDRITE GRV 021710

X. Zhao ${ }^{1}$, C. Floss ${ }^{2}$, F. J. Stadermann ${ }^{2}$, Y. Lin ${ }^{1}$ and M. Bose $^{2}$. ${ }^{1}$ Laboratory of Earth's Deep Interior, Institute of Geology and Geophysics, Chinese Academy of Sciences, Beijing 100029, China. E-mail: xzhao@mail.iggcas.ac.cn. ${ }^{2}$ Laboratory for Space Sciences and Physics Department, Washington University, St. Louis, MO 63130, USA.

Introduction: Abundant presolar grains have been identified in CR3 chondrites [1,2], but studies of CR2 chondrites so far have not shown very high abundances of presolar material [3-5]. We are investigating the presolar grain abundance of GRV 021710, which is a very fresh CR2 chondrite collected from Grove Mountains, East Antarctica [6].

Experimental: We used the Washington University Cameca NanoSIMS for isotopic mapping. Two $\mathrm{C}$ and three $\mathrm{O}$ isotopes were collected simultaneously. The total area of matrix analyzed was $10,700 \mu \mathrm{m}^{2}$. Isotopically anomalous grains were analyzed for their elemental compositions using the Auger Nanoprobe.

Results and Discussion: A total of 35 O-anomalous grains and $32 \mathrm{C}$ anomalous grains were found. Nine of the $35 \mathrm{O}$-anomalous grains are ${ }^{18} \mathrm{O}$-rich and belong to Group 4, with likely origins in supernovae [7]. The remaining $\mathrm{O}$-anomalous grains belong to Group 1, with enrichments in ${ }^{17} \mathrm{O}$ and close to solar ${ }^{18} \mathrm{O} /{ }^{16} \mathrm{O}$ ratios [7]. Most of the $\mathrm{C}$-anomalous grains (25 out of 32) have ${ }^{12} \mathrm{C} /{ }^{13} \mathrm{C}$ ratios range from 25 to 76 , similar to the compositions of mainstream $\mathrm{SiC}$ grains [8]. Six $\mathrm{C}$-anomalous grains are ${ }^{13} \mathrm{C}$-depleted, whereas the remaining one has a very low ${ }^{12} \mathrm{C} /{ }^{13} \mathrm{C}$ ratio $\left({ }^{12} \mathrm{C} /{ }^{13} \mathrm{C}=4\right)$ like $\mathrm{SiC} \mathrm{A}+\mathrm{B}$ grains.

Auger elemental results show that $35 \mathrm{O}$-anomalous grains consist of $33 \mathrm{Fe}$ - and/or Mg-bearing silicates and 2 oxides, with an average grain size of $260 \mathrm{~nm}$. Based on the $(\mathrm{Fe}+\mathrm{Mg}+\mathrm{Ca}) / \mathrm{Si}$ ratios, these 33 silicate grains can be classified into five types: olivine-like grains (10), pyroxenelike grains (7), intermediate grains (6), Si-rich (6) and Si-poor (4) grains. Most of these silicate grains are Mg-rich (mg\#s: 29-100). The two oxides include one spinel-like grain and one $\mathrm{SiO}_{2}$ grain. More structural data is needed to confirm the crystallinity of the $\mathrm{SiO}_{2}$ grain.

Auger spectra and elemental maps of the $32 \mathrm{C}$-anomalous grains show $15 \mathrm{SiC}$ and 16 carbonaceous grains. The remaining one was sputtered away during the NanoSIMS measurement and could not be located in the Auger Nanoprobe. Except one $\mathrm{SiC} \mathrm{A}+\mathrm{B}$ grain and one probable $\mathrm{SiC}$ Y grain, remaining grains are mainstream $\mathrm{SiC}$ grains. Most of the carbonaceous grains are dark in secondary electron images, could be graphites or insoluble organic matter.

Based on the total matrix area analyzed and the surface areas of the grains, we can calculate the abundances of $\mathrm{O}$-anomalous and $\mathrm{C}$ anomalous grains to be $174 \pm 30$ and $203 \pm 36 \mathrm{ppm}$ respectively, which are similar to the results from CR 3 chondrites $[1,2]$. We can also calculate an abundances of $165 \pm 29 \mathrm{ppm}$ for presolar silicates, $9 \pm 6 \mathrm{ppm}$ for presolar oxides, $135 \pm 35 \mathrm{ppm}$ for $\mathrm{SiC}$ grains and $50 \pm 12$ for carbonaceous grains.

References: [1] Floss C. and Stadermann F. J. 2009. Geochimica et Cosmochimica Acta 73:2415-2440. [2] Floss C. and Stadermann F. J. 2009. The Astrophysical Journal 697:1242-1255. [3] Nagashima K. et al. 2004. Nature 428:921-924. [4] Floss C. and Stadermann F. J. 2005. Abstract \#1390. 36th Lunar and Planetary Science Conference. [5] Leitner J. et al. 2011. Abstract \#1713. 42nd Lunar and Planetary Science Conference. [6] Miao B. et al. 2007. Meteoritics \& Planetary Science 42:A106. [7] Nittler L. R. et al. 2008. The Astrophysical Journal 682:14501478. [8] Zinner E. 2004. In Meteorites, comets, and planets. Treatise on Geochemistry, vol. 1 (ed. A. M. Davis), pp. 17-39.

\section{3}

ON THE QUESTION OF INTRINSIC ISOTOPIC HETEROGENEITY WITHIN PRESOLAR GRAINS

E. Zinner ${ }^{1}$ and M. Jadhav ${ }^{1,2}$. ${ }^{1}$ Laboratory for Space Sciences and Physics Department, Washington University, St. Louis, MO, USA. E-mail: ekz@wustl.edu. ${ }^{2}$ Institute of Geophysics and Planetology, University of Hawaii, Honolulu, HI 96822, USA.

Introduction: Detection of isotopic heterogeneity at the time of grain formation could provide information on the astrophysical environment where presolar grains formed. Isotopic heterogeneity has been found in many cases but has been generally attributed to contamination [1, Fig. 8] or isotopic equilibration [2]. Strictly speaking, isotopic contamination or equilibration is always a possibility if only two isotopes have been measured and the resulting ratios in a given grain are not both larger and smaller than solar. But even measurements of 3-isotope system cannot unequivocally identify isotopic heterogeneity. An example is the Oisotopic measurements inside of a TEM slice of a low-density graphite grain from Murchison [2]. Because the ${ }^{17} \mathrm{O} /{ }^{16} \mathrm{O}$ ratios are close to solar, the large variations in ${ }^{18} \mathrm{O} /{ }^{16} \mathrm{O}$ can still be explained by isotopic equilibration. The situation is similar for Fe-rich subgrains within a $\mathrm{SiC}$ $\mathrm{X}$ grain that show differences in the ${ }^{57} \mathrm{Fe} /{ }^{56} \mathrm{Fe}$ ratios but solar ${ }^{54} \mathrm{Fe} /{ }^{56} \mathrm{Fe}$ ratios [3]. These considerations can be extended to apparent variations in inferred ${ }^{26} \mathrm{Al} /{ }^{27} \mathrm{Al}$ ratios in $\mathrm{X}$ grains that can be explained by contamination with $\mathrm{Al}[4,5]$.

Results: The best candidate for a grain with intrinsic isotopic heterogeneity is ORG1f3m-25 from the high density graphite fraction $\left(2.02-2.04 \mathrm{~g} \mathrm{~cm}^{-3}\right)$ from Orgueil [6]. This $5 \mu \mathrm{m}$ large grain has ${ }^{12} \mathrm{C} /{ }^{13} \mathrm{C}=486, \delta^{29} \mathrm{Si}=-55 \%, \delta^{30} \mathrm{Si}=-30 \%$, normal $\mathrm{N}, \mathrm{O}$, and $\mathrm{Mg}$, but large anomalies in $\mathrm{Ca}\left(\delta^{42} \mathrm{Ca}=-69 \%, \delta^{43} \mathrm{Ca}=487 \%, \quad \delta^{44} \mathrm{Ca}=\right.$ $1193 \%)$ and $\mathrm{Ti} \quad\left(\delta^{46} \mathrm{Ti}=-792 \%, \quad \delta^{47} \mathrm{Ti}=-122 \%, \quad \delta^{49} \mathrm{Ti}=1802 \%\right.$, $\delta^{50} \mathrm{Ti}=5300 \%$ ). Large variations in the $\mathrm{Ti}$ signal during grain analysis indicate the presence of Ti-rich subgrains (TiC). However, while the ${ }^{49} \mathrm{Ti} /{ }^{48} \mathrm{Ti}$ and ${ }^{50} \mathrm{Ti} /{ }^{48} \mathrm{Ti}$ ratios are fairly uniform throughout the graphite grain, the ${ }^{47} \mathrm{Ti} /{ }^{48} \mathrm{Ti}$ ratio shows large variations (Fig. 1). While the fact that $\delta^{44} \mathrm{Ca}>\delta^{43} \mathrm{Ca}$ and the association of the largest ${ }^{44} \mathrm{Ca}$ excesses with the highest ${ }^{48} \mathrm{Ti} /{ }^{40} \mathrm{Ca}$ ratios (in the middle of Ti-rich subgrain 2 ) indicate that some ${ }^{44} \mathrm{Ca}$ is from ${ }^{44} \mathrm{Ti}$ decay, Ti-rich subgrain 1 seems to have no or only little radiogenic ${ }^{44} \mathrm{Ca}$, but a ${ }^{47} \mathrm{Ti}$ excess comparable to that in subgrain 2 .

References: [1] Zinner E. et al. 1995. Meteoritics 30:209-226. [2] Stadermann F. J. et al. 2005. Geochimica et Cosmochimica Acta 69:177-188. [3] Marhas K. K. et al. 2008. The Astrophysical Journal 689:622-645. [4] Lin Y. et al. 2010. The Astrophysical Journal 709:1157-1173. [5] Zinner E. et al. 2011. Abstract \#1070. 42nd Lunar and Planetary Science Conference. [6] Jadhav M. et al. 2006. New Astronomical Review 50:591-595.

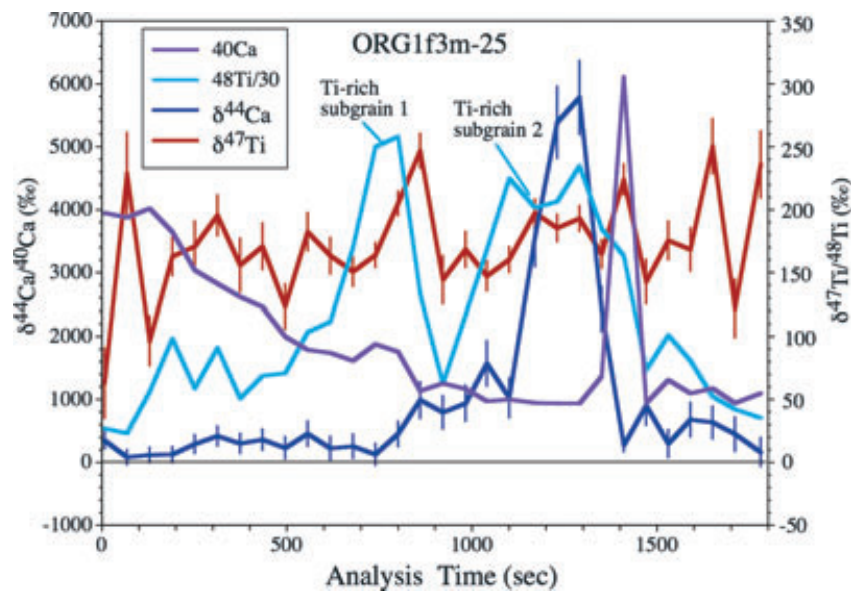


5375

\section{LITHOPHILE TRACE ELEMENTS IN MAGMATIC IRON METEORITES}

J. Zipfel ${ }^{1}$, M. Chaussidon ${ }^{2}$, C. Rollion-Bard ${ }^{2}$, H. Palme ${ }^{1}$ and A. Pack $^{3}$. ${ }^{1}$ Senckenberg Forschungsinstitut Frankfurt, Senckenberganlage 25,D-60325 Frankfurt, Germany. E-mail: jzipfel@ senckenberg.de. ${ }^{2}$ CRPG-Nancy Université-CNRS, UPR2300, BP 20, 54501 Vandoeuvrelès-Nancy, France. ${ }^{3}$ Geowissenschaftliches Zentrum, Universität Göttingen, D-37077 Göttingen, Germany.

Introduction: The chemical composition of iron meteorites reflects the conditions and processes under which they formed, such as condenzation, oxidation and fractional crystallization. Magmatic irons are characterized by steep trends of Ir versus Ni consistent with formation by fractional crystallization. Assuming that magmatic irons formed in the cores of molten asteroids in the presence of coexisting silicates we expect lithophile trace elements, e.g., $\mathrm{Si}$ to be dissolved in metal melts in concentrations of the order of 10-100 ppm [1]. Earlier work has shown that concentrations of $\mathrm{Si}$ are extremely low in all groups of iron meteorites, magmatic and non-magmatic. Concentrations around approximately $0.12 \mathrm{ppm}$ apparently reflecting low temperature processes rather than high temperature metal/silicate melt equilibrium [1]. We have started systematic analyses of lithophile elements, Si and Al, as well as $\mathrm{Cr}$ and $\mathrm{P}$ in IVB, IVA and IIIAB iron meteorites.

Analyses: Trace concentrations of $\mathrm{Si}, \mathrm{Al}, \mathrm{Cr}$ and $\mathrm{P}$ were analyzed with a Cameca ims1280 HR2 at the CRPG in Nancy at high mass resolution $(\mathrm{M} / \Delta \mathrm{M}$ approximately 7000$)$. Sensitivity factors for all elements were calculated relative to the intensity of ${ }^{56} \mathrm{Fe}$ and based on intensities in known standards from the Bundesanstalt für Rohstoffe (BAM). The spot size was about $20 \mu \mathrm{m}$ allowing to separate between Nirich and low-Ni lamellae if Widmanstätten patterns were wide enough.

Samples: We analyzed two to eight spots in Ni-rich or Ni-poor areas in the IVB iron Hoba, IVA-an iron Steinbach, one IC iron Bendego and three IIIAB irons Turtle River, Grant and Acuña. Trace concentrations of $\mathrm{Si}$ and $\mathrm{Al}$ are highest in metal of Hoba $(\mathrm{Si}=0.75 \mathrm{ppm}$ and $\mathrm{Al}=0.05 \mathrm{ppm})$ and Steinbach $(\mathrm{Si}=0.25 \mathrm{ppm}$ and $\mathrm{Al}=0.02 \mathrm{ppm})$, respectively. Concentrations in metal of the IIIAB are identical and extremely low with $\mathrm{Si}=0.04 \mathrm{ppm}$ and $\mathrm{Al}=0.007 \mathrm{ppm}$. The aluminum concentration in Bendego is slightly higher with $\mathrm{Al}=0.013 \mathrm{ppm}$ while $\mathrm{Si}$ is as low $(\mathrm{Si}=0.04 \mathrm{ppm})$ as in IIIAB irons. No difference in concentrations of these two elements between Ni-rich and Ni-poor metal was observed. $\mathrm{P}$ and $\mathrm{Cr}$ concentrations are high and variable in Hoba $(\mathrm{P}=4100-7300 \mathrm{ppm}, \quad \mathrm{Cr}=180-475 \mathrm{ppm} \quad$ in Hoba $)$ and low in Steinbach, Bendego and all IIIAB irons. In addition, there is a clear anticorrelation of $\mathrm{P}$ with $\mathrm{Cr}$; kamacite being high in $\mathrm{Cr}$ and low in $\mathrm{P}$ and taenite being low in $\mathrm{Cr}$ and high in $\mathrm{P}$.

Conclusion: Our results confirm previous studies [1] that $\mathrm{Si}$ concentrations in iron meteorites are significantly lower than expected from metal/silicate melt equilibria. This is also confirmed by low $\mathrm{Al}$ concentrations. Apparently lithophile elements do not reflect the high temperature history of iron meteorites. Concentrations of $\mathrm{Cr}$ and $\mathrm{P}$ are lower than bulk metal INAA data [e.g., 2]. This indicates that bulk analyses may be dominated by inclusions rich in such elements.

References: [1] Pack A. et al. 2011. Meteoritics \& Planetary Science, in revision. [2] Wasson J. 1999. Geochimica et Cosmochimica Acta 63:2875-2889.
5181

HOW REPRESENTATIVE OF AN ASTEROID'S MINERALOGY ARE SAMPLES FROM ITS PONDED DEPOSITS?

M. E. Zolensky ${ }^{1}$, W. Cribbs ${ }^{2}$, L. Le ${ }^{3}$ and D. K. Ross ${ }^{3}$. ${ }^{1}$ NASA Johnson Space Center, Houston, TX 77058, USA. ${ }^{2}$ University of Houston, Clear Lake, TX 77058, USA. ${ }^{3}$ Jacobs ESCG, Houston, TX 77058, USA. E-mail: michael.e.zolensky@nasa.gov.

Introduction: An unexpected observation of asteroid 433 Eros by the NEAR mission was the ponded deposits [1, 2]. Hayabusa recently returned to Earth with dust from the surface of a ponded deposit on asteroid Itokawa [3-5]. Thus the nature of asteroid ponds is of considerable interest. While ponded deposits should initially be global samples, various asteroid surface processes can modify them. Thus, how representative of a bulk asteroid is a sample from a ponded deposit?

Pond Samples: Compared to the bulk asteroid, ponds are distinctly bluer, and depleted in $\mathrm{S}$ and $\mathrm{Fe}$, possibly due to loss of species by settling or sputtering $[1,2,6]$. We have previously proposed that we have meteoritic samples of ponds from at least one asteroid, the parent body of Vigarano (CV3) [7, 8]. There are clasts found in Vigarano which consist nearly entirely of fine-grained olivine, of a composition that is identical to that of the host meteorite. The most distinctive features of these clasts are cross-bedded, arcuate bands which contain a high proportion of the finest-grained, iron-rich olivine. The relatively finegrained bands are located at the bottom of each bed. Given that the Vigarano clast mineralogies are consistent with what we already know about ponds on Eros, we think these crossbeds formed from impactinduced seismic shaking. On a suitably small, airless body, this process can result in grain-size separation with fine-grained materials "percolating" through a coarse matrix to the bottom [9] which is exactly what we observe in the Vigarano clasts. Thus the mineralogy of a sampled pond varies according to where in the pond's vertical column the sampling is made.

We have recently found another clast we believe to be from a ponded deposit. The clast is in the SAH 98328 L4 chondrite, and is a porous agglomeration of anhedral to euhedral crystals of olivine (Fa2129, peak at Fa25-26), low Ca pyroxene (Fs17-35, no peak), plagioclase, troilite, metal and minor phases. The host meteorite has the same mineralogy and mineral compositions, except that the host has a narrower range of olivine and pyroxene compositions. Unlike the Vigarano clast there is no mineralogical layering in the SAH clast, which would suggest that this clast derives from within a single pond layer.

Implications for Sampling Missions: How representative are sample return missions that sample only smooth, flat, pond deposits? The smallest asteroids have low G, and so settling may be minimized. Largest asteroids have sufficient mass to prevent ponds from forming. The midrange is where the setting effects are most pronounced, and mineralogical differentiation will be greatest. The implication of these results will be discussed.

Acknowledgment: We thank the Muséum National d'Histoire Naturelle (Paris) for the loan of the section of SAH 98328.

References: [1] Cheng et al. 2002. Meteoritics \& Planetary Science 37:1095-1106. [2] Veverka et al. 2001. Science 292:484-488. [3] Tsuchiyama et al. 2011. Science, in press. [4] Nakamura et al. 2011. Science, in press. [5] Noguchi et al. 2011. Science, in press. [6] Trombka et al. 2001. Meteoritics \& Planetary Science 36:1605-1616. [7] Zolensky et al. 2002. 33rd Lunar and Planetary Science Conference. [8] Zolensky et al. 2004. 35th Lunar and Planetary Science Conference. [9] Horz and Schaal. 1981. Icarus 46:337353. 
5524

\section{AUTHENTIC REPLICAS OF CHONDRITES FOR BASIC EDUCATION PROGRAMS}

M. E. Zucolotto ${ }^{1}{ }^{1}$ Museu Nacional/UFRJ, RJ, Brazil. E-mail: mezucolotto@globo.com.

As very few meteorites are found by geologist one of the purpose of our divulgation program "Is there an ET in your backyard?" is to teach people to identify meteorites. A proper way for identify meteorites requires a study of a large variety of samples. Indeed meteorites are not abundantly available for loan to schools or astronomy clubs and only few museums have a representative collection for public exhibition.

The meteorites on displays at museum are many times polished and etched and the meteorites are not found like this in the country so that it is need show them as they are real find. Many students of geology or astronomy never picked up a meteorite.

The reproduction of meteorite masses before cutting was done since the XIX century whether for exhibitions or for study, is show to be very important for activities of any meteorite divulgation program.

The ability to recognize a possible meteorite in the country will require some degree of familiarity with their general appearance.

To make a replica of the meteorite before cutting it can preserve most of the important features of this appearance, but replica are not quite equal to real object.

To account for postulations of private and public schools, as well as the request of the community in general, there were developed at the Seção de Meteoritica/DGP Museu Nacional Rio de Janeiro a didactic kit of authentic replica of meteorites for loan to the schools, astronomy clubs and museums pretend to promote the elementary education in Meteoritics at the schools with the objective to develop strategies, that allow the recognization of meteorites.

Particularly the molding technique has achieved a notable advance, which was made possible due to the development of new products such as silicone. There are several articles describing how molds and casts are made but very few present the steps that precede the realization of the mold.

The basic technique for molding three-dimensional meteorites. When the mold is made of two or more parts it is necessary to separate each part of the surface through the application of clay (or plastiline) around the meteorite, according to a previously established molding plan.

Here we present a variation of the classical technique using resin, cement, sand and iron fillings to make a real authentic replica of a chondrite that can compose a meteorite kit for schools and small museums.
5119

VARRE-SAI (L5) - A RECENT METEORITE FALL FROM BRAZIL

M. E. Zucolotto ${ }^{2}$, L. L. Antonello ${ }^{1}$, R. B. Scorzelli ${ }^{1}$, M. E. Varela ${ }^{3}$, D. E. Magnelli $^{3}$, P. Munayco ${ }^{1}$, E. dos Santos ${ }^{1}$ and I. P. Ludka ${ }^{4}$. ${ }^{1}$ Centro Brasileiro de Pesquisas Físicas (CBPF/MCT), Brazil. ${ }^{2}$ Museu Nacional/ UFRJ, RJ, Brazil. ${ }^{3}$ ICATE- CONICET, San Juan, Argentina. ${ }^{4}$ IGEO/ UFRJ, RJ, Brazil. E-mail: mezucolotto@globo.com.

A bright fireball was observed at noon, on June 19, 2010, north of Rio de Janeiro and south of Espirito Santo state (Brazil). After detonations a whistling sound of the stone falling was perceived by a farmer, about $17 \mathrm{~km}$ from Varre-Sai (RJ) and $8 \mathrm{~km}$ from Guaçui (ES). Due to the meteorite divulgation program sponsored by Faperj and $\mathrm{CNPq}$ on Brazilian schools, the Museu Nacional was contacted. At least five masses (total $\sim 2.5 \mathrm{~kg}$ ) were recovered in the next days at both states. The name Varre-Sai was proposed to the NomCom as the first piece was recovered in the RJ State. Most of the stones are fusion crusted with the exposed interior surfaces light gray with few dark shock veins.

Three thin polished and etched sections from a slice of $35 \mathrm{~g}$ on deposit at MNRJ were investigated by optical and scanning electron microscope, microprobe and Mössbauer spectroscopy.

The meteorite has a poor defined chondritic texture. The discernible chondrules have sub-spherical, ellipsoidal or irregular shapes with variable amounts of metal and/or sulfide. Their apparent diameter range in size from $350 \mu \mathrm{m}$ to $2.2 \mathrm{~mm}$ (mean size of $\sim 800 \mu \mathrm{m}$ ). Clearly visible are large radial pyroxene $(\mathrm{RP})(\sim 1.8 \mathrm{~mm})$ and barred olivine $(\mathrm{BO})(\sim 2.2 \mathrm{~mm})$ types with the porphyritic ones (mean size $800 \mu \mathrm{m}$ ) being the more abundants. Chondrules are imbedded in a recrystallized matrix, being sometimes difficult to discern chondrule fragments from dispersed crystals of the matrix. The later is composed by anhedral silicates crystals $(\sim 80 \mu \mathrm{m})$ and fresh glass.

The essential minerals are the ferromagnesian olivine and pyroxene; the accessories are plagioclase (almost transformed into maskelinite) and the opaque minerals sulfides and $\mathrm{Fe}-\mathrm{Ni}$ metal. Olivine crystals have undulatory to mosaic extinctions, and are fractured with PDFs occurring in larger grains. Pyroxenes and olivine exhibit similar features. Plagioclase grains are mainly $<50 \mu \mathrm{m}$ in size, mostly with undulatory extinction. Opaque minerals are represented by troilite and metal phases. Kamacite and taenite can develop plessitic texture. The troilite show a slight polygonization and undulatory extinction and taenite grains are zoned or with martensitic interior. The kamacite occurs as single crystals with many displaying the shock-hatched $\varepsilon$ structure, although some recrystallized polycrystalline grains occur near the heat affected zone close to fusion crust.

Microprobe analysis yielded olivine $\left(\mathrm{Fa}_{0.25}, \mathrm{Fo}_{0.75}\right)$ and low-Ca pyroxene $\left(\mathrm{Fs}_{21.66} \mathrm{En}_{76.48}\right.$ Wo $\left.{ }_{1.49}\right)$. Mössbauer analysis of the bulk exhibit troilite, olivine and pyroxene; and $\mathrm{Fe}-\mathrm{Ni}$ metal in the magnetic fraction.

The poorly delineated chondritic texture, the occurrence of very little clinopyroxene grain and the plagioclase size indicates petrologic type 5. The presence of planar fractures in olivine and mosaicism indicates that the chondrite is weakly shocked (S4 $[2,3])$. The degree of weathering is W0.

References: [1] Brearley A. J. and Jones R. H. 1988. In Planetary Materials, edited by J. Papike. pp. 3-1-3-398. [2] Stöffler et al. 1991. Geochimica et Cosmochimica Acta 55:3845-3867. [3] Bischoff A. and Stöffler D. 1992. European Journal of Mineral 4:707-755. 
5214

\section{MECHANICAL DISASSEMBLING AND TESSELLATION REASSEMBLING, TOOLS FOR UNDERSTANDING THE WIDMANSTÄTTEN STRUCTURE}

M. E. Zucolotto ${ }^{1}$, O. Grillo ${ }^{1}$ and L. L. Antonello ${ }^{2}$ H.S. Amorim ${ }^{3}$. ${ }^{1}$ Museu Nacional/UFRJ, ${ }^{3} \mathrm{IF} / \mathrm{UFRJ}, \mathrm{RJ}$, Brazil. ${ }^{2} \mathrm{CBPF} /$ MCT, RJ, Brazil. E-mail: mezucolotto@globo.com.

Although the Widmanstätten structure has been studied for more than two centuries with many mechanism been proposed to explain this pattern, there is still much about this transformation that is not well understood. The EBSD and use of orientation mapping techniques applied to meteorites [1-3] has proved very useful in better understanding of its crystallographic interrelations.

The three-dimensional (3-D) microstructural analyses provides a new insights into the widmanstätten pattern transformations not aboarded previously by any two-dimensional (2-D) techniques.

Tessellations or tilling, the repeating arrangements of crystallographic solids, combined with the X-ray crystallography techniques in special electron backscatter diffraction (EBSD) is a very powerful tool for three-dimensional characterization of the materials. The tessellation can help on understand of the intricate arrangement of bars, needles, plates and lath shapes lamellae of widmanstätten pattern on meteorites.

A recently arrived sample for identification at Museu Nacional/ UFRJ, found in Descoberto, MG, probably another mass of São João Nepomuceno, an anomalous IVA silicate rich iron, exhibits near the surface plates so loose that can easily be disassembled and broken into interpenetrating triangle plates, acute rhombic prisms and tetrahedrons in steady of the expected octahedrons.

The sample has been studied by stereo and metallographic microscope, electron microprobe, crystallographic macrotexture optical goniometer and microtexture by x-ray diffraction and EBSD.

The Kepler's Stella Octangula (an octahedron with a tetrahedron on every face) in a cubic unitcell, which is also a compound of two tetrahedras from the fcc cubic alternating vertices is the cell observed in steady of expected octahedric fcc cubic cell. These structure could also been present in the high-pressure phases in the closest packing cubic or hexahedral cells.

The goal of the use of tessellation is to link the observed 3-D morphology and crystallography compared with other techiniques concerning 3-D in 2-D observations, providing new insights into the widmanstätten formation mechanism.

The tessellation of a tetrahedron is formed by tetra hedrons plus rhombohedrons (a combination of tetrahedra and octahedra). In a tetrahedral site the interstitial is in the center of a tetrahedra formed by four lattice atoms. Three atoms, touching each other, are in plane; the fourth atom sits in the symmetrical position on top.

It is therefore of interest to raise the question of whether the crystallographic data gathered in the (taenite + kamacite) region on the one hand and the plessite regions on the other can improve our understanding of the transformation mechanisms that govern the formation of these microstructures.

References: [1] Zucolotto M. E. and Pinto A. L. 2000. Meteoritics \& Planetary Science 35:A180. [2] Nolze G. and Geist V. 2004. Crystallographic Research and Technology 39:343-352. [3] Goldstein J. I. and Michael J. R. 2006. Meteoritics \& Planetary Science 41:553-570.
5229

NEW INSIGHTS INTO THE STRONTIUM CONTAMINATION OF METEORITES

F. J. Zurfluh ${ }^{1}$, B. A. Hofmann ${ }^{2}$, E. Gnos ${ }^{3}$, U. Eggenberger ${ }^{1}$, I. M. Villa $^{1,4}$, N. D. Greber ${ }^{1,2}$ and A. J. T. Jull ${ }^{5}$. ${ }^{1}$ Institut für Geologie, Universität Bern. E-mail: florian.zurfluh@geo.unibe.ch. ${ }^{2}$ Naturhistorisches Museum der Burgergemeinde Bern. ${ }^{3}$ Muséum d'histoire naturelle de la Ville de Genève. ${ }^{4}$ Università di Milano Bicocca, Milano, Italy. ${ }^{5} \mathrm{NSF}$ Arizona AMS Laboratory, The University of Arizona, Tucson, AZ 85721, USA.

Introduction: Since the discovery of meteorites in hot deserts, studies on their weathering and contamination have become important to understand the terrestrial overprint of these samples. One of the early recognized features is the continuous uptake of $\mathrm{Ba}$ and $\mathrm{Sr}$ of meteorites in hot deserts [e.g., 1-4]. Our studies focus on the quantification of the contamination patterns and on the determination of the source of contaminants and their correlation with terrestrial ages.

Methods: Meteorites found during the joint Omani-Swiss meteorite search project are routinely classified by standard procedures. Some selected samples (covering all weathering degrees and all LL chondrites) were dated by ${ }^{14} \mathrm{C}$ [e.g., 5]. Currently, the elemental concentrations of all samples (excluding samples from large strewn fields) are systematically analyzed by handheld XRF (HHXRF) with special focus on $\mathrm{Ba}$ and $\mathrm{Sr}$ contamination. Three $\mathrm{L}$ chondrites from selected sites representing three distinct typical geographical provenances of meteorites were analyzed for their ${ }^{87} \mathrm{Sr} /{ }^{86} \mathrm{Sr}$ isotopic ratio. Strontium isotopes were measured on aqueous and acidic leaches from meteorites and corresponding soil samples.

Results: The terrestrial age pattern of Omani meteorites is characterized by an abundance peak at $20 \mathrm{ka}$ and a lack of young $(<10 \mathrm{ka})$ meteorites. The correlation of terrestrial age and weathering degree with $\mathrm{Ba}$ and $\mathrm{Sr}$ contamination is obvious. An estimation of the terrestrial age of meteorites by a combination of HHXRF measurements, weathering degree, geographical find location and physical weathering signatures such as fragmentation and wind ablation will likely be possible. Strontium isotope ratios of corresponding meteorite-soil pairs obtained by leaching with water are very similar, but characteristic for each of the three locations. Acidic leaches from meteorites are also similar to the former, while the leachates of soil samples with strong acid are less radiogenic, probably because leaching with strong acid taps Sr reservoirs that are insoluble in rainwater.

Discussion: Based on our observations there is strong evidence that the source of $\mathrm{Sr}$ contamination is the local soil and not sea spray, since the strontium isotope ratio of Miocene and recent ocean-water is significantly more radiogenic [6]. By measuring the contamination pattern ( $\mathrm{Ba}$ and $\mathrm{Sr}$ concentration, $\mathrm{Sr}$ isotopes and alteration mineralogy) of meteorites it is possible to trace back the find location, to some extent even within the homogeneous Omani desert.

References: [1] Gibson E. K. and Bogard D. D. 1978. Meteoritics 13:277-289. [2] Stelzner T. et al. 1999. Meteoritics \& Planetary Science 34:787-794. [3] Al-Kathiri A. F. et al. 2005. Meteoritics \& Planetary Science 40:1215-1239. [4] Lee M. R. and Bland P. A. 2004. Geochimica et Cosmochimica Acta 68:893-916. [5] Jull T. 2006. In Meteorites and the early solar system II, eds. D. S. Lauretta and H. Y. McSween pp. 889905. [6] Palmer M. R. and Elderfield H. 1985 Nature 314:526-528. 